Argonne

\title{
Summary of Operations and Performance of the Murdock Site Restoration Project in June 2005-December 2006
}

\section{Environmental Science Division}




\begin{abstract}
About Argonne National Laboratory
Argonne is a U.S. Department of Energy laboratory managed by UChicago Argonne, LLC under contract DE-AC02-06CH11357. The Laboratory's main facility is outside Chicago, at 9700 South Cass Avenue, Argonne, Illinois 60439. For information about Argonne, see www.anl.gov.
\end{abstract}

\title{
Availability of This Report
}

This report is available, at no cost, at http://www.osti.gov/bridge. It is also available on paper to the U.S. Department of Energy and its contractors, for a processing fee, from:

U.S. Department of Energy

Office of Scientific and Technical Information

P.O. Box 62

Oak Ridge, TN 37831-0062

phone (865) 576-8401

fax (865) 576-5728

reports@adonis.osti.gov

\section{Disclaimer}

This report was prepared as an account of work sponsored by an agency of the United States Government. Reference herein to any specific commercial product, process, or service by trade name, trademark, manufacturer, or otherwise, does not necessarily constitute or imply its endorsement, recommendation, or favoring by the United States Government or any agency thereof. The views and opinions of document authors expressed herein do not necessarily state or reflect those of the United States Government or any agency thereof, Argonne National Laboratory, or UChicago Argonne, LLC. 


\section{Summary of Operations and Performance of the Murdock Site Restoration Project in June 2005-December 2006}

by

Applied Geosciences and Environmental Management Section

Environmental Science Division, Argonne National Laboratory

May 2007

USDA 


\section{Contents}

Notation.

1 Introduction

2 Overview of the Treatment Facilities at Murdock

2.1 Well GWEX-1 and the Spray Irrigation Treatment Unit.

2.2 Phytoremediation and Buffer Plantation Area ....................................................... 2- 2-1

2.3 Constructed Wetlands Area ……………………............................................. 2-2

2.4 Monitoring Well Network.......................................................................... 2-2

3 Overview of System Operations and Site Activities...................................................... 3-1

3.1 GWEX-1 and the Spray Irrigation Treatment Unit............................................ 3-1

3.2 Phytoremediation and Buffer Areas................................................................ 3-1

3.3 Constructed Wetlands Area ……………...................................................... 3-3

3.4 Monitoring Well Sampling ……………....................................................... 3-4

3.5 Monitoring of Surface Waters ........................................................................ 3-4

3.6 Monitoring of Ambient Air............................................................................... 3-5

3.7 Installation of Visitor Amenities...................................................................... 3-5

3.8 2006 National CREP Conference Visitation.......................................................... 3-6

4 Results of Groundwater, Surface Water, Vegetation, and Atmospheric Monitoring ......... 4-1

4.1 Results for GWEX-1 and Compliance Monitoring of the Spray Irrigation Treatment Unit ...................................................................................... 4-

4.2 Results for the Phytoremediation Area …………............................................. 4-2

4.2.1 Evidence of Possible Hydraulic "Pumping" Effects..................................... 4-3

4.2.2 Evidence of Carbon Tetrachloride Uptake and Possible Degradation by the Phytoremediation Plantings

4.2.3 Evidence of Possible Enhanced Microbial Degradation................................. 4-7

4.2.4 Summary of Results for the Phytoremediation Area .................................... 4-9

4.3 Results of Vegetation Analyses in the Wetlands Area .......................................... 4-10

4.4 Results for Compliance Monitoring at Surface Water Locations SWM1, SMW2,

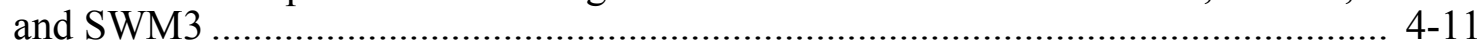

4.5 Results for Atmospheric Compliance Monitoring ....................................................... 4-11

4.6 Quality Control for Monitoring Activities ................................................................. 4-13

5 Operation, Maintenance, and System Modifications ....................................................... 5-1

5.1 Well GWEX-1 and the Spray Irrigation Treatment Unit....................................... 5-1

5.2 Phytoremediation Planting and Buffer Areas ……………...................................... 5- 5-1

5.3 Constructed Wetlands Area ……………………............................................. $5-2$

5.4 Monitoring Well Network.............................................................................. $5-2$ 
6 Summary

6.1 Performance of the Removal Action Systems

6.2 Regulatory Compliance of the Removal Action Systems.

7 References

Appendix A: GWEX-1 Registration Form and Construction Diagram.

Appendix B: Trail Signs

B-1

Appendix C: Visitor's Guide

C-1

Appendix D: August 2006 National CREP Conference CCC/USDA Summary

D-1

Appendix E: Vegetation Analysis Results

E-1

Supplement 1: Water Level Recorded at Murdock, August 2005 through December 2006 (on CD)

\section{Figures}

1.1 Locations of Murdock, Nebraska, the carbon tetrachloride plume in groundwater, and the treatment facilities

2.1 Locations of the GWEX-1 well at Murdock, the spray irrigation treatment area, the shallow and deep phytoremediation planting areas, the constructed wetlands, the former CCC/USDA grain storage facility, and the carbon tetrachloride plume in groundwater

2.2 Specially designed mobile spray treatment unit on the athletic field at Murdock........

2.3 Distribution of the 19 monitoring wells at Murdock that are used for twice yearly groundwater sampling and the 16 wells that are used for water level monitoring .......

3.1 Surface water monitoring locations

3.2 Air sampling locations

4.1 Hydrographs for upgradient wells, August 2005 to December 2006 
4.3 Analytical results for carbon tetrachloride in branch tissue samples, July 2005.

4.4 Analytical results for carbon tetrachloride in branch tissue samples, September 2005

4.5 Analytical results for carbon tetrachloride in branch tissue samples, January 2006 .... 4-33

4.6 Analytical results for carbon tetrachloride in branch tissue samples, March 2006 ...... 4-34

4.7 Analytical results for carbon tetrachloride in branch tissue samples, August 2006 .... 4-35

4.8 Tree growth for eastern cottonwood and hybrid poplar, Niobe willow, green ash, and northern catalpa trees planted in the shallow or deep phytoremediation zone, based on measurements of diameter and height before planting and in winter 2006

4.9 Correlation of carbon tetrachloride and chloroform concentrations in branch tissue samples in July 2005, September 2005, and August 2006, in two concentration ranges.

4.10 Maximum carbon tetrachloride concentrations in groundwater samples collected from permanent monitoring points in April 2006.

4.11 Maximum carbon tetrachloride concentrations in groundwater samples collected from permanent monitoring points in August 2006

4.12 Established plants in the wetlands in August 2006

5.1 Canker lesion on a willow branch in May 2006, showing Cytospora spp. pichnydia carrying maturing fungal spores.

\section{Tables}

3.1 Plantings in the Murdock phytoremediation and wetlands areas

4.1 Analytical results for volatile organic compounds in samples of untreated water collected from extraction well GWEX-1 at Murdock, June 2005 through 2006

4.2 Analytical results for volatile organic compounds in samples of treated spray collected at Murdock in June 2005 through 2006. 
4.3 Analytical results for $\mathrm{pH}$ in samples of treated spray collected at Murdock in June 2005 through 2006

4.4 Analytical results for volatile organic compounds in groundwater samples collected from monitoring wells at Murdock in July 2005, April 2006, and August 2006

4.5 Geochemical parameters measured in water samples collected at Murdock in 2005 and 2006

4.6 Analytical results for volatile organic compounds in surface water samples collected at Murdock in October 2005 through 2006.

4.7 Analytical results from Severn-Trent Laboratories for volatile organic compounds in ambient air at Murdock in 2005 and 2006.

5.1 O\&M costs for the Murdock site restoration project, June 2005 through December 2007

E.1 Results of analyses for volatile organic compounds in vegetation samples collected at Murdock, Nebraska, in September 2005 through December 2006 


\section{Notation}

AGEM Applied Geosciences and Environmental Management

BGL below ground level

CCC Commodity Credit Corporation

CD compact disc

$\mathrm{cm} \quad$ centimeter(s)

CREP Conservation Reserve Enhancement Program

DO dissolved oxygen

EPA U.S. Environmental Protection Agency

$\mathrm{Fe}^{2+} \quad$ reduced iron

$\mathrm{ft} \quad$ foot (feet)

gal gallon(s)

gpm gallon(s) per minute

GWEX groundwater extraction well

hr hour(s)

in. $\quad \operatorname{inch}(\mathrm{es})$

$\mu \mathrm{g} / \mathrm{kg} \quad$ microgram(s) per kilogram

$\mu \mathrm{g} / \mathrm{L} \quad$ microgram(s) per liter

$\mu \mathrm{g} / \mathrm{m}^{3} \quad$ microgram(s) per kilogram

MRL minimal risk level

NDEQ Nebraska Department of Environmental Quality

NPDES National Pollutant Discharge Elimination System

O\&M operating and maintenance

ORP oxidation-reduction potential

USDA U.S. Department of Agriculture

VOC volatile organic compound 


\section{Summary of Operations and Performance of the Murdock Site Restoration Project in June 2005-December 2006}

\section{Introduction}

This document summarizes the performance of the groundwater and surface water restoration systems installed by the Commodity Credit Corporation of the U.S. Department of Agriculture (CCC/USDA) at the former CCC/USDA grain storage facility in Murdock, Nebraska, during the initial period of systems operation, from June 2005 through December 2006.

In the Murdock project, several innovative technologies are being used to remove carbon tetrachloride contamination from a shallow aquifer underlying the town, as well as from water naturally discharged to the surface at the headwaters of a small creek (a tributary to Pawnee Creek) north of the town (Figure 1.1). The restoration activities at Murdock are being conducted by the CCC/USDA as a non-time-critical removal action under the regulatory authority and supervision of the U.S. Environmental Protection Agency (EPA), Region VII. Argonne National Laboratory assisted the CCC/USDA by providing technical oversight for the restoration effort and facilities during this review period.

Included in this report are the results of all sampling and monitoring activities performed in accord with the EPA-approved Monitoring Plan for this site (Argonne 2006), as well as additional investigative activities conducted during the review period.

This document presents overviews of the treatment facilities (Section 2) and site operations and activities (Section 3), then describes the groundwater, surface water, vegetation, and atmospheric monitoring results (Section 4) and modifications and costs during the review period (Section 5). Section 6 summarizes the initial period of operation. 


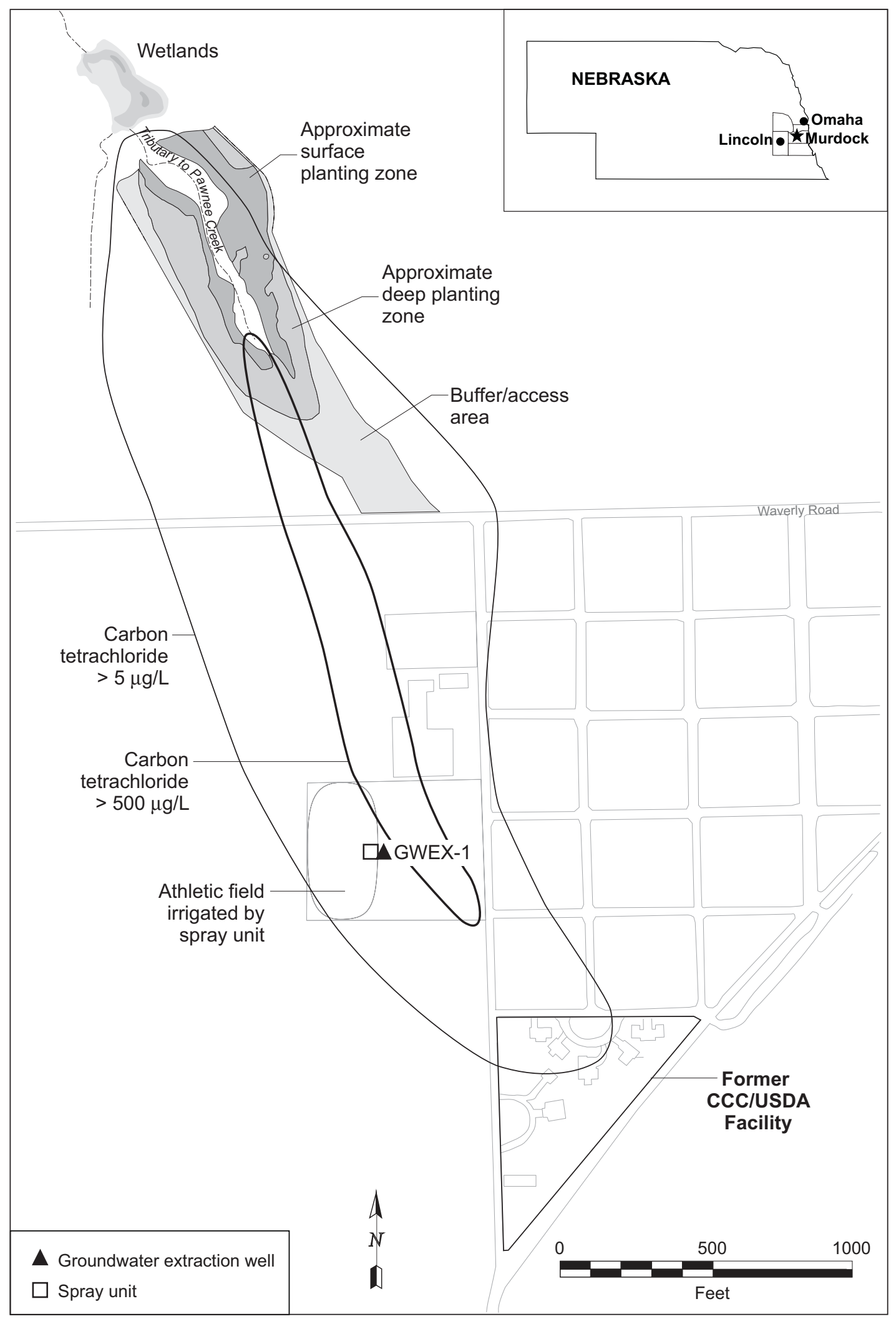

FIGURE 1.1 Locations of Murdock, Nebraska, the carbon tetrachloride plume in groundwater (2005 values), and the treatment facilities. 


\section{Overview of the Treatment Facilities at Murdock}

The removal action implemented at Murdock employs several innovative technologies in sequence along the contaminant migration pathway to decrease carbon tetrachloride levels in groundwater and in water naturally discharged to the surface at the headwaters of the tributary creek. The principal elements of this restoration approach are described below. The facilities include (1) a groundwater extraction well and spray irrigation treatment system, (2) a phytoremediation and buffer vegetation planting area, and (3) constructed wetlands (Figure 2.1).

\subsection{Well GWEX-1 and the Spray Irrigation Treatment Unit}

Groundwater extraction well GWEX-1 (Figure 2.1) removes contaminated groundwater from the upgradient, more concentrated portion of the carbon tetrachloride plume, before the high contaminant concentrations reach the headwaters of the tributary creek. Construction and registration data for the well are in Appendix A. A specially designed mobile spray treatment unit (Figure 2.2) is used to simultaneously volatilize carbon tetrachloride from the produced groundwater and irrigate property owned by the Elmwood-Murdock Public School system.

Well GWEX-1 is operated seasonally, subject to local weather conditions and the irrigation needs of the school system. Routine operation of the well and spray unit is performed by representatives of the school system, under Argonne supervision.

\subsection{Phytoremediation and Buffer Plantation Area}

Selected vegetation types planted in the headwaters area of the tributary creek reduce carbon tetrachloride concentrations in the relatively shallow groundwater and surface waters via phytoremediation processes. Approximately 2,000 trees representing six species (Niobe willow, black willow, eastern cottonwood, hybrid poplar, green ash, and northern catalpa) cover an area of approximately 4.5 acres (Figure 2.1). Removal of the carbon tetrachloride occurs as a result of uptake, transpiration, and volatilization of the contaminated groundwater by the trees and degradation of the carbon tetrachloride within the plant tissues, as well as via enhanced microbial activity in the root zone created by the plantings. At locations where the static groundwater level is typically more than 4-5 ft below ground level (BGL) - in the "deep" planting zone (Figure 2.1) - a special technique was used to plant the trees in 24-in.-diameter boreholes lined 
with plastic sleeves. This TreeWell ${ }^{\circledR}$ technique limits the availability of shallow soil water and direct precipitation to the trees, and hence it promotes vertical root growth and uptake of the deeper contaminated groundwater. In the remaining "surface" planting zone (Figure 2.1), the trees were installed without borehole liners to give the roots access to contaminated groundwater typically encountered at depths of less than $4 \mathrm{ft} \mathrm{BGL.}$

Additional vegetation, including a mixture of native prairie grasses, wildflowers, and other species, was planted between the trees and in the adjacent areas (Figure 2.1). These cover plantings (1) enhance erosion control, (2) intercept local precipitation and runoff and hence promote the uptake of deeper contaminated groundwater by the trees, (3) help protect the trees from physical damage, (4) provide a transitional buffer zone between the tree planting areas and the surrounding croplands, and (5) create a barrier to herbicide drift.

\subsection{Constructed Wetlands Area}

Shallow wetlands constructed downstream from the main planting area provide an additional phytoremediation "polishing" stage for the water discharged to the tributary creek (Figure 2.1). In the wetlands, surface water flow is directed through a sequence of shallow ponds of various depths before it returns to the natural tributary creek channel via an adjustable water control structure. By increasing the residence time and surface exposure area of the flow entering the downstream portion of the tributary, the wetlands promote further carbon tetrachloride evaporation and degradation by water-loving plants and associated microbes.

\subsection{Monitoring Well Network}

A suite of 19 permanent monitoring wells established at the Murdock restoration site facilitates sampling of the groundwater for volatile organic compounds (VOCs) analyses and for the measurement of selected geochemical parameters indicative of possible contaminant degradation within the aquifer (Figure 2.3; see also Section 4.2.3.2). At each location, wells have been installed either individually or as clusters screened to permit sampling from multiple vertical intervals through the saturated zone. Construction information for these wells is in the Monitoring Plan (Appendix A in Argonne 2006). 
Groundwater levels are measured automatically and continuously, at 1-hr intervals (for wells in the phytoremediation area until April 2006) or 4-hr intervals (for wells in the upgradient area and for wells in the phytoremediation area after June 2006). The measurements are made by using downhole pressure transducers and data loggers installed at 16 locations along the contaminant migration pathway. These locations are highlighted in Figure 2.3. 


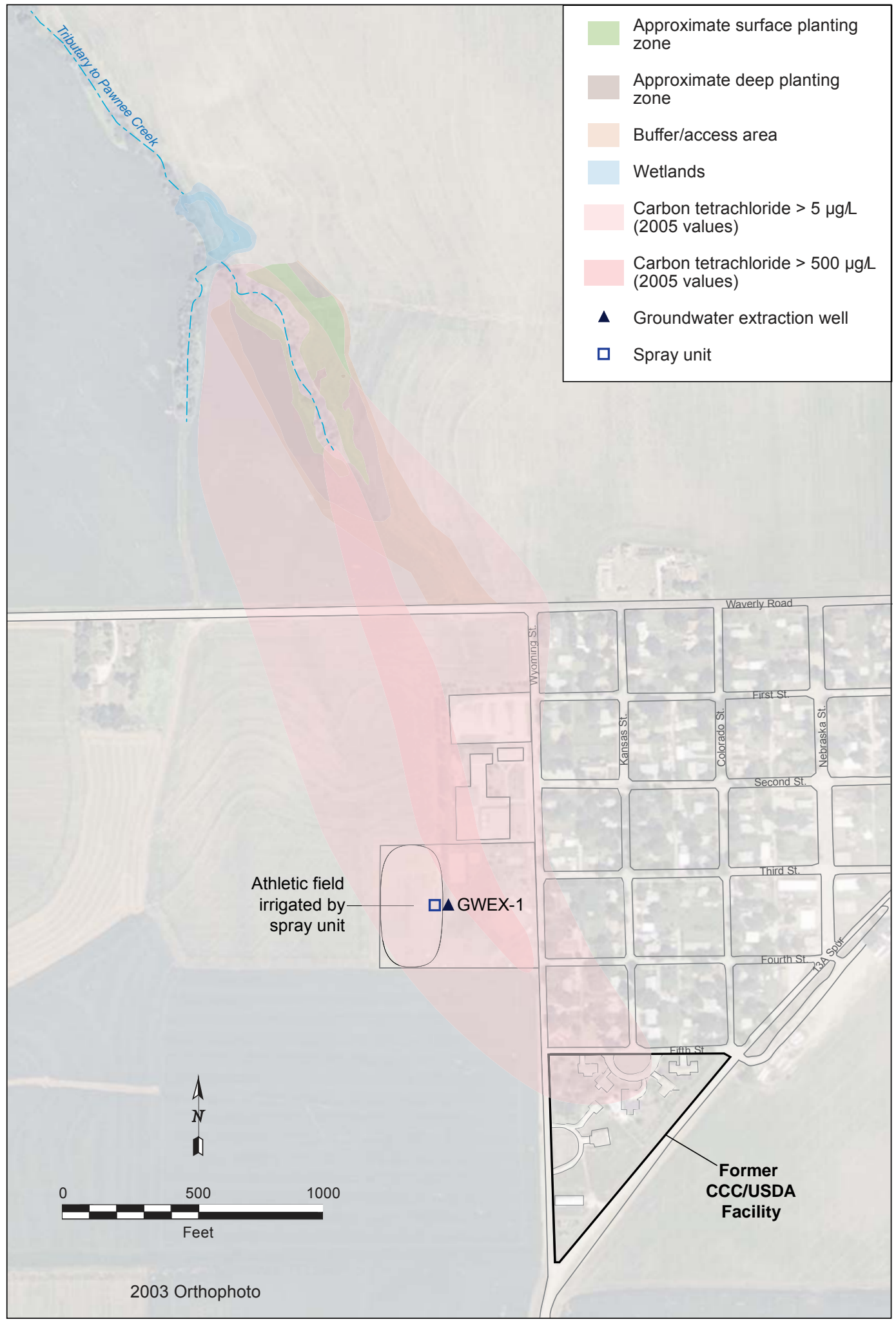

FIGURE 2.1 Locations of the GWEX-1 well at Murdock, the spray irrigation treatment area, the shallow and deep phytoremediation planting areas, the constructed wetlands, the former CCC/USDA grain storage facility, and the carbon tetrachloride plume in groundwater (2005 values). Source of photograph: NAIP (2003). 


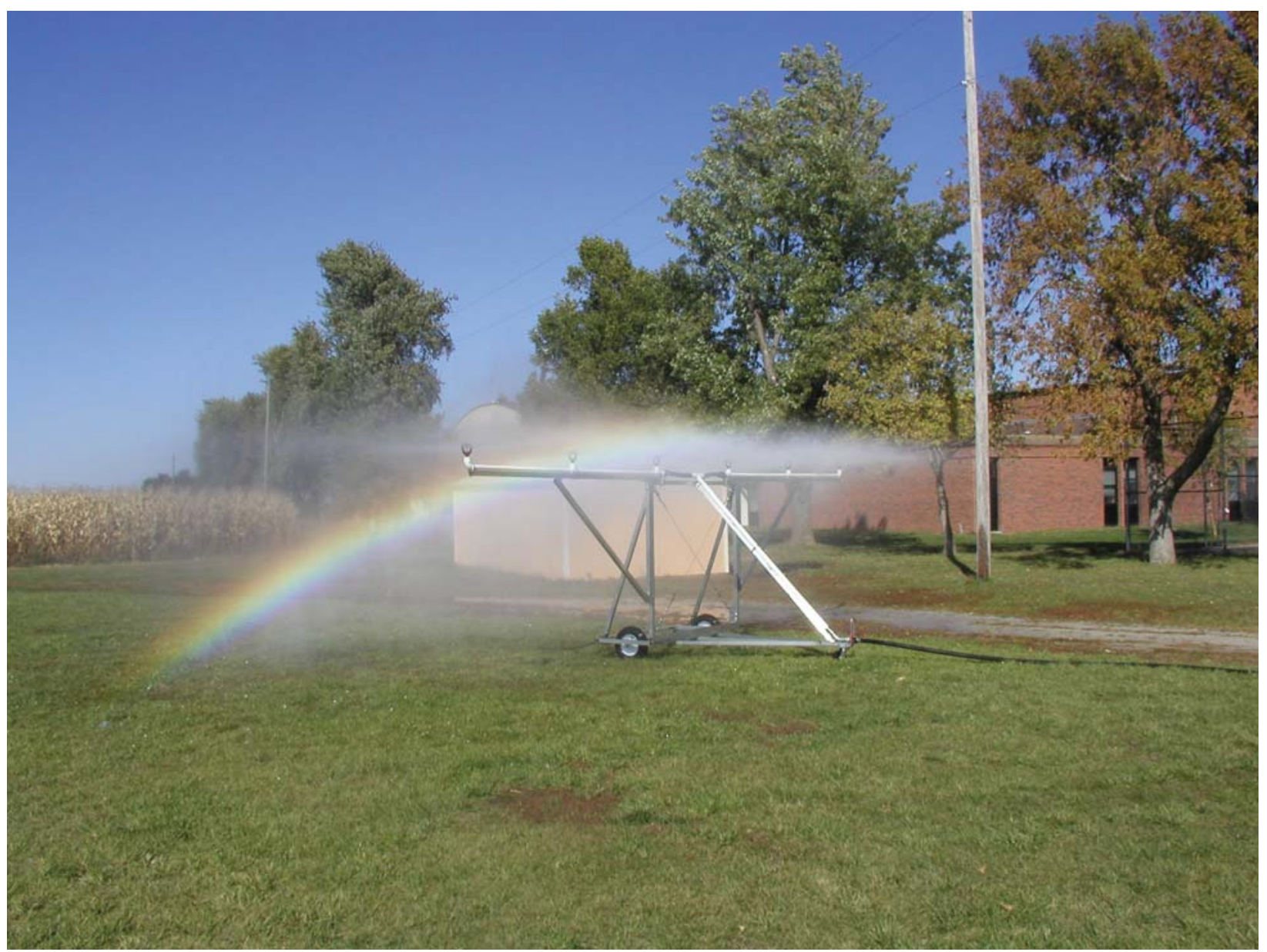

FIGURE 2.2 Specially designed mobile spray treatment unit on the athletic field at Murdock. 


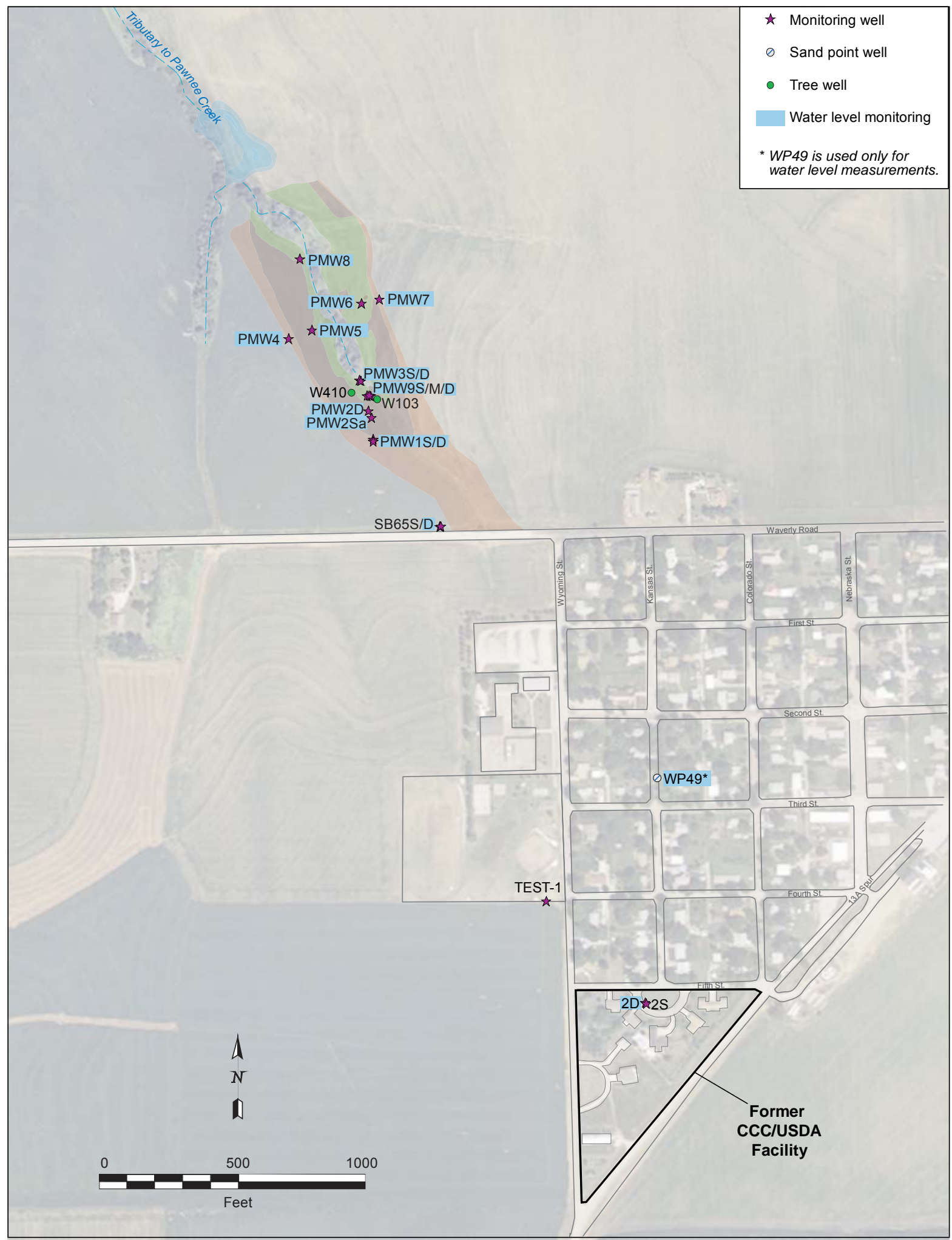

FIGURE 2.3 Distribution of the 19 monitoring wells at Murdock that are used for twice yearly groundwater sampling and the 16 wells that are used for water level monitoring. Source of photograph: NAIP (2003). 


\section{Overview of System Operations and Site Activities}

This section briefly summarizes the operations and related activities at the Murdock restoration site during the review period.

\subsection{GWEX-1 and the Spray Irrigation Treatment Unit}

Well GWEX-1 was installed on August 2, 2004. Pilot testing was carried out in October 2004 to determine the well's sustainable production capacity and to demonstrate the viability of the spray irrigation treatment technology at this site (Argonne 2006). With the approval of the EPA, the use of GWEX-1 and the spray irrigation treatment unit as one component of the removal action at Murdock began in 2005.

The extraction well is pumped at an approximate rate of $35 \mathrm{gpm}$, to maintain a critical operating pressure for the spray irrigation treatment unit. Routine operation of GWEX-1 and the spray treatment unit (for watering of the Elmwood-Murdock Public School system property) began on June 2, 2005, and was terminated at the end of the irrigation season on September 30, 2005. Well GWEX-1 and the spray treatment unit were reactivated for irrigation use on June 8, 2006, and remained in operation until September 30, 2006.

During the seasonal operating periods, samples of the untreated groundwater and the treated effluent from the spray irrigation unit are collected quarterly for VOCs analyses (Argonne 2006). The quarterly monitoring events during the present review period occurred on June 2 and September 22, 2005, and on June 8 and August 15, 2006. The analytical results are discussed in Section 4.1.

\subsection{Phytoremediation and Buffer Areas}

Planting of trees in the main phytoremediation area was performed in April and May 2005. Preliminary sampling data obtained during baseline studies in the phytoremediation area in July 2005 indicated that carbon tetrachloride uptake from the contaminated groundwater and surface waters by these plantings had begun by the summer of 2005. (The complete results of the July 2005 baseline studies were reported previously [Argonne 2006]; see also Section 4.2.) 
Monitoring of vegetation in the phytoremediation planting area in accord with the EPA-approved Monitoring Plan (Argonne 2006) began in August 2005.

Because of unfavorable weather conditions, the required plantings in the phytoremediation and buffer zone areas could not be completed until spring 2006. Additional activities to establish the permanent configuration of the phytoremediation and buffer zones continued into early summer 2006, as follows:

- Fall 2005. Initial seeding of a fast-growing groundcover species (winter rye) in the phytoremediation and buffer areas for temporary erosion control, weed control, and protection of the newly planted trees.

- Spring 2006. Mowing of the phytoremediation and buffer areas and seeding with a mixture of selected native prairie grasses, wildflowers, and other desirable species to establish a permanent groundcover in these areas. The grass and groundcover species planted are listed in Table 3.1 .

- Spring 2006. Removal of temporary border rings installed to contain surface soils and gravel mounded around each of the tree planting sites; removal of the "rabbit guard" tubing originally placed around each tree.

- Early Summer 2006. Installation of markers to permanently identify the location and species of each tree planted in the phytoremediation area.

Twice yearly vegetation sampling (for VOCs analyses) from a representative suite of trees in the phytoremediation area, on an approximate grid pattern, was recommended in the approved Monitoring Plan (Argonne 2006) to assess the seasonal uptake of groundwater and carbon tetrachloride by the plantings. Vegetation sampling was proposed (1) in early spring, before the onset of significant plant growth, and (2) in late summer or early fall, at the peak or near the end of the growth cycle.

Sampling on the proposed grid pattern was performed during the peak growth period immediately following planting of the trees, as part of the baseline sampling event conducted in July 2005 (Argonne 2006), and again in August 2006. Between these two main sampling events, vegetation samples were collected for VOCs analyses from selected plantings and preexisting 
native trees in the phytoremediation area. Results of the sampling event on September 27, 2005, confirmed the initial contaminant detections observed during the baseline event in July 2005. Additional sampling on January 9, March 2, and April 3-4, 2006, was intended to monitor for possible changes in the contaminant levels of the plantings while they were dormant. These events focused on selected areas to accomplish the stated objectives. The results of these analyses and a comparison with the baseline data from July 2005 reported previously (Argonne 2006) are in Section 4.2.2.

Measurements of tree height and trunk diameter were collected for a representative suite of the trees in the July 2005 baseline sampling event (Argonne 2006), as well as in the September 2005, September 2006, and January 2007 events. The purpose was to correlate tree growth with (1) planting method and (2) the observed patterns of carbon tetrachloride uptake. The resulting growth data are discussed in Section 4.2.2.

The general health of the trees was monitored closely throughout the review period. A particular concern was a canker outbreak that preferentially affected Niobe willows. This outbreak was first noticed in late winter 2005. No corrective measures were required. Further information on this outbreak is in Section 5.2.

\subsection{Constructed Wetlands Area}

Construction of the wetlands treatment area started in the summer of 2005, after the design phase was complete. Wetlands construction included the following activities:

- General site grading.

- Placement of stream crossings (culverts).

- Excavation of the wetlands pool (open water area).

- Construction of the concrete outlet structure, auxiliary spillway, and rock weir.

- Placement of stop logs. 
- Inundation of the wetlands pool (open water area).

Growth milestones were observed as follows:

- February 2006. Initial growth of upland vegetation cover.

- May 2006. Initial growth of native riparian and wetlands vegetation.

- June 2006. Flourishing riparian/wetlands vegetation downstream from the outlet structure.

- August 2006. Flourishing vegetation throughout the wetlands area, as a result of transplanting of rushes and sedges from existing (off-site) wetlands in July 2006, plus seeding by Stock Seed Farms.

The wetlands species planted are listed in Table 3.1. Modifications in the wetlands during the spring and early summer of 2006 are reported in Section 5.3.

\subsection{Monitoring Well Sampling}

Groundwater samples are collected twice annually from the full suite of 19 permanent monitoring wells shown in Figure 2.3, for VOCs analyses and the determination of selected inorganic geochemical parameters including nitrate (Argonne 2006). During the current review period, the monitoring wells were sampled on April 5-18 and August 15-24, 2006. The results of the analyses are presented in Section 4.2.3.1.

\subsection{Monitoring of Surface Waters}

Surface water samples for VOCs analyses are collected quarterly (Argonne 2006) along the tributary creek at three locations. One location (SWM1) is near the upgradient margin of the phytoremediation area, the second (SWM2) is at the upgradient margin of the wetlands area, and the third (SWM3) is directly downgradient from the outfall from the wetlands area (Figure 3.1). Because the required plantings in the phytoremediation and wetlands areas were not completed 
until spring 2006, routine quarterly sampling at the designated monitoring points did not begin until April 2006. Surface water was sampled on October 19, 2005, at only the SWM3 location (the identified compliance point). No sampling was performed during the subsequent winter months. Surface water samples were collected at all three designated monitoring points on April 10, August 14, and November 8, 2006. The results of the analyses are in Section 4.4.

\subsection{Monitoring of Ambient Air}

Ambient air samples for VOCs analyses are collected twice annually (Argonne 2006) at three locations (Figure 3.2). Two locations (AA1, AA2) are in the phytoremediation area, and one (AA3) is in the wetlands area. Sampling at these locations occurred on May 17-19, 2006, and August 14-16, 2006. In each of these events, a comparison sample of "background" air was collected at location BA1, to the southwest and upgradient/upstream from the tributary creek headwaters area. In a supplemental sampling event on August 23, 2006, samples were collected at a height of $10 \mathrm{ft}$ above ground level (among the leaves), rather than the specified monitoring height of $5.5 \mathrm{ft}$. The analytical results for all three events are discussed in Section 4.5.

\subsection{Installation of Visitor Amenities}

To increase public awareness of the Murdock site restoration project and facilitate public recreational and educational access to the phytoremediation and constructed wetlands areas, a number of visitor amenities were installed at the site in the late fall of 2005 and spring of 2006. These facilities include the following:

- A paved (gravel) parking area off Waverly Road, at the entrance to the tributary headwaters and phytoremediation planting areas.

- Landscaping around the parking area.

- A paved (gravel) walking trail leading from the parking area and through the phytoremediation and wetlands areas.

- Park benches and refuse receptacles at several points along the walking trail. 
- A series of 19 permanent, illustrated signs (Appendix B) along the trail through the phytoremediation and wetlands areas. These signs provide information on the CCC/USDA program, the issues of remedial concern at Murdock, and the technologies being employed to restore the groundwater and surface waters at and around the site.

- A brief Visitor's Guide brochure, available from several weatherproof boxes along the walking trail. The brochure (Appendix C) provides an overview of the site and summarizes the more detailed information on the trail signs.

\subsection{National CREP Conference Visitation}

As part of the 2006 National Conservation Reserve Enhancement Program (CREP) Forum held in Lincoln, Nebraska, the CCC/USDA hosted a field visit for conference attendees at the Murdock site on August 16, 2006. Brief presentations by the CCC/USDA, Murdock property owners, the superintendent of the Elmwood-Murdock Public School system, and Argonne staff were followed by a walking tour of the Murdock phytoremediation and constructed wetlands areas. A summary prepared by the CCC/USDA for the visit is in Appendix D. 
TABLE 3.1 Plantings in the Murdock phytoremediation and wetlands areas.

\begin{tabular}{|c|c|}
\hline Common Name & Scientific Name \\
\hline \multicolumn{2}{|c|}{ Buffer, Upland, and Tree Area Seeding } \\
\hline Big Bluestem & Andropogon gerardii \\
\hline Indiangrass & Sorghastrum nutans \\
\hline Switchgrass & Panicum virgatum \\
\hline Little Bluestem & Schizachyrium scoparium \\
\hline Sideoats Grama & Bouteloua curtipendula \\
\hline Western Wheatgrass & Agropyron smithii \\
\hline Virginia Wildrye & Elymus virginicus \\
\hline Blue Grama & Bouteloua gracilis \\
\hline Buffalograss & Buchloe dactyloides \\
\hline Canada Wildrye & Elymus Canadensis \\
\hline Hard Fescue & Festuca longifolia \\
\hline Sheep Fescue & Festuca ovina \\
\hline Annual Ryegrass & Lolium multiflorum \\
\hline Baby's Breath & Gypsophila elegans \\
\hline Blackeyed Susan & Rudbeckia hirta \\
\hline Blanket Flower & Gaillardia aristata \\
\hline Blue Flax & Linum lewisii \\
\hline California Poppy & Eschscholzia californica \\
\hline Clarkia & Clarkia amoena \\
\hline Clasping Coneflower & Rudbeckia amplexicaulis \\
\hline Cornflower & Centaurea cyanus \\
\hline Corn Poppy & Papaver rhoeas \\
\hline Dwarf Red Coreopsis & Coreopsis tintoria, red \\
\hline Illinois Bundleflower & Desmanthus illinoensis \\
\hline Indian Blanket & Gaillardia pulchella \\
\hline Lance Leaved & Coreopsis lanceolata \\
\hline \multicolumn{2}{|l|}{ Coreopsis } \\
\hline Lead Plant & Amorpha canescens \\
\hline Lemon Mint & Monarda citriodora \\
\hline Mexican Red Hat & Ratibida columnifera, red \\
\hline \multicolumn{2}{|l|}{ Coneflower } \\
\hline Missouri Primrose & Oenothera missouriensis \\
\hline Partridgepea & Cassia chamaecrista \\
\hline Purple Prairieclover & $\begin{array}{l}\text { Petalostemum or Dalea } \\
\text { purpurea }\end{array}$ \\
\hline Plains Coreopsis & Coreopsis tinctoria \\
\hline Perennial Lupine & Lupinus perennis \\
\hline Purple Coneflower & Echinacea purpurea \\
\hline Rosy Red Yarrow & Achillea millefolium, rubra \\
\hline Shasta Daisy & $\begin{array}{l}\text { Chrysanthemum } \\
\text { maximum }\end{array}$ \\
\hline Upright Coneflower & Ratibida columnifera \\
\hline Catchfly & Silene ameria \\
\hline Drummond Phlox & Phlox drummondii \\
\hline Siberian Wallflower & Cheiranthus allioni \\
\hline Scarlet Flax & $\begin{array}{l}\text { Linum grandiflorum } \\
\text { rubrum }\end{array}$ \\
\hline Alfalfa & Medicago sativa \\
\hline Red Clover & Trifolium pratense \\
\hline
\end{tabular}

Common Name Scientific Name

Wetlands Seeding

Alumroot
Aster, New England
Aster, Silky
Aster, Swamp
Blackeyed Susan,
Sweet
Blue Flag Iris
Blue Vervain
Boneset
Bottle Gentian
Cardinal Flower
Coreopsis, Tall
Duck Potato
False Dragonhead
Fowl Mannagrass
Great Blue Lobelia
Great Angelica
Joe Pye Weed
Marsh Betony
Marsh Blazing Star
Marsh Milkweed
Nodding Bur Marigold
Primrose, Showy
Evening
Sneezeweed

Heuchera richardsonii

Aster novae-angliae

Aster sericeus

Aster puniceus

Rudbeckia subtromentosa

Iris veriscolor

Verbena hastate

Eupatorium perfoliatum

Gentiana andrewsii

Lobelia cardinalis

Coreopsis tripteris

Sagittaria latifolia

Physostegia virginiana

Glyceria striata

Lobelia siphiltica

Angelica atropurpurea

Eupatorium maculatum

Stachys palustris

Liatris spicata

Ascelpias incarnata

Bidens cernua

Oenothera speciosa

Helenium autumnale

Blue Joint Grass

Bulrush, Hardstem

Bulrush, Dark Green

Bulrush, Softstem

Bur Reed

Rush, Inland

Rush, Creeping Spike

Rush, Spike

Rush, Soft

Sedge, Bebb's

Sedge, Bristly

Sedge, Long Beaked

Sedge, Porcupine

Sedge, Three Way

Sedge, Tussock

Woolgrass

Wetlands Transplanting

Broadleaf

Arrowhead

Bulrush, Green

Bulrush, Hardstem

Bulrush, River

Bulrush, Softstem

Flatstem Spikerush
Calamagrostis canadensis

Scirpus acutus

Scirpus atrovirens

Scirpus validus

Sparganium eurycarpum

Juncus interior

Eleocharis palustris

Eleocharis calva

Juncus effuses

Carex bebbii

Carex comosa

Carex sprengelii

Carex hystericina

Dulichium arundinaceum

Carex stricta

Scirpus cyperinus

\section{Sagittaria latifolia}

Scirpus atrovirens

Scirpus acutus

Scirpus fluviatilis

Scirpus validus

Eleocharis compressa 


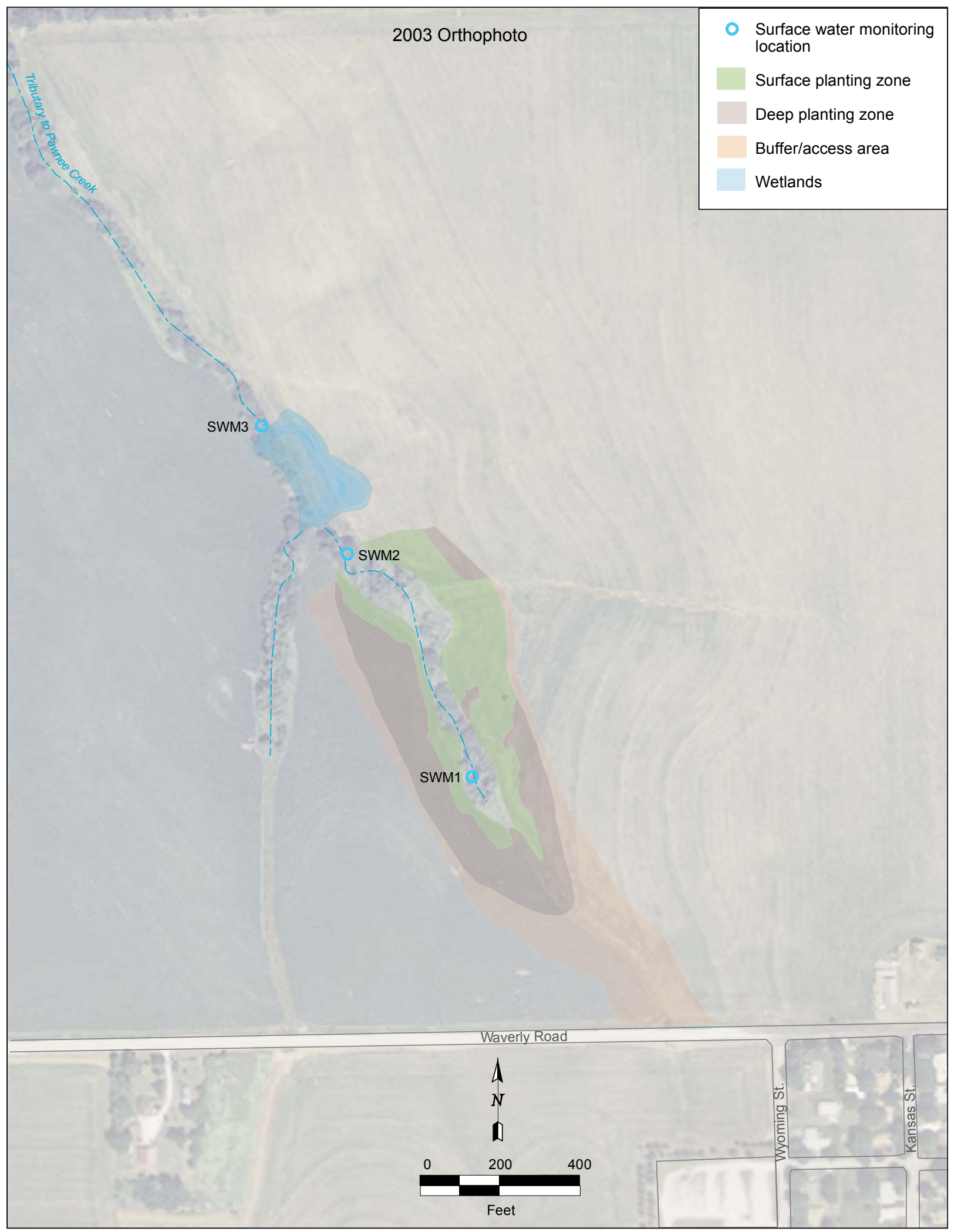

FIGURE 3.1 Surface water monitoring locations. Source of photograph: NAIP (2003). 


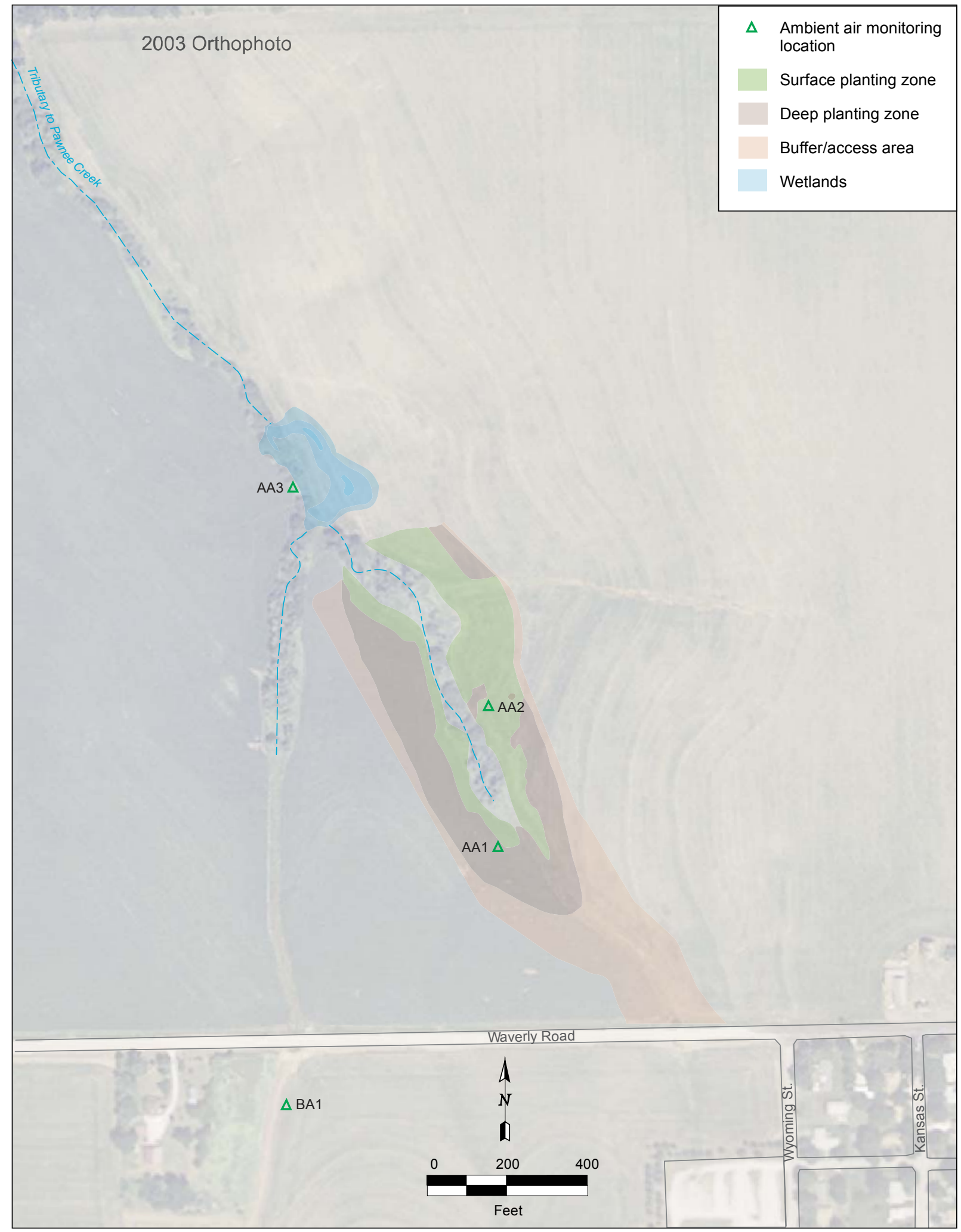

FIGURE 3.2 Air sampling locations. Source of photograph: NAIP (2003). 


\section{Results of Groundwater, Surface Water, Vegetation, and Atmospheric Monitoring}

\subsection{Results for GWEX-1 and Compliance Monitoring of the Spray Irrigation Treatment Unit}

Well GWEX-1 and the spray irrigation unit at Murdock extracted, treated, and discharged totals of 627,402 gal of groundwater during the 2005 operating period (June-September) and 400,757 gal during the 2006 operating period. The treated effluent was used for irrigation of the Elmwood-Murdock Public School system's athletic fields.

The spray irrigation treatment unit and GWEX-1 are operated in accord with a statement of discharge requirements (National Pollutant Discharge Elimination System [NPDES] Tracking No. NE0137464) issued by the Nebraska Department of Environmental Quality (NDEQ) (Appendix B in Argonne 2006). In keeping with the NDEQ requirements, samples of the treated effluent are analyzed for carbon tetrachloride, chloroform, and $\mathrm{pH}$ levels. The NDEQ statement of discharge requirements places no restrictions on the quality of the groundwater supplied to the spray irrigation unit before treatment occurs, and it does not require analysis of the untreated flow. Nevertheless, well GWEX-1 is sampled for VOCs analyses to document the concentrations of carbon tetrachloride in the groundwater captured by the well.

Analytical results for the untreated groundwater from GWEX-1 in the July 2005 baseline sampling event and the September 2005, June 2006, and August 2006 quarterly sampling events are in Table 4.1. The data tables are grouped at the end of this section, before the figures.

Concentrations of carbon tetrachloride in the untreated groundwater from GWEX-1 ranged from $179 \mu \mathrm{g} / \mathrm{L}$ to $270 \mu \mathrm{g} / \mathrm{L}$ during the reporting period. The concentrations detected at this well showed an apparent increase during the 2005 irrigation season and the early portion of the 2006 season, and then they remained stable through the remainder of 2006. The observed concentrations in the untreated groundwater indicate that GWEX-1 is effectively capturing relatively high contaminant concentrations in the plume before they reach the phytoremediation and wetlands treatment areas. Low levels of chloroform, ranging from $2.3 \mu \mathrm{g} / \mathrm{L}$ to $4.5 \mu \mathrm{g} / \mathrm{L}$, were also detected in the untreated groundwater.

The outfall from the spray irrigation treatment unit is identified by the NDEQ as a land application; no specific targets for the removal of carbon tetrachloride (or chloroform) have 
therefore been established. The NDEQ requirements qualitatively note, however, that the discharge may not be toxic to aquatic life in surface waters of the state outside the mixing zones allowed under NDEQ Title 177 - Nebraska Surface Water Quality Standard. This standard defines a maximum acceptable level of $44.2 \mu \mathrm{g} / \mathrm{L}$ for carbon tetrachloride in surface water bodies. The NDEQ requirements further identify an acceptable $\mathrm{pH}$ range of 6.0 to 9.0 for the treated groundwater. These targets were accepted by the EPA (2005) in conjunction with the Murdock Monitoring Plan (Argonne 2006).

Samples of treated groundwater discharged by the irrigation treatment unit are collected from multiple locations throughout the spray cloud during each sampling event. Spray accumulation rates at each collection point may differ for each event, depending on the local wind and site conditions at the time of sample collection. A minimum of four individual spray samples are analyzed, however, for each event, to account for the potential variability.

Carbon tetrachloride concentrations detected in individual samples of the spray discharge are in Table 4.2. The carbon tetrachloride levels in the spray discharge ranged from $<1 \mu \mathrm{g} / \mathrm{L}$ to $5.9 \mu \mathrm{g} / \mathrm{L}$ during the 2005 irrigation season and from $<1 \mu \mathrm{g} / \mathrm{L}$ to $5.2 \mu \mathrm{g} / \mathrm{L}$ during the 2006 season. The average concentrations determined from the analytical results, considered to approximate the levels of carbon tetrachloride in the net spray discharged to the land surface, were $2.4 \mu \mathrm{g} / \mathrm{L}$ for the 2005 irrigation season and $2.8 \mu \mathrm{g} / \mathrm{L}$ for the 2006 season. These results indicate that the levels of carbon tetrachloride in the spray outfall to date have been far below the acceptable limit established by the EPA and the NDEQ. Chloroform was not detected in any of the spray samples analyzed.

The maximum and minimum $\mathrm{pH}$ levels detected in samples of the treated spray discharge during this reporting period are in Table 4.3. The observed levels have ranged from 6.54 to 7.88, and thus they comply with the $\mathrm{pH}$ requirements (6.0 to 9.0) specified by the NDEQ.

\subsection{Results for the Phytoremediation Area}

Carbon tetrachloride removal in the phytoremediation treatment area is expected to occur through the combined effects of 
- Hydraulic "pumping" of the contaminated groundwater as a result of plant uptake,

- Transpiration and degradation of carbon tetrachloride within plant tissues, and

- Enhanced microbial degradation (reductive dechlorination) in the root zone created by the plants.

The results of the monitoring activities targeted to investigate these remedial processes (Argonne 2006, Section 3.1.2) are summarized below.

\subsubsection{Evidence of Possible Hydraulic "Pumping" Effects}

Groundwater levels are recorded automatically at 16 locations at Murdock (Figure 2.3) to monitor the patterns of water level variations along the contaminant migration pathway, as well as within the phytoremediation treatment zone, that might influence the fate and transport of the carbon tetrachloride. Complete data recovered from the water level recorders are in Supplement 1 (on a compact disc [CD] inside the back cover of this report); these data are summarized in Figures 4.1 and 4.2.

The gaps (April to late June 2006) in the hydrographs shown in Figures 4.1 and 4.2 resulted because of a temporary equipment shortage. Monitoring at Murdock began in summer 2005 with In-Situ MiniTroll WL recorders. A need for these recorders at other CCC/USDA sites required their removal from Murdock in April 2006. The replacement recorders for Murdock (Instrumentation Northwest model PT2X) were not received and installed until late June 2006.

The groundwater levels at Murdock showed a slow, steady decline (by as much as several feet) throughout the aquifer during the later part of 2005 and spring of 2006. After spring 2006, the levels remained relatively stable. Frequent, small-scale oscillations are apparent in the hydrographs for many of the wells completed at shallow depths (PMW5, PMW6, PMW7, PMW8, PMW9S) in the phytoremediation area during the summer and early fall of 2005. Similar oscillations, of generally greater amplitude (up to approximately $0.2 \mathrm{ft}$ ), were again observed for all of the shallow wells and several of the deeper wells (PMW2D, PMW3D) in the phytoremediation area during the 2006 growing period. The observed pattern strongly suggests 
that the fluctuations are associated with the diurnal cycle of groundwater uptake by the phytoremediation plantings (and other vegetation) during the annual growing season.

The large apparent water level fluctuations in the hydrographs for PMW1S and PMW3S during late 2006 (Figure 4.2) are believed to be an artifact of an unexpected change in the electronic recorder response at these locations during the monitoring period. See Section 5.4 for further discussion.

\subsubsection{Evidence of Carbon Tetrachloride Uptake and Possible Degradation by the Phytoremediation Plantings}

Quantitative estimation of contaminant transpiration and degradation rates occurring in plants, although theoretically possible, is logistically difficult and impractical for use as a routine monitoring technique. The detection and measured levels of volatile solvents in plant tissues have, however, been shown to be positively linked to the presence and concentrations of these contaminants in the water source(s) used to support plant growth. At Murdock, carbon tetrachloride has been identified in the subsurface groundwater and in groundwater seepage from the contaminated aquifer to the surface waters of the tributary creek. At this site, vegetation sampling (primarily of branch and leaf tissues) for VOCs analyses is therefore employed in accord with the EPA-approved Monitoring Plan (Argonne 2006) as an indicator of groundwater utilization and contaminant uptake by the phytoremediation plantings.

Complete results of the vegetation sampling and VOCs analyses during the current review period are in Appendix E, Table E.1.

Review of the analysis data for the July and September 2005 and August 2006 sampling events indicated that samples of leaf tissues collected during the growth seasons were relatively insensitive to uptake of carbon tetrachloride by the trees, in comparison to the corresponding branch tissue samples. Carbon tetrachloride concentrations identified in branch samples are therefore summarized for individual sampling events (July 2005, September 2005, January 2006, March 2006, and August 2006, respectively) in Figures 4.3-4.7. The results of analyses of the branch tissue samples for carbon tetrachloride are reviewed in Section 4.2.2.1; results for chloroform are discussed in Section 4.2.2.2. 


\subsubsection{Results of Vegetation Analyses for Carbon Tetrachloride}

Figure 4.3 shows that in July 2005 quantifiable levels of carbon tetrachloride $(1.5 \mu \mathrm{g} / \mathrm{kg}$ to $43 \mu \mathrm{g} / \mathrm{kg}$ ) were detected in tree branch samples at six locations in the more upgradient (and upstream) shallow planting zone of the phytoremediation area. Complete data for the July 2005 baseline sampling event were reported previously (Table 2.2 in Argonne 2006). The confirmation sampling in September 2005 verified these initial detections and also demonstrated (Figure 4.4) that by the approximate end of the 2005 growing season, both the number of new planting locations having quantifiable levels of carbon tetrachloride (22) and the contaminant levels identified at these locations (generally $\geq 11 \mu \mathrm{g} / \mathrm{kg}$; maximum $314 \mu \mathrm{g} / \mathrm{kg}$ in new plantings) had increased significantly in comparison to the July findings. Figure 4.4 shows that the increases occurred primarily in the upgradient portion of the shallow planting zone; however, carbon tetrachloride was also identified at several deep planting locations near the upgradient margin of the phytoremediation area, as well as in a small cluster of trees near the northwest corner of the area. The latter location approximately corresponds to the outfall of agricultural tile drain TD2; previous Argonne sampling demonstrated that the discharge from this tile drain may carry elevated levels of carbon tetrachloride contamination $(88 \mu \mathrm{g} / \mathrm{L}$; Argonne 2004).

Concentrations of carbon tetrachloride in the tree branch tissues fell dramatically, generally to nondetectable levels, during the winter and early spring of 2006 (Figures 4.5-4.6). This effect was noted in the phytoremediation plantings, as well as in a number of large, established native trees in the phytoremediation area. The analyses clearly demonstrate that carbon tetrachloride uptake by the vegetation in the phytoremediation area is seasonal; the contaminant does not accumulate in the plant tissues from year to year, but instead it is eliminated fairly rapidly from the plant tissue when the annual growing season ends and the trees become dormant.

The results of the August 2006 (growth season) vegetation sampling event (Figure 4.7) show further increases (relative to July and September 2005) in the number (49) and areal distribution of new planting locations having quantifiable levels of carbon tetrachloride in branch tissues, as well as in the concentrations detected at those locations (10 locations with $>75 \mu \mathrm{g} / \mathrm{kg}$; maximum $455 \mu \mathrm{g} / \mathrm{kg}$ in new plantings).

The patterns of carbon tetrachloride uptake outlined above are qualitatively consistent with measurements of tree growth (height and trunk diameter) recorded in the phytoremediation 
area in July and September 2005 and late in 2006. These data, summarized in Figure 4.8, indicate that the most pronounced growth, for all tree species, has occurred in the shallow planting zone, where a relatively unlimited supply of water is readily available. The increasing uptake of carbon tetrachloride observed in this planting zone during the review period further indicates that $a$ significant component of the water being used by the trees in the shallow planting zone is already being derived from the contaminated aquifer.

The relatively slower tree growth (and less frequent detections of carbon tetrachloride uptake) observed in the deep planting areas upslope from the creek suggests that the specialized planting technique (TreeWell ${ }^{\circledR}$ ) and the seeding of groundcover species in this phytoremediation zone are achieving the intended effect of limiting the availability of direct precipitation and surface runoff to these trees; hence, these techniques should promote development of the deeper rooting systems necessary for the trees to access the contaminated groundwater resource. Continued monitoring of carbon tetrachloride uptake by the trees in the shallow and deep planting zones, in accord with the approved Monitoring Plan (Argonne 2006), will be required to document the anticipated further utilization of contaminated groundwater by the trees.

\subsubsection{Results of Vegetation Analyses for Chloroform}

Reductive dechlorination of carbon tetrachloride will result in the generation of degradation products, including chloroform and dichloromethane, that are progressively more reduced. The identification of elevated levels (compared to the carbon tetrachloride concentration) of these daughter species in groundwater or plant materials at Murdock might therefore provide empirical evidence for possible reductive dechlorination, on either the macroscale or the microscale, in the phytoremediation environment.

Relatively low concentrations of chloroform (ranging from $<1 \mu \mathrm{g} / \mathrm{kg}$ to approximately $6 \mu \mathrm{g} / \mathrm{kg}$ ) were detected fairly ubiquitously in both branch and leaf tissue samples collected from locations throughout the phytoremediation area, as well as from several background locations outside this area, during each monitoring event (Table E.1 in Appendix E). In numerous cases, values in this range were detected in the absence of associated carbon tetrachloride. The interpretive significance of these low values is therefore questionable. 
With one exception, chloroform concentrations exceeding approximately $6 \mu \mathrm{g} / \mathrm{kg}$ were identified in branch samples only at locations in the phytoremediation area. In some cases, the higher chloroform values were found in association with little or no detectable carbon tetrachloride. Figure 4.9 illustrates, however, that chloroform concentrations above approximately $6 \mu \mathrm{g} / \mathrm{kg}$ were detected (with two exceptions only) in all branch tissue samples having carbon tetrachloride levels of $\geq 6 \mu \mathrm{g} / \mathrm{kg}$. These observations empirically suggest that reductive dechlorination of carbon tetrachloride may be taking place in the remedial environment created by the phytoremediation plantings (and native trees) at Murdock. On the basis of the present data, however, it is not possible to discern whether this degradation is occurring in the plant tissues themselves, in the possible microenvironment associated with the root zones of these plantings (see Section 4.2.3), or both.

\subsubsection{Evidence of Possible Enhanced Microbial Degradation}

Direct measurement of the microbial activity within the root zone created by the phytoremediation plantings is neither easy nor economical to employ as a periodic monitoring tool. In accordance with the approved Monitoring Plan (Argonne 2006), twice yearly sampling and analysis of the groundwater at 19 permanent monitoring wells within and upgradient of the phytoremediation area (Figure 2.3) is performed as a logistically viable alternative for monitoring for (1) changes in the concentrations of carbon tetrachloride in groundwater and (2) the possible development of reducing/anaerobic conditions within the aquifer required for the microbial degradation of carbon tetrachloride by reductive dechlorination.

\subsubsection{Groundwater VOCs Analysis Results}

The analytical results for VOCs in groundwater samples collected during the review period are summarized in Table 4.4 and in Figures 4.10 and 4.11. Table 4.4 includes selected results from the baseline sampling in July 2005. The complete data set from the baseline sampling was reported previously (Argonne 2006).

Table 4.4 indicates that the observed concentrations of carbon tetrachloride in the Murdock aquifer have remained relatively stable at most of the monitoring well locations, showing no consistent pattern of increase or decrease. The highest concentrations of carbon tetrachloride in groundwater (since July 2005) have consistently been identified in several wells 
(PMW1D, PMW2D, PMW3S, PMW3D, SB65D) completed primarily in the deeper portion of the aquifer, at locations near or upgradient (south) of the phytoremediation planting area. The highest maximum concentrations detected during all sampling events have occurred at monitoring wells PMW1D $(1,233 \mu \mathrm{g} / \mathrm{L}$ to $3,077 \mu \mathrm{g} / \mathrm{L})$ and PMW2D $(1,475 \mu \mathrm{g} / \mathrm{L}$ to $3,827 \mu \mathrm{g} / \mathrm{L})$. Figures 4.10 and 4.11 illustrate that measured carbon tetrachloride concentrations in groundwater have decreased rapidly northward and downgradient from the above well locations throughout the review period. The maximum concentrations identified at the northernmost groundwater sampling location, PMW8, have consistently ranged from $16 \mu \mathrm{g} / \mathrm{L}$ to $23 \mu \mathrm{g} / \mathrm{L}$ since the July 2005 baseline sampling event (Table 4.4).

The presently available groundwater sampling results suggest a possible cyclicity in carbon tetrachloride concentrations at monitoring wells PMW1D and PMW2D. This cyclicity appears, empirically, to correlate with the seasonal growth patterns of the phytoremediation plantings. Table 4.4 shows that the concentrations at these two locations increased dramatically during the April 2006 sampling event (early in the spring 2006 growth period of the trees; Figure 4.10), in comparison to the levels observed later in the tree growth cycles of both July 2005 and August 2006 (Figure 4.11). The increased levels of carbon tetrachloride observed at PMW1D and PMW2D in spring 2006 coincide (empirically) with the decreases in concentrations observed in the phytoremediation plantings before the April 2006 sampling event. No similar temporal pattern was observed in carbon tetrachloride concentrations at other monitoring wells also completed in the deeper part of the aquifer, however, or in the shallower monitoring wells also installed at locations PMW1 and PMW2. Continued groundwater sampling in accord with the approved Monitoring Plan (Argonne 2006) will be required to investigate the potential seasonal concentration variations suggested by these initial data.

Chloroform concentrations identified in the groundwater samples from each of the monitoring events during this review period were generally low $(\leq 10 \mu \mathrm{g} / \mathrm{L})$, with the consistent exception of the TEST-1 well on the Murdock public school property. Maximum chloroform levels of $49 \mu \mathrm{g} / \mathrm{L}, 24 \mu \mathrm{g} / \mathrm{L}$, and $24 \mu \mathrm{g} / \mathrm{L}$ were detected at this location, respectively, during the July 2005, April 2006, and August 2006 sampling events (Table 4.4).

\subsubsection{Groundwater Geochemical Analysis Results}

The inorganic geochemical parameters dissolved oxygen (DO), reduced iron $\left(\mathrm{Fe}^{2+}\right)$, and oxidation-reduction potential (ORP) are measured in each of the monitoring wells as possible 
indicators of the development of oxygen-depleted, chemically reducing conditions that would promote anaerobic degradation of the carbon tetrachloride within the Murdock aquifer. The results of these measurements for the present review period are in Table 4.5, together with data from the July 2005 baseline investigation. The results show no clear patterns of significantly decreased DO levels or increased $\mathrm{Fe}^{2+}$ concentrations in conjunction with the 2005 or 2006 growing seasons that would suggest the widespread onset of anaerobic conditions in the groundwater as a result of plant growth or microbial activity. Data for the August 2006 monitoring event do, however, show an apparent decrease in the ORP values measured at all well locations in the phytoremediation area in comparison to the April 2006 event. These results suggest the possible development of generally more reducing conditions in groundwater during the summer growth period. Continued monitoring in accord with the approved Monitoring Plan (Argonne 2006) will again be required to investigate the potential seasonal variations suggested in these geochemical parameters by the present data.

\subsubsection{Summary of Results for the Phytoremediation Area}

The results of the phytoremediation monitoring activities during the present review period support the following observations:

- Small, but distinct, seasonal fluctuations in groundwater levels identified at multiple monitoring well locations in the phytoremedation area appear linked to the diurnal effects of groundwater "pumping" by the phytoremediation plantings and native vegetation during the annual growing season.

- Uptake of carbon tetrachloride by the native and planted trees in the phytoremediation area has been confirmed by the presence of the contaminant in branch tissues.

- Evidence of groundwater and carbon tetrachloride uptake by the phytoremediation plantings was first detected in July 2005. The number of trees showing contaminant uptake, the areal extent of these trees in the phytoremediation area, and the concentrations of carbon tetrachloride in branch tissues all showed significant increases during the 2006 growing season. 
- Carbon tetrachloride taken up by the phytoremediation plantings during each growing season is not retained in the plant tissues from year to year; observed contaminant concentrations in the branch tissues dropped to generally nondetectable levels during the 2005-2006 winter period.

- The analytical results suggest a localized, transient reduction in the maximum concentrations of carbon tetrachloride in groundwater near the upgradient margin of the phytoremediation area in response to the seasonal plant growth cycle.

- Elevated chloroform concentrations identified in association with carbon tetrachloride in many branch samples suggest that reductive dechlorination of the carbon tetrachloride is occurring in the plant tissues themselves, within the microenvironment associated with the tree root zones, or both.

- Geochemical data suggest the possible development of generally more reducing conditions in the Murdock aquifer during the summer 2006 growing period. This finding is in qualitative agreement with the inferred degradation of carbon tetrachloride by reductive dechlorination.

\subsection{Results of Vegetation Analyses in the Wetlands Area}

As soon as the wetlands construction was completed and plants were established (Figure 4.12), a limited sampling event was conducted (August 16, 2006) to establish a tissue VOCs concentration baseline in plants in the wetlands area growing in contact with the surface water entering/leaving the wetlands cells. Three locations were selected for this study in the wetlands area. These locations were (1) the outfall of tile drain 5 (bulrush sample); (2) the east bank of the southernmost wetlands cell, at the partition line between the north and south cells (bulrush and cattail samples); and (3) a point immediately north of the wetlands outfall, just past the riprap on the west side (bulrush sample). Carbon tetrachloride concentrations were below the detection limit at all of these locations, while chloroform concentrations were $20 \mu \mathrm{g} / \mathrm{kg}$ (in bulrush) at the tile drain 5 outfall, $2.6 \mu \mathrm{g} / \mathrm{kg}$ (in bulrush) and $1.8 \mu \mathrm{g} / \mathrm{kg}$ (in cattail) at the partition line, and $6.4 \mu \mathrm{g} / \mathrm{kg}$ (in bulrush) north of the wetlands outfall (Table E.1, Appendix E). 


\subsection{Results for Compliance Monitoring at Surface Water Locations SWM1, SMW2, and SWM3}

Surface water sampling point SMW3 lies directly downstream of the outfall from the phytoremediation and wetlands treatment zones (Figure 3.1), and hence it reflects the final contaminant levels achieved in the effluent from the combined treatment processes. Location SWM3 was therefore identified in the EPA-accepted Monitoring Plan (Argonne 2006) as the primary point for quantitative assessment of the net performance of the Murdock treatment efforts. A maximum target carbon tetrachloride concentration of $44.2 \mu \mathrm{g} / \mathrm{L}$ was adopted for this discharge, as established by the NDEQ for surface waters of the state under NDEQ Title 117 Nebraska Surface Water Quality Standard.

The results of the surface water sampling and analyses are in Table 4.6. No carbon tetrachloride (or chloroform) contamination was detected (at an instrument detection limit of $0.1 \mu \mathrm{g} / \mathrm{L}$ ) in any of the surface waters sampled at SWM3 during the 2005-2006 review period, except for a trace concentration of carbon tetrachloride in the November 2006 sampling. Carbon tetrachloride levels detected at upstream sampling location SWM2 (ranging from $1.4 \mu \mathrm{g} / \mathrm{L}$ to $6.1 \mu \mathrm{g} / \mathrm{L}$ ) also fell well below the identified target level for surface waters during this time. These values contrast markedly with corresponding carbon tetrachloride levels of $151 \mu \mathrm{g} / \mathrm{L}$ to $380 \mu \mathrm{g} / \mathrm{L}$ that were identified in the surface waters sampled at location SWM1, at the upstream edge of the phytoremediation area. The results demonstrate complete removal of carbon tetrachloride contamination from the surface water, as well as the groundwater seepage, that passed through the phytoremediation and wetlands treatment zones during the review period.

\subsection{Results for Atmospheric Compliance Monitoring}

The EPA has adopted the Agency for Toxic Substances and Disease Registry's intermediate inhalation minimal risk level (MRL) of $192 \mu \mathrm{g} / \mathrm{m}^{3}$ as a target maximum concentration for carbon tetrachloride in air in the phytoremediation and wetlands treatment areas. This concentration will be protective of health for both workers and members of the general public who might periodically visit these areas. The intermediate MRL is defined as an estimate of the daily human exposure that is likely to be without appreciable risk of adverse noncancer health effects for an exposure duration of 15 to 364 days (EPA 2005). 
Ambient air is sampled at three locations (Figure 3.2) - two in the phytoremediation area (AA1, AA2) and one in the wetlands (AA3) - as specified in the EPA-approved Monitoring Plan (Argonne 2006). Samples are collected by using special evacuated canisters fitted with metered inlet valves. The valves allow air to enter the canisters at a preset, slow rate. At Murdock, an air sample is collected at each location over an 8-hr period, on each of three consecutive days, for each sampling event. This procedure yields "average" samples of the air to which a visitor at the site might be exposed. The purpose is to compensate for unusual wind or other weather conditions that might affect the air sampled on any one given day. For comparison, samples of "background" air are collected during each sampling event from a location southwest of the phytoremediation and wetlands areas (BA1; Figure 3.2), by using the same procedures.

The air samplers are normally placed at an inlet height of approximately $5.5 \mathrm{ft}$ above ground level, roughly corresponding to the breathing zone. In August 2006, however, additional samples were collected at a height of $10 \mathrm{ft}$ above ground, within the vegetation canopy in the phytoremediation area, at locations AA1 and AA2.

The results of the air sampling and analyses are in Table 4.7. With one exception, carbon tetrachloride has not been identified (at an indicated reporting limit of $1.3 \mu \mathrm{g} / \mathrm{m}^{3}$ ) in the ambient air at Murdock. The exception is the carbon tetrachloride detected in one air sample collected on May 18, 2006, at location AA2, at a concentration of $1.4 \mu \mathrm{g} / \mathrm{m}^{3}$. Carbon tetrachloride was not identified in the related air samples collected at AA2 (on May 17 and May 19, 2006) during this sampling event. Chloroform has not been detected in the air at Murdock, at an indicated reporting limit of $0.98 \mu \mathrm{g} / \mathrm{m}^{3}$.

Several other compounds, including chloromethane, acetone, benzene, toluene, and chlorofluoromethanes were identified at low or trace concentrations in many of the air samples collected in the phytoremediation and wetlands areas, at levels comparable to those found at background sampling location BA1. The presence of these compounds appears unrelated to the former CCC/USDA grain storage activities at Murdock or to the groundwater and surface water restoration processes that are presently in progress. 


\subsection{Quality Control for Monitoring Activities}

A comprehensive quality assurance/quality control program was implemented to confirm the reliability and representativeness of all analytical results as they were accumulated. Sample collection and handling activities were monitored by documenting samples as they were collected and using chain-of-custody forms and custody seals to ensure sample integrity during handling and shipment. Field blanks, equipment rinsates, and trip blanks were collected as appropriate to ensure that cross-contamination did not occur during sample collection and handling. To monitor the consistency of the sampling methodology and provide a measure of analytical precision, blind replicate samples were collected, and other samples were selected for duplicate analyses.

Groundwater, surface water, and spray irrigation effluent samples were analyzed for VOCs at the Applied Geosciences and Environmental Management (AGEM) Laboratory at Argonne, Illinois. The AGEM Laboratory used a modification of EPA Method 524.2 to achieve a quantitation limit of $1.0 \mu \mathrm{g} / \mathrm{L}$. In this purge-and-trap method with analysis on a gas chromatograph-mass spectrometer system, VOCs are extracted (purged) from the sample matrix by bubbling an inert gas through each sample. The purged components are then trapped in a specified sorbent tube. After the purging, the sorbent tube is heated and backflushed with an inert gas to desorb the components into the analytical system. The compounds eluting from the gas chromatography column are identified by retention time and through comparison with reference library spectra. The concentration of each component is calculated by comparison of the mass spectrometer response for the quantitation ion to corresponding calibration curves, the responses for internal standards, or both.

Vegetation samples were analyzed for carbon tetrachloride and chloroform at the AGEM Laboratory by using a modification of the protocol in EPA Method 5021 (headspace analysis on a gas chromatograph with electron capture detection) to achieve the low detection limits required. An 11-point calibration of the gas chromatograph system was established on the basis of the mass of known quantities of carbon tetrachloride and chloroform. Consistency in the results for secondary quality control analyses provides support for the sampling and analytical methodologies.

To aid in characterization of the water-bearing zone, groundwater samples were analyzed for geochemical parameters including nitrate, $\mathrm{DO}, \mathrm{Fe}^{2+}, \mathrm{ORP}, \mathrm{pH}$, and conductivity. Samples 
were analyzed for nitrate/nitrite-nitrogen by Severn-Trent Laboratories with EPA Method 353.2, an automated colorimetric method based on cadmium reduction. Commercial field test kits/instruments were used for measurement of the other geochemical parameters of interest.

Air monitoring samples were analyzed at Severn-Trent Laboratories by EPA Method TO-15 on a gas chromatograph-mass spectrometer system. The atmospheric air samples were drawn into specially prepared, preevacuated stainless steel canisters. For the analysis, the sample (a known volume of air) was directed from the canister through a multisorbent concentrator. After the concentration and drying steps were complete, the VOCs were thermally desorbed, entrained in a carrier gas stream, and directed through a multisorbent trap. The sample was then released by thermal desorption and carried onto the gas chromatography column for separation. 
TABLE 4.1 Analytical results for volatile organic compounds in samples of untreated water collected from extraction well GWEX-1 at Murdock, June 2005 through 2006.

\begin{tabular}{|c|c|c|c|c|c|}
\hline \multirow[b]{2}{*}{ Sample } & \multirow[b]{2}{*}{$\begin{array}{c}\text { Sample } \\
\text { Date }\end{array}$} & \multirow[b]{2}{*}{$\begin{array}{l}\text { Depth } \\
\text { (ft BGL) }\end{array}$} & \multicolumn{3}{|c|}{ Concentration $(\mu \mathrm{g} / \mathrm{L})$} \\
\hline & & & $\begin{array}{c}\text { Carbon } \\
\text { Tetrachloride }\end{array}$ & Chloroform & $\begin{array}{c}\text { Methylene } \\
\text { Chloride }\end{array}$ \\
\hline MUGWEX-W-18312 & $7 / 18 / 05$ & $47.0-77.0$ & 205 & 2.3 & $N D^{a}$ \\
\hline MUTAP-W-15717 & $9 / 22 / 05$ & $47.0-77.0$ & 232 & 2.7 & ND \\
\hline MUTAP-W-15718 & $9 / 22 / 05$ & $47.0-77.0$ & 223 & 2.4 & ND \\
\hline MUTAP-W-18128 & $6 / 8 / 06$ & $47.0-77.0$ & 262 & 3.2 & ND \\
\hline MUTAP-W-18129b & $6 / 8 / 06$ & $47.0-77.0$ & 270 & 3.3 & ND \\
\hline MUGWX1-W-18151 & $8 / 15 / 06$ & $47.0-77.0$ & 262 & 4.5 & ND \\
\hline MUGWX1-W-18152 & $8 / 15 / 06$ & $47.0-77.0$ & 179 & 2.7 & ND \\
\hline
\end{tabular}

a ND, not detected at instrument detection limit of $0.1 \mu \mathrm{g} / \mathrm{L}$.

b Quality control replicate. 
TABLE 4.2 Analytical results for volatile organic compounds in samples of treated spray collected at Murdock in June 2005 through 2006.

\begin{tabular}{|c|c|c|c|c|}
\hline Sample & $\begin{array}{c}\text { Sample } \\
\text { Date }\end{array}$ & $\begin{array}{c}\text { Carbon } \\
\text { Tetrachloride }\end{array}$ & Chloroform & $\begin{array}{c}\text { Methylene } \\
\text { Chloride }\end{array}$ \\
\hline MUTEST1-W-B & $6 / 2 / 05$ & $0.8 \mathrm{~J}^{\mathrm{a}}$ & $N D^{b}$ & ND \\
\hline MUTEST1-W-C & $6 / 2 / 05$ & 1.8 & ND & ND \\
\hline MUTEST1-W-D & $6 / 2 / 05$ & 1.1 & ND & ND \\
\hline MUTEST1-W-E & $6 / 2 / 05$ & $0.7 \mathrm{~J}$ & ND & ND \\
\hline MUTEST2-W-B & $6 / 2 / 05$ & 1.3 & ND & ND \\
\hline MUTEST2-W-C & $6 / 2 / 05$ & 2.1 & ND & ND \\
\hline MUTEST2-W-D & $6 / 2 / 05$ & 2.2 & ND & ND \\
\hline MUTEST2-W-E & $6 / 2 / 05$ & $5.9 \mathrm{~S}^{\mathrm{c}}$ & ND & ND \\
\hline MU-C-W-15722 & $9 / 22 / 05$ & 1.9 & ND & ND \\
\hline MU-D-W-15723 & $9 / 22 / 05$ & 2.9 & ND & ND \\
\hline MU-E-W-15719 & $9 / 22 / 05$ & 4.7 & ND & ND \\
\hline MU-F-W-15720 & $9 / 22 / 05$ & 3.0 & ND & ND \\
\hline MU-G-W-15721 & $9 / 22 / 05$ & 4.0 & ND & ND \\
\hline MUB-W-18134 & $6 / 8 / 06$ & 5.2 & ND & ND \\
\hline MUC-W-18133 & 6/8/06 & 1.7 & ND & ND \\
\hline MUD-W-18130 & 6/8/06 & 3.0 & ND & ND \\
\hline MUD-W-18131d & $6 / 8 / 06$ & 3.1 & ND & ND \\
\hline MUE-W-18132 & $6 / 8 / 06$ & 2.7 & ND & ND \\
\hline MUB-W-18153 & $8 / 15 / 06$ & 5.2 & ND & ND \\
\hline MUC-W-18154 & $8 / 15 / 06$ & $0.9 \mathrm{~J}$ & ND & ND \\
\hline MUD-W-18155 & $8 / 15 / 06$ & 2.6 & ND & ND \\
\hline MUD-W-18156 & 8/15/06 & 1.7 & ND & ND \\
\hline MUE-W-18157 & $8 / 15 / 06$ & 2.5 & ND & ND \\
\hline MUF-W-18158 & $8 / 15 / 06$ & 2.3 & ND & ND \\
\hline
\end{tabular}

a Qualifier J indicates an estimated concentration below the method quantitation limit of $1.0 \mu \mathrm{g} / \mathrm{L}$.

b ND, not detected at instrument detection limit of $0.1 \mu \mathrm{g} / \mathrm{L}$.

c Qualifier S indicates that the surrogate recovery was outside the quality control range.

d Quality control replicate. 
TABLE 4.3 Analytical results for $\mathrm{pH}$ in samples of treated spray collected at Murdock in June 2005 through 2006.

\begin{tabular}{ccc} 
& \multicolumn{2}{c}{$\mathrm{pH}$} \\
\cline { 2 - 3 } $\begin{array}{c}\text { Sample } \\
\text { Date }\end{array}$ & Minimum & Maximum \\
\hline 6/2/05 & 7.76 & 7.88 \\
$9 / 22 / 05$ & 6.57 & 7.33 \\
$6 / 8 / 06$ & 6.54 & 6.55 \\
$8 / 15 / 06$ & 7.60 & 7.66 \\
\hline
\end{tabular}


TABLE 4.4 Analytical results for volatile organic compounds in groundwater samples collected from monitoring wells at Murdock in July 2005, April 2006, and August 2006.

\begin{tabular}{|c|c|c|c|c|c|c|}
\hline \multirow[b]{2}{*}{ Location } & \multirow[b]{2}{*}{ Sample } & \multirow[b]{2}{*}{$\begin{array}{l}\text { Sample } \\
\text { Date }\end{array}$} & \multirow[b]{2}{*}{$\begin{array}{l}\text { Depth } \\
\text { (ft BGL) }\end{array}$} & \multicolumn{3}{|c|}{ Concentration $(\mu \mathrm{g} / \mathrm{L})$} \\
\hline & & & & $\begin{array}{c}\text { Carbon } \\
\text { Tetrachloride }\end{array}$ & Chloroform & $\begin{array}{l}\text { Methylene } \\
\text { Chloride }\end{array}$ \\
\hline \multicolumn{7}{|c|}{ Wells in phytoremediation treatment area } \\
\hline PMW1S & MUPMW1S-W-18318 & $7 / 19 / 05$ & $4.6-14.6$ & 9.4 & $0.4 \mathrm{~J}^{\mathrm{a}}$ & $N D^{b}$ \\
\hline PMW1S & MUPMW1S-W-15720 & $4 / 6 / 06$ & $4.6-14.6$ & 4.1 & $0.3 \mathrm{~J}$ & ND \\
\hline PMW1S & MUPMW1S-W-18168 & $8 / 17 / 06$ & $4.6-14.6$ & $0.2 \mathrm{~J}$ & ND & ND \\
\hline PMW1D & MUPMW1D-W-18313 & $7 / 19 / 05$ & $24.6-34.6$ & 1233 & 5.2 & ND \\
\hline PMW1D & MUPMW1D-W-15721 & $4 / 6 / 06$ & $24.6-34.6$ & 3077 & 1.6 & ND \\
\hline PMW1D & MUPMW1D-W-18166 & $8 / 17 / 06$ & $24.6-34.6$ & 2241 & 9.3 & ND \\
\hline PMW2SA & MUPMW2SA-W-15717 & $4 / 6 / 06$ & $4.6-14.6$ & 359 & 2.8 & ND \\
\hline PMW2SA & MUPMW2SA-W-18163 & $8 / 17 / 06$ & $4.6-14.6$ & 385 & 3.5 & ND \\
\hline PMW2SB & MUPMW2SB-W-18334 & $7 / 21 / 05$ & $4.6-14.6$ & 377 & 6.4 & ND \\
\hline PMW2D & MUPMW2D-W-18314 & $7 / 19 / 05$ & $19.6-29.6$ & 1564 & 4.8 & ND \\
\hline PMW2D & MUPMW2D-W-15718 & $4 / 6 / 06$ & $19.6-29.6$ & 3353 & 9.6 & ND \\
\hline PMW2D & MUPMW2D-W-15719c & $4 / 6 / 06$ & $19.6-29.6$ & 3827 & 10 & ND \\
\hline PMW2D & MUPMW2D-W-18164 & $8 / 17 / 06$ & $19.6-29.6$ & 1336 & 7.4 & ND \\
\hline PMW2D & MUPMW2D-W-18165 & $8 / 17 / 06$ & $19.6-29.6$ & 1475 & 7.6 & ND \\
\hline PMW3S & MUPMW3S-W-18331 & $7 / 21 / 05$ & $4.5-14.5$ & 394 & 2.4 & ND \\
\hline PMW3S & MUPMW3S-W-15673 & $4 / 5 / 06$ & $4.5-14.5$ & 65 & $0.7 \mathrm{~J}$ & ND \\
\hline PMW3S & MUPMW3S-W-18159 & $8 / 15 / 06$ & $4.5-14.5$ & 85 & 1.0 & ND \\
\hline PMW3S & MUPMW3S-W-18160 & $8 / 15 / 06$ & $4.5-14.5$ & 140 & 1.5 & ND \\
\hline PMW3D & MUPMW3D-W-18316 & $7 / 19 / 05$ & $19.5-24.5$ & 801 & 4.9 & ND \\
\hline PMW3D & MUPMW3D-W-15671 & $4 / 5 / 06$ & $19.5-24.5$ & 346 & ND & ND \\
\hline PMW3D & MUPMW3D-W-18161 & $8 / 15 / 06$ & $19.5-24.5$ & 444 & 4.4 & ND \\
\hline PMW4 & MUPMW4-W-18321 & $7 / 20 / 05$ & $19.5-24.5$ & 52 & 1.8 & ND \\
\hline PMW4 & MUPMW4-W-15728 & $4 / 10 / 06$ & $19.5-24.5$ & 31 & $0.6 \mathrm{~J}$ & ND \\
\hline PMW4 & MUPMW4-W-18346 & $8 / 21 / 06$ & $19.5-24.5$ & 35 & $0.6 \mathrm{~J}$ & ND \\
\hline PMW5 & MUPMW5-W-18322 & $7 / 20 / 05$ & $4.5-14.5$ & 118 & 1.0 & ND \\
\hline PMW5 & MUPMW5-W-15727 & $4 / 10 / 06$ & $4.5-14.5$ & 178 & 1.5 & ND \\
\hline PMW5 & MUPMW5-W-18347 & $8 / 21 / 06$ & $4.5-14.5$ & 34 & $0.2 \mathrm{~J}$ & ND \\
\hline PMW6 & MUPMW6-W-18333 & $7 / 21 / 05$ & $4.5-14.5$ & 9.6 & $0.4 \mathrm{~J}$ & ND \\
\hline PMW6 & MUPMW6-W-15724 & $4 / 10 / 06$ & $4.5-14.5$ & 8.7 & $0.4 \mathrm{~J}$ & ND \\
\hline PMW6 & MUPMW6-W-15725c & $4 / 10 / 06$ & $4.5-14.5$ & 8.1 & $0.3 \mathrm{~J}$ & ND \\
\hline PMW6 & MUPMW6-W-18174 & $8 / 23 / 06$ & $4.5-14.5$ & 9.4 & $0.6 \mathrm{~J}$ & ND \\
\hline PMW7 & MUPMW7-W-18332 & $7 / 21 / 05$ & $14.5-19.5$ & 3.5 & $0.6 \mathrm{~J}$ & ND \\
\hline PMW7 & MUPMW7-W-15722 & $4 / 10 / 06$ & $14.5-19.5$ & 3.7 & $0.9 \mathrm{~J}$ & ND \\
\hline PMW7 & MUPMW7-W-18172 & $8 / 23 / 06$ & $14.5-19.5$ & 5.4 & 1.1 & ND \\
\hline PMW7 & MUPMW7-W-18173' & $8 / 23 / 06$ & $14.5-19.5$ & 5.1 & 1.0 & ND \\
\hline
\end{tabular}


TABLE 4.4 (Cont.)

\begin{tabular}{|c|c|c|c|c|c|c|}
\hline \multirow[b]{2}{*}{ Location } & \multirow[b]{2}{*}{ Sample } & \multirow[b]{2}{*}{$\begin{array}{l}\text { Sample } \\
\text { Date }\end{array}$} & \multirow[b]{2}{*}{$\begin{array}{l}\text { Depth } \\
\text { (ft BGL) }\end{array}$} & \multicolumn{3}{|c|}{ Concentration $(\mu \mathrm{g} / \mathrm{L})$} \\
\hline & & & & $\begin{array}{c}\text { Carbon } \\
\text { Tetrachloride }\end{array}$ & Chloroform & $\begin{array}{c}\text { Methylene } \\
\text { Chloride }\end{array}$ \\
\hline \multicolumn{7}{|c|}{ Wells in phytoremediation treatment area (cont.) } \\
\hline PMW8 & MUPMW8-W-18323 & $7 / 20 / 05$ & $4.5-14.5$ & 23 & $0.8 \mathrm{~J}$ & ND \\
\hline PMW8 & MUPMW8-W-15726 & 4/10/06 & $4.5-14.5$ & 16 & $0.6 \mathrm{~J}$ & ND \\
\hline PMW8 & MUPMW8-W-18169 & 8/21/06 & $4.5-14.5$ & 19 & $0.7 \mathrm{~J}$ & ND \\
\hline PMW9S & MUPMW9S-W-18328 & 7/20/05 & $5.0-9.0$ & 10 & 14 & ND \\
\hline PMW9S & MUPMW9S-W-15712 & $4 / 5 / 06$ & $5.0-9.0$ & 8.1 & 14 & ND \\
\hline PMW9S & MUPMW9S-W-17206 & 8/16/06 & $5.0-9.0$ & 11 & 8.7 & ND \\
\hline PMW9M & MUPMW9M-W-18329 & $7 / 20 / 05$ & $11.0-15.0$ & 60 & 2.0 & ND \\
\hline PMW9M & MUPMW9M-W-15674 & $4 / 5 / 06$ & $11.0-15.0$ & 60 & 1.8 & ND \\
\hline PMW9M & MUPMW9M-W-17207 & 8/16/06 & $11.0-15.0$ & 44 & 1.6 & ND \\
\hline PMW9D & MUPMW9D-W-18315 & 7/20/05 & $20-30$ & 4.6 & $0.9 \mathrm{~J}$ & ND \\
\hline PMW9D & MUPMW9D-W-15713 & $4 / 6 / 06$ & $19.5-29.5$ & ND & $0.4 \mathrm{~J}$ & ND \\
\hline PMW9D & MUPMW9D-W-18345 & 8/16/06 & $19.5-29.5$ & $0.5 \mathrm{~J}$ & $0.3 \mathrm{~J}$ & ND \\
\hline W103 & MU578-W-18260 & 7/19/05 & $5.66-7.78$ & ND & ND & ND \\
\hline W103 & MUW103-W-17200 & 4/13/06 & $2.9-5.0$ & ND & ND & ND \\
\hline W103 & MUW103-W-18182 & $8 / 24 / 06$ & $2.9-5.0$ & ND & ND & ND \\
\hline W410 & MUTW444-W-18261 & $7 / 25 / 05$ & $2.5-4.9$ & 38 & 31 & ND \\
\hline W410 & MUW410-W-18127 & $4 / 13 / 06$ & $2.5-4.9$ & 1.6 & 3.6 & ND \\
\hline \multicolumn{7}{|c|}{ Wells in upgradient plume area } \\
\hline $2 S$ & MU2S-W-18308 & 7/18/05 & $70.5-80.5$ & 85 & 2.5 & ND \\
\hline $2 S$ & MUS2-W-17202 & 4/18/06 & $70.5-80.5$ & 88 & 1.6 & ND \\
\hline $2 S$ & MUS2-W-18179 & 8/24/06 & $70.5-80.5$ & 130 & 2.8 & ND \\
\hline $2 S$ & MUS2-W-18180 & 8/24/06 & $70.5-80.5$ & 124 & 2.8 & ND \\
\hline $2 \mathrm{D}$ & MUD2-W-18344 & 7/22/05 & $85.0-95.0$ & 10 & 3.4 & ND \\
\hline $2 \mathrm{D}$ & MUD2-W-17201 & 4/17/06 & $85.0-95.0$ & 2.2 & 4.6 & ND \\
\hline $2 \mathrm{D}$ & MUD2-W-18181 & $8 / 24 / 06$ & $85.0-95.0$ & 36 & 4.1 & ND \\
\hline SB65S & MUSB65S-W-18335 & 7/21/05 & $23.7-38.7$ & ND & ND & ND \\
\hline SB65S & MUSB65S-W-15732 & 4/13/06 & $23.7-38.7$ & $0.5 \mathrm{~J}$ & ND & ND \\
\hline SB65S & MUSB65S-W-18176 & 8/23/06 & $23.7-38.7$ & $0.7 \mathrm{~J}$ & ND & ND \\
\hline SB65D & MU65D-W-18309 & 7/18/05 & $38.0-53.0$ & 201 & 1.0 & ND \\
\hline SB65D & MUSB65D-W-15733 & 4/13/06 & $38.0-53.0$ & 582 & 3.6 & ND \\
\hline SB65D & MUSB65D-W-18175 & 8/23/06 & $38.0-53.0$ & 595 & 4.9 & ND \\
\hline TEST-1 & MUTEST1-W-18311 & 7/18/05 & $60.0-65.0$ & 81 & 49 & ND \\
\hline TEST-1 & MUTEST1-W-18125 & 4/13/06 & $60.0-65.0$ & 79 & 24 & ND \\
\hline TEST-1 & MUTST1-W-18177 & 8/23/06 & $60.0-65.0$ & 84 & 24 & $0.4 \mathrm{~J}$ \\
\hline TEST-1 & MUTST1-W-18178C & 8/23/06 & $60.0-65.0$ & 88 & 23 & $0.3 \mathrm{~J}$ \\
\hline
\end{tabular}




\section{TABLE 4.4 Footnotes}

a Qualifier $\mathrm{J}$ indicates an estimated concentration below the method quantitation limit of $1.0 \mu \mathrm{g} / \mathrm{L}$.

b ND, not detected at instrument detection limit of $0.1 \mu \mathrm{g} / \mathrm{L}$.

c Quality control replicate. 
TABLE 4.5 Geochemical parameters measured in water samples collected at Murdock in 2005 and 2006.

\begin{tabular}{|c|c|c|c|c|c|c|c|c|c|c|}
\hline Location & Sample & $\begin{array}{l}\text { Sample } \\
\text { Date }\end{array}$ & $\begin{array}{l}\text { Depth } \\
\text { (ft BGL) }\end{array}$ & $\begin{array}{c}\text { Temperature } \\
\left({ }^{\circ} \mathrm{C}\right)\end{array}$ & $\mathrm{pH}$ & $\begin{array}{l}\text { Conductivity } \\
(\mu \mathrm{S} / \mathrm{cm})\end{array}$ & $\begin{array}{c}\text { Dissolved } \\
\text { Oxygen } \\
(\mathrm{mg} / \mathrm{L})\end{array}$ & $\begin{array}{l}\text { Iron II } \\
\text { (mg/L) }\end{array}$ & $\begin{array}{l}\text { Oxygen } \\
\text { Reduction } \\
\text { Potential } \\
(\mathrm{mV})\end{array}$ & $\begin{array}{l}\text { Nitrate- } \\
\text { Nitrite } \\
\text { Nitrogen }^{a} \\
\text { (mg/L) }\end{array}$ \\
\hline \multicolumn{11}{|c|}{ Monitoring wells in phytoremediation treatment area } \\
\hline PMW1S & MUPMW1S-W-18318 & $7 / 19 / 05$ & $4.6-14.6$ & 19.8 & 6.45 & 463 & $N R^{b}$ & NR & NR & $N^{c}$ \\
\hline PMW1S & MUPMW1S-W-15720 & 4/6/06 & $4.6-14.6$ & 10.7 & 6.81 & 470 & 8.29 & 0.09 & 177 & 15.4 \\
\hline PMW1S & MUPMW1S-W-18168 & $8 / 17 / 06$ & $4.6-14.6$ & 18.7 & 6.68 & 377 & 0.13 & 0.07 & 27 & 1.98 \\
\hline PMW1D & MUPMW1D-W-18313 & $7 / 19 / 05$ & $24.6-34.6$ & 14.6 & 6.85 & 416 & 8.31 & 0.00 & 215 & $3.84^{a}$ \\
\hline PMW1D & MUPMW1D-W-15721 & 4/6/06 & $24.6-34.6$ & 12.2 & 6.74 & 430 & 8.04 & 0.12 & 188 & 4.25 \\
\hline PMW1D & MUPMW1D-W-18166 & $8 / 17 / 06$ & $24.6-34.6$ & 14.9 & 6.42 & 403 & 7.17 & 0.15 & 84 & 4.23 \\
\hline PMW2SA & MUPMW2SA-W-15717 & 4/6/06 & $4.6-14.6$ & 10.0 & 6.87 & 439 & 8.91 & 0.09 & 189 & 8.28 \\
\hline PMW2SA & MUPMW2SA-W-18163 & $8 / 17 / 06$ & $4.6-14.6$ & 17.8 & 6.42 & 443 & 5.62 & 0.06 & 96 & 7.45 \\
\hline PMW2SB & MUPMW2SB-W-18334 & $7 / 21 / 05$ & $4.6-14.6$ & 16.0 & 6.54 & 540 & 7.67 & 0.55 & NR & $13.0^{a}$ \\
\hline PMW2D & MUPMW2D-W-18314 & $7 / 19 / 05$ & $19.6-29.6$ & 12.0 & 6.56 & 500 & 8.36 & 0.00 & 220 & $4.48^{a}$ \\
\hline PMW2D & MUPMW2D-W-15718 & 4/6/06 & $19.6-29.6$ & 11.8 & 6.68 & 435 & 7.26 & 0.13 & 202 & 5.75 \\
\hline PMW2D & MUPMW2D-W-18164 & $8 / 17 / 06$ & $19.6-29.6$ & 14.9 & 6.40 & 461 & 7.00 & 0.23 & 73 & 5.45 \\
\hline PMW3S & MUPMW3S-W-18331 & $7 / 21 / 05$ & $4.5-14.5$ & 13.0 & 6.16 & 407 & 8.17 & 0.20 & NR & $6.12^{a}$ \\
\hline PMW3S & MUPMW3S-W-15673 & 4/5/06 & $4.5-14.5$ & 10.6 & 6.45 & 419 & 8.49 & 1.51 & 207 & 8.80 \\
\hline PMW3S & MUPMW3S-W-18159 & $8 / 15 / 06$ & $4.5-14.5$ & 15.8 & 6.96 & 403 & 7.48 & 0.13 & 72 & 6.20 \\
\hline PMW3D & MUPMW3D-W-18316 & $7 / 19 / 05$ & $19.5-24.5$ & 12.1 & 6.44 & 595 & 7.17 & 0.00 & 215 & $5.06^{a}$ \\
\hline PMW3D & MUPMW3D-W-15671 & 4/5/06 & $19.5-24.5$ & 11.6 & 6.86 & 638 & 5.13 & 0.00 & 196 & 5.76 \\
\hline PMW3D & MUPMW3D-W-18161 & $8 / 15 / 06$ & $19.5-24.5$ & 14.0 & 7.32 & 620 & 5.01 & 0.15 & 77 & 5.45 \\
\hline PMW4 & MUPMW4-W-18321 & $7 / 20 / 05$ & $19.5-24.5$ & 13.0 & 6.36 & 395 & 7.97 & NR & 210 & $9.08^{a}$ \\
\hline PMW4 & MUPMW4-W-15728 & $4 / 10 / 06$ & $19.5-24.5$ & 11.3 & 6.80 & 411 & 8.05 & 0.31 & 207 & 9.98 \\
\hline PMW4 & MUPMW4-W-18346 & $8 / 21 / 06$ & $19.5-24.5$ & 14.9 & 6.37 & 410 & 2.97 & 0.05 & 102 & 11.8 \\
\hline PMW5 & MUPMW5-W-18322 & $7 / 20 / 05$ & $4.5-14.5$ & 14.6 & 6.56 & 520 & 6.74 & 0.00 & NR & $4.58^{a}$ \\
\hline PMW5 & MUPMW5-W-15727 & $4 / 10 / 06$ & $4.5-14.5$ & 10.2 & 6.86 & 591 & 7.16 & 0.26 & 191 & 5.17 \\
\hline PMW5 & MUPMW5-W-18347 & $8 / 21 / 06$ & $4.5-14.5$ & 16.6 & 6.58 & 572 & 6.42 & 0.38 & 86 & 5.02 \\
\hline
\end{tabular}


Monitoring wells in phytoremediation treatment area (cont)

\begin{tabular}{|c|c|c|c|c|c|c|c|c|c|c|}
\hline PMW6 & MUPMW6-W-18333 & $7 / 21 / 05$ & $4.5-14.5$ & 18.4 & 6.52 & 884 & NR & NR & NR & NA \\
\hline PMW6 & MUPMW6-W-15724 & $4 / 10 / 06$ & $4.5-14.5$ & 10.7 & 6.72 & 863 & 5.15 & 0.17 & 222 & 10.7 \\
\hline PMW6 & MUPMW6-W-18174 & 8/23/06 & $4.5-14.5$ & 17.3 & 6.58 & 835 & 3.16 & 0.82 & 68 & 9.33 \\
\hline PMW7 & MUPMW7-W-18332 & $7 / 21 / 05$ & $14.5-19.5$ & 14.6 & 6.31 & 691 & NR & NR & NR & NA \\
\hline PMW7 & MUPMW7-W-15722 & $4 / 10 / 06$ & $14.5-19.5$ & 11.3 & 6.36 & 604 & 8.65 & 0.00 & 174 & 10.8 \\
\hline PMW7 & MUPMW7-W-18172 & 8/23/06 & $14.5-19.5$ & 13.9 & 6.41 & 642 & 9.26 & 0.07 & 96 & 14.9 \\
\hline PMW8 & MUPMW8-W-18323 & 7/20/05 & $4.5-14.5$ & 15.8 & 6.46 & 418 & 2.79 & 0.60 & NR & $4.63^{a}$ \\
\hline PMW8 & MUPMW8-W-15726 & $4 / 10 / 06$ & $4.5-14.5$ & 10.1 & 6.82 & 435 & 5.42 & 0.07 & 181 & 5.37 \\
\hline PMW8 & MUPMW8-W-18169 & $8 / 21 / 06$ & $4.5-14.5$ & 16.2 & 6.52 & 425 & 5.93 & 0.00 & 100 & 6.43 \\
\hline PMW9S & MUPMW9S-W-18328 & 7/20/05 & $5.0-9.0$ & 21.3 & 6.41 & 508 & NR & NR & NR & NA \\
\hline PMW9S & MUPMW9S-W-15712 & $4 / 5 / 06$ & $5.0-9.0$ & 10.1 & 6.87 & 474 & 5.36 & 0.13 & 203 & 12.3 \\
\hline PMW9S & MUPMW9S-W-17206 & 8/16/06 & $5.0-9.0$ & 19.5 & 6.30 & 454 & 4.54 & 0.05 & 111 & 11.2 \\
\hline PMW9M & MUPMW9M-W-18329 & $7 / 20 / 05$ & $11.0-15.0$ & 14.8 & 6.24 & 466 & NR & NR & NR & NA \\
\hline PMW9M & MUPMW9M-W-15674 & $4 / 5 / 06$ & $11.0-15.0$ & 10.3 & 6.62 & 502 & 4.55 & 0.06 & 206 & 12.3 \\
\hline PMW9M & MUPMW9M-W-17207 & $8 / 16 / 06$ & $11.0-15.0$ & 16.2 & 6.41 & 461 & 4.19 & 0.12 & 203 & 12.7 \\
\hline PMW9D & MUPMW9D-W-18315 & 7/20/05 & $19.5-29.5$ & 25.1 & 7.13 & 643 & NR & NR & NR & $3.85^{\mathrm{a}}$ \\
\hline PMW9D & MUPMW9D-W-15713 & $4 / 6 / 06$ & $19.5-29.5$ & 12.0 & 7.23 & 646 & 3.52 & 0.00 & 189 & 0.17 \\
\hline PMW9D & MUPMW9D-W-18345 & 8/16/06 & $19.5-29.5$ & 14.9 & 7.06 & 659 & 3.48 & 0.09 & 177 & 2.25 \\
\hline W103 & MU578-W-18260 & $7 / 19 / 05$ & $2.9-5.0$ & 24.5 & 6.57 & 4100 & NR & NR & NR & NA \\
\hline W103 & MUW103-W-17200 & $4 / 13 / 06$ & $2.9-5.0$ & 12.5 & 6.66 & 2860 & NR & NR & NR & 13.1 \\
\hline W103 & MUW103-W-18182 & $8 / 24 / 06$ & $2.9-5.0$ & 25.3 & 6.79 & 3450 & NR & 3.30 & -145 & 0.143 \\
\hline
\end{tabular}


Monitoring wells in upgradient plume area

\begin{tabular}{|c|c|c|c|c|c|c|c|c|c|c|}
\hline $2 S$ & MUS2-W-18308 & 7/18/05 & $70.5-80.5$ & 16.3 & 6.20 & 552 & 6.76 & 0.00 & 275 & $5.00^{a}$ \\
\hline $2 S$ & MUS2-W-17202 & 4/18/06 & $70.5-80.5$ & 13.4 & 6.43 & 566 & 6.48 & 0.00 & 61 & 5.16 \\
\hline $2 S$ & MUS2-W-18179 & $8 / 24 / 06$ & $70.5-80.5$ & 16.5 & 6.30 & 552 & 6.32 & 0.15 & 141 & 4.18 \\
\hline $2 \mathrm{D}$ & MUD2-W-18344 & $7 / 22 / 05$ & $85.0-95.0$ & d & $d$ & d & $d$ & $d$ & $d$ & d \\
\hline 2D & MUD2-W-17201 & 4/17/06 & $85.0-95.0$ & 13.1 & 8.21 & 481 & 0.95 & 0.12 & 140 & 1.64 \\
\hline $2 \mathrm{D}$ & MUD2-W-18181 & 8/24/06 & $85.0-95.0$ & 21.2 & 6.92 & 598 & 6.69 & 0.33 & 113 & 2.61 \\
\hline SB65S & MUSB65S-W-18335 & $7 / 21 / 05$ & $23.7-38.7$ & 15.3 & 6.28 & 403 & NR & NR & NR & NA \\
\hline SB65S & MUSB65S-W-15732 & 4/13/06 & $23.7-38.7$ & 12.5 & 6.59 & 371 & 7.24 & 0.00 & 228 & 9.17 \\
\hline SB65S & MUSB65S-W-18176 & 8/23/06 & $23.7-38.7$ & 17.7 & 6.18 & 403 & 7.17 & 0.17 & 120 & 9.98 \\
\hline SB65D & MU65D-W-18309 & $7 / 18 / 05$ & $38.0-53.0$ & 15.1 & 6.28 & 510 & 8.76 & 0.00 & 235 & $4.74^{a}$ \\
\hline SB65D & MUSB65D-W-15733 & 4/13/06 & $38.0-53.0$ & 16.2 & 6.59 & 494 & NR & NR & 203 & 5.96 \\
\hline SB65D & MUSB65D-W-18175 & 8/23/06 & $38.0-53.0$ & 17.2 & 6.51 & 489 & 8.06 & 0.00 & 102 & 5.67 \\
\hline TEST-1 & MUTEST1-W-18311 & $7 / 18 / 05$ & $60.0-65.0$ & 12.7 & 6.56 & 663 & 5.14 & NR & 245 & $6.13^{a}$ \\
\hline TEST-1 & MUTEST1-W-18125 & 4/13/06 & $60.0-65.0$ & 16.0 & 6.71 & 631 & NR & NR & NR & 7.12 \\
\hline TEST-1 & MUTST1-W-18177 & 8/23/06 & $60.0-65.0$ & 16.5 & 6.61 & 616 & 6.82 & 0.02 & 126 & 7.15 \\
\hline \multicolumn{11}{|c|}{ Surface water } \\
\hline SWM1 & MUSWM1-W-15730 & $4 / 10 / 06$ & - & NR & NR & NR & NR & NR & NR & 2.35 \\
\hline SWM1 & MUSWM1-W-18147 & $8 / 14 / 06$ & - & NR & NR & NR & NR & NR & NR & 3.51 \\
\hline SWM2 & MUSWM2-W-15729 & 4/10/06 & - & NR & NR & NR & NR & NR & NR & 9.65 \\
\hline SWM2 & MUSWM2-W-18148 & $8 / 14 / 06$ & - & NR & NR & NR & NR & NR & NR & 7.57 \\
\hline SWM3 & MU-W-15670 & $10 / 19 / 05$ & - & 14.3 & 7.58 & 555 & NR & NR & NR & NA \\
\hline SWM3 & MUSWM3-W-15731 & $4 / 10 / 06$ & - & NR & NR & NR & NR & NR & NR & 7.79 \\
\hline SWM3 & MUSWM3-W-18149 & $8 / 14 / 06$ & - & NR & NR & NR & NR & NR & NR & 1.09 \\
\hline
\end{tabular}


TABLE 4.5 Footnotes

a July 2005 samples analyzed for nitrate as nitrogen.

b NR, not recorded

c NA, not analyzed.

d Well dry. 
TABLE 4.6 Analytical results for volatile organic compounds in surface water samples collected at Murdock in October 2005 through 2006.

\begin{tabular}{|c|c|c|c|c|c|}
\hline \multirow[b]{2}{*}{ Location } & \multirow[b]{2}{*}{ Sample } & \multirow[b]{2}{*}{$\begin{array}{l}\text { Sample } \\
\text { Date }\end{array}$} & \multicolumn{3}{|c|}{ Concentration $(\mu \mathrm{g} / \mathrm{L})$} \\
\hline & & & $\begin{array}{c}\text { Carbon } \\
\text { Tetrachloride }\end{array}$ & Chloroform & $\begin{array}{c}\text { Methylene } \\
\text { Chloride }\end{array}$ \\
\hline SWM1 & MUSWM1-W-15730 & $4 / 10 / 06$ & 151 & 8.3 & $0.4 \mathrm{~J}^{\mathrm{a}}$ \\
\hline SWM1 & MUSWM1-W-18147 & 8/14/06 & 380 & 6.3 & $N D^{b}$ \\
\hline SWM1 & MUSWM1-W-15724 & $11 / 8 / 06$ & 259 & 12 & 1.6 \\
\hline SWM2 & MUSWM2-W-15729 & $4 / 10 / 06$ & 3.6 & $0.4 \mathrm{~J}$ & ND \\
\hline SWM2 & MUSWM2-W-18148 & 8/14/06 & 1.4 & $0.2 \mathrm{~J}$ & ND \\
\hline SWM2 & MUSWM2-W-15725 & $11 / 8 / 06$ & 6.1 & $0.6 \mathrm{~J}$ & ND \\
\hline SWM3 & MU-W-15670 & $10 / 19 / 05$ & ND & ND & ND \\
\hline SWM3 & MUSWM3-W-15731 & 4/10/06 & ND & ND & ND \\
\hline SWM3 & MUSWM3-W-18149 & 8/14/06 & ND & ND & ND \\
\hline SWM3 & MUSWM3-W-18150C & 8/14/06 & ND & ND & ND \\
\hline SWM3 & MUSWM3-W-15726 & $11 / 8 / 06$ & $0.7 \mathrm{~J}$ & ND & ND \\
\hline
\end{tabular}

a Qualifier J indicates an estimated concentration below the method quantitation limit of $1.0 \mu \mathrm{g} / \mathrm{L}$.

b ND, not detected at instrument detection limit of $0.1 \mu \mathrm{g} / \mathrm{L}$.

c Quality control replicate. 
TABLE 4.7 Analytical results from Severn-Trent Laboratories for volatile organic compounds in ambient air at Murdock in 2005 and 2006.

\begin{tabular}{|c|c|c|c|c|c|c|c|c|c|c|}
\hline \multirow[b]{2}{*}{ Locationa $^{a}$} & \multirow[b]{2}{*}{ Sample } & \multirow[b]{2}{*}{$\begin{array}{l}\text { Sample } \\
\text { Date }\end{array}$} & \multicolumn{8}{|c|}{ Concentrations $\left(\mu \mathrm{g} / \mathrm{m}^{3}\right)$} \\
\hline & & & $\begin{array}{l}\text { 1,2,4-Trimethyl- } \\
\text { benzene }\end{array}$ & $\begin{array}{l}\text { 2,2,4-Trimethyl- } \\
\text { pentane }\end{array}$ & $\begin{array}{c}2- \\
\text { Butanone }\end{array}$ & $\begin{array}{l}\text { 4-Ethyl- } \\
\text { toluene }\end{array}$ & Acetone & Benzene & $\begin{array}{l}\text { Carbon } \\
\text { Disulfide }\end{array}$ & $\begin{array}{c}\text { Carbon } \\
\text { Tetrachloride }\end{array}$ \\
\hline \multicolumn{11}{|c|}{ Ambient air monitoring in May 2006} \\
\hline $\begin{array}{l}\text { AA1 } \\
\text { AA2 } \\
\text { AA3 } \\
\text { BA1 }\end{array}$ & $\begin{array}{l}\text { MUAA1-G-15785 } \\
\text { MUAA2-G-15791 } \\
\text { MUAA3-G-15788 } \\
\text { MUBA1-G-17203 }\end{array}$ & $\begin{array}{l}5 / 17 / 06 \\
5 / 17 / 06 \\
5 / 17 / 06 \\
5 / 17 / 06\end{array}$ & $\begin{array}{l}0.98 U^{b} \\
0.98 U \\
0.98 U \\
0.98 U\end{array}$ & $\begin{array}{l}0.93 U \\
0.93 U \\
0.93 U \\
0.93 U\end{array}$ & $\begin{array}{l}1.5 U \\
1.5 U \\
1.5 U \\
1.5 U\end{array}$ & $\begin{array}{l}0.98 U \\
0.98 U \\
0.98 U \\
0.98 U\end{array}$ & $\begin{array}{l}12 U \\
12 U \\
12 U \\
12 U\end{array}$ & $\begin{array}{l}0.64 U \\
0.64 U \\
0.64 U \\
0.64 U\end{array}$ & $\begin{array}{l}1.6 U \\
1.6 U \\
1.6 U \\
1.6 U\end{array}$ & $\begin{array}{l}1.3 U \\
1.3 U \\
1.3 U \\
1.3 U\end{array}$ \\
\hline $\begin{array}{l}\text { AA1 } \\
\text { AA2 } \\
\text { AA3 } \\
\text { BA1 }\end{array}$ & $\begin{array}{l}\text { MUAA1-G-15786 } \\
\text { MUAA2-G-15792 } \\
\text { MUAA3-G-15789 } \\
\text { MUBA1-G-17204 }\end{array}$ & $\begin{array}{l}5 / 18 / 06 \\
5 / 18 / 06 \\
5 / 18 / 06 \\
5 / 18 / 06\end{array}$ & $\begin{array}{l}0.98 \mathrm{U} \\
0.98 \mathrm{U} \\
0.98 \mathrm{U} \\
0.98 \mathrm{U}\end{array}$ & $\begin{array}{l}0.93 U \\
0.93 U \\
0.93 U \\
0.93 U\end{array}$ & $\begin{array}{l}1.5 U \\
1.5 U \\
1.5 U \\
1.5 U\end{array}$ & $\begin{array}{l}0.98 U \\
0.98 U \\
0.98 U \\
0.98 U\end{array}$ & $\begin{array}{l}12 U \\
12 U \\
12 U \\
12 U\end{array}$ & $\begin{array}{l}0.64 U \\
0.64 U \\
0.64 U \\
0.64 U\end{array}$ & $\begin{array}{l}1.6 U \\
1.6 U \\
1.6 U \\
1.6 U\end{array}$ & $\begin{array}{l}1.3 \mathrm{U} \\
1.4 \\
1.3 \mathrm{U} \\
1.3 \mathrm{U}\end{array}$ \\
\hline $\begin{array}{l}\text { AA1 } \\
\text { AA2 } \\
\text { AA3 } \\
\text { BA1 }\end{array}$ & $\begin{array}{l}\text { MUAA1-G-15787 } \\
\text { MUAA2-G-15793 } \\
\text { MUAA3-G-15790 } \\
\text { MUBA1-G-17205 }\end{array}$ & $\begin{array}{l}5 / 19 / 06 \\
5 / 19 / 06 \\
5 / 19 / 06 \\
5 / 19 / 06\end{array}$ & $\begin{array}{l}0.98 U \\
0.98 U \\
0.98 U \\
0.98 U\end{array}$ & $\begin{array}{l}0.93 U \\
0.93 U \\
0.93 U \\
0.93 U\end{array}$ & $\begin{array}{l}1.5 \mathrm{U} \\
1.5 \mathrm{U} \\
1.5 \\
1.7\end{array}$ & $\begin{array}{l}0.98 U \\
0.98 U \\
0.98 U \\
0.98 U\end{array}$ & $\begin{array}{l}12 U \\
12 U \\
12 U \\
18\end{array}$ & $\begin{array}{l}0.64 \mathrm{U} \\
0.64 \mathrm{U} \\
0.77 \\
0.64 \mathrm{U}\end{array}$ & $\begin{array}{l}1.6 U \\
1.6 U \\
1.6 U \\
1.6 U\end{array}$ & $\begin{array}{l}1.3 U \\
1.3 U \\
1.3 U \\
1.3 U\end{array}$ \\
\hline \multicolumn{11}{|c|}{ Ambient air monitoring in August 2006} \\
\hline $\begin{array}{l}\text { AA1 } \\
\text { AA2 } \\
\text { AA3 } \\
\text { BA1 }\end{array}$ & $\begin{array}{l}\text { MUAA1-G-18138 } \\
\text { MUAA2-G-18144 } \\
\text { MUAA3-G-18141 } \\
\text { MUBA1-G-18135 }\end{array}$ & $\begin{array}{l}8 / 14 / 06 \\
8 / 14 / 06 \\
8 / 14 / 06 \\
8 / 14 / 06\end{array}$ & $\begin{array}{l}0.98 \mathrm{U} \\
1.7 \\
0.98 \mathrm{U} \\
0.98 \mathrm{U}\end{array}$ & $\begin{array}{l}1.1 \\
1.1 \\
0.93 \mathrm{U} \\
0.93 \mathrm{U}\end{array}$ & $\begin{array}{l}2.1 \\
1.5 \mathrm{U} \\
1.5 \mathrm{U} \\
7.7\end{array}$ & $\begin{array}{l}0.98 \mathrm{U} \\
1.4 \\
0.98 \mathrm{U} \\
0.98 \mathrm{U}\end{array}$ & $\begin{array}{l}15 \\
22 \\
12 U \\
50\end{array}$ & $\begin{array}{l}1.2 \\
1.2 \\
0.64 \mathrm{U} \\
0.64 \mathrm{U}\end{array}$ & $\begin{array}{l}1.6 U \\
1.6 U \\
1.6 U \\
1.6 U\end{array}$ & $\begin{array}{l}1.3 U \\
1.3 U \\
1.3 U \\
1.3 U\end{array}$ \\
\hline $\begin{array}{l}\text { AA1 } \\
\text { AA2 } \\
\text { AA3 } \\
\text { BA1 }\end{array}$ & $\begin{array}{l}\text { MUAA1-G-18139 } \\
\text { MUAA2-G-18145 } \\
\text { MUAA3-G-18142 } \\
\text { MUBA1-G-18136 }\end{array}$ & $\begin{array}{l}8 / 15 / 06 \\
8 / 15 / 06 \\
8 / 15 / 06 \\
8 / 15 / 06\end{array}$ & $\begin{array}{l}0.98 U \\
0.98 U \\
0.98 U \\
0.98 U\end{array}$ & $\begin{array}{l}0.93 U \\
0.93 U \\
0.93 U \\
0.93 U\end{array}$ & $\begin{array}{l}1.5 U \\
1.5 U \\
1.5 U \\
1.5 U\end{array}$ & $\begin{array}{l}0.98 \mathrm{U} \\
0.98 \mathrm{U} \\
0.98 \mathrm{U} \\
0.98 \mathrm{U}\end{array}$ & $\begin{array}{l}12 U \\
12 U \\
12 U \\
12 U\end{array}$ & $\begin{array}{l}0.64 \mathrm{U} \\
0.64 \mathrm{U} \\
0.64 \mathrm{U} \\
0.64 \mathrm{U}\end{array}$ & $\begin{array}{l}1.6 U \\
1.6 U \\
1.6 U \\
1.6 U\end{array}$ & $\begin{array}{l}1.3 U \\
1.3 U \\
1.3 U \\
1.3 U\end{array}$ \\
\hline $\begin{array}{l}\text { AA1 } \\
\text { AA2 } \\
\text { AA3 } \\
\text { BA1 }\end{array}$ & $\begin{array}{l}\text { MUAA1-G-18140 } \\
\text { MUAA2-G-18146 } \\
\text { MUAA3-G-18143 } \\
\text { MUBA1-G-18137 }\end{array}$ & $\begin{array}{l}8 / 16 / 06 \\
8 / 16 / 06 \\
8 / 16 / 06 \\
8 / 16 / 06\end{array}$ & $\begin{array}{l}0.98 U \\
0.98 U \\
2 U \\
0.98 U\end{array}$ & $\begin{array}{l}0.93 \mathrm{U} \\
0.93 \mathrm{U} \\
1.9 \mathrm{U} \\
0.93 \mathrm{U}\end{array}$ & $\begin{array}{c}2.8 \\
2.3 \\
27 \\
1.5\end{array}$ & $\begin{array}{l}0.98 U \\
0.98 U \\
2 U \\
0.98 U\end{array}$ & $\begin{array}{r}15 \\
19 \\
170 \\
13\end{array}$ & $\begin{array}{l}0.64 \mathrm{U} \\
0.64 \mathrm{U} \\
1.3 \mathrm{U} \\
0.64 \mathrm{U}\end{array}$ & $\begin{array}{l}1.6 \\
1.6 U \\
3.1 U \\
1.6 U\end{array}$ & $\begin{array}{l}1.3 U \\
1.3 U \\
2.5 U \\
1.3 U\end{array}$ \\
\hline
\end{tabular}

Supplemental samples collected at a height of $10 \mathrm{ft}$ rather than the specified monitoring height of $5.5 \mathrm{ft}$ above ground level

$\begin{array}{llllllllll}\text { AA1 } & \text { MUAA1-G-18170 } & 8 / 23 / 06 & 0.98 U & 0.93 U & 3.8 & 0.98 U & 36 & 0.64 U & 1.6 U \\ \text { AA2 } & \text { MUAA2-G-18171 } & 8 / 23 / 06 & 0.98 U & 0.93 U & 4.1 & 0.98 U & 38 & 0.64 U & 1.6 U\end{array}$


Ambient air monitoring in May 2006

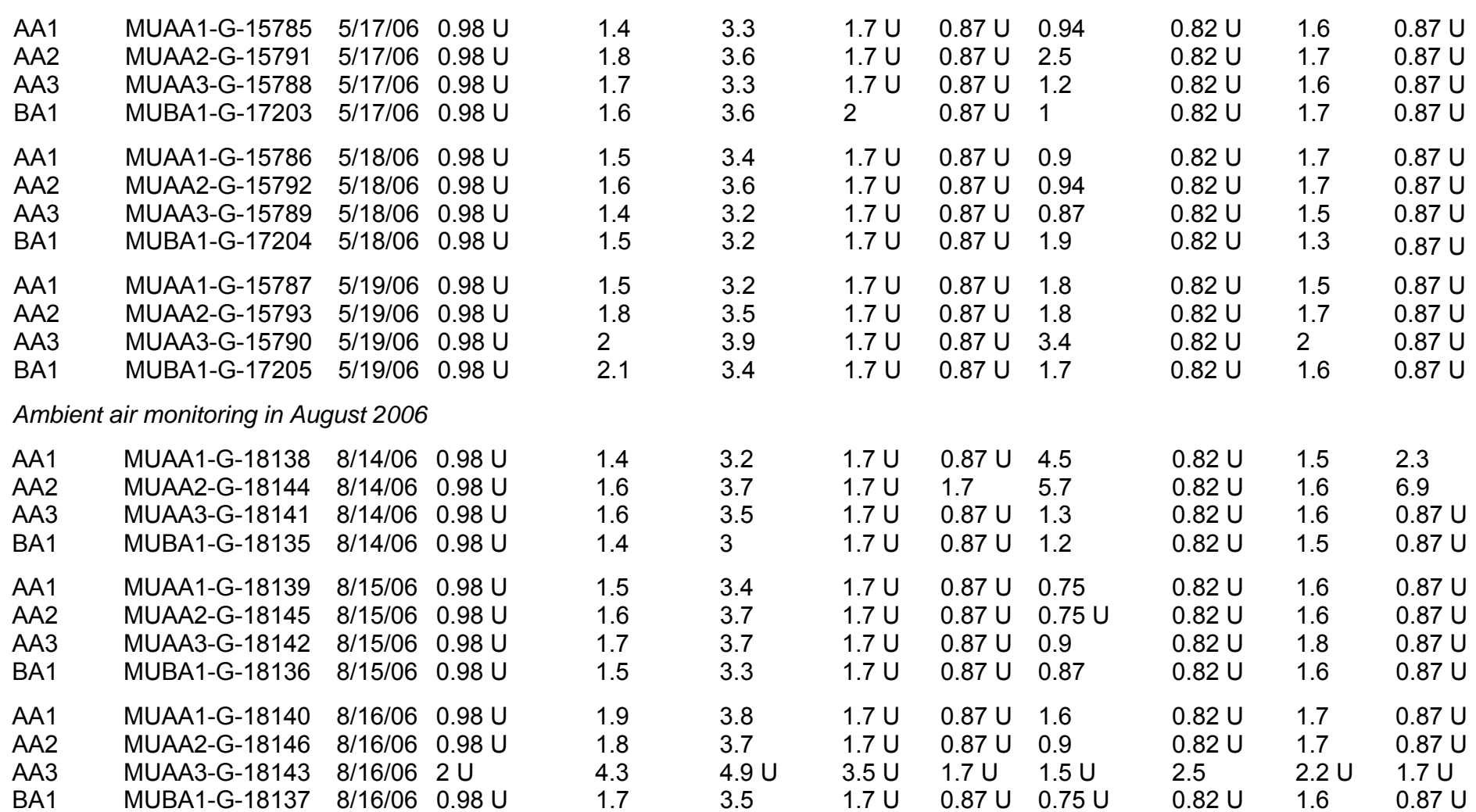

Supplemental samples collected at a height of $10 \mathrm{ft}$ rather than the specified monitoring height of $5.5 \mathrm{ft}$ above ground level

$\begin{array}{llllllllllll}\text { AA1 } & \text { MUAA1-G-18170 } & 8 / 23 / 06 & 0.98 \mathrm{U} & 1.8 & 3.8 & 1.7 \mathrm{U} & 0.87 \mathrm{U} & 0.87 & 0.82 \mathrm{U} & 1.6 & 0.87 \mathrm{U} \\ \mathrm{AA2} & \text { MUAA2-G-18171 } & 8 / 23 / 06 & 0.98 \mathrm{U} & 1.9 & 3.9 & 1.7 \mathrm{U} & 0.87 \mathrm{U} & 0.79 & 0.82 \mathrm{U} & 1.7 & 0.87 \mathrm{U}\end{array}$


a Locations (see Figure 3.2):

AA1 Headwaters area, west side of creek along path in phytoremediation area.

AA2 Phytoremediation area, east side of creek, west of monitoring well PMW6.

AA3 West side of wetlands area.

BA1 Background location in drainageway on south side of Waverly Road.

b $U$, not detected at the indicated reporting limit. 


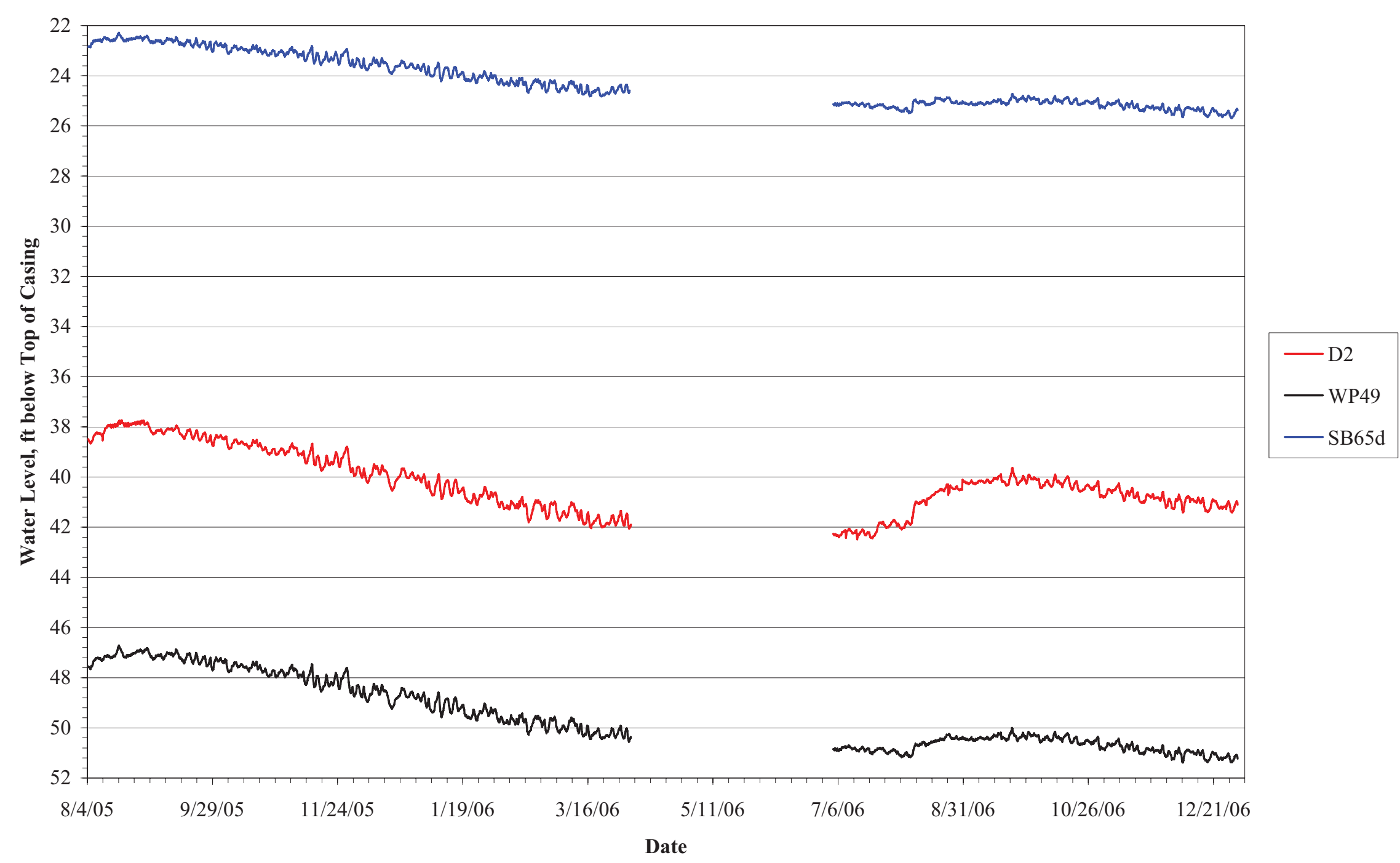

FIGURE 4.1 Hydrographs for upgradient wells, August 2005 to December 2006. 


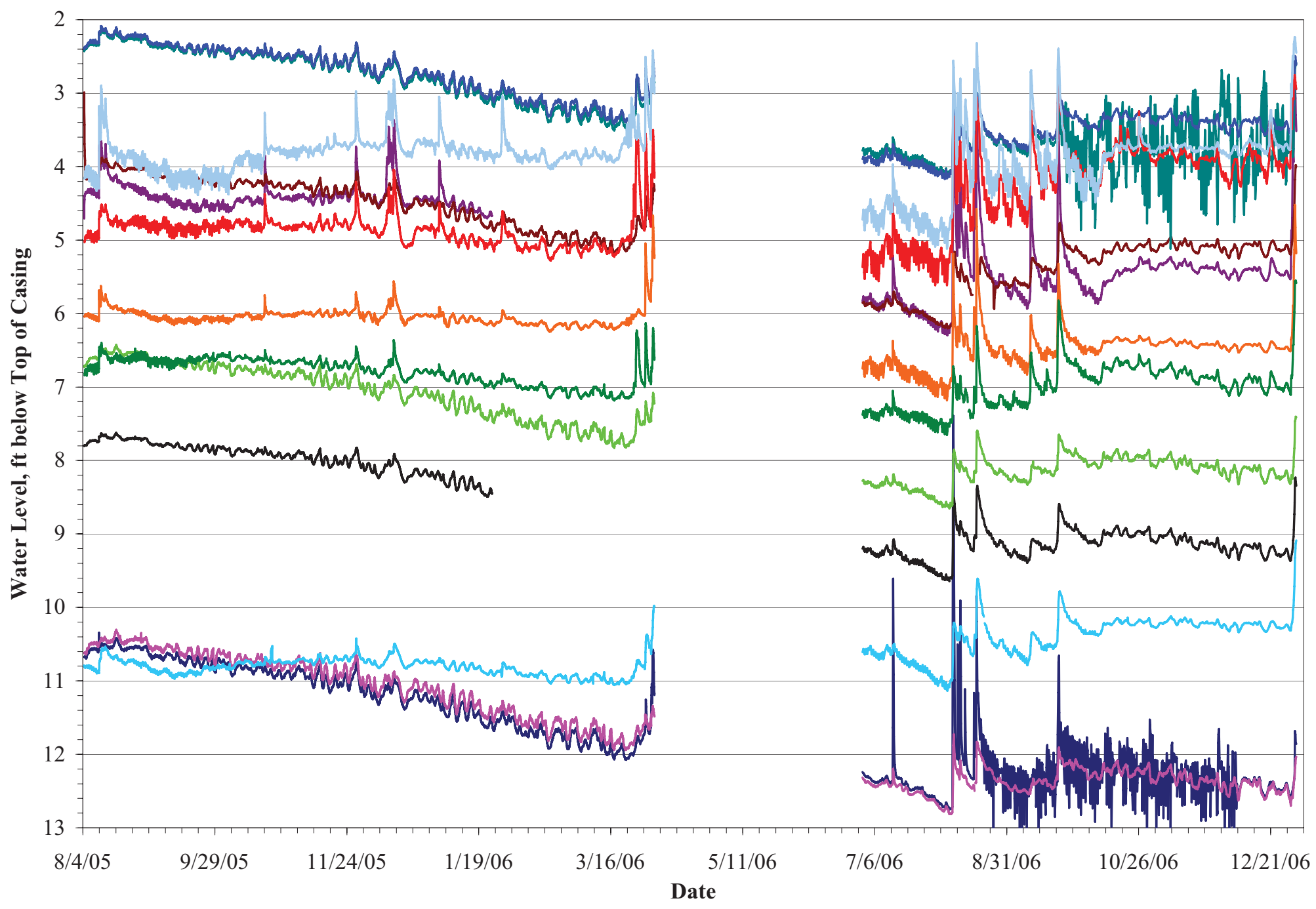

FIGURE 4.2 Hydrographs for phytoremediation area wells, August 2005 to December 2006. 


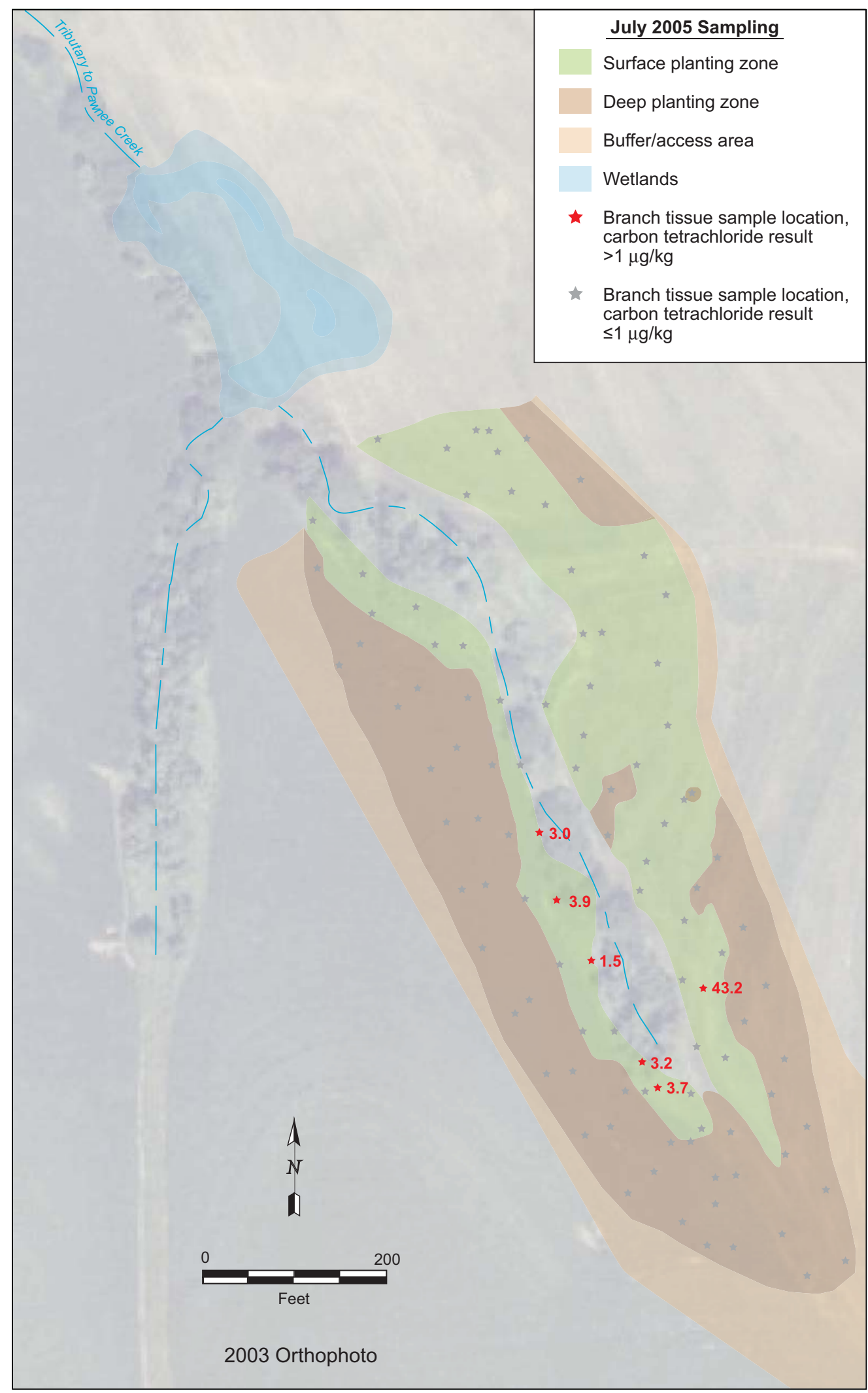

FIGURE 4.3 Analytical results for carbon tetrachloride in branch tissue samples, July 2005. Source of photograph: NAIP (2003). 


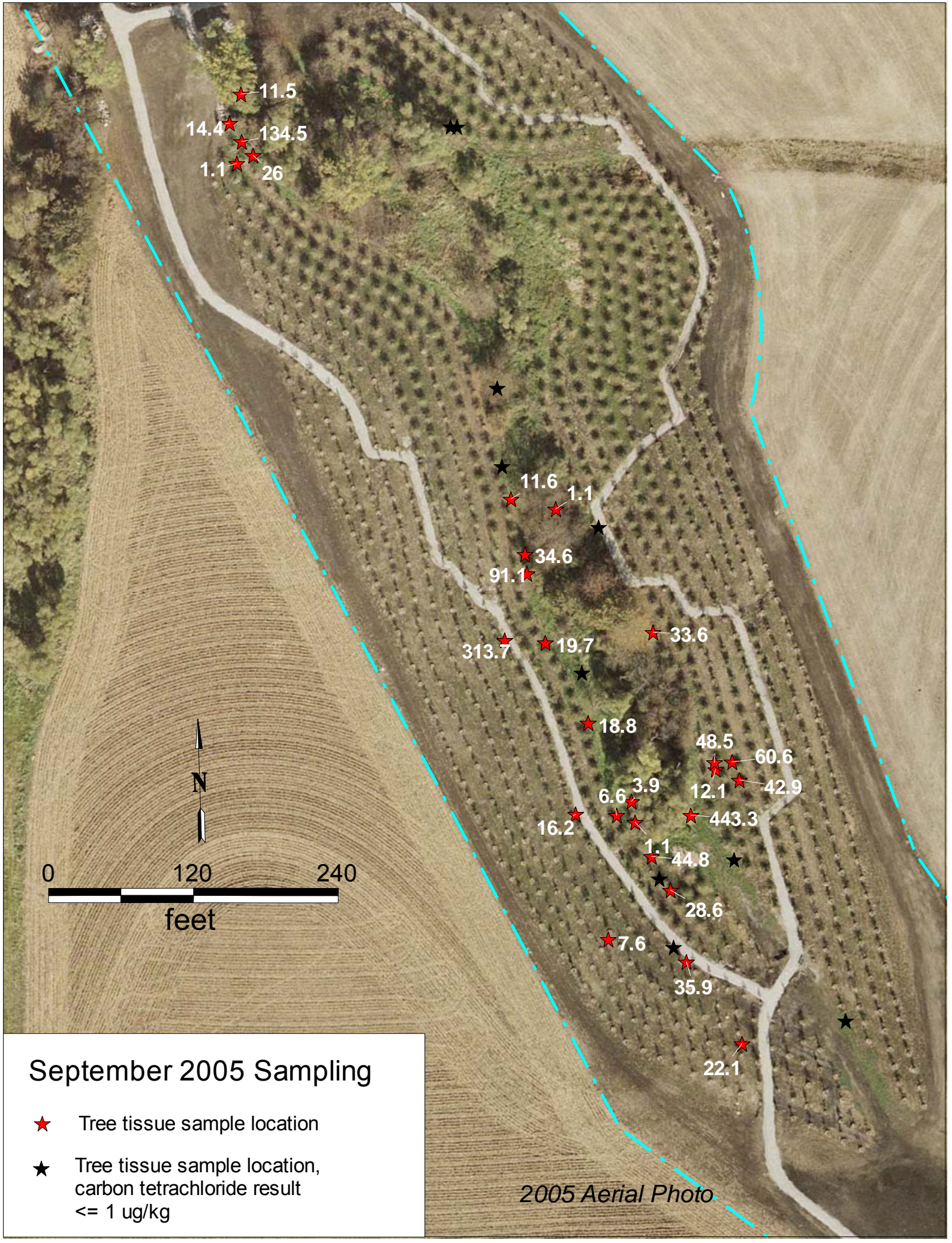

FIGURE 4.4 Analytical results for carbon tetrachloride in branch tissue samples, September 2005. Source of photograph: Olsson (2005). 


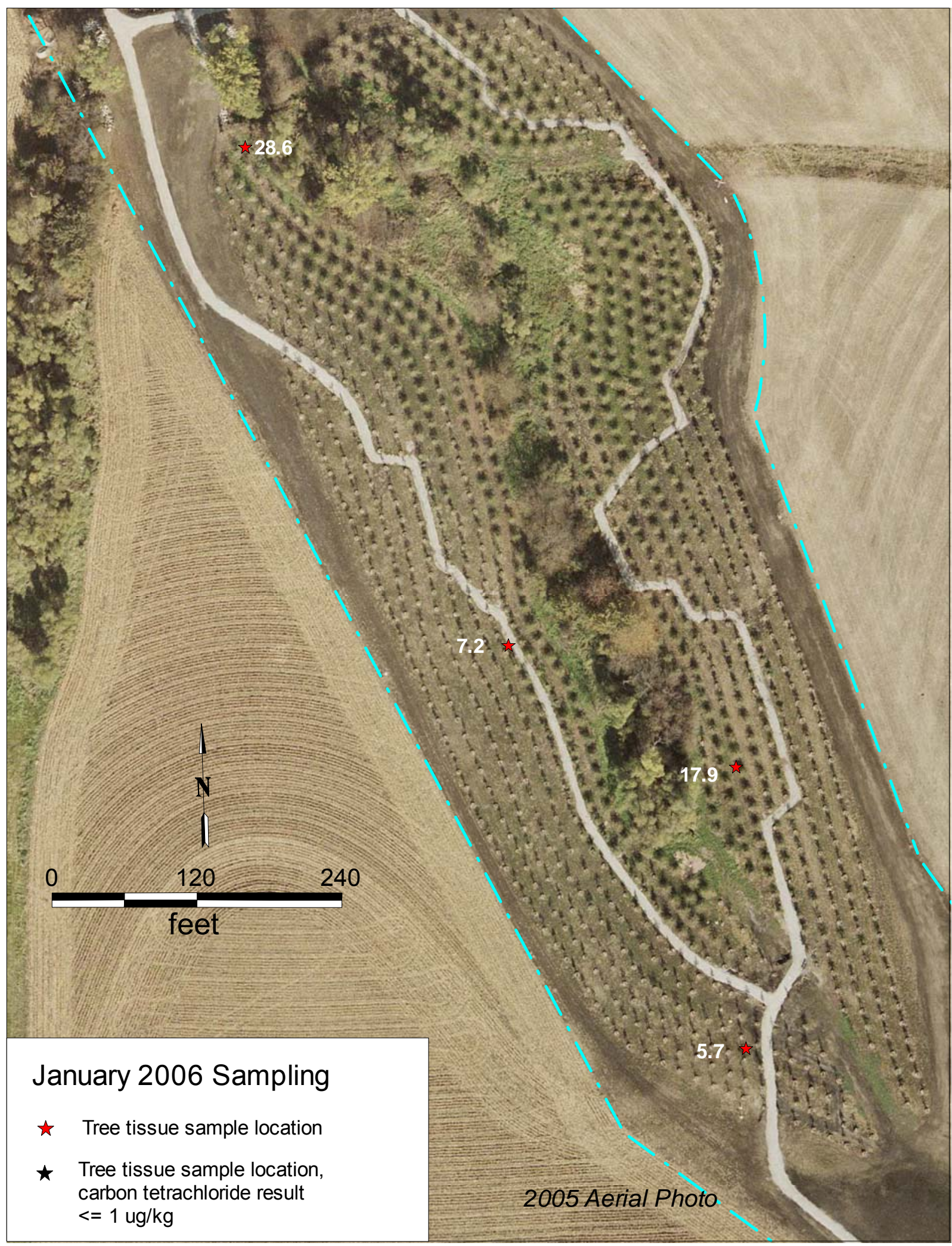

FIGURE 4.5 Analytical results for carbon tetrachloride in branch tissue samples, January 2006. Source of photograph: Olsson (2005). 


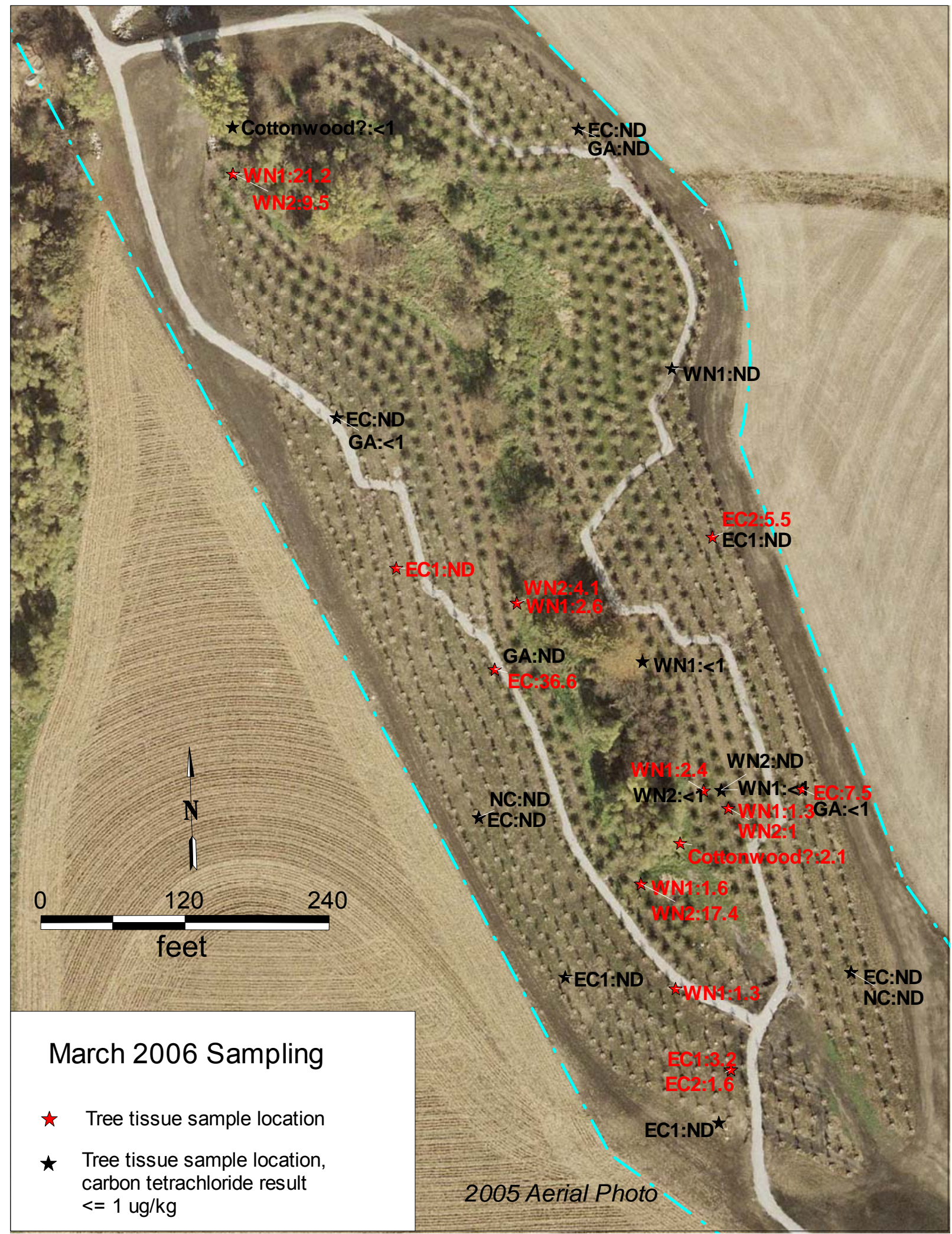

FIGURE 4.6 Analytical results for carbon tetrachloride in branch tissue samples, March 2006. Source of photograph: Olsson (2005). 


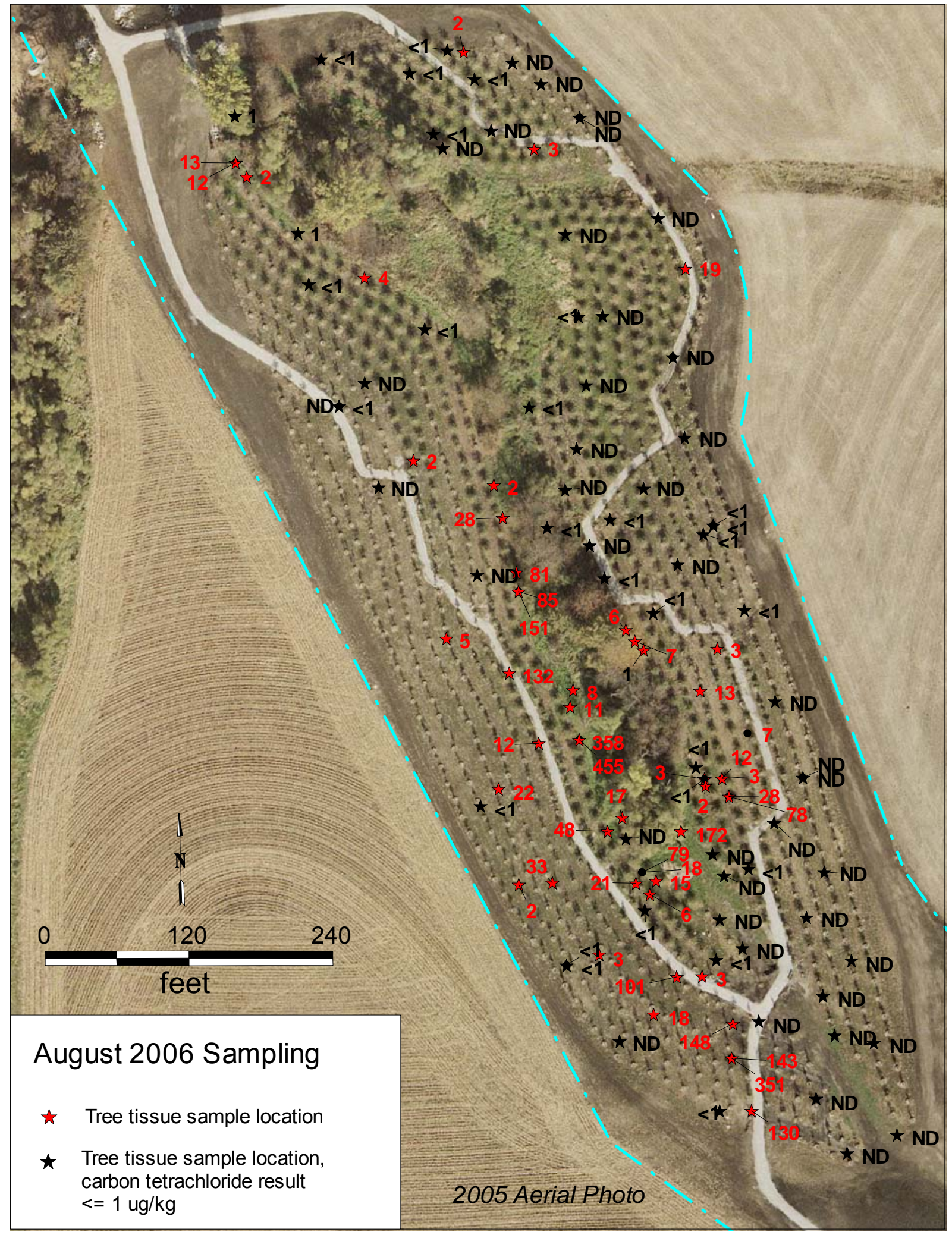

FIGURE 4.7 Analytical results for carbon tetrachloride in branch tissue samples, August 2006. Source of photograph: Olsson (2005). 

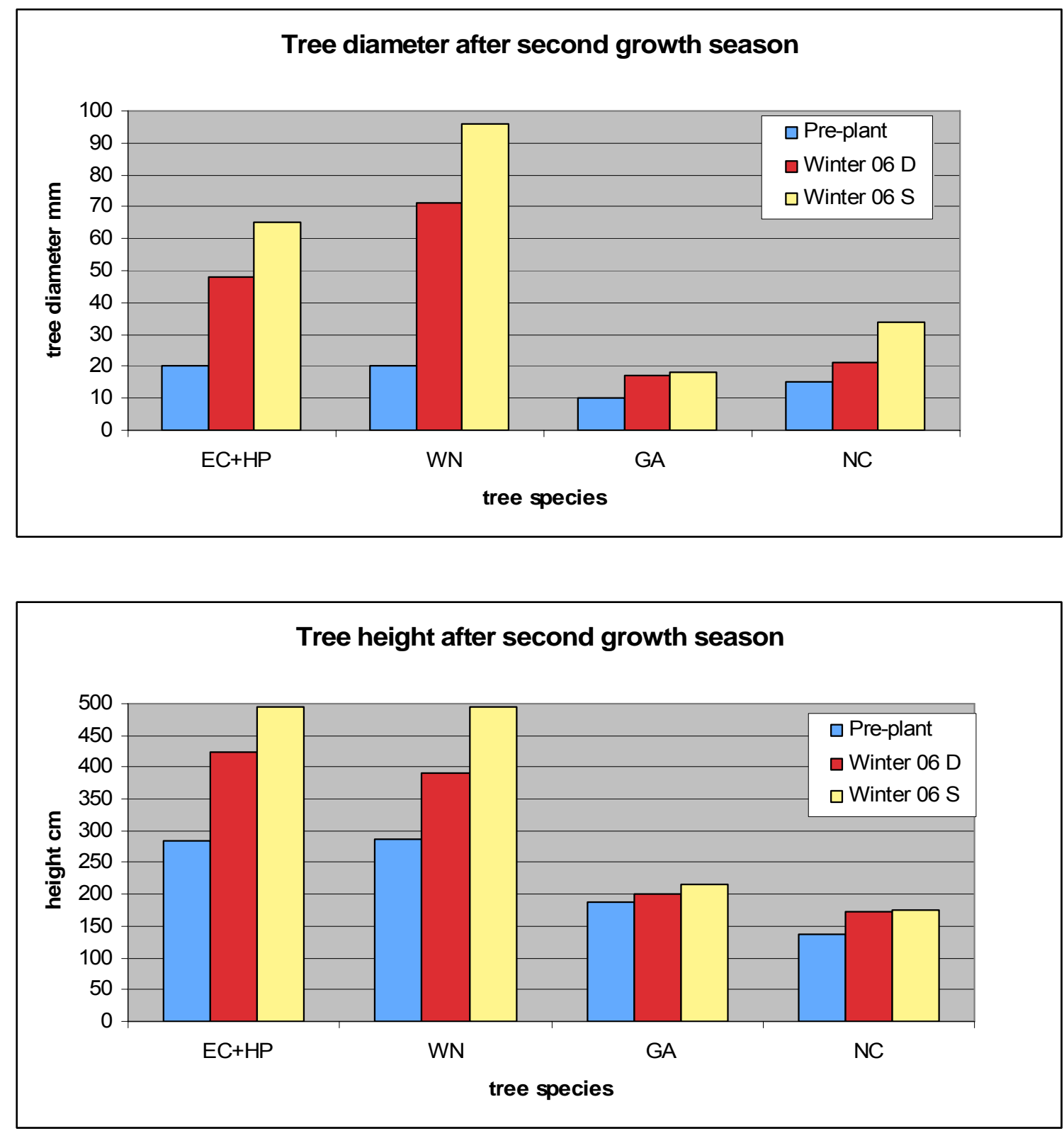

FIGURE 4.8 Tree growth for eastern cottonwood and hybrid poplar (EC+HP), Niobe willow $(\mathrm{WN})$, green ash (GN), and northern catalpa (NC) trees planted in the shallow (S) or deep (D) phytoremediation zone, based on measurements of diameter (top) and height (bottom) before planting and in winter 2006. Black willow was planted only in the shallow zone and is not shown. 

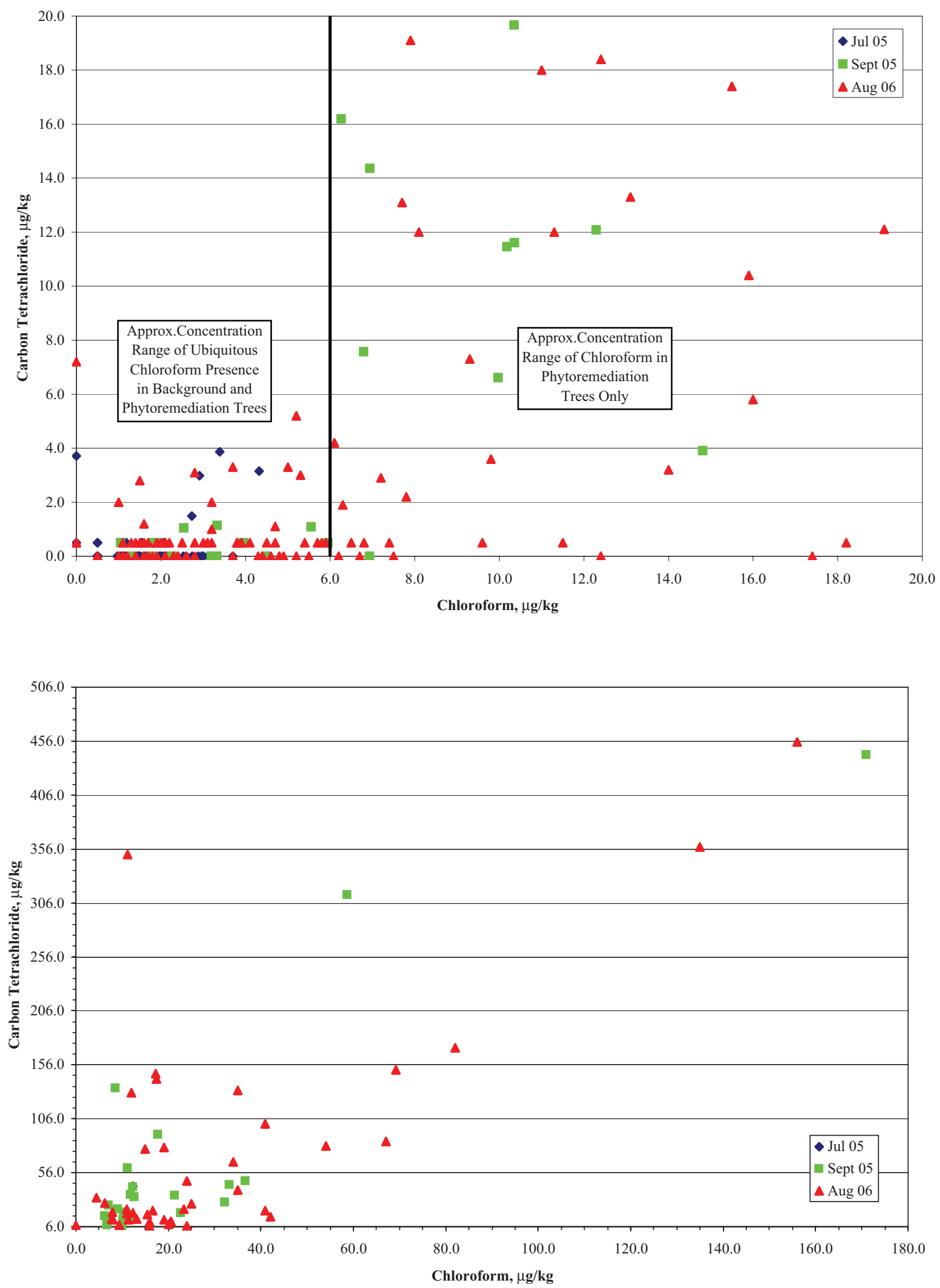

FIGURE 4.9 Correlation of carbon tetrachloride and chloroform concentrations in branch tissue samples in July 2005, September 2005, and August 2006, in two concentration ranges (lower values at top and higher values at bottom). 


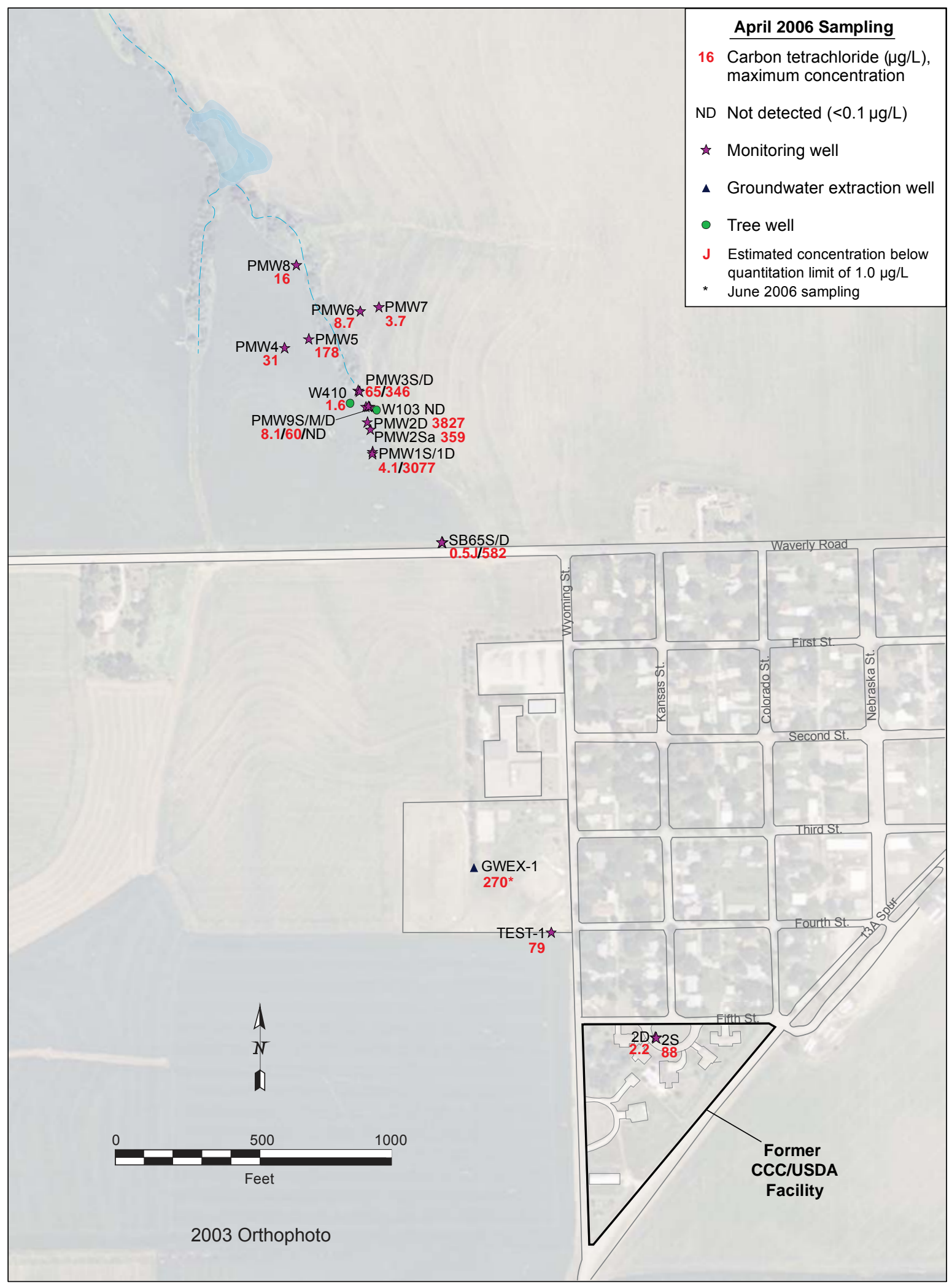

FIGURE 4.10 Maximum carbon tetrachloride concentrations in groundwater samples collected from permanent monitoring points in April 2006. 


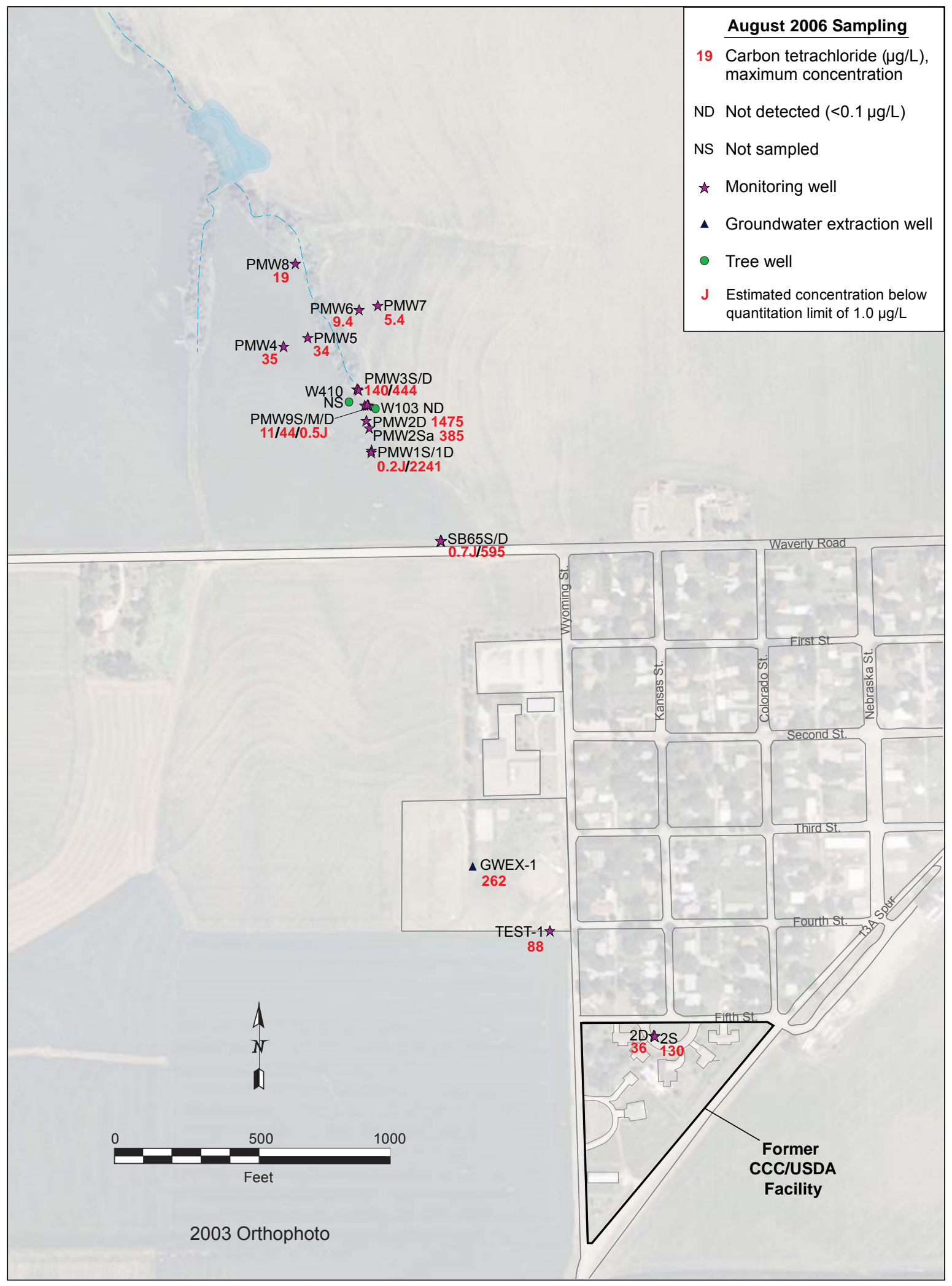

FIGURE 4.11 Maximum carbon tetrachloride concentrations in groundwater samples collected from permanent monitoring points in August 2006. 

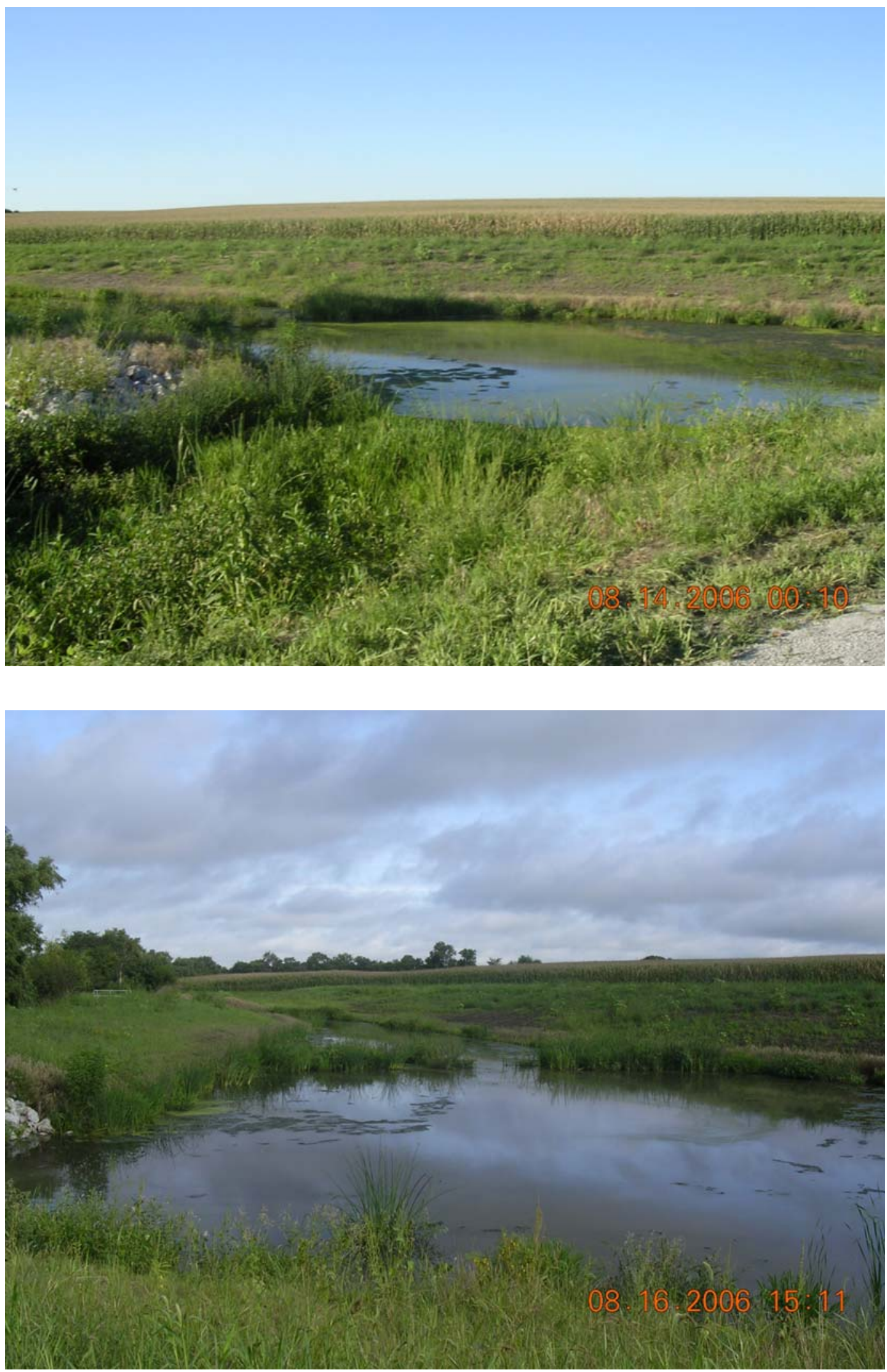

FIGURE 4.12 Established plants in the wetlands in August 2006. 


\section{Operation, Maintenance, and System Modifications}

\subsection{Well GWEX-1 and the Spray Irrigation Treatment Unit}

No service or maintenance was required for the GWEX-1 extraction well or the spray irrigation treatment unit during the review period. A slight decrease in the flow rate achieved by GWEX-1 was observed at the end of the 2006 irrigation season. Further monitoring will be performed when the well is reactivated in 2007, to determine if any corrective action is necessary.

\subsection{Phytoremediation Planting and Buffer Areas}

Tree health observations were conducted at various times during the review period. Upon discovery of a possibly significant tree disease, a survey of all trees was conducted between April 26 and May 12, 2006, and several experts were called to inspect the diseased trees either in person or by observing photographs or infected specimens. The infected trees were inspected by Argonne personnel, Kansas State University faculty, and a forester from the Nebraska Forest Service. In addition, infected plant material was submitted to the Morton Arboretum Plant Clinic in Lisle, Illinois, and photographs were forwarded to the University of Illinois at UrbanaChampaign Extension Service for diagnostic purposes.

The conclusion was that a number of the Niobe willows had been infected with a cankerinducing opportunistic fungus (Cytospora spp.; Figure 5.1). Such a fungus targets trees of the Salix and Populus genera, typically when insects, hail, or mechanical trauma create entryways. Evidence of hail damage was found on the trunk and limb bark of many of the willows at Murdock, especially in the more unprotected areas that are most exposed to the elements.

Although the canker infestation was relatively extensive in early spring, the disease was easily contained by the rapidly growing trees during the summer, and no corrective measures were required. Inspections are planned for winter and early spring of 2007. No action was recommended by the experts other than removing the severely diseased limbs, if appropriate, during the driest parts of the year. A photograph of the canker, which mostly affected willow trees, is in Figure 5.1. 


\subsection{Constructed Wetlands Area}

Site visits were conducted after wetlands construction was complete to monitor the site for potentially needed maintenance actions such as erosion control, as well as to evaluate vegetation establishment and growth. At the recommendation of Olsson Associates, the engineering firm that designed and oversaw construction of the wetlands, several modifications were implemented to control the excessive surface erosion observed along the southern margins of the wetlands basin area. The erosion had resulted from winter and early spring precipitation and runoff. The modifications included the following:

- Removal of excess sediment deposited in the southern wetlands subbasin.

- Regrading of the southern basin margin to eliminate channeling that had developed.

- Installation of erosion-control matting and planting of additional protective groundcover species, to reduce or eliminate future runoff effects.

The above modifications were completed during the spring and early summer of 2006.

\subsection{Monitoring Well Network}

No repairs or modifications of the monitoring well network were required during the review period. Routine data recovery and battery replacement for the 16 water level recorders installed at the site were performed quarterly. Data obtained from the recorders in wells PMW1S and PMW3S suggested an unexpected increase in the response of the transducers or signal processors in these units during the later part of the review period. The recorder in well PMW1S was reset and reprogrammed after the data recovery event in November 2006 and has been functioning normally since that time. Further investigation has indicated that the fault at PMW3S cannot be corrected in the field. Both recorders were returned to the manufacturer for inspection and repair. 


\subsection{Operating and Maintenance Costs for the Current Review Period}

The operating and maintenance (O\&M) costs for the 19-month period from June 2005 to December 2006 are summarized in Table 5.1. The costs for this initial period of operation include one-time expenses associated with the following:

- Seeding of permanent groundcover species, removal of plant guards, and installation of permanent identification markers in the phytoremediation planting area (Section 3.2).

- Investigation of the canker outbreak identified in the phytoremediation planting area (Sections 3.2 and 5.2).

- Installation of the on-site visitor amenities (Section 3.7).

- Implementation of erosion control measures in the constructed wetlands area (Sections 3.3 and 5.3).

Costs in subsequent years are expected to be lower.

TABLE 5.1 O\&M costs for the Murdock site restoration project, June 2005 through

December 2007.

\begin{tabular}{ll}
\hline Item & Cost (\$) \\
\hline Routine O\&M & \\
General Management & \\
Logistics Support & 33,094 \\
Remediation Monitoring and & 44,581 \\
$\quad$ Reporting & 357,430 \\
Technical Oversight & \\
Total Routine O\&M & 29,721 \\
$\quad$ & 464,827 \\
One-Time Expenses & \\
Visitor Amenities & 66,107 \\
Erosion Control Measures & 87,222 \\
Seeding, Plant Guards, Markers & 90,493 \\
Canker Investigation & 13,895 \\
$\quad$ Total One-Time Expenses & 257,716 \\
$\quad$ Grand Total & 722,544 \\
\hline
\end{tabular}




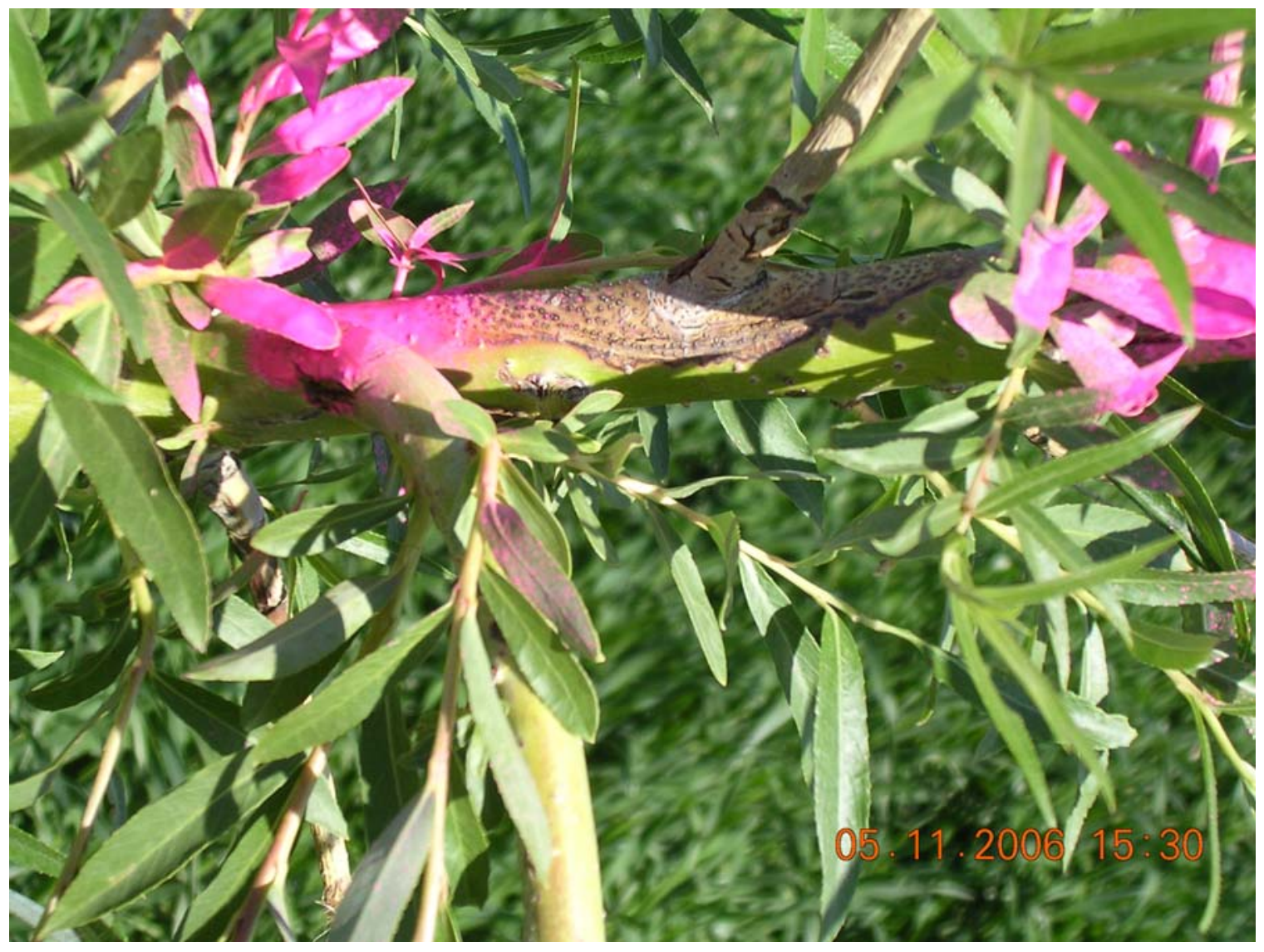

FIGURE 5.1 Canker lesion on a willow branch (between the pink paint marks) in May 2006, showing Cytospora spp. pichnydia carrying maturing fungal spores. 


\section{Summary}

\subsection{Performance of the Removal Action Systems}

The results of the baseline sampling in July 2005 indicated that the intended outcome of the integrated remedial approach designed for the Murdock site is achievable. Subsequent monitoring conducted during the first full year of operation (2005-2006) of the remediation systems demonstrated the following:

- Seasonal operation of GWEX-1 is capturing elevated concentrations of carbon tetrachloride in the upgradient portion of the groundwater plume and reducing the contaminant load approaching the tributary creek headwaters.

- The spray irrigation treatment unit is decreasing contaminant concentrations in the spray discharge to acceptable levels and is improving the quality of the Elmwood-Murdock public school athletic fields.

- Highly contaminated water in the more downgradient portion of the groundwater plume continues to approach the phytoremediation and wetlands treatment areas.

- Carbon tetrachloride uptake and removal from the contaminated groundwater were first observed in the phytoremediation vegetation shortly after the plantings were installed; the influence of this treatment zone has increased since that time.

- Carbon tetrachloride levels in surface waters show a rapid drop along the flow pathway established through the phytoremediation and wetlands treatment areas; no unacceptable levels of this contaminant have been detected in the final surface discharge from the wetlands.

- With one trace-level exception, carbon tetrachloride has not been detected in ambient air in the phytoremediation and wetlands treatment zones, at the 
"breathing" or canopy levels among the newly planted and preexisting native trees.

- The total costs for operation and maintenance of the removal action systems at Murdock during the review period were \$722,544. This amount includes several unexpected one-time costs and exceeds anticipated ongoing expenses.

\subsection{Regulatory Compliance of the Removal Action Systems}

Under the EPA-approved Monitoring Plan (Argonne 2006), site-specific monitoring requirements and compliance criteria governing the removal action at Murdock have been established by the EPA and the NDEQ. These requirements are as follows:

- For GWEX-1 and the spray irrigation treatment system:

- Sample the discharge quarterly during periods of seasonal operation.

- Demonstrate an acceptable carbon tetrachloride level $(44.2 \mu \mathrm{g} / \mathrm{L})$ in the discharge.

- Demonstrate an acceptable pH range (6.0-9.0) in the discharge.

- Report the actual volume of groundwater treated and discharged.

- Report results quarterly.

- For surface water discharged from the combined phytoremediation and wetlands treatment zones:

- Sample surface flow quarterly at location SWM1-SWM3.

- Demonstrate an acceptable carbon tetrachloride level $(44.2 \mu \mathrm{g} / \mathrm{L})$ at location SWM3. 
- Report results quarterly.

- For ambient air in the phytoremediation and wetlands treatment zones:

- Sample twice yearly at locations AA1-AA3.

- Demonstrate an acceptable carbon tetrachloride level $\left(192 \mu \mathrm{g} / \mathrm{m}^{3}\right)$ in the ambient air.

- Report results annually.

The results of all compliance monitoring performed during the current review period are summarized as follows:

- Carbon tetrachloride concentrations in samples of treated spray discharge ranged from $<1 \mu \mathrm{g} / \mathrm{L}$ to $5.9 \mu \mathrm{g} / \mathrm{L}$ (average $2.4 \mu \mathrm{g} / \mathrm{L}$ ) during the 2005 irrigation season and from $<1 \mu \mathrm{g} / \mathrm{L}$ to $5.2 \mu \mathrm{g} / \mathrm{L}$ (average $2.8 \mu \mathrm{g} / \mathrm{L}$ ) during the 2006 season, below the acceptable limit of $44.2 \mu \mathrm{g} / \mathrm{L}$ established by the EPA and the NDEQ.

- The $\mathrm{pH}$ levels detected in samples of treated spray discharge ranged from 6.54 to 7.88 , in compliance with the required range $(\mathrm{pH}=6.0-9.0)$ specified by the NDEQ.

- Except for a trace concentration $(<1 \mu \mathrm{g} / \mathrm{L})$ in the November 2006 sampling, no carbon tetrachloride contamination was detected (at an instrument detection limit of $0.1 \mu \mathrm{g} / \mathrm{L}$ ) in any of the surface water samples collected at the surface water compliance point, location SWM3. The acceptable limit is $44.2 \mu \mathrm{g} / \mathrm{L}$.

- Except for a concentration of $1.4 \mu \mathrm{g} / \mathrm{m}^{3}$ detected in one air sample collected on May 18, 2006, carbon tetrachloride has not been identified (at a reporting limit of $1.3 \mu \mathrm{g} / \mathrm{m}^{3}$ ) in the ambient air at Murdock. The target maximum concentration is $192 \mu \mathrm{g} / \mathrm{m}^{3}$. 
All monitoring and reporting requirements and compliance criteria were satisfied during the review period. 


\section{References}

Argonne, 2004, Results of Aquifer Testing at Murdock, Nebraska, in October 2005, ANL/ER/AGEM/CHRON-767, letter from R. Sedivy (Environmental Research Division, Argonne National Laboratory, Argonne, Illinois) to R. Asch (Wastewater Section, Nebraska Department of Environmental Quality, Lincoln, Nebraska), November 4.

Argonne, 2006, Final Monitoring Plan for Site Restoration at Murdock, Nebraska, ANL/eVS/AGEM/TR-05-04, prepared for the Commodity Credit Corporation, U.S. Department of Agriculture, by Argonne National Laboratory, Argonne, Illinois, February.

EPA, 2005, memorandum from M. Beringer (Data Interpretation and Support Operations Branch, Environmental Services Division, U.S. Environmental Protection Agency Region VII, Kansas City, Kansas) to J. Field (Drinking Water Management Branch; Water, Wetlands, and Pesticides Division, U.S. Environmental Protection Agency Region VII, Kansas City, Kansas), December 21.

NAIP, 2003, aerial photograph of Murdock, Nebraska, National Agricultural Imagery Program, U.S. Department of Agriculture, http://www.apfo.usda.gov/NAIP.html.

Olsson, 2005, aerial photograph of Murdock, Nebraska, phytoremediation project, flown by Western Air for Olsson Associates, Lincoln, Nebraska, October (orthorectified by Olsson Associates, November 23, 2005). 


\section{Appendix A:}

\section{GWEX-1 Registration Form and Construction Diagram}


State of Nebraska

\section{NOTICE OF WATER WELL REGISTRATION UPDATE}

Registration Number

Sequence Number

Daie

Ferson frocessing Update
$\underline{G-132761}$

$\underline{166436}$

March 15,2005

Christine Southwick

Information regarding the water well referenced above has веeл changed in the Department's water well registration records. Please note the following changes and the -ea-r changes were made:

Section/Township/Range/County Location (Item 3A): Accotding to the aerial photorraph and the location given in item $3 A$ on the registration form, the woll is located in Cass County.

The following information was provided by Griffin DeWatering N.C.. LLC:

Pumping water level (Item 9c): 62'.

This change has modified item(s) $3 a$ and $9 c$ of DNR Form \#145. If these change: are inaccurate, please contact the Department of Natural Resources at P.O. Box 941276 , lincoln, NE, 68509-4676. Phone (402)471-2363.

I certify that this update has been forwarded to the owner of the referenced water well and is now a part of the registration records.

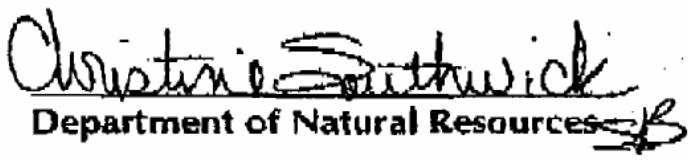




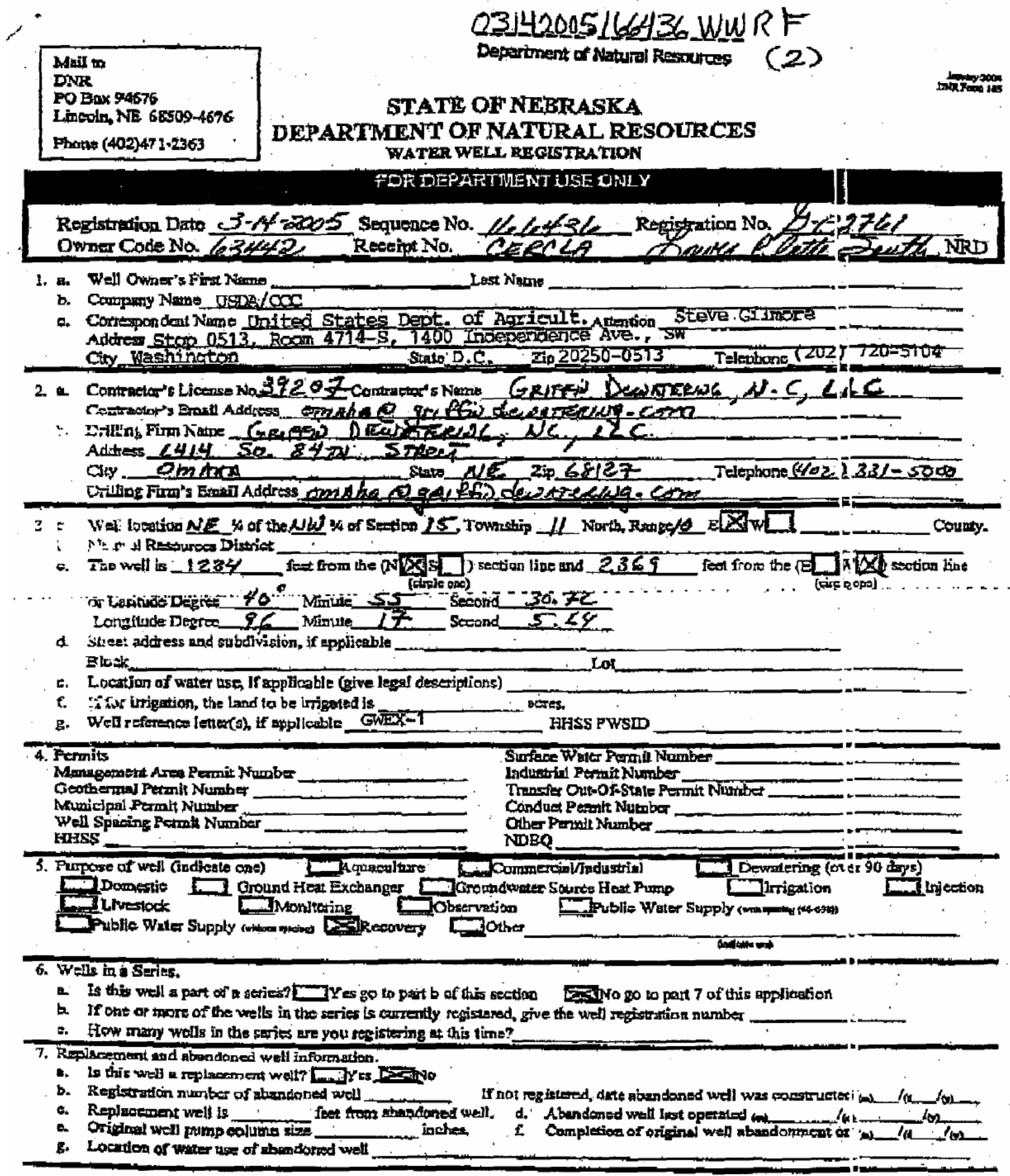


3. Print informedion.

2 Is poup installed at this tim Soryes

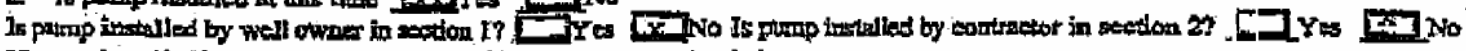
Ifpump installed by pump installex, pleses fill ont fibenss number below

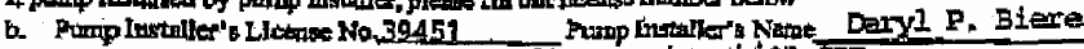

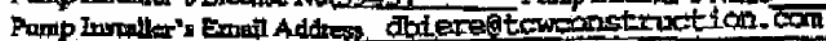

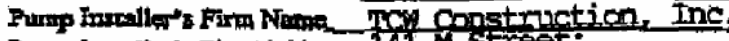

Pump imaliers Firm Addrese 141 M street;

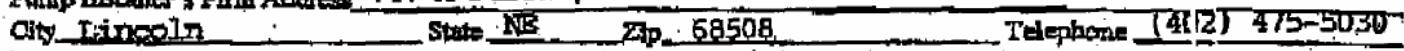

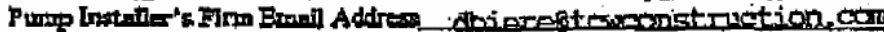

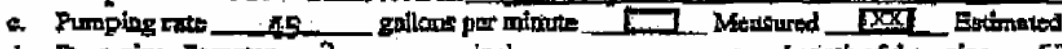

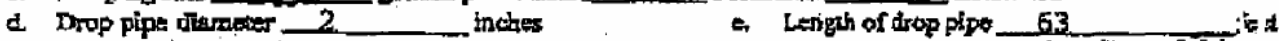

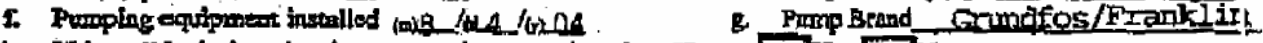

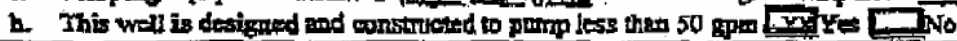

9. Well Corstruetion In thatratian.
2. Tetal well depth
B2

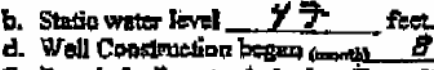

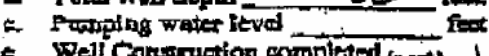

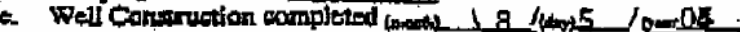

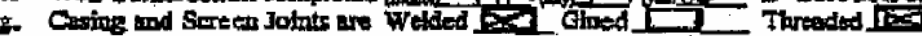 Other

0.3276

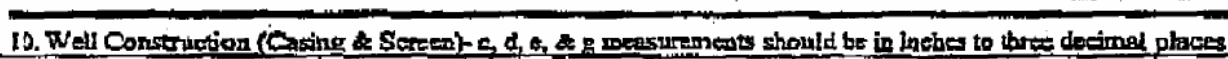

\begin{tabular}{|c|c|c|c|c|c|c|c|c|}
\hline 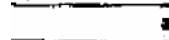 & & b & 0 & d & $c$ & 5 & 8 & $\overline{4}$ \\
\hline Prista & thent & $\begin{array}{l}\text { Casing or } \\
\text { sareos }\end{array}$ & $\begin{array}{l}\text { Insido } \\
\text { Dinmater }\end{array}$ & $\begin{array}{l}\text { Dartside } \\
\text { Diameter. }\end{array}$ & $\begin{array}{c}\text { Wall } \\
\text { Tripknesp }\end{array}$ & $\begin{array}{c}\text { Sertot } \\
\text { Sitot }\end{array}$ & $\begin{array}{l}\text { Typs of } \\
\text { Matminl }\end{array}$ & Trade Nime \\
\hline Fram & To & & & & & & & \\
\hline 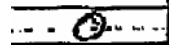 & $-4-7$ & Essith & $-6.66^{\circ}$ & 6.625 & - 280 & & Evere & \\
\hline 47 & $\Rightarrow 7$ & screas & 605 & 6,425 & $B \in O$ & $0.030^{N}$ & 5.57 eed & $\sqrt{3} / 450 \mathrm{~d}$ \\
\hline 77 & $22^{-}$ & CAsrac & $\cos x$ & 6.625 & 0.210 & & Eve & \\
\hline
\end{tabular}

11. Girnu and Ginvel Pack

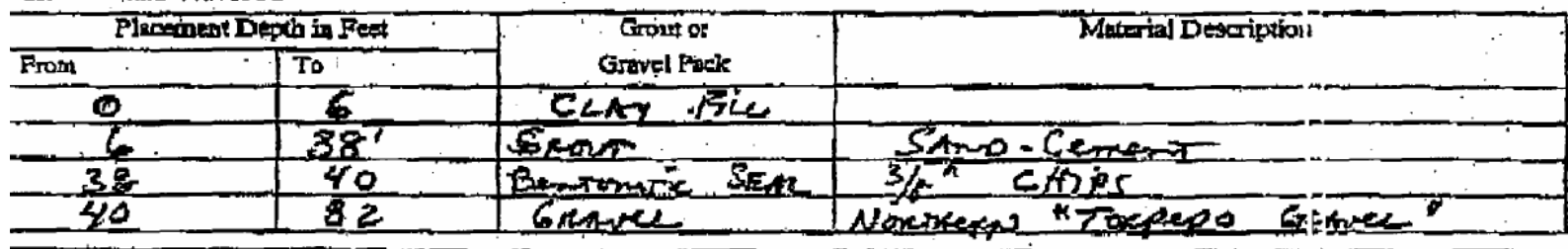

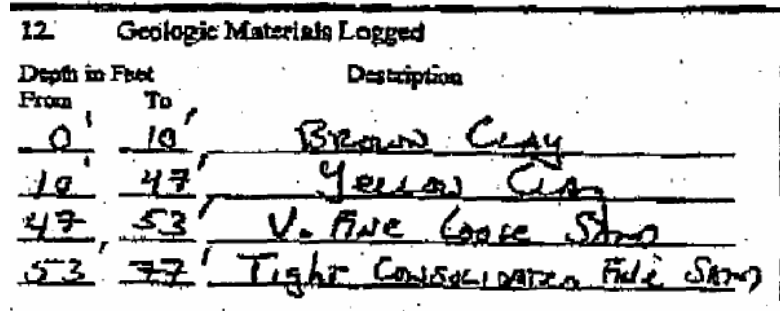

(Additional shects may bo natmitited)

$$
\begin{aligned}
& \text { Depth in Feet } \\
& \text { From to }
\end{aligned}
$$

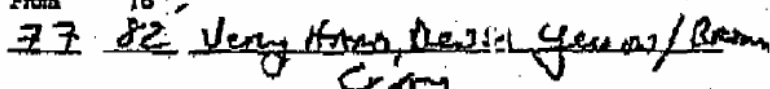$$
-0 \text { - Con }
$$$$
\text { क }
$$

bo matonitited)

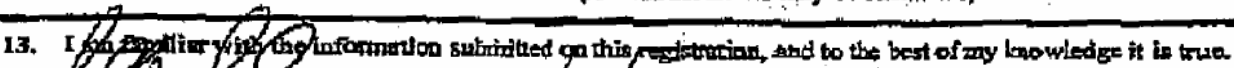

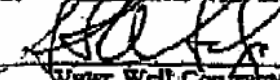

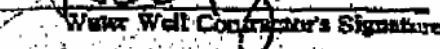

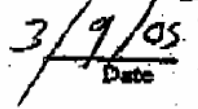




\section{GWEX: Murdock, NE}

NE 1/4 of NW 1/4 of Section 15, Twp. 11 North, Range 10 East, Cass County, State of Nebraska

Drilled: August 2, 2004

Location: N40.92518, W96.28483

Elevation: 1301' AMSL

Estimated Pumping Rate: 35 GPM

Tested Pumping Rate: 46 GPM

\section{PITLESS ADAPTOR}

6' pitless adaptor; 1' above ground level, 5' below ground level.

\section{IMPERVIOUS GROUT}

The well is grouted with cement grout as required, mixed with clean fresh water.

\section{WELL CASING}

Well casing is Schedule 40 steel casing, from $5^{\prime}$ BGL to $47^{\prime}$.

Screen is 6" x $30^{\prime}$ stainless steel ( 0.030 slot $)$ from $47^{\prime}$ to $77^{\prime}$.

\section{HOLE SIZE}

The hole is $26^{\prime \prime}$ in diameter to $82^{\prime}$ T.D.

\section{GRAVEL / SAND PACK}

Gravel/sand pack is designed to stabilize the aquifer material and permit the Pne fraction to move into the well during development. Gravel/sand pack extends to at least 2' above screen.

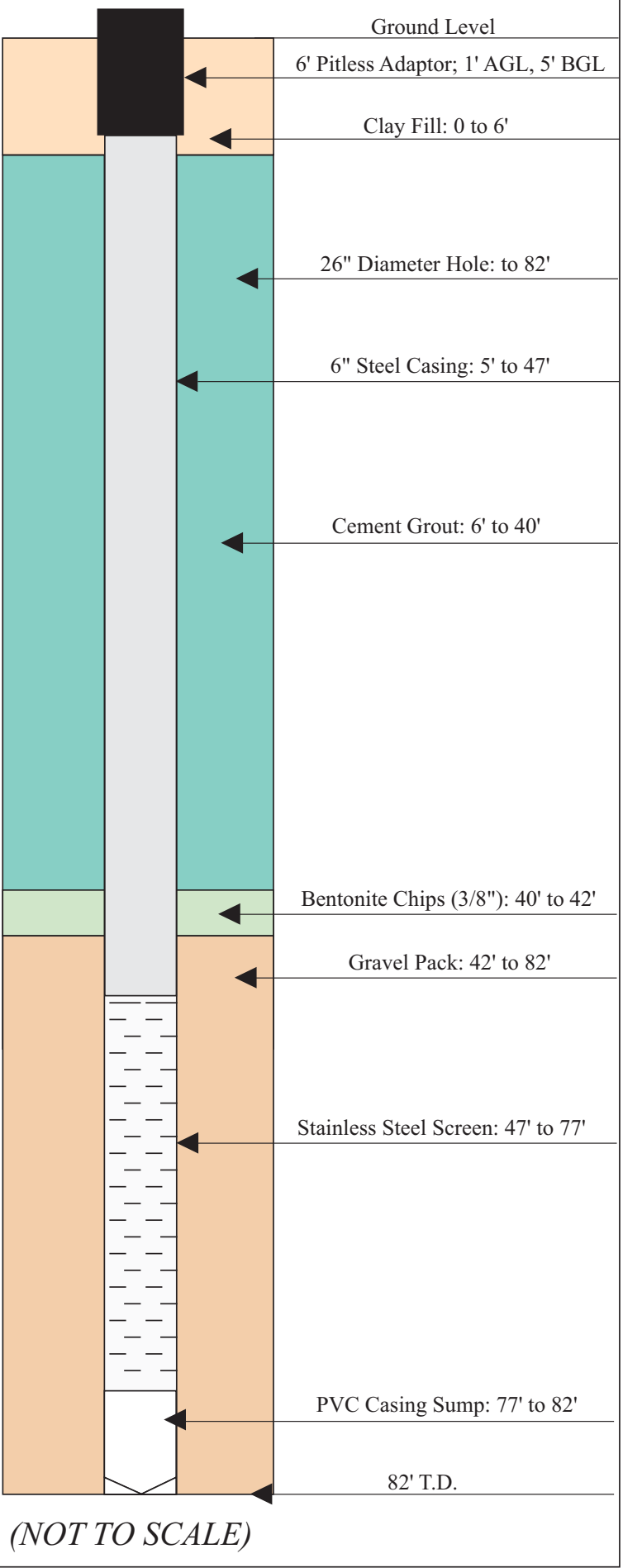




\section{Appendix B:}

\section{Trail Signs}




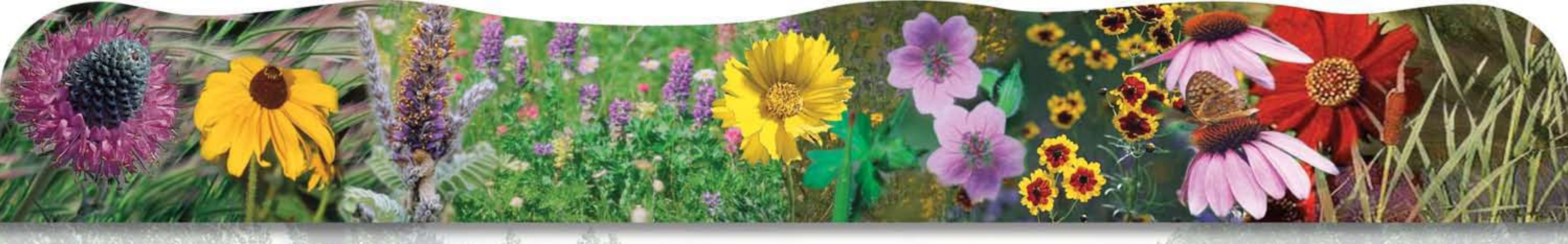

\section{THANKS TO THE USDA's Cooperative Conservation Partners}

The USDA acknowledges its partners in implementing this cooperative conservation project:

- U. S. Environmental Protection Agency

- Argonne National Laboratory, Argonne, Illinois

- TCW Construction, Inc., Lincoln, Nebraska

- Olsson Associates, Lincoln, Nebraska

- Stock Seed Farms, Murdock, Nebraska

- Applied Natural Sciences, Hamilton, Ohio

Special Thanks to the Murdock Community The USDA thanks the citizens of Murdock, the ElmwoodMurdock Public Schools, Stock Seed Farms, and the Bruttig family for their hospitality and support and for the land access needed to make this project a reality.

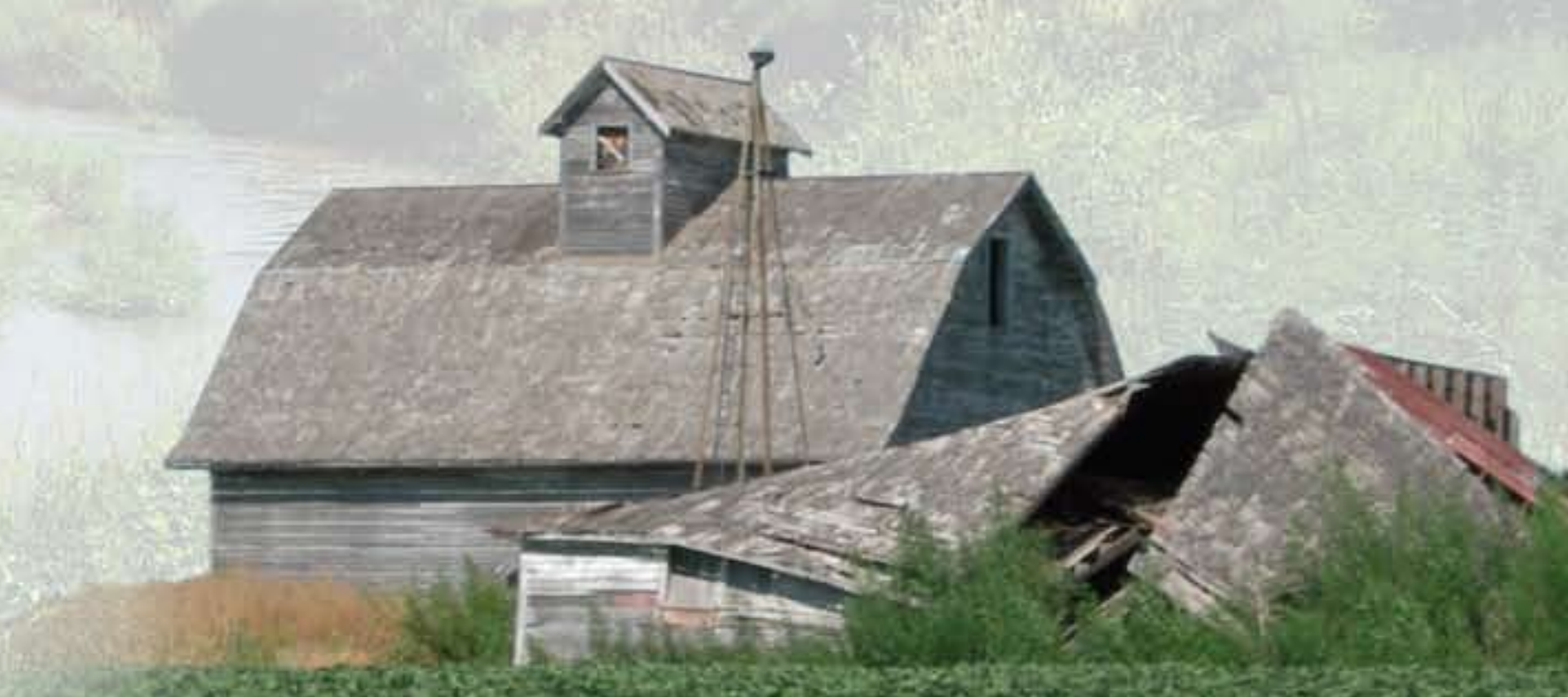

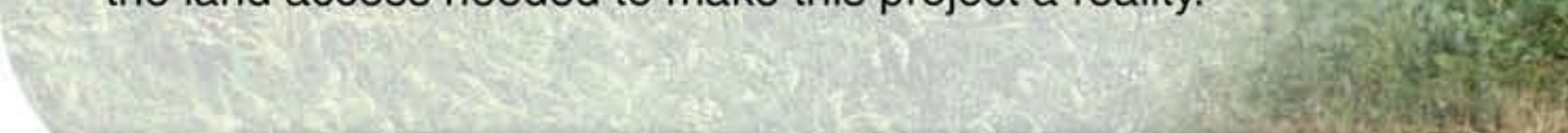




\section{Welcome to the}

\section{MURDOCK \\ GroundWATER \\ Cleanup Project}

A Cooperative Conservation Effort of

U.S. Department of Agriculture

Farm Service Agency

Commodity Credit Corporation

with

U.S. Environmental Protection Agency Argonne National Laboratory

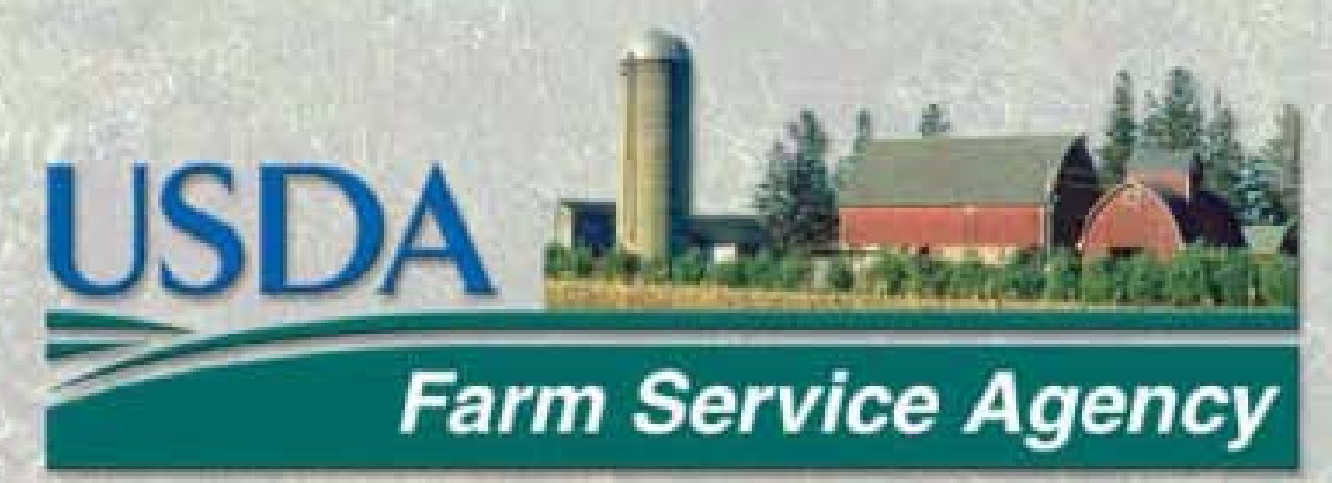

(5) (P) ARGonNE 


\section{Phytoremediation Wetlands Trail}

Please enjoy this self-guided trail through the phytoremediation and wetlands areas of the Murdock Groundwater Cleanup Project.

- Trail guides are in the box next to this sign.

- Thank you for taking the trail guide home with you or returning it to the box for the next visitor.

This trail and the parking area are designed to be accessible to people with disabilities.

Please use this public access area with care. The landowner and the USDA cannot accept responsibility for personal injuries to visitors.

This area is open to the public between dawn and dusk. The trail is designed for walking or jogging. Swimming and motorized vehicles are not permitted.
"That land is a community is the basic concept of ecology, but that land is to be loved and respected is an extension of etfics. A land etfic, then, reflects the existence of an ecological conscience, and that in turn reflects a conviction of individual responsibility for the fiealth of land."

Aldo Leopold Pioneer in American Conservation

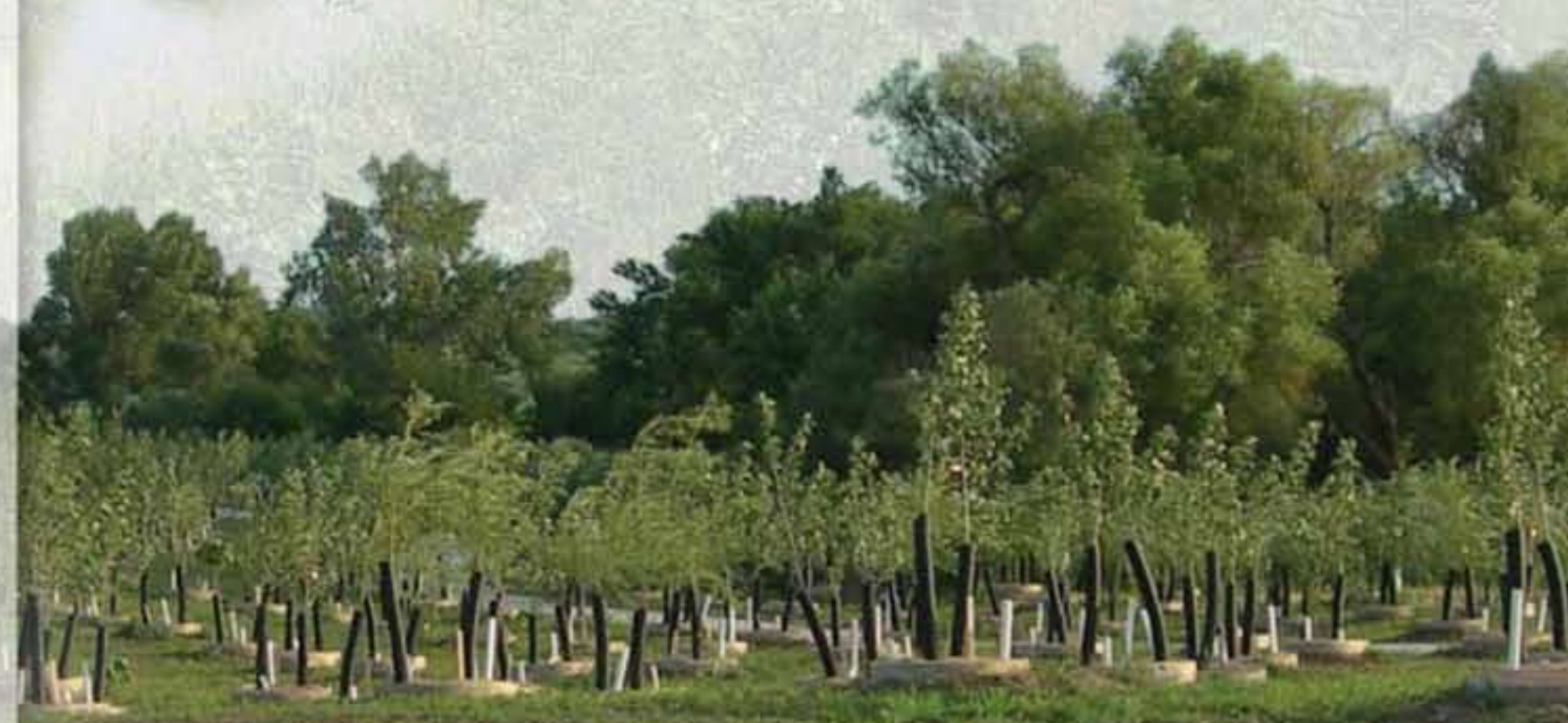





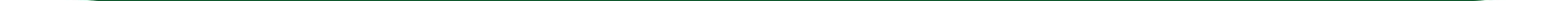




\section{How Does the Spray IRRIGATION SYSTEM WORK?}

- The spray irrigation part of this project is south of Waverly Road, on school property.

- Near the school, the groundwater is deep below the surface. During warm months, a pump removes water from the aquifer and delivers it to a spray irrigation system. The system

- Sprays water into the air, allowing the contaminant to evaporate;

- Irrigates the school's athletic fields; and

- Decreases carbon tetrachloride concentrations in the groundwater to levels that the phytoremediation system can handle.
- Periodic testing of the spray irrigation system ensures that

- Contaminant concentrations in the sprayed water pose no human health risk,

- The system effectively removes contaminant from the aquifer, and

- The treated water can be a community resource.

A mobile spray rig is used to treat and distribute the groundwater.
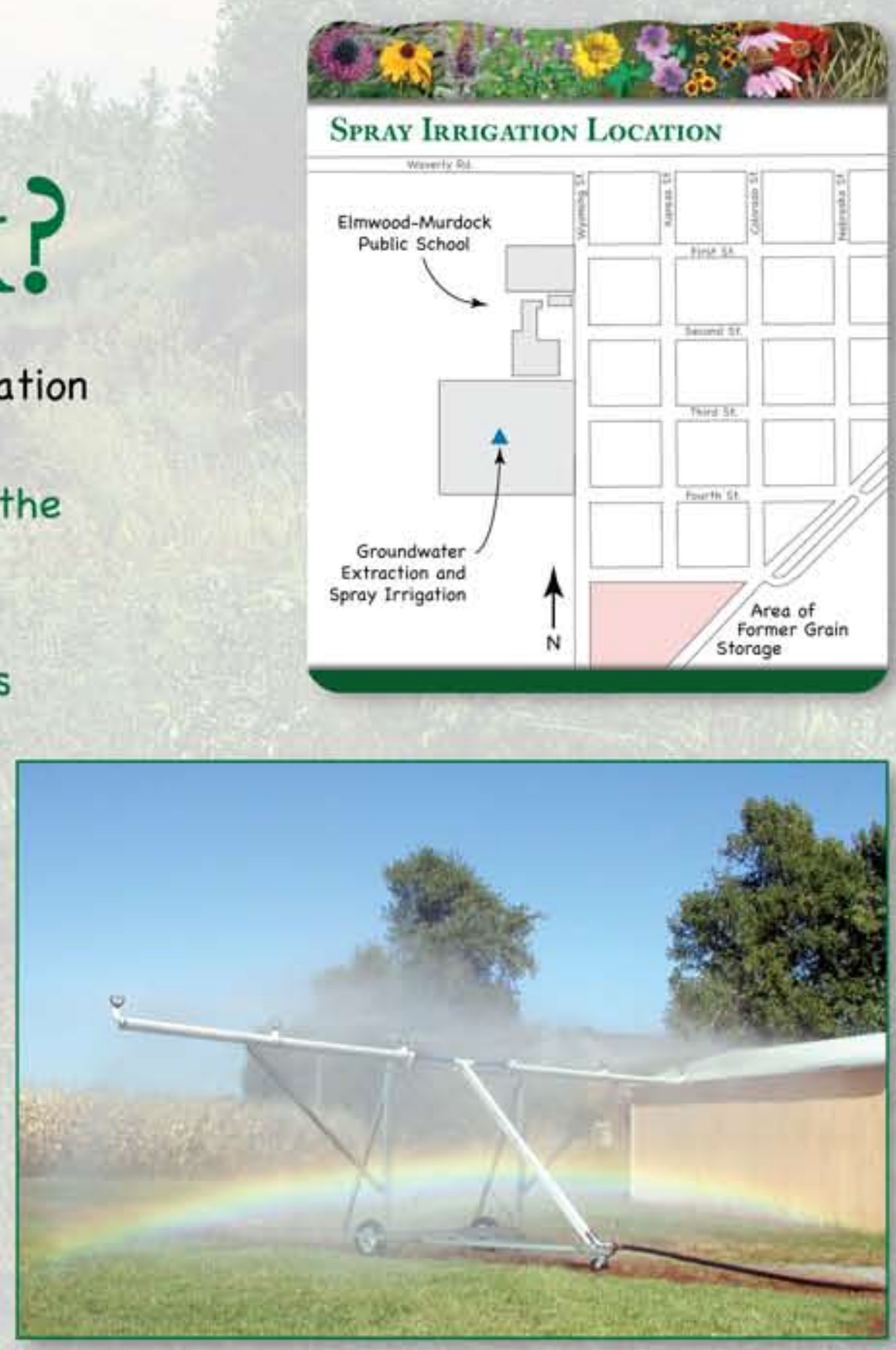



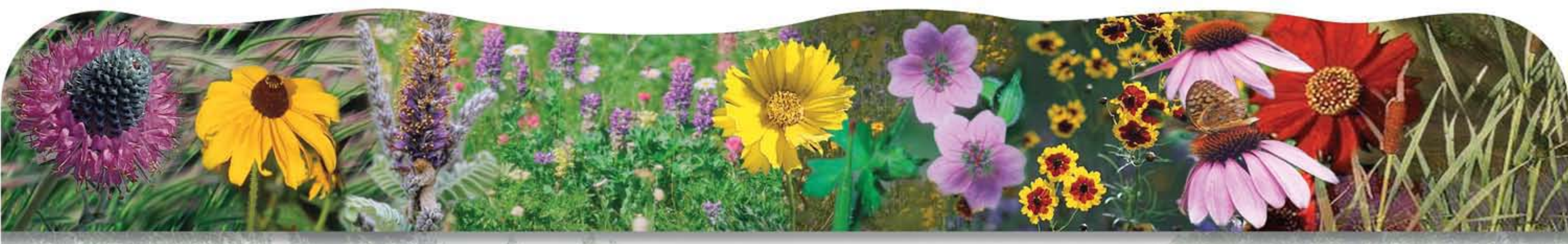

\section{Phytoremediation: How Does IT Work?}

Plants take up the fumigant carbon tetrachloride (CT) dissolved in water. Plants can store $C T$, release it to the atmosphere, or break it down chemically.

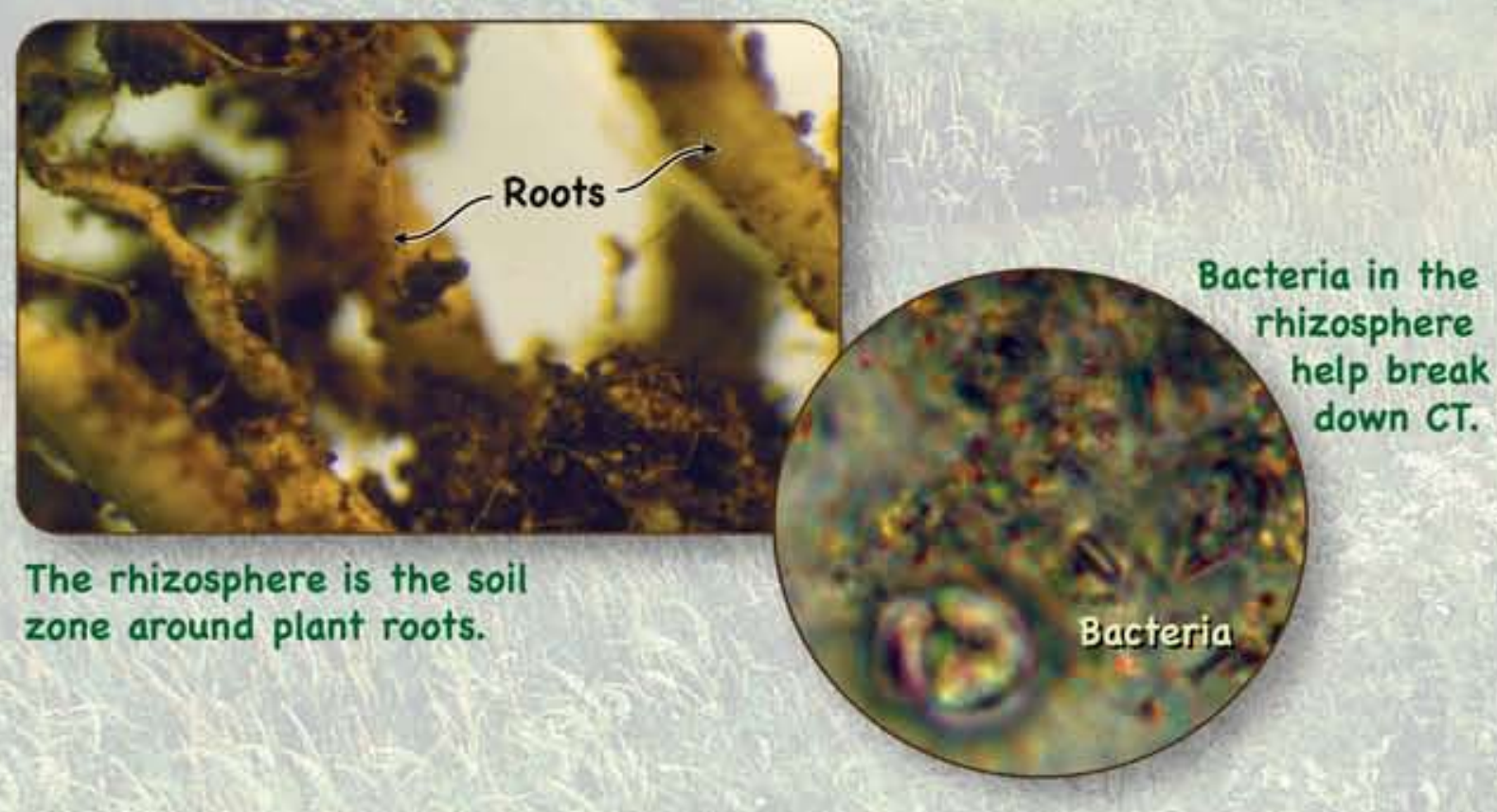

Microbes in the soil around the roots (in the rhizosphere) also help degrade CT.

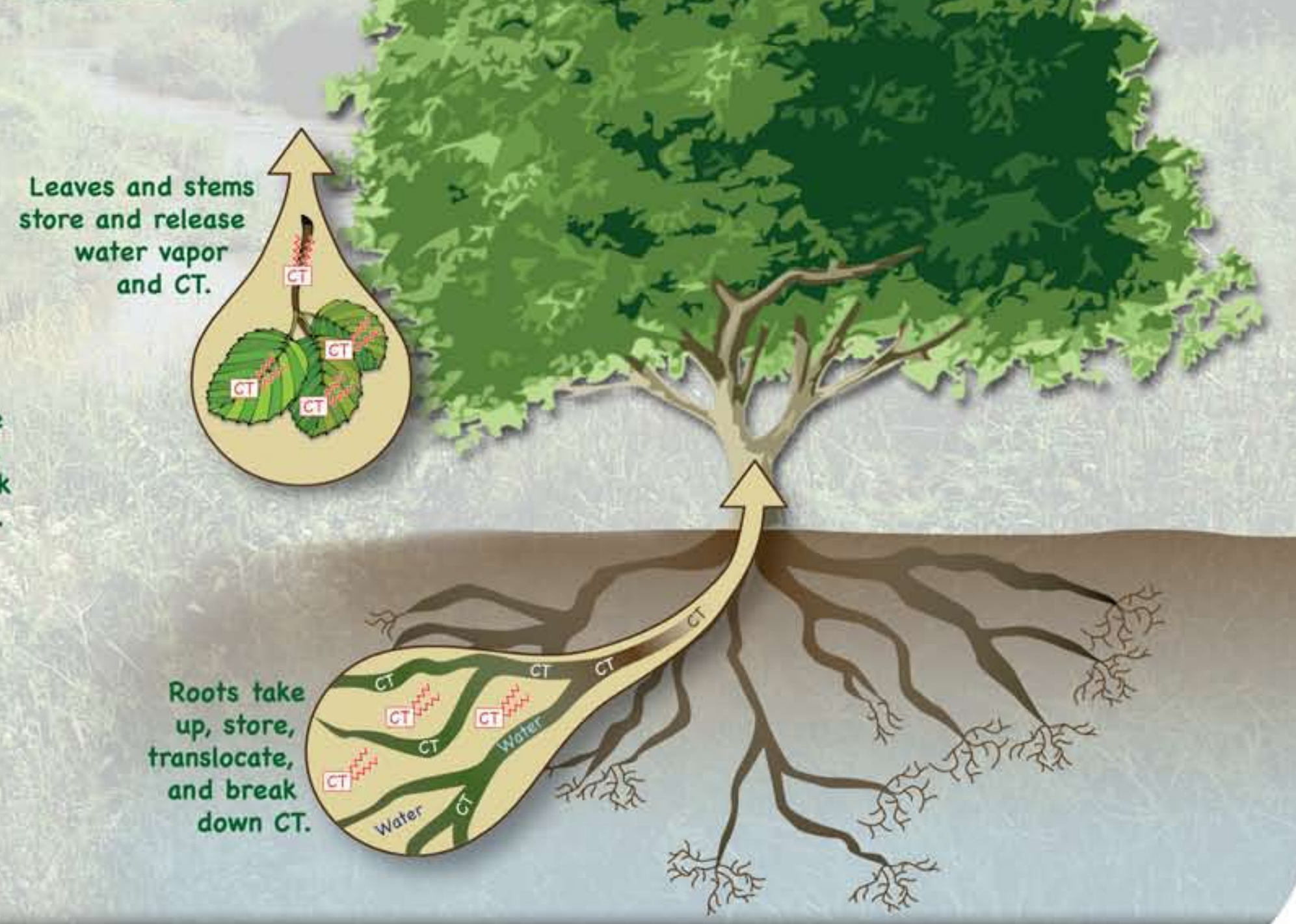




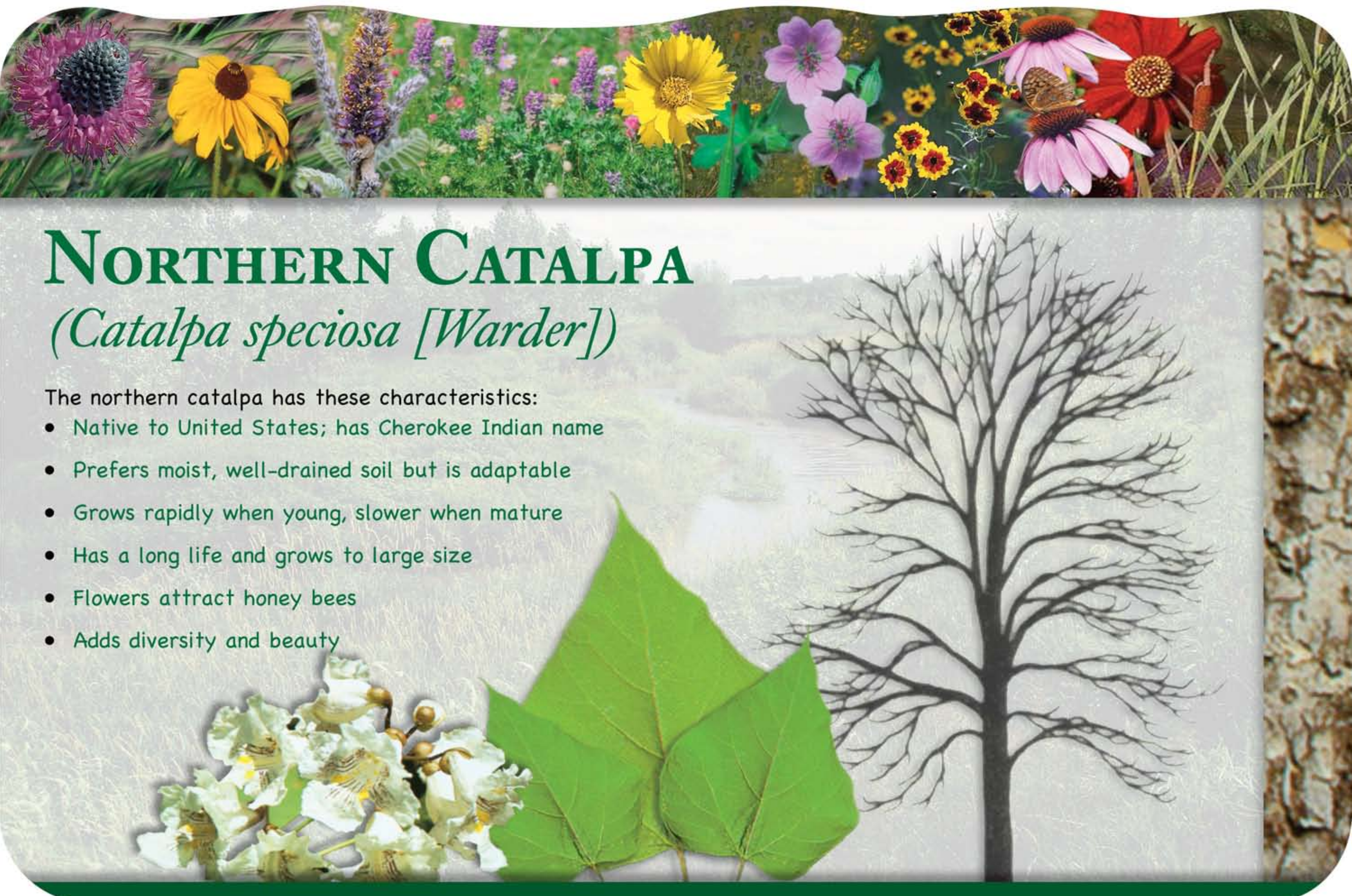




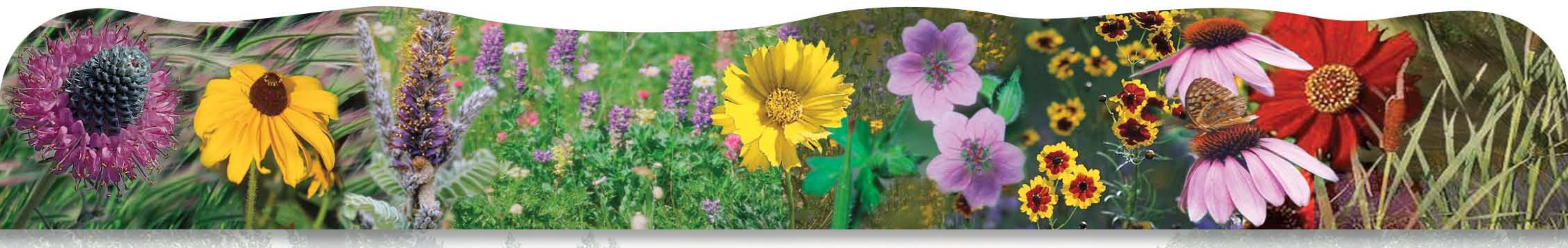

\section{Who Lives Here - Besides People?}

The groundwater cleanup project is enhancing the existing ecosystem. The project introduced the wetlands habitat and increased the diversity of native plant and animal life in the area.

Phytoremediation plantation

- The trees provide food and shelter for mammals, birds, and insects, including deer, wild turkeys, pheasants, grouse, woodpeckers, songbirds, and honeybees. The trees also provide building material for beavers.

- As time passes, the shorter-lived trees (willows and poplars) die, and the longer-lived trees (catalpas and green ashes) create a shaded area and continue their remediation task.

- Over time, the animal populations change as the habitat changes.

The trail guide tells how you can use the project's visitor log to report unusual species that you see in the phytoremediation area and the wetlands.
Wetlands

- Attract a variety of birds (egrets, herons, ducks, geese, songbirds).

- Provide habitat and food supply for mammals and other animals.

- Invite repopulation of the creek by fish.

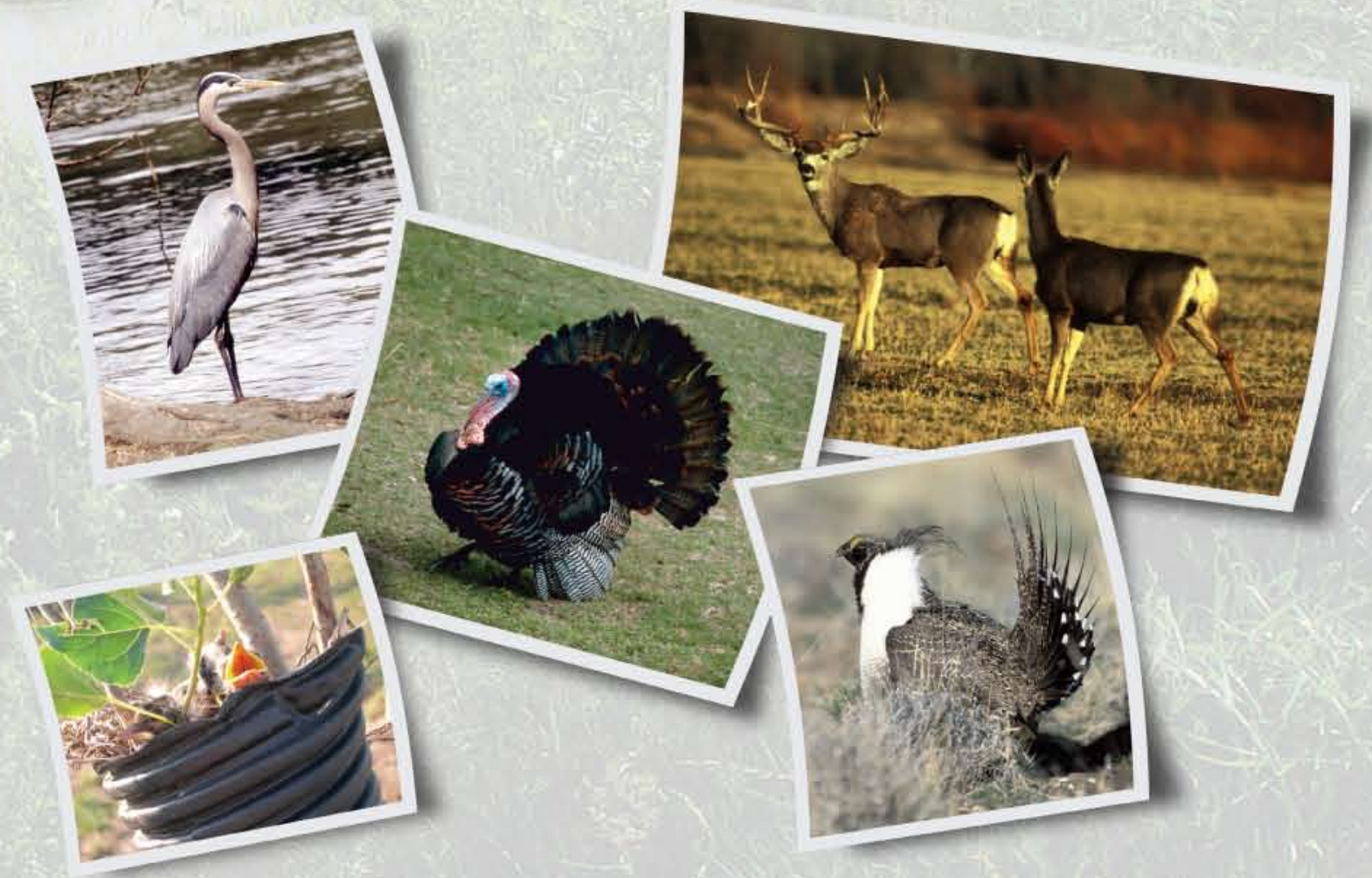






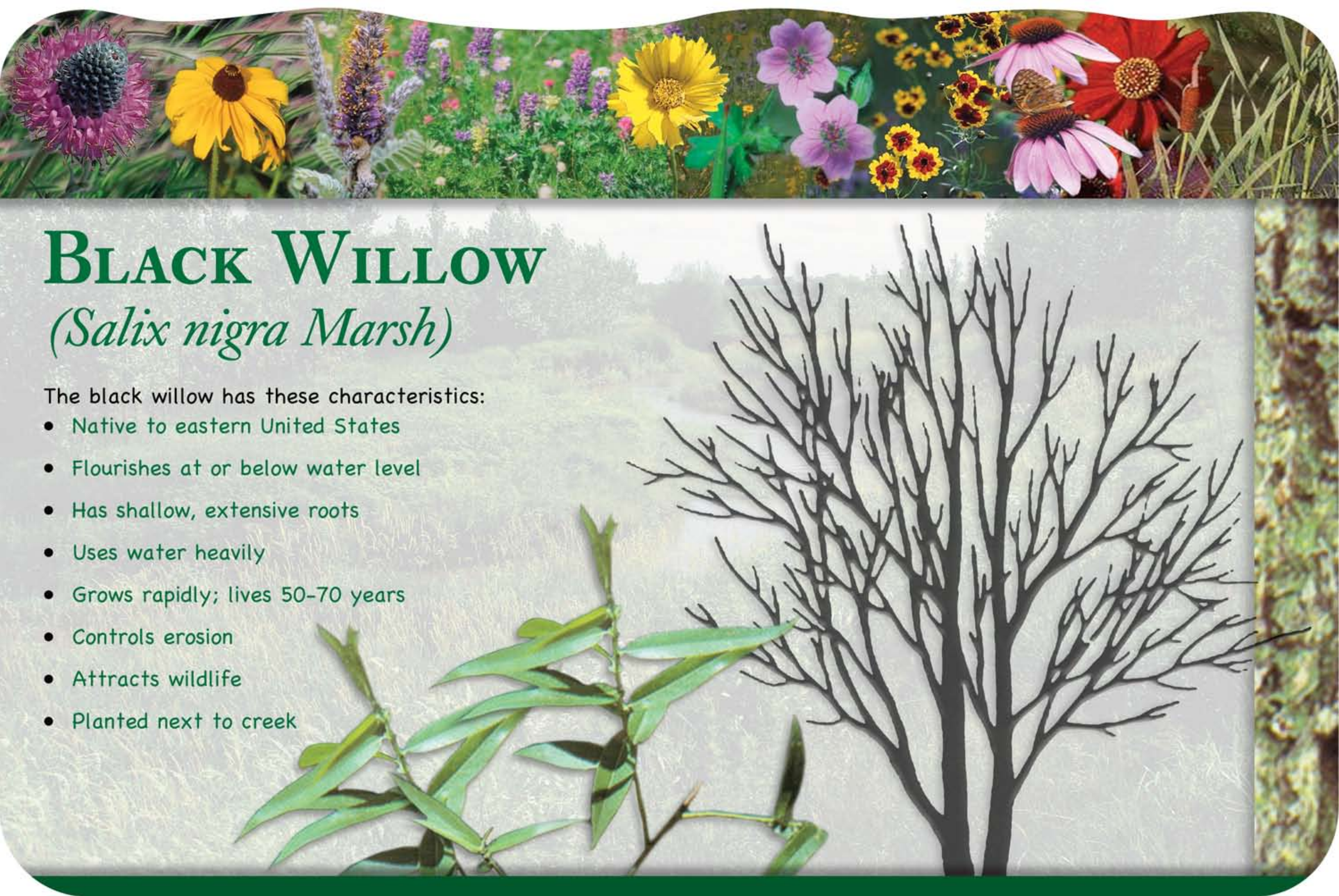




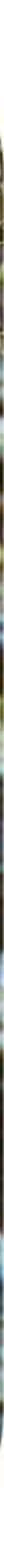




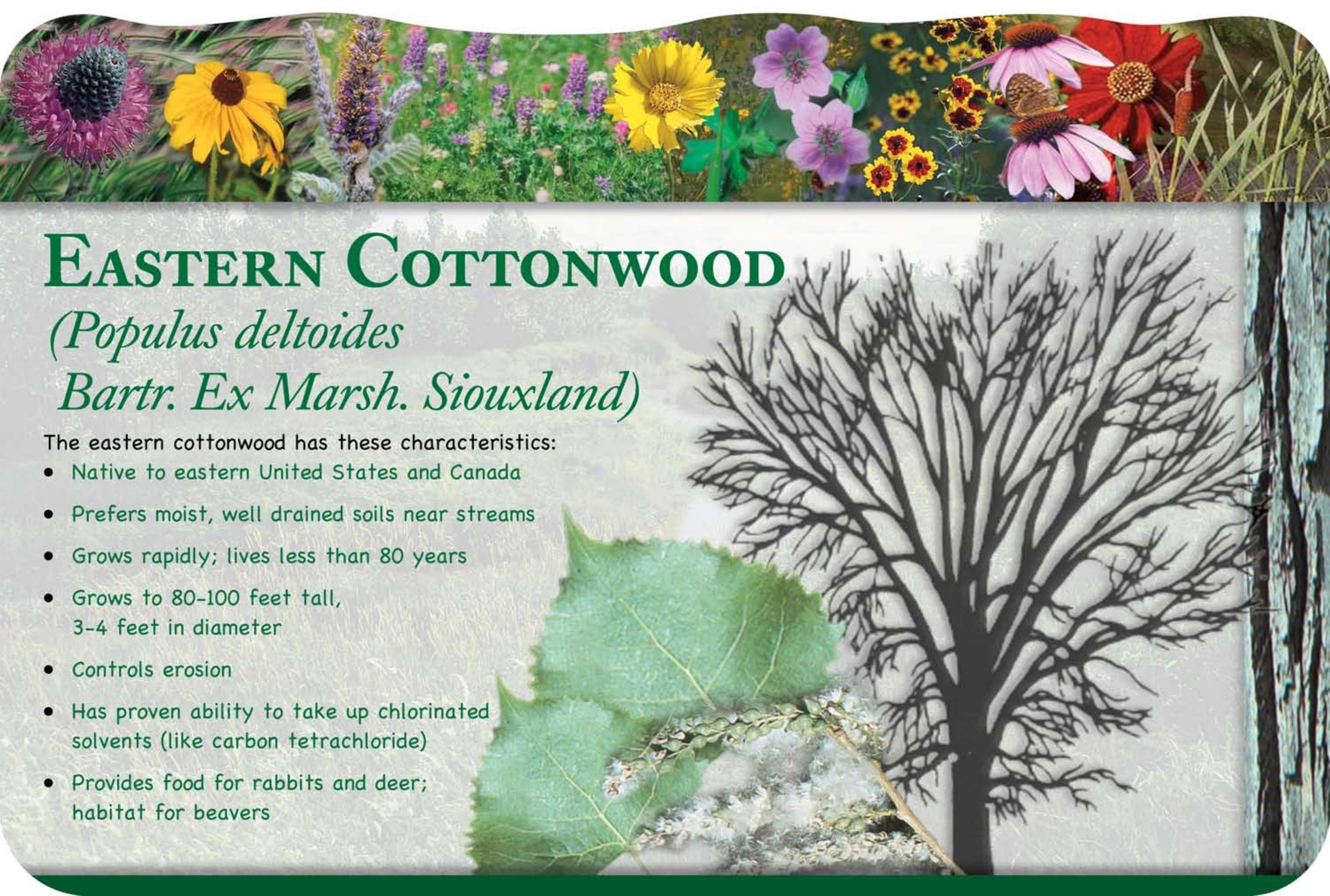





\section{What Does The Weather}

\section{Station Tell Us?}

- The weather station near this sign is helping project managers understand the balance between

- Addition of water to the aquifer by precipitation and

- Removal of water from the aquifer and shallow soil by plant uptake and other processes.

- The weather station is self-contained and solar powered. It can store data for six months with no operator attention.

- Every two hours, the weather station automatically measures and records temperature, barometric pressure, relative humidity, wind speed and direction, rainfall, the intensity of sunlight and ultraviolet radiation, and moisture levels in shallow and deeper soil.

- The data from the weather station are used to

- Calculate the rate of water loss from near-surface soils by direct evaporation and by plant uptake and

- Determine how effectively the grasses between the trees capture precipitation.

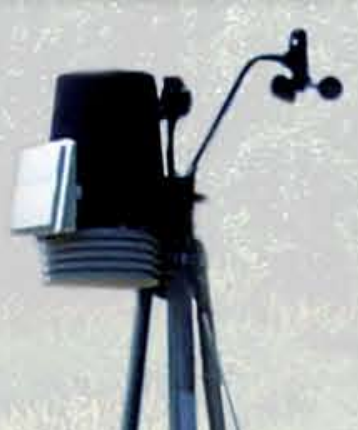

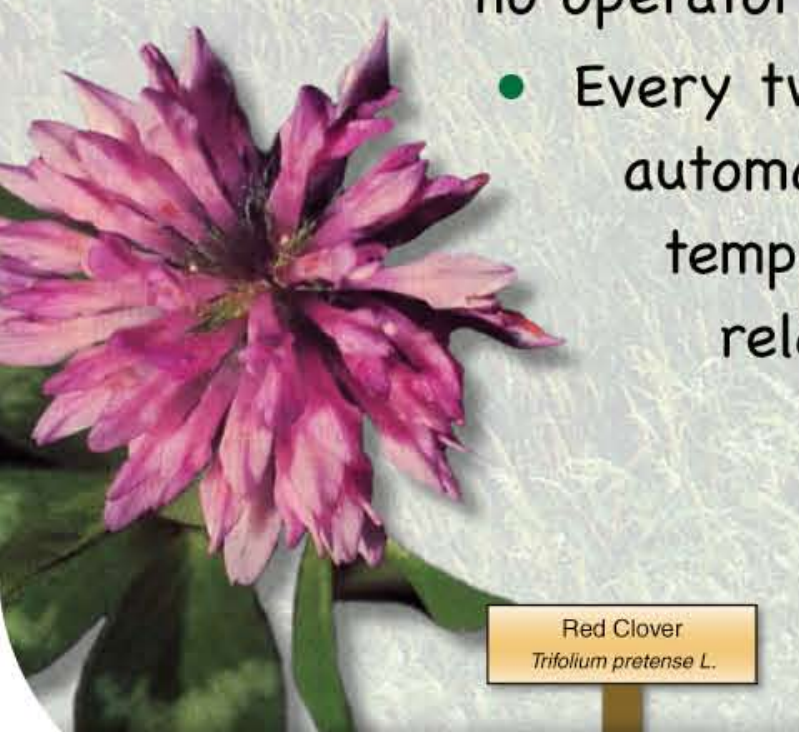$$
\text { deper soll. }
$$ 


\section{Appendix C:}

\section{Visitor's Guide}



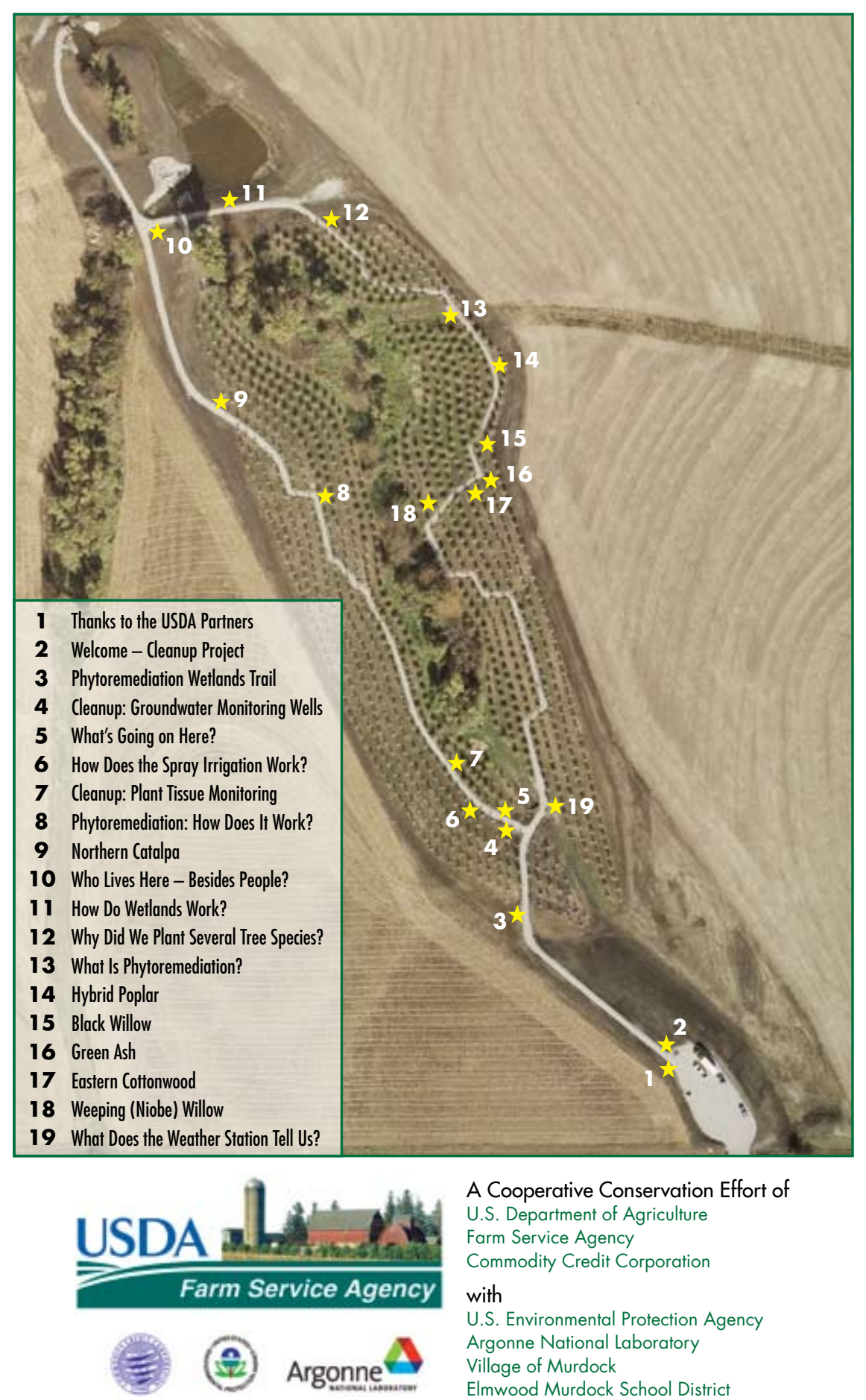

For information about the cleanup project, contact Steve Gilmore/Steve.Gilmore@wdc.usda.gov

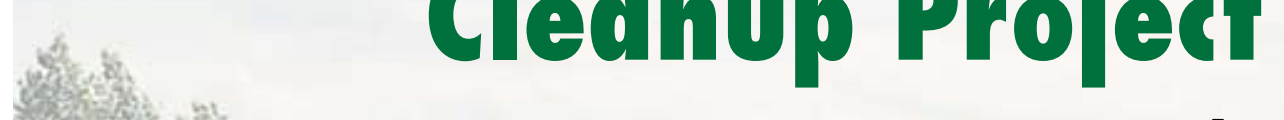

Visitor's Guide

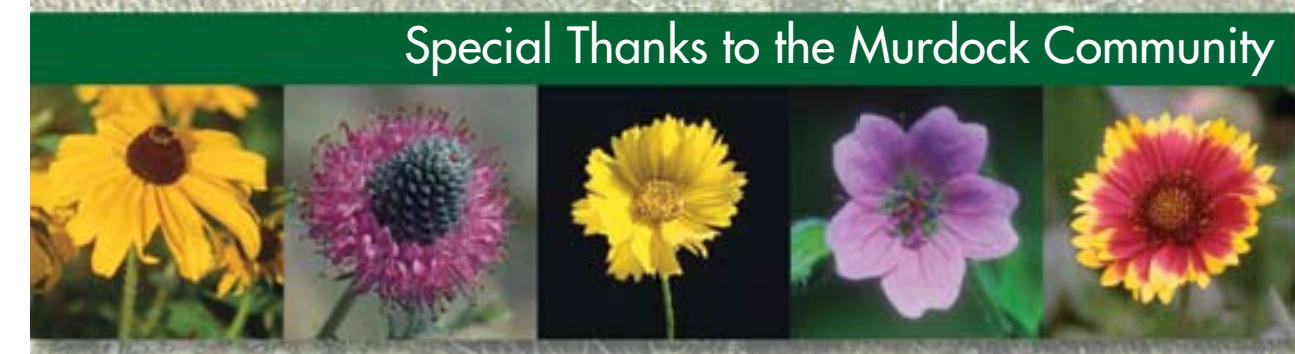




\section{Appendix D:}

\section{August 2006 National CREP Conference CCC/USDA Summary}




\title{
Murdock Clean \\ Water Partnership
}

\section{Trees Help Clean Up Rural Water Contamination}

\author{
Location: Midwest/Northern High Plains Region: \\ Nebraska
}

Project Summary: The community of Murdock, Nebraska, and state and federal partners, used innovative technologies to solve ground and surface water contamination.

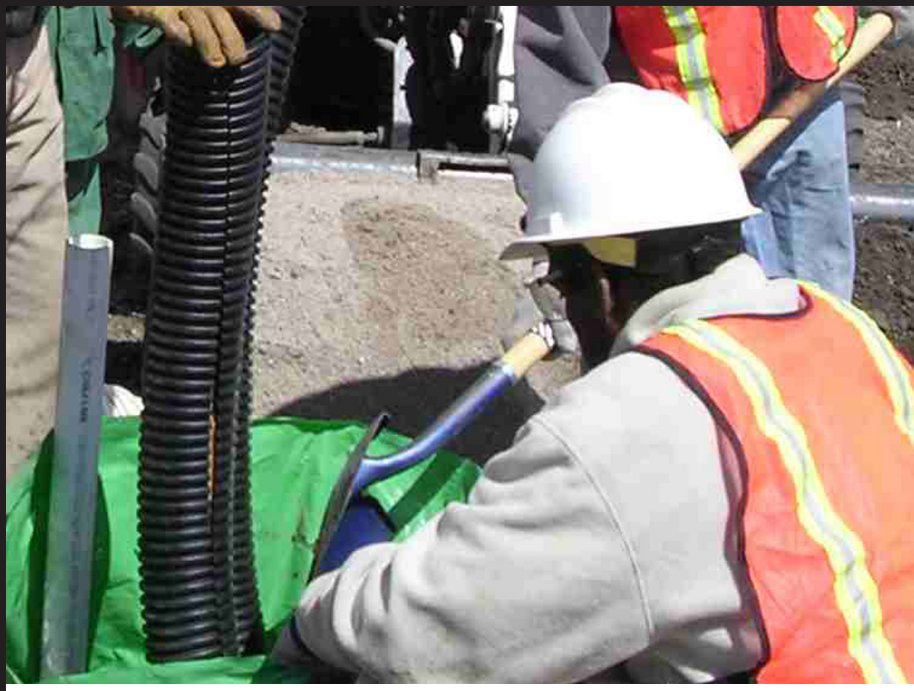

Workers planting trees in a deep hole for the phytoremediation project at Murdock, Nebraska - April 2005. (Photo courtesy Argonne National Laboratory)

\section{Resource Challenge}

Not long ago, children who used Murdock, Nebraska's school athletic fields in the summertime played on parched grass and exposed gravel. At the same time, a nearby creek was virtually unusable because of limited public access and because carbon tetrachloride, used decades ago to fumigate stored grain, had entered the aquifer that lay beneath the town and that fed the creek.

Two problems turned out to have one very creative solution. Faced with the need to treat contaminated groundwater and protect the creek, State and Federal governments, regulators, the local school district, the village, and private citizens set up an innovative system that is dramatically improving the community's recreational and educational opportunities while at the same time ridding the town of its contaminated water.

\section{Examples of Key Partners}

U.S. Environmental Protection Agency, USDA Farm Service Agency, U.S. Department of Energy Argonne National Laboratory, Nebraska Department of Environmental Quality, Stock Seed Co., Village of Murdock, Elmwood-Murdock Public School, local landowners, and others.

\section{Results and Accomplishments}

The Murdock Partnership used an innovative system that combines multiple technologies to address surface and groundwater contamination. Near the contamination source, pumps extract contaminated ground water, which goes to a spray irrigation system that dissipates carbon tetrachloride harmlessly into the air. The treated water is reused on the school's athletic fields, nurturing a healthy, grassy surface for the children.

To supplement the spray technology, partners worked with landowners, the town, and local farmers, planting more than 2,000 trees downstream from where the groundwater enters the creek. These trees take up contaminated water and break down polluting chemicals naturally, a process called phytoremediation. Native prairie plants around and between the trees intercept rainwater and force the trees to draw most of their water from the aquifer.

This year, partners are restoring a downstream wetland to intercept lingering traces of the polluting chemical before it enters the creek. They are also installing an ADA-accessible trail at both the tree plantation and the wetland for public use. Interpretive signs will enhance the visitor's experience and facilitate use of the site as an outdoor "living" classroom.

Further partnerships will be formed as installation is completed and the community begins to fully use the new resources.

\section{Project Contact}

Steve and Bonnie Bruttig

Nebraska Landowners

\section{Innovation/Highlight}

A combination of tree plantings and other natural treatments, including an innovative new spray technology, helped resolve ground and surface water contamination, while enhancing recreational opportunities for local residents.
402-867-2274

Spbruttig@aol.com

Website:

www.fsa.usda.gov/dafp/cepd/epb/hazardous_waste.htm 


\section{Appendix E:}

\section{Vegetation Analysis Results}


TABLE E.1 Results of analyses for volatile organic compounds in vegetation samples collected at Murdock, Nebraska, in September 2005 through December 2006.

\begin{tabular}{|c|c|c|c|c|c|c|c|c|}
\hline & & & Concentrati & $n(\mu \mathrm{g} / \mathrm{kg})$ & & & & \\
\hline Location & Sample & $\begin{array}{l}\text { Sample } \\
\text { Date }\end{array}$ & $\begin{array}{c}\text { Carbon } \\
\text { Tetrachloride }\end{array}$ & Chloroform & $\begin{array}{c}\text { Tree } \\
\text { Species }\end{array}$ & $\begin{array}{c}\text { Sample } \\
\text { Type }^{a}\end{array}$ & $\begin{array}{l}\text { (cm) } \\
\text { Height }\end{array}$ & Sample Description \\
\hline
\end{tabular}

Tree branch samples collected in September 2005

\begin{tabular}{|c|c|c|c|c|c|c|c|}
\hline E100 & MUE100WN1-B-18655 & 9/27/05 & $N D^{c}$ & 1.3 & WN1 & Branch & $N R^{d}$ \\
\hline E111 & MUE111WN1-B-18645 & 9/27/05 & 34 & 13 & WN1 & Branch & NR \\
\hline E118 & MUE118WN1-B-18641 & $9 / 27 / 05$ & ND & 3.2 & WN1 & Branch & NR \\
\hline E128 & MUE128WN1-B-18637 & $9 / 27 / 05$ & $<1$ & 4.0 & WN1 & Branch & NR \\
\hline E206 & MUE206WN2-B-18649 & $9 / 27 / 05$ & 12 & 12 & WN2 & Branch & NR \\
\hline E207 & MUE207WN2-B-18647 & $9 / 27 / 05$ & 48 & 37 & WN2 & Branch & NR \\
\hline E308 & MUE308WN1-B-18651 & $9 / 27 / 05$ & 43 & 12 & WN1 & Branch & NR \\
\hline E309 & MUE309WN2-B-18643 & $9 / 27 / 05$ & 61 & 11 & WN2 & Branch & NR \\
\hline E500 & MUE500EC1-B-18657 & $9 / 27 / 05$ & ND & 1.8 & EC1 & Branch & NR \\
\hline W111 & MUW111WN2-B-18579 & $9 / 27 / 05$ & 45 & 33 & WN2 & Branch & NR \\
\hline W114 & MUW114WN2-B-18581 & $9 / 27 / 05$ & 6.6 & 10 & WN2 & Branch & NR \\
\hline W119 & MUW119WN2-B-18583 & 9/27/05 & 19 & 23 & WN2 & Branch & NR \\
\hline W124 & MUW124WN2-B-18585 & $9 / 27 / 05$ & 20 & 10 & WN2 & Branch & NR \\
\hline W128 & MUW128WN2-B-18601 & $9 / 27 / 05$ & 91 & 18 & WN2 & Branch & NR \\
\hline W129 & MUW129WN2-B-18587 & $9 / 27 / 05$ & 35 & 21 & WN2 & Branch & NR \\
\hline W132 & MUW132WN1-B-18599 & $9 / 27 / 05$ & 12 & 10 & WN1 & Branch & NR \\
\hline W134 & MUW134WN1-B-18589 & $9 / 27 / 05$ & ND & 6.9 & WN1 & Branch & NR \\
\hline W157 & MUW157WN1-B-18593 & $9 / 27 / 05$ & 26 & 7.1 & WN1 & Branch & NR \\
\hline W158 & MUW158WN2-B-18591 & $9 / 27 / 05$ & 135 & 8.5 & WN2 & Branch & NR \\
\hline W159 & MUW159EC2-B-18597 & $9 / 27 / 05$ & 14 & 6.9 & $\mathrm{EC} 2$ & Branch & NR \\
\hline W210 & MUW210WN2-B-18603 & 9/27/05 & 29 & 32 & WN2 & Branch & NR \\
\hline W211 & MUW211EC-B-18605 & $9 / 27 / 05$ & $<1$ & 1.9 & EC & Branch & NR \\
\hline W211 & MUW211GA-B-18607 & $9 / 27 / 05$ & ND & 3.3 & GA & Branch & NR \\
\hline W357 & MUW357WN1-B-18595 & $9 / 27 / 05$ & 1.1 & 3.3 & WN1 & Branch & NR \\
\hline W407 & MUW407WN1-B-18609 & $9 / 27 / 05$ & 36 & 12 & WN1 & Branch & NR \\
\hline W408 & MUW408EC1-B-18611 & $9 / 27 / 05$ & ND & ND & EC1 & Branch & NR \\
\hline W417 & MUW417EC1-B-18617 & 9/27/05 & 16 & 6.3 & EC1 & Branch & NR \\
\hline W427 & MUW427EC-B-18619 & $9 / 27 / 05$ & 314 & 59 & EC & Branch & NR \\
\hline W427 & MUW427GA-B-18620 & $9 / 27 / 05$ & $<1$ & 6.0 & $\mathrm{GA}$ & Branch & NR \\
\hline W606 & MUW606EC1-B-18613 & $9 / 27 / 05$ & 22 & 9.0 & EC1 & Branch & NR \\
\hline W616 & MUW616EC2-B-18615 & $9 / 27 / 05$ & 7.6 & 6.8 & $\mathrm{EC} 2$ & Branch & NR \\
\hline
\end{tabular}


TABLE E.1 (Cont.)

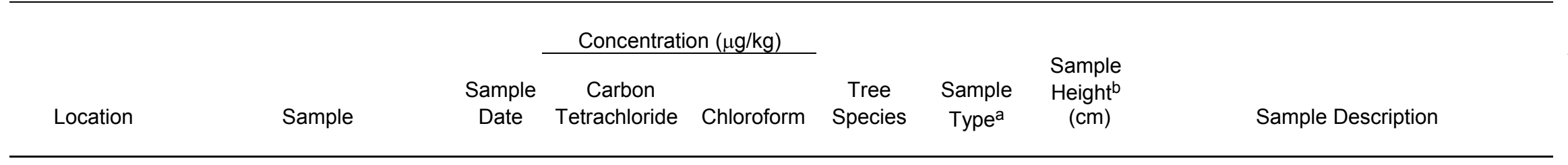

Tree branch samples collected in September 2005 (cont.)

\begin{tabular}{|c|c|c|c|c|c|c|c|}
\hline PREW001 & MUPREW001-B-18623 & 9/27/05 & 3.9 & 15 & Willow & Branch & NR \\
\hline PREW002 & MUPREW002-B-18625 & 9/27/05 & 1.1 & 5.6 & Ash & Branch & $N R$ \\
\hline PREW003 & MUPREW003-B-18627 & 9/27/05 & $<1$ & 1.8 & Willow & Branch & NR \\
\hline PREW004 & MUPREW004-B-18629 & 9/27/05 & 1.1 & 2.5 & Ash & Branch & NR \\
\hline PREW005 & MUPREW005-B-18631 & 9/27/05 & $<1$ & 1.1 & Locust & Branch & NR \\
\hline PREW006 & MUPREW006-B-18633 & 9/27/05 & 11 & 10 & EC & Branch & NR \\
\hline PREW007 & MUPREW007-B-18634 & 9/27/05 & ND & 4.5 & Willow & Branch & NR \\
\hline PREE008 & MUPRE008-B-18639 & 9/27/05 & ND & 2.3 & & Branch & NR \\
\hline PREE009 & MUPRE009-B-18653 & 9/27/05 & 443 & 171 & EC & Branch & NR \\
\hline \multicolumn{8}{|c|}{ Tree leaf samples collected in September 2005} \\
\hline E100 & MUE100WN1-L-18654 & 9/27/05 & ND & 7.6 & WN1 & Leaf & NR \\
\hline E111 & MUE111WN1-L-18644 & 9/27/05 & ND & 1.7 & WN1 & Leaf & $N R$ \\
\hline E118 & MUE118WN1-L-18640 & 9/27/05 & ND & 5.1 & WN1 & Leaf & NR \\
\hline E128 & MUE128WN1-L-18636 & 9/27/05 & ND & 7.7 & WN1 & Leaf & NR \\
\hline E206 & MUE206WN2-L-18648 & 9/27/05 & ND & 2.2 & WN2 & Leaf & NR \\
\hline E207 & MUE207WN2-L-18646 & 9/27/05 & ND & 2.3 & WN2 & Leaf & NR \\
\hline E308 & MUE308WN1-L-18650 & 9/27/05 & $<1$ & 2.4 & WN1 & Leaf & NR \\
\hline E309 & MUE309WN2-L-18642 & 9/27/05 & $<1$ & 1.8 & WN2 & Leaf & NR \\
\hline E500 & MUE500EC1-L-18656 & 9/27/05 & ND & 19 & EC1 & Leaf & NR \\
\hline W111 & MUW111WN2-L-18578 & 9/27/05 & $<1$ & 2.6 & WN2 & Leaf & NR \\
\hline W114 & MUW114WN2-L-18580 & 9/27/05 & ND & 2.3 & WN2 & Leaf & NR \\
\hline W119 & MUW119WN2-L-18582 & 9/27/05 & ND & 1.3 & WN2 & Leaf & NR \\
\hline W124 & MUW124WN2-L-18584 & 9/27/05 & $<1$ & 2.0 & WN2 & Leaf & $\mathrm{R}$ \\
\hline
\end{tabular}

Preexisting willow tree east of location W114.

Preexisting ash tree east of location W113.

Preexisting willow tree east of location W122. Has old yellow and black ribbon marker.

Preexisting ash tree east of location W131. Almost in creek bed.

Preexisting locust tree east of location W138.

Preexisting cottonwood.

Preexisting willow tree, west of wetland between wetland and path.

Preexisting (unspecified type) tree near E128.

Preexisting cottonwood tree west of E102. 
TABLE E.1 (Cont.)

\begin{tabular}{|c|c|c|c|c|c|c|c|c|}
\hline \multirow[b]{2}{*}{ Location } & \multirow[b]{2}{*}{ Sample } & \multirow[b]{2}{*}{$\begin{array}{l}\text { Sample } \\
\text { Date }\end{array}$} & \multicolumn{2}{|c|}{ Concentration $(\mu \mathrm{g} / \mathrm{kg})$} & \multirow[b]{2}{*}{$\begin{array}{c}\text { Tree } \\
\text { Species }\end{array}$} & \multirow[b]{2}{*}{$\begin{array}{c}\text { Sample } \\
\text { Type }^{a}\end{array}$} & \multirow[b]{2}{*}{$\begin{array}{l}\text { Sample } \\
\text { Height }^{b} \\
\quad(\mathrm{~cm})\end{array}$} & \multirow[b]{2}{*}{ Sample Description } \\
\hline & & & $\begin{array}{c}\text { Carbon } \\
\text { Tetrachloride }\end{array}$ & Chloroform & & & & \\
\hline \multicolumn{9}{|c|}{ Tree leaf samples collected in September 2005 (cont.) } \\
\hline W128 & MUW128WN2-L-18600 & 9/27/05 & $<1$ & 5.7 & WN2 & Leaf & NR & \\
\hline W129 & MUW129WN2-L-18586 & 9/27/05 & $<1$ & 2.1 & WN2 & Leaf & NR & \\
\hline W132 & MUW132WN1-L-18598 & $9 / 27 / 05$ & ND & 4.5 & WN1 & Leaf & NR & \\
\hline W134 & MUW134WN1-L-18588 & $9 / 27 / 05$ & ND & 3.6 & WN1 & Leaf & NR & \\
\hline W157 & MUW157WN1-L-18592 & $9 / 27 / 05$ & $<1$ & 1.6 & WN1 & Leaf & NR & \\
\hline W158 & MUW158WN2-L-18590 & $9 / 27 / 05$ & 2.9 & 2.9 & WN2 & Leaf & NR & \\
\hline W159 & MUW159EC2-L-18596 & $9 / 27 / 05$ & $<1$ & 2.8 & EC2 & Leaf & NR & \\
\hline W210 & MUW210WN2-L-18602 & $9 / 27 / 05$ & $<1$ & 5.0 & WN2 & Leaf & NR & \\
\hline W211 & MUW211EC-L-18604 & 9/27/05 & ND & 3.1 & EC & Leaf & NR & \\
\hline W211 & MUW211GA-L-18606 & $9 / 27 / 05$ & ND & 6.8 & GA & Leaf & NR & \\
\hline W357 & MUW357WN1-L-18594 & $9 / 27 / 05$ & ND & 5.1 & WN1 & Leaf & NR & \\
\hline W407 & MUW407WN1-L-18608 & $9 / 27 / 05$ & $<1$ & 2.5 & WN1 & Leaf & NR & \\
\hline W408 & MUW408EC1-L-18610 & 9/27/05 & ND & 2.6 & EC1 & Leaf & NR & \\
\hline W417 & MUW417EC1-L-18616 & 9/27/05 & $<1$ & 4.6 & EC1 & Leaf & NR & \\
\hline W427 & MUW427EC-L-18618 & $9 / 27 / 05$ & $<1$ & 4.0 & $\mathrm{EC}$ & Leaf & NR & \\
\hline W427 & MUW427GA-L-18621 & 9/27/05 & ND & 9.2 & GA & Leaf & NR & \\
\hline W606 & MUW606EC1-L-18612 & $9 / 27 / 05$ & ND & 4.4 & EC1 & Leaf & NR & \\
\hline W616 & MUW616EC2-L-18614 & 9/27/05 & ND & 5.1 & EC2 & Leaf & NR & \\
\hline PREW001 & MUPREW001-L-18622 & $9 / 27 / 05$ & ND & 2.0 & Willow & Leaf & NR & $\begin{array}{l}\text { Leaf from preexisting willow tree east of } \\
\text { location } \mathrm{W} 114 \text {. }\end{array}$ \\
\hline PREW002 & MUPREW002-L-18624 & $9 / 27 / 05$ & $<1$ & 6.0 & Ash & Leaf & NR & $\begin{array}{l}\text { Leaf from preexisting ash tree east of } \\
\text { location W113. }\end{array}$ \\
\hline PREW003 & MUPREW003-L-18626 & $9 / 27 / 05$ & ND & 1.8 & Willow & Leaf & NR & $\begin{array}{l}\text { Leaf from preexisting willow tree east of } \\
\text { location W122. Has old yellow and } \\
\text { black ribbon marker. }\end{array}$ \\
\hline PREW004 & MUPREW004-L-18628 & $9 / 27 / 05$ & $<1$ & 6.3 & Ash & Leaf & NR & $\begin{array}{l}\text { Leaf from preexisting ash tree east of } \\
\text { location W131. Almost in creek bed. }\end{array}$ \\
\hline PREW005 & MUPREW005-L-18630 & $9 / 27 / 05$ & ND & 2.3 & Locust & Leaf & NR & $\begin{array}{l}\text { Leaf from preexisting locust tree east of } \\
\text { location W138. }\end{array}$ \\
\hline PREW006 & MUPREW006-L-18632 & $9 / 27 / 05$ & ND & 2.9 & EC & Leaf & NR & Leaf from preexisting cottonwood. \\
\hline
\end{tabular}


Tree branch samples collected in January 2006

$\begin{array}{llccccrr}\text { E309 } & \text { MUE309-18085 } & 1 / 9 / 06 & 18 & 3.7 & \text { WN2 } & \text { Branch } & 122 \\ & & & & & & & \\ \text { W158 } & \text { MUW158-18083 } & 1 / 9 / 06 & 29 & 4.5 & \text { WN1 } & \text { Branch } & 137 \\ \text { W427 } & \text { MUW427-18084 } & 1 / 9 / 06 & 7.2 & 5.4 & \text { EC } & \text { Branch } & 137 \\ \text { W606 } & \text { MUW606-18086 } & 1 / 9 / 06 & 5.7 & 8.1 & \text { EC1 } & \text { Branch } & 137\end{array}$

Tree branch samples collected in March 2006

$\begin{array}{llllcrrr}\text { E111 } & \text { MU-E111-WN1-18087 } & 3 / 2 / 06 & <1 & 1.1 & \text { WN1 } & \text { Branch } & 86 \\ \text { E207 } & \text { MU-E207-WN1-18088 } & 3 / 2 / 06 & 2.4 & 2.2 & \text { WN1 } & \text { Branch } & 84 \\ \text { E207 } & \text { MU-E207-WN2-18089 } & 3 / 2 / 06 & <1 & 6.9 & \text { WN2 } & \text { Branch } & 84 \\ \text { E308 } & \text { MU-E308-WN1-18090 } & 3 / 2 / 06 & 1.3 & 8.0 & \text { WN1 } & \text { Branch } & 69 \\ \text { E308 } & \text { MU-E308-WN2-18124 } & 3 / 2 / 06 & 1.0 & \text { ND } & \text { WN2 } & \text { Branch } & 104 \\ \text { E309 } & \text { MU-E309-WN1-18091 } & 3 / 2 / 06 & <1 & 19 & \text { WN1 } & \text { Branch } & 109 \\ \text { E309 } & \text { MU-E309-WN2-18092 } & 3 / 2 / 06 & \text { ND } & \text { ND } & \text { WN2 } & \text { Branch } & 112 \\ \text { E710 } & \text { MU-E710-EC-18107 } & 3 / 2 / 06 & \text { ND } & 1.2 & \text { EC } & \text { Branch } & 91 \\ \text { E710 } & \text { MU-E710-NC-18108 } & 3 / 2 / 06 & \text { ND } & 5.6 & \text { NC } & \text { Branch } & 99 \\ \text { E720 } & \text { MU-E720-EC-18110 } & 3 / 2 / 06 & 7.5 & 2.6 & \text { EC } & \text { Branch } & 99 \\ \text { E720 } & \text { MU-E720-GA-18111 } & 3 / 2 / 06 & <1 & \text { ND } & \text { GA } & \text { Branch } & 97 \\ \text { E735 } & \text { MU-E735-EC1-18112 } & 3 / 2 / 06 & \text { ND } & 1.5 & \text { EC1 } & \text { Branch } & 97 \\ \text { E735 } & \text { MU-E735-EC2-18113 } & 3 / 2 / 06 & 5.5 & 2.5 & \text { EC2 } & \text { Branch } & 99 \\ \text { E845 } & \text { MU-E845-WN1-18106 } & 3 / 2 / 06 & \text { ND } & 3.4 & \text { WN1 } & \text { Branch } & 76 \\ \text { E866 } & \text { MU-E866-EC-18115 } & 3 / 2 / 06 & \text { ND } & 3.1 & \text { EC } & \text { Branch } & 130 \\ \text { E866 } & \text { MU-E866-GA-18116 } & 3 / 2 / 06 & \text { ND } & 1.3 & \text { GA } & \text { Branch } & 145\end{array}$

South side of tree. No leaves on branches. South side of tree. No leaves on branches. South side of tree. No leaves on branches. South side of tree. No leaves on branches. South side of tree. No leaves on branches. West side of tree. No leaves on branches. South side of tree. No leaves on branches. North side of tree. No leaves on branches. Sample collected from top of tree.

West side of tree. No leaves on branches. South side of tree. No leaves on branches. South side of tree. No leaves on branches. South side of tree. No leaves on branches. South side of tree. No leaves on branches. South side of tree. No leaves on branches. Sample collected from top of tree. 


\begin{tabular}{|c|c|c|c|c|c|c|c|c|}
\hline W606 & MU-W606-EC1-18102 & $3 / 2 / 06$ & 3.2 & ND & EC1 & Branch & 142 & $\begin{array}{l}\text { of tree. } \\
\text { South side of tree. No leaves on branches. }\end{array}$ \\
\hline W606 & MU-W606-EC2-18103 & $3 / 2 / 06$ & 1.6 & 7.4 & EC2 & Branch & 140 & South side of tree. No leaves on branches. \\
\hline W820 & MU-W820-EC1-18114 & $3 / 2 / 06$ & ND & ND & EC1 & Branch & 112 & North side of tree. No leaves on branches. \\
\hline W830 & MU-W830-EC-18117 & $3 / 2 / 06$ & ND & ND & EC & Branch & 109 & South side of tree. No leaves on branches. \\
\hline W830 & MU-W830-NC-18118 & $3 / 2 / 06$ & ND & $<1$ & $\mathrm{NC}$ & Branch & 122 & North side of tree. No leaves on branches. \\
\hline W845 & MU-W845-EC1-18119 & $3 / 2 / 06$ & ND & ND & EC1 & Branch & 84 & South side of tree. No leaves on branches. \\
\hline W854 & MU-W854-EC-18120 & $3 / 2 / 06$ & ND & $<1$ & EC & Branch & 102 & South side of tree. No leaves on branches. \\
\hline W854 & MU-W854-GA-18121 & $3 / 2 / 06$ & $<1$ & 1.0 & $\mathrm{GA}$ & Branch & 107 & East side of tree. No leaves on branches. \\
\hline W908 & MU-W908-EC1-18109 & $3 / 2 / 06$ & ND & 2.8 & EC1 & Branch & 132 & South side of tree. No leaves on branches. \\
\hline PREE009 & MU-PREE009-18104 & $3 / 2 / 06$ & 2.1 & 4.7 & & Branch & 94 & South side of tree. No leaves on branches. \\
\hline PREW006 & MU-PREW006-18105 & $3 / 2 / 06$ & $<1$ & 1.2 & & Branch & 211 & South side of tree. No leaves on branches. \\
\hline Bruttig-2 & MU-BRUTTIG-18122 & $3 / 2 / 06$ & ND & 2.4 & & Branch & 165 & $\begin{array}{l}\text { Tree located in front yard at southwest } \\
\text { corner of house, east of the driveway. } \\
\text { Sample collected on south side of tree. }\end{array}$ \\
\hline School-1 & MU-SCHOOL-18123 & $3 / 2 / 06$ & 8.4 & 2.1 & & Branch & 193 & $\begin{array}{l}\text { Tree located south of school parking lot, } \\
\text { southwest from the corner of the school } \\
\text { building. Sample collected on south } \\
\text { side of tree. }\end{array}$ \\
\hline
\end{tabular}


TABLE E.1 (Cont.)

Location

Sample

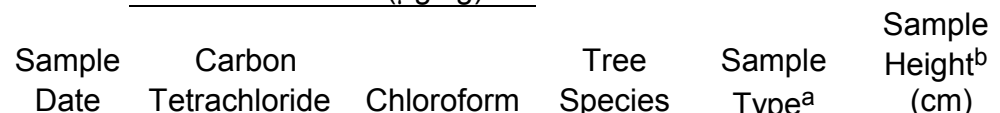

Sample Description

Tree branch samples collected in April 2006

$\begin{array}{llll}\text { E101 } & \text { MUE101-WN1-B-18658 } & 4 / 3 / 06 & \text { ND } \\ \text { E106 } & \text { MUE106-WN1-B-18659 } & 4 / 3 / 06 & \text { ND } \\ \text { E111 } & \text { MUE111-WN1-B-18660 } & 4 / 3 / 06 & \text { ND } \\ \text { E116 } & \text { MUE116-WN2-B-18661 } & 4 / 3 / 06 & \text { ND } \\ \text { E126 } & \text { MUE126-WN1-B-18663 } & 4 / 3 / 06 & \text { ND } \\ \text { E129 } & \text { MUE129-WN1-B-18664 } & 4 / 3 / 06 & \text { ND } \\ \text { E245 } & \text { MUE245-WN1-B-18665 } & 4 / 3 / 06 & <1 \\ \text { E304 } & \text { MUE304-WN2-B-18673 } & 4 / 3 / 06 & \text { ND } \\ \text { E309 } & \text { MUE309-WN1-B-18672 } & 4 / 3 / 06 & <1 \\ \text { E314 } & \text { MUE314-HP1-B-18671 } & 4 / 3 / 06 & 34 \\ \text { E319 } & \text { MUE319-WN2-B-18670 } & 4 / 3 / 06 & \text { ND } \\ \text { E324 } & \text { MUE324-WN1-B-18669 } & 4 / 3 / 06 & \text { ND } \\ \text { E329 } & \text { MUE329-WN1-B-18668 } & 4 / 3 / 06 & \text { ND } \\ \text { E340 } & \text { MUE340-WN2-B-18667 } & 4 / 3 / 06 & \text { ND } \\ \text { E345 } & \text { MUE345-WN1-B-18666 } & 4 / 3 / 06 & \text { ND } \\ \text { E438 } & \text { MUE438-WN1-B-18674 } & 4 / 3 / 06 & \text { ND } \\ \text { E502 } & \text { MUE502-WN1-B-18678 } & 4 / 3 / 06 & \text { ND } \\ \text { E507 } & \text { MUE507-EC-B-18679 } & 4 / 3 / 06 & \text { ND } \\ \text { E512 } & \text { MUE512-WN2-B-18680 } & 4 / 3 / 06 & \text { ND } \\ \text { E517 } & \text { MUE517-WN1-B-18681 } & 4 / 3 / 06 & \text { ND } \\ \text { E522 } & \text { MUE522-EC-B-18682 } & 4 / 3 / 06 & <1 \\ \text { E527 } & \text { MUE527-WN2-B-18683 } & 4 / 3 / 06 & \text { ND } \\ \text { E532 } & \text { MUE532-WN1-B-18684 } & 4 / 3 / 06 & \text { ND } \\ \text { E537 } & \text { MUE537-WN1-B-18685 } & 4 / 3 / 06 & \text { ND } \\ \text { E542 } & \text { MUE542-WN-B-18675 } & 4 / 3 / 06 & \text { ND } \\ \text { E547 } & \text { MUE547-WN2-B-18676 } & 4 / 3 / 06 & \text { ND } \\ \text { E550 } & \text { MUE550-WN1-B-18677 } & 4 / 3 / 06 & \text { ND } \\ \text { E550 } & \text { MUE550-WN1-B-18686 } & 4 / 3 / 06 & \text { ND } \\ \text { E555 } & \text { MUE555-WN1-B-18687 } & 4 / 3 / 06 & \text { ND } \\ \text { E557 } & \text { MUE557-WN1-B-18688 } & 4 / 3 / 06 & \text { ND } \\ \text { E659 } & \text { MUE659-WN1-B-18689 } & 4 / 3 / 06 & \text { ND } \\ \text { E700 } & \text { MUE700-EC1-B-18690 } & 4 / 4 / 06 & \text { ND } \\ \text { E705 } & \text { MUE705-EC2-B-18691 } & 4 / 4 / 06 & \text { ND } \\ \text { E710 } & \text { MUE710-EC-B-18692 } & 4 / 4 / 06 & \text { ND }\end{array}$

$\begin{array}{cccc}\text { ND } & \text { WN1 } & \text { Branch } & 76 \\ \text { ND } & \text { WN1 } & \text { Branch } & 113 \\ 1.1 & \text { WN1 } & \text { Branch } & 100 \\ \text { ND } & \text { WN2 } & \text { Branch } & \text { NR } \\ \text { ND } & \text { WN1 } & \text { Branch } & 78 \\ \text { ND } & \text { WN1 } & \text { Branch } & 78 \\ \text { ND } & \text { WN1 } & \text { Branch } & 85 \\ \text { ND } & \text { WN2 } & \text { Branch } & 73 \\ \text { ND } & \text { WN1 } & \text { Branch } & 117 \\ \text { ND } & \text { HP1 } & \text { Branch } & 64 \\ \text { ND } & \text { WN2 } & \text { Branch } & 105 \\ \text { ND } & \text { WN1 } & \text { Branch } & 92 \\ \text { ND } & \text { WN1 } & \text { Branch } & \text { NR } \\ \text { ND } & \text { WN2 } & \text { Branch } & 87 \\ \text { ND } & \text { WN1 } & \text { Branch } & 72 \\ \text { ND } & \text { WN1 } & \text { Branch } & 69 \\ \text { ND } & \text { WN1 } & \text { Branch } & 81 \\ \text { ND } & \text { EC } & \text { Branch } & 47 \\ \text { ND } & \text { WN2 } & \text { Branch } & 74 \\ \text { ND } & \text { WN1 } & \text { Branch } & 86 \\ \text { ND } & \text { EC } & \text { Branch } & 81 \\ \text { ND } & \text { WN2 } & \text { Branch } & 64 \\ \text { ND } & \text { WN1 } & \text { Branch } & 64 \\ \text { 3.6 } & \text { WN1 } & \text { Branch } & 116 \\ \text { ND } & \text { WN } & \text { Branch } & 81 \\ \text { ND } & \text { WN2 } & \text { Branch } & 68 \\ \text { ND } & \text { WN1 } & \text { Branch } & 90 \\ \text { ND } & \text { WN1 } & \text { Branch } & 88 \\ \text { ND } & \text { WN1 } & \text { Branch } & 88 \\ \text { ND } & \text { WN1 } & \text { Branch } & 79 \\ \text { ND } & \text { WN1 } & \text { Branch } & 86 \\ 5.3 & \text { EC1 } & \text { Branch } & 98 \\ \text { ND } & \text { EC2 } & \text { Branch } & 84 \\ \text { ND } & \text { EC } & \text { Branch } & 127\end{array}$


TABLE E.1 (Cont.)

Location

Sample

Sample Carbon

Tetrachloride

Type ${ }^{a}$

Sample

(cm)

Sample Description

Tree branch samples collected in April 2006 (cont.)

$\begin{array}{lll}\text { E715 } & \text { MUE715-EC2-B-18693 } & 4 / 4 / 06 \\ \text { E720 } & \text { MUE720-EC-B-18694 } & 4 / 4 / 06 \\ \text { E725 } & \text { MUE725-EC-B-18695 } & 4 / 4 / 06 \\ \text { E730 } & \text { MUE730-EC-B-18696 } & 4 / 4 / 06 \\ \text { E735 } & \text { MUE735-EC1-B-18697 } & 4 / 4 / 06 \\ \text { E740 } & \text { MUE740-EC-B-18698 } & 4 / 4 / 06 \\ \text { E758 } & \text { MUE758-EC1-B-18699 } & 4 / 4 / 06 \\ \text { E845 } & \text { MUE845-WN2-B-18700 } & 4 / 4 / 06 \\ \text { E858 } & \text { MUE858-HP-B-18701 } & 4 / 4 / 06 \\ \text { E862 } & \text { MUE862-EC-B-18702 } & 4 / 4 / 06 \\ \text { E869 } & \text { MUE869-EC-B-18705 } & 4 / 4 / 06 \\ \text { E871 } & \text { MUE871-EC-B-18704 } & 4 / 4 / 06 \\ & & \\ \text { W103 } & \text { MUW103-EC1-B-18706 } & 4 / 4 / 06 \\ \text { W107 } & \text { MUW107-WN2-B-18707 } & 4 / 4 / 06 \\ \text { W111 } & \text { MUW111-WN1-B-18709 } & 4 / 4 / 06 \\ \text { W111 } & \text { MUW111-WN2-B-18708 } & 4 / 4 / 06 \\ \text { W114 } & \text { MUW114-WN2-B-18710 } & 4 / 4 / 06 \\ \text { W119 } & \text { MUW119-WN1-B-18711 } & 4 / 4 / 06 \\ \text { W119 } & \text { MUW119-WN2-B-18712 } & 4 / 4 / 06 \\ \text { W121 } & \text { MUW121-WN2-B-18662 } & 4 / 3 / 06 \\ \text { W124 } & \text { MUW124-WN2-B-18713 } & 4 / 4 / 06 \\ \text { W128 } & \text { MUW128-WN1-B-18715 } & 4 / 4 / 06 \\ \text { W128 } & \text { MUW128-WN2-B-18714 } & 4 / 4 / 06 \\ \text { W129 } & \text { MUW129-WN1-B-18717 } & 4 / 4 / 06 \\ \text { W129 } & \text { MUW129-WN2-B-18716 } & 4 / 4 / 06 \\ \text { W132 } & \text { MUW132-WN1-B-18718 } & 4 / 4 / 06 \\ \text { W134 } & \text { MUW134-WN1-B-18719 } & 4 / 4 / 06 \\ \text { W139 } & \text { MUW139-WN2-B-18720 } & 4 / 4 / 06 \\ \text { W144 } & \text { MUW144-WN2-B-18721 } & 4 / 4 / 06 \\ \text { W148 } & \text { MUW148-WN2-B-18722 } & 4 / 4 / 06 \\ \text { W153 } & \text { MUW153-WN2-B-18723 } & 4 / 4 / 06 \\ \text { W157 } & \text { MUW157-WN1-B-18724 } & 4 / 4 / 06 \\ \text { W158 } & \text { MUW158-WN2-B-18726 } & 4 / 4 / 06 \\ & & \\ & & \end{array}$

$\begin{array}{lcclr}\text { ND } & \text { ND } & \text { EC2 } & \text { Branch } & 84 \\ \text { ND } & \text { ND } & \text { EC } & \text { Branch } & 104 \\ \text { ND } & \text { ND } & \text { EC } & \text { Branch } & 77 \\ \text { ND } & \text { ND } & \text { EC } & \text { Branch } & 96 \\ \text { ND } & \text { ND } & \text { EC1 } & \text { Branch } & 108 \\ \text { ND } & 2.7 & \text { EC } & \text { Branch } & 75 \\ \text { ND } & \text { ND } & \text { EC1 } & \text { Branch } & 78 \\ \text { ND } & \text { ND } & \text { WN2 } & \text { Branch } & 67 \\ \text { ND } & <1 & \text { HP } & \text { Branch } & 61 \\ \text { ND } & \text { ND } & \text { EC } & \text { Branch } & 69 \\ \text { ND } & 1.7 & \text { EC } & \text { Branch } & 105 \\ \text { ND } & \text { ND } & \text { EC } & \text { Branch } & 90 \\ & & & & \\ \text { ND } & 2.3 & \text { EC1 } & \text { Branch } & 83 \\ \text { ND } & \text { ND } & \text { WN2 } & \text { Branch } & 98 \\ <1 & \text { ND } & \text { WN1 } & \text { Branch } & 83 \\ <1 & \text { ND } & \text { WN2 } & \text { Branch } & 77 \\ \text { ND } & \text { ND } & \text { WN2 } & \text { Branch } & 87 \\ \text { ND } & \text { ND } & \text { WN1 } & \text { Branch } & 81 \\ \text { ND } & 1.1 & \text { WN2 } & \text { Branch } & 75 \\ \text { ND } & \text { ND } & \text { WN2 } & \text { Branch } & 77 \\ \text { ND } & \text { ND } & \text { WN2 } & \text { Branch } & 81 \\ \text { ND } & 2.0 & \text { WN1 } & \text { Branch } & 60 \\ <1 & \text { ND } & \text { WN2 } & \text { Branch } & 70 \\ <1 & \text { ND } & \text { WN1 } & \text { Branch } & 62 \\ <1 & \text { ND } & \text { WN2 } & \text { Branch } & 85 \\ <1 & \text { ND } & \text { WN1 } & \text { Branch } & 89 \\ \text { ND } & \text { ND } & \text { WN1 } & \text { Branch } & 76 \\ \text { ND } & \text { ND } & \text { WN2 } & \text { Branch } & 91 \\ \text { ND } & \text { ND } & \text { WN2 } & \text { Branch } & 72 \\ \text { ND } & \text { ND } & \text { WN2 } & \text { Branch } & 86 \\ \text { ND } & \text { ND } & \text { WN2 } & \text { Branch } & 89 \\ \text { ND } & \text { ND } & \text { WN1 } & \text { Branch } & 92 \\ <1 & \text { ND } & \text { WN2 } & \text { Branch } & 89\end{array}$


TABLE E.1 (Cont.)

Location

Sample

Sample Carbon

Tree

Sample

ample

Date Tetrachloride Chloroform Species

Type $^{a}$

Sample Description

Tree branch samples collected in April 2006 (cont.)

W159

W203

W210

W210

W211

W303

W308

W357

W402

W407

W412

W417

W422

W426

W432

W436

W442

W447

W452

W457

W505

W601

W606

W611

W616

W621

W626

W636

W641

W646

W651

N656

W801

W806

$\begin{array}{ll}\text { MUW159-EC2-B-18727 } & 4 / 4 / 06 \\ \text { MUW203-WN1-B-18728 } & 4 / 4 / 06 \\ \text { MUW210-WN1-B-18730 } & 4 / 4 / 06 \\ \text { MUW210-WN2-B-18729 } & 4 / 4 / 06 \\ \text { MUW211-GA-B-18731 } & 4 / 4 / 06 \\ \text { MUW303-EC1-B-18732 } & 4 / 4 / 06 \\ \text { MUW308-EC-B-18733 } & 4 / 4 / 06 \\ \text { MUW357-WN1-B-18734 } & 4 / 4 / 06 \\ \text { MUW402-EC2-B-18736 } & 4 / 4 / 06 \\ \text { MUW407-WN2-B-18737 } & 4 / 4 / 06 \\ \text { MUW412-EC2-B-18747 } & 4 / 4 / 06 \\ \text { MUW417-EC1-B-18746 } & 4 / 4 / 06 \\ \text { MUW422-EC2-B-18745 } & 4 / 4 / 06 \\ \text { MUW426-EC2-B-18744 } & 4 / 4 / 06 \\ \text { MUW432-EC-B-18743 } & 4 / 4 / 06 \\ \text { MUW436-WN2-B-18742 } & 4 / 4 / 06 \\ \text { MUW442-WN-B-18741 } & 4 / 4 / 06 \\ \text { MUW447-WN-B-18740 } & 4 / 4 / 06 \\ \text { MUW452-WN2-B-18739 } & 4 / 4 / 06 \\ \text { MUW457-EC-B-18738 } & 4 / 4 / 06 \\ \text { MUW505-EC2-B-18748 } & 4 / 4 / 06 \\ \text { MUW601-EC-B-18749 } & 4 / 4 / 06 \\ \text { MUW606-EC2-B-18750 } & 4 / 4 / 06 \\ \text { MUW611-EC1-B-18751 } & 4 / 4 / 06 \\ \text { MUW616-EC1-B-18752 } & 4 / 4 / 06 \\ \text { MUW621-EC-B-18753 } & 4 / 4 / 06 \\ \text { MUW626-EC-B-18754 } & 4 / 4 / 06 \\ \text { MUW636-EC-B-18755 } & 4 / 4 / 06 \\ \text { MUW641-EC1-B-18756 } & 4 / 4 / 06 \\ \text { MUW646-WN1-B-18757 } & 4 / 4 / 06 \\ \text { MUW651-WN2-B-18758 } & 4 / 4 / 06 \\ \text { MUW656-EC1-B-18759 } & 4 / 4 / 06 \\ \text { MUW801-EC2-B-18772 } & 4 / 4 / 06 \\ \text { MUW806-EC2-B-18771 } & 4 / 4 / 06 \\ \end{array}$

$\begin{array}{lcclr}<1 & 3.5 & \text { EC2 } & \text { Branch } & 44 \\ \text { ND } & 1.6 & \text { WN1 } & \text { Branch } & 109 \\ \text { ND } & \text { ND } & \text { WN1 } & \text { Branch } & 76 \\ <1 & 3.5 & \text { WN2 } & \text { Branch } & 84 \\ \text { ND } & \text { ND } & \text { GA } & \text { Branch } & 91 \\ \text { ND } & 1.4 & \text { EC1 } & \text { Branch } & 85 \\ \text { ND } & \text { ND } & \text { EC } & \text { Branch } & 71 \\ \text { ND } & \text { ND } & \text { WN1 } & \text { Branch } & 98 \\ \text { ND } & \text { ND } & \text { EC2 } & \text { Branch } & 111 \\ <1 & \text { ND } & \text { WN2 } & \text { Branch } & \text { NR } \\ \text { ND } & 3.2 & \text { EC2 } & \text { Branch } & 85 \\ \text { ND } & \text { ND } & \text { EC1 } & \text { Branch } & 80 \\ 1.6 & \text { ND } & \text { EC2 } & \text { Branch } & 116 \\ <1 & \text { ND } & \text { EC2 } & \text { Branch } & 113 \\ \text { ND } & \text { ND } & \text { EC } & \text { Branch } & 99 \\ \text { ND } & \text { ND } & \text { WN2 } & \text { Branch } & 107 \\ \text { ND } & 5.2 & \text { WN } & \text { Branch } & 74 \\ \text { ND } & \text { ND } & \text { WN } & \text { Branch } & 98 \\ \text { ND } & 2.0 & \text { WN2 } & \text { Branch } & 109 \\ \text { ND } & \text { ND } & \text { EC } & \text { Branch } & 90 \\ \text { ND } & \text { ND } & \text { EC2 } & \text { Branch } & 102 \\ \text { ND } & 1.0 & \text { EC } & \text { Branch } & 122 \\ <1 & \text { ND } & \text { EC2 } & \text { Branch } & 122 \\ \text { ND } & \text { ND } & \text { EC1 } & \text { Branch } & 86 \\ <1 & \text { ND } & \text { EC1 } & \text { Branch } & 99 \\ \text { ND } & \text { ND } & \text { EC } & \text { Branch } & 72 \\ <1 & \text { ND } & \text { EC } & \text { Branch } & 85 \\ <1 & \text { ND } & \text { EC } & \text { Branch } & 80 \\ \text { ND } & 0.9 & \text { EC1 } & \text { Branch } & 95 \\ \text { ND } & \text { ND } & \text { WN1 } & \text { Branch } & 97 \\ \text { ND } & \text { ND } & \text { WN2 } & \text { Branch } & 103 \\ \text { ND } & \text { ND } & \text { EC1 } & \text { Branch } & 83 \\ \text { ND } & \text { ND } & \text { EC2 } & \text { Branch } & 109 \\ <1 & \text { ND } & \text { EC2 } & \text { Branch } & 81\end{array}$


TABLE E.1 (Cont.)

\begin{tabular}{|c|c|c|c|c|c|c|c|c|}
\hline & & & Concentrati & $\mathrm{n}(\mu \mathrm{g} / \mathrm{kg})$ & & & & \\
\hline Location & Sample & $\begin{array}{c}\text { Sample } \\
\text { Date }\end{array}$ & $\begin{array}{c}\text { Carbon } \\
\text { Tetrachloride }\end{array}$ & Chloroform & $\begin{array}{c}\text { Tree } \\
\text { Species }\end{array}$ & $\begin{array}{c}\text { Sample } \\
\text { Type }^{a}\end{array}$ & $\begin{array}{l}\text { Height }^{\mathrm{b}} \\
(\mathrm{cm})\end{array}$ & Sample Description \\
\hline
\end{tabular}

Tree branch samples collected in April 2006 (cont.)

\begin{tabular}{|c|c|c|c|c|c|c|c|}
\hline W810 & MUW810-EC2-B-18770 & $4 / 4 / 06$ & ND & ND & EC2 & Branch & 97 \\
\hline W815 & MUW815-EC2-B-18769 & $4 / 4 / 06$ & ND & ND & EC2 & Branch & 98 \\
\hline W820 & MUW820-EC2-B-18768 & $4 / 4 / 06$ & ND & ND & EC2 & Branch & 99 \\
\hline W825 & MUW825-EC1-B-18767 & $4 / 4 / 06$ & ND & ND & EC1 & Branch & 90 \\
\hline W830 & MUW830-EC-B-18766 & $4 / 4 / 06$ & ND & ND & EC & Branch & 110 \\
\hline W835 & MUW835-EC-B-18765 & $4 / 4 / 06$ & ND & ND & EC & Branch & 80 \\
\hline W840 & MUW840-WN1-B-18764 & $4 / 4 / 06$ & ND & ND & WN1 & Branch & 92 \\
\hline W845 & MUW845-EC1-B-18763 & $4 / 4 / 06$ & ND & 3.4 & EC1 & Branch & 82 \\
\hline W849 & MUW849-EC1-B-18762 & $4 / 4 / 06$ & ND & 0.9 & EC1 & Branch & 95 \\
\hline W854 & MUW854-EC-B-18761 & $4 / 4 / 06$ & ND & 22 & EC & Branch & 105 \\
\hline W859 & MUW859-EC-B-18760 & $4 / 4 / 06$ & ND & ND & EC & Branch & 97 \\
\hline W908 & MUW908-EC1-B-18773 & $4 / 4 / 06$ & ND & 1.2 & EC1 & Branch & 106 \\
\hline PREW001 & MUPREW001-B-18777 & $4 / 4 / 06$ & ND & 1.5 & & Branch & 100 \\
\hline PREW002 & MUPREW002-B-18778 & $4 / 4 / 06$ & ND & 3.8 & & Branch & 180 \\
\hline PREW005 & MUPREW005-B-18781 & $4 / 4 / 06$ & ND & 2.2 & & Branch & 150 \\
\hline PREW006 & MUPREW006-B-18779 & $4 / 4 / 06$ & 1.5 & 1.0 & & Branch & 180 \\
\hline PREE008 & MUPREE008-B-18782 & $4 / 4 / 06$ & ND & ND & & Branch & 150 \\
\hline PREE009 & MUPREE009-B-18780 & $4 / 4 / 06$ & 1.9 & ND & & Branch & \\
\hline Bruttig-2 & MUBRUTTIG1-B-18774 & $4 / 4 / 06$ & ND & 11 & & Branch & NR \\
\hline Bruttig-3 & MUBRUTTIG2-B-18775 & $4 / 4 / 06$ & ND & 1.4 & & Branch & NR \\
\hline School-1 & MUSCHOOLASH-N-18776 & $4 / 4 / 06$ & ND & 1.6 & & Branch & NR \\
\hline \multicolumn{8}{|c|}{ Tree branch samples collected in August 2006} \\
\hline E100 & MUE100-WN1-B-18923 & $8 / 16 / 06$ & ND & 4.4 & WN1 & Branch & 102 \\
\hline E101 & MUE101-WN1-B-18924 & $8 / 16 / 06$ & ND & 4.6 & WN1 & Branch & 99 \\
\hline E106 & MUE106-WN1-B-18925 & $8 / 16 / 06$ & $<1$ & 3.9 & WN1 & Branch & 117 \\
\hline E111 & MUE111-WN1-B-18926 & $8 / 16 / 06$ & $<1$ & 12 & WN1 & Branch & 112 \\
\hline E112 & MUE112-WN2-B-18864 & $8 / 15 / 06$ & 7.3 & 9.3 & WN2 & Branch & 84 \\
\hline E113 & MUE113-WN1-B-18866 & $8 / 15 / 06$ & 5.8 & 16 & WN1 & Branch & 107 \\
\hline
\end{tabular}

Near air monitoring location AA2.

Shaded, near air monitoring location AA2. 
Location

Sample

\begin{tabular}{|c|c|c|c|c|c|}
\hline & Concentrat & $(\mu \mathrm{g} / \mathrm{kg})$ & & & \\
\hline $\begin{array}{l}\text { Sample } \\
\text { Date }\end{array}$ & $\begin{array}{c}\text { Carbon } \\
\text { Tetrachloride }\end{array}$ & Chloroform & $\begin{array}{c}\text { Tree } \\
\text { Species }\end{array}$ & $\begin{array}{c}\text { Sample } \\
\text { Type }^{a}\end{array}$ & $\begin{array}{l}\text { Height }^{b} \\
\text { (cm) }\end{array}$ \\
\hline
\end{tabular}

Sample Description

Tree branch samples collected in August 2006 (cont.)

\begin{tabular}{|c|c|c|c|c|c|c|c|}
\hline E116 & MUE116-WN2-B-18927 & $8 / 16 / 06$ & ND & 4.8 & WN2 & Branch & 117 \\
\hline E118 & MUE118-WN1-B-18928 & $8 / 16 / 06$ & ND & 2.4 & WN1 & Branch & 76 \\
\hline E121 & MUE121-WN1-B-18929 & $8 / 16 / 06$ & ND & 2.4 & WN1 & Branch & 102 \\
\hline E126 & MUE126-WN1-B-18930 & $8 / 16 / 06$ & $<1$ & 9.6 & WN1 & Branch & 97 \\
\hline E129 & MUE129-WN1-B-18931 & $8 / 16 / 06$ & $<1$ & 2.5 & WN1 & Branch & NR \\
\hline E206 & MUE206-WN2-B-18932 & $8 / 16 / 06$ & 2.2 & 7.8 & WN2 & Branch & 86 \\
\hline E207 & MUE207-WN1-B-18933 & $8 / 16 / 06$ & $<1$ & 5.8 & WN1 & Branch & 91 \\
\hline E207 & MUE207-WN2-B-18934 & $8 / 16 / 06$ & 3.2 & 14 & WN2 & Branch & 76 \\
\hline E245 & MUE245-WN1-B-18935 & $8 / 16 / 06$ & $<1$ & 5.4 & WN1 & Branch & 84 \\
\hline E304 & MUE304-WN2-B-18917 & $8 / 16 / 06$ & $<1$ & 2.1 & WN2 & Branch & 122 \\
\hline E308 & MUE308-WN1-B-18915 & $8 / 16 / 06$ & 28 & 6.3 & WN1 & Branch & 84 \\
\hline E308 & MUE308-WN2-B-18916 & $8 / 16 / 06$ & 78 & 15 & WN2 & Branch & 109 \\
\hline E309 & MUE309-WN1-B-18914 & $8 / 16 / 06$ & 3.3 & 3.7 & WN1 & Branch & 58 \\
\hline E309 & MUE309-WN2-B-18913 & $8 / 16 / 06$ & 12 & 8.1 & WN2 & Branch & 124 \\
\hline E314 & MUE314-HP1-B-18912 & $8 / 16 / 06$ & 13 & 13 & HP1 & Branch & 130 \\
\hline E319 & MUE319-WN2-B-18911 & $8 / 16 / 06$ & $<1$ & 3.1 & WN2 & Branch & 84 \\
\hline E324 & MUE324-WN1-B-18910 & $8 / 16 / 06$ & $<1$ & ND & WN1 & Branch & 51 \\
\hline E329 & MUE329-WN1-B-18909 & $8 / 16 / 06$ & ND & ND & WN1 & Branch & 102 \\
\hline E340 & MUE340-WN2-B-18908 & $8 / 16 / 06$ & ND & ND & WN2 & Branch & 107 \\
\hline E345 & MUE345-WN1-B-18907 & $8 / 16 / 06$ & $<1$ & 2.1 & WN1 & Branch & 107 \\
\hline E438 & MUE438-WN1-B-18906 & $8 / 16 / 06$ & $<1$ & 1.6 & WN1 & Branch & 109 \\
\hline E500 & MUE500-EC1-B-18890 & $8 / 16 / 06$ & ND & ND & EC1 & Branch & 99 \\
\hline E502 & MUE502-WN1-B-18891 & $8 / 16 / 06$ & ND & 4.8 & WN1 & Branch & 86 \\
\hline E507 & MUE507-EC-B-18892 & $8 / 16 / 06$ & ND & 1.1 & $\mathrm{EC}$ & Branch & 89 \\
\hline E512 & MUE512-WN1-B-18893 & $8 / 16 / 06$ & ND & 1.1 & WN1 & Branch & 8 \\
\hline E517 & MUE517-WN1-B-18894 & $8 / 16 / 06$ & 7.2 & ND & WN1 & Branch & 20 \\
\hline E522 & MUE522-EC-B-18896 & 8/16/06 & 3.1 & 2.8 & EC & Branch & 74 \\
\hline E527 & MUE527-WN2-B-18897 & $8 / 16 / 06$ & ND & 1.6 & WN2 & Branch & 99 \\
\hline E532 & MUE532-WN1-B-18898 & $8 / 16 / 06$ & ND & 4.3 & WN1 & Branch & 99 \\
\hline
\end{tabular}

Weak tree, few large leaves, many new small leaves but not as vigorous. Much weedy vine.

Vigorous.

Perfect branches.

Only branch of right size with north orientation.

Tree suffering. Few leaves. Cankered.

Branch reddish.

Secondary branch sampled.

New sappling. No WN2 tree existing at location.

Reddish goo on branch. 
TABLE E.1 (Cont.)

\begin{tabular}{|c|c|c|c|c|c|c|c|c|}
\hline & & & Concentrati & $\mathrm{n}(\mu \mathrm{g} / \mathrm{kg})$ & & & & \\
\hline Location & Sample & $\begin{array}{l}\text { Sample } \\
\text { Date }\end{array}$ & $\begin{array}{c}\text { Carbon } \\
\text { Tetrachloride }\end{array}$ & Chloroform & $\begin{array}{c}\text { Tree } \\
\text { Species }\end{array}$ & $\begin{array}{c}\text { Sample } \\
\text { Type }^{a}\end{array}$ & $\begin{array}{c}\text { Height }^{\mathrm{b}} \\
(\mathrm{cm})\end{array}$ & Sample Description \\
\hline
\end{tabular}

Tree branch samples collected in August 2006 (cont.)

\begin{tabular}{|c|c|c|c|c|c|c|}
\hline E537 & MUE537-WN1-B-18899 & $8 / 16 / 06$ & ND & 1.9 & WN1 & Branch \\
\hline E542 & MUE542-WN-B-18900 & $8 / 16 / 06$ & ND & 1.4 & WN & Branch \\
\hline E547 & MUE547-WN2-B-18901 & $8 / 16 / 06$ & ND & 6.2 & WN2 & Branch \\
\hline E550 & MUE550-WN1-B-18902 & $8 / 16 / 06$ & 2.8 & 1.5 & WN1 & Branch \\
\hline E555 & MUE555-WN1-B-18903 & $8 / 16 / 06$ & $<1$ & ND & WN1 & Branch \\
\hline E557 & MUE557-WN1-B-18904 & $8 / 16 / 06$ & $<1$ & 1.1 & WN1 & Branch \\
\hline E659 & MUE659-WN1-B-18905 & $8 / 16 / 06$ & 2.0 & 1.0 & WN1 & Branch \\
\hline E700 & MUE700-EC1-B-18887 & $8 / 16 / 06$ & ND & ND & EC1 & Branch \\
\hline E705 & MUE705-EC2-B-18886 & $8 / 16 / 06$ & ND & ND & EC2 & Branch \\
\hline E710 & MUE710-EC-B-18885 & $8 / 16 / 06$ & ND & 1.0 & $\mathrm{EC}$ & Branch \\
\hline E715 & MUE715-EC2-B-18884 & $8 / 16 / 06$ & ND & $<1$ & EC2 & Branch \\
\hline E720 & MUE720-EC-B-18883 & $8 / 16 / 06$ & ND & 2.1 & $\mathrm{EC}$ & Branch \\
\hline E720 & MUE720-GA-B-18882 & $8 / 16 / 06$ & ND & 2.6 & $\mathrm{GA}$ & Branch \\
\hline E725 & MUE725-EC-B-18881 & 8/16/06 & ND & 1.4 & $\mathrm{EC}$ & Branch \\
\hline E730 & MUE730-EC-B-18880 & $8 / 16 / 06$ & $<1$ & 5.9 & EC & Branch \\
\hline E735 & MUE735-EC1-B-18879 & $8 / 16 / 06$ & $<1$ & 2.1 & EC1 & Branch \\
\hline E735 & MUE735-EC2-B-18878 & $8 / 16 / 06$ & $<1$ & 1.4 & EC2 & Branch \\
\hline E740 & MUE740-EC-B-18877 & $8 / 16 / 06$ & ND & 1.9 & $\mathrm{EC}$ & Branch \\
\hline E758 & MUE758-EC1-B-18876 & $8 / 16 / 06$ & $<1$ & 4.7 & EC1 & Branch \\
\hline E845 & MUE845-WN1-B-18868 & $8 / 16 / 06$ & ND & 1.4 & WN1 & Branch \\
\hline E845 & MUE845-WN2-B-18869 & $8 / 16 / 06$ & ND & 1.2 & WN2 & Branch \\
\hline E858 & MUE858-HP-B-18870 & $8 / 16 / 06$ & 19 & 7.9 & $\mathrm{HP}$ & Branch \\
\hline E862 & MUE862-EC-B-18871 & $8 / 16 / 06$ & ND & 1.7 & EC & Branch \\
\hline E866 & MUE866-EC-B-18872 & $8 / 16 / 06$ & ND & 5.2 & $\mathrm{EC}$ & Branch \\
\hline E866 & MUE866-GA-B-18873 & $8 / 16 / 06$ & ND & 1.2 & GA & Branch \\
\hline E869 & MUE869-EC-B-18874 & $8 / 16 / 06$ & ND & 1.0 & EC & Branch \\
\hline E871 & MUE871-EC-B-18875 & $8 / 16 / 06$ & ND & 1.2 & EC & Branch \\
\hline
\end{tabular}

Mislabeled tree location at E556 was changed to E550. Saw original tag.

Eaten by grasshoppers?

Sample from two secondary branches from same branchlet.

New sucker sampled.

Vigorous tree.

Other tree at this location is not marked by species.

New sucker sampled. Tree significantly defoliated by insects.

Small tree.

New sucker sampled. 
Location

Sample

\begin{tabular}{|c|c|c|c|c|c|}
\hline & entra & $(\mu \mathrm{g} / \mathrm{kg})$ & & & \\
\hline $\begin{array}{l}\text { Sample } \\
\text { Date }\end{array}$ & $\begin{array}{c}\text { Carbon } \\
\text { Tetrachloride }\end{array}$ & Chloroform & $\begin{array}{c}\text { Tree } \\
\text { Species }\end{array}$ & $\begin{array}{c}\text { Sample } \\
\text { Type }^{a}\end{array}$ & $\begin{array}{l}\text { Height }^{b} \\
\text { (cm) }\end{array}$ \\
\hline
\end{tabular}

Tree branch samples collected in August 2006 (cont.)

\begin{tabular}{|c|c|c|c|c|c|c|}
\hline W103 & MUW103-EC1-B-18826 & $8 / 15 / 06$ & ND & 1.8 & EC1 & Branch \\
\hline W104 & MUW104-WN1-B-18888 & $8 / 16 / 06$ & ND & 2.3 & WN1 & Branch \\
\hline W106 & MUW106-WN1-B-18889 & $8 / 16 / 06$ & ND & 2.4 & WN1 & Branch \\
\hline W107 & MUW107-WN2-B-18827 & $8 / 15 / 06$ & $<1$ & 4.5 & WN2 & Branch \\
\hline W110 & MUW110-WN1-B-18859 & $8 / 15 / 06$ & 15 & 42 & WN1 & Branch \\
\hline W111 & MUW111-WN1-B-18828 & $8 / 15 / 06$ & 18 & 12 & WN1 & Branch \\
\hline W111 & MUW111-WN2-B-18829 & $8 / 15 / 06$ & 79 & 19 & WN2 & Branch \\
\hline W114 & MUW114-WN2-B-18830 & $8 / 15 / 06$ & 48 & 24 & WN2 & Branch \\
\hline W119 & MUW119-WN1-B-18831 & $8 / 15 / 06$ & 358 & 135 & WN1 & Branch \\
\hline W119 & MUW119-WN2-B-18832 & $8 / 15 / 06$ & 455 & 156 & WN2 & Branch \\
\hline W121 & MUW121-WN2-B-18833 & $8 / 15 / 06$ & 11 & 21 & WN2 & Branch \\
\hline W124 & MUW124-WN2-B-18834 & $8 / 15 / 06$ & 66 & 34 & WN2 & Branch \\
\hline W128 & MUW128-WN1-B-18835 & $8 / 15 / 06$ & 151 & 69 & WN1 & Branch \\
\hline W128 & MUW128-WN2-B-18836 & $8 / 15 / 06$ & 85 & 67 & WN2 & Branch \\
\hline W129 & MUW129-WN2-B-18837 & $8 / 15 / 06$ & 81 & 54 & WN2 & Branch \\
\hline W132 & MUW132-WN1-B-18838 & $8 / 15 / 06$ & 27 & 25 & WN1 & Branch \\
\hline W134 & MUW134-WN1-B-18839 & $8 / 15 / 06$ & 1.9 & 6.3 & WN1 & Branch \\
\hline W139 & MUW139-WN2-B-18840 & $8 / 15 / 06$ & 1.0 & 3.2 & WN2 & Branch \\
\hline W144 & MUW144-WN2-B-18841 & $8 / 15 / 06$ & $<1$ & 3.8 & WN2 & Branch \\
\hline W148 & MUW148-WN2-B-18842 & $8 / 15 / 06$ & 4.2 & 6.1 & WN2 & Branch \\
\hline W153 & MUW153-WN2-B-18843 & $8 / 15 / 06$ & 1.2 & 1.6 & WN2 & Branch \\
\hline W157 & MUW157-WN1-B-18844 & $8 / 15 / 06$ & 2.0 & 40 & WN1 & Branch \\
\hline W158 & MUW158-WN1-B-18846 & $8 / 15 / 06$ & 12 & 19 & WN1 & Branch \\
\hline W158 & MUW158-WN2-B-18845 & $8 / 15 / 06$ & 13 & 7.7 & WN2 & Branch \\
\hline W159 & MUW159-EC2-B-18847 & $8 / 15 / 06$ & 3.3 & 5.0 & EC2 & Branch \\
\hline W203 & MUW203-WN1-B-18848 & $8 / 15 / 06$ & $<1$ & 7.4 & WN1 & Branch \\
\hline W210 & MUW210-WN2-B-18849 & $8 / 15 / 06$ & 21 & 41 & WN2 & Branch \\
\hline W211 & MUW211-EC-B-18850 & $8 / 15 / 06$ & 6.4 & 24 & EC & Branch \\
\hline
\end{tabular}

Tree is suffering

Tree is suffering.

Also collected leaf sample as \#18855 near air monitoring location.

Also collected leaf sample as \#18856 near air monitoring location.

Also collected leaf sample as \#18857 near air monitoring AA1.

Also collected leaf sample as \#18858 near air monitoring AA1. 
Location

Sample

\begin{tabular}{|c|c|c|c|c|c|}
\hline & entra & $(\mu \mathrm{g} / \mathrm{kg})$ & & & \\
\hline $\begin{array}{l}\text { Sample } \\
\text { Date }\end{array}$ & $\begin{array}{c}\text { Carbon } \\
\text { Tetrachloride }\end{array}$ & Chloroform & $\begin{array}{c}\text { Tree } \\
\text { Species }\end{array}$ & $\begin{array}{c}\text { Sample } \\
\text { Type }^{a}\end{array}$ & $\begin{array}{l}\text { Height }^{b} \\
\text { (cm) }\end{array}$ \\
\hline
\end{tabular}

Tree branch samples collected in August 2006 (cont.)

\begin{tabular}{|c|c|c|c|c|c|c|c|}
\hline W212 & MUW212-WN2-B-18861 & $8 / 15 / 06$ & 21 & 17 & WN2 & Branch & 94 \\
\hline $\begin{array}{l}\text { W303 } \\
\text { W308 }\end{array}$ & $\begin{array}{l}\text { MUW303-EC1-B-18851 } \\
\text { MUW308-EC-B-18852 }\end{array}$ & $\begin{array}{l}8 / 15 / 06 \\
8 / 15 / 06\end{array}$ & $\begin{aligned} & 3.0 \\
< & 1\end{aligned}$ & $\begin{array}{l}5.3 \\
18\end{array}$ & $\begin{array}{l}\mathrm{EC} 1 \\
\mathrm{EC}\end{array}$ & $\begin{array}{l}\text { Branch } \\
\text { Branch }\end{array}$ & $\begin{array}{r}119 \\
8\end{array}$ \\
\hline W357 & MUW357-WN1-B-18853 & $8 / 15 / 06$ & $<1$ & 3.8 & WN1 & Branch & 20 \\
\hline W402 & MUW402-EC2-B-18810 & $8 / 15 / 06$ & ND & $<1$ & EC2 & Branch & 127 \\
\hline W407 & MUW407-WN1-B-18811 & $8 / 15 / 06$ & 101 & 41 & WN1 & Branch & 97 \\
\hline W407 & MUW407-WN2-B-18812 & $8 / 15 / 06$ & 10 & 16 & WN2 & Branch & 0 \\
\hline W412 & MUW412-EC2-B-18813 & $8 / 15 / 06$ & $<1$ & 1.3 & EC2 & Branch & 89 \\
\hline W417 & MUW417-EC1-B-18814 & $8 / 15 / 06$ & 22 & 11 & EC1 & Branch & 86 \\
\hline W422 & MUW422-EC2-B-18815 & $8 / 15 / 06$ & 12 & 11 & EC2 & Branch & 124 \\
\hline W426 & MUW426-EC2-B-18816 & $8 / 15 / 06$ & 132 & 35 & $\mathrm{EC} 2$ & Branch & 137 \\
\hline W427 & MUW427-EC-B-18817 & $8 / 15 / 06$ & 40 & 35 & EC & Branch & 132 \\
\hline W427 & MUW427-GA-B-18818 & $8 / 15 / 06$ & 3.6 & 9.8 & GA & Branch & 74 \\
\hline W432 & MUW432-EC-B-18819 & $8 / 15 / 06$ & ND & 3.7 & EC & Branch & 109 \\
\hline W436 & MUW436-WN2-B-18820 & $8 / 15 / 06$ & ND & 5.5 & WN2 & Branch & 41 \\
\hline W442 & MUW442-WN-B-18821 & $8 / 15 / 06$ & $<1$ & 2.1 & WN & Branch & 18 \\
\hline W447 & MUW447-WN-B-18822 & $8 / 15 / 06$ & $<1$ & 3.9 & WN & Branch & 107 \\
\hline W452 & MUW452-WN2-B-18823 & $8 / 15 / 06$ & $<1$ & 4.1 & WN2 & Branch & 109 \\
\hline W457 & MUW457-EC-B-18824 & $8 / 15 / 06$ & ND & 4.4 & EC & Branch & 109 \\
\hline W505 & MUW505-EC2-B-18825 & $8 / 15 / 06$ & 148 & 17 & EC2 & Branch & 119 \\
\hline W601 & MUW601-EC-B-18799 & $8 / 15 / 06$ & ND & 2.8 & $\mathrm{EC}$ & Branch & 117 \\
\hline W606 & MUW606-EC1-B-18800 & $8 / 15 / 06$ & 351 & 11 & EC1 & Branch & 10 \\
\hline W606 & MUW606-EC2-B-18801 & $8 / 15 / 06$ & 143 & 17 & EC2 & Branch & 10 \\
\hline W611 & MUW611-EC1-B-18802 & $8 / 15 / 06$ & 18 & 11 & EC1 & Branch & 89 \\
\hline
\end{tabular}

Close to air monitoring point AA1. Sketch of sample location in log.

Sampled low sucker. Also collected leaf sample as \#18863 near air monitoring point \#1 W308-W309, $6 \mathrm{ft}$ west of W211.

Sucker at ground level sampled.

$8 / 15 / 06$

Sampled only branch available.

Cankered tree. Many low-bedded branches at bottom, regrowth from bottom.

GA tree at this location incorrectly labeled EC2. Tree sampled was EC, labeled EC2.

Small branch. Only one available to sample.

Base sprout sampled.

Base sprout sampled. 
TABLE E.1 (Cont.)

\begin{tabular}{|c|c|c|c|c|c|c|c|c|}
\hline & & & Concentratic & n $(\mu \mathrm{g} / \mathrm{kg})$ & & & & \\
\hline Location & Sample & $\begin{array}{l}\text { Sample } \\
\text { Date }\end{array}$ & $\begin{array}{c}\text { Carbon } \\
\text { Tetrachloride }\end{array}$ & Chloroform & $\begin{array}{c}\text { Tree } \\
\text { Species }\end{array}$ & $\begin{array}{c}\text { Sample } \\
\text { Type }^{a}\end{array}$ & $\begin{array}{l}\text { Height }{ }^{b} \\
\text { (cm) }\end{array}$ & Sample Description \\
\hline
\end{tabular}

Tree branch samples collected in August 2006 (cont.)

\begin{tabular}{|c|c|c|c|c|c|c|c|c|}
\hline $\begin{array}{l}\text { W616 } \\
\text { W621 }\end{array}$ & $\begin{array}{l}\text { MUW616-EC1-B-18803 } \\
\text { MUW621-EC-B-18804 }\end{array}$ & $\begin{array}{l}8 / 15 / 06 \\
8 / 15 / 06\end{array}$ & $\begin{array}{l}2.9 \\
33\end{array}$ & $\begin{array}{l}7.2 \\
4.4\end{array}$ & $\begin{array}{c}\text { EC1 } \\
\text { EC }\end{array}$ & $\begin{array}{l}\text { Branch } \\
\text { Branch }\end{array}$ & $\begin{array}{r}109 \\
33\end{array}$ & $\begin{array}{l}\text { Base sprout sampled. GA tree mislabeled } \\
\text { as EC1. Sample collected from EC tree. }\end{array}$ \\
\hline $\begin{array}{l}\text { W626 } \\
\text { W636 } \\
\text { W641 }\end{array}$ & $\begin{array}{l}\text { MUW626-EC-B-18805 } \\
\text { MUW636-EC-B-18806 } \\
\text { MUW641-EC1-B-18807 }\end{array}$ & $\begin{array}{l}8 / 15 / 06 \\
8 / 15 / 06 \\
8 / 15 / 06\end{array}$ & $\begin{array}{l}22 \\
5.2 \\
<1\end{array}$ & $\begin{array}{r}23 \\
5.2 \\
3.2\end{array}$ & $\begin{array}{l}E C \\
E C \\
E C 1\end{array}$ & $\begin{array}{l}\text { Branch } \\
\text { Branch } \\
\text { Branch }\end{array}$ & $\begin{array}{r}86 \\
84 \\
102\end{array}$ & $\begin{array}{l}\text { Weeds overpowering planted trees. } \\
\text { Location is incorrectly labeled as W642. } \\
\text { Location sampled was actually W641. }\end{array}$ \\
\hline $\begin{array}{l}\text { W646 } \\
\text { W651 } \\
\text { W801 } \\
\text { W806 } \\
\text { W810 } \\
\text { W815 } \\
\text { W820 } \\
\text { W820 } \\
\text { W825 }\end{array}$ & $\begin{array}{l}\text { MUW646-WN1-B-18808 } \\
\text { MUW651-WN2-B-18809 } \\
\text { MUW801-EC2-B-18798 } \\
\text { MUW806-EC2-B-18797 } \\
\text { MUW810-EC2-B-18796 } \\
\text { MUW815-EC2-B-18795 } \\
\text { MUW820-EC1-B-18793 } \\
\text { MUW820-EC2-B-18794 } \\
\text { MUW825-EC1-B-18792 }\end{array}$ & $\begin{array}{l}8 / 15 / 06 \\
8 / 15 / 06 \\
8 / 15 / 06 \\
8 / 15 / 06 \\
8 / 15 / 06 \\
8 / 15 / 06 \\
8 / 15 / 06 \\
8 / 15 / 06 \\
8 / 15 / 06\end{array}$ & $\begin{array}{l}2.0 \\
\text { ND } \\
\text { ND } \\
130 \\
<1 \\
\text { ND } \\
<1 \\
<1 \\
2.3\end{array}$ & $\begin{array}{c}3.2 \\
2.3 \\
12 \\
12 \\
1.7 \\
N D \\
6.8 \\
2.0 \\
21\end{array}$ & $\begin{array}{l}\text { WN1 } \\
\text { WN2 } \\
\text { EC2 } \\
\text { EC2 } \\
\text { EC2 } \\
\text { EC2 } \\
\text { EC1 } \\
\text { EC2 } \\
\text { EC1 }\end{array}$ & $\begin{array}{l}\text { Branch } \\
\text { Branch } \\
\text { Branch } \\
\text { Branch } \\
\text { Branch } \\
\text { Branch } \\
\text { Branch } \\
\text { Branch } \\
\text { Branch }\end{array}$ & $\begin{array}{r}104 \\
124 \\
115 \\
83 \\
168 \\
115 \\
113 \\
120 \\
105\end{array}$ & \\
\hline W830 & MUW830-EC-B-18791 & $8 / 15 / 06$ & $<1$ & 3.8 & EC & Branch & 115 & NC tree too small to sample. \\
\hline W835 & MUW835-EC-B-18790 & $8 / 15 / 06$ & $<1$ & 23 & EC & Branch & 98 & \\
\hline W840 & MUW840-WN1-B-18789 & $8 / 15 / 06$ & $<1$ & 2.2 & WN1 & Branch & 107 & \\
\hline W845 & MUW845-EC1-B-18788 & $8 / 15 / 06$ & $<1$ & 3.0 & EC1 & Branch & 84 & \\
\hline W849 & MUW849-EC1-B-18787 & 8/15/06 & ND & 1.2 & EC1 & Branch & 70 & \\
\hline W854 & MUW854-EC-B-18786 & $8 / 15 / 06$ & $<1$ & 6.5 & EC & Branch & 65 & \\
\hline W854 & MUW854-GA-B-18785 & 8/15/06 & ND & 6.7 & GA & Branch & 80 & \\
\hline W859 & MUW859-EC-B-18784 & 8/15/06 & ND & 7.5 & EC & Branch & 108 & \\
\hline W908 & MUW908-EC1-B-18783 & $8 / 15 / 06$ & $<1$ & 2.8 & EC1 & Branch & 115 & Well developed tree. \\
\hline PREW001 & MUPREW001-B-18946 & $8 / 17 / 06$ & 17 & 16 & & Branch & 213 & $\begin{array}{l}\text { Willow branch height } 7 \mathrm{ft} \text {. Yellow ribbon to } \\
\text { identify previously sampled tree not } \\
\text { located. }\end{array}$ \\
\hline PREW002 & MUPREW002-B-18947 & $8 / 17 / 06$ & ND & 1.2 & & Branch & 122 & $\begin{array}{l}\text { Mullberry positively identified by yellow } \\
\text { ribbon as previously sampled tree. }\end{array}$ \\
\hline
\end{tabular}




\begin{tabular}{|c|c|c|c|c|c|c|c|c|}
\hline & & \multicolumn{3}{|c|}{ Concentration $(\mu \mathrm{g} / \mathrm{kg})$} & \multirow[b]{2}{*}{$\begin{array}{c}\text { Tree } \\
\text { Species }\end{array}$} & \multirow[b]{2}{*}{$\begin{array}{c}\text { Sample } \\
\text { Type }^{a}\end{array}$} & \multirow[b]{2}{*}{$\begin{array}{l}\text { Sample } \\
\text { Height }^{\mathrm{b}} \\
\quad(\mathrm{cm})\end{array}$} & \multirow[b]{2}{*}{ Sample Description } \\
\hline Location & Sample & $\begin{array}{c}\text { Sample } \\
\text { Date }\end{array}$ & $\begin{array}{c}\text { Carbon } \\
\text { Tetrachloride }\end{array}$ & Chloroform & & & & \\
\hline
\end{tabular}

Tree branch samples collected in August 2006 (cont.)

\begin{tabular}{|c|c|c|c|c|c|c|c|c|}
\hline PREW003 & MUPREW003-B-18948 & $8 / 17 / 06$ & 7.8 & 20 & & Branch & 152 & $\begin{array}{l}\text { Ash tree. Yellow ribbon to identify } \\
\text { previously sampled tree not located. }\end{array}$ \\
\hline PREW004 & MUPREW004-B-18949 & $8 / 17 / 06$ & $<1$ & 1.9 & & Branch & 183 & $\begin{array}{l}\text { Locust tree. Yellow ribbon to identify } \\
\text { previously sampled tree not located. }\end{array}$ \\
\hline PREW006 & MUPREW006-B-18943 & $8 / 16 / 06$ & 1.1 & 4.7 & & Branch & 122 & Cottonwood tree. \\
\hline PREE008 & MUPRE008-B-18936 & $8 / 16 / 06$ & ND & 17 & & Branch & 152 & $\begin{array}{l}\text { Large native tree near E130, close to } \\
\text { PRE008. }\end{array}$ \\
\hline PREE009 & MUPRE009-B-18953 & $8 / 17 / 06$ & 172 & 82 & & Branch & 244 & Willow (?). \\
\hline PREE010 & MUPRE010-B-18951 & $8 / 17 / 06$ & $<1$ & 1.5 & & Branch & 213 & $\begin{array}{l}\text { Locust tree. No ribbon ID. Immediately } \\
\text { west of E112 near air monitoring AA2. }\end{array}$ \\
\hline PREE011 & MUPRE011-B-18952 & $8 / 17 / 06$ & $<1$ & 5.7 & & Branch & 213 & $\begin{array}{l}\text { Elm straight west of E113. Found pink } \\
\text { ribbon from previous sampling. }\end{array}$ \\
\hline PREWET1 & MUPREWET1-B-18942 & $8 / 16 / 06$ & ND & 4.9 & & Branch & NR & $\begin{array}{l}\text { Branch sample from locust tree close to air } \\
\text { sampler AA3 at wetlands. Collected } \\
\text { from branch at same height as air } \\
\text { sampler. }\end{array}$ \\
\hline BUTTIG-2 & MUBRUTIG-B-18945 & $8 / 16 / 06$ & ND & 1.9 & & Branch & 122 & $\begin{array}{l}\text { Ash on Bruttig property on north side of } \\
\text { Waverly Road. East end of grass near } \\
\text { cornfield. }\end{array}$ \\
\hline SCHOOL-1 & MUSCHOOLASH-B-18944 & $8 / 16 / 06$ & ND & 1.6 & & Branch & 213 & $\begin{array}{l}\text { Ash on south side of gravelly street near } \\
\text { school. }\end{array}$ \\
\hline \multicolumn{9}{|c|}{ Tree leaf samples collected in August 2006} \\
\hline E112 & MUE112-WN2-L-18865 & $8 / 15 / 06$ & ND & 3.2 & WN2 & Leaf & 84 & Shaded, near air monitoring location AA2. \\
\hline E113 & MUE113-WN1-L-18867 & $8 / 15 / 06$ & ND & 1.7 & WN1 & Leaf & 107 & Shaded, near air monitoring location AA2. \\
\hline W110 & MUW110-WN1-L-18860 & $8 / 15 / 06$ & ND & 4.4 & WN1 & Leaf & 97 & Branch sample collected as \#18859. \\
\hline
\end{tabular}




\begin{tabular}{|c|c|c|c|c|c|c|c|c|}
\hline & & & Concentrati & on $(\mu \mathrm{g} / \mathrm{kg})$ & & & & \\
\hline Location & Sample & $\begin{array}{c}\text { Sample } \\
\text { Date }\end{array}$ & $\begin{array}{c}\text { Carbon } \\
\text { Tetrachloride }\end{array}$ & Chloroform & $\begin{array}{c}\text { Tree } \\
\text { Species }\end{array}$ & $\begin{array}{l}\text { Sample } \\
\text { Type }^{a}\end{array}$ & $\begin{array}{c}\text { Height }^{\mathrm{b}} \\
\text { (cm) }\end{array}$ & Sample Description \\
\hline
\end{tabular}

Existing grass samples collected in August 2006

\begin{tabular}{|c|c|c|c|c|c|c|c|}
\hline Groundcover & MUGRASS1-S-18918 & $8 / 16 / 06$ & $<1$ & 3.4 & Grass & - & $\begin{array}{l}\text { Virginia wild rye } 3 \mathrm{ft} \text { south of W318 straight } \\
\text { south toward W317 along path. Stalk } \\
\text { sampled. }\end{array}$ \\
\hline Groundcover & MUGRASS2-S-18919 & $8 / 16 / 06$ & ND & 2.3 & Grass & - & $\begin{array}{l}\text { Switch grass south of W321 } 3 \mathrm{ft} \text { south } \\
\text { along path. }\end{array}$ \\
\hline Groundcover & MUGRASS3-S-18920 & 8/16/06 & 1.1 & 3.7 & Grass & - & Canada wild rye $2 \mathrm{ft}$ south of W220. \\
\hline Groundcover & MUGRASS4-S-18921 & 8/16/06 & ND & ND & Grass & - & Big bluestem $1 \mathrm{ft}$ east of W643. \\
\hline Groundcover & MUGRASS5-S-18922 & $8 / 16 / 06$ & ND & $<1$ & Grass & - & Illinois bundleflower $1 \mathrm{ft}$ east of W744. \\
\hline Wetlands & MUGRASS6-S-18937 & 8/16/06 & ND & 20 & Grass & - & $\begin{array}{l}\text { Bulrush at outfall of tile drain } 5 \text { at south } \\
\text { end of wetland. }\end{array}$ \\
\hline Wetlands & MUGRASS7-S-18938 & $8 / 16 / 06$ & ND & 2.6 & Grass & - & $\begin{array}{l}\text { Bulrush at east bank of southernmost cell } \\
\text { of wetland at partitioning between south } \\
\text { and north cells. }\end{array}$ \\
\hline Wetlands & MUGRASS8-S-18939 & $8 / 16 / 06$ & ND & 1.8 & Grass & - & $\begin{array}{l}\text { Cattail (?) collected at same location as } \\
\quad \# 18938 \text {. }\end{array}$ \\
\hline Wetlands & MUGRASS9-S-18940 & $8 / 16 / 06$ & ND & 6.4 & Grass & - & $\begin{array}{l}\text { Bulrush, north of outfall of wetland just } \\
\text { past riprap on west side. }\end{array}$ \\
\hline
\end{tabular}

Close to air monitoring point AA1. Branch sample collected as \#18828.

Branch sample collected as \#18829.

Branch sample collected as \#18849.

Branch sample collected as \#18850.

Close to air monitoring location AA1.

Branch sample collected as \#18852. Close to air monitoring location AA1.

NR Leaf sample from locust tree close to air sampler AA3 at wetlands. itch grass

along path.

.

attail (?) collected at same location as 
Tree species: EC, eastern cottonwood; GA, green ash; HP, hybrid poplar; NC, northern catalpa; WN, Niobe willow. Numerals 1 and 2 distinguish two trees of the same species at a single location.

b Sample height is measured from the branch insertion point to ground level.

c ND, contaminant not detected at a method detection limit of $0.1 \mu \mathrm{g} / \mathrm{kg}$.

d NR, not recorded.

e $<1$, concentration below the method quantitation limit of $1.0 \mu \mathrm{g} / \mathrm{kg}$. 


\section{Supplement 1:}

Water Levels Recorded at Murdock, August 2005 through December 2006 
TABLE S1.1 Automatically measured water levels in August 2005 through December 2006 in the upgradient wells at Murdock.

\begin{tabular}{|c|c|c|c|c|}
\hline \multirow[b]{2}{*}{ Date } & \multicolumn{4}{|c|}{$\begin{array}{c}\text { Water Level (ft below top of } \\
\text { casing) at Indicated Well }\end{array}$} \\
\hline & Time & $\mathrm{D} 2$ & WP49 & SB65D \\
\hline 8/4/2005 & $12: 00$ & 38.491 & 47.549 & 22.849 \\
\hline 8/4/2005 & $16: 00$ & 38.561 & 47.572 & 22.822 \\
\hline 8/4/2005 & $20: 00$ & 38.576 & 47.574 & 22.818 \\
\hline 8/5/2005 & 0:00 & 38.6 & 47.615 & 22.841 \\
\hline 8/5/2005 & $4: 00$ & 38.614 & 47.621 & 22.841 \\
\hline 8/5/2005 & $8: 00$ & 38.651 & 47.66 & 22.859 \\
\hline 8/5/2005 & $12: 00$ & 38.663 & 47.641 & 22.83 \\
\hline 8/5/2005 & $16: 00$ & 38.641 & 47.572 & 22.734 \\
\hline 8/5/2005 & $20: 00$ & 38.59 & 47.525 & 22.699 \\
\hline 8/6/2005 & $0: 00$ & 38.59 & 47.559 & 22.732 \\
\hline 8/6/2005 & $4: 00$ & 38.552 & 47.519 & 22.703 \\
\hline 8/6/2005 & $8: 00$ & 38.535 & 47.519 & 22.716 \\
\hline 8/6/2005 & $12: 00$ & 38.506 & 47.444 & 22.672 \\
\hline 8/6/2005 & $16: 00$ & 38.429 & 47.365 & 22.601 \\
\hline 8/6/2005 & $20: 00$ & 38.366 & 47.32 & 22.609 \\
\hline 8/7/2005 & $0: 00$ & 38.351 & 47.314 & 22.617 \\
\hline 8/7/2005 & $4: 00$ & 38.318 & 47.288 & 22.595 \\
\hline 8/7/2005 & $8: 00$ & 38.318 & 47.314 & 22.636 \\
\hline 8/7/2005 & $12: 00$ & 38.313 & 47.292 & 22.622 \\
\hline 8/7/2005 & $16: 00$ & 38.267 & 47.228 & 22.568 \\
\hline 8/7/2005 & $20: 00$ & 38.231 & 47.209 & 22.576 \\
\hline 8/8/2005 & $0: 00$ & 38.238 & 47.237 & 22.613 \\
\hline 8/8/2005 & $4: 00$ & 38.226 & 47.213 & 22.595 \\
\hline 8/8/2005 & $8: 00$ & 38.221 & 47.217 & 22.591 \\
\hline 8/8/2005 & $12: 00$ & 38.26 & 47.23 & 22.603 \\
\hline 8/8/2005 & $16: 00$ & 38.264 & 47.198 & 22.556 \\
\hline 8/8/2005 & $20: 00$ & 38.245 & 47.202 & 22.558 \\
\hline 8/9/2005 & $0: 00$ & 38.262 & 47.243 & 22.589 \\
\hline 8/9/2005 & $4: 00$ & 38.236 & 47.207 & 22.562 \\
\hline 8/9/2005 & $8: 00$ & 38.255 & 47.249 & 22.605 \\
\hline 8/9/2005 & $12: 00$ & 38.277 & 47.239 & 22.583 \\
\hline 8/9/2005 & $16: 00$ & 38.279 & 47.224 & 22.55 \\
\hline 8/9/2005 & $20: 00$ & 38.248 & 47.217 & 22.562 \\
\hline $8 / 10 / 2005$ & $0: 00$ & 38.293 & 47.296 & 22.634 \\
\hline $8 / 10 / 2005$ & $4: 00$ & 38.296 & 47.294 & 22.634 \\
\hline $8 / 10 / 2005$ & $8: 00$ & 38.315 & 47.322 & 22.661 \\
\hline $8 / 10 / 2005$ & $12: 00$ & 38.351 & 47.322 & 22.642 \\
\hline $8 / 10 / 2005$ & $16: 00$ & 38.344 & 47.269 & 22.57 \\
\hline $8 / 10 / 2005$ & $20: 00$ & 38.535 & 47.301 & 22.583 \\
\hline
\end{tabular}

TABLE S1.1 (Cont.)

\begin{tabular}{|c|c|c|c|c|}
\hline \multirow[b]{2}{*}{ Date } & \multicolumn{4}{|c|}{$\begin{array}{l}\text { Water Level (ft below top of } \\
\text { casing) at Indicated Well }\end{array}$} \\
\hline & Time & D2 & WP49 & SB65D \\
\hline $8 / 11 / 2005$ & $0: 00$ & 38.313 & 47.29 & 22.535 \\
\hline $8 / 11 / 2005$ & 4:00 & 38.26 & 47.281 & 22.527 \\
\hline $8 / 11 / 2005$ & $8: 00$ & 38.238 & 47.239 & 22.507 \\
\hline $8 / 11 / 2005$ & $12: 00$ & 38.231 & 47.185 & 22.47 \\
\hline $8 / 11 / 2005$ & $16: 00$ & 38.168 & 47.155 & 22.468 \\
\hline $8 / 11 / 2005$ & $20: 00$ & 38.12 & 47.128 & 22.455 \\
\hline 8/12/2005 & 0:00 & 38.067 & 47.147 & 22.482 \\
\hline $8 / 12 / 2005$ & 4:00 & 38.04 & 47.147 & 22.496 \\
\hline $8 / 12 / 2005$ & 8:00 & 38.04 & 47.14 & 22.497 \\
\hline $8 / 12 / 2005$ & $12: 00$ & 38.021 & 47.177 & 22.544 \\
\hline 8/12/2005 & $16: 00$ & 38.026 & 47.138 & 22.519 \\
\hline $8 / 12 / 2005$ & $20: 00$ & 38.004 & 47.104 & 22.491 \\
\hline 8/13/2005 & 0:00 & 37.958 & 47.147 & 22.538 \\
\hline $8 / 13 / 2005$ & 4:00 & 37.97 & 47.098 & 22.493 \\
\hline $8 / 13 / 2005$ & $8: 00$ & 37.927 & 47.143 & 22.55 \\
\hline $8 / 13 / 2005$ & $12: 00$ & 37.924 & 47.166 & 22.567 \\
\hline $8 / 13 / 2005$ & $16: 00$ & 37.96 & 47.132 & 22.542 \\
\hline $8 / 13 / 2005$ & $20: 00$ & 37.922 & 47.134 & 22.566 \\
\hline $8 / 14 / 2005$ & 0:00 & 37.931 & 47.171 & 22.589 \\
\hline $8 / 14 / 2005$ & 4:00 & 37.927 & 47.192 & 22.608 \\
\hline 8/14/2005 & $8: 00$ & 37.912 & 47.222 & 22.634 \\
\hline 8/14/2005 & $12: 00$ & 37.997 & 47.202 & 22.606 \\
\hline $8 / 14 / 2005$ & $16: 00$ & 38.033 & 47.183 & 22.575 \\
\hline 8/14/2005 & $20: 00$ & 37.968 & 47.16 & 22.571 \\
\hline $8 / 15 / 2005$ & $0: 00$ & 37.931 & 47.19 & 22.601 \\
\hline $8 / 15 / 2005$ & 4:00 & 37.915 & 47.2 & 22.608 \\
\hline $8 / 15 / 2005$ & 8:00 & 37.91 & 47.196 & 22.616 \\
\hline $8 / 15 / 2005$ & $12: 00$ & 37.989 & 47.183 & 22.591 \\
\hline $8 / 15 / 2005$ & $16: 00$ & 38.018 & 47.132 & 22.546 \\
\hline $8 / 15 / 2005$ & $20: 00$ & 37.946 & 47.132 & 22.56 \\
\hline 8/16/2005 & $0: 00$ & 37.912 & 47.145 & 22.575 \\
\hline 8/16/2005 & 4:00 & 37.898 & 47.126 & 22.562 \\
\hline $8 / 16 / 2005$ & $8: 00$ & 37.886 & 47.16 & 22.595 \\
\hline 8/16/2005 & $12: 00$ & 37.97 & 47.132 & 22.567 \\
\hline 8/16/2005 & $16: 00$ & 37.997 & 47.066 & 22.511 \\
\hline $8 / 16 / 2005$ & $20: 00$ & 37.929 & 47.053 & 22.515 \\
\hline 8/17/2005 & $0: 00$ & 37.907 & 47.038 & 22.499 \\
\hline $8 / 17 / 2005$ & 4:00 & 37.893 & 46.986 & 22.466 \\
\hline 8/17/2005 & $8: 00$ & 37.907 & 46.937 & 22.429 \\
\hline $8 / 17 / 2005$ & $12: 00$ & 37.96 & 46.89 & 22.396 \\
\hline 8/17/2005 & $16: 00$ & 37.869 & 46.811 & 22.329 \\
\hline $8 / 17 / 2005$ & $20: 00$ & 37.816 & 46.79 & 22.326 \\
\hline
\end{tabular}


TABLE S1.1 (Cont.)

\begin{tabular}{|c|c|c|c|c|}
\hline \multirow[b]{2}{*}{ Date } & \multicolumn{4}{|c|}{$\begin{array}{l}\text { Water Level (ft below top of } \\
\text { casing) at Indicated Well }\end{array}$} \\
\hline & Time & D2 & WP49 & SB65D \\
\hline 8/18/2005 & $0: 00$ & 37.794 & 46.773 & 22.322 \\
\hline $8 / 18 / 2005$ & 4:00 & 37.777 & 46.713 & 22.285 \\
\hline 8/18/2005 & 8:00 & 37.748 & 46.779 & 22.361 \\
\hline $8 / 18 / 2005$ & $12: 00$ & 37.837 & 46.818 & 22.39 \\
\hline $8 / 18 / 2005$ & $16: 00$ & 37.888 & 46.83 & 22.39 \\
\hline $8 / 18 / 2005$ & $20: 00$ & 37.83 & 46.843 & 22.399 \\
\hline $8 / 19 / 2005$ & $0: 00$ & 37.775 & 46.903 & 22.448 \\
\hline $8 / 19 / 2005$ & 4:00 & 37.753 & 46.918 & 22.46 \\
\hline $8 / 19 / 2005$ & 8:00 & 37.738 & 46.969 & 22.505 \\
\hline $8 / 19 / 2005$ & $12: 00$ & 37.808 & 46.993 & 22.516 \\
\hline $8 / 19 / 2005$ & $16: 00$ & 37.854 & 47.008 & 22.501 \\
\hline $8 / 19 / 2005$ & $20: 00$ & 37.857 & 47.057 & 22.528 \\
\hline 8/20/2005 & $0: 00$ & 37.852 & 47.113 & 22.573 \\
\hline $8 / 20 / 2005$ & 4:00 & 37.847 & 47.132 & 22.583 \\
\hline $8 / 20 / 2005$ & 8:00 & 37.849 & 47.172 & 22.62 \\
\hline $8 / 20 / 2005$ & $12: 00$ & 37.941 & 47.192 & 22.626 \\
\hline $8 / 20 / 2005$ & $16: 00$ & 37.985 & 47.158 & 22.581 \\
\hline 8/20/2005 & $20: 00$ & 37.902 & 47.164 & 22.596 \\
\hline $8 / 21 / 2005$ & 0:00 & 37.871 & 47.185 & 22.612 \\
\hline $8 / 21 / 2005$ & 4:00 & 37.859 & 47.166 & 22.606 \\
\hline $8 / 21 / 2005$ & 8:00 & 37.854 & 47.205 & 22.63 \\
\hline $8 / 21 / 2005$ & $12: 00$ & 37.946 & 47.177 & 22.6 \\
\hline $8 / 21 / 2005$ & $16: 00$ & 37.985 & 47.1 & 22.53 \\
\hline $8 / 21 / 2005$ & $20: 00$ & 37.912 & 47.102 & 22.544 \\
\hline $8 / 22 / 2005$ & 0:00 & 37.871 & 47.128 & 22.569 \\
\hline 8/22/2005 & 4:00 & 37.861 & 47.098 & 22.547 \\
\hline 8/22/2005 & 8:00 & 37.835 & 47.147 & 22.598 \\
\hline 8/22/2005 & $12: 00$ & 37.987 & 47.145 & 22.557 \\
\hline 8/22/2005 & $16: 00$ & 37.951 & 47.093 & 22.514 \\
\hline 8/22/2005 & $20: 00$ & 37.917 & 47.092 & 22.514 \\
\hline 8/23/2005 & & 37.888 & 47.122 & 22.547 \\
\hline 8/23/2005 & 4:00 & 37.888 & 47.096 & 22.526 \\
\hline 8/23/2005 & 8:00 & 37.864 & 47.122 & 22.553 \\
\hline $8 / 23 / 2005$ & $12: 00$ & 37.902 & 47.115 & 22.549 \\
\hline 8/23/2005 & $16: 00$ & 37.943 & 47.053 & 22.5 \\
\hline 8/23/2005 & $20: 00$ & 37.89 & 47.051 & 22.508 \\
\hline $8 / 24 / 2005$ & 0:00 & 37.857 & 47.081 & 22.543 \\
\hline 8/24/2005 & 4:00 & 37.861 & 47.053 & 22.52 \\
\hline 8/24/2005 & 8:00 & 37.857 & 47.03 & 22.506 \\
\hline $8 / 24 / 2005$ & $12: 00$ & 37.864 & 47.055 & 22.536 \\
\hline $8 / 24 / 2005$ & $16: 00$ & 37.886 & 47.017 & 22.506 \\
\hline 8/24/2005 & 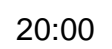 & 37.854 & 017 & 22.512 \\
\hline
\end{tabular}

TABLE S1.1 (Cont.)

\begin{tabular}{|c|c|c|c|c|}
\hline \multirow[b]{2}{*}{ Date } & \multicolumn{4}{|c|}{$\begin{array}{l}\text { Water Level (ft below top of } \\
\text { casing) at Indicated Well }\end{array}$} \\
\hline & Time & D2 & WP49 & SB65D \\
\hline 8/25/2005 & $0: 00$ & 37.842 & 47.027 & 22.52 \\
\hline $8 / 25 / 2005$ & $4: 00$ & 37.842 & 47.006 & 22.504 \\
\hline $8 / 25 / 2005$ & $8: 00$ & 37.82 & 47.036 & 22.539 \\
\hline 8/25/2005 & $12: 00$ & 37.9 & 47.021 & 22.518 \\
\hline $8 / 25 / 2005$ & $16: 00$ & 37.912 & 46.976 & 22.477 \\
\hline 8/25/2005 & $20: 00$ & 37.845 & 46.98 & 22.497 \\
\hline 8/26/2005 & $0: 00$ & 37.859 & 46.963 & 22.471 \\
\hline 8/26/2005 & $4: 00$ & 37.84 & 46.916 & 22.436 \\
\hline 8/26/2005 & $8: 00$ & 37.791 & 46.968 & 22.502 \\
\hline 8/26/2005 & $12: 00$ & 37.874 & 46.944 & 22.479 \\
\hline 8/26/2005 & 16:00 & 37.881 & 46.91 & 22.445 \\
\hline 8/26/2005 & $20: 00$ & 37.816 & 46.923 & 22.477 \\
\hline $8 / 27 / 2005$ & $0: 00$ & 37.791 & 46.955 & 22.504 \\
\hline 8/27/2005 & $4: 00$ & 37.794 & 46.916 & 22.467 \\
\hline 8/27/2005 & $8: 00$ & 37.787 & 46.946 & 22.497 \\
\hline $8 / 27 / 2005$ & $12: 00$ & 37.859 & 46.938 & 22.488 \\
\hline 8/27/2005 & $16: 00$ & 37.893 & 46.876 & 22.43 \\
\hline 8/27/2005 & $20: 00$ & 37.811 & 46.886 & 22.451 \\
\hline 8/28/2005 & $0: 00$ & 37.787 & 46.893 & 22.463 \\
\hline 8/28/2005 & $4: 00$ & 37.762 & 46.912 & 22.481 \\
\hline 8/28/2005 & $8: 00$ & 37.758 & 46.944 & 22.514 \\
\hline 8/28/2005 & 12:00 & 37.832 & 46.944 & 22.504 \\
\hline 8/28/2005 & $16: 00$ & 37.784 & 47.032 & 22.596 \\
\hline 8/28/2005 & $20: 00$ & 37.77 & 46.94 & 22.5 \\
\hline 8/29/2005 & $0: 00$ & 37.753 & 46.985 & 22.531 \\
\hline 8/29/2005 & $4: 00$ & 37.758 & 46.965 & 22.518 \\
\hline 8/29/2005 & 8:00 & 37.758 & 46.98 & 22.529 \\
\hline 8/29/2005 & $12: 00$ & 37.84 & 46.97 & 22.516 \\
\hline 8/29/2005 & $16: 00$ & 37.919 & 46.891 & 22.442 \\
\hline 8/29/2005 & $20: 00$ & 37.893 & 46.884 & 22.453 \\
\hline 8/30/2005 & $0: 00$ & 37.89 & 46.88 & 22.449 \\
\hline 8/30/2005 & $4: 00$ & 37.874 & 46.867 & 22.443 \\
\hline 8/30/2005 & $8: 00$ & 37.883 & 46.886 & 22.463 \\
\hline 8/30/2005 & $12: 00$ & 37.881 & 46.865 & 22.445 \\
\hline 8/30/2005 & $16: 00$ & 37.842 & 46.82 & 22.408 \\
\hline 8/30/2005 & $20: 00$ & 37.825 & 46.829 & 22.43 \\
\hline $8 / 31 / 2005$ & $0: 00$ & 37.847 & 46.854 & 22.453 \\
\hline $8 / 31 / 2005$ & $4: 00$ & 37.876 & 46.891 & 22.486 \\
\hline 8/31/2005 & $8: 00$ & 37.915 & 46.944 & 22.531 \\
\hline $8 / 31 / 2005$ & $12: 00$ & 37.972 & 47 & 22.578 \\
\hline 8/31/2005 & $16: 00$ & 37.992 & 47 & 22.57 \\
\hline $8 / 31 / 2005$ & 20 & 38.023 & 47.047 & $22.60 \varsigma$ \\
\hline
\end{tabular}


TABLE S1.1 (Cont.)

\begin{tabular}{|c|c|c|c|c|}
\hline \multirow[b]{2}{*}{ Date } & \multicolumn{4}{|c|}{$\begin{array}{l}\text { Water Level (ft below top of } \\
\text { casing) at Indicated Well }\end{array}$} \\
\hline & Time & D2 & WP49 & SB65D \\
\hline 9/1/2005 & $0: 00$ & 38.071 & 47.096 & 22.642 \\
\hline 9/1/2005 & 4:00 & 38.096 & 47.113 & 22.648 \\
\hline 9/1/2005 & 8:00 & 38.146 & 47.169 & 22.687 \\
\hline 9/1/2005 & $12: 00$ & 38.194 & 47.186 & 22.68 \\
\hline 9/1/2005 & $16: 00$ & 38.199 & 47.145 & 22.609 \\
\hline 9/1/2005 & $20: 00$ & 38.207 & 47.171 & 22.625 \\
\hline 9/2/2005 & $0: 00$ & 38.24 & 47.211 & 22.65 \\
\hline 9/2/2005 & 4:00 & 38.245 & 47.228 & 22.664 \\
\hline 9/2/2005 & 8:00 & 38.284 & 47.282 & 22.706 \\
\hline 9/2/2005 & $12: 00$ & 38.305 & 47.286 & 22.699 \\
\hline 9/2/2005 & $16: 00$ & 38.255 & 47.211 & 22.631 \\
\hline 9/2/2005 & $20: 00$ & 38.238 & 47.213 & 22.648 \\
\hline 9/3/2005 & $0: 00$ & 38.264 & 47.245 & 22.68 \\
\hline 9/3/2005 & 4:00 & 38.26 & 47.237 & 22.672 \\
\hline 9/3/2005 & 8:00 & 38.26 & 47.233 & 22.67 \\
\hline 9/3/2005 & $12: 00$ & 38.25 & 47.205 & 22.644 \\
\hline 9/3/2005 & $16: 00$ & 38.182 & 47.122 & 22.572 \\
\hline 9/3/2005 & $20: 00$ & 38.144 & 47.109 & 22.584 \\
\hline 9/4/2005 & $0: 00$ & 38.149 & 47.119 & 22.594 \\
\hline 9/4/2005 & 4:00 & 38.149 & 47.132 & 22.613 \\
\hline 9/4/2005 & 8:00 & 38.158 & 47.143 & 22.625 \\
\hline 9/4/2005 & $12: 00$ & 38.163 & 47.119 & 22.611 \\
\hline $9 / 4 / 2005$ & $16: 00$ & 38.122 & 47.083 & 22.574 \\
\hline 9/4/2005 & $20: 00$ & 38.115 & 47.098 & 22.601 \\
\hline 9/5/2005 & $0: 00$ & 38.141 & 47.128 & 22.634 \\
\hline 9/5/2005 & 4:00 & 38.139 & 47.122 & 22.628 \\
\hline 9/5/2005 & 8:00 & 38.144 & 47.132 & 22.64 \\
\hline 9/5/2005 & $12: 00$ & 38.166 & 47.145 & 22.654 \\
\hline 9/5/2005 & $16: 00$ & 38.117 & 47.068 & 22.578 \\
\hline 9/5/2005 & $20: 00$ & 38.098 & 47.07 & 22.598 \\
\hline 9/6/2005 & $0: 00$ & 38.103 & 47.074 & 22.605 \\
\hline 9/6/2005 & 4:00 & 38.115 & 47.102 & 22.628 \\
\hline 9/6/2005 & 8:00 & 38.187 & 47.211 & 22.73 \\
\hline 9/6/2005 & $12: 00$ & 38.236 & 47.198 & 22.693 \\
\hline 9/6/2005 & $16: 00$ & 38.238 & 47.196 & 22.677 \\
\hline 9/6/2005 & $20: 00$ & 38.26 & 47.235 & 22.701 \\
\hline 9/7/2005 & $0: 00$ & 38.296 & 47.28 & 22.734 \\
\hline 9/7/2005 & 4:00 & 38.281 & 47.241 & 22.687 \\
\hline 9/7/2005 & 8:00 & 38.277 & 47.239 & 22.689 \\
\hline 9/7/2005 & $12: 00$ & 38.269 & 47.222 & 22.675 \\
\hline 9/7/2005 & $16: 00$ & 38.211 & 47.141 & 22.607 \\
\hline 9/7/2005 & 00 & 38.182 & 136 & 22.619 \\
\hline
\end{tabular}

TABLE S1.1 (Cont.)

\begin{tabular}{|c|c|c|c|c|}
\hline \multirow[b]{2}{*}{ Date } & \multicolumn{4}{|c|}{$\begin{array}{l}\text { Water Level (ft below top of } \\
\text { casing) at Indicated Well }\end{array}$} \\
\hline & Time & D2 & WP49 & SB65D \\
\hline 9/8/2005 & $0: 00$ & 38.185 & 47.141 & 22.626 \\
\hline 9/8/2005 & 4:00 & 38.149 & 47.096 & 22.587 \\
\hline 9/8/2005 & 8:00 & 38.12 & 47.072 & 22.576 \\
\hline 9/8/2005 & $12: 00$ & 38.112 & 47.038 & 22.546 \\
\hline 9/8/2005 & $16: 00$ & 38.086 & 46.987 & 22.49 \\
\hline 9/8/2005 & $20: 00$ & 38.076 & 47.017 & 22.525 \\
\hline 9/9/2005 & $0: 00$ & 38.067 & 47.019 & 22.525 \\
\hline 9/9/2005 & 4:00 & 38.067 & 47.027 & 22.541 \\
\hline 9/9/2005 & 8:00 & 38.079 & 47.053 & 22.568 \\
\hline 9/9/2005 & $12: 00$ & 38.096 & 47.042 & 22.548 \\
\hline 9/9/2005 & $16: 00$ & 38.091 & 47.008 & 22.517 \\
\hline 9/9/2005 & $20: 00$ & 38.093 & 47.038 & 22.548 \\
\hline 9/10/2005 & 0:00 & 38.103 & 47.07 & 22.564 \\
\hline $9 / 10 / 2005$ & 4:00 & 38.098 & 47.06 & 22.558 \\
\hline $9 / 10 / 2005$ & 8:00 & 38.105 & 47.081 & 22.587 \\
\hline 9/10/2005 & $12: 00$ & 38.088 & 47.049 & 22.56 \\
\hline 9/10/2005 & $16: 00$ & 38.059 & 47.01 & 22.529 \\
\hline 9/10/2005 & $20: 00$ & 38.071 & 47.055 & 22.585 \\
\hline $9 / 11 / 2005$ & $0: 00$ & 38.103 & 47.096 & 22.623 \\
\hline $9 / 11 / 2005$ & 4:00 & 38.118 & 47.109 & 22.632 \\
\hline $9 / 11 / 2005$ & 8:00 & 38.151 & 47.151 & 22.671 \\
\hline 9/11/2005 & $12: 00$ & 38.156 & 47.126 & 22.64 \\
\hline $9 / 11 / 2005$ & $16: 00$ & 38.106 & 47.06 & 22.593 \\
\hline 9/11/2005 & $20: 00$ & 38.096 & 47.055 & 22.607 \\
\hline $9 / 12 / 2005$ & $0: 00$ & 38.11 & 47.072 & 22.625 \\
\hline $9 / 12 / 2005$ & 4:00 & 38.093 & 47.051 & 22.615 \\
\hline 9/12/2005 & 8:00 & 38.074 & 47.026 & 22.589 \\
\hline $9 / 12 / 2005$ & $12: 00$ & 38.038 & 46.948 & 22.519 \\
\hline $9 / 12 / 2005$ & $16: 00$ & 37.972 & 46.88 & 22.459 \\
\hline 9/12/2005 & $20: 00$ & 37.948 & 46.884 & 22.468 \\
\hline 9/13/2005 & $0: 00$ & 3 & 46.91 & 22.494 \\
\hline 9/13/2005 & 4:00 & 37.968 & 46.936 & 22.517 \\
\hline 9/13/2005 & 8:00 & 38.004 & 46.978 & 22.574 \\
\hline 9/13/2005 & $12: 00$ & 38.045 & 47.025 & 22.603 \\
\hline 9/13/2005 & $16: 00$ & 38.057 & 47.053 & 22.63 \\
\hline 9/13/2005 & $20: 00$ & 38.098 & 47.098 & 22.669 \\
\hline $9 / 14 / 2005$ & $0: 00$ & 38.166 & 47.166 & 22.736 \\
\hline 9/14/2005 & 4:00 & 38.202 & 47.186 & 22.74 \\
\hline 9/14/2005 & 8:00 & 38.245 & 47.233 & 22.773 \\
\hline 9/14/2005 & $12: 00$ & 38.284 & 47.241 & 22.747 \\
\hline 9/14/2005 & $16: 00$ & 38.277 & 47.192 & 22.673 \\
\hline 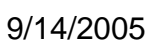 & 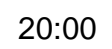 & 38.289 & 1 & 22.708 \\
\hline
\end{tabular}


TABLE S1.1 (Cont.)

\begin{tabular}{|c|c|c|c|c|}
\hline \multirow[b]{2}{*}{ Date } & \multicolumn{4}{|c|}{$\begin{array}{l}\text { Water Level (ft below top of } \\
\text { casing) at Indicated Well }\end{array}$} \\
\hline & Time & D2 & WP49 & SB65D \\
\hline 9/15/2005 & $0: 00$ & 38.313 & 47.252 & 22.714 \\
\hline $9 / 15 / 2005$ & 4:00 & 38.301 & 47.237 & 22.695 \\
\hline $9 / 15 / 2005$ & 8:00 & 38.349 & 47.322 & 22.783 \\
\hline $9 / 15 / 2005$ & $12: 00$ & 38.378 & 47.307 & 22.747 \\
\hline $9 / 15 / 2005$ & $16: 00$ & 38.371 & 47.288 & 22.712 \\
\hline $9 / 15 / 2005$ & $20: 00$ & 38.404 & 47.348 & 22.767 \\
\hline $9 / 16 / 2005$ & $0: 00$ & 38.441 & 47.391 & 22.796 \\
\hline $9 / 16 / 2005$ & 4:00 & 38.445 & 47.395 & 22.794 \\
\hline $9 / 16 / 2005$ & 8:00 & 38.474 & 47.434 & 22.831 \\
\hline $9 / 16 / 2005$ & $12: 00$ & 38.474 & 47.406 & 22.798 \\
\hline $9 / 16 / 2005$ & $16: 00$ & 38.397 & 47.301 & 22.706 \\
\hline $9 / 16 / 2005$ & $20: 00$ & 38.351 & 47.271 & 22.699 \\
\hline 9/17/2005 & $0: 00$ & 38.356 & 47.297 & 22.73 \\
\hline 9/17/2005 & 4:00 & 38.301 & 47.207 & 22.656 \\
\hline $9 / 17 / 2005$ & 8:00 & 38.272 & 47.188 & 22.656 \\
\hline 9/17/2005 & $12: 00$ & 38.226 & 47.122 & 22.601 \\
\hline $9 / 17 / 2005$ & $16: 00$ & 38.149 & 47.049 & 22.556 \\
\hline $9 / 17 / 2005$ & $20: 00$ & 38.132 & 47.066 & 22.589 \\
\hline 9/18/2005 & 0:00 & 38.144 & 47.077 & 22.607 \\
\hline 9/18/2005 & 4:00 & 38.115 & 47.057 & 22.603 \\
\hline 9/18/2005 & 8:00 & 38.144 & 47.094 & 22.648 \\
\hline 9/18/2005 & $12: 00$ & 38.153 & 47.068 & 22.623 \\
\hline 9/18/2005 & $16: 00$ & 38.11 & 47.023 & 22.589 \\
\hline 9/18/2005 & $20: 00$ & 38.1 & 47.034 & 22.593 \\
\hline 9/19/2005 & 0:00 & 38.1 & 47.041 & 22.603 \\
\hline 9/19/2005 & 4:00 & 38.149 & 47.134 & 22.697 \\
\hline 9/19/2005 & 8:00 & 38.226 & 47.216 & 22.763 \\
\hline 9/19/2005 & $12: 00$ & 38.305 & 47.265 & 22.788 \\
\hline 9/19/2005 & $16: 00$ & 38.356 & 47.307 & 22.796 \\
\hline 9/19/2005 & $20: 00$ & 38.421 & 47.359 & 22.823 \\
\hline 9/20/2005 & & 38.4 & 47.399 & 22.841 \\
\hline 9/20/2005 & 4:00 & 38.467 & 47.416 & 22.847 \\
\hline 9/20/2005 & 8:00 & 38.489 & 47.446 & 22.872 \\
\hline 9/20/2005 & $12: 00$ & 38.486 & 47.408 & 22.827 \\
\hline 9/20/2005 & $16: 00$ & 38.414 & 47.312 & 22.74 \\
\hline 9/20/2005 & $20: 00$ & 38.375 & 47.301 & 22.745 \\
\hline 9/21/2005 & 0:00 & 38.375 & 47.297 & 22.742 \\
\hline 9/21/2005 & 4:00 & 38.337 & 47.241 & 22.7 \\
\hline 9/21/2005 & 8:00 & 38.284 & 47.188 & 22.661 \\
\hline $9 / 21 / 2005$ & $12: 00$ & 38.236 & 47.102 & 22.585 \\
\hline 9/21/2005 & $16: 00$ & 38.166 & 47.006 & 22.501 \\
\hline $9 / 21 / 2005$ & . & 38.139 & 5 & 22.531 \\
\hline
\end{tabular}

TABLE S1.1 (Cont.)

\begin{tabular}{|c|c|c|c|c|}
\hline \multirow[b]{2}{*}{ Date } & \multicolumn{4}{|c|}{$\begin{array}{l}\text { Water Level (ft below top of } \\
\text { casing) at Indicated Well }\end{array}$} \\
\hline & Time & D2 & WP49 & SB65D \\
\hline 9/22/2005 & 0:00 & 38.127 & 47.066 & 22.58 \\
\hline 9/22/2005 & 4:00 & 38.19 & 47.149 & 22.66 \\
\hline 9/22/2005 & 8:00 & 38.272 & 47.256 & 22.778 \\
\hline 9/22/2005 & $12: 00$ & 38.351 & 47.31 & 22.782 \\
\hline 9/22/2005 & $16: 00$ & 38.417 & 47.361 & 22.798 \\
\hline 9/22/2005 & $20: 00$ & 38.472 & 47.423 & 22.835 \\
\hline 9/23/2005 & 0:00 & 38.511 & 47.453 & 22.841 \\
\hline 9/23/2005 & 4:00 & 38.525 & 47.466 & 22.848 \\
\hline 9/23/2005 & 8:00 & 38.535 & 47.479 & 22.86 \\
\hline 9/23/2005 & $12: 00$ & 38.537 & 47.449 & 22.815 \\
\hline 9/23/2005 & $16: 00$ & 38.511 & 47.387 & 22.747 \\
\hline 9/23/2005 & $20: 00$ & 38.496 & 47.393 & 22.757 \\
\hline 9/24/2005 & 0:00 & 38.477 & 47.37 & 22.735 \\
\hline 9/24/2005 & 4:00 & 38.433 & 47.327 & 22.706 \\
\hline 9/24/2005 & 8:00 & 39 & 47.297 & 22.7 \\
\hline 9/24/2005 & $12: 00$ & 38.375 & 47.263 & 22.669 \\
\hline 9/24/2005 & $16: 00$ & 38.293 & 47.175 & 22.609 \\
\hline 9/24/2005 & $20: 00$ & 38.274 & 47.186 & 22.654 \\
\hline $9 / 25 / 2005$ & 0:00 & 38.277 & 47.18 & 22.646 \\
\hline 9/25/2005 & 4:00 & 38.267 & 47.214 & 22.692 \\
\hline 9/25/2005 & 8:00 & 38.284 & 47.216 & 22.689 \\
\hline 9/25/2005 & $12: 00$ & 38.274 & 47.197 & 22.673 \\
\hline $9 / 25 / 2005$ & $16: 00$ & 38.226 & 47.145 & 22.636 \\
\hline 9/25/2005 & $20: 00$ & 38.286 & 47.263 & 22.755 \\
\hline 9/26/2005 & $0: 00$ & 38.373 & 47.359 & 22.837 \\
\hline 9/26/2005 & 4:00 & 38.429 & 47.41 & 22.888 \\
\hline 9/26/2005 & 8:00 & 38.532 & 47.522 & 22.956 \\
\hline 9/26/2005 & $12: 00$ & 38.598 & 47.547 & 22.952 \\
\hline 9/26/2005 & $16: 00$ & 38.61 & 47.524 & 22.907 \\
\hline 9/26/2005 & $20: 00$ & 38.605 & 47.515 & 22.899 \\
\hline 9/27/2005 & $0: 00$ & 38.593 & 47.483 & 22.866 \\
\hline 9/27/2005 & 4:00 & 38.576 & 47.468 & 22.852 \\
\hline 9/27/2005 & 8:00 & 38.559 & 47.462 & 22.837 \\
\hline 9/27/2005 & $12: 00$ & 38.525 & 47.381 & 22.759 \\
\hline 9/27/2005 & $16: 00$ & 38.448 & 47.28 & 22.669 \\
\hline 9/27/2005 & $20: 00$ & 38.421 & 47.278 & 22.669 \\
\hline 9/28/2005 & $0: 00$ & 38.385 & 47.237 & 22.648 \\
\hline 9/28/2005 & 4:00 & 38.334 & 47.205 & 22.642 \\
\hline 9/28/2005 & 8:00 & 38.39 & 47.338 & 22.774 \\
\hline 9/28/2005 & $12: 00$ & 38.559 & 47.547 & 22.95 \\
\hline 9/28/2005 & $16: 00$ & 38.619 & 47.592 & 22.971 \\
\hline 9/28/2005 & $20: 00$ & 38.699 & 47.669 & 23.03 \\
\hline
\end{tabular}


TABLE S1.1 (Cont.)

\begin{tabular}{|c|c|c|c|c|}
\hline \multirow[b]{2}{*}{ Date } & \multicolumn{4}{|c|}{$\begin{array}{l}\text { Water Level (ft below top of } \\
\text { casing) at Indicated Well }\end{array}$} \\
\hline & Time & D2 & WP49 & SB65D \\
\hline 9/29/2005 & 0:00 & 38.75 & 47.705 & 23.044 \\
\hline 9/29/2005 & 4:00 & 38.745 & 47.675 & 23.01 \\
\hline 9/29/2005 & 8:00 & 38.764 & 47.678 & 23.01 \\
\hline 9/29/2005 & $12: 00$ & 38.721 & 47.584 & 22.911 \\
\hline 9/29/2005 & $16: 00$ & 38.612 & 47.421 & 22.757 \\
\hline 9/29/2005 & $20: 00$ & 38.547 & 47.381 & 22.729 \\
\hline 9/30/2005 & 0:00 & 38.508 & 47.352 & 22.714 \\
\hline 9/30/2005 & 4:00 & 38.453 & 47.301 & 22.689 \\
\hline 9/30/2005 & 8:00 & 38.421 & 47.284 & 22.693 \\
\hline 9/30/2005 & $12: 00$ & 38.388 & 47.25 & 22.66 \\
\hline 9/30/2005 & $16: 00$ & 38.32 & 47.19 & 22.628 \\
\hline 9/30/2005 & $20: 00$ & 38.332 & 47.244 & 22.695 \\
\hline 10/1/2005 & $0: 00$ & 38.349 & 47.265 & 22.718 \\
\hline $10 / 1 / 2005$ & 4:00 & 38.378 & 47.316 & 22.772 \\
\hline 10/1/2005 & 8:00 & 38.414 & 47.344 & 22.792 \\
\hline 10/1/2005 & $12: 00$ & 38.431 & 47.348 & 22.794 \\
\hline 10/1/2005 & $16: 00$ & 38.407 & 47.31 & 22.761 \\
\hline 10/1/2005 & $20: 00$ & 38.419 & 47.342 & 22.798 \\
\hline $10 / 2 / 2005$ & 0:00 & 38.412 & 47.312 & 22.767 \\
\hline $10 / 2 / 2$ & 4:00 & 38.388 & 47.289 & 22.747 \\
\hline $10 / 2 / 2005$ & 8:00 & 38.388 & 47.293 & 22.759 \\
\hline $10 / 2 / 2005$ & $12: 00$ & 38.363 & 47.244 & 22.712 \\
\hline 10/2/2005 & $16: 00$ & 38.325 & 47.212 & 22.69 \\
\hline 10/2/2005 & $20: 00$ & 38.351 & 47.28 & 22.761 \\
\hline $10 / 3 / 2005$ & 0:00 & 38.407 & 47.325 & 22.806 \\
\hline 10/3/2005 & 4:00 & 38.419 & 47.333 & 22.81 \\
\hline 10/3/2005 & 8:00 & 38.445 & 47.374 & 22.837 \\
\hline 10/3/2005 & $12: 00$ & 38.474 & 47.398 & 22.849 \\
\hline 10/3/2005 & $16: 00$ & 38.433 & 47.329 & 22.783 \\
\hline $10 / 3 / 2005$ & $20: 00$ & 38.455 & 47.381 & 22.837 \\
\hline 10/4/2005 & $0: 00$ & 38.494 & 47.419 & 22.866 \\
\hline $10 / 4 / 2005$ & 4:00 & 38.499 & 47.415 & 22.855 \\
\hline $10 / 4 / 2005$ & 8:00 & 38.491 & 47.4 & 22.843 \\
\hline 10/4/2005 & $12: 00$ & 38.462 & 47.344 & 22.792 \\
\hline $10 / 4 / 2005$ & $16: 00$ & 38.407 & 47.265 & 22.722 \\
\hline 10/4/2005 & $20: 00$ & 38.39 & 47.286 & 22.757 \\
\hline $10 / 5 / 2005$ & 0:00 & 38.39 & 47.295 & 22.767 \\
\hline 10/5/2005 & 4:00 & 38.385 & 47.299 & 22.781 \\
\hline 10/5/2005 & 8:00 & 38.491 & 47.47 & 22.95 \\
\hline $10 / 5 / 2005$ & $12: 00$ & 38.605 & 47.581 & 23.013 \\
\hline 10/5/2005 & $16: 00$ & 38.665 & 47.626 & 23.044 \\
\hline & & & & \\
\hline
\end{tabular}

TABLE S1.1 (Cont.)

\begin{tabular}{|c|c|c|c|c|}
\hline \multirow[b]{2}{*}{ Date } & \multicolumn{4}{|c|}{$\begin{array}{l}\text { Water Level (ft below top of } \\
\text { casing) at Indicated Well }\end{array}$} \\
\hline & Time & D2 & WP49 & SB65D \\
\hline 10/6/2005 & $0: 00$ & 38.815 & 47.748 & 23.12 \\
\hline 10/6/2005 & $4: 00$ & 38.839 & 47.75 & 23.106 \\
\hline 10/6/2005 & $8: 00$ & 38.868 & 47.778 & 23.122 \\
\hline 10/6/2005 & $12: 00$ & 38.88 & 47.757 & 23.083 \\
\hline $10 / 6 / 2005$ & $16: 00$ & 38.836 & 47.716 & 23.036 \\
\hline 10/6/2005 & $20: 00$ & 38.829 & 47.72 & 23.042 \\
\hline 10/7/2005 & $0: 00$ & 38.844 & 47.729 & 23.046 \\
\hline $10 / 7 / 2005$ & $4: 00$ & 38.844 & 47.731 & 23.044 \\
\hline $10 / 7 / 2005$ & $8: 00$ & 38.846 & 47.735 & 23.046 \\
\hline 10/7/2005 & $12: 00$ & 38.807 & 47.661 & 22.974 \\
\hline 10/7/2005 & 16:00 & 38.711 & 47.543 & 22.878 \\
\hline 10/7/2005 & $20: 00$ & 38.67 & 47.53 & 22.882 \\
\hline 10/8/2005 & $0: 00$ & 38.663 & 47.534 & 22.894 \\
\hline 10/8/2005 & 4:00 & 38.643 & 47.511 & 22.88 \\
\hline 10/8/2005 & $8: 00$ & 38.643 & 47.541 & 22.907 \\
\hline 10/8/2005 & $12: 00$ & 38.624 & 47.49 & 22.859 \\
\hline 10/8/2005 & $16: 00$ & 38.547 & 47.402 & 22.792 \\
\hline 10/8/2005 & $20: 00$ & 38.515 & 47.381 & 22.802 \\
\hline $10 / 9 / 2005$ & $0: 00$ & 38.515 & 47.398 & 22.814 \\
\hline $10 / 9 / 2005$ & $4: 00$ & 38.523 & 47.413 & 22.831 \\
\hline 10/9/2005 & $8: 00$ & 38.53 & 47.43 & 22.847 \\
\hline 10/9/2005 & 12:00 & 38.53 & 47.419 & 22.833 \\
\hline 10/9/2005 & 16:00 & 38.496 & 47.385 & 22.806 \\
\hline 10/9/2005 & $20: 00$ & 38.542 & 47.464 & 22.89 \\
\hline $10 / 10 / 2005$ & $0: 00$ & 38.585 & 47.513 & 22.923 \\
\hline 10/10/2005 & $4: 00$ & 38.607 & 47.532 & 22.941 \\
\hline 10/10/2005 & 8:00 & 38.66 & 47.588 & 22.983 \\
\hline 10/10/2005 & $12: 00$ & 38.68 & 47.575 & 22.972 \\
\hline $10 / 10 / 2005$ & $16: 00$ & 38.653 & 47.543 & 22.927 \\
\hline $10 / 10 / 2005$ & $20: 00$ & 38.66 & 47.562 & 22.948 \\
\hline $10 / 11 / 2005$ & $0: 00$ & 38.672 & 47.564 & 22.942 \\
\hline $10 / 11 / 2005$ & $4: 00$ & 38.663 & 47.539 & 22.929 \\
\hline 10/11/2005 & $8: 00$ & 38.663 & 47.541 & 22.933 \\
\hline 10/11/2005 & $12: 00$ & 38.653 & 47.524 & 22.907 \\
\hline $10 / 11 / 2005$ & $16: 00$ & 38.602 & 47.47 & 22.863 \\
\hline 10/11/2005 & $20: 00$ & 38.593 & 47.472 & 22.874 \\
\hline 10/12/2005 & 0:00 & 38.588 & 47.466 & 22.866 \\
\hline $10 / 12 / 2005$ & $4: 00$ & 38.583 & 47.466 & 22.868 \\
\hline 10/12/2005 & $8: 00$ & 38.6 & 47.5 & 22.903 \\
\hline $10 / 12 / 2005$ & $12: 00$ & 38.629 & 47.528 & 22.927 \\
\hline 10/12/2005 & 16:00 & 38.629 & 47.532 & 22.929 \\
\hline 10 & 00 & 38.665 & 47.573 & 22.964 \\
\hline
\end{tabular}


TABLE S1.1 (Cont.)

\begin{tabular}{|c|c|c|c|c|}
\hline \multirow[b]{2}{*}{ Date } & \multicolumn{4}{|c|}{$\begin{array}{l}\text { Water Level (ft below top of } \\
\text { casing) at Indicated Well }\end{array}$} \\
\hline & Time & D2 & WP49 & SB65D \\
\hline 10/13/2005 & $0: 00$ & 38.68 & 47.581 & 22.966 \\
\hline $10 / 13 / 2005$ & 4:00 & 38.689 & 47.584 & 22.964 \\
\hline $10 / 13 / 2005$ & $8: 00$ & 38.704 & 47.599 & 22.98 \\
\hline $10 / 13 / 2005$ & $12: 00$ & 38.723 & 47.611 & 22.978 \\
\hline 10/13/2005 & 16:00 & 38.692 & 47.562 & 22.931 \\
\hline $10 / 13 / 2005$ & $20: 00$ & 38.689 & 47.573 & 22.946 \\
\hline $10 / 14 / 2005$ & $0: 00$ & 38.684 & 47.564 & 22.935 \\
\hline 10/14/2005 & 4:00 & 38.672 & 47.551 & 22.929 \\
\hline $10 / 14 / 2005$ & 8:00 & 38.706 & 47.616 & 22.989 \\
\hline $10 / 14 / 2005$ & $12: 00$ & 38.747 & 47.641 & 23.007 \\
\hline $10 / 14 / 2005$ & 16:00 & 38.738 & 47.624 & 22.987 \\
\hline $10 / 14 / 2005$ & $20: 00$ & 38.771 & 47.675 & 23.038 \\
\hline $10 / 15 / 2005$ & $0: 00$ & 38.815 & 47.712 & 23.063 \\
\hline $10 / 15 / 2005$ & $4: 00$ & 38.829 & 47.716 & 23.061 \\
\hline $10 / 15 / 2005$ & 8:00 & 38.861 & 47.757 & 23.091 \\
\hline 10/15/2005 & 12:00 & 38.861 & 47.703 & 23.04 \\
\hline $10 / 15 / 2005$ & $16: 00$ & 38.783 & 47.618 & 22.96 \\
\hline $10 / 15 / 2005$ & $20: 00$ & 38.759 & 47.605 & 22.962 \\
\hline 10/16/2005 & $0: 00$ & 38.759 & 47.609 & 22.966 \\
\hline $10 / 16 / 20$ & 4:00 & 38.754 & 47.616 & 22.964 \\
\hline $10 / 16 / 2005$ & 8:00 & 38.757 & 47.631 & 22.982 \\
\hline $10 / 16 / 2005$ & $12: 00$ & 38.747 & 47.601 & 22.952 \\
\hline 10/16/2005 & $16: 00$ & 38.672 & 47.496 & 22.863 \\
\hline $10 / 16 / 2005$ & $20: 00$ & 38.626 & 47.464 & 22.847 \\
\hline $10 / 17 / 2005$ & $0: 00$ & 38.585 & 47.41 & 22.808 \\
\hline $10 / 17 / 2005$ & $4: 00$ & 38.53 & 47.361 & 22.781 \\
\hline 10/17/2005 & 8:00 & 38.544 & 47.41 & 22.847 \\
\hline 10/17/2005 & 12:00 & 38.61 & 47.485 & 22.911 \\
\hline $10 / 17 / 2005$ & $16: 00$ & 38.607 & 47.47 & 22.892 \\
\hline $10 / 17 / 2005$ & $20: 00$ & 38.622 & 47.49 & 22.914 \\
\hline 10/18/2005 & $0: 00$ & 38.641 & 47.515 & 22.919 \\
\hline $10 / 18 / 2005$ & $4: 00$ & 38.624 & 47.49 & 22.898 \\
\hline $10 / 18 / 2005$ & $8: 00$ & 38.626 & 47.5 & 22.907 \\
\hline 10/18/2005 & $12: 00$ & 38.607 & 47.449 & 22.855 \\
\hline $10 / 18 / 2005$ & $16: 00$ & 38.535 & 47.368 & 22.789 \\
\hline 10/18/2005 & 20:00 & 38.52 & 47.376 & 22.81 \\
\hline $10 / 19 / 2005$ & $0: 00$ & 38.614 & 47.53 & 22.96 \\
\hline 10/19/2005 & 4:00 & 38.701 & 47.613 & 23.021 \\
\hline 10/19/2005 & 8:00 & 38.757 & 47.669 & 23.06 \\
\hline $10 / 19 / 2005$ & $12: 00$ & 38.776 & 47.648 & 23.021 \\
\hline 10/19/2005 & 16:00 & 38.752 & 47.607 & 22.98 \\
\hline & & & & \\
\hline
\end{tabular}

TABLE S1.1 (Cont.)

\begin{tabular}{|c|c|c|c|c|}
\hline \multirow[b]{2}{*}{ Date } & \multicolumn{4}{|c|}{$\begin{array}{l}\text { Water Level (ft below top of } \\
\text { casing) at Indicated Well }\end{array}$} \\
\hline & Time & D2 & WP49 & SB65D \\
\hline 10/20/2005 & $0: 00$ & 38.723 & 47.594 & 22.972 \\
\hline $10 / 20 / 2005$ & $4: 00$ & 38.648 & 47.492 & 22.88 \\
\hline $10 / 20 / 2005$ & $8: 00$ & 38.672 & 47.56 & 22.943 \\
\hline 10/20/2005 & $12: 00$ & 38.713 & 47.594 & 22.966 \\
\hline $10 / 20 / 2005$ & $16: 00$ & 38.725 & 47.611 & 22.984 \\
\hline 10/20/2005 & $20: 00$ & 38.781 & 47.678 & 23.042 \\
\hline $10 / 21 / 2005$ & $0: 00$ & 38.827 & 47.716 & 23.066 \\
\hline $10 / 21 / 2005$ & 4:00 & 38.849 & 47.731 & 23.074 \\
\hline 10/21/2005 & $8: 00$ & 38.897 & 47.799 & 23.132 \\
\hline $10 / 21 / 2005$ & $12: 00$ & 38.928 & 47.797 & 23.109 \\
\hline 10/21/2005 & 16:00 & 38.892 & 47.727 & 23.04 \\
\hline $10 / 21 / 2005$ & $20: 00$ & 38.906 & 47.767 & 23.079 \\
\hline $10 / 22 / 2005$ & $0: 00$ & 38.911 & 47.757 & 23.064 \\
\hline 10/22/2005 & 4:00 & 38.894 & 47.725 & 23.031 \\
\hline $10 / 22 / 2 C$ & $8: 00$ & 38.868 & 47.72 & 23.037 \\
\hline $10 / 22 / 2005$ & $12: 00$ & 38.839 & 47.656 & 22.976 \\
\hline 10/22/2005 & 16:00 & 38.798 & 47.633 & 22.962 \\
\hline 10/22/2005 & $20: 00$ & 38.829 & 47.682 & 23.021 \\
\hline $10 / 23 / 2005$ & 0:00 & 38.87 & 47.735 & 23.066 \\
\hline $10 / 23 / 2005$ & $4: 00$ & 38.914 & 47.802 & 23.109 \\
\hline $10 / 23 / 2005$ & $8: 00$ & 38.972 & 47.866 & 23.159 \\
\hline 10/23/2005 & 12:00 & 39.003 & 47.881 & 23.157 \\
\hline 10/23/2005 & $16: 00$ & 39.03 & 47.915 & 23.187 \\
\hline $10 / 23 / 2005$ & $20: 00$ & 39.063 & 47.938 & 23.193 \\
\hline $10 / 24 / 2005$ & $0: 00$ & 39.085 & 47.947 & 23.189 \\
\hline $10 / 24 / 2$ & $4: 00$ & 39.09 & 47.938 & 23.177 \\
\hline 10/24/2005 & 8:00 & 39.1 & 47.964 & 23.193 \\
\hline $10 / 24 / 2005$ & $12: 00$ & 39.102 & 47.936 & 23.156 \\
\hline $10 / 24 / 2005$ & $16: 00$ & 39.049 & 47.87 & 23.099 \\
\hline 10/24/2005 & $20: 00$ & 39.056 & 47.885 & 23.115 \\
\hline 10/25/2005 & $0: 00$ & 39.037 & 47.87 & 23.103 \\
\hline $10 / 25 / 2005$ & $4: 00$ & 39.03 & 47.87 & 23.109 \\
\hline 10/25/2005 & 8:00 & 39.046 & 47.906 & 23.14 \\
\hline $10 / 25 / 2005$ & $12: 00$ & 39.03 & 47.842 & 23.085 \\
\hline $10 / 25 / 2005$ & $16: 00$ & 38.947 & 47.748 & 23.009 \\
\hline $10 / 25 / 2005$ & $20: 00$ & 38.911 & 47.731 & 22.997 \\
\hline $10 / 26 / 2005$ & 0:00 & 38.882 & 47.705 & 22.98 \\
\hline $10 / 26 / 2005$ & $4: 00$ & 38.877 & 47.714 & 22.999 \\
\hline 10/26/2005 & 8:00 & 38.877 & 47.725 & 23.015 \\
\hline $10 / 26 / 2005$ & $12: 00$ & 38.89 & 47.729 & 23.015 \\
\hline 10/26/2005 & 16:00 & 38.868 & 47.72 & 23.013 \\
\hline & & & 12 & \\
\hline
\end{tabular}


TABLE S1.1 (Cont.)

\begin{tabular}{|c|c|c|c|c|}
\hline \multirow[b]{2}{*}{ Date } & \multicolumn{4}{|c|}{$\begin{array}{l}\text { Water Level (ft below top of } \\
\text { casing) at Indicated Well }\end{array}$} \\
\hline & Time & D2 & WP49 & SB65D \\
\hline $10 / 27 / 2005$ & $0: 00$ & 38.991 & 47.885 & 23.162 \\
\hline $10 / 27 / 2005$ & $4: 00$ & 39.037 & 47.921 & 23.187 \\
\hline 10/27/2005 & 8:00 & 39.085 & 47.977 & 23.228 \\
\hline 10/27/2005 & $12: 00$ & 39.119 & 47.979 & 23.211 \\
\hline $10 / 27 / 2005$ & $16: 00$ & 39.071 & 47.913 & 23.142 \\
\hline 10/27/2005 & $20: 00$ & 39.075 & 47.928 & 23.164 \\
\hline $10 / 28 / 2005$ & $0: 00$ & 39.09 & 47.93 & 23.175 \\
\hline $10 / 28 / 2005$ & $4: 00$ & 39.092 & 47.93 & 23.173 \\
\hline 10/28/2005 & 8:00 & 39.095 & 47.932 & 23.171 \\
\hline $10 / 28 / 2005$ & $12: 00$ & 39.071 & 47.872 & 23.111 \\
\hline $10 / 28 / 2005$ & $16: 00$ & 38.996 & 47.793 & 23.051 \\
\hline $10 / 28 / 2005$ & $20: 00$ & 38.981 & 47.812 & 23.066 \\
\hline 10/29/2005 & $0: 00$ & 38.976 & 47.81 & 23.068 \\
\hline 10/29/2005 & $4: 00$ & 38.955 & 47.778 & 23.045 \\
\hline 10/29/2005 & $8: 00$ & 38.938 & 47.772 & 23.047 \\
\hline $10 / 29 / 2005$ & $12: 00$ & 38.924 & 47.744 & 23.021 \\
\hline $10 / 29 / 2005$ & $16: 00$ & 38.866 & 47.675 & 22.986 \\
\hline 10/29/2005 & $20: 00$ & 38.892 & 47.74 & 23.041 \\
\hline $10 / 30 / 2005$ & $0: 00$ & 38.89 & 47.72 & 23.025 \\
\hline $10 / 30 / 2005$ & 4:00 & 38.863 & 47.684 & 22.992 \\
\hline $10 / 30 / 2005$ & $8: 00$ & 38.887 & 47.74 & 23.052 \\
\hline $10 / 30 / 2005$ & $12: 00$ & 38.914 & 47.759 & 23.062 \\
\hline $10 / 30 / 2005$ & $16: 00$ & 38.902 & 47.75 & 23.056 \\
\hline $10 / 30 / 2005$ & $20: 00$ & 38.943 & 47.806 & 23.107 \\
\hline $10 / 31 / 2005$ & $0: 00$ & 39.013 & 47.885 & 23.175 \\
\hline $10 / 31 / 2005$ & $4: 00$ & 39. & 47.913 & 23.185 \\
\hline $10 / 31 /$ & $8: 00$ & 39.104 & 47.979 & 23.242 \\
\hline $10 / 31 / 2005$ & $12: 00$ & 39.126 & 47.97 & 23.214 \\
\hline $10 / 31 / 2005$ & $16: 00$ & 39.073 & 47.9 & 23.15 \\
\hline $10 / 31 / 2005$ & $20: 00$ & 39.087 & 47.921 & 23.171 \\
\hline $11 / 1 / 2005$ & $0: 00$ & 39.078 & 47.893 & 23.136 \\
\hline $11 / 1 / 2005$ & $4: 00$ & 39.034 & 47.844 & 23.093 \\
\hline $11 / 1 / 2005$ & $8: 00$ & 38.986 & 47.799 & 23.062 \\
\hline $11 / 1 / 2005$ & $12: 00$ & 38.989 & 47.81 & 23.07 \\
\hline $11 / 1 / 2005$ & $16: 00$ & 38.976 & 47.819 & 23.093 \\
\hline $11 / 1 / 2005$ & $20: 00$ & 39.017 & 47.872 & 23.14 \\
\hline 11/2/2005 & $0: 00$ & 39.015 & 47.842 & 23.109 \\
\hline $11 / 2 / 2005$ & $4: 00$ & 38.972 & 47.776 & 23.047 \\
\hline $11 / 2 / 2005$ & 8:00 & 38.931 & 47.731 & 23.015 \\
\hline 11/2/2005 & $12: 00$ & 38.88 & 47.667 & 22.965 \\
\hline $11 / 2 / 2005$ & $16: 00$ & 38.8 & 47.594 & 22.92 \\
\hline $11 / 2 / 2005$ & 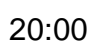 & 38.798 & 2 & 22.957 \\
\hline
\end{tabular}

TABLE S1.1 (Cont.)

\begin{tabular}{|c|c|c|c|c|}
\hline \multirow[b]{2}{*}{ Date } & \multicolumn{4}{|c|}{$\begin{array}{l}\text { Water Level (ft below top of } \\
\text { casing) at Indicated Well }\end{array}$} \\
\hline & Time & D2 & WP49 & SB65D \\
\hline $11 / 3 / 2005$ & $0: 00$ & 38.807 & 47.637 & 22.979 \\
\hline $11 / 3 / 2005$ & $4: 00$ & 38.769 & 47.577 & 22.92 \\
\hline $11 / 3 / 2005$ & $8: 00$ & 38.745 & 47.566 & 22.926 \\
\hline $11 / 3 / 2005$ & $12: 00$ & 38.701 & 47.5 & 22.862 \\
\hline $11 / 3 / 2005$ & $16: 00$ & 38.663 & 47.483 & 22.863 \\
\hline $11 / 3 / 2005$ & $20: 00$ & 38.721 & 47.59 & 22.973 \\
\hline $11 / 4 / 2005$ & $0: 00$ & 38.762 & 47.616 & 22.992 \\
\hline $11 / 4 / 2005$ & $4: 00$ & 38.817 & 47.684 & 23.039 \\
\hline $11 / 4 / 2005$ & $8: 00$ & 38.854 & 47.735 & 23.084 \\
\hline $11 / 4 / 2005$ & $12: 00$ & 38.863 & 47.705 & 23.039 \\
\hline $11 / 4 / 2005$ & $16: 00$ & 38.81 & 47.631 & 22.977 \\
\hline $11 / 4 / 2005$ & $20: 00$ & 38.836 & 47.69 & 23.037 \\
\hline $11 / 5 / 2005$ & $0: 00$ & 38.824 & 47.652 & 22.998 \\
\hline $11 / 5 / 2005$ & $4: 00$ & 38.82 & 47.667 & 23.018 \\
\hline $11 / 5 / 2005$ & $8: 00$ & 38.836 & 47.682 & 23.022 \\
\hline $11 / 5 / 2005$ & $12: 00$ & 38.841 & 47.686 & 23.022 \\
\hline $11 / 5 / 2005$ & $16: 00$ & 38.844 & 47.718 & 23.063 \\
\hline $11 / 5 / 2005$ & $20: 00$ & 38.897 & 47.748 & 23.092 \\
\hline $11 / 6 / 2005$ & $0: 00$ & 38.957 & 47.842 & 23.17 \\
\hline $11 / 6 / 2005$ & $4: 00$ & 39.039 & 47.913 & 23.217 \\
\hline $11 / 6 / 2005$ & 8:00 & 39.102 & & 23.271 \\
\hline $11 / 6 / 2005$ & $12: 00$ & 39.143 & 47.998 & 23.242 \\
\hline $11 / 6 / 2005$ & $16: 00$ & 39.085 & 47.896 & 23.154 \\
\hline $11 / 6 / 2005$ & $20: 00$ & 39.068 & 47.876 & 23.141 \\
\hline $11 / 7 / 2005$ & $0: 00$ & 39.071 & 47.885 & 23.15 \\
\hline $11 / 7 / 2005$ & $4: 00$ & 39.073 & 47.891 & 23.143 \\
\hline $11 / 7 / 2005$ & $8: 00$ & 39.073 & 47.911 & 23.162 \\
\hline $11 / 7 / 2005$ & $12: 00$ & 39.075 & 47.896 & 23.141 \\
\hline $11 / 7 / 2005$ & $16: 00$ & 39.056 & 47.889 & 23.139 \\
\hline $11 / 7 / 2005$ & $20: 00$ & 39.1 & 47.934 & 23.193 \\
\hline $11 / 8 / 2005$ & $0: 00$ & 39.128 & 47.96 & 23.209 \\
\hline $11 / 8 / 2005$ & $4: 00$ & 39.107 & 47.915 & 23.164 \\
\hline $11 / 8 / 2005$ & $8: 00$ & 39.078 & 47.866 & 23.119 \\
\hline $11 / 8 / 2005$ & $12: 00$ & 39.027 & 47.808 & 23.059 \\
\hline $11 / 8 / 2005$ & $16: 00$ & 38.964 & 47.755 & 23.027 \\
\hline $11 / 8 / 2005$ & $20: 00$ & 38.974 & 47.795 & 23.074 \\
\hline $11 / 9 / 2005$ & $0: 00$ & 39.102 & 48.015 & 23.287 \\
\hline $11 / 9 / 2005$ & $4: 00$ & 39.24 & 48.135 & 23.361 \\
\hline $11 / 9 / 2005$ & $8: 00$ & 39.324 & 48.216 & 23.41 \\
\hline $11 / 9 / 2005$ & $12: 00$ & 39.408 & 48.27 & 23.42 \\
\hline $11 / 9 / 2005$ & $16: 00$ & 39.425 & 48.268 & 23.402 \\
\hline $11 / 9 / 2005$ & 0 & 2 & 287 & 23.402 \\
\hline
\end{tabular}


TABLE S1.1 (Cont.)

\begin{tabular}{|c|c|c|c|c|}
\hline \multirow[b]{2}{*}{ Date } & \multicolumn{4}{|c|}{$\begin{array}{l}\text { Water Level (ft below top of } \\
\text { casing) at Indicated Well }\end{array}$} \\
\hline & Time & D2 & WP49 & SB65D \\
\hline $11 / 10 / 2005$ & $0: 00$ & 39.464 & 48.278 & 23.383 \\
\hline $11 / 10 / 2005$ & $4: 00$ & 39.45 & 48.238 & 23.342 \\
\hline $11 / 10 / 2005$ & 8:00 & 39.425 & 48.208 & 23.309 \\
\hline $11 / 10 / 2005$ & $12: 00$ & 39.372 & 48.116 & 23.223 \\
\hline $11 / 10 / 2005$ & $16: 00$ & 39.271 & 48.007 & 23.152 \\
\hline $11 / 10 / 2005$ & $20: 00$ & 39.235 & 47.983 & 23.145 \\
\hline $11 / 11 / 2005$ & $0: 00$ & 39.189 & 47.947 & 23.11 \\
\hline $11 / 11 / 2005$ & $4: 00$ & 39.136 & 47.893 & 23.077 \\
\hline $11 / 11 / 2005$ & 8:00 & 39.114 & 47.889 & 23.082 \\
\hline $11 / 11 / 2005$ & $12: 00$ & 39.073 & 47.834 & 23.04 \\
\hline $11 / 11 / 2005$ & $16: 00$ & 38.984 & 47.742 & 22.977 \\
\hline $11 / 11 / 2005$ & $20: 00$ & 38.957 & 47.734 & 22.985 \\
\hline $11 / 12 / 2005$ & $0: 00$ & 38.919 & 47.684 & 22.952 \\
\hline $11 / 12 / 2005$ & $4: 00$ & 38.846 & 47.605 & 22.897 \\
\hline $11 / 12 / 2005$ & $8: 00$ & 38.786 & 47.558 & 22.88 \\
\hline $11 / 12 / 2005$ & $12: 00$ & 38.721 & 47.47 & 22.809 \\
\hline $11 / 12 / 2005$ & $16: 00$ & 38.67 & 47.483 & 22.866 \\
\hline $11 / 12 / 2005$ & $20: 00$ & 38.861 & 47.789 & 23.139 \\
\hline $11 / 13 / 2005$ & $0: 00$ & 39.046 & 47.988 & 23.287 \\
\hline $11 / 13 / 2005$ & 4:00 & 39.194 & 48.126 & 23.369 \\
\hline $11 / 13 / 2005$ & $8: 00$ & 39.336 & 48.259 & 23.451 \\
\hline $11 / 13 / 2005$ & $12: 00$ & 39.476 & 48.375 & 23.51 \\
\hline $11 / 13 / 2005$ & $16: 00$ & 39.51 & 48.362 & 23.469 \\
\hline $11 / 13 / 2005$ & $20: 00$ & 39.534 & 48.362 & 23.451 \\
\hline $11 / 14 / 2005$ & $0: 00$ & 39.536 & 48.334 & 23.412 \\
\hline $11 / 14 / 2005$ & $4: 00$ & 39.505 & & 23.362 \\
\hline $11 / 14 / 2005$ & $8: 00$ & 39.425 & 48.159 & 23.247 \\
\hline $11 / 14 / 2005$ & $12: 00$ & 39.319 & 48.009 & 23.137 \\
\hline $11 / 14 / 2005$ & $16: 00$ & 39.206 & 47.93 & 23.087 \\
\hline $11 / 14 / 2005$ & $20: 00$ & 39.189 & 47.951 & 23.12 \\
\hline $11 / 15 / 2005$ & $0: 00$ & 39.194 & 47.979 & 23.149 \\
\hline $11 / 15 / 2005$ & $4: 00$ & 39.15 & 47.928 & 23.106 \\
\hline $11 / 15 / 2005$ & $8: 00$ & 39.189 & 48.005 & 23.182 \\
\hline $11 / 15 / 2005$ & $12: 00$ & 39.218 & 48.035 & 23.206 \\
\hline $11 / 15 / 2005$ & $16: 00$ & 39.295 & 48.157 & 23.317 \\
\hline $11 / 15 / 2005$ & $20: 00$ & & 48.276 & 23.407 \\
\hline $11 / 16 / 2005$ & 0:00 & 39.476 & 48.349 & 23.455 \\
\hline $11 / 16 / 2005$ & $4: 00$ & 39.534 & 48.377 & 23.461 \\
\hline $11 / 16 / 2005$ & $8: 00$ & 39.611 & 48.466 & 23.532 \\
\hline $11 / 16 / 2005$ & $12: 00$ & 39.72 & 48.554 & 23.576 \\
\hline $11 / 16 / 2005$ & $16: 00$ & 39.734 & 48.552 & 23.549 \\
\hline $11 / 16 / 2005$ & $20: 00$ & 39.749 & 48.543 & 23.524 \\
\hline
\end{tabular}

TABLE S1.1 (Cont.)

\begin{tabular}{|c|c|c|c|c|}
\hline \multirow[b]{2}{*}{ Date } & \multicolumn{4}{|c|}{$\begin{array}{l}\text { Water Level (ft below top of } \\
\text { casing) at Indicated Well }\end{array}$} \\
\hline & Time & D2 & WP49 & SB65D \\
\hline 11/17/2005 & $0: 00$ & 39.739 & 48.492 & 23.483 \\
\hline $11 / 17 / 2005$ & $4: 00$ & 39.717 & 48.477 & 23.463 \\
\hline 11/17/2005 & $8: 00$ & 39.708 & 48.466 & 23.452 \\
\hline $11 / 17 / 2005$ & $12: 00$ & 39.686 & 48.424 & 23.413 \\
\hline 11/17/2005 & 16:00 & 39.611 & 48.342 & 23.35 \\
\hline 11/17/2005 & $20: 00$ & 39.616 & 48.349 & 23.368 \\
\hline $11 / 18 / 2005$ & $0: 00$ & 39.604 & 48.342 & 23.366 \\
\hline $11 / 18 / 2005$ & $4: 00$ & 39.57 & 48.317 & 23.344 \\
\hline $11 / 18 / 2005$ & $8: 00$ & 39.539 & 48.294 & 23.327 \\
\hline $11 / 18 / 2005$ & $12: 00$ & 39.481 & 48.2 & 23.245 \\
\hline $11 / 18 / 2005$ & 16:00 & 39.338 & 48.035 & 23.124 \\
\hline $11 / 18 / 2005$ & $20: 00$ & 39.227 & 47.932 & 23.063 \\
\hline $11 / 19 / 2005$ & $0: 00$ & 39.157 & 47.883 & 23.05 \\
\hline 11/19/2005 & 4:00 & 9.16 & 47.934 & 23.112 \\
\hline $11 / 19 / 2$ & $8: 00$ & 39.208 & 48.031 & 23.204 \\
\hline $11 / 19 / 2005$ & $12: 00$ & 39.281 & 48.118 & 23.276 \\
\hline $11 / 19 / 2005$ & 16:00 & 39.348 & 48.202 & 23.341 \\
\hline $11 / 19 / 2005$ & $20: 00$ & 39.428 & 48.27 & 23.393 \\
\hline $11 / 20 / 2005$ & 0:00 & 39.491 & 48.33 & 23.425 \\
\hline $11 / 20 / 2005$ & $4: 00$ & 39.529 & 48.347 & 23.427 \\
\hline $11 / 20 / 2005$ & $8: 00$ & 39.536 & 48.341 & 23.417 \\
\hline 11/20/2005 & 12:00 & 39.534 & 48.313 & 23.384 \\
\hline 11/20/2005 & $16: 00$ & 39.483 & 48.242 & 23.329 \\
\hline $11 / 20 / 2005$ & $20: 00$ & 39.471 & 48.24 & 23.321 \\
\hline $11 / 21 / 2005$ & 0:00 & 39.45 & 48.219 & 23.304 \\
\hline $11 / 21 / 2005$ & $4: 00$ & 39.404 & 48.161 & 23.261 \\
\hline 11/21/2005 & 8:00 & 39.404 & 48.195 & 23.304 \\
\hline $11 / 21 / 2005$ & $12: 00$ & 39.454 & 48.253 & 23.354 \\
\hline $11 / 21 / 2005$ & $16: 00$ & 39.466 & 48.27 & 23.368 \\
\hline $11 / 21 / 2005$ & $20: 00$ & 39.495 & 48.304 & 23.393 \\
\hline $11 / 22 / 2005$ & 0:00 & 39.471 & 48.289 & 23.384 \\
\hline $11 / 22 / 2005$ & $4: 00$ & 39.425 & 48.235 & 23.345 \\
\hline 11/22/2005 & 8:00 & 39.392 & 48.219 & 23.333 \\
\hline 11/22/2005 & $12: 00$ & 39.336 & 48.146 & 23.272 \\
\hline $11 / 22 / 2005$ & $16: 00$ & 39.232 & 48.032 & 23.185 \\
\hline 11/22/2005 & $20: 00$ & 39.17 & 47.96 & 23.136 \\
\hline 11/23/2005 & 0:00 & 39.08 & 47.858 & 23.072 \\
\hline $11 / 23 / 2005$ & $4: 00$ & 39.02 & 47.81 & 23.048 \\
\hline 11/23/2005 & $8: 00$ & 39.008 & 47.834 & 23.087 \\
\hline $11 / 23 / 2005$ & $12: 00$ & 39.044 & 47.891 & 23.142 \\
\hline $11 / 23$ & 16:00 & 39.063 & 47.915 & 23.163 \\
\hline $11 / 23 / 26$ & 20 & 39.121 & 47.99 & 23.224 \\
\hline
\end{tabular}


TABLE S1.1 (Cont.)

\begin{tabular}{|c|c|c|c|c|}
\hline \multirow[b]{2}{*}{ Date } & \multicolumn{4}{|c|}{$\begin{array}{l}\text { Water Level (ft below top of } \\
\text { casing) at Indicated Well }\end{array}$} \\
\hline & Time & D2 & WP49 & SB65D \\
\hline $11 / 24 / 2005$ & $0: 00$ & 39.201 & 48.082 & 23.3 \\
\hline $11 / 24 / 2005$ & $4: 00$ & 39.278 & 48.17 & 23.365 \\
\hline $11 / 24 / 2005$ & 8:00 & 39.411 & 48.33 & 23.493 \\
\hline $11 / 24 / 2005$ & $12: 00$ & 39.546 & 48.445 & 23.56 \\
\hline $11 / 24 / 2005$ & $16: 00$ & 39.568 & 48.424 & 23.513 \\
\hline $11 / 24 / 2005$ & $20: 00$ & 39.604 & 48.45 & 23.525 \\
\hline $11 / 25 / 2005$ & $0: 00$ & 39.594 & 48.409 & 23.47 \\
\hline $11 / 25 / 2005$ & $4: 00$ & 39.551 & 48.343 & 23.411 \\
\hline $11 / 25 / 2005$ & 8:00 & 39.491 & 48.253 & 23.331 \\
\hline $11 / 25 / 2005$ & $12: 00$ & 39.394 & 48.108 & 23.212 \\
\hline $11 / 25 / 2005$ & $16: 00$ & 39.266 & 48.018 & 23.158 \\
\hline $11 / 25 / 2005$ & $20: 00$ & 39.24 & 48.014 & 23.171 \\
\hline $11 / 26 / 2005$ & $0: 00$ & 39.247 & 48.054 & 23.216 \\
\hline $11 / 26 / 2005$ & $4: 00$ & 39.242 & 48.033 & 23.199 \\
\hline $11 / 26 / 2005$ & $8: 00$ & 39.227 & 48.018 & 23.193 \\
\hline $11 / 26 / 2005$ & $12: 00$ & 39.186 & 47.941 & 23.125 \\
\hline $11 / 26 / 2005$ & $16: 00$ & 39.107 & 47.87 & 23.082 \\
\hline $11 / 26 / 2005$ & $20: 00$ & 39.071 & 47.836 & 23.078 \\
\hline $11 / 27 / 2005$ & $0: 00$ & 39.051 & 47.819 & 23.07 \\
\hline 11/27/2005 & 4:00 & 39.003 & 47.778 & 23.035 \\
\hline $11 / 27 / 2005$ & $8: 00$ & 38.991 & 47.766 & 23.041 \\
\hline $11 / 27 / 2005$ & $12: 00$ & 38.969 & 47.755 & 23.023 \\
\hline $11 / 27 / 2005$ & $16: 00$ & 38.873 & 47.676 & 22.974 \\
\hline $11 / 27 / 2005$ & $20: 00$ & 38.846 & 47.667 & 22.98 \\
\hline $11 / 28 / 2005$ & $0: 00$ & 38.817 & 47.614 & 22.939 \\
\hline $11 / 28 / 2005$ & $4: 00$ & 38.7 & 47.603 & 22.938 \\
\hline $11 / 28 / 2005$ & $8: 00$ & 38.82 & 47.661 & 22.986 \\
\hline $11 / 28 / 2005$ & $12: 00$ & 38.887 & 47.763 & 23.078 \\
\hline $11 / 28 / 2005$ & $16: 00$ & 38.998 & 47.905 & 23.197 \\
\hline $11 / 28 / 2005$ & $20: 00$ & 39.136 & 48.039 & 23.291 \\
\hline $11 / 29 / 2005$ & $0: 00$ & 39.256 & 48.174 & 23.371 \\
\hline $11 / 29 / 2005$ & $4: 00$ & 39.394 & 48.315 & 23.464 \\
\hline $11 / 29 / 2005$ & $8: 00$ & 39.507 & 48.413 & 23.515 \\
\hline $11 / 29 / 2005$ & $12: 00$ & 39.626 & 48.505 & 23.568 \\
\hline $11 / 29 / 2005$ & $16: 00$ & 39.696 & 48.569 & 23.593 \\
\hline $11 / 29 / 2005$ & $20: 00$ & 39.768 & 48.61 & 23.603 \\
\hline $11 / 30 / 2005$ & $0: 00$ & 39.802 & 48.614 & 23.591 \\
\hline $11 / 30 / 2005$ & $4: 00$ & 39.792 & 48.578 & 23.542 \\
\hline $11 / 30 / 2005$ & 8:00 & 39.771 & 48.535 & 23.5 \\
\hline $11 / 30 / 2005$ & $12: 00$ & 39.734 & 48.473 & 23.437 \\
\hline $11 / 30 / 2005$ & $16: 00$ & 39.645 & 48.366 & 23.357 \\
\hline $11 / 30 / 2005$ & $20: 00$ & 39.655 & 48.424 & 23.412 \\
\hline
\end{tabular}

TABLE S1.1 (Cont.)

\begin{tabular}{ccccc}
\hline & & & & \\
& & Water Level (ft below top of \\
& casing) at Indicated Well \\
\cline { 2 - 5 } Date & Time & D2 & WP49 & SB65D \\
& & & & \\
\hline & & & & \\
$12 / 1 / 2005$ & $0: 00$ & 39.71 & 48.535 & 23.527 \\
$12 / 1 / 2005$ & $4: 00$ & 39.845 & 48.68 & 23.636 \\
$12 / 1 / 2005$ & $8: 00$ & 39.913 & 48.721 & 23.65 \\
$12 / 1 / 2005$ & $12: 00$ & 39.968 & 48.757 & 23.659 \\
$12 / 1 / 2005$ & $16: 00$ & 39.947 & 48.689 & 23.597 \\
$12 / 1 / 2005$ & $20: 00$ & 39.913 & 48.638 & 23.552 \\
$12 / 2 / 2005$ & $0: 00$ & 39.918 & 48.648 & 23.55 \\
$12 / 2 / 2005$ & $4: 00$ & 39.886 & 48.601 & 23.514 \\
$12 / 2 / 2005$ & $8: 00$ & 39.836 & 48.542 & 23.463 \\
$12 / 2 / 2005$ & $12: 00$ & 39.758 & 48.443 & 23.385 \\
$12 / 2 / 2005$ & $16: 00$ & 39.635 & 48.319 & 23.301 \\
$12 / 2 / 2005$ & $20: 00$ & 39.618 & 48.338 & 23.34 \\
$12 / 3 / 2005$ & $0: 00$ & 39.597 & 48.33 & 23.346 \\
$12 / 3 / 2005$ & $4: 00$ & 39.577 & 48.291 & 23.32 \\
$12 / 3 / 2005$ & $8: 00$ & 39.585 & 48.323 & 23.359 \\
$12 / 3 / 2005$ & $12: 00$ & 39.604 & 48.353 & 23.383 \\
$12 / 3 / 2005$ & $16: 00$ & 39.621 & 48.405 & 23.432 \\
$12 / 3 / 2005$ & $20: 00$ & 39.698 & 48.509 & 23.521 \\
$12 / 4 / 2005$ & $0: 00$ & 39.778 & 48.584 & 23.568 \\
$12 / 4 / 2005$ & $4: 00$ & 39.831 & 48.631 & 23.603 \\
$12 / 4 / 2005$ & $8: 00$ & 39.894 & 48.704 & 23.658 \\
$12 / 4 / 2005$ & $12: 00$ & 39.961 & 48.755 & 23.679 \\
$12 / 4 / 2005$ & $16: 00$ & 39.971 & 48.753 & 23.668 \\
$12 / 4 / 2005$ & $20: 00$ & 40.002 & 48.772 & 23.674 \\
$12 / 5 / 2005$ & $0: 00$ & 40.024 & 48.779 & 23.67 \\
$12 / 5 / 2005$ & $4: 00$ & 39.997 & 48.721 & 23.609 \\
$12 / 5 / 2005$ & $8: 00$ & 39.932 & 48.627 & 23.525 \\
$12 / 7 / 2005$ & $12: 00$ & 40.229 & 48.965 & 23.76 \\
$12 / 5 / 2005$ & $12: 00$ & 39.845 & 48.509 & 23.426 \\
$12 / 5 / 2005$ & $16: 00$ & 39.698 & 48.353 & 23.307 \\
$12 / 5 / 2005$ & $20: 00$ & 39.65 & 48.379 & 23.365 \\
$12 / 6 / 2005$ & $0: 00$ & 39.78 & 48.601 & 23.574 \\
$12 / 6 / 2005$ & $4: 00$ & 39.85 & 48.638 & 23.596 \\
$12 / 6 / 2005$ & $8: 00$ & 39.937 & 48.749 & 23.679 \\
$12 / 6 / 2005$ & $12: 00$ & 40.024 & 48.811 & 23.715 \\
$12 / 6 / 2005$ & $16: 00$ & 40.036 & 48.815 & 23.705 \\
$12 / 6 / 2005$ & $20: 00$ & 40.096 & 48.873 & 23.754 \\
$12 / 7 / 2005$ & $0: 00$ & 40.154 & 48.913 & 23.768 \\
12005 & $4: 00$ & 40.174 & 48.92 & 23.752 \\
1200.185 & 48.905 & 23.703
\end{tabular}


TABLE S1.1 (Cont.)

\begin{tabular}{|c|c|c|c|c|}
\hline \multirow[b]{2}{*}{ Date } & \multicolumn{4}{|c|}{$\begin{array}{l}\text { Water Level (ft below top of } \\
\text { casing) at Indicated Well }\end{array}$} \\
\hline & Time & D2 & WP49 & SB65D \\
\hline $12 / 8 / 2005$ & $0: 00$ & 40.142 & 48.843 & 23.647 \\
\hline 12/8/2005 & $4: 00$ & 40.084 & 48.77 & 23.586 \\
\hline 12/8/2005 & 8:00 & 40.031 & 48.73 & 23.569 \\
\hline 12/8/2005 & $12: 00$ & 39.983 & 48.683 & 23.537 \\
\hline $12 / 8 / 2005$ & $16: 00$ & 39.923 & 48.642 & 23.518 \\
\hline 12/8/2005 & $20: 00$ & 39.935 & 48.678 & 23.559 \\
\hline 12/9/2005 & $0: 00$ & 39.939 & 48.678 & 23.576 \\
\hline 12/9/2005 & $4: 00$ & 39.925 & 48.659 & 23.547 \\
\hline 12/9/2005 & 8:00 & 39.898 & 48.642 & 23.541 \\
\hline 12/9/2005 & $12: 00$ & 39.877 & 48.614 & 23.52 \\
\hline 12/9/2005 & $16: 00$ & 39.79 & 48.501 & 23.43 \\
\hline 12/9/2005 & $20: 00$ & 39.703 & 48.409 & 23.37 \\
\hline $12 / 10 / 2005$ & $0: 00$ & 39.628 & 48.347 & 23.341 \\
\hline $12 / 10 / 2005$ & $4: 00$ & 39.529 & 48.247 & 23.272 \\
\hline $12 / 10 / 2005$ & $8: 00$ & 39.495 & 48.249 & 23.295 \\
\hline $12 / 10 / 2005$ & $12: 00$ & 39.517 & 48.302 & 23.356 \\
\hline $12 / 10 / 2005$ & $16: 00$ & 39.561 & 48.388 & 23.44 \\
\hline $12 / 10 / 2005$ & $20: 00$ & 39.633 & 48.456 & 23.485 \\
\hline $12 / 11 / 2005$ & $0: 00$ & 39.664 & 48.471 & 23.489 \\
\hline $12 / 11 / 2005$ & 4:00 & 39.674 & 48.484 & 23.495 \\
\hline $12 / 11 / 2005$ & $8: 00$ & 39.657 & 48.443 & 23.454 \\
\hline $12 / 11 / 2005$ & $12: 00$ & 39.606 & 48.347 & 23.364 \\
\hline $12 / 11 / 2005$ & $16: 00$ & 39.563 & 48.345 & 23.382 \\
\hline $12 / 11 / 2005$ & $20: 00$ & 39.616 & 48.42 & 23.452 \\
\hline $12 / 12 / 2005$ & $0: 00$ & 39.645 & 48.443 & 23.466 \\
\hline $12 / 12 / 2005$ & $4: 00$ & 39.696 & 48.516 & 23.528 \\
\hline $12 / 12 / 2005$ & $8: 00$ & 39.79 & 48.623 & 23.608 \\
\hline $12 / 12 / 2005$ & $12: 00$ & 39.86 & 48.67 & 23.627 \\
\hline $12 / 12 / 2005$ & $16: 00$ & 39.869 & 48.661 & 23.608 \\
\hline $12 / 12 / 2005$ & $20: 00$ & 39.898 & 48.674 & 23.618 \\
\hline $12 / 13 / 2005$ & $0: 00$ & & 48.631 & 23.567 \\
\hline $12 / 13 / 2005$ & $4: 00$ & 39.848 & 48.576 & 23.52 \\
\hline $12 / 13 / 2005$ & $8: 00$ & 39.792 & 48.516 & 23.454 \\
\hline $12 / 13 / 2005$ & $12: 00$ & 39.715 & 48.409 & 23.374 \\
\hline $12 / 13 / 2005$ & $16: 00$ & 39.611 & 48.33 & 23.325 \\
\hline $12 / 13 / 2005$ & $20: 00$ & 39.556 & 48.283 & 23.302 \\
\hline $12 / 14 / 2005$ & $0: 00$ & 39.541 & 48.302 & 23.333 \\
\hline $12 / 14 / 2005$ & $4: 00$ & 39.563 & 48.343 & 23.387 \\
\hline $12 / 14 / 2005$ & 8:00 & 39.652 & 48.471 & 23.475 \\
\hline $12 / 14 / 2005$ & $12: 00$ & 39.708 & 48.514 & 23.489 \\
\hline $12 / 14 / 2005$ & $16: 00$ & 39.732 & 48.537 & 23.499 \\
\hline $12 / 14 / 2005$ & 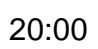 & 39.744 & 3 & 23.481 \\
\hline
\end{tabular}

TABLE S1.1 (Cont.)

\begin{tabular}{|c|c|c|c|c|}
\hline \multirow[b]{2}{*}{ Date } & \multicolumn{4}{|c|}{$\begin{array}{l}\text { Water Level (ft below top of } \\
\text { casing) at Indicated Well }\end{array}$} \\
\hline & Time & D2 & WP49 & SB65D \\
\hline $12 / 15 / 2005$ & $0: 00$ & 39.761 & 48.542 & 23.481 \\
\hline $12 / 15 / 2005$ & $4: 00$ & 39.739 & 48.501 & 23.444 \\
\hline $12 / 15 / 2005$ & $8: 00$ & 39.737 & 48.509 & 23.458 \\
\hline $12 / 15 / 2005$ & $12: 00$ & 39.758 & 48.535 & 23.478 \\
\hline $12 / 15 / 2005$ & $16: 00$ & 39.771 & 48.542 & 23.499 \\
\hline $12 / 15 / 2005$ & $20: 00$ & 39.802 & 48.582 & 23.513 \\
\hline $12 / 16 / 2005$ & $0: 00$ & 39.816 & 48.595 & 23.522 \\
\hline $12 / 16 / 2005$ & $4: 00$ & 39.857 & 48.644 & 23.561 \\
\hline $12 / 16 / 2005$ & $8: 00$ & 39.901 & 48.7 & 23.606 \\
\hline $12 / 16 / 2005$ & $12: 00$ & 39.981 & 48.777 & 23.659 \\
\hline $12 / 16 / 2005$ & 16:00 & 40.012 & 48.811 & 23.68 \\
\hline $12 / 16 / 2005$ & $20: 00$ & 40.092 & 48.894 & 23.743 \\
\hline $12 / 17 / 2005$ & $0: 00$ & 40.176 & 48.973 & 23.794 \\
\hline 12/17/2005 & 4:00 & 40.244 & 49.044 & 23.842 \\
\hline $12 / 17 / 2$ & $8: 00$ & 40.3 & 49.093 & 23.868 \\
\hline $12 / 17 / 2005$ & $12: 00$ & 40.369 & 49.121 & 23.866 \\
\hline $12 / 17 / 2005$ & 16:00 & 40.372 & 49.108 & 23.848 \\
\hline $12 / 17 / 2005$ & $20: 00$ & 40.417 & 49.153 & 23.881 \\
\hline $12 / 18 / 2005$ & 0:00 & 40.471 & 49.202 & 23.913 \\
\hline $12 / 18 / 2005$ & $4: 00$ & 40.504 & 49.215 & 23.909 \\
\hline $12 / 18 / 2005$ & $8: 00$ & 40.526 & 49.243 & 23.926 \\
\hline 12/18/2005 & 12:00 & 40.541 & 49.208 & 23.882 \\
\hline $12 / 18 / 2005$ & 16:00 & 40.497 & 49.17 & 23.843 \\
\hline $12 / 18 / 2005$ & $20: 00$ & 40.485 & 49.159 & 23.835 \\
\hline $12 / 19 / 2005$ & $0: 00$ & 40.463 & 49.131 & 23.814 \\
\hline 12/19/2005 & $4: 00$ & 40.432 & 49.11 & 23.792 \\
\hline 12/19/2005 & 8:00 & 40.398 & 49.061 & 23.773 \\
\hline $12 / 19 / 2005$ & $12: 00$ & 40.36 & 48.993 & 23.708 \\
\hline $12 / 19 / 2005$ & $16: 00$ & 40.239 & 48.892 & 23.632 \\
\hline $12 / 19 / 2005$ & $20: 00$ & 40.198 & 48.875 & 23.638 \\
\hline $12 / 20 / 2005$ & $0: 00$ & 40.162 & 48.847 & 23.624 \\
\hline $12 / 20 / 2005$ & $4: 00$ & 40.133 & 48.834 & 23.622 \\
\hline 12/20/2005 & 8:00 & 40.106 & 48.817 & 23.622 \\
\hline 12/20/2005 & $12: 00$ & 40.116 & 48.83 & 23.636 \\
\hline $12 / 20 / 2005$ & $16: 00$ & 40.075 & 48.804 & 23.622 \\
\hline $12 / 20 / 2005$ & $20: 00$ & 40.065 & 48.804 & 23.634 \\
\hline $12 / 21 / 2005$ & 0:00 & 40.051 & 48.775 & 23.609 \\
\hline $12 / 21 / 2005$ & $4: 00$ & 40.012 & 48.745 & 23.593 \\
\hline $12 / 21 / 2005$ & $8: 00$ & 39.997 & 48.745 & 23.601 \\
\hline $12 / 21 / 2005$ & $12: 00$ & 40.012 & 48.757 & 23.618 \\
\hline $2 / 21$ & 16:00 & 39.978 & 48.73 & 23.599 \\
\hline 12 & 20 & 39.968 & 48.727 & 23.607 \\
\hline
\end{tabular}


TABLE S1.1 (Cont.)

\begin{tabular}{|c|c|c|c|c|}
\hline \multirow[b]{2}{*}{ Date } & \multicolumn{4}{|c|}{$\begin{array}{l}\text { Water Level (ft below top of } \\
\text { casing) at Indicated Well }\end{array}$} \\
\hline & Time & D2 & WP49 & SB65D \\
\hline $12 / 22 / 2005$ & $0: 00$ & 39.937 & 48.665 & 23.55 \\
\hline $12 / 22 / 2005$ & $4: 00$ & 39.879 & 48.601 & 23.51 \\
\hline $12 / 22 / 2005$ & 8:00 & 39.812 & 48.544 & 23.478 \\
\hline $12 / 22 / 2005$ & $12: 00$ & 39.775 & 48.497 & 23.437 \\
\hline $12 / 22 / 2005$ & $16: 00$ & 39.674 & 48.409 & 23.392 \\
\hline $12 / 22 / 2005$ & $20: 00$ & 39.669 & 48.422 & 23.422 \\
\hline $12 / 23 / 2005$ & $0: 00$ & 39.65 & 48.415 & 23.424 \\
\hline $12 / 23 / 2005$ & $4: 00$ & 39.664 & 48.45 & 23.461 \\
\hline $12 / 23 / 2005$ & 8:00 & 39.686 & 48.477 & 23.488 \\
\hline $12 / 23 / 2005$ & $12: 00$ & 39.713 & 48.486 & 23.482 \\
\hline $12 / 23 / 2005$ & $16: 00$ & 39.664 & 48.443 & 23.455 \\
\hline $12 / 23 / 2005$ & $20: 00$ & 39.703 & 48.494 & 23.504 \\
\hline $12 / 24 / 2005$ & $0: 00$ & 39.744 & 48.533 & 23.533 \\
\hline $12 / 24 / 2005$ & $4: 00$ & 39.766 & 48.563 & 23.556 \\
\hline $12 / 24 / 2005$ & $8: 00$ & 39.848 & 48.67 & 23.646 \\
\hline $12 / 24 / 2005$ & $12: 00$ & 39.935 & 48.736 & 23.687 \\
\hline $12 / 24 / 2005$ & $16: 00$ & 39.956 & 48.734 & 23.671 \\
\hline $12 / 24 / 2005$ & $20: 00$ & 39.995 & 48.775 & 23.699 \\
\hline $12 / 25 / 2005$ & $0: 00$ & 40.019 & 48.772 & 23.683 \\
\hline $12 / 25 / 2005$ & 4:00 & 40.014 & 48.757 & 23.66 \\
\hline $12 / 25 / 2005$ & $8: 00$ & 40.034 & 48.787 & 23.687 \\
\hline $12 / 25 / 2005$ & $12: 00$ & 40.053 & 48.785 & 23.676 \\
\hline $12 / 25 / 2005$ & $16: 00$ & 40.026 & 48.766 & 23.658 \\
\hline $12 / 25 / 2005$ & $20: 00$ & 40.036 & 48.775 & 23.666 \\
\hline $12 / 26 / 2005$ & $0: 00$ & 40.024 & 48.749 & 23.641 \\
\hline $12 / 26 / 2005$ & $4: 00$ & & 48 & 23.607 \\
\hline $12 / 26 / 2005$ & $8: 00$ & 39.968 & 48.67 & 23.584 \\
\hline $12 / 26 / 2005$ & $12: 00$ & 39.937 & 48.631 & 23.555 \\
\hline $12 / 26 / 2005$ & $16: 00$ & 39.872 & 48.582 & 23.512 \\
\hline $12 / 26 / 2005$ & $20: 00$ & 39.877 & 48.608 & 23.559 \\
\hline $12 / 27 / 2005$ & $0: 00$ & 39.867 & 48.586 & 23.541 \\
\hline $12 / 27 / 2005$ & $4: 00$ & 39.831 & 48.556 & 23.508 \\
\hline $12 / 27 / 2005$ & $8: 00$ & 39.814 & 48.546 & 23.508 \\
\hline $12 / 27 / 2005$ & $12: 00$ & 39.821 & 48.574 & 23.539 \\
\hline $12 / 27 / 2005$ & $16: 00$ & 39.821 & 48.601 & 23.576 \\
\hline $12 / 27 / 2005$ & $20: 00$ & & 48.713 & 23.666 \\
\hline $12 / 28 / 2005$ & $0: 00$ & 39.944 & 48.717 & 23.654 \\
\hline $12 / 28 / 2005$ & $4: 00$ & 39.973 & 48.745 & 23.672 \\
\hline $12 / 28 / 2005$ & 8:00 & 40.019 & 48.787 & 23.701 \\
\hline $12 / 28 / 2005$ & $12: 00$ & 40.053 & 48.804 & 23.703 \\
\hline $12 / 28 / 2005$ & $16: 00$ & 40.053 & 48.807 & 23.703 \\
\hline $12 / 28 / 2005$ & $20: 00$ & 40.077 & 48.824 & 23.713 \\
\hline
\end{tabular}

TABLE S1.1 (Cont.)

\begin{tabular}{|c|c|c|c|c|}
\hline \multirow[b]{2}{*}{ Date } & \multicolumn{4}{|c|}{$\begin{array}{l}\text { Water Level (ft below top of } \\
\text { casing) at Indicated Well }\end{array}$} \\
\hline & Time & D2 & WP49 & SB65D \\
\hline 12/29/2005 & $0: 00$ & 40.116 & 48.856 & 23.734 \\
\hline 12/29/2005 & $4: 00$ & 40.101 & 48.839 & 23.717 \\
\hline 12/29/2005 & $8: 00$ & 40.094 & 48.824 & 23.696 \\
\hline 12/29/2005 & $12: 00$ & 40.077 & 48.781 & 23.653 \\
\hline 12/29/2005 & 16:00 & 40.017 & 48.715 & 23.596 \\
\hline 12/29/2005 & $20: 00$ & 39.968 & 48.661 & 23.565 \\
\hline 12/30/2005 & $0: 00$ & 39.937 & 48.653 & 23.571 \\
\hline $12 / 30 / 2005$ & $4: 00$ & 39.954 & 48.68 & 23.61 \\
\hline 12/30/2005 & $8: 00$ & 39.985 & 48.732 & 23.647 \\
\hline 12/30/2005 & $12: 00$ & 40.029 & 48.779 & 23.682 \\
\hline 12/30/2005 & 16:00 & 40.043 & 48.789 & 23.69 \\
\hline 12/30/2005 & $20: 00$ & 40.092 & 48.858 & 23.746 \\
\hline $12 / 31 / 2005$ & $0: 00$ & 40.142 & 48.905 & 23.778 \\
\hline 12/31/2005 & 4:00 & 40.171 & 48.92 & 23.781 \\
\hline 12/31/2005 & $8: 00$ & 40.2 & 48.948 & 23.801 \\
\hline $12 / 31 / 2005$ & $12: 00$ & 40.215 & 48.909 & 23.744 \\
\hline 12/31/2005 & 16:00 & 40.116 & 48.8 & 23.655 \\
\hline 12/31/2005 & $20: 00$ & 40.084 & 48.781 & 23.645 \\
\hline $1 / 1 / 2006$ & 0:00 & 40.06 & 48.755 & 23.629 \\
\hline $1 / 1 / 2006$ & 4:00 & 40.022 & 48.715 & 23.602 \\
\hline $1 / 1 / 2006$ & $8: 00$ & 39.985 & 48.663 & 23.571 \\
\hline 1/1/2006 & 12:00 & 39.947 & 48.608 & 23.524 \\
\hline $1 / 1 / 2006$ & $16: 00$ & 39.889 & 48.591 & 23.54 \\
\hline $1 / 1 / 2006$ & $20: 00$ & 39.954 & 48.7 & 23.631 \\
\hline 1/2/2006 & 0:00 & 40.005 & 48.775 & 23.694 \\
\hline $1 / 2 / 2006$ & 4:00 & 40.07 & 48.854 & 23.76 \\
\hline 1/2/2006 & 8:00 & 40.203 & 48.999 & 23.87 \\
\hline 1/2/2006 & $12: 00$ & 40.285 & 49.05 & 23.877 \\
\hline $1 / 2 / 2006$ & $16: 00$ & 40.352 & 49.142 & 23.952 \\
\hline $1 / 2 / 2006$ & $20: 00$ & 40.437 & 49.191 & 23.959 \\
\hline $1 / 3 / 2006$ & 0:00 & 40.446 & 49.161 & 23.909 \\
\hline $1 / 3 / 2006$ & $4: 00$ & 40.442 & 49.138 & 23.872 \\
\hline 1/3/2006 & 8:00 & 40.41 & 49.089 & 23.827 \\
\hline 1/3/2006 & $12: 00$ & 40.347 & 48.982 & 23.729 \\
\hline $1 / 3 / 2006$ & $16: 00$ & 40.207 & 48.841 & 23.628 \\
\hline $1 / 3 / 2006$ & $20: 00$ & 40.176 & 48.847 & 23.649 \\
\hline $1 / 4 / 2006$ & 0:00 & 40.265 & 49.018 & 23.827 \\
\hline $1 / 4 / 2006$ & $4: 00$ & 40.396 & 49.168 & 23.938 \\
\hline $1 / 4 / 2006$ & $8: 00$ & 40.495 & 49.249 & 23.987 \\
\hline $1 / 4 / 2006$ & $12: 00$ & 40.557 & 49.273 & 23.979 \\
\hline 24 & 16:00 & 40.562 & 49.273 & 23.973 \\
\hline $1 / 4 / 2006$ & $20: 00$ & 40.62 & 49.33 & 24.02 \\
\hline
\end{tabular}


TABLE S1.1 (Cont.)

\begin{tabular}{|c|c|c|c|c|}
\hline \multirow[b]{2}{*}{ Date } & \multicolumn{4}{|c|}{$\begin{array}{l}\text { Water Level (ft below top of } \\
\text { casing) at Indicated Well }\end{array}$} \\
\hline & Time & D2 & WP49 & SB65D \\
\hline $1 / 5 / 2006$ & $0: 00$ & 40.656 & 49.345 & 24.018 \\
\hline $1 / 5 / 2006$ & 4:00 & 40.681 & 49.367 & 24.028 \\
\hline $1 / 5 / 2006$ & 8:00 & 40.702 & 49.379 & 24.033 \\
\hline $1 / 5 / 2006$ & $12: 00$ & 40.734 & 49.382 & 24.032 \\
\hline $1 / 5 / 2006$ & $16: 00$ & 40.717 & 49.36 & 23.997 \\
\hline $1 / 5 / 2006$ & $20: 00$ & 40.719 & 49.371 & 24.009 \\
\hline 1/6/2006 & $0: 00$ & 40.724 & 49.364 & 24.001 \\
\hline 1/6/2006 & 4:00 & 40.695 & 49.322 & 23.958 \\
\hline 1/6/2006 & 8:00 & 40.661 & 49.29 & 23.932 \\
\hline 1/6/2006 & $12: 00$ & 40.574 & 49.157 & 23.815 \\
\hline 1/6/2006 & $16: 00$ & 40.422 & 49.01 & 23.708 \\
\hline $1 / 6 / 2006$ & $20: 00$ & 40.355 & 48.971 & 23.702 \\
\hline 1/7/2006 & $0: 00$ & 40.323 & 48.956 & 23.712 \\
\hline 1/7/2006 & 4:00 & 40.285 & 48.931 & 23.691 \\
\hline 1/7/2006 & 8:00 & 40.241 & 48.888 & 23.677 \\
\hline 1/7/2006 & $12: 00$ & 40.207 & 48.86 & 23.65 \\
\hline 1/7/2006 & $16: 00$ & 40.096 & 48.738 & 23.566 \\
\hline 1/7/2006 & $20: 00$ & 40.041 & 48.702 & 23.556 \\
\hline $1 / 8 / 2006$ & $0: 00$ & 39.954 & 48.601 & 23.486 \\
\hline $1 / 8 / 2006$ & 4:00 & 39.901 & 48.591 & 23.505 \\
\hline 1/8/2006 & 8:00 & 39.888 & 48.618 & 23.55 \\
\hline 1/8/2006 & $12: 00$ & 39.993 & 48.77 & 23.687 \\
\hline $1 / 8 / 2006$ & $16: 00$ & 40.154 & 48.98 & 23.868 \\
\hline $1 / 8 / 2006$ & $20: 00$ & 40.369 & 49.204 & 24.028 \\
\hline 1/9/2006 & $0: 00$ & 40.528 & 49.337 & 24.1 \\
\hline 1/9/2006 & 4:00 & 40.635 & 49.409 & 24.129 \\
\hline 1/9/2006 & 8:00 & 40.77 & 49.548 & 24.222 \\
\hline 1/9/2006 & $12: 00$ & 40.876 & 49.578 & 24.196 \\
\hline 1/9/2006 & $16: 00$ & 40.869 & 49.527 & 24.128 \\
\hline 1/9/2006 & $20: 00$ & 40.852 & 49.484 & 24.081 \\
\hline $1 / 10 / 2006$ & $0: 00$ & 40.835 & 49.459 & 24.054 \\
\hline $1 / 10 / 2006$ & 4:00 & 40.767 & 49.367 & 23.98 \\
\hline $1 / 10 / 2006$ & 8:00 & 40.702 & 49.307 & 23.933 \\
\hline $1 / 10 / 2006$ & $12: 00$ & 40.64 & 49.213 & 23.849 \\
\hline 1/10/2006 & $16: 00$ & 40.5 & 49.072 & 23.765 \\
\hline $1 / 10 / 2006$ & $20: 00$ & 40.42 & 49.023 & 23.736 \\
\hline $1 / 11 / 2006$ & 0:00 & 40.364 & 48.98 & 23.718 \\
\hline $1 / 11 / 2006$ & 4:00 & 40.275 & 48.907 & 23.669 \\
\hline $1 / 11 / 2006$ & 8:00 & 40.222 & 48.866 & 23.669 \\
\hline $1 / 11 / 2006$ & $12: 00$ & 40.215 & 48.869 & 23.681 \\
\hline $1 / 11 / 2006$ & $16: 00$ & 40.169 & 48.841 & 23.669 \\
\hline 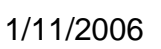 & 00 & 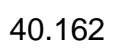 & 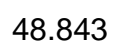 & 23.685 \\
\hline
\end{tabular}

TABLE S1.1 (Cont.)

\begin{tabular}{|c|c|c|c|c|}
\hline \multirow[b]{2}{*}{ Date } & \multicolumn{4}{|c|}{$\begin{array}{l}\text { Water Level (ft below top of } \\
\text { casing) at Indicated Well }\end{array}$} \\
\hline & Time & D2 & WP49 & SB65D \\
\hline $1 / 12 / 2006$ & $0: 00$ & 40.147 & 48.841 & 23.681 \\
\hline $1 / 12 / 2006$ & $4: 00$ & 40.128 & 48.826 & 23.671 \\
\hline $1 / 12 / 2006$ & $8: 00$ & 40.121 & 48.832 & 23.689 \\
\hline $1 / 12 / 2006$ & $12: 00$ & 40.149 & 48.864 & 23.719 \\
\hline $1 / 12 / 2006$ & $16: 00$ & 40.2 & 48.95 & 23.798 \\
\hline $1 / 12 / 2006$ & $20: 00$ & 40.335 & 49.106 & 23.931 \\
\hline $1 / 13 / 2006$ & $0: 00$ & 40.41 & 49.146 & 23.943 \\
\hline $1 / 13 / 2006$ & $4: 00$ & 40.5 & 49.251 & 24.019 \\
\hline $1 / 13 / 2006$ & $8: 00$ & 40.611 & 49.36 & 24.093 \\
\hline $1 / 13 / 2006$ & $12: 00$ & 40.695 & 49.403 & 24.103 \\
\hline $1 / 13 / 2006$ & $16: 00$ & 40.717 & 49.412 & 24.097 \\
\hline $1 / 13 / 2006$ & $20: 00$ & 40.741 & 49.418 & 24.089 \\
\hline $1 / 14 / 2006$ & $0: 00$ & 40.748 & 49.424 & 24.087 \\
\hline $1 / 14 / 2006$ & $4: 00$ & 40.734 & 49.373 & 24.029 \\
\hline $1 / 14 / 2006$ & $8: 00$ & 40.707 & 49.343 & 24.003 \\
\hline $1 / 14 / 2006$ & $12: 00$ & 40.652 & 49.253 & 23.923 \\
\hline $1 / 14 / 2006$ & $16: 00$ & 40.526 & 49.113 & 23.822 \\
\hline $1 / 14 / 2006$ & $20: 00$ & 40.446 & 49.059 & 23.785 \\
\hline $1 / 15 / 2006$ & $0: 00$ & 40.362 & 48.971 & 23.721 \\
\hline $1 / 15 / 2006$ & $4: 00$ & 40.265 & 48.869 & 23.662 \\
\hline $1 / 15 / 2006$ & 8:00 & 40.207 & 48.858 & 23.668 \\
\hline $1 / 15 / 2006$ & $12: 00$ & 40.174 & 48.824 & 23.65 \\
\hline $1 / 15 / 2006$ & $16: 00$ & 40.111 & 48.787 & 23.642 \\
\hline $1 / 15 / 2006$ & $20: 00$ & 40.113 & 48.813 & 23.678 \\
\hline $1 / 16 / 2006$ & $0: 00$ & 40.128 & 48.828 & 23.697 \\
\hline $1 / 16 / 2006$ & $4: 00$ & 40.183 & 48.928 & 23.793 \\
\hline $1 / 16 / 2006$ & $8: 00$ & 40.287 & 49.04 & 23.885 \\
\hline $1 / 16 / 2006$ & $12: 00$ & 40.381 & 49.131 & 23.949 \\
\hline $1 / 16 / 2006$ & $16: 00$ & 40.456 & 49.205 & 24 \\
\hline $1 / 16 / 2006$ & $20: 00$ & 40.555 & 49.29 & 24.048 \\
\hline $1 / 17 / 2006$ & & 40.623 & 49.33 & 24.06 \\
\hline $1 / 17 / 2006$ & $4: 00$ & 40.64 & 49.328 & 24.045 \\
\hline $1 / 17 / 2006$ & $8: 00$ & 40.64 & 49.311 & 24.021 \\
\hline $1 / 17 / 2006$ & $12: 00$ & 40.615 & 49.249 & 23.955 \\
\hline $1 / 17 / 2006$ & $16: 00$ & 40.577 & 49.236 & 23.949 \\
\hline $1 / 17 / 2006$ & $20: 00$ & 40.579 & 49.236 & 23.947 \\
\hline $1 / 18 / 2006$ & $0: 00$ & 40.574 & 49.23 & 23.945 \\
\hline $1 / 18 / 2006$ & $4: 00$ & 40.545 & 49.183 & 23.904 \\
\hline $1 / 18 / 2006$ & $8: 00$ & 40.531 & 49.191 & 23.922 \\
\hline $1 / 18 / 2006$ & $12: 00$ & 40.535 & 49.179 & 23.908 \\
\hline $1 / 18 / 2006$ & $16: 00$ & 40.468 & 49.106 & 23.846 \\
\hline $1 / 18 / 2006$ & 0 & 1 & 17 & 23.869 \\
\hline
\end{tabular}


TABLE S1.1 (Cont.)

\begin{tabular}{ccccc}
\hline & & & & \\
& & Water Level (ft below top of \\
& casing) at Indicated Well \\
\cline { 2 - 5 } Date & Time & D2 & WP49 & SB65D \\
& & & & \\
\hline & & & & \\
$1 / 19 / 2006$ & $0: 00$ & 40.437 & 49.104 & 23.861 \\
$1 / 19 / 2006$ & $4: 00$ & 40.42 & 49.08 & 23.857 \\
$1 / 19 / 2006$ & $8: 00$ & 40.415 & 49.091 & 23.877 \\
$1 / 19 / 2006$ & $12: 00$ & 40.516 & 49.208 & 23.978 \\
$1 / 19 / 2006$ & $16: 00$ & 40.577 & 49.283 & 24.039 \\
$1 / 19 / 2006$ & $20: 00$ & 40.697 & 49.431 & 24.154 \\
$1 / 20 / 2006$ & $0: 00$ & 40.794 & 49.497 & 24.17 \\
$1 / 20 / 2006$ & $4: 00$ & 40.83 & 49.527 & 24.183 \\
$1 / 20 / 2006$ & $8: 00$ & 40.866 & 49.546 & 24.187 \\
$1 / 20 / 2006$ & $12: 00$ & 40.866 & 49.491 & 24.117 \\
$1 / 20 / 2006$ & $16: 00$ & 40.854 & 49.503 & 24.133 \\
$1 / 20 / 2006$ & $20: 00$ & 40.905 & 49.555 & 24.172 \\
$1 / 21 / 2006$ & $0: 00$ & 40.949 & 49.595 & 24.203 \\
$1 / 21 / 2006$ & $4: 00$ & 40.973 & 49.594 & 24.188 \\
$1 / 21 / 2006$ & $8: 00$ & 40.987 & 49.612 & 24.201 \\
$1 / 21 / 2006$ & $12: 00$ & 41.006 & 49.595 & 24.172 \\
$1 / 21 / 2006$ & $16: 00$ & 40.965 & 49.553 & 24.135 \\
$1 / 21 / 2006$ & $20: 00$ & 40.977 & 49.58 & 24.166 \\
$1 / 22 / 2006$ & $0: 00$ & 40.99 & 49.589 & 24.17 \\
$1 / 22 / 2006$ & $4: 00$ & 41.004 & 49.598 & 24.178 \\
$1 / 22 / 2006$ & $8: 00$ & 41.019 & 49.63 & 24.219 \\
$1 / 22 / 2006$ & $12: 00$ & 41.052 & 49.642 & 24.205 \\
$1 / 22 / 2006$ & $16: 00$ & 41.014 & 49.611 & 24.18 \\
$1 / 22 / 2006$ & $20: 00$ & 41.028 & 49.619 & 24.182 \\
$1 / 25 / 2006$ & $12: 00$ & 41.113 & 49.709 & 24.264 \\
$1 / 25 / 2006$ & $16: 00$ & 41.033 & 49.594 & 24.155 \\
$1 / 25 / 2006$ & $20: 00$ & 40.973 & 49.536 & 24.11
\end{tabular}

TABLE S1.1 (Cont.)

\begin{tabular}{|c|c|c|c|c|}
\hline \multirow[b]{2}{*}{ Date } & \multicolumn{4}{|c|}{$\begin{array}{l}\text { Water Level (ft below top of } \\
\text { casing) at Indicated Well }\end{array}$} \\
\hline & Time & D2 & WP49 & SB65D \\
\hline 1/26/2006 & $0: 00$ & 40.927 & 49.503 & 24.09 \\
\hline $1 / 26 / 2006$ & $4: 00$ & 40.888 & 49.476 & 24.073 \\
\hline $1 / 26 / 2006$ & $8: 00$ & 40.849 & 49.441 & 24.049 \\
\hline $1 / 26 / 2006$ & $12: 00$ & 40.82 & 49.407 & 24.024 \\
\hline $1 / 26 / 2006$ & $16: 00$ & 40.733 & 49.33 & 23.975 \\
\hline $1 / 26 / 2006$ & $20: 00$ & 40.709 & 49.331 & 23.993 \\
\hline 1/27/2006 & $0: 00$ & 40.705 & 49.332 & 24.003 \\
\hline $1 / 27 / 2006$ & $4: 00$ & 40.714 & 49.354 & 24.028 \\
\hline $1 / 27 / 2006$ & $8: 00$ & 40.738 & 49.391 & 24.061 \\
\hline $1 / 27 / 2006$ & $12: 00$ & 40.777 & 49.418 & 24.081 \\
\hline $1 / 27 / 2006$ & $16: 00$ & 40.724 & 49.352 & 24.024 \\
\hline $1 / 27 / 2006$ & $20: 00$ & 40.717 & 49.35 & 24.028 \\
\hline $1 / 28 / 2006$ & $0: 00$ & 40.705 & 49.337 & 24.02 \\
\hline $1 / 28 / 2006$ & $4: 00$ & 40.664 & 49.27 & 23.962 \\
\hline 1/28/2006 & $8: 00$ & 40.594 & 49.2 & 23.911 \\
\hline 1/28/2006 & $12: 00$ & 40.524 & 49.129 & 23.859 \\
\hline $1 / 28 / 2006$ & $16: 00$ & 40.429 & 49.042 & 23.817 \\
\hline $1 / 28 / 2006$ & $20: 00$ & 40.391 & 49.04 & 23.835 \\
\hline $1 / 29 / 2006$ & $0: 00$ & 40.403 & 49.068 & 23.866 \\
\hline $1 / 29 / 2006$ & $4: 00$ & 40.415 & 49.097 & 23.898 \\
\hline $1 / 29 / 2006$ & $8: 00$ & 40.483 & 49.198 & 23.976 \\
\hline $1 / 29 / 2006$ & $12: 00$ & 40.541 & 49.226 & 23.987 \\
\hline $1 / 29 / 2006$ & $16: 00$ & 40.567 & 49.273 & 24.024 \\
\hline $1 / 29 / 2006$ & $20: 00$ & 40.644 & 49.343 & 24.077 \\
\hline $1 / 30 / 2006$ & $0: 00$ & 40.695 & 49.373 & 24.091 \\
\hline 1/30/2006 & $4: 00$ & 40.722 & 49.388 & 24.089 \\
\hline 1/30/2006 & $8: 00$ & 40.78 & 49.465 & 24.153 \\
\hline $1 / 30 / 2006$ & $12: 00$ & 40.862 & 49.531 & 24.196 \\
\hline $1 / 30 / 2006$ & $16: 00$ & 40.874 & 49.51 & 24.161 \\
\hline $1 / 30 / 2006$ & $20: 00$ & 40.866 & 49.495 & 24.136 \\
\hline $1 / 31 / 2006$ & & 40.837 & 49.45 & 24.095 \\
\hline $1 / 31 / 2006$ & $4: 00$ & 40.799 & 49.388 & 24.038 \\
\hline $1 / 31 / 2006$ & $8: 00$ & 40.729 & 49.333 & 23.997 \\
\hline $1 / 31 / 2006$ & $12: 00$ & 40.69 & 49.29 & 23.966 \\
\hline 1/31/2006 & $16: 00$ & 40.596 & 49.194 & 23.89 \\
\hline $1 / 31 / 2006$ & $20: 00$ & 40.611 & 49.258 & 23.97 \\
\hline 2/1/2006 & $0: 00$ & 40.695 & 49.371 & 24.075 \\
\hline 2/1/2006 & $4: 00$ & 40.717 & 49.373 & 24.071 \\
\hline 2/1/2006 & $8: 00$ & 40.751 & 49.405 & 24.093 \\
\hline 2/1/2006 & $12: 00$ & 40.777 & 49.423 & 24.099 \\
\hline 2/1/2006 & $16: 00$ & 40.722 & 49.332 & 24.013 \\
\hline $2 / 1 / 2006$ & 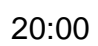 & 7 & 41 & 24.024 \\
\hline
\end{tabular}


TABLE S1.1 (Cont.)

\begin{tabular}{|c|c|c|c|c|}
\hline \multirow[b]{2}{*}{ Date } & \multicolumn{4}{|c|}{$\begin{array}{l}\text { Water Level (ft below top of } \\
\text { casing) at Indicated Well }\end{array}$} \\
\hline & Time & D2 & WP49 & SB65D \\
\hline 2/2/2006 & 0:00 & 40.668 & 49.279 & 23.976 \\
\hline 2/2/2006 & 4:00 & 40.644 & 49.277 & 23.986 \\
\hline 2/2/2006 & 8:00 & 40.627 & 49.264 & 23.98 \\
\hline 2/2/2006 & $12: 00$ & 40.635 & 49.273 & 23.99 \\
\hline 2/2/2006 & $16: 00$ & 40.613 & 49.258 & 23.986 \\
\hline 2/2/2006 & $20: 00$ & 40.666 & 49.335 & 24.058 \\
\hline 2/3/2006 & 0:00 & 40.719 & 49.392 & 24.111 \\
\hline 2/3/2006 & 4:00 & 40.794 & 49.474 & 24.169 \\
\hline 2/3/2006 & 8:00 & 40.902 & 49.591 & 24.263 \\
\hline 2/3/2006 & $12: 00$ & 40.994 & 49.638 & 24.29 \\
\hline 2/3/2006 & $16: 00$ & 41.009 & 49.63 & 24.267 \\
\hline 2/3/2006 & $20: 00$ & 41.074 & 49.719 & 24.325 \\
\hline 2/4/2006 & $0: 00$ & 41.134 & 49.767 & 24.349 \\
\hline 2/4/2006 & 4:00 & 41.166 & 49.769 & 24.337 \\
\hline $2 / 4 / 2006$ & 8:00 & 41.197 & 49.792 & 24.351 \\
\hline 2/4/2006 & $12: 00$ & 41.202 & 49.756 & 24.294 \\
\hline 2/4/2006 & $16: 00$ & 41.127 & 49.659 & 24.21 \\
\hline $2 / 4 / 2006$ & $20: 00$ & 41.086 & 49.636 & 24.189 \\
\hline $2 / 5 / 2006$ & 0:00 & 41.05 & 49.611 & 24.169 \\
\hline 2/5/2006 & 4:00 & 41.004 & 49.553 & 24.126 \\
\hline 2/5/2006 & 8:00 & 40.99 & 49.589 & 24.173 \\
\hline 2/5/2006 & $12: 00$ & 41.031 & 49.632 & 24.209 \\
\hline 2/5/2006 & $16: 00$ & 40.997 & 49.6 & 24.183 \\
\hline 2/5/2006 & $20: 00$ & 41.074 & 49.713 & 24.29 \\
\hline 2/6/2006 & 0:00 & 41.139 & 49.766 & 24.331 \\
\hline 2/6/2006 & 4:00 & 41.171 & 49.775 & 24.333 \\
\hline 2/6/2006 & 8:00 & 41.214 & 49.826 & 24.374 \\
\hline 2/6/2006 & $12: 00$ & 41.272 & 49.846 & 24.382 \\
\hline 2/6/2006 & $16: 00$ & 41.238 & 49.781 & 24.313 \\
\hline 2/6/2006 & $20: 00$ & 41.235 & 49.801 & 24.328 \\
\hline 2/7/2006 & 0:00 & 41.238 & 49.797 & 24.322 \\
\hline 2/7/2006 & 4:00 & 41.233 & 49.788 & 24.314 \\
\hline 2/7/2006 & 8:00 & 41.238 & 49.809 & 24.335 \\
\hline 2/7/2006 & $12: 00$ & 41.243 & 49.799 & 24.322 \\
\hline 2/7/2006 & $16: 00$ & 41.175 & 49.713 & 24.248 \\
\hline 2/7/2006 & $20: 00$ & 41.139 & 49.688 & 24.23 \\
\hline 2/8/2006 & 0:00 & 41.149 & 49.724 & 24.273 \\
\hline 2/8/2006 & 4:00 & 41.158 & 49.745 & 24.296 \\
\hline 2/8/2006 & 8:00 & 41.219 & 49.827 & 24.37 \\
\hline 2/8/2006 & $12: 00$ & 41.277 & 49.863 & 24.392 \\
\hline 2/8/2006 & $16: 00$ & 41.255 & 49.824 & 24.351 \\
\hline & & & 856 & \\
\hline
\end{tabular}

TABLE S1.1 (Cont.)

\begin{tabular}{|c|c|c|c|c|}
\hline \multirow[b]{2}{*}{ Date } & \multicolumn{4}{|c|}{$\begin{array}{l}\text { Water Level (ft below top of } \\
\text { casing) at Indicated Well }\end{array}$} \\
\hline & Time & D2 & WP49 & SB65D \\
\hline 2/9/2006 & 0:00 & 41.291 & 49.848 & 24.367 \\
\hline 2/9/2006 & 4:00 & 41.241 & 49.767 & 24.287 \\
\hline 2/9/2006 & 8:00 & 41.146 & 49.672 & 24.209 \\
\hline 2/9/2006 & $12: 00$ & 41.055 & 49.574 & 24.125 \\
\hline 2/9/2006 & $16: 00$ & 40.953 & 49.491 & 24.084 \\
\hline 2/9/2006 & $20: 00$ & 40.98 & 49.585 & 24.185 \\
\hline 2/10/2006 & 0:00 & 41.004 & 49.628 & 24.221 \\
\hline 2/10/2006 & 4:00 & 41.014 & 49.623 & 24.212 \\
\hline 2/10/2006 & 8:00 & 41.033 & 49.641 & 24.244 \\
\hline 2/10/2006 & $12: 00$ & 41.067 & 49.681 & 24.265 \\
\hline 2/10/2006 & $16: 00$ & 41.052 & 49.666 & 24.251 \\
\hline 2/10/2006 & $20: 00$ & 41.113 & 49.741 & 24.32 \\
\hline 2/11/2006 & 0:00 & 41.137 & 49.748 & 24.314 \\
\hline 2/11/2006 & 4:00 & 41.142 & 49.748 & 24.31 \\
\hline 2/11/2006 & 8:00 & 41.178 & 49.801 & 24.361 \\
\hline 2/11/2006 & 12:00 & 41.243 & 49.867 & 24.41 \\
\hline $2 / 11 / 2006$ & $16: 00$ & 41.245 & 49.837 & 24.373 \\
\hline 2/11/2006 & $20: 00$ & 41.229 & 49.81 & 24.343 \\
\hline 2/12/2006 & 0:00 & 41.171 & 49.722 & 24.26 \\
\hline 2/12/2006 & 4:00 & 41.091 & 49.641 & 24.191 \\
\hline 2/12/2006 & 8:00 & 41.076 & 49.683 & 24.262 \\
\hline 2/12/2006 & $12: 00$ & 41.178 & 49.818 & 24.377 \\
\hline 2/12/2006 & $16: 00$ & 41.236 & 49.859 & 24.406 \\
\hline 2/12/2006 & $20: 00$ & 41.258 & 49.861 & 24.4 \\
\hline 2/13/2006 & $0: 00$ & 41.209 & 49.762 & 24.299 \\
\hline 2/13/2006 & 4:00 & 41.108 & 49.643 & 24.189 \\
\hline 2/13/2006 & 8:00 & 40.98 & 49.508 & 24.092 \\
\hline 2/13/2006 & $12: 00$ & 40.949 & 49.534 & 24.133 \\
\hline 2/13/2006 & $16: 00$ & 41.154 & 49.553 & 24.115 \\
\hline 2/13/2006 & $20: 00$ & 40.985 & 49.587 & 24.184 \\
\hline 2/14/2006 & 0:00 & 40.953 & 49.544 & 24.146 \\
\hline 2/14/2006 & 4:00 & 40.9 & 49.487 & 24.104 \\
\hline 2/14/2006 & 8:00 & 40.852 & 49.471 & 24.105 \\
\hline 2/14/2006 & $12: 00$ & 40.845 & 49.453 & 24.094 \\
\hline 2/14/2006 & $16: 00$ & 40.794 & 49.42 & 24.078 \\
\hline 2/14/2006 & $20: 00$ & 40.927 & 49.626 & 24.275 \\
\hline 2/15/2006 & 0:00 & 41.06 & 49.735 & 24.355 \\
\hline 2/15/2006 & 4:00 & 41.108 & 49.737 & 24.338 \\
\hline 2/15/2006 & 8:00 & 41.141 & 49.773 & 24.365 \\
\hline 2/15/2006 & $12: 00$ & 41.178 & 49.784 & 24.363 \\
\hline 2/15/2006 & $16: 00$ & 41.105 & 49.671 & 24.25 \\
\hline $2 / 15 / 2006$ & 20:00 & 41.125 & 49.735 & 319 \\
\hline
\end{tabular}


TABLE S1.1 (Cont.)

\begin{tabular}{|c|c|c|c|c|}
\hline \multirow[b]{2}{*}{ Date } & \multicolumn{4}{|c|}{$\begin{array}{l}\text { Water Level (ft below top of } \\
\text { casing) at Indicated Well }\end{array}$} \\
\hline & Time & D2 & WP49 & SB65D \\
\hline 2/16/2006 & $0: 00$ & 41.125 & 49.726 & 24.304 \\
\hline 2/16/2006 & 4:00 & 41.081 & 49.641 & 24.239 \\
\hline 2/16/2006 & 8:00 & 41.079 & 49.688 & 24.283 \\
\hline 2/16/2006 & $12: 00$ & 41.159 & 49.792 & 24.375 \\
\hline 2/16/2006 & $16: 00$ & 41.281 & 49.942 & 24.502 \\
\hline 2/16/2006 & $20: 00$ & 41.472 & 50.13 & 24.627 \\
\hline $2 / 17 / 2006$ & $0: 00$ & 41.591 & 50.169 & 24.625 \\
\hline $2 / 17 / 2006$ & 4:00 & 41.666 & 50.194 & 24.635 \\
\hline $2 / 17 / 2006$ & 8:00 & 41.716 & 50.252 & 24.681 \\
\hline $2 / 17 / 2006$ & $12: 00$ & 41.803 & 50.273 & 24.67 \\
\hline $2 / 17 / 2006$ & $16: 00$ & 41.755 & 50.173 & 24.574 \\
\hline $2 / 17 / 2006$ & $20: 00$ & 41.736 & 50.164 & 24.566 \\
\hline 2/18/2006 & $0: 00$ & 41.723 & 50.132 & 24.537 \\
\hline 2/18/2006 & 4:00 & 41.69 & 50.117 & 24.521 \\
\hline 2/18/2006 & 8:00 & 41.665 & 50.107 & 24.519 \\
\hline 2/18/2006 & $12: 00$ & 41.646 & 50.085 & 24.496 \\
\hline 2/18/2006 & $16: 00$ & 41.538 & 49.959 & 24.391 \\
\hline 2/18/2006 & $20: 00$ & 41.472 & 49.931 & 24.379 \\
\hline 2/19/2006 & 0:00 & 41.436 & 49.916 & 24.375 \\
\hline 2/19/2006 & 4:00 & & 49.839 & 24.313 \\
\hline 2/19/2006 & 8:00 & & 49.814 & 24.309 \\
\hline 2/19/2006 & $12: 00$ & 41.274 & 49.788 & 24.287 \\
\hline 2/19/2006 & $16: 00$ & 41.163 & 49.675 & 24.208 \\
\hline 2/19/2006 & $20: 00$ & 41.118 & 49.671 & 24.217 \\
\hline 2/20/2006 & 0:00 & 41.103 & 49.681 & 24.239 \\
\hline 2/20/2006 & 4:00 & 41. & 49.613 & 24.18 \\
\hline 2/20/2006 & 8:00 & 41.014 & 49.594 & 24.176 \\
\hline 2/20/2006 & $12: 00$ & 40.975 & 49.555 & 24.155 \\
\hline 2/20/2006 & $16: 00$ & 40.924 & 49.529 & 24.139 \\
\hline 2/20/2006 & $20: 00$ & 40.941 & 49.585 & 24.2 \\
\hline $2 / 21 / 2006$ & $0: 00$ & & 49.617 & 24.237 \\
\hline $2 / 21 / 2006$ & 4:00 & 41.011 & 49.649 & 24.253 \\
\hline $2 / 21 / 2006$ & 8:00 & 41.014 & 49.632 & 24.237 \\
\hline $2 / 21 / 2006$ & $12: 00$ & 41.011 & 49.613 & 24.217 \\
\hline 2/21/2006 & $16: 00$ & 40.932 & 49.519 & 24.139 \\
\hline $2 / 21 / 2006$ & $20: 00$ & 40.924 & 49.57 & 24.202 \\
\hline 2/22/2006 & 0:00 & 40.999 & 49.634 & 24.251 \\
\hline 2/22/2006 & 4:00 & 41.004 & 49.632 & 24.249 \\
\hline 2/22/2006 & 8:00 & 41.042 & 49.671 & 24.278 \\
\hline 2/22/2006 & $12: 00$ & 41.048 & 49.636 & 24.241 \\
\hline 2/22/2006 & $16: 00$ & 40.996 & 49.598 & 24.214 \\
\hline 2/22/2006 & . & 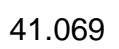 & 4 & 24.309 \\
\hline
\end{tabular}

TABLE S1.1 (Cont.)

\begin{tabular}{|c|c|c|c|c|}
\hline \multirow[b]{2}{*}{ Date } & \multicolumn{4}{|c|}{$\begin{array}{l}\text { Water Level (ft below top of } \\
\text { casing) at Indicated Well }\end{array}$} \\
\hline & Time & D2 & WP49 & SB65D \\
\hline 2/23/2006 & $0: 00$ & 41.127 & 49.748 & 24.342 \\
\hline 2/23/2006 & $4: 00$ & 41.146 & 49.76 & 24.352 \\
\hline 2/23/2006 & $8: 00$ & 41.253 & 49.878 & 24.459 \\
\hline 2/23/2006 & $12: 00$ & 41.361 & 49.961 & 24.504 \\
\hline 2/23/2006 & $16: 00$ & 41.347 & 49.912 & 24.444 \\
\hline 2/23/2006 & $20: 00$ & 41.33 & 49.876 & 24.407 \\
\hline 2/24/2006 & $0: 00$ & 41.315 & 49.859 & 24.389 \\
\hline 2/24/2006 & $4: 00$ & 41.262 & 49.784 & 24.321 \\
\hline 2/24/2006 & $8: 00$ & 41.207 & 49.743 & 24.284 \\
\hline 2/24/2006 & $12: 00$ & 41.161 & 49.709 & 24.262 \\
\hline 2/24/2006 & 16:00 & 41.105 & 49.675 & 24.245 \\
\hline 2/24/2006 & $20: 00$ & 41.175 & 49.81 & 24.377 \\
\hline 2/25/2006 & $0: 00$ & 41.357 & 50 & 24.543 \\
\hline 2/25/2006 & 4:00 & 41.47 & 50.092 & 24.608 \\
\hline 2/25/2006 & $8: 00$ & 41.581 & 50.173 & 24.656 \\
\hline 2/25/2006 & 12:00 & 41.668 & 50.218 & 24.67 \\
\hline 2/25/2006 & $16: 00$ & 41.641 & 50.152 & 24.596 \\
\hline 2/25/2006 & $20: 00$ & 41.648 & 50.158 & 24.598 \\
\hline 2/26/2006 & $0: 00$ & 41.656 & 50.158 & 24.596 \\
\hline 2/26/2006 & $4: 00$ & 41.634 & 50.109 & 24.545 \\
\hline 2/26/2006 & $8: 00$ & 41.596 & 50.072 & 24.512 \\
\hline 2/26/2006 & 12:00 & 41.532 & 49.987 & 24.44 \\
\hline 2/26/2006 & $16: 00$ & 41.443 & 49.846 & 24.3 \\
\hline 2/26/2006 & $20: 00$ & 41.291 & 49.777 & 24.276 \\
\hline 2/27/2006 & $0: 00$ & 41.188 & 49.689 & 24.216 \\
\hline 2/27/2006 & $4: 00$ & 41.083 & 49.613 & 24.169 \\
\hline 2/27/2006 & 8:00 & 41.062 & 49.647 & 24.216 \\
\hline 2/27/2006 & $12: 00$ & 41.052 & 49.649 & 24.224 \\
\hline 2/27/2006 & $16: 00$ & 41.013 & 49.611 & 24.208 \\
\hline 2/27/2006 & $20: 00$ & 41.04 & 49.649 & 24.251 \\
\hline 2/28/2006 & $0: 00$ & 41.084 & 49.703 & 24.296 \\
\hline 2/28/2006 & $4: 00$ & 41.083 & 49.686 & 24.274 \\
\hline 2/28/2006 & $8: 00$ & 41.103 & 49.715 & 24.302 \\
\hline 2/28/2006 & $12: 00$ & 41.115 & 49.7 & 24.296 \\
\hline 2/28/2006 & $16: 00$ & 41.052 & 49.628 & 24.224 \\
\hline 2/28/2006 & $20: 00$ & 41.031 & 49.623 & 24.226 \\
\hline $3 / 1 / 2006$ & $0: 00$ & 41.009 & 49.598 & 24.204 \\
\hline $3 / 1 / 2006$ & $4: 00$ & 40.968 & 49.559 & 24.181 \\
\hline $3 / 1 / 2006$ & $8: 00$ & 40.99 & 49.615 & 24.237 \\
\hline $3 / 1 / 2006$ & $12: 00$ & 41.052 & 49.681 & 24.298 \\
\hline $3 / 1 / 2006$ & $16: 00$ & 41.11 & 49.756 & 24.366 \\
\hline 3/1/2006 & $20: 00$ & 41.274 & 49.933 & 24.516 \\
\hline
\end{tabular}


TABLE S1.1 (Cont.)

\begin{tabular}{|c|c|c|c|c|}
\hline \multirow[b]{2}{*}{ Date } & \multicolumn{4}{|c|}{$\begin{array}{l}\text { Water Level (ft below top of } \\
\text { casing) at Indicated Well }\end{array}$} \\
\hline & Time & D2 & WP49 & SB65D \\
\hline $3 / 2 / 2006$ & $0: 00$ & 41.385 & 49.985 & 24.54 \\
\hline 3/2/2006 & 4:00 & 41.419 & 49.985 & 24.526 \\
\hline 3/2/2006 & 8:00 & 41.499 & 50.077 & 24.598 \\
\hline $3 / 2 / 2006$ & $12: 00$ & 41.593 & 50.143 & 24.639 \\
\hline $3 / 2 / 2006$ & $16: 00$ & 41.608 & 50.119 & 24.606 \\
\hline $3 / 2 / 2006$ & $20: 00$ & 41.649 & 50.154 & 24.633 \\
\hline 3/3/2006 & $0: 00$ & 41.68 & 50.169 & 24.635 \\
\hline 3/3/2006 & 4:00 & 41.711 & 50.19 & 24.653 \\
\hline 3/3/2006 & 8:00 & 41.74 & 50.214 & 24.667 \\
\hline $3 / 3 / 2006$ & $12: 00$ & 41.733 & 50.175 & 24.616 \\
\hline 3/3/2006 & $16: 00$ & 41.66 & 50.085 & 24.528 \\
\hline $3 / 3 / 2006$ & $20: 00$ & 41.61 & 50.068 & 24.505 \\
\hline $3 / 4 / 2006$ & $0: 00$ & 41.598 & 50.079 & 24.522 \\
\hline $3 / 4 / 2006$ & 4:00 & 41.583 & 50.066 & 24.514 \\
\hline 3/4/2006 & 8:00 & 41.564 & 50.055 & 24.511 \\
\hline $3 / 4 / 2006$ & $12: 00$ & 41.528 & 50.002 & 24.46 \\
\hline $3 / 4 / 2006$ & $16: 00$ & 41.431 & 49.908 & 24.384 \\
\hline $3 / 4 / 2006$ & $20: 00$ & 41.409 & 49.938 & 24.419 \\
\hline $3 / 5 / 2006$ & $0: 00$ & 41.373 & 49.895 & 24.384 \\
\hline $3 / 5 / 2006$ & 4:00 & 41.366 & 49.91 & 24.409 \\
\hline $3 / 5 / 2006$ & 8:00 & 41.356 & 49.948 & 24.454 \\
\hline $3 / 5 / 2006$ & $12: 00$ & 41.407 & 49.959 & 24.468 \\
\hline $3 / 5 / 2006$ & $16: 00$ & 41.451 & 50.008 & 24.515 \\
\hline $3 / 5 / 2006$ & $20: 00$ & 41.494 & 50.064 & 24.55 \\
\hline $3 / 6 / 2006$ & $0: 00$ & 41.513 & 50.051 & 24.532 \\
\hline 3/6/2006 & 4:00 & 41.533 & 50.087 & 24.565 \\
\hline 3/6/2006 & 8:00 & 41.583 & 50.132 & 24.601 \\
\hline 3/6/2006 & $12: 00$ & 41.615 & 50.139 & 24.599 \\
\hline 3/6/2006 & $16: 00$ & 41.557 & 50.06 & 24.521 \\
\hline 3/6/2006 & $20: 00$ & 41.554 & 50.079 & 24.544 \\
\hline 3/7/2006 & $0: 00$ & 41.53 & 50.038 & 24.505 \\
\hline 3/7/2006 & 4:00 & 41.479 & 49.963 & 24.441 \\
\hline 3/7/2006 & 8:00 & 41.395 & 49.876 & 24.363 \\
\hline $3 / 7 / 2006$ & $12: 00$ & 41.291 & 49.79 & 24.306 \\
\hline 3/7/2006 & $16: 00$ & 41.185 & 49.711 & 24.253 \\
\hline 3/7/2006 & $20: 00$ & 41.19 & 49.773 & 24.327 \\
\hline 3/8/2006 & $0: 00$ & 41.182 & 49.748 & 24.302 \\
\hline $3 / 8 / 2006$ & 4:00 & 41.163 & 49.735 & 24.296 \\
\hline 3/8/2006 & 8:00 & 41.141 & 49.694 & 24.275 \\
\hline $3 / 8 / 2006$ & $12: 00$ & 41.071 & 49.604 & 24.201 \\
\hline 3/8/2006 & $16: 00$ & 41.006 & 49.589 & 24.203 \\
\hline $3 / 8 / 2006$ & 00 & 57 & . & 24.298 \\
\hline
\end{tabular}

TABLE S1.1 (Cont.)

\begin{tabular}{|c|c|c|c|c|}
\hline \multirow[b]{2}{*}{ Date } & \multicolumn{4}{|c|}{$\begin{array}{l}\text { Water Level (ft below top of } \\
\text { casing) at Indicated Well }\end{array}$} \\
\hline & Time & D2 & WP49 & SB65D \\
\hline 3/9/2006 & $0: 00$ & 41.11 & 49.733 & 24.329 \\
\hline 3/9/2006 & 4:00 & 41.074 & 49.688 & 24.294 \\
\hline 3/9/2006 & 8:00 & 41.11 & 49.715 & 24.312 \\
\hline 3/9/2006 & $12: 00$ & 41.108 & 49.7 & 24.298 \\
\hline 3/9/2006 & $16: 00$ & 41.054 & 49.641 & 24.247 \\
\hline 3/9/2006 & $20: 00$ & 41.129 & 49.762 & 24.368 \\
\hline $3 / 10 / 2006$ & $0: 00$ & 41.199 & 49.803 & 24.394 \\
\hline $3 / 10 / 2006$ & 4:00 & 41.24 & 49.833 & 24.415 \\
\hline $3 / 10 / 2006$ & 8:00 & 41.315 & 49.912 & 24.474 \\
\hline $3 / 10 / 2006$ & $12: 00$ & 41.368 & 49.927 & 24.472 \\
\hline $3 / 10 / 2006$ & $16: 00$ & 41.315 & 49.839 & 24.39 \\
\hline $3 / 10 / 2006$ & $20: 00$ & 41.347 & 49.904 & 24.451 \\
\hline $3 / 11 / 2006$ & $0: 00$ & 41.339 & 49.857 & 24.398 \\
\hline $3 / 11 / 2006$ & 4:00 & 41.323 & 49.865 & 24.413 \\
\hline $3 / 11 / 2006$ & 8:00 & 41.414 & 50.004 & 24.544 \\
\hline $3 / 11 / 2006$ & $12: 00$ & 41.554 & 50.122 & 24.63 \\
\hline $3 / 11 / 2006$ & $16: 00$ & 41.595 & 50.128 & 24.616 \\
\hline $3 / 11 / 2006$ & $20: 00$ & 41.668 & 50.214 & 24.685 \\
\hline 3/12/2006 & $0: 00$ & 41.709 & 50.19 & 24.659 \\
\hline $3 / 12 / 2006$ & 4:00 & 41.672 & 50.137 & \\
\hline $3 / 12 / 2006$ & 8:00 & 41.605 & 50.008 & 24.476 \\
\hline $3 / 12 / 2006$ & $12: 00$ & 41.513 & 49.951 & 24.409 \\
\hline $3 / 12 / 2006$ & $16: 00$ & 41.385 & 49.859 & 24.347 \\
\hline $3 / 12 / 2006$ & $20: 00$ & 41.354 & 49.859 & 24.361 \\
\hline $3 / 13 / 2006$ & $0: 00$ & 41.33 & 49.837 & 24.355 \\
\hline $3 / 13 / 2006$ & 4:00 & 41.375 & 49.94 & 24.449 \\
\hline $3 / 13 / 2006$ & 8:00 & 41.537 & 50.143 & 24.63 \\
\hline $3 / 13 / 2006$ & $12: 00$ & 41.68 & 50.258 & 24.716 \\
\hline $3 / 13 / 2006$ & $16: 00$ & 41.747 & 50.28 & 24.718 \\
\hline $3 / 13 / 2006$ & $20: 00$ & 41.822 & 50.335 & 24.753 \\
\hline $3 / 14 / 2006$ & $0: 00$ & & 50.335 & 24.741 \\
\hline $3 / 14 / 2006$ & 4:00 & 41.875 & 50.32 & 24.724 \\
\hline 3/14/2006 & 8:00 & 41.897 & 50.327 & 24.737 \\
\hline $3 / 14 / 2006$ & $12: 00$ & 41.885 & 50.278 & 24.683 \\
\hline $3 / 14 / 2006$ & $16: 00$ & 41.825 & 50.229 & 24.64 \\
\hline $3 / 14 / 2006$ & $20: 00$ & 41.841 & 50.271 & 24.681 \\
\hline $3 / 15 / 2006$ & $0: 00$ & 41.856 & 50.28 & 24.685 \\
\hline 3/15/2006 & 4:00 & 41.837 & 50.263 & 24.671 \\
\hline $3 / 15 / 2006$ & 8:00 & 41.808 & 50.216 & 24.628 \\
\hline 3/15/2006 & $12: 00$ & 41.685 & 50.032 & 24.459 \\
\hline $3 / 15 / 2006$ & $16: 00$ & 41.508 & 49.906 & 24.377 \\
\hline $3 / 15 / 2006$ & 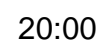 & 41.467 & 9 & 24.406 \\
\hline
\end{tabular}


TABLE S1.1 (Cont.)

\begin{tabular}{|c|c|c|c|c|}
\hline \multirow[b]{2}{*}{ Date } & \multicolumn{4}{|c|}{$\begin{array}{l}\text { Water Level (ft below top of } \\
\text { casing) at Indicated Well }\end{array}$} \\
\hline & Time & D2 & WP49 & SB65D \\
\hline 3/16/2006 & $0: 00$ & 41.443 & 49.936 & 24.406 \\
\hline 3/16/2006 & 4:00 & 41.404 & 49.936 & 24.423 \\
\hline $3 / 16 / 2006$ & 8:00 & 41.561 & 50.164 & 24.634 \\
\hline 3/16/2006 & $12: 00$ & 41.716 & 50.284 & 24.734 \\
\hline 3/16/2006 & $16: 00$ & 41.82 & 50.361 & 24.784 \\
\hline $3 / 16 / 2006$ & $20: 00$ & 41.914 & 50.425 & 24.817 \\
\hline $3 / 17 / 2006$ & $0: 00$ & 41.979 & 50.436 & 24.81 \\
\hline $3 / 17 / 2006$ & 4:00 & 42.003 & 50.432 & 24.8 \\
\hline $3 / 17 / 2006$ & 8:00 & 42.015 & 50.436 & 24.806 \\
\hline $3 / 17 / 2006$ & $12: 00$ & 42.037 & 50.427 & 24.788 \\
\hline $3 / 17 / 2006$ & $16: 00$ & 41.936 & 50.295 & 24.661 \\
\hline $3 / 17 / 2006$ & $20: 00$ & 41.88 & 50.278 & 24.656 \\
\hline $3 / 18 / 2006$ & $0: 00$ & 41.88 & 50.303 & 24.675 \\
\hline 3/18/2006 & 4:00 & 41.846 & 50.265 & 24.64 \\
\hline $3 / 18 / 2006$ & 8:00 & 41.858 & 50.301 & 24.679 \\
\hline 3/18/2006 & $12: 00$ & 41.842 & 50.273 & 24.65 \\
\hline $3 / 18 / 2006$ & $16: 00$ & 41.777 & 50.203 & 24.595 \\
\hline 3/18/2006 & $20: 00$ & 41.752 & 50.199 & 24.593 \\
\hline 3/19/2006 & 0:00 & 41.76 & 50.218 & 24.628 \\
\hline $3 / 19 / 2006$ & 4:00 & 41.736 & 50.167 & 24.581 \\
\hline $3 / 19 / 2006$ & 8:00 & 41.735 & 50.224 & 24.63 \\
\hline 3/19/2006 & $12: 00$ & 41.745 & 50.222 & 24.626 \\
\hline $3 / 19 / 2006$ & $16: 00$ & 41.695 & 50.156 & 24.566 \\
\hline 3/19/2006 & $20: 00$ & 41.68 & 50.156 & 24.581 \\
\hline $3 / 20 / 2006$ & 0:00 & 41.646 & 50.139 & 24.564 \\
\hline $3 / 20 / 2006$ & 4:00 & 41.631 & 50.115 & 24.542 \\
\hline 3/20/2006 & 8:00 & 41.6 & 50.096 & 24.527 \\
\hline 3/20/2006 & $12: 00$ & 41.535 & 50.023 & 24.474 \\
\hline 3/20/2006 & $16: 00$ & 41.499 & 50.025 & 24.486 \\
\hline 3/20/2006 & $20: 00$ & 41.535 & 50.092 & 24.552 \\
\hline $3 / 21 / 2006$ & & 41.571 & 50.109 & 24.566 \\
\hline $3 / 21 / 2006$ & 4:00 & 41.624 & 50.19 & 24.638 \\
\hline $3 / 21 / 2006$ & 8:00 & 41.728 & 50.293 & 24.728 \\
\hline $3 / 21 / 2006$ & $12: 00$ & 41.829 & 50.37 & 24.782 \\
\hline $3 / 21 / 2006$ & $16: 00$ & 41.875 & 50.385 & 24.786 \\
\hline $3 / 21 / 2006$ & $20: 00$ & 41.907 & 50.391 & 24.786 \\
\hline 3/22/2006 & 0:00 & 41.953 & 50.425 & 24.816 \\
\hline 3/22/2006 & 4:00 & 41.984 & 50.429 & 24.812 \\
\hline $3 / 22 / 2006$ & 8:00 & 42.003 & 50.447 & 24.821 \\
\hline 3/22/2006 & $12: 00$ & 42.003 & 50.427 & 24.798 \\
\hline 3/22/2006 & $16: 00$ & 41.969 & 50.378 & 24.753 \\
\hline 3/22/2006 & 20:00 & 41.965 & 389 & 24.761 \\
\hline
\end{tabular}

TABLE S1.1 (Cont.)

\begin{tabular}{|c|c|c|c|c|}
\hline \multirow[b]{2}{*}{ Date } & \multicolumn{4}{|c|}{$\begin{array}{l}\text { Water Level (ft below top of } \\
\text { casing) at Indicated Well }\end{array}$} \\
\hline & Time & D2 & WP49 & SB65D \\
\hline 3/23/2006 & 0:00 & 41.974 & 50.4 & 24.771 \\
\hline 3/23/2006 & 4:00 & 41.96 & 50.382 & 24.752 \\
\hline 3/23/2006 & 8:00 & 41.967 & 50.406 & 24.777 \\
\hline 3/23/2006 & $12: 00$ & 41.97 & 50.4 & 24.765 \\
\hline 3/23/2006 & $16: 00$ & 41.931 & 50.357 & 24.726 \\
\hline 3/23/2006 & $20: 00$ & 41.914 & 50.346 & 24.72 \\
\hline 3/24/2006 & 0:00 & 41.89 & 50.323 & 24.695 \\
\hline 3/24/2006 & 4:00 & 41.851 & 50.293 & 24.673 \\
\hline 3/24/2006 & 8:00 & 41.849 & 50.316 & 24.697 \\
\hline $3 / 24 / 2006$ & $12: 00$ & 41.861 & 50.32 & 24.695 \\
\hline 3/24/2006 & $16: 00$ & 41.822 & 50.276 & 24.662 \\
\hline 3/24/2006 & $20: 00$ & 41.825 & 50.301 & 24.691 \\
\hline $3 / 25 / 2006$ & 0:00 & 41.846 & 50.327 & 24.71 \\
\hline 3/25/2006 & 4:00 & 41.849 & 50.329 & 24.714 \\
\hline 3/25/2006 & 8:00 & 41.878 & 50.365 & 24.744 \\
\hline 3/25/2006 & $12: 00$ & 41.895 & 50.365 & 24.742 \\
\hline 3/25/2006 & $16: 00$ & 41.856 & 50.31 & 24.687 \\
\hline 3/25/2006 & $20: 00$ & 41.812 & 50.271 & 24.658 \\
\hline 3/26/2006 & 0:00 & 41.793 & 50.263 & 24.648 \\
\hline 3/26/2006 & 4:00 & 41.745 & 50.216 & 24.613 \\
\hline 3/26/2006 & 8:00 & 41.718 & 50.199 & 24.597 \\
\hline 3/26/2006 & $12: 00$ & 41.653 & 50.122 & 24.533 \\
\hline 3/26/2006 & $16: 00$ & 41.54 & 50.013 & 24.451 \\
\hline 3/26/2006 & $20: 00$ & 41.557 & 50.081 & 24.51 \\
\hline 3/27/2006 & $0: 00$ & 41.586 & 50.102 & 24.525 \\
\hline 3/27/2006 & 4:00 & 41.525 & 50.036 & 24.451 \\
\hline 3/27/2006 & 8:00 & 41.53 & 50.066 & 24.474 \\
\hline 3/27/2006 & $12: 00$ & 41.583 & 50.149 & 24.545 \\
\hline 3/27/2006 & $16: 00$ & 41.66 & 50.226 & 24.601 \\
\hline 3/27/2006 & $20: 00$ & 41.755 & 50.301 & 24.656 \\
\hline 3/28/2006 & 0:00 & 41.82 & 50.342 & 24.675 \\
\hline 3/28/2006 & 4:00 & 41.846 & 50.342 & 24.662 \\
\hline 3/28/2006 & 8:00 & 41.902 & 50.406 & 24.709 \\
\hline 3/28/2006 & $12: 00$ & 41.933 & 50.4 & 24.691 \\
\hline 3/28/2006 & $16: 00$ & 41.885 & 50.34 & 24.625 \\
\hline 3/28/2006 & $20: 00$ & 41.849 & 50.303 & 24.609 \\
\hline 3/29/2006 & $0: 00$ & 41.832 & 50.271 & 24.58 \\
\hline 3/29/2006 & 4:00 & 41.798 & 50.258 & 24.562 \\
\hline 3/29/2006 & 8:00 & 41.771 & 50.243 & 24.554 \\
\hline 3/29/2006 & $12: 00$ & 41.735 & 50.192 & 24.506 \\
\hline 3/29/2006 & $16: 00$ & 41.639 & 50.09 & 24.432 \\
\hline $3 / 29 / 2006$ & $20: 00$ & 41.581 & 50.077 & 24.434 \\
\hline
\end{tabular}


TABLE S1.1 (Cont.)

\begin{tabular}{|c|c|c|c|c|}
\hline \multirow[b]{2}{*}{ Date } & \multicolumn{4}{|c|}{$\begin{array}{l}\text { Water Level (ft below top of } \\
\text { casing) at Indicated Well }\end{array}$} \\
\hline & Time & D2 & WP49 & SB65D \\
\hline 3/30/2006 & 0:00 & 41.578 & 50.068 & 24.435 \\
\hline $3 / 30 / 2006$ & 4:00 & 41.523 & 50.006 & 24.394 \\
\hline 3/30/2006 & 8:00 & 41.513 & 50.021 & 24.414 \\
\hline $3 / 30 / 2006$ & $12: 00$ & 41.465 & 49.976 & 24.361 \\
\hline 3/30/2006 & $16: 00$ & 41.354 & 49.925 & 24.359 \\
\hline 3/30/2006 & $20: 00$ & 41.349 & 49.933 & 24.355 \\
\hline $3 / 31 / 2006$ & 0:00 & 41.491 & 50.111 & 24.48 \\
\hline $3 / 31 / 2006$ & 4:00 & 41.583 & 50.179 & 24.519 \\
\hline 3/31/2006 & 8:00 & 41.658 & 50.239 & 24.556 \\
\hline $3 / 31 / 2006$ & $12: 00$ & 41.755 & 50.318 & 24.613 \\
\hline $3 / 31 / 2006$ & $16: 00$ & 41.812 & 50.353 & 24.631 \\
\hline $3 / 31 / 2006$ & $20: 00$ & 41.87 & 50.404 & 24.666 \\
\hline 4/1/2006 & $0: 00$ & 41.916 & 50.423 & 24.673 \\
\hline 4/1/2006 & 4:00 & 41.919 & 50.397 & 24.638 \\
\hline 4/1/2006 & 8:00 & 41.931 & 50.412 & 24.65 \\
\hline 4/1/2006 & $12: 00$ & 41.904 & 50.361 & 24.597 \\
\hline 4/1/2006 & $16: 00$ & 41.784 & 50.229 & 24.482 \\
\hline 4/1/2006 & $20: 00$ & 41.694 & 50.152 & 24.426 \\
\hline 4/2/2006 & 0:00 & 41.631 & 50.102 & 24.396 \\
\hline 4/2/2006 & 4:00 & 41.52 & 50.032 & 24.35 \\
\hline 4/2/2006 & 8:00 & 41.489 & 50.025 & 24.35 \\
\hline 4/2/2006 & $12: 00$ & 41.462 & 50.025 & 24.357 \\
\hline 4/2/2006 & $16: 00$ & 41.511 & 50.122 & 24.443 \\
\hline 4/2/2006 & $20: 00$ & 41.641 & 50.286 & 24.556 \\
\hline 4/3/2006 & 0:00 & 41.806 & 50.425 & 24.617 \\
\hline 4/3/2006 & 4:00 & 41.911 & 50.491 & 24.646 \\
\hline 4/3/2006 & 8:00 & 42.01 & 50.562 & 24.685 \\
\hline 4/3/2006 & $12: 00$ & 42.059 & 50.541 & 24.666 \\
\hline 4/3/2006 & $16: 00$ & 42.02 & 50.459 & 24.586 \\
\hline 4/3/2006 & $20: 00$ & 41.981 & 50.447 & \\
\hline 4/4/2006 & $0: 00$ & 41.962 & 50.415 & \\
\hline $4 / 4 / 2006$ & 4:00 & 41.911 & 50.37 & \\
\hline $4 / 4 / 2006$ & 8:00 & 41.897 & 50.37 & \\
\hline 7/3/2006 & $20: 00$ & 42.259 & 50.847 & 25.135 \\
\hline 7/4/2006 & $0: 00$ & 42.277 & 50.854 & 25.138 \\
\hline 7/4/2006 & 4:00 & 42.294 & 50.868 & 25.152 \\
\hline 7/4/2006 & 8:00 & 42.324 & 50.894 & 25.171 \\
\hline 7/4/2006 & $12: 00$ & 42.322 & 50.875 & 25.15 \\
\hline 7/4/2006 & $16: 00$ & 42.275 & 50.814 & 25.098 \\
\hline 7/4/2006 & $20: 00$ & 42.28 & 50.858 & 25.147 \\
\hline & & & 50.882 & \\
\hline
\end{tabular}

TABLE S1.1 (Cont.)

\begin{tabular}{|c|c|c|c|c|}
\hline \multirow[b]{2}{*}{ Date } & \multicolumn{4}{|c|}{$\begin{array}{l}\text { Water Level (ft below top of } \\
\text { casing) at Indicated Well }\end{array}$} \\
\hline & Time & D2 & WP49 & SB65D \\
\hline 7/5/2006 & 4:00 & 42.308 & 50.884 & 25.171 \\
\hline $7 / 5 / 2006$ & 8:00 & 42.35 & 50.915 & 25.194 \\
\hline $7 / 5 / 2006$ & $12: 00$ & 42.345 & 50.861 & 25.138 \\
\hline $7 / 5 / 2006$ & $16: 00$ & 42.315 & 50.826 & 25.098 \\
\hline $7 / 5 / 2006$ & $20: 00$ & 42.319 & 50.879 & 25.15 \\
\hline 7/6/2006 & 0:00 & 42.347 & 50.903 & 25.175 \\
\hline 7/6/2006 & 4:00 & 42.343 & 50.894 & 25.168 \\
\hline 7/6/2006 & 8:00 & 42.406 & 50.929 & 25.199 \\
\hline 7/6/2006 & $12: 00$ & 42.368 & 50.877 & 25.15 \\
\hline 7/6/2006 & $16: 00$ & 42.333 & 50.842 & 25.107 \\
\hline 7/6/2006 & $20: 00$ & 42.319 & 50.861 & 25.126 \\
\hline 7/7/2006 & 0:00 & 42.329 & 50.884 & 25.15 \\
\hline $7 / 7 / 2006$ & 4:00 & 42.317 & 50.875 & 25.143 \\
\hline 7/7/2006 & 8:00 & 42.324 & 50.882 & 25.152 \\
\hline 7/7/2006 & $12: 00$ & 42.282 & 50.826 & 25. \\
\hline 7/7/2006 & $16: 00$ & 42.219 & 50.776 & 25.065 \\
\hline 7/7/2006 & $20: 00$ & 42.191 & 50.786 & 25.086 \\
\hline 7/8/2006 & 0:00 & 42.194 & 50.781 & 25.086 \\
\hline 7/8/2006 & 4:00 & 42.182 & 50.797 & 25.112 \\
\hline 7/8/2006 & 8:00 & 42.198 & 50.795 & 25.093 \\
\hline 7/8/2006 & $12: 00$ & 42.182 & 50.781 & 25.091 \\
\hline 7/8/2006 & $16: 00$ & 42.156 & 50.758 & 25.072 \\
\hline $7 / 8 / 2006$ & $20: 00$ & 42.138 & 50.758 & 25.079 \\
\hline 7/9/2006 & 0:00 & 42.135 & 50.746 & 25.07 \\
\hline 7/9/2006 & 4:00 & 42.114 & 50.734 & 25.068 \\
\hline 7/9/2006 & 8:00 & 42.131 & 50.76 & 25.086 \\
\hline $7 / 9 / 2006$ & $12: 00$ & 42.422 & 50.816 & 25.068 \\
\hline 7/9/2006 & $16: 00$ & 42.31 & 50.769 & 25.049 \\
\hline 7/9/2006 & $20: 00$ & 42.175 & 50.767 & 25.072 \\
\hline $7 / 10 / 2006$ & 0:00 & 42.152 & 50.755 & 25.07 \\
\hline 7/10/2006 & 4:00 & 42.135 & 50.758 & 25.079 \\
\hline $7 / 10 / 2006$ & 8:00 & 42.14 & 50.774 & 25.096 \\
\hline 7/10/2006 & $12: 00$ & 42.126 & 50.751 & 25.075 \\
\hline 7/10/2006 & $16: 00$ & 42.082 & 50.706 & 25.04 \\
\hline $7 / 10 / 2006$ & $20: 00$ & 42.068 & 50.715 & 25.056 \\
\hline $7 / 11 / 2006$ & $0: 00$ & 42.07 & 50.715 & 25.054 \\
\hline $7 / 11 / 2006$ & 4:00 & 42.075 & 50.732 & 25.075 \\
\hline $7 / 11 / 2006$ & 8:00 & 42.112 & 50.772 & 25.11 \\
\hline $7 / 11 / 2006$ & $12: 00$ & 42.138 & 50.779 & 25.119 \\
\hline $7 / 11 / 2006$ & $16: 00$ & 42.145 & 50.781 & 25.122 \\
\hline $7 / 11 / 2006$ & $20: 00$ & 42.154 & 50.8 & 25.143 \\
\hline $7 / 12 / 2006$ & 0:00 & 42.184 & 50.821 & 25.161 \\
\hline
\end{tabular}


TABLE S1.1 (Cont.)

\begin{tabular}{ccccc} 
& & & & \\
& \multicolumn{4}{c}{ Water Level (ft below top of } \\
& casing) at Indicated Well \\
\cline { 2 - 5 } Date & Time & D2 & WP49 & SB65D \\
& & & & \\
\hline & & & & \\
$7 / 12 / 2006$ & $4: 00$ & 42.194 & 50.833 & 25.173 \\
$7 / 12 / 2006$ & $8: 00$ & 42.24 & 50.868 & 25.194 \\
$7 / 12 / 2006$ & $12: 00$ & 42.25 & 50.837 & 25.154 \\
$7 / 12 / 2006$ & $16: 00$ & 42.236 & 50.809 & 25.117 \\
$7 / 12 / 2006$ & $20: 00$ & 42.238 & 50.835 & 25.143 \\
$7 / 13 / 2006$ & $0: 00$ & 42.231 & 50.821 & 25.129 \\
$7 / 13 / 2006$ & $4: 00$ & 42.217 & 50.819 & 25.126 \\
$7 / 13 / 2006$ & $8: 00$ & 42.215 & 50.816 & 25.122 \\
$7 / 13 / 2006$ & $12: 00$ & 42.212 & 50.783 & 25.086 \\
$7 / 13 / 2006$ & $16: 00$ & 42.222 & 50.79 & 25.065 \\
$7 / 13 / 2006$ & $20: 00$ & 42.138 & 50.842 & 25.1 \\
$7 / 14 / 2006$ & $0: 00$ & 42.154 & 50.851 & 25.091 \\
$7 / 14 / 2006$ & $4: 00$ & 42.105 & 50.915 & 25.15 \\
$7 / 14 / 2006$ & $8: 00$ & 42.28 & 50.922 & 25.152 \\
$7 / 14 / 2006$ & $12: 00$ & 42.48 & 50.912 & 25.15 \\
$7 / 14 / 2006$ & $16: 00$ & 42.403 & 50.894 & 25.147 \\
$7 / 14 / 2006$ & $20: 00$ & 42.271 & 50.908 & 25.166 \\
$7 / 15 / 2006$ & $0: 00$ & 42.289 & 50.919 & 25.185 \\
$7 / 15 / 2006$ & $4: 00$ & 42.305 & 50.938 & 25.203 \\
$7 / 15 / 2006$ & $8: 00$ & 42.315 & 50.94 & 25.203 \\
$7 / 15 / 2006$ & $12: 00$ & 42.298 & 50.901 & 25.171 \\
$7 / 15 / 2006$ & $16: 00$ & 42.25 & 50.847 & 25.126 \\
$7 / 15 / 2006$ & $20: 00$ & 42.222 & 50.856 & 25.143 \\
$7 / 16 / 2006$ & $0: 00$ & 42.208 & 50.835 & 25.126 \\
$7 / 16 / 2006$ & $4: 00$ & 42.194 & 50.835 & 25.133 \\
$7 / 18 / 2006$ & $16: 00$ & 42.277 & 50.856 & 25.14 \\
$7 / 16 / 2006$ & $20: 00$ & 42.247 & 50.851 & 25.14 \\
$7 / 19 / 2006$ & $0: 00$ & 42.229 & 50.84 & 25.129
\end{tabular}

TABLE S1.1 (Cont.)

\begin{tabular}{|c|c|c|c|c|}
\hline \multirow[b]{2}{*}{ Date } & \multicolumn{4}{|c|}{$\begin{array}{l}\text { Water Level (ft below top of } \\
\text { casing) at Indicated Well }\end{array}$} \\
\hline & Time & D2 & WP49 & SB65D \\
\hline 7/19/2006 & 4:00 & 42.191 & 50.816 & 25.112 \\
\hline $7 / 19 / 2006$ & 8:00 & 42.243 & 50.863 & 25.14 \\
\hline 7/19/2006 & $12: 00$ & 42.222 & 50.833 & 25.124 \\
\hline 7/19/2006 & $16: 00$ & 42.215 & 50.819 & 25.105 \\
\hline 7/19/2006 & $20: 00$ & 42.203 & 50.835 & 25.122 \\
\hline 7/20/2006 & 0:00 & 42.233 & 50.903 & 25.194 \\
\hline 7/20/2006 & 4:00 & 42.296 & 50.957 & 25.241 \\
\hline 7/20/2006 & 8:00 & 42.385 & 51.013 & 25.281 \\
\hline 7/20/2006 & $12: 00$ & 42.413 & 50.999 & 25.264 \\
\hline 7/20/2006 & $16: 00$ & 42.41 & 50.964 & 25.229 \\
\hline 7/20/2006 & $20: 00$ & 42.399 & 50.983 & 25.246 \\
\hline $7 / 21 / 2006$ & 0:00 & 42.417 & 51.034 & 25.283 \\
\hline $7 / 21 / 2006$ & 4:00 & 42.415 & 50.994 & 25.264 \\
\hline 7/21/2006 & 8:00 & 42.441 & 51.053 & 25.309 \\
\hline 7/21/2006 & $12: 00$ & 42.42 & 51.001 & 25.267 \\
\hline 7/21/2006 & $16: 00$ & 42.371 & 50.952 & 25.229 \\
\hline 7/21/2006 & $20: 00$ & 42.35 & 50.964 & 25.243 \\
\hline 7/22/2006 & 0:00 & 42.35 & 50.962 & 25.243 \\
\hline 7/22/2006 & 4:00 & 42.338 & 50.959 & 25.25 \\
\hline 7/22/2006 & 8:00 & 42.34 & 50.964 & 25.253 \\
\hline 7/22/2006 & $12: 00$ & 42.315 & 50.933 & 25.224 \\
\hline 7/22/2006 & $16: 00$ & 42.259 & 50.879 & 25.187 \\
\hline 7/22/2006 & $20: 00$ & 42.231 & 50.889 & 25.201 \\
\hline $7 / 23 / 2006$ & 0:00 & 42.219 & 50.884 & 25.201 \\
\hline 7/23/2006 & 4:00 & 42.189 & 50.884 & 25.203 \\
\hline 7/23/2006 & 8:00 & 42.156 & 50.891 & 25.21 \\
\hline 7/23/2006 & $12: 00$ & 42.084 & 50.849 & 25.173 \\
\hline 7/23/2006 & $16: 00$ & 41.977 & 50.814 & 25.147 \\
\hline 7/23/2006 & $20: 00$ & 41.944 & 50.828 & 25.166 \\
\hline 7/24/2006 & 0:00 & 41.911 & 50.823 & 25.166 \\
\hline 7/24/2006 & 4:00 & 41.874 & 50.828 & 25.173 \\
\hline $7 / 24 / 2006$ & 8:00 & 41.827 & 50.83 & 25.175 \\
\hline 7/24/2006 & $12: 00$ & 41.834 & 50.819 & 25.157 \\
\hline 7/24/2006 & $16: 00$ & 41.818 & 50.795 & 25.136 \\
\hline $7 / 24 / 2006$ & $20: 00$ & 41.806 & 50.807 & 25.15 \\
\hline 7/25/2006 & $0: 00$ & 41.827 & 50.823 & 25.161 \\
\hline 7/25/2006 & 4:00 & 41.825 & 50.83 & 25.168 \\
\hline $7 / 25 / 2006$ & 8:00 & 41.848 & 50.851 & 25.189 \\
\hline 7/25/2006 & $12: 00$ & 41.862 & 50.844 & 25.178 \\
\hline $7 / 25 / 2006$ & $16: 00$ & 41.832 & 50.809 & 25.14 \\
\hline $7 / 25 / 2006$ & $20: 00$ & 41.79 & 50.8 & 25.131 \\
\hline $7 / 26 / 2006$ & 000 & 41.783 & 50.797 & 25.133 \\
\hline
\end{tabular}


TABLE S1.1 (Cont.)

\begin{tabular}{|c|c|c|c|c|}
\hline \multirow[b]{2}{*}{ Date } & \multicolumn{4}{|c|}{$\begin{array}{l}\text { Water Level (ft below top of } \\
\text { casing) at Indicated Well }\end{array}$} \\
\hline & Time & D2 & WP49 & SB65D \\
\hline $7 / 26 / 2006$ & 4:00 & 41.785 & 50.826 & 25.164 \\
\hline $7 / 26 / 2006$ & 8:00 & 41.858 & 50.903 & 25.229 \\
\hline 7/26/2006 & $12: 00$ & 41.902 & 50.903 & 25.224 \\
\hline $7 / 26 / 2006$ & $16: 00$ & 41.904 & 50.889 & 25.206 \\
\hline 7/26/2006 & $20: 00$ & 41.909 & 50.919 & 25.236 \\
\hline $7 / 27 / 2006$ & 0:00 & 41.932 & 50.933 & 25.248 \\
\hline $7 / 27 / 2006$ & 4:00 & 41.942 & 50.952 & 25.267 \\
\hline 7/27/2006 & 8:00 & 41.981 & 50.99 & 25.297 \\
\hline $7 / 27 / 2006$ & $12: 00$ & 42.026 & 51.004 & 25.297 \\
\hline $7 / 27 / 2006$ & $16: 00$ & 42.03 & 50.987 & 25.281 \\
\hline 7/27/2006 & $20: 00$ & 41.984 & 51.011 & 25.302 \\
\hline $7 / 28 / 2006$ & 0:00 & 41.991 & 51.023 & 25.311 \\
\hline $7 / 28 / 2006$ & 4:00 & 41.988 & 51.02 & 25.313 \\
\hline $7 / 28 / 2006$ & 8:00 & 42.016 & 51.044 & 25.33 \\
\hline $7 / 28 / 2006$ & $12: 00$ & 42.009 & 51.018 & 25.306 \\
\hline 7/28/2006 & $16: 00$ & 41.967 & 50.973 & 25.271 \\
\hline $7 / 28 / 2006$ & $20: 00$ & 41.942 & 50.983 & 25.285 \\
\hline $7 / 29 / 2006$ & 0:00 & 41.928 & 50.959 & 25.264 \\
\hline 7/29/2006 & 4:00 & 41.918 & 50.971 & 25.278 \\
\hline 7/29/2006 & 8:00 & 41.918 & 50.973 & 25.288 \\
\hline $7 / 29 / 2006$ & $12: 00$ & 41.914 & 50.955 & 25.269 \\
\hline 7/29/2006 & $16: 00$ & 41.858 & 50.908 & 25.234 \\
\hline $7 / 29 / 2006$ & $20: 00$ & 41.811 & 50.926 & 25.257 \\
\hline 7/30/2006 & $0: 00$ & 41.811 & 50.933 & 25.264 \\
\hline $7 / 30 / 2006$ & 4:00 & 41.788 & 50.901 & 25.241 \\
\hline $7 / 30 / 2006$ & 8:00 & 41.785 & 50.917 & 25.26 \\
\hline 7/30/2006 & $12: 00$ & 41.769 & 50.887 & 25.231 \\
\hline 7/30/2006 & $16: 00$ & 41.72 & 50.84 & 25.201 \\
\hline $7 / 30 / 2006$ & $20: 00$ & 41.713 & 50.877 & 25.241 \\
\hline $7 / 31 / 2006$ & $0: 00$ & 41.736 & 50.884 & 25.248 \\
\hline $7 / 31 / 2006$ & $4: 00$ & 41.713 & 50.865 & 25.234 \\
\hline $7 / 31 / 2006$ & 8:00 & 41.746 & 50.901 & 25.262 \\
\hline $7 / 31 / 2006$ & $12: 00$ & 41.771 & 50.896 & 25.255 \\
\hline $7 / 31 / 2006$ & $16: 00$ & 41.776 & 50.891 & 25.248 \\
\hline 7/31/2006 & $20: 00$ & 41.813 & 50.955 & 25.302 \\
\hline 8/1/2006 & 0:00 & 41.834 & 50.959 & 25.302 \\
\hline $8 / 1 / 2006$ & 4:00 & 41.86 & 51.001 & 25.337 \\
\hline 8/1/2006 & 8:00 & 41.944 & 51.03 & 25.344 \\
\hline 8/1/2006 & $12: 00$ & 41.914 & 50.987 & 25.302 \\
\hline $8 / 1 / 2006$ & $16: 00$ & 41.865 & 50.947 & 25.262 \\
\hline 8/1/2006 & $20: 00$ & 41.851 & 51.004 & 25.33 \\
\hline & & & & \\
\hline
\end{tabular}

TABLE S1.1 (Cont.)

\begin{tabular}{|c|c|c|c|c|}
\hline \multirow[b]{2}{*}{ Date } & \multicolumn{4}{|c|}{$\begin{array}{l}\text { Water Level (ft below top of } \\
\text { casing) at Indicated Well }\end{array}$} \\
\hline & Time & D2 & WP49 & SB65D \\
\hline 8/2/2006 & 4:00 & 41.909 & 51.053 & 25.37 \\
\hline $8 / 2 / 2006$ & 8:00 & 41.949 & 51.074 & 25.381 \\
\hline 8/2/2006 & $12: 00$ & 42.005 & 51.105 & 25.4 \\
\hline $8 / 2 / 2006$ & $16: 00$ & 42.019 & 51.083 & 25.374 \\
\hline $8 / 2 / 2006$ & $20: 00$ & 42.03 & 51.112 & 25.4 \\
\hline $8 / 3 / 2006$ & 0:00 & 42.021 & 51.086 & 25.379 \\
\hline $8 / 3 / 2006$ & 4:00 & 42.035 & 51.123 & 25.416 \\
\hline 8/3/2006 & 8:00 & 42.086 & 51.168 & 25.444 \\
\hline 8/3/2006 & $12: 00$ & 42.084 & 51.133 & 25.409 \\
\hline $8 / 3 / 2006$ & $16: 00$ & 42.054 & 51.112 & 25.393 \\
\hline 8/3/2006 & $20: 00$ & 42.03 & 51.142 & 25.423 \\
\hline $8 / 4 / 2006$ & 0:00 & 42.026 & 51.135 & 25.421 \\
\hline $8 / 4 / 2006$ & 4:00 & 42.005 & 51.121 & 25.414 \\
\hline $8 / 4 / 2006$ & 8:00 & 42.021 & 51.142 & 25.428 \\
\hline $8 / 4 / 2006$ & $12: 00$ & 41.998 & 51.086 & 25.374 \\
\hline $8 / 4 / 2006$ & $16: 00$ & 42.028 & 51.053 & 25.32 \\
\hline 8/4/2006 & $20: 00$ & 41.956 & 51.072 & 25.346 \\
\hline 8/5/2006 & 0:00 & 41.907 & 51.032 & 25.316 \\
\hline 8/5/2006 & 4:00 & 41.853 & 50.994 & 25.302 \\
\hline 8/5/2006 & 8:00 & 41.832 & 51.001 & 25.299 \\
\hline $8 / 5 / 2006$ & $12: 00$ & 41.881 & 51.004 & 25.292 \\
\hline 8/5/2006 & $16: 00$ & 41.767 & 50.962 & 25.276 \\
\hline 8/5/2006 & $20: 00$ & 41.75 & 50.997 & 25.32 \\
\hline $8 / 6 / 2006$ & 0:00 & 41.816 & 51.1 & 25.419 \\
\hline 8/6/2006 & 4:00 & 41.781 & 51.058 & 25.393 \\
\hline 8/6/2006 & 8:00 & 41.778 & 51.095 & 25.423 \\
\hline 8/6/2006 & 12:00 & 41.816 & 51.114 & 25.437 \\
\hline 8/6/2006 & $16: 00$ & 41.848 & 51.161 & 25.487 \\
\hline $8 / 6 / 2006$ & $20: 00$ & 41.82 & 51.102 & 25.421 \\
\hline 8/7/2006 & $0: 00$ & 41.858 & 51.128 & 25.451 \\
\hline 8/7/2006 & 4:00 & 41.869 & 51.144 & 25.47 \\
\hline $8 / 7 / 2006$ & 8:00 & 41.902 & 51.17 & 25.482 \\
\hline 8/7/2006 & $12: 00$ & 41.895 & 51.149 & 25.463 \\
\hline 8/7/2006 & $16: 00$ & 41.862 & 51.109 & 25.433 \\
\hline $8 / 7 / 2006$ & $20: 00$ & 41.853 & 51.126 & 25.451 \\
\hline 8/8/2006 & $0: 00$ & 41.823 & 51.095 & 25.423 \\
\hline 8/8/2006 & 4:00 & 41.604 & 51.093 & 25.379 \\
\hline 8/8/2006 & 8:00 & 41.643 & 51.058 & 25.234 \\
\hline 8/8/2006 & $12: 00$ & 41.555 & 51.004 & 25.108 \\
\hline 8/8/2006 & $16: 00$ & 41.398 & 50.887 & 25.019 \\
\hline 8/8/2006 & $20: 00$ & 41.289 & 50.849 & 25.007 \\
\hline $8 / 9 / 2006$ & $0: 00$ & 41.209 & 50.788 & 24.974 \\
\hline
\end{tabular}


TABLE S1.1 (Cont.)

\begin{tabular}{|c|c|c|c|c|}
\hline \multirow[b]{2}{*}{ Date } & \multicolumn{4}{|c|}{$\begin{array}{l}\text { Water Level (ft below top of } \\
\text { casing) at Indicated Well }\end{array}$} \\
\hline & Time & D2 & WP49 & SB65D \\
\hline 8/9/2006 & 4:00 & 41.144 & 50.753 & 24.974 \\
\hline 8/9/2006 & 8:00 & 41.109 & 50.725 & 24.969 \\
\hline 8/9/2006 & $12: 00$ & 41.077 & 50.706 & 24.979 \\
\hline 8/9/2006 & $16: 00$ & 40.988 & 50.631 & 24.946 \\
\hline 8/9/2006 & $20: 00$ & 40.967 & 50.65 & 24.981 \\
\hline $8 / 10 / 2006$ & $0: 00$ & 40.979 & 50.666 & 25.019 \\
\hline $8 / 10 / 2006$ & 4:00 & 41.004 & 50.694 & 25.054 \\
\hline $8 / 10 / 2006$ & 8:00 & 41.004 & 50.694 & 25.054 \\
\hline $8 / 10 / 2006$ & $12: 00$ & 41.046 & 50.708 & 25.072 \\
\hline $8 / 10 / 2006$ & $16: 00$ & 41.023 & 50.661 & 25.04 \\
\hline $8 / 10 / 2006$ & $20: 00$ & 41.035 & 50.692 & 25.077 \\
\hline $8 / 11 / 2006$ & $0: 00$ & 41.074 & 50.72 & 25.105 \\
\hline $8 / 11 / 2006$ & 4:00 & 41.049 & 50.683 & 25.075 \\
\hline $8 / 11 / 2006$ & 8:00 & 41.065 & 50.715 & 25.084 \\
\hline $8 / 11 / 2006$ & $12: 00$ & 41.063 & 50.687 & 25.049 \\
\hline $8 / 11 / 2006$ & $16: 00$ & 41.009 & 50.633 & 25.007 \\
\hline $8 / 11 / 2006$ & $20: 00$ & 40.986 & 50.647 & 25.023 \\
\hline $8 / 12 / 2006$ & $0: 00$ & 41.018 & 50.671 & 25.054 \\
\hline $8 / 12 / 2006$ & 4:00 & 40.983 & 50.636 & 25.028 \\
\hline $8 / 12 / 2006$ & 8:00 & 41.009 & 50.666 & 25.068 \\
\hline 8/12/2006 & $12: 00$ & 41.007 & 50.636 & 25.044 \\
\hline 8/12/2006 & $16: 00$ & 40.941 & 50.565 & 24.998 \\
\hline $8 / 12 / 2006$ & $20: 00$ & 40.927 & 50.593 & 25.035 \\
\hline 8/13/2006 & $0: 00$ & 40.948 & 50.6 & 25.047 \\
\hline $8 / 13 / 2006$ & 4:00 & 40.916 & 50.558 & 25.016 \\
\hline 8/13/2006 & 8:00 & 40.925 & 50.577 & 25.028 \\
\hline 8/13/2006 & $12: 00$ & 40.944 & 50.603 & 25.072 \\
\hline $8 / 13 / 2006$ & $16: 00$ & 40.927 & 50.577 & 25.044 \\
\hline $8 / 13 / 2006$ & $20: 00$ & 40.958 & 50.619 & 25.089 \\
\hline $8 / 14 / 2006$ & $0: 00$ & 41.023 & 50.678 & 25.138 \\
\hline $8 / 14 / 2006$ & 4:00 & & 50.6 & 25.15 \\
\hline $8 / 14 / 2006$ & 8:00 & 41.116 & 50.729 & 25.178 \\
\hline $8 / 14 / 2006$ & $12: 00$ & 41.116 & 50.697 & 25.152 \\
\hline $8 / 14 / 2006$ & $16: 00$ & 41.07 & 50.645 & 25.115 \\
\hline $8 / 14 / 2006$ & $20: 00$ & 40.846 & 50.654 & 25.133 \\
\hline $8 / 15 / 2006$ & & 40.874 & 50.671 & 25.152 \\
\hline $8 / 15 / 2006$ & 4:00 & 40.864 & 50.661 & 25.15 \\
\hline $8 / 15 / 2006$ & 8:00 & 40.878 & 50.678 & 25.166 \\
\hline $8 / 15 / 2006$ & $12: 00$ & 40.876 & 50.652 & 25.147 \\
\hline $8 / 15 / 2006$ & $16: 00$ & 40.836 & 50.605 & 25.115 \\
\hline $8 / 15 / 2006$ & $20: 00$ & 40.825 & 50.629 & 25.14 \\
\hline $8 / 16 / 2006$ & $0: 00$ & 40.832 & 50.624 & 25.143 \\
\hline
\end{tabular}

TABLE S1.1 (Cont.)

\begin{tabular}{|c|c|c|c|c|}
\hline \multirow[b]{2}{*}{ Date } & \multicolumn{4}{|c|}{$\begin{array}{l}\text { Water Level (ft below top of } \\
\text { casing) at Indicated Well }\end{array}$} \\
\hline & Time & D2 & WP49 & SB65D \\
\hline 8/16/2006 & 4:00 & 40.82 & 50.615 & 25.14 \\
\hline 8/16/2006 & 8:00 & 40.832 & 50.624 & 25.147 \\
\hline 8/16/2006 & $12: 00$ & 40.813 & 50.593 & 25.124 \\
\hline 8/16/2006 & $16: 00$ & 40.757 & 50.542 & 25.093 \\
\hline $8 / 16 / 2006$ & $20: 00$ & 40.764 & 50.584 & 25.131 \\
\hline 8/17/2006 & 0:00 & 40.738 & 50.563 & 25.119 \\
\hline 8/17/2006 & 4:00 & 40.72 & 50.549 & 25.105 \\
\hline $8 / 17 / 2006$ & 8:00 & 40.731 & 50.575 & 25.112 \\
\hline $8 / 17 / 2006$ & $12: 00$ & 40.727 & 50.558 & 25.086 \\
\hline 8/17/2006 & $16: 00$ & 40.699 & 50.528 & 25.058 \\
\hline 8/17/2006 & $20: 00$ & 40.708 & 50.565 & 25.089 \\
\hline 8/18/2006 & 0:00 & 40.699 & 50.535 & 25.065 \\
\hline 8/18/2006 & 4:00 & 40.682 & 50.563 & 25.077 \\
\hline $8 / 18 / 2006$ & 8:00 & 40.671 & 50.547 & 24.993 \\
\hline 8/18/2006 & $12: 00$ & 40.652 & 50.533 & 24.927 \\
\hline 8/18/2006 & $16: 00$ & 40.605 & 50.486 & 24.864 \\
\hline 8/18/2006 & $20: 00$ & 40.591 & 50.516 & 24.892 \\
\hline $8 / 19 / 2006$ & 0:00 & 40.608 & 50.547 & 24.913 \\
\hline $8 / 19 / 2006$ & 4:00 & 40.598 & 50.521 & 24.89 \\
\hline $8 / 19 / 2006$ & 8:00 & 40.598 & 50.518 & 24.897 \\
\hline 8/19/2006 & $12: 00$ & 40.601 & 50.516 & 24.899 \\
\hline 8/19/2006 & $16: 00$ & 40.577 & 50.488 & 24.885 \\
\hline $8 / 19 / 2006$ & $20: 00$ & 40.556 & 50.497 & 24.904 \\
\hline 8/20/2006 & 0:00 & 40.549 & 50.49 & 24.904 \\
\hline $8 / 20 / 2006$ & 4:00 & 40.533 & 50.483 & 24.906 \\
\hline 8/20/2006 & 8:00 & 40.55 & 50.507 & 24.934 \\
\hline 8/20/2006 & 12:00 & 40.531 & 50.472 & 24.911 \\
\hline $8 / 20 / 2006$ & $16: 00$ & 40.48 & 50.42 & 24.881 \\
\hline 8/20/2006 & $20: 00$ & 40.475 & 50.465 & 24.932 \\
\hline $8 / 21 / 2006$ & 0:00 & 40.501 & 50.462 & 24.93 \\
\hline $8 / 21 / 2006$ & 4:00 & 40.463 & 50.439 & 24.925 \\
\hline $8 / 21 / 2006$ & 8:00 & 40.522 & 50.488 & 24.969 \\
\hline 8/21/2006 & $12: 00$ & 40.529 & 50.469 & 24.958 \\
\hline $8 / 21 / 2006$ & $16: 00$ & 40.496 & 50.436 & 24.939 \\
\hline 8/21/2006 & $20: 00$ & 40.515 & 50.481 & 24.988 \\
\hline $8 / 22 / 2006$ & $0: 00$ & 40.533 & 50.472 & 24.981 \\
\hline $8 / 22 / 2006$ & 4:00 & 40.535 & 50.469 & 24.991 \\
\hline 8/22/2006 & 8:00 & 40.57 & 50.502 & 25.019 \\
\hline 8/22/2006 & $12: 00$ & 40.563 & 50.465 & 24.988 \\
\hline 8/22/2006 & $16: 00$ & 40.487 & 50.389 & 24.937 \\
\hline 8/22/2006 & $20: 00$ & 40.461 & 50.406 & 24.96 \\
\hline $8 / 23 / 200$ & 000 & 40.456 & 50.397 & \\
\hline
\end{tabular}


TABLE S1.1 (Cont.)

\begin{tabular}{|c|c|c|c|c|}
\hline \multirow[b]{2}{*}{ Date } & \multicolumn{4}{|c|}{$\begin{array}{l}\text { Water Level (ft below top of } \\
\text { casing) at Indicated Well }\end{array}$} \\
\hline & Time & D2 & WP49 & SB65D \\
\hline 8/23/2006 & 4:00 & 40.414 & 50.364 & 24.93 \\
\hline $8 / 23 / 2006$ & 8:00 & 40.405 & 50.368 & 24.934 \\
\hline $8 / 23 / 2006$ & $12: 00$ & 40.365 & 50.319 & \\
\hline $8 / 23 / 2006$ & $16: 00$ & 40.302 & 50.263 & 24.85 \\
\hline 8/23/2006 & $20: 00$ & 40.288 & 50.291 & 24.876 \\
\hline $8 / 24 / 2006$ & $0: 00$ & 40.291 & 50.286 & 24.874 \\
\hline $8 / 24 / 2006$ & 4:00 & 40.293 & 50.296 & 24.89 \\
\hline $8 / 24 / 2006$ & 8:00 & 40.715 & 50.293 & 24.885 \\
\hline $8 / 24 / 2006$ & $12: 00$ & & 50.263 & 24.867 \\
\hline $8 / 24 / 2006$ & $16: 00$ & & 50.244 & 24.857 \\
\hline $8 / 24 / 2006$ & $20: 00$ & 40.626 & 50.258 & 24.878 \\
\hline $8 / 25 / 2006$ & $0: 00$ & 40.37 & 50.305 & 24.92 \\
\hline 8/25/2006 & 4:00 & 40.333 & 50.345 & 24.958 \\
\hline $8 / 25 / 2006$ & 8:00 & 40.358 & 50.387 & 24.993 \\
\hline $8 / 25 / 2006$ & $12: 00$ & & 50.385 & 25.028 \\
\hline $8 / 25 / 2006$ & $16: 00$ & 40.41 & 50.399 & 25.042 \\
\hline $8 / 25 / 2006$ & $20: 00$ & 40.421 & 50.441 & 25.079 \\
\hline $8 / 26 / 2006$ & $0: 00$ & 40.419 & 50.401 & 25.049 \\
\hline $8 / 26 / 2006$ & 4:00 & 40.433 & 50.408 & 25.054 \\
\hline $8 / 26 / 2006$ & & 40.459 & 50.436 & 25.079 \\
\hline 8/26/2006 & $12: 00$ & 40.473 & 50.422 & 25.061 \\
\hline 8/26/2006 & $16: 00$ & 40.466 & 50.415 & 25.061 \\
\hline $8 / 26 / 2006$ & $20: 00$ & 40.466 & 50.439 & 25.091 \\
\hline $8 / 27 / 2006$ & $0: 00$ & 40.482 & 50.42 & 25.075 \\
\hline $8 / 27 / 2006$ & 4:00 & 40.477 & 50.432 & 25.086 \\
\hline $8 / 27 / 2006$ & 8:00 & 40.477 & 50.425 & 25.079 \\
\hline 8/27/2006 & $12: 00$ & 40.461 & 50.399 & 25.061 \\
\hline $8 / 27 / 2006$ & $16: 00$ & 40.407 & 50.354 & 25.023 \\
\hline 8/27/2006 & $20: 00$ & 40.389 & 50.366 & 25.044 \\
\hline $8 / 28 / 2006$ & $0: 00$ & 40.375 & 50.326 & 25.007 \\
\hline 8/28/2006 & 4:00 & 40.377 & 50.371 & 25.049 \\
\hline 8/28/2006 & 8:00 & 40.41 & 50.399 & 25.075 \\
\hline 8/28/2006 & $12: 00$ & 40.459 & 50.448 & 25.115 \\
\hline $8 / 28 / 2006$ & $16: 00$ & 40.477 & 50.446 & 25.11 \\
\hline $8 / 28 / 2006$ & $20: 00$ & 40.496 & 50.46 & 25.122 \\
\hline $8 / 29 / 2006$ & & 40.505 & 50.446 & 25.11 \\
\hline $8 / 29 / 2006$ & 4:00 & 40.508 & 50.45 & 25.112 \\
\hline $8 / 29 / 2006$ & 8:00 & 40.521 & 50.462 & 25.122 \\
\hline $8 / 29 / 2006$ & $12: 00$ & 40.512 & 50.427 & 25.089 \\
\hline 8/29/2006 & $16: 00$ & 40.48 & 50.399 & 25.07 \\
\hline $8 / 29 / 2006$ & $20: 00$ & 40.473 & 50.422 & 25.094 \\
\hline $8 / 30 / 2006$ & $0: 00$ & 40.482 & 2 & 25.096 \\
\hline
\end{tabular}

TABLE S1.1 (Cont.)

\begin{tabular}{|c|c|c|c|c|}
\hline \multirow[b]{2}{*}{ Date } & \multicolumn{4}{|c|}{$\begin{array}{l}\text { Water Level (ft below top of } \\
\text { casing) at Indicated Well }\end{array}$} \\
\hline & Time & D2 & WP49 & SB65D \\
\hline 8/30/2006 & 4:00 & 40.487 & 50.434 & 25.108 \\
\hline 8/30/2006 & 8:00 & 40.503 & 50.448 & 25.119 \\
\hline 8/30/2006 & $12: 00$ & 40.494 & 50.411 & 25.087 \\
\hline 8/30/2006 & $16: 00$ & 40.113 & 50.357 & 25.012 \\
\hline 8/30/2006 & $20: 00$ & 40.13 & 50.401 & 25.056 \\
\hline $8 / 31 / 2006$ & 0:00 & 40.151 & 50.411 & 25.065 \\
\hline $8 / 31 / 2006$ & 4:00 & 40.162 & 50.422 & 25.077 \\
\hline $8 / 31 / 2006$ & 8:00 & 40.186 & 50.443 & 25.091 \\
\hline $8 / 31 / 2006$ & $12: 00$ & 40.19 & 50.432 & 25.082 \\
\hline $8 / 31 / 2006$ & $16: 00$ & 40.176 & 50.411 & 25.065 \\
\hline 8/31/2006 & $20: 00$ & 40.188 & 50.45 & 25.108 \\
\hline 9/1/2006 & 0:00 & 40.221 & 50.479 & 25.126 \\
\hline 9/1/2006 & 4:00 & 40.242 & 50.476 & 25.122 \\
\hline $9 / 1 / 2006$ & 8:00 & 40.249 & 50.486 & 25.133 \\
\hline 9/1/2006 & $12: 00$ & 40.239 & 50.457 & 25.108 \\
\hline $9 / 1 / 2006$ & $16: 00$ & 40.216 & 50.441 & 25.1 \\
\hline 9/1/2006 & $20: 00$ & 40.228 & 50.467 & 25.124 \\
\hline $9 / 2 / 2006$ & 0:00 & 40.235 & 50.465 & 25.126 \\
\hline $9 / 2 / 2006$ & 4:00 & 40.26 & 50.495 & 25.147 \\
\hline $9 / 2 / 2006$ & 8:00 & 40.277 & 50.5 & 25.154 \\
\hline 9/2/2006 & $12: 00$ & 40.274 & 50.502 & 25.152 \\
\hline 9/2/2006 & $16: 00$ & 40.246 & 50.457 & 25.108 \\
\hline 9/2/2006 & $20: 00$ & 40.237 & 50.469 & 25.124 \\
\hline 9/3/2006 & 0:00 & 40.235 & 50.462 & 25.112 \\
\hline $9 / 3 / 2006$ & 4:00 & 40.216 & 50.45 & 25.103 \\
\hline 9/3/2006 & 8:00 & 40.221 & 50.458 & 25.108 \\
\hline $9 / 3 / 2006$ & $12: 00$ & 40.195 & 50.413 & 25.07 \\
\hline 9/3/2006 & $16: 00$ & 40.134 & 50.371 & 25.04 \\
\hline 9/3/2006 & $20: 00$ & 40.167 & 50.45 & 25.115 \\
\hline $9 / 4 / 2006$ & 0:00 & 40.186 & 50.446 & 25.112 \\
\hline $9 / 4 / 2006$ & 4:00 & 40.2 & 50.46 & 25.122 \\
\hline 9/4/2006 & 8:00 & 40.256 & 50.511 & 25.166 \\
\hline 9/4/2006 & $12: 00$ & 40.265 & 50.488 & 25.145 \\
\hline $9 / 4 / 2006$ & $16: 00$ & 40.239 & 50.462 & 25.124 \\
\hline 9/4/2006 & $20: 00$ & 40.258 & 50.504 & 25.164 \\
\hline 9/5/2006 & $0: 00$ & 40.26 & 50.481 & 25.145 \\
\hline $9 / 5 / 2006$ & 4:00 & 40.263 & 50.497 & 25.159 \\
\hline $9 / 5 / 2006$ & 8:00 & 40.288 & 50.521 & 25.178 \\
\hline 9/5/2006 & $12: 00$ & 40.277 & 50.479 & 25.138 \\
\hline 9/5/2006 & $16: 00$ & 40.228 & 50.434 & 25.103 \\
\hline 9/5/2006 & $20: 00$ & 40.23 & 50.474 & 25.145 \\
\hline 9/6/2006 & 0:00 & 40.235 & 50.462 & 25.133 \\
\hline
\end{tabular}


TABLE S1.1 (Cont.)

\begin{tabular}{|c|c|c|c|c|}
\hline \multirow[b]{2}{*}{ Date } & \multicolumn{4}{|c|}{$\begin{array}{l}\text { Water Level (ft below top of } \\
\text { casing) at Indicated Well }\end{array}$} \\
\hline & Time & D2 & WP49 & SB65D \\
\hline 9/6/2006 & 4:00 & 40.225 & 50.455 & 25.131 \\
\hline 9/6/2006 & 8:00 & 40.246 & 50.483 & 25.152 \\
\hline $9 / 6 / 2006$ & $12: 00$ & 40.232 & 50.441 & 25.11 \\
\hline 9/6/2006 & $16: 00$ & 40.179 & 50.397 & 25.079 \\
\hline 9/6/2006 & $20: 00$ & 40.165 & 50.413 & 25.101 \\
\hline 9/7/2006 & 0:00 & 40.155 & 50.397 & 25.087 \\
\hline 9/7/2006 & 4:00 & 40.141 & 50.394 & 25.087 \\
\hline 9/7/2006 & 8:00 & 40.162 & 50.422 & 25.11 \\
\hline 9/7/2006 & $12: 00$ & 40.155 & 50.392 & 25.07 \\
\hline 9/7/2006 & $16: 00$ & 40.179 & 50.359 & 25.04 \\
\hline 9/7/2006 & $20: 00$ & 40.144 & 50.392 & 25.079 \\
\hline 9/8/2006 & 0:00 & 40.141 & 50.397 & 25.089 \\
\hline 9/8/2006 & 4:00 & 40.141 & 50.401 & 25.098 \\
\hline 9/8/2006 & 8:00 & 40.172 & 50.429 & 25.119 \\
\hline 9/8/2006 & $12: 00$ & 40.172 & 50.406 & 25.098 \\
\hline 9/8/2006 & $16: 00$ & 40.153 & 50.392 & 25.096 \\
\hline 9/8/2006 & $20: 00$ & 40.172 & 50.432 & 25.136 \\
\hline 9/9/2006 & 0:00 & 40.193 & 50.434 & 25.138 \\
\hline 9/9/2006 & 4:00 & 40.207 & 50.441 & 25.145 \\
\hline 9/9/2006 & 8:00 & 40.232 & 50.462 & 25.161 \\
\hline 9/9/2006 & $12: 00$ & 40.242 & 50.453 & 25.147 \\
\hline 9/9/2006 & $16: 00$ & 40.214 & 50.427 & 25.133 \\
\hline 9/9/2006 & $20: 00$ & 40.214 & 50.441 & 25.14 \\
\hline 9/10/2006 & $0: 00$ & 40.211 & 50.443 & 25.14 \\
\hline 9/10/2006 & 4:00 & 40.16 & 50.392 & 25.089 \\
\hline 9/10/2006 & 8:00 & 40.139 & 50.397 & 25.082 \\
\hline 9/10/2006 & $12: 00$ & 40.125 & 50.383 & 25.056 \\
\hline 9/10/2006 & $16: 00$ & 40.097 & 50.359 & 25.03 \\
\hline 9/10/2006 & $20: 00$ & 40.109 & 50.397 & 25.058 \\
\hline 9/11/2006 & $0: 00$ & 40.109 & 50.38 & 25.04 \\
\hline 9/11/2006 & 4:00 & 40.116 & 50.399 & 25.058 \\
\hline 9/11/2006 & 8:00 & 40.167 & 50.453 & 25.105 \\
\hline $9 / 11 / 2006$ & $12: 00$ & 40.207 & 50.469 & 25.115 \\
\hline 9/11/2006 & $16: 00$ & 40.195 & 50.436 & 25.087 \\
\hline $9 / 11 / 2006$ & $20: 00$ & 40.223 & 50.474 & 25.119 \\
\hline 9/12/2006 & 0:00 & 40.237 & 50.455 & 25.101 \\
\hline 9/12/2006 & 4:00 & 40.223 & 50.453 & 25.101 \\
\hline 9/12/2006 & 8:00 & 40.237 & 50.46 & 25.108 \\
\hline 9/12/2006 & $12: 00$ & 40.223 & 50.429 & 25.079 \\
\hline $9 / 12 / 2006$ & $16: 00$ & 40.169 & 50.378 & 25.04 \\
\hline 9/12/2006 & $20: 00$ & 40.16 & 50.401 & 25.063 \\
\hline & & & & \\
\hline
\end{tabular}

TABLE S1.1 (Cont.)

\begin{tabular}{|c|c|c|c|c|}
\hline \multirow[b]{2}{*}{ Date } & \multicolumn{4}{|c|}{$\begin{array}{l}\text { Water Level (ft below top of } \\
\text { casing) at Indicated Well }\end{array}$} \\
\hline & Time & D2 & WP49 & SB65D \\
\hline 9/13/2006 & 4:00 & 40.137 & 50.373 & 25.044 \\
\hline $9 / 13 / 2006$ & 8:00 & 40.137 & 50.378 & 25.044 \\
\hline 9/13/2006 & $12: 00$ & 40.095 & 50.314 & 24.993 \\
\hline $9 / 13 / 2006$ & $16: 00$ & 40.032 & 50.27 & 24.965 \\
\hline $9 / 13 / 2006$ & $20: 00$ & 40.027 & 50.305 & 25 \\
\hline 9/14/2006 & 0:00 & 40.036 & 50.31 & 25.009 \\
\hline $9 / 14 / 2006$ & 4:00 & 40.041 & 50.312 & 25.009 \\
\hline $9 / 14 / 2006$ & 8:00 & 40.069 & 50.34 & 25.03 \\
\hline $9 / 14 / 2006$ & $12: 00$ & 40.057 & 50.307 & 25.005 \\
\hline $9 / 14 / 2006$ & $16: 00$ & 40.039 & 50.296 & 25 \\
\hline 9/14/2006 & $20: 00$ & 40.06 & 50.336 & 25.042 \\
\hline 9/15/2006 & 0:00 & 40.081 & 50.343 & 25.044 \\
\hline $9 / 15 / 2006$ & 4:00 & 40.083 & 50.331 & 25.035 \\
\hline $9 / 15 / 2006$ & 8:00 & 40.102 & 50.357 & 25.056 \\
\hline 9/15/2006 & $12: 00$ & 40.055 & 50.27 & 24.981 \\
\hline $9 / 15 / 2006$ & $16: 00$ & 39.983 & 50.209 & 24.937 \\
\hline 9/15/2006 & $20: 00$ & 39.964 & 50.261 & 24.993 \\
\hline 9/16/2006 & 0:00 & 39.964 & 50.258 & 24.986 \\
\hline $9 / 16 / 2006$ & 4:00 & 39.962 & 50.249 & 24.974 \\
\hline $9 / 16 / 2006$ & 8:00 & 39.976 & 50.256 & 24.979 \\
\hline 9/16/2006 & $12: 00$ & 39.943 & 50.211 & 24.939 \\
\hline 9/16/2006 & $16: 00$ & 39.908 & 50.195 & 24.927 \\
\hline $9 / 16 / 2006$ & $20: 00$ & 39.887 & 50.197 & 24.988 \\
\hline $9 / 17 / 2006$ & 0:00 & 39.997 & 50.347 & 25.063 \\
\hline $9 / 17 / 2006$ & 4:00 & 40.12 & 50.443 & 25.129 \\
\hline 9/17/2006 & 8:00 & 40.186 & 50.429 & 25.098 \\
\hline 9/17/2006 & $12: 00$ & 40.19 & 50.406 & 25.072 \\
\hline 9/17/2006 & $16: 00$ & 40.153 & 50.357 & 25.035 \\
\hline 9/17/2006 & $20: 00$ & 40.151 & 50.373 & 25.049 \\
\hline $9 / 18 / 2006$ & 0:00 & 40.167 & 50.397 & 25.07 \\
\hline $9 / 18 / 2006$ & 4:00 & 40.176 & 50.406 & 25.079 \\
\hline 9/18/2006 & 8:00 & 40.19 & 50.408 & 25.077 \\
\hline 9/18/2006 & $12: 00$ & 40.2 & 50.408 & 25.077 \\
\hline 9/18/2006 & $16: 00$ & 40.216 & 50.436 & 25.108 \\
\hline 9/18/2006 & $20: 00$ & 40.263 & 50.472 & 25.136 \\
\hline 9/19/2006 & $0: 00$ & 40.274 & 50.467 & 25.131 \\
\hline $9 / 19 / 2006$ & 4:00 & 40.295 & 50.49 & 25.15 \\
\hline 9/19/2006 & 8:00 & 40.344 & 50.523 & 25.175 \\
\hline 9/19/2006 & $12: 00$ & 40.342 & 50.49 & 25.143 \\
\hline 9/19/2006 & $16: 00$ & 40.291 & 50.434 & 25.098 \\
\hline $9 / 19 / 2006$ & $20: 00$ & 40.3 & 50.476 & 25.138 \\
\hline 9/20/200€ & 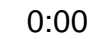 & 40.3 & 50.469 & 25.133 \\
\hline
\end{tabular}


TABLE S1.1 (Cont.)

\begin{tabular}{|c|c|c|c|c|}
\hline \multirow[b]{2}{*}{ Date } & \multicolumn{4}{|c|}{$\begin{array}{l}\text { Water Level (ft below top of } \\
\text { casing) at Indicated Well }\end{array}$} \\
\hline & Time & D2 & WP49 & SB65D \\
\hline 9/20/2006 & 4:00 & 40.298 & 50.455 & 25.122 \\
\hline $9 / 20 / 2006$ & 8:00 & 40.298 & 50.46 & 25.119 \\
\hline $9 / 20 / 2006$ & $12: 00$ & 40.209 & 50.338 & 25.014 \\
\hline $9 / 20 / 2006$ & $16: 00$ & 40.111 & 50.284 & 24.981 \\
\hline 9/20/2006 & $20: 00$ & 40.064 & 50.272 & 24.979 \\
\hline $9 / 21 / 2006$ & $0: 00$ & 40.008 & 50.216 & 24.932 \\
\hline $9 / 21 / 2006$ & 4:00 & 39.943 & 50.193 & 24.923 \\
\hline $9 / 21 / 2006$ & 8:00 & 39.939 & 50.223 & 24.953 \\
\hline $9 / 21 / 2006$ & $12: 00$ & 39.871 & 50.134 & 24.878 \\
\hline $9 / 21 / 2006$ & $16: 00$ & 39.796 & 50.094 & 24.846 \\
\hline $9 / 21 / 2006$ & $20: 00$ & 39.677 & 50 & 24.757 \\
\hline $9 / 22 / 2006$ & $0: 00$ & 39.628 & 50.028 & 24.719 \\
\hline 9/22/2006 & 4:00 & 39.698 & 50.139 & 24.785 \\
\hline 9/22/2006 & 8:00 & 39.785 & 50.216 & 24.824 \\
\hline $9 / 22 / 2006$ & $12: 00$ & 39.841 & 50.232 & 24.82 \\
\hline $9 / 22 / 2006$ & $16: 00$ & 39.871 & 50.244 & 24.827 \\
\hline $9 / 22 / 2006$ & $20: 00$ & 39.946 & 50.312 & 24.878 \\
\hline 9/23/2006 & $0: 00$ & 39.969 & 50.296 & 24.855 \\
\hline 9/23/2006 & 4:00 & 39.985 & 50.315 & 24.874 \\
\hline 9/23/2006 & 8:00 & 40.011 & 50.31 & 24.867 \\
\hline 9/23/2006 & $12: 00$ & 40.034 & 50.326 & 24.885 \\
\hline 9/23/2006 & $16: 00$ & 40.076 & 50.375 & 24.934 \\
\hline 9/23/2006 & $20: 00$ & 40.144 & 50.427 & 24.97 \\
\hline 9/24/2006 & $0: 00$ & 40.211 & 50.467 & 25.005 \\
\hline 9/24/2006 & 4:00 & 40.27 & 50.507 & 25.03 \\
\hline 9/24/2006 & 8:00 & 40.316 & 50.528 & 25.047 \\
\hline 9/24/2006 & $12: 00$ & 40.312 & 50.479 & 24.998 \\
\hline 9/24/2006 & $16: 00$ & 40.258 & 50.427 & 24.955 \\
\hline 9/24/2006 & $20: 00$ & 40.253 & 50.439 & 24.967 \\
\hline 9/25/2006 & $0: 00$ & 40.228 & 50.404 & 24.932 \\
\hline 9/25/2006 & 4:00 & 40.1 & 50.387 & 24.927 \\
\hline 9/25/2006 & 8:00 & 40.193 & 50.401 & 24.941 \\
\hline 9/25/2006 & $12: 00$ & 40.179 & 50.375 & 24.918 \\
\hline $9 / 25 / 2006$ & $16: 00$ & 40.137 & 50.343 & 24.897 \\
\hline 9/25/2006 & $20: 00$ & 40.148 & 50.378 & 24.932 \\
\hline 9/26/2006 & & 40.153 & 50.373 & 24.93 \\
\hline 9/26/2006 & 4:00 & 40.13 & 50.347 & 24.913 \\
\hline 9/26/2006 & 8:00 & 40.116 & 50.333 & 24.897 \\
\hline 9/26/2006 & $12: 00$ & 40.057 & 50.261 & 24.836 \\
\hline 9/26/2006 & $16: 00$ & 39.969 & 50.193 & 24.796 \\
\hline 9/26/2006 & $20: 00$ & 39.943 & 50.204 & 24.815 \\
\hline 9/27/2006 & $0: 00$ & 39.992 & 50.277 & 24.881 \\
\hline
\end{tabular}

TABLE S1.1 (Cont.)

\begin{tabular}{|c|c|c|c|c|}
\hline \multirow[b]{2}{*}{ Date } & \multicolumn{4}{|c|}{$\begin{array}{l}\text { Water Level (ft below top of } \\
\text { casing) at Indicated Well }\end{array}$} \\
\hline & Time & D2 & WP49 & SB65D \\
\hline 9/27/2006 & 4:00 & 40.027 & 50.307 & 24.909 \\
\hline 9/27/2006 & 8:00 & 40.083 & 50.361 & 24.955 \\
\hline 9/27/2006 & $12: 00$ & 40.099 & 50.336 & 24.927 \\
\hline 9/27/2006 & $16: 00$ & 40.09 & 50.326 & 24.918 \\
\hline 9/27/2006 & $20: 00$ & 40.155 & 50.408 & 24.993 \\
\hline 9/28/2006 & 0:00 & 40.207 & 50.434 & 25.009 \\
\hline 9/28/2006 & 4:00 & 40.237 & 50.443 & 25.016 \\
\hline 9/28/2006 & 8:00 & 40.265 & 50.462 & 25.026 \\
\hline 9/28/2006 & $12: 00$ & 40.225 & 50.394 & 24.953 \\
\hline 9/28/2006 & $16: 00$ & 40.153 & 50.326 & 24.906 \\
\hline 9/28/2006 & $20: 00$ & 40.085 & 50.258 & 24.855 \\
\hline 9/29/2006 & 0:00 & 40.011 & 50.211 & 24.824 \\
\hline 9/29/2006 & 4:00 & 39.929 & 50.164 & 24.792 \\
\hline 9/29/2006 & 8:00 & 39.911 & 50.183 & 24.817 \\
\hline 9/29/2006 & $12: 00$ & 39.922 & 50.2 & 24.831 \\
\hline 9/29/2006 & $16: 00$ & 39.92 & 50.204 & 24.838 \\
\hline 9/29/2006 & $20: 00$ & 39.964 & 50.256 & 24.883 \\
\hline 9/30/2006 & 0:00 & 40.011 & 50.286 & 24.909 \\
\hline 9/30/2006 & 4:00 & 40.029 & 50.293 & 24.911 \\
\hline 9/30/2006 & 8:00 & 40.078 & 50.34 & 24.951 \\
\hline 9/30/2006 & $12: 00$ & 40.085 & 50.319 & 24.92 \\
\hline 9/30/2006 & $16: 00$ & 40.062 & 50.282 & 24.899 \\
\hline 9/30/2006 & $20: 00$ & 40.078 & 50.314 & 24.93 \\
\hline $10 / 1 / 2006$ & 0:00 & 40.085 & 50.312 & 24.932 \\
\hline $10 / 1 / 2006$ & 4:00 & 40.09 & 50.312 & 24.93 \\
\hline 10/1/2006 & 8:00 & 40.097 & 50.305 & 24.923 \\
\hline 10/1/2006 & 12:00 & 40.055 & 50.251 & 24.869 \\
\hline $10 / 1 / 2006$ & $16: 00$ & 40.004 & 50.214 & 24.853 \\
\hline 10/1/2006 & $20: 00$ & 40.02 & 50.268 & 24.902 \\
\hline $10 / 2 / 2006$ & 0:00 & 40.034 & 50.263 & 24.897 \\
\hline $10 / 2 / 2006$ & 4:00 & 40.043 & 50.277 & 24.909 \\
\hline $10 / 2 / 2006$ & 8:00 & 40.078 & 50.322 & 24.951 \\
\hline 10/2/2006 & $12: 00$ & 40.085 & 50.3 & 24.92 \\
\hline $10 / 2 / 2006$ & $16: 00$ & 40.083 & 50.307 & 24.939 \\
\hline 10/2/2006 & $20: 00$ & 40.116 & 50.338 & 24.962 \\
\hline 10/3/2006 & $0: 00$ & 40.111 & 50.31 & 24.934 \\
\hline $10 / 3 / 2006$ & 4:00 & 40.102 & 50.307 & 24.934 \\
\hline $10 / 3 / 2006$ & 8:00 & 40.118 & 50.333 & 24.955 \\
\hline 10/3/2006 & $12: 00$ & 40.102 & 50.291 & 24.913 \\
\hline 10/3/2006 & $16: 00$ & 40.074 & 50.286 & 24.916 \\
\hline $10 / 3 / 2006$ & $20: 00$ & 40.092 & 50.322 & 24.946 \\
\hline $10 / 4 / 2006$ & 0:00 & 40.193 & 50.42 & 25.037 \\
\hline
\end{tabular}


TABLE S1.1 (Cont.)

\begin{tabular}{|c|c|c|c|c|}
\hline \multirow[b]{2}{*}{ Date } & \multicolumn{4}{|c|}{$\begin{array}{l}\text { Water Level (ft below top of } \\
\text { casing) at Indicated Well }\end{array}$} \\
\hline & Time & D2 & WP49 & SB65D \\
\hline $10 / 4 / 2006$ & $4: 00$ & 40.284 & 50.497 & 25.091 \\
\hline $10 / 4 / 2006$ & $8: 00$ & 40.379 & 50.565 & 25.14 \\
\hline $10 / 4 / 2006$ & $12: 00$ & 40.438 & 50.577 & 25.136 \\
\hline $10 / 4 / 2006$ & $16: 00$ & 40.433 & 50.556 & 25.115 \\
\hline 10/4/2006 & $20: 00$ & 40.442 & 50.563 & 25.124 \\
\hline 10/5/2006 & $0: 00$ & 40.431 & 50.547 & 25.105 \\
\hline $10 / 5 / 2006$ & $4: 00$ & 40.412 & 50.525 & 25.087 \\
\hline $10 / 5 / 2006$ & $8: 00$ & 40.424 & 50.54 & 25.098 \\
\hline $10 / 5 / 2006$ & $12: 00$ & 40.393 & 50.493 & 25.051 \\
\hline $10 / 5 / 2006$ & $16: 00$ & 40.326 & 50.441 & 25.016 \\
\hline 10/5/2006 & $20: 00$ & 40.328 & 50.479 & 25.054 \\
\hline $10 / 6 / 2006$ & $0: 00$ & 40.342 & 50.49 & 25.063 \\
\hline $10 / 6 / 2006$ & $4: 00$ & 40.335 & 50.479 & 25.054 \\
\hline $10 / 6 / 2006$ & $8: 00$ & 40.34 & 50.488 & 25.061 \\
\hline $10 / 6 / 2006$ & $12: 00$ & 40.291 & 50.418 & 24.991 \\
\hline 10/6/2006 & $16: 00$ & 40.209 & 50.352 & 24.948 \\
\hline $10 / 6 / 2006$ & $20: 00$ & 40.209 & 50.392 & 24.988 \\
\hline 10/7/2006 & $0: 00$ & 40.202 & 50.378 & 24.977 \\
\hline 10/7/2006 & $4: 00$ & 40.183 & 50.371 & 24.972 \\
\hline 10/7/2006 & $8: 00$ & 40.19 & 50.382 & 24.986 \\
\hline $10 / 7 / 2006$ & $12: 00$ & 40.158 & 50.331 & 24.934 \\
\hline 10/7/2006 & $16: 00$ & 40.116 & 50.315 & 24.932 \\
\hline 10/7/2006 & $20: 00$ & 40.137 & 50.357 & 24.974 \\
\hline $10 / 8 / 2006$ & $0: 00$ & 40.155 & 50.366 & 24.981 \\
\hline 10/8/2006 & $4: 00$ & 40.188 & 50.415 & 25.03 \\
\hline 10/8/2006 & $8: 00$ & 40.267 & 50.488 & 25.089 \\
\hline $10 / 8 / 2006$ & $12: 00$ & 40.295 & 50.472 & 25.061 \\
\hline 10/8/2006 & $16: 00$ & 40.279 & 50.455 & 25.054 \\
\hline $10 / 8 / 2006$ & $20: 00$ & 40.328 & 50.511 & 25.103 \\
\hline 10/9/2006 & $0: 00$ & 40.335 & 50.495 & 25.084 \\
\hline 10/9/2006 & $4: 00$ & 40.358 & 50.516 & 25.101 \\
\hline 10/9/2006 & $8: 00$ & 40.384 & 50.54 & 25.119 \\
\hline 10/9/2006 & $12: 00$ & 40.386 & 50.511 & 25.087 \\
\hline 10/9/2006 & $16: 00$ & 40.344 & 50.483 & 25.063 \\
\hline 10/9/2006 & $20: 00$ & 40.349 & 50.507 & 25.089 \\
\hline $10 / 10 / 2006$ & $0: 00$ & 40.333 & 50.472 & 25.049 \\
\hline $10 / 10 / 2006$ & $4: 00$ & 40.277 & 50.418 & 25.005 \\
\hline $10 / 10 / 2006$ & $8: 00$ & 40.237 & 50.39 & 24.984 \\
\hline 10/10/2006 & $12: 00$ & 40.144 & 50.291 & 24.902 \\
\hline $10 / 10 / 2006$ & $16: 00$ & 40.057 & 50.237 & 24.864 \\
\hline $10 / 10 / 2006$ & $20: 00$ & 39.997 & 50.207 & 24.848 \\
\hline & & & 50.16 & \\
\hline
\end{tabular}

TABLE S1.1 (Cont.)

\begin{tabular}{|c|c|c|c|c|}
\hline \multirow[b]{2}{*}{ Date } & \multicolumn{4}{|c|}{$\begin{array}{l}\text { Water Level (ft below top of } \\
\text { casing) at Indicated Well }\end{array}$} \\
\hline & Time & D2 & WP49 & SB65D \\
\hline $10 / 11 / 2006$ & $4: 00$ & 39.899 & 50.153 & 24.813 \\
\hline $10 / 11 / 2006$ & $8: 00$ & 39.969 & 50.289 & 24.934 \\
\hline $10 / 11 / 2006$ & $12: 00$ & 40.081 & 50.366 & 24.984 \\
\hline $10 / 11 / 2006$ & $16: 00$ & 40.109 & 50.352 & 24.97 \\
\hline $10 / 11 / 2006$ & $20: 00$ & 40.132 & 50.364 & 24.981 \\
\hline $10 / 12 / 2006$ & $0: 00$ & 40.16 & 50.378 & 24.991 \\
\hline $10 / 12 / 2006$ & $4: 00$ & 40.209 & 50.42 & 25.021 \\
\hline $10 / 12 / 2006$ & $8: 00$ & 40.218 & 50.404 & 24.998 \\
\hline $10 / 12 / 2006$ & $12: 00$ & 40.183 & 50.35 & 24.941 \\
\hline $10 / 12 / 2006$ & $16: 00$ & 40.144 & 50.333 & 24.941 \\
\hline $10 / 12 / 2006$ & $20: 00$ & 40.181 & 50.401 & 24.993 \\
\hline $10 / 13 / 2006$ & $0: 00$ & 40.207 & 50.408 & 25 \\
\hline $10 / 13 / 2006$ & $4: 00$ & 40.235 & 50.429 & 25.021 \\
\hline $10 / 13 / 2006$ & $8: 00$ & 40.27 & 50.462 & 25.04 \\
\hline $10 / 13 / 2006$ & $12: 00$ & 40.263 & 50.422 & 24.995 \\
\hline $10 / 13 / 2006$ & $16: 00$ & 40.228 & 50.399 & 24.981 \\
\hline $10 / 13 / 2006$ & $20: 00$ & 40.26 & 50.448 & 25.03 \\
\hline $10 / 14 / 2006$ & $0: 00$ & 40.312 & 50.493 & 25.065 \\
\hline $10 / 14 / 2006$ & $4: 00$ & 40.356 & 50.533 & 25.087 \\
\hline $10 / 14 / 2006$ & $8: 00$ & & 50.563 & 25.11 \\
\hline $10 / 14 / 2006$ & $12: 00$ & 40.375 & 50.495 & 25.04 \\
\hline $10 / 14 / 2006$ & $16: 00$ & 40.307 & 50.441 & 25.002 \\
\hline $10 / 14 / 2006$ & $20: 00$ & 40.265 & 50.415 & 24.979 \\
\hline $10 / 15 / 2006$ & $0: 00$ & 40.223 & 50.378 & 24.948 \\
\hline $10 / 15 / 2006$ & $4: 00$ & 40.2 & 50.361 & 24.934 \\
\hline $10 / 15 / 2006$ & $8: 00$ & 40.174 & 50.359 & 24.937 \\
\hline $10 / 15$ & $12: 00$ & 40.146 & 50.326 & 24.909 \\
\hline $10 / 15 / 2006$ & $16: 00$ & 40.102 & 50.3 & 24.892 \\
\hline $10 / 15 / 2006$ & $20: 00$ & 40.097 & 50.312 & 24.909 \\
\hline $10 / 16 / 2006$ & 0:00 & 40.074 & 50.289 & 24.892 \\
\hline $10 / 16 / 2006$ & 4:00 & 40.041 & 50.258 & 24.869 \\
\hline $10 / 16 / 2006$ & $8: 00$ & 40.043 & 50.272 & 24.883 \\
\hline $10 / 16 / 2006$ & $12: 00$ & 40.011 & 50.223 & 24.838 \\
\hline $10 / 16 / 2006$ & $16: 00$ & 39.971 & 50.211 & 24.838 \\
\hline $10 / 16 / 2006$ & $20: 00$ & 39.978 & 50.228 & 24.853 \\
\hline $10 / 17 / 2006$ & $0: 00$ & 39.994 & 50.256 & 24.881 \\
\hline $10 / 17 / 2006$ & $4: 00$ & 40.025 & 50.279 & 24.902 \\
\hline $10 / 17 / 2006$ & $8: 00$ & 40.123 & 50.387 & 24.984 \\
\hline $10 / 17 / 2006$ & $12: 00$ & 40.202 & 50.415 & 24.998 \\
\hline $10 / 17 / 2006$ & $16: 00$ & 40.253 & 50.458 & 25.03 \\
\hline $10 / 17 / 2006$ & $20: 00$ & 40.344 & 50.551 & 25.105 \\
\hline $10 / 18 / 2006$ & $0: 00$ & 40.379 & .533 & 25.077 \\
\hline
\end{tabular}


TABLE S1.1 (Cont.)

\begin{tabular}{|c|c|c|c|c|}
\hline \multirow[b]{2}{*}{ Date } & \multicolumn{4}{|c|}{$\begin{array}{l}\text { Water Level (ft below top of } \\
\text { casing) at Indicated Well }\end{array}$} \\
\hline & Time & D2 & WP49 & SB65D \\
\hline $10 / 18 / 2006$ & 4:00 & 40.412 & 50.558 & 25.101 \\
\hline $10 / 18 / 2006$ & $8: 00$ & 40.468 & 50.608 & 25.136 \\
\hline $10 / 18 / 2006$ & $12: 00$ & 40.484 & 50.582 & 25.103 \\
\hline $10 / 18 / 2006$ & $16: 00$ & 40.473 & 50.582 & 25.101 \\
\hline $10 / 18 / 2006$ & $20: 00$ & 40.517 & 50.61 & 25.122 \\
\hline $10 / 19 / 2006$ & $0: 00$ & 40.487 & 50.57 & 25.079 \\
\hline $10 / 19 / 2006$ & 4:00 & 40.44 & 50.535 & 25.054 \\
\hline $10 / 19 / 2006$ & $8: 00$ & 40.431 & 50.54 & 25.051 \\
\hline $10 / 19 / 2006$ & $12: 00$ & 40.361 & 50.448 & 24.974 \\
\hline $10 / 19 / 2006$ & $16: 00$ & 40.286 & 50.411 & 24.951 \\
\hline $10 / 19 / 2006$ & $20: 00$ & 40.279 & 50.434 & 24.972 \\
\hline $10 / 20 / 2006$ & $0: 00$ & 40.244 & 50.401 & 24.946 \\
\hline $10 / 20 / 2006$ & $4: 00$ & 40.223 & 50.392 & 24.941 \\
\hline $10 / 20 / 2006$ & $8: 00$ & 40.209 & 50.382 & 24.939 \\
\hline $10 / 20 / 2006$ & $12: 00$ & 40.172 & 50.343 & 24.902 \\
\hline $10 / 20 / 2006$ & $16: 00$ & 40.162 & 50.357 & 24.916 \\
\hline $10 / 20 / 2006$ & $20: 00$ & 40.207 & 50.415 & 24.972 \\
\hline $10 / 21 / 2006$ & $0: 00$ & 40.27 & 50.483 & 25.023 \\
\hline $10 / 21 / 2006$ & $4: 00$ & 40.347 & 50.558 & 25.091 \\
\hline $10 / 21 / 2006$ & 8:00 & & 50.629 & 25.147 \\
\hline $10 / 21 / 2006$ & $12: 00$ & 40.482 & 50.615 & 25.122 \\
\hline $10 / 21 / 2006$ & $16: 00$ & 40.515 & 50.64 & 25.143 \\
\hline $10 / 21 / 2006$ & $20: 00$ & 40.556 & 50.666 & 25.152 \\
\hline $10 / 22 / 2006$ & $0: 00$ & 40.568 & 50.676 & 25.154 \\
\hline $10 / 22 / 2006$ & $4: 00$ & 40.561 & 50.652 & 25.131 \\
\hline 10/22/2006 & $8: 00$ & & & 25.166 \\
\hline $10 / 22 / 2006$ & $12: 00$ & 40.577 & 50.659 & 25.133 \\
\hline $10 / 22 / 2006$ & $16: 00$ & 40.54 & 50.624 & 25.103 \\
\hline 10/22/2006 & $20: 00$ & 40.552 & 50.662 & 25.138 \\
\hline $10 / 23 / 2006$ & $0: 00$ & 40.543 & 50.64 & 25.115 \\
\hline $10 / 23 / 2006$ & 4:00 & 40.538 & 50.652 & 25.129 \\
\hline $10 / 23 / 2006$ & 8:00 & 40.543 & 50.657 & 25.133 \\
\hline $10 / 23 / 2006$ & $12: 00$ & 40.522 & 50.617 & 25.089 \\
\hline $10 / 23 / 2006$ & $16: 00$ & 40.484 & 50.598 & 25.079 \\
\hline $10 / 23 / 2006$ & $20: 00$ & 40.494 & 50.629 & 25.112 \\
\hline $10 / 24 / 2006$ & $0: 00$ & & 50.626 & 25.108 \\
\hline $10 / 24 / 2006$ & $4: 00$ & 40.489 & 50.619 & 25.101 \\
\hline $10 / 24 / 2006$ & $8: 00$ & 40.489 & 50.626 & 25.105 \\
\hline $10 / 24 / 2006$ & $12: 00$ & 40.438 & 50.547 & 25.023 \\
\hline $10 / 24 / 2006$ & $16: 00$ & 40.375 & 50.511 & 25.007 \\
\hline $10 / 24 / 2006$ & $20: 00$ & 40.375 & 50.528 & 25.026 \\
\hline 10/25/2006 & $0: 00$ & 40.351 & 504 & 25.007 \\
\hline
\end{tabular}

TABLE S1.1 (Cont.)

\begin{tabular}{|c|c|c|c|c|}
\hline \multirow[b]{2}{*}{ Date } & \multicolumn{4}{|c|}{$\begin{array}{l}\text { Water Level (ft below top of } \\
\text { casing) at Indicated Well }\end{array}$} \\
\hline & Time & D2 & WP49 & SB65D \\
\hline $10 / 25 / 2006$ & 4:00 & 40.33 & 50.497 & 25.005 \\
\hline $10 / 25 / 2006$ & 8:00 & 40.33 & 50.511 & 25.016 \\
\hline $10 / 25 / 2006$ & $12: 00$ & 40.316 & 50.472 & 24.981 \\
\hline $10 / 25 / 2006$ & $16: 00$ & 40.3 & 50.483 & 25.002 \\
\hline $10 / 25 / 2006$ & 20:00 & 40.309 & 50.497 & 25.009 \\
\hline 10/26/2006 & 0:00 & 40.321 & 50.511 & 25.023 \\
\hline $10 / 26 / 2006$ & 4:00 & 40.33 & 50.516 & 25.028 \\
\hline $10 / 26 / 2006$ & 8:00 & 40.372 & 50.563 & 25.063 \\
\hline 10/26/2006 & $12: 00$ & 40.396 & 50.565 & 25.056 \\
\hline $10 / 26 / 2006$ & $16: 00$ & 40.405 & 50.577 & 25.068 \\
\hline 10/26/2006 & $20: 00$ & 40.445 & 50.605 & 25.089 \\
\hline $10 / 27 / 2006$ & 0:00 & 40.449 & 50.582 & 25.063 \\
\hline $10 / 27 / 2006$ & 4:00 & 40.463 & 50.619 & 25.096 \\
\hline 10/27/2006 & 8:00 & 40.503 & 50.657 & 25.126 \\
\hline 10/27/2006 & $12: 00$ & 40.482 & 50.598 & 25.065 \\
\hline $10 / 27 / 2006$ & $16: 00$ & 40.428 & 50.561 & 25.047 \\
\hline 10/27/2006 & $20: 00$ & 40.468 & 50.624 & 25.108 \\
\hline 10/28/2006 & 0:00 & 40.503 & 50.643 & 25.115 \\
\hline $10 / 28 / 2006$ & $4: 00$ & 40.51 & 50.64 & 25.11 \\
\hline $10 / 28 / 2006$ & 8:00 & 40.498 & 50.622 & 25.089 \\
\hline $10 / 28 / 2006$ & $12: 00$ & 40.433 & 50.533 & 25.009 \\
\hline 10/28/2006 & $16: 00$ & 40.368 & 50.504 & 24.998 \\
\hline $10 / 28 / 2006$ & $20: 00$ & 40.368 & 50.519 & 25.014 \\
\hline $10 / 29 / 2006$ & 0:00 & 40.365 & 50.53 & 25.026 \\
\hline 10/29/2006 & 4:00 & 40.37 & 50.525 & 25.016 \\
\hline 10/29/2006 & 8:00 & 40.363 & 50.53 & 25.021 \\
\hline 10/29/2006 & $12: 00$ & 40.349 & 50.481 & 24.97 \\
\hline 10/29/2006 & $16: 00$ & 40.286 & 50.434 & 24.944 \\
\hline 10/29/2006 & $20: 00$ & 40.258 & 50.427 & 24.941 \\
\hline $10 / 30 / 2006$ & $0: 00$ & 40.216 & 50.382 & 24.911 \\
\hline $10 / 30 / 2006$ & $4: 00$ & 40.167 & 50.352 & 24.89 \\
\hline $10 / 30 / 2006$ & 8:00 & 40.16 & 50.364 & 24.904 \\
\hline 10/30/2006 & $12: 00$ & 40.183 & 50.408 & 24.948 \\
\hline 10/30/2006 & $16: 00$ & 40.379 & 50.643 & 25.154 \\
\hline 10/30/2006 & $20: 00$ & 40.531 & 50.72 & 25.206 \\
\hline 10/31/2006 & $0: 00$ & 40.671 & 50.833 & 25.276 \\
\hline 10/31/2006 & $4: 00$ & 40.762 & 50.87 & 25.295 \\
\hline $10 / 31 / 2006$ & 8:00 & 40.811 & 50.873 & 25.285 \\
\hline 10/31/2006 & $12: 00$ & 40.771 & 50.793 & 25.215 \\
\hline $10 / 31 /$ & $16: 00$ & 40.715 & 50.748 & 25.173 \\
\hline 10/31/ & 20:00 & 40.717 & 50.781 & 25.201 \\
\hline 1200 & & 727 & 50.793 & 25.213 \\
\hline
\end{tabular}


TABLE S1.1 (Cont.)

\begin{tabular}{ccccc}
\hline & & & & \\
& Water Level (ft below top of \\
& casing) at Indicated Well \\
\cline { 2 - 5 } Date & Time & D2 & WP49 & SB65D \\
& & & & \\
\hline & & & & \\
$11 / 1 / 2006$ & $4: 00$ & 40.734 & 50.8 & 25.229 \\
$11 / 1 / 2006$ & $8: 00$ & 40.773 & 50.847 & 25.271 \\
$11 / 1 / 2006$ & $12: 00$ & 40.771 & 50.814 & 25.234 \\
$11 / 1 / 2006$ & $16: 00$ & 40.818 & 50.823 & 25.229 \\
$11 / 1 / 2006$ & $20: 00$ & 40.776 & 50.828 & 25.25 \\
$11 / 2 / 2006$ & $0: 00$ & 40.78 & 50.84 & 25.26 \\
$11 / 2 / 2006$ & $4: 00$ & 40.771 & 50.84 & 25.257 \\
$11 / 2 / 2006$ & $8: 00$ & 40.811 & 50.894 & 25.304 \\
$11 / 2 / 2006$ & $12: 00$ & 40.797 & 50.837 & 25.239 \\
$11 / 2 / 2006$ & $16: 00$ & 40.743 & 50.793 & 25.21 \\
$11 / 2 / 2006$ & $20: 00$ & 40.722 & 50.786 & 25.203 \\
$11 / 3 / 2006$ & $0: 00$ & 40.706 & 50.776 & 25.192 \\
$11 / 3 / 2006$ & $4: 00$ & 40.668 & 50.739 & 25.166 \\
$11 / 3 / 2006$ & $8: 00$ & 40.643 & 50.73 & 25.154 \\
$11 / 3 / 2006$ & $12: 00$ & 40.559 & 50.633 & 25.072 \\
$11 / 3 / 2006$ & $16: 00$ & 40.491 & 50.601 & 25.056 \\
$11 / 3 / 2006$ & $20: 00$ & 40.484 & 50.619 & 25.075 \\
$11 / 4 / 2006$ & $0: 00$ & 40.48 & 50.626 & 25.082 \\
$11 / 4 / 2006$ & $4: 00$ & 40.466 & 50.622 & 25.084 \\
$11 / 4 / 2006$ & $8: 00$ & 40.501 & 50.664 & 25.117 \\
$11 / 4 / 2006$ & $12: 00$ & 40.505 & 50.652 & 25.103 \\
$11 / 4 / 2006$ & $16: 00$ & 40.517 & 50.68 & 25.133 \\
$11 / 4 / 2006$ & $20: 00$ & 40.559 & 50.711 & 25.157 \\
$11 / 5 / 2006$ & $0: 00$ & 40.575 & 50.713 & 25.157 \\
$11 / 5 / 2006$ & $4: 00$ & 40.577 & 50.708 & 25.152 \\
$11 / 5 / 2006$ & $8: 00$ & 40.598 & 50.737 & 25.175 \\
$11 / 5 / 2006$ & $12: 00$ & 40.636 & 50.694 & 25.112 \\
$11 / 7 / 7 / 2006$ & $16: 00$ & 40.379 & 50.526 & 25.012 \\
$11 / 2006$ & $20: 00$ & 40.377 & 50.535 & 25.023 \\
$11 / 5 / 2006$ & $16: 00$ & 40.526 & 50.633 & 25.084 \\
$11 / 6 / 2006$ & $20: 00$ & 40.526 & 50.662 & 25.11 \\
$11 / 6 / 2006$ & $4: 00$ & 40.536 & 50.671 & 25.122 \\
$11 / 6 / 2006$ & $8: 00$ & 40.526 & 50.657 & 25.108 \\
$11 / 6 / 2006$ & $12: 00$ & 40.526 & 50.652 & 25.094 \\
$11 / 6 / 2006$ & $16: 00$ & 40.505 & 50.638 & 25.096 \\
$11 / 6 / 2006$ & $20: 00$ & 40.51 & 50.659 & 25.117 \\
$11 / 7 / 2006$ & $0: 00$ & 40.517 & 50.645 & 25.1 \\
$11 / 7 / 2006$ & $4: 00$ & 40.466 & 50.582 & 25.042 \\
$117 / 2006$ & $8: 00$ & 40.47 & 50.612 & 25.075 \\
$11 / 2006$ & $12: 00$ & 40.41 & 50.528 & 25 \\
11354 & 50.514 & 25.005
\end{tabular}

TABLE S1.1 (Cont.)

\begin{tabular}{ccccc}
\hline & & & & \\
& Water Level (ft below top of \\
& casing) at & Indicated Well \\
\cline { 2 - 5 } Date & Time & D2 & WP49 & SB65D \\
& & & & \\
\hline & & & & \\
$11 / 8 / 2006$ & $4: 00$ & 40.328 & 50.488 & 24.988 \\
$11 / 8 / 2006$ & $8: 00$ & 40.316 & 50.495 & 24.995 \\
$11 / 8 / 2006$ & $12: 00$ & 40.272 & 50.429 & 24.953 \\
$11 / 8 / 2006$ & $16: 00$ & 40.256 & 50.451 & 24.972 \\
$11 / 8 / 2006$ & $20: 00$ & 40.34 & 50.556 & 25.061 \\
$11 / 9 / 2006$ & $0: 00$ & 40.442 & 50.652 & 25.14 \\
$11 / 9 / 2006$ & $4: 00$ & 40.529 & 50.701 & 25.166 \\
$11 / 9 / 2006$ & $8: 00$ & 40.587 & 50.739 & 25.194 \\
$11 / 9 / 2006$ & $12: 00$ & 40.626 & 50.715 & 25.159 \\
$11 / 9 / 2006$ & $16: 00$ & 40.605 & 50.699 & 25.145 \\
$11 / 9 / 2006$ & $20: 00$ & 40.619 & 50.72 & 25.164 \\
$11 / 10 / 2006$ & $0: 00$ & 40.652 & 50.76 & 25.203 \\
$11 / 10 / 2006$ & $4: 00$ & 40.692 & 50.786 & 25.215 \\
$11 / 10 / 2006$ & $8: 00$ & 40.738 & 50.861 & 25.281 \\
$11 / 10 / 2006$ & $12: 00$ & 40.808 & 50.884 & 25.285 \\
$11 / 10 / 2006$ & $16: 00$ & 40.883 & 50.962 & 25.349 \\
$11 / 10 / 2006$ & $20: 00$ & 40.937 & 50.987 & 25.36 \\
$11 / 11 / 2006$ & $0: 00$ & 40.944 & 50.969 & 25.341 \\
$11 / 11 / 2006$ & $4: 00$ & 40.941 & 50.955 & 25.327 \\
$11 / 11 / 2006$ & $8: 00$ & 40.923 & 50.929 & 25.304 \\
$11 / 11 / 2006$ & $12: 00$ & 40.857 & 50.856 & 25.241 \\
$11 / 11 / 2006$ & $16: 00$ & 40.755 & 50.76 & 25.164 \\
$11 / 11 / 2006$ & $20: 00$ & 40.687 & 50.734 & 25.145 \\
$11 / 12 / 2006$ & $0: 00$ & 40.661 & 50.711 & 25.129 \\
$11 / 14 / 14 / 2006$ & $16: 00$ & 40.438 & 50.617 & 25.082 \\
$11 / 15 / 2006$ & $20: 00$ & 40.51 & 50.685 & 25.136 \\
$11 / 12 / 2006$ & $4: 00$ & 40.584 & 50.647 & 25.082 \\
$11 / 12 / 2006$ & $12: 00$ & 40.568 & 50.673 & 25.11 \\
$11 / 14 / 2006$ & 40.554 & 50.657 & 25.091 \\
$11 / 1200$ & 40.594 & 50.758 & 25.189
\end{tabular}


TABLE S1.1 (Cont.)

\begin{tabular}{|c|c|c|c|c|}
\hline \multirow[b]{2}{*}{ Date } & \multicolumn{4}{|c|}{$\begin{array}{l}\text { Water Level (ft below top of } \\
\text { casing) at Indicated Well }\end{array}$} \\
\hline & Time & D2 & WP49 & SB65D \\
\hline $11 / 15 / 2006$ & $4: 00$ & 40.638 & 50.788 & 25.215 \\
\hline $11 / 15 / 2006$ & $8: 00$ & 40.822 & 50.91 & 25.292 \\
\hline $11 / 15 / 2006$ & $12: 00$ & 40.808 & 50.889 & 25.283 \\
\hline $11 / 15 / 2006$ & $16: 00$ & 40.806 & 50.882 & 25.271 \\
\hline $11 / 15 / 2006$ & $20: 00$ & 40.832 & 50.903 & 25.29 \\
\hline $11 / 16 / 2006$ & $0: 00$ & 40.815 & 50.873 & 25.264 \\
\hline $11 / 16 / 2006$ & 4:00 & 40.766 & 50.819 & 25.213 \\
\hline $11 / 16 / 2006$ & $8: 00$ & 40.764 & 50.844 & 25.234 \\
\hline $11 / 16 / 2006$ & $12: 00$ & 40.717 & 50.772 & 25.164 \\
\hline $11 / 16 / 2006$ & $16: 00$ & 40.643 & 50.722 & 25.131 \\
\hline $11 / 16 / 2006$ & $20: 00$ & 40.629 & 50.715 & 25.131 \\
\hline $11 / 17 / 2006$ & $0: 00$ & 40.608 & 50.711 & 25.124 \\
\hline $11 / 17 / 2006$ & $4: 00$ & 40.626 & 50.765 & 25.185 \\
\hline $11 / 17 / 2006$ & $8: 00$ & 40.722 & 50.868 & 25.267 \\
\hline $11 / 17 / 2006$ & $12: 00$ & 40.797 & 50.917 & 25.304 \\
\hline $11 / 17 / 2006$ & $16: 00$ & 40.869 & 50.976 & 25.353 \\
\hline $11 / 17 / 2006$ & $20: 00$ & 40.937 & 51.023 & 25.386 \\
\hline $11 / 18 / 2006$ & $0: 00$ & 40.965 & 51.013 & 25.379 \\
\hline $11 / 18 / 2006$ & $4: 00$ & 40.979 & 51.023 & 25.384 \\
\hline $11 / 18 / 2006$ & 8:00 & 40.997 & 51.032 & 25.398 \\
\hline $11 / 18 / 2006$ & $12: 00$ & 41.011 & 51.004 & 25.358 \\
\hline $11 / 18 / 2006$ & $16: 00$ & 40.981 & 50.999 & 25.358 \\
\hline $11 / 18 / 2006$ & $20: 00$ & 41.011 & 51.039 & 25.398 \\
\hline $11 / 19 / 2006$ & $0: 00$ & 41.009 & 51.032 & 25.393 \\
\hline $11 / 19 / 2006$ & $4: 00$ & 41.014 & 51.032 & 25.384 \\
\hline $11 / 19 / 2006$ & $8: 00$ & 41.018 & 51.041 & 25.393 \\
\hline $11 / 19 / 2006$ & $12: 00$ & 40.981 & 50.978 & 25.332 \\
\hline $11 / 19 / 2006$ & $16: 00$ & 40.965 & 51.011 & 25.377 \\
\hline $11 / 19 / 2006$ & $20: 00$ & 41.011 & 51.065 & 25.419 \\
\hline $11 / 20 / 2006$ & $0: 00$ & 41.023 & 51.053 & 25.407 \\
\hline $11 / 20 / 2006$ & 4:00 & 40.99 & 51.011 & 25.372 \\
\hline $11 / 20 / 2006$ & 8:00 & 40.976 & 50.999 & 25.353 \\
\hline $11 / 20 / 2006$ & $12: 00$ & 40.925 & 50.931 & 25.292 \\
\hline $11 / 20 / 2006$ & $16: 00$ & 40.911 & 50.875 & 25.243 \\
\hline $11 / 20 / 2006$ & $20: 00$ & 40.79 & 50.842 & 25.229 \\
\hline $11 / 21 / 2006$ & 0:00 & 40.748 & 50.812 & 25.203 \\
\hline $11 / 21 / 2006$ & $4: 00$ & 40.703 & 50.798 & 25.199 \\
\hline $11 / 21 / 2006$ & $8: 00$ & 40.71 & 50.828 & 25.225 \\
\hline $11 / 21 / 2006$ & $12: 00$ & 40.706 & 50.814 & 25.213 \\
\hline $11 / 21 / 2006$ & $16: 00$ & 40.703 & 50.821 & 25.217 \\
\hline $11 / 21 / 2006$ & $20: 00$ & 40.738 & 50.861 & 25.255 \\
\hline $11 / 22 / 2006$ & $0: 00$ & 40.736 & 50.835 & 25.23 \\
\hline
\end{tabular}

TABLE S1.1 (Cont.)

\begin{tabular}{|c|c|c|c|c|}
\hline \multirow[b]{2}{*}{ Date } & \multicolumn{4}{|c|}{$\begin{array}{l}\text { Water Level (ft below top of } \\
\text { casing) at Indicated Well }\end{array}$} \\
\hline & Time & D2 & WP49 & SB65D \\
\hline 11/22/2006 & 4:00 & 40.729 & 50.84 & 25.239 \\
\hline $11 / 22 / 2006$ & 8:00 & 40.757 & 50.882 & 25.274 \\
\hline 11/22/2006 & $12: 00$ & 40.766 & 50.861 & 25.25 \\
\hline $11 / 22 / 2006$ & $16: 00$ & 40.762 & 50.868 & 25.257 \\
\hline 11/22/2006 & 20:00 & 40.797 & 50.908 & 25.306 \\
\hline 11/23/2006 & 0:00 & 40.818 & 50.905 & 25.292 \\
\hline $11 / 23 / 2006$ & 4:00 & 40.801 & 50.88 & 25.26 \\
\hline $11 / 23 / 2006$ & 8:00 & 40.797 & 50.884 & 25.267 \\
\hline $11 / 23 / 2006$ & $12: 00$ & 40.766 & 50.826 & 25.213 \\
\hline $11 / 23 / 2006$ & $16: 00$ & 40.699 & 50.783 & 25.189 \\
\hline 11/23/2006 & $20: 00$ & 40.68 & 50.783 & 25.189 \\
\hline $11 / 24 / 2006$ & 0:00 & 40.699 & 50.814 & 25.21 \\
\hline $11 / 24 / 2006$ & 4:00 & 40.752 & 50.891 & 25.285 \\
\hline $11 / 24 / 2006$ & 8:00 & 40.867 & 50.971 & 25.344 \\
\hline $11 / 24 / 2006$ & $12: 00$ & 40.864 & 50.934 & 25.299 \\
\hline $11 / 24 / 2006$ & $16: 00$ & 40. & 50. & 25.295 \\
\hline $11 / 24 / 2006$ & $20: 00$ & 40.853 & 50.926 & 25.297 \\
\hline $11 / 25 / 2006$ & 0:00 & 40.834 & 50.903 & 25.278 \\
\hline $11 / 25 / 2006$ & $4: 00$ & 40.834 & 50.896 & 25.278 \\
\hline $11 / 25 / 2006$ & 8:00 & 40.836 & 50.924 & 25.297 \\
\hline $11 / 25 / 2006$ & $12: 00$ & 40.815 & 50.882 & 25.26 \\
\hline $11 / 25 / 2006$ & $16: 00$ & 40.822 & 50.901 & 25.26 \\
\hline $11 / 25 / 2006$ & $20: 00$ & 40.82 & 50.896 & 25.267 \\
\hline $11 / 26 / 2006$ & $0: 00$ & 40.808 & 50.884 & 25.26 \\
\hline $11 / 26 / 2006$ & 4:00 & 40.799 & 50.882 & 25.26 \\
\hline $11 / 26 / 2006$ & 8:00 & 40.832 & 50.929 & 25.299 \\
\hline 11/26/2006 & $12: 00$ & 40.825 & 50.894 & 25.262 \\
\hline $11 / 26 / 2006$ & $16: 00$ & 40.841 & 50.938 & 25.313 \\
\hline $11 / 26 / 2006$ & $20: 00$ & 40.897 & 50.98 & 25.351 \\
\hline $11 / 27 / 2006$ & 0:00 & 40.932 & 51.009 & 25.365 \\
\hline $11 / 27 / 2006$ & 4:00 & 40.937 & 50.997 & 25.356 \\
\hline $11 / 27 / 2006$ & 8:00 & 40.939 & 50.992 & 25.351 \\
\hline 11/27/2006 & $12: 00$ & 40.885 & 50.91 & 25.271 \\
\hline 11/27/2006 & $16: 00$ & 40.822 & 50.875 & 25.239 \\
\hline 11/27/2006 & $20: 00$ & 40.773 & 50.828 & 25.21 \\
\hline 11/28/2006 & $0: 00$ & 40.696 & 50.76 & 25.154 \\
\hline 11/28/2006 & $4: 00$ & 40.612 & 50.69 & 25.103 \\
\hline $11 / 28 / 2006$ & 8:00 & 40.584 & 50.711 & 25.124 \\
\hline $11 / 28 / 2006$ & $12: 00$ & 40.594 & 50.727 & 25.145 \\
\hline $11 / 28 / 2006$ & $16: 00$ & 40.736 & 50.91 & 25.283 \\
\hline $11 / 28 / 2$ & 20:00 & 40.848 & 50.987 & 25.353 \\
\hline $11 / 29 / 20$ & & 40.913 & 51.02 & 25.381 \\
\hline
\end{tabular}


TABLE S1.1 (Cont.)

\begin{tabular}{|c|c|c|c|c|}
\hline \multirow[b]{2}{*}{ Date } & \multicolumn{4}{|c|}{$\begin{array}{l}\text { Water Level (ft below top of } \\
\text { casing) at Indicated Well }\end{array}$} \\
\hline & Time & D2 & WP49 & SB65D \\
\hline $11 / 29 / 2006$ & 4:00 & 40.983 & 51.048 & 25.395 \\
\hline $11 / 29 / 2006$ & $8: 00$ & 41.044 & 51.128 & 25.456 \\
\hline $11 / 29 / 2006$ & $12: 00$ & 41.072 & 51.086 & 25.419 \\
\hline $11 / 29 / 2006$ & $16: 00$ & 41.223 & 51.152 & 25.465 \\
\hline $11 / 29 / 2006$ & $20: 00$ & 41.165 & 51.163 & 25.473 \\
\hline $11 / 30 / 2006$ & $0: 00$ & 41.16 & 51.137 & 25.451 \\
\hline $11 / 30 / 2006$ & 4:00 & 41.158 & 51.137 & 25.463 \\
\hline $11 / 30 / 2006$ & $8: 00$ & 41.16 & 51.128 & 25.447 \\
\hline $11 / 30 / 2006$ & $12: 00$ & 41.098 & 51.053 & 25.374 \\
\hline $11 / 30 / 2006$ & $16: 00$ & 40.983 & 50.966 & 25.304 \\
\hline $11 / 30 / 2006$ & $20: 00$ & 40.916 & 50.945 & 25.292 \\
\hline $12 / 1 / 2006$ & $0: 00$ & 40.878 & 50.917 & 25.269 \\
\hline $12 / 1 / 2006$ & $4: 00$ & 40.862 & 50.938 & 25.292 \\
\hline $12 / 1 / 2006$ & $8: 00$ & 40.892 & 50.976 & 25.33 \\
\hline $12 / 1 / 2006$ & $12: 00$ & 40.916 & 50.99 & 25.33 \\
\hline $12 / 1 / 2006$ & $16: 00$ & 40.941 & 51.03 & 25.37 \\
\hline $12 / 1 / 2006$ & $20: 00$ & 40.995 & 51.074 & 25.405 \\
\hline 12/2/2006 & $0: 00$ & 41.025 & 51.091 & 25.421 \\
\hline 12/2/2006 & $4: 00$ & 41.091 & 51.168 & 25.484 \\
\hline $12 / 2 / 2006$ & 8:00 & 41.244 & 51.273 & 25.561 \\
\hline $12 / 2 / 2006$ & $12: 00$ & 41.235 & 51.22 & 25.508 \\
\hline 12/2/2006 & $16: 00$ & 41.226 & 51.222 & 25.524 \\
\hline $12 / 2 / 2006$ & $20: 00$ & 41.261 & 51.25 & 25.552 \\
\hline $12 / 3 / 2006$ & $0: 00$ & 41.268 & 51.243 & 25.543 \\
\hline 12/3/2006 & $4: 00$ & 41.254 & 51.231 & 25.533 \\
\hline 12/3/2006 & $8: 00$ & 41.272 & 51.257 & 25.557 \\
\hline $12 / 3 / 2006$ & $12: 00$ & 41.235 & 51.187 & 25.489 \\
\hline $12 / 3 / 2006$ & $16: 00$ & 41.207 & 51.126 & 25.435 \\
\hline 12/3/2006 & $20: 00$ & 41.074 & 51.034 & 25.36 \\
\hline $12 / 4 / 2006$ & $0: 00$ & 40.958 & 50.934 & 25.278 \\
\hline $12 / 4 / 2006$ & 4:00 & 40.883 & 50.919 & 25.278 \\
\hline $12 / 4 / 2006$ & $8: 00$ & 40.895 & 50.98 & 25.332 \\
\hline $12 / 4 / 2006$ & $12: 00$ & 40.925 & 51.004 & 25.346 \\
\hline $12 / 4 / 2006$ & $16: 00$ & 40.918 & 51.004 & 25.353 \\
\hline $12 / 4 / 2006$ & $20: 00$ & 40.944 & 51.025 & 25.37 \\
\hline $12 / 5 / 2006$ & $0: 00$ & 40.918 & 50.966 & 25.318 \\
\hline $12 / 5 / 2006$ & $4: 00$ & 40.846 & 50.896 & 25.26 \\
\hline $12 / 5 / 2006$ & $8: 00$ & 40.771 & 50.847 & 25.229 \\
\hline $12 / 5 / 2006$ & $12: 00$ & 40.703 & 50.779 & 25.168 \\
\hline $12 / 5 / 2006$ & $16: 00$ & 40.75 & 50.83 & 25.203 \\
\hline $12 / 5 / 2006$ & $20: 00$ & 40.778 & 50.919 & 25.288 \\
\hline $12 / 6 / 2006$ & 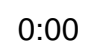 & 40.848 & 978 & 25.337 \\
\hline
\end{tabular}

TABLE S1.1 (Cont.)

\begin{tabular}{ccccc}
\hline & & & & \\
& & Water Level (ft below top of \\
& casing) at & Indicated Well \\
\cline { 2 - 5 } Date & Time & D2 & WP49 & SB65D \\
& & & & \\
\hline & & & & \\
$12 / 6 / 2006$ & $4: 00$ & 40.892 & 51.006 & 25.358 \\
$12 / 6 / 2006$ & $8: 00$ & 40.946 & 51.051 & 25.393 \\
$12 / 6 / 2006$ & $12: 00$ & 40.965 & 51.032 & 25.377 \\
$12 / 6 / 2006$ & $16: 00$ & 40.972 & 51.065 & 25.412 \\
$12 / 6 / 2006$ & $20: 00$ & 41.14 & 51.262 & 25.566 \\
$12 / 7 / 2006$ & $0: 00$ & 41.296 & 51.353 & 25.627 \\
$12 / 7 / 2006$ & $4: 00$ & 41.37 & 51.379 & 25.641 \\
$12 / 7 / 2006$ & $8: 00$ & 41.412 & 51.386 & 25.65 \\
$12 / 7 / 2006$ & $12: 00$ & 41.387 & 51.304 & 25.575 \\
$12 / 7 / 2006$ & $16: 00$ & 41.289 & 51.21 & 25.496 \\
$12 / 7 / 2006$ & $20: 00$ & 41.221 & 51.145 & 25.449 \\
$12 / 8 / 2006$ & $0: 00$ & 41.126 & 51.074 & 25.393 \\
$12 / 8 / 2006$ & $4: 00$ & 41.025 & 51.006 & 25.337 \\
$12 / 8 / 2006$ & $8: 00$ & 40.972 & 50.99 & 25.332 \\
$12 / 8 / 2006$ & $12: 00$ & 40.904 & 50.931 & 25.292 \\
$12 / 8 / 2006$ & $16: 00$ & 40.848 & 50.912 & 25.288 \\
$12 / 8 / 2006$ & $20: 00$ & 40.834 & 50.915 & 25.288 \\
$12 / 9 / 2006$ & $0: 00$ & 40.804 & 50.884 & 25.264 \\
$12 / 9 / 2006$ & $4: 00$ & 40.778 & 50.873 & 25.255 \\
$12 / 9 / 2006$ & $8: 00$ & 40.785 & 50.903 & 25.278 \\
$12 / 9 / 2006$ & $12: 00$ & 40.815 & 50.88 & 25.248 \\
$12 / 9 / 2006$ & $16: 00$ & 40.759 & 50.861 & 25.241 \\
$12 / 9 / 2006$ & $20: 00$ & 40.79 & 50.912 & 25.292 \\
$12 / 10 / 2006$ & $0: 00$ & 40.801 & 50.894 & 25.271 \\
$12 / 12 / 2006$ & $16: 00$ & 40.972 & 51.023 & 25.37 \\
$12 / 13 / 2006$ & $20: 00$ & 40.976 & 51.027 & 25.37 \\
$12 / 10 / 2006$ & $4: 00$ & 40.792 & 50.891 & 25.271 \\
$12 / 10 / 2006$ & $8: 00$ & 40.815 & 50.922 & 25.297 \\
$12 / 12 / 12006$ & 40.972 & 51.02 & 25.377
\end{tabular}


TABLE S1.1 (Cont.)

\begin{tabular}{|c|c|c|c|c|}
\hline \multirow[b]{2}{*}{ Date } & \multicolumn{4}{|c|}{$\begin{array}{l}\text { Water Level (ft below top of } \\
\text { casing) at Indicated Well }\end{array}$} \\
\hline & Time & D2 & WP49 & SB65D \\
\hline $12 / 13 / 2006$ & 4:00 & 40.995 & 51.051 & 25.388 \\
\hline $12 / 13 / 2006$ & $8: 00$ & 41.025 & 51.081 & 25.412 \\
\hline $12 / 13 / 2006$ & $12: 00$ & 41.009 & 51.016 & 25.341 \\
\hline $12 / 13 / 2006$ & $16: 00$ & 41.004 & 50.969 & 25.302 \\
\hline $12 / 13 / 2006$ & $20: 00$ & 40.911 & 50.941 & 25.285 \\
\hline $12 / 14 / 2006$ & $0: 00$ & 40.855 & 50.884 & 25.243 \\
\hline $12 / 14 / 2006$ & 4:00 & 40.815 & 50.894 & 25.262 \\
\hline $12 / 14 / 2006$ & $8: 00$ & 40.874 & 50.978 & 25.339 \\
\hline $12 / 14 / 2006$ & $12: 00$ & 40.911 & 50.978 & 25.332 \\
\hline $12 / 14 / 2006$ & $16: 00$ & 40.906 & 50.98 & 25.337 \\
\hline $12 / 14 / 2006$ & $20: 00$ & 40.983 & 51.058 & 25.4 \\
\hline $12 / 15 / 2006$ & $0: 00$ & 41.023 & 51.088 & 25.416 \\
\hline $12 / 15 / 2006$ & $4: 00$ & 41.039 & 51.081 & 25.407 \\
\hline $12 / 15 / 2006$ & $8: 00$ & 41. & 51.084 & 25.409 \\
\hline $12 / 15 / 2006$ & $12: 00$ & 41.016 & 51.018 & 25.353 \\
\hline $12 / 15 / 2006$ & $16: 00$ & 40.946 & 50.962 & 25.311 \\
\hline $12 / 15 / 2006$ & $20: 00$ & 40.918 & 50.936 & 25.29 \\
\hline $12 / 16 / 2006$ & $0: 00$ & 40.869 & 50.91 & 25.269 \\
\hline $12 / 16 / 2006$ & $4: 00$ & 40.834 & 50.896 & 25.26 \\
\hline $12 / 16 / 2006$ & 8:00 & 40.876 & & 25.337 \\
\hline $12 / 16 / 2006$ & $12: 00$ & & 51.009 & 25.348 \\
\hline $12 / 16 / 2006$ & $16: 00$ & 41.025 & 51.128 & 25.444 \\
\hline $12 / 16 / 2006$ & $20: 00$ & 41.142 & 51.217 & 25.519 \\
\hline $12 / 17 / 2006$ & $0: 00$ & 41.209 & 51.238 & 25.533 \\
\hline $12 / 17 / 2006$ & $4: 00$ & 41.249 & 51.257 & 25.54 \\
\hline $12 / 17 / 2006$ & $8: 00$ & 41.296 & 51.302 & 25.582 \\
\hline $12 / 17 / 2006$ & $12: 00$ & 41.356 & 51.283 & 25.538 \\
\hline $12 / 17 / 2006$ & $16: 00$ & 41.305 & 51.278 & 25.552 \\
\hline $12 / 17 / 2006$ & $20: 00$ & 41.338 & 51.323 & 25.599 \\
\hline $12 / 18 / 2006$ & $0: 00$ & 41.352 & 51.318 & 25.599 \\
\hline $12 / 18 / 2006$ & 4:00 & & 51.337 & 25.62 \\
\hline $12 / 18 / 2006$ & 8:00 & 41.401 & 51.37 & 25.648 \\
\hline $12 / 18 / 2006$ & $12: 00$ & 41.403 & 51.332 & 25.613 \\
\hline $12 / 18 / 2006$ & $16: 00$ & 41.356 & 51.292 & 25.587 \\
\hline $12 / 18 / 2006$ & $20: 00$ & 41.359 & 51.302 & 25.589 \\
\hline $12 / 19 / 2006$ & 0:00 & 41.342 & 51.288 & 25.578 \\
\hline $12 / 19 / 2006$ & $4: 00$ & 41.305 & 51.259 & 25.545 \\
\hline $12 / 19 / 2006$ & $8: 00$ & 41.284 & 51.245 & 25.517 \\
\hline $12 / 19 / 2006$ & $12: 00$ & 41.249 & 51.194 & 25.479 \\
\hline $12 / 19 / 2006$ & $16: 00$ & 41.258 & 51.177 & 25.468 \\
\hline $12 / 19 / 2006$ & $20: 00$ & 41.156 & 51.128 & 25.433 \\
\hline $12 / 20 / 2006$ & $0: 00$ & 41.084 & .067 & 25.386 \\
\hline
\end{tabular}

TABLE S1.1 (Cont.)

\begin{tabular}{ccccc}
\hline & & & & \\
& & Water Level (ft below top of \\
& casing) at & Indicated Well \\
\cline { 2 - 5 } Date & Time & D2 & WP49 & SB65D \\
& & & & \\
\hline & & & & \\
$12 / 20 / 2006$ & $4: 00$ & 41.016 & 51.03 & 25.358 \\
$12 / 20 / 2006$ & $8: 00$ & 40.972 & 51.009 & 25.327 \\
$12 / 20 / 2006$ & $12: 00$ & 40.925 & 50.957 & 25.288 \\
$12 / 20 / 2006$ & $16: 00$ & 40.895 & 50.978 & 25.316 \\
$12 / 20 / 2006$ & $20: 00$ & 40.913 & 50.987 & 25.297 \\
$12 / 21 / 2006$ & $0: 00$ & 40.911 & 50.997 & 25.327 \\
$12 / 21 / 2006$ & $4: 00$ & 40.927 & 51.018 & 25.365 \\
$12 / 21 / 2006$ & $8: 00$ & 40.967 & 51.062 & 25.416 \\
$12 / 21 / 2006$ & $12: 00$ & 40.965 & 51.018 & 25.377 \\
$12 / 21 / 2006$ & $16: 00$ & 40.953 & 51.025 & 25.386 \\
$12 / 21 / 2006$ & $20: 00$ & 40.965 & 51.03 & 25.407 \\
$12 / 22 / 2006$ & $0: 00$ & 40.953 & 51.006 & 25.386 \\
$12 / 22 / 2006$ & $4: 00$ & 40.969 & 51.044 & 25.388 \\
$12 / 22 / 2006$ & $8: 00$ & 41.009 & 51.081 & 25.421 \\
$12 / 22 / 2006$ & $12: 00$ & 41.051 & 51.109 & 25.444 \\
$12 / 22 / 2006$ & $16: 00$ & 41.098 & 51.159 & 25.479 \\
$12 / 22 / 2006$ & $20: 00$ & 41.142 & 51.177 & 25.517 \\
$12 / 23 / 2006$ & $0: 00$ & 41.156 & 51.182 & 25.519 \\
$12 / 23 / 2006$ & $4: 00$ & 41.193 & 51.229 & 25.566 \\
$12 / 23 / 2006$ & $8: 00$ & 41.247 & 51.278 & 25.589 \\
$12 / 23 / 2006$ & $12: 00$ & 41.258 & 51.252 & 25.552 \\
$12 / 23 / 2006$ & $16: 00$ & 41.256 & 51.262 & 25.564 \\
$12 / 26 / 2006$ & $16: 00$ & 41.272 & 51.182 & 25.519 \\
$12 / 2006$ & $20: 00$ & 41.16 & 51.173 & 25.524 \\
$12 / 24 / 2006$ & $20: 00$ & 41.254 & 51.243 & 25.536 \\
$12 / 24 / 2006$ & $4: 00$ & 41.242 & 51.234 & 25.564 \\
$12 / 2606$ & $0: 00$ & 41.121 & 51.13 & 25.491
\end{tabular}


TABLE S1.1 (Cont.)

\begin{tabular}{|c|c|c|c|c|}
\hline \multirow[b]{2}{*}{ Date } & \multicolumn{4}{|c|}{$\begin{array}{l}\text { Water Level (ft below top of } \\
\text { casing) at Indicated Well }\end{array}$} \\
\hline & Time & D2 & WP49 & SB65D \\
\hline $12 / 27 / 2006$ & $4: 00$ & 41.063 & 51.084 & 25.456 \\
\hline $12 / 27 / 2006$ & $8: 00$ & 41.035 & 51.074 & 25.442 \\
\hline $12 / 27 / 2006$ & $12: 00$ & 40.997 & 51.025 & 25.395 \\
\hline $12 / 27 / 2006$ & $16: 00$ & 40.96 & 51.023 & 25.4 \\
\hline 12/27/2006 & $20: 00$ & 41.004 & 51.093 & 25.463 \\
\hline $12 / 28 / 2006$ & $0: 00$ & 41.037 & 51.107 & 25.465 \\
\hline $12 / 28 / 2006$ & 4:00 & 41.072 & 51.142 & 25.493 \\
\hline $12 / 28 / 2006$ & 8:00 & 41.151 & 51.231 & 25.568 \\
\hline $12 / 28 / 2006$ & $12: 00$ & 41.216 & 51.271 & 25.601 \\
\hline $12 / 28 / 2006$ & $16: 00$ & 41.34 & 51.356 & 25.648 \\
\hline $12 / 28 / 2006$ & $20: 00$ & 41.363 & 51.365 & 25.671 \\
\hline 12/29/2006 & $0: 00$ & 41.391 & 51.372 & 25.681 \\
\hline $12 / 29 / 2006$ & 4:00 & 41.396 & 51.358 & 25.674 \\
\hline $12 / 29 / 2006$ & 8:00 & 41.408 & 51.372 & 25.685 \\
\hline $12 / 29 / 2006$ & $12: 00$ & 41.382 & 51.32 & 25.634 \\
\hline 12/29/2006 & $16: 00$ & 41.352 & 51.311 & 25.629 \\
\hline 12/29/2006 & $20: 00$ & 41.342 & 51.306 & 25.622 \\
\hline $12 / 30 / 2006$ & $0: 00$ & 41.286 & 51.245 & 25.571 \\
\hline $12 / 30 / 2006$ & 4:00 & 41.254 & 51.234 & 25.566 \\
\hline $12 / 30 / 2006$ & 8:00 & 41.223 & 51.227 & 25.554 \\
\hline $12 / 30 / 2006$ & $12: 00$ & 41.163 & 51.14 & 25.472 \\
\hline 12/30/2006 & $16: 00$ & 41.112 & 51.133 & 25.475 \\
\hline $12 / 30 / 2006$ & $20: 00$ & 41.079 & 51.121 & 25.461 \\
\hline $12 / 31 / 2006$ & $0: 00$ & 41.046 & 51.095 & 25.409 \\
\hline 12/31/2006 & 4:00 & 41.009 & 51.086 & 25.372 \\
\hline $12 / 31 / 2006$ & 8:00 & 40.965 & 51.072 & 25.327 \\
\hline 12/31/2006 & $12: 00$ & 40.979 & 51.1 & 25.316 \\
\hline $12 / 31 / 2006$ & $16: 00$ & 41.021 & 51.159 & 25.348 \\
\hline $12 / 31 / 2006$ & $20: 00$ & 41.105 & 51.229 & 25.379 \\
\hline
\end{tabular}


TABLE S1.2 Automatically measured water levels in August 2005 through December 2006 in the phytoremediation area wells at Murdock.

Water Level (ft below top of casing) at Indicated Well

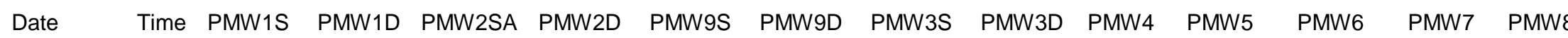

\begin{tabular}{|c|c|c|c|c|c|c|c|c|c|c|c|c|c|c|}
\hline $8 / 4 / 2005$ & $12: 00$ & & & & & & & & 2.379 & 10.79 & & & & \\
\hline 8/4/2005 & $13: 00$ & & & & & & & 2.414 & 2.393 & 10.794 & & & & \\
\hline 8/4/2005 & $14: 00$ & & & & & 4.706 & 2.991 & 2.42 & 2.399 & 10.798 & & & & \\
\hline 8/4/2005 & $15: 00$ & & & 7.804 & 6.726 & 4.521 & 3.246 & 2.427 & 2.399 & 10.804 & & & & \\
\hline 8/4/2005 & $16: 00$ & 10.68 & 10.625 & 7.803 & 6.724 & 4.449 & 3.432 & 2.417 & 2.393 & 10.807 & & & & \\
\hline 8/4/2005 & $17: 00$ & 10.675 & 10.618 & 7.799 & 6.712 & 4.413 & 3.576 & 2.399 & 2.382 & 10.812 & 6.036 & & & 4.163 \\
\hline $8 / 4 / 2005$ & $18: 00$ & 10.672 & 10.617 & 7.795 & 6.715 & 4.402 & 3.691 & 2.407 & 2.38 & 10.814 & 6.041 & 5.031 & 6.841 & 4.159 \\
\hline 8/4/2005 & $19: 00$ & 10.67 & 10.614 & 7.791 & 6.708 & 4.393 & 3.782 & 2.402 & 2.376 & 10.815 & 6.036 & 5.017 & 6.821 & 4.155 \\
\hline 8/4/2005 & $20: 00$ & 10.67 & 10.614 & 7.791 & 6.71 & 4.389 & 3.857 & 2.4 & 2.373 & 10.815 & 6.037 & 5.005 & 6.807 & 4.145 \\
\hline 8/4/2005 & $21: 00$ & 10.67 & 10.616 & 7.791 & 6.71 & 4.383 & 3.917 & 2.392 & 2.373 & 10.817 & 6.035 & 4.995 & 6.796 & 4.131 \\
\hline $8 / 4 / 2005$ & $22: 00$ & 10.672 & 10.623 & 7.793 & 6.715 & 4.384 & 3.968 & 2.397 & 2.376 & 10.819 & 6.034 & 4.989 & 6.788 & 4.123 \\
\hline 8/4/2005 & $23: 00$ & 10.675 & 10.621 & 7.792 & 6.71 & 4.376 & 4.008 & 2.395 & 2.369 & 10.817 & 6.028 & 4.977 & 6.78 & 4.103 \\
\hline $8 / 5 / 2005$ & $0: 00$ & 10.675 & 10.621 & 7.79 & 6.706 & 4.37 & 4.041 & 2.39 & 2.365 & 10.815 & 6.024 & 4.97 & 6.777 & 4.087 \\
\hline $8 / 5 / 2005$ & $1: 00$ & 10.677 & 10.629 & 7.794 & 6.715 & 4.368 & 4.067 & 2.395 & 2.371 & 10.815 & 6.024 & 4.966 & 6.773 & 4.078 \\
\hline 8/5/2005 & $2: 00$ & 10.68 & 10.629 & 7.792 & 6.71 & 4.365 & 4.089 & 2.393 & 2.367 & 10.814 & 6.019 & 4.958 & 6.767 & 4.065 \\
\hline $8 / 5 / 2005$ & $3: 00$ & 10.68 & 10.628 & 7.794 & 6.712 & 4.359 & 4.107 & 2.383 & 2.365 & 10.812 & 6.015 & 4.954 & 6.766 & 4.052 \\
\hline $8 / 5 / 2005$ & $4: 00$ & 10.682 & 10.627 & 7.792 & 6.71 & 4.353 & 4.123 & 2.388 & 2.363 & 10.81 & 6.01 & 4.947 & 6.76 & 4.04 \\
\hline $8 / 5 / 2005$ & $5: 00$ & 10.682 & 10.629 & 7.792 & 6.71 & 4.35 & 4.134 & 2.385 & 2.363 & 10.808 & 6.011 & 4.945 & 6.76 & 4.03 \\
\hline 8/5/2005 & $6: 00$ & 10.685 & 10.63 & 7.794 & 6.708 & 4.348 & 4.143 & 2.385 & 2.363 & 10.804 & 6.008 & 4.941 & 6.756 & 4.022 \\
\hline 8/5/2005 & $7: 00$ & 10.685 & 10.63 & 7.792 & 6.706 & 4.342 & 4.151 & 2.383 & 2.361 & 10.8 & 6.006 & 4.937 & 6.754 & 4.012 \\
\hline 8/5/2005 & $8: 00$ & 10.688 & 10.638 & 7.794 & 6.712 & 4.346 & 4.158 & 2.388 & 2.367 & 10.8 & 6.004 & 4.939 & 6.764 & 4.01 \\
\hline $8 / 5 / 2005$ & $9: 00$ & 10.69 & 10.636 & 7.796 & 6.712 & 4.344 & 4.165 & 2.38 & 2.365 & 10.796 & 6.004 & 4.937 & 6.783 & 4.004 \\
\hline 8/5/2005 & $10: 00$ & 10.69 & 10.638 & 7.796 & 6.71 & 4.344 & 4.169 & 2.391 & 2.368 & 10.796 & 6.004 & 4.943 & 6.792 & 4.002 \\
\hline $8 / 5 / 2005$ & $11: 00$ & 10.688 & 10.629 & 7.796 & 6.706 & 4.344 & 4.171 & 2.391 & 2.368 & 10.795 & 6.004 & 4.941 & 6.802 & 4.006 \\
\hline $8 / 5 / 2005$ & $12: 00$ & 10.69 & 10.627 & 7.796 & 6.706 & 4.348 & 4.174 & 2.396 & 2.372 & 10.795 & 6.009 & 4.949 & 6.804 & 4.022 \\
\hline $8 / 5 / 2005$ & $13: 00$ & 10.685 & 10.621 & 7.796 & 6.704 & 4.35 & 4.176 & 2.398 & 2.374 & 10.795 & 6.011 & 4.951 & 6.807 & 4.034 \\
\hline 8/5/2005 & $14: 00$ & 10.68 & 10.612 & 7.792 & 6.699 & 4.35 & 4.176 & 2.388 & 2.37 & 10.796 & 6.016 & 4.961 & 6.817 & 4.049 \\
\hline $8 / 5 / 2005$ & $15: 00$ & 10.671 & 10.601 & 7.786 & 6.69 & 4.35 & 4.176 & 2.391 & 2.365 & 10.8 & 6.025 & 4.966 & 6.838 & 4.075 \\
\hline $8 / 5 / 2005$ & $16: 00$ & 10.663 & 10.59 & 7.782 & 6.681 & 4.352 & 4.174 & 2.38 & 2.361 & 10.802 & 6.029 & 4.974 & 6.846 & 4.102 \\
\hline 8/5/2005 & $17: 00$ & 10.653 & 10.579 & 7.778 & 6.675 & 4.354 & 4.174 & 2.385 & 2.357 & 10.806 & 6.033 & 4.978 & 6.825 & 4.122 \\
\hline $8 / 5 / 2005$ & $18: 00$ & 10.646 & 10.57 & 7.774 & 6.673 & 4.354 & 4.172 & 2.381 & 2.353 & 10.814 & 6.038 & 4.974 & 6.809 & 4.14 \\
\hline 8/5/2005 & $19: 00$ & 10.636 & 10.563 & 7.771 & 6.664 & 4.356 & 4.169 & 2.376 & 2.349 & 10.815 & 6.04 & 4.972 & 6.796 & 4.148 \\
\hline $8 / 5 / 2005$ & $20: 00$ & 10.634 & 10.557 & 7.767 & 6.662 & 4.356 & 4.17 & 2.371 & 2.342 & 10.818 & 6.04 & 4.965 & 6.781 & 4.144 \\
\hline 8/5/2005 & $21: 00$ & 10.629 & 10.556 & 7.765 & 6.659 & 4.354 & 4.169 & 2.358 & 2.338 & 10.82 & 6.04 & 4.955 & 6.767 & 4.132 \\
\hline
\end{tabular}


TABLE S1.2 (Cont.)

Water Level (ft below top of casing) at Indicated Well

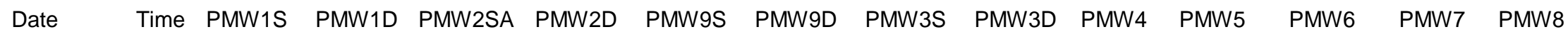

\begin{tabular}{|c|c|c|c|c|c|c|c|c|c|c|c|c|c|c|}
\hline 8/5/2005 & $22: 00$ & 10.631 & 10.561 & 7.767 & 6.666 & 4.356 & 4.171 & 2.368 & 2.34 & 10.822 & 6.036 & 4.949 & 6.76 & 4.118 \\
\hline $8 / 5 / 2005$ & $23: 00$ & 10.632 & 10.563 & 7.767 & 6.668 & 4.354 & 4.171 & 2.361 & 2.342 & 10.822 & 6.032 & 4.939 & 6.752 & 4.104 \\
\hline 8/6/2005 & $0: 00$ & 10.637 & 10.568 & 7.769 & 6.668 & 4.351 & 4.171 & 2.361 & 2.342 & 10.822 & 6.027 & 4.93 & 6.746 & 4.092 \\
\hline 8/6/2005 & $1: 00$ & 10.639 & 10.568 & 7.767 & 6.668 & 4.345 & 4.171 & 2.364 & 2.338 & 10.82 & 6.023 & 4.92 & 6.741 & 4.074 \\
\hline $8 / 6 / 2005$ & $2: 00$ & 10.634 & 10.563 & 7.763 & 6.659 & 4.335 & 4.169 & 2.349 & 2.33 & 10.816 & 6.017 & 4.908 & 6.733 & 4.054 \\
\hline 8/6/2005 & $3: 00$ & 10.629 & 10.556 & 7.761 & 6.653 & 4.326 & 4.167 & 2.349 & 2.323 & 10.812 & 6.012 & 4.9 & 6.728 & 4.04 \\
\hline $8 / 6 / 2005$ & $4: 00$ & 10.627 & 10.555 & 7.759 & 6.653 & 4.322 & 4.167 & 2.341 & 2.323 & 10.808 & 6.01 & 4.892 & 6.724 & 4.029 \\
\hline 8/6/2005 & $5: 00$ & 10.624 & 10.552 & 7.755 & 6.648 & 4.318 & 4.163 & 2.341 & 2.317 & 10.802 & 6.006 & 4.883 & 6.718 & 4.015 \\
\hline $8 / 6 / 2005$ & $6: 00$ & 10.624 & 10.55 & 7.754 & 6.65 & 4.312 & 4.16 & 2.334 & 2.315 & 10.801 & 6.001 & 4.879 & 6.714 & 4.005 \\
\hline 8/6/2005 & $7: 00$ & 10.625 & 10.55 & 7.754 & 6.648 & 4.309 & 4.158 & 2.336 & 2.313 & 10.797 & 5.999 & 4.875 & 6.711 & 3.997 \\
\hline $8 / 6 / 2005$ & $8: 00$ & 10.622 & 10.552 & 7.756 & 6.646 & 4.31 & 4.156 & 2.331 & 2.313 & 10.793 & 5.997 & 4.871 & 6.709 & 3.989 \\
\hline 8/6/2005 & $9: 00$ & 10.625 & 10.556 & 7.758 & 6.65 & 4.31 & 4.154 & 2.346 & 2.319 & 10.791 & 5.997 & 4.871 & 6.722 & 3.989 \\
\hline $8 / 6 / 2005$ & $10: 00$ & 10.625 & 10.556 & 7.758 & 6.648 & 4.312 & 4.151 & 2.349 & 2.321 & 10.789 & 5.999 & 4.881 & 6.722 & 3.995 \\
\hline 8/6/2005 & $11: 00$ & 10.622 & 10.552 & 7.758 & 6.646 & 4.317 & 4.149 & 2.349 & 2.323 & 10.787 & 6.004 & 4.891 & 6.735 & 4.014 \\
\hline 8/6/2005 & $12: 00$ & 10.618 & 10.545 & 7.756 & 6.642 & 4.319 & 4.147 & 2.344 & 2.323 & 10.789 & 6.01 & 4.905 & 6.743 & 4.036 \\
\hline $8 / 6 / 2005$ & $13: 00$ & 10.615 & 10.537 & 7.754 & 6.639 & 4.324 & 4.145 & 2.344 & 2.321 & 10.793 & 6.02 & 4.918 & 6.754 & 4.066 \\
\hline 8/6/2005 & $14: 00$ & 10.61 & 10.53 & 7.752 & 6.633 & 4.332 & 4.143 & 2.351 & 2.323 & 10.797 & 6.026 & 4.934 & 6.762 & 4.101 \\
\hline 8/6/2005 & $15: 00$ & 10.603 & 10.521 & 7.748 & 6.628 & 4.336 & 4.138 & 2.341 & 2.319 & 10.802 & 6.035 & 4.944 & 6.772 & 4.129 \\
\hline 8/6/2005 & $16: 00$ & 10.596 & 10.512 & 7.744 & 6.624 & 4.342 & 4.138 & 2.346 & 2.317 & 10.808 & 6.044 & 4.958 & 6.775 & 4.156 \\
\hline $8 / 6 / 2005$ & $17: 00$ & 10.591 & 10.506 & 7.748 & 6.619 & 4.348 & 4.136 & 2.341 & 2.315 & 10.814 & 6.049 & 4.968 & 6.777 & 4.176 \\
\hline 8/6/2005 & $18: 00$ & 10.584 & 10.501 & 7.738 & 6.618 & 4.352 & 4.136 & 2.346 & 2.313 & 10.822 & 6.05 & 4.975 & 6.775 & 4.194 \\
\hline 8/6/2005 & $19: 00$ & 10.579 & 10.495 & 7.737 & 6.613 & 4.353 & 4.134 & 2.339 & 2.309 & 10.823 & 6.053 & 4.977 & 6.77 & 4.201 \\
\hline 8/6/2005 & $20: 00$ & 10.576 & 10.494 & 7.735 & 6.613 & 4.355 & 4.134 & 2.332 & 2.309 & 10.827 & 6.055 & 4.972 & 6.758 & 4.199 \\
\hline $8 / 6 / 2005$ & $21: 00$ & 10.576 & 10.499 & 7.744 & 6.619 & 4.359 & 4.138 & 2.332 & 2.311 & 10.829 & 6.055 & 4.964 & 6.745 & 4.185 \\
\hline 8/6/2005 & $22: 00$ & 10.579 & 10.503 & 7.738 & 6.62 & 4.355 & 4.138 & 2.329 & 2.309 & 10.829 & 6.046 & 4.952 & 6.733 & 4.165 \\
\hline $8 / 6 / 2005$ & $23: 00$ & 10.579 & 10.503 & 7.737 & 6.62 & 4.349 & 4.14 & 2.324 & 2.305 & 10.829 & 6.04 & 4.94 & 6.724 & 4.144 \\
\hline 8/7/2005 & $0: 00$ & 10.577 & 10.503 & 7.735 & 6.618 & 4.34 & 4.138 & 2.327 & 2.298 & 10.827 & 6.033 & 4.926 & 6.716 & 4.124 \\
\hline 8/7/2005 & $1: 00$ & 10.577 & 10.501 & 7.735 & 6.613 & 4.334 & 4.138 & 2.322 & 2.294 & 10.825 & 6.029 & 4.915 & 6.712 & 4.104 \\
\hline 8/7/2005 & $2: 00$ & 10.577 & 10.499 & 7.733 & 6.613 & 4.325 & 4.136 & 2.317 & 2.29 & 10.822 & 6.023 & 4.905 & 6.705 & 4.088 \\
\hline 8/7/2005 & $3: 00$ & 10.574 & 10.497 & 7.731 & 6.611 & 4.317 & 4.134 & 2.315 & 2.288 & 10.818 & 6.02 & 4.895 & 6.699 & 4.072 \\
\hline 8/7/2005 & $4: 00$ & 10.572 & 10.494 & 7.729 & 6.604 & 4.309 & 4.132 & 2.307 & 2.282 & 10.812 & 6.016 & 4.886 & 6.696 & 4.057 \\
\hline 8/7/2005 & 5:00 & 10.57 & 10.49 & 7.725 & 6.6 & 4.303 & 4.127 & 2.297 & 2.276 & 10.808 & 6.012 & 4.88 & 6.692 & 4.045 \\
\hline 8/7/2005 & $6: 00$ & 10.569 & 10.492 & 7.725 & 6.602 & 4.3 & 4.125 & 2.302 & 2.278 & 10.804 & 6.009 & 4.876 & 6.692 & 4.035 \\
\hline 8/7/2005 & 7:00 & 10.57 & 10.495 & 7.727 & 6.604 & 4.298 & 4.125 & 2.307 & 2.28 & 10.801 & 6.009 & 4.88 & 6.694 & 4.029 \\
\hline
\end{tabular}


TABLE S1.2 (Cont.)

Water Level (ft below top of casing) at Indicated Well

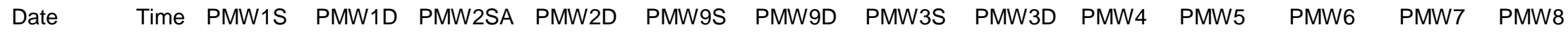

\begin{tabular}{|c|c|c|c|c|c|c|c|c|c|c|c|c|c|c|}
\hline 8/7/2005 & $8: 00$ & 10.575 & 10.499 & 7.729 & 6.606 & 4.3 & 4.125 & 2.31 & 2.284 & 10.799 & 6.007 & 4.888 & 6.699 & 4.023 \\
\hline 8/7/2005 & $9: 00$ & 10.577 & 10.503 & 7.733 & 6.611 & 4.304 & 4.123 & 2.315 & 2.288 & 10.797 & 6.007 & 4.9 & 6.705 & 4.025 \\
\hline 8/7/2005 & $10: 00$ & 10.578 & 10.505 & 7.733 & 6.613 & 4.308 & 4.12 & 2.318 & 2.292 & 10.795 & 6.01 & 4.911 & 6.718 & 4.035 \\
\hline 8/7/2005 & $11: 00$ & 10.58 & 10.506 & 7.735 & 6.613 & 4.313 & 4.12 & 2.328 & 2.299 & 10.797 & 6.016 & 4.931 & 6.73 & 4.057 \\
\hline 8/7/2005 & $12: 00$ & 10.58 & 10.505 & 7.737 & 6.611 & 4.321 & 4.12 & 2.325 & 2.301 & 10.801 & 6.025 & 4.947 & 6.745 & 4.084 \\
\hline 8/7/2005 & $13: 00$ & 10.578 & 10.503 & 7.737 & 6.613 & 4.331 & 4.12 & 2.338 & 2.307 & 10.805 & 6.032 & 4.966 & 6.758 & 4.12 \\
\hline $8 / 7 / 2005$ & $14: 00$ & 10.575 & 10.499 & 7.737 & 6.613 & 4.343 & 4.12 & 2.343 & 2.309 & 10.81 & 6.045 & 4.986 & 6.772 & 4.157 \\
\hline 8/7/2005 & $15: 00$ & 10.573 & 10.494 & 7.735 & 6.609 & 4.352 & 4.12 & 2.343 & 2.313 & 10.82 & 6.052 & 4.998 & 6.781 & 4.191 \\
\hline 8/7/2005 & $16: 00$ & 10.568 & 10.486 & 7.733 & 6.606 & 4.358 & 4.12 & 2.34 & 2.313 & 10.826 & 6.061 & 5.008 & 6.787 & 4.216 \\
\hline 8/7/2005 & $17: 00$ & 10.56 & 10.481 & 7.731 & 6.604 & 4.366 & 4.12 & 2.34 & 2.311 & 10.833 & 6.065 & 5.015 & 6.789 & 4.234 \\
\hline 8/7/2005 & $18: 00$ & 10.56 & 10.479 & 7.731 & 6.604 & 4.373 & 4.123 & 2.341 & 2.311 & 10.837 & 6.067 & 5.019 & 6.787 & 4.246 \\
\hline 8/7/2005 & $19: 00$ & 10.558 & 10.477 & 7.729 & 6.604 & 4.379 & 4.125 & 2.341 & 2.309 & 10.843 & 6.07 & 5.024 & 6.781 & 4.254 \\
\hline 8/7/2005 & $20: 00$ & 10.558 & 10.479 & 7.729 & 6.606 & 4.379 & 4.125 & 2.338 & 2.307 & 10.845 & 6.07 & 5.018 & 6.768 & 4.249 \\
\hline 8/7/2005 & $21: 00$ & 10.558 & 10.481 & 7.731 & 6.606 & 4.377 & 4.127 & 2.328 & 2.305 & 10.848 & 6.067 & 5.006 & 6.757 & 4.232 \\
\hline 8/7/2005 & $22: 00$ & 10.561 & 10.486 & 7.732 & 6.609 & 4.373 & 4.132 & 2.331 & 2.305 & 10.848 & 6.061 & 4.996 & 6.747 & 4.21 \\
\hline 8/7/2005 & $23: 00$ & 10.566 & 10.49 & 7.734 & 6.613 & 4.37 & 4.134 & 2.333 & 2.305 & 10.85 & 6.057 & 4.986 & 6.738 & 4.19 \\
\hline 8/8/2005 & $0: 00$ & 10.568 & 10.495 & 7.736 & 6.613 & 4.364 & 4.134 & 2.331 & 2.303 & 10.848 & 6.055 & 4.977 & 6.73 & 4.172 \\
\hline 8/8/2005 & $1: 00$ & 10.571 & 10.497 & 7.734 & 6.611 & 4.356 & 4.134 & 2.328 & 2.299 & 10.848 & 6.05 & 4.967 & 6.724 & 4.152 \\
\hline 8/8/2005 & $2: 00$ & 10.571 & 10.495 & 7.732 & 6.609 & 4.345 & 4.134 & 2.313 & 2.292 & 10.845 & 6.041 & 4.957 & 6.717 & 4.131 \\
\hline 8/8/2005 & $3: 00$ & 10.569 & 10.492 & 7.728 & 6.602 & 4.335 & 4.132 & 2.308 & 2.286 & 10.841 & 6.035 & 4.947 & 6.711 & 4.116 \\
\hline 8/8/2005 & $4: 00$ & 10.571 & 10.494 & 7.728 & 6.604 & 4.331 & 4.129 & 2.316 & 2.286 & 10.837 & 6.033 & 4.94 & 6.707 & 4.103 \\
\hline 8/8/2005 & $5: 00$ & 10.566 & 10.49 & 7.726 & 6.598 & 4.324 & 4.127 & 2.301 & 2.28 & 10.833 & 6.028 & 4.93 & 6.701 & 4.087 \\
\hline 8/8/2005 & $6: 00$ & 10.571 & 10.495 & 7.728 & 6.604 & 4.322 & 4.127 & 2.304 & 2.284 & 10.829 & 6.026 & 4.926 & 6.699 & 4.079 \\
\hline 8/8/2005 & $7: 00$ & 10.571 & 10.494 & 7.726 & 6.598 & 4.314 & 4.125 & 2.306 & 2.28 & 10.826 & 6.022 & 4.92 & 6.698 & 4.065 \\
\hline 8/8/2005 & $8: 00$ & 10.569 & 10.494 & 7.722 & 6.598 & 4.308 & 4.12 & 2.296 & 2.276 & 10.822 & 6.02 & 4.916 & 6.734 & 4.055 \\
\hline 8/8/2005 & $9: 00$ & 10.572 & 10.497 & 7.728 & 6.602 & 4.311 & 4.12 & 2.306 & 2.282 & 10.818 & 6.02 & 4.924 & 6.762 & 4.057 \\
\hline 8/8/2005 & $10: 00$ & 10.574 & 10.501 & 7.73 & 6.606 & 4.318 & 4.118 & 2.317 & 2.292 & 10.818 & 6.024 & 4.936 & 6.778 & 4.071 \\
\hline 8/8/2005 & $11: 00$ & 10.577 & 10.505 & 7.734 & 6.609 & 4.328 & 4.12 & 2.324 & 2.301 & 10.82 & 6.031 & 4.95 & 6.799 & 4.096 \\
\hline 8/8/2005 & $12: 00$ & 10.577 & 10.497 & 7.734 & 6.609 & 4.336 & 4.12 & 2.329 & 2.305 & 10.822 & 6.042 & 4.966 & 6.795 & 4.124 \\
\hline 8/8/2005 & $13: 00$ & 10.575 & 10.501 & 7.736 & 6.606 & 4.344 & 4.118 & 2.334 & 2.31 & 10.83 & 6.053 & 4.977 & 6.791 & 4.162 \\
\hline 8/8/2005 & $14: 00$ & 10.572 & 10.497 & 7.736 & 6.609 & 4.357 & 4.118 & 2.344 & 2.314 & 10.837 & 6.064 & 4.997 & 6.82 & 4.205 \\
\hline 8/8/2005 & $15: 00$ & 10.57 & 10.492 & 7.734 & 6.606 & 4.369 & 4.118 & 2.349 & 2.318 & 10.845 & 6.073 & 5.013 & 6.821 & 4.236 \\
\hline 8/8/2005 & $16: 00$ & 10.565 & 10.483 & 7.732 & 6.602 & 4.378 & 4.118 & 2.342 & 2.316 & 10.851 & 6.08 & 5.028 & 6.821 & 4.26 \\
\hline 8/8/2005 & $17: 00$ & 10.56 & 10.483 & 7.736 & 6.606 & 4.388 & 4.12 & 2.344 & 2.32 & 10.856 & 6.084 & 5.034 & 6.827 & 4.281 \\
\hline
\end{tabular}


TABLE S1.2 (Cont.)

Water Level (ft below top of casing) at Indicated Well

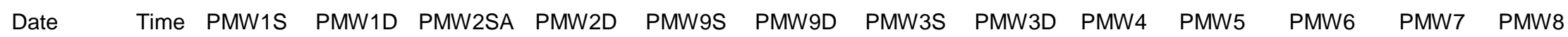

\begin{tabular}{|c|c|c|c|c|c|c|c|c|c|c|c|c|c|c|}
\hline 8/8/2005 & $18: 00$ & 10.555 & 10.472 & 7.728 & 6.6 & 4.388 & 4.123 & 2.344 & 2.312 & 10.86 & 6.08 & 5.026 & 6.799 & 4.279 \\
\hline 8/8/2005 & 19:00 & 10.553 & 10.472 & 7.728 & 6.598 & 4.388 & 4.123 & 2.337 & 2.305 & 10.864 & 6.082 & 5.015 & 6.78 & 4.269 \\
\hline 8/8/2005 & $20: 00$ & 10.553 & 10.472 & 7.728 & 6.598 & 4.388 & 4.125 & 2.329 & 2.303 & 10.868 & 6.08 & 5.005 & 6.764 & 4.26 \\
\hline 8/8/2005 & $21: 00$ & 10.553 & 10.475 & 7.727 & 6.6 & 4.386 & 4.127 & 2.332 & 2.301 & 10.868 & 6.078 & 4.997 & 6.753 & 4.245 \\
\hline 8/8/2005 & $22: 00$ & 10.558 & 10.483 & 7.729 & 6.604 & 4.384 & 4.127 & 2.324 & 2.301 & 10.87 & 6.073 & 4.983 & 6.741 & 4.226 \\
\hline 8/8/2005 & $23: 00$ & 10.561 & 10.484 & 7.729 & 6.604 & 4.375 & 4.129 & 2.33 & 2.299 & 10.87 & 6.067 & 4.97 & 6.732 & 4.206 \\
\hline $8 / 9 / 2005$ & $0: 00$ & 10.563 & 10.488 & 7.731 & 6.605 & 4.367 & 4.129 & 2.32 & 2.297 & 10.868 & 6.062 & 4.958 & 6.722 & 4.188 \\
\hline 8/9/2005 & $1: 00$ & 10.566 & 10.488 & 7.727 & 6.605 & 4.361 & 4.129 & 2.322 & 2.295 & 10.866 & 6.058 & 4.946 & 6.713 & 4.17 \\
\hline 8/9/2005 & $2: 00$ & 10.563 & 10.484 & 7.727 & 6.598 & 4.349 & 4.127 & 2.31 & 2.286 & 10.862 & 6.051 & 4.933 & 6.705 & 4.15 \\
\hline 8/9/2005 & $3: 00$ & 10.561 & 10.479 & 7.721 & 6.591 & 4.338 & 4.125 & 2.307 & 2.278 & 10.856 & 6.047 & 4.923 & 6.698 & 4.131 \\
\hline 8/9/2005 & $4: 00$ & 10.559 & 10.479 & 7.719 & 6.589 & 4.331 & 4.123 & 2.305 & 2.274 & 10.853 & 6.042 & 4.909 & 6.694 & 4.115 \\
\hline $8 / 9 / 2005$ & $5: 00$ & 10.559 & 10.477 & 7.719 & 6.587 & 4.325 & 4.118 & 2.302 & 2.272 & 10.847 & 6.036 & 4.903 & 6.688 & 4.103 \\
\hline 8/9/2005 & $6: 00$ & 10.564 & 10.488 & 7.723 & 6.595 & 4.329 & 4.118 & 2.3 & 2.279 & 10.847 & 6.039 & 4.899 & 6.688 & 4.099 \\
\hline 8/9/2005 & $7: 00$ & 10.569 & 10.494 & 7.725 & 6.598 & 4.329 & 4.118 & 2.313 & 2.283 & 10.843 & 6.034 & 4.896 & 6.684 & 4.091 \\
\hline 8/9/2005 & $8: 00$ & 10.571 & 10.495 & 7.725 & 6.598 & 4.323 & 4.116 & 2.308 & 2.279 & 10.839 & 6.03 & 4.888 & 6.705 & 4.079 \\
\hline 8/9/2005 & $9: 00$ & 10.574 & 10.501 & 7.725 & 6.598 & 4.325 & 4.116 & 2.315 & 2.285 & 10.836 & 6.032 & 4.898 & 6.73 & 4.077 \\
\hline 8/9/2005 & $10: 00$ & 10.571 & 10.494 & 7.727 & 6.602 & 4.327 & 4.114 & 2.321 & 2.291 & 10.834 & 6.034 & 4.904 & 6.746 & 4.091 \\
\hline 8/9/2005 & $11: 00$ & 10.576 & 10.499 & 7.733 & 6.605 & 4.339 & 4.114 & 2.331 & 2.302 & 10.836 & 6.043 & 4.915 & 6.746 & 4.118 \\
\hline 8/9/2005 & $12: 00$ & 10.571 & 10.494 & 7.729 & 6.602 & 4.346 & 4.112 & 2.326 & 2.302 & 10.837 & 6.052 & 4.921 & 6.753 & 4.15 \\
\hline 8/9/2005 & $13: 00$ & 10.569 & 10.49 & 7.731 & 6.6 & 4.362 & 4.112 & 2.338 & 2.306 & 10.845 & 6.066 & 4.939 & 6.759 & 4.194 \\
\hline 8/9/2005 & $14: 00$ & 10.569 & 10.488 & 7.733 & 6.605 & 4.377 & 4.114 & 2.348 & 2.314 & 10.855 & 6.079 & 4.96 & 6.789 & 4.239 \\
\hline 8/9/2005 & $15: 00$ & 10.567 & 10.486 & 7.735 & 6.605 & 4.393 & 4.114 & 2.353 & 2.321 & 10.864 & 6.086 & 4.986 & 6.808 & 4.273 \\
\hline $8 / 9 / 2005$ & $16: 00$ & 10.567 & 10.484 & 7.735 & 6.607 & 4.404 & 4.116 & 2.358 & 2.321 & 10.872 & 6.094 & 5.004 & 6.82 & 4.302 \\
\hline 8/9/2005 & $17: 00$ & 10.562 & 10.475 & 7.733 & 6.602 & 4.408 & 4.116 & 2.348 & 2.321 & 10.878 & 6.097 & 5.014 & 6.81 & 4.319 \\
\hline 8/9/2005 & $18: 00$ & 10.557 & 10.475 & 7.733 & 6.6 & 4.416 & 4.12 & 2.345 & 2.321 & 10.882 & 6.103 & 5.022 & 6.799 & 4.333 \\
\hline 8/9/2005 & $19: 00$ & 10.555 & 10.472 & 7.731 & 6.598 & 4.416 & 4.12 & 2.348 & 2.314 & 10.885 & 6.103 & 5.014 & 6.776 & 4.331 \\
\hline 8/9/2005 & $20: 00$ & 10.555 & 10.474 & 7.729 & 6.6 & 4.416 & 4.125 & 2.338 & 2.312 & 10.889 & 6.103 & 5.002 & 6.757 & 4.321 \\
\hline 8/9/2005 & $21: 00$ & 10.562 & 10.483 & 7.733 & 6.605 & 4.416 & 4.127 & 2.336 & 2.31 & 10.891 & 6.101 & 4.99 & 6.744 & 4.303 \\
\hline 8/9/2005 & $22: 00$ & 10.57 & 10.492 & 7.737 & 6.611 & 4.416 & 4.132 & 2.346 & 2.314 & 10.893 & 6.095 & 4.977 & 6.73 & 4.283 \\
\hline 8/9/2005 & 23:00 & 10.58 & 10.506 & 7.743 & 6.62 & 4.416 & 4.136 & 2.351 & 2.321 & 10.893 & 6.093 & 4.967 & 6.725 & 4.268 \\
\hline 8/10/2005 & 0:00 & 10.587 & 10.512 & 7.745 & 6.625 & 4.406 & 4.141 & 2.341 & 2.321 & 10.895 & 6.086 & 4.953 & 6.715 & 4.248 \\
\hline $8 / 10 / 2005$ & $1: 00$ & 10.59 & 10.515 & 7.745 & 6.62 & 4.401 & 4.14 & 2.344 & 2.314 & 10.891 & 6.079 & 4.939 & 6.705 & 4.226 \\
\hline $8 / 10 / 2005$ & $2: 00$ & 10.592 & 10.515 & 7.745 & 6.62 & 4.391 & 4.14 & 2.339 & 2.31 & 10.889 & 6.07 & 4.926 & 6.698 & 4.206 \\
\hline $8 / 10 / 2005$ & $3: 00$ & 10.592 & 10.514 & 7.743 & 6.614 & 4.379 & 4.138 & 2.334 & 2.302 & 10.883 & 6.066 & 4.914 & 6.69 & 4.185 \\
\hline
\end{tabular}


TABLE S1.2 (Cont.)

Water Level (ft below top of casing) at Indicated Well

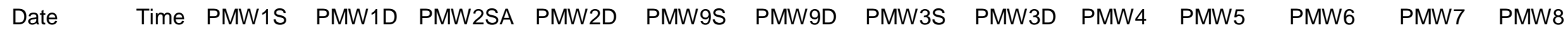

\begin{tabular}{|c|c|c|c|c|c|c|c|c|c|c|c|c|c|c|}
\hline $8 / 10 / 2005$ & $4: 00$ & 10.592 & 10.515 & 7.741 & 6.614 & 4.372 & 4.138 & 2.332 & 2.302 & 10.88 & 6.062 & 4.902 & 6.686 & 4.171 \\
\hline $8 / 10 / 2005$ & $5: 00$ & 10.595 & 10.519 & 7.741 & 6.616 & 4.368 & 4.139 & 2.324 & 2.302 & 10.878 & 6.058 & 4.895 & 6.681 & 4.157 \\
\hline $8 / 10 / 2005$ & $6: 00$ & 10.6 & 10.521 & 7.741 & 6.616 & 4.362 & 4.136 & 2.327 & 2.297 & 10.874 & 6.055 & 4.887 & 6.675 & 4.143 \\
\hline $8 / 10 / 2005$ & $7: 00$ & 10.6 & 10.525 & 7.743 & 6.618 & 4.359 & 4.136 & 2.327 & 2.3 & 10.868 & 6.053 & 4.879 & 6.671 & 4.133 \\
\hline $8 / 10 / 2005$ & 8:00 & 10.602 & 10.526 & 7.743 & 6.618 & 4.357 & 4.136 & 2.327 & 2.3 & 10.866 & 6.049 & 4.875 & 6.677 & 4.121 \\
\hline $8 / 10 / 2005$ & $9: 00$ & 10.608 & 10.534 & 7.745 & 6.622 & 4.357 & 4.134 & 2.322 & 2.302 & 10.863 & 6.051 & 4.871 & 6.677 & 4.115 \\
\hline $8 / 10 / 2005$ & $10: 00$ & 10.607 & 10.534 & 7.747 & 6.62 & 4.353 & 4.132 & 2.332 & 2.304 & 10.861 & 6.047 & 4.873 & 6.687 & 4.113 \\
\hline $8 / 10 / 2005$ & $11: 00$ & 10.613 & 10.537 & 7.751 & 6.627 & 4.359 & 4.134 & 2.34 & 2.31 & 10.859 & 6.051 & 4.885 & 6.715 & 4.127 \\
\hline $8 / 10 / 2005$ & $12: 00$ & 10.61 & 10.534 & 7.751 & 6.625 & 4.367 & 4.132 & 2.337 & 2.314 & 10.861 & 6.06 & 4.903 & 6.74 & 4.143 \\
\hline $8 / 10 / 2005$ & $13: 00$ & 10.61 & 10.53 & 7.751 & 6.62 & 4.374 & 4.132 & 2.347 & 2.317 & 10.861 & 6.066 & 4.917 & 6.74 & 4.17 \\
\hline $8 / 10 / 2005$ & $14: 00$ & 10.603 & 10.521 & 7.749 & 6.616 & 4.384 & 4.13 & 2.347 & 2.317 & 10.867 & 6.073 & 4.934 & 6.748 & 4.204 \\
\hline $8 / 10 / 2005$ & $15: 00$ & 10.6 & 10.517 & 7.749 & 6.616 & 4.4 & 4.13 & 2.352 & 2.321 & 10.872 & 6.087 & 4.952 & 6.773 & 4.238 \\
\hline $8 / 10 / 2005$ & $16: 00$ & 10.593 & 10.51 & 7.745 & 6.611 & 4.407 & 4.128 & 2.35 & 2.316 & 10.88 & 6.096 & 4.973 & 6.801 & 4.265 \\
\hline $8 / 10 / 2005$ & $17: 00$ & 10.586 & 10.497 & 7.743 & 6.602 & 4.411 & 4.128 & 2.342 & 2.315 & 10.888 & 6.1 & 4.985 & 6.795 & 4.289 \\
\hline $8 / 10 / 2005$ & $18: 00$ & 10.581 & 10.495 & 7.739 & 6.602 & 4.419 & 4.128 & 2.337 & 2.308 & 10.893 & 6.105 & 4.983 & 6.765 & 4.298 \\
\hline $8 / 10 / 2005$ & $19: 00$ & 10.586 & 10.503 & 7.743 & 6.614 & 4.427 & 4.132 & 2.342 & 2.315 & 10.897 & 6.1 & 4.973 & 6.748 & 4.29 \\
\hline $8 / 10 / 2005$ & $20: 00$ & 10.345 & 10.497 & 7.705 & 6.6 & 4.196 & 4.141 & 2.229 & 2.229 & 10.888 & 5.684 & 4.785 & 6.622 & 3.56 \\
\hline $8 / 10 / 2005$ & $21: 00$ & 10.531 & 10.486 & 7.68 & 6.538 & 3.786 & 4.116 & 2.2 & 2.176 & 10.857 & 5.702 & 4.713 & 6.509 & 3.177 \\
\hline $8 / 10 / 2005$ & $22: 00$ & 10.544 & 10.486 & 7.672 & 6.538 & 3.702 & 4.081 & 2.19 & 2.172 & 10.821 & 5.757 & 4.68 & 6.456 & 3.184 \\
\hline $8 / 10 / 2005$ & $23: 00$ & 10.545 & 10.483 & 7.67 & 6.532 & 3.712 & 4.05 & 2.192 & 2.168 & 10.77 & 5.789 & 4.65 & 6.458 & 3.236 \\
\hline $8 / 11 / 2005$ & $0: 00$ & 10.543 & 10.477 & 7.67 & 6.527 & 3.749 & 4.021 & 2.187 & 2.163 & 10.755 & 5.807 & 4.631 & 6.458 & 3.286 \\
\hline $8 / 11 / 2005$ & $1: 00$ & 10.54 & 10.475 & 7.67 & 6.527 & 3.792 & 4.001 & 2.185 & 2.163 & 10.743 & 5.827 & 4.619 & 6.464 & 3.339 \\
\hline $8 / 11 / 2005$ & $2: 00$ & 10.54 & 10.472 & 7.67 & 6.523 & 3.828 & 3.984 & 2.183 & 2.161 & 10.736 & 5.84 & 4.611 & 6.467 & 3.376 \\
\hline $8 / 11 / 2005$ & $3: 00$ & 10.537 & 10.472 & 7.676 & 6.523 & 3.861 & 3.973 & 2.185 & 2.161 & 10.726 & 5.851 & 4.607 & 6.473 & 3.41 \\
\hline $8 / 11 / 2005$ & $4: 00$ & 10.54 & 10.477 & 7.678 & 6.532 & 3.896 & 3.968 & 2.193 & 2.17 & 10.724 & 5.863 & 4.614 & 6.485 & 3.447 \\
\hline $8 / 11 / 2005$ & $5: 00$ & 10.538 & 10.474 & 7.68 & 6.529 & 3.921 & 3.961 & 2.198 & 2.172 & 10.718 & 5.865 & 4.612 & 6.488 & 3.467 \\
\hline $8 / 11 / 2005$ & $6: 00$ & 10.54 & 10.474 & 7.68 & 6.529 & 3.942 & 3.959 & 2.2 & 2.172 & 10.715 & 5.872 & 4.615 & 6.496 & 3.488 \\
\hline $8 / 11 / 2005$ & $7: 00$ & 10.54 & 10.474 & 7.682 & 6.532 & 3.963 & 3.957 & 2.196 & 2.174 & 10.709 & 5.876 & 4.619 & 6.506 & 3.508 \\
\hline $8 / 11 / 2005$ & $8: 00$ & 10.535 & 10.47 & 7.68 & 6.529 & 3.979 & 3.955 & 2.196 & 2.172 & 10.705 & 5.881 & 4.621 & 6.512 & 3.521 \\
\hline $8 / 11 / 2005$ & 9:00 & 10.533 & 10.463 & 7.678 & 6.523 & 3.988 & 3.953 & 2.198 & 2.17 & 10.701 & 5.878 & 4.627 & 6.531 & 3.533 \\
\hline $8 / 11 / 2005$ & $10: 00$ & 10.533 & 10.461 & 7.678 & 6.523 & 4 & 3.953 & 2.196 & 2.168 & 10.697 & 5.883 & 4.633 & 6.538 & 3.547 \\
\hline $8 / 11 / 2005$ & $11: 00$ & 10.528 & 10.455 & 7.678 & 6.521 & 4.012 & 3.953 & 2.191 & 2.168 & 10.694 & 5.885 & 4.637 & 6.538 & 3.559 \\
\hline $8 / 11 / 2005$ & $12: 00$ & 10.526 & 10.452 & 7.674 & 6.516 & 4.021 & 3.953 & 2.193 & 2.168 & 10.692 & 5.889 & 4.647 & 6.552 & 3.577 \\
\hline $8 / 11 / 2005$ & $13: 00$ & 10.523 & 10.446 & 7.672 & 6.516 & 4.031 & 3.95 & 2.193 & 2.168 & 10.69 & 5.896 & 4.661 & 6.567 & 3.599 \\
\hline
\end{tabular}


TABLE S1.2 (Cont.)

Water Level (ft below top of casing) at Indicated Well

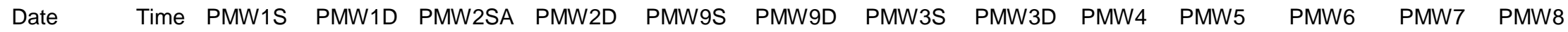

\begin{tabular}{|c|c|c|c|c|c|c|c|c|c|c|c|c|c|c|}
\hline $8 / 11 / 2005$ & $14: 00$ & 10.518 & 10.443 & 7.672 & 6.514 & 4.043 & 3.95 & 2.203 & 2.17 & 10.692 & 5.901 & 4.677 & 6.576 & 3.632 \\
\hline $8 / 11 / 2005$ & $15: 00$ & 10.513 & 10.437 & 7.666 & 6.505 & 4.035 & 3.95 & 2.176 & 2.151 & 10.686 & 5.844 & 4.663 & 6.559 & 3.535 \\
\hline $8 / 11 / 2005$ & $16: 00$ & 10.487 & 10.432 & 7.651 & 6.476 & 3.938 & 3.953 & 2.113 & 2.092 & 10.663 & 5.63 & 4.61 & 6.46 & 3.131 \\
\hline $8 / 11 / 2005$ & $17: 00$ & 10.486 & 10.426 & 7.641 & 6.472 & 3.738 & 3.95 & 2.113 & 2.086 & 10.64 & 5.627 & 4.567 & 6.43 & 2.923 \\
\hline $8 / 11 / 2005$ & $18: 00$ & 10.492 & 10.419 & 7.634 & 6.465 & 3.661 & 3.937 & 2.106 & 2.084 & 10.622 & 5.649 & 4.539 & 6.424 & 2.895 \\
\hline $8 / 11 / 2005$ & $19: 00$ & 10.49 & 10.415 & 7.632 & 6.461 & 3.655 & 3.922 & 2.113 & 2.084 & 10.607 & 5.671 & 4.526 & 6.428 & 2.915 \\
\hline $8 / 11 / 2005$ & $20: 00$ & 10.487 & 10.413 & 7.634 & 6.465 & 3.681 & 3.908 & 2.111 & 2.09 & 10.597 & 5.691 & 4.522 & 6.431 & 2.948 \\
\hline $8 / 11 / 2005$ & $21: 00$ & 10.485 & 10.413 & 7.634 & 6.468 & 3.708 & 3.897 & 2.114 & 2.092 & 10.588 & 5.7 & 4.518 & 6.399 & 2.939 \\
\hline $8 / 11 / 2005$ & $22: 00$ & 10.49 & 10.421 & 7.64 & 6.474 & 3.733 & 3.891 & 2.129 & 2.101 & 10.582 & 5.711 & 4.522 & 6.43 & 2.954 \\
\hline $8 / 11 / 2005$ & $23: 00$ & 10.49 & 10.422 & 7.64 & 6.476 & 3.747 & 3.886 & 2.124 & 2.103 & 10.574 & 5.723 & 4.522 & 6.447 & 2.984 \\
\hline $8 / 12 / 2005$ & $0: 00$ & 10.492 & 10.424 & 7.642 & 6.479 & 3.768 & 3.882 & 2.134 & 2.107 & 10.57 & 5.729 & 4.524 & 6.452 & 3.019 \\
\hline $8 / 12 / 2005$ & $1: 00$ & 10.495 & 10.426 & 7.643 & 6.481 & 3.787 & 3.882 & 2.129 & 2.109 & 10.567 & 5.74 & 4.528 & 6.458 & 3.053 \\
\hline $8 / 12 / 2005$ & $2: 00$ & 10.495 & 10.424 & 7.643 & 6.481 & 3.805 & 3.88 & 2.137 & 2.109 & 10.563 & 5.747 & 4.53 & 6.464 & 3.084 \\
\hline $8 / 12 / 2005$ & $3: 00$ & 10.49 & 10.421 & 7.643 & 6.476 & 3.818 & 3.877 & 2.129 & 2.107 & 10.559 & 5.752 & 4.534 & 6.466 & 3.11 \\
\hline $8 / 12 / 2005$ & $4: 00$ & 10.49 & 10.422 & 7.643 & 6.479 & 3.836 & 3.88 & 2.139 & 2.109 & 10.557 & 5.76 & 4.54 & 6.473 & 3.139 \\
\hline $8 / 12 / 2005$ & $5: 00$ & 10.495 & 10.428 & 7.647 & 6.488 & 3.857 & 3.882 & 2.147 & 2.12 & 10.557 & 5.769 & 4.55 & 6.481 & 3.171 \\
\hline $8 / 12 / 2005$ & $6: 00$ & 10.497 & 10.43 & 7.649 & 6.49 & 3.872 & 3.884 & 2.152 & 2.124 & 10.557 & 5.774 & 4.554 & 6.487 & 3.198 \\
\hline $8 / 12 / 2005$ & $7: 00$ & 10.505 & 10.439 & 7.655 & 6.499 & 3.891 & 3.888 & 2.16 & 2.134 & 10.557 & 5.782 & 4.566 & 6.498 & 3.226 \\
\hline $8 / 12 / 2005$ & $8: 00$ & 10.51 & 10.443 & 7.659 & 6.496 & 3.903 & 3.893 & 2.165 & 2.134 & 10.559 & 5.782 & 4.57 & 6.502 & 3.247 \\
\hline $8 / 12 / 2005$ & $9: 00$ & 10.5 & 10.432 & 7.651 & 6.485 & 3.903 & 3.891 & 2.152 & 2.124 & 10.576 & 5.781 & 4.566 & 6.531 & 3.247 \\
\hline $8 / 12 / 2005$ & $10: 00$ & 10.513 & 10.448 & 7.661 & 6.505 & 3.926 & 3.899 & 2.165 & 2.143 & 10.589 & 5.796 & 4.581 & 6.517 & 3.274 \\
\hline $8 / 12 / 2005$ & $11: 00$ & 10.515 & 10.452 & 7.665 & 6.508 & 3.938 & 3.904 & 2.167 & 2.145 & 10.563 & 5.798 & 4.587 & 6.529 & 3.287 \\
\hline $8 / 12 / 2005$ & $12: 00$ & 10.518 & 10.452 & 7.665 & 6.508 & 3.946 & 3.908 & 2.175 & 2.147 & 10.561 & 5.8 & 4.591 & 6.525 & 3.302 \\
\hline $8 / 12 / 2005$ & $13: 00$ & 10.518 & 10.45 & 7.665 & 6.505 & 3.952 & 3.911 & 2.168 & 2.147 & 10.563 & 5.805 & 4.593 & 6.527 & 3.316 \\
\hline $8 / 12 / 2005$ & $14: 00$ & 10.52 & 10.448 & 7.663 & 6.503 & 3.959 & 3.913 & 2.17 & 2.145 & 10.563 & 5.805 & 4.601 & 6.531 & 3.334 \\
\hline $8 / 12 / 2005$ & $15: 00$ & 10.518 & 10.448 & 7.665 & 6.505 & 3.967 & 3.915 & 2.178 & 2.147 & 10.565 & 5.812 & 4.607 & 6.537 & 3.353 \\
\hline $8 / 12 / 2005$ & $16: 00$ & 10.516 & 10.441 & 7.661 & 6.499 & 3.971 & 3.915 & 2.168 & 2.143 & 10.567 & 5.814 & 4.607 & 6.535 & 3.369 \\
\hline $8 / 12 / 2005$ & $17: 00$ & 10.513 & 10.437 & 7.661 & 6.499 & 3.979 & 3.919 & 2.17 & 2.143 & 10.569 & 5.816 & 4.611 & 6.541 & 3.387 \\
\hline $8 / 12 / 2005$ & $18: 00$ & 10.508 & 10.433 & 7.659 & 6.496 & 3.983 & 3.922 & 2.168 & 2.143 & 10.569 & 5.818 & 4.613 & 6.539 & 3.399 \\
\hline $8 / 12 / 2005$ & $19: 00$ & 10.506 & 10.43 & 7.657 & 6.492 & 3.985 & 3.919 & 2.168 & 2.139 & 10.569 & 5.818 & 4.611 & 6.52 & 3.405 \\
\hline $8 / 12 / 2005$ & $20: 00$ & 10.501 & 10.424 & 7.656 & 6.488 & 3.987 & 3.922 & 2.16 & 2.137 & 10.569 & 5.818 & 4.605 & 6.535 & 3.411 \\
\hline $8 / 12 / 2005$ & $21: 00$ & 10.501 & 10.424 & 7.654 & 6.492 & 3.992 & 3.924 & 2.168 & 2.137 & 10.57 & 5.821 & 4.605 & 6.537 & 3.424 \\
\hline $8 / 12 / 2005$ & $22: 00$ & 10.503 & 10.432 & 7.659 & 6.501 & 4.004 & 3.926 & 2.176 & 2.147 & 10.575 & 5.825 & 4.613 & 6.541 & 3.442 \\
\hline $8 / 12 / 2005$ & $23: 00$ & 10.503 & 10.432 & 7.659 & 6.496 & 4.006 & 3.928 & 2.176 & 2.143 & 10.573 & 5.825 & 4.613 & 6.541 & 3.446 \\
\hline
\end{tabular}


TABLE S1.2 (Cont.)

Water Level (ft below top of casing) at Indicated Well

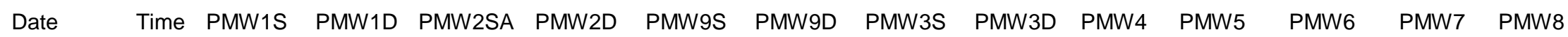

\begin{tabular}{|c|c|c|c|c|c|c|c|c|c|c|c|c|c|c|}
\hline $8 / 13 / 2005$ & $0: 00$ & 10.504 & 10.433 & 7.659 & 6.499 & 4.012 & 3.928 & 2.178 & 2.145 & 10.574 & 5.828 & 4.615 & 6.542 & 3.456 \\
\hline $8 / 13 / 2005$ & $1: 00$ & 10.509 & 10.441 & 7.664 & 6.505 & 4.022 & 3.933 & 2.186 & 2.153 & 10.578 & 5.83 & 4.619 & 6.546 & 3.468 \\
\hline $8 / 13 / 2005$ & $2: 00$ & 10.511 & 10.437 & 7.665 & 6.505 & 4.025 & 3.937 & 2.178 & 2.153 & 10.578 & 5.83 & 4.621 & 6.544 & 3.472 \\
\hline $8 / 13 / 2005$ & $3: 00$ & 10.506 & 10.417 & 7.66 & 6.496 & 4.02 & 3.935 & 2.176 & 2.147 & 10.578 & 5.824 & 4.615 & 6.539 & 3.46 \\
\hline $8 / 13 / 2005$ & $4: 00$ & 10.501 & 10.406 & 7.657 & 6.492 & 4.018 & 3.933 & 2.166 & 2.137 & 10.565 & 5.824 & 4.611 & 6.533 & 3.402 \\
\hline $8 / 13 / 2005$ & $5: 00$ & 10.496 & 10.404 & 7.652 & 6.485 & 4.014 & 3.93 & 2.153 & 2.129 & 10.558 & 5.819 & 4.604 & 6.531 & 3.349 \\
\hline $8 / 13 / 2005$ & $6: 00$ & 10.496 & 10.401 & 7.654 & 6.488 & 4.006 & 3.933 & 2.159 & 2.126 & 10.548 & 5.813 & 4.598 & 6.521 & 3.309 \\
\hline $8 / 13 / 2005$ & $7: 00$ & 10.497 & 10.419 & 7.654 & 6.49 & 3.999 & 3.933 & 2.161 & 2.129 & 10.552 & 5.813 & 4.596 & 6.529 & 3.297 \\
\hline $8 / 13 / 2005$ & $8: 00$ & 10.504 & 10.43 & 7.66 & 6.496 & 3.993 & 3.935 & 2.167 & 2.135 & 10.561 & 5.813 & 4.596 & 6.539 & 3.299 \\
\hline $8 / 13 / 2005$ & 9:00 & 10.509 & 10.426 & 7.664 & 6.501 & 3.991 & 3.935 & 2.172 & 2.139 & 10.554 & 5.811 & 4.596 & 6.541 & 3.299 \\
\hline $8 / 13 / 2005$ & $10: 00$ & 10.514 & 10.419 & 7.666 & 6.503 & 3.985 & 3.938 & 2.164 & 2.141 & 10.548 & 5.804 & 4.592 & 6.539 & 3.253 \\
\hline $8 / 13 / 2005$ & $11: 00$ & 10.517 & 10.417 & 7.662 & 6.503 & 3.949 & 3.939 & 2.162 & 2.137 & 10.537 & 5.78 & 4.577 & 6.533 & 3.179 \\
\hline $8 / 13 / 2005$ & $12: 00$ & 10.519 & 10.422 & 7.656 & 6.499 & 3.864 & 3.942 & 2.154 & 2.129 & 10.535 & 5.76 & 4.551 & 6.537 & 3.121 \\
\hline $8 / 13 / 2005$ & $13: 00$ & 10.516 & 10.446 & 7.65 & 6.495 & 3.775 & 3.939 & 2.149 & 2.124 & 10.559 & 5.74 & 4.528 & 6.516 & 3.075 \\
\hline $8 / 13 / 2005$ & $14: 00$ & 10.512 & 10.439 & 7.647 & 6.483 & 3.71 & 3.93 & 2.139 & 2.116 & 10.554 & 5.736 & 4.516 & 6.518 & 3.073 \\
\hline $8 / 13 / 2005$ & $15: 00$ & 10.504 & 10.43 & 7.646 & 6.474 & 3.693 & 3.917 & 2.137 & 2.114 & 10.55 & 5.74 & 4.516 & 6.527 & 3.099 \\
\hline $8 / 13 / 2005$ & $16: 00$ & 10.504 & 10.43 & 7.646 & 6.477 & 3.711 & 3.911 & 2.142 & 2.118 & 10.55 & 5.751 & 4.53 & 6.539 & 3.145 \\
\hline $8 / 13 / 2005$ & $17: 00$ & 10.507 & 10.433 & 7.65 & 6.485 & 3.744 & 3.904 & 2.157 & 2.126 & 10.55 & 5.765 & 4.547 & 6.547 & 3.198 \\
\hline $8 / 13 / 2005$ & $18: 00$ & 10.504 & 10.435 & 7.652 & 6.488 & 3.775 & 3.897 & 2.157 & 2.131 & 10.552 & 5.774 & 4.561 & 6.552 & 3.242 \\
\hline $8 / 13 / 2005$ & $19: 00$ & 10.507 & 10.432 & 7.652 & 6.49 & 3.801 & 3.895 & 2.159 & 2.135 & 10.554 & 5.778 & 4.573 & 6.554 & 3.283 \\
\hline $8 / 13 / 2005$ & $20: 00$ & 10.51 & 10.439 & 7.658 & 6.499 & 3.831 & 3.897 & 2.175 & 2.143 & 10.558 & 5.789 & 4.584 & 6.554 & 3.319 \\
\hline $8 / 13 / 2005$ & $21: 00$ & 10.512 & 10.443 & 7.66 & 6.501 & 3.854 & 3.9 & 2.175 & 2.147 & 10.561 & 5.794 & 4.589 & 6.552 & 3.341 \\
\hline $8 / 13 / 2005$ & $22: 00$ & 10.52 & 10.45 & 7.666 & 6.51 & 3.877 & 3.902 & 2.185 & 2.154 & 10.563 & 5.802 & 4.599 & 6.554 & 3.364 \\
\hline $8 / 13 / 2005$ & $23: 00$ & 10.522 & 10.455 & 7.67 & 6.512 & 3.894 & 3.906 & 2.183 & 2.16 & 10.567 & 5.802 & 4.602 & 6.554 & 3.38 \\
\hline $8 / 14 / 2005$ & $0: 00$ & 10.525 & 10.455 & 7.67 & 6.51 & 3.906 & 3.909 & 2.188 & 2.158 & 10.569 & 5.807 & 4.604 & 6.552 & 3.39 \\
\hline $8 / 14 / 2005$ & $1: 00$ & 10.527 & 10.457 & 7.672 & 6.512 & 3.917 & 3.911 & 2.19 & 2.158 & 10.571 & 5.807 & 4.606 & 6.552 & 3.402 \\
\hline $8 / 14 / 2005$ & $2: 00$ & 10.527 & 10.459 & 7.672 & 6.515 & 3.929 & 3.915 & 2.185 & 2.16 & 10.571 & 5.811 & 4.61 & 6.554 & 3.413 \\
\hline $8 / 14 / 2005$ & $3: 00$ & 10.527 & 10.457 & 7.672 & 6.512 & 3.939 & 3.919 & 2.185 & 2.161 & 10.573 & 5.811 & 4.61 & 6.554 & 3.422 \\
\hline $8 / 14 / 2005$ & $4: 00$ & 10.532 & 10.463 & 7.674 & 6.519 & 3.952 & 3.924 & 2.198 & 2.166 & 10.575 & 5.814 & 4.616 & 6.558 & 3.435 \\
\hline $8 / 14 / 2005$ & $5: 00$ & 10.535 & 10.466 & 7.678 & 6.521 & 3.962 & 3.928 & 2.195 & 2.171 & 10.575 & 5.816 & 4.618 & 6.56 & 3.446 \\
\hline $8 / 14 / 2005$ & $6: 00$ & 10.54 & 10.47 & 7.68 & 6.523 & 3.972 & 3.93 & 2.205 & 2.173 & 10.577 & 5.82 & 4.622 & 6.562 & 3.456 \\
\hline $8 / 14 / 2005$ & 7:00 & 10.545 & 10.474 & 7.682 & 6.526 & 3.983 & 3.935 & 2.208 & 2.177 & 10.579 & 5.825 & 4.626 & 6.564 & 3.466 \\
\hline $8 / 14 / 2005$ & $8: 00$ & 10.545 & 10.475 & 7.69 & 6.53 & 3.991 & 3.939 & 2.205 & 2.182 & 10.581 & 5.825 & 4.63 & 6.564 & 3.476 \\
\hline $8 / 14 / 2005$ & $9: 00$ & 10.55 & 10.481 & 7.686 & 6.534 & 4.002 & 3.942 & 2.215 & 2.184 & 10.583 & 5.83 & 4.638 & 6.568 & 3.488 \\
\hline
\end{tabular}


TABLE S1.2 (Cont.)

Water Level (ft below top of casing) at Indicated Well

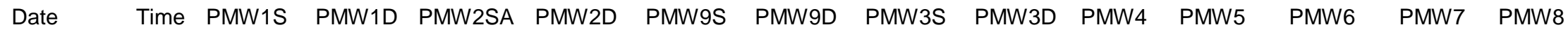

\begin{tabular}{|c|c|c|c|c|c|c|c|c|c|c|c|c|c|c|}
\hline $8 / 14 / 2005$ & $10: 00$ & 10.55 & 10.483 & 7.69 & 6.537 & 4.01 & 3.946 & 2.22 & 2.186 & 10.584 & 5.834 & 4.644 & 6.574 & 3.5 \\
\hline 8/14/2005 & $11: 00$ & 10.555 & 10.486 & 7.692 & 6.539 & 4.022 & 3.948 & 2.225 & 2.194 & 10.588 & 5.841 & 4.656 & 6.585 & 3.518 \\
\hline $8 / 14 / 2005$ & $12: 00$ & 10.555 & 10.484 & 7.694 & 6.537 & 4.03 & 3.953 & 2.223 & 2.196 & 10.59 & 5.843 & 4.667 & 6.596 & 3.538 \\
\hline 8/14/2005 & $13: 00$ & 10.553 & 10.477 & 7.69 & 6.532 & 4.037 & 3.955 & 2.228 & 2.194 & 10.592 & 5.847 & 4.679 & 6.608 & 3.56 \\
\hline $8 / 14 / 2005$ & $14: 00$ & 10.548 & 10.474 & 7.69 & 6.53 & 4.049 & 3.955 & 2.225 & 2.196 & 10.596 & 5.856 & 4.693 & 6.616 & 3.586 \\
\hline 8/14/2005 & $15: 00$ & 10.545 & 10.468 & 7.69 & 6.53 & 4.059 & 3.959 & 2.225 & 2.198 & 10.603 & 5.863 & 4.707 & 6.623 & 3.611 \\
\hline $8 / 14 / 2005$ & $16: 00$ & 10.543 & 10.464 & 7.686 & 6.528 & 4.069 & 3.961 & 2.233 & 2.2 & 10.609 & 5.87 & 4.719 & 6.627 & 3.635 \\
\hline 8/14/2005 & $17: 00$ & 10.54 & 10.461 & 7.684 & 6.528 & 4.076 & 3.964 & 2.228 & 2.198 & 10.613 & 5.872 & 4.727 & 6.629 & 3.656 \\
\hline 8/14/2005 & $18: 00$ & 10.536 & 10.457 & 7.684 & 6.526 & 4.084 & 3.966 & 2.236 & 2.2 & 10.621 & 5.879 & 4.735 & 6.629 & 3.672 \\
\hline 8/14/2005 & $19: 00$ & 10.533 & 10.453 & 7.682 & 6.523 & 4.09 & 3.968 & 2.233 & 2.198 & 10.626 & 5.881 & 4.738 & 6.627 & 3.682 \\
\hline 8/14/2005 & $20: 00$ & 10.533 & 10.455 & 7.682 & 6.528 & 4.096 & 3.973 & 2.233 & 2.198 & 10.63 & 5.881 & 4.736 & 6.619 & 3.686 \\
\hline $8 / 14 / 2005$ & $21: 00$ & 10.536 & 10.459 & 7.684 & 6.53 & 4.099 & 3.977 & 2.233 & 2.198 & 10.634 & 5.883 & 4.736 & 6.614 & 3.686 \\
\hline $8 / 14 / 2005$ & $22: 00$ & 10.543 & 10.466 & 7.688 & 6.537 & 4.107 & 3.981 & 2.241 & 2.207 & 10.638 & 5.888 & 4.736 & 6.614 & 3.69 \\
\hline 8/14/2005 & $23: 00$ & 10.546 & 10.47 & 7.694 & 6.537 & 4.109 & 3.986 & 2.233 & 2.205 & 10.642 & 5.885 & 4.735 & 6.61 & 3.686 \\
\hline 8/15/2005 & $0: 00$ & 10.546 & 10.47 & 7.694 & 6.539 & 4.107 & 3.988 & 2.241 & 2.205 & 10.643 & 5.885 & 4.731 & 6.608 & 3.68 \\
\hline $8 / 15 / 2005$ & $1: 00$ & 10.546 & 10.472 & 7.694 & 6.539 & 4.109 & 3.99 & 2.233 & 2.205 & 10.643 & 5.885 & 4.729 & 6.604 & 3.678 \\
\hline 8/15/2005 & $2: 00$ & 10.549 & 10.472 & 7.694 & 6.539 & 4.107 & 3.99 & 2.231 & 2.205 & 10.643 & 5.886 & 4.727 & 6.602 & 3.674 \\
\hline 8/15/2005 & $3: 00$ & 10.554 & 10.475 & 7.696 & 6.541 & 4.113 & 3.992 & 2.234 & 2.209 & 10.645 & 5.888 & 4.725 & 6.604 & 3.676 \\
\hline 8/15/2005 & $4: 00$ & 10.556 & 10.481 & 7.698 & 6.546 & 4.117 & 3.997 & 2.238 & 2.211 & 10.648 & 5.89 & 4.729 & 6.602 & 3.676 \\
\hline 8/15/2005 & 5:00 & 10.554 & 10.477 & 7.694 & 6.541 & 4.114 & 3.997 & 2.241 & 2.207 & 10.646 & 5.883 & 4.725 & 6.601 & 3.674 \\
\hline 8/15/2005 & $6: 00$ & 10.554 & 10.477 & 7.692 & 6.543 & 4.116 & 3.997 & 2.241 & 2.205 & 10.644 & 5.886 & 4.725 & 6.601 & 3.672 \\
\hline $8 / 15 / 2005$ & 7:00 & 10.556 & 10.479 & 7.694 & 6.543 & 4.117 & 3.999 & 2.241 & 2.207 & 10.645 & 5.886 & 4.723 & 6.601 & 3.672 \\
\hline $8 / 15 / 2005$ & $8: 00$ & 10.554 & 10.479 & 7.694 & 6.546 & 4.119 & 4.002 & 2.241 & 2.209 & 10.644 & 5.884 & 4.725 & 6.618 & 3.67 \\
\hline 8/15/2005 & 9:00 & 10.559 & 10.481 & 7.698 & 6.546 & 4.121 & 4.002 & 2.236 & 2.211 & 10.644 & 5.881 & 4.729 & 6.623 & 3.674 \\
\hline $8 / 15 / 2005$ & $10: 00$ & 10.559 & 10.481 & 7.698 & 6.548 & 4.126 & 4.001 & 2.242 & 2.213 & 10.645 & 5.886 & 4.733 & 6.625 & 3.676 \\
\hline 8/15/2005 & $11: 00$ & 10.561 & 10.481 & 7.698 & 6.548 & 4.131 & 4.001 & 2.254 & 2.218 & 10.646 & 5.89 & 4.739 & 6.641 & 3.691 \\
\hline $8 / 15 / 2005$ & $12: 00$ & 10.559 & 10.479 & 7.7 & 6.548 & 4.137 & 4.004 & 2.257 & 2.222 & 10.648 & 5.893 & 4.751 & 6.644 & 3.707 \\
\hline 8/15/2005 & $13: 00$ & 10.557 & 10.474 & 7.696 & 6.543 & 4.141 & 4.004 & 2.257 & 2.222 & 10.651 & 5.899 & 4.759 & 6.648 & 3.723 \\
\hline $8 / 15 / 2005$ & $14: 00$ & 10.552 & 10.468 & 7.698 & 6.541 & 4.145 & 4.004 & 2.249 & 2.222 & 10.653 & 5.902 & 4.769 & 6.656 & 3.743 \\
\hline 8/15/2005 & $15: 00$ & 10.547 & 10.461 & 7.696 & 6.534 & 4.149 & 4.004 & 2.249 & 2.22 & 10.655 & 5.904 & 4.778 & 6.664 & 3.757 \\
\hline $8 / 15 / 2005$ & $16: 00$ & 10.542 & 10.455 & 7.692 & 6.532 & 4.155 & 4.004 & 2.246 & 2.218 & 10.661 & 5.911 & 4.784 & 6.677 & 3.772 \\
\hline 8/15/2005 & $17: 00$ & 10.539 & 10.453 & 7.692 & 6.532 & 4.161 & 4.006 & 2.252 & 2.218 & 10.665 & 5.918 & 4.79 & 6.671 & 3.786 \\
\hline $8 / 15 / 2005$ & $18: 00$ & 10.535 & 10.448 & 7.69 & 6.53 & 4.162 & 4.006 & 2.246 & 2.218 & 10.669 & 5.92 & 4.792 & 6.662 & 3.796 \\
\hline 8/15/2005 & $19: 00$ & 10.532 & 10.446 & 7.69 & 6.532 & 4.168 & 4.008 & 2.246 & 2.218 & 10.672 & 5.92 & 4.794 & 6.652 & 3.802 \\
\hline
\end{tabular}


TABLE S1.2 (Cont.)

Water Level (ft below top of casing) at Indicated Well

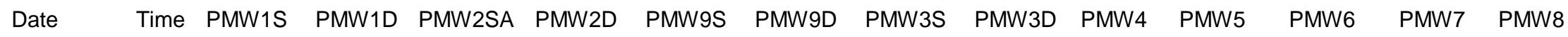

\begin{tabular}{|c|c|c|c|c|c|c|c|c|c|c|c|c|c|c|}
\hline $8 / 15 / 2005$ & $20: 00$ & 10.532 & 10.448 & 7.691 & 6.534 & 4.168 & 4.01 & 2.252 & 2.216 & 10.676 & 5.922 & 4.79 & 6.643 & 3.802 \\
\hline $8 / 15 / 2005$ & $21: 00$ & 10.537 & 10.453 & 7.692 & 6.539 & 4.174 & 4.011 & 2.247 & 2.22 & 10.68 & 5.92 & 4.785 & 6.635 & 3.798 \\
\hline $8 / 15 / 2005$ & $22: 00$ & 10.542 & 10.459 & 7.694 & 6.541 & 4.174 & 4.015 & 2.247 & 2.22 & 10.684 & 5.92 & 4.781 & 6.629 & 3.792 \\
\hline $8 / 15 / 2005$ & $23: 00$ & 10.542 & 10.459 & 7.694 & 6.539 & 4.169 & 4.015 & 2.252 & 2.216 & 10.684 & 5.918 & 4.773 & 6.624 & 3.782 \\
\hline $8 / 16 / 2005$ & $0: 00$ & 10.543 & 10.459 & 7.694 & 6.539 & 4.166 & 4.017 & 2.249 & 2.213 & 10.684 & 5.916 & 4.765 & 6.618 & 3.772 \\
\hline $8 / 16 / 2005$ & $1: 00$ & 10.545 & 10.463 & 7.694 & 6.541 & 4.165 & 4.017 & 2.244 & 2.216 & 10.684 & 5.916 & 4.761 & 6.616 & 3.768 \\
\hline $8 / 16 / 2005$ & $2: 00$ & 10.545 & 10.461 & 7.695 & 6.534 & 4.161 & 4.017 & 2.247 & 2.211 & 10.682 & 5.913 & 4.753 & 6.61 & 3.76 \\
\hline $8 / 16 / 2005$ & $3: 00$ & 10.543 & 10.459 & 7.693 & 6.532 & 4.157 & 4.015 & 2.242 & 2.207 & 10.68 & 5.909 & 4.747 & 6.609 & 3.752 \\
\hline $8 / 16 / 2005$ & $4: 00$ & 10.543 & 10.457 & 7.691 & 6.533 & 4.155 & 4.015 & 2.235 & 2.21 & 10.68 & 5.909 & 4.743 & 6.603 & 3.748 \\
\hline $8 / 16 / 2005$ & $5: 00$ & 10.54 & 10.457 & 7.691 & 6.53 & 4.155 & 4.015 & 2.235 & 2.207 & 10.678 & 5.907 & 4.738 & 6.601 & 3.742 \\
\hline $8 / 16 / 2005$ & $6: 00$ & 10.541 & 10.457 & 7.691 & 6.532 & 4.154 & 4.015 & 2.243 & 2.207 & 10.676 & 5.907 & 4.736 & 6.599 & 3.74 \\
\hline $8 / 16 / 2005$ & $7: 00$ & 10.546 & 10.463 & 7.695 & 6.537 & 4.159 & 4.015 & 2.238 & 2.211 & 10.674 & 5.907 & 4.736 & 6.601 & 3.738 \\
\hline $8 / 16 / 2005$ & $8: 00$ & 10.551 & 10.466 & 7.699 & 6.537 & 4.161 & 4.015 & 2.243 & 2.213 & 10.673 & 5.905 & 4.74 & 6.62 & 3.738 \\
\hline $8 / 16 / 2005$ & $9: 00$ & 10.553 & 10.472 & 7.701 & 6.544 & 4.165 & 4.017 & 2.248 & 2.22 & 10.674 & 5.909 & 4.742 & 6.616 & 3.736 \\
\hline $8 / 16 / 2005$ & $10: 00$ & 10.555 & 10.472 & 7.701 & 6.544 & 4.167 & 4.015 & 2.258 & 2.222 & 10.673 & 5.907 & 4.746 & 6.626 & 3.736 \\
\hline $8 / 16 / 2005$ & $11: 00$ & 10.558 & 10.472 & 7.703 & 6.546 & 4.173 & 4.017 & 2.263 & 2.226 & 10.673 & 5.909 & 4.758 & 6.626 & 3.748 \\
\hline $8 / 16 / 2005$ & $12: 00$ & 10.555 & 10.472 & 7.703 & 6.541 & 4.175 & 4.019 & 2.263 & 2.229 & 10.673 & 5.912 & 4.765 & 6.63 & 3.762 \\
\hline $8 / 16 / 2005$ & $13: 00$ & 10.553 & 10.464 & 7.701 & 6.539 & 4.177 & 4.017 & 2.263 & 2.226 & 10.675 & 5.916 & 4.773 & 6.656 & 3.783 \\
\hline $8 / 16 / 2005$ & $14: 00$ & 10.549 & 10.457 & 7.701 & 6.533 & 4.181 & 4.017 & 2.263 & 2.226 & 10.68 & 5.92 & 4.789 & 6.671 & 3.795 \\
\hline $8 / 16 / 2005$ & $15: 00$ & 10.541 & 10.448 & 7.697 & 6.53 & 4.183 & 4.015 & 2.263 & 2.224 & 10.684 & 5.925 & 4.797 & 6.668 & 3.807 \\
\hline $8 / 16 / 2005$ & $16: 00$ & 10.536 & 10.439 & 7.693 & 6.524 & 4.183 & 4.015 & 2.25 & 2.22 & 10.688 & 5.927 & 4.799 & 6.662 & 3.82 \\
\hline $8 / 16 / 2005$ & $17: 00$ & 10.531 & 10.437 & 7.691 & 6.524 & 4.187 & 4.013 & 2.253 & 2.22 & 10.69 & 5.934 & 4.805 & 6.666 & 3.834 \\
\hline $8 / 16 / 2005$ & $18: 00$ & 10.529 & 10.435 & 7.691 & 6.526 & 4.193 & 4.015 & 2.261 & 2.222 & 10.695 & 5.934 & 4.81 & 6.664 & 3.846 \\
\hline $8 / 16 / 2005$ & 19:00 & 10.526 & 10.432 & 7.691 & 6.522 & 4.191 & 4.013 & 2.248 & 2.218 & 10.699 & 5.934 & 4.81 & 6.65 & 3.85 \\
\hline $8 / 16 / 2005$ & $20: 00$ & 10.524 & 10.432 & 7.691 & 6.526 & 4.193 & 4.015 & 2.253 & 2.216 & 10.705 & 5.937 & 4.805 & 6.643 & 3.85 \\
\hline $8 / 16 / 2005$ & $21: 00$ & 10.524 & 10.433 & 7.691 & 6.526 & 4.193 & 4.017 & 2.253 & 2.216 & 10.705 & 5.934 & 4.797 & 6.633 & 3.844 \\
\hline $8 / 16 / 2005$ & $22: 00$ & 10.529 & 10.441 & 7.691 & 6.53 & 4.195 & 4.019 & 2.248 & 2.218 & 10.707 & 5.934 & 4.793 & 6.63 & 3.838 \\
\hline $8 / 16 / 2005$ & $23: 00$ & 10.529 & 10.437 & 7.691 & 6.526 & 4.189 & 4.017 & 2.251 & 2.214 & 10.707 & 5.93 & 4.781 & 6.622 & 3.824 \\
\hline $8 / 17 / 2005$ & $0: 00$ & 10.524 & 10.433 & 7.687 & 6.522 & 4.179 & 4.017 & 2.236 & 2.205 & 10.705 & 5.928 & 4.772 & 6.615 & 3.81 \\
\hline $8 / 17 / 2005$ & $1: 00$ & 10.522 & 10.428 & 7.683 & 6.517 & 4.17 & 4.015 & 2.228 & 2.199 & 10.701 & 5.926 & 4.762 & 6.607 & 3.798 \\
\hline $8 / 17 / 2005$ & $2: 00$ & 10.517 & 10.424 & 7.682 & 6.51 & 4.164 & 4.013 & 2.223 & 2.193 & 10.697 & 5.919 & 4.752 & 6.601 & 3.786 \\
\hline $8 / 17 / 2005$ & $3: 00$ & 10.515 & 10.421 & 7.68 & 6.513 & 4.158 & 4.008 & 2.226 & 2.189 & 10.695 & 5.919 & 4.746 & 6.597 & 3.776 \\
\hline $8 / 17 / 2005$ & $4: 00$ & 10.51 & 10.415 & 7.674 & 6.506 & 4.152 & 4.006 & 2.213 & 2.184 & 10.69 & 5.913 & 4.736 & 6.592 & 3.768 \\
\hline $8 / 17 / 2005$ & $5: 00$ & 10.505 & 10.412 & 7.672 & 6.502 & 4.148 & 4.002 & 2.213 & 2.178 & 10.688 & 5.911 & 4.731 & 6.588 & 3.758 \\
\hline
\end{tabular}


TABLE S1.2 (Cont.)

Water Level (ft below top of casing) at Indicated Well

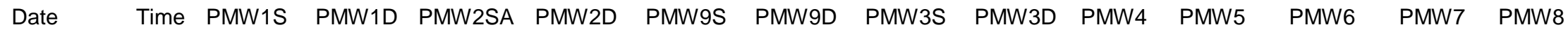

\begin{tabular}{|c|c|c|c|c|c|c|c|c|c|c|c|c|c|c|}
\hline $8 / 17 / 2005$ & $6: 00$ & 10.502 & 10.408 & 7.672 & 6.502 & 4.147 & 3.999 & 2.206 & 2.176 & 10.684 & 5.904 & 4.725 & 6.584 & 3.752 \\
\hline 8/17/2005 & $7: 00$ & 10.497 & 10.402 & 7.668 & 6.495 & 4.141 & 3.995 & 2.204 & 2.17 & 10.678 & 5.902 & 4.719 & 6.58 & 3.743 \\
\hline $8 / 17 / 2005$ & 8:00 & 10.495 & 10.399 & 7.67 & 6.495 & 4.141 & 3.993 & 2.199 & 2.17 & 10.676 & 5.9 & 4.717 & 6.584 & 3.737 \\
\hline 8/17/2005 & $9: 00$ & 10.491 & 10.393 & 7.662 & 6.486 & 4.133 & 3.986 & 2.196 & 2.161 & 10.673 & 5.902 & 4.709 & 6.594 & 3.732 \\
\hline $8 / 17 / 2005$ & $10: 00$ & 10.491 & 10.39 & 7.664 & 6.491 & 4.137 & 3.984 & 2.199 & 2.168 & 10.669 & 5.9 & 4.717 & 6.594 & 3.737 \\
\hline $8 / 17 / 2005$ & $11: 00$ & 10.491 & 10.388 & 7.662 & 6.484 & 4.137 & 3.98 & 2.206 & 2.168 & 10.667 & 5.9 & 4.721 & 6.592 & 3.744 \\
\hline $8 / 17 / 2005$ & $12: 00$ & 10.486 & 10.386 & 7.662 & 6.486 & 4.145 & 3.977 & 2.204 & 2.17 & 10.665 & 5.902 & 4.729 & 6.596 & 3.758 \\
\hline $8 / 17 / 2005$ & $13: 00$ & 10.481 & 10.379 & 7.66 & 6.479 & 4.143 & 3.973 & 2.201 & 2.164 & 10.667 & 5.904 & 4.731 & 6.597 & 3.768 \\
\hline $8 / 17 / 2005$ & $14: 00$ & 10.471 & 10.366 & 7.652 & 6.471 & 4.137 & 3.966 & 2.189 & 2.151 & 10.664 & 5.903 & 4.729 & 6.594 & 3.764 \\
\hline $8 / 17 / 2005$ & $15: 00$ & 10.466 & 10.36 & 7.651 & 6.468 & 4.139 & 3.964 & 2.184 & 2.156 & 10.665 & 5.907 & 4.735 & 6.601 & 3.78 \\
\hline $8 / 17 / 2005$ & $16: 00$ & 10.462 & 10.353 & 7.647 & 6.466 & 4.145 & 3.962 & 2.191 & 2.153 & 10.667 & 5.911 & 4.743 & 6.603 & 3.795 \\
\hline $8 / 17 / 2005$ & $17: 00$ & 10.454 & 10.346 & 7.647 & 6.462 & 4.147 & 3.957 & 2.189 & 2.151 & 10.669 & 5.914 & 4.753 & 6.615 & 3.809 \\
\hline $8 / 17 / 2005$ & $18: 00$ & 10.452 & 10.342 & 7.643 & 6.46 & 4.151 & 3.955 & 2.186 & 2.147 & 10.671 & 5.915 & 4.753 & 6.601 & 3.817 \\
\hline $8 / 17 / 2005$ & $19: 00$ & 10.447 & 10.337 & 7.643 & 6.455 & 4.153 & 3.955 & 2.176 & 2.145 & 10.675 & 5.915 & 4.751 & 6.592 & 3.819 \\
\hline 8/17/2005 & $20: 00$ & 10.442 & 10.331 & 7.639 & 6.455 & 4.151 & 3.953 & 2.176 & 2.141 & 10.675 & 5.916 & 4.745 & 6.582 & 3.815 \\
\hline $8 / 17 / 2005$ & $21: 00$ & 10.437 & 10.333 & 7.641 & 6.457 & 4.155 & 3.955 & 2.176 & 2.141 & 10.677 & 5.914 & 4.739 & 6.575 & 3.813 \\
\hline 8/17/2005 & $22: 00$ & 10.437 & 10.331 & 7.639 & 6.455 & 4.151 & 3.953 & 2.174 & 2.139 & 10.675 & 5.914 & 4.729 & 6.567 & 3.803 \\
\hline $8 / 17 / 2005$ & 23:00 & 10.435 & 10.331 & 7.639 & 6.453 & 4.147 & 3.953 & 2.171 & 2.137 & 10.675 & 5.909 & 4.72 & 6.562 & 3.795 \\
\hline 8/18/2005 & $0: 00$ & 10.432 & 10.329 & 7.637 & 6.451 & 4.141 & 3.951 & 2.166 & 2.132 & 10.671 & 5.903 & 4.712 & 6.555 & 3.783 \\
\hline $8 / 18 / 2005$ & $1: 00$ & 10.432 & 10.326 & 7.635 & 6.448 & 4.136 & 3.949 & 2.164 & 2.128 & 10.667 & 5.901 & 4.702 & 6.552 & 3.773 \\
\hline 8/18/2005 & $2: 00$ & 10.43 & 10.324 & 7.632 & 6.442 & 4.128 & 3.946 & 2.154 & 2.12 & 10.664 & 5.901 & 4.694 & 6.55 & 3.761 \\
\hline $8 / 18 / 2005$ & $3: 00$ & 10.42 & 10.313 & 7.628 & 6.435 & 4.116 & 3.942 & 2.142 & 2.114 & 10.66 & 5.894 & 4.684 & 6.544 & 3.749 \\
\hline $8 / 18 / 2005$ & $4: 00$ & 10.415 & 10.304 & 7.624 & 6.424 & 4.107 & 3.935 & 2.137 & 2.103 & 10.654 & 5.888 & 4.671 & 6.539 & 3.733 \\
\hline 8/18/2005 & $5: 00$ & 10.415 & 10.309 & 7.628 & 6.435 & 4.117 & 3.935 & 2.142 & 2.111 & 10.65 & 5.889 & 4.673 & 6.539 & 3.737 \\
\hline 8/18/2005 & $6: 00$ & 10.42 & 10.318 & 7.632 & 6.444 & 4.126 & 3.935 & 2.155 & 2.12 & 10.65 & 5.892 & 4.675 & 6.541 & 3.743 \\
\hline $8 / 18 / 2005$ & $7: 00$ & 10.42 & 10.318 & 7.632 & 6.442 & 4.124 & 3.935 & 2.155 & 2.12 & 10.648 & 5.89 & 4.673 & 6.541 & 3.739 \\
\hline $8 / 18 / 2005$ & $8: 00$ & 10.425 & 10.329 & 7.636 & 6.448 & 4.134 & 3.938 & 2.155 & 2.131 & 10.648 & 5.892 & 4.677 & 6.552 & 3.745 \\
\hline $8 / 18 / 2005$ & 9:00 & 10.433 & 10.335 & 7.64 & 6.457 & 4.14 & 3.94 & 2.165 & 2.137 & 10.648 & 5.897 & 4.689 & 6.552 & 3.755 \\
\hline $8 / 18 / 2005$ & $10: 00$ & 10.435 & 10.34 & 7.643 & 6.462 & 4.149 & 3.94 & 2.182 & 2.148 & 10.65 & 5.901 & 4.7 & 6.56 & 3.769 \\
\hline $8 / 18 / 2005$ & $11: 00$ & 10.443 & 10.348 & 7.648 & 6.468 & 4.163 & 3.944 & 2.185 & 2.156 & 10.652 & 5.906 & 4.716 & 6.571 & 3.791 \\
\hline 8/18/2005 & $12: 00$ & 10.448 & 10.351 & 7.649 & 6.471 & 4.173 & 3.949 & 2.2 & 2.164 & 10.658 & 5.914 & 4.738 & 6.586 & 3.816 \\
\hline 8/18/2005 & $13: 00$ & 10.448 & 10.351 & 7.651 & 6.468 & 4.178 & 3.951 & 2.2 & 2.166 & 10.664 & 5.919 & 4.759 & 6.598 & 3.84 \\
\hline $8 / 18 / 2005$ & $14: 00$ & 10.45 & 10.353 & 7.651 & 6.473 & 4.188 & 3.953 & 2.208 & 2.173 & 10.671 & 5.926 & 4.777 & 6.619 & 3.863 \\
\hline $8 / 18 / 2005$ & $15: 00$ & 10.453 & 10.355 & 7.653 & 6.475 & 4.198 & 3.957 & 2.213 & 2.177 & 10.681 & 5.935 & 4.801 & 6.63 & 3.887 \\
\hline
\end{tabular}


TABLE S1.2 (Cont.)

Water Level (ft below top of casing) at Indicated Well

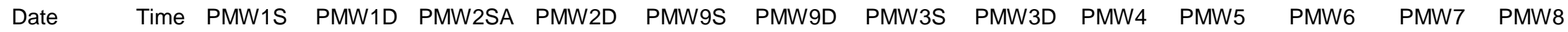

\begin{tabular}{|c|c|c|c|c|c|c|c|c|c|c|c|c|c|c|}
\hline $8 / 18 / 2005$ & $16: 00$ & 10.453 & 10.353 & 7.655 & 6.475 & 4.204 & 3.96 & 2.218 & 2.179 & 10.688 & 5.939 & 4.812 & 6.638 & 3.905 \\
\hline $8 / 18 / 2005$ & $17: 00$ & 10.453 & 10.353 & 7.655 & 6.478 & 4.211 & 3.964 & 2.218 & 2.183 & 10.696 & 5.946 & 4.82 & 6.634 & 3.92 \\
\hline $8 / 18 / 2005$ & $18: 00$ & 10.456 & 10.355 & 7.657 & 6.48 & 4.215 & 3.969 & 2.223 & 2.185 & 10.703 & 5.948 & 4.826 & 6.632 & 3.932 \\
\hline $8 / 18 / 2005$ & $19: 00$ & 10.453 & 10.355 & 7.656 & 6.48 & 4.219 & 3.971 & 2.218 & 2.181 & 10.707 & 5.95 & 4.822 & 6.624 & 3.932 \\
\hline $8 / 18 / 2005$ & $20: 00$ & 10.453 & 10.355 & 7.656 & 6.478 & 4.215 & 3.973 & 2.213 & 2.177 & 10.713 & 5.95 & 4.812 & 6.611 & 3.924 \\
\hline $8 / 18 / 2005$ & $21: 00$ & 10.458 & 10.36 & 7.656 & 6.482 & 4.213 & 3.977 & 2.213 & 2.177 & 10.715 & 5.95 & 4.801 & 6.6 & 3.914 \\
\hline $8 / 18 / 2005$ & $22: 00$ & 10.463 & 10.366 & 7.66 & 6.486 & 4.211 & 3.982 & 2.208 & 2.179 & 10.717 & 5.946 & 4.793 & 6.596 & 3.906 \\
\hline $8 / 18 / 2005$ & $23: 00$ & 10.466 & 10.371 & 7.66 & 6.486 & 4.205 & 3.984 & 2.205 & 2.177 & 10.719 & 5.944 & 4.785 & 6.59 & 3.892 \\
\hline $8 / 19 / 2005$ & $0: 00$ & 10.473 & 10.379 & 7.663 & 6.493 & 4.206 & 3.986 & 2.211 & 2.183 & 10.721 & 5.944 & 4.779 & 6.588 & 3.886 \\
\hline $8 / 19 / 2005$ & $1: 00$ & 10.478 & 10.384 & 7.663 & 6.493 & 4.202 & 3.988 & 2.218 & 2.181 & 10.721 & 5.942 & 4.769 & 6.585 & 3.874 \\
\hline $8 / 19 / 2005$ & $2: 00$ & 10.481 & 10.388 & 7.663 & 6.493 & 4.196 & 3.988 & 2.216 & 2.179 & 10.719 & 5.939 & 4.764 & 6.579 & 3.862 \\
\hline $8 / 19 / 2005$ & $3: 00$ & 10.481 & 10.386 & 7.665 & 6.493 & 4.19 & 3.991 & 2.205 & 2.177 & 10.717 & 5.937 & 4.758 & 6.577 & 3.852 \\
\hline $8 / 19 / 2005$ & $4: 00$ & 10.486 & 10.391 & 7.665 & 6.495 & 4.188 & 3.991 & 2.211 & 2.181 & 10.717 & 5.938 & 4.75 & 6.575 & 3.845 \\
\hline $8 / 19 / 2005$ & $5: 00$ & 10.486 & 10.393 & 7.665 & 6.495 & 4.184 & 3.991 & 2.211 & 2.177 & 10.713 & 5.931 & 4.744 & 6.577 & 3.835 \\
\hline $8 / 19 / 2005$ & $6: 00$ & 10.488 & 10.399 & 7.667 & 6.5 & 4.183 & 3.991 & 2.206 & 2.179 & 10.711 & 5.931 & 4.742 & 6.575 & 3.831 \\
\hline $8 / 19 / 2005$ & $7: 00$ & 10.491 & 10.401 & 7.67 & 6.498 & 4.183 & 3.991 & 2.214 & 2.179 & 10.71 & 5.931 & 4.738 & 6.577 & 3.825 \\
\hline $8 / 19 / 2005$ & 8:00 & 10.499 & 10.408 & 7.668 & 6.504 & 4.186 & 3.993 & 2.219 & 2.181 & 10.708 & 5.929 & 4.74 & 6.583 & 3.821 \\
\hline $8 / 19 / 2005$ & $9: 00$ & 10.504 & 10.413 & 7.672 & 6.506 & 4.188 & 3.995 & 2.219 & 2.19 & 10.708 & 5.929 & 4.74 & 6.581 & 3.821 \\
\hline $8 / 19 / 2005$ & $10: 00$ & 10.509 & 10.417 & 7.673 & 6.511 & 4.192 & 3.995 & 2.229 & 2.194 & 10.706 & 5.933 & 4.746 & 6.585 & 3.823 \\
\hline $8 / 19 / 2005$ & $11: 00$ & 10.511 & 10.419 & 7.676 & 6.513 & 4.198 & 3.995 & 2.234 & 2.2 & 10.706 & 5.935 & 4.756 & 6.621 & 3.831 \\
\hline $8 / 19 / 2005$ & $12: 00$ & 10.509 & 10.415 & 7.676 & 6.511 & 4.202 & 3.995 & 2.229 & 2.2 & 10.708 & 5.936 & 4.776 & 6.623 & 3.844 \\
\hline $8 / 19 / 2005$ & $13: 00$ & 10.516 & 10.426 & 7.679 & 6.522 & 4.218 & 3.999 & 2.242 & 2.211 & 10.715 & 5.945 & 4.786 & 6.621 & 3.864 \\
\hline $8 / 19 / 2005$ & $14: 00$ & 10.516 & 10.422 & 7.681 & 6.517 & 4.221 & 4.002 & 2.247 & 2.209 & 10.717 & 5.942 & 4.793 & 6.651 & 3.87 \\
\hline $8 / 19 / 2005$ & $15: 00$ & 10.521 & 10.433 & 7.685 & 6.529 & 4.229 & 4.006 & 2.247 & 2.219 & 10.721 & 5.951 & 4.799 & 6.651 & 3.882 \\
\hline $8 / 19 / 2005$ & $16: 00$ & 10.516 & 10.421 & 7.679 & 6.513 & 4.221 & 4.004 & 2.235 & 2.205 & 10.721 & 5.947 & 4.79 & 6.632 & 3.876 \\
\hline 8/19/2005 & $17: 00$ & 10.516 & 10.421 & 7.679 & 6.517 & 4.221 & 4.006 & 2.24 & 2.207 & 10.721 & 5.947 & 4.784 & 6.573 & 3.878 \\
\hline $8 / 19 / 2005$ & $18: 00$ & 10.516 & 10.39 & 7.679 & 6.515 & 4.218 & 4.008 & 2.235 & 2.173 & 10.723 & 5.945 & 4.776 & 6.568 & 3.866 \\
\hline $8 / 19 / 2005$ & $19: 00$ & 10.514 & 10.386 & 7.678 & 6.509 & 4.212 & 4.006 & 2.222 & 2.158 & 10.721 & 5.938 & 4.76 & 6.606 & 3.852 \\
\hline $8 / 19 / 2005$ & $20: 00$ & 10.514 & 10.415 & 7.674 & 6.504 & 4.204 & 4.006 & 2.222 & 2.19 & 10.719 & 5.936 & 4.75 & 6.596 & 3.84 \\
\hline $8 / 19 / 2005$ & $21: 00$ & 10.524 & 10.43 & 7.682 & 6.522 & 4.211 & 4.011 & 2.23 & 2.205 & 10.719 & 5.938 & 4.75 & 6.598 & 3.842 \\
\hline $8 / 19 / 2005$ & $22: 00$ & 10.524 & 10.433 & 7.682 & 6.522 & 4.21 & 4.011 & 2.235 & 2.2 & 10.717 & 5.938 & 4.745 & 6.592 & 3.83 \\
\hline $8 / 19 / 2005$ & $23: 00$ & 10.527 & 10.433 & 7.682 & 6.522 & 4.206 & 4.011 & 2.232 & 2.198 & 10.716 & 5.934 & 4.739 & 6.589 & 3.82 \\
\hline $8 / 20 / 2005$ & $0: 00$ & 10.532 & 10.441 & 7.686 & 6.526 & 4.206 & 4.013 & 2.23 & 2.203 & 10.714 & 5.936 & 4.737 & 6.589 & 3.816 \\
\hline $8 / 20 / 2005$ & $1: 00$ & 10.539 & 10.453 & 7.692 & 6.542 & 4.216 & 4.017 & 2.251 & 2.213 & 10.716 & 5.939 & 4.741 & 6.592 & 3.822 \\
\hline
\end{tabular}


TABLE S1.2 (Cont.)

Water Level (ft below top of casing) at Indicated Well

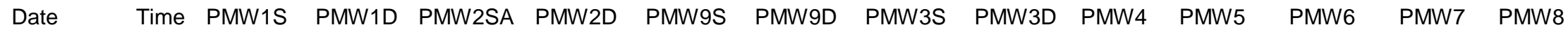

\begin{tabular}{|c|c|c|c|c|c|c|c|c|c|c|c|c|c|c|}
\hline 8/20/2005 & $2: 00$ & 10.544 & 10.455 & 7.693 & 6.537 & 4.212 & 4.019 & 2.24 & 2.213 & 10.714 & 5.934 & 4.735 & 6.589 & 3.81 \\
\hline $8 / 20 / 2005$ & $3: 00$ & 10.546 & 10.455 & 7.693 & 6.54 & 4.21 & 4.019 & 2.248 & 2.213 & 10.712 & 5.932 & 4.733 & 6.589 & 3.804 \\
\hline 8/20/2005 & $4: 00$ & 10.544 & 10.452 & 7.69 & 6.533 & 4.2 & 4.019 & 2.24 & 2.205 & 10.706 & 5.928 & 4.727 & 6.583 & 3.794 \\
\hline 8/20/2005 & $5: 00$ & 10.549 & 10.463 & 7.696 & 6.542 & 4.208 & 4.022 & 2.245 & 2.213 & 10.706 & 5.932 & 4.729 & 6.587 & 3.794 \\
\hline 8/20/2005 & $6: 00$ & 10.557 & 10.47 & 7.7 & 6.549 & 4.214 & 4.024 & 2.253 & 2.219 & 10.706 & 5.932 & 4.733 & 6.589 & 3.796 \\
\hline 8/20/2005 & 7:00 & 10.561 & 10.475 & 7.703 & 6.551 & 4.216 & 4.026 & 2.259 & 2.224 & 10.706 & 5.932 & 4.733 & 6.591 & 3.794 \\
\hline $8 / 20 / 2005$ & $8: 00$ & 10.564 & 10.475 & 7.703 & 6.551 & 4.214 & 4.028 & 2.259 & 2.224 & 10.706 & 5.93 & 4.731 & 6.587 & 3.786 \\
\hline 8/20/2005 & 9:00 & 10.569 & 10.481 & 7.705 & 6.553 & 4.216 & 4.028 & 2.261 & 2.228 & 10.704 & 5.928 & 4.737 & 6.593 & 3.788 \\
\hline 8/20/2005 & $10: 00$ & 10.574 & 10.488 & 7.711 & 6.562 & 4.225 & 4.033 & 2.274 & 2.238 & 10.706 & 5.935 & 4.751 & 6.602 & 3.798 \\
\hline 8/20/2005 & $11: 00$ & 10.579 & 10.494 & 7.715 & 6.566 & 4.235 & 4.035 & 2.279 & 2.247 & 10.706 & 5.941 & 4.765 & 6.614 & 3.812 \\
\hline $8 / 20 / 2005$ & $12: 00$ & 10.582 & 10.494 & 7.717 & 6.566 & 4.239 & 4.038 & 2.276 & 2.251 & 10.71 & 5.944 & 4.779 & 6.623 & 3.829 \\
\hline 8/20/2005 & $13: 00$ & 10.582 & 10.494 & 7.717 & 6.562 & 4.245 & 4.037 & 2.286 & 2.253 & 10.716 & 5.95 & 4.794 & 6.637 & 3.849 \\
\hline $8 / 20 / 2005$ & $14: 00$ & 10.582 & 10.492 & 7.719 & 6.562 & 4.25 & 4.04 & 2.289 & 2.255 & 10.719 & 5.957 & 4.81 & 6.646 & 3.875 \\
\hline 8/20/2005 & $15: 00$ & 10.582 & 10.488 & 7.719 & 6.562 & 4.26 & 4.04 & 2.286 & 2.257 & 10.727 & 5.963 & 4.824 & 6.656 & 3.897 \\
\hline 8/20/2005 & $16: 00$ & 10.579 & 10.481 & 7.715 & 6.56 & 4.264 & 4.042 & 2.294 & 2.258 & 10.731 & 5.966 & 4.837 & 6.661 & 3.918 \\
\hline 8/20/2005 & $17: 00$ & 10.574 & 10.475 & 7.713 & 6.555 & 4.266 & 4.042 & 2.289 & 2.255 & 10.735 & 5.97 & 4.843 & 6.661 & 3.934 \\
\hline 8/20/2005 & $18: 00$ & 10.569 & 10.47 & 7.711 & 6.553 & 4.271 & 4.044 & 2.289 & 2.253 & 10.74 & 5.975 & 4.849 & 6.663 & 3.949 \\
\hline 8/20/2005 & $19: 00$ & 10.569 & 10.474 & 7.713 & 6.558 & 4.279 & 4.046 & 2.289 & 2.255 & 10.746 & 5.979 & 4.855 & 6.659 & 3.961 \\
\hline 8/20/2005 & 20:00 & 10.572 & 10.475 & 7.715 & 6.558 & 4.281 & 4.049 & 2.281 & 2.253 & 10.75 & 5.979 & 4.849 & 6.65 & 3.961 \\
\hline $8 / 20 / 2005$ & $21: 00$ & 10.58 & 10.486 & 7.719 & 6.564 & 4.285 & 4.053 & 2.291 & 2.257 & 10.754 & 5.977 & 4.843 & 6.644 & 3.953 \\
\hline 8/20/2005 & $22: 00$ & 10.582 & 10.492 & 7.721 & 6.571 & 4.281 & 4.057 & 2.294 & 2.259 & 10.756 & 5.977 & 4.835 & 6.637 & 3.941 \\
\hline 8/20/2005 & $23: 00$ & 10.584 & 10.492 & 7.721 & 6.569 & 4.273 & 4.06 & 2.284 & 2.257 & 10.757 & 5.973 & 4.824 & 6.631 & 3.927 \\
\hline 8/21/2005 & $0: 00$ & 10.584 & 10.492 & 7.721 & 6.567 & 4.266 & 4.06 & 2.287 & 2.253 & 10.758 & 5.968 & 4.812 & 6.625 & 3.911 \\
\hline $8 / 21 / 2005$ & $1: 00$ & 10.584 & 10.494 & 7.721 & 6.567 & 4.26 & 4.062 & 2.277 & 2.253 & 10.757 & 5.968 & 4.806 & 6.623 & 3.901 \\
\hline $8 / 21 / 2005$ & $2: 00$ & 10.584 & 10.492 & 7.719 & 6.564 & 4.252 & 4.06 & 2.28 & 2.247 & 10.756 & 5.964 & 4.796 & 6.617 & 3.886 \\
\hline $8 / 21 / 2005$ & $3: 00$ & 10.587 & 10.492 & 7.719 & 6.562 & 4.246 & 4.062 & 2.28 & 2.247 & 10.752 & 5.962 & 4.791 & 6.614 & 3.874 \\
\hline 8/21/2005 & $4: 00$ & 10.587 & 10.492 & 7.719 & 6.562 & 4.241 & 4.06 & 2.269 & 2.242 & 10.752 & 5.96 & 4.783 & 6.612 & 3.864 \\
\hline $8 / 21 / 2005$ & $5: 00$ & 10.587 & 10.492 & 7.717 & 6.562 & 4.239 & 4.06 & 2.277 & 2.243 & 10.75 & 5.962 & 4.779 & 6.61 & 3.856 \\
\hline $8 / 21 / 2005$ & $6: 00$ & 10.587 & 10.494 & 7.72 & 6.562 & 4.237 & 4.057 & 2.277 & 2.243 & 10.746 & 5.955 & 4.775 & 6.608 & 3.85 \\
\hline 8/21/2005 & $7: 00$ & 10.59 & 10.497 & 7.72 & 6.567 & 4.237 & 4.057 & 2.28 & 2.245 & 10.746 & 5.956 & 4.769 & 6.606 & 3.844 \\
\hline $8 / 21 / 2005$ & $8: 00$ & 10.592 & 10.501 & 7.723 & 6.571 & 4.237 & 4.057 & 2.282 & 2.247 & 10.744 & 5.956 & 4.767 & 6.606 & 3.838 \\
\hline $8 / 21 / 2005$ & 9:00 & 10.592 & 10.503 & 7.723 & 6.569 & 4.239 & 4.057 & 2.28 & 2.251 & 10.741 & 5.956 & 4.769 & 6.61 & 3.836 \\
\hline $8 / 21 / 2005$ & $10: 00$ & 10.595 & 10.501 & 7.726 & 6.569 & 4.239 & 4.055 & 2.29 & 2.251 & 10.741 & 5.957 & 4.775 & 6.612 & 3.838 \\
\hline $8 / 21 / 2005$ & $11: 00$ & 10.595 & 10.503 & 7.728 & 6.573 & 4.247 & 4.055 & 2.295 & 2.258 & 10.741 & 5.96 & 4.787 & 6.625 & 3.848 \\
\hline
\end{tabular}


TABLE S1.2 (Cont.)

Water Level (ft below top of casing) at Indicated Well

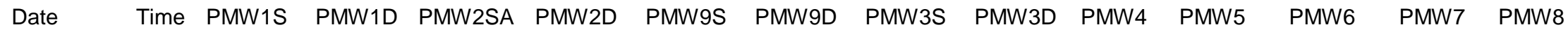

\begin{tabular}{|c|c|c|c|c|c|c|c|c|c|c|c|c|c|c|}
\hline $8 / 21 / 2005$ & $12: 00$ & 10.595 & 10.501 & 7.728 & 6.571 & 4.255 & 4.055 & 2.3 & 2.26 & 10.744 & 5.967 & 4.801 & 6.639 & 3.868 \\
\hline $8 / 21 / 2005$ & $13: 00$ & 10.593 & 10.494 & 7.727 & 6.567 & 4.262 & 4.055 & 2.3 & 2.26 & 10.748 & 5.971 & 4.819 & 6.65 & 3.89 \\
\hline $8 / 21 / 2005$ & $14: 00$ & 10.585 & 10.486 & 7.723 & 6.562 & 4.272 & 4.053 & 2.293 & 2.262 & 10.752 & 5.978 & 4.832 & 6.658 & 3.913 \\
\hline 8/21/2005 & $15: 00$ & 10.58 & 10.481 & 7.723 & 6.564 & 4.281 & 4.055 & 2.295 & 2.264 & 10.758 & 5.984 & 4.85 & 6.669 & 3.939 \\
\hline 8/21/2005 & $16: 00$ & 10.573 & 10.47 & 7.722 & 6.553 & 4.283 & 4.053 & 2.292 & 2.258 & 10.762 & 5.987 & 4.86 & 6.673 & 3.958 \\
\hline 8/21/2005 & $17: 00$ & 10.566 & 10.463 & 7.718 & 6.551 & 4.285 & 4.051 & 2.292 & 2.253 & 10.767 & 5.991 & 4.868 & 6.669 & 3.974 \\
\hline $8 / 21 / 2005$ & $18: 00$ & 10.563 & 10.461 & 7.716 & 6.551 & 4.293 & 4.053 & 2.287 & 2.256 & 10.769 & 5.995 & 4.873 & 6.671 & 3.986 \\
\hline $8 / 21 / 2005$ & $19: 00$ & 10.563 & 10.464 & 7.72 & 6.558 & 4.301 & 4.055 & 2.293 & 2.26 & 10.775 & 5.998 & 4.879 & 6.667 & 3.999 \\
\hline $8 / 21 / 2005$ & $20: 00$ & 10.561 & 10.461 & 7.718 & 6.553 & 4.295 & 4.057 & 2.285 & 2.253 & 10.777 & 5.995 & 4.864 & 6.652 & 3.989 \\
\hline 8/21/2005 & $21: 00$ & 10.563 & 10.464 & 7.718 & 6.556 & 4.293 & 4.057 & 2.288 & 2.249 & 10.779 & 5.993 & 4.854 & 6.644 & 3.975 \\
\hline $8 / 21 / 2005$ & $22: 00$ & 10.569 & 10.47 & 7.718 & 6.558 & 4.289 & 4.06 & 2.288 & 2.249 & 10.781 & 5.989 & 4.844 & 6.637 & 3.96 \\
\hline $8 / 21 / 2005$ & $23: 00$ & 10.571 & 10.474 & 7.72 & 6.56 & 4.285 & 4.062 & 2.288 & 2.249 & 10.781 & 5.985 & 4.835 & 6.631 & 3.947 \\
\hline $8 / 22 / 2005$ & $0: 00$ & 10.574 & 10.475 & 7.718 & 6.558 & 4.28 & 4.062 & 2.278 & 2.247 & 10.781 & 5.983 & 4.824 & 6.626 & 3.932 \\
\hline 8/22/2005 & $1: 00$ & 10.576 & 10.477 & 7.718 & 6.56 & 4.27 & 4.062 & 2.283 & 2.245 & 10.779 & 5.983 & 4.815 & 6.62 & 3.92 \\
\hline 8/22/2005 & $2: 00$ & 10.574 & 10.477 & 7.718 & 6.556 & 4.264 & 4.062 & 2.27 & 2.241 & 10.777 & 5.981 & 4.805 & 6.616 & 3.906 \\
\hline 8/22/2005 & 3:00 & 10.569 & 10.472 & 7.714 & 6.549 & 4.255 & 4.057 & 2.273 & 2.235 & 10.773 & 5.976 & 4.795 & 6.61 & 3.892 \\
\hline 8/22/2005 & 4:00 & 10.569 & 10.47 & 7.712 & 6.549 & 4.249 & 4.057 & 2.27 & 2.233 & 10.769 & 5.974 & 4.788 & 6.609 & 3.882 \\
\hline 8/22/2005 & $5: 00$ & 10.569 & 10.468 & 7.71 & 6.547 & 4.241 & 4.055 & 2.265 & 2.229 & 10.763 & 5.97 & 4.782 & 6.605 & 3.872 \\
\hline 8/22/2005 & $6: 00$ & 10.574 & 10.475 & 7.714 & 6.553 & 4.245 & 4.055 & 2.27 & 2.233 & 10.762 & 5.97 & 4.78 & 6.605 & 3.868 \\
\hline 8/22/2005 & $7: 00$ & 10.574 & 10.479 & 7.716 & 6.556 & 4.243 & 4.053 & 2.268 & 2.237 & 10.76 & 5.967 & 4.776 & 6.605 & 3.863 \\
\hline 8/22/2005 & 8:00 & 10.581 & 10.484 & 7.718 & 6.56 & 4.245 & 4.053 & 2.278 & 2.241 & 10.76 & 5.967 & 4.782 & 6.641 & 3.859 \\
\hline $8 / 22 / 2005$ & $9: 00$ & 10.584 & 10.488 & 7.72 & 6.563 & 4.247 & 4.053 & 2.283 & 2.245 & 10.758 & 5.967 & 4.784 & 6.641 & 3.859 \\
\hline 8/22/2005 & $10: 00$ & 10.584 & 10.49 & 7.722 & 6.564 & 4.249 & 4.051 & 2.283 & 2.245 & 10.756 & 5.968 & 4.784 & 6.633 & 3.857 \\
\hline $8 / 22 / 2005$ & $11: 00$ & 10.587 & 10.492 & 7.724 & 6.567 & 4.253 & 4.051 & 2.288 & 2.25 & 10.754 & 5.968 & 4.79 & 6.637 & 3.863 \\
\hline 8/22/2005 & $12: 00$ & 10.584 & 10.486 & 7.722 & 6.56 & 4.251 & 4.049 & 2.278 & 2.248 & 10.754 & 5.968 & 4.791 & 6.641 & 3.866 \\
\hline $8 / 22 / 2005$ & $13: 00$ & 10.577 & 10.474 & 7.716 & 6.549 & 4.247 & 4.046 & 2.271 & 2.241 & 10.752 & 5.968 & 4.798 & 6.633 & 3.87 \\
\hline 8/22/2005 & $14: 00$ & 10.57 & 10.468 & 7.713 & 6.547 & 4.251 & 4.044 & 2.276 & 2.239 & 10.752 & 5.974 & 4.808 & 6.643 & 3.88 \\
\hline $8 / 22 / 2005$ & $15: 00$ & 10.565 & 10.463 & 7.711 & 6.544 & 4.249 & 4.042 & 2.271 & 2.233 & 10.752 & 5.97 & 4.802 & 6.637 & 3.874 \\
\hline 8/22/2005 & $16: 00$ & 10.56 & 10.453 & 7.707 & 6.536 & 4.245 & 4.038 & 2.256 & 2.222 & 10.751 & 5.966 & 4.796 & 6.631 & 3.858 \\
\hline 8/22/2005 & $17: 00$ & 10.555 & 10.45 & 7.703 & 6.533 & 4.241 & 4.035 & 2.254 & 2.222 & 10.749 & 5.968 & 4.796 & 6.63 & 3.852 \\
\hline 8/22/2005 & $18: 00$ & 10.552 & 10.444 & 7.701 & 6.529 & 4.241 & 4.033 & 2.256 & 2.22 & 10.747 & 5.966 & 4.794 & 6.628 & 3.848 \\
\hline 8/22/2005 & 19:00 & 10.545 & 10.439 & 7.697 & 6.527 & 4.238 & 4.031 & 2.252 & 2.212 & 10.745 & 5.961 & 4.788 & 6.62 & 3.84 \\
\hline 8/22/2005 & $20: 00$ & 10.547 & 10.444 & 7.699 & 6.532 & 4.241 & 4.031 & 2.246 & 2.216 & 10.745 & 5.963 & 4.784 & 6.615 & 3.836 \\
\hline 8/22/2005 & $21: 00$ & 10.548 & 10.446 & 7.699 & 6.532 & 4.241 & 4.029 & 2.252 & 2.214 & 10.743 & 5.962 & 4.778 & 6.605 & 3.83 \\
\hline
\end{tabular}


TABLE S1.2 (Cont.)

Water Level (ft below top of casing) at Indicated Well

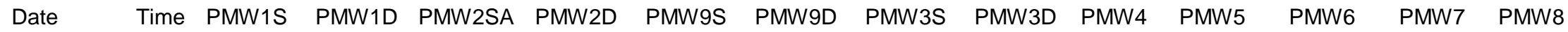

\begin{tabular}{|c|c|c|c|c|c|c|c|c|c|c|c|c|c|c|}
\hline $8 / 22 / 2005$ & $22: 00$ & 10.55 & 10.45 & 7.701 & 6.536 & 4.239 & 4.031 & 2.249 & 2.218 & 10.743 & 5.959 & 4.772 & 6.603 & 3.826 \\
\hline $8 / 22 / 2005$ & $23: 00$ & 10.552 & 10.452 & 7.703 & 6.536 & 4.239 & 4.031 & 2.247 & 2.218 & 10.739 & 5.955 & 4.768 & 6.601 & 3.82 \\
\hline $8 / 23 / 2005$ & $0: 00$ & 10.555 & 10.457 & 7.705 & 6.538 & 4.241 & 4.031 & 2.252 & 2.222 & 10.739 & 5.957 & 4.764 & 6.599 & 3.816 \\
\hline $8 / 23 / 2005$ & $1: 00$ & 10.555 & 10.457 & 7.705 & 6.538 & 4.236 & 4.029 & 2.257 & 2.219 & 10.737 & 5.953 & 4.761 & 6.597 & 3.808 \\
\hline $8 / 23 / 2005$ & $2: 00$ & 10.555 & 10.455 & 7.703 & 6.536 & 4.23 & 4.031 & 2.252 & 2.216 & 10.733 & 5.951 & 4.755 & 6.593 & 3.8 \\
\hline $8 / 23 / 2005$ & $3: 00$ & 10.553 & 10.452 & 7.701 & 6.532 & 4.226 & 4.029 & 2.242 & 2.212 & 10.73 & 5.946 & 4.749 & 6.59 & 3.793 \\
\hline $8 / 23 / 2005$ & $4: 00$ & 10.55 & 10.45 & 7.697 & 6.529 & 4.222 & 4.026 & 2.244 & 2.208 & 10.728 & 5.948 & 4.745 & 6.586 & 3.788 \\
\hline $8 / 23 / 2005$ & $5: 00$ & 10.548 & 10.448 & 7.697 & 6.527 & 4.22 & 4.024 & 2.237 & 2.206 & 10.724 & 5.946 & 4.741 & 6.586 & 3.784 \\
\hline $8 / 23 / 2005$ & $6: 00$ & 10.553 & 10.453 & 7.7 & 6.532 & 4.224 & 4.024 & 2.247 & 2.21 & 10.722 & 5.944 & 4.741 & 6.586 & 3.784 \\
\hline 8/23/2005 & $7: 00$ & 10.553 & 10.455 & 7.701 & 6.534 & 4.224 & 4.024 & 2.242 & 2.212 & 10.72 & 5.942 & 4.737 & 6.586 & 3.782 \\
\hline $8 / 23 / 2005$ & 8:00 & 10.558 & 10.459 & 7.703 & 6.536 & 4.226 & 4.024 & 2.252 & 2.214 & 10.718 & 5.942 & 4.745 & 6.636 & 3.782 \\
\hline $8 / 23 / 2005$ & $9: 00$ & 10.558 & 10.459 & 7.703 & 6.534 & 4.226 & 4.026 & 2.249 & 2.214 & 10.717 & 5.94 & 4.747 & 6.649 & 3.78 \\
\hline $8 / 23 / 2005$ & $10: 00$ & 10.558 & 10.457 & 7.703 & 6.536 & 4.226 & 4.024 & 2.252 & 2.214 & 10.717 & 5.942 & 4.745 & 6.624 & 3.778 \\
\hline 8/23/2005 & $11: 00$ & 10.561 & 10.463 & 7.707 & 6.54 & 4.232 & 4.024 & 2.257 & 2.219 & 10.715 & 5.944 & 4.745 & 6.616 & 3.784 \\
\hline $8 / 23 / 2005$ & $12: 00$ & 10.564 & 10.464 & 7.707 & 6.54 & 4.236 & 4.024 & 2.26 & 2.225 & 10.715 & 5.945 & 4.749 & 6.624 & 3.79 \\
\hline $8 / 23 / 2005$ & $13: 00$ & 10.558 & 10.459 & 7.707 & 6.534 & 4.238 & 4.024 & 2.255 & 2.223 & 10.713 & 5.949 & 4.753 & 6.637 & 3.798 \\
\hline $8 / 23 / 2005$ & $14: 00$ & 10.556 & 10.455 & 7.707 & 6.536 & 4.244 & 4.024 & 2.255 & 2.223 & 10.715 & 5.954 & 4.773 & 6.662 & 3.816 \\
\hline $8 / 23 / 2005$ & $15: 00$ & 10.551 & 10.448 & 7.703 & 6.532 & 4.251 & 4.022 & 2.265 & 2.225 & 10.719 & 5.958 & 4.781 & 6.678 & 3.835 \\
\hline $8 / 23 / 2005$ & $16: 00$ & 10.546 & 10.443 & 7.702 & 6.527 & 4.257 & 4.022 & 2.262 & 2.223 & 10.72 & 5.961 & 4.793 & 6.683 & 3.853 \\
\hline $8 / 23 / 2005$ & $17: 00$ & 10.542 & 10.435 & 7.699 & 6.525 & 4.263 & 4.022 & 2.254 & 2.223 & 10.724 & 5.967 & 4.797 & 6.666 & 3.869 \\
\hline $8 / 23 / 2005$ & $18: 00$ & 10.542 & 10.433 & 7.699 & 6.527 & 4.274 & 4.022 & 2.262 & 2.223 & 10.728 & 5.969 & 4.799 & 6.651 & 3.881 \\
\hline 8/23/2005 & $19: 00$ & 10.539 & 10.435 & 7.699 & 6.527 & 4.278 & 4.024 & 2.26 & 2.221 & 10.732 & 5.971 & 4.795 & 6.636 & 3.886 \\
\hline $8 / 23 / 2005$ & $20: 00$ & 10.539 & 10.433 & 7.7 & 6.525 & 4.276 & 4.026 & 2.257 & 2.219 & 10.736 & 5.967 & 4.785 & 6.624 & 3.878 \\
\hline $8 / 23 / 2005$ & $21: 00$ & 10.542 & 10.437 & 7.698 & 6.529 & 4.274 & 4.029 & 2.26 & 2.219 & 10.736 & 5.967 & 4.777 & 6.616 & 3.872 \\
\hline $8 / 23 / 2005$ & $22: 00$ & 10.546 & 10.446 & 7.704 & 6.538 & 4.276 & 4.031 & 2.263 & 2.225 & 10.738 & 5.969 & 4.773 & 6.613 & 3.869 \\
\hline $8 / 23 / 2005$ & 23:00 & 10.549 & 10.452 & 7.706 & 6.54 & 4.274 & 4.033 & 2.258 & 2.225 & 10.741 & 5.965 & 4.767 & 6.609 & 3.861 \\
\hline 8/24/2005 & $0: 00$ & 10.552 & 10.45 & 7.704 & 6.536 & 4.267 & 4.033 & 2.253 & 2.221 & 10.736 & 5.962 & 4.76 & 6.601 & 3.847 \\
\hline $8 / 24 / 2005$ & 1:00 & 10.554 & 10.453 & 7.704 & 6.54 & 4.265 & 4.033 & 2.253 & 2.223 & 10.736 & 5.962 & 4.756 & 6.6 & 3.841 \\
\hline 8/24/2005 & $2: 00$ & 10.549 & 10.45 & 7.702 & 6.534 & 4.257 & 4.033 & 2.253 & 2.217 & 10.732 & 5.958 & 4.744 & 6.594 & 3.829 \\
\hline $8 / 24 / 2005$ & $3: 00$ & 10.547 & 10.443 & 7.698 & 6.527 & 4.249 & 4.031 & 2.246 & 2.209 & 10.728 & 5.949 & 4.736 & 6.586 & 3.815 \\
\hline 8/24/2005 & $4: 00$ & 10.549 & 10.448 & 7.7 & 6.532 & 4.251 & 4.031 & 2.251 & 2.213 & 10.726 & 5.954 & 4.734 & 6.586 & 3.813 \\
\hline $8 / 24 / 2005$ & $5: 00$ & 10.547 & 10.444 & 7.698 & 6.53 & 4.244 & 4.029 & 2.241 & 2.209 & 10.722 & 5.949 & 4.726 & 6.581 & 3.803 \\
\hline $8 / 24 / 2005$ & $6: 00$ & 10.547 & 10.446 & 7.698 & 6.527 & 4.244 & 4.026 & 2.244 & 2.209 & 10.72 & 5.949 & 4.726 & 6.579 & 3.799 \\
\hline $8 / 24 / 2005$ & $7: 00$ & 10.542 & 10.437 & 7.694 & 6.523 & 4.236 & 4.024 & 2.239 & 2.2 & 10.717 & 5.943 & 4.717 & 6.573 & 3.789 \\
\hline
\end{tabular}


TABLE S1.2 (Cont.)

Water Level (ft below top of casing) at Indicated Well

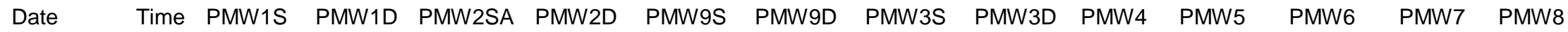

\begin{tabular}{|c|c|c|c|c|c|c|c|c|c|c|c|c|c|c|}
\hline $8 / 24 / 2005$ & $8: 00$ & 10.542 & 10.439 & 7.692 & 6.521 & 4.236 & 4.022 & 2.239 & 2.2 & 10.715 & 5.943 & 4.717 & 6.579 & 3.787 \\
\hline 8/24/2005 & 9:00 & 10.54 & 10.437 & 7.692 & 6.523 & 4.236 & 4.02 & 2.239 & 2.2 & 10.711 & 5.945 & 4.719 & 6.581 & 3.785 \\
\hline $8 / 24 / 2005$ & $10: 00$ & 10.547 & 10.443 & 7.694 & 6.53 & 4.244 & 4.02 & 2.249 & 2.211 & 10.711 & 5.945 & 4.726 & 6.581 & 3.795 \\
\hline $8 / 24 / 2005$ & $11: 00$ & 10.547 & 10.444 & 7.698 & 6.53 & 4.247 & 4.02 & 2.249 & 2.213 & 10.713 & 5.95 & 4.734 & 6.588 & 3.805 \\
\hline $8 / 24 / 2005$ & $12: 00$ & 10.55 & 10.448 & 7.7 & 6.534 & 4.257 & 4.022 & 2.259 & 2.219 & 10.713 & 5.954 & 4.75 & 6.609 & 3.819 \\
\hline 8/24/2005 & $13: 00$ & 10.55 & 10.45 & 7.7 & 6.534 & 4.263 & 4.02 & 2.256 & 2.221 & 10.715 & 5.956 & 4.756 & 6.613 & 3.828 \\
\hline 8/24/2005 & $14: 00$ & 10.548 & 10.441 & 7.7 & 6.527 & 4.267 & 4.02 & 2.251 & 2.219 & 10.717 & 5.959 & 4.768 & 6.657 & 3.84 \\
\hline $8 / 24 / 2005$ & $15: 00$ & 10.543 & 10.437 & 7.698 & 6.527 & 4.271 & 4.02 & 2.256 & 2.221 & 10.719 & 5.959 & 4.776 & 6.638 & 3.856 \\
\hline $8 / 24 / 2005$ & $16: 00$ & 10.54 & 10.435 & 7.698 & 6.525 & 4.279 & 4.02 & 2.259 & 2.221 & 10.721 & 5.966 & 4.784 & 6.657 & 3.87 \\
\hline 8/24/2005 & $17: 00$ & 10.543 & 10.437 & 7.7 & 6.53 & 4.286 & 4.022 & 2.261 & 2.223 & 10.727 & 5.968 & 4.786 & 6.659 & 3.884 \\
\hline $8 / 24 / 2005$ & $18: 00$ & 10.54 & 10.433 & 7.698 & 6.527 & 4.286 & 4.024 & 2.254 & 2.223 & 10.728 & 5.97 & 4.786 & 6.647 & 3.889 \\
\hline $8 / 24 / 2005$ & $19: 00$ & 10.538 & 10.432 & 7.698 & 6.527 & 4.288 & 4.024 & 2.252 & 2.221 & 10.732 & 5.97 & 4.776 & 6.624 & 3.889 \\
\hline $8 / 24 / 2005$ & $20: 00$ & 10.538 & 10.435 & 7.698 & 6.53 & 4.288 & 4.026 & 2.252 & 2.219 & 10.732 & 5.97 & 4.766 & 6.611 & 3.883 \\
\hline $8 / 24 / 2005$ & $21: 00$ & 10.54 & 10.437 & 7.698 & 6.53 & 4.284 & 4.026 & 2.257 & 2.219 & 10.734 & 5.966 & 4.756 & 6.6 & 3.873 \\
\hline 8/24/2005 & $22: 00$ & 10.548 & 10.444 & 7.7 & 6.536 & 4.283 & 4.031 & 2.259 & 2.222 & 10.732 & 5.963 & 4.75 & 6.592 & 3.865 \\
\hline $8 / 24 / 2005$ & $23: 00$ & 10.55 & 10.448 & 7.702 & 6.539 & 4.281 & 4.031 & 2.252 & 2.223 & 10.732 & 5.961 & 4.739 & 6.584 & 3.853 \\
\hline $8 / 25 / 2005$ & $0: 00$ & 10.553 & 10.45 & 7.702 & 6.536 & 4.275 & 4.033 & 2.25 & 2.219 & 10.732 & 5.959 & 4.731 & 6.579 & 3.843 \\
\hline $8 / 25 / 2005$ & $1: 00$ & 10.551 & 10.444 & 7.698 & 6.532 & 4.269 & 4.031 & 2.244 & 2.213 & 10.728 & 5.953 & 4.719 & 6.571 & 3.828 \\
\hline $8 / 25 / 2005$ & $2: 00$ & 10.551 & 10.446 & 7.698 & 6.53 & 4.261 & 4.031 & 2.25 & 2.211 & 10.725 & 5.953 & 4.711 & 6.566 & 3.818 \\
\hline $8 / 25 / 2005$ & $3: 00$ & 10.546 & 10.441 & 7.694 & 6.525 & 4.254 & 4.029 & 2.237 & 2.205 & 10.721 & 5.948 & 4.702 & 6.56 & 3.806 \\
\hline $8 / 25 / 2005$ & $4: 00$ & 10.546 & 10.441 & 7.693 & 6.523 & 4.25 & 4.027 & 2.239 & 2.203 & 10.717 & 5.947 & 4.696 & 6.558 & 3.798 \\
\hline $8 / 25 / 2005$ & $5: 00$ & 10.543 & 10.437 & 7.691 & 6.521 & 4.242 & 4.024 & 2.234 & 2.198 & 10.713 & 5.942 & 4.688 & 6.55 & 3.788 \\
\hline $8 / 25 / 2005$ & $6: 00$ & 10.546 & 10.441 & 7.693 & 6.523 & 4.242 & 4.025 & 2.239 & 2.203 & 10.711 & 5.942 & 4.686 & 6.552 & 3.784 \\
\hline $8 / 25 / 2005$ & 7:00 & 10.549 & 10.446 & 7.697 & 6.527 & 4.244 & 4.025 & 2.239 & 2.205 & 10.711 & 5.944 & 4.684 & 6.558 & 3.784 \\
\hline $8 / 25 / 2005$ & $8: 00$ & 10.551 & 10.448 & 7.697 & 6.527 & 4.242 & 4.022 & 2.242 & 2.205 & 10.709 & 5.942 & 4.686 & 6.606 & 3.776 \\
\hline $8 / 25 / 2005$ & $9: 00$ & 10.554 & 10.45 & 7.699 & 6.53 & 4.244 & 4.022 & 2.239 & 2.209 & 10.708 & 5.938 & 4.688 & 6.632 & 3.772 \\
\hline $8 / 25 / 2005$ & $10: 00$ & 10.556 & 10.453 & 7.702 & 6.532 & 4.246 & 4.022 & 2.247 & 2.211 & 10.706 & 5.938 & 4.68 & 6.592 & 3.772 \\
\hline $8 / 25 / 2005$ & $11: 00$ & 10.556 & 10.455 & 7.702 & 6.534 & 4.25 & 4.022 & 2.258 & 2.217 & 10.704 & 5.944 & 4.684 & 6.606 & 3.784 \\
\hline $8 / 25 / 2005$ & $12: 00$ & 10.556 & 10.45 & 7.702 & 6.532 & 4.256 & 4.02 & 2.258 & 2.219 & 10.704 & 5.951 & 4.698 & 6.608 & 3.806 \\
\hline $8 / 25 / 2005$ & $13: 00$ & 10.551 & 10.443 & 7.702 & 6.532 & 4.27 & 4.02 & 2.257 & 2.224 & 10.708 & 5.955 & 4.717 & 6.617 & 3.838 \\
\hline $8 / 25 / 2005$ & $14: 00$ & 10.549 & 10.441 & 7.702 & 6.532 & 4.285 & 4.02 & 2.265 & 2.23 & 10.713 & 5.966 & 4.737 & 6.621 & 3.875 \\
\hline $8 / 25 / 2005$ & $15: 00$ & 10.549 & 10.439 & 7.704 & 6.532 & 4.299 & 4.022 & 2.272 & 2.234 & 10.719 & 5.977 & 4.758 & 6.627 & 3.908 \\
\hline $8 / 25 / 2005$ & $16: 00$ & 10.544 & 10.432 & 7.701 & 6.527 & 4.302 & 4.02 & 2.272 & 2.232 & 10.727 & 5.984 & 4.775 & 6.636 & 3.93 \\
\hline $8 / 25 / 2005$ & $17: 00$ & 10.539 & 10.426 & 7.701 & 6.527 & 4.31 & 4.025 & 2.275 & 2.232 & 10.734 & 5.989 & 4.79 & 6.64 & 3.959 \\
\hline
\end{tabular}


TABLE S1.2 (Cont.)

Water Level (ft below top of casing) at Indicated Well

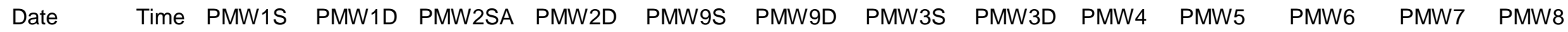

\begin{tabular}{|c|c|c|c|c|c|c|c|c|c|c|c|c|c|c|}
\hline $8 / 25 / 2005$ & $18: 00$ & 10.537 & 10.424 & 7.701 & 6.527 & 4.32 & 4.025 & 2.277 & 2.234 & 10.742 & 5.995 & 4.804 & 6.638 & 3.977 \\
\hline 8/25/2005 & $19: 00$ & 10.537 & 10.424 & 7.702 & 6.527 & 4.324 & 4.027 & 2.265 & 2.232 & 10.75 & 5.993 & 4.796 & 6.619 & 3.977 \\
\hline $8 / 25 / 2005$ & $20: 00$ & 10.534 & 10.426 & 7.701 & 6.525 & 4.319 & 4.031 & 2.268 & 2.228 & 10.755 & 5.991 & 4.784 & 6.604 & 3.967 \\
\hline 8/25/2005 & $21: 00$ & 10.542 & 10.433 & 7.703 & 6.534 & 4.322 & 4.033 & 2.27 & 2.23 & 10.759 & 5.989 & 4.775 & 6.592 & 3.957 \\
\hline $8 / 25 / 2005$ & $22: 00$ & 10.547 & 10.441 & 7.705 & 6.541 & 4.32 & 4.038 & 2.275 & 2.235 & 10.761 & 5.989 & 4.765 & 6.585 & 3.945 \\
\hline 8/25/2005 & $23: 00$ & 10.55 & 10.444 & 7.705 & 6.539 & 4.312 & 4.04 & 2.263 & 2.232 & 10.761 & 5.985 & 4.755 & 6.579 & 3.931 \\
\hline $8 / 26 / 2005$ & $0: 00$ & 10.549 & 10.441 & 7.701 & 6.532 & 4.299 & 4.04 & 2.263 & 2.224 & 10.759 & 5.978 & 4.741 & 6.572 & 3.911 \\
\hline 8/26/2005 & $1: 00$ & 10.539 & 10.426 & 7.695 & 6.516 & 4.281 & 4.033 & 2.243 & 2.211 & 10.755 & 5.967 & 4.722 & 6.558 & 3.887 \\
\hline $8 / 26 / 2005$ & $2: 00$ & 10.537 & 10.426 & 7.691 & 6.519 & 4.275 & 4.036 & 2.24 & 2.207 & 10.752 & 5.967 & 4.716 & 6.555 & 3.875 \\
\hline 8/26/2005 & $3: 00$ & 10.525 & 10.408 & 7.683 & 6.501 & 4.256 & 4.027 & 2.221 & 2.19 & 10.742 & 5.958 & 4.698 & 6.543 & 3.852 \\
\hline $8 / 26 / 2005$ & $4: 00$ & 10.54 & 10.393 & 7.695 & 6.53 & 4.277 & 4.031 & 2.253 & 2.174 & 10.746 & 5.969 & 4.708 & 6.478 & 3.866 \\
\hline 8/26/2005 & $5: 00$ & 10.525 & 10.41 & 7.679 & 6.499 & 4.252 & 4.02 & 2.218 & 2.188 & 10.738 & 5.954 & 4.681 & 6.547 & 3.832 \\
\hline $8 / 26 / 2005$ & $6: 00$ & 10.518 & 10.415 & 7.677 & 6.494 & 4.241 & 4.016 & 2.218 & 2.193 & 10.731 & 5.954 & 4.671 & 6.528 & 3.822 \\
\hline 8/26/2005 & $7: 00$ & 10.523 & 10.406 & 7.685 & 6.508 & 4.249 & 4.016 & 2.224 & 2.182 & 10.731 & 5.954 & 4.669 & 6.528 & 3.826 \\
\hline 8/26/2005 & 8:00 & 10.533 & 10.428 & 7.691 & 6.521 & 4.257 & 4.018 & 2.239 & 2.203 & 10.731 & 5.954 & 4.677 & 6.577 & 3.828 \\
\hline 8/26/2005 & 9:00 & 10.538 & 10.433 & 7.695 & 6.521 & 4.259 & 4.018 & 2.241 & 2.203 & 10.729 & 5.952 & 4.673 & 6.574 & 3.82 \\
\hline 8/26/2005 & $10: 00$ & 10.543 & 10.437 & 7.699 & 6.526 & 4.26 & 4.018 & 2.249 & 2.211 & 10.727 & 5.954 & 4.673 & 6.591 & 3.82 \\
\hline 8/26/2005 & $11: 00$ & 10.543 & 10.437 & 7.699 & 6.523 & 4.26 & 4.016 & 2.249 & 2.211 & 10.725 & 5.956 & 4.681 & 6.602 & 3.83 \\
\hline 8/26/2005 & $12: 00$ & 10.535 & 10.426 & 7.695 & 6.517 & 4.264 & 4.014 & 2.251 & 2.209 & 10.725 & 5.963 & 4.695 & 6.6 & 3.846 \\
\hline $8 / 26 / 2005$ & $13: 00$ & 10.538 & 10.428 & 7.697 & 6.521 & 4.28 & 4.014 & 2.256 & 2.216 & 10.729 & 5.972 & 4.716 & 6.61 & 3.876 \\
\hline 8/26/2005 & $14: 00$ & 10.53 & 10.421 & 7.697 & 6.517 & 4.291 & 4.014 & 2.259 & 2.218 & 10.733 & 5.979 & 4.738 & 6.627 & 3.909 \\
\hline $8 / 26 / 2005$ & $15: 00$ & 10.531 & 10.421 & 7.697 & 6.519 & 4.307 & 4.014 & 2.256 & 2.222 & 10.74 & 5.988 & 4.759 & 6.652 & 3.939 \\
\hline 8/26/2005 & $16: 00$ & 10.526 & 10.417 & 7.695 & 6.519 & 4.314 & 4.016 & 2.266 & 2.224 & 10.752 & 5.994 & 4.777 & 6.621 & 3.966 \\
\hline $8 / 26 / 2005$ & $17: 00$ & 10.526 & 10.415 & 7.699 & 6.521 & 4.326 & 4.018 & 2.269 & 2.227 & 10.755 & 6.003 & 4.793 & 6.65 & 3.99 \\
\hline 8/26/2005 & $18: 00$ & 10.524 & 10.415 & 7.699 & 6.521 & 4.334 & 4.02 & 2.269 & 2.227 & 10.763 & 6.007 & 4.802 & 6.643 & 4.004 \\
\hline $8 / 26 / 2005$ & $19: 00$ & 10.526 & 10.413 & 7.699 & 6.521 & 4.337 & 4.025 & 2.266 & 2.227 & 10.769 & 6.01 & 4.803 & 6.629 & 4.013 \\
\hline 8/26/2005 & 20:00 & 10.526 & 10.417 & 7.699 & 6.523 & 4.339 & 4.027 & 2.261 & 2.227 & 10.775 & 6.008 & 4.795 & 6.612 & 4.007 \\
\hline $8 / 26 / 2005$ & $21: 00$ & 10.533 & 10.428 & 7.703 & 6.532 & 4.341 & 4.033 & 2.269 & 2.229 & 10.779 & 6.008 & 4.781 & 6.601 & 3.993 \\
\hline 8/26/2005 & $22: 00$ & 10.538 & 10.433 & 7.705 & 6.534 & 4.336 & 4.036 & 2.269 & 2.231 & 10.779 & 6.001 & 4.767 & 6.587 & 3.975 \\
\hline 8/26/2005 & 23:00 & 10.543 & 10.439 & 7.705 & 6.539 & 4.328 & 4.038 & 2.262 & 2.229 & 10.779 & 5.997 & 4.754 & 6.578 & 3.958 \\
\hline 8/27/2005 & $0: 00$ & 10.546 & 10.443 & 7.705 & 6.537 & 4.32 & 4.04 & 2.264 & 2.227 & 10.779 & 5.992 & 4.74 & 6.57 & 3.94 \\
\hline 8/27/2005 & $1: 00$ & 10.549 & 10.443 & 7.706 & 6.537 & 4.312 & 4.04 & 2.262 & 2.224 & 10.775 & 5.988 & 4.73 & 6.564 & 3.924 \\
\hline 8/27/2005 & $2: 00$ & 10.549 & 10.441 & 7.703 & 6.53 & 4.303 & 4.04 & 2.257 & 2.218 & 10.771 & 5.983 & 4.717 & 6.557 & 3.908 \\
\hline 8/27/2005 & 3:00 & 10.546 & 10.437 & 7.7 & 6.526 & 4.291 & 4.04 & 2.249 & 2.214 & 10.765 & 5.979 & 4.703 & 6.551 & 3.894 \\
\hline
\end{tabular}


TABLE S1.2 (Cont.)

Water Level (ft below top of casing) at Indicated Well

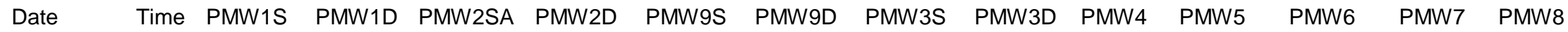

\begin{tabular}{|c|c|c|c|c|c|c|c|c|c|c|c|c|c|c|}
\hline 8/27/2005 & $4: 00$ & 10.541 & 10.432 & 7.698 & 6.521 & 4.284 & 4.038 & 2.245 & 2.206 & 10.762 & 5.973 & 4.693 & 6.547 & 3.88 \\
\hline $8 / 27 / 2005$ & $5: 00$ & 10.536 & 10.43 & 7.694 & 6.517 & 4.274 & 4.033 & 2.24 & 2.201 & 10.758 & 5.968 & 4.683 & 6.54 & 3.868 \\
\hline $8 / 27 / 2005$ & $6: 00$ & 10.539 & 10.43 & 7.694 & 6.517 & 4.272 & 4.031 & 2.24 & 2.201 & 10.754 & 5.966 & 4.677 & 6.536 & 3.861 \\
\hline $8 / 27 / 2005$ & $7: 00$ & 10.544 & 10.441 & 7.698 & 6.526 & 4.274 & 4.031 & 2.245 & 2.208 & 10.75 & 5.966 & 4.677 & 6.536 & 3.861 \\
\hline $8 / 27 / 2005$ & $8: 00$ & 10.549 & 10.443 & 7.702 & 6.528 & 4.274 & 4.031 & 2.245 & 2.216 & 10.748 & 5.964 & 4.674 & 6.534 & 3.853 \\
\hline $8 / 27 / 2005$ & $9: 00$ & 10.546 & 10.439 & 7.7 & 6.523 & 4.267 & 4.029 & 2.237 & 2.206 & 10.746 & 5.962 & 4.668 & 6.53 & 3.845 \\
\hline $8 / 27 / 2005$ & $10: 00$ & 10.549 & 10.441 & 7.7 & 6.523 & 4.268 & 4.027 & 2.242 & 2.21 & 10.742 & 5.962 & 4.678 & 6.538 & 3.849 \\
\hline $8 / 27 / 2005$ & $11: 00$ & 10.551 & 10.445 & 7.702 & 6.53 & 4.28 & 4.027 & 2.26 & 2.22 & 10.744 & 5.969 & 4.697 & 6.557 & 3.869 \\
\hline $8 / 27 / 2005$ & $12: 00$ & 10.549 & 10.441 & 7.704 & 6.53 & 4.292 & 4.025 & 2.265 & 2.224 & 10.746 & 5.975 & 4.723 & 6.572 & 3.895 \\
\hline 8/27/2005 & $13: 00$ & 10.547 & 10.437 & 7.704 & 6.53 & 4.307 & 4.025 & 2.27 & 2.229 & 10.75 & 5.986 & 4.748 & 6.591 & 3.932 \\
\hline $8 / 27 / 2005$ & $14: 00$ & 10.544 & 10.433 & 7.704 & 6.528 & 4.325 & 4.025 & 2.272 & 2.233 & 10.758 & 6 & 4.776 & 6.608 & 3.971 \\
\hline 8/27/2005 & $15: 00$ & 10.537 & 10.424 & 7.7 & 6.523 & 4.336 & 4.025 & 2.275 & 2.233 & 10.765 & 6.008 & 4.799 & 6.624 & 4.005 \\
\hline $8 / 27 / 2005$ & $16: 00$ & 10.534 & 10.419 & 7.7 & 6.521 & 4.346 & 4.025 & 2.277 & 2.235 & 10.773 & 6.015 & 4.821 & 6.633 & 4.034 \\
\hline 8/27/2005 & $17: 00$ & 10.529 & 10.415 & 7.699 & 6.521 & 4.355 & 4.027 & 2.277 & 2.235 & 10.781 & 6.022 & 4.834 & 6.639 & 4.056 \\
\hline $8 / 27 / 2005$ & $18: 00$ & 10.529 & 10.415 & 7.702 & 6.523 & 4.365 & 4.029 & 2.272 & 2.238 & 10.786 & 6.024 & 4.842 & 6.633 & 4.071 \\
\hline 8/27/2005 & $19: 00$ & 10.527 & 10.415 & 7.698 & 6.521 & 4.367 & 4.033 & 2.265 & 2.231 & 10.79 & 6.022 & 4.832 & 6.614 & 4.058 \\
\hline $8 / 27 / 2005$ & $20: 00$ & 10.529 & 10.419 & 7.7 & 6.523 & 4.365 & 4.036 & 2.27 & 2.229 & 10.792 & 6.02 & 4.815 & 6.599 & 4.043 \\
\hline 8/27/2005 & $21: 00$ & 10.532 & 10.425 & 7.7 & 6.528 & 4.359 & 4.038 & 2.263 & 2.227 & 10.794 & 6.013 & 4.799 & 6.588 & 4.025 \\
\hline $8 / 27 / 2005$ & $22: 00$ & 10.53 & 10.421 & 7.696 & 6.521 & 4.346 & 4.04 & 2.253 & 2.219 & 10.796 & 6.009 & 4.782 & 6.576 & 4 \\
\hline 8/27/2005 & $23: 00$ & 10.537 & 10.43 & 7.7 & 6.528 & 4.342 & 4.04 & 2.261 & 2.223 & 10.794 & 6.007 & 4.772 & 6.57 & 3.984 \\
\hline 8/28/2005 & $0: 00$ & 10.537 & 10.428 & 7.696 & 6.523 & 4.332 & 4.04 & 2.251 & 2.219 & 10.792 & 6.002 & 4.756 & 6.563 & 3.964 \\
\hline $8 / 28 / 2005$ & $1: 00$ & 10.54 & 10.43 & 7.698 & 6.521 & 4.322 & 4.04 & 2.253 & 2.214 & 10.788 & 6 & 4.747 & 6.557 & 3.948 \\
\hline 8/28/2005 & $2: 00$ & 10.54 & 10.43 & 7.694 & 6.521 & 4.313 & 4.038 & 2.243 & 2.21 & 10.785 & 5.996 & 4.737 & 6.551 & 3.933 \\
\hline 8/28/2005 & $3: 00$ & 10.54 & 10.434 & 7.696 & 6.526 & 4.309 & 4.038 & 2.243 & 2.212 & 10.783 & 5.991 & 4.727 & 6.548 & 3.924 \\
\hline $8 / 28 / 2005$ & $4: 00$ & 10.542 & 10.432 & 7.696 & 6.522 & 4.303 & 4.038 & 2.246 & 2.208 & 10.779 & 5.989 & 4.717 & 6.542 & 3.909 \\
\hline $8 / 28 / 2005$ & $5: 00$ & 10.545 & 10.437 & 7.696 & 6.526 & 4.3 & 4.038 & 2.248 & 2.21 & 10.775 & 5.983 & 4.711 & 6.54 & 3.901 \\
\hline 8/28/2005 & $6: 00$ & 10.545 & 10.437 & 7.697 & 6.526 & 4.296 & 4.036 & 2.246 & 2.208 & 10.773 & 5.981 & 4.704 & 6.536 & 3.891 \\
\hline $8 / 28 / 2005$ & $7: 00$ & 10.547 & 10.444 & 7.702 & 6.53 & 4.296 & 4.036 & 2.249 & 2.21 & 10.771 & 5.981 & 4.7 & 6.536 & 3.885 \\
\hline $8 / 28 / 2005$ & $8: 00$ & 10.552 & 10.446 & 7.702 & 6.526 & 4.292 & 4.036 & 2.248 & 2.21 & 10.766 & 5.978 & 4.692 & 6.53 & 3.875 \\
\hline $8 / 28 / 2005$ & $9: 00$ & 10.555 & 10.452 & 7.706 & 6.533 & 4.294 & 4.033 & 2.251 & 2.214 & 10.764 & 5.976 & 4.69 & 6.532 & 3.871 \\
\hline 8/28/2005 & $10: 00$ & 10.56 & 10.457 & 7.708 & 6.537 & 4.292 & 4.033 & 2.251 & 2.217 & 10.762 & 5.976 & 4.692 & 6.534 & 3.869 \\
\hline $8 / 28 / 2005$ & $11: 00$ & 10.56 & 10.455 & 7.708 & 6.535 & 4.29 & 4.033 & 2.254 & 2.215 & 10.76 & 5.976 & 4.69 & 6.538 & 3.863 \\
\hline $8 / 28 / 2005$ & $12: 00$ & 10.56 & 10.452 & 7.708 & 6.533 & 4.292 & 4.031 & 2.256 & 2.219 & 10.758 & 5.981 & 4.704 & 6.551 & 3.871 \\
\hline $8 / 28 / 2005$ & $13: 00$ & 10.56 & 10.452 & 7.71 & 6.533 & 4.304 & 4.031 & 2.259 & 2.227 & 10.758 & 5.987 & 4.731 & 6.574 & 3.895 \\
\hline
\end{tabular}


TABLE S1.2 (Cont.)

Water Level (ft below top of casing) at Indicated Well

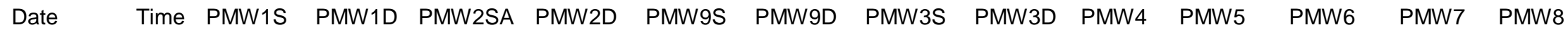

\begin{tabular}{|c|c|c|c|c|c|c|c|c|c|c|c|c|c|c|}
\hline 8/28/2005 & $14: 00$ & 10.555 & 10.446 & 7.71 & 6.533 & 4.321 & 4.029 & 2.272 & 2.234 & 10.762 & 5.998 & 4.761 & 6.595 & 3.929 \\
\hline $8 / 28 / 2005$ & $15: 00$ & 10.553 & 10.441 & 7.712 & 6.533 & 4.338 & 4.029 & 2.277 & 2.236 & 10.768 & 6.007 & 4.79 & 6.61 & 3.97 \\
\hline 8/28/2005 & $16: 00$ & 10.57 & 10.477 & 7.729 & 6.573 & 4.385 & 4.042 & 2.312 & 2.269 & 10.783 & 6.034 & 4.831 & 6.63 & 4.029 \\
\hline 8/28/2005 & $17: 00$ & 10.578 & 10.349 & 7.732 & 6.546 & 4.387 & 4.045 & 2.304 & 2.206 & 10.79 & 6.012 & 4.81 & 6.515 & 4.009 \\
\hline 8/28/2005 & $18: 00$ & 10.561 & 10.472 & 7.714 & 6.533 & 4.344 & 4.036 & 2.259 & 2.215 & 10.819 & 5.996 & 4.78 & 6.58 & 3.942 \\
\hline 8/28/2005 & 19:00 & 10.553 & 10.435 & 7.704 & 6.519 & 4.327 & 4.033 & 2.249 & 2.211 & 10.781 & 5.992 & 4.763 & 6.567 & 3.91 \\
\hline $8 / 28 / 2005$ & $20: 00$ & 10.548 & 10.436 & 7.702 & 6.522 & 4.319 & 4.033 & 2.24 & 2.209 & 10.779 & 5.992 & 4.753 & 6.561 & 3.892 \\
\hline 8/28/2005 & $21: 00$ & 10.553 & 10.448 & 7.708 & 6.533 & 4.323 & 4.036 & 2.257 & 2.217 & 10.779 & 5.992 & 4.747 & 6.557 & 3.884 \\
\hline 8/28/2005 & $22: 00$ & 10.568 & 10.468 & 7.718 & 6.553 & 4.333 & 4.04 & 2.265 & 2.234 & 10.781 & 5.997 & 4.749 & 6.563 & 3.885 \\
\hline 8/28/2005 & $23: 00$ & 10.573 & 10.47 & 7.719 & 6.55 & 4.329 & 4.04 & 2.27 & 2.234 & 10.779 & 5.99 & 4.737 & 6.557 & 3.87 \\
\hline $8 / 29 / 2005$ & $0: 00$ & 10.575 & 10.468 & 7.716 & 6.546 & 4.319 & 4.042 & 2.26 & 2.227 & 10.776 & 5.986 & 4.727 & 6.55 & 3.853 \\
\hline 8/29/2005 & $1: 00$ & 10.573 & 10.464 & 7.714 & 6.544 & 4.312 & 4.04 & 2.258 & 2.221 & 10.772 & 5.979 & 4.716 & 6.544 & 3.841 \\
\hline $8 / 29 / 2005$ & $2: 00$ & 10.578 & 10.472 & 7.716 & 6.546 & 4.31 & 4.042 & 2.26 & 2.223 & 10.77 & 5.981 & 4.714 & 6.544 & 3.835 \\
\hline 8/29/2005 & $3: 00$ & 10.573 & 10.47 & 7.716 & 6.546 & 4.304 & 4.042 & 2.258 & 2.221 & 10.766 & 5.977 & 4.704 & 6.54 & 3.827 \\
\hline 8/29/2005 & $4: 00$ & 10.573 & 10.464 & 7.712 & 6.539 & 4.296 & 4.04 & 2.245 & 2.217 & 10.762 & 5.975 & 4.696 & 6.536 & 3.815 \\
\hline 8/29/2005 & $5: 00$ & 10.568 & 10.459 & 7.708 & 6.535 & 4.288 & 4.038 & 2.248 & 2.211 & 10.758 & 5.971 & 4.69 & 6.533 & 3.807 \\
\hline 8/29/2005 & $6: 00$ & 10.568 & 10.459 & 7.709 & 6.535 & 4.287 & 4.036 & 2.245 & 2.211 & 10.755 & 5.968 & 4.685 & 6.531 & 3.803 \\
\hline 8/29/2005 & $7: 00$ & 10.573 & 10.464 & 7.711 & 6.537 & 4.288 & 4.036 & 2.248 & 2.213 & 10.753 & 5.966 & 4.685 & 6.533 & 3.803 \\
\hline 8/29/2005 & 8:00 & 10.569 & 10.461 & 7.709 & 6.533 & 4.285 & 4.033 & 2.246 & 2.211 & 10.749 & 5.962 & 4.679 & 6.527 & 3.795 \\
\hline 8/29/2005 & $9: 00$ & 10.571 & 10.464 & 7.711 & 6.537 & 4.285 & 4.033 & 2.243 & 2.213 & 10.747 & 5.964 & 4.679 & 6.529 & 3.793 \\
\hline 8/29/2005 & $10: 00$ & 10.569 & 10.464 & 7.711 & 6.537 & 4.285 & 4.031 & 2.246 & 2.213 & 10.743 & 5.964 & 4.683 & 6.531 & 3.797 \\
\hline 8/29/2005 & $11: 00$ & 10.576 & 10.472 & 7.716 & 6.546 & 4.295 & 4.032 & 2.258 & 2.226 & 10.743 & 5.971 & 4.704 & 6.55 & 3.815 \\
\hline 8/29/2005 & $12: 00$ & 10.573 & 10.468 & 7.717 & 6.544 & 4.302 & 4.031 & 2.261 & 2.23 & 10.745 & 5.978 & 4.72 & 6.562 & 3.835 \\
\hline $8 / 29 / 2005$ & $13: 00$ & 10.571 & 10.461 & 7.714 & 6.537 & 4.31 & 4.029 & 2.261 & 2.23 & 10.747 & 5.985 & 4.74 & 6.577 & 3.857 \\
\hline 8/29/2005 & $14: 00$ & 10.564 & 10.45 & 7.712 & 6.533 & 4.322 & 4.029 & 2.268 & 2.228 & 10.749 & 5.991 & 4.759 & 6.588 & 3.884 \\
\hline $8 / 29 / 2005$ & $15: 00$ & 10.557 & 10.443 & 7.711 & 6.53 & 4.333 & 4.027 & 2.266 & 2.228 & 10.757 & 5.997 & 4.779 & 6.617 & 3.908 \\
\hline 8/29/2005 & $16: 00$ & 10.549 & 10.428 & 7.709 & 6.524 & 4.345 & 4.027 & 2.261 & 2.226 & 10.76 & 6.004 & 4.798 & 6.617 & 3.935 \\
\hline $8 / 29 / 2005$ & $17: 00$ & 10.544 & 10.428 & 7.708 & 6.537 & 4.356 & 4.023 & 2.268 & 2.226 & 10.77 & 6.013 & 4.812 & 6.628 & 3.961 \\
\hline 8/29/2005 & $18: 00$ & 10.542 & 10.424 & 7.704 & 6.537 & 4.366 & 4.023 & 2.26 & 2.228 & 10.777 & 6.015 & 4.822 & 6.623 & 3.979 \\
\hline 8/29/2005 & $19: 00$ & 10.539 & 10.424 & 7.707 & 6.537 & 4.374 & 4.027 & 2.26 & 2.226 & 10.783 & 6.02 & 4.824 & 6.609 & 3.99 \\
\hline 8/29/2005 & $20: 00$ & 10.539 & 10.424 & 7.705 & 6.539 & 4.372 & 4.029 & 2.256 & 2.224 & 10.789 & 6.02 & 4.81 & 6.596 & 3.982 \\
\hline 8/29/2005 & $21: 00$ & 10.539 & 10.43 & 7.705 & 6.542 & 4.37 & 4.032 & 2.256 & 2.222 & 10.791 & 6.013 & 4.797 & 6.583 & 3.966 \\
\hline 8/29/2005 & $22: 00$ & 10.544 & 10.435 & 7.705 & 6.544 & 4.364 & 4.033 & 2.261 & 2.224 & 10.793 & 6.011 & 4.783 & 6.573 & 3.952 \\
\hline 8/29/2005 & 23:00 & 10.547 & 10.435 & 7.705 & 6.542 & 4.354 & 4.033 & 2.251 & 2.219 & 10.791 & 6.007 & 4.769 & 6.565 & 3.933 \\
\hline
\end{tabular}


TABLE S1.2 (Cont.)

Water Level (ft below top of casing) at Indicated Well

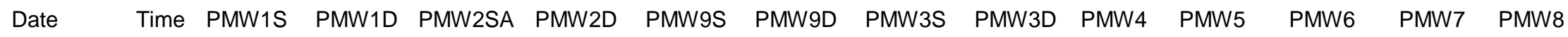

\begin{tabular}{|c|c|c|c|c|c|c|c|c|c|c|c|c|c|c|}
\hline $8 / 30 / 2005$ & $0: 00$ & 10.544 & 10.432 & 7.701 & 6.54 & 4.343 & 4.034 & 2.249 & 2.215 & 10.787 & 6 & 4.754 & 6.558 & 3.915 \\
\hline $8 / 30 / 2005$ & $1: 00$ & 10.542 & 10.428 & 7.699 & 6.533 & 4.329 & 4.034 & 2.246 & 2.207 & 10.784 & 5.993 & 4.738 & 6.548 & 3.899 \\
\hline $8 / 30 / 2005$ & $2: 00$ & 10.537 & 10.426 & 7.697 & 6.531 & 4.318 & 4.029 & 2.241 & 2.203 & 10.779 & 5.991 & 4.726 & 6.542 & 3.885 \\
\hline $8 / 30 / 2005$ & $3: 00$ & 10.535 & 10.421 & 7.693 & 6.529 & 4.31 & 4.027 & 2.236 & 2.196 & 10.774 & 5.987 & 4.716 & 6.537 & 3.873 \\
\hline $8 / 30 / 2005$ & $4: 00$ & 10.535 & 10.421 & 7.694 & 6.526 & 4.306 & 4.027 & 2.233 & 2.194 & 10.77 & 5.985 & 4.705 & 6.531 & 3.863 \\
\hline $8 / 30 / 2005$ & $5: 00$ & 10.535 & 10.422 & 7.694 & 6.524 & 4.3 & 4.025 & 2.226 & 2.194 & 10.766 & 5.976 & 4.697 & 6.527 & 3.855 \\
\hline $8 / 30 / 2005$ & $6: 00$ & 10.535 & 10.424 & 7.692 & 6.526 & 4.296 & 4.023 & 2.231 & 2.194 & 10.763 & 5.976 & 4.691 & 6.524 & 3.847 \\
\hline $8 / 30 / 2005$ & $7: 00$ & 10.54 & 10.43 & 7.694 & 6.529 & 4.296 & 4.023 & 2.236 & 2.199 & 10.761 & 5.976 & 4.687 & 6.525 & 3.843 \\
\hline $8 / 30 / 2005$ & $8: 00$ & 10.542 & 10.432 & 7.696 & 6.531 & 4.295 & 4.021 & 2.229 & 2.199 & 10.759 & 5.971 & 4.682 & 6.521 & 3.836 \\
\hline $8 / 30 / 2005$ & 9:00 & 10.54 & 10.432 & 7.695 & 6.529 & 4.291 & 4.018 & 2.229 & 2.197 & 10.755 & 5.971 & 4.678 & 6.524 & 3.829 \\
\hline $8 / 30 / 2005$ & $10: 00$ & 10.545 & 10.436 & 7.697 & 6.533 & 4.291 & 4.016 & 2.241 & 2.203 & 10.751 & 5.974 & 4.684 & 6.552 & 3.83 \\
\hline $8 / 30 / 2005$ & $11: 00$ & 10.543 & 10.434 & 7.699 & 6.533 & 4.291 & 4.016 & 2.239 & 2.207 & 10.749 & 5.976 & 4.699 & 6.565 & 3.841 \\
\hline $8 / 30 / 2005$ & $12: 00$ & 10.54 & 10.43 & 7.699 & 6.531 & 4.301 & 4.014 & 2.251 & 2.212 & 10.749 & 5.983 & 4.725 & 6.59 & 3.863 \\
\hline $8 / 30 / 2005$ & $13: 00$ & 10.538 & 10.422 & 7.7 & 6.529 & 4.313 & 4.012 & 2.254 & 2.216 & 10.753 & 5.992 & 4.748 & 6.604 & 3.893 \\
\hline $8 / 30 / 2005$ & $14: 00$ & 10.533 & 10.421 & 7.699 & 6.531 & 4.33 & 4.01 & 2.259 & 2.22 & 10.761 & 6.005 & 4.772 & 6.628 & 3.934 \\
\hline $8 / 30 / 2005$ & $15: 00$ & 10.531 & 10.415 & 7.699 & 6.526 & 4.349 & 4.01 & 2.257 & 2.222 & 10.768 & 6.016 & 4.795 & 6.634 & 3.974 \\
\hline $8 / 30 / 2005$ & $16: 00$ & 10.528 & 10.41 & 7.699 & 6.526 & 4.365 & 4.012 & 2.259 & 2.224 & 10.776 & 6.024 & 4.815 & 6.634 & 4.009 \\
\hline $8 / 30 / 2005$ & $17: 00$ & 10.526 & 10.408 & 7.699 & 6.529 & 4.378 & 4.014 & 2.266 & 2.228 & 10.785 & 6.032 & 4.834 & 6.64 & 4.037 \\
\hline $8 / 30 / 2005$ & $18: 00$ & 10.526 & 10.406 & 7.699 & 6.529 & 4.386 & 4.016 & 2.264 & 2.228 & 10.793 & 6.036 & 4.846 & 6.642 & 4.055 \\
\hline $8 / 30 / 2005$ & $19: 00$ & 10.523 & 10.408 & 7.702 & 6.531 & 4.393 & 4.021 & 2.264 & 2.23 & 10.799 & 6.038 & 4.848 & 6.629 & 4.062 \\
\hline $8 / 30 / 2005$ & $20: 00$ & 10.526 & 10.412 & 7.702 & 6.533 & 4.395 & 4.023 & 2.267 & 2.228 & 10.803 & 6.036 & 4.838 & 6.613 & 4.054 \\
\hline $8 / 30 / 2005$ & $21: 00$ & 10.531 & 10.417 & 7.703 & 6.537 & 4.393 & 4.029 & 2.267 & 2.228 & 10.806 & 6.034 & 4.823 & 6.602 & 4.038 \\
\hline $8 / 30 / 2005$ & $22: 00$ & 10.536 & 10.423 & 7.705 & 6.54 & 4.389 & 4.032 & 2.267 & 2.228 & 10.806 & 6.029 & 4.811 & 6.594 & 4.022 \\
\hline $8 / 30 / 2005$ & $23: 00$ & 10.538 & 10.426 & 7.706 & 6.54 & 4.38 & 4.036 & 2.264 & 2.226 & 10.806 & 6.025 & 4.798 & 6.587 & 4 \\
\hline $8 / 31 / 2005$ & $0: 00$ & 10.541 & 10.428 & 7.703 & 6.537 & 4.372 & 4.036 & 2.262 & 2.222 & 10.806 & 6.018 & 4.786 & 6.579 & 3.983 \\
\hline $8 / 31 / 2005$ & $1: 00$ & 10.543 & 10.432 & 7.705 & 6.542 & 4.362 & 4.036 & 2.259 & 2.222 & 10.805 & 6.016 & 4.774 & 6.573 & 3.967 \\
\hline $8 / 31 / 2005$ & $2: 00$ & 10.546 & 10.435 & 7.706 & 6.542 & 4.357 & 4.038 & 2.259 & 2.222 & 10.803 & 6.012 & 4.764 & 6.569 & 3.953 \\
\hline $8 / 31 / 2005$ & $3: 00$ & 10.548 & 10.437 & 7.706 & 6.544 & 4.351 & 4.038 & 2.252 & 2.22 & 10.801 & 6.006 & 4.754 & 6.566 & 3.941 \\
\hline $8 / 31 / 2005$ & $4: 00$ & 10.553 & 10.446 & 7.709 & 6.551 & 4.351 & 4.041 & 2.262 & 2.227 & 10.799 & 6.008 & 4.751 & 6.564 & 3.933 \\
\hline $8 / 31 / 2005$ & $5: 00$ & 10.556 & 10.446 & 7.71 & 6.549 & 4.345 & 4.041 & 2.259 & 2.222 & 10.795 & 6.001 & 4.741 & 6.56 & 3.919 \\
\hline $8 / 31 / 2005$ & $6: 00$ & 10.561 & 10.456 & 7.712 & 6.553 & 4.343 & 4.043 & 2.257 & 2.227 & 10.793 & 5.999 & 4.735 & 6.558 & 3.913 \\
\hline $8 / 31 / 2005$ & 7:00 & 10.566 & 10.459 & 7.714 & 6.557 & 4.341 & 4.043 & 2.267 & 2.228 & 10.791 & 5.999 & 4.731 & 6.556 & 3.905 \\
\hline $8 / 31 / 2005$ & $8: 00$ & 10.571 & 10.465 & 7.717 & 6.555 & 4.34 & 4.043 & 2.265 & 2.229 & 10.786 & 5.997 & 4.731 & 6.579 & 3.899 \\
\hline $8 / 31 / 2005$ & $9: 00$ & 10.576 & 10.47 & 7.719 & 6.564 & 4.34 & 4.045 & 2.267 & 2.239 & 10.786 & 6.001 & 4.737 & 6.583 & 3.901 \\
\hline
\end{tabular}


TABLE S1.2 (Cont.)

Water Level (ft below top of casing) at Indicated Well

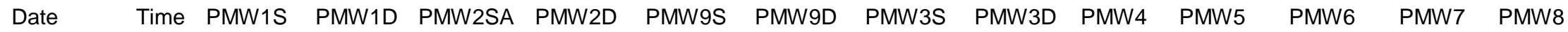

\begin{tabular}{|c|c|c|c|c|c|c|c|c|c|c|c|c|c|c|}
\hline 8/31/2005 & $10: 00$ & 10.583 & 10.481 & 7.727 & 6.573 & 4.351 & 4.047 & 2.288 & 2.25 & 10.788 & 6.008 & 4.752 & 6.59 & 3.913 \\
\hline $8 / 31 / 2005$ & $11: 00$ & 10.588 & 10.488 & 7.732 & 6.58 & 4.361 & 4.049 & 2.298 & 2.26 & 10.791 & 6.01 & 4.77 & 6.613 & 3.933 \\
\hline $8 / 31 / 2005$ & $12: 00$ & 10.596 & 10.492 & 7.733 & 6.584 & 4.375 & 4.054 & 2.3 & 2.269 & 10.795 & 6.019 & 4.79 & 6.623 & 3.959 \\
\hline $8 / 31 / 2005$ & $13: 00$ & 10.596 & 10.496 & 7.737 & 6.588 & 4.39 & 4.058 & 2.315 & 2.279 & 10.799 & 6.03 & 4.818 & 6.648 & 3.996 \\
\hline $8 / 31 / 2005$ & $14: 00$ & 10.601 & 10.499 & 7.743 & 6.595 & 4.405 & 4.06 & 2.325 & 2.288 & 10.805 & 6.041 & 4.841 & 6.665 & 4.032 \\
\hline $8 / 31 / 2005$ & $15: 00$ & 10.606 & 10.501 & 7.745 & 6.597 & 4.415 & 4.063 & 2.323 & 2.29 & 10.812 & 6.046 & 4.859 & 6.68 & 4.061 \\
\hline $8 / 31 / 2005$ & $16: 00$ & 10.606 & 10.499 & 7.745 & 6.597 & 4.423 & 4.067 & 2.33 & 2.29 & 10.818 & 6.052 & 4.874 & 6.705 & 4.087 \\
\hline $8 / 31 / 2005$ & $17: 00$ & 10.608 & 10.499 & 7.747 & 6.6 & 4.432 & 4.072 & 2.336 & 2.296 & 10.822 & 6.057 & 4.892 & 6.719 & 4.105 \\
\hline 8/31/2005 & $18: 00$ & 10.608 & 10.505 & 7.751 & 6.604 & 4.44 & 4.076 & 2.336 & 2.298 & 10.83 & 6.062 & 4.894 & 6.701 & 4.12 \\
\hline $8 / 31 / 2005$ & $19: 00$ & 10.613 & 10.507 & 7.753 & 6.606 & 4.444 & 4.08 & 2.333 & 2.3 & 10.835 & 6.064 & 4.89 & 6.688 & 4.12 \\
\hline $8 / 31 / 2005$ & $20: 00$ & 10.615 & 10.512 & 7.755 & 6.611 & 4.448 & 4.085 & 2.338 & 2.3 & 10.839 & 6.063 & 4.878 & 6.673 & 4.112 \\
\hline $8 / 31 / 2005$ & $21: 00$ & 10.621 & 10.519 & 7.757 & 6.615 & 4.444 & 4.094 & 2.338 & 2.3 & 10.841 & 6.059 & 4.865 & 6.658 & 4.094 \\
\hline $8 / 31 / 2005$ & $22: 00$ & 10.626 & 10.525 & 7.757 & 6.617 & 4.438 & 4.096 & 2.331 & 2.3 & 10.845 & 6.055 & 4.851 & 6.646 & 4.074 \\
\hline $8 / 31 / 2005$ & $23: 00$ & 10.631 & 10.529 & 7.759 & 6.617 & 4.432 & 4.1 & 2.336 & 2.298 & 10.845 & 6.05 & 4.837 & 6.637 & 4.052 \\
\hline $9 / 1 / 2005$ & $0: 00$ & 10.633 & 10.534 & 7.759 & 6.62 & 4.424 & 4.103 & 2.328 & 2.298 & 10.843 & 6.046 & 4.824 & 6.629 & 4.033 \\
\hline $9 / 1 / 2005$ & $1: 00$ & 10.638 & 10.536 & 7.759 & 6.622 & 4.419 & 4.105 & 2.328 & 2.296 & 10.843 & 6.043 & 4.814 & 6.621 & 4.017 \\
\hline $9 / 1 / 2005$ & $2: 00$ & 10.64 & 10.54 & 7.761 & 6.62 & 4.411 & 4.107 & 2.333 & 2.296 & 10.841 & 6.039 & 4.802 & 6.616 & 4.001 \\
\hline $9 / 1 / 2005$ & $3: 00$ & 10.64 & 10.541 & 7.759 & 6.62 & 4.402 & 4.107 & 2.324 & 2.294 & 10.837 & 6.035 & 4.792 & 6.61 & 3.985 \\
\hline $9 / 1 / 2005$ & $4: 00$ & 10.645 & 10.54 & 7.759 & 6.618 & 4.396 & 4.105 & 2.321 & 2.292 & 10.833 & 6.031 & 4.783 & 6.606 & 3.973 \\
\hline $9 / 1 / 2005$ & $5: 00$ & 10.645 & 10.545 & 7.761 & 6.62 & 4.392 & 4.105 & 2.324 & 2.292 & 10.832 & 6.031 & 4.777 & 6.602 & 3.962 \\
\hline $9 / 1 / 2005$ & $6: 00$ & 10.648 & 10.547 & 7.761 & 6.62 & 4.386 & 4.105 & 2.329 & 2.29 & 10.828 & 6.026 & 4.769 & 6.6 & 3.951 \\
\hline $9 / 1 / 2005$ & $7: 00$ & 10.653 & 10.552 & 7.756 & 6.629 & 4.386 & 4.107 & 2.329 & 2.296 & 10.826 & 6.029 & 4.765 & 6.604 & 3.948 \\
\hline $9 / 1 / 2005$ & $8: 00$ & 10.656 & 10.558 & 7.769 & 6.631 & 4.388 & 4.107 & 2.339 & 2.301 & 10.824 & 6.026 & 4.767 & 6.61 & 3.942 \\
\hline $9 / 1 / 2005$ & $9: 00$ & 10.661 & 10.561 & 7.771 & 6.633 & 4.386 & 4.109 & 2.334 & 2.305 & 10.822 & 6.024 & 4.767 & 6.612 & 3.934 \\
\hline $9 / 1 / 2005$ & $10: 00$ & 10.663 & 10.565 & 7.775 & 6.638 & 4.392 & 4.107 & 2.341 & 2.311 & 10.82 & 6.027 & 4.777 & 6.623 & 3.942 \\
\hline $9 / 1 / 2005$ & $11: 00$ & 10.666 & 10.565 & 7.777 & 6.638 & 4.398 & 4.107 & 2.357 & 2.317 & 10.822 & 6.036 & 4.794 & 6.642 & 3.967 \\
\hline $9 / 1 / 2005$ & $12: 00$ & 10.668 & 10.569 & 7.779 & 6.642 & 4.408 & 4.107 & 2.362 & 2.324 & 10.826 & 6.046 & 4.816 & 6.659 & 3.997 \\
\hline $9 / 1 / 2005$ & $13: 00$ & 10.668 & 10.567 & 7.781 & 6.642 & 4.415 & 4.109 & 2.367 & 2.33 & 10.832 & 6.056 & 4.839 & 6.696 & 4.033 \\
\hline $9 / 1 / 2005$ & $14: 00$ & 10.663 & 10.558 & 7.779 & 6.638 & 4.421 & 4.107 & 2.367 & 2.328 & 10.839 & 6.064 & 4.863 & 6.719 & 4.066 \\
\hline $9 / 1 / 2005$ & $15: 00$ & 10.658 & 10.55 & 7.777 & 6.633 & 4.429 & 4.11 & 2.364 & 2.328 & 10.847 & 6.071 & 4.879 & 6.728 & 4.094 \\
\hline $9 / 1 / 2005$ & $16: 00$ & 10.653 & 10.541 & 7.775 & 6.629 & 4.435 & 4.107 & 2.367 & 2.326 & 10.851 & 6.075 & 4.89 & 6.736 & 4.117 \\
\hline $9 / 1 / 2005$ & $17: 00$ & 10.646 & 10.541 & 7.777 & 6.629 & 4.446 & 4.11 & 2.359 & 2.328 & 10.855 & 6.082 & 4.898 & 6.73 & 4.137 \\
\hline $9 / 1 / 2005$ & $18: 00$ & 10.643 & 10.536 & 7.773 & 6.626 & 4.45 & 4.111 & 2.357 & 2.326 & 10.86 & 6.084 & 4.901 & 6.717 & 4.15 \\
\hline $9 / 1 / 2005$ & $19: 00$ & 10.638 & 10.532 & 7.771 & 6.622 & 4.452 & 4.111 & 2.352 & 2.322 & 10.864 & 6.084 & 4.893 & 6.702 & 4.15 \\
\hline
\end{tabular}


TABLE S1.2 (Cont.)

Water Level (ft below top of casing) at Indicated Well

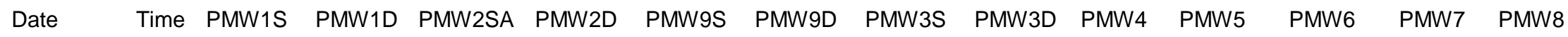

\begin{tabular}{|c|c|c|c|c|c|c|c|c|c|c|c|c|c|c|}
\hline 9/1/2005 & $20: 00$ & 10.646 & 10.536 & 7.773 & 6.629 & 4.452 & 4.116 & 2.352 & 2.319 & 10.868 & 6.084 & 4.881 & 6.685 & 4.14 \\
\hline $9 / 1 / 2005$ & $21: 00$ & 10.659 & 10.545 & 7.775 & 6.633 & 4.452 & 4.118 & 2.36 & 2.324 & 10.87 & 6.082 & 4.865 & 6.671 & 4.126 \\
\hline 9/1/2005 & $22: 00$ & 10.664 & 10.55 & 7.777 & 6.638 & 4.446 & 4.122 & 2.36 & 2.322 & 10.872 & 6.073 & 4.85 & 6.66 & 4.105 \\
\hline 9/1/2005 & $23: 00$ & 10.666 & 10.552 & 7.775 & 6.638 & 4.438 & 4.122 & 2.358 & 2.319 & 10.87 & 6.069 & 4.834 & 6.648 & 4.083 \\
\hline 9/2/2005 & $0: 00$ & 10.673 & 10.556 & 7.777 & 6.638 & 4.431 & 4.125 & 2.352 & 2.317 & 10.87 & 6.065 & 4.82 & 6.639 & 4.065 \\
\hline 9/2/2005 & $1: 00$ & 10.676 & 10.56 & 7.777 & 6.64 & 4.425 & 4.127 & 2.347 & 2.318 & 10.868 & 6.062 & 4.81 & 6.633 & 4.049 \\
\hline 9/2/2005 & $2: 00$ & 10.676 & 10.56 & 7.777 & 6.638 & 4.415 & 4.125 & 2.35 & 2.316 & 10.864 & 6.058 & 4.797 & 6.625 & 4.03 \\
\hline 9/2/2005 & $3: 00$ & 10.681 & 10.565 & 7.777 & 6.64 & 4.411 & 4.127 & 2.345 & 2.314 & 10.862 & 6.056 & 4.789 & 6.622 & 4.019 \\
\hline 9/2/2005 & $4: 00$ & 10.686 & 10.565 & 7.778 & 6.64 & 4.408 & 4.125 & 2.353 & 2.316 & 10.862 & 6.047 & 4.779 & 6.618 & 4.006 \\
\hline 9/2/2005 & $5: 00$ & 10.689 & 10.567 & 7.777 & 6.64 & 4.402 & 4.125 & 2.343 & 2.314 & 10.856 & 6.047 & 4.771 & 6.612 & 3.994 \\
\hline $9 / 2 / 2005$ & $6: 00$ & 10.706 & 10.574 & 7.782 & 6.649 & 4.404 & 4.127 & 2.353 & 2.322 & 10.856 & 6.052 & 4.767 & 6.612 & 3.99 \\
\hline 9/2/2005 & $7: 00$ & 10.674 & 10.578 & 7.784 & 6.649 & 4.402 & 4.127 & 2.35 & 2.322 & 10.855 & 6.047 & 4.761 & 6.608 & 3.98 \\
\hline 9/2/2005 & $8: 00$ & 10.676 & 10.58 & 7.785 & 6.649 & 4.396 & 4.127 & 2.356 & 2.32 & 10.851 & 6.043 & 4.761 & 6.646 & 3.97 \\
\hline 9/2/2005 & $9: 00$ & 10.679 & 10.581 & 7.786 & 6.655 & 4.396 & 4.127 & 2.351 & 2.324 & 10.851 & 6.043 & 4.762 & 6.652 & 3.964 \\
\hline 9/2/2005 & $10: 00$ & 10.684 & 10.589 & 7.79 & 6.66 & 4.396 & 4.129 & 2.366 & 2.333 & 10.847 & 6.041 & 4.759 & 6.647 & 3.966 \\
\hline 9/2/2005 & $11: 00$ & 10.684 & 10.589 & 7.79 & 6.656 & 4.398 & 4.127 & 2.363 & 2.326 & 10.847 & 6.045 & 4.754 & 6.637 & 3.962 \\
\hline 9/2/2005 & $12: 00$ & 10.687 & 10.589 & 7.793 & 6.657 & 4.4 & 4.129 & 2.368 & 2.333 & 10.844 & 6.048 & 4.76 & 6.639 & 3.966 \\
\hline 9/2/2005 & $13: 00$ & 10.684 & 10.583 & 7.791 & 6.655 & 4.402 & 4.127 & 2.363 & 2.335 & 10.845 & 6.054 & 4.772 & 6.652 & 3.982 \\
\hline 9/2/2005 & $14: 00$ & 10.679 & 10.574 & 7.787 & 6.646 & 4.402 & 4.123 & 2.371 & 2.333 & 10.845 & 6.058 & 4.789 & 6.664 & 4.006 \\
\hline 9/2/2005 & $15: 00$ & 10.674 & 10.567 & 7.785 & 6.644 & 4.412 & 4.121 & 2.374 & 2.335 & 10.849 & 6.065 & 4.811 & 6.675 & 4.039 \\
\hline 9/2/2005 & $16: 00$ & 10.669 & 10.561 & 7.785 & 6.644 & 4.421 & 4.121 & 2.374 & 2.335 & 10.857 & 6.072 & 4.83 & 6.685 & 4.071 \\
\hline 9/2/2005 & $17: 00$ & 10.667 & 10.554 & 7.783 & 6.638 & 4.429 & 4.121 & 2.374 & 2.335 & 10.864 & 6.078 & 4.846 & 6.689 & 4.1 \\
\hline 9/2/2005 & $18: 00$ & 10.664 & 10.552 & 7.783 & 6.64 & 4.437 & 4.121 & 2.374 & 2.337 & 10.874 & 6.083 & 4.858 & 6.689 & 4.122 \\
\hline 9/2/2005 & $19: 00$ & 10.664 & 10.554 & 7.785 & 6.642 & 4.446 & 4.121 & 2.374 & 2.337 & 10.878 & 6.087 & 4.86 & 6.681 & 4.131 \\
\hline 9/2/2005 & $20: 00$ & 10.662 & 10.554 & 7.785 & 6.645 & 4.448 & 4.125 & 2.366 & 2.335 & 10.88 & 6.085 & 4.852 & 6.668 & 4.125 \\
\hline $9 / 2 / 2005$ & $21: 00$ & 10.669 & 10.563 & 7.788 & 6.649 & 4.448 & 4.129 & 2.366 & 2.335 & 10.882 & 6.083 & 4.842 & 6.66 & 4.111 \\
\hline 9/2/2005 & $22: 00$ & 10.677 & 10.574 & 7.792 & 6.656 & 4.452 & 4.134 & 2.371 & 2.341 & 10.883 & 6.083 & 4.832 & 6.654 & 4.095 \\
\hline $9 / 2 / 2005$ & $23: 00$ & 10.679 & 10.578 & 7.792 & 6.658 & 4.445 & 4.136 & 2.374 & 2.339 & 10.883 & 6.077 & 4.819 & 6.645 & 4.074 \\
\hline 9/3/2005 & $0: 00$ & 10.684 & 10.581 & 7.792 & 6.658 & 4.437 & 4.138 & 2.374 & 2.339 & 10.882 & 6.072 & 4.807 & 6.639 & 4.056 \\
\hline 9/3/2005 & $1: 00$ & 10.684 & 10.58 & 7.792 & 6.656 & 4.429 & 4.138 & 2.369 & 2.335 & 10.878 & 6.066 & 4.795 & 6.631 & 4.036 \\
\hline 9/3/2005 & $2: 00$ & 10.684 & 10.581 & 7.79 & 6.656 & 4.421 & 4.138 & 2.366 & 2.333 & 10.874 & 6.061 & 4.785 & 6.628 & 4.02 \\
\hline 9/3/2005 & $3: 00$ & 10.687 & 10.58 & 7.788 & 6.653 & 4.412 & 4.136 & 2.364 & 2.329 & 10.87 & 6.057 & 4.774 & 6.62 & 4.003 \\
\hline 9/3/2005 & $4: 00$ & 10.685 & 10.58 & 7.788 & 6.651 & 4.406 & 4.136 & 2.352 & 2.327 & 10.868 & 6.048 & 4.764 & 6.614 & 3.989 \\
\hline $9 / 3 / 2005$ & $5: 00$ & 10.685 & 10.576 & 7.786 & 6.649 & 4.398 & 4.134 & 2.352 & 2.322 & 10.863 & 6.046 & 4.754 & 6.609 & 3.975 \\
\hline
\end{tabular}


TABLE S1.2 (Cont.)

Water Level (ft below top of casing) at Indicated Well

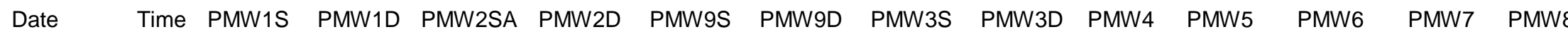

\begin{tabular}{|c|c|c|c|c|c|c|c|c|c|c|c|c|c|c|}
\hline 9/3/2005 & $6: 00$ & 10.682 & 10.576 & 7.784 & 6.645 & 4.391 & 4.132 & 2.344 & 2.318 & 10.859 & 6.042 & 4.745 & 6.603 & 3.963 \\
\hline 9/3/2005 & 7:00 & 10.685 & 10.576 & 7.784 & 6.645 & 4.387 & 4.132 & 2.344 & 2.318 & 10.853 & 6.04 & 4.739 & 6.597 & 3.953 \\
\hline $9 / 3 / 2005$ & $8: 00$ & 10.685 & 10.578 & 7.784 & 6.649 & 4.385 & 4.129 & 2.355 & 2.318 & 10.851 & 6.037 & 4.735 & 6.597 & 3.945 \\
\hline 9/3/2005 & $9: 00$ & 10.682 & 10.574 & 7.784 & 6.647 & 4.379 & 4.127 & 2.35 & 2.316 & 10.845 & 6.035 & 4.733 & 6.597 & 3.939 \\
\hline $9 / 3 / 2005$ & $10: 00$ & 10.682 & 10.578 & 7.784 & 6.647 & 4.383 & 4.127 & 2.36 & 2.322 & 10.844 & 6.04 & 4.745 & 6.613 & 3.949 \\
\hline 9/3/2005 & $11: 00$ & 10.682 & 10.574 & 7.788 & 6.649 & 4.387 & 4.125 & 2.364 & 2.329 & 10.846 & 6.044 & 4.764 & 6.63 & 3.969 \\
\hline $9 / 3 / 2005$ & $12: 00$ & 10.682 & 10.572 & 7.788 & 6.647 & 4.397 & 4.123 & 2.369 & 2.333 & 10.848 & 6.053 & 4.786 & 6.649 & 3.999 \\
\hline 9/3/2005 & $13: 00$ & 10.675 & 10.565 & 7.788 & 6.647 & 4.404 & 4.121 & 2.371 & 2.333 & 10.852 & 6.062 & 4.812 & 6.672 & 4.034 \\
\hline 9/3/2005 & $14: 00$ & 10.67 & 10.556 & 7.788 & 6.642 & 4.416 & 4.118 & 2.366 & 2.335 & 10.859 & 6.073 & 4.839 & 6.691 & 4.077 \\
\hline 9/3/2005 & $15: 00$ & 10.665 & 10.547 & 7.782 & 6.638 & 4.428 & 4.116 & 2.366 & 2.335 & 10.867 & 6.079 & 4.859 & 6.7 & 4.113 \\
\hline 9/3/2005 & $16: 00$ & 10.658 & 10.538 & 7.78 & 6.633 & 4.439 & 4.116 & 2.371 & 2.333 & 10.874 & 6.086 & 4.878 & 6.71 & 4.142 \\
\hline 9/3/2005 & $17: 00$ & 10.653 & 10.532 & 7.778 & 6.631 & 4.449 & 4.116 & 2.371 & 2.333 & 10.884 & 6.095 & 4.893 & 6.716 & 4.168 \\
\hline 9/3/2005 & $18: 00$ & 10.648 & 10.525 & 7.776 & 6.631 & 4.455 & 4.116 & 2.369 & 2.331 & 10.888 & 6.097 & 4.902 & 6.714 & 4.18 \\
\hline 9/3/2005 & $19: 00$ & 10.641 & 10.523 & 7.774 & 6.627 & 4.456 & 4.116 & 2.366 & 2.329 & 10.893 & 6.099 & 4.9 & 6.704 & 4.187 \\
\hline 9/3/2005 & $20: 00$ & 10.641 & 10.523 & 7.774 & 6.629 & 4.46 & 4.118 & 2.361 & 2.327 & 10.895 & 6.097 & 4.888 & 6.687 & 4.179 \\
\hline $9 / 3 / 2005$ & $21: 00$ & 10.648 & 10.532 & 7.776 & 6.636 & 4.462 & 4.123 & 2.367 & 2.329 & 10.899 & 6.095 & 4.876 & 6.676 & 4.163 \\
\hline 9/3/2005 & $22: 00$ & 10.651 & 10.538 & 7.779 & 6.64 & 4.458 & 4.125 & 2.359 & 2.329 & 10.899 & 6.088 & 4.861 & 6.664 & 4.138 \\
\hline 9/3/2005 & $23: 00$ & 10.653 & 10.54 & 7.777 & 6.638 & 4.451 & 4.127 & 2.362 & 2.325 & 10.899 & 6.082 & 4.845 & 6.653 & 4.118 \\
\hline 9/4/2005 & $0: 00$ & 10.654 & 10.54 & 7.775 & 6.633 & 4.439 & 4.127 & 2.357 & 2.319 & 10.897 & 6.078 & 4.829 & 6.641 & 4.092 \\
\hline 9/4/2005 & 1:00 & 10.656 & 10.54 & 7.773 & 6.633 & 4.43 & 4.127 & 2.345 & 2.314 & 10.891 & 6.073 & 4.815 & 6.636 & 4.071 \\
\hline 9/4/2005 & $2: 00$ & 10.651 & 10.536 & 7.771 & 6.629 & 4.42 & 4.125 & 2.345 & 2.31 & 10.888 & 6.069 & 4.802 & 6.624 & 4.053 \\
\hline 9/4/2005 & $3: 00$ & 10.656 & 10.543 & 7.771 & 6.633 & 4.414 & 4.127 & 2.35 & 2.312 & 10.886 & 6.065 & 4.792 & 6.622 & 4.039 \\
\hline 9/4/2005 & $4: 00$ & 10.656 & 10.545 & 7.771 & 6.636 & 4.403 & 4.125 & 2.34 & 2.312 & 10.882 & 6.062 & 4.782 & 6.615 & 4.027 \\
\hline 9/4/2005 & $5: 00$ & 10.658 & 10.547 & 7.773 & 6.638 & 4.404 & 4.125 & 2.345 & 2.312 & 10.878 & 6.058 & 4.772 & 6.609 & 4.017 \\
\hline 9/4/2005 & $6: 00$ & 10.661 & 10.549 & 7.771 & 6.636 & 4.399 & 4.123 & 2.337 & 2.31 & 10.872 & 6.054 & 4.765 & 6.605 & 4.003 \\
\hline 9/4/2005 & $7: 00$ & 10.663 & 10.55 & 7.771 & 6.633 & 4.393 & 4.121 & 2.345 & 2.306 & 10.871 & 6.051 & 4.757 & 6.602 & 3.991 \\
\hline 9/4/2005 & $8: 00$ & 10.666 & 10.552 & 7.773 & 6.638 & 4.389 & 4.121 & 2.345 & 2.31 & 10.867 & 6.047 & 4.749 & 6.597 & 3.983 \\
\hline 9/4/2005 & 9:00 & 10.666 & 10.552 & 7.773 & 6.636 & 4.385 & 4.121 & 2.337 & 2.31 & 10.863 & 6.049 & 4.751 & 6.602 & 3.976 \\
\hline 9/4/2005 & $10: 00$ & 10.669 & 10.556 & 7.777 & 6.642 & 4.389 & 4.118 & 2.353 & 2.319 & 10.863 & 6.054 & 4.765 & 6.617 & 3.986 \\
\hline 9/4/2005 & $11: 00$ & 10.671 & 10.56 & 7.781 & 6.645 & 4.397 & 4.116 & 2.365 & 2.329 & 10.863 & 6.06 & 4.784 & 6.636 & 4.013 \\
\hline 9/4/2005 & $12: 00$ & 10.671 & 10.556 & 7.783 & 6.645 & 4.407 & 4.118 & 2.37 & 2.333 & 10.867 & 6.067 & 4.81 & 6.659 & 4.045 \\
\hline 9/4/2005 & $13: 00$ & 10.664 & 10.549 & 7.783 & 6.643 & 4.416 & 4.114 & 2.365 & 2.333 & 10.873 & 6.076 & 4.835 & 6.678 & 4.082 \\
\hline 9/4/2005 & $14: 00$ & 10.659 & 10.543 & 7.781 & 6.643 & 4.43 & 4.114 & 2.368 & 2.335 & 10.879 & 6.085 & 4.861 & 6.695 & 4.122 \\
\hline 9/4/2005 & $15: 00$ & 10.654 & 10.54 & 7.781 & 6.64 & 4.443 & 4.117 & 2.378 & 2.34 & 10.89 & 6.096 & 4.884 & 6.708 & 4.159 \\
\hline
\end{tabular}


TABLE S1.2 (Cont.)

Water Level (ft below top of casing) at Indicated Well

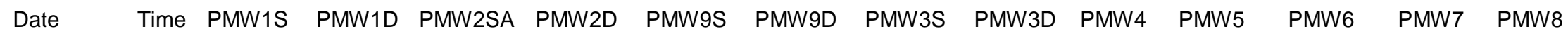

\begin{tabular}{|c|c|c|c|c|c|c|c|c|c|c|c|c|c|c|}
\hline 9/4/2005 & $16: 00$ & 10.649 & 10.536 & 7.781 & 6.64 & 4.455 & 4.116 & 2.373 & 2.342 & 10.898 & 6.102 & 4.902 & 6.72 & 4.187 \\
\hline 9/4/2005 & $17: 00$ & 10.649 & 10.534 & 7.781 & 6.64 & 4.466 & 4.118 & 2.383 & 2.346 & 10.905 & 6.109 & 4.916 & 6.727 & 4.21 \\
\hline 9/4/2005 & $18: 00$ & 10.649 & 10.534 & 7.783 & 6.643 & 4.474 & 4.123 & 2.385 & 2.346 & 10.909 & 6.111 & 4.926 & 6.724 & 4.222 \\
\hline 9/4/2005 & $19: 00$ & 10.649 & 10.538 & 7.783 & 6.645 & 4.482 & 4.125 & 2.385 & 2.349 & 10.913 & 6.113 & 4.926 & 6.716 & 4.228 \\
\hline 9/4/2005 & $20: 00$ & 10.654 & 10.541 & 7.785 & 6.647 & 4.484 & 4.129 & 2.385 & 2.346 & 10.917 & 6.113 & 4.916 & 6.703 & 4.216 \\
\hline 9/4/2005 & $21: 00$ & 10.656 & 10.547 & 7.787 & 6.652 & 4.482 & 4.134 & 2.383 & 2.346 & 10.918 & 6.107 & 4.902 & 6.689 & 4.198 \\
\hline $9 / 4 / 2005$ & $22: 00$ & 10.664 & 10.556 & 7.789 & 6.656 & 4.478 & 4.139 & 2.378 & 2.346 & 10.92 & 6.103 & 4.886 & 6.678 & 4.18 \\
\hline 9/4/2005 & $23: 00$ & 10.667 & 10.558 & 7.787 & 6.656 & 4.47 & 4.141 & 2.373 & 2.344 & 10.92 & 6.096 & 4.871 & 6.668 & 4.156 \\
\hline 9/5/2005 & $0: 00$ & 10.669 & 10.563 & 7.789 & 6.656 & 4.464 & 4.143 & 2.378 & 2.342 & 10.918 & 6.09 & 4.855 & 6.661 & 4.135 \\
\hline $9 / 5 / 2005$ & $1: 00$ & 10.672 & 10.565 & 7.789 & 6.658 & 4.455 & 4.145 & 2.376 & 2.34 & 10.917 & 6.087 & 4.843 & 6.655 & 4.115 \\
\hline $9 / 5 / 2005$ & $2: 00$ & 10.674 & 10.565 & 7.787 & 6.656 & 4.445 & 4.145 & 2.371 & 2.338 & 10.913 & 6.083 & 4.832 & 6.645 & 4.095 \\
\hline $9 / 5 / 2005$ & $3: 00$ & 10.674 & 10.565 & 7.786 & 6.654 & 4.435 & 4.143 & 2.361 & 2.332 & 10.907 & 6.076 & 4.82 & 6.64 & 4.077 \\
\hline $9 / 5 / 2005$ & $4: 00$ & 10.674 & 10.561 & 7.784 & 6.649 & 4.426 & 4.141 & 2.364 & 2.328 & 10.903 & 6.072 & 4.81 & 6.632 & 4.058 \\
\hline $9 / 5 / 2005$ & $5: 00$ & 10.674 & 10.563 & 7.784 & 6.649 & 4.42 & 4.141 & 2.353 & 2.328 & 10.898 & 6.07 & 4.801 & 6.629 & 4.046 \\
\hline 9/5/2005 & $6: 00$ & 10.675 & 10.565 & 7.784 & 6.647 & 4.414 & 4.138 & 2.358 & 2.325 & 10.892 & 6.066 & 4.791 & 6.623 & 4.032 \\
\hline 9/5/2005 & $7: 00$ & 10.674 & 10.567 & 7.784 & 6.652 & 4.413 & 4.137 & 2.351 & 2.325 & 10.89 & 6.064 & 4.787 & 6.621 & 4.022 \\
\hline 9/5/2005 & $8: 00$ & 10.674 & 10.565 & 7.784 & 6.647 & 4.405 & 4.137 & 2.356 & 2.321 & 10.884 & 6.061 & 4.779 & 6.617 & 4.012 \\
\hline 9/5/2005 & $9: 00$ & 10.675 & 10.567 & 7.784 & 6.649 & 4.405 & 4.134 & 2.356 & 2.33 & 10.882 & 6.061 & 4.783 & 6.626 & 4.014 \\
\hline $9 / 5 / 2005$ & $10: 00$ & 10.682 & 10.574 & 7.79 & 6.654 & 4.411 & 4.134 & 2.369 & 2.334 & 10.882 & 6.066 & 4.789 & 6.631 & 4.02 \\
\hline 9/5/2005 & $11: 00$ & 10.689 & 10.583 & 7.794 & 6.665 & 4.418 & 4.137 & 2.377 & 2.343 & 10.881 & 6.068 & 4.793 & 6.631 & 4.022 \\
\hline $9 / 5 / 2005$ & $12: 00$ & 10.687 & 10.578 & 7.792 & 6.66 & 4.411 & 4.134 & 2.364 & 2.336 & 10.879 & 6.059 & 4.785 & 6.627 & 4.014 \\
\hline 9/5/2005 & $13: 00$ & 10.684 & 10.574 & 7.79 & 6.656 & 4.407 & 4.134 & 2.361 & 2.334 & 10.877 & 6.064 & 4.787 & 6.629 & 4.012 \\
\hline $9 / 5 / 2005$ & $14: 00$ & 10.675 & 10.563 & 7.786 & 6.645 & 4.401 & 4.13 & 2.359 & 2.324 & 10.875 & 6.061 & 4.781 & 6.627 & 4.006 \\
\hline $9 / 5 / 2005$ & $15: 00$ & 10.67 & 10.556 & 7.782 & 6.643 & 4.401 & 4.128 & 2.354 & 2.326 & 10.871 & 6.064 & 4.795 & 6.638 & 4.018 \\
\hline $9 / 5 / 2005$ & $16: 00$ & 10.66 & 10.547 & 7.78 & 6.634 & 4.401 & 4.123 & 2.359 & 2.324 & 10.869 & 6.068 & 4.808 & 6.65 & 4.031 \\
\hline $9 / 5 / 2005$ & $17: 00$ & 10.657 & 10.54 & 7.778 & 6.636 & 4.409 & 4.121 & 2.364 & 2.328 & 10.873 & 6.079 & 4.83 & 6.669 & 4.065 \\
\hline $9 / 5 / 2005$ & $18: 00$ & 10.652 & 10.54 & 7.78 & 6.636 & 4.422 & 4.119 & 2.369 & 2.332 & 10.879 & 6.088 & 4.851 & 6.682 & 4.101 \\
\hline $9 / 5 / 2005$ & $19: 00$ & 10.65 & 10.538 & 7.78 & 6.638 & 4.434 & 4.121 & 2.372 & 2.336 & 10.885 & 6.093 & 4.864 & 6.68 & 4.128 \\
\hline $9 / 5 / 2005$ & $20: 00$ & 10.65 & 10.54 & 7.782 & 6.64 & 4.441 & 4.121 & 2.369 & 2.334 & 10.89 & 6.095 & 4.863 & 6.676 & 4.132 \\
\hline 9/5/2005 & $21: 00$ & 10.653 & 10.547 & 7.782 & 6.647 & 4.449 & 4.125 & 2.372 & 2.334 & 10.894 & 6.093 & 4.86 & 6.667 & 4.124 \\
\hline 9/5/2005 & $22: 00$ & 10.658 & 10.552 & 7.786 & 6.649 & 4.449 & 4.13 & 2.372 & 2.336 & 10.9 & 6.088 & 4.852 & 6.661 & 4.11 \\
\hline $9 / 5 / 2005$ & $23: 00$ & 10.663 & 10.554 & 7.786 & 6.649 & 4.443 & 4.13 & 2.362 & 2.334 & 10.898 & 6.086 & 4.84 & 6.654 & 4.094 \\
\hline 9/6/2005 & $0: 00$ & 10.66 & 10.549 & 7.78 & 6.643 & 4.434 & 4.13 & 2.357 & 2.326 & 10.896 & 6.079 & 4.83 & 6.646 & 4.072 \\
\hline $9 / 6 / 2005$ & $1: 00$ & 10.66 & 10.552 & 7.78 & 6.643 & 4.43 & 4.132 & 2.359 & 2.326 & 10.892 & 6.078 & 4.822 & 6.642 & 4.059 \\
\hline
\end{tabular}


TABLE S1.2 (Cont.)

Water Level (ft below top of casing) at Indicated Well

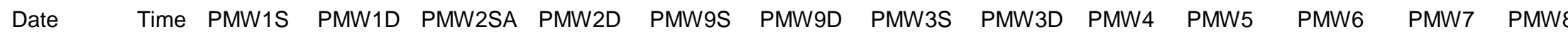

\begin{tabular}{|c|c|c|c|c|c|c|c|c|c|c|c|c|c|c|}
\hline 9/6/2005 & $2: 00$ & 10.663 & 10.556 & 7.782 & 6.647 & 4.428 & 4.132 & 2.357 & 2.326 & 10.89 & 6.075 & 4.815 & 6.64 & 4.049 \\
\hline 9/6/2005 & $3: 00$ & 10.66 & 10.554 & 7.78 & 6.643 & 4.42 & 4.13 & 2.352 & 2.324 & 10.888 & 6.068 & 4.803 & 6.632 & 4.033 \\
\hline 9/6/2005 & $4: 00$ & 10.665 & 10.56 & 7.783 & 6.647 & 4.418 & 4.13 & 2.36 & 2.324 & 10.885 & 6.064 & 4.797 & 6.632 & 4.025 \\
\hline 9/6/2005 & $5: 00$ & 10.668 & 10.563 & 7.784 & 6.65 & 4.416 & 4.13 & 2.36 & 2.326 & 10.883 & 6.062 & 4.793 & 6.629 & 4.015 \\
\hline 9/6/2005 & $6: 00$ & 10.67 & 10.565 & 7.784 & 6.649 & 4.411 & 4.13 & 2.357 & 2.326 & 10.879 & 6.06 & 4.785 & 6.625 & 4.003 \\
\hline 9/6/2005 & $7: 00$ & 10.685 & 10.585 & 7.794 & 6.67 & 4.42 & 4.134 & 2.368 & 2.341 & 10.879 & 6.064 & 4.792 & 6.631 & 4.007 \\
\hline 9/6/2005 & $8: 00$ & 10.698 & 10.598 & 7.803 & 6.679 & 4.43 & 4.141 & 2.383 & 2.351 & 10.881 & 6.064 & 4.791 & 6.598 & 4.005 \\
\hline 9/6/2005 & $9: 00$ & 10.702 & 10.603 & 7.807 & 6.683 & 4.428 & 4.143 & 2.38 & 2.356 & 10.879 & 6.06 & 4.79 & 6.629 & 3.995 \\
\hline 9/6/2005 & $10: 00$ & 10.705 & 10.607 & 7.807 & 6.683 & 4.426 & 4.145 & 2.38 & 2.355 & 10.877 & 6.058 & 4.785 & 6.631 & 3.987 \\
\hline 9/6/2005 & $11: 00$ & 10.71 & 10.611 & 7.805 & 6.681 & 4.424 & 4.145 & 2.39 & 2.358 & 10.875 & 6.058 & 4.797 & 6.669 & 3.989 \\
\hline 9/6/2005 & $12: 00$ & 10.71 & 10.605 & 7.809 & 6.681 & 4.424 & 4.148 & 2.393 & 2.36 & 10.871 & 6.062 & 4.809 & 6.679 & 3.999 \\
\hline $9 / 6 / 2005$ & $13: 00$ & 10.702 & 10.596 & 7.805 & 6.674 & 4.424 & 4.143 & 2.385 & 2.358 & 10.871 & 6.065 & 4.825 & 6.699 & 4.017 \\
\hline 9/6/2005 & $14: 00$ & 10.695 & 10.587 & 7.802 & 6.667 & 4.428 & 4.143 & 2.39 & 2.356 & 10.874 & 6.069 & 4.835 & 6.715 & 4.035 \\
\hline 9/6/2005 & $15: 00$ & 10.69 & 10.58 & 7.8 & 6.665 & 4.43 & 4.141 & 2.385 & 2.349 & 10.875 & 6.076 & 4.837 & 6.703 & 4.052 \\
\hline 9/6/2005 & $16: 00$ & 10.69 & 10.583 & 7.802 & 6.67 & 4.438 & 4.143 & 2.385 & 2.354 & 10.879 & 6.078 & 4.835 & 6.724 & 4.06 \\
\hline 9/6/2005 & $17: 00$ & 10.696 & 10.591 & 7.807 & 6.674 & 4.446 & 4.145 & 2.385 & 2.358 & 10.881 & 6.078 & 4.831 & 6.73 & 4.06 \\
\hline 9/6/2005 & $18: 00$ & 10.7 & 10.591 & 7.807 & 6.679 & 4.443 & 4.148 & 2.391 & 2.358 & 10.883 & 6.076 & 4.821 & 6.711 & 4.052 \\
\hline 9/6/2005 & $19: 00$ & 10.7 & 10.592 & 7.807 & 6.674 & 4.439 & 4.148 & 2.383 & 2.356 & 10.881 & 6.071 & 4.803 & 6.69 & 4.04 \\
\hline 9/6/2005 & $20: 00$ & 10.7 & 10.594 & 7.807 & 6.676 & 4.438 & 4.15 & 2.378 & 2.354 & 10.879 & 6.072 & 4.786 & 6.671 & 4.026 \\
\hline 9/6/2005 & $21: 00$ & 10.708 & 10.603 & 7.809 & 6.683 & 4.439 & 4.152 & 2.388 & 2.356 & 10.879 & 6.067 & 4.774 & 6.659 & 4.017 \\
\hline 9/6/2005 & $22: 00$ & 10.71 & 10.605 & 7.809 & 6.683 & 4.434 & 4.154 & 2.388 & 2.356 & 10.879 & 6.061 & 4.76 & 6.65 & 4.002 \\
\hline 9/6/2005 & $23: 00$ & 10.71 & 10.607 & 7.811 & 6.683 & 4.428 & 4.154 & 2.381 & 2.354 & 10.875 & 6.056 & 4.748 & 6.642 & 3.987 \\
\hline 9/7/2005 & $0: 00$ & 10.715 & 10.612 & 7.811 & 6.687 & 4.426 & 4.154 & 2.389 & 2.358 & 10.872 & 6.056 & 4.743 & 6.637 & 3.977 \\
\hline 9/7/2005 & $1: 00$ & 10.72 & 10.616 & 7.813 & 6.69 & 4.424 & 4.156 & 2.391 & 2.358 & 10.87 & 6.052 & 4.735 & 6.633 & 3.969 \\
\hline 9/7/2005 & $2: 00$ & 10.718 & 10.612 & 7.811 & 6.683 & 4.414 & 4.156 & 2.376 & 2.354 & 10.866 & 6.045 & 4.723 & 6.627 & 3.955 \\
\hline 9/7/2005 & $3: 00$ & 10.718 & 10.612 & 7.811 & 6.681 & 4.411 & 4.156 & 2.376 & 2.349 & 10.862 & 6.043 & 4.717 & 6.623 & 3.945 \\
\hline 9/7/2005 & $4: 00$ & 10.71 & 10.602 & 7.803 & 6.665 & 4.395 & 4.152 & 2.371 & 2.339 & 10.856 & 6.034 & 4.704 & 6.618 & 3.927 \\
\hline 9/7/2005 & $5: 00$ & 10.706 & 10.598 & 7.801 & 6.665 & 4.39 & 4.15 & 2.361 & 2.335 & 10.853 & 6.032 & 4.698 & 6.61 & 3.919 \\
\hline 9/7/2005 & $6: 00$ & 10.706 & 10.596 & 7.799 & 6.663 & 4.386 & 4.145 & 2.359 & 2.333 & 10.849 & 6.032 & 4.692 & 6.608 & 3.912 \\
\hline 9/7/2005 & $7: 00$ & 10.706 & 10.596 & 7.799 & 6.665 & 4.384 & 4.143 & 2.359 & 2.333 & 10.847 & 6.032 & 4.69 & 6.604 & 3.908 \\
\hline 9/7/2005 & $8: 00$ & 10.706 & 10.598 & 7.801 & 6.667 & 4.382 & 4.143 & 2.366 & 2.335 & 10.841 & 6.028 & 4.69 & 6.624 & 3.904 \\
\hline 9/7/2005 & $9: 00$ & 10.703 & 10.594 & 7.799 & 6.661 & 4.378 & 4.139 & 2.359 & 2.331 & 10.839 & 6.026 & 4.684 & 6.612 & 3.897 \\
\hline 9/7/2005 & $10: 00$ & 10.703 & 10.598 & 7.799 & 6.663 & 4.38 & 4.139 & 2.359 & 2.335 & 10.836 & 6.028 & 4.692 & 6.616 & 3.897 \\
\hline 9/7/2005 & $11: 00$ & 10.708 & 10.598 & 7.801 & 6.667 & 4.386 & 4.137 & 2.374 & 2.339 & 10.836 & 6.032 & 4.702 & 6.622 & 3.905 \\
\hline
\end{tabular}


Water Level (ft below top of casing) at Indicated Well

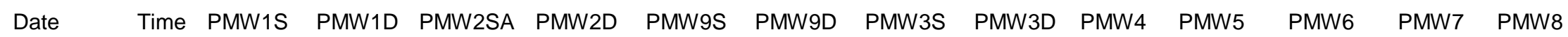

\begin{tabular}{|c|c|c|c|c|c|c|c|c|c|c|c|c|c|c|}
\hline 9/7/2005 & $12: 00$ & 10.706 & 10.598 & 7.803 & 6.665 & 4.386 & 4.134 & 2.374 & 2.341 & 10.834 & 6.034 & 4.714 & 6.629 & 3.913 \\
\hline 9/7/2005 & $13: 00$ & 10.698 & 10.589 & 7.799 & 6.659 & 4.388 & 4.134 & 2.367 & 2.339 & 10.832 & 6.037 & 4.727 & 6.643 & 3.927 \\
\hline 9/7/2005 & $14: 00$ & 10.694 & 10.581 & 7.799 & 6.659 & 4.396 & 4.132 & 2.372 & 2.343 & 10.834 & 6.048 & 4.757 & 6.664 & 3.957 \\
\hline 9/7/2005 & $15: 00$ & 10.686 & 10.572 & 7.797 & 6.654 & 4.405 & 4.13 & 2.379 & 2.343 & 10.838 & 6.057 & 4.782 & 6.677 & 3.998 \\
\hline 9/7/2005 & $16: 00$ & 10.676 & 10.563 & 7.795 & 6.65 & 4.419 & 4.126 & 2.379 & 2.343 & 10.845 & 6.068 & 4.808 & 6.69 & 4.036 \\
\hline 9/7/2005 & $17: 00$ & 10.671 & 10.558 & 7.795 & 6.65 & 4.434 & 4.126 & 2.381 & 2.343 & 10.853 & 6.077 & 4.827 & 6.694 & 4.069 \\
\hline 9/7/2005 & $18: 00$ & 10.669 & 10.556 & 7.793 & 6.65 & 4.446 & 4.126 & 2.381 & 2.346 & 10.861 & 6.081 & 4.839 & 6.694 & 4.095 \\
\hline 9/7/2005 & $19: 00$ & 10.667 & 10.554 & 7.793 & 6.65 & 4.453 & 4.128 & 2.382 & 2.346 & 10.866 & 6.086 & 4.841 & 6.686 & 4.105 \\
\hline 9/7/2005 & $20: 00$ & 10.665 & 10.554 & 7.793 & 6.652 & 4.457 & 4.13 & 2.377 & 2.343 & 10.87 & 6.081 & 4.835 & 6.675 & 4.093 \\
\hline 9/7/2005 & $21: 00$ & 10.669 & 10.56 & 7.793 & 6.655 & 4.457 & 4.134 & 2.372 & 2.343 & 10.874 & 6.079 & 4.824 & 6.664 & 4.077 \\
\hline 9/7/2005 & $22: 00$ & 10.672 & 10.563 & 7.794 & 6.657 & 4.453 & 4.137 & 2.374 & 2.342 & 10.874 & 6.077 & 4.812 & 6.654 & 4.055 \\
\hline 9/7/2005 & $23: 00$ & 10.672 & 10.565 & 7.794 & 6.657 & 4.444 & 4.139 & 2.372 & 2.339 & 10.874 & 6.068 & 4.798 & 6.646 & 4.037 \\
\hline 9/8/2005 & 0:00 & 10.674 & 10.567 & 7.794 & 6.657 & 4.436 & 4.139 & 2.37 & 2.338 & 10.872 & 6.064 & 4.786 & 6.639 & 4.019 \\
\hline 9/8/2005 & $1: 00$ & 10.674 & 10.563 & 7.79 & 6.65 & 4.424 & 4.137 & 2.364 & 2.331 & 10.87 & 6.057 & 4.773 & 6.629 & 3.999 \\
\hline 9/8/2005 & $2: 00$ & 10.669 & 10.56 & 7.786 & 6.646 & 4.415 & 4.137 & 2.357 & 2.325 & 10.864 & 6.053 & 4.759 & 6.624 & 3.978 \\
\hline 9/8/2005 & $3: 00$ & 10.665 & 10.552 & 7.782 & 6.637 & 4.403 & 4.132 & 2.349 & 2.317 & 10.859 & 6.046 & 4.745 & 6.614 & 3.962 \\
\hline 9/8/2005 & $4: 00$ & 10.66 & 10.549 & 7.778 & 6.635 & 4.396 & 4.13 & 2.344 & 2.312 & 10.855 & 6.042 & 4.733 & 6.609 & 3.95 \\
\hline 9/8/2005 & $5: 00$ & 10.658 & 10.543 & 7.778 & 6.628 & 4.388 & 4.125 & 2.337 & 2.308 & 10.849 & 6.035 & 4.722 & 6.601 & 3.936 \\
\hline 9/8/2005 & $6: 00$ & 10.653 & 10.54 & 7.774 & 6.626 & 4.38 & 4.121 & 2.335 & 2.302 & 10.843 & 6.031 & 4.71 & 6.595 & 3.924 \\
\hline 9/8/2005 & $7: 00$ & 10.65 & 10.536 & 7.77 & 6.621 & 4.374 & 4.119 & 2.322 & 2.298 & 10.84 & 6.029 & 4.7 & 6.591 & 3.914 \\
\hline 9/8/2005 & $8: 00$ & 10.648 & 10.536 & 7.77 & 6.626 & 4.372 & 4.115 & 2.33 & 2.298 & 10.836 & 6.027 & 4.696 & 6.591 & 3.91 \\
\hline 9/8/2005 & $9: 00$ & 10.645 & 10.532 & 7.768 & 6.621 & 4.369 & 4.113 & 2.322 & 2.296 & 10.83 & 6.024 & 4.694 & 6.591 & 3.904 \\
\hline 9/8/2005 & $10: 00$ & 10.645 & 10.534 & 7.768 & 6.624 & 4.371 & 4.11 & 2.333 & 2.3 & 10.828 & 6.029 & 4.706 & 6.605 & 3.916 \\
\hline 9/8/2005 & $11: 00$ & 10.643 & 10.53 & 7.77 & 6.621 & 4.375 & 4.106 & 2.34 & 2.306 & 10.828 & 6.035 & 4.728 & 6.624 & 3.94 \\
\hline 9/8/2005 & $12: 00$ & 10.638 & 10.525 & 7.768 & 6.619 & 4.386 & 4.104 & 2.337 & 2.308 & 10.83 & 6.047 & 4.757 & 6.645 & 3.981 \\
\hline 9/8/2005 & $13: 00$ & 10.636 & 10.522 & 7.772 & 6.619 & 4.398 & 4.101 & 2.35 & 2.315 & 10.834 & 6.06 & 4.789 & 6.668 & 4.029 \\
\hline 9/8/2005 & $14: 00$ & 10.633 & 10.519 & 7.774 & 6.619 & 4.419 & 4.101 & 2.347 & 2.319 & 10.84 & 6.071 & 4.822 & 6.702 & 4.08 \\
\hline 9/8/2005 & $15: 00$ & 10.631 & 10.512 & 7.774 & 6.619 & 4.438 & 4.099 & 2.357 & 2.323 & 10.845 & 6.077 & 4.846 & 6.712 & 4.118 \\
\hline 9/8/2005 & $16: 00$ & 10.626 & 10.505 & 7.772 & 6.615 & 4.454 & 4.099 & 2.352 & 2.325 & 10.857 & 6.086 & 4.865 & 6.721 & 4.157 \\
\hline $9 / 8 / 2005$ & $17: 00$ & 10.621 & 10.498 & 7.77 & 6.615 & 4.465 & 4.101 & 2.357 & 2.325 & 10.864 & 6.095 & 4.887 & 6.729 & 4.183 \\
\hline 9/8/2005 & $18: 00$ & 10.621 & 10.5 & 7.774 & 6.619 & 4.479 & 4.104 & 2.364 & 2.329 & 10.876 & 6.104 & 4.896 & 6.731 & 4.206 \\
\hline 9/8/2005 & $19: 00$ & 10.619 & 10.5 & 7.772 & 6.621 & 4.486 & 4.108 & 2.364 & 2.327 & 10.883 & 6.104 & 4.893 & 6.712 & 4.212 \\
\hline 9/8/2005 & $20: 00$ & 10.618 & 10.503 & 7.774 & 6.624 & 4.486 & 4.113 & 2.362 & 2.327 & 10.889 & 6.102 & 4.883 & 6.694 & 4.202 \\
\hline 9/8/2005 & $21: 00$ & 10.624 & 10.509 & 7.774 & 6.624 & 4.484 & 4.117 & 2.354 & 2.325 & 10.893 & 6.097 & 4.867 & 6.681 & 4.178 \\
\hline
\end{tabular}


TABLE S1.2 (Cont.)

Water Level (ft below top of casing) at Indicated Well

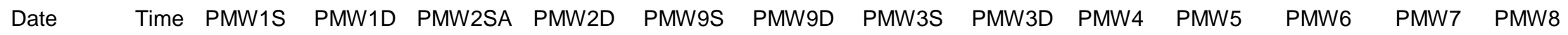

\begin{tabular}{|c|c|c|c|c|c|c|c|c|c|c|c|c|c|c|}
\hline 9/8/2005 & $22: 00$ & 10.624 & 10.511 & 7.773 & 6.624 & 4.476 & 4.119 & 2.354 & 2.321 & 10.893 & 6.089 & 4.849 & 6.668 & 4.152 \\
\hline 9/8/2005 & $23: 00$ & 10.621 & 10.511 & 7.771 & 6.621 & 4.465 & 4.119 & 2.349 & 2.317 & 10.893 & 6.082 & 4.834 & 6.656 & 4.125 \\
\hline 9/9/2005 & $0: 00$ & 10.624 & 10.51 & 7.769 & 6.621 & 4.458 & 4.121 & 2.337 & 2.311 & 10.891 & 6.075 & 4.82 & 6.647 & 4.105 \\
\hline 9/9/2005 & $1: 00$ & 10.624 & 10.512 & 7.769 & 6.619 & 4.45 & 4.119 & 2.34 & 2.307 & 10.888 & 6.071 & 4.806 & 6.639 & 4.083 \\
\hline 9/9/2005 & $2: 00$ & 10.624 & 10.512 & 7.767 & 6.619 & 4.44 & 4.119 & 2.332 & 2.304 & 10.884 & 6.065 & 4.795 & 6.631 & 4.065 \\
\hline 9/9/2005 & $3: 00$ & 10.624 & 10.512 & 7.765 & 6.617 & 4.429 & 4.117 & 2.327 & 2.302 & 10.878 & 6.06 & 4.783 & 6.624 & 4.048 \\
\hline 9/9/2005 & $4: 00$ & 10.627 & 10.514 & 7.765 & 6.615 & 4.423 & 4.117 & 2.335 & 2.3 & 10.874 & 6.058 & 4.771 & 6.62 & 4.034 \\
\hline 9/9/2005 & $5: 00$ & 10.627 & 10.514 & 7.765 & 6.615 & 4.417 & 4.115 & 2.333 & 2.298 & 10.872 & 6.054 & 4.764 & 6.615 & 4.02 \\
\hline 9/9/2005 & $6: 00$ & 10.627 & 10.518 & 7.765 & 6.617 & 4.413 & 4.115 & 2.325 & 2.298 & 10.865 & 6.049 & 4.753 & 6.609 & 4.008 \\
\hline 9/9/2005 & $7: 00$ & 10.632 & 10.521 & 7.767 & 6.619 & 4.409 & 4.115 & 2.325 & 2.298 & 10.863 & 6.047 & 4.748 & 6.605 & 4 \\
\hline 9/9/2005 & 8:00 & 10.634 & 10.525 & 7.767 & 6.621 & 4.406 & 4.113 & 2.333 & 2.3 & 10.859 & 6.045 & 4.744 & 6.605 & 3.992 \\
\hline 9/9/2005 & $9: 00$ & 10.634 & 10.529 & 7.769 & 6.624 & 4.404 & 4.113 & 2.338 & 2.304 & 10.857 & 6.045 & 4.748 & 6.613 & 3.992 \\
\hline 9/9/2005 & $10: 00$ & 10.639 & 10.53 & 7.771 & 6.624 & 4.407 & 4.11 & 2.346 & 2.311 & 10.855 & 6.052 & 4.768 & 6.63 & 4.008 \\
\hline 9/9/2005 & $11: 00$ & 10.641 & 10.533 & 7.775 & 6.628 & 4.419 & 4.108 & 2.356 & 2.321 & 10.857 & 6.061 & 4.791 & 6.651 & 4.04 \\
\hline 9/9/2005 & $12: 00$ & 10.639 & 10.529 & 7.779 & 6.628 & 4.433 & 4.108 & 2.356 & 2.328 & 10.863 & 6.074 & 4.822 & 6.674 & 4.081 \\
\hline 9/9/2005 & $13: 00$ & 10.636 & 10.525 & 7.78 & 6.628 & 4.442 & 4.108 & 2.366 & 2.33 & 10.867 & 6.081 & 4.85 & 6.691 & 4.127 \\
\hline 9/9/2005 & $14: 00$ & 10.637 & 10.522 & 7.781 & 6.628 & 4.462 & 4.11 & 2.376 & 2.338 & 10.876 & 6.094 & 4.885 & 6.712 & 4.174 \\
\hline 9/9/2005 & $15: 00$ & 10.629 & 10.516 & 7.783 & 6.628 & 4.479 & 4.108 & 2.378 & 2.342 & 10.888 & 6.105 & 4.911 & 6.727 & 4.211 \\
\hline 9/9/2005 & $16: 00$ & 10.63 & 10.514 & 7.781 & 6.628 & 4.493 & 4.113 & 2.376 & 2.346 & 10.893 & 6.111 & 4.928 & 6.741 & 4.237 \\
\hline 9/9/2005 & $17: 00$ & 10.629 & 10.509 & 7.783 & 6.633 & 4.5 & 4.117 & 2.378 & 2.349 & 10.905 & 6.116 & 4.946 & 6.767 & 4.255 \\
\hline 9/9/2005 & $18: 00$ & 10.63 & 10.514 & 7.783 & 6.633 & 4.51 & 4.119 & 2.383 & 2.349 & 10.914 & 6.122 & 4.95 & 6.75 & 4.27 \\
\hline 9/9/2005 & $19: 00$ & 10.63 & 10.514 & 7.785 & 6.635 & 4.516 & 4.124 & 2.386 & 2.349 & 10.922 & 6.125 & 4.948 & 6.739 & 4.276 \\
\hline 9/9/2005 & $20: 00$ & 10.632 & 10.518 & 7.785 & 6.637 & 4.517 & 4.128 & 2.376 & 2.347 & 10.924 & 6.121 & 4.938 & 6.723 & 4.264 \\
\hline 9/9/2005 & $21: 00$ & 10.639 & 10.529 & 7.787 & 6.642 & 4.515 & 4.132 & 2.381 & 2.347 & 10.927 & 6.118 & 4.924 & 6.71 & 4.246 \\
\hline 9/9/2005 & $22: 00$ & 10.642 & 10.529 & 7.787 & 6.642 & 4.508 & 4.137 & 2.378 & 2.343 & 10.928 & 6.112 & 4.905 & 6.695 & 4.22 \\
\hline 9/9/2005 & 23:00 & 10.644 & 10.534 & 7.787 & 6.644 & 4.5 & 4.139 & 2.376 & 2.341 & 10.929 & 6.107 & 4.891 & 6.687 & 4.2 \\
\hline 9/10/2005 & 0:00 & 10.649 & 10.536 & 7.785 & 6.642 & 4.49 & 4.141 & 2.371 & 2.338 & 10.926 & 6.099 & 4.875 & 6.676 & 4.179 \\
\hline 9/10/2005 & $1: 00$ & 10.65 & 10.538 & 7.785 & 6.642 & 4.481 & 4.141 & 2.369 & 2.336 & 10.924 & 6.094 & 4.862 & 6.668 & 4.157 \\
\hline 9/10/2005 & $2: 00$ & 10.652 & 10.54 & 7.785 & 6.639 & 4.471 & 4.141 & 2.359 & 2.332 & 10.92 & 6.09 & 4.848 & 6.657 & 4.139 \\
\hline 9/10/2005 & $3: 00$ & 10.652 & 10.538 & 7.783 & 6.637 & 4.461 & 4.141 & 2.361 & 2.328 & 10.918 & 6.083 & 4.834 & 6.651 & 4.121 \\
\hline 9/10/2005 & $4: 00$ & 10.65 & 10.538 & 7.781 & 6.635 & 4.452 & 4.139 & 2.351 & 2.324 & 10.911 & 6.079 & 4.825 & 6.644 & 4.104 \\
\hline 9/10/2005 & $5: 00$ & 10.65 & 10.536 & 7.778 & 6.633 & 4.444 & 4.137 & 2.346 & 2.317 & 10.907 & 6.077 & 4.813 & 6.638 & 4.088 \\
\hline 9/10/2005 & $6: 00$ & 10.652 & 10.54 & 7.779 & 6.635 & 4.438 & 4.135 & 2.346 & 2.317 & 10.903 & 6.072 & 4.803 & 6.63 & 4.076 \\
\hline $9 / 10 / 2005$ & $7: 00$ & 10.652 & 10.54 & 7.781 & 6.633 & 4.437 & 4.133 & 2.354 & 2.32 & 10.897 & 6.07 & 4.795 & 6.626 & 4.064 \\
\hline
\end{tabular}


TABLE S1.2 (Cont.)

Water Level (ft below top of casing) at Indicated Well

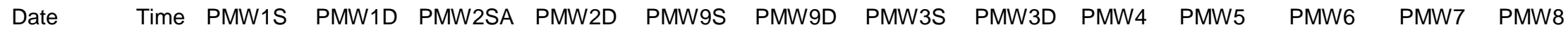

\begin{tabular}{|c|c|c|c|c|c|c|c|c|c|c|c|c|c|c|}
\hline 9/10/2005 & $8: 00$ & 10.657 & 10.545 & 7.782 & 6.637 & 4.435 & 4.133 & 2.349 & 2.322 & 10.896 & 6.068 & 4.793 & 6.625 & 4.056 \\
\hline 9/10/2005 & $9: 00$ & 10.657 & 10.547 & 7.784 & 6.639 & 4.433 & 4.13 & 2.36 & 2.324 & 10.892 & 6.068 & 4.795 & 6.632 & 4.053 \\
\hline 9/10/2005 & $10: 00$ & 10.659 & 10.549 & 7.787 & 6.639 & 4.437 & 4.13 & 2.367 & 2.33 & 10.892 & 6.073 & 4.807 & 6.642 & 4.066 \\
\hline 9/10/2005 & $11: 00$ & 10.652 & 10.543 & 7.782 & 6.637 & 4.441 & 4.126 & 2.365 & 2.339 & 10.888 & 6.077 & 4.827 & 6.663 & 4.088 \\
\hline 9/10/2005 & $12: 00$ & 10.655 & 10.54 & 7.783 & 6.637 & 4.45 & 4.126 & 2.375 & 2.341 & 10.894 & 6.088 & 4.854 & 6.68 & 4.125 \\
\hline $9 / 10 / 2005$ & $13: 00$ & 10.655 & 10.536 & 7.785 & 6.639 & 4.469 & 4.126 & 2.379 & 2.347 & 10.901 & 6.095 & 4.882 & 6.699 & 4.169 \\
\hline $9 / 10 / 2005$ & $14: 00$ & 10.65 & 10.534 & 7.787 & 6.642 & 4.487 & 4.126 & 2.389 & 2.352 & 10.909 & 6.108 & 4.909 & 6.714 & 4.21 \\
\hline $9 / 10 / 2005$ & $15: 00$ & 10.65 & 10.534 & 7.789 & 6.646 & 4.504 & 4.128 & 2.394 & 2.358 & 10.918 & 6.121 & 4.933 & 6.729 & 4.246 \\
\hline 9/10/2005 & $16: 00$ & 10.653 & 10.534 & 7.793 & 6.648 & 4.518 & 4.131 & 2.392 & 2.362 & 10.928 & 6.128 & 4.952 & 6.737 & 4.273 \\
\hline 9/10/2005 & $17: 00$ & 10.648 & 10.529 & 7.789 & 6.644 & 4.523 & 4.132 & 2.399 & 2.362 & 10.932 & 6.13 & 4.962 & 6.741 & 4.289 \\
\hline $9 / 10 / 2005$ & $18: 00$ & 10.653 & 10.533 & 7.793 & 6.648 & 4.537 & 4.135 & 2.404 & 2.366 & 10.939 & 6.141 & 4.974 & 6.747 & 4.305 \\
\hline 9/10/2005 & $19: 00$ & 10.655 & 10.538 & 7.797 & 6.653 & 4.541 & 4.142 & 2.399 & 2.368 & 10.943 & 6.139 & 4.972 & 6.733 & 4.309 \\
\hline $9 / 10 / 2005$ & $20: 00$ & 10.66 & 10.547 & 7.801 & 6.66 & 4.548 & 4.151 & 2.399 & 2.37 & 10.947 & 6.139 & 4.964 & 6.726 & 4.3 \\
\hline 9/10/2005 & $21: 00$ & 10.667 & 10.554 & 7.803 & 6.664 & 4.545 & 4.153 & 2.399 & 2.368 & 10.949 & 6.132 & 4.95 & 6.716 & 4.281 \\
\hline 9/10/2005 & $22: 00$ & 10.672 & 10.562 & 7.805 & 6.668 & 4.539 & 4.157 & 2.397 & 2.37 & 10.953 & 6.128 & 4.937 & 6.708 & 4.261 \\
\hline 9/10/2005 & $23: 00$ & 10.677 & 10.567 & 7.806 & 6.671 & 4.533 & 4.162 & 2.402 & 2.368 & 10.951 & 6.122 & 4.921 & 6.699 & 4.241 \\
\hline $9 / 11 / 2005$ & $0: 00$ & 10.68 & 10.571 & 7.804 & 6.671 & 4.523 & 4.164 & 2.395 & 2.366 & 10.951 & 6.119 & 4.909 & 6.691 & 4.219 \\
\hline 9/11/2005 & $1: 00$ & 10.685 & 10.574 & 7.806 & 6.671 & 4.518 & 4.166 & 2.392 & 2.364 & 10.949 & 6.115 & 4.894 & 6.686 & 4.202 \\
\hline $9 / 11 / 2005$ & $2: 00$ & 10.69 & 10.576 & 7.806 & 6.671 & 4.51 & 4.166 & 2.39 & 2.362 & 10.947 & 6.11 & 4.882 & 6.678 & 4.186 \\
\hline 9/11/2005 & $3: 00$ & 10.69 & 10.58 & 7.804 & 6.669 & 4.502 & 4.166 & 2.395 & 2.36 & 10.943 & 6.106 & 4.872 & 6.672 & 4.168 \\
\hline $9 / 11 / 2005$ & $4: 00$ & 10.692 & 10.58 & 7.804 & 6.669 & 4.493 & 4.166 & 2.385 & 2.358 & 10.939 & 6.102 & 4.858 & 6.665 & 4.152 \\
\hline $9 / 11 / 2005$ & $5: 00$ & 10.692 & 10.583 & 7.804 & 6.671 & 4.489 & 4.166 & 2.39 & 2.356 & 10.936 & 6.1 & 4.851 & 6.659 & 4.138 \\
\hline $9 / 11 / 2005$ & $6: 00$ & 10.697 & 10.589 & 7.808 & 6.677 & 4.485 & 4.168 & 2.393 & 2.36 & 10.934 & 6.098 & 4.843 & 6.655 & 4.129 \\
\hline $9 / 11 / 2005$ & $7: 00$ & 10.7 & 10.593 & 7.808 & 6.677 & 4.481 & 4.168 & 2.393 & 2.36 & 10.932 & 6.093 & 4.835 & 6.652 & 4.115 \\
\hline 9/11/2005 & $8: 00$ & 10.705 & 10.596 & 7.81 & 6.68 & 4.477 & 4.168 & 2.393 & 2.36 & 10.93 & 6.091 & 4.829 & 6.65 & 4.105 \\
\hline $9 / 11 / 2005$ & $9: 00$ & 10.707 & 10.602 & 7.812 & 6.682 & 4.475 & 4.168 & 2.393 & 2.366 & 10.924 & 6.093 & 4.833 & 6.655 & 4.103 \\
\hline 9/11/2005 & $10: 00$ & 10.712 & 10.604 & 7.816 & 6.684 & 4.477 & 4.166 & 2.398 & 2.373 & 10.923 & 6.095 & 4.843 & 6.667 & 4.113 \\
\hline $9 / 11 / 2005$ & $11: 00$ & 10.712 & 10.602 & 7.818 & 6.684 & 4.483 & 4.166 & 2.408 & 2.379 & 10.923 & 6.102 & 4.86 & 6.676 & 4.138 \\
\hline 9/11/2005 & $12: 00$ & 10.712 & 10.604 & 7.822 & 6.689 & 4.493 & 4.166 & 2.418 & 2.383 & 10.926 & 6.113 & 4.88 & 6.695 & 4.166 \\
\hline 9/11/2005 & $13: 00$ & 10.705 & 10.595 & 7.822 & 6.682 & 4.502 & 4.164 & 2.421 & 2.383 & 10.93 & 6.12 & 4.9 & 6.705 & 4.195 \\
\hline 9/11/2005 & $14: 00$ & 10.702 & 10.587 & 7.821 & 6.68 & 4.516 & 4.162 & 2.42 & 2.386 & 10.94 & 6.129 & 4.923 & 6.72 & 4.229 \\
\hline $9 / 11 / 2005$ & $15: 00$ & 10.702 & 10.582 & 7.817 & 6.677 & 4.528 & 4.162 & 2.426 & 2.388 & 10.945 & 6.14 & 4.943 & 6.734 & 4.26 \\
\hline 9/11/2005 & $16: 00$ & 10.695 & 10.574 & 7.815 & 6.673 & 4.541 & 4.162 & 2.418 & 2.386 & 10.951 & 6.142 & 4.96 & 6.739 & 4.286 \\
\hline $9 / 11 / 2005$ & $17: 00$ & 10.695 & 10.573 & 7.815 & 6.675 & 4.549 & 4.164 & 2.418 & 2.386 & 10.957 & 6.149 & 4.976 & 6.745 & 4.306 \\
\hline
\end{tabular}


TABLE S1.2 (Cont.)

Water Level (ft below top of casing) at Indicated Well

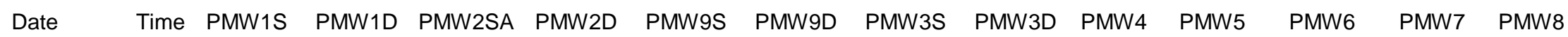

\begin{tabular}{|c|c|c|c|c|c|c|c|c|c|c|c|c|c|c|}
\hline 9/11/2005 & $18: 00$ & 10.69 & 10.571 & 7.815 & 6.675 & 4.554 & 4.166 & 2.423 & 2.388 & 10.961 & 6.151 & 4.974 & 6.737 & 4.315 \\
\hline $9 / 11 / 2005$ & $19: 00$ & 10.695 & 10.574 & 7.815 & 6.68 & 4.558 & 4.171 & 2.423 & 2.388 & 10.964 & 6.149 & 4.972 & 6.734 & 4.315 \\
\hline $9 / 11 / 2005$ & $20: 00$ & 10.693 & 10.576 & 7.815 & 6.677 & 4.558 & 4.173 & 2.41 & 2.381 & 10.968 & 6.145 & 4.961 & 6.722 & 4.301 \\
\hline 9/11/2005 & $21: 00$ & 10.695 & 10.578 & 7.816 & 6.677 & 4.554 & 4.175 & 2.413 & 2.379 & 10.968 & 6.14 & 4.947 & 6.709 & 4.28 \\
\hline $9 / 11 / 2005$ & $22: 00$ & 10.7 & 10.584 & 7.816 & 6.682 & 4.549 & 4.179 & 2.416 & 2.379 & 10.968 & 6.136 & 4.933 & 6.699 & 4.26 \\
\hline 9/11/2005 & $23: 00$ & 10.703 & 10.585 & 7.816 & 6.68 & 4.537 & 4.179 & 2.406 & 2.375 & 10.966 & 6.127 & 4.916 & 6.69 & 4.238 \\
\hline $9 / 12 / 2005$ & $0: 00$ & 10.705 & 10.589 & 7.816 & 6.68 & 4.533 & 4.182 & 2.403 & 2.375 & 10.964 & 6.127 & 4.904 & 6.682 & 4.222 \\
\hline 9/12/2005 & $1: 00$ & 10.705 & 10.589 & 7.814 & 6.677 & 4.52 & 4.179 & 2.404 & 2.371 & 10.959 & 6.114 & 4.886 & 6.673 & 4.201 \\
\hline $9 / 12 / 2005$ & $2: 00$ & 10.703 & 10.584 & 7.808 & 6.671 & 4.508 & 4.177 & 2.396 & 2.362 & 10.955 & 6.112 & 4.873 & 6.665 & 4.181 \\
\hline 9/12/2005 & $3: 00$ & 10.7 & 10.578 & 7.806 & 6.669 & 4.497 & 4.175 & 2.381 & 2.354 & 10.949 & 6.107 & 4.859 & 6.658 & 4.161 \\
\hline $9 / 12 / 2005$ & $4: 00$ & 10.703 & 10.584 & 7.806 & 6.669 & 4.493 & 4.175 & 2.389 & 2.357 & 10.947 & 6.107 & 4.851 & 6.656 & 4.151 \\
\hline $9 / 12 / 2005$ & $5: 00$ & 10.698 & 10.58 & 7.805 & 6.662 & 4.485 & 4.173 & 2.387 & 2.352 & 10.94 & 6.096 & 4.837 & 6.625 & 4.133 \\
\hline $9 / 12 / 2005$ & $6: 00$ & 10.693 & 10.582 & 7.8 & 6.66 & 4.47 & 4.168 & 2.371 & 2.344 & 10.934 & 6.089 & 4.824 & 6.638 & 4.114 \\
\hline 9/12/2005 & $7: 00$ & 10.69 & 10.571 & 7.797 & 6.657 & 4.464 & 4.164 & 2.366 & 2.34 & 10.93 & 6.087 & 4.816 & 6.631 & 4.098 \\
\hline $9 / 12 / 2005$ & 8:00 & 10.688 & 10.565 & 7.793 & 6.655 & 4.456 & 4.159 & 2.366 & 2.333 & 10.923 & 6.081 & 4.804 & 6.621 & 4.084 \\
\hline $9 / 12 / 2005$ & 9:00 & 10.686 & 10.564 & 7.791 & 6.655 & 4.449 & 4.157 & 2.356 & 2.331 & 10.919 & 6.079 & 4.794 & 6.618 & 4.074 \\
\hline $9 / 12 / 2005$ & $10: 00$ & 10.681 & 10.558 & 7.789 & 6.644 & 4.443 & 4.151 & 2.359 & 2.327 & 10.913 & 6.072 & 4.79 & 6.616 & 4.062 \\
\hline $9 / 12 / 2005$ & $11: 00$ & 10.674 & 10.553 & 7.787 & 6.642 & 4.439 & 4.144 & 2.361 & 2.327 & 10.91 & 6.077 & 4.798 & 6.631 & 4.066 \\
\hline $9 / 12 / 2005$ & $12: 00$ & 10.669 & 10.545 & 7.783 & 6.638 & 4.441 & 4.14 & 2.359 & 2.329 & 10.906 & 6.088 & 4.814 & 6.644 & 4.086 \\
\hline $9 / 12 / 2005$ & $13: 00$ & 10.659 & 10.534 & 7.783 & 6.626 & 4.447 & 4.135 & 2.367 & 2.327 & 10.908 & 6.092 & 4.837 & 6.661 & 4.114 \\
\hline $9 / 12 / 2005$ & $14: 00$ & 10.649 & 10.522 & 7.781 & 6.626 & 4.457 & 4.128 & 2.364 & 2.327 & 10.908 & 6.105 & 4.861 & 6.68 & 4.157 \\
\hline $9 / 12 / 2005$ & $15: 00$ & 10.639 & 10.514 & 7.775 & 6.62 & 4.472 & 4.127 & 2.359 & 2.329 & 10.91 & 6.11 & 4.885 & 6.688 & 4.2 \\
\hline 9/12/2005 & $16: 00$ & 10.629 & 10.503 & 7.777 & 6.616 & 4.487 & 4.117 & 2.366 & 2.327 & 10.915 & 6.121 & 4.91 & 6.698 & 4.238 \\
\hline $9 / 12 / 2005$ & $17: 00$ & 10.627 & 10.496 & 7.773 & 6.62 & 4.511 & 4.12 & 2.374 & 2.333 & 10.921 & 6.13 & 4.928 & 6.703 & 4.273 \\
\hline $9 / 12 / 2005$ & $18: 00$ & 10.62 & 10.496 & 7.775 & 6.622 & 4.528 & 4.122 & 2.366 & 2.336 & 10.93 & 6.137 & 4.941 & 6.701 & 4.295 \\
\hline $9 / 12 / 2005$ & $19: 00$ & 10.617 & 10.492 & 7.773 & 6.618 & 4.534 & 4.12 & 2.364 & 2.331 & 10.938 & 6.137 & 4.938 & 6.692 & 4.307 \\
\hline 9/12/2005 & 20:00 & 10.612 & 10.492 & 7.771 & 6.62 & 4.535 & 4.124 & 2.364 & 2.329 & 10.94 & 6.137 & 4.928 & 6.684 & 4.302 \\
\hline $9 / 12 / 2005$ & $21: 00$ & 10.617 & 10.5 & 7.771 & 6.622 & 4.537 & 4.129 & 2.359 & 2.327 & 10.944 & 6.137 & 4.914 & 6.669 & 4.285 \\
\hline $9 / 12 / 2005$ & $22: 00$ & 10.622 & 10.503 & 7.771 & 6.627 & 4.531 & 4.129 & 2.366 & 2.331 & 10.944 & 6.128 & 4.902 & 6.661 & 4.263 \\
\hline $9 / 12 / 2005$ & $23: 00$ & 10.627 & 10.509 & 7.773 & 6.629 & 4.526 & 4.133 & 2.357 & 2.327 & 10.944 & 6.123 & 4.885 & 6.662 & 4.249 \\
\hline 9/13/2005 & $0: 00$ & 10.62 & 10.511 & 7.775 & 6.622 & 4.512 & 4.133 & 2.349 & 2.321 & 10.942 & 6.112 & 4.867 & 6.642 & 4.227 \\
\hline 9/13/2005 & $1: 00$ & 10.625 & 10.511 & 7.772 & 6.622 & 4.502 & 4.133 & 2.352 & 2.317 & 10.936 & 6.106 & 4.855 & 6.639 & 4.204 \\
\hline $9 / 13 / 2005$ & $2: 00$ & 10.622 & 10.509 & 7.769 & 6.62 & 4.491 & 4.131 & 2.347 & 2.313 & 10.932 & 6.101 & 4.842 & 6.631 & 4.188 \\
\hline 9/13/2005 & 3:00 & 10.627 & 10.514 & 7.77 & 6.625 & 4.489 & 4.133 & 2.342 & 2.315 & 10.929 & 6.103 & 4.832 & 6.629 & 4.176 \\
\hline
\end{tabular}


TABLE S1.2 (Cont.)

Water Level (ft below top of casing) at Indicated Well

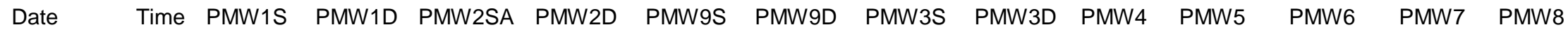

\begin{tabular}{|c|c|c|c|c|c|c|c|c|c|c|c|c|c|c|}
\hline 9/13/2005 & $4: 00$ & 10.63 & 10.516 & 7.762 & 6.625 & 4.481 & 4.131 & 2.347 & 2.312 & 10.927 & 6.095 & 4.82 & 6.622 & 4.158 \\
\hline 9/13/2005 & $5: 00$ & 10.63 & 10.52 & 7.762 & 6.625 & 4.477 & 4.131 & 2.337 & 2.313 & 10.921 & 6.091 & 4.81 & 6.614 & 4.144 \\
\hline 9/13/2005 & $6: 00$ & 10.63 & 10.516 & 7.76 & 6.62 & 4.464 & 4.129 & 2.332 & 2.305 & 10.915 & 6.081 & 4.795 & 6.606 & 4.125 \\
\hline 9/13/2005 & $7: 00$ & 10.633 & 10.523 & 7.762 & 6.625 & 4.46 & 4.129 & 2.339 & 2.307 & 10.913 & 6.084 & 4.787 & 6.603 & 4.115 \\
\hline 9/13/2005 & 8:00 & 10.645 & 10.536 & 7.768 & 6.638 & 4.466 & 4.131 & 2.347 & 2.319 & 10.91 & 6.084 & 4.789 & 6.633 & 4.111 \\
\hline 9/13/2005 & $9: 00$ & 10.648 & 10.542 & 7.774 & 6.64 & 4.462 & 4.131 & 2.353 & 2.321 & 10.908 & 6.075 & 4.783 & 6.616 & 4.101 \\
\hline $9 / 13 / 2005$ & $10: 00$ & 10.657 & 10.547 & 7.778 & 6.651 & 4.466 & 4.133 & 2.363 & 2.33 & 10.906 & 6.081 & 4.791 & 6.648 & 4.097 \\
\hline $9 / 13 / 2005$ & $11: 00$ & 10.665 & 10.56 & 7.782 & 6.656 & 4.466 & 4.138 & 2.37 & 2.336 & 10.902 & 6.081 & 4.791 & 6.645 & 4.095 \\
\hline 9/13/2005 & $12: 00$ & 10.66 & 10.545 & 7.78 & 6.645 & 4.457 & 4.133 & 2.355 & 2.328 & 10.898 & 6.073 & 4.783 & 6.629 & 4.083 \\
\hline 9/13/2005 & $13: 00$ & 10.665 & 10.554 & 7.78 & 6.647 & 4.457 & 4.135 & 2.36 & 2.33 & 10.897 & 6.064 & 4.775 & 6.622 & 4.079 \\
\hline $9 / 13 / 2005$ & $14: 00$ & 10.662 & 10.553 & 7.778 & 6.645 & 4.453 & 4.133 & 2.355 & 2.33 & 10.891 & 6.075 & 4.789 & 6.633 & 4.085 \\
\hline 9/13/2005 & $15: 00$ & 10.665 & 10.556 & 7.782 & 6.651 & 4.462 & 4.133 & 2.371 & 2.34 & 10.891 & 6.084 & 4.811 & 6.65 & 4.113 \\
\hline $9 / 13 / 2005$ & $16: 00$ & 10.673 & 10.564 & 7.788 & 6.658 & 4.482 & 4.135 & 2.383 & 2.349 & 10.895 & 6.095 & 4.834 & 6.664 & 4.148 \\
\hline 9/13/2005 & $17: 00$ & 10.675 & 10.573 & 7.792 & 6.667 & 4.495 & 4.138 & 2.395 & 2.362 & 10.9 & 6.104 & 4.854 & 6.673 & 4.178 \\
\hline $9 / 13 / 2005$ & $18: 00$ & 10.68 & 10.576 & 7.8 & 6.673 & 4.513 & 4.142 & 2.393 & 2.365 & 10.908 & 6.113 & 4.867 & 6.677 & 4.201 \\
\hline 9/13/2005 & $19: 00$ & 10.685 & 10.582 & 7.803 & 6.678 & 4.516 & 4.147 & 2.395 & 2.37 & 10.912 & 6.115 & 4.873 & 6.675 & 4.213 \\
\hline 9/13/2005 & $20: 00$ & 10.69 & 10.587 & 7.806 & 6.68 & 4.52 & 4.151 & 2.403 & 2.372 & 10.918 & 6.115 & 4.865 & 6.664 & 4.207 \\
\hline 9/13/2005 & $21: 00$ & 10.697 & 10.595 & 7.808 & 6.687 & 4.521 & 4.158 & 2.398 & 2.374 & 10.919 & 6.111 & 4.858 & 6.654 & 4.193 \\
\hline $9 / 13 / 2005$ & $22: 00$ & 10.707 & 10.605 & 7.812 & 6.693 & 4.52 & 4.164 & 2.4 & 2.376 & 10.921 & 6.109 & 4.848 & 6.65 & 4.175 \\
\hline 9/13/2005 & $23: 00$ & 10.714 & 10.613 & 7.814 & 6.693 & 4.516 & 4.169 & 2.403 & 2.378 & 10.919 & 6.104 & 4.838 & 6.643 & 4.159 \\
\hline $9 / 14 / 2005$ & $0: 00$ & 10.719 & 10.616 & 7.816 & 6.698 & 4.511 & 4.173 & 2.411 & 2.381 & 10.918 & 6.097 & 4.828 & 6.639 & 4.143 \\
\hline $9 / 14 / 2005$ & $1: 00$ & 10.727 & 10.626 & 7.82 & 6.704 & 4.509 & 4.178 & 2.413 & 2.383 & 10.916 & 6.098 & 4.822 & 6.637 & 4.128 \\
\hline $9 / 14 / 2005$ & $2: 00$ & 10.729 & 10.627 & 7.82 & 6.7 & 4.501 & 4.178 & 2.408 & 2.376 & 10.912 & 6.091 & 4.811 & 6.632 & 4.108 \\
\hline $9 / 14 / 2005$ & $3: 00$ & 10.732 & 10.629 & 7.82 & 6.703 & 4.494 & 4.18 & 2.408 & 2.376 & 10.908 & 6.082 & 4.803 & 6.626 & 4.094 \\
\hline 9/14/2005 & $4: 00$ & 10.737 & 10.633 & 7.82 & 6.702 & 4.488 & 4.182 & 2.409 & 2.378 & 10.904 & 6.08 & 4.795 & 6.624 & 4.08 \\
\hline $9 / 14 / 2005$ & $5: 00$ & 10.739 & 10.636 & 7.822 & 6.705 & 4.482 & 4.182 & 2.406 & 2.376 & 10.9 & 6.078 & 4.789 & 6.622 & 4.069 \\
\hline 9/14/2005 & $6: 00$ & 10.744 & 10.64 & 7.824 & 6.707 & 4.48 & 4.184 & 2.404 & 2.378 & 10.899 & 6.08 & 4.787 & 6.624 & 4.061 \\
\hline $9 / 14 / 2005$ & $7: 00$ & 10.747 & 10.647 & 7.824 & 6.711 & 4.48 & 4.184 & 2.411 & 2.383 & 10.899 & 6.073 & 4.784 & 6.62 & 4.049 \\
\hline 9/14/2005 & $8: 00$ & 10.749 & 10.646 & 7.824 & 6.707 & 4.472 & 4.184 & 2.409 & 2.376 & 10.893 & 6.073 & 4.779 & 6.618 & 4.041 \\
\hline 9/14/2005 & 9:00 & 10.754 & 10.647 & 7.828 & 6.709 & 4.465 & 4.18 & 2.409 & 2.385 & 10.891 & 6.069 & 4.776 & 6.62 & 4.033 \\
\hline 9/14/2005 & $10: 00$ & 10.756 & 10.653 & 7.831 & 6.714 & 4.469 & 4.18 & 2.419 & 2.387 & 10.889 & 6.074 & 4.787 & 6.63 & 4.037 \\
\hline $9 / 14 / 2005$ & $11: 00$ & 10.757 & 10.64 & 7.831 & 6.714 & 4.476 & 4.18 & 2.427 & 2.395 & 10.887 & 6.076 & 4.807 & 6.643 & 4.051 \\
\hline 9/14/2005 & $12: 00$ & 10.754 & 10.651 & 7.834 & 6.714 & 4.482 & 4.178 & 2.422 & 2.397 & 10.887 & 6.087 & 4.831 & 6.658 & 4.076 \\
\hline $9 / 14 / 2005$ & $13: 00$ & 10.752 & 10.647 & 7.836 & 6.689 & 4.494 & 4.175 & 2.429 & 2.397 & 10.891 & 6.089 & 4.854 & 6.673 & 4.108 \\
\hline
\end{tabular}


TABLE S1.2 (Cont.)

Water Level (ft below top of casing) at Indicated Well

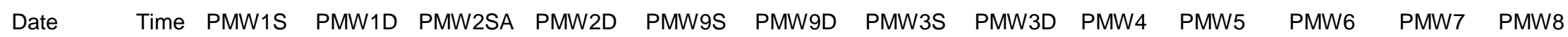

\begin{tabular}{|c|c|c|c|c|c|c|c|c|c|c|c|c|c|c|}
\hline 9/14/2005 & $14: 00$ & 10.747 & 10.636 & 7.833 & 6.703 & 4.507 & 4.173 & 2.424 & 2.399 & 10.893 & 6.096 & 4.876 & 6.687 & 4.145 \\
\hline $9 / 14 / 2005$ & $15: 00$ & 10.735 & 10.624 & 7.832 & 6.703 & 4.519 & 4.173 & 2.427 & 2.399 & 10.899 & 6.1 & 4.895 & 6.694 & 4.179 \\
\hline 9/14/2005 & $16: 00$ & 10.727 & 10.624 & 7.83 & 6.698 & 4.53 & 4.173 & 2.427 & 2.402 & 10.906 & 6.107 & 4.913 & 6.7 & 4.214 \\
\hline 9/14/2005 & $17: 00$ & 10.723 & 10.613 & 7.827 & 6.698 & 4.542 & 4.173 & 2.434 & 2.399 & 10.91 & 6.114 & 4.929 & 6.704 & 4.24 \\
\hline 9/14/2005 & $18: 00$ & 10.72 & 10.611 & 7.825 & 6.698 & 4.55 & 4.175 & 2.432 & 2.4 & 10.916 & 6.12 & 4.94 & 6.704 & 4.26 \\
\hline $9 / 14 / 2005$ & $19: 00$ & 10.723 & 10.615 & 7.828 & 6.703 & 4.561 & 4.18 & 2.437 & 2.402 & 10.923 & 6.123 & 4.938 & 6.696 & 4.267 \\
\hline $9 / 14 / 2005$ & $20: 00$ & 10.727 & 10.618 & 7.828 & 6.705 & 4.563 & 4.184 & 2.432 & 2.4 & 10.927 & 6.118 & 4.927 & 6.685 & 4.251 \\
\hline $9 / 14 / 2005$ & $21: 00$ & 10.735 & 10.627 & 7.83 & 6.709 & 4.565 & 4.191 & 2.437 & 2.404 & 10.933 & 6.116 & 4.915 & 6.677 & 4.235 \\
\hline 9/14/2005 & $22: 00$ & 10.74 & 10.635 & 7.834 & 6.714 & 4.557 & 4.195 & 2.437 & 2.404 & 10.933 & 6.112 & 4.899 & 6.672 & 4.214 \\
\hline 9/14/2005 & $23: 00$ & 10.742 & 10.636 & 7.832 & 6.711 & 4.552 & 4.195 & 2.432 & 2.402 & 10.933 & 6.107 & 4.885 & 6.664 & 4.192 \\
\hline $9 / 15 / 2005$ & $0: 00$ & 10.742 & 10.633 & 7.831 & 6.709 & 4.54 & 4.197 & 2.427 & 2.398 & 10.931 & 6.101 & 4.87 & 6.657 & 4.17 \\
\hline 9/15/2005 & $1: 00$ & 10.742 & 10.633 & 7.829 & 6.705 & 4.53 & 4.197 & 2.422 & 2.391 & 10.927 & 6.094 & 4.858 & 6.651 & 4.152 \\
\hline $9 / 15 / 2005$ & $2: 00$ & 10.74 & 10.631 & 7.826 & 6.705 & 4.523 & 4.195 & 2.417 & 2.387 & 10.922 & 6.092 & 4.848 & 6.645 & 4.135 \\
\hline 9/15/2005 & $3: 00$ & 10.737 & 10.629 & 7.824 & 6.7 & 4.513 & 4.195 & 2.414 & 2.383 & 10.92 & 6.085 & 4.834 & 6.638 & 4.119 \\
\hline $9 / 15 / 2005$ & $4: 00$ & 10.742 & 10.633 & 7.824 & 6.703 & 4.507 & 4.196 & 2.415 & 2.383 & 10.916 & 6.086 & 4.829 & 6.638 & 4.109 \\
\hline 9/15/2005 & $5: 00$ & 10.735 & 10.627 & 7.821 & 6.696 & 4.498 & 4.193 & 2.407 & 2.375 & 10.91 & 6.079 & 4.817 & 6.63 & 4.093 \\
\hline 9/15/2005 & $6: 00$ & 10.74 & 10.635 & 7.823 & 6.701 & 4.498 & 4.191 & 2.412 & 2.379 & 10.908 & 6.083 & 4.813 & 6.628 & 4.087 \\
\hline 9/15/2005 & $7: 00$ & 10.748 & 10.642 & 7.827 & 6.709 & 4.5 & 4.193 & 2.415 & 2.385 & 10.906 & 6.083 & 4.807 & 6.626 & 4.079 \\
\hline $9 / 15 / 2005$ & $8: 00$ & 10.755 & 10.653 & 7.833 & 6.711 & 4.5 & 4.196 & 2.423 & 2.394 & 10.906 & 6.09 & 4.805 & 6.628 & 4.073 \\
\hline 9/15/2005 & $9: 00$ & 10.762 & 10.662 & 7.837 & 6.727 & 4.498 & 4.197 & 2.42 & 2.398 & 10.903 & 6.094 & 4.804 & 6.63 & 4.069 \\
\hline $9 / 15 / 2005$ & $10: 00$ & 10.768 & 10.662 & 7.836 & 6.727 & 4.5 & 4.198 & 2.435 & 2.404 & 10.903 & 6.099 & 4.813 & 6.643 & 4.067 \\
\hline $9 / 15 / 2005$ & $11: 00$ & 10.765 & 10.662 & 7.835 & 6.718 & 4.5 & 4.198 & 2.43 & 2.406 & 10.901 & 6.097 & 4.821 & 6.651 & 4.076 \\
\hline $9 / 15 / 2005$ & $12: 00$ & 10.762 & 10.657 & 7.839 & 6.723 & 4.502 & 4.193 & 2.433 & 2.406 & 10.899 & 6.098 & 4.837 & 6.664 & 4.093 \\
\hline $9 / 15 / 2005$ & $13: 00$ & 10.76 & 10.642 & 7.839 & 6.718 & 4.508 & 4.191 & 2.438 & 2.406 & 10.901 & 6.098 & 4.856 & 6.674 & 4.119 \\
\hline 9/15/2005 & $14: 00$ & 10.752 & 10.642 & 7.837 & 6.714 & 4.517 & 4.191 & 2.438 & 2.406 & 10.903 & 6.105 & 4.874 & 6.681 & 4.152 \\
\hline $9 / 15 / 2005$ & $15: 00$ & 10.748 & 10.635 & 7.837 & 6.712 & 4.527 & 4.187 & 2.44 & 2.409 & 10.908 & 6.112 & 4.898 & 6.693 & 4.188 \\
\hline 9/15/2005 & $16: 00$ & 10.745 & 10.64 & 7.836 & 6.707 & 4.542 & 4.189 & 2.448 & 2.413 & 10.914 & 6.121 & 4.921 & 6.712 & 4.222 \\
\hline $9 / 15 / 2005$ & $17: 00$ & 10.745 & 10.633 & 7.838 & 6.714 & 4.556 & 4.191 & 2.443 & 2.417 & 10.922 & 6.13 & 4.939 & 6.712 & 4.253 \\
\hline 9/15/2005 & $18: 00$ & 10.748 & 10.638 & 7.841 & 6.721 & 4.569 & 4.196 & 2.451 & 2.423 & 10.929 & 6.137 & 4.954 & 6.718 & 4.275 \\
\hline 9/15/2005 & $19: 00$ & 10.75 & 10.644 & 7.845 & 6.725 & 4.579 & 4.198 & 2.458 & 2.426 & 10.935 & 6.138 & 4.952 & 6.71 & 4.282 \\
\hline 9/15/2005 & $20: 00$ & 10.758 & 10.653 & 7.847 & 6.73 & 4.583 & 4.204 & 2.46 & 2.43 & 10.941 & 6.139 & 4.943 & 6.697 & 4.27 \\
\hline 9/15/2005 & $21: 00$ & 10.765 & 10.662 & 7.849 & 6.738 & 4.583 & 4.211 & 2.461 & 2.43 & 10.943 & 6.138 & 4.931 & 6.693 & 4.252 \\
\hline 9/15/2005 & $22: 00$ & 10.77 & 10.666 & 7.853 & 6.738 & 4.577 & 4.216 & 2.461 & 2.43 & 10.945 & 6.134 & 4.917 & 6.684 & 4.232 \\
\hline $9 / 15 / 2005$ & $23: 00$ & 10.773 & 10.669 & 7.851 & 6.741 & 4.571 & 4.22 & 2.451 & 2.426 & 10.943 & 6.13 & 4.903 & 6.676 & 4.211 \\
\hline
\end{tabular}


TABLE S1.2 (Cont.)

Water Level (ft below top of casing) at Indicated Well

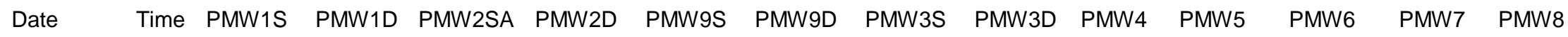

\begin{tabular}{|c|c|c|c|c|c|c|c|c|c|c|c|c|c|c|}
\hline 9/16/2005 & $0: 00$ & 10.778 & 10.671 & 7.851 & 6.741 & 4.564 & 4.22 & 2.448 & 2.426 & 10.941 & 6.13 & 4.89 & 6.67 & 4.189 \\
\hline 9/16/2005 & $1: 00$ & 10.778 & 10.675 & 7.851 & 6.741 & 4.554 & 4.222 & 2.451 & 2.423 & 10.937 & 6.126 & 4.878 & 6.664 & 4.171 \\
\hline 9/16/2005 & $2: 00$ & 10.782 & 10.678 & 7.852 & 6.741 & 4.547 & 4.224 & 2.453 & 2.421 & 10.933 & 6.126 & 4.867 & 6.659 & 4.152 \\
\hline 9/16/2005 & $3: 00$ & 10.782 & 10.677 & 7.852 & 6.738 & 4.539 & 4.224 & 2.448 & 2.419 & 10.93 & 6.121 & 4.855 & 6.653 & 4.136 \\
\hline 9/16/2005 & $4: 00$ & 10.78 & 10.675 & 7.848 & 6.736 & 4.527 & 4.222 & 2.443 & 2.413 & 10.924 & 6.115 & 4.845 & 6.649 & 4.118 \\
\hline $9 / 16 / 2005$ & $5: 00$ & 10.785 & 10.677 & 7.85 & 6.738 & 4.522 & 4.222 & 2.436 & 2.413 & 10.92 & 6.114 & 4.835 & 6.644 & 4.106 \\
\hline $9 / 16 / 2005$ & $6: 00$ & 10.788 & 10.682 & 7.85 & 6.741 & 4.518 & 4.222 & 2.444 & 2.413 & 10.916 & 6.115 & 4.829 & 6.642 & 4.096 \\
\hline 9/16/2005 & $7: 00$ & 10.79 & 10.684 & 7.852 & 6.743 & 4.514 & 4.222 & 2.439 & 2.415 & 10.914 & 6.082 & 4.825 & 6.64 & 4.086 \\
\hline 9/16/2005 & $8: 00$ & 10.793 & 10.684 & 7.856 & 6.747 & 4.51 & 4.222 & 2.439 & 2.415 & 10.911 & 6.077 & 4.818 & 6.638 & 4.074 \\
\hline 9/16/2005 & 9:00 & 10.795 & 10.69 & 7.854 & 6.747 & 4.508 & 4.222 & 2.446 & 2.417 & 10.907 & 6.075 & 4.814 & 6.636 & 4.068 \\
\hline $9 / 16 / 2005$ & $10: 00$ & 10.8 & 10.688 & 7.854 & 6.752 & 4.508 & 4.22 & 2.451 & 2.422 & 10.903 & 6.077 & 4.818 & 6.642 & 4.064 \\
\hline 9/16/2005 & $11: 00$ & 10.798 & 10.692 & 7.86 & 6.747 & 4.508 & 4.22 & 2.449 & 2.424 & 10.901 & 6.082 & 4.831 & 6.655 & 4.074 \\
\hline $9 / 16 / 2005$ & $12: 00$ & 10.796 & 10.689 & 7.859 & 6.745 & 4.514 & 4.216 & 2.451 & 2.426 & 10.901 & 6.088 & 4.849 & 6.67 & 4.098 \\
\hline 9/16/2005 & $13: 00$ & 10.791 & 10.682 & 7.855 & 6.741 & 4.52 & 4.213 & 2.459 & 2.43 & 10.901 & 6.1 & 4.87 & 6.68 & 4.13 \\
\hline 9/16/2005 & $14: 00$ & 10.78 & 10.67 & 7.855 & 6.734 & 4.528 & 4.211 & 2.451 & 2.428 & 10.905 & 6.106 & 4.892 & 6.693 & 4.167 \\
\hline 9/16/2005 & $15: 00$ & 10.771 & 10.657 & 7.853 & 6.73 & 4.539 & 4.207 & 2.459 & 2.426 & 10.907 & 6.113 & 4.914 & 6.724 & 4.206 \\
\hline $9 / 16 / 2005$ & $16: 00$ & 10.763 & 10.651 & 7.849 & 6.725 & 4.549 & 4.204 & 2.459 & 2.424 & 10.913 & 6.122 & 4.933 & 6.722 & 4.238 \\
\hline 9/16/2005 & $17: 00$ & 10.756 & 10.64 & 7.847 & 6.718 & 4.564 & 4.204 & 2.454 & 2.424 & 10.916 & 6.126 & 4.949 & 6.72 & 4.264 \\
\hline 9/16/2005 & $18: 00$ & 10.749 & 10.635 & 7.843 & 6.716 & 4.572 & 4.204 & 2.453 & 2.422 & 10.922 & 6.133 & 4.959 & 6.718 & 4.287 \\
\hline 9/16/2005 & $19: 00$ & 10.746 & 10.631 & 7.843 & 6.718 & 4.58 & 4.207 & 2.454 & 2.42 & 10.928 & 6.133 & 4.959 & 6.71 & 4.297 \\
\hline $9 / 16 / 2005$ & $20: 00$ & 10.744 & 10.63 & 7.841 & 6.716 & 4.58 & 4.207 & 2.441 & 2.415 & 10.933 & 6.126 & 4.949 & 6.699 & 4.285 \\
\hline $9 / 16 / 2005$ & $21: 00$ & 10.744 & 10.631 & 7.839 & 6.716 & 4.578 & 4.211 & 2.441 & 2.411 & 10.935 & 6.122 & 4.933 & 6.687 & 4.267 \\
\hline 9/16/2005 & $22: 00$ & 10.744 & 10.635 & 7.839 & 6.719 & 4.572 & 4.213 & 2.441 & 2.409 & 10.935 & 6.118 & 4.919 & 6.682 & 4.247 \\
\hline $9 / 16 / 2005$ & $23: 00$ & 10.747 & 10.637 & 7.837 & 6.717 & 4.564 & 4.213 & 2.439 & 2.407 & 10.936 & 6.111 & 4.906 & 6.672 & 4.222 \\
\hline 9/17/2005 & $0: 00$ & 10.749 & 10.642 & 7.838 & 6.721 & 4.557 & 4.213 & 2.431 & 2.409 & 10.932 & 6.107 & 4.894 & 6.667 & 4.206 \\
\hline $9 / 17 / 2005$ & $1: 00$ & 10.749 & 10.642 & 7.838 & 6.721 & 4.549 & 4.213 & 2.434 & 2.405 & 10.93 & 6.1 & 4.881 & 6.661 & 4.186 \\
\hline 9/17/2005 & $2: 00$ & 10.744 & 10.635 & 7.832 & 6.71 & 4.533 & 4.211 & 2.427 & 2.394 & 10.922 & 6.091 & 4.865 & 6.65 & 4.162 \\
\hline $9 / 17 / 2005$ & $3: 00$ & 10.739 & 10.626 & 7.826 & 6.699 & 4.518 & 4.207 & 2.414 & 2.382 & 10.917 & 6.084 & 4.849 & 6.644 & 4.14 \\
\hline 9/17/2005 & $4: 00$ & 10.732 & 10.616 & 7.82 & 6.69 & 4.505 & 4.2 & 2.399 & 2.369 & 10.911 & 6.078 & 4.834 & 6.632 & 4.119 \\
\hline 9/17/2005 & $5: 00$ & 10.724 & 10.611 & 7.814 & 6.686 & 4.495 & 4.196 & 2.396 & 2.365 & 10.905 & 6.076 & 4.824 & 6.627 & 4.105 \\
\hline 9/17/2005 & $6: 00$ & 10.722 & 10.609 & 7.812 & 6.686 & 4.487 & 4.194 & 2.387 & 2.365 & 10.9 & 6.073 & 4.816 & 6.623 & 4.093 \\
\hline 9/17/2005 & 7:00 & 10.717 & 10.606 & 7.81 & 6.681 & 4.481 & 4.187 & 2.389 & 2.359 & 10.892 & 6.069 & 4.808 & 6.619 & 4.081 \\
\hline 9/17/2005 & $8: 00$ & 10.717 & 10.604 & 7.808 & 6.677 & 4.474 & 4.185 & 2.382 & 2.357 & 10.888 & 6.063 & 4.796 & 6.613 & 4.068 \\
\hline $9 / 17 / 2005$ & $9: 00$ & 10.712 & 10.602 & 7.806 & 6.679 & 4.472 & 4.18 & 2.379 & 2.357 & 10.886 & 6.061 & 4.794 & 6.613 & 4.06 \\
\hline
\end{tabular}


TABLE S1.2 (Cont.)

Water Level (ft below top of casing) at Indicated Well

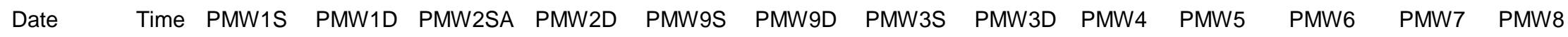

\begin{tabular}{|c|c|c|c|c|c|c|c|c|c|c|c|c|c|c|}
\hline 9/17/2005 & $10: 00$ & 10.712 & 10.595 & 7.803 & 6.668 & 4.464 & 4.174 & 2.382 & 2.351 & 10.879 & 6.058 & 4.794 & 6.617 & 4.058 \\
\hline 9/17/2005 & $11: 00$ & 10.705 & 10.589 & 7.802 & 6.668 & 4.466 & 4.167 & 2.387 & 2.355 & 10.879 & 6.065 & 4.806 & 6.621 & 4.081 \\
\hline 9/17/2005 & $12: 00$ & 10.702 & 10.586 & 7.804 & 6.668 & 4.474 & 4.165 & 2.382 & 2.357 & 10.875 & 6.072 & 4.822 & 6.634 & 4.099 \\
\hline 9/17/2005 & $13: 00$ & 10.697 & 10.577 & 7.8 & 6.662 & 4.474 & 4.158 & 2.379 & 2.355 & 10.875 & 6.072 & 4.836 & 6.638 & 4.125 \\
\hline 9/17/2005 & $14: 00$ & 10.69 & 10.569 & 7.794 & 6.657 & 4.48 & 4.154 & 2.384 & 2.353 & 10.875 & 6.078 & 4.851 & 6.642 & 4.154 \\
\hline $9 / 17 / 2005$ & $15: 00$ & 10.683 & 10.562 & 7.792 & 6.653 & 4.491 & 4.152 & 2.376 & 2.349 & 10.877 & 6.103 & 4.863 & 6.64 & 4.178 \\
\hline $9 / 17 / 2005$ & $16: 00$ & 10.678 & 10.557 & 7.79 & 6.648 & 4.501 & 4.147 & 2.381 & 2.349 & 10.881 & 6.091 & 4.877 & 6.642 & 4.201 \\
\hline 9/17/2005 & $17: 00$ & 10.67 & 10.551 & 7.79 & 6.65 & 4.509 & 4.147 & 2.384 & 2.351 & 10.881 & 6.096 & 4.887 & 6.646 & 4.227 \\
\hline 9/17/2005 & $18: 00$ & 10.673 & 10.555 & 7.79 & 6.655 & 4.524 & 4.147 & 2.386 & 2.355 & 10.888 & 6.1 & 4.89 & 6.642 & 4.239 \\
\hline 9/17/2005 & $19: 00$ & 10.668 & 10.551 & 7.79 & 6.65 & 4.526 & 4.15 & 2.376 & 2.351 & 10.888 & 6.098 & 4.885 & 6.636 & 4.235 \\
\hline $9 / 17 / 2005$ & $20: 00$ & 10.668 & 10.553 & 7.789 & 6.65 & 4.524 & 4.15 & 2.379 & 2.349 & 10.89 & 6.094 & 4.871 & 6.631 & 4.219 \\
\hline 9/17/2005 & $21: 00$ & 10.671 & 10.557 & 7.789 & 6.655 & 4.526 & 4.154 & 2.379 & 2.349 & 10.89 & 6.089 & 4.859 & 6.621 & 4.205 \\
\hline $9 / 17 / 2005$ & $22: 00$ & 10.673 & 10.562 & 7.789 & 6.657 & 4.52 & 4.154 & 2.379 & 2.349 & 10.89 & 6.083 & 4.846 & 6.615 & 4.184 \\
\hline 9/17/2005 & $23: 00$ & 10.678 & 10.566 & 7.791 & 6.662 & 4.516 & 4.156 & 2.371 & 2.349 & 10.886 & 6.078 & 4.836 & 6.61 & 4.167 \\
\hline $9 / 18 / 2005$ & $0: 00$ & 10.678 & 10.566 & 7.789 & 6.659 & 4.507 & 4.156 & 2.374 & 2.344 & 10.884 & 6.072 & 4.822 & 6.604 & 4.146 \\
\hline 9/18/2005 & $1: 00$ & 10.676 & 10.564 & 7.787 & 6.653 & 4.495 & 4.154 & 2.364 & 2.34 & 10.879 & 6.068 & 4.809 & 6.6 & 4.126 \\
\hline 9/18/2005 & $2: 00$ & 10.673 & 10.562 & 7.783 & 6.65 & 4.484 & 4.152 & 2.364 & 2.334 & 10.875 & 6.061 & 4.797 & 6.596 & 4.108 \\
\hline 9/18/2005 & $3: 00$ & 10.671 & 10.555 & 7.779 & 6.642 & 4.472 & 4.147 & 2.354 & 2.326 & 10.869 & 6.052 & 4.781 & 6.591 & 4.088 \\
\hline $9 / 18 / 2005$ & $4: 00$ & 10.666 & 10.549 & 7.778 & 6.642 & 4.464 & 4.145 & 2.349 & 2.322 & 10.865 & 6.048 & 4.771 & 6.585 & 4.071 \\
\hline 9/18/2005 & $5: 00$ & 10.669 & 10.557 & 7.78 & 6.646 & 4.464 & 4.143 & 2.354 & 2.328 & 10.863 & 6.048 & 4.769 & 6.585 & 4.061 \\
\hline $9 / 18 / 2005$ & $6: 00$ & 10.674 & 10.562 & 7.78 & 6.648 & 4.461 & 4.143 & 2.354 & 2.326 & 10.86 & 6.048 & 4.762 & 6.581 & 4.053 \\
\hline $9 / 18 / 2005$ & $7: 00$ & 10.676 & 10.567 & 7.784 & 6.653 & 4.461 & 4.143 & 2.359 & 2.33 & 10.856 & 6.043 & 4.758 & 6.581 & 4.043 \\
\hline 9/18/2005 & $8: 00$ & 10.676 & 10.567 & 7.783 & 6.653 & 4.455 & 4.141 & 2.357 & 2.328 & 10.854 & 6.042 & 4.752 & 6.579 & 4.035 \\
\hline $9 / 18 / 2005$ & $9: 00$ & 10.684 & 10.577 & 7.788 & 6.66 & 4.457 & 4.141 & 2.362 & 2.336 & 10.852 & 6.044 & 4.756 & 6.583 & 4.031 \\
\hline 9/18/2005 & $10: 00$ & 10.691 & 10.586 & 7.794 & 6.666 & 4.463 & 4.143 & 2.377 & 2.347 & 10.851 & 6.048 & 4.771 & 6.597 & 4.041 \\
\hline $9 / 18 / 2005$ & $11: 00$ & 10.693 & 10.584 & 7.798 & 6.666 & 4.465 & 4.141 & 2.375 & 2.351 & 10.851 & 6.052 & 4.787 & 6.606 & 4.055 \\
\hline 9/18/2005 & $12: 00$ & 10.688 & 10.577 & 7.8 & 6.662 & 4.469 & 4.141 & 2.382 & 2.353 & 10.851 & 6.057 & 4.811 & 6.618 & 4.077 \\
\hline $9 / 18 / 2005$ & $13: 00$ & 10.686 & 10.573 & 7.797 & 6.662 & 4.476 & 4.136 & 2.387 & 2.355 & 10.849 & 6.068 & 4.84 & 6.631 & 4.118 \\
\hline 9/18/2005 & $14: 00$ & 10.681 & 10.562 & 7.795 & 6.655 & 4.488 & 4.136 & 2.387 & 2.355 & 10.854 & 6.081 & 4.867 & 6.644 & 4.167 \\
\hline 9/18/2005 & $15: 00$ & 10.676 & 10.558 & 7.797 & 6.653 & 4.511 & 4.134 & 2.392 & 2.36 & 10.862 & 6.099 & 4.895 & 6.658 & 4.217 \\
\hline 9/18/2005 & $16: 00$ & 10.674 & 10.553 & 7.797 & 6.655 & 4.53 & 4.134 & 2.395 & 2.364 & 10.871 & 6.105 & 4.91 & 6.658 & 4.254 \\
\hline 9/18/2005 & $17: 00$ & 10.672 & 10.558 & 7.799 & 6.662 & 4.551 & 4.138 & 2.4 & 2.368 & 10.875 & 6.114 & 4.926 & 6.658 & 4.278 \\
\hline 9/18/2005 & $18: 00$ & 10.672 & 10.555 & 7.797 & 6.657 & 4.561 & 4.143 & 2.397 & 2.366 & 10.883 & 6.114 & 4.922 & 6.65 & 4.287 \\
\hline $9 / 18 / 2005$ & $19: 00$ & 10.672 & 10.558 & 7.801 & 6.664 & 4.567 & 4.147 & 2.392 & 2.366 & 10.889 & 6.112 & 4.916 & 6.642 & 4.283 \\
\hline
\end{tabular}


TABLE S1.2 (Cont.)

Water Level (ft below top of casing) at Indicated Well

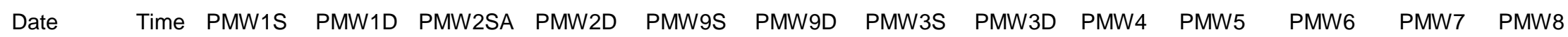

\begin{tabular}{|c|c|c|c|c|c|c|c|c|c|c|c|c|c|c|}
\hline 9/18/2005 & $20: 00$ & 10.674 & 10.558 & 7.797 & 6.66 & 4.563 & 4.15 & 2.389 & 2.362 & 10.89 & 6.104 & 4.897 & 6.633 & 4.258 \\
\hline 9/18/2005 & $21: 00$ & 10.674 & 10.558 & 7.795 & 6.66 & 4.555 & 4.152 & 2.387 & 2.356 & 10.889 & 6.095 & 4.879 & 6.623 & 4.232 \\
\hline 9/18/2005 & $22: 00$ & 10.671 & 10.547 & 7.787 & 6.646 & 4.536 & 4.15 & 2.372 & 2.343 & 10.889 & 6.082 & 4.856 & 6.612 & 4.202 \\
\hline 9/18/2005 & $23: 00$ & 10.672 & 10.558 & 7.789 & 6.651 & 4.538 & 4.152 & 2.375 & 2.343 & 10.885 & 6.09 & 4.85 & 6.612 & 4.19 \\
\hline 9/19/2005 & $0: 00$ & 10.677 & 10.566 & 7.795 & 6.662 & 4.538 & 4.156 & 2.375 & 2.352 & 10.887 & 6.082 & 4.836 & 6.61 & 4.177 \\
\hline 9/19/2005 & $1: 00$ & 10.677 & 10.562 & 7.792 & 6.653 & 4.525 & 4.154 & 2.37 & 2.341 & 10.881 & 6.075 & 4.823 & 6.601 & 4.155 \\
\hline 9/19/2005 & $2: 00$ & 10.684 & 10.575 & 7.792 & 6.664 & 4.526 & 4.156 & 2.372 & 2.35 & 10.879 & 6.075 & 4.817 & 6.598 & 4.145 \\
\hline 9/19/2005 & $3: 00$ & 10.684 & 10.575 & 7.792 & 6.662 & 4.515 & 4.156 & 2.375 & 2.345 & 10.875 & 6.066 & 4.803 & 6.593 & 4.127 \\
\hline 9/19/2005 & $4: 00$ & 10.694 & 10.589 & 7.798 & 6.675 & 4.517 & 4.161 & 2.385 & 2.356 & 10.875 & 6.068 & 4.801 & 6.595 & 4.119 \\
\hline 9/19/2005 & $5: 00$ & 10.702 & 10.6 & 7.802 & 6.682 & 4.517 & 4.161 & 2.391 & 2.362 & 10.873 & 6.066 & 4.793 & 6.593 & 4.109 \\
\hline 9/19/2005 & $6: 00$ & 10.712 & 10.609 & 7.808 & 6.686 & 4.513 & 4.165 & 2.393 & 2.364 & 10.871 & 6.062 & 4.787 & 6.593 & 4.096 \\
\hline 9/19/2005 & $7: 00$ & 10.716 & 10.617 & 7.812 & 6.691 & 4.509 & 4.165 & 2.396 & 2.368 & 10.87 & 6.057 & 4.783 & 6.591 & 4.084 \\
\hline 9/19/2005 & $8: 00$ & 10.726 & 10.63 & 7.816 & 6.702 & 4.511 & 4.169 & 2.403 & 2.375 & 10.868 & 6.06 & 4.781 & 6.597 & 4.076 \\
\hline 9/19/2005 & 9:00 & 10.734 & 10.635 & 7.821 & 6.704 & 4.505 & 4.172 & 2.401 & 2.379 & 10.868 & 6.055 & 4.78 & 6.606 & 4.064 \\
\hline 9/19/2005 & $10: 00$ & 10.743 & 10.65 & 7.828 & 6.713 & 4.511 & 4.177 & 2.411 & 2.387 & 10.866 & 6.062 & 4.789 & 6.612 & 4.066 \\
\hline 9/19/2005 & $11: 00$ & 10.748 & 10.653 & 7.832 & 6.717 & 4.513 & 4.177 & 2.423 & 2.396 & 10.864 & 6.064 & 4.801 & 6.624 & 4.072 \\
\hline 9/19/2005 & $12: 00$ & 10.754 & 10.657 & 7.836 & 6.72 & 4.517 & 4.181 & 2.426 & 2.402 & 10.866 & 6.073 & 4.821 & 6.637 & 4.094 \\
\hline 9/19/2005 & $13: 00$ & 10.756 & 10.659 & 7.84 & 6.72 & 4.527 & 4.183 & 2.431 & 2.408 & 10.868 & 6.082 & 4.842 & 6.645 & 4.125 \\
\hline 9/19/2005 & $14: 00$ & 10.761 & 10.659 & 7.844 & 6.727 & 4.538 & 4.183 & 2.436 & 2.415 & 10.872 & 6.091 & 4.864 & 6.654 & 4.16 \\
\hline 9/19/2005 & $15: 00$ & 10.761 & 10.661 & 7.846 & 6.724 & 4.554 & 4.185 & 2.449 & 2.419 & 10.879 & 6.1 & 4.878 & 6.658 & 4.192 \\
\hline 9/19/2005 & $16: 00$ & 10.766 & 10.662 & 7.849 & 6.733 & 4.571 & 4.19 & 2.456 & 2.428 & 10.885 & 6.113 & 4.899 & 6.666 & 4.224 \\
\hline 9/19/2005 & $17: 00$ & 10.768 & 10.668 & 7.854 & 6.74 & 4.586 & 4.196 & 2.464 & 2.434 & 10.893 & 6.121 & 4.915 & 6.673 & 4.253 \\
\hline 9/19/2005 & $18: 00$ & 10.773 & 10.671 & 7.858 & 6.742 & 4.596 & 4.201 & 2.464 & 2.438 & 10.898 & 6.128 & 4.929 & 6.673 & 4.271 \\
\hline 9/19/2005 & $19: 00$ & 10.773 & 10.668 & 7.855 & 6.738 & 4.594 & 4.205 & 2.456 & 2.434 & 10.902 & 6.128 & 4.925 & 6.667 & 4.275 \\
\hline 9/19/2005 & $20: 00$ & 10.778 & 10.675 & 7.857 & 6.744 & 4.598 & 4.21 & 2.464 & 2.434 & 10.906 & 6.124 & 4.913 & 6.66 & 4.262 \\
\hline 9/19/2005 & $21: 00$ & 10.783 & 10.682 & 7.86 & 6.751 & 4.598 & 4.216 & 2.466 & 2.438 & 10.91 & 6.118 & 4.901 & 6.654 & 4.241 \\
\hline 9/19/2005 & $22: 00$ & 10.788 & 10.688 & 7.864 & 6.751 & 4.594 & 4.221 & 2.459 & 2.436 & 10.912 & 6.111 & 4.888 & 6.65 & 4.221 \\
\hline 9/19/2005 & $23: 00$ & 10.793 & 10.693 & 7.862 & 6.753 & 4.584 & 4.225 & 2.464 & 2.436 & 10.91 & 6.104 & 4.874 & 6.645 & 4.199 \\
\hline 9/20/2005 & $0: 00$ & 10.793 & 10.695 & 7.862 & 6.753 & 4.575 & 4.227 & 2.454 & 2.434 & 10.906 & 6.1 & 4.862 & 6.639 & 4.179 \\
\hline 9/20/2005 & $1: 00$ & 10.795 & 10.697 & 7.862 & 6.751 & 4.567 & 4.227 & 2.457 & 2.43 & 10.902 & 6.093 & 4.848 & 6.635 & 4.158 \\
\hline 9/20/2005 & $2: 00$ & 10.795 & 10.697 & 7.862 & 6.751 & 4.559 & 4.232 & 2.449 & 2.43 & 10.9 & 6.089 & 4.839 & 6.633 & 4.142 \\
\hline 9/20/2005 & $3: 00$ & 10.798 & 10.699 & 7.862 & 6.751 & 4.55 & 4.232 & 2.449 & 2.428 & 10.898 & 6.082 & 4.829 & 6.63 & 4.126 \\
\hline 9/20/2005 & $4: 00$ & 10.8 & 10.701 & 7.863 & 6.749 & 4.542 & 4.232 & 2.447 & 2.428 & 10.895 & 6.08 & 4.821 & 6.628 & 4.111 \\
\hline 9/20/2005 & $5: 00$ & 10.801 & 10.701 & 7.863 & 6.749 & 4.534 & 4.23 & 2.444 & 2.426 & 10.891 & 6.076 & 4.813 & 6.626 & 4.095 \\
\hline
\end{tabular}


TABLE S1.2 (Cont.)

Water Level (ft below top of casing) at Indicated Well

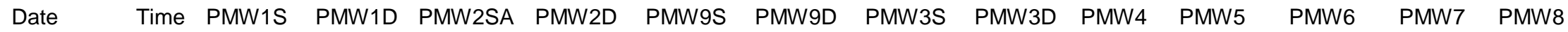

\begin{tabular}{|c|c|c|c|c|c|c|c|c|c|c|c|c|c|c|}
\hline 9/20/2005 & $6: 00$ & 10.803 & 10.701 & 7.86 & 6.746 & 4.526 & 4.227 & 2.447 & 2.421 & 10.887 & 6.074 & 4.805 & 6.624 & 4.081 \\
\hline 9/20/2005 & $7: 00$ & 10.803 & 10.702 & 7.862 & 6.751 & 4.521 & 4.227 & 2.45 & 2.422 & 10.883 & 6.071 & 4.801 & 6.624 & 4.071 \\
\hline 9/20/2005 & $8: 00$ & 10.808 & 10.71 & 7.865 & 6.758 & 4.523 & 4.23 & 2.45 & 2.43 & 10.881 & 6.071 & 4.803 & 6.626 & 4.065 \\
\hline 9/20/2005 & 9:00 & 10.808 & 10.71 & 7.867 & 6.758 & 4.519 & 4.227 & 2.457 & 2.43 & 10.878 & 6.069 & 4.801 & 6.628 & 4.055 \\
\hline 9/20/2005 & $10: 00$ & 10.811 & 10.713 & 7.869 & 6.758 & 4.521 & 4.225 & 2.455 & 2.436 & 10.878 & 6.076 & 4.815 & 6.635 & 4.063 \\
\hline 9/20/2005 & $11: 00$ & 10.811 & 10.712 & 7.873 & 6.762 & 4.527 & 4.223 & 2.47 & 2.442 & 10.878 & 6.089 & 4.835 & 6.645 & 4.091 \\
\hline 9/20/2005 & $12: 00$ & 10.808 & 10.706 & 7.872 & 6.758 & 4.534 & 4.221 & 2.473 & 2.447 & 10.879 & 6.103 & 4.862 & 6.656 & 4.136 \\
\hline 9/20/2005 & $13: 00$ & 10.803 & 10.697 & 7.872 & 6.753 & 4.55 & 4.219 & 2.48 & 2.449 & 10.883 & 6.118 & 4.888 & 6.672 & 4.187 \\
\hline 9/20/2005 & $14: 00$ & 10.796 & 10.686 & 7.871 & 6.749 & 4.565 & 4.216 & 2.477 & 2.447 & 10.889 & 6.126 & 4.913 & 6.679 & 4.236 \\
\hline 9/20/2005 & $15: 00$ & 10.786 & 10.67 & 7.863 & 6.74 & 4.577 & 4.216 & 2.472 & 2.443 & 10.893 & 6.133 & 4.933 & 6.681 & 4.272 \\
\hline 9/20/2005 & $16: 00$ & 10.779 & 10.664 & 7.863 & 6.738 & 4.592 & 4.214 & 2.477 & 2.443 & 10.902 & 6.142 & 4.955 & 6.691 & 4.307 \\
\hline 9/20/2005 & $17: 00$ & 10.774 & 10.657 & 7.859 & 6.735 & 4.606 & 4.214 & 2.477 & 2.444 & 10.908 & 6.146 & 4.968 & 6.691 & 4.329 \\
\hline 9/20/2005 & $18: 00$ & 10.769 & 10.653 & 7.859 & 6.736 & 4.615 & 4.216 & 2.474 & 2.445 & 10.916 & 6.153 & 4.976 & 6.689 & 4.347 \\
\hline 9/20/2005 & $19: 00$ & 10.766 & 10.651 & 7.859 & 6.733 & 4.621 & 4.219 & 2.472 & 2.443 & 10.922 & 6.153 & 4.972 & 6.683 & 4.354 \\
\hline 9/20/2005 & $20: 00$ & 10.764 & 10.65 & 7.857 & 6.734 & 4.621 & 4.221 & 2.459 & 2.434 & 10.925 & 6.146 & 4.955 & 6.67 & 4.337 \\
\hline 9/20/2005 & $21: 00$ & 10.766 & 10.653 & 7.857 & 6.733 & 4.621 & 4.225 & 2.457 & 2.434 & 10.929 & 6.14 & 4.941 & 6.664 & 4.319 \\
\hline 9/20/2005 & $22: 00$ & 10.766 & 10.657 & 7.856 & 6.734 & 4.615 & 4.225 & 2.462 & 2.43 & 10.927 & 6.131 & 4.923 & 6.656 & 4.295 \\
\hline 9/20/2005 & $23: 00$ & 10.769 & 10.659 & 7.854 & 6.733 & 4.607 & 4.227 & 2.452 & 2.428 & 10.927 & 6.125 & 4.91 & 6.651 & 4.273 \\
\hline $9 / 21 / 2005$ & $0: 00$ & 10.766 & 10.657 & 7.852 & 6.731 & 4.598 & 4.227 & 2.452 & 2.422 & 10.925 & 6.118 & 4.896 & 6.643 & 4.253 \\
\hline 9/21/2005 & $1: 00$ & 10.764 & 10.651 & 7.848 & 6.727 & 4.584 & 4.227 & 2.442 & 2.415 & 10.92 & 6.111 & 4.876 & 6.638 & 4.23 \\
\hline 9/21/2005 & $2: 00$ & 10.762 & 10.65 & 7.844 & 6.72 & 4.573 & 4.225 & 2.432 & 2.409 & 10.916 & 6.107 & 4.865 & 6.634 & 4.214 \\
\hline $9 / 21 / 2005$ & $3: 00$ & 10.759 & 10.644 & 7.84 & 6.716 & 4.561 & 4.221 & 2.422 & 2.403 & 10.91 & 6.099 & 4.851 & 6.626 & 4.194 \\
\hline 9/21/2005 & $4: 00$ & 10.754 & 10.64 & 7.836 & 6.709 & 4.55 & 4.216 & 2.425 & 2.394 & 10.906 & 6.092 & 4.837 & 6.62 & 4.175 \\
\hline $9 / 21 / 2005$ & $5: 00$ & 10.749 & 10.631 & 7.832 & 6.703 & 4.536 & 4.212 & 2.415 & 2.386 & 10.899 & 6.087 & 4.823 & 6.615 & 4.157 \\
\hline 9/21/2005 & $6: 00$ & 10.745 & 10.628 & 7.829 & 6.698 & 4.53 & 4.206 & 2.41 & 2.38 & 10.893 & 6.081 & 4.814 & 6.611 & 4.145 \\
\hline $9 / 21 / 2005$ & $7: 00$ & 10.742 & 10.62 & 7.822 & 6.691 & 4.515 & 4.199 & 2.395 & 2.371 & 10.887 & 6.078 & 4.804 & 6.603 & 4.129 \\
\hline 9/21/2005 & $8: 00$ & 10.735 & 10.615 & 7.819 & 6.685 & 4.505 & 4.197 & 2.387 & 2.366 & 10.884 & 6.072 & 4.794 & 6.601 & 4.115 \\
\hline $9 / 21 / 2005$ & $9: 00$ & 10.732 & 10.609 & 7.813 & 6.68 & 4.503 & 4.19 & 2.395 & 2.363 & 10.878 & 6.074 & 4.792 & 6.607 & 4.109 \\
\hline 9/21/2005 & $10: 00$ & 10.725 & 10.602 & 7.811 & 6.676 & 4.499 & 4.184 & 2.385 & 2.361 & 10.874 & 6.072 & 4.8 & 6.605 & 4.113 \\
\hline 9/21/2005 & $11: 00$ & 10.72 & 10.595 & 7.811 & 6.672 & 4.503 & 4.177 & 2.39 & 2.368 & 10.872 & 6.083 & 4.814 & 6.613 & 4.135 \\
\hline 9/21/2005 & $12: 00$ & 10.713 & 10.584 & 7.809 & 6.667 & 4.509 & 4.168 & 2.395 & 2.366 & 10.872 & 6.09 & 4.839 & 6.618 & 4.174 \\
\hline 9/21/2005 & $13: 00$ & 10.705 & 10.575 & 7.807 & 6.663 & 4.527 & 4.161 & 2.402 & 2.37 & 10.874 & 6.107 & 4.871 & 6.634 & 4.226 \\
\hline 9/21/2005 & $14: 00$ & 10.7 & 10.566 & 7.804 & 6.658 & 4.546 & 4.157 & 2.392 & 2.37 & 10.878 & 6.116 & 4.898 & 6.632 & 4.275 \\
\hline $9 / 21 / 2005$ & $15: 00$ & 10.69 & 10.558 & 7.8 & 6.654 & 4.563 & 4.155 & 2.402 & 2.368 & 10.884 & 6.123 & 4.921 & 6.641 & 4.316 \\
\hline
\end{tabular}


TABLE S1.2 (Cont.)

Water Level (ft below top of casing) at Indicated Well

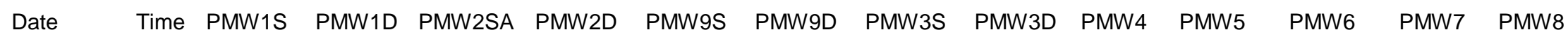

\begin{tabular}{|c|c|c|c|c|c|c|c|c|c|c|c|c|c|c|}
\hline 9/21/2005 & $16: 00$ & 10.68 & 10.546 & 7.797 & 6.649 & 4.581 & 4.15 & 2.402 & 2.368 & 10.887 & 6.134 & 4.941 & 6.643 & 4.35 \\
\hline 9/21/2005 & $17: 00$ & 10.673 & 10.54 & 7.794 & 6.649 & 4.594 & 4.15 & 2.394 & 2.366 & 10.893 & 6.138 & 4.955 & 6.645 & 4.371 \\
\hline $9 / 21 / 2005$ & $18: 00$ & 10.668 & 10.535 & 7.798 & 6.647 & 4.609 & 4.153 & 2.404 & 2.37 & 10.901 & 6.147 & 4.961 & 6.634 & 4.383 \\
\hline 9/21/2005 & $19: 00$ & 10.664 & 10.535 & 7.795 & 6.649 & 4.617 & 4.155 & 2.404 & 2.37 & 10.907 & 6.14 & 4.957 & 6.628 & 4.389 \\
\hline 9/21/2005 & 20:00 & 10.661 & 10.531 & 7.793 & 6.647 & 4.615 & 4.157 & 2.387 & 2.361 & 10.912 & 6.136 & 4.937 & 6.618 & 4.377 \\
\hline 9/21/2005 & $21: 00$ & 10.661 & 10.533 & 7.793 & 6.649 & 4.613 & 4.161 & 2.392 & 2.359 & 10.912 & 6.128 & 4.922 & 6.613 & 4.359 \\
\hline $9 / 21 / 2005$ & $22: 00$ & 10.659 & 10.533 & 7.789 & 6.645 & 4.603 & 4.161 & 2.382 & 2.351 & 10.912 & 6.121 & 4.902 & 6.605 & 4.336 \\
\hline 9/21/2005 & $23: 00$ & 10.659 & 10.533 & 7.785 & 6.643 & 4.592 & 4.161 & 2.372 & 2.347 & 10.909 & 6.114 & 4.886 & 6.6 & 4.316 \\
\hline 9/22/2005 & $0: 00$ & 10.656 & 10.533 & 7.783 & 6.643 & 4.582 & 4.159 & 2.372 & 2.342 & 10.907 & 6.11 & 4.871 & 6.594 & 4.298 \\
\hline 9/22/2005 & $1: 00$ & 10.664 & 10.547 & 7.788 & 6.658 & 4.586 & 4.164 & 2.38 & 2.355 & 10.907 & 6.11 & 4.867 & 6.598 & 4.29 \\
\hline $9 / 22 / 2005$ & $2: 00$ & 10.677 & 10.56 & 7.792 & 6.66 & 4.586 & 4.166 & 2.387 & 2.355 & 10.907 & 6.108 & 4.859 & 6.592 & 4.277 \\
\hline 9/22/2005 & $3: 00$ & 10.679 & 10.562 & 7.794 & 6.66 & 4.574 & 4.166 & 2.385 & 2.355 & 10.903 & 6.099 & 4.843 & 6.586 & 4.259 \\
\hline $9 / 22 / 2005$ & $4: 00$ & 10.686 & 10.567 & 7.796 & 6.663 & 4.568 & 4.168 & 2.38 & 2.355 & 10.901 & 6.095 & 4.836 & 6.586 & 4.243 \\
\hline 9/22/2005 & $5: 00$ & 10.696 & 10.582 & 7.8 & 6.674 & 4.568 & 4.172 & 2.388 & 2.364 & 10.899 & 6.095 & 4.83 & 6.588 & 4.235 \\
\hline 9/22/2005 & $6: 00$ & 10.706 & 10.593 & 7.806 & 6.68 & 4.565 & 4.175 & 2.396 & 2.368 & 10.897 & 6.09 & 4.822 & 6.586 & 4.221 \\
\hline 9/22/2005 & 7:00 & 10.716 & 10.606 & 7.81 & 6.689 & 4.563 & 4.177 & 2.396 & 2.374 & 10.895 & 6.088 & 4.822 & 6.588 & 4.211 \\
\hline $9 / 22 / 2005$ & $8: 00$ & 10.729 & 10.619 & 7.816 & 6.7 & 4.561 & 4.181 & 2.403 & 2.385 & 10.895 & 6.086 & 4.818 & 6.594 & 4.198 \\
\hline 9/22/2005 & $9: 00$ & 10.736 & 10.624 & 7.822 & 6.705 & 4.557 & 4.184 & 2.411 & 2.385 & 10.892 & 6.082 & 4.814 & 6.594 & 4.191 \\
\hline 9/22/2005 & $10: 00$ & 10.746 & 10.637 & 7.826 & 6.71 & 4.559 & 4.186 & 2.423 & 2.395 & 10.892 & 6.086 & 4.824 & 6.605 & 4.191 \\
\hline 9/22/2005 & 11:00 & 10.753 & 10.644 & 7.832 & 6.716 & 4.563 & 4.188 & 2.426 & 2.404 & 10.892 & 6.095 & 4.841 & 6.615 & 4.199 \\
\hline 9/22/2005 & $12: 00$ & 10.76 & 10.652 & 7.836 & 6.723 & 4.571 & 4.19 & 2.444 & 2.412 & 10.893 & 6.104 & 4.861 & 6.621 & 4.221 \\
\hline $9 / 22 / 2005$ & $13: 00$ & 10.765 & 10.653 & 7.842 & 6.725 & 4.577 & 4.192 & 2.449 & 2.416 & 10.895 & 6.106 & 4.873 & 6.623 & 4.242 \\
\hline 9/22/2005 & $14: 00$ & 10.768 & 10.655 & 7.848 & 6.727 & 4.582 & 4.197 & 2.444 & 2.421 & 10.897 & 6.11 & 4.881 & 6.623 & 4.255 \\
\hline $9 / 22 / 2005$ & $15: 00$ & 10.773 & 10.664 & 7.851 & 6.736 & 4.592 & 4.201 & 2.456 & 2.427 & 10.899 & 6.121 & 4.895 & 6.636 & 4.272 \\
\hline 9/22/2005 & $16: 00$ & 10.778 & 10.668 & 7.856 & 6.736 & 4.598 & 4.206 & 2.461 & 2.431 & 10.907 & 6.117 & 4.897 & 6.636 & 4.282 \\
\hline $9 / 22 / 2005$ & $17: 00$ & 10.78 & 10.668 & 7.857 & 6.738 & 4.603 & 4.206 & 2.461 & 2.431 & 10.907 & 6.121 & 4.904 & 6.64 & 4.29 \\
\hline 9/22/2005 & $18: 00$ & 10.785 & 10.673 & 7.859 & 6.743 & 4.605 & 4.212 & 2.466 & 2.437 & 10.912 & 6.128 & 4.912 & 6.642 & 4.303 \\
\hline $9 / 22 / 2005$ & $19: 00$ & 10.79 & 10.677 & 7.863 & 6.745 & 4.609 & 4.217 & 2.471 & 2.439 & 10.912 & 6.13 & 4.916 & 6.642 & 4.309 \\
\hline 9/22/2005 & 20:00 & 10.795 & 10.681 & 7.864 & 6.752 & 4.611 & 4.221 & 2.469 & 2.439 & 10.914 & 6.121 & 4.908 & 6.636 & 4.298 \\
\hline 9/22/2005 & $21: 00$ & 10.8 & 10.688 & 7.868 & 6.752 & 4.609 & 4.226 & 2.462 & 2.442 & 10.916 & 6.117 & 4.897 & 6.634 & 4.282 \\
\hline 9/22/2005 & $22: 00$ & 10.805 & 10.69 & 7.868 & 6.752 & 4.603 & 4.228 & 2.467 & 2.439 & 10.914 & 6.111 & 4.887 & 6.632 & 4.262 \\
\hline 9/22/2005 & 23:00 & 10.812 & 10.697 & 7.872 & 6.758 & 4.599 & 4.232 & 2.462 & 2.442 & 10.912 & 6.108 & 4.879 & 6.632 & 4.246 \\
\hline 9/23/2005 & $0: 00$ & 10.812 & 10.695 & 7.87 & 6.754 & 4.586 & 4.234 & 2.457 & 2.436 & 10.911 & 6.099 & 4.863 & 6.627 & 4.226 \\
\hline 9/23/2005 & $1: 00$ & 10.812 & 10.697 & 7.868 & 6.749 & 4.578 & 4.234 & 2.46 & 2.431 & 10.907 & 6.095 & 4.854 & 6.623 & 4.207 \\
\hline
\end{tabular}


TABLE S1.2 (Cont.)

Water Level (ft below top of casing) at Indicated Well

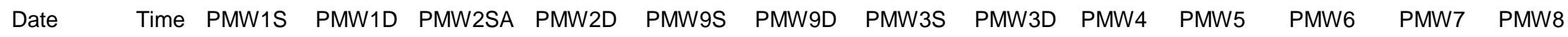

\begin{tabular}{|c|c|c|c|c|c|c|c|c|c|c|c|c|c|c|}
\hline 9/23/2005 & $2: 00$ & 10.817 & 10.702 & 7.868 & 6.756 & 4.576 & 4.237 & 2.462 & 2.436 & 10.903 & 6.097 & 4.852 & 6.625 & 4.199 \\
\hline 9/23/2005 & $3: 00$ & 10.82 & 10.705 & 7.868 & 6.756 & 4.57 & 4.237 & 2.455 & 2.434 & 10.901 & 6.093 & 4.842 & 6.621 & 4.181 \\
\hline 9/23/2005 & $4: 00$ & 10.817 & 10.701 & 7.864 & 6.749 & 4.557 & 4.234 & 2.447 & 2.427 & 10.896 & 6.084 & 4.83 & 6.617 & 4.167 \\
\hline 9/23/2005 & $5: 00$ & 10.822 & 10.703 & 7.864 & 6.752 & 4.553 & 4.234 & 2.455 & 2.427 & 10.894 & 6.084 & 4.828 & 6.619 & 4.159 \\
\hline 9/23/2005 & $6: 00$ & 10.82 & 10.703 & 7.864 & 6.749 & 4.545 & 4.232 & 2.447 & 2.427 & 10.89 & 6.08 & 4.82 & 6.615 & 4.149 \\
\hline 9/23/2005 & $7: 00$ & 10.82 & 10.701 & 7.862 & 6.747 & 4.54 & 4.23 & 2.447 & 2.423 & 10.886 & 6.078 & 4.814 & 6.613 & 4.137 \\
\hline 9/23/2005 & $8: 00$ & 10.822 & 10.705 & 7.864 & 6.749 & 4.536 & 4.23 & 2.452 & 2.425 & 10.884 & 6.073 & 4.815 & 6.619 & 4.128 \\
\hline 9/23/2005 & $9: 00$ & 10.825 & 10.705 & 7.864 & 6.752 & 4.534 & 4.23 & 2.452 & 2.425 & 10.881 & 6.073 & 4.815 & 6.617 & 4.122 \\
\hline 9/23/2005 & $10: 00$ & 10.825 & 10.71 & 7.868 & 6.754 & 4.538 & 4.23 & 2.458 & 2.431 & 10.881 & 6.078 & 4.821 & 6.621 & 4.124 \\
\hline 9/23/2005 & $11: 00$ & 10.82 & 10.706 & 7.862 & 6.752 & 4.534 & 4.228 & 2.455 & 2.429 & 10.879 & 6.073 & 4.819 & 6.619 & 4.118 \\
\hline $9 / 23 / 2005$ & $12: 00$ & 10.813 & 10.692 & 7.86 & 6.739 & 4.522 & 4.224 & 2.448 & 2.419 & 10.873 & 6.073 & 4.817 & 6.617 & 4.11 \\
\hline 9/23/2005 & $13: 00$ & 10.806 & 10.686 & 7.856 & 6.734 & 4.52 & 4.219 & 2.432 & 2.415 & 10.869 & 6.069 & 4.817 & 6.617 & 4.108 \\
\hline 9/23/2005 & $14: 00$ & 10.798 & 10.675 & 7.854 & 6.732 & 4.516 & 4.215 & 2.44 & 2.413 & 10.867 & 6.074 & 4.823 & 6.617 & 4.112 \\
\hline 9/23/2005 & $15: 00$ & 10.793 & 10.67 & 7.848 & 6.725 & 4.516 & 4.208 & 2.435 & 2.408 & 10.865 & 6.073 & 4.832 & 6.619 & 4.118 \\
\hline 9/23/2005 & $16: 00$ & 10.786 & 10.661 & 7.844 & 6.717 & 4.516 & 4.204 & 2.43 & 2.402 & 10.863 & 6.078 & 4.838 & 6.619 & 4.128 \\
\hline 9/23/2005 & $17: 00$ & 10.779 & 10.652 & 7.842 & 6.714 & 4.52 & 4.202 & 2.428 & 2.398 & 10.863 & 6.08 & 4.846 & 6.621 & 4.135 \\
\hline 9/23/2005 & $18: 00$ & 10.774 & 10.65 & 7.84 & 6.712 & 4.524 & 4.199 & 2.427 & 2.398 & 10.862 & 6.082 & 4.848 & 6.619 & 4.141 \\
\hline 9/23/2005 & $19: 00$ & 10.769 & 10.646 & 7.836 & 6.71 & 4.524 & 4.197 & 2.425 & 2.396 & 10.862 & 6.082 & 4.846 & 6.612 & 4.143 \\
\hline 9/23/2005 & $20: 00$ & 10.769 & 10.644 & 7.834 & 6.708 & 4.526 & 4.197 & 2.42 & 2.394 & 10.858 & 6.078 & 4.836 & 6.61 & 4.133 \\
\hline 9/23/2005 & $21: 00$ & 10.769 & 10.648 & 7.836 & 6.712 & 4.524 & 4.197 & 2.42 & 2.392 & 10.858 & 6.074 & 4.828 & 6.606 & 4.121 \\
\hline $9 / 23 / 2005$ & $22: 00$ & 10.769 & 10.644 & 7.834 & 6.71 & 4.516 & 4.195 & 2.415 & 2.388 & 10.856 & 6.067 & 4.815 & 6.6 & 4.106 \\
\hline 9/23/2005 & $23: 00$ & 10.766 & 10.646 & 7.832 & 6.706 & 4.513 & 4.195 & 2.407 & 2.388 & 10.846 & 6.067 & 4.809 & 6.598 & 4.093 \\
\hline 9/24/2005 & $0: 00$ & 10.761 & 10.641 & 7.831 & 6.701 & 4.503 & 4.191 & 2.407 & 2.379 & 10.842 & 6.06 & 4.795 & 6.593 & 4.076 \\
\hline 9/24/2005 & $1: 00$ & 10.756 & 10.637 & 7.826 & 6.697 & 4.495 & 4.188 & 2.402 & 2.375 & 10.841 & 6.058 & 4.787 & 6.591 & 4.064 \\
\hline 9/24/2005 & $2: 00$ & 10.756 & 10.635 & 7.825 & 6.694 & 4.489 & 4.186 & 2.395 & 2.373 & 10.837 & 6.056 & 4.781 & 6.589 & 4.058 \\
\hline $9 / 24 / 2005$ & $3: 00$ & 10.752 & 10.624 & 7.818 & 6.688 & 4.48 & 4.182 & 2.383 & 2.365 & 10.833 & 6.05 & 4.77 & 6.583 & 4.04 \\
\hline 9/24/2005 & $4: 00$ & 10.747 & 10.621 & 7.817 & 6.683 & 4.474 & 4.177 & 2.388 & 2.36 & 10.83 & 6.047 & 4.762 & 6.581 & 4.031 \\
\hline $9 / 24 / 2005$ & $5: 00$ & 10.742 & 10.619 & 7.815 & 6.681 & 4.47 & 4.173 & 2.383 & 2.356 & 10.827 & 6.045 & 4.756 & 6.579 & 4.023 \\
\hline 9/24/2005 & $6: 00$ & 10.737 & 10.612 & 7.809 & 6.674 & 4.463 & 4.168 & 2.375 & 2.35 & 10.822 & 6.041 & 4.746 & 6.574 & 4.012 \\
\hline 9/24/2005 & $7: 00$ & 10.729 & 10.608 & 7.805 & 6.67 & 4.455 & 4.164 & 2.37 & 2.344 & 10.818 & 6.039 & 4.74 & 6.579 & 4.001 \\
\hline 9/24/2005 & $8: 00$ & 10.727 & 10.61 & 7.801 & 6.679 & 4.451 & 4.16 & 2.37 & 2.342 & 10.814 & 6.037 & 4.736 & 6.598 & 3.995 \\
\hline 9/24/2005 & $9: 00$ & 10.727 & 10.608 & 7.803 & 6.672 & 4.453 & 4.16 & 2.373 & 2.346 & 10.812 & 6.037 & 4.735 & 6.57 & 3.993 \\
\hline 9/24/2005 & $10: 00$ & 10.727 & 10.608 & 7.803 & 6.67 & 4.451 & 4.155 & 2.373 & 2.346 & 10.809 & 6.034 & 4.732 & 6.566 & 3.985 \\
\hline $9 / 24 / 2005$ & $11: 00$ & 10.727 & 10.606 & 7.803 & 6.67 & 4.447 & 4.153 & 2.371 & 2.344 & 10.807 & 6.032 & 4.731 & 6.566 & 3.983 \\
\hline
\end{tabular}


TABLE S1.2 (Cont.)

Water Level (ft below top of casing) at Indicated Well

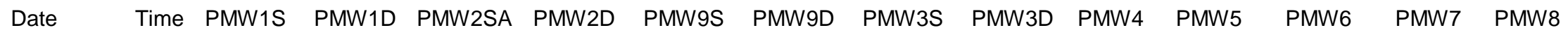

\begin{tabular}{|c|c|c|c|c|c|c|c|c|c|c|c|c|c|c|}
\hline 9/24/2005 & $12: 00$ & 10.725 & 10.604 & 7.803 & 6.67 & 4.449 & 4.151 & 2.368 & 2.348 & 10.807 & 6.034 & 4.743 & 6.57 & 3.995 \\
\hline $9 / 24 / 2005$ & $13: 00$ & 10.718 & 10.595 & 7.801 & 6.663 & 4.447 & 4.144 & 2.371 & 2.348 & 10.803 & 6.043 & 4.762 & 6.578 & 4.019 \\
\hline 9/24/2005 & $14: 00$ & 10.71 & 10.584 & 7.799 & 6.657 & 4.455 & 4.14 & 2.373 & 2.35 & 10.803 & 6.054 & 4.79 & 6.581 & 4.06 \\
\hline 9/24/2005 & $15: 00$ & 10.7 & 10.579 & 7.797 & 6.655 & 4.472 & 4.135 & 2.386 & 2.354 & 10.803 & 6.067 & 4.817 & 6.589 & 4.111 \\
\hline 9/24/2005 & $16: 00$ & 10.696 & 10.571 & 7.797 & 6.657 & 4.491 & 4.133 & 2.388 & 2.354 & 10.811 & 6.078 & 4.845 & 6.597 & 4.16 \\
\hline 9/24/2005 & $17: 00$ & 10.693 & 10.568 & 7.799 & 6.657 & 4.511 & 4.133 & 2.393 & 2.363 & 10.816 & 6.091 & 4.868 & 6.6 & 4.198 \\
\hline $9 / 24 / 2005$ & $18: 00$ & 10.688 & 10.564 & 7.799 & 6.657 & 4.53 & 4.133 & 2.388 & 2.363 & 10.824 & 6.1 & 4.884 & 6.6 & 4.227 \\
\hline 9/24/2005 & $19: 00$ & 10.686 & 10.564 & 7.801 & 6.661 & 4.543 & 4.14 & 2.395 & 2.365 & 10.83 & 6.096 & 4.882 & 6.6 & 4.243 \\
\hline 9/24/2005 & $20: 00$ & 10.688 & 10.57 & 7.803 & 6.666 & 4.555 & 4.142 & 2.395 & 2.367 & 10.833 & 6.094 & 4.872 & 6.595 & 4.237 \\
\hline 9/24/2005 & $21: 00$ & 10.693 & 10.577 & 7.805 & 6.67 & 4.557 & 4.149 & 2.395 & 2.367 & 10.835 & 6.092 & 4.858 & 6.589 & 4.221 \\
\hline $9 / 24 / 2005$ & $22: 00$ & 10.698 & 10.581 & 7.805 & 6.67 & 4.553 & 4.153 & 2.388 & 2.365 & 10.837 & 6.079 & 4.841 & 6.581 & 4.199 \\
\hline 9/24/2005 & $23: 00$ & 10.698 & 10.581 & 7.801 & 6.666 & 4.541 & 4.153 & 2.388 & 2.359 & 10.837 & 6.074 & 4.829 & 6.579 & 4.177 \\
\hline $9 / 25 / 2005$ & $0: 00$ & 10.698 & 10.581 & 7.801 & 6.668 & 4.535 & 4.157 & 2.378 & 2.359 & 10.833 & 6.068 & 4.813 & 6.576 & 4.156 \\
\hline 9/25/2005 & $1: 00$ & 10.693 & 10.575 & 7.795 & 6.657 & 4.518 & 4.156 & 2.368 & 2.346 & 10.83 & 6.059 & 4.796 & 6.568 & 4.132 \\
\hline 9/25/2005 & $2: 00$ & 10.688 & 10.571 & 7.794 & 6.655 & 4.508 & 4.153 & 2.363 & 2.34 & 10.826 & 6.055 & 4.784 & 6.566 & 4.116 \\
\hline 9/25/2005 & 3:00 & 10.691 & 10.573 & 7.792 & 6.657 & 4.505 & 4.153 & 2.368 & 2.34 & 10.822 & 6.05 & 4.776 & 6.566 & 4.101 \\
\hline 9/25/2005 & $4: 00$ & 10.689 & 10.573 & 7.79 & 6.655 & 4.495 & 4.151 & 2.366 & 2.336 & 10.818 & 6.044 & 4.762 & 6.561 & 4.085 \\
\hline 9/25/2005 & $5: 00$ & 10.696 & 10.586 & 7.796 & 6.666 & 4.497 & 4.153 & 2.368 & 2.346 & 10.816 & 6.048 & 4.762 & 6.564 & 4.081 \\
\hline 9/25/2005 & $6: 00$ & 10.701 & 10.588 & 7.794 & 6.664 & 4.491 & 4.151 & 2.363 & 2.342 & 10.814 & 6.042 & 4.752 & 6.559 & 4.063 \\
\hline 9/25/2005 & 7:00 & 10.698 & 10.582 & 7.794 & 6.659 & 4.48 & 4.149 & 2.363 & 2.336 & 10.811 & 6.035 & 4.737 & 6.553 & 4.049 \\
\hline 9/25/2005 & 8:00 & 10.703 & 10.592 & 7.796 & 6.664 & 4.48 & 4.151 & 2.364 & 2.342 & 10.809 & 6.035 & 4.735 & 6.555 & 4.041 \\
\hline $9 / 25 / 2005$ & $9: 00$ & 10.701 & 10.588 & 7.792 & 6.659 & 4.47 & 4.147 & 2.356 & 2.336 & 10.805 & 6.03 & 4.727 & 6.551 & 4.024 \\
\hline 9/25/2005 & $10: 00$ & 10.704 & 10.59 & 7.794 & 6.662 & 4.466 & 4.147 & 2.359 & 2.338 & 10.801 & 6.031 & 4.727 & 6.555 & 4.02 \\
\hline $9 / 25 / 2005$ & $11: 00$ & 10.701 & 10.59 & 7.794 & 6.662 & 4.464 & 4.144 & 2.366 & 2.338 & 10.799 & 6.031 & 4.729 & 6.555 & 4.022 \\
\hline 9/25/2005 & $12: 00$ & 10.701 & 10.586 & 7.794 & 6.655 & 4.46 & 4.14 & 2.364 & 2.336 & 10.798 & 6.035 & 4.733 & 6.557 & 4.029 \\
\hline $9 / 25 / 2005$ & $13: 00$ & 10.696 & 10.582 & 7.794 & 6.657 & 4.466 & 4.138 & 2.361 & 2.34 & 10.795 & 6.042 & 4.749 & 6.561 & 4.051 \\
\hline 9/25/2005 & $14: 00$ & 10.689 & 10.571 & 7.792 & 6.65 & 4.468 & 4.131 & 2.369 & 2.34 & 10.796 & 6.048 & 4.767 & 6.566 & 4.081 \\
\hline $9 / 25 / 2005$ & $15: 00$ & 10.684 & 10.566 & 7.788 & 6.648 & 4.48 & 4.129 & 2.364 & 2.34 & 10.798 & 6.057 & 4.788 & 6.574 & 4.118 \\
\hline 9/25/2005 & $16: 00$ & 10.679 & 10.564 & 7.79 & 6.65 & 4.497 & 4.131 & 2.371 & 2.34 & 10.799 & 6.062 & 4.8 & 6.572 & 4.142 \\
\hline 9/25/2005 & $17: 00$ & 10.679 & 10.564 & 7.792 & 6.653 & 4.512 & 4.131 & 2.371 & 2.347 & 10.805 & 6.073 & 4.815 & 6.576 & 4.167 \\
\hline 9/25/2005 & $18: 00$ & 10.684 & 10.573 & 7.796 & 6.662 & 4.528 & 4.136 & 2.376 & 2.353 & 10.809 & 6.075 & 4.823 & 6.576 & 4.181 \\
\hline 9/25/2005 & 19:00 & 10.692 & 10.582 & 7.8 & 6.668 & 4.537 & 4.14 & 2.381 & 2.359 & 10.813 & 6.071 & 4.819 & 6.576 & 4.181 \\
\hline $9 / 25 / 2005$ & $20: 00$ & 10.704 & 10.599 & 7.808 & 6.682 & 4.543 & 4.147 & 2.397 & 2.366 & 10.817 & 6.07 & 4.817 & 6.576 & 4.175 \\
\hline 9/25/2005 & $21: 00$ & 10.717 & 10.612 & 7.814 & 6.693 & 4.547 & 4.153 & 2.397 & 2.378 & 10.817 & 6.066 & 4.812 & 6.576 & 4.165 \\
\hline
\end{tabular}


TABLE S1.2 (Cont.)

Water Level (ft below top of casing) at Indicated Well

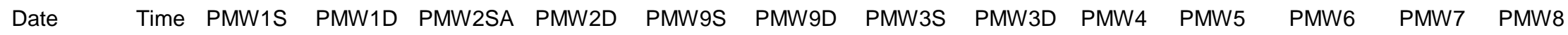

\begin{tabular}{|c|c|c|c|c|c|c|c|c|c|c|c|c|c|c|}
\hline 9/25/2005 & $22: 00$ & 10.726 & 10.623 & 7.82 & 6.697 & 4.545 & 4.16 & 2.405 & 2.378 & 10.819 & 6.062 & 4.804 & 6.576 & 4.147 \\
\hline $9 / 25 / 2005$ & $23: 00$ & 10.736 & 10.633 & 7.824 & 6.704 & 4.543 & 4.164 & 2.41 & 2.384 & 10.819 & 6.059 & 4.798 & 6.578 & 4.129 \\
\hline 9/26/2005 & $0: 00$ & 10.748 & 10.646 & 7.83 & 6.715 & 4.543 & 4.171 & 2.42 & 2.393 & 10.82 & 6.058 & 4.794 & 6.58 & 4.118 \\
\hline 9/26/2005 & $1: 00$ & 10.758 & 10.657 & 7.835 & 6.72 & 4.541 & 4.178 & 2.417 & 2.397 & 10.819 & 6.058 & 4.79 & 6.584 & 4.1 \\
\hline $9 / 26 / 2005$ & $2: 00$ & 10.769 & 10.668 & 7.841 & 6.728 & 4.539 & 4.182 & 2.428 & 2.403 & 10.819 & 6.058 & 4.788 & 6.588 & 4.092 \\
\hline 9/26/2005 & $3: 00$ & 10.768 & 10.661 & 7.837 & 6.717 & 4.524 & 4.184 & 2.413 & 2.393 & 10.815 & 6.044 & 4.772 & 6.58 & 4.072 \\
\hline $9 / 26 / 2005$ & $4: 00$ & 10.778 & 10.675 & 7.843 & 6.728 & 4.528 & 4.189 & 2.43 & 2.403 & 10.817 & 6.049 & 4.774 & 6.588 & 4.066 \\
\hline 9/26/2005 & $5: 00$ & 10.786 & 10.681 & 7.847 & 6.737 & 4.524 & 4.191 & 2.435 & 2.41 & 10.815 & 6.046 & 4.77 & 6.588 & 4.053 \\
\hline 9/26/2005 & $6: 00$ & 10.798 & 10.699 & 7.855 & 6.751 & 4.528 & 4.198 & 2.445 & 2.418 & 10.815 & 6.053 & 4.775 & 6.593 & 4.052 \\
\hline 9/26/2005 & $7: 00$ & 10.813 & 10.714 & 7.862 & 6.759 & 4.533 & 4.204 & 2.456 & 2.431 & 10.815 & 6.056 & 4.779 & 6.597 & 4.049 \\
\hline 9/26/2005 & 8:00 & 10.82 & 10.719 & 7.867 & 6.764 & 4.532 & 4.209 & 2.458 & 2.433 & 10.817 & 6.053 & 4.777 & 6.601 & 4.042 \\
\hline 9/26/2005 & $9: 00$ & 10.825 & 10.723 & 7.87 & 6.762 & 4.526 & 4.211 & 2.453 & 2.433 & 10.819 & 6.051 & 4.779 & 6.603 & 4.029 \\
\hline 9/26/2005 & $10: 00$ & 10.832 & 10.728 & 7.873 & 6.768 & 4.528 & 4.215 & 2.466 & 2.439 & 10.817 & 6.056 & 4.79 & 6.609 & 4.033 \\
\hline 9/26/2005 & $11: 00$ & 10.837 & 10.732 & 7.877 & 6.771 & 4.53 & 4.218 & 2.471 & 2.444 & 10.819 & 6.06 & 4.802 & 6.616 & 4.048 \\
\hline 9/26/2005 & $12: 00$ & 10.84 & 10.734 & 7.879 & 6.775 & 4.539 & 4.22 & 2.481 & 2.454 & 10.82 & 6.071 & 4.824 & 6.624 & 4.076 \\
\hline 9/26/2005 & $13: 00$ & 10.842 & 10.732 & 7.885 & 6.775 & 4.547 & 4.222 & 2.486 & 2.458 & 10.824 & 6.078 & 4.843 & 6.63 & 4.11 \\
\hline 9/26/2005 & $14: 00$ & 10.84 & 10.73 & 7.887 & 6.779 & 4.558 & 4.222 & 2.486 & 2.46 & 10.828 & 6.087 & 4.861 & 6.635 & 4.145 \\
\hline 9/26/2005 & $15: 00$ & 10.837 & 10.728 & 7.888 & 6.777 & 4.57 & 4.222 & 2.491 & 2.463 & 10.832 & 6.1 & 4.884 & 6.643 & 4.182 \\
\hline 9/26/2005 & $16: 00$ & 10.837 & 10.725 & 7.888 & 6.777 & 4.58 & 4.227 & 2.493 & 2.465 & 10.838 & 6.108 & 4.904 & 6.654 & 4.21 \\
\hline 9/26/2005 & $17: 00$ & 10.835 & 10.723 & 7.89 & 6.777 & 4.591 & 4.229 & 2.493 & 2.465 & 10.844 & 6.115 & 4.92 & 6.653 & 4.235 \\
\hline 9/26/2005 & $18: 00$ & 10.832 & 10.719 & 7.886 & 6.775 & 4.599 & 4.231 & 2.493 & 2.465 & 10.849 & 6.117 & 4.928 & 6.654 & 4.249 \\
\hline 9/26/2005 & $19: 00$ & 10.832 & 10.717 & 7.886 & 6.773 & 4.605 & 4.233 & 2.491 & 2.463 & 10.853 & 6.117 & 4.924 & 6.651 & 4.249 \\
\hline 9/26/2005 & $20: 00$ & 10.83 & 10.716 & 7.884 & 6.771 & 4.601 & 4.236 & 2.483 & 2.456 & 10.855 & 6.111 & 4.91 & 6.645 & 4.231 \\
\hline 9/26/2005 & $21: 00$ & 10.832 & 10.717 & 7.884 & 6.773 & 4.599 & 4.24 & 2.481 & 2.454 & 10.857 & 6.102 & 4.898 & 6.641 & 4.211 \\
\hline 9/26/2005 & $22: 00$ & 10.832 & 10.716 & 7.882 & 6.768 & 4.591 & 4.242 & 2.471 & 2.45 & 10.855 & 6.095 & 4.884 & 6.636 & 4.188 \\
\hline 9/26/2005 & 23:00 & 10.83 & 10.716 & 7.878 & 6.766 & 4.579 & 4.24 & 2.466 & 2.446 & 10.855 & 6.089 & 4.873 & 6.634 & 4.166 \\
\hline 9/27/2005 & $0: 00$ & 10.825 & 10.71 & 7.874 & 6.759 & 4.568 & 4.24 & 2.466 & 2.437 & 10.849 & 6.083 & 4.861 & 6.628 & 4.146 \\
\hline 9/27/2005 & 1:00 & 10.82 & 10.703 & 7.87 & 6.755 & 4.56 & 4.238 & 2.458 & 2.431 & 10.847 & 6.078 & 4.851 & 6.624 & 4.13 \\
\hline 9/27/2005 & $2: 00$ & 10.82 & 10.705 & 7.87 & 6.755 & 4.554 & 4.238 & 2.451 & 2.432 & 10.845 & 6.074 & 4.845 & 6.622 & 4.12 \\
\hline 9/27/2005 & $3: 00$ & 10.818 & 10.703 & 7.868 & 6.755 & 4.549 & 4.236 & 2.456 & 2.429 & 10.842 & 6.069 & 4.837 & 6.62 & 4.105 \\
\hline 9/27/2005 & $4: 00$ & 10.813 & 10.695 & 7.864 & 6.747 & 4.537 & 4.231 & 2.448 & 2.421 & 10.836 & 6.067 & 4.83 & 6.615 & 4.089 \\
\hline 9/27/2005 & $5: 00$ & 10.81 & 10.695 & 7.86 & 6.744 & 4.533 & 4.229 & 2.446 & 2.419 & 10.834 & 6.065 & 4.82 & 6.611 & 4.075 \\
\hline 9/27/2005 & $6: 00$ & 10.808 & 10.692 & 7.861 & 6.744 & 4.527 & 4.229 & 2.444 & 2.417 & 10.832 & 6.056 & 4.814 & 6.611 & 4.067 \\
\hline 9/27/2005 & $7: 00$ & 10.806 & 10.692 & 7.859 & 6.742 & 4.52 & 4.225 & 2.441 & 2.415 & 10.826 & 6.052 & 4.808 & 6.607 & 4.055 \\
\hline
\end{tabular}


TABLE S1.2 (Cont.)

Water Level (ft below top of casing) at Indicated Well

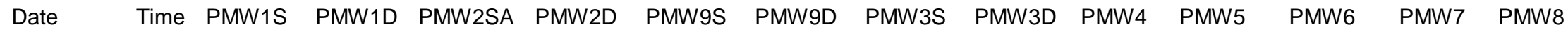

\begin{tabular}{|c|c|c|c|c|c|c|c|c|c|c|c|c|c|c|}
\hline 9/27/2005 & $8: 00$ & 10.803 & 10.69 & 7.857 & 6.739 & 4.512 & 4.222 & 2.431 & 2.411 & 10.823 & 6.052 & 4.802 & 6.607 & 4.04 \\
\hline 9/27/2005 & $9: 00$ & 10.799 & 10.683 & 7.853 & 6.733 & 4.504 & 4.218 & 2.426 & 2.406 & 10.819 & 6.047 & 4.795 & 6.601 & 4.028 \\
\hline 9/27/2005 & $10: 00$ & 10.796 & 10.679 & 7.853 & 6.729 & 4.5 & 4.214 & 2.424 & 2.404 & 10.815 & 6.047 & 4.801 & 6.601 & 4.028 \\
\hline 9/27/2005 & $11: 00$ & 10.789 & 10.674 & 7.849 & 6.727 & 4.498 & 4.207 & 2.436 & 2.406 & 10.815 & 6.054 & 4.812 & 6.605 & 4.046 \\
\hline 9/27/2005 & $12: 00$ & 10.779 & 10.663 & 7.848 & 6.722 & 4.504 & 4.202 & 2.436 & 2.408 & 10.813 & 6.063 & 4.832 & 6.609 & 4.085 \\
\hline 9/27/2005 & $13: 00$ & 10.773 & 10.652 & 7.844 & 6.715 & 4.512 & 4.196 & 2.431 & 2.408 & 10.815 & 6.076 & 4.857 & 6.617 & 4.132 \\
\hline 9/27/2005 & $14: 00$ & 10.757 & 10.632 & 7.84 & 6.702 & 4.52 & 4.187 & 2.431 & 2.402 & 10.817 & 6.09 & 4.877 & 6.617 & 4.17 \\
\hline 9/27/2005 & $15: 00$ & 10.746 & 10.623 & 7.836 & 6.698 & 4.539 & 4.183 & 2.431 & 2.402 & 10.821 & 6.105 & 4.9 & 6.619 & 4.217 \\
\hline 9/27/2005 & $16: 00$ & 10.739 & 10.613 & 7.834 & 6.698 & 4.554 & 4.18 & 2.433 & 2.402 & 10.826 & 6.114 & 4.916 & 6.622 & 4.256 \\
\hline 9/27/2005 & $17: 00$ & 10.73 & 10.608 & 7.834 & 6.693 & 4.574 & 4.18 & 2.433 & 2.404 & 10.83 & 6.123 & 4.93 & 6.626 & 4.284 \\
\hline 9/27/2005 & $18: 00$ & 10.725 & 10.601 & 7.832 & 6.693 & 4.585 & 4.18 & 2.433 & 2.4 & 10.836 & 6.125 & 4.936 & 6.622 & 4.304 \\
\hline 9/27/2005 & $19: 00$ & 10.72 & 10.597 & 7.83 & 6.693 & 4.593 & 4.183 & 2.428 & 2.398 & 10.842 & 6.123 & 4.926 & 6.617 & 4.312 \\
\hline 9/27/2005 & $20: 00$ & 10.717 & 10.597 & 7.828 & 6.693 & 4.595 & 4.185 & 2.415 & 2.394 & 10.845 & 6.116 & 4.908 & 6.609 & 4.295 \\
\hline 9/27/2005 & $21: 00$ & 10.717 & 10.593 & 7.824 & 6.689 & 4.589 & 4.185 & 2.413 & 2.386 & 10.845 & 6.107 & 4.891 & 6.601 & 4.27 \\
\hline 9/27/2005 & $22: 00$ & 10.715 & 10.593 & 7.821 & 6.687 & 4.581 & 4.185 & 2.411 & 2.382 & 10.844 & 6.099 & 4.877 & 6.596 & 4.25 \\
\hline 9/27/2005 & $23: 00$ & 10.713 & 10.59 & 7.817 & 6.68 & 4.57 & 4.185 & 2.398 & 2.375 & 10.84 & 6.092 & 4.859 & 6.592 & 4.228 \\
\hline 9/28/2005 & $0: 00$ & 10.708 & 10.588 & 7.813 & 6.676 & 4.56 & 4.183 & 2.395 & 2.367 & 10.836 & 6.085 & 4.848 & 6.588 & 4.207 \\
\hline 9/28/2005 & $1: 00$ & 10.703 & 10.582 & 7.809 & 6.671 & 4.546 & 4.178 & 2.381 & 2.358 & 10.833 & 6.076 & 4.83 & 6.581 & 4.185 \\
\hline 9/28/2005 & $2: 00$ & 10.7 & 10.577 & 7.803 & 6.667 & 4.537 & 4.174 & 2.381 & 2.352 & 10.827 & 6.074 & 4.818 & 6.579 & 4.167 \\
\hline 9/28/2005 & $3: 00$ & 10.693 & 10.573 & 7.801 & 6.66 & 4.525 & 4.171 & 2.376 & 2.346 & 10.825 & 6.066 & 4.804 & 6.571 & 4.146 \\
\hline 9/28/2005 & $4: 00$ & 10.688 & 10.564 & 7.795 & 6.651 & 4.512 & 4.165 & 2.363 & 2.335 & 10.819 & 6.063 & 4.791 & 6.565 & 4.131 \\
\hline 9/28/2005 & $5: 00$ & 10.69 & 10.577 & 7.795 & 6.66 & 4.517 & 4.165 & 2.366 & 2.342 & 10.817 & 6.07 & 4.791 & 6.571 & 4.131 \\
\hline 9/28/2005 & $6: 00$ & 10.698 & 10.592 & 7.804 & 6.678 & 4.525 & 4.165 & 2.386 & 2.358 & 10.815 & 6.068 & 4.789 & 6.571 & 4.128 \\
\hline 9/28/2005 & $7: 00$ & 10.701 & 10.559 & 7.804 & 6.649 & 4.517 & 4.165 & 2.384 & 2.354 & 10.814 & 6.059 & 4.779 & 6.535 & 4.114 \\
\hline 9/28/2005 & $8: 00$ & 10.706 & 10.555 & 7.806 & 6.649 & 4.512 & 4.163 & 2.381 & 2.35 & 10.8 & 6.054 & 4.769 & 6.537 & 4.098 \\
\hline 9/28/2005 & $9: 00$ & 10.723 & 10.624 & 7.818 & 6.7 & 4.525 & 4.169 & 2.399 & 2.373 & 10.806 & 6.059 & 4.781 & 6.571 & 4.102 \\
\hline 9/28/2005 & $10: 00$ & 10.748 & 10.659 & 7.837 & 6.731 & 4.546 & 4.178 & 2.429 & 2.405 & 10.815 & 6.07 & 4.799 & 6.588 & 4.116 \\
\hline 9/28/2005 & $11: 00$ & 10.767 & 10.677 & 7.849 & 6.747 & 4.55 & 4.187 & 2.447 & 2.42 & 10.819 & 6.07 & 4.804 & 6.596 & 4.114 \\
\hline 9/28/2005 & $12: 00$ & 10.785 & 10.694 & 7.859 & 6.756 & 4.554 & 4.196 & 2.455 & 2.428 & 10.814 & 6.072 & 4.812 & 6.6 & 4.114 \\
\hline 9/28/2005 & $13: 00$ & 10.8 & 10.705 & 7.866 & 6.762 & 4.556 & 4.201 & 2.465 & 2.437 & 10.821 & 6.073 & 4.821 & 6.605 & 4.112 \\
\hline 9/28/2005 & $14: 00$ & 10.81 & 10.714 & 7.871 & 6.771 & 4.56 & 4.209 & 2.472 & 2.445 & 10.823 & 6.073 & 4.826 & 6.613 & 4.12 \\
\hline 9/28/2005 & $15: 00$ & 10.817 & 10.721 & 7.875 & 6.773 & 4.562 & 4.214 & 2.475 & 2.451 & 10.823 & 6.079 & 4.836 & 6.617 & 4.13 \\
\hline 9/28/2005 & $16: 00$ & 10.824 & 10.73 & 7.885 & 6.78 & 4.569 & 4.218 & 2.477 & 2.458 & 10.825 & 6.083 & 4.848 & 6.623 & 4.15 \\
\hline 9/28/2005 & $17: 00$ & 10.832 & 10.736 & 7.885 & 6.782 & 4.577 & 4.223 & 2.493 & 2.464 & 10.838 & 6.088 & 4.864 & 6.627 & 4.175 \\
\hline
\end{tabular}


TABLE S1.2 (Cont.)

Water Level (ft below top of casing) at Indicated Well

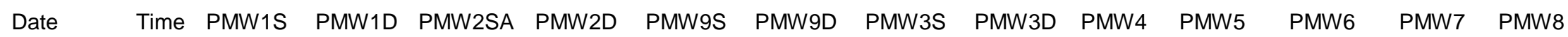

\begin{tabular}{|c|c|c|c|c|c|c|c|c|c|c|c|c|c|c|}
\hline 9/28/2005 & $18: 00$ & 10.842 & 10.743 & 7.895 & 6.793 & 4.585 & 4.229 & 2.495 & 2.474 & 10.829 & 6.099 & 4.877 & 6.634 & 4.195 \\
\hline 9/28/2005 & 19:00 & 10.849 & 10.752 & 7.901 & 6.802 & 4.594 & 4.238 & 2.508 & 2.483 & 10.84 & 6.104 & 4.885 & 6.642 & 4.208 \\
\hline 9/28/2005 & $20: 00$ & 10.856 & 10.759 & 7.907 & 6.807 & 4.602 & 4.245 & 2.513 & 2.487 & 10.846 & 6.104 & 4.885 & 6.644 & 4.204 \\
\hline 9/28/2005 & $21: 00$ & 10.864 & 10.765 & 7.909 & 6.811 & 4.6 & 4.252 & 2.513 & 2.487 & 10.848 & 6.099 & 4.881 & 6.646 & 4.192 \\
\hline 9/28/2005 & $22: 00$ & 10.869 & 10.769 & 7.911 & 6.811 & 4.598 & 4.258 & 2.513 & 2.489 & 10.848 & 6.095 & 4.875 & 6.646 & 4.175 \\
\hline 9/28/2005 & $23: 00$ & 10.876 & 10.776 & 7.913 & 6.816 & 4.594 & 4.262 & 2.506 & 2.489 & 10.85 & 6.09 & 4.87 & 6.648 & 4.159 \\
\hline 9/29/2005 & $0: 00$ & 10.878 & 10.778 & 7.913 & 6.816 & 4.589 & 4.267 & 2.508 & 2.487 & 10.85 & 6.088 & 4.864 & 6.646 & 4.141 \\
\hline 9/29/2005 & $1: 00$ & 10.883 & 10.779 & 7.915 & 6.816 & 4.579 & 4.269 & 2.511 & 2.487 & 10.848 & 6.081 & 4.858 & 6.646 & 4.127 \\
\hline 9/29/2005 & $2: 00$ & 10.883 & 10.78 & 7.913 & 6.811 & 4.571 & 4.271 & 2.508 & 2.483 & 10.846 & 6.079 & 4.85 & 6.646 & 4.109 \\
\hline 9/29/2005 & $3: 00$ & 10.883 & 10.774 & 7.911 & 6.807 & 4.56 & 4.269 & 2.501 & 2.474 & 10.84 & 6.073 & 4.838 & 6.64 & 4.093 \\
\hline 9/29/2005 & $4: 00$ & 10.881 & 10.772 & 7.907 & 6.8 & 4.55 & 4.269 & 2.488 & 2.47 & 10.839 & 6.07 & 4.832 & 6.638 & 4.076 \\
\hline 9/29/2005 & $5: 00$ & 10.881 & 10.772 & 7.908 & 6.802 & 4.546 & 4.267 & 2.496 & 2.47 & 10.835 & 6.068 & 4.826 & 6.638 & 4.07 \\
\hline 9/29/2005 & $6: 00$ & 10.883 & 10.774 & 7.909 & 6.802 & 4.54 & 4.267 & 2.493 & 2.469 & 10.833 & 6.068 & 4.823 & 6.636 & 4.058 \\
\hline 9/29/2005 & $7: 00$ & 10.886 & 10.778 & 7.909 & 6.804 & 4.539 & 4.267 & 2.491 & 2.471 & 10.831 & 6.066 & 4.819 & 6.636 & 4.052 \\
\hline 9/29/2005 & 8:00 & 10.888 & 10.778 & 7.911 & 6.804 & 4.535 & 4.267 & 2.496 & 2.471 & 10.827 & 6.064 & 4.815 & 6.636 & 4.042 \\
\hline 9/29/2005 & $9: 00$ & 10.886 & 10.776 & 7.911 & 6.802 & 4.529 & 4.265 & 2.496 & 2.469 & 10.825 & 6.062 & 4.809 & 6.634 & 4.032 \\
\hline 9/29/2005 & $10: 00$ & 10.883 & 10.772 & 7.909 & 6.798 & 4.525 & 4.263 & 2.486 & 2.466 & 10.823 & 6.06 & 4.809 & 6.632 & 4.024 \\
\hline 9/29/2005 & $11: 00$ & 10.874 & 10.758 & 7.902 & 6.789 & 4.517 & 4.258 & 2.478 & 2.46 & 10.82 & 6.062 & 4.815 & 6.632 & 4.026 \\
\hline 9/29/2005 & $12: 00$ & 10.861 & 10.745 & 7.894 & 6.78 & 4.515 & 4.249 & 2.476 & 2.454 & 10.816 & 6.064 & 4.823 & 6.631 & 4.044 \\
\hline 9/29/2005 & $13: 00$ & 10.849 & 10.732 & 7.888 & 6.771 & 4.515 & 4.245 & 2.476 & 2.45 & 10.816 & 6.066 & 4.831 & 6.629 & 4.062 \\
\hline 9/29/2005 & $14: 00$ & 10.832 & 10.71 & 7.878 & 6.754 & 4.512 & 4.236 & 2.466 & 2.435 & 10.814 & 6.073 & 4.841 & 6.625 & 4.085 \\
\hline 9/29/2005 & $15: 00$ & 10.82 & 10.692 & 7.867 & 6.742 & 4.51 & 4.227 & 2.448 & 2.427 & 10.814 & 6.076 & 4.85 & 6.621 & 4.109 \\
\hline 9/29/2005 & $16: 00$ & 10.802 & 10.676 & 7.861 & 6.73 & 4.515 & 4.221 & 2.443 & 2.42 & 10.812 & 6.08 & 4.86 & 6.621 & 4.139 \\
\hline 9/29/2005 & $17: 00$ & 10.79 & 10.659 & 7.855 & 6.72 & 4.519 & 4.214 & 2.435 & 2.414 & 10.814 & 6.082 & 4.868 & 6.617 & 4.164 \\
\hline 9/29/2005 & $18: 00$ & 10.777 & 10.652 & 7.851 & 6.716 & 4.527 & 4.209 & 2.433 & 2.41 & 10.816 & 6.088 & 4.874 & 6.617 & 4.186 \\
\hline 9/29/2005 & 19:00 & 10.768 & 10.647 & 7.848 & 6.711 & 4.532 & 4.207 & 2.435 & 2.403 & 10.82 & 6.091 & 4.868 & 6.612 & 4.193 \\
\hline 9/29/2005 & 20:00 & 10.763 & 10.641 & 7.843 & 6.712 & 4.532 & 4.205 & 2.428 & 2.399 & 10.82 & 6.084 & 4.854 & 6.606 & 4.181 \\
\hline 9/29/2005 & 21:00 & 10.758 & 10.636 & 7.84 & 6.705 & 4.53 & 4.201 & 2.415 & 2.393 & 10.818 & 6.078 & 4.839 & 6.598 & 4.162 \\
\hline 9/29/2005 & $22: 00$ & 10.756 & 10.632 & 7.836 & 6.703 & 4.525 & 4.198 & 2.413 & 2.389 & 10.816 & 6.073 & 4.827 & 6.596 & 4.146 \\
\hline 9/29/2005 & 23:00 & 10.748 & 10.628 & 7.832 & 6.7 & 4.519 & 4.196 & 2.41 & 2.385 & 10.814 & 6.066 & 4.817 & 6.591 & 4.13 \\
\hline 9/30/2005 & 0:00 & 10.746 & 10.625 & 7.83 & 6.696 & 4.511 & 4.194 & 2.405 & 2.379 & 10.81 & 6.064 & 4.807 & 6.587 & 4.115 \\
\hline 9/30/2005 & $1: 00$ & 10.738 & 10.619 & 7.824 & 6.689 & 4.504 & 4.19 & 2.401 & 2.372 & 10.806 & 6.058 & 4.793 & 6.583 & 4.1 \\
\hline 9/30/2005 & $2: 00$ & 10.736 & 10.616 & 7.82 & 6.685 & 4.494 & 4.185 & 2.388 & 2.366 & 10.804 & 6.055 & 4.786 & 6.579 & 4.085 \\
\hline 9/30/2005 & 3:00 & 10.731 & 10.612 & 7.82 & 6.681 & 4.488 & 4.181 & 2.393 & 2.364 & 10.801 & 6.049 & 4.774 & 6.574 & 4.071 \\
\hline
\end{tabular}


TABLE S1.2 (Cont.)

Water Level (ft below top of casing) at Indicated Well

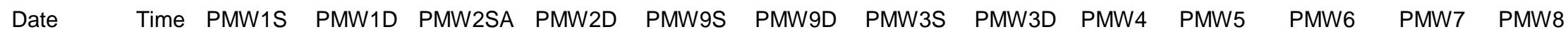

\begin{tabular}{|c|c|c|c|c|c|c|c|c|c|c|c|c|c|c|}
\hline 9/30/2005 & $4: 00$ & 10.726 & 10.605 & 7.813 & 6.676 & 4.48 & 4.177 & 2.378 & 2.358 & 10.797 & 6.045 & 4.762 & 6.57 & 4.059 \\
\hline 9/30/2005 & $5: 00$ & 10.721 & 10.601 & 7.811 & 6.674 & 4.475 & 4.174 & 2.38 & 2.353 & 10.793 & 6.042 & 4.754 & 6.566 & 4.045 \\
\hline 9/30/2005 & $6: 00$ & 10.717 & 10.599 & 7.809 & 6.67 & 4.471 & 4.17 & 2.378 & 2.349 & 10.79 & 6.04 & 4.748 & 6.564 & 4.032 \\
\hline 9/30/2005 & $7: 00$ & 10.717 & 10.595 & 7.805 & 6.67 & 4.465 & 4.165 & 2.375 & 2.347 & 10.786 & 6.036 & 4.741 & 6.56 & 4.022 \\
\hline 9/30/2005 & 8:00 & 10.714 & 10.594 & 7.805 & 6.667 & 4.463 & 4.163 & 2.368 & 2.347 & 10.784 & 6.034 & 4.735 & 6.558 & 4.02 \\
\hline 9/30/2005 & $9: 00$ & 10.712 & 10.594 & 7.805 & 6.67 & 4.465 & 4.159 & 2.371 & 2.349 & 10.782 & 6.032 & 4.741 & 6.56 & 4.022 \\
\hline 9/30/2005 & $10: 00$ & 10.709 & 10.592 & 7.807 & 6.672 & 4.469 & 4.157 & 2.378 & 2.356 & 10.782 & 6.039 & 4.756 & 6.566 & 4.047 \\
\hline 9/30/2005 & $11: 00$ & 10.709 & 10.592 & 7.807 & 6.672 & 4.475 & 4.152 & 2.391 & 2.36 & 10.788 & 6.047 & 4.776 & 6.568 & 4.081 \\
\hline 9/30/2005 & $12: 00$ & 10.707 & 10.588 & 7.807 & 6.67 & 4.486 & 4.148 & 2.388 & 2.362 & 10.782 & 6.061 & 4.799 & 6.574 & 4.119 \\
\hline 9/30/2005 & $13: 00$ & 10.707 & 10.586 & 7.807 & 6.67 & 4.498 & 4.146 & 2.395 & 2.366 & 10.786 & 6.072 & 4.823 & 6.579 & 4.164 \\
\hline 9/30/2005 & $14: 00$ & 10.697 & 10.577 & 7.807 & 6.665 & 4.513 & 4.148 & 2.393 & 2.37 & 10.79 & 6.087 & 4.845 & 6.579 & 4.209 \\
\hline 9/30/2005 & $15: 00$ & 10.695 & 10.572 & 7.807 & 6.665 & 4.53 & 4.141 & 2.395 & 2.37 & 10.797 & 6.098 & 4.868 & 6.587 & 4.244 \\
\hline 9/30/2005 & $16: 00$ & 10.695 & 10.568 & 7.809 & 6.667 & 4.552 & 4.146 & 2.4 & 2.377 & 10.801 & 6.109 & 4.886 & 6.587 & 4.274 \\
\hline 9/30/2005 & $17: 00$ & 10.69 & 10.564 & 7.809 & 6.667 & 4.567 & 4.146 & 2.41 & 2.379 & 10.807 & 6.114 & 4.898 & 6.589 & 4.3 \\
\hline 9/30/2005 & $18: 00$ & 10.693 & 10.57 & 7.813 & 6.674 & 4.586 & 4.15 & 2.418 & 2.385 & 10.816 & 6.12 & 4.906 & 6.589 & 4.323 \\
\hline 9/30/2005 & $19: 00$ & 10.693 & 10.575 & 7.815 & 6.676 & 4.594 & 4.157 & 2.41 & 2.385 & 10.822 & 6.116 & 4.894 & 6.587 & 4.323 \\
\hline 9/30/2005 & $20: 00$ & 10.698 & 10.583 & 7.817 & 6.683 & 4.598 & 4.163 & 2.418 & 2.387 & 10.824 & 6.107 & 4.878 & 6.583 & 4.307 \\
\hline 9/30/2005 & $21: 00$ & 10.7 & 10.585 & 7.817 & 6.683 & 4.594 & 4.168 & 2.408 & 2.383 & 10.829 & 6.098 & 4.86 & 6.579 & 4.283 \\
\hline 9/30/2005 & $22: 00$ & 10.705 & 10.59 & 7.815 & 6.685 & 4.584 & 4.172 & 2.41 & 2.381 & 10.826 & 6.096 & 4.847 & 6.576 & 4.26 \\
\hline 9/30/2005 & $23: 00$ & 10.707 & 10.594 & 7.817 & 6.685 & 4.578 & 4.174 & 2.408 & 2.379 & 10.824 & 6.089 & 4.833 & 6.574 & 4.24 \\
\hline $10 / 1 / 2005$ & $0: 00$ & 10.707 & 10.595 & 7.815 & 6.685 & 4.569 & 4.177 & 2.398 & 2.375 & 10.822 & 6.08 & 4.819 & 6.57 & 4.22 \\
\hline $10 / 1 / 2005$ & $1: 00$ & 10.71 & 10.595 & 7.814 & 6.685 & 4.561 & 4.177 & 2.401 & 2.375 & 10.82 & 6.076 & 4.808 & 6.566 & 4.202 \\
\hline $10 / 1 / 2005$ & $2: 00$ & 10.712 & 10.597 & 7.814 & 6.683 & 4.553 & 4.177 & 2.395 & 2.371 & 10.816 & 6.072 & 4.794 & 6.562 & 4.183 \\
\hline $10 / 1 / 2005$ & $3: 00$ & 10.717 & 10.606 & 7.818 & 6.692 & 4.553 & 4.179 & 2.406 & 2.377 & 10.816 & 6.068 & 4.786 & 6.566 & 4.171 \\
\hline $10 / 1 / 2005$ & $4: 00$ & 10.72 & 10.612 & 7.818 & 6.692 & 4.544 & 4.179 & 2.396 & 2.375 & 10.812 & 6.065 & 4.778 & 6.564 & 4.153 \\
\hline $10 / 1 / 2005$ & $5: 00$ & 10.728 & 10.619 & 7.82 & 6.699 & 4.544 & 4.181 & 2.406 & 2.379 & 10.81 & 6.061 & 4.772 & 6.564 & 4.143 \\
\hline $10 / 1 / 2005$ & $6: 00$ & 10.73 & 10.623 & 7.824 & 6.697 & 4.536 & 4.181 & 2.398 & 2.379 & 10.809 & 6.057 & 4.763 & 6.563 & 4.129 \\
\hline $10 / 1 / 2005$ & $7: 00$ & 10.735 & 10.628 & 7.824 & 6.701 & 4.532 & 4.181 & 2.409 & 2.379 & 10.805 & 6.052 & 4.755 & 6.564 & 4.117 \\
\hline $10 / 1 / 2005$ & $8: 00$ & 10.737 & 10.63 & 7.826 & 6.701 & 4.522 & 4.181 & 2.399 & 2.379 & 10.803 & 6.05 & 4.747 & 6.563 & 4.104 \\
\hline $10 / 1 / 2005$ & 9:00 & 10.737 & 10.63 & 7.824 & 6.701 & 4.518 & 4.181 & 2.406 & 2.379 & 10.799 & 6.046 & 4.739 & 6.563 & 4.092 \\
\hline $10 / 1 / 2005$ & $10: 00$ & 10.745 & 10.639 & 7.83 & 6.708 & 4.522 & 4.181 & 2.417 & 2.385 & 10.799 & 6.054 & 4.751 & 6.568 & 4.096 \\
\hline $10 / 1 / 2005$ & $11: 00$ & 10.747 & 10.639 & 7.832 & 6.71 & 4.522 & 4.181 & 2.419 & 2.392 & 10.797 & 6.054 & 4.757 & 6.564 & 4.106 \\
\hline $10 / 1 / 2005$ & $12: 00$ & 10.745 & 10.634 & 7.834 & 6.703 & 4.522 & 4.179 & 2.422 & 2.392 & 10.797 & 6.061 & 4.772 & 6.572 & 4.131 \\
\hline $10 / 1 / 2005$ & $13: 00$ & 10.75 & 10.637 & 7.836 & 6.712 & 4.536 & 4.179 & 2.427 & 2.402 & 10.799 & 6.072 & 4.792 & 6.574 & 4.167 \\
\hline
\end{tabular}


TABLE S1.2 (Cont.)

Water Level (ft below top of casing) at Indicated Well

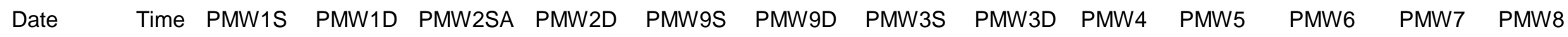

\begin{tabular}{|c|c|c|c|c|c|c|c|c|c|c|c|c|c|c|}
\hline $10 / 1 / 2005$ & $14: 00$ & 10.745 & 10.632 & 7.835 & 6.706 & 4.542 & 4.179 & 2.429 & 2.4 & 10.803 & 6.083 & 4.808 & 6.574 & 4.193 \\
\hline $10 / 1 / 2005$ & $15: 00$ & 10.747 & 10.632 & 7.835 & 6.71 & 4.555 & 4.179 & 2.429 & 2.403 & 10.805 & 6.09 & 4.817 & 6.585 & 4.214 \\
\hline $10 / 1 / 2005$ & $16: 00$ & 10.742 & 10.626 & 7.835 & 6.706 & 4.559 & 4.181 & 2.431 & 2.403 & 10.809 & 6.094 & 4.822 & 6.576 & 4.233 \\
\hline $10 / 1 / 2005$ & $17: 00$ & 10.74 & 10.626 & 7.837 & 6.708 & 4.564 & 4.181 & 2.426 & 2.404 & 10.815 & 6.101 & 4.831 & 6.584 & 4.255 \\
\hline $10 / 1 / 2005$ & $18: 00$ & 10.74 & 10.625 & 7.837 & 6.703 & 4.568 & 4.183 & 2.421 & 2.4 & 10.817 & 6.098 & 4.825 & 6.582 & 4.257 \\
\hline $10 / 1 / 2005$ & $19: 00$ & 10.742 & 10.626 & 7.835 & 6.708 & 4.57 & 4.186 & 2.426 & 2.398 & 10.818 & 6.09 & 4.815 & 6.578 & 4.245 \\
\hline $10 / 1 / 2005$ & $20: 00$ & 10.747 & 10.636 & 7.836 & 6.71 & 4.57 & 4.19 & 2.422 & 2.4 & 10.818 & 6.085 & 4.806 & 6.576 & 4.229 \\
\hline $10 / 1 / 2005$ & $21: 00$ & 10.75 & 10.632 & 7.84 & 6.706 & 4.566 & 4.192 & 2.422 & 2.398 & 10.818 & 6.079 & 4.792 & 6.557 & 4.211 \\
\hline $10 / 1 / 2005$ & $22: 00$ & 10.75 & 10.621 & 7.836 & 6.699 & 4.555 & 4.192 & 2.419 & 2.382 & 10.815 & 6.072 & 4.777 & 6.546 & 4.184 \\
\hline $10 / 1 / 2005$ & $23: 00$ & 10.748 & 10.625 & 7.832 & 6.708 & 4.543 & 4.192 & 2.409 & 2.382 & 10.818 & 6.068 & 4.763 & 6.569 & 4.16 \\
\hline $10 / 2 / 2005$ & $0: 00$ & 10.748 & 10.632 & 7.83 & 6.703 & 4.533 & 4.19 & 2.409 & 2.388 & 10.809 & 6.059 & 4.749 & 6.564 & 4.139 \\
\hline $10 / 2 / 2005$ & $1: 00$ & 10.743 & 10.628 & 7.826 & 6.697 & 4.52 & 4.188 & 2.404 & 2.375 & 10.805 & 6.054 & 4.737 & 6.561 & 4.119 \\
\hline $10 / 2 / 2005$ & $2: 00$ & 10.743 & 10.628 & 7.824 & 6.697 & 4.514 & 4.186 & 2.394 & 2.371 & 10.799 & 6.055 & 4.728 & 6.557 & 4.103 \\
\hline $10 / 2 / 2005$ & $3: 00$ & 10.743 & 10.625 & 7.822 & 6.692 & 4.501 & 4.181 & 2.387 & 2.367 & 10.796 & 6.048 & 4.716 & 6.553 & 4.085 \\
\hline $10 / 2 / 2005$ & $4: 00$ & 10.738 & 10.619 & 7.816 & 6.686 & 4.491 & 4.177 & 2.389 & 2.363 & 10.792 & 6.044 & 4.708 & 6.553 & 4.071 \\
\hline $10 / 2 / 2005$ & $5: 00$ & 10.735 & 10.616 & 7.812 & 6.681 & 4.483 & 4.172 & 2.387 & 2.358 & 10.788 & 6.039 & 4.7 & 6.548 & 4.055 \\
\hline $10 / 2 / 2005$ & $6: 00$ & 10.73 & 10.614 & 7.812 & 6.681 & 4.476 & 4.17 & 2.379 & 2.352 & 10.782 & 6.037 & 4.694 & 6.548 & 4.042 \\
\hline $10 / 2 / 2005$ & $7: 00$ & 10.73 & 10.616 & 7.812 & 6.679 & 4.474 & 4.168 & 2.379 & 2.354 & 10.78 & 6.035 & 4.69 & 6.544 & 4.032 \\
\hline $10 / 2 / 2005$ & $8: 00$ & 10.733 & 10.617 & 7.812 & 6.681 & 4.468 & 4.166 & 2.375 & 2.354 & 10.779 & 6.035 & 4.685 & 6.544 & 4.02 \\
\hline $10 / 2 / 2005$ & $9: 00$ & 10.73 & 10.616 & 7.812 & 6.681 & 4.464 & 4.162 & 2.372 & 2.352 & 10.777 & 6.03 & 4.681 & 6.542 & 4.012 \\
\hline $10 / 2 / 2005$ & $10: 00$ & 10.73 & 10.619 & 7.812 & 6.683 & 4.462 & 4.162 & 2.375 & 2.355 & 10.773 & 6.03 & 4.683 & 6.544 & 4.004 \\
\hline $10 / 2 / 2005$ & $11: 00$ & 10.728 & 10.612 & 7.812 & 6.677 & 4.454 & 4.157 & 2.38 & 2.351 & 10.769 & 6.026 & 4.685 & 6.542 & 4.002 \\
\hline $10 / 2 / 2005$ & $12: 00$ & 10.726 & 10.606 & 7.81 & 6.677 & 4.454 & 4.155 & 2.382 & 2.352 & 10.769 & 6.033 & 4.698 & 6.544 & 4.02 \\
\hline $10 / 2 / 2005$ & $13: 00$ & 10.718 & 10.599 & 7.81 & 6.672 & 4.462 & 4.148 & 2.38 & 2.359 & 10.771 & 6.051 & 4.724 & 6.553 & 4.06 \\
\hline $10 / 2 / 2005$ & $14: 00$ & 10.713 & 10.594 & 7.808 & 6.67 & 4.477 & 4.144 & 2.387 & 2.363 & 10.771 & 6.066 & 4.753 & 6.559 & 4.115 \\
\hline $10 / 2 / 2005$ & $15: 00$ & 10.708 & 10.588 & 7.812 & 6.674 & 4.494 & 4.142 & 2.397 & 2.367 & 10.779 & 6.075 & 4.777 & 6.561 & 4.162 \\
\hline $10 / 2 / 2005$ & $16: 00$ & 10.708 & 10.59 & 7.814 & 6.679 & 4.518 & 4.144 & 2.4 & 2.376 & 10.786 & 6.086 & 4.798 & 6.571 & 4.206 \\
\hline $10 / 2 / 2005$ & $17: 00$ & 10.711 & 10.588 & 7.82 & 6.677 & 4.533 & 4.144 & 2.412 & 2.382 & 10.788 & 6.093 & 4.816 & 6.576 & 4.237 \\
\hline $10 / 2 / 2005$ & $18: 00$ & 10.713 & 10.592 & 7.82 & 6.688 & 4.554 & 4.148 & 2.422 & 2.386 & 10.794 & 6.106 & 4.831 & 6.582 & 4.272 \\
\hline $10 / 2 / 2005$ & $19: 00$ & 10.718 & 10.595 & 7.824 & 6.688 & 4.571 & 4.157 & 2.419 & 2.39 & 10.802 & 6.106 & 4.828 & 6.58 & 4.28 \\
\hline $10 / 2 / 2005$ & $20: 00$ & 10.721 & 10.605 & 7.826 & 6.697 & 4.577 & 4.164 & 2.417 & 2.393 & 10.805 & 6.104 & 4.824 & 6.586 & 4.27 \\
\hline $10 / 2 / 2005$ & $21: 00$ & 10.729 & 10.616 & 7.828 & 6.704 & 4.583 & 4.17 & 2.422 & 2.399 & 10.811 & 6.097 & 4.816 & 6.578 & 4.258 \\
\hline $10 / 2 / 2005$ & $22: 00$ & 10.736 & 10.625 & 7.834 & 6.708 & 4.579 & 4.175 & 2.43 & 2.401 & 10.813 & 6.095 & 4.808 & 6.58 & 4.241 \\
\hline $10 / 2 / 2005$ & $23: 00$ & 10.741 & 10.634 & 7.836 & 6.715 & 4.575 & 4.181 & 2.425 & 2.403 & 10.815 & 6.09 & 4.798 & 6.576 & 4.226 \\
\hline
\end{tabular}


TABLE S1.2 (Cont.)

Water Level (ft below top of casing) at Indicated Well

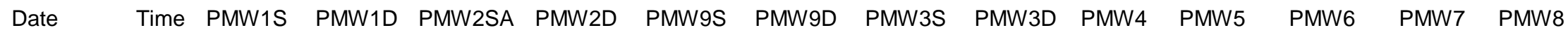

\begin{tabular}{|c|c|c|c|c|c|c|c|c|c|c|c|c|c|c|}
\hline $10 / 3 / 2005$ & $0: 00$ & 10.746 & 10.637 & 7.836 & 6.715 & 4.567 & 4.184 & 2.43 & 2.401 & 10.813 & 6.084 & 4.785 & 6.567 & 4.207 \\
\hline $10 / 3 / 2005$ & $1: 00$ & 10.751 & 10.641 & 7.839 & 6.715 & 4.56 & 4.186 & 2.43 & 2.401 & 10.815 & 6.08 & 4.775 & 6.571 & 4.189 \\
\hline $10 / 3 / 2005$ & $2: 00$ & 10.756 & 10.645 & 7.839 & 6.715 & 4.552 & 4.19 & 2.427 & 2.399 & 10.811 & 6.075 & 4.767 & 6.569 & 4.175 \\
\hline $10 / 3 / 2005$ & $3: 00$ & 10.758 & 10.645 & 7.837 & 6.717 & 4.544 & 4.193 & 2.42 & 2.397 & 10.809 & 6.071 & 4.755 & 6.561 & 4.158 \\
\hline $10 / 3 / 2005$ & $4: 00$ & 10.758 & 10.645 & 7.837 & 6.713 & 4.535 & 4.19 & 2.425 & 2.395 & 10.807 & 6.071 & 4.748 & 6.565 & 4.145 \\
\hline $10 / 3 / 2005$ & $5: 00$ & 10.76 & 10.647 & 7.837 & 6.713 & 4.529 & 4.19 & 2.42 & 2.393 & 10.805 & 6.066 & 4.738 & 6.563 & 4.13 \\
\hline $10 / 3 / 2005$ & $6: 00$ & 10.758 & 10.643 & 7.833 & 6.71 & 4.519 & 4.19 & 2.41 & 2.389 & 10.804 & 6.062 & 4.726 & 6.559 & 4.116 \\
\hline $10 / 3 / 2005$ & $7: 00$ & 10.761 & 10.65 & 7.837 & 6.71 & 4.513 & 4.188 & 2.415 & 2.389 & 10.8 & 6.062 & 4.726 & 6.558 & 4.108 \\
\hline $10 / 3 / 2005$ & $8: 00$ & 10.763 & 10.652 & 7.837 & 6.715 & 4.513 & 4.19 & 2.413 & 2.393 & 10.798 & 6.062 & 4.722 & 6.558 & 4.1 \\
\hline $10 / 3 / 2005$ & $9: 00$ & 10.766 & 10.656 & 7.841 & 6.717 & 4.513 & 4.193 & 2.426 & 2.397 & 10.798 & 6.062 & 4.728 & 6.561 & 4.104 \\
\hline $10 / 3 / 2005$ & $10: 00$ & 10.771 & 10.661 & 7.851 & 6.726 & 4.519 & 4.19 & 2.436 & 2.408 & 10.8 & 6.068 & 4.742 & 6.567 & 4.122 \\
\hline $10 / 3 / 2005$ & $11: 00$ & 10.773 & 10.665 & 7.849 & 6.728 & 4.525 & 4.19 & 2.443 & 2.414 & 10.802 & 6.078 & 4.755 & 6.575 & 4.151 \\
\hline $10 / 3 / 2005$ & $12: 00$ & 10.776 & 10.667 & 7.853 & 6.73 & 4.532 & 4.193 & 2.443 & 2.416 & 10.805 & 6.08 & 4.765 & 6.578 & 4.169 \\
\hline $10 / 3 / 2005$ & $13: 00$ & 10.778 & 10.665 & 7.853 & 6.732 & 4.54 & 4.195 & 2.449 & 2.42 & 10.807 & 6.084 & 4.775 & 6.58 & 4.185 \\
\hline $10 / 3 / 2005$ & $14: 00$ & 10.773 & 10.654 & 7.849 & 6.721 & 4.536 & 4.193 & 2.441 & 2.412 & 10.811 & 6.091 & 4.789 & 6.582 & 4.212 \\
\hline $10 / 3 / 2005$ & $15: 00$ & 10.766 & 10.649 & 7.846 & 6.721 & 4.544 & 4.188 & 2.449 & 2.416 & 10.811 & 6.1 & 4.808 & 6.578 & 4.252 \\
\hline $10 / 3 / 2005$ & $16: 00$ & 10.763 & 10.645 & 7.848 & 6.717 & 4.559 & 4.188 & 2.451 & 2.418 & 10.817 & 6.111 & 4.824 & 6.588 & 4.287 \\
\hline $10 / 3 / 2005$ & $17: 00$ & 10.763 & 10.645 & 7.85 & 6.721 & 4.576 & 4.193 & 2.458 & 2.424 & 10.821 & 6.122 & 4.846 & 6.592 & 4.317 \\
\hline $10 / 3 / 2005$ & $18: 00$ & 10.761 & 10.641 & 7.852 & 6.724 & 4.59 & 4.193 & 2.463 & 2.429 & 10.828 & 6.126 & 4.853 & 6.592 & 4.338 \\
\hline $10 / 3 / 2005$ & 19:00 & 10.766 & 10.649 & 7.856 & 6.733 & 4.605 & 4.199 & 2.456 & 2.433 & 10.834 & 6.128 & 4.855 & 6.592 & 4.344 \\
\hline $10 / 3 / 2005$ & $20: 00$ & 10.776 & 10.658 & 7.858 & 6.739 & 4.611 & 4.204 & 2.458 & 2.433 & 10.836 & 6.122 & 4.848 & 6.59 & 4.332 \\
\hline $10 / 3 / 2005$ & $21: 00$ & 10.778 & 10.667 & 7.861 & 6.742 & 4.615 & 4.21 & 2.463 & 2.433 & 10.842 & 6.117 & 4.838 & 6.592 & 4.312 \\
\hline $10 / 3 / 2005$ & $22: 00$ & 10.781 & 10.672 & 7.861 & 6.744 & 4.609 & 4.215 & 2.456 & 2.433 & 10.842 & 6.111 & 4.826 & 6.588 & 4.287 \\
\hline $10 / 3 / 2005$ & $23: 00$ & 10.785 & 10.676 & 7.863 & 6.748 & 4.605 & 4.219 & 2.463 & 2.433 & 10.842 & 6.104 & 4.817 & 6.588 & 4.269 \\
\hline $10 / 4 / 2005$ & $0: 00$ & 10.791 & 10.68 & 7.863 & 6.748 & 4.595 & 4.221 & 2.454 & 2.431 & 10.842 & 6.098 & 4.802 & 6.59 & 4.249 \\
\hline $10 / 4 / 2005$ & $1: 00$ & 10.791 & 10.681 & 7.863 & 6.748 & 4.585 & 4.221 & 2.454 & 2.429 & 10.838 & 6.096 & 4.795 & 6.586 & 4.228 \\
\hline $10 / 4 / 2005$ & $2: 00$ & 10.793 & 10.683 & 7.863 & 6.748 & 4.578 & 4.224 & 2.451 & 2.429 & 10.836 & 6.089 & 4.785 & 6.586 & 4.21 \\
\hline $10 / 4 / 2005$ & $3: 00$ & 10.793 & 10.683 & 7.861 & 6.744 & 4.568 & 4.224 & 2.451 & 2.424 & 10.832 & 6.085 & 4.773 & 6.583 & 4.192 \\
\hline $10 / 4 / 2005$ & $4: 00$ & 10.793 & 10.68 & 7.859 & 6.739 & 4.557 & 4.221 & 2.441 & 2.419 & 10.829 & 6.078 & 4.764 & 6.579 & 4.174 \\
\hline $10 / 4 / 2005$ & $5: 00$ & 10.793 & 10.68 & 7.857 & 6.737 & 4.547 & 4.221 & 2.444 & 2.416 & 10.826 & 6.074 & 4.756 & 6.577 & 4.157 \\
\hline $10 / 4 / 2005$ & $6: 00$ & 10.788 & 10.674 & 7.853 & 6.733 & 4.539 & 4.217 & 2.431 & 2.41 & 10.821 & 6.067 & 4.744 & 6.575 & 4.139 \\
\hline $10 / 4 / 2005$ & $7: 00$ & 10.788 & 10.674 & 7.853 & 6.733 & 4.531 & 4.217 & 2.436 & 2.408 & 10.817 & 6.062 & 4.736 & 6.569 & 4.127 \\
\hline $10 / 4 / 2005$ & $8: 00$ & 10.786 & 10.671 & 7.849 & 6.731 & 4.522 & 4.213 & 2.426 & 2.402 & 10.813 & 6.058 & 4.73 & 6.567 & 4.113 \\
\hline $10 / 4 / 2005$ & $9: 00$ & 10.783 & 10.671 & 7.849 & 6.728 & 4.52 & 4.211 & 2.436 & 2.408 & 10.812 & 6.065 & 4.73 & 6.571 & 4.109 \\
\hline
\end{tabular}


TABLE S1.2 (Cont.)

Water Level (ft below top of casing) at Indicated Well

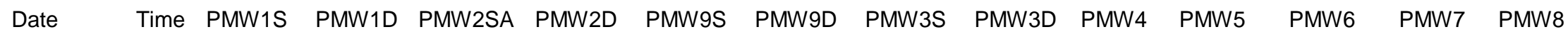

\begin{tabular}{|c|c|c|c|c|c|c|c|c|c|c|c|c|c|c|}
\hline $10 / 4 / 2005$ & $10: 00$ & 10.783 & 10.667 & 7.851 & 6.728 & 4.52 & 4.208 & 2.431 & 2.41 & 10.81 & 6.067 & 4.738 & 6.565 & 4.119 \\
\hline $10 / 4 / 2005$ & $11: 00$ & 10.779 & 10.658 & 7.847 & 6.724 & 4.518 & 4.202 & 2.431 & 2.408 & 10.81 & 6.072 & 4.75 & 6.577 & 4.141 \\
\hline $10 / 4 / 2005$ & $12: 00$ & 10.776 & 10.658 & 7.851 & 6.726 & 4.529 & 4.202 & 2.446 & 2.416 & 10.812 & 6.087 & 4.781 & 6.583 & 4.182 \\
\hline $10 / 4 / 2005$ & $13: 00$ & 10.774 & 10.649 & 7.851 & 6.722 & 4.541 & 4.2 & 2.449 & 2.416 & 10.813 & 6.098 & 4.805 & 6.583 & 4.228 \\
\hline $10 / 4 / 2005$ & $14: 00$ & 10.766 & 10.64 & 7.85 & 6.717 & 4.556 & 4.195 & 2.451 & 2.421 & 10.819 & 6.113 & 4.828 & 6.585 & 4.271 \\
\hline $10 / 4 / 2005$ & $15: 00$ & 10.766 & 10.636 & 7.85 & 6.72 & 4.577 & 4.193 & 2.459 & 2.425 & 10.825 & 6.127 & 4.854 & 6.59 & 4.314 \\
\hline $10 / 4 / 2005$ & $16: 00$ & 10.756 & 10.627 & 7.848 & 6.717 & 4.594 & 4.193 & 2.456 & 2.425 & 10.831 & 6.133 & 4.871 & 6.598 & 4.344 \\
\hline $10 / 4 / 2005$ & $17: 00$ & 10.752 & 10.619 & 7.846 & 6.713 & 4.604 & 4.191 & 2.454 & 2.421 & 10.84 & 6.138 & 4.883 & 6.585 & 4.365 \\
\hline $10 / 4 / 2005$ & $18: 00$ & 10.746 & 10.616 & 7.846 & 6.711 & 4.612 & 4.193 & 2.449 & 2.421 & 10.84 & 6.138 & 4.879 & 6.594 & 4.375 \\
\hline $10 / 4 / 2005$ & $19: 00$ & 10.749 & 10.621 & 7.846 & 6.715 & 4.623 & 4.2 & 2.454 & 2.421 & 10.846 & 6.136 & 4.879 & 6.594 & 4.377 \\
\hline $10 / 4 / 2005$ & $20: 00$ & 10.749 & 10.623 & 7.847 & 6.717 & 4.621 & 4.204 & 2.449 & 2.419 & 10.85 & 6.125 & 4.864 & 6.591 & 4.355 \\
\hline $10 / 4 / 2005$ & $21: 00$ & 10.752 & 10.625 & 7.845 & 6.717 & 4.617 & 4.204 & 2.438 & 2.414 & 10.846 & 6.118 & 4.85 & 6.585 & 4.332 \\
\hline $10 / 4 / 2005$ & $22: 00$ & 10.754 & 10.629 & 7.843 & 6.717 & 4.608 & 4.206 & 2.436 & 2.41 & 10.848 & 6.107 & 4.838 & 6.579 & 4.308 \\
\hline $10 / 4 / 2005$ & $23: 00$ & 10.754 & 10.63 & 7.843 & 6.715 & 4.6 & 4.208 & 2.436 & 2.409 & 10.844 & 6.1 & 4.824 & 6.575 & 4.284 \\
\hline $10 / 5 / 2005$ & $0: 00$ & 10.754 & 10.63 & 7.841 & 6.715 & 4.592 & 4.208 & 2.434 & 2.404 & 10.84 & 6.094 & 4.809 & 6.575 & 4.266 \\
\hline $10 / 5 / 2005$ & $1: 00$ & 10.754 & 10.629 & 7.837 & 6.711 & 4.58 & 4.206 & 2.421 & 2.398 & 10.838 & 6.087 & 4.797 & 6.573 & 4.243 \\
\hline $10 / 5 / 2005$ & $2: 00$ & 10.754 & 10.625 & 7.833 & 6.707 & 4.567 & 4.206 & 2.424 & 2.394 & 10.832 & 6.08 & 4.781 & 6.57 & 4.223 \\
\hline $10 / 5 / 2005$ & $3: 00$ & 10.75 & 10.623 & 7.832 & 6.702 & 4.559 & 4.202 & 2.417 & 2.388 & 10.831 & 6.076 & 4.773 & 6.566 & 4.207 \\
\hline $10 / 5 / 2005$ & $4: 00$ & 10.752 & 10.616 & 7.832 & 6.691 & 4.553 & 4.2 & 2.409 & 2.385 & 10.825 & 6.074 & 4.766 & 6.53 & 4.192 \\
\hline $10 / 5 / 2005$ & $5: 00$ & 10.757 & 10.636 & 7.832 & 6.711 & 4.555 & 4.2 & 2.414 & 2.39 & 10.821 & 6.079 & 4.762 & 6.566 & 4.182 \\
\hline $10 / 5 / 2005$ & $6: 00$ & 10.774 & 10.656 & 7.846 & 6.733 & 4.565 & 4.204 & 2.432 & 2.409 & 10.82 & 6.076 & 4.764 & 6.573 & 4.174 \\
\hline $10 / 5 / 2005$ & $7: 00$ & 10.784 & 10.621 & 7.851 & 6.698 & 4.561 & 4.206 & 2.437 & 2.402 & 10.789 & 6.065 & 4.752 & 6.539 & 4.154 \\
\hline $10 / 5 / 2005$ & $8: 00$ & 10.794 & 10.612 & 7.853 & 6.682 & 4.551 & 4.208 & 2.44 & 2.419 & 10.77 & 6.061 & 4.738 & 6.522 & 4.116 \\
\hline $10 / 5 / 2005$ & $9: 00$ & 10.814 & 10.702 & 7.867 & 6.762 & 4.559 & 4.213 & 2.455 & 2.434 & 10.818 & 6.07 & 4.75 & 6.581 & 4.116 \\
\hline $10 / 5 / 2005$ & $10: 00$ & 10.819 & 10.711 & 7.873 & 6.766 & 4.553 & 4.215 & 2.453 & 2.432 & 10.817 & 6.063 & 4.744 & 6.583 & 4.1 \\
\hline $10 / 5 / 2005$ & $11: 00$ & 10.836 & 10.729 & 7.881 & 6.782 & 4.561 & 4.224 & 2.476 & 2.451 & 10.821 & 6.072 & 4.757 & 6.594 & 4.1 \\
\hline $10 / 5 / 2005$ & $12: 00$ & 10.841 & 10.734 & 7.888 & 6.786 & 4.557 & 4.228 & 2.478 & 2.455 & 10.82 & 6.068 & 4.76 & 6.596 & 4.094 \\
\hline $10 / 5 / 2005$ & $13: 00$ & 10.851 & 10.738 & 7.89 & 6.791 & 4.555 & 4.231 & 2.486 & 2.459 & 10.82 & 6.072 & 4.77 & 6.602 & 4.093 \\
\hline $10 / 5 / 2005$ & $14: 00$ & 10.856 & 10.745 & 7.895 & 6.791 & 4.555 & 4.235 & 2.481 & 2.461 & 10.821 & 6.074 & 4.778 & 6.606 & 4.097 \\
\hline $10 / 5 / 2005$ & $15: 00$ & 10.86 & 10.751 & 7.897 & 6.795 & 4.557 & 4.237 & 2.486 & 2.465 & 10.82 & 6.076 & 4.79 & 6.608 & 4.103 \\
\hline $10 / 5 / 2005$ & $16: 00$ & 10.868 & 10.758 & 7.905 & 6.802 & 4.566 & 4.244 & 2.499 & 2.474 & 10.827 & 6.086 & 4.804 & 6.615 & 4.118 \\
\hline $10 / 5 / 2005$ & $17: 00$ & 10.868 & 10.767 & 7.908 & 6.811 & 4.576 & 4.251 & 2.511 & 2.485 & 10.825 & 6.094 & 4.817 & 6.619 & 4.134 \\
\hline $10 / 5 / 2005$ & $18: 00$ & 10.876 & 10.778 & 7.914 & 6.817 & 4.583 & 4.255 & 2.511 & 2.49 & 10.829 & 6.096 & 4.825 & 6.625 & 4.144 \\
\hline $10 / 5 / 2005$ & $19: 00$ & 10.881 & 10.78 & 7.916 & 6.822 & 4.585 & 4.259 & 2.521 & 2.493 & 10.833 & 6.099 & 4.831 & 6.629 & 4.144 \\
\hline
\end{tabular}


TABLE S1.2 (Cont.)

Water Level (ft below top of casing) at Indicated Well

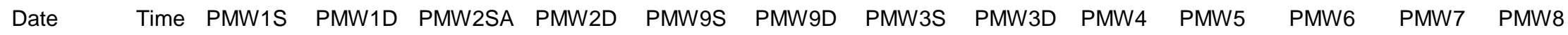

\begin{tabular}{|c|c|c|c|c|c|c|c|c|c|c|c|c|c|c|}
\hline $10 / 5 / 2005$ & $20: 00$ & 10.888 & 10.791 & 7.924 & 6.831 & 4.595 & 4.268 & 2.529 & 2.501 & 10.837 & 6.101 & 4.835 & 6.634 & 4.142 \\
\hline $10 / 5 / 2005$ & $21: 00$ & 10.898 & 10.8 & 7.928 & 6.84 & 4.601 & 4.273 & 2.529 & 2.508 & 10.84 & 6.101 & 4.837 & 6.638 & 4.136 \\
\hline $10 / 5 / 2005$ & $22: 00$ & 10.903 & 10.811 & 7.929 & 6.844 & 4.603 & 4.281 & 2.539 & 2.512 & 10.84 & 6.103 & 4.839 & 6.642 & 4.128 \\
\hline $10 / 5 / 2005$ & $23: 00$ & 10.91 & 10.813 & 7.935 & 6.846 & 4.597 & 4.286 & 2.539 & 2.514 & 10.842 & 6.096 & 4.833 & 6.642 & 4.116 \\
\hline $10 / 6 / 2005$ & $0: 00$ & 10.913 & 10.815 & 7.935 & 6.84 & 4.589 & 4.289 & 2.536 & 2.51 & 10.842 & 6.092 & 4.827 & 6.64 & 4.098 \\
\hline $10 / 6 / 2005$ & $1: 00$ & 10.915 & 10.816 & 7.935 & 6.842 & 4.581 & 4.291 & 2.529 & 2.51 & 10.839 & 6.09 & 4.823 & 6.64 & 4.085 \\
\hline $10 / 6 / 2005$ & $2: 00$ & 10.92 & 10.82 & 7.936 & 6.847 & 4.579 & 4.293 & 2.539 & 2.51 & 10.837 & 6.092 & 4.821 & 6.64 & 4.079 \\
\hline $10 / 6 / 2005$ & $3: 00$ & 10.922 & 10.822 & 7.938 & 6.847 & 4.575 & 4.297 & 2.537 & 2.512 & 10.839 & 6.088 & 4.819 & 6.644 & 4.069 \\
\hline $10 / 6 / 2005$ & $4: 00$ & 10.922 & 10.818 & 7.936 & 6.842 & 4.568 & 4.295 & 2.532 & 2.506 & 10.835 & 6.083 & 4.813 & 6.639 & 4.059 \\
\hline $10 / 6 / 2005$ & $5: 00$ & 10.925 & 10.818 & 7.936 & 6.84 & 4.564 & 4.297 & 2.532 & 2.506 & 10.835 & 6.081 & 4.809 & 6.64 & 4.051 \\
\hline $10 / 6 / 2005$ & $6: 00$ & 10.925 & 10.822 & 7.936 & 6.844 & 4.56 & 4.297 & 2.527 & 2.508 & 10.831 & 6.081 & 4.808 & 6.642 & 4.043 \\
\hline $10 / 6 / 2005$ & $7: 00$ & 10.93 & 10.826 & 7.94 & 6.847 & 4.56 & 4.297 & 2.534 & 2.51 & 10.831 & 6.079 & 4.806 & 6.642 & 4.039 \\
\hline $10 / 6 / 2005$ & $8: 00$ & 10.932 & 10.829 & 7.94 & 6.847 & 4.56 & 4.3 & 2.537 & 2.512 & 10.829 & 6.079 & 4.806 & 6.644 & 4.033 \\
\hline $10 / 6 / 2005$ & $9: 00$ & 10.935 & 10.829 & 7.942 & 6.847 & 4.556 & 4.297 & 2.537 & 2.51 & 10.827 & 6.079 & 4.804 & 6.644 & 4.025 \\
\hline $10 / 6 / 2005$ & $10: 00$ & 10.937 & 10.831 & 7.943 & 6.851 & 4.556 & 4.302 & 2.534 & 2.514 & 10.825 & 6.077 & 4.808 & 6.644 & 4.023 \\
\hline $10 / 6 / 2005$ & $11: 00$ & 10.937 & 10.833 & 7.943 & 6.851 & 4.554 & 4.3 & 2.534 & 2.514 & 10.827 & 6.081 & 4.814 & 6.648 & 4.025 \\
\hline $10 / 6 / 2005$ & $12: 00$ & 10.935 & 10.826 & 7.942 & 6.844 & 4.55 & 4.295 & 2.532 & 2.51 & 10.823 & 6.079 & 4.816 & 6.644 & 4.031 \\
\hline $10 / 6 / 2005$ & $13: 00$ & 10.93 & 10.816 & 7.944 & 6.838 & 4.552 & 4.295 & 2.537 & 2.508 & 10.825 & 6.083 & 4.818 & 6.642 & 4.041 \\
\hline $10 / 6 / 2005$ & $14: 00$ & 10.922 & 10.807 & 7.936 & 6.831 & 4.548 & 4.293 & 2.522 & 2.501 & 10.825 & 6.077 & 4.824 & 6.644 & 4.053 \\
\hline $10 / 6 / 2005$ & $15: 00$ & 10.915 & 10.798 & 7.929 & 6.822 & 4.548 & 4.289 & 2.524 & 2.495 & 10.821 & 6.078 & 4.827 & 6.639 & 4.063 \\
\hline $10 / 6 / 2005$ & $16: 00$ & 10.913 & 10.796 & 7.931 & 6.827 & 4.558 & 4.289 & 2.529 & 2.499 & 10.825 & 6.086 & 4.839 & 6.644 & 4.082 \\
\hline $10 / 6 / 2005$ & $17: 00$ & 10.91 & 10.796 & 7.931 & 6.824 & 4.561 & 4.289 & 2.522 & 2.5 & 10.825 & 6.088 & 4.843 & 6.646 & 4.092 \\
\hline $10 / 6 / 2005$ & $18: 00$ & 10.91 & 10.797 & 7.931 & 6.824 & 4.567 & 4.289 & 2.522 & 2.502 & 10.831 & 6.09 & 4.845 & 6.646 & 4.094 \\
\hline $10 / 6 / 2005$ & 19:00 & 10.908 & 10.793 & 7.929 & 6.824 & 4.565 & 4.289 & 2.524 & 2.498 & 10.829 & 6.088 & 4.841 & 6.643 & 4.08 \\
\hline $10 / 6 / 2005$ & $20: 00$ & 10.908 & 10.789 & 7.929 & 6.822 & 4.561 & 4.289 & 2.517 & 2.495 & 10.829 & 6.082 & 4.835 & 6.64 & 4.066 \\
\hline $10 / 6 / 2005$ & $21: 00$ & 10.91 & 10.796 & 7.929 & 6.829 & 4.563 & 4.289 & 2.524 & 2.498 & 10.829 & 6.082 & 4.831 & 6.643 & 4.054 \\
\hline $10 / 6 / 2005$ & $22: 00$ & 10.913 & 10.8 & 7.93 & 6.831 & 4.561 & 4.291 & 2.527 & 2.498 & 10.827 & 6.079 & 4.826 & 6.643 & 4.039 \\
\hline $10 / 6 / 2005$ & $23: 00$ & 10.915 & 10.802 & 7.932 & 6.831 & 4.557 & 4.291 & 2.527 & 2.498 & 10.827 & 6.075 & 4.818 & 6.64 & 4.025 \\
\hline $10 / 7 / 2005$ & $0: 00$ & 10.913 & 10.802 & 7.93 & 6.829 & 4.551 & 4.291 & 2.524 & 2.495 & 10.826 & 6.071 & 4.81 & 6.64 & 4.011 \\
\hline $10 / 7 / 2005$ & $1: 00$ & 10.913 & 10.796 & 7.926 & 6.827 & 4.54 & 4.289 & 2.509 & 2.491 & 10.822 & 6.064 & 4.802 & 6.639 & 3.997 \\
\hline $10 / 7 / 2005$ & $2: 00$ & 10.913 & 10.8 & 7.928 & 6.829 & 4.54 & 4.291 & 2.517 & 2.491 & 10.82 & 6.062 & 4.798 & 6.639 & 3.989 \\
\hline $10 / 7 / 2005$ & $3: 00$ & 10.911 & 10.795 & 7.924 & 6.822 & 4.532 & 4.289 & 2.512 & 2.485 & 10.816 & 6.06 & 4.792 & 6.637 & 3.977 \\
\hline $10 / 7 / 2005$ & $4: 00$ & 10.911 & 10.793 & 7.924 & 6.824 & 4.526 & 4.286 & 2.512 & 2.485 & 10.814 & 6.057 & 4.786 & 6.635 & 3.971 \\
\hline $10 / 7 / 2005$ & $5: 00$ & 10.908 & 10.793 & 7.924 & 6.82 & 4.522 & 4.284 & 2.51 & 2.485 & 10.812 & 6.056 & 4.784 & 6.637 & 3.964 \\
\hline
\end{tabular}


TABLE S1.2 (Cont.)

Water Level (ft below top of casing) at Indicated Well

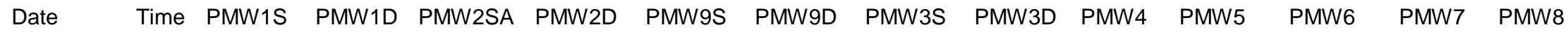

\begin{tabular}{|c|c|c|c|c|c|c|c|c|c|c|c|c|c|c|}
\hline 10/7/2005 & $6: 00$ & 10.906 & 10.793 & 7.922 & 6.82 & 4.518 & 4.284 & 2.512 & 2.483 & 10.81 & 6.053 & 4.78 & 6.635 & 3.958 \\
\hline $10 / 7 / 2005$ & $7: 00$ & 10.906 & 10.791 & 7.922 & 6.82 & 4.515 & 4.282 & 2.505 & 2.481 & 10.807 & 6.04 & 4.777 & 6.635 & 3.952 \\
\hline $10 / 7 / 2005$ & $8: 00$ & 10.906 & 10.793 & 7.922 & 6.823 & 4.514 & 4.282 & 2.51 & 2.483 & 10.807 & 6.039 & 4.777 & 6.635 & 3.949 \\
\hline $10 / 7 / 2005$ & $9: 00$ & 10.906 & 10.793 & 7.922 & 6.82 & 4.51 & 4.28 & 2.51 & 2.483 & 10.805 & 6.038 & 4.775 & 6.635 & 3.943 \\
\hline $10 / 7 / 2005$ & $10: 00$ & 10.906 & 10.789 & 7.921 & 6.818 & 4.507 & 4.276 & 2.5 & 2.479 & 10.803 & 6.036 & 4.771 & 6.633 & 3.938 \\
\hline $10 / 7 / 2005$ & $11: 00$ & 10.901 & 10.784 & 7.918 & 6.809 & 4.501 & 4.273 & 2.5 & 2.474 & 10.799 & 6.034 & 4.767 & 6.629 & 3.932 \\
\hline $10 / 7 / 2005$ & $12: 00$ & 10.893 & 10.773 & 7.91 & 6.798 & 4.491 & 4.269 & 2.492 & 2.467 & 10.795 & 6.027 & 4.765 & 6.626 & 3.926 \\
\hline $10 / 7 / 2005$ & $13: 00$ & 10.886 & 10.762 & 7.906 & 6.794 & 4.487 & 4.262 & 2.487 & 2.46 & 10.793 & 6.028 & 4.763 & 6.624 & 3.932 \\
\hline $10 / 7 / 2005$ & $14: 00$ & 10.874 & 10.747 & 7.9 & 6.778 & 4.48 & 4.255 & 2.47 & 2.45 & 10.788 & 6.028 & 4.761 & 6.62 & 3.936 \\
\hline 10/7/2005 & $15: 00$ & 10.864 & 10.733 & 7.891 & 6.769 & 4.476 & 4.249 & 2.462 & 2.441 & 10.786 & 6.03 & 4.761 & 6.616 & 3.943 \\
\hline $10 / 7 / 2005$ & $16: 00$ & 10.854 & 10.724 & 7.885 & 6.763 & 4.474 & 4.242 & 2.457 & 2.437 & 10.782 & 6.034 & 4.763 & 6.612 & 3.951 \\
\hline $10 / 7 / 2005$ & $17: 00$ & 10.844 & 10.715 & 7.879 & 6.758 & 4.475 & 4.24 & 2.459 & 2.431 & 10.78 & 6.036 & 4.763 & 6.61 & 3.953 \\
\hline $10 / 7 / 2005$ & $18: 00$ & 10.839 & 10.707 & 7.875 & 6.754 & 4.478 & 4.234 & 2.449 & 2.429 & 10.778 & 6.038 & 4.761 & 6.61 & 3.955 \\
\hline 10/7/2005 & $19: 00$ & 10.834 & 10.706 & 7.873 & 6.754 & 4.477 & 4.231 & 2.457 & 2.427 & 10.778 & 6.038 & 4.761 & 6.606 & 3.951 \\
\hline $10 / 7 / 2005$ & $20: 00$ & 10.829 & 10.702 & 7.872 & 6.752 & 4.475 & 4.229 & 2.444 & 2.425 & 10.776 & 6.034 & 4.755 & 6.605 & 3.939 \\
\hline $10 / 7 / 2005$ & $21: 00$ & 10.827 & 10.702 & 7.87 & 6.752 & 4.473 & 4.229 & 2.452 & 2.422 & 10.776 & 6.034 & 4.751 & 6.603 & 3.929 \\
\hline $10 / 7 / 2005$ & $22: 00$ & 10.825 & 10.7 & 7.868 & 6.752 & 4.47 & 4.225 & 2.449 & 2.42 & 10.774 & 6.031 & 4.746 & 6.601 & 3.921 \\
\hline $10 / 7 / 2005$ & $23: 00$ & 10.822 & 10.7 & 7.868 & 6.754 & 4.472 & 4.223 & 2.447 & 2.422 & 10.772 & 6.029 & 4.744 & 6.599 & 3.914 \\
\hline $10 / 8 / 2005$ & $0: 00$ & 10.82 & 10.698 & 7.866 & 6.749 & 4.468 & 4.223 & 2.447 & 2.418 & 10.772 & 6.025 & 4.738 & 6.597 & 3.906 \\
\hline $10 / 8 / 2005$ & $1: 00$ & 10.822 & 10.698 & 7.866 & 6.749 & 4.466 & 4.22 & 2.445 & 2.418 & 10.769 & 6.025 & 4.736 & 6.597 & 3.905 \\
\hline $10 / 8 / 2005$ & $2: 00$ & 10.817 & 10.696 & 7.866 & 6.747 & 4.462 & 4.218 & 2.442 & 2.416 & 10.769 & 6.025 & 4.734 & 6.595 & 3.899 \\
\hline $10 / 8 / 2005$ & $3: 00$ & 10.82 & 10.696 & 7.864 & 6.749 & 4.46 & 4.216 & 2.435 & 2.416 & 10.767 & 6.023 & 4.73 & 6.593 & 3.894 \\
\hline $10 / 8 / 2005$ & $4: 00$ & 10.815 & 10.693 & 7.862 & 6.743 & 4.454 & 4.214 & 2.432 & 2.41 & 10.763 & 6.021 & 4.728 & 6.592 & 3.888 \\
\hline $10 / 8 / 2005$ & $5: 00$ & 10.815 & 10.691 & 7.86 & 6.743 & 4.45 & 4.211 & 2.43 & 2.41 & 10.763 & 6.02 & 4.726 & 6.592 & 3.884 \\
\hline $10 / 8 / 2005$ & $6: 00$ & 10.813 & 10.689 & 7.858 & 6.741 & 4.448 & 4.211 & 2.435 & 2.408 & 10.761 & 6.018 & 4.722 & 6.59 & 3.88 \\
\hline $10 / 8 / 2005$ & $7: 00$ & 10.813 & 10.691 & 7.861 & 6.743 & 4.448 & 4.209 & 2.437 & 2.408 & 10.759 & 6.018 & 4.722 & 6.59 & 3.88 \\
\hline $10 / 8 / 2005$ & $8: 00$ & 10.813 & 10.693 & 7.861 & 6.745 & 4.45 & 4.209 & 2.437 & 2.41 & 10.758 & 6.018 & 4.722 & 6.59 & 3.879 \\
\hline $10 / 8 / 2005$ & 9:00 & 10.815 & 10.696 & 7.861 & 6.747 & 4.452 & 4.209 & 2.435 & 2.414 & 10.758 & 6.016 & 4.724 & 6.59 & 3.881 \\
\hline $10 / 8 / 2005$ & $10: 00$ & 10.818 & 10.7 & 7.865 & 6.749 & 4.452 & 4.207 & 2.445 & 2.419 & 10.758 & 6.014 & 4.726 & 6.592 & 3.879 \\
\hline $10 / 8 / 2005$ & $11: 00$ & 10.815 & 10.695 & 7.863 & 6.745 & 4.448 & 4.207 & 2.435 & 2.414 & 10.756 & 6.014 & 4.724 & 6.588 & 3.877 \\
\hline $10 / 8 / 2005$ & $12: 00$ & 10.813 & 10.689 & 7.859 & 6.741 & 4.444 & 4.203 & 2.44 & 2.412 & 10.756 & 6.018 & 4.728 & 6.59 & 3.885 \\
\hline $10 / 8 / 2005$ & $13: 00$ & 10.808 & 10.68 & 7.855 & 6.732 & 4.44 & 4.198 & 2.43 & 2.408 & 10.752 & 6.016 & 4.732 & 6.59 & 3.894 \\
\hline $10 / 8 / 2005$ & $14: 00$ & 10.798 & 10.671 & 7.85 & 6.723 & 4.436 & 4.192 & 2.43 & 2.4 & 10.75 & 6.018 & 4.734 & 6.588 & 3.907 \\
\hline $10 / 8 / 2005$ & $15: 00$ & 10.793 & 10.664 & 7.848 & 6.718 & 4.438 & 4.189 & 2.42 & 2.398 & 10.748 & 6.023 & 4.742 & 6.59 & 3.927 \\
\hline
\end{tabular}


Water Level (ft below top of casing) at Indicated Well

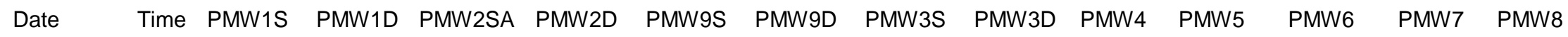

\begin{tabular}{|c|c|c|c|c|c|c|c|c|c|c|c|c|c|c|}
\hline $10 / 8 / 2005$ & $16: 00$ & 10.786 & 10.656 & 7.844 & 6.717 & 4.442 & 4.187 & 2.427 & 2.396 & 10.75 & 6.027 & 4.75 & 6.586 & 3.943 \\
\hline $10 / 8 / 2005$ & $17: 00$ & 10.781 & 10.651 & 7.842 & 6.714 & 4.448 & 4.183 & 2.427 & 2.396 & 10.752 & 6.034 & 4.758 & 6.586 & 3.96 \\
\hline $10 / 8 / 2005$ & $18: 00$ & 10.776 & 10.647 & 7.842 & 6.714 & 4.455 & 4.183 & 2.419 & 2.396 & 10.752 & 6.041 & 4.764 & 6.586 & 3.974 \\
\hline $10 / 8 / 2005$ & $19: 00$ & 10.771 & 10.645 & 7.84 & 6.712 & 4.459 & 4.183 & 2.417 & 2.396 & 10.754 & 6.039 & 4.764 & 6.586 & 3.972 \\
\hline $10 / 8 / 2005$ & $20: 00$ & 10.771 & 10.642 & 7.84 & 6.712 & 4.461 & 4.183 & 2.42 & 2.391 & 10.754 & 6.034 & 4.76 & 6.582 & 3.958 \\
\hline $10 / 8 / 2005$ & $21: 00$ & 10.769 & 10.643 & 7.84 & 6.714 & 4.463 & 4.183 & 2.42 & 2.391 & 10.754 & 6.032 & 4.758 & 6.582 & 3.948 \\
\hline $10 / 8 / 2005$ & $22: 00$ & 10.769 & 10.645 & 7.841 & 6.714 & 4.461 & 4.183 & 2.42 & 2.391 & 10.754 & 6.027 & 4.752 & 6.578 & 3.936 \\
\hline $10 / 8 / 2005$ & $23: 00$ & 10.769 & 10.645 & 7.839 & 6.714 & 4.457 & 4.183 & 2.417 & 2.389 & 10.752 & 6.025 & 4.748 & 6.58 & 3.923 \\
\hline $10 / 9 / 2005$ & 0:00 & 10.769 & 10.645 & 7.837 & 6.714 & 4.457 & 4.183 & 2.415 & 2.387 & 10.752 & 6.025 & 4.744 & 6.578 & 3.913 \\
\hline $10 / 9 / 2005$ & $1: 00$ & 10.767 & 10.643 & 7.837 & 6.714 & 4.453 & 4.183 & 2.407 & 2.385 & 10.75 & 6.021 & 4.738 & 6.58 & 3.899 \\
\hline $10 / 9 / 2005$ & $2: 00$ & 10.769 & 10.645 & 7.837 & 6.714 & 4.451 & 4.183 & 2.412 & 2.385 & 10.748 & 6.021 & 4.738 & 6.58 & 3.891 \\
\hline $10 / 9 / 2005$ & $3: 00$ & 10.769 & 10.647 & 7.837 & 6.714 & 4.447 & 4.183 & 2.413 & 2.385 & 10.748 & 6.019 & 4.734 & 6.58 & 3.885 \\
\hline $10 / 9 / 2005$ & $4: 00$ & 10.769 & 10.649 & 7.837 & 6.712 & 4.444 & 4.183 & 2.41 & 2.385 & 10.744 & 6.018 & 4.73 & 6.578 & 3.875 \\
\hline $10 / 9 / 2005$ & $5: 00$ & 10.769 & 10.647 & 7.835 & 6.712 & 4.438 & 4.181 & 2.402 & 2.383 & 10.744 & 6.014 & 4.726 & 6.576 & 3.869 \\
\hline $10 / 9 / 2005$ & $6: 00$ & 10.769 & 10.649 & 7.835 & 6.714 & 4.436 & 4.181 & 2.405 & 2.383 & 10.742 & 6.012 & 4.722 & 6.575 & 3.867 \\
\hline $10 / 9 / 2005$ & $7: 00$ & 10.771 & 10.654 & 7.837 & 6.717 & 4.436 & 4.181 & 2.413 & 2.385 & 10.74 & 6.014 & 4.722 & 6.577 & 3.863 \\
\hline $10 / 9 / 2005$ & $8: 00$ & 10.771 & 10.656 & 7.836 & 6.714 & 4.434 & 4.181 & 2.407 & 2.385 & 10.74 & 6.01 & 4.719 & 6.577 & 3.859 \\
\hline $10 / 9 / 2005$ & $9: 00$ & 10.771 & 10.654 & 7.836 & 6.714 & 4.432 & 4.179 & 2.405 & 2.385 & 10.737 & 6.012 & 4.715 & 6.575 & 3.855 \\
\hline $10 / 9 / 2005$ & $10: 00$ & 10.774 & 10.654 & 7.838 & 6.719 & 4.428 & 4.179 & 2.41 & 2.385 & 10.739 & 6.008 & 4.715 & 6.575 & 3.851 \\
\hline $10 / 9 / 2005$ & $11: 00$ & 10.776 & 10.656 & 7.838 & 6.717 & 4.428 & 4.176 & 2.415 & 2.388 & 10.735 & 6.008 & 4.715 & 6.575 & 3.849 \\
\hline $10 / 9 / 2005$ & $12: 00$ & 10.776 & 10.656 & 7.839 & 6.721 & 4.43 & 4.176 & 2.413 & 2.392 & 10.737 & 6.014 & 4.726 & 6.577 & 3.871 \\
\hline $10 / 9 / 2005$ & $13: 00$ & 10.771 & 10.649 & 7.838 & 6.714 & 4.43 & 4.174 & 2.42 & 2.392 & 10.735 & 6.014 & 4.736 & 6.575 & 3.887 \\
\hline $10 / 9 / 2005$ & $14: 00$ & 10.767 & 10.642 & 7.835 & 6.708 & 4.432 & 4.17 & 2.415 & 2.388 & 10.737 & 6.014 & 4.744 & 6.578 & 3.907 \\
\hline $10 / 9 / 2005$ & $15: 00$ & 10.767 & 10.64 & 7.835 & 6.708 & 4.439 & 4.168 & 2.415 & 2.39 & 10.739 & 6.021 & 4.758 & 6.58 & 3.932 \\
\hline $10 / 9 / 2005$ & $16: 00$ & 10.762 & 10.64 & 7.833 & 6.71 & 4.449 & 4.17 & 2.415 & 2.396 & 10.741 & 6.03 & 4.773 & 6.586 & 3.956 \\
\hline $10 / 9 / 2005$ & $17: 00$ & 10.762 & 10.636 & 7.835 & 6.71 & 4.454 & 4.168 & 2.418 & 2.394 & 10.743 & 6.035 & 4.781 & 6.584 & 3.975 \\
\hline $10 / 9 / 2005$ & $18: 00$ & 10.762 & 10.638 & 7.837 & 6.712 & 4.46 & 4.17 & 2.42 & 2.398 & 10.746 & 6.035 & 4.787 & 6.586 & 3.988 \\
\hline $10 / 9 / 2005$ & $19: 00$ & 10.767 & 10.647 & 7.843 & 6.721 & 4.472 & 4.176 & 2.425 & 2.404 & 10.75 & 6.041 & 4.795 & 6.59 & 3.991 \\
\hline $10 / 9 / 2005$ & $20: 00$ & 10.777 & 10.662 & 7.851 & 6.734 & 4.485 & 4.183 & 2.435 & 2.415 & 10.754 & 6.041 & 4.799 & 6.594 & 3.987 \\
\hline $10 / 9 / 2005$ & $21: 00$ & 10.782 & 10.669 & 7.854 & 6.739 & 4.485 & 4.187 & 2.438 & 2.417 & 10.756 & 6.039 & 4.793 & 6.592 & 3.969 \\
\hline $10 / 9 / 2005$ & $22: 00$ & 10.789 & 10.675 & 7.856 & 6.739 & 4.483 & 4.19 & 2.446 & 2.417 & 10.756 & 6.035 & 4.785 & 6.594 & 3.951 \\
\hline $10 / 9 / 2005$ & $23: 00$ & 10.792 & 10.68 & 7.858 & 6.746 & 4.479 & 4.194 & 2.446 & 2.419 & 10.758 & 6.032 & 4.781 & 6.592 & 3.936 \\
\hline $10 / 10 / 2005$ & $0: 00$ & 10.796 & 10.685 & 7.86 & 6.748 & 4.477 & 4.199 & 2.441 & 2.419 & 10.756 & 6.03 & 4.775 & 6.594 & 3.922 \\
\hline $10 / 10 / 2005$ & $1: 00$ & 10.804 & 10.691 & 7.862 & 6.75 & 4.475 & 4.201 & 2.448 & 2.424 & 10.758 & 6.026 & 4.772 & 6.594 & 3.912 \\
\hline
\end{tabular}


Water Level (ft below top of casing) at Indicated Well

$\begin{array}{lllllllllllllll}\text { Date } & \text { Time } & \text { PMW1S } & \text { PMW1D } & \text { PMW2SA } & \text { PMW2D } & \text { PMW9S } & \text { PMW9D } & \text { PMW3S } & \text { PMW3D } & \text { PMW4 } & \text { PMW5 } & \text { PMW6 } & \text { PMW7 } & \text { PMW8 }\end{array}$

\begin{tabular}{|c|c|c|c|c|c|c|c|c|c|c|c|c|c|c|}
\hline $10 / 10 / 2005$ & $2: 00$ & 10.806 & 10.695 & 7.862 & 6.748 & 4.471 & 4.203 & 2.448 & 2.422 & 10.756 & 6.026 & 4.768 & 6.596 & 3.9 \\
\hline $10 / 10 / 2005$ & $3: 00$ & 10.806 & 10.693 & 7.86 & 6.748 & 4.464 & 4.203 & 2.446 & 2.419 & 10.754 & 6.023 & 4.758 & 6.594 & 3.886 \\
\hline $10 / 10 / 2005$ & $4: 00$ & 10.809 & 10.696 & 7.862 & 6.75 & 4.461 & 4.205 & 2.441 & 2.419 & 10.752 & 6.021 & 4.752 & 6.594 & 3.88 \\
\hline $10 / 10 / 2005$ & $5: 00$ & 10.814 & 10.702 & 7.866 & 6.755 & 4.461 & 4.207 & 2.451 & 2.424 & 10.75 & 6.021 & 4.752 & 6.596 & 3.876 \\
\hline $10 / 10 / 2005$ & $6: 00$ & 10.819 & 10.711 & 7.868 & 6.761 & 4.463 & 4.21 & 2.456 & 2.43 & 10.752 & 6.024 & 4.752 & 6.598 & 3.874 \\
\hline $10 / 10 / 2005$ & $7: 00$ & 10.826 & 10.718 & 7.874 & 6.768 & 4.465 & 4.212 & 2.464 & 2.436 & 10.752 & 6.024 & 4.752 & 6.602 & 3.872 \\
\hline $10 / 10 / 2005$ & $8: 00$ & 10.831 & 10.72 & 7.875 & 6.766 & 4.46 & 4.216 & 2.461 & 2.436 & 10.752 & 6.019 & 4.75 & 6.602 & 3.864 \\
\hline $10 / 10 / 2005$ & $9: 00$ & 10.833 & 10.724 & 7.877 & 6.77 & 4.458 & 4.216 & 2.456 & 2.436 & 10.75 & 6.019 & 4.748 & 6.604 & 3.858 \\
\hline $10 / 10 / 2005$ & $10: 00$ & 10.838 & 10.727 & 7.879 & 6.772 & 4.458 & 4.216 & 2.459 & 2.438 & 10.75 & 6.02 & 4.748 & 6.604 & 3.856 \\
\hline $10 / 10 / 2005$ & $11: 00$ & 10.841 & 10.731 & 7.883 & 6.772 & 4.458 & 4.216 & 2.469 & 2.443 & 10.75 & 6.024 & 4.756 & 6.606 & 3.86 \\
\hline $10 / 10 / 2005$ & $12: 00$ & 10.844 & 10.731 & 7.883 & 6.777 & 4.459 & 4.216 & 2.476 & 2.449 & 10.75 & 6.026 & 4.766 & 6.611 & 3.882 \\
\hline $10 / 10 / 2005$ & $13: 00$ & 10.841 & 10.727 & 7.884 & 6.772 & 4.461 & 4.217 & 2.474 & 2.447 & 10.75 & 6.028 & 4.778 & 6.611 & 3.898 \\
\hline $10 / 10 / 2005$ & $14: 00$ & 10.839 & 10.722 & 7.884 & 6.77 & 4.465 & 4.216 & 2.471 & 2.447 & 10.752 & 6.035 & 4.789 & 6.613 & 3.922 \\
\hline $10 / 10 / 2005$ & $15: 00$ & 10.836 & 10.716 & 7.882 & 6.766 & 4.471 & 4.217 & 2.468 & 2.447 & 10.756 & 6.037 & 4.797 & 6.613 & 3.937 \\
\hline $10 / 10 / 2005$ & $16: 00$ & 10.831 & 10.713 & 7.88 & 6.766 & 4.474 & 4.217 & 2.476 & 2.447 & 10.758 & 6.042 & 4.807 & 6.617 & 3.957 \\
\hline $10 / 10 / 2005$ & $17: 00$ & 10.829 & 10.709 & 7.882 & 6.766 & 4.48 & 4.216 & 2.468 & 2.447 & 10.76 & 6.048 & 4.817 & 6.617 & 3.973 \\
\hline $10 / 10 / 2005$ & $18: 00$ & 10.831 & 10.713 & 7.882 & 6.768 & 4.488 & 4.219 & 2.479 & 2.451 & 10.762 & 6.05 & 4.823 & 6.619 & 3.983 \\
\hline $10 / 10 / 2005$ & 19:00 & 10.831 & 10.715 & 7.882 & 6.768 & 4.492 & 4.221 & 2.471 & 2.449 & 10.765 & 6.05 & 4.821 & 6.617 & 3.973 \\
\hline $10 / 10 / 2005$ & $20: 00$ & 10.834 & 10.715 & 7.882 & 6.77 & 4.493 & 4.223 & 2.471 & 2.449 & 10.768 & 6.048 & 4.813 & 6.615 & 3.957 \\
\hline $10 / 10 / 2005$ & $21: 00$ & 10.833 & 10.718 & 7.884 & 6.772 & 4.492 & 4.225 & 2.474 & 2.449 & 10.767 & 6.044 & 4.807 & 6.615 & 3.941 \\
\hline $10 / 10 / 2005$ & $22: 00$ & 10.839 & 10.724 & 7.884 & 6.775 & 4.489 & 4.226 & 2.474 & 2.451 & 10.768 & 6.044 & 4.801 & 6.615 & 3.929 \\
\hline $10 / 10 / 2005$ & $23: 00$ & 10.841 & 10.728 & 7.886 & 6.779 & 4.487 & 4.23 & 2.479 & 2.451 & 10.77 & 6.039 & 4.795 & 6.615 & 3.919 \\
\hline $10 / 11 / 2005$ & $0: 00$ & 10.841 & 10.724 & 7.884 & 6.772 & 4.48 & 4.23 & 2.474 & 2.445 & 10.766 & 6.032 & 4.786 & 6.613 & 3.902 \\
\hline $10 / 11 / 2005$ & $1: 00$ & 10.836 & 10.716 & 7.88 & 6.766 & 4.47 & 4.228 & 2.467 & 2.439 & 10.764 & 6.028 & 4.776 & 6.611 & 3.887 \\
\hline $10 / 11 / 2005$ & $2: 00$ & 10.839 & 10.719 & 7.88 & 6.768 & 4.464 & 4.226 & 2.466 & 2.439 & 10.762 & 6.028 & 4.774 & 6.609 & 3.88 \\
\hline $10 / 11 / 2005$ & $3: 00$ & 10.836 & 10.717 & 7.878 & 6.766 & 4.46 & 4.226 & 2.464 & 2.437 & 10.76 & 6.026 & 4.77 & 6.608 & 3.872 \\
\hline $10 / 11 / 2005$ & $4: 00$ & 10.831 & 10.711 & 7.874 & 6.759 & 4.454 & 4.221 & 2.459 & 2.43 & 10.758 & 6.02 & 4.764 & 6.606 & 3.866 \\
\hline $10 / 11 / 2005$ & $5: 00$ & 10.829 & 10.711 & 7.874 & 6.759 & 4.452 & 4.221 & 2.457 & 2.43 & 10.756 & 6.02 & 4.76 & 6.606 & 3.86 \\
\hline $10 / 11 / 2005$ & $6: 00$ & 10.826 & 10.706 & 7.87 & 6.757 & 4.448 & 4.219 & 2.454 & 2.424 & 10.753 & 6.017 & 4.752 & 6.602 & 3.85 \\
\hline $10 / 11 / 2005$ & $7: 00$ & 10.829 & 10.709 & 7.873 & 6.762 & 4.45 & 4.219 & 2.457 & 2.428 & 10.751 & 6.017 & 4.752 & 6.604 & 3.85 \\
\hline $10 / 11 / 2005$ & $8: 00$ & 10.829 & 10.711 & 7.873 & 6.759 & 4.446 & 4.217 & 2.454 & 2.428 & 10.751 & 6.015 & 4.748 & 6.602 & 3.844 \\
\hline $10 / 11 / 2005$ & $9: 00$ & 10.826 & 10.711 & 7.873 & 6.759 & 4.444 & 4.217 & 2.454 & 2.428 & 10.749 & 6.015 & 4.748 & 6.602 & 3.844 \\
\hline $10 / 11 / 2005$ & $10: 00$ & 10.829 & 10.711 & 7.873 & 6.759 & 4.444 & 4.217 & 2.454 & 2.433 & 10.749 & 6.017 & 4.756 & 6.604 & 3.852 \\
\hline $10 / 11 / 2005$ & $11: 00$ & 10.831 & 10.713 & 7.877 & 6.766 & 4.448 & 4.214 & 2.464 & 2.437 & 10.749 & 6.02 & 4.766 & 6.606 & 3.868 \\
\hline
\end{tabular}


Water Level (ft below top of casing) at Indicated Well

$\begin{array}{lllllllllllllll}\text { Date } & \text { Time } & \text { PMW1S } & \text { PMW1D } & \text { PMW2SA } & \text { PMW2D } & \text { PMW9S } & \text { PMW9D } & \text { PMW3S } & \text { PMW3D } & \text { PMW4 } & \text { PMW5 } & \text { PMW6 } & \text { PMW7 } & \text { PMW8 }\end{array}$

\begin{tabular}{|c|c|c|c|c|c|c|c|c|c|c|c|c|c|c|}
\hline $10 / 11 / 2005$ & $12: 00$ & 10.831 & 10.713 & 7.877 & 6.766 & 4.452 & 4.214 & 2.469 & 2.439 & 10.749 & 6.026 & 4.774 & 6.608 & 3.88 \\
\hline $10 / 11 / 2005$ & $13: 00$ & 10.821 & 10.7 & 7.87 & 6.753 & 4.444 & 4.21 & 2.454 & 2.43 & 10.754 & 6.017 & 4.766 & 6.604 & 3.871 \\
\hline $10 / 11 / 2005$ & $14: 00$ & 10.817 & 10.693 & 7.866 & 6.744 & 4.44 & 4.206 & 2.449 & 2.418 & 10.747 & 6.017 & 4.762 & 6.602 & 3.865 \\
\hline $10 / 11 / 2005$ & $15: 00$ & 10.809 & 10.684 & 7.862 & 6.74 & 4.436 & 4.203 & 2.437 & 2.414 & 10.745 & 6.015 & 4.764 & 6.598 & 3.867 \\
\hline $10 / 11 / 2005$ & $16: 00$ & 10.804 & 10.68 & 7.86 & 6.74 & 4.438 & 4.201 & 2.444 & 2.416 & 10.745 & 6.02 & 4.774 & 6.6 & 3.882 \\
\hline $10 / 11 / 2005$ & $17: 00$ & 10.802 & 10.677 & 7.86 & 6.737 & 4.44 & 4.199 & 2.437 & 2.414 & 10.745 & 6.024 & 4.782 & 6.6 & 3.893 \\
\hline $10 / 11 / 2005$ & $18: 00$ & 10.797 & 10.673 & 7.858 & 6.735 & 4.441 & 4.197 & 2.439 & 2.412 & 10.745 & 6.024 & 4.782 & 6.598 & 3.895 \\
\hline $10 / 11 / 2005$ & $19: 00$ & 10.797 & 10.673 & 7.858 & 6.735 & 4.449 & 4.197 & 2.439 & 2.412 & 10.747 & 6.022 & 4.782 & 6.596 & 3.889 \\
\hline $10 / 11 / 2005$ & $20: 00$ & 10.797 & 10.675 & 7.856 & 6.735 & 4.449 & 4.197 & 2.434 & 2.41 & 10.747 & 6.02 & 4.778 & 6.596 & 3.879 \\
\hline $10 / 11 / 2005$ & $21: 00$ & 10.797 & 10.673 & 7.857 & 6.737 & 4.447 & 4.197 & 2.439 & 2.41 & 10.747 & 6.017 & 4.772 & 6.592 & 3.871 \\
\hline $10 / 11 / 2005$ & $22: 00$ & 10.794 & 10.649 & 7.855 & 6.711 & 4.445 & 4.195 & 2.437 & 2.401 & 10.724 & 6.009 & 4.762 & 6.564 & 3.861 \\
\hline $10 / 11 / 2005$ & $23: 00$ & 10.797 & 10.653 & 7.855 & 6.717 & 4.441 & 4.195 & 2.434 & 2.408 & 10.736 & 6.004 & 4.753 & 6.595 & 3.847 \\
\hline $10 / 12 / 2005$ & $0: 00$ & 10.797 & 10.655 & 7.853 & 6.731 & 4.431 & 4.192 & 2.427 & 2.395 & 10.724 & 6.005 & 4.743 & 6.587 & 3.83 \\
\hline $10 / 12 / 2005$ & $1: 00$ & 10.795 & 10.671 & 7.851 & 6.728 & 4.421 & 4.19 & 2.424 & 2.395 & 10.738 & 6.002 & 4.733 & 6.583 & 3.82 \\
\hline $10 / 12 / 2005$ & $2: 00$ & 10.792 & 10.669 & 7.849 & 6.728 & 4.418 & 4.188 & 2.415 & 2.395 & 10.734 & 5.998 & 4.731 & 6.583 & 3.816 \\
\hline $10 / 12 / 2005$ & $3: 00$ & 10.79 & 10.671 & 7.849 & 6.726 & 4.416 & 4.188 & 2.415 & 2.395 & 10.734 & 5.998 & 4.725 & 6.581 & 3.814 \\
\hline $10 / 12 / 2005$ & $4: 00$ & 10.792 & 10.68 & 7.849 & 6.74 & 4.414 & 4.183 & 2.415 & 2.397 & 10.736 & 5.985 & 4.717 & 6.594 & 3.806 \\
\hline $10 / 12 / 2005$ & $5: 00$ & 10.788 & 10.68 & 7.847 & 6.735 & 4.406 & 4.181 & 2.415 & 2.389 & 10.734 & 5.985 & 4.713 & 6.595 & 3.796 \\
\hline $10 / 12 / 2005$ & $6: 00$ & 10.792 & 10.677 & 7.845 & 6.731 & 4.406 & 4.181 & 2.41 & 2.389 & 10.721 & 5.985 & 4.711 & 6.581 & 3.796 \\
\hline $10 / 12 / 2005$ & $7: 00$ & 10.792 & 10.675 & 7.847 & 6.728 & 4.409 & 4.179 & 2.413 & 2.393 & 10.721 & 5.987 & 4.711 & 6.581 & 3.798 \\
\hline $10 / 12 / 2005$ & $8: 00$ & 10.795 & 10.678 & 7.849 & 6.733 & 4.409 & 4.179 & 2.42 & 2.397 & 10.728 & 5.987 & 4.709 & 6.581 & 3.798 \\
\hline $10 / 12 / 2005$ & $9: 00$ & 10.797 & 10.682 & 7.851 & 6.733 & 4.409 & 4.179 & 2.418 & 2.398 & 10.728 & 5.985 & 4.709 & 6.583 & 3.796 \\
\hline $10 / 12 / 2005$ & $10: 00$ & 10.8 & 10.684 & 7.854 & 6.738 & 4.411 & 4.182 & 2.425 & 2.399 & 10.724 & 5.987 & 4.709 & 6.583 & 3.796 \\
\hline $10 / 12 / 2005$ & $11: 00$ & 10.805 & 10.693 & 7.856 & 6.742 & 4.413 & 4.184 & 2.43 & 2.404 & 10.726 & 5.992 & 4.717 & 6.587 & 3.802 \\
\hline $10 / 12 / 2005$ & $12: 00$ & 10.81 & 10.695 & 7.858 & 6.746 & 4.413 & 4.184 & 2.428 & 2.408 & 10.726 & 5.992 & 4.721 & 6.589 & 3.804 \\
\hline $10 / 12 / 2005$ & $13: 00$ & 10.81 & 10.695 & 7.86 & 6.747 & 4.413 & 4.184 & 2.435 & 2.408 & 10.728 & 5.994 & 4.723 & 6.589 & 3.81 \\
\hline $10 / 12 / 2005$ & $14: 00$ & 10.805 & 10.688 & 7.857 & 6.74 & 4.409 & 4.184 & 2.433 & 2.406 & 10.726 & 5.996 & 4.725 & 6.589 & 3.814 \\
\hline $10 / 12 / 2005$ & $15: 00$ & 10.805 & 10.688 & 7.857 & 6.74 & 4.412 & 4.184 & 2.436 & 2.406 & 10.726 & 6.001 & 4.731 & 6.593 & 3.825 \\
\hline $10 / 12 / 2005$ & $16: 00$ & 10.807 & 10.693 & 7.861 & 6.747 & 4.418 & 4.186 & 2.438 & 2.41 & 10.726 & 6.002 & 4.737 & 6.593 & 3.835 \\
\hline $10 / 12 / 2005$ & $17: 00$ & 10.813 & 10.697 & 7.864 & 6.749 & 4.422 & 4.186 & 2.443 & 2.414 & 10.73 & 6.005 & 4.741 & 6.595 & 3.839 \\
\hline $10 / 12 / 2005$ & $18: 00$ & 10.815 & 10.7 & 7.866 & 6.753 & 4.426 & 4.188 & 2.448 & 2.419 & 10.73 & 6.009 & 4.747 & 6.597 & 3.845 \\
\hline $10 / 12 / 2005$ & $19: 00$ & 10.82 & 10.708 & 7.87 & 6.758 & 4.429 & 4.193 & 2.443 & 2.425 & 10.732 & 6.009 & 4.749 & 6.6 & 3.847 \\
\hline $10 / 12 / 2005$ & $20: 00$ & 10.825 & 10.711 & 7.87 & 6.76 & 4.431 & 4.195 & 2.446 & 2.427 & 10.734 & 6.007 & 4.747 & 6.6 & 3.839 \\
\hline $10 / 12 / 2005$ & $21: 00$ & 10.827 & 10.715 & 7.874 & 6.764 & 4.429 & 4.199 & 2.446 & 2.429 & 10.734 & 6.005 & 4.745 & 6.6 & 3.833 \\
\hline
\end{tabular}


Water Level (ft below top of casing) at Indicated Well

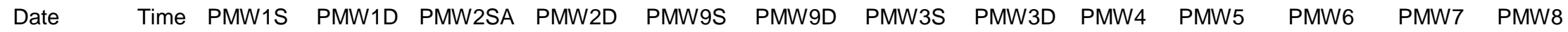

\begin{tabular}{|c|c|c|c|c|c|c|c|c|c|c|c|c|c|c|}
\hline $10 / 12 / 2005$ & $22: 00$ & 10.83 & 10.717 & 7.874 & 6.762 & 4.427 & 4.199 & 2.453 & 2.429 & 10.734 & 6.001 & 4.741 & 6.6 & 3.823 \\
\hline $10 / 12 / 2005$ & $23: 00$ & 10.83 & 10.717 & 7.872 & 6.762 & 4.423 & 4.202 & 2.446 & 2.427 & 10.734 & 5.996 & 4.737 & 6.6 & 3.813 \\
\hline $10 / 13 / 2005$ & $0: 00$ & 10.832 & 10.719 & 7.872 & 6.764 & 4.419 & 4.202 & 2.446 & 2.425 & 10.732 & 5.996 & 4.733 & 6.6 & 3.807 \\
\hline $10 / 13 / 2005$ & $1: 00$ & 10.832 & 10.719 & 7.872 & 6.762 & 4.417 & 4.204 & 2.446 & 2.425 & 10.732 & 5.996 & 4.729 & 6.6 & 3.803 \\
\hline $10 / 13 / 2005$ & $2: 00$ & 10.835 & 10.72 & 7.874 & 6.764 & 4.417 & 4.204 & 2.453 & 2.427 & 10.73 & 5.994 & 4.727 & 6.6 & 3.801 \\
\hline $10 / 13 / 2005$ & 3:00 & 10.835 & 10.72 & 7.874 & 6.764 & 4.415 & 4.204 & 2.446 & 2.427 & 10.73 & 5.994 & 4.726 & 6.6 & 3.799 \\
\hline $10 / 13 / 2005$ & $4: 00$ & 10.835 & 10.72 & 7.872 & 6.762 & 4.411 & 4.204 & 2.443 & 2.423 & 10.728 & 5.994 & 4.724 & 6.601 & 3.795 \\
\hline $10 / 13 / 2005$ & $5: 00$ & 10.835 & 10.72 & 7.872 & 6.762 & 4.411 & 4.204 & 2.451 & 2.423 & 10.726 & 5.994 & 4.722 & 6.599 & 3.791 \\
\hline $10 / 13 / 2005$ & $6: 00$ & 10.835 & 10.717 & 7.87 & 6.76 & 4.411 & 4.204 & 2.448 & 2.423 & 10.726 & 5.99 & 4.722 & 6.601 & 3.789 \\
\hline $10 / 13 / 2005$ & 7:00 & 10.835 & 10.72 & 7.872 & 6.764 & 4.411 & 4.204 & 2.446 & 2.425 & 10.726 & 5.99 & 4.722 & 6.601 & 3.789 \\
\hline $10 / 13 / 2005$ & $8: 00$ & 10.837 & 10.722 & 7.872 & 6.764 & 4.408 & 4.206 & 2.451 & 2.432 & 10.726 & 5.99 & 4.722 & 6.603 & 3.789 \\
\hline $10 / 13 / 2005$ & $9: 00$ & 10.84 & 10.726 & 7.876 & 6.769 & 4.414 & 4.206 & 2.451 & 2.431 & 10.726 & 5.994 & 4.726 & 6.605 & 3.791 \\
\hline $10 / 13 / 2005$ & $10: 00$ & 10.845 & 10.735 & 7.881 & 6.778 & 4.418 & 4.208 & 2.456 & 2.438 & 10.727 & 5.998 & 4.731 & 6.606 & 3.797 \\
\hline $10 / 13 / 2005$ & $11: 00$ & 10.847 & 10.735 & 7.883 & 6.778 & 4.418 & 4.208 & 2.466 & 2.442 & 10.729 & 6.001 & 4.741 & 6.608 & 3.797 \\
\hline $10 / 13 / 2005$ & $12: 00$ & 10.847 & 10.735 & 7.883 & 6.778 & 4.421 & 4.208 & 2.471 & 2.444 & 10.73 & 6.007 & 4.753 & 6.612 & 3.817 \\
\hline $10 / 13 / 2005$ & $13: 00$ & 10.845 & 10.731 & 7.884 & 6.776 & 4.423 & 4.208 & 2.466 & 2.446 & 10.73 & 6.014 & 4.765 & 6.614 & 3.843 \\
\hline $10 / 13 / 2005$ & $14: 00$ & 10.842 & 10.722 & 7.884 & 6.771 & 4.429 & 4.208 & 2.473 & 2.444 & 10.734 & 6.02 & 4.776 & 6.616 & 3.873 \\
\hline $10 / 13 / 2005$ & $15: 00$ & 10.838 & 10.717 & 7.882 & 6.771 & 4.436 & 4.206 & 2.476 & 2.446 & 10.738 & 6.03 & 4.794 & 6.622 & 3.908 \\
\hline $10 / 13 / 2005$ & $16: 00$ & 10.835 & 10.711 & 7.882 & 6.769 & 4.444 & 4.209 & 2.476 & 2.449 & 10.742 & 6.036 & 4.808 & 6.622 & 3.938 \\
\hline $10 / 13 / 2005$ & $17: 00$ & 10.832 & 10.709 & 7.884 & 6.767 & 4.455 & 4.208 & 2.471 & 2.451 & 10.748 & 6.045 & 4.82 & 6.622 & 3.965 \\
\hline $10 / 13 / 2005$ & $18: 00$ & 10.83 & 10.709 & 7.884 & 6.771 & 4.465 & 4.21 & 2.481 & 2.451 & 10.751 & 6.049 & 4.823 & 6.624 & 3.979 \\
\hline $10 / 13 / 2005$ & $19: 00$ & 10.83 & 10.709 & 7.884 & 6.771 & 4.469 & 4.21 & 2.476 & 2.451 & 10.755 & 6.049 & 4.82 & 6.622 & 3.969 \\
\hline 10/13/2005 & $20: 00$ & 10.832 & 10.715 & 7.884 & 6.773 & 4.475 & 4.215 & 2.476 & 2.449 & 10.757 & 6.045 & 4.814 & 6.62 & 3.951 \\
\hline $10 / 13 / 2005$ & $21: 00$ & 10.835 & 10.715 & 7.884 & 6.773 & 4.471 & 4.217 & 2.473 & 2.449 & 10.759 & 6.041 & 4.806 & 6.618 & 3.931 \\
\hline $10 / 13 / 2005$ & $22: 00$ & 10.835 & 10.717 & 7.882 & 6.773 & 4.469 & 4.222 & 2.473 & 2.449 & 10.759 & 6.038 & 4.8 & 6.618 & 3.915 \\
\hline $10 / 13 / 2005$ & $23: 00$ & 10.837 & 10.719 & 7.882 & 6.773 & 4.465 & 4.222 & 2.474 & 2.446 & 10.759 & 6.036 & 4.794 & 6.616 & 3.903 \\
\hline $10 / 14 / 2005$ & $0: 00$ & 10.833 & 10.713 & 7.88 & 6.769 & 4.459 & 4.221 & 2.466 & 2.44 & 10.759 & 6.032 & 4.786 & 6.612 & 3.886 \\
\hline $10 / 14 / 2005$ & $1: 00$ & 10.83 & 10.709 & 7.878 & 6.765 & 4.451 & 4.22 & 2.456 & 2.436 & 10.757 & 6.027 & 4.778 & 6.61 & 3.876 \\
\hline $10 / 14 / 2005$ & $2: 00$ & 10.828 & 10.706 & 7.874 & 6.762 & 4.445 & 4.22 & 2.458 & 2.434 & 10.755 & 6.023 & 4.775 & 6.61 & 3.866 \\
\hline $10 / 14 / 2005$ & $3: 00$ & 10.825 & 10.706 & 7.874 & 6.762 & 4.441 & 4.217 & 2.461 & 2.432 & 10.753 & 6.021 & 4.769 & 6.608 & 3.858 \\
\hline $10 / 14 / 2005$ & $4: 00$ & 10.823 & 10.704 & 7.87 & 6.76 & 4.437 & 4.215 & 2.448 & 2.428 & 10.749 & 6.019 & 4.761 & 6.606 & 3.846 \\
\hline $10 / 14 / 2005$ & $5: 00$ & 10.825 & 10.704 & 7.87 & 6.758 & 4.435 & 4.215 & 2.456 & 2.428 & 10.749 & 6.019 & 4.759 & 6.608 & 3.842 \\
\hline $10 / 14 / 2005$ & $6: 00$ & 10.825 & 10.706 & 7.869 & 6.762 & 4.433 & 4.215 & 2.456 & 2.428 & 10.748 & 6.019 & 4.755 & 6.607 & 3.836 \\
\hline $10 / 14 / 2005$ & $7: 00$ & 10.83 & 10.713 & 7.875 & 6.767 & 4.437 & 4.215 & 2.459 & 2.434 & 10.746 & 6.019 & 4.755 & 6.608 & 3.836 \\
\hline
\end{tabular}


TABLE S1.2 (Cont.)

Water Level (ft below top of casing) at Indicated Well

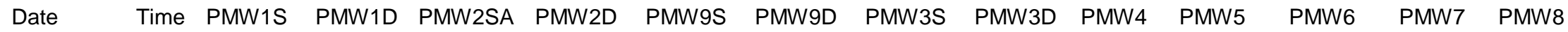

\begin{tabular}{|c|c|c|c|c|c|c|c|c|c|c|c|c|c|c|}
\hline $10 / 14 / 2005$ & $8: 00$ & 10.835 & 10.72 & 7.877 & 6.771 & 4.438 & 4.215 & 2.466 & 2.438 & 10.748 & 6.016 & 4.753 & 6.611 & 3.832 \\
\hline $10 / 14 / 2005$ & $9: 00$ & 10.843 & 10.73 & 7.883 & 6.78 & 4.442 & 4.217 & 2.471 & 2.447 & 10.748 & 6.019 & 4.757 & 6.611 & 3.832 \\
\hline $10 / 14 / 2005$ & $10: 00$ & 10.847 & 10.737 & 7.889 & 6.785 & 4.446 & 4.222 & 2.482 & 2.453 & 10.749 & 6.027 & 4.769 & 6.618 & 3.84 \\
\hline $10 / 14 / 2005$ & $11: 00$ & 10.853 & 10.74 & 7.893 & 6.789 & 4.451 & 4.22 & 2.484 & 2.462 & 10.751 & 6.032 & 4.782 & 6.62 & 3.862 \\
\hline $10 / 14 / 2005$ & $12: 00$ & 10.857 & 10.746 & 7.899 & 6.798 & 4.465 & 4.224 & 2.494 & 2.472 & 10.755 & 6.043 & 4.802 & 6.628 & 3.898 \\
\hline $10 / 14 / 2005$ & $13: 00$ & 10.86 & 10.746 & 7.903 & 6.8 & 4.472 & 4.222 & 2.499 & 2.478 & 10.759 & 6.054 & 4.82 & 6.632 & 3.937 \\
\hline $10 / 14 / 2005$ & $14: 00$ & 10.86 & 10.742 & 7.905 & 6.8 & 4.48 & 4.224 & 2.509 & 2.48 & 10.765 & 6.063 & 4.84 & 6.637 & 3.977 \\
\hline $10 / 14 / 2005$ & $15: 00$ & 10.86 & 10.74 & 7.904 & 6.798 & 4.491 & 4.226 & 2.507 & 2.485 & 10.77 & 6.074 & 4.853 & 6.641 & 4.014 \\
\hline $10 / 14 / 2005$ & $16: 00$ & 10.86 & 10.74 & 7.908 & 6.8 & 4.503 & 4.229 & 2.509 & 2.487 & 10.776 & 6.078 & 4.869 & 6.643 & 4.04 \\
\hline $10 / 14 / 2005$ & $17: 00$ & 10.863 & 10.744 & 7.912 & 6.805 & 4.515 & 4.235 & 2.521 & 2.493 & 10.784 & 6.087 & 4.878 & 6.647 & 4.06 \\
\hline $10 / 14 / 2005$ & $18: 00$ & 10.865 & 10.748 & 7.914 & 6.809 & 4.524 & 4.24 & 2.524 & 2.495 & 10.792 & 6.089 & 4.882 & 6.649 & 4.067 \\
\hline $10 / 14 / 2005$ & $19: 00$ & 10.87 & 10.751 & 7.916 & 6.814 & 4.528 & 4.244 & 2.516 & 2.495 & 10.795 & 6.087 & 4.879 & 6.649 & 4.047 \\
\hline $10 / 14 / 2005$ & $20: 00$ & 10.872 & 10.759 & 7.918 & 6.818 & 4.532 & 4.251 & 2.516 & 2.497 & 10.799 & 6.085 & 4.871 & 6.649 & 4.024 \\
\hline $10 / 14 / 2005$ & $21: 00$ & 10.877 & 10.766 & 7.921 & 6.823 & 4.532 & 4.257 & 2.527 & 2.499 & 10.799 & 6.083 & 4.867 & 6.651 & 4.002 \\
\hline $10 / 14 / 2005$ & $22: 00$ & 10.882 & 10.771 & 7.923 & 6.827 & 4.53 & 4.262 & 2.527 & 2.501 & 10.801 & 6.076 & 4.859 & 6.651 & 3.984 \\
\hline $10 / 14 / 2005$ & $23: 00$ & 10.887 & 10.777 & 7.925 & 6.827 & 4.524 & 4.268 & 2.529 & 2.501 & 10.801 & 6.076 & 4.853 & 6.651 & 3.964 \\
\hline $10 / 15 / 2005$ & $0: 00$ & 10.892 & 10.779 & 7.923 & 6.827 & 4.516 & 4.271 & 2.527 & 2.499 & 10.801 & 6.072 & 4.843 & 6.649 & 3.945 \\
\hline $10 / 15 / 2005$ & $1: 00$ & 10.895 & 10.781 & 7.925 & 6.827 & 4.51 & 4.271 & 2.524 & 2.5 & 10.799 & 6.067 & 4.838 & 6.647 & 3.928 \\
\hline $10 / 15 / 2005$ & $2: 00$ & 10.895 & 10.782 & 7.924 & 6.825 & 4.504 & 4.271 & 2.522 & 2.496 & 10.797 & 6.063 & 4.83 & 6.645 & 3.913 \\
\hline $10 / 15 / 2005$ & $3: 00$ & 10.897 & 10.786 & 7.926 & 6.825 & 4.5 & 4.273 & 2.522 & 2.496 & 10.795 & 6.059 & 4.824 & 6.645 & 3.901 \\
\hline $10 / 15 / 2005$ & $4: 00$ & 10.899 & 10.784 & 7.924 & 6.823 & 4.492 & 4.273 & 2.512 & 2.493 & 10.792 & 6.056 & 4.818 & 6.645 & 3.889 \\
\hline $10 / 15 / 2005$ & $5: 00$ & 10.899 & 10.786 & 7.924 & 6.825 & 4.486 & 4.273 & 2.512 & 2.491 & 10.792 & 6.052 & 4.814 & 6.643 & 3.879 \\
\hline $10 / 15 / 2005$ & $6: 00$ & 10.902 & 10.79 & 7.926 & 6.829 & 4.486 & 4.273 & 2.522 & 2.496 & 10.79 & 6.054 & 4.814 & 6.643 & 3.873 \\
\hline $10 / 15 / 2005$ & $7: 00$ & 10.904 & 10.792 & 7.928 & 6.834 & 4.484 & 4.275 & 2.522 & 2.498 & 10.79 & 6.054 & 4.81 & 6.645 & 3.865 \\
\hline $10 / 15 / 2005$ & $8: 00$ & 10.909 & 10.795 & 7.928 & 6.834 & 4.482 & 4.275 & 2.524 & 2.5 & 10.788 & 6.052 & 4.806 & 6.645 & 3.859 \\
\hline $10 / 15 / 2005$ & $9: 00$ & 10.912 & 10.799 & 7.93 & 6.834 & 4.478 & 4.275 & 2.524 & 2.498 & 10.792 & 6.05 & 4.804 & 6.647 & 3.851 \\
\hline $10 / 15 / 2005$ & $10: 00$ & 10.914 & 10.801 & 7.932 & 6.834 & 4.476 & 4.273 & 2.522 & 2.502 & 10.79 & 6.052 & 4.81 & 6.653 & 3.847 \\
\hline $10 / 15 / 2005$ & $11: 00$ & 10.914 & 10.801 & 7.934 & 6.836 & 4.476 & 4.273 & 2.532 & 2.506 & 10.79 & 6.057 & 4.822 & 6.657 & 3.859 \\
\hline $10 / 15 / 2005$ & $12: 00$ & 10.912 & 10.794 & 7.932 & 6.832 & 4.476 & 4.273 & 2.532 & 2.504 & 10.788 & 6.059 & 4.834 & 6.659 & 3.881 \\
\hline $10 / 15 / 2005$ & $13: 00$ & 10.904 & 10.781 & 7.929 & 6.823 & 4.475 & 4.268 & 2.527 & 2.5 & 10.79 & 6.063 & 4.847 & 6.659 & 3.905 \\
\hline $10 / 15 / 2005$ & $14: 00$ & 10.895 & 10.768 & 7.923 & 6.814 & 4.475 & 4.262 & 2.519 & 2.493 & 10.79 & 6.063 & 4.857 & 6.655 & 3.932 \\
\hline $10 / 15 / 2005$ & $15: 00$ & 10.885 & 10.755 & 7.919 & 6.807 & 4.479 & 4.259 & 2.517 & 2.489 & 10.792 & 6.07 & 4.869 & 6.655 & 3.964 \\
\hline $10 / 15 / 2005$ & $16: 00$ & 10.877 & 10.75 & 7.916 & 6.805 & 4.489 & 4.257 & 2.517 & 2.487 & 10.795 & 6.076 & 4.881 & 6.655 & 3.994 \\
\hline $10 / 15 / 2005$ & $17: 00$ & 10.872 & 10.744 & 7.914 & 6.803 & 4.498 & 4.255 & 2.512 & 2.489 & 10.797 & 6.081 & 4.889 & 6.657 & 4.014 \\
\hline
\end{tabular}


TABLE S1.2 (Cont.)

Water Level (ft below top of casing) at Indicated Well

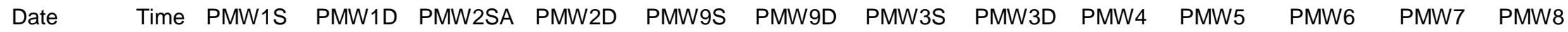

\begin{tabular}{|c|c|c|c|c|c|c|c|c|c|c|c|c|c|c|}
\hline $10 / 15 / 2005$ & $18: 00$ & 10.868 & 10.74 & 7.914 & 6.8 & 4.506 & 4.255 & 2.516 & 2.487 & 10.801 & 6.081 & 4.89 & 6.657 & 4.021 \\
\hline $10 / 15 / 2005$ & $19: 00$ & 10.863 & 10.737 & 7.91 & 6.799 & 4.51 & 4.255 & 2.504 & 2.481 & 10.803 & 6.077 & 4.887 & 6.653 & 4.006 \\
\hline $10 / 15 / 2005$ & $20: 00$ & 10.863 & 10.737 & 7.91 & 6.796 & 4.51 & 4.257 & 2.509 & 2.479 & 10.803 & 6.07 & 4.879 & 6.649 & 3.986 \\
\hline $10 / 15 / 2005$ & $21: 00$ & 10.863 & 10.739 & 7.908 & 6.799 & 4.508 & 4.258 & 2.507 & 2.479 & 10.803 & 6.068 & 4.869 & 6.647 & 3.966 \\
\hline $10 / 15 / 2005$ & $22: 00$ & 10.865 & 10.741 & 7.909 & 6.801 & 4.506 & 4.26 & 2.507 & 2.477 & 10.802 & 6.066 & 4.861 & 6.645 & 3.952 \\
\hline $10 / 15 / 2005$ & $23: 00$ & 10.865 & 10.744 & 7.909 & 6.801 & 4.501 & 4.258 & 2.502 & 2.477 & 10.801 & 6.059 & 4.855 & 6.643 & 3.938 \\
\hline $10 / 16 / 2005$ & $0: 00$ & 10.865 & 10.739 & 7.905 & 6.799 & 4.492 & 4.258 & 2.499 & 2.473 & 10.8 & 6.055 & 4.843 & 6.64 & 3.922 \\
\hline $10 / 16 / 2005$ & $1: 00$ & 10.863 & 10.739 & 7.903 & 6.796 & 4.486 & 4.258 & 2.494 & 2.468 & 10.796 & 6.052 & 4.84 & 6.64 & 3.908 \\
\hline $10 / 16 / 2005$ & $2: 00$ & 10.863 & 10.739 & 7.901 & 6.794 & 4.482 & 4.255 & 2.497 & 2.467 & 10.794 & 6.048 & 4.832 & 6.638 & 3.897 \\
\hline $10 / 16 / 2005$ & 3:00 & 10.863 & 10.741 & 7.901 & 6.794 & 4.478 & 4.255 & 2.495 & 2.467 & 10.792 & 6.048 & 4.826 & 6.634 & 3.887 \\
\hline $10 / 16 / 2005$ & $4: 00$ & 10.86 & 10.737 & 7.899 & 6.792 & 4.47 & 4.253 & 2.49 & 2.462 & 10.79 & 6.046 & 4.818 & 6.632 & 3.877 \\
\hline $10 / 16 / 2005$ & $5: 00$ & 10.86 & 10.735 & 7.897 & 6.788 & 4.464 & 4.249 & 2.487 & 2.462 & 10.784 & 6.041 & 4.81 & 6.63 & 3.867 \\
\hline $10 / 16 / 2005$ & $6: 00$ & 10.858 & 10.737 & 7.897 & 6.79 & 4.462 & 4.249 & 2.479 & 2.46 & 10.783 & 6.039 & 4.808 & 6.626 & 3.861 \\
\hline $10 / 16 / 2005$ & $7: 00$ & 10.858 & 10.739 & 7.897 & 6.79 & 4.46 & 4.247 & 2.487 & 2.46 & 10.783 & 6.037 & 4.806 & 6.626 & 3.857 \\
\hline $10 / 16 / 2005$ & $8: 00$ & 10.858 & 10.737 & 7.897 & 6.79 & 4.456 & 4.244 & 2.485 & 2.458 & 10.779 & 6.037 & 4.8 & 6.624 & 3.855 \\
\hline $10 / 16 / 2005$ & 9:00 & 10.858 & 10.739 & 7.898 & 6.79 & 4.456 & 4.247 & 2.49 & 2.46 & 10.779 & 6.037 & 4.8 & 6.628 & 3.853 \\
\hline $10 / 16 / 2005$ & $10: 00$ & 10.861 & 10.739 & 7.898 & 6.792 & 4.455 & 4.244 & 2.492 & 2.464 & 10.777 & 6.039 & 4.804 & 6.628 & 3.859 \\
\hline $10 / 16 / 2005$ & $11: 00$ & 10.863 & 10.741 & 7.898 & 6.794 & 4.457 & 4.242 & 2.497 & 2.469 & 10.775 & 6.048 & 4.812 & 6.628 & 3.877 \\
\hline $10 / 16 / 2005$ & $12: 00$ & 10.858 & 10.735 & 7.9 & 6.792 & 4.459 & 4.24 & 2.495 & 2.467 & 10.777 & 6.046 & 4.82 & 6.632 & 3.895 \\
\hline $10 / 16 / 2005$ & $13: 00$ & 10.853 & 10.73 & 7.899 & 6.785 & 4.461 & 4.238 & 2.495 & 2.464 & 10.779 & 6.05 & 4.83 & 6.634 & 3.916 \\
\hline $10 / 16 / 2005$ & $14: 00$ & 10.846 & 10.717 & 7.893 & 6.776 & 4.459 & 4.233 & 2.484 & 2.462 & 10.779 & 6.057 & 4.843 & 6.636 & 3.944 \\
\hline $10 / 16 / 2005$ & $15: 00$ & 10.839 & 10.708 & 7.892 & 6.772 & 4.464 & 4.231 & 2.489 & 2.46 & 10.781 & 6.059 & 4.853 & 6.636 & 3.976 \\
\hline $10 / 16 / 2005$ & $16: 00$ & 10.834 & 10.701 & 7.89 & 6.768 & 4.472 & 4.227 & 2.489 & 2.458 & 10.784 & 6.068 & 4.865 & 6.636 & 4.003 \\
\hline $10 / 16 / 2005$ & $17: 00$ & 10.826 & 10.693 & 7.886 & 6.765 & 4.478 & 4.224 & 2.477 & 2.454 & 10.784 & 6.072 & 4.873 & 6.632 & 4.019 \\
\hline $10 / 16 / 2005$ & $18: 00$ & 10.821 & 10.688 & 7.882 & 6.761 & 4.484 & 4.224 & 2.471 & 2.45 & 10.788 & 6.07 & 4.869 & 6.63 & 4.015 \\
\hline $10 / 16 / 2005$ & $19: 00$ & 10.816 & 10.684 & 7.88 & 6.756 & 4.482 & 4.222 & 2.471 & 2.444 & 10.786 & 6.068 & 4.861 & 6.628 & 3.997 \\
\hline $10 / 16 / 2005$ & $20: 00$ & 10.812 & 10.681 & 7.875 & 6.752 & 4.478 & 4.222 & 2.466 & 2.438 & 10.786 & 6.059 & 4.85 & 6.623 & 3.977 \\
\hline $10 / 16 / 2005$ & $21: 00$ & 10.809 & 10.677 & 7.873 & 6.75 & 4.474 & 4.22 & 2.459 & 2.431 & 10.784 & 6.055 & 4.838 & 6.619 & 3.955 \\
\hline $10 / 16 / 2005$ & $22: 00$ & 10.804 & 10.673 & 7.869 & 6.746 & 4.468 & 4.218 & 2.454 & 2.427 & 10.779 & 6.048 & 4.828 & 6.613 & 3.934 \\
\hline $10 / 16 / 2005$ & $23: 00$ & 10.801 & 10.668 & 7.865 & 6.741 & 4.458 & 4.218 & 2.447 & 2.421 & 10.777 & 6.044 & 4.816 & 6.609 & 3.918 \\
\hline $10 / 17 / 2005$ & $0: 00$ & 10.797 & 10.664 & 7.861 & 6.732 & 4.45 & 4.211 & 2.442 & 2.415 & 10.773 & 6.037 & 4.806 & 6.606 & 3.9 \\
\hline $10 / 17 / 2005$ & $1: 00$ & 10.792 & 10.657 & 7.855 & 6.728 & 4.437 & 4.207 & 2.427 & 2.406 & 10.769 & 6.033 & 4.794 & 6.602 & 3.884 \\
\hline $10 / 17 / 2005$ & $2: 00$ & 10.782 & 10.648 & 7.849 & 6.719 & 4.425 & 4.201 & 2.424 & 2.396 & 10.763 & 6.026 & 4.783 & 6.596 & 3.868 \\
\hline $10 / 17 / 2005$ & $3: 00$ & 10.78 & 10.646 & 7.847 & 6.719 & 4.423 & 4.198 & 2.424 & 2.396 & 10.762 & 6.026 & 4.779 & 6.596 & 3.859 \\
\hline
\end{tabular}


Water Level (ft below top of casing) at Indicated Well

$\begin{array}{lllllllllllllll}\text { Date } & \text { Time } & \text { PMW1S } & \text { PMW1D } & \text { PMW2SA } & \text { PMW2D } & \text { PMW9S } & \text { PMW9D } & \text { PMW3S } & \text { PMW3D } & \text { PMW4 } & \text { PMW5 } & \text { PMW6 } & \text { PMW7 } & \text { PMW8 }\end{array}$

\begin{tabular}{|c|c|c|c|c|c|c|c|c|c|c|c|c|c|c|}
\hline $10 / 17 / 2005$ & $4: 00$ & 10.775 & 10.642 & 7.843 & 6.715 & 4.415 & 4.194 & 2.411 & 2.389 & 10.758 & 6.022 & 4.771 & 6.589 & 3.849 \\
\hline $10 / 17 / 2005$ & $5: 00$ & 10.77 & 10.637 & 7.841 & 6.71 & 4.411 & 4.187 & 2.414 & 2.385 & 10.754 & 6.02 & 4.762 & 6.586 & 3.842 \\
\hline $10 / 17 / 2005$ & $6: 00$ & 10.767 & 10.635 & 7.837 & 6.713 & 4.409 & 4.187 & 2.414 & 2.383 & 10.752 & 6.02 & 4.758 & 6.585 & 3.835 \\
\hline $10 / 17 / 2005$ & $7: 00$ & 10.767 & 10.639 & 7.838 & 6.715 & 4.411 & 4.185 & 2.414 & 2.387 & 10.75 & 6.018 & 4.752 & 6.583 & 3.833 \\
\hline $10 / 17 / 2005$ & $8: 00$ & 10.772 & 10.644 & 7.842 & 6.719 & 4.411 & 4.185 & 2.419 & 2.389 & 10.748 & 6.017 & 4.75 & 6.583 & 3.829 \\
\hline $10 / 17 / 2005$ & $9: 00$ & 10.775 & 10.653 & 7.844 & 6.726 & 4.415 & 4.185 & 2.417 & 2.394 & 10.748 & 6.015 & 4.748 & 6.585 & 3.827 \\
\hline $10 / 17 / 2005$ & $10: 00$ & 10.782 & 10.666 & 7.85 & 6.737 & 4.423 & 4.185 & 2.435 & 2.404 & 10.746 & 6.02 & 4.762 & 6.588 & 3.831 \\
\hline $10 / 17 / 2005$ & $11: 00$ & 10.792 & 10.679 & 7.858 & 6.748 & 4.431 & 4.187 & 2.45 & 2.421 & 10.751 & 6.029 & 4.773 & 6.596 & 3.853 \\
\hline $10 / 17 / 2005$ & $12: 00$ & 10.8 & 10.684 & 7.866 & 6.75 & 4.438 & 4.189 & 2.452 & 2.427 & 10.752 & 6.033 & 4.789 & 6.6 & 3.882 \\
\hline $10 / 17 / 2005$ & $13: 00$ & 10.805 & 10.686 & 7.868 & 6.755 & 4.447 & 4.192 & 2.47 & 2.438 & 10.756 & 6.042 & 4.811 & 6.61 & 3.924 \\
\hline $10 / 17 / 2005$ & $14: 00$ & 10.802 & 10.682 & 7.87 & 6.755 & 4.457 & 4.192 & 2.47 & 2.44 & 10.76 & 6.046 & 4.83 & 6.615 & 3.96 \\
\hline $10 / 17 / 2005$ & $15: 00$ & 10.805 & 10.684 & 7.874 & 6.757 & 4.468 & 4.194 & 2.47 & 2.446 & 10.762 & 6.057 & 4.85 & 6.621 & 3.999 \\
\hline $10 / 17 / 2005$ & $16: 00$ & 10.807 & 10.684 & 7.875 & 6.761 & 4.479 & 4.198 & 2.479 & 2.451 & 10.77 & 6.066 & 4.863 & 6.623 & 4.025 \\
\hline $10 / 17 / 2005$ & $17: 00$ & 10.807 & 10.684 & 7.877 & 6.759 & 4.487 & 4.203 & 2.477 & 2.453 & 10.775 & 6.073 & 4.873 & 6.625 & 4.041 \\
\hline $10 / 17 / 2005$ & $18: 00$ & 10.807 & 10.684 & 7.879 & 6.761 & 4.493 & 4.205 & 2.482 & 2.451 & 10.779 & 6.077 & 4.877 & 6.627 & 4.043 \\
\hline $10 / 17 / 2005$ & $19: 00$ & 10.81 & 10.686 & 7.877 & 6.761 & 4.497 & 4.209 & 2.472 & 2.449 & 10.781 & 6.073 & 4.871 & 6.625 & 4.023 \\
\hline $10 / 17 / 2005$ & $20: 00$ & 10.814 & 10.693 & 7.88 & 6.768 & 4.499 & 4.216 & 2.475 & 2.451 & 10.783 & 6.066 & 4.861 & 6.625 & 4.002 \\
\hline $10 / 17 / 2005$ & $21: 00$ & 10.817 & 10.693 & 7.878 & 6.764 & 4.493 & 4.216 & 2.475 & 2.446 & 10.781 & 6.057 & 4.85 & 6.623 & 3.975 \\
\hline $10 / 17 / 2005$ & $22: 00$ & 10.819 & 10.699 & 7.88 & 6.766 & 4.489 & 4.22 & 2.475 & 2.446 & 10.781 & 6.055 & 4.842 & 6.623 & 3.955 \\
\hline $10 / 17 / 2005$ & $23: 00$ & 10.824 & 10.702 & 7.878 & 6.768 & 4.483 & 4.223 & 2.47 & 2.444 & 10.781 & 6.051 & 4.83 & 6.621 & 3.937 \\
\hline $10 / 18 / 2005$ & $0: 00$ & 10.824 & 10.702 & 7.878 & 6.764 & 4.474 & 4.223 & 2.465 & 2.444 & 10.779 & 6.046 & 4.822 & 6.615 & 3.916 \\
\hline $10 / 18 / 2005$ & $1: 00$ & 10.822 & 10.699 & 7.874 & 6.761 & 4.466 & 4.223 & 2.46 & 2.438 & 10.775 & 6.042 & 4.814 & 6.615 & 3.901 \\
\hline $10 / 18 / 2005$ & $2: 00$ & 10.819 & 10.695 & 7.872 & 6.757 & 4.458 & 4.22 & 2.455 & 2.432 & 10.771 & 6.037 & 4.805 & 6.612 & 3.884 \\
\hline $10 / 18 / 2005$ & $3: 00$ & 10.817 & 10.693 & 7.87 & 6.752 & 4.45 & 4.218 & 2.45 & 2.428 & 10.77 & 6.035 & 4.799 & 6.61 & 3.872 \\
\hline $10 / 18 / 2005$ & $4: 00$ & 10.812 & 10.688 & 7.866 & 6.748 & 4.442 & 4.216 & 2.445 & 2.423 & 10.764 & 6.033 & 4.791 & 6.606 & 3.86 \\
\hline $10 / 18 / 2005$ & $5: 00$ & 10.812 & 10.688 & 7.864 & 6.748 & 4.439 & 4.214 & 2.45 & 2.421 & 10.762 & 6.033 & 4.787 & 6.606 & 3.854 \\
\hline $10 / 18 / 2005$ & $6: 00$ & 10.81 & 10.686 & 7.864 & 6.748 & 4.434 & 4.212 & 2.448 & 2.421 & 10.76 & 6.031 & 4.785 & 6.604 & 3.85 \\
\hline $10 / 18 / 2005$ & $7: 00$ & 10.812 & 10.688 & 7.864 & 6.748 & 4.434 & 4.209 & 2.45 & 2.421 & 10.758 & 6.029 & 4.779 & 6.604 & 3.844 \\
\hline $10 / 18 / 2005$ & $8: 00$ & 10.81 & 10.686 & 7.863 & 6.748 & 4.43 & 4.209 & 2.44 & 2.419 & 10.754 & 6.024 & 4.775 & 6.6 & 3.838 \\
\hline $10 / 18 / 2005$ & $9: 00$ & 10.812 & 10.69 & 7.864 & 6.751 & 4.432 & 4.207 & 2.453 & 2.421 & 10.752 & 6.029 & 4.781 & 6.604 & 3.844 \\
\hline $10 / 18 / 2005$ & $10: 00$ & 10.812 & 10.69 & 7.866 & 6.751 & 4.434 & 4.205 & 2.458 & 2.428 & 10.752 & 6.033 & 4.791 & 6.604 & 3.86 \\
\hline $10 / 18 / 2005$ & $11: 00$ & 10.81 & 10.682 & 7.868 & 6.748 & 4.436 & 4.201 & 2.45 & 2.43 & 10.752 & 6.037 & 4.801 & 6.606 & 3.884 \\
\hline $10 / 18 / 2005$ & $12: 00$ & 10.807 & 10.679 & 7.87 & 6.746 & 4.443 & 4.198 & 2.463 & 2.432 & 10.754 & 6.044 & 4.816 & 6.614 & 3.925 \\
\hline $10 / 18 / 2005$ & $13: 00$ & 10.802 & 10.668 & 7.868 & 6.742 & 4.451 & 4.196 & 2.458 & 2.432 & 10.758 & 6.055 & 4.836 & 6.618 & 3.969 \\
\hline
\end{tabular}


Water Level (ft below top of casing) at Indicated Well

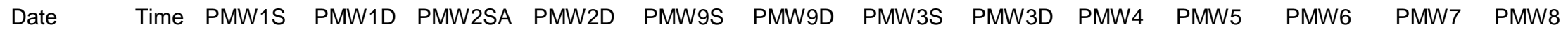

\begin{tabular}{|c|c|c|c|c|c|c|c|c|c|c|c|c|c|c|}
\hline $10 / 18 / 2005$ & $14: 00$ & 10.792 & 10.659 & 7.861 & 6.735 & 4.456 & 4.194 & 2.46 & 2.43 & 10.76 & 6.062 & 4.856 & 6.62 & 4.005 \\
\hline 10/18/2005 & $15: 00$ & 10.785 & 10.648 & 7.861 & 6.733 & 4.464 & 4.192 & 2.457 & 2.428 & 10.764 & 6.068 & 4.869 & 6.62 & 4.04 \\
\hline $10 / 18 / 2005$ & $16: 00$ & 10.78 & 10.642 & 7.859 & 6.728 & 4.478 & 4.189 & 2.457 & 2.426 & 10.77 & 6.079 & 4.881 & 6.62 & 4.068 \\
\hline $10 / 18 / 2005$ & $17: 00$ & 10.775 & 10.639 & 7.859 & 6.733 & 4.489 & 4.192 & 2.455 & 2.428 & 10.777 & 6.084 & 4.891 & 6.618 & 4.089 \\
\hline $10 / 18 / 2005$ & $18: 00$ & 10.773 & 10.639 & 7.859 & 6.733 & 4.499 & 4.192 & 2.46 & 2.428 & 10.779 & 6.084 & 4.893 & 6.618 & 4.099 \\
\hline $10 / 18 / 2005$ & $19: 00$ & 10.773 & 10.639 & 7.857 & 6.728 & 4.505 & 4.196 & 2.45 & 2.426 & 10.783 & 6.081 & 4.883 & 6.618 & 4.085 \\
\hline $10 / 18 / 2005$ & $20: 00$ & 10.773 & 10.64 & 7.855 & 6.731 & 4.505 & 4.198 & 2.45 & 2.42 & 10.781 & 6.07 & 4.869 & 6.614 & 4.054 \\
\hline $10 / 18 / 2005$ & $21: 00$ & 10.773 & 10.64 & 7.854 & 6.728 & 4.501 & 4.201 & 2.445 & 2.415 & 10.781 & 6.062 & 4.856 & 6.608 & 4.028 \\
\hline $10 / 18 / 2005$ & $22: 00$ & 10.773 & 10.644 & 7.852 & 6.728 & 4.495 & 4.201 & 2.442 & 2.413 & 10.779 & 6.051 & 4.844 & 6.604 & 4.002 \\
\hline $10 / 18 / 2005$ & $23: 00$ & 10.787 & 10.671 & 7.864 & 6.755 & 4.51 & 4.207 & 2.462 & 2.432 & 10.779 & 6.06 & 4.85 & 6.612 & 4 \\
\hline $10 / 19 / 2005$ & $0: 00$ & 10.802 & 10.69 & 7.873 & 6.764 & 4.512 & 4.214 & 2.477 & 2.447 & 10.785 & 6.059 & 4.852 & 6.612 & 3.991 \\
\hline 10/19/2005 & $1: 00$ & 10.814 & 10.705 & 7.879 & 6.775 & 4.514 & 4.22 & 2.485 & 2.457 & 10.785 & 6.06 & 4.85 & 6.62 & 3.982 \\
\hline $10 / 19 / 2005$ & $2: 00$ & 10.827 & 10.719 & 7.889 & 6.789 & 4.518 & 4.227 & 2.493 & 2.466 & 10.789 & 6.06 & 4.848 & 6.623 & 3.971 \\
\hline 10/19/2005 & $3: 00$ & 10.835 & 10.726 & 7.892 & 6.79 & 4.51 & 4.23 & 2.493 & 2.464 & 10.787 & 6.053 & 4.842 & 6.623 & 3.955 \\
\hline $10 / 19 / 2005$ & $4: 00$ & 10.842 & 10.736 & 7.895 & 6.795 & 4.506 & 4.234 & 2.495 & 2.466 & 10.787 & 6.053 & 4.844 & 6.627 & 3.945 \\
\hline $10 / 19 / 2005$ & $5: 00$ & 10.852 & 10.739 & 7.898 & 6.797 & 4.502 & 4.239 & 2.49 & 2.472 & 10.787 & 6.049 & 4.836 & 6.629 & 3.931 \\
\hline $10 / 19 / 2005$ & $6: 00$ & 10.857 & 10.745 & 7.899 & 6.8 & 4.496 & 4.243 & 2.49 & 2.472 & 10.785 & 6.047 & 4.832 & 6.629 & 3.917 \\
\hline $10 / 19 / 2005$ & $7: 00$ & 10.862 & 10.754 & 7.904 & 6.806 & 4.496 & 4.245 & 2.496 & 2.476 & 10.785 & 6.049 & 4.83 & 6.631 & 3.911 \\
\hline $10 / 19 / 2005$ & $8: 00$ & 10.866 & 10.755 & 7.906 & 6.804 & 4.49 & 4.247 & 2.496 & 2.476 & 10.781 & 6.044 & 4.826 & 6.631 & 3.899 \\
\hline $10 / 19 / 2005$ & $9: 00$ & 10.871 & 10.763 & 7.91 & 6.811 & 4.49 & 4.252 & 2.509 & 2.483 & 10.783 & 6.044 & 4.828 & 6.637 & 3.894 \\
\hline $10 / 19 / 2005$ & $10: 00$ & 10.881 & 10.774 & 7.914 & 6.82 & 4.496 & 4.254 & 2.509 & 2.489 & 10.783 & 6.049 & 4.83 & 6.641 & 3.892 \\
\hline $10 / 19 / 2005$ & $11: 00$ & 10.881 & 10.772 & 7.916 & 6.817 & 4.488 & 4.256 & 2.514 & 2.487 & 10.781 & 6.044 & 4.832 & 6.643 & 3.888 \\
\hline $10 / 19 / 2005$ & $12: 00$ & 10.879 & 10.763 & 7.912 & 6.806 & 4.478 & 4.254 & 2.506 & 2.479 & 10.778 & 6.042 & 4.834 & 6.641 & 3.888 \\
\hline $10 / 19 / 2005$ & $13: 00$ & 10.876 & 10.759 & 7.911 & 6.804 & 4.478 & 4.254 & 2.506 & 2.477 & 10.778 & 6.044 & 4.838 & 6.643 & 3.903 \\
\hline $10 / 19 / 2005$ & $14: 00$ & 10.866 & 10.744 & 7.903 & 6.793 & 4.47 & 4.25 & 2.496 & 2.468 & 10.774 & 6.036 & 4.83 & 6.639 & 3.899 \\
\hline $10 / 19 / 2005$ & $15: 00$ & 10.869 & 10.747 & 7.903 & 6.795 & 4.474 & 4.247 & 2.491 & 2.47 & 10.774 & 6.04 & 4.834 & 6.637 & 3.905 \\
\hline $10 / 19 / 2005$ & $16: 00$ & 10.866 & 10.745 & 7.901 & 6.793 & 4.474 & 4.247 & 2.488 & 2.466 & 10.772 & 6.042 & 4.838 & 6.637 & 3.905 \\
\hline $10 / 19 / 2005$ & $17: 00$ & 10.864 & 10.743 & 7.901 & 6.791 & 4.472 & 4.247 & 2.493 & 2.464 & 10.774 & 6.042 & 4.836 & 6.639 & 3.905 \\
\hline $10 / 19 / 2005$ & $18: 00$ & 10.857 & 10.726 & 7.895 & 6.78 & 4.462 & 4.241 & 2.478 & 2.458 & 10.77 & 6.031 & 4.824 & 6.629 & 3.893 \\
\hline $10 / 19 / 2005$ & $19: 00$ & 10.849 & 10.725 & 7.893 & 6.775 & 4.458 & 4.241 & 2.478 & 2.449 & 10.768 & 6.033 & 4.824 & 6.629 & 3.887 \\
\hline $10 / 19 / 2005$ & $20: 00$ & 10.847 & 10.706 & 7.885 & 6.753 & 4.452 & 4.236 & 2.47 & 2.433 & 10.764 & 6.027 & 4.814 & 6.614 & 3.872 \\
\hline $10 / 19 / 2005$ & $21: 00$ & 10.844 & 10.716 & 7.885 & 6.769 & 4.45 & 4.234 & 2.461 & 2.458 & 10.762 & 6.02 & 4.807 & 6.62 & 3.86 \\
\hline $10 / 19 / 2005$ & $22: 00$ & 10.835 & 10.701 & 7.879 & 6.755 & 4.44 & 4.227 & 2.456 & 2.433 & 10.757 & 6.015 & 4.795 & 6.61 & 3.842 \\
\hline $10 / 19 / 2005$ & $23: 00$ & 10.835 & 10.699 & 7.879 & 6.758 & 4.439 & 4.227 & 2.461 & 2.437 & 10.743 & 6.016 & 4.791 & 6.62 & 3.832 \\
\hline
\end{tabular}


Water Level (ft below top of casing) at Indicated Well

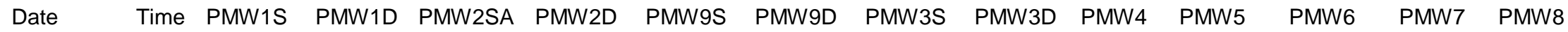

\begin{tabular}{|c|c|c|c|c|c|c|c|c|c|c|c|c|c|c|}
\hline 10/20/2005 & 0:00 & 10.849 & 10.703 & 7.881 & 6.766 & 4.444 & 4.225 & 2.466 & 2.439 & 10.743 & 6.016 & 4.793 & 6.627 & 3.83 \\
\hline $10 / 20 / 2005$ & 1:00 & 10.852 & 10.717 & 7.884 & 6.771 & 4.442 & 4.223 & 2.464 & 2.439 & 10.747 & 5.996 & 4.777 & 6.627 & 3.804 \\
\hline 10/20/2005 & $2: 00$ & 10.835 & 10.745 & 7.87 & 6.78 & 4.338 & 4.219 & 2.411 & 2.441 & 10.763 & 5.857 & 4.57 & 6.606 & 3.519 \\
\hline $10 / 20 / 2005$ & 3:00 & 10.808 & 10.761 & 7.845 & 6.786 & 4.082 & 4.208 & 2.359 & 2.399 & 10.778 & 5.747 & 4.399 & 6.597 & 3.315 \\
\hline $10 / 20 / 2005$ & $4: 00$ & 10.808 & 10.752 & 7.829 & 6.771 & 3.921 & 4.19 & 2.346 & 2.336 & 10.761 & 5.762 & 4.374 & 6.572 & 3.268 \\
\hline $10 / 20 / 2005$ & 5:00 & 10.798 & 10.756 & 7.82 & 6.74 & 3.852 & 4.168 & 2.346 & 2.324 & 10.761 & 5.786 & 4.388 & 6.57 & 3.296 \\
\hline $10 / 20 / 2005$ & $6: 00$ & 10.798 & 10.677 & 7.819 & 6.696 & 3.862 & 4.148 & 2.351 & 2.328 & 10.683 & 5.817 & 4.411 & 6.496 & 3.356 \\
\hline $10 / 20 / 2005$ & 7:00 & 10.8 & 10.679 & 7.819 & 6.7 & 3.897 & 4.132 & 2.359 & 2.334 & 10.677 & 5.842 & 4.433 & 6.505 & 3.414 \\
\hline $10 / 20 / 2005$ & $8: 00$ & 10.805 & 10.685 & 7.823 & 6.707 & 3.937 & 4.121 & 2.369 & 2.343 & 10.675 & 5.861 & 4.452 & 6.515 & 3.46 \\
\hline $10 / 20 / 2005$ & 9:00 & 10.817 & 10.694 & 7.831 & 6.716 & 3.978 & 4.115 & 2.382 & 2.355 & 10.673 & 5.879 & 4.476 & 6.523 & 3.502 \\
\hline $10 / 20 / 2005$ & $10: 00$ & 10.83 & 10.703 & 7.837 & 6.724 & 4.012 & 4.111 & 2.392 & 2.366 & 10.675 & 5.89 & 4.494 & 6.53 & 3.536 \\
\hline 10/20/2005 & $11: 00$ & 10.835 & 10.708 & 7.841 & 6.729 & 4.039 & 4.108 & 2.397 & 2.372 & 10.675 & 5.899 & 4.509 & 6.538 & 3.561 \\
\hline $10 / 20 / 2005$ & $12: 00$ & 10.837 & 10.712 & 7.847 & 6.731 & 4.066 & 4.108 & 2.402 & 2.378 & 10.677 & 5.908 & 4.527 & 6.547 & 3.586 \\
\hline $10 / 20 / 2005$ & $13: 00$ & 10.835 & 10.71 & 7.847 & 6.734 & 4.087 & 4.106 & 2.405 & 2.378 & 10.677 & 5.916 & 4.539 & 6.553 & 3.609 \\
\hline $10 / 20 / 2005$ & $14: 00$ & 10.83 & 10.706 & 7.849 & 6.734 & 4.104 & 4.108 & 2.407 & 2.383 & 10.679 & 5.921 & 4.552 & 6.557 & 3.632 \\
\hline $10 / 20 / 2005$ & $15: 00$ & 10.842 & 10.716 & 7.853 & 6.738 & 4.129 & 4.113 & 2.409 & 2.389 & 10.681 & 5.934 & 4.572 & 6.563 & 3.658 \\
\hline $10 / 20 / 2005$ & $16: 00$ & 10.837 & 10.712 & 7.855 & 6.742 & 4.146 & 4.115 & 2.42 & 2.393 & 10.683 & 5.939 & 4.584 & 6.566 & 3.677 \\
\hline $10 / 20 / 2005$ & $17: 00$ & 10.847 & 10.717 & 7.859 & 6.749 & 4.168 & 4.119 & 2.427 & 2.399 & 10.687 & 5.947 & 4.601 & 6.574 & 3.699 \\
\hline $10 / 20 / 2005$ & 18:00 & 10.85 & 10.721 & 7.861 & 6.751 & 4.183 & 4.124 & 2.425 & 2.406 & 10.689 & 5.952 & 4.611 & 6.578 & 3.712 \\
\hline $10 / 20 / 2005$ & 19:00 & 10.872 & 10.734 & 7.869 & 6.767 & 4.206 & 4.133 & 2.445 & 2.418 & 10.692 & 5.958 & 4.627 & 6.584 & 3.727 \\
\hline $10 / 20 / 2005$ & 20:00 & 10.85 & 10.741 & 7.875 & 6.769 & 4.22 & 4.139 & 2.45 & 2.425 & 10.698 & 5.958 & 4.633 & 6.587 & 3.731 \\
\hline $10 / 20 / 2005$ & $21: 00$ & 10.857 & 10.748 & 7.879 & 6.776 & 4.233 & 4.146 & 2.455 & 2.429 & 10.7 & 5.963 & 4.642 & 6.593 & 3.736 \\
\hline $10 / 20 / 2005$ & $22: 00$ & 10.867 & 10.761 & 7.888 & 6.789 & 4.25 & 4.155 & 2.46 & 2.439 & 10.704 & 5.967 & 4.652 & 6.601 & 3.747 \\
\hline $10 / 20 / 2005$ & 23:00 & 10.872 & 10.763 & 7.889 & 6.789 & 4.256 & 4.159 & 2.47 & 2.444 & 10.706 & 5.968 & 4.658 & 6.601 & 3.749 \\
\hline $10 / 21 / 2005$ & 0:00 & 10.872 & 10.761 & 7.889 & 6.787 & 4.26 & 4.164 & 2.465 & 2.441 & 10.708 & 5.967 & 4.658 & 6.601 & 3.747 \\
\hline $10 / 21 / 2005$ & $1: 00$ & 10.877 & 10.765 & 7.891 & 6.791 & 4.269 & 4.17 & 2.473 & 2.446 & 10.709 & 5.969 & 4.666 & 6.605 & 3.751 \\
\hline $10 / 21 / 2005$ & $2: 00$ & 10.879 & 10.77 & 7.895 & 6.793 & 4.277 & 4.175 & 2.472 & 2.448 & 10.711 & 5.972 & 4.67 & 6.607 & 3.753 \\
\hline $10 / 21 / 2005$ & 3:00 & 10.882 & 10.772 & 7.897 & 6.796 & 4.286 & 4.181 & 2.478 & 2.45 & 10.711 & 5.976 & 4.676 & 6.611 & 3.757 \\
\hline $10 / 21 / 2005$ & 4:00 & 10.882 & 10.77 & 7.895 & 6.791 & 4.286 & 4.181 & 2.472 & 2.446 & 10.711 & 5.976 & 4.68 & 6.611 & 3.753 \\
\hline $10 / 21 / 2005$ & 5:00 & 10.884 & 10.774 & 7.899 & 6.798 & 4.296 & 4.186 & 2.477 & 2.452 & 10.713 & 5.976 & 4.682 & 6.614 & 3.757 \\
\hline $10 / 21 / 2005$ & $6: 00$ & 10.891 & 10.785 & 7.906 & 6.809 & 4.309 & 4.192 & 2.491 & 2.463 & 10.717 & 5.985 & 4.691 & 6.62 & 3.767 \\
\hline $10 / 21 / 2005$ & 7:00 & 10.896 & 10.789 & 7.908 & 6.812 & 4.314 & 4.197 & 2.49 & 2.465 & 10.717 & 5.983 & 4.695 & 6.62 & 3.767 \\
\hline $10 / 21 / 2005$ & $8: 00$ & 10.899 & 10.79 & 7.91 & 6.812 & 4.318 & 4.201 & 2.485 & 2.465 & 10.719 & 5.985 & 4.699 & 6.622 & 3.767 \\
\hline $10 / 21 / 2005$ & 9:00 & 10.909 & 10.803 & 7.916 & 6.825 & 4.331 & 4.208 & 2.503 & 2.478 & 10.721 & 5.994 & 4.711 & 6.63 & 3.775 \\
\hline
\end{tabular}


Water Level (ft below top of casing) at Indicated Well

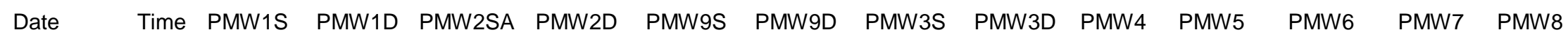

\begin{tabular}{|c|c|c|c|c|c|c|c|c|c|c|c|c|c|c|}
\hline 10/21/2005 & $10: 00$ & 10.911 & 10.801 & 7.918 & 6.823 & 4.333 & 4.21 & 2.503 & 2.48 & 10.723 & 5.987 & 4.713 & 6.632 & 3.773 \\
\hline 10/21/2005 & $11: 00$ & 10.919 & 10.809 & 7.922 & 6.827 & 4.339 & 4.215 & 2.51 & 2.482 & 10.727 & 5.992 & 4.721 & 6.633 & 3.775 \\
\hline $10 / 21 / 2005$ & $12: 00$ & 10.916 & 10.805 & 7.922 & 6.82 & 4.337 & 4.217 & 2.505 & 2.48 & 10.727 & 5.989 & 4.723 & 6.635 & 3.773 \\
\hline $10 / 21 / 2005$ & $13: 00$ & 10.914 & 10.801 & 7.921 & 6.82 & 4.338 & 4.217 & 2.506 & 2.478 & 10.727 & 5.996 & 4.733 & 6.637 & 3.785 \\
\hline $10 / 21 / 2005$ & $14: 00$ & 10.906 & 10.79 & 7.917 & 6.812 & 4.34 & 4.215 & 2.5 & 2.471 & 10.727 & 5.996 & 4.736 & 6.638 & 3.797 \\
\hline 10/21/2005 & $15: 00$ & 10.899 & 10.781 & 7.913 & 6.809 & 4.344 & 4.217 & 2.498 & 2.467 & 10.727 & 5.998 & 4.742 & 6.638 & 3.803 \\
\hline 10/21/2005 & $16: 00$ & 10.894 & 10.774 & 7.911 & 6.803 & 4.347 & 4.215 & 2.495 & 2.465 & 10.729 & 6.002 & 4.75 & 6.637 & 3.813 \\
\hline $10 / 21 / 2005$ & $17: 00$ & 10.887 & 10.77 & 7.91 & 6.803 & 4.353 & 4.215 & 2.493 & 2.463 & 10.731 & 6.005 & 4.754 & 6.635 & 3.819 \\
\hline $10 / 21 / 2005$ & $18: 00$ & 10.889 & 10.774 & 7.912 & 6.805 & 4.361 & 4.219 & 2.495 & 2.467 & 10.731 & 6.009 & 4.764 & 6.638 & 3.825 \\
\hline 10/21/2005 & $19: 00$ & 10.891 & 10.779 & 7.914 & 6.814 & 4.369 & 4.221 & 2.5 & 2.471 & 10.735 & 6.011 & 4.766 & 6.641 & 3.823 \\
\hline $10 / 21 / 2005$ & $20: 00$ & 10.899 & 10.785 & 7.917 & 6.818 & 4.374 & 4.223 & 2.498 & 2.478 & 10.738 & 6.007 & 4.77 & 6.641 & 3.819 \\
\hline 10/21/2005 & $21: 00$ & 10.901 & 10.787 & 7.919 & 6.818 & 4.374 & 4.226 & 2.503 & 2.476 & 10.738 & 6.007 & 4.77 & 6.643 & 3.811 \\
\hline $10 / 21 / 2005$ & $22: 00$ & 10.904 & 10.79 & 7.921 & 6.82 & 4.376 & 4.23 & 2.5 & 2.48 & 10.738 & 6.007 & 4.772 & 6.645 & 3.805 \\
\hline $10 / 21 / 2005$ & $23: 00$ & 10.906 & 10.794 & 7.921 & 6.82 & 4.378 & 4.233 & 2.5 & 2.48 & 10.74 & 6.007 & 4.772 & 6.647 & 3.801 \\
\hline $10 / 22 / 2005$ & $0: 00$ & 10.904 & 10.789 & 7.919 & 6.816 & 4.374 & 4.232 & 2.498 & 2.478 & 10.74 & 6.002 & 4.77 & 6.643 & 3.795 \\
\hline $10 / 22 / 2005$ & $1: 00$ & 10.899 & 10.783 & 7.915 & 6.812 & 4.366 & 4.233 & 2.498 & 2.47 & 10.738 & 5.998 & 4.766 & 6.641 & 3.785 \\
\hline $10 / 22 / 2005$ & $2: 00$ & 10.894 & 10.778 & 7.913 & 6.807 & 4.366 & 4.233 & 2.495 & 2.467 & 10.736 & 5.996 & 4.762 & 6.64 & 3.779 \\
\hline $10 / 22 / 2005$ & $3: 00$ & 10.891 & 10.776 & 7.911 & 6.807 & 4.366 & 4.23 & 2.493 & 2.465 & 10.736 & 5.994 & 4.762 & 6.64 & 3.777 \\
\hline $10 / 22 / 2005$ & $4: 00$ & 10.889 & 10.772 & 7.909 & 6.803 & 4.365 & 4.23 & 2.49 & 2.463 & 10.735 & 5.994 & 4.76 & 6.638 & 3.771 \\
\hline $10 / 22 / 2005$ & $5: 00$ & 10.879 & 10.761 & 7.903 & 6.794 & 4.359 & 4.228 & 2.483 & 2.455 & 10.733 & 5.985 & 4.75 & 6.632 & 3.761 \\
\hline $10 / 22 / 2005$ & $6: 00$ & 10.874 & 10.758 & 7.899 & 6.789 & 4.355 & 4.226 & 2.47 & 2.449 & 10.729 & 5.985 & 4.75 & 6.632 & 3.759 \\
\hline 10/22/2005 & $7: 00$ & 10.877 & 10.761 & 7.901 & 6.796 & 4.361 & 4.226 & 2.483 & 2.453 & 10.727 & 5.992 & 4.754 & 6.636 & 3.763 \\
\hline $10 / 22 / 2005$ & $8: 00$ & 10.869 & 10.75 & 7.895 & 6.783 & 4.352 & 4.219 & 2.47 & 2.442 & 10.723 & 5.985 & 4.748 & 6.628 & 3.753 \\
\hline $10 / 22 / 2005$ & 9:00 & 10.874 & 10.761 & 7.899 & 6.798 & 4.36 & 4.222 & 2.483 & 2.457 & 10.725 & 5.983 & 4.75 & 6.641 & 3.761 \\
\hline $10 / 22 / 2005$ & $10: 00$ & 10.867 & 10.798 & 7.895 & 6.787 & 4.356 & 4.217 & 2.475 & 2.472 & 10.765 & 5.983 & 4.746 & 6.685 & 3.755 \\
\hline $10 / 22 / 2005$ & $11: 00$ & 10.864 & 10.747 & 7.893 & 6.785 & 4.354 & 4.215 & 2.47 & 2.444 & 10.721 & 5.979 & 4.74 & 6.624 & 3.749 \\
\hline $10 / 22 / 2005$ & $12: 00$ & 10.86 & 10.743 & 7.891 & 6.778 & 4.35 & 4.211 & 2.462 & 2.44 & 10.721 & 5.981 & 4.736 & 6.624 & 3.745 \\
\hline $10 / 22 / 2005$ & $13: 00$ & 10.852 & 10.736 & 7.887 & 6.772 & 4.346 & 4.208 & 2.46 & 2.432 & 10.716 & 5.979 & 4.729 & 6.62 & 3.741 \\
\hline $10 / 22 / 2005$ & $14: 00$ & 10.845 & 10.725 & 7.881 & 6.765 & 4.341 & 4.204 & 2.455 & 2.426 & 10.714 & 5.976 & 4.725 & 6.619 & 3.734 \\
\hline $10 / 22 / 2005$ & $15: 00$ & 10.84 & 10.716 & 7.876 & 6.758 & 4.34 & 4.202 & 2.445 & 2.421 & 10.712 & 5.976 & 4.725 & 6.601 & 3.736 \\
\hline $10 / 22 / 2005$ & $16: 00$ & 10.837 & 10.709 & 7.876 & 6.749 & 4.343 & 4.199 & 2.445 & 2.409 & 10.706 & 5.976 & 4.719 & 6.59 & 3.734 \\
\hline 10/22/2005 & $17: 00$ & 10.837 & 10.708 & 7.877 & 6.75 & 4.345 & 4.197 & 2.443 & 2.4 & 10.706 & 5.976 & 4.715 & 6.594 & 3.73 \\
\hline $10 / 22 / 2005$ & $18: 00$ & 10.837 & 10.725 & 7.879 & 6.759 & 4.348 & 4.195 & 2.443 & 2.381 & 10.687 & 5.974 & 4.709 & 6.613 & 3.722 \\
\hline $10 / 22 / 2005$ & $19: 00$ & 10.84 & 10.721 & 7.881 & 6.765 & 4.352 & 4.195 & 2.453 & 2.401 & 10.706 & 5.972 & 4.701 & 6.598 & 3.721 \\
\hline
\end{tabular}


Water Level (ft below top of casing) at Indicated Well

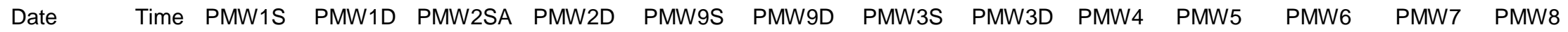

\begin{tabular}{|c|c|c|c|c|c|c|c|c|c|c|c|c|c|c|}
\hline $10 / 22 / 2005$ & $20: 00$ & 10.847 & 10.737 & 7.885 & 6.776 & 4.356 & 4.197 & 2.458 & 2.432 & 10.619 & 5.974 & 4.703 & 6.615 & 3.719 \\
\hline $10 / 22 / 2005$ & $21: 00$ & 10.855 & 10.745 & 7.888 & 6.783 & 4.361 & 4.199 & 2.466 & 2.432 & 10.584 & 5.974 & 4.701 & 6.617 & 3.719 \\
\hline $10 / 22 / 2005$ & $22: 00$ & 10.86 & 10.752 & 7.892 & 6.787 & 4.363 & 4.202 & 2.468 & 2.461 & 10.604 & 5.972 & 4.701 & 6.619 & 3.716 \\
\hline $10 / 22 / 2005$ & $23: 00$ & 10.864 & 10.756 & 7.894 & 6.794 & 4.365 & 4.204 & 2.473 & 2.42 & 10.568 & 5.974 & 4.703 & 6.623 & 3.719 \\
\hline $10 / 23 / 2005$ & $0: 00$ & 10.869 & 10.765 & 7.898 & 6.799 & 4.367 & 4.206 & 2.478 & 2.392 & 10.573 & 5.976 & 4.705 & 6.625 & 3.721 \\
\hline $10 / 23 / 2005$ & $1: 00$ & 10.877 & 10.767 & 7.902 & 6.801 & 4.367 & 4.208 & 2.478 & 2.449 & 10.558 & 5.974 & 4.707 & 6.627 & 3.721 \\
\hline $10 / 23 / 2005$ & $2: 00$ & 10.882 & 10.772 & 7.904 & 6.805 & 4.37 & 4.211 & 2.483 & 2.439 & 10.579 & 5.978 & 4.711 & 6.628 & 3.723 \\
\hline $10 / 23 / 2005$ & $3: 00$ & 10.887 & 10.781 & 7.908 & 6.81 & 4.374 & 4.218 & 2.493 & 2.43 & 10.573 & 5.98 & 4.717 & 6.634 & 3.728 \\
\hline $10 / 23 / 2005$ & $4: 00$ & 10.891 & 10.785 & 7.912 & 6.816 & 4.377 & 4.219 & 2.496 & 2.418 & 10.56 & 5.983 & 4.719 & 6.636 & 3.731 \\
\hline $10 / 23 / 2005$ & $5: 00$ & 10.897 & 10.791 & 7.916 & 6.821 & 4.379 & 4.224 & 2.501 & 2.485 & 10.564 & 5.98 & 4.725 & 6.638 & 3.732 \\
\hline $10 / 23 / 2005$ & $6: 00$ & 10.909 & 10.799 & 7.921 & 6.827 & 4.385 & 4.228 & 2.504 & 2.489 & 10.558 & 5.985 & 4.731 & 6.642 & 3.738 \\
\hline $10 / 23 / 2005$ & $7: 00$ & 10.912 & 10.805 & 7.924 & 6.832 & 4.387 & 4.233 & 2.506 & 2.468 & 10.533 & 5.985 & 4.735 & 6.646 & 3.741 \\
\hline $10 / 23 / 2005$ & $8: 00$ & 10.916 & 10.813 & 7.929 & 6.836 & 4.39 & 4.235 & 2.519 & 2.462 & 10.527 & 5.99 & 4.742 & 6.647 & 3.743 \\
\hline $10 / 23 / 2005$ & $9: 00$ & 10.921 & 10.818 & 7.933 & 6.841 & 4.393 & 4.242 & 2.524 & 2.493 & 10.585 & 5.989 & 4.748 & 6.651 & 3.749 \\
\hline $10 / 23 / 2005$ & $10: 00$ & 10.926 & 10.82 & 7.937 & 6.843 & 4.395 & 4.244 & 2.526 & 2.495 & 10.723 & 5.994 & 4.75 & 6.651 & 3.747 \\
\hline $10 / 23 / 2005$ & $11: 00$ & 10.929 & 10.823 & 7.935 & 6.845 & 4.395 & 4.244 & 2.524 & 2.499 & 10.725 & 5.992 & 4.754 & 6.653 & 3.749 \\
\hline $10 / 23 / 2005$ & $12: 00$ & 10.931 & 10.825 & 7.938 & 6.845 & 4.395 & 4.249 & 2.521 & 2.495 & 10.727 & 5.994 & 4.758 & 6.655 & 3.749 \\
\hline $10 / 23 / 2005$ & $13: 00$ & 10.934 & 10.825 & 7.938 & 6.845 & 4.396 & 4.251 & 2.521 & 2.497 & 10.725 & 5.996 & 4.76 & 6.653 & 3.751 \\
\hline $10 / 23 / 2005$ & $14: 00$ & 10.934 & 10.825 & 7.94 & 6.845 & 4.398 & 4.253 & 2.521 & 2.497 & 10.729 & 5.996 & 4.764 & 6.655 & 3.753 \\
\hline $10 / 23 / 2005$ & $15: 00$ & 10.936 & 10.827 & 7.94 & 6.845 & 4.4 & 4.253 & 2.529 & 2.499 & 10.729 & 5.996 & 4.77 & 6.655 & 3.759 \\
\hline $10 / 23 / 2005$ & $16: 00$ & 10.941 & 10.834 & 7.943 & 6.852 & 4.41 & 4.257 & 2.536 & 2.508 & 10.733 & 6.003 & 4.776 & 6.659 & 3.767 \\
\hline $10 / 23 / 2005$ & $17: 00$ & 10.943 & 10.838 & 7.949 & 6.858 & 4.412 & 4.262 & 2.536 & 2.51 & 10.735 & 6.003 & 4.78 & 6.663 & 3.769 \\
\hline $10 / 23 / 2005$ & $18: 00$ & 10.948 & 10.842 & 7.949 & 6.861 & 4.413 & 4.264 & 2.541 & 2.512 & 10.735 & 6.005 & 4.782 & 6.663 & 3.771 \\
\hline $10 / 23 / 2005$ & 19:00 & 10.951 & 10.844 & 7.951 & 6.858 & 4.413 & 4.266 & 2.536 & 2.515 & 10.739 & 6.007 & 4.784 & 6.667 & 3.771 \\
\hline $10 / 23 / 2005$ & $20: 00$ & 10.953 & 10.845 & 7.953 & 6.858 & 4.415 & 4.271 & 2.546 & 2.517 & 10.741 & 6.007 & 4.783 & 6.667 & 3.771 \\
\hline $10 / 23 / 2005$ & $21: 00$ & 10.956 & 10.845 & 7.953 & 6.863 & 4.415 & 4.273 & 2.541 & 2.517 & 10.743 & 6.005 & 4.786 & 6.668 & 3.771 \\
\hline $10 / 23 / 2005$ & $22: 00$ & 10.961 & 10.849 & 7.955 & 6.863 & 4.418 & 4.273 & 2.549 & 2.521 & 10.746 & 6.007 & 4.787 & 6.671 & 3.771 \\
\hline $10 / 23 / 2005$ & $23: 00$ & 10.961 & 10.851 & 7.957 & 6.865 & 4.418 & 4.277 & 2.549 & 2.521 & 10.744 & 6.005 & 4.787 & 6.671 & 3.769 \\
\hline $10 / 24 / 2005$ & $0: 00$ & 10.963 & 10.853 & 7.957 & 6.865 & 4.417 & 4.28 & 2.543 & 2.521 & 10.744 & 6.005 & 4.789 & 6.673 & 3.769 \\
\hline $10 / 24 / 2005$ & $1: 00$ & 10.963 & 10.851 & 7.955 & 6.863 & 4.414 & 4.28 & 2.543 & 2.521 & 10.746 & 6.003 & 4.785 & 6.671 & 3.765 \\
\hline $10 / 24 / 2005$ & $2: 00$ & 10.966 & 10.851 & 7.957 & 6.863 & 4.413 & 4.28 & 2.549 & 2.521 & 10.746 & 6.005 & 4.787 & 6.673 & 3.763 \\
\hline $10 / 24 / 2005$ & $3: 00$ & 10.966 & 10.853 & 7.959 & 6.868 & 4.415 & 4.282 & 2.546 & 2.523 & 10.748 & 6.007 & 4.787 & 6.674 & 3.763 \\
\hline $10 / 24 / 2005$ & $4: 00$ & 10.963 & 10.849 & 7.957 & 6.863 & 4.411 & 4.282 & 2.549 & 2.519 & 10.746 & 6.005 & 4.787 & 6.671 & 3.759 \\
\hline $10 / 24 / 2005$ & $5: 00$ & 10.963 & 10.847 & 7.955 & 6.863 & 4.409 & 4.282 & 2.541 & 2.517 & 10.746 & 6.005 & 4.785 & 6.671 & 3.758 \\
\hline
\end{tabular}


Water Level (ft below top of casing) at Indicated Well

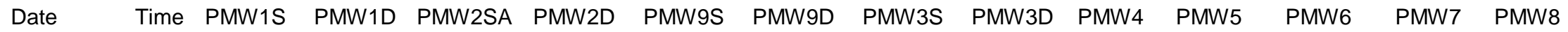

\begin{tabular}{|c|c|c|c|c|c|c|c|c|c|c|c|c|c|c|}
\hline $10 / 24 / 2005$ & $6: 00$ & 10.961 & 10.847 & 7.955 & 6.861 & 4.411 & 4.282 & 2.538 & 2.517 & 10.746 & 6.005 & 4.788 & 6.673 & 3.757 \\
\hline $10 / 24 / 2005$ & $7: 00$ & 10.966 & 10.849 & 7.955 & 6.863 & 4.41 & 4.282 & 2.541 & 2.519 & 10.746 & 6.009 & 4.787 & 6.673 & 3.758 \\
\hline $10 / 24 / 2005$ & $8: 00$ & 10.966 & 10.851 & 7.957 & 6.865 & 4.413 & 4.284 & 2.543 & 2.521 & 10.746 & 6.009 & 4.789 & 6.673 & 3.76 \\
\hline $10 / 24 / 2005$ & $9: 00$ & 10.966 & 10.854 & 7.959 & 6.87 & 4.417 & 4.284 & 2.554 & 2.538 & 10.747 & 6.012 & 4.789 & 6.674 & 3.762 \\
\hline $10 / 24 / 2005$ & $10: 00$ & 10.97 & 10.858 & 7.963 & 6.87 & 4.419 & 4.284 & 2.554 & 2.525 & 10.749 & 6.014 & 4.793 & 6.676 & 3.762 \\
\hline $10 / 24 / 2005$ & $11: 00$ & 10.968 & 10.856 & 7.961 & 6.868 & 4.417 & 4.284 & 2.556 & 2.525 & 10.75 & 6.014 & 4.791 & 6.676 & 3.76 \\
\hline $10 / 24 / 2005$ & $12: 00$ & 10.968 & 10.853 & 7.961 & 6.865 & 4.415 & 4.284 & 2.546 & 2.523 & 10.747 & 6.014 & 4.793 & 6.674 & 3.76 \\
\hline $10 / 24 / 2005$ & $13: 00$ & 10.958 & 10.842 & 7.955 & 6.859 & 4.409 & 4.28 & 2.543 & 2.512 & 10.744 & 6.009 & 4.791 & 6.671 & 3.76 \\
\hline $10 / 24 / 2005$ & $14: 00$ & 10.948 & 10.829 & 7.948 & 6.845 & 4.407 & 4.277 & 2.536 & 2.506 & 10.747 & 6.009 & 4.791 & 6.669 & 3.768 \\
\hline $10 / 24 / 2005$ & $15: 00$ & 10.943 & 10.822 & 7.944 & 6.841 & 4.407 & 4.273 & 2.533 & 2.502 & 10.743 & 6.012 & 4.793 & 6.667 & 3.778 \\
\hline $10 / 24 / 2005$ & $16: 00$ & 10.936 & 10.816 & 7.942 & 6.839 & 4.406 & 4.273 & 2.526 & 2.5 & 10.743 & 6.012 & 4.797 & 6.667 & 3.786 \\
\hline $10 / 24 / 2005$ & $17: 00$ & 10.931 & 10.811 & 7.94 & 6.837 & 4.41 & 4.271 & 2.531 & 2.5 & 10.743 & 6.012 & 4.799 & 6.667 & 3.79 \\
\hline $10 / 24 / 2005$ & $18: 00$ & 10.929 & 10.809 & 7.938 & 6.835 & 4.412 & 4.271 & 2.531 & 2.498 & 10.744 & 6.012 & 4.799 & 6.667 & 3.792 \\
\hline $10 / 24 / 2005$ & $19: 00$ & 10.926 & 10.809 & 7.938 & 6.837 & 4.414 & 4.271 & 2.531 & 2.5 & 10.745 & 6.014 & 4.799 & 6.667 & 3.788 \\
\hline $10 / 24 / 2005$ & $20: 00$ & 10.929 & 10.811 & 7.94 & 6.839 & 4.416 & 4.271 & 2.531 & 2.5 & 10.747 & 6.011 & 4.799 & 6.665 & 3.782 \\
\hline $10 / 24 / 2005$ & $21: 00$ & 10.931 & 10.813 & 7.94 & 6.844 & 4.415 & 4.271 & 2.533 & 2.502 & 10.747 & 6.012 & 4.797 & 6.665 & 3.778 \\
\hline $10 / 24 / 2005$ & $22: 00$ & 10.929 & 10.813 & 7.94 & 6.844 & 4.411 & 4.271 & 2.526 & 2.502 & 10.747 & 6.009 & 4.795 & 6.665 & 3.772 \\
\hline $10 / 24 / 2005$ & $23: 00$ & 10.926 & 10.807 & 7.937 & 6.839 & 4.407 & 4.271 & 2.528 & 2.498 & 10.745 & 6.005 & 4.789 & 6.665 & 3.768 \\
\hline $10 / 25 / 2005$ & $0: 00$ & 10.924 & 10.805 & 7.936 & 6.835 & 4.403 & 4.269 & 2.526 & 2.496 & 10.743 & 6.003 & 4.785 & 6.663 & 3.76 \\
\hline $10 / 25 / 2005$ & $1: 00$ & 10.921 & 10.805 & 7.934 & 6.837 & 4.403 & 4.269 & 2.526 & 2.496 & 10.743 & 6.003 & 4.785 & 6.661 & 3.758 \\
\hline $10 / 25 / 2005$ & $2: 00$ & 10.921 & 10.805 & 7.935 & 6.835 & 4.401 & 4.267 & 2.523 & 2.494 & 10.743 & 6.003 & 4.785 & 6.663 & 3.758 \\
\hline $10 / 25 / 2005$ & $3: 00$ & 10.921 & 10.805 & 7.935 & 6.835 & 4.399 & 4.267 & 2.526 & 2.494 & 10.743 & 6.001 & 4.783 & 6.663 & 3.756 \\
\hline $10 / 25 / 2005$ & $4: 00$ & 10.921 & 10.803 & 7.932 & 6.833 & 4.397 & 4.264 & 2.521 & 2.492 & 10.739 & 6.001 & 4.782 & 6.66 & 3.751 \\
\hline $10 / 25 / 2005$ & $5: 00$ & 10.921 & 10.805 & 7.933 & 6.835 & 4.398 & 4.267 & 2.523 & 2.496 & 10.741 & 6.001 & 4.782 & 6.661 & 3.753 \\
\hline $10 / 25 / 2005$ & $6: 00$ & 10.926 & 10.809 & 7.935 & 6.839 & 4.402 & 4.267 & 2.528 & 2.498 & 10.739 & 6.003 & 4.787 & 6.663 & 3.753 \\
\hline $10 / 25 / 2005$ & $7: 00$ & 10.926 & 10.813 & 7.937 & 6.837 & 4.402 & 4.267 & 2.528 & 2.5 & 10.741 & 6.002 & 4.785 & 6.663 & 3.753 \\
\hline $10 / 25 / 2005$ & $8: 00$ & 10.929 & 10.813 & 7.939 & 6.841 & 4.401 & 4.267 & 2.528 & 2.5 & 10.739 & 6.002 & 4.785 & 6.663 & 3.751 \\
\hline $10 / 25 / 2005$ & $9: 00$ & 10.934 & 10.82 & 7.943 & 6.848 & 4.407 & 4.269 & 2.536 & 2.519 & 10.741 & 6.003 & 4.791 & 6.667 & 3.758 \\
\hline $10 / 25 / 2005$ & $10: 00$ & 10.934 & 10.816 & 7.943 & 6.844 & 4.403 & 4.267 & 2.533 & 2.504 & 10.741 & 6 & 4.787 & 6.667 & 3.751 \\
\hline $10 / 25 / 2005$ & $11: 00$ & 10.931 & 10.813 & 7.939 & 6.841 & 4.398 & 4.267 & 2.531 & 2.5 & 10.737 & 5.998 & 4.785 & 6.663 & 3.747 \\
\hline $10 / 25 / 2005$ & $12: 00$ & 10.931 & 10.811 & 7.941 & 6.841 & 4.399 & 4.264 & 2.523 & 2.501 & 10.737 & 6.001 & 4.785 & 6.663 & 3.747 \\
\hline $10 / 25 / 2005$ & $13: 00$ & 10.919 & 10.8 & 7.935 & 6.83 & 4.391 & 4.26 & 2.521 & 2.492 & 10.735 & 5.998 & 4.783 & 6.661 & 3.749 \\
\hline $10 / 25 / 2005$ & $14: 00$ & 10.907 & 10.785 & 7.926 & 6.817 & 4.385 & 4.256 & 2.506 & 2.48 & 10.733 & 6.001 & 4.782 & 6.656 & 3.756 \\
\hline $10 / 25 / 2005$ & $15: 00$ & 10.899 & 10.774 & 7.92 & 6.81 & 4.384 & 4.251 & 2.506 & 2.473 & 10.73 & 5.996 & 4.783 & 6.654 & 3.762 \\
\hline
\end{tabular}


Water Level (ft below top of casing) at Indicated Well

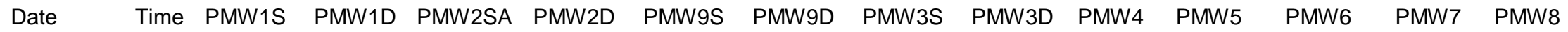

\begin{tabular}{|c|c|c|c|c|c|c|c|c|c|c|c|c|c|c|}
\hline $10 / 25 / 2005$ & $16: 00$ & 10.892 & 10.769 & 7.916 & 6.806 & 4.386 & 4.247 & 2.503 & 2.473 & 10.73 & 6.001 & 4.787 & 6.652 & 3.772 \\
\hline $10 / 25 / 2005$ & $17: 00$ & 10.885 & 10.761 & 7.914 & 6.802 & 4.388 & 4.245 & 2.5 & 2.469 & 10.728 & 6 & 4.787 & 6.652 & 3.774 \\
\hline $10 / 25 / 2005$ & $18: 00$ & 10.877 & 10.751 & 7.91 & 6.797 & 4.388 & 4.242 & 2.495 & 2.465 & 10.728 & 6 & 4.787 & 6.65 & 3.772 \\
\hline $10 / 25 / 2005$ & $19: 00$ & 10.872 & 10.745 & 7.906 & 6.795 & 4.386 & 4.24 & 2.493 & 2.461 & 10.726 & 5.996 & 4.783 & 6.646 & 3.766 \\
\hline $10 / 25 / 2005$ & $20: 00$ & 10.872 & 10.745 & 7.906 & 6.797 & 4.389 & 4.24 & 2.493 & 2.463 & 10.726 & 5.996 & 4.783 & 6.646 & 3.762 \\
\hline $10 / 25 / 2005$ & $21: 00$ & 10.867 & 10.743 & 7.904 & 6.795 & 4.385 & 4.236 & 2.49 & 2.459 & 10.726 & 5.998 & 4.78 & 6.642 & 3.756 \\
\hline $10 / 25 / 2005$ & $22: 00$ & 10.865 & 10.74 & 7.9 & 6.793 & 4.381 & 4.236 & 2.48 & 2.455 & 10.724 & 5.994 & 4.776 & 6.64 & 3.752 \\
\hline $10 / 25 / 2005$ & $23: 00$ & 10.86 & 10.736 & 7.898 & 6.786 & 4.375 & 4.231 & 2.483 & 2.452 & 10.724 & 5.99 & 4.77 & 6.639 & 3.746 \\
\hline $10 / 26 / 2005$ & 0:00 & 10.855 & 10.733 & 7.896 & 6.784 & 4.373 & 4.231 & 2.475 & 2.448 & 10.72 & 5.987 & 4.768 & 6.637 & 3.744 \\
\hline $10 / 26 / 2005$ & $1: 00$ & 10.855 & 10.729 & 7.892 & 6.782 & 4.369 & 4.229 & 2.478 & 2.446 & 10.72 & 5.987 & 4.768 & 6.637 & 3.74 \\
\hline $10 / 26 / 2005$ & $2: 00$ & 10.855 & 10.73 & 7.892 & 6.782 & 4.369 & 4.227 & 2.473 & 2.446 & 10.719 & 5.987 & 4.768 & 6.637 & 3.74 \\
\hline $10 / 26 / 2005$ & $3: 00$ & 10.853 & 10.73 & 7.893 & 6.786 & 4.37 & 4.225 & 2.478 & 2.448 & 10.717 & 5.987 & 4.768 & 6.637 & 3.74 \\
\hline $10 / 26 / 2005$ & 4:00 & 10.855 & 10.733 & 7.894 & 6.789 & 4.37 & 4.225 & 2.478 & 2.45 & 10.719 & 6.001 & 4.77 & 6.637 & 3.74 \\
\hline $10 / 26 / 2005$ & $5: 00$ & 10.853 & 10.732 & 7.893 & 6.786 & 4.369 & 4.222 & 2.478 & 2.448 & 10.715 & 5.998 & 4.768 & 6.637 & 3.738 \\
\hline $10 / 26 / 2005$ & $6: 00$ & 10.853 & 10.733 & 7.893 & 6.786 & 4.368 & 4.222 & 2.48 & 2.45 & 10.715 & 6 & 4.768 & 6.635 & 3.738 \\
\hline $10 / 26 / 2005$ & $7: 00$ & 10.853 & 10.734 & 7.893 & 6.786 & 4.368 & 4.223 & 2.475 & 2.448 & 10.717 & 5.998 & 4.766 & 6.635 & 3.736 \\
\hline $10 / 26 / 2005$ & $8: 00$ & 10.853 & 10.732 & 7.893 & 6.786 & 4.366 & 4.22 & 2.48 & 2.448 & 10.713 & 5.994 & 4.762 & 6.633 & 3.734 \\
\hline $10 / 26 / 2005$ & $9: 00$ & 10.855 & 10.734 & 7.895 & 6.789 & 4.367 & 4.222 & 2.48 & 2.45 & 10.713 & 5.996 & 4.764 & 6.631 & 3.736 \\
\hline $10 / 26 / 2005$ & $10: 00$ & 10.855 & 10.736 & 7.895 & 6.789 & 4.366 & 4.221 & 2.48 & 2.452 & 10.711 & 5.994 & 4.764 & 6.633 & 3.734 \\
\hline 10/26/2005 & $11: 00$ & 10.86 & 10.741 & 7.899 & 6.793 & 4.374 & 4.221 & 2.483 & 2.459 & 10.715 & 6.001 & 4.772 & 6.637 & 3.742 \\
\hline $10 / 26 / 2005$ & $12: 00$ & 10.862 & 10.747 & 7.903 & 6.797 & 4.38 & 4.223 & 2.496 & 2.465 & 10.715 & 6.003 & 4.78 & 6.639 & 3.756 \\
\hline $10 / 26 / 2005$ & $13: 00$ & 10.858 & 10.738 & 7.899 & 6.791 & 4.376 & 4.221 & 2.491 & 2.461 & 10.713 & 6.003 & 4.783 & 6.639 & 3.766 \\
\hline $10 / 26 / 2005$ & $14: 00$ & 10.855 & 10.732 & 7.897 & 6.786 & 4.38 & 4.218 & 2.483 & 2.453 & 10.715 & 6.005 & 4.787 & 6.639 & 3.778 \\
\hline $10 / 26 / 2005$ & $15: 00$ & 10.853 & 10.733 & 7.898 & 6.789 & 4.387 & 4.218 & 2.483 & 2.455 & 10.717 & 6.011 & 4.799 & 6.643 & 3.796 \\
\hline $10 / 26 / 2005$ & $16: 00$ & 10.855 & 10.734 & 7.9 & 6.793 & 4.397 & 4.221 & 2.496 & 2.459 & 10.719 & 6.018 & 4.807 & 6.645 & 3.81 \\
\hline $10 / 26 / 2005$ & $17: 00$ & 10.855 & 10.738 & 7.902 & 6.796 & 4.406 & 4.223 & 2.493 & 2.463 & 10.722 & 6.025 & 4.815 & 6.647 & 3.82 \\
\hline $10 / 26 / 2005$ & $18: 00$ & 10.86 & 10.747 & 7.908 & 6.805 & 4.416 & 4.227 & 2.501 & 2.472 & 10.726 & 6.027 & 4.821 & 6.65 & 3.828 \\
\hline $10 / 26 / 2005$ & $19: 00$ & 10.87 & 10.754 & 7.913 & 6.813 & 4.423 & 4.234 & 2.508 & 2.478 & 10.73 & 6.027 & 4.823 & 6.652 & 3.824 \\
\hline $10 / 26 / 2005$ & $20: 00$ & 10.877 & 10.769 & 7.919 & 6.824 & 4.433 & 4.238 & 2.523 & 2.486 & 10.734 & 6.029 & 4.829 & 6.658 & 3.822 \\
\hline $10 / 26 / 2005$ & $21: 00$ & 10.887 & 10.778 & 7.925 & 6.831 & 4.432 & 4.243 & 2.521 & 2.493 & 10.736 & 6.027 & 4.829 & 6.66 & 3.816 \\
\hline $10 / 26 / 2005$ & $22: 00$ & 10.895 & 10.785 & 7.929 & 6.836 & 4.433 & 4.249 & 2.526 & 2.497 & 10.738 & 6.025 & 4.829 & 6.664 & 3.81 \\
\hline $10 / 26 / 2005$ & $23: 00$ & 10.902 & 10.796 & 7.934 & 6.844 & 4.437 & 4.254 & 2.538 & 2.503 & 10.739 & 6.025 & 4.831 & 6.666 & 3.806 \\
\hline $10 / 27 / 2005$ & $0: 00$ & 10.912 & 10.806 & 7.94 & 6.851 & 4.437 & 4.26 & 2.546 & 2.51 & 10.743 & 6.027 & 4.832 & 6.669 & 3.802 \\
\hline $10 / 27 / 2005$ & $1: 00$ & 10.919 & 10.811 & 7.944 & 6.855 & 4.438 & 4.265 & 2.543 & 2.512 & 10.743 & 6.025 & 4.832 & 6.671 & 3.798 \\
\hline
\end{tabular}


Water Level (ft below top of casing) at Indicated Well

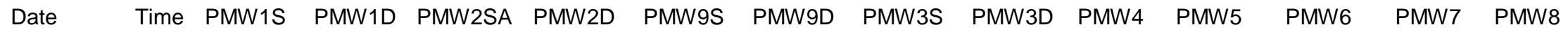

\begin{tabular}{|c|c|c|c|c|c|c|c|c|c|c|c|c|c|c|}
\hline $10 / 27 / 2005$ & $2: 00$ & 10.924 & 10.816 & 7.946 & 6.86 & 4.436 & 4.269 & 2.546 & 2.516 & 10.745 & 6.025 & 4.831 & 6.673 & 3.79 \\
\hline $10 / 27 / 2005$ & $3: 00$ & 10.932 & 10.824 & 7.95 & 6.862 & 4.436 & 4.274 & 2.556 & 2.52 & 10.745 & 6.025 & 4.832 & 6.675 & 3.788 \\
\hline $10 / 27 / 2005$ & $4: 00$ & 10.934 & 10.826 & 7.954 & 6.862 & 4.434 & 4.276 & 2.549 & 2.52 & 10.747 & 6.023 & 4.831 & 6.677 & 3.782 \\
\hline $10 / 27 / 2005$ & $5: 00$ & 10.941 & 10.831 & 7.956 & 6.867 & 4.433 & 4.278 & 2.559 & 2.522 & 10.747 & 6.025 & 4.831 & 6.677 & 3.78 \\
\hline $10 / 27 / 2005$ & $6: 00$ & 10.944 & 10.837 & 7.958 & 6.869 & 4.434 & 4.283 & 2.559 & 2.526 & 10.747 & 6.025 & 4.829 & 6.679 & 3.776 \\
\hline $10 / 27 / 2005$ & $7: 00$ & 10.949 & 10.842 & 7.96 & 6.871 & 4.436 & 4.285 & 2.564 & 2.528 & 10.749 & 6.025 & 4.829 & 6.681 & 3.776 \\
\hline $10 / 27 / 2005$ & $8: 00$ & 10.956 & 10.846 & 7.962 & 6.875 & 4.437 & 4.289 & 2.559 & 2.535 & 10.749 & 6.022 & 4.831 & 6.681 & 3.774 \\
\hline $10 / 27 / 2005$ & $9: 00$ & 10.964 & 10.853 & 7.966 & 6.882 & 4.441 & 4.294 & 2.566 & 2.537 & 10.751 & 6.029 & 4.832 & 6.685 & 3.776 \\
\hline $10 / 27 / 2005$ & $10: 00$ & 10.969 & 10.858 & 7.972 & 6.884 & 4.442 & 4.296 & 2.576 & 2.541 & 10.753 & 6.029 & 4.834 & 6.685 & 3.774 \\
\hline $10 / 27 / 2005$ & $11: 00$ & 10.971 & 10.86 & 7.972 & 6.884 & 4.44 & 4.298 & 2.576 & 2.541 & 10.751 & 6.029 & 4.834 & 6.683 & 3.77 \\
\hline $10 / 27 / 2005$ & $12: 00$ & 10.973 & 10.86 & 7.974 & 6.884 & 4.44 & 4.298 & 2.579 & 2.544 & 10.753 & 6.033 & 4.838 & 6.685 & 3.778 \\
\hline $10 / 27 / 2005$ & $13: 00$ & 10.968 & 10.855 & 7.97 & 6.878 & 4.441 & 4.296 & 2.574 & 2.539 & 10.753 & 6.033 & 4.838 & 6.687 & 3.792 \\
\hline $10 / 27 / 2005$ & $14: 00$ & 10.963 & 10.846 & 7.968 & 6.874 & 4.438 & 4.296 & 2.571 & 2.535 & 10.753 & 6.036 & 4.842 & 6.687 & 3.8 \\
\hline $10 / 27 / 2005$ & $15: 00$ & 10.953 & 10.835 & 7.963 & 6.867 & 4.438 & 4.291 & 2.566 & 2.529 & 10.753 & 6.038 & 4.846 & 6.685 & 3.81 \\
\hline $10 / 27 / 2005$ & $16: 00$ & 10.951 & 10.829 & 7.961 & 6.862 & 4.439 & 4.291 & 2.561 & 2.527 & 10.755 & 6.038 & 4.85 & 6.683 & 3.819 \\
\hline $10 / 27 / 2005$ & $17: 00$ & 10.946 & 10.827 & 7.959 & 6.862 & 4.442 & 4.291 & 2.556 & 2.525 & 10.755 & 6.04 & 4.852 & 6.685 & 3.825 \\
\hline $10 / 27 / 2005$ & $18: 00$ & 10.943 & 10.826 & 7.959 & 6.86 & 4.446 & 4.291 & 2.556 & 2.525 & 10.757 & 6.044 & 4.854 & 6.685 & 3.827 \\
\hline $10 / 27 / 2005$ & $19: 00$ & 10.946 & 10.827 & 7.961 & 6.865 & 4.449 & 4.292 & 2.561 & 2.525 & 10.759 & 6.043 & 4.854 & 6.685 & 3.819 \\
\hline $10 / 27 / 2005$ & $20: 00$ & 10.946 & 10.831 & 7.963 & 6.867 & 4.449 & 4.294 & 2.556 & 2.527 & 10.759 & 6.042 & 4.854 & 6.687 & 3.812 \\
\hline $10 / 27 / 2005$ & $21: 00$ & 10.948 & 10.835 & 7.963 & 6.871 & 4.45 & 4.296 & 2.566 & 2.529 & 10.761 & 6.04 & 4.852 & 6.687 & 3.804 \\
\hline $10 / 27 / 2005$ & $22: 00$ & 10.953 & 10.84 & 7.965 & 6.874 & 4.451 & 4.298 & 2.564 & 2.531 & 10.761 & 6.04 & 4.852 & 6.687 & 3.802 \\
\hline $10 / 27 / 2005$ & $23: 00$ & 10.956 & 10.842 & 7.965 & 6.874 & 4.45 & 4.298 & 2.561 & 2.533 & 10.761 & 6.038 & 4.85 & 6.689 & 3.796 \\
\hline $10 / 28 / 2005$ & 0:00 & 10.956 & 10.84 & 7.965 & 6.871 & 4.445 & 4.301 & 2.566 & 2.531 & 10.763 & 6.036 & 4.848 & 6.689 & 3.788 \\
\hline $10 / 28 / 2005$ & $1: 00$ & 10.958 & 10.842 & 7.965 & 6.874 & 4.444 & 4.301 & 2.566 & 2.531 & 10.761 & 6.034 & 4.846 & 6.689 & 3.784 \\
\hline $10 / 28 / 2005$ & $2: 00$ & 10.956 & 10.844 & 7.966 & 6.874 & 4.44 & 4.301 & 2.566 & 2.531 & 10.761 & 6.034 & 4.842 & 6.689 & 3.778 \\
\hline $10 / 28 / 2005$ & $3: 00$ & 10.956 & 10.842 & 7.965 & 6.871 & 4.437 & 4.303 & 2.566 & 2.531 & 10.761 & 6.031 & 4.838 & 6.685 & 3.774 \\
\hline $10 / 28 / 2005$ & $4: 00$ & 10.956 & 10.84 & 7.965 & 6.871 & 4.434 & 4.301 & 2.564 & 2.527 & 10.757 & 6.031 & 4.834 & 6.685 & 3.77 \\
\hline $10 / 28 / 2005$ & $5: 00$ & 10.956 & 10.844 & 7.964 & 6.874 & 4.434 & 4.303 & 2.564 & 2.529 & 10.757 & 6.031 & 4.834 & 6.687 & 3.77 \\
\hline $10 / 28 / 2005$ & $6: 00$ & 10.953 & 10.838 & 7.964 & 6.869 & 4.43 & 4.301 & 2.554 & 2.527 & 10.757 & 6.027 & 4.831 & 6.687 & 3.766 \\
\hline $10 / 28 / 2005$ & $7: 00$ & 10.953 & 10.838 & 7.964 & 6.869 & 4.428 & 4.301 & 2.561 & 2.527 & 10.755 & 6.027 & 4.831 & 6.685 & 3.762 \\
\hline $10 / 28 / 2005$ & $8: 00$ & 10.953 & 10.837 & 7.962 & 6.867 & 4.425 & 4.301 & 2.561 & 2.525 & 10.755 & 6.025 & 4.825 & 6.681 & 3.76 \\
\hline $10 / 28 / 2005$ & $9: 00$ & 10.956 & 10.84 & 7.964 & 6.871 & 4.429 & 4.298 & 2.561 & 2.527 & 10.755 & 6.027 & 4.826 & 6.685 & 3.76 \\
\hline $10 / 28 / 2005$ & $10: 00$ & 10.958 & 10.84 & 7.964 & 6.871 & 4.43 & 4.301 & 2.561 & 2.529 & 10.751 & 6.027 & 4.825 & 6.683 & 3.76 \\
\hline $10 / 28 / 2005$ & $11: 00$ & 10.953 & 10.833 & 7.962 & 6.865 & 4.423 & 4.296 & 2.559 & 2.525 & 10.749 & 6.025 & 4.825 & 6.681 & 3.762 \\
\hline
\end{tabular}


Water Level (ft below top of casing) at Indicated Well

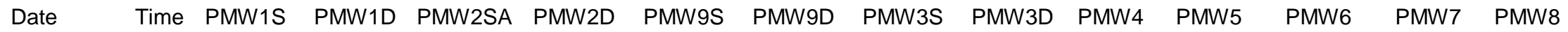

\begin{tabular}{|c|c|c|c|c|c|c|c|c|c|c|c|c|c|c|}
\hline $10 / 28 / 2005$ & $12: 00$ & 10.943 & 10.824 & 7.959 & 6.856 & 4.423 & 4.294 & 2.556 & 2.519 & 10.747 & 6.027 & 4.829 & 6.678 & 3.772 \\
\hline $10 / 28 / 2005$ & $13: 00$ & 10.939 & 10.816 & 7.953 & 6.852 & 4.422 & 4.29 & 2.543 & 2.517 & 10.747 & 6.034 & 4.836 & 6.681 & 3.788 \\
\hline $10 / 28 / 2005$ & $14: 00$ & 10.929 & 10.804 & 7.949 & 6.845 & 4.423 & 4.285 & 2.546 & 2.51 & 10.745 & 6.036 & 4.844 & 6.676 & 3.804 \\
\hline $10 / 28 / 2005$ & $15: 00$ & 10.921 & 10.791 & 7.945 & 6.838 & 4.427 & 4.281 & 2.535 & 2.504 & 10.749 & 6.036 & 4.85 & 6.676 & 3.821 \\
\hline $10 / 28 / 2005$ & $16: 00$ & 10.914 & 10.787 & 7.943 & 6.834 & 4.428 & 4.279 & 2.541 & 2.502 & 10.749 & 6.038 & 4.856 & 6.678 & 3.833 \\
\hline $10 / 28 / 2005$ & $17: 00$ & 10.907 & 10.782 & 7.938 & 6.834 & 4.435 & 4.276 & 2.533 & 2.502 & 10.749 & 6.038 & 4.858 & 6.676 & 3.843 \\
\hline $10 / 28 / 2005$ & $18: 00$ & 10.907 & 10.78 & 7.937 & 6.831 & 4.438 & 4.276 & 2.535 & 2.5 & 10.753 & 6.04 & 4.861 & 6.678 & 3.845 \\
\hline $10 / 28 / 2005$ & $19: 00$ & 10.904 & 10.78 & 7.937 & 6.834 & 4.443 & 4.276 & 2.531 & 2.5 & 10.755 & 6.038 & 4.862 & 6.674 & 3.841 \\
\hline $10 / 28 / 2005$ & $20: 00$ & 10.904 & 10.78 & 7.937 & 6.834 & 4.444 & 4.276 & 2.536 & 2.5 & 10.755 & 6.038 & 4.858 & 6.674 & 3.833 \\
\hline $10 / 28 / 2005$ & $21: 00$ & 10.904 & 10.782 & 7.937 & 6.834 & 4.44 & 4.276 & 2.528 & 2.5 & 10.755 & 6.033 & 4.852 & 6.67 & 3.823 \\
\hline $10 / 28 / 2005$ & $22: 00$ & 10.907 & 10.785 & 7.937 & 6.836 & 4.442 & 4.276 & 2.531 & 2.502 & 10.755 & 6.033 & 4.85 & 6.67 & 3.819 \\
\hline $10 / 28 / 2005$ & $23: 00$ & 10.909 & 10.785 & 7.935 & 6.834 & 4.438 & 4.276 & 2.538 & 2.5 & 10.755 & 6.031 & 4.848 & 6.67 & 3.81 \\
\hline $10 / 29 / 2005$ & $0: 00$ & 10.904 & 10.785 & 7.935 & 6.834 & 4.434 & 4.276 & 2.533 & 2.498 & 10.753 & 6.027 & 4.842 & 6.668 & 3.803 \\
\hline $10 / 29 / 2005$ & $1: 00$ & 10.904 & 10.782 & 7.931 & 6.83 & 4.428 & 4.274 & 2.526 & 2.496 & 10.751 & 6.025 & 4.838 & 6.666 & 3.797 \\
\hline $10 / 29 / 2005$ & $2: 00$ & 10.902 & 10.78 & 7.931 & 6.83 & 4.424 & 4.274 & 2.528 & 2.492 & 10.749 & 6.02 & 4.832 & 6.664 & 3.79 \\
\hline $10 / 29 / 2005$ & $3: 00$ & 10.899 & 10.776 & 7.929 & 6.827 & 4.42 & 4.272 & 2.526 & 2.489 & 10.744 & 6.018 & 4.83 & 6.659 & 3.786 \\
\hline $10 / 29 / 2005$ & $4: 00$ & 10.899 & 10.771 & 7.925 & 6.823 & 4.415 & 4.27 & 2.513 & 2.487 & 10.744 & 6.016 & 4.824 & 6.653 & 3.784 \\
\hline 10/29/2005 & $5: 00$ & 10.894 & 10.769 & 7.923 & 6.819 & 4.413 & 4.266 & 2.513 & 2.481 & 10.74 & 6.011 & 4.822 & 6.659 & 3.78 \\
\hline $10 / 29 / 2005$ & $6: 00$ & 10.892 & 10.769 & 7.923 & 6.821 & 4.409 & 4.266 & 2.513 & 2.481 & 10.738 & 6.013 & 4.816 & 6.657 & 3.78 \\
\hline $10 / 29 / 2005$ & $7: 00$ & 10.889 & 10.765 & 7.921 & 6.816 & 4.407 & 4.261 & 2.516 & 2.479 & 10.738 & 6.011 & 4.814 & 6.653 & 3.778 \\
\hline $10 / 29 / 2005$ & $8: 00$ & 10.887 & 10.762 & 7.919 & 6.816 & 4.405 & 4.261 & 2.513 & 2.477 & 10.738 & 6.011 & 4.812 & 6.655 & 3.776 \\
\hline $10 / 29 / 2005$ & $9: 00$ & 10.889 & 10.764 & 7.921 & 6.816 & 4.409 & 4.259 & 2.518 & 2.479 & 10.74 & 6.013 & 4.816 & 6.655 & 3.78 \\
\hline $10 / 29 / 2005$ & $10: 00$ & 10.889 & 10.765 & 7.921 & 6.821 & 4.41 & 4.257 & 2.518 & 2.481 & 10.738 & 6.016 & 4.818 & 6.655 & 3.788 \\
\hline $10 / 29 / 2005$ & $11: 00$ & 10.887 & 10.76 & 7.921 & 6.821 & 4.415 & 4.255 & 2.523 & 2.484 & 10.738 & 6.018 & 4.822 & 6.651 & 3.802 \\
\hline $10 / 29 / 2005$ & $12: 00$ & 10.889 & 10.76 & 7.927 & 6.819 & 4.416 & 4.252 & 2.526 & 2.488 & 10.744 & 6.022 & 4.834 & 6.655 & 3.825 \\
\hline $10 / 29 / 2005$ & $13: 00$ & 10.882 & 10.754 & 7.921 & 6.812 & 4.421 & 4.25 & 2.523 & 2.486 & 10.744 & 6.022 & 4.84 & 6.657 & 3.841 \\
\hline $10 / 29 / 2005$ & $14: 00$ & 10.877 & 10.747 & 7.917 & 6.805 & 4.42 & 4.25 & 2.51 & 2.477 & 10.74 & 6.022 & 4.842 & 6.657 & 3.849 \\
\hline $10 / 29 / 2005$ & $15: 00$ & 10.872 & 10.738 & 7.911 & 6.797 & 4.424 & 4.248 & 2.515 & 2.471 & 10.738 & 6.022 & 4.846 & 6.649 & 3.857 \\
\hline $10 / 29 / 2005$ & $16: 00$ & 10.87 & 10.734 & 7.912 & 6.799 & 4.43 & 4.246 & 2.508 & 2.475 & 10.738 & 6.029 & 4.852 & 6.651 & 3.871 \\
\hline $10 / 29 / 2005$ & $17: 00$ & 10.867 & 10.737 & 7.914 & 6.803 & 4.436 & 4.246 & 2.515 & 2.477 & 10.742 & 6.031 & 4.86 & 6.655 & 3.881 \\
\hline $10 / 29 / 2005$ & $18: 00$ & 10.867 & 10.742 & 7.915 & 6.808 & 4.442 & 4.248 & 2.513 & 2.479 & 10.748 & 6.031 & 4.861 & 6.655 & 3.885 \\
\hline $10 / 29 / 2005$ & $19: 00$ & 10.87 & 10.744 & 7.917 & 6.81 & 4.447 & 4.252 & 2.515 & 2.481 & 10.748 & 6.033 & 4.86 & 6.657 & 3.878 \\
\hline $10 / 29 / 2005$ & $20: 00$ & 10.875 & 10.753 & 7.919 & 6.819 & 4.447 & 4.255 & 2.518 & 2.488 & 10.752 & 6.031 & 4.858 & 6.657 & 3.869 \\
\hline $10 / 29 / 2005$ & $21: 00$ & 10.877 & 10.754 & 7.921 & 6.819 & 4.445 & 4.257 & 2.523 & 2.488 & 10.748 & 6.029 & 4.852 & 6.653 & 3.857 \\
\hline
\end{tabular}


Water Level (ft below top of casing) at Indicated Well

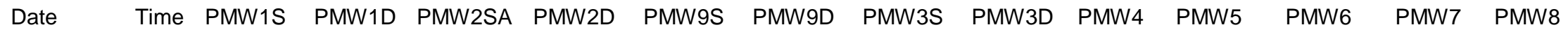

\begin{tabular}{|c|c|c|c|c|c|c|c|c|c|c|c|c|c|c|}
\hline $10 / 29 / 2005$ & $22: 00$ & 10.88 & 10.756 & 7.919 & 6.817 & 4.44 & 4.257 & 2.523 & 2.486 & 10.746 & 6.025 & 4.848 & 6.651 & 3.847 \\
\hline $10 / 29 / 2005$ & $23: 00$ & 10.882 & 10.756 & 7.921 & 6.817 & 4.436 & 4.257 & 2.515 & 2.486 & 10.746 & 6.022 & 4.846 & 6.653 & 3.837 \\
\hline $10 / 30 / 2005$ & $0: 00$ & 10.877 & 10.753 & 7.917 & 6.814 & 4.429 & 4.257 & 2.515 & 2.479 & 10.746 & 6.022 & 4.84 & 6.651 & 3.827 \\
\hline $10 / 30 / 2005$ & $1: 00$ & 10.877 & 10.751 & 7.917 & 6.81 & 4.423 & 4.255 & 2.508 & 2.477 & 10.744 & 6.016 & 4.834 & 6.647 & 3.817 \\
\hline $10 / 30 / 2005$ & $2: 00$ & 10.877 & 10.747 & 7.913 & 6.808 & 4.419 & 4.255 & 2.503 & 2.473 & 10.742 & 6.013 & 4.83 & 6.647 & 3.81 \\
\hline $10 / 30 / 2005$ & 3:00 & 10.872 & 10.744 & 7.911 & 6.803 & 4.415 & 4.252 & 2.506 & 2.469 & 10.74 & 6.009 & 4.824 & 6.644 & 3.804 \\
\hline $10 / 30 / 2005$ & $4: 00$ & 10.867 & 10.738 & 7.909 & 6.801 & 4.409 & 4.25 & 2.495 & 2.465 & 10.74 & 6.007 & 4.818 & 6.644 & 3.798 \\
\hline $10 / 30 / 2005$ & $5: 00$ & 10.862 & 10.735 & 7.905 & 6.797 & 4.403 & 4.248 & 2.498 & 2.459 & 10.736 & 6.005 & 4.812 & 6.642 & 3.79 \\
\hline $10 / 30 / 2005$ & $6: 00$ & 10.867 & 10.745 & 7.911 & 6.806 & 4.412 & 4.248 & 2.508 & 2.469 & 10.738 & 6.011 & 4.816 & 6.646 & 3.797 \\
\hline $10 / 30 / 2005$ & 7:00 & 10.87 & 10.749 & 7.913 & 6.806 & 4.41 & 4.248 & 2.506 & 2.467 & 10.738 & 6.006 & 4.812 & 6.644 & 3.79 \\
\hline $10 / 30 / 2005$ & $8: 00$ & 10.872 & 10.749 & 7.912 & 6.808 & 4.407 & 4.246 & 2.506 & 2.467 & 10.737 & 6.005 & 4.805 & 6.642 & 3.782 \\
\hline $10 / 30 / 2005$ & $9: 00$ & 10.877 & 10.757 & 7.916 & 6.814 & 4.411 & 4.248 & 2.511 & 2.473 & 10.736 & 6.005 & 4.805 & 6.646 & 3.782 \\
\hline $10 / 30 / 2005$ & $10: 00$ & 10.882 & 10.76 & 7.918 & 6.817 & 4.412 & 4.25 & 2.511 & 2.478 & 10.739 & 6.005 & 4.805 & 6.646 & 3.782 \\
\hline $10 / 30 / 2005$ & $11: 00$ & 10.885 & 10.769 & 7.922 & 6.823 & 4.415 & 4.248 & 2.516 & 2.482 & 10.737 & 6.007 & 4.809 & 6.647 & 3.788 \\
\hline $10 / 30 / 2005$ & $12: 00$ & 10.887 & 10.771 & 7.924 & 6.823 & 4.416 & 4.25 & 2.518 & 2.484 & 10.741 & 6.011 & 4.811 & 6.649 & 3.796 \\
\hline $10 / 30 / 2005$ & $13: 00$ & 10.889 & 10.768 & 7.924 & 6.819 & 4.416 & 4.251 & 2.523 & 2.486 & 10.739 & 6.013 & 4.814 & 6.649 & 3.81 \\
\hline $10 / 30 / 2005$ & $14: 00$ & 10.887 & 10.762 & 7.923 & 6.819 & 4.42 & 4.25 & 2.518 & 2.486 & 10.739 & 6.018 & 4.82 & 6.652 & 3.825 \\
\hline $10 / 30 / 2005$ & $15: 00$ & 10.882 & 10.757 & 7.921 & 6.814 & 4.421 & 4.248 & 2.521 & 2.482 & 10.739 & 6.02 & 4.826 & 6.653 & 3.839 \\
\hline $10 / 30 / 2005$ & $16: 00$ & 10.882 & 10.757 & 7.923 & 6.817 & 4.43 & 4.248 & 2.518 & 2.486 & 10.741 & 6.025 & 4.836 & 6.653 & 3.853 \\
\hline $10 / 30 / 2005$ & $17: 00$ & 10.887 & 10.764 & 7.927 & 6.823 & 4.444 & 4.253 & 2.525 & 2.492 & 10.744 & 6.029 & 4.844 & 6.657 & 3.867 \\
\hline $10 / 30 / 2005$ & $18: 00$ & 10.892 & 10.769 & 7.929 & 6.828 & 4.449 & 4.255 & 2.528 & 2.497 & 10.748 & 6.031 & 4.846 & 6.657 & 3.867 \\
\hline $10 / 30 / 2005$ & $19: 00$ & 10.897 & 10.777 & 7.932 & 6.833 & 4.453 & 4.259 & 2.538 & 2.499 & 10.748 & 6.031 & 4.844 & 6.659 & 3.857 \\
\hline $10 / 30 / 2005$ & $20: 00$ & 10.902 & 10.784 & 7.936 & 6.839 & 4.455 & 4.264 & 2.541 & 2.503 & 10.75 & 6.027 & 4.84 & 6.659 & 3.843 \\
\hline $10 / 30 / 2005$ & $21: 00$ & 10.904 & 10.788 & 7.94 & 6.841 & 4.451 & 4.266 & 2.538 & 2.505 & 10.75 & 6.022 & 4.838 & 6.659 & 3.829 \\
\hline $10 / 30 / 2005$ & $22: 00$ & 10.914 & 10.799 & 7.944 & 6.85 & 4.455 & 4.273 & 2.549 & 2.511 & 10.752 & 6.025 & 4.838 & 6.665 & 3.823 \\
\hline $10 / 30 / 2005$ & $23: 00$ & 10.921 & 10.81 & 7.948 & 6.857 & 4.455 & 4.277 & 2.546 & 2.515 & 10.754 & 6.022 & 4.838 & 6.667 & 3.815 \\
\hline $10 / 31 / 2005$ & $0: 00$ & 10.929 & 10.82 & 7.955 & 6.866 & 4.456 & 4.282 & 2.554 & 2.524 & 10.756 & 6.025 & 4.836 & 6.669 & 3.81 \\
\hline $10 / 31 / 2005$ & $1: 00$ & 10.934 & 10.822 & 7.956 & 6.868 & 4.451 & 4.286 & 2.561 & 2.524 & 10.756 & 6.02 & 4.834 & 6.669 & 3.802 \\
\hline $10 / 31 / 2005$ & $2: 00$ & 10.943 & 10.839 & 7.963 & 6.879 & 4.457 & 4.29 & 2.566 & 2.534 & 10.758 & 6.024 & 4.838 & 6.675 & 3.802 \\
\hline $10 / 31 / 2005$ & $3: 00$ & 10.951 & 10.842 & 7.967 & 6.881 & 4.455 & 4.295 & 2.571 & 2.537 & 10.758 & 6.022 & 4.834 & 6.676 & 3.797 \\
\hline $10 / 31 / 2005$ & $4: 00$ & 10.956 & 10.846 & 7.969 & 6.884 & 4.45 & 4.297 & 2.566 & 2.539 & 10.758 & 6.02 & 4.832 & 6.676 & 3.793 \\
\hline $10 / 31 / 2005$ & $5: 00$ & 10.953 & 10.835 & 7.965 & 6.872 & 4.439 & 4.295 & 2.564 & 2.528 & 10.756 & 6.011 & 4.822 & 6.675 & 3.778 \\
\hline $10 / 31 / 2005$ & $6: 00$ & 10.958 & 10.848 & 7.968 & 6.881 & 4.443 & 4.299 & 2.564 & 2.535 & 10.756 & 6.02 & 4.828 & 6.68 & 3.78 \\
\hline $10 / 31 / 2005$ & $7: 00$ & 10.963 & 10.853 & 7.97 & 6.888 & 4.446 & 4.301 & 2.571 & 2.541 & 10.758 & 6.022 & 4.83 & 6.682 & 3.784 \\
\hline
\end{tabular}


Water Level (ft below top of casing) at Indicated Well

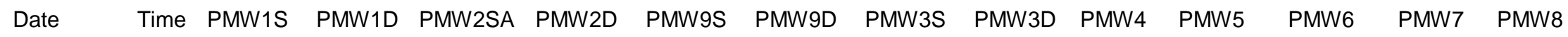

\begin{tabular}{|c|c|c|c|c|c|c|c|c|c|c|c|c|c|c|}
\hline $10 / 31 / 2005$ & $8: 00$ & 10.968 & 10.861 & 7.975 & 6.895 & 4.449 & 4.306 & 2.584 & 2.547 & 10.76 & 6.02 & 4.83 & 6.682 & 3.782 \\
\hline $10 / 31 / 2005$ & $9: 00$ & 10.973 & 10.866 & 7.979 & 6.897 & 4.451 & 4.308 & 2.579 & 2.549 & 10.76 & 6.022 & 4.832 & 6.684 & 3.78 \\
\hline $10 / 31 / 2005$ & $10: 00$ & 10.978 & 10.868 & 7.979 & 6.897 & 4.446 & 4.31 & 2.579 & 2.552 & 10.76 & 6.022 & 4.83 & 6.686 & 3.778 \\
\hline $10 / 31 / 2005$ & $11: 00$ & 10.978 & 10.868 & 7.981 & 6.897 & 4.446 & 4.31 & 2.584 & 2.549 & 10.76 & 6.022 & 4.83 & 6.686 & 3.778 \\
\hline $10 / 31 / 2005$ & $12: 00$ & 10.98 & 10.868 & 7.983 & 6.892 & 4.448 & 4.31 & 2.586 & 2.549 & 10.761 & 6.024 & 4.836 & 6.686 & 3.786 \\
\hline $10 / 31 / 2005$ & $13: 00$ & 10.978 & 10.859 & 7.981 & 6.89 & 4.445 & 4.31 & 2.584 & 2.547 & 10.761 & 6.024 & 4.838 & 6.686 & 3.8 \\
\hline $10 / 31 / 2005$ & $14: 00$ & 10.963 & 10.842 & 7.972 & 6.872 & 4.435 & 4.304 & 2.568 & 2.531 & 10.756 & 6.024 & 4.843 & 6.68 & 3.81 \\
\hline $10 / 31 / 2005$ & $15: 00$ & 10.96 & 10.839 & 7.968 & 6.872 & 4.444 & 4.306 & 2.571 & 2.533 & 10.756 & 6.031 & 4.853 & 6.682 & 3.832 \\
\hline $10 / 31 / 2005$ & $16: 00$ & 10.958 & 10.833 & 7.97 & 6.87 & 4.451 & 4.304 & 2.571 & 2.533 & 10.76 & 6.033 & 4.857 & 6.684 & 3.847 \\
\hline $10 / 31 / 2005$ & $17: 00$ & 10.956 & 10.833 & 7.967 & 6.873 & 4.456 & 4.304 & 2.566 & 2.535 & 10.761 & 6.037 & 4.865 & 6.686 & 3.859 \\
\hline $10 / 31 / 2005$ & $18: 00$ & 10.956 & 10.831 & 7.969 & 6.872 & 4.464 & 4.304 & 2.568 & 2.537 & 10.763 & 6.037 & 4.869 & 6.688 & 3.863 \\
\hline $10 / 31 / 2005$ & $19: 00$ & 10.961 & 10.841 & 7.973 & 6.882 & 4.473 & 4.306 & 2.581 & 2.543 & 10.767 & 6.041 & 4.873 & 6.692 & 3.861 \\
\hline $10 / 31 / 2005$ & $20: 00$ & 10.961 & 10.841 & 7.976 & 6.882 & 4.47 & 4.308 & 2.578 & 2.541 & 10.767 & 6.037 & 4.869 & 6.69 & 3.847 \\
\hline $10 / 31 / 2005$ & $21: 00$ & 10.963 & 10.842 & 7.974 & 6.882 & 4.466 & 4.31 & 2.578 & 2.539 & 10.769 & 6.035 & 4.867 & 6.69 & 3.835 \\
\hline $10 / 31 / 2005$ & $22: 00$ & 10.965 & 10.844 & 7.976 & 6.882 & 4.464 & 4.313 & 2.578 & 2.541 & 10.767 & 6.031 & 4.863 & 6.688 & 3.823 \\
\hline $10 / 31 / 2005$ & $23: 00$ & 10.965 & 10.844 & 7.974 & 6.882 & 4.46 & 4.313 & 2.576 & 2.541 & 10.769 & 6.026 & 4.857 & 6.688 & 3.812 \\
\hline $11 / 1 / 2005$ & $0: 00$ & 10.955 & 10.831 & 7.966 & 6.868 & 4.446 & 4.31 & 2.563 & 2.526 & 10.763 & 6.02 & 4.847 & 6.682 & 3.797 \\
\hline $11 / 1 / 2005$ & $1: 00$ & 10.953 & 10.824 & 7.962 & 6.864 & 4.437 & 4.306 & 2.551 & 2.52 & 10.76 & 6.018 & 4.84 & 6.679 & 3.786 \\
\hline $11 / 1 / 2005$ & $2: 00$ & 10.945 & 10.819 & 7.956 & 6.857 & 4.427 & 4.302 & 2.551 & 2.514 & 10.756 & 6.015 & 4.832 & 6.677 & 3.776 \\
\hline $11 / 1 / 2005$ & $3: 00$ & 10.941 & 10.815 & 7.956 & 6.855 & 4.425 & 4.301 & 2.548 & 2.512 & 10.754 & 6.013 & 4.83 & 6.677 & 3.772 \\
\hline $11 / 1 / 2005$ & $4: 00$ & 10.938 & 10.808 & 7.95 & 6.846 & 4.418 & 4.297 & 2.543 & 2.506 & 10.75 & 6.009 & 4.822 & 6.671 & 3.764 \\
\hline $11 / 1 / 2005$ & $5: 00$ & 10.933 & 10.802 & 7.946 & 6.842 & 4.413 & 4.293 & 2.535 & 2.497 & 10.747 & 6.006 & 4.816 & 6.667 & 3.756 \\
\hline $11 / 1 / 2005$ & $6: 00$ & 10.928 & 10.793 & 7.94 & 6.835 & 4.404 & 4.286 & 2.53 & 2.491 & 10.745 & 6.006 & 4.812 & 6.665 & 3.748 \\
\hline $11 / 1 / 2005$ & $7: 00$ & 10.923 & 10.788 & 7.937 & 6.833 & 4.402 & 4.282 & 2.52 & 2.489 & 10.741 & 6.002 & 4.804 & 6.663 & 3.746 \\
\hline $11 / 1 / 2005$ & $8: 00$ & 10.914 & 10.78 & 7.932 & 6.824 & 4.394 & 4.277 & 2.513 & 2.481 & 10.739 & 6 & 4.798 & 6.66 & 3.74 \\
\hline $11 / 1 / 2005$ & $9: 00$ & 10.911 & 10.78 & 7.932 & 6.826 & 4.397 & 4.275 & 2.513 & 2.483 & 10.737 & 6.002 & 4.798 & 6.658 & 3.742 \\
\hline $11 / 1 / 2005$ & $10: 00$ & 10.916 & 10.778 & 7.934 & 6.833 & 4.402 & 4.273 & 2.525 & 2.489 & 10.735 & 6.004 & 4.8 & 6.658 & 3.75 \\
\hline $11 / 1 / 2005$ & $11: 00$ & 10.914 & 10.78 & 7.938 & 6.833 & 4.402 & 4.271 & 2.523 & 2.491 & 10.735 & 6.004 & 4.8 & 6.658 & 3.758 \\
\hline $11 / 1 / 2005$ & $12: 00$ & 10.916 & 10.786 & 7.938 & 6.835 & 4.409 & 4.269 & 2.53 & 2.495 & 10.737 & 6.011 & 4.808 & 6.661 & 3.774 \\
\hline $11 / 1 / 2005$ & $13: 00$ & 10.909 & 10.778 & 7.938 & 6.831 & 4.407 & 4.266 & 2.53 & 2.493 & 10.737 & 6.015 & 4.814 & 6.665 & 3.788 \\
\hline $11 / 1 / 2005$ & $14: 00$ & 10.901 & 10.773 & 7.926 & 6.827 & 4.412 & 4.262 & 2.523 & 2.491 & 10.735 & 6.019 & 4.822 & 6.663 & 3.802 \\
\hline $11 / 1 / 2005$ & $15: 00$ & 10.906 & 10.771 & 7.935 & 6.831 & 4.418 & 4.262 & 2.528 & 2.495 & 10.741 & 6.028 & 4.833 & 6.665 & 3.825 \\
\hline $11 / 1 / 2005$ & $16: 00$ & 10.904 & 10.778 & 7.94 & 6.836 & 4.43 & 4.266 & 2.535 & 2.502 & 10.745 & 6.035 & 4.843 & 6.671 & 3.845 \\
\hline $11 / 1 / 2005$ & $17: 00$ & 10.914 & 10.788 & 7.944 & 6.844 & 4.446 & 4.269 & 2.543 & 2.51 & 10.745 & 6.039 & 4.855 & 6.681 & 3.863 \\
\hline
\end{tabular}


Water Level (ft below top of casing) at Indicated Well

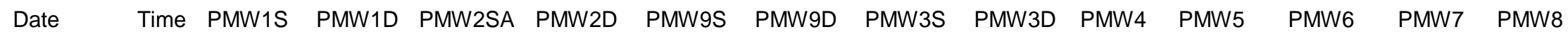

\begin{tabular}{|c|c|c|c|c|c|c|c|c|c|c|c|c|c|c|}
\hline $11 / 1 / 2005$ & $18: 00$ & 10.916 & 10.793 & 7.948 & 6.849 & 4.453 & 4.271 & 2.553 & 2.514 & 10.75 & 6.042 & 4.861 & 6.681 & 3.867 \\
\hline $11 / 1 / 2005$ & 19:00 & 10.926 & 10.8 & 7.952 & 6.858 & 4.462 & 4.275 & 2.558 & 2.521 & 10.754 & 6.044 & 4.865 & 6.682 & 3.861 \\
\hline $11 / 1 / 2005$ & $20: 00$ & 10.928 & 10.802 & 7.954 & 6.858 & 4.457 & 4.28 & 2.556 & 2.521 & 10.756 & 6.037 & 4.863 & 6.679 & 3.845 \\
\hline $11 / 1 / 2005$ & $21: 00$ & 10.936 & 10.811 & 7.956 & 6.862 & 4.461 & 4.284 & 2.558 & 2.525 & 10.756 & 6.037 & 4.861 & 6.684 & 3.835 \\
\hline $11 / 1 / 2005$ & $22: 00$ & 10.933 & 10.808 & 7.954 & 6.858 & 4.452 & 4.286 & 2.551 & 2.518 & 10.754 & 6.033 & 4.853 & 6.679 & 3.821 \\
\hline $11 / 1 / 2005$ & $23: 00$ & 10.931 & 10.808 & 7.954 & 6.855 & 4.447 & 4.286 & 2.551 & 2.516 & 10.754 & 6.029 & 4.847 & 6.677 & 3.81 \\
\hline $11 / 2 / 2005$ & $0: 00$ & 10.931 & 10.806 & 7.95 & 6.853 & 4.441 & 4.289 & 2.551 & 2.513 & 10.752 & 6.026 & 4.841 & 6.673 & 3.8 \\
\hline $11 / 2 / 2005$ & $1: 00$ & 10.926 & 10.8 & 7.948 & 6.847 & 4.434 & 4.286 & 2.543 & 2.506 & 10.75 & 6.018 & 4.833 & 6.669 & 3.79 \\
\hline $11 / 2 / 2005$ & $2: 00$ & 10.926 & 10.797 & 7.944 & 6.844 & 4.429 & 4.284 & 2.533 & 2.504 & 10.75 & 6.015 & 4.827 & 6.669 & 3.784 \\
\hline $11 / 2 / 2005$ & $3: 00$ & 10.921 & 10.791 & 7.942 & 6.838 & 4.421 & 4.28 & 2.535 & 2.498 & 10.745 & 6.011 & 4.823 & 6.665 & 3.778 \\
\hline $11 / 2 / 2005$ & $4: 00$ & 10.911 & 10.781 & 7.936 & 6.829 & 4.413 & 4.277 & 2.52 & 2.489 & 10.741 & 6.006 & 4.814 & 6.658 & 3.77 \\
\hline $11 / 2 / 2005$ & $5: 00$ & 10.904 & 10.772 & 7.93 & 6.82 & 4.407 & 4.273 & 2.52 & 2.483 & 10.739 & 6.002 & 4.808 & 6.658 & 3.764 \\
\hline $11 / 2 / 2005$ & $6: 00$ & 10.899 & 10.766 & 7.928 & 6.818 & 4.402 & 4.269 & 2.515 & 2.479 & 10.735 & 5.999 & 4.802 & 6.654 & 3.76 \\
\hline $11 / 2 / 2005$ & $7: 00$ & 10.891 & 10.762 & 7.924 & 6.813 & 4.401 & 4.264 & 2.513 & 2.475 & 10.735 & 5.998 & 4.798 & 6.652 & 3.758 \\
\hline $11 / 2 / 2005$ & $8: 00$ & 10.887 & 10.759 & 7.92 & 6.813 & 4.398 & 4.262 & 2.503 & 2.473 & 10.733 & 5.997 & 4.794 & 6.648 & 3.756 \\
\hline $11 / 2 / 2005$ & $9: 00$ & 10.882 & 10.753 & 7.918 & 6.805 & 4.393 & 4.258 & 2.498 & 2.466 & 10.729 & 5.993 & 4.788 & 6.645 & 3.75 \\
\hline $11 / 2 / 2005$ & $10: 00$ & 10.882 & 10.751 & 7.916 & 6.807 & 4.393 & 4.253 & 2.498 & 2.466 & 10.728 & 5.993 & 4.788 & 6.643 & 3.754 \\
\hline $11 / 2 / 2005$ & $11: 00$ & 10.877 & 10.746 & 7.914 & 6.8 & 4.391 & 4.251 & 2.495 & 2.464 & 10.726 & 5.997 & 4.788 & 6.641 & 3.766 \\
\hline $11 / 2 / 2005$ & $12: 00$ & 10.872 & 10.737 & 7.913 & 6.798 & 4.396 & 4.245 & 2.503 & 2.464 & 10.724 & 5.999 & 4.794 & 6.641 & 3.792 \\
\hline $11 / 2 / 2005$ & $13: 00$ & 10.864 & 10.728 & 7.911 & 6.793 & 4.399 & 4.24 & 2.498 & 2.462 & 10.726 & 6.004 & 4.8 & 6.643 & 3.818 \\
\hline $11 / 2 / 2005$ & $14: 00$ & 10.852 & 10.72 & 7.907 & 6.785 & 4.405 & 4.234 & 2.497 & 2.458 & 10.726 & 6.006 & 4.812 & 6.645 & 3.845 \\
\hline $11 / 2 / 2005$ & $15: 00$ & 10.85 & 10.709 & 7.905 & 6.78 & 4.412 & 4.231 & 2.49 & 2.454 & 10.726 & 6.013 & 4.82 & 6.643 & 3.871 \\
\hline $11 / 2 / 2005$ & $16: 00$ & 10.844 & 10.704 & 7.9 & 6.778 & 4.422 & 4.229 & 2.49 & 2.454 & 10.728 & 6.017 & 4.829 & 6.643 & 3.887 \\
\hline $11 / 2 / 2005$ & $17: 00$ & 10.839 & 10.704 & 7.9 & 6.78 & 4.43 & 4.229 & 2.49 & 2.456 & 10.73 & 6.024 & 4.843 & 6.647 & 3.905 \\
\hline $11 / 2 / 2005$ & $18: 00$ & 10.837 & 10.704 & 7.903 & 6.785 & 4.441 & 4.231 & 2.492 & 2.46 & 10.733 & 6.026 & 4.859 & 6.652 & 3.913 \\
\hline $11 / 2 / 2005$ & $19: 00$ & 10.837 & 10.706 & 7.903 & 6.789 & 4.445 & 4.234 & 2.5 & 2.458 & 10.734 & 6.026 & 4.863 & 6.652 & 3.905 \\
\hline $11 / 2 / 2005$ & $20: 00$ & 10.835 & 10.706 & 7.901 & 6.785 & 4.444 & 4.234 & 2.497 & 2.456 & 10.734 & 6.019 & 4.863 & 6.65 & 3.889 \\
\hline $11 / 2 / 2005$ & $21: 00$ & 10.84 & 10.709 & 7.901 & 6.789 & 4.442 & 4.236 & 2.497 & 2.458 & 10.734 & 6.017 & 4.861 & 6.65 & 3.879 \\
\hline $11 / 2 / 2005$ & $22: 00$ & 10.837 & 10.711 & 7.901 & 6.787 & 4.441 & 4.238 & 2.497 & 2.458 & 10.734 & 6.015 & 4.859 & 6.648 & 3.867 \\
\hline $11 / 2 / 2005$ & 23:00 & 10.839 & 10.713 & 7.899 & 6.789 & 4.436 & 4.238 & 2.495 & 2.458 & 10.733 & 6.01 & 4.855 & 6.648 & 3.857 \\
\hline $11 / 3 / 2005$ & 0:00 & 10.842 & 10.713 & 7.9 & 6.789 & 4.433 & 4.238 & 2.49 & 2.456 & 10.732 & 6.01 & 4.853 & 6.648 & 3.847 \\
\hline $11 / 3 / 2005$ & $1: 00$ & 10.842 & 10.715 & 7.9 & 6.789 & 4.43 & 4.238 & 2.495 & 2.456 & 10.73 & 6.006 & 4.847 & 6.647 & 3.836 \\
\hline $11 / 3 / 2005$ & $2: 00$ & 10.839 & 10.713 & 7.898 & 6.785 & 4.423 & 4.238 & 2.482 & 2.45 & 10.728 & 6.002 & 4.839 & 6.643 & 3.82 \\
\hline $11 / 3 / 2005$ & $3: 00$ & 10.835 & 10.706 & 7.893 & 6.778 & 4.411 & 4.234 & 2.477 & 2.444 & 10.726 & 5.997 & 4.829 & 6.641 & 3.804 \\
\hline
\end{tabular}


TABLE S1.2 (Cont.)

Water Level (ft below top of casing) at Indicated Well

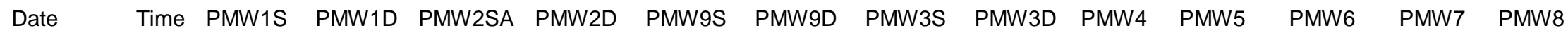

\begin{tabular}{|c|c|c|c|c|c|c|c|c|c|c|c|c|c|c|}
\hline $11 / 3 / 2005$ & $4: 00$ & 10.832 & 10.7 & 7.89 & 6.772 & 4.402 & 4.231 & 2.477 & 2.437 & 10.722 & 5.993 & 4.822 & 6.639 & 3.79 \\
\hline $11 / 3 / 2005$ & $5: 00$ & 10.827 & 10.693 & 7.884 & 6.765 & 4.39 & 4.225 & 2.467 & 2.429 & 10.718 & 5.988 & 4.812 & 6.635 & 3.78 \\
\hline $11 / 3 / 2005$ & $6: 00$ & 10.822 & 10.691 & 7.881 & 6.763 & 4.389 & 4.22 & 2.464 & 2.427 & 10.715 & 5.988 & 4.808 & 6.632 & 3.772 \\
\hline $11 / 3 / 2005$ & $7: 00$ & 10.822 & 10.691 & 7.879 & 6.761 & 4.386 & 4.22 & 2.462 & 2.425 & 10.713 & 5.99 & 4.804 & 6.627 & 3.77 \\
\hline $11 / 3 / 2005$ & $8: 00$ & 10.817 & 10.686 & 7.876 & 6.758 & 4.381 & 4.218 & 2.46 & 2.423 & 10.711 & 5.986 & 4.798 & 6.626 & 3.764 \\
\hline $11 / 3 / 2005$ & $9: 00$ & 10.817 & 10.689 & 7.876 & 6.763 & 4.382 & 4.214 & 2.457 & 2.423 & 10.709 & 5.986 & 4.798 & 6.627 & 3.762 \\
\hline $11 / 3 / 2005$ & $10: 00$ & 10.815 & 10.684 & 7.876 & 6.756 & 4.375 & 4.211 & 2.452 & 2.421 & 10.709 & 5.979 & 4.79 & 6.622 & 3.758 \\
\hline $11 / 3 / 2005$ & $11: 00$ & 10.815 & 10.682 & 7.876 & 6.756 & 4.375 & 4.207 & 2.452 & 2.421 & 10.707 & 5.988 & 4.794 & 6.624 & 3.764 \\
\hline $11 / 3 / 2005$ & $12: 00$ & 10.81 & 10.677 & 7.871 & 6.747 & 4.373 & 4.203 & 2.454 & 2.416 & 10.705 & 5.986 & 4.796 & 6.624 & 3.78 \\
\hline $11 / 3 / 2005$ & $13: 00$ & 10.805 & 10.666 & 7.867 & 6.741 & 4.372 & 4.198 & 2.449 & 2.41 & 10.701 & 5.988 & 4.794 & 6.62 & 3.798 \\
\hline $11 / 3 / 2005$ & $14: 00$ & 10.798 & 10.662 & 7.865 & 6.741 & 4.378 & 4.194 & 2.449 & 2.41 & 10.703 & 5.992 & 4.8 & 6.62 & 3.818 \\
\hline $11 / 3 / 2005$ & $15: 00$ & 10.798 & 10.66 & 7.867 & 6.743 & 4.387 & 4.192 & 2.454 & 2.414 & 10.703 & 5.997 & 4.807 & 6.62 & 3.839 \\
\hline $11 / 3 / 2005$ & $16: 00$ & 10.795 & 10.658 & 7.867 & 6.741 & 4.398 & 4.192 & 2.449 & 2.412 & 10.705 & 6.006 & 4.815 & 6.622 & 3.854 \\
\hline $11 / 3 / 2005$ & $17: 00$ & 10.793 & 10.657 & 7.867 & 6.743 & 4.404 & 4.192 & 2.457 & 2.414 & 10.705 & 6.004 & 4.819 & 6.62 & 3.863 \\
\hline $11 / 3 / 2005$ & $18: 00$ & 10.795 & 10.664 & 7.871 & 6.752 & 4.413 & 4.194 & 2.462 & 2.423 & 10.707 & 6.006 & 4.823 & 6.622 & 3.858 \\
\hline $11 / 3 / 2005$ & $19: 00$ & 10.805 & 10.677 & 7.873 & 6.761 & 4.421 & 4.2 & 2.469 & 2.427 & 10.709 & 6.008 & 4.825 & 6.624 & 3.853 \\
\hline $11 / 3 / 2005$ & $20: 00$ & 10.81 & 10.686 & 7.88 & 6.768 & 4.425 & 4.203 & 2.475 & 2.435 & 10.711 & 6.006 & 4.823 & 6.626 & 3.843 \\
\hline $11 / 3 / 2005$ & $21: 00$ & 10.82 & 10.697 & 7.884 & 6.776 & 4.428 & 4.21 & 2.48 & 2.442 & 10.711 & 6.008 & 4.823 & 6.628 & 3.832 \\
\hline $11 / 3 / 2005$ & $22: 00$ & 10.822 & 10.7 & 7.886 & 6.774 & 4.423 & 4.211 & 2.48 & 2.442 & 10.711 & 6.004 & 4.815 & 6.626 & 3.818 \\
\hline $11 / 3 / 2005$ & $23: 00$ & 10.827 & 10.706 & 7.886 & 6.779 & 4.418 & 4.216 & 2.48 & 2.442 & 10.711 & 6.001 & 4.811 & 6.626 & 3.806 \\
\hline $11 / 4 / 2005$ & $0: 00$ & 10.83 & 10.709 & 7.886 & 6.779 & 4.415 & 4.219 & 2.475 & 2.442 & 10.709 & 5.999 & 4.807 & 6.626 & 3.792 \\
\hline $11 / 4 / 2005$ & $1: 00$ & 10.832 & 10.711 & 7.888 & 6.781 & 4.411 & 4.221 & 2.48 & 2.442 & 10.707 & 5.997 & 4.802 & 6.626 & 3.786 \\
\hline $11 / 4 / 2005$ & $2: 00$ & 10.839 & 10.72 & 7.893 & 6.79 & 4.412 & 4.223 & 2.487 & 2.45 & 10.709 & 5.997 & 4.802 & 6.63 & 3.782 \\
\hline $11 / 4 / 2005$ & $3: 00$ & 10.844 & 10.728 & 7.896 & 6.794 & 4.411 & 4.227 & 2.49 & 2.454 & 10.709 & 5.997 & 4.8 & 6.63 & 3.775 \\
\hline $11 / 4 / 2005$ & $4: 00$ & 10.849 & 10.733 & 7.899 & 6.799 & 4.41 & 4.23 & 2.487 & 2.457 & 10.709 & 5.995 & 4.8 & 6.632 & 3.771 \\
\hline $11 / 4 / 2005$ & $5: 00$ & 10.854 & 10.735 & 7.899 & 6.794 & 4.404 & 4.23 & 2.492 & 2.455 & 10.709 & 5.992 & 4.796 & 6.634 & 3.762 \\
\hline $11 / 4 / 2005$ & $6: 00$ & 10.857 & 10.741 & 7.901 & 6.799 & 4.401 & 4.232 & 2.492 & 2.455 & 10.707 & 5.992 & 4.794 & 6.637 & 3.758 \\
\hline $11 / 4 / 2005$ & $7: 00$ & 10.859 & 10.742 & 7.903 & 6.801 & 4.402 & 4.232 & 2.497 & 2.459 & 10.705 & 5.992 & 4.794 & 6.637 & 3.753 \\
\hline $11 / 4 / 2005$ & $8: 00$ & 10.862 & 10.748 & 7.904 & 6.805 & 4.401 & 4.234 & 2.5 & 2.461 & 10.707 & 5.994 & 4.792 & 6.637 & 3.751 \\
\hline $11 / 4 / 2005$ & $9: 00$ & 10.869 & 10.755 & 7.911 & 6.812 & 4.402 & 4.236 & 2.505 & 2.467 & 10.709 & 5.995 & 4.794 & 6.639 & 3.751 \\
\hline $11 / 4 / 2005$ & $10: 00$ & 10.871 & 10.753 & 7.913 & 6.807 & 4.397 & 4.236 & 2.495 & 2.463 & 10.705 & 5.988 & 4.792 & 6.639 & 3.743 \\
\hline $11 / 4 / 2005$ & $11: 00$ & 10.874 & 10.753 & 7.911 & 6.805 & 4.393 & 4.236 & 2.495 & 2.463 & 10.705 & 5.99 & 4.794 & 6.639 & 3.739 \\
\hline $11 / 4 / 2005$ & $12: 00$ & 10.871 & 10.751 & 7.909 & 6.803 & 4.39 & 4.234 & 2.495 & 2.461 & 10.705 & 5.988 & 4.792 & 6.639 & 3.747 \\
\hline $11 / 4 / 2005$ & $13: 00$ & 10.866 & 10.742 & 7.908 & 6.799 & 4.39 & 4.234 & 2.49 & 2.459 & 10.703 & 5.988 & 4.794 & 6.639 & 3.759 \\
\hline
\end{tabular}


Water Level (ft below top of casing) at Indicated Well

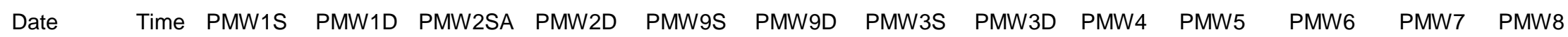

\begin{tabular}{|c|c|c|c|c|c|c|c|c|c|c|c|c|c|c|}
\hline $11 / 4 / 2005$ & $14: 00$ & 10.859 & 10.733 & 7.904 & 6.792 & 4.388 & 4.23 & 2.485 & 2.453 & 10.704 & 5.988 & 4.798 & 6.636 & 3.771 \\
\hline $11 / 4 / 2005$ & $15: 00$ & 10.854 & 10.726 & 7.9 & 6.785 & 4.391 & 4.227 & 2.49 & 2.448 & 10.704 & 5.99 & 4.8 & 6.636 & 3.784 \\
\hline $11 / 4 / 2005$ & $16: 00$ & 10.849 & 10.717 & 7.896 & 6.776 & 4.392 & 4.225 & 2.485 & 2.446 & 10.702 & 5.988 & 4.802 & 6.636 & 3.794 \\
\hline $11 / 4 / 2005$ & $17: 00$ & 10.844 & 10.715 & 7.893 & 6.779 & 4.397 & 4.225 & 2.487 & 2.444 & 10.704 & 5.992 & 4.807 & 6.638 & 3.804 \\
\hline $11 / 4 / 2005$ & $18: 00$ & 10.846 & 10.715 & 7.896 & 6.781 & 4.405 & 4.225 & 2.487 & 2.446 & 10.704 & 5.992 & 4.811 & 6.636 & 3.81 \\
\hline $11 / 4 / 2005$ & $19: 00$ & 10.851 & 10.728 & 7.902 & 6.794 & 4.417 & 4.23 & 2.497 & 2.457 & 10.707 & 5.997 & 4.817 & 6.638 & 3.81 \\
\hline $11 / 4 / 2005$ & $20: 00$ & 10.854 & 10.73 & 7.902 & 6.794 & 4.415 & 4.23 & 2.487 & 2.455 & 10.707 & 5.99 & 4.809 & 6.636 & 3.797 \\
\hline $11 / 4 / 2005$ & $21: 00$ & 10.861 & 10.739 & 7.906 & 6.801 & 4.416 & 4.232 & 2.499 & 2.461 & 10.707 & 5.994 & 4.811 & 6.64 & 3.794 \\
\hline $11 / 4 / 2005$ & $22: 00$ & 10.859 & 10.733 & 7.904 & 6.797 & 4.411 & 4.232 & 2.49 & 2.457 & 10.705 & 5.983 & 4.805 & 6.636 & 3.78 \\
\hline $11 / 4 / 2005$ & $23: 00$ & 10.851 & 10.722 & 7.896 & 6.783 & 4.395 & 4.23 & 2.482 & 2.444 & 10.704 & 5.977 & 4.793 & 6.63 & 3.761 \\
\hline $11 / 5 / 2005$ & $0: 00$ & 10.854 & 10.728 & 7.896 & 6.786 & 4.397 & 4.232 & 2.485 & 2.446 & 10.702 & 5.983 & 4.797 & 6.634 & 3.763 \\
\hline $11 / 5 / 2005$ & $1: 00$ & 10.851 & 10.724 & 7.898 & 6.786 & 4.394 & 4.23 & 2.482 & 2.442 & 10.702 & 5.977 & 4.789 & 6.632 & 3.757 \\
\hline $11 / 5 / 2005$ & $2: 00$ & 10.854 & 10.728 & 7.9 & 6.788 & 4.395 & 4.23 & 2.487 & 2.448 & 10.702 & 5.979 & 4.787 & 6.634 & 3.755 \\
\hline $11 / 5 / 2005$ & $3: 00$ & 10.846 & 10.719 & 7.894 & 6.777 & 4.384 & 4.227 & 2.477 & 2.438 & 10.7 & 5.97 & 4.778 & 6.626 & 3.739 \\
\hline $11 / 5 / 2005$ & $4: 00$ & 10.846 & 10.719 & 7.894 & 6.779 & 4.382 & 4.228 & 2.477 & 2.438 & 10.698 & 5.974 & 4.776 & 6.628 & 3.737 \\
\hline $11 / 5 / 2005$ & $5: 00$ & 10.849 & 10.728 & 7.896 & 6.786 & 4.387 & 4.227 & 2.477 & 2.445 & 10.698 & 5.977 & 4.778 & 6.628 & 3.741 \\
\hline $11 / 5 / 2005$ & $6: 00$ & 10.851 & 10.724 & 7.896 & 6.786 & 4.382 & 4.226 & 2.482 & 2.445 & 10.696 & 5.97 & 4.772 & 6.626 & 3.733 \\
\hline $11 / 5 / 2005$ & $7: 00$ & 10.854 & 10.728 & 7.896 & 6.786 & 4.38 & 4.226 & 2.475 & 2.442 & 10.694 & 5.97 & 4.77 & 6.628 & 3.729 \\
\hline $11 / 5 / 2005$ & $8: 00$ & 10.854 & 10.728 & 7.896 & 6.788 & 4.378 & 4.226 & 2.475 & 2.445 & 10.694 & 5.97 & 4.766 & 6.624 & 3.727 \\
\hline $11 / 5 / 2005$ & $9: 00$ & 10.851 & 10.728 & 7.898 & 6.786 & 4.374 & 4.223 & 2.48 & 2.442 & 10.694 & 5.968 & 4.762 & 6.626 & 3.723 \\
\hline $11 / 5 / 2005$ & $10: 00$ & 10.851 & 10.722 & 7.894 & 6.779 & 4.368 & 4.221 & 2.472 & 2.436 & 10.692 & 5.967 & 4.756 & 6.624 & 3.714 \\
\hline $11 / 5 / 2005$ & $11: 00$ & 10.854 & 10.728 & 7.896 & 6.788 & 4.373 & 4.221 & 2.482 & 2.442 & 10.692 & 5.97 & 4.76 & 6.626 & 3.723 \\
\hline $11 / 5 / 2005$ & $12: 00$ & 10.856 & 10.731 & 7.9 & 6.79 & 4.377 & 4.221 & 2.485 & 2.447 & 10.692 & 5.972 & 4.762 & 6.628 & 3.733 \\
\hline $11 / 5 / 2005$ & $13: 00$ & 10.856 & 10.731 & 7.9 & 6.788 & 4.379 & 4.219 & 2.482 & 2.447 & 10.692 & 5.97 & 4.762 & 6.624 & 3.739 \\
\hline $11 / 5 / 2005$ & $14: 00$ & 10.849 & 10.717 & 7.89 & 6.777 & 4.369 & 4.217 & 2.475 & 2.434 & 10.69 & 5.965 & 4.756 & 6.62 & 3.735 \\
\hline $11 / 5 / 2005$ & $15: 00$ & 10.849 & 10.72 & 7.892 & 6.781 & 4.376 & 4.217 & 2.469 & 2.438 & 10.688 & 5.972 & 4.762 & 6.624 & 3.745 \\
\hline $11 / 5 / 2005$ & $16: 00$ & 10.854 & 10.728 & 7.898 & 6.788 & 4.388 & 4.219 & 2.48 & 2.451 & 10.692 & 5.974 & 4.766 & 6.624 & 3.761 \\
\hline $11 / 5 / 2005$ & $17: 00$ & 10.864 & 10.744 & 7.906 & 6.803 & 4.4 & 4.223 & 2.492 & 2.461 & 10.696 & 5.983 & 4.776 & 6.632 & 3.771 \\
\hline $11 / 5 / 2005$ & $18: 00$ & 10.871 & 10.754 & 7.912 & 6.81 & 4.407 & 4.228 & 2.5 & 2.468 & 10.698 & 5.985 & 4.78 & 6.634 & 3.773 \\
\hline $11 / 5 / 2005$ & $19: 00$ & 10.876 & 10.755 & 7.912 & 6.812 & 4.406 & 4.23 & 2.5 & 2.468 & 10.698 & 5.981 & 4.78 & 6.634 & 3.767 \\
\hline $11 / 5 / 2005$ & $20: 00$ & 10.881 & 10.761 & 7.917 & 6.817 & 4.41 & 4.234 & 2.505 & 2.474 & 10.7 & 5.981 & 4.778 & 6.615 & 3.763 \\
\hline $11 / 5 / 2005$ & $21: 00$ & 10.886 & 10.764 & 7.918 & 6.803 & 4.406 & 4.237 & 2.505 & 2.472 & 10.7 & 5.981 & 4.778 & 6.601 & 3.755 \\
\hline $11 / 5 / 2005$ & $22: 00$ & 10.888 & 10.768 & 7.918 & 6.819 & 4.405 & 4.239 & 2.505 & 2.472 & 10.7 & 5.981 & 4.776 & 6.64 & 3.749 \\
\hline $11 / 5 / 2005$ & $23: 00$ & 10.893 & 10.775 & 7.925 & 6.828 & 4.407 & 4.245 & 2.512 & 2.482 & 10.702 & 5.983 & 4.78 & 6.644 & 3.751 \\
\hline
\end{tabular}


Water Level (ft below top of casing) at Indicated Well

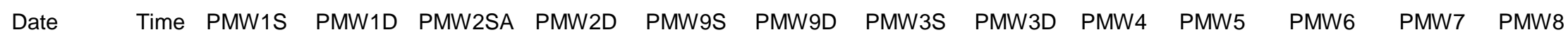

\begin{tabular}{|c|c|c|c|c|c|c|c|c|c|c|c|c|c|c|}
\hline $11 / 6 / 2005$ & $0: 00$ & 10.9 & 10.786 & 7.929 & 6.837 & 4.412 & 4.25 & 2.52 & 2.489 & 10.705 & 5.985 & 4.782 & 6.648 & 3.751 \\
\hline $11 / 6 / 2005$ & $1: 00$ & 10.908 & 10.797 & 7.935 & 6.844 & 4.417 & 4.254 & 2.53 & 2.497 & 10.71 & 5.99 & 4.786 & 6.651 & 3.751 \\
\hline $11 / 6 / 2005$ & $2: 00$ & 10.918 & 10.806 & 7.941 & 6.855 & 4.421 & 4.261 & 2.535 & 2.503 & 10.711 & 5.992 & 4.787 & 6.655 & 3.753 \\
\hline $11 / 6 / 2005$ & $3: 00$ & 10.925 & 10.817 & 7.947 & 6.861 & 4.424 & 4.265 & 2.543 & 2.512 & 10.713 & 5.994 & 4.791 & 6.663 & 3.753 \\
\hline $11 / 6 / 2005$ & $4: 00$ & 10.932 & 10.825 & 7.951 & 6.868 & 4.426 & 4.272 & 2.55 & 2.516 & 10.715 & 5.994 & 4.793 & 6.665 & 3.751 \\
\hline $11 / 6 / 2005$ & $5: 00$ & 10.937 & 10.828 & 7.955 & 6.866 & 4.425 & 4.274 & 2.547 & 2.502 & 10.717 & 5.994 & 4.793 & 6.667 & 3.745 \\
\hline $11 / 6 / 2005$ & $6: 00$ & 10.945 & 10.834 & 7.957 & 6.87 & 4.424 & 4.279 & 2.553 & 2.502 & 10.719 & 5.996 & 4.793 & 6.668 & 3.743 \\
\hline $11 / 6 / 2005$ & $7: 00$ & 10.949 & 10.839 & 7.961 & 6.875 & 4.425 & 4.283 & 2.558 & 2.487 & 10.719 & 5.996 & 4.793 & 6.67 & 3.743 \\
\hline $11 / 6 / 2005$ & $8: 00$ & 10.954 & 10.847 & 7.967 & 6.881 & 4.429 & 4.288 & 2.563 & 2.479 & 10.723 & 6.003 & 4.799 & 6.674 & 3.747 \\
\hline $11 / 6 / 2005$ & $9: 00$ & 10.962 & 10.856 & 7.973 & 6.89 & 4.436 & 4.292 & 2.573 & 2.539 & 10.725 & 6.005 & 4.803 & 6.678 & 3.749 \\
\hline $11 / 6 / 2005$ & $10: 00$ & 10.969 & 10.861 & 7.977 & 6.892 & 4.436 & 4.296 & 2.573 & 2.544 & 10.727 & 6.005 & 4.803 & 6.68 & 3.747 \\
\hline $11 / 6 / 2005$ & $11: 00$ & 10.974 & 10.867 & 7.979 & 6.895 & 4.435 & 4.299 & 2.578 & 2.546 & 10.729 & 6.007 & 4.803 & 6.682 & 3.745 \\
\hline $11 / 6 / 2005$ & $12: 00$ & 10.976 & 10.863 & 7.979 & 6.892 & 4.431 & 4.299 & 2.575 & 2.542 & 10.729 & 6 & 4.801 & 6.684 & 3.741 \\
\hline $11 / 6 / 2005$ & $13: 00$ & 10.976 & 10.858 & 7.979 & 6.886 & 4.425 & 4.299 & 2.57 & 2.539 & 10.727 & 6 & 4.801 & 6.682 & 3.749 \\
\hline $11 / 6 / 2005$ & $14: 00$ & 10.969 & 10.845 & 7.972 & 6.875 & 4.423 & 4.299 & 2.562 & 2.529 & 10.725 & 5.998 & 4.803 & 6.676 & 3.761 \\
\hline $11 / 6 / 2005$ & $15: 00$ & 10.961 & 10.832 & 7.966 & 6.866 & 4.42 & 4.292 & 2.555 & 2.523 & 10.723 & 5.994 & 4.805 & 6.676 & 3.771 \\
\hline $11 / 6 / 2005$ & $16: 00$ & 10.954 & 10.825 & 7.964 & 6.859 & 4.42 & 4.292 & 2.55 & 2.514 & 10.725 & 5.996 & 4.809 & 6.672 & 3.781 \\
\hline $11 / 6 / 2005$ & $17: 00$ & 10.949 & 10.819 & 7.962 & 6.857 & 4.426 & 4.29 & 2.55 & 2.518 & 10.723 & 5.998 & 4.815 & 6.676 & 3.793 \\
\hline $11 / 6 / 2005$ & $18: 00$ & 10.944 & 10.814 & 7.957 & 6.855 & 4.426 & 4.29 & 2.545 & 2.512 & 10.723 & 5.998 & 4.816 & 6.672 & 3.793 \\
\hline $11 / 6 / 2005$ & $19: 00$ & 10.942 & 10.816 & 7.957 & 6.857 & 4.433 & 4.29 & 2.545 & 2.512 & 10.725 & 6 & 4.82 & 6.676 & 3.793 \\
\hline $11 / 6 / 2005$ & $20: 00$ & 10.942 & 10.816 & 7.962 & 6.857 & 4.435 & 4.29 & 2.547 & 2.514 & 10.725 & 5.998 & 4.82 & 6.674 & 3.789 \\
\hline $11 / 6 / 2005$ & $21: 00$ & 10.942 & 10.814 & 7.96 & 6.855 & 4.432 & 4.29 & 2.542 & 2.51 & 10.723 & 5.994 & 4.82 & 6.674 & 3.783 \\
\hline $11 / 6 / 2005$ & $22: 00$ & 10.942 & 10.816 & 7.96 & 6.859 & 4.433 & 4.29 & 2.545 & 2.514 & 10.725 & 5.996 & 4.824 & 6.675 & 3.781 \\
\hline $11 / 6 / 2005$ & $23: 00$ & 10.942 & 10.817 & 7.96 & 6.86 & 4.434 & 4.288 & 2.547 & 2.516 & 10.725 & 5.994 & 4.824 & 6.676 & 3.779 \\
\hline $11 / 7 / 2005$ & $0: 00$ & 10.944 & 10.817 & 7.96 & 6.86 & 4.432 & 4.29 & 2.547 & 2.514 & 10.725 & 5.994 & 4.822 & 6.673 & 3.777 \\
\hline $11 / 7 / 2005$ & $1: 00$ & 10.942 & 10.816 & 7.958 & 6.857 & 4.433 & 4.288 & 2.545 & 2.512 & 10.725 & 5.994 & 4.818 & 6.67 & 3.773 \\
\hline $11 / 7 / 2005$ & $2: 00$ & 10.944 & 10.816 & 7.958 & 6.862 & 4.432 & 4.29 & 2.547 & 2.515 & 10.725 & 5.993 & 4.818 & 6.675 & 3.777 \\
\hline $11 / 7 / 2005$ & $3: 00$ & 10.944 & 10.816 & 7.956 & 6.857 & 4.431 & 4.29 & 2.547 & 2.515 & 10.725 & 5.994 & 4.818 & 6.674 & 3.771 \\
\hline $11 / 7 / 2005$ & $4: 00$ & 10.944 & 10.817 & 7.956 & 6.86 & 4.431 & 4.29 & 2.547 & 2.515 & 10.725 & 5.994 & 4.816 & 6.672 & 3.771 \\
\hline $11 / 7 / 2005$ & $5: 00$ & 10.942 & 10.814 & 7.954 & 6.857 & 4.428 & 4.288 & 2.542 & 2.513 & 10.723 & 5.991 & 4.813 & 6.673 & 3.769 \\
\hline $11 / 7 / 2005$ & $6: 00$ & 10.939 & 10.812 & 7.954 & 6.855 & 4.427 & 4.288 & 2.542 & 2.51 & 10.723 & 5.991 & 4.81 & 6.672 & 3.771 \\
\hline $11 / 7 / 2005$ & $7: 00$ & 10.939 & 10.814 & 7.956 & 6.857 & 4.431 & 4.288 & 2.545 & 2.513 & 10.725 & 5.991 & 4.811 & 6.673 & 3.771 \\
\hline $11 / 7 / 2005$ & $8: 00$ & 10.942 & 10.814 & 7.956 & 6.86 & 4.432 & 4.288 & 2.545 & 2.515 & 10.723 & 5.991 & 4.809 & 6.673 & 3.769 \\
\hline $11 / 7 / 2005$ & $9: 00$ & 10.944 & 10.819 & 7.958 & 6.862 & 4.433 & 4.29 & 2.55 & 2.517 & 10.723 & 5.993 & 4.81 & 6.673 & 3.769 \\
\hline
\end{tabular}


TABLE S1.2 (Cont.)

Water Level (ft below top of casing) at Indicated Well

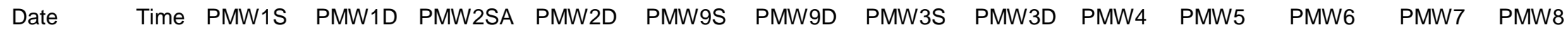

\begin{tabular}{|c|c|c|c|c|c|c|c|c|c|c|c|c|c|c|}
\hline $11 / 7 / 2005$ & $10: 00$ & 10.947 & 10.821 & 7.96 & 6.864 & 4.434 & 4.288 & 2.552 & 2.519 & 10.725 & 5.993 & 4.81 & 6.673 & 3.773 \\
\hline $11 / 7 / 2005$ & $11: 00$ & 10.947 & 10.819 & 7.962 & 6.864 & 4.437 & 4.29 & 2.555 & 2.521 & 10.727 & 5.995 & 4.814 & 6.675 & 3.787 \\
\hline $11 / 7 / 2005$ & $12: 00$ & 10.949 & 10.819 & 7.964 & 6.864 & 4.444 & 4.288 & 2.557 & 2.523 & 10.727 & 5.998 & 4.82 & 6.676 & 3.805 \\
\hline $11 / 7 / 2005$ & $13: 00$ & 10.944 & 10.814 & 7.962 & 6.86 & 4.446 & 4.288 & 2.555 & 2.521 & 10.729 & 6.002 & 4.826 & 6.676 & 3.821 \\
\hline $11 / 7 / 2005$ & $14: 00$ & 10.941 & 10.808 & 7.962 & 6.86 & 4.451 & 4.288 & 2.554 & 2.521 & 10.731 & 6 & 4.832 & 6.676 & 3.835 \\
\hline $11 / 7 / 2005$ & $15: 00$ & 10.939 & 10.805 & 7.959 & 6.857 & 4.456 & 4.286 & 2.554 & 2.519 & 10.733 & 6.006 & 4.838 & 6.676 & 3.85 \\
\hline $11 / 7 / 2005$ & $16: 00$ & 10.939 & 10.806 & 7.961 & 6.86 & 4.463 & 4.288 & 2.557 & 2.523 & 10.734 & 6.009 & 4.84 & 6.678 & 3.864 \\
\hline $11 / 7 / 2005$ & $17: 00$ & 10.941 & 10.81 & 7.964 & 6.866 & 4.47 & 4.29 & 2.562 & 2.527 & 10.737 & 6.013 & 4.849 & 6.68 & 3.874 \\
\hline $11 / 7 / 2005$ & $18: 00$ & 10.946 & 10.816 & 7.967 & 6.868 & 4.474 & 4.295 & 2.562 & 2.529 & 10.738 & 6.015 & 4.851 & 6.682 & 3.868 \\
\hline $11 / 7 / 2005$ & $19: 00$ & 10.951 & 10.823 & 7.97 & 6.873 & 4.478 & 4.299 & 2.567 & 2.534 & 10.74 & 6.015 & 4.853 & 6.684 & 3.856 \\
\hline $11 / 7 / 2005$ & $20: 00$ & 10.956 & 10.832 & 7.974 & 6.882 & 4.479 & 4.303 & 2.572 & 2.54 & 10.742 & 6.013 & 4.853 & 6.686 & 3.843 \\
\hline $11 / 7 / 2005$ & $21: 00$ & 10.963 & 10.837 & 7.976 & 6.884 & 4.475 & 4.308 & 2.575 & 2.54 & 10.744 & 6.011 & 4.851 & 6.688 & 3.831 \\
\hline $11 / 7 / 2005$ & $22: 00$ & 10.968 & 10.848 & 7.98 & 6.888 & 4.476 & 4.31 & 2.58 & 2.546 & 10.744 & 6.013 & 4.851 & 6.69 & 3.823 \\
\hline $11 / 7 / 2005$ & $23: 00$ & 10.971 & 10.852 & 7.98 & 6.893 & 4.474 & 4.313 & 2.582 & 2.548 & 10.744 & 6.006 & 4.847 & 6.692 & 3.813 \\
\hline $11 / 8 / 2005$ & $0: 00$ & 10.973 & 10.848 & 7.98 & 6.886 & 4.463 & 4.314 & 2.575 & 2.542 & 10.742 & 6.002 & 4.84 & 6.688 & 3.799 \\
\hline $11 / 8 / 2005$ & $1: 00$ & 10.973 & 10.848 & 7.978 & 6.889 & 4.458 & 4.315 & 2.575 & 2.542 & 10.742 & 6.002 & 4.836 & 6.688 & 3.791 \\
\hline $11 / 8 / 2005$ & $2: 00$ & 10.971 & 10.843 & 7.976 & 6.882 & 4.451 & 4.315 & 2.567 & 2.536 & 10.74 & 5.995 & 4.824 & 6.684 & 3.779 \\
\hline $11 / 8 / 2005$ & $3: 00$ & 10.966 & 10.834 & 7.97 & 6.875 & 4.441 & 4.313 & 2.562 & 2.531 & 10.739 & 5.993 & 4.816 & 6.682 & 3.769 \\
\hline $11 / 8 / 2005$ & $4: 00$ & 10.963 & 10.83 & 7.968 & 6.871 & 4.435 & 4.313 & 2.557 & 2.525 & 10.737 & 5.991 & 4.814 & 6.68 & 3.762 \\
\hline $11 / 8 / 2005$ & $5: 00$ & 10.961 & 10.827 & 7.966 & 6.869 & 4.432 & 4.308 & 2.554 & 2.521 & 10.733 & 5.991 & 4.808 & 6.676 & 3.758 \\
\hline $11 / 8 / 2005$ & $6: 00$ & 10.953 & 10.819 & 7.962 & 6.862 & 4.424 & 4.306 & 2.547 & 2.517 & 10.731 & 5.984 & 4.798 & 6.673 & 3.75 \\
\hline $11 / 8 / 2005$ & $7: 00$ & 10.951 & 10.817 & 7.958 & 6.86 & 4.422 & 4.301 & 2.544 & 2.513 & 10.729 & 5.986 & 4.795 & 6.671 & 3.746 \\
\hline $11 / 8 / 2005$ & $8: 00$ & 10.944 & 10.81 & 7.954 & 6.853 & 4.414 & 4.299 & 2.537 & 2.505 & 10.727 & 5.984 & 4.789 & 6.669 & 3.738 \\
\hline $11 / 8 / 2005$ & $9: 00$ & 10.941 & 10.803 & 7.95 & 6.849 & 4.409 & 4.295 & 2.534 & 2.5 & 10.723 & 5.98 & 4.781 & 6.665 & 3.734 \\
\hline $11 / 8 / 2005$ & $10: 00$ & 10.934 & 10.799 & 7.948 & 6.842 & 4.406 & 4.291 & 2.532 & 2.498 & 10.721 & 5.98 & 4.777 & 6.661 & 3.728 \\
\hline $11 / 8 / 2005$ & $11: 00$ & 10.926 & 10.79 & 7.942 & 6.836 & 4.398 & 4.284 & 2.524 & 2.49 & 10.716 & 5.98 & 4.773 & 6.658 & 3.728 \\
\hline $11 / 8 / 2005$ & $12: 00$ & 10.919 & 10.787 & 7.94 & 6.836 & 4.4 & 4.279 & 2.524 & 2.492 & 10.716 & 5.975 & 4.773 & 6.655 & 3.74 \\
\hline $11 / 8 / 2005$ & $13: 00$ & 10.911 & 10.776 & 7.936 & 6.824 & 4.395 & 4.275 & 2.514 & 2.482 & 10.714 & 5.977 & 4.769 & 6.652 & 3.74 \\
\hline $11 / 8 / 2005$ & $14: 00$ & 10.901 & 10.766 & 7.929 & 6.818 & 4.39 & 4.271 & 2.506 & 2.475 & 10.712 & 5.977 & 4.769 & 6.652 & 3.748 \\
\hline $11 / 8 / 2005$ & $15: 00$ & 10.894 & 10.759 & 7.925 & 6.813 & 4.394 & 4.264 & 2.506 & 2.473 & 10.71 & 5.982 & 4.773 & 6.654 & 3.768 \\
\hline $11 / 8 / 2005$ & $16: 00$ & 10.889 & 10.752 & 7.923 & 6.809 & 4.4 & 4.262 & 2.506 & 2.471 & 10.712 & 5.984 & 4.777 & 6.65 & 3.787 \\
\hline $11 / 8 / 2005$ & $17: 00$ & 10.887 & 10.754 & 7.925 & 6.816 & 4.412 & 4.26 & 2.509 & 2.475 & 10.71 & 5.988 & 4.78 & 6.65 & 3.805 \\
\hline $11 / 8 / 2005$ & $18: 00$ & 10.887 & 10.757 & 7.928 & 6.82 & 4.419 & 4.26 & 2.514 & 2.479 & 10.712 & 5.992 & 4.786 & 6.65 & 3.811 \\
\hline $11 / 8 / 2005$ & $19: 00$ & 10.887 & 10.754 & 7.924 & 6.818 & 4.42 & 4.26 & 2.509 & 2.477 & 10.712 & 5.988 & 4.784 & 6.648 & 3.799 \\
\hline
\end{tabular}


Water Level (ft below top of casing) at Indicated Well

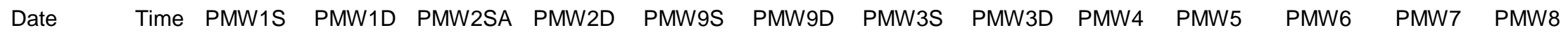

\begin{tabular}{|c|c|c|c|c|c|c|c|c|c|c|c|c|c|c|}
\hline $11 / 8 / 2005$ & $20: 00$ & 10.887 & 10.758 & 7.926 & 6.82 & 4.422 & 4.262 & 2.514 & 2.479 & 10.714 & 5.986 & 4.786 & 6.652 & 3.793 \\
\hline $11 / 8 / 2005$ & $21: 00$ & 10.892 & 10.765 & 7.928 & 6.824 & 4.426 & 4.264 & 2.516 & 2.484 & 10.71 & 5.988 & 4.79 & 6.654 & 3.787 \\
\hline $11 / 8 / 2005$ & $22: 00$ & 10.892 & 10.765 & 7.928 & 6.822 & 4.42 & 4.262 & 2.514 & 2.482 & 10.71 & 5.984 & 4.786 & 6.652 & 3.776 \\
\hline $11 / 8 / 2005$ & $23: 00$ & 10.914 & 10.796 & 7.942 & 6.855 & 4.441 & 4.271 & 2.542 & 2.505 & 10.714 & 6.006 & 4.81 & 6.665 & 3.793 \\
\hline $11 / 9 / 2005$ & $0: 00$ & 10.939 & 10.827 & 7.959 & 6.88 & 4.467 & 4.282 & 2.567 & 2.534 & 10.72 & 6.008 & 4.818 & 6.671 & 3.807 \\
\hline $11 / 9 / 2005$ & $1: 00$ & 10.963 & 10.856 & 7.975 & 6.905 & 4.475 & 4.295 & 2.592 & 2.555 & 10.723 & 6.012 & 4.831 & 6.684 & 3.819 \\
\hline $11 / 9 / 2005$ & $2: 00$ & 10.983 & 10.874 & 7.989 & 6.916 & 4.483 & 4.304 & 2.599 & 2.568 & 10.733 & 6.01 & 4.837 & 6.69 & 3.825 \\
\hline $11 / 9 / 2005$ & $3: 00$ & 11 & 10.891 & 7.998 & 6.927 & 4.491 & 4.313 & 2.61 & 2.578 & 10.737 & 6.017 & 4.847 & 6.696 & 3.827 \\
\hline $11 / 9 / 2005$ & $4: 00$ & 11.008 & 10.9 & 8.004 & 6.936 & 4.494 & 4.322 & 2.617 & 2.587 & 10.746 & 6.017 & 4.851 & 6.703 & 3.827 \\
\hline $11 / 9 / 2005$ & $5: 00$ & 11.017 & 10.905 & 8.006 & 6.936 & 4.495 & 4.33 & 2.619 & 2.587 & 10.75 & 6.019 & 4.851 & 6.703 & 3.823 \\
\hline $11 / 9 / 2005$ & $6: 00$ & 11.025 & 10.914 & 8.014 & 6.942 & 4.497 & 4.337 & 2.625 & 2.595 & 10.752 & 6.021 & 4.857 & 6.707 & 3.823 \\
\hline $11 / 9 / 2005$ & $7: 00$ & 11.032 & 10.92 & 8.018 & 6.949 & 4.498 & 4.344 & 2.63 & 2.597 & 10.756 & 6.021 & 4.859 & 6.713 & 3.821 \\
\hline $11 / 9 / 2005$ & $8: 00$ & 11.037 & 10.924 & 8.019 & 6.947 & 4.494 & 4.35 & 2.63 & 2.597 & 10.756 & 6.019 & 4.859 & 6.713 & 3.813 \\
\hline $11 / 9 / 2005$ & $9: 00$ & 11.044 & 10.934 & 8.026 & 6.958 & 4.501 & 4.357 & 2.637 & 2.606 & 10.759 & 6.023 & 4.863 & 6.717 & 3.815 \\
\hline $11 / 9 / 2005$ & $10: 00$ & 11.056 & 10.942 & 8.028 & 6.96 & 4.503 & 4.361 & 2.642 & 2.61 & 10.762 & 6.025 & 4.867 & 6.719 & 3.819 \\
\hline $11 / 9 / 2005$ & $11: 00$ & 11.059 & 10.947 & 8.032 & 6.962 & 4.505 & 4.366 & 2.645 & 2.61 & 10.761 & 6.025 & 4.871 & 6.723 & 3.823 \\
\hline $11 / 9 / 2005$ & $12: 00$ & 11.064 & 10.955 & 8.038 & 6.969 & 4.513 & 4.37 & 2.652 & 2.621 & 10.767 & 6.028 & 4.873 & 6.726 & 3.833 \\
\hline $11 / 9 / 2005$ & $13: 00$ & 11.068 & 10.955 & 8.04 & 6.967 & 4.509 & 4.375 & 2.65 & 2.618 & 10.769 & 6.027 & 4.873 & 6.727 & 3.835 \\
\hline $11 / 9 / 2005$ & $14: 00$ & 11.068 & 10.949 & 8.039 & 6.962 & 4.505 & 4.375 & 2.645 & 2.614 & 10.767 & 6.028 & 4.872 & 6.724 & 3.835 \\
\hline $11 / 9 / 2005$ & $15: 00$ & 11.073 & 10.956 & 8.041 & 6.967 & 4.511 & 4.379 & 2.652 & 2.618 & 10.773 & 6.032 & 4.88 & 6.73 & 3.843 \\
\hline $11 / 9 / 2005$ & $16: 00$ & 11.073 & 10.953 & 8.041 & 6.962 & 4.518 & 4.397 & 2.647 & 2.616 & 10.773 & 6.032 & 4.88 & 6.73 & 3.843 \\
\hline $11 / 9 / 2005$ & $17: 00$ & 11.076 & 10.958 & 8.045 & 6.969 & 4.527 & 4.408 & 2.655 & 2.623 & 10.777 & 6.036 & 4.888 & 6.736 & 3.853 \\
\hline $11 / 9 / 2005$ & $18: 00$ & 11.083 & 10.962 & 8.047 & 6.969 & 4.525 & 4.408 & 2.657 & 2.625 & 10.778 & 6.038 & 4.888 & 6.736 & 3.855 \\
\hline $11 / 9 / 2005$ & 19:00 & 11.088 & 10.964 & 8.047 & 6.971 & 4.522 & 4.408 & 2.66 & 2.629 & 10.78 & 6.036 & 4.89 & 6.738 & 3.847 \\
\hline $11 / 9 / 2005$ & $20: 00$ & 11.091 & 10.969 & 8.051 & 6.975 & 4.524 & 4.41 & 2.662 & 2.631 & 10.782 & 6.038 & 4.894 & 6.74 & 3.843 \\
\hline $11 / 9 / 2005$ & $21: 00$ & 11.093 & 10.969 & 8.05 & 6.971 & 4.517 & 4.408 & 2.66 & 2.627 & 10.782 & 6.036 & 4.892 & 6.742 & 3.827 \\
\hline $11 / 9 / 2005$ & $22: 00$ & 11.093 & 10.965 & 8.053 & 6.971 & 4.513 & 4.408 & 2.66 & 2.627 & 10.783 & 6.034 & 4.888 & 6.74 & 3.821 \\
\hline $11 / 9 / 2005$ & $23: 00$ & 11.09 & 10.964 & 8.049 & 6.967 & 4.505 & 4.403 & 2.655 & 2.623 & 10.783 & 6.032 & 4.882 & 6.738 & 3.811 \\
\hline $11 / 10 / 2005$ & $0: 00$ & 11.09 & 10.96 & 8.046 & 6.965 & 4.499 & 4.402 & 2.65 & 2.618 & 10.781 & 6.032 & 4.88 & 6.736 & 3.802 \\
\hline $11 / 10 / 2005$ & $1: 00$ & 11.095 & 10.964 & 8.048 & 6.967 & 4.499 & 4.399 & 2.652 & 2.618 & 10.781 & 6.032 & 4.88 & 6.738 & 3.798 \\
\hline $11 / 10 / 2005$ & $2: 00$ & 11.083 & 10.951 & 8.044 & 6.956 & 4.485 & 4.395 & 2.642 & 2.61 & 10.777 & 6.023 & 4.868 & 6.732 & 3.786 \\
\hline $11 / 10 / 2005$ & $3: 00$ & 11.085 & 10.953 & 8.042 & 6.956 & 4.488 & 4.395 & 2.644 & 2.61 & 10.777 & 6.025 & 4.868 & 6.732 & 3.784 \\
\hline $11 / 10 / 2005$ & $4: 00$ & 11.088 & 10.953 & 8.042 & 6.958 & 4.484 & 4.393 & 2.642 & 2.612 & 10.777 & 6.021 & 4.864 & 6.732 & 3.78 \\
\hline $11 / 10 / 2005$ & $5: 00$ & 11.078 & 10.945 & 8.038 & 6.951 & 4.477 & 4.388 & 2.637 & 2.604 & 10.773 & 6.021 & 4.86 & 6.73 & 3.772 \\
\hline
\end{tabular}


Water Level (ft below top of casing) at Indicated Well

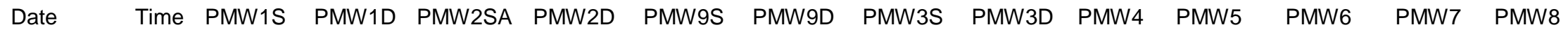

\begin{tabular}{|c|c|c|c|c|c|c|c|c|c|c|c|c|c|c|}
\hline $11 / 10 / 2005$ & $6: 00$ & 11.07 & 10.934 & 8.034 & 6.943 & 4.472 & 4.384 & 2.629 & 2.598 & 10.773 & 6.014 & 4.853 & 6.725 & 3.764 \\
\hline $11 / 10 / 2005$ & $7: 00$ & 11.063 & 10.927 & 8.028 & 6.938 & 4.465 & 4.382 & 2.624 & 2.591 & 10.769 & 6.014 & 4.85 & 6.721 & 3.762 \\
\hline $11 / 10 / 2005$ & 8:00 & 11.061 & 10.925 & 8.028 & 6.94 & 4.466 & 4.379 & 2.624 & 2.591 & 10.767 & 6.014 & 4.845 & 6.721 & 3.76 \\
\hline $11 / 10 / 2005$ & $9: 00$ & 11.058 & 10.924 & 8.026 & 6.936 & 4.462 & 4.375 & 2.622 & 2.592 & 10.767 & 6.012 & 4.844 & 6.719 & 3.756 \\
\hline $11 / 10 / 2005$ & $10: 00$ & 11.056 & 10.922 & 8.024 & 6.934 & 4.462 & 4.373 & 2.619 & 2.589 & 10.764 & 6.011 & 4.842 & 6.717 & 3.756 \\
\hline $11 / 10 / 2005$ & $11: 00$ & 11.048 & 10.911 & 8.024 & 6.927 & 4.457 & 4.366 & 2.614 & 2.583 & 10.76 & 6.012 & 4.837 & 6.713 & 3.754 \\
\hline $11 / 10 / 2005$ & $12: 00$ & 11.041 & 10.902 & 8.02 & 6.918 & 4.45 & 4.362 & 2.606 & 2.575 & 10.756 & 6.007 & 4.831 & 6.713 & 3.754 \\
\hline $11 / 10 / 2005$ & $13: 00$ & 11.026 & 10.885 & 8.011 & 6.905 & 4.441 & 4.353 & 2.596 & 2.562 & 10.756 & 6.007 & 4.825 & 6.706 & 3.76 \\
\hline $11 / 10 / 2005$ & $14: 00$ & 11.013 & 10.867 & 8.001 & 6.892 & 4.437 & 4.346 & 2.584 & 2.552 & 10.747 & 6.005 & 4.823 & 6.7 & 3.766 \\
\hline $11 / 10 / 2005$ & $15: 00$ & 11.001 & 10.854 & 7.993 & 6.878 & 4.434 & 4.337 & 2.573 & 2.539 & 10.747 & 6.009 & 4.817 & 6.694 & 3.772 \\
\hline $11 / 10 / 2005$ & $16: 00$ & 10.996 & 10.849 & 7.989 & 6.874 & 4.436 & 4.335 & 2.573 & 2.539 & 10.745 & 6.009 & 4.821 & 6.692 & 3.78 \\
\hline $11 / 10 / 2005$ & $17: 00$ & 10.991 & 10.843 & 7.987 & 6.876 & 4.44 & 4.331 & 2.574 & 2.539 & 10.745 & 6.009 & 4.821 & 6.692 & 3.79 \\
\hline $11 / 10 / 2005$ & $18: 00$ & 10.984 & 10.84 & 7.983 & 6.872 & 4.44 & 4.326 & 2.568 & 2.535 & 10.745 & 6.007 & 4.821 & 6.69 & 3.79 \\
\hline $11 / 10 / 2005$ & $19: 00$ & 10.979 & 10.836 & 7.979 & 6.872 & 4.438 & 4.322 & 2.566 & 2.533 & 10.743 & 6.009 & 4.819 & 6.688 & 3.784 \\
\hline $11 / 10 / 2005$ & $20: 00$ & 10.977 & 10.832 & 7.975 & 6.87 & 4.437 & 4.32 & 2.566 & 2.531 & 10.741 & 6.007 & 4.817 & 6.685 & 3.778 \\
\hline $11 / 10 / 2005$ & $21: 00$ & 10.972 & 10.83 & 7.973 & 6.867 & 4.436 & 4.317 & 2.563 & 2.529 & 10.739 & 6.005 & 4.813 & 6.683 & 3.774 \\
\hline $11 / 10 / 2005$ & $22: 00$ & 10.969 & 10.827 & 7.971 & 6.865 & 4.432 & 4.313 & 2.558 & 2.526 & 10.739 & 6.003 & 4.807 & 6.683 & 3.767 \\
\hline $11 / 10 / 2005$ & $23: 00$ & 10.964 & 10.821 & 7.967 & 6.858 & 4.427 & 4.313 & 2.553 & 2.52 & 10.735 & 5.998 & 4.803 & 6.679 & 3.761 \\
\hline $11 / 11 / 2005$ & $0: 00$ & 10.957 & 10.816 & 7.965 & 6.856 & 4.421 & 4.307 & 2.551 & 2.516 & 10.735 & 5.996 & 4.799 & 6.677 & 3.757 \\
\hline $11 / 11 / 2005$ & $1: 00$ & 10.952 & 10.81 & 7.96 & 6.852 & 4.417 & 4.304 & 2.543 & 2.512 & 10.731 & 5.991 & 4.793 & 6.675 & 3.749 \\
\hline $11 / 11 / 2005$ & $2: 00$ & 10.945 & 10.805 & 7.957 & 6.845 & 4.412 & 4.3 & 2.538 & 2.505 & 10.729 & 5.989 & 4.788 & 6.671 & 3.743 \\
\hline $11 / 11 / 2005$ & $3: 00$ & 10.942 & 10.803 & 7.954 & 6.843 & 4.409 & 4.296 & 2.538 & 2.503 & 10.727 & 5.989 & 4.788 & 6.671 & 3.741 \\
\hline $11 / 11 / 2005$ & $4: 00$ & 10.934 & 10.796 & 7.949 & 6.838 & 4.404 & 4.291 & 2.53 & 2.497 & 10.724 & 5.985 & 4.78 & 6.666 & 3.733 \\
\hline $11 / 11 / 2005$ & $5: 00$ & 10.93 & 10.79 & 7.947 & 6.832 & 4.4 & 4.287 & 2.528 & 2.493 & 10.722 & 5.982 & 4.774 & 6.664 & 3.733 \\
\hline $11 / 11 / 2005$ & $6: 00$ & 10.927 & 10.79 & 7.946 & 6.836 & 4.4 & 4.285 & 2.528 & 2.495 & 10.72 & 5.985 & 4.772 & 6.664 & 3.733 \\
\hline $11 / 11 / 2005$ & $7: 00$ & 10.922 & 10.785 & 7.942 & 6.83 & 4.395 & 4.28 & 2.523 & 2.491 & 10.718 & 5.98 & 4.766 & 6.66 & 3.727 \\
\hline $11 / 11 / 2005$ & $8: 00$ & 10.922 & 10.785 & 7.942 & 6.83 & 4.395 & 4.278 & 2.523 & 2.491 & 10.716 & 5.98 & 4.766 & 6.66 & 3.727 \\
\hline $11 / 11 / 2005$ & $9: 00$ & 10.917 & 10.783 & 7.94 & 6.828 & 4.395 & 4.276 & 2.523 & 2.487 & 10.716 & 5.98 & 4.762 & 6.656 & 3.727 \\
\hline $11 / 11 / 2005$ & $10: 00$ & 10.915 & 10.779 & 7.938 & 6.825 & 4.392 & 4.274 & 2.52 & 2.485 & 10.712 & 5.978 & 4.76 & 6.654 & 3.727 \\
\hline $11 / 11 / 2005$ & $11: 00$ & 10.912 & 10.778 & 7.938 & 6.823 & 4.392 & 4.269 & 2.518 & 2.484 & 10.71 & 5.976 & 4.754 & 6.654 & 3.733 \\
\hline $11 / 11 / 2005$ & $12: 00$ & 10.91 & 10.772 & 7.934 & 6.821 & 4.392 & 4.267 & 2.515 & 2.483 & 10.71 & 5.975 & 4.754 & 6.65 & 3.741 \\
\hline $11 / 11 / 2005$ & $13: 00$ & 10.905 & 10.765 & 7.932 & 6.812 & 4.389 & 4.263 & 2.51 & 2.477 & 10.708 & 5.973 & 4.75 & 6.649 & 3.751 \\
\hline $11 / 11 / 2005$ & $14: 00$ & 10.895 & 10.754 & 7.929 & 6.803 & 4.383 & 4.256 & 2.505 & 2.468 & 10.705 & 5.973 & 4.75 & 6.645 & 3.757 \\
\hline $11 / 11 / 2005$ & $15: 00$ & 10.89 & 10.747 & 7.921 & 6.797 & 4.382 & 4.251 & 2.5 & 2.466 & 10.703 & 5.973 & 4.748 & 6.643 & 3.769 \\
\hline
\end{tabular}


Water Level (ft below top of casing) at Indicated Well

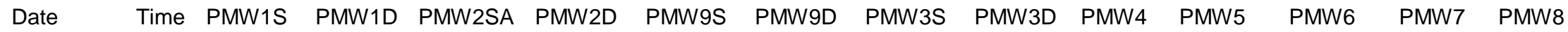

\begin{tabular}{|c|c|c|c|c|c|c|c|c|c|c|c|c|c|c|}
\hline $11 / 11 / 2005$ & $16: 00$ & 10.883 & 10.741 & 7.919 & 6.794 & 4.386 & 4.249 & 2.5 & 2.462 & 10.703 & 5.975 & 4.752 & 6.641 & 3.779 \\
\hline $11 / 11 / 2005$ & $17: 00$ & 10.876 & 10.734 & 7.917 & 6.79 & 4.394 & 4.258 & 2.495 & 2.46 & 10.641 & 5.975 & 4.754 & 6.641 & 3.787 \\
\hline $11 / 11 / 2005$ & $18: 00$ & 10.87 & 10.73 & 7.915 & 6.792 & 4.402 & 4.263 & 2.495 & 2.458 & 10.681 & 5.975 & 4.754 & 6.637 & 3.789 \\
\hline $11 / 11 / 2005$ & $19: 00$ & 10.866 & 10.73 & 7.915 & 6.79 & 4.402 & 4.26 & 2.492 & 2.46 & 10.68 & 5.975 & 4.752 & 6.635 & 3.785 \\
\hline $11 / 11 / 2005$ & $20: 00$ & 10.865 & 10.728 & 7.913 & 6.79 & 4.398 & 4.258 & 2.492 & 2.456 & 10.678 & 5.975 & 4.748 & 6.634 & 3.777 \\
\hline $11 / 11 / 2005$ & $21: 00$ & 10.863 & 10.725 & 7.909 & 6.786 & 4.393 & 4.254 & 2.487 & 2.453 & 10.677 & 5.971 & 4.742 & 6.628 & 3.767 \\
\hline $11 / 11 / 2005$ & $22: 00$ & 10.86 & 10.723 & 7.909 & 6.786 & 4.388 & 4.247 & 2.485 & 2.451 & 10.675 & 5.973 & 4.74 & 6.63 & 3.761 \\
\hline $11 / 11 / 2005$ & $23: 00$ & 10.855 & 10.721 & 7.907 & 6.781 & 4.383 & 4.245 & 2.482 & 2.449 & 10.673 & 5.969 & 4.734 & 6.626 & 3.753 \\
\hline $11 / 12 / 2005$ & 0:00 & 10.853 & 10.716 & 7.903 & 6.777 & 4.376 & 4.238 & 2.474 & 2.443 & 10.669 & 5.966 & 4.726 & 6.62 & 3.747 \\
\hline $11 / 12 / 2005$ & $1: 00$ & 10.848 & 10.71 & 7.894 & 6.77 & 4.369 & 4.232 & 2.469 & 2.435 & 10.669 & 5.964 & 4.721 & 6.618 & 3.737 \\
\hline $11 / 12 / 2005$ & $2: 00$ & 10.843 & 10.705 & 7.891 & 6.766 & 4.365 & 4.23 & 2.467 & 2.43 & 10.667 & 5.962 & 4.717 & 6.616 & 3.732 \\
\hline $11 / 12 / 2005$ & $3: 00$ & 10.838 & 10.698 & 7.888 & 6.764 & 4.359 & 4.223 & 2.462 & 2.426 & 10.665 & 5.96 & 4.709 & 6.611 & 3.726 \\
\hline $11 / 12 / 2005$ & $4: 00$ & 10.833 & 10.688 & 7.882 & 6.752 & 4.35 & 4.216 & 2.452 & 2.418 & 10.661 & 5.958 & 4.704 & 6.607 & 3.718 \\
\hline $11 / 12 / 2005$ & $5: 00$ & 10.828 & 10.683 & 7.877 & 6.748 & 4.347 & 4.212 & 2.447 & 2.414 & 10.657 & 5.958 & 4.695 & 6.603 & 3.714 \\
\hline $11 / 12 / 2005$ & $6: 00$ & 10.823 & 10.678 & 7.876 & 6.744 & 4.343 & 4.205 & 2.442 & 2.409 & 10.655 & 5.958 & 4.689 & 6.603 & 3.71 \\
\hline $11 / 12 / 2005$ & $7: 00$ & 10.819 & 10.67 & 7.869 & 6.737 & 4.335 & 4.201 & 2.437 & 2.401 & 10.653 & 5.953 & 4.683 & 6.597 & 3.704 \\
\hline $11 / 12 / 2005$ & $8: 00$ & 10.811 & 10.667 & 7.868 & 6.735 & 4.335 & 4.197 & 2.432 & 2.399 & 10.651 & 5.953 & 4.681 & 6.593 & 3.702 \\
\hline $11 / 12 / 2005$ & $9: 00$ & 10.809 & 10.663 & 7.866 & 6.735 & 4.336 & 4.194 & 2.432 & 2.399 & 10.65 & 5.955 & 4.677 & 6.593 & 3.704 \\
\hline $11 / 12 / 2005$ & $10: 00$ & 10.804 & 10.656 & 7.861 & 6.726 & 4.327 & 4.188 & 2.427 & 2.39 & 10.644 & 5.946 & 4.667 & 6.588 & 3.696 \\
\hline $11 / 12 / 2005$ & $11: 00$ & 10.799 & 10.65 & 7.856 & 6.724 & 4.327 & 4.181 & 2.424 & 2.389 & 10.642 & 5.946 & 4.665 & 6.58 & 3.696 \\
\hline $11 / 12 / 2005$ & $12: 00$ & 10.796 & 10.648 & 7.856 & 6.719 & 4.324 & 4.181 & 2.419 & 2.384 & 10.636 & 5.944 & 4.661 & 6.582 & 3.695 \\
\hline $11 / 12 / 2005$ & $13: 00$ & 10.786 & 10.636 & 7.846 & 6.706 & 4.31 & 4.172 & 2.411 & 2.374 & 10.636 & 5.942 & 4.65 & 6.573 & 3.692 \\
\hline $11 / 12 / 2005$ & $14: 00$ & 10.779 & 10.627 & 7.844 & 6.699 & 4.309 & 4.163 & 2.404 & 2.366 & 10.634 & 5.938 & 4.648 & 6.574 & 3.698 \\
\hline $11 / 12 / 2005$ & $15: 00$ & 10.774 & 10.619 & 7.839 & 6.693 & 4.31 & 4.161 & 2.401 & 2.364 & 10.631 & 5.94 & 4.648 & 6.577 & 3.714 \\
\hline $11 / 12 / 2005$ & $16: 00$ & 10.771 & 10.621 & 7.846 & 6.706 & 4.325 & 4.154 & 2.411 & 2.372 & 10.631 & 5.946 & 4.654 & 6.573 & 3.738 \\
\hline $11 / 12 / 2005$ & $17: 00$ & 10.771 & 10.63 & 7.854 & 6.719 & 4.343 & 4.163 & 2.426 & 2.387 & 10.634 & 5.949 & 4.665 & 6.574 & 3.754 \\
\hline $11 / 12 / 2005$ & $18: 00$ & 10.776 & 10.634 & 7.862 & 6.735 & 4.363 & 4.166 & 2.439 & 2.401 & 10.636 & 5.955 & 4.677 & 6.58 & 3.766 \\
\hline $11 / 12 / 2005$ & $19: 00$ & 10.798 & 10.679 & 7.876 & 6.764 & 4.384 & 4.181 & 2.466 & 2.431 & 10.642 & 5.97 & 4.697 & 6.592 & 3.785 \\
\hline $11 / 12 / 2005$ & $20: 00$ & 10.826 & 10.712 & 7.895 & 6.793 & 4.406 & 4.194 & 2.492 & 2.454 & 10.651 & 5.977 & 4.714 & 6.611 & 3.797 \\
\hline $11 / 12 / 2005$ & $21: 00$ & 10.848 & 10.741 & 7.911 & 6.815 & 4.422 & 4.208 & 2.512 & 2.479 & 10.663 & 5.986 & 4.728 & 6.617 & 3.803 \\
\hline $11 / 12 / 2005$ & $22: 00$ & 10.86 & 10.76 & 7.921 & 6.823 & 4.425 & 4.219 & 2.522 & 2.487 & 10.667 & 5.99 & 4.736 & 6.624 & 3.803 \\
\hline $11 / 12 / 2005$ & $23: 00$ & 10.88 & 10.776 & 7.933 & 6.844 & 4.438 & 4.232 & 2.537 & 2.502 & 10.671 & 5.997 & 4.748 & 6.634 & 3.805 \\
\hline $11 / 13 / 2005$ & $0: 00$ & 10.892 & 10.802 & 7.947 & 6.859 & 4.445 & 4.245 & 2.55 & 2.521 & 10.677 & 6.003 & 4.753 & 6.638 & 3.805 \\
\hline $11 / 13 / 2005$ & $1: 00$ & 10.912 & 10.831 & 7.961 & 6.877 & 4.456 & 4.259 & 2.567 & 2.534 & 10.68 & 6.01 & 4.765 & 6.651 & 3.811 \\
\hline
\end{tabular}


Water Level (ft below top of casing) at Indicated Well

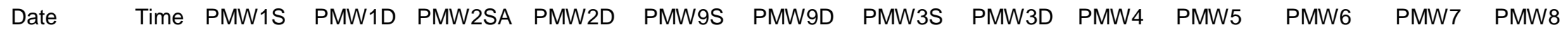

\begin{tabular}{|c|c|c|c|c|c|c|c|c|c|c|c|c|c|c|}
\hline $11 / 13 / 2005$ & $2: 00$ & 10.927 & 10.833 & 7.967 & 6.881 & 4.457 & 4.267 & 2.575 & 2.54 & 10.686 & 6.003 & 4.775 & 6.655 & 3.809 \\
\hline $11 / 13 / 2005$ & $3: 00$ & 10.941 & 10.851 & 7.975 & 6.892 & 4.461 & 4.278 & 2.582 & 2.55 & 10.69 & 6.01 & 4.784 & 6.666 & 3.807 \\
\hline $11 / 13 / 2005$ & $4: 00$ & 10.956 & 10.865 & 7.982 & 6.903 & 4.468 & 4.289 & 2.593 & 2.559 & 10.694 & 6.014 & 4.793 & 6.67 & 3.811 \\
\hline $11 / 13 / 2005$ & $5: 00$ & 10.968 & 10.88 & 7.993 & 6.915 & 4.474 & 4.301 & 2.603 & 2.571 & 10.701 & 6.021 & 4.802 & 6.68 & 3.811 \\
\hline $11 / 13 / 2005$ & $6: 00$ & 10.983 & 10.889 & 7.999 & 6.923 & 4.477 & 4.309 & 2.61 & 2.578 & 10.705 & 6.014 & 4.806 & 6.683 & 3.811 \\
\hline $11 / 13 / 2005$ & $7: 00$ & 10.993 & 10.902 & 8.007 & 6.932 & 4.483 & 4.321 & 2.62 & 2.588 & 10.707 & 6.021 & 4.814 & 6.689 & 3.813 \\
\hline $11 / 13 / 2005$ & $8: 00$ & 11.008 & 10.915 & 8.017 & 6.941 & 4.487 & 4.329 & 2.63 & 2.597 & 10.711 & 6.023 & 4.82 & 6.695 & 3.815 \\
\hline $11 / 13 / 2005$ & $9: 00$ & 11.02 & 10.926 & 8.025 & 6.952 & 4.495 & 4.338 & 2.638 & 2.607 & 10.72 & 6.023 & 4.826 & 6.704 & 3.819 \\
\hline $11 / 13 / 2005$ & $10: 00$ & 11.032 & 10.944 & 8.031 & 6.966 & 4.505 & 4.347 & 2.653 & 2.622 & 10.718 & 6.032 & 4.837 & 6.71 & 3.825 \\
\hline $11 / 13 / 2005$ & $11: 00$ & 11.049 & 10.96 & 8.045 & 6.979 & 4.514 & 4.359 & 2.663 & 2.632 & 10.726 & 6.036 & 4.845 & 6.716 & 3.835 \\
\hline $11 / 13 / 2005$ & $12: 00$ & 11.059 & 10.968 & 8.045 & 6.984 & 4.515 & 4.367 & 2.666 & 2.634 & 10.726 & 6.036 & 4.849 & 6.722 & 3.835 \\
\hline $11 / 13 / 2005$ & $13: 00$ & 11.069 & 10.975 & 8.055 & 6.988 & 4.518 & 4.374 & 2.671 & 2.641 & 10.732 & 6.038 & 4.851 & 6.725 & 3.839 \\
\hline $11 / 13 / 2005$ & $14: 00$ & 11.076 & 10.979 & 8.057 & 6.986 & 4.515 & 4.378 & 2.673 & 2.641 & 10.737 & 6.038 & 4.853 & 6.727 & 3.841 \\
\hline $11 / 13 / 2005$ & $15: 00$ & 11.084 & 10.98 & 8.058 & 6.984 & 4.515 & 4.383 & 2.673 & 2.641 & 10.739 & 6.04 & 4.857 & 6.731 & 3.843 \\
\hline $11 / 13 / 2005$ & $16: 00$ & 11.086 & 10.982 & 8.058 & 6.984 & 4.513 & 4.385 & 2.67 & 2.641 & 10.745 & 6.04 & 4.859 & 6.733 & 3.843 \\
\hline $11 / 13 / 2005$ & $17: 00$ & 11.091 & 10.984 & 8.06 & 6.984 & 4.517 & 4.39 & 2.673 & 2.641 & 10.745 & 6.043 & 4.863 & 6.733 & 3.843 \\
\hline $11 / 13 / 2005$ & $18: 00$ & 11.093 & 10.984 & 8.062 & 6.984 & 4.515 & 4.392 & 2.673 & 2.641 & 10.747 & 6.045 & 4.865 & 6.733 & 3.841 \\
\hline $11 / 13 / 2005$ & $19: 00$ & 11.096 & 10.986 & 8.061 & 6.984 & 4.516 & 4.396 & 2.673 & 2.641 & 10.749 & 6.045 & 4.866 & 6.737 & 3.833 \\
\hline $11 / 13 / 2005$ & $20: 00$ & 11.098 & 10.986 & 8.063 & 6.986 & 4.515 & 4.398 & 2.675 & 2.643 & 10.749 & 6.045 & 4.866 & 6.739 & 3.825 \\
\hline $11 / 13 / 2005$ & $21: 00$ & 11.098 & 10.986 & 8.067 & 6.986 & 4.511 & 4.401 & 2.67 & 2.641 & 10.751 & 6.043 & 4.864 & 6.739 & 3.817 \\
\hline $11 / 13 / 2005$ & $22: 00$ & 11.103 & 10.988 & 8.063 & 6.986 & 4.509 & 4.403 & 2.673 & 2.641 & 10.753 & 6.042 & 4.864 & 6.739 & 3.811 \\
\hline $11 / 13 / 2005$ & $23: 00$ & 11.098 & 10.982 & 8.063 & 6.981 & 4.503 & 4.403 & 2.67 & 2.637 & 10.751 & 6.04 & 4.859 & 6.739 & 3.805 \\
\hline $11 / 14 / 2005$ & $0: 00$ & 11.098 & 10.98 & 8.061 & 6.979 & 4.497 & 4.403 & 2.665 & 2.633 & 10.751 & 6.04 & 4.859 & 6.737 & 3.794 \\
\hline $11 / 14 / 2005$ & $1: 00$ & 11.096 & 10.977 & 8.059 & 6.975 & 4.495 & 4.401 & 2.663 & 2.631 & 10.749 & 6.036 & 4.853 & 6.735 & 3.79 \\
\hline $11 / 14 / 2005$ & $2: 00$ & 11.091 & 10.968 & 8.055 & 6.966 & 4.489 & 4.398 & 2.655 & 2.624 & 10.749 & 6.033 & 4.847 & 6.731 & 3.782 \\
\hline $11 / 14 / 2005$ & $3: 00$ & 11.083 & 10.96 & 8.051 & 6.964 & 4.482 & 4.394 & 2.65 & 2.618 & 10.745 & 6.031 & 4.845 & 6.729 & 3.776 \\
\hline $11 / 14 / 2005$ & $4: 00$ & 11.08 & 10.955 & 8.049 & 6.959 & 4.479 & 4.392 & 2.645 & 2.614 & 10.745 & 6.031 & 4.841 & 6.727 & 3.772 \\
\hline $11 / 14 / 2005$ & $5: 00$ & 11.078 & 10.951 & 8.047 & 6.955 & 4.477 & 4.39 & 2.643 & 2.61 & 10.741 & 6.029 & 4.837 & 6.727 & 3.768 \\
\hline $11 / 14 / 2005$ & $6: 00$ & 11.071 & 10.946 & 8.043 & 6.95 & 4.473 & 4.385 & 2.64 & 2.605 & 10.739 & 6.027 & 4.835 & 6.723 & 3.766 \\
\hline $11 / 14 / 2005$ & $7: 00$ & 11.061 & 10.933 & 8.037 & 6.939 & 4.461 & 4.381 & 2.63 & 2.597 & 10.737 & 6.025 & 4.827 & 6.72 & 3.756 \\
\hline $11 / 14 / 2005$ & $8: 00$ & 11.046 & 10.915 & 8.026 & 6.924 & 4.45 & 4.374 & 2.612 & 2.58 & 10.732 & 6.016 & 4.815 & 6.712 & 3.744 \\
\hline $11 / 14 / 2005$ & $9: 00$ & 11.036 & 10.9 & 8.017 & 6.913 & 4.441 & 4.367 & 2.602 & 2.568 & 10.728 & 6.014 & 4.81 & 6.708 & 3.735 \\
\hline $11 / 14 / 2005$ & $10: 00$ & 11.021 & 10.884 & 8.004 & 6.895 & 4.425 & 4.359 & 2.587 & 2.553 & 10.722 & 6 & 4.796 & 6.695 & 3.725 \\
\hline $11 / 14 / 2005$ & $11: 00$ & 11.014 & 10.876 & 7.999 & 6.891 & 4.426 & 4.354 & 2.585 & 2.551 & 10.715 & 6.005 & 4.794 & 6.691 & 3.727 \\
\hline
\end{tabular}


Water Level (ft below top of casing) at Indicated Well

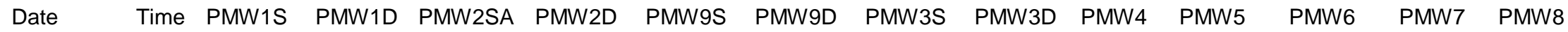

\begin{tabular}{|c|c|c|c|c|c|c|c|c|c|c|c|c|c|c|}
\hline $11 / 14 / 2005$ & $12: 00$ & 11.001 & 10.864 & 7.988 & 6.877 & 4.414 & 4.341 & 2.574 & 2.538 & 10.711 & 6 & 4.786 & 6.689 & 3.733 \\
\hline $11 / 14 / 2005$ & $13: 00$ & 10.987 & 10.845 & 7.982 & 6.868 & 4.409 & 4.334 & 2.564 & 2.53 & 10.707 & 5.994 & 4.784 & 6.68 & 3.725 \\
\hline $11 / 14 / 2005$ & $14: 00$ & 10.979 & 10.834 & 7.976 & 6.862 & 4.406 & 4.325 & 2.554 & 2.519 & 10.703 & 5.994 & 4.78 & 6.676 & 3.729 \\
\hline $11 / 14 / 2005$ & $15: 00$ & 10.962 & 10.818 & 7.966 & 6.844 & 4.394 & 4.316 & 2.544 & 2.509 & 10.697 & 5.993 & 4.77 & 6.668 & 3.725 \\
\hline $11 / 14 / 2005$ & $16: 00$ & 10.957 & 10.811 & 7.96 & 6.842 & 4.396 & 4.31 & 2.539 & 2.503 & 10.696 & 5.994 & 4.774 & 6.668 & 3.729 \\
\hline $11 / 14 / 2005$ & $17: 00$ & 10.95 & 10.807 & 7.957 & 6.842 & 4.401 & 4.305 & 2.539 & 2.505 & 10.699 & 5.991 & 4.774 & 6.666 & 3.737 \\
\hline $11 / 14 / 2005$ & $18: 00$ & 10.942 & 10.803 & 7.955 & 6.837 & 4.401 & 4.299 & 2.536 & 2.5 & 10.696 & 5.991 & 4.776 & 6.664 & 3.737 \\
\hline $11 / 14 / 2005$ & $19: 00$ & 10.94 & 10.798 & 7.951 & 6.84 & 4.399 & 4.297 & 2.534 & 2.503 & 10.694 & 5.987 & 4.77 & 6.663 & 3.735 \\
\hline $11 / 14 / 2005$ & $20: 00$ & 10.94 & 10.805 & 7.953 & 6.844 & 4.406 & 4.294 & 2.541 & 2.507 & 10.694 & 5.993 & 4.776 & 6.665 & 3.741 \\
\hline $11 / 14 / 2005$ & $21: 00$ & 10.938 & 10.804 & 7.955 & 6.844 & 4.406 & 4.292 & 2.541 & 2.505 & 10.694 & 5.991 & 4.776 & 6.665 & 3.737 \\
\hline $11 / 14 / 2005$ & $22: 00$ & 10.935 & 10.806 & 7.955 & 6.847 & 4.407 & 4.292 & 2.541 & 2.507 & 10.69 & 5.993 & 4.776 & 6.664 & 3.739 \\
\hline $11 / 14 / 2005$ & $23: 00$ & 10.938 & 10.809 & 7.957 & 6.851 & 4.41 & 4.292 & 2.546 & 2.513 & 10.692 & 5.993 & 4.778 & 6.664 & 3.739 \\
\hline $11 / 15 / 2005$ & $0: 00$ & 10.935 & 10.804 & 7.953 & 6.847 & 4.404 & 4.29 & 2.541 & 2.507 & 10.692 & 5.989 & 4.776 & 6.664 & 3.731 \\
\hline $11 / 15 / 2005$ & $1: 00$ & 10.938 & 10.807 & 7.956 & 6.851 & 4.407 & 4.29 & 2.544 & 2.511 & 10.692 & 5.989 & 4.774 & 6.667 & 3.733 \\
\hline $11 / 15 / 2005$ & $2: 00$ & 10.93 & 10.795 & 7.949 & 6.836 & 4.392 & 4.284 & 2.529 & 2.497 & 10.688 & 5.982 & 4.764 & 6.661 & 3.719 \\
\hline $11 / 15 / 2005$ & $3: 00$ & 10.928 & 10.793 & 7.947 & 6.836 & 4.393 & 4.281 & 2.529 & 2.495 & 10.685 & 5.982 & 4.764 & 6.661 & 3.717 \\
\hline $11 / 15 / 2005$ & $4: 00$ & 10.923 & 10.787 & 7.943 & 6.831 & 4.388 & 4.279 & 2.526 & 2.492 & 10.685 & 5.976 & 4.756 & 6.659 & 3.715 \\
\hline $11 / 15 / 2005$ & $5: 00$ & 10.923 & 10.782 & 7.943 & 6.827 & 4.389 & 4.279 & 2.526 & 2.49 & 10.683 & 5.98 & 4.758 & 6.649 & 3.715 \\
\hline $11 / 15 / 2005$ & $6: 00$ & 10.933 & 10.761 & 7.953 & 6.809 & 4.408 & 4.284 & 2.546 & 2.474 & 10.667 & 5.993 & 4.772 & 6.636 & 3.729 \\
\hline $11 / 15 / 2005$ & $7: 00$ & 10.94 & 10.811 & 7.958 & 6.782 & 4.406 & 4.281 & 2.546 & 2.513 & 10.652 & 5.985 & 4.762 & 6.613 & 3.716 \\
\hline $11 / 15 / 2005$ & $8: 00$ & 10.94 & 10.817 & 7.958 & 6.858 & 4.401 & 4.279 & 2.543 & 2.509 & 10.683 & 5.984 & 4.756 & 6.661 & 3.704 \\
\hline $11 / 15 / 2005$ & $9: 00$ & 10.945 & 10.817 & 7.956 & 6.86 & 4.399 & 4.277 & 2.543 & 2.509 & 10.679 & 5.98 & 4.75 & 6.659 & 3.702 \\
\hline $11 / 15 / 2005$ & $10: 00$ & 10.947 & 10.809 & 7.964 & 6.867 & 4.404 & 4.279 & 2.546 & 2.513 & 10.669 & 5.984 & 4.75 & 6.663 & 3.7 \\
\hline $11 / 15 / 2005$ & $11: 00$ & 10.952 & 10.831 & 7.966 & 6.869 & 4.406 & 4.284 & 2.55 & 2.518 & 10.681 & 5.975 & 4.742 & 6.659 & 3.696 \\
\hline $11 / 15 / 2005$ & $12: 00$ & 10.955 & 10.829 & 7.964 & 6.869 & 4.401 & 4.281 & 2.55 & 2.528 & 10.677 & 5.975 & 4.734 & 6.663 & 3.688 \\
\hline $11 / 15 / 2005$ & $13: 00$ & 10.952 & 10.833 & 7.962 & 6.873 & 4.395 & 4.279 & 2.542 & 2.53 & 10.675 & 5.971 & 4.728 & 6.668 & 3.682 \\
\hline $11 / 15 / 2005$ & $14: 00$ & 10.965 & 10.835 & 7.97 & 6.88 & 4.395 & 4.281 & 2.542 & 2.518 & 10.667 & 5.969 & 4.721 & 6.663 & 3.645 \\
\hline $11 / 15 / 2005$ & $15: 00$ & 10.965 & 10.837 & 7.968 & 6.882 & 4.401 & 4.275 & 2.551 & 2.52 & 10.673 & 5.964 & 4.719 & 6.655 & 3.629 \\
\hline $11 / 15 / 2005$ & $16: 00$ & 10.972 & 10.862 & 7.972 & 6.893 & 4.399 & 4.29 & 2.559 & 2.528 & 10.673 & 5.953 & 4.719 & 6.665 & 3.619 \\
\hline $11 / 15 / 2005$ & $17: 00$ & 10.984 & 10.88 & 7.982 & 6.906 & 4.4 & 4.292 & 2.569 & 2.539 & 10.673 & 5.953 & 4.719 & 6.667 & 3.601 \\
\hline $11 / 15 / 2005$ & $18: 00$ & 10.994 & 10.895 & 7.991 & 6.911 & 4.402 & 4.301 & 2.579 & 2.551 & 10.675 & 5.957 & 4.723 & 6.667 & 3.598 \\
\hline $11 / 15 / 2005$ & $19: 00$ & 11.009 & 10.91 & 8 & 6.926 & 4.41 & 4.306 & 2.594 & 2.564 & 10.673 & 5.955 & 4.725 & 6.688 & 3.606 \\
\hline $11 / 15 / 2005$ & $20: 00$ & 11.019 & 10.926 & 8.006 & 6.937 & 4.411 & 4.312 & 2.6 & 2.573 & 10.679 & 5.957 & 4.723 & 6.689 & 3.614 \\
\hline $11 / 15 / 2005$ & $21: 00$ & 11.028 & 10.931 & 8.014 & 6.942 & 4.413 & 4.319 & 2.608 & 2.577 & 10.685 & 5.957 & 4.723 & 6.693 & 3.622 \\
\hline
\end{tabular}


Water Level (ft below top of casing) at Indicated Well

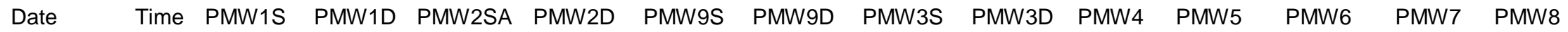

\begin{tabular}{|c|c|c|c|c|c|c|c|c|c|c|c|c|c|c|}
\hline $11 / 15 / 2005$ & $22: 00$ & 11.033 & 10.942 & 8.018 & 6.949 & 4.413 & 4.323 & 2.613 & 2.583 & 10.688 & 5.962 & 4.725 & 6.691 & 3.633 \\
\hline $11 / 15 / 2005$ & $23: 00$ & 11.046 & 10.948 & 8.024 & 6.949 & 4.419 & 4.33 & 2.621 & 2.589 & 10.688 & 5.971 & 4.728 & 6.701 & 3.647 \\
\hline $11 / 16 / 2005$ & $0: 00$ & 11.05 & 10.953 & 8.03 & 6.958 & 4.425 & 4.335 & 2.626 & 2.596 & 10.692 & 5.975 & 4.734 & 6.705 & 3.657 \\
\hline $11 / 16 / 2005$ & $1: 00$ & 11.06 & 10.966 & 8.036 & 6.969 & 4.431 & 4.343 & 2.637 & 2.606 & 10.694 & 5.982 & 4.744 & 6.703 & 3.671 \\
\hline $11 / 16 / 2005$ & $2: 00$ & 11.065 & 10.966 & 8.042 & 6.969 & 4.433 & 4.348 & 2.64 & 2.611 & 10.702 & 5.984 & 4.744 & 6.712 & 3.677 \\
\hline $11 / 16 / 2005$ & $3: 00$ & 11.075 & 10.975 & 8.046 & 6.975 & 4.438 & 4.354 & 2.645 & 2.617 & 10.705 & 5.988 & 4.752 & 6.72 & 3.685 \\
\hline $11 / 16 / 2005$ & $4: 00$ & 11.08 & 10.979 & 8.05 & 6.978 & 4.441 & 4.361 & 2.649 & 2.619 & 10.707 & 5.995 & 4.758 & 6.72 & 3.693 \\
\hline $11 / 16 / 2005$ & $5: 00$ & 11.084 & 10.984 & 8.052 & 6.98 & 4.442 & 4.363 & 2.652 & 2.619 & 10.711 & 5.999 & 4.762 & 6.722 & 3.698 \\
\hline $11 / 16 / 2005$ & $6: 00$ & 11.092 & 10.988 & 8.057 & 6.984 & 4.448 & 4.37 & 2.661 & 2.629 & 10.713 & 6.004 & 4.77 & 6.73 & 3.705 \\
\hline $11 / 16 / 2005$ & $7: 00$ & 11.102 & 10.995 & 8.063 & 6.991 & 4.454 & 4.375 & 2.664 & 2.636 & 10.719 & 6.01 & 4.777 & 6.732 & 3.714 \\
\hline $11 / 16 / 2005$ & $8: 00$ & 11.104 & 11.001 & 8.067 & 7.002 & 4.461 & 4.384 & 2.671 & 2.642 & 10.723 & 6.015 & 4.787 & 6.736 & 3.722 \\
\hline $11 / 16 / 2005$ & $9: 00$ & 11.109 & 11.012 & 8.073 & 7.009 & 4.471 & 4.388 & 2.681 & 2.65 & 10.728 & 6.021 & 4.799 & 6.741 & 3.732 \\
\hline $11 / 16 / 2005$ & $10: 00$ & 11.119 & 11.026 & 8.079 & 7.02 & 4.482 & 4.397 & 2.693 & 2.663 & 10.732 & 6.027 & 4.811 & 6.751 & 3.742 \\
\hline $11 / 16 / 2005$ & $11: 00$ & 11.131 & 11.032 & 8.087 & 7.029 & 4.492 & 4.403 & 2.701 & 2.669 & 10.74 & 6.034 & 4.822 & 6.755 & 3.752 \\
\hline $11 / 16 / 2005$ & $12: 00$ & 11.138 & 11.039 & 8.091 & 7.031 & 4.497 & 4.41 & 2.709 & 2.678 & 10.742 & 6.036 & 4.828 & 6.757 & 3.758 \\
\hline $11 / 16 / 2005$ & $13: 00$ & 11.143 & 11.039 & 8.095 & 7.031 & 4.492 & 4.41 & 2.703 & 2.671 & 10.751 & 6.039 & 4.832 & 6.755 & 3.754 \\
\hline $11 / 16 / 2005$ & $14: 00$ & 11.146 & 11.043 & 8.095 & 7.029 & 4.491 & 4.417 & 2.703 & 2.673 & 10.747 & 6.039 & 4.832 & 6.76 & 3.754 \\
\hline $11 / 16 / 2005$ & $15: 00$ & 11.146 & 11.035 & 8.092 & 7.024 & 4.484 & 4.417 & 2.698 & 2.667 & 10.747 & 6.039 & 4.832 & 6.76 & 3.75 \\
\hline $11 / 16 / 2005$ & $16: 00$ & 11.151 & 11.039 & 8.094 & 7.024 & 4.486 & 4.419 & 2.701 & 2.667 & 10.751 & 6.039 & 4.834 & 6.76 & 3.752 \\
\hline $11 / 16 / 2005$ & $17: 00$ & 11.153 & 11.043 & 8.096 & 7.029 & 4.492 & 4.421 & 2.703 & 2.671 & 10.753 & 6.043 & 4.838 & 6.766 & 3.756 \\
\hline $11 / 16 / 2005$ & $18: 00$ & 11.153 & 11.041 & 8.098 & 7.026 & 4.492 & 4.423 & 2.703 & 2.671 & 10.755 & 6.04 & 4.838 & 6.766 & 3.754 \\
\hline $11 / 16 / 2005$ & $19: 00$ & 11.155 & 11.043 & 8.098 & 7.025 & 4.491 & 4.426 & 2.703 & 2.671 & 10.755 & 6.045 & 4.84 & 6.768 & 3.754 \\
\hline $11 / 16 / 2005$ & $20: 00$ & 11.158 & 11.045 & 8.1 & 7.027 & 4.493 & 4.428 & 2.705 & 2.676 & 10.757 & 6.043 & 4.842 & 6.77 & 3.758 \\
\hline $11 / 16 / 2005$ & $21: 00$ & 11.158 & 11.045 & 8.1 & 7.027 & 4.492 & 4.43 & 2.705 & 2.673 & 10.759 & 6.045 & 4.844 & 6.77 & 3.756 \\
\hline $11 / 16 / 2005$ & $22: 00$ & 11.155 & 11.041 & 8.1 & 7.025 & 4.491 & 4.43 & 2.702 & 2.671 & 10.757 & 6.045 & 4.846 & 6.77 & 3.756 \\
\hline $11 / 16 / 2005$ & $23: 00$ & 11.15 & 11.034 & 8.098 & 7.02 & 4.486 & 4.428 & 2.696 & 2.667 & 10.757 & 6.043 & 4.848 & 6.768 & 3.754 \\
\hline $11 / 17 / 2005$ & $0: 00$ & 11.148 & 11.03 & 8.096 & 7.018 & 4.486 & 4.428 & 2.697 & 2.668 & 10.759 & 6.04 & 4.85 & 6.766 & 3.754 \\
\hline $11 / 17 / 2005$ & $1: 00$ & 11.148 & 11.028 & 8.096 & 7.016 & 4.484 & 4.428 & 2.694 & 2.663 & 10.757 & 6.045 & 4.851 & 6.768 & 3.754 \\
\hline $11 / 17 / 2005$ & $2: 00$ & 11.143 & 11.024 & 8.093 & 7.014 & 4.484 & 4.43 & 2.693 & 2.661 & 10.757 & 6.043 & 4.853 & 6.768 & 3.754 \\
\hline $11 / 17 / 2005$ & $3: 00$ & 11.14 & 11.019 & 8.09 & 7.011 & 4.482 & 4.426 & 2.691 & 2.659 & 10.759 & 6.043 & 4.853 & 6.766 & 3.752 \\
\hline $11 / 17 / 2005$ & $4: 00$ & 11.14 & 11.019 & 8.09 & 7.014 & 4.484 & 4.426 & 2.691 & 2.661 & 10.759 & 6.045 & 4.855 & 6.766 & 3.755 \\
\hline $11 / 17 / 2005$ & $5: 00$ & 11.138 & 11.017 & 8.09 & 7.011 & 4.483 & 4.426 & 2.691 & 2.661 & 10.759 & 6.047 & 4.859 & 6.766 & 3.758 \\
\hline $11 / 17 / 2005$ & $6: 00$ & 11.138 & 11.015 & 8.088 & 7.011 & 4.483 & 4.426 & 2.691 & 2.661 & 10.761 & 6.045 & 4.859 & 6.766 & 3.755 \\
\hline $11 / 17 / 2005$ & $7: 00$ & 11.133 & 11.01 & 8.086 & 7.005 & 4.479 & 4.423 & 2.685 & 2.655 & 10.759 & 6.04 & 4.861 & 6.762 & 3.751 \\
\hline
\end{tabular}


Water Level (ft below top of casing) at Indicated Well

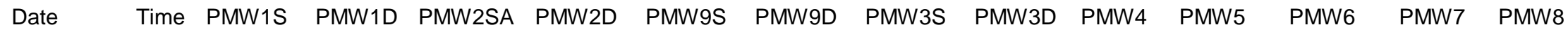

\begin{tabular}{|c|c|c|c|c|c|c|c|c|c|c|c|c|c|c|}
\hline $11 / 17 / 2005$ & 8:00 & 11.13 & 11.008 & 8.087 & 7.005 & 4.476 & 4.423 & 2.688 & 2.655 & 10.759 & 6.043 & 4.863 & 6.766 & 3.753 \\
\hline $11 / 17 / 2005$ & $9: 00$ & 11.135 & 11.012 & 8.088 & 7.009 & 4.484 & 4.423 & 2.691 & 2.659 & 10.759 & 6.043 & 4.869 & 6.766 & 3.755 \\
\hline $11 / 17 / 2005$ & $10: 00$ & 11.13 & 11.008 & 8.086 & 7.005 & 4.481 & 4.423 & 2.688 & 2.655 & 10.757 & 6.04 & 4.867 & 6.764 & 3.753 \\
\hline $11 / 17 / 2005$ & $11: 00$ & 11.125 & 11.001 & 8.084 & 7 & 4.478 & 4.419 & 2.685 & 2.653 & 10.755 & 6.04 & 4.867 & 6.762 & 3.749 \\
\hline $11 / 17 / 2005$ & $12: 00$ & 11.125 & 11.001 & 8.082 & 6.998 & 4.476 & 4.419 & 2.683 & 2.651 & 10.753 & 6.04 & 4.869 & 6.764 & 3.751 \\
\hline $11 / 17 / 2005$ & $13: 00$ & 11.118 & 10.99 & 8.078 & 6.994 & 4.472 & 4.415 & 2.677 & 2.647 & 10.751 & 6.036 & 4.865 & 6.76 & 3.745 \\
\hline $11 / 17 / 2005$ & $14: 00$ & 11.108 & 10.981 & 8.071 & 6.982 & 4.465 & 4.413 & 2.667 & 2.634 & 10.751 & 6.036 & 4.859 & 6.758 & 3.739 \\
\hline $11 / 17 / 2005$ & $15: 00$ & 11.101 & 10.97 & 8.065 & 6.974 & 4.457 & 4.406 & 2.658 & 2.624 & 10.75 & 6.031 & 4.851 & 6.755 & 3.733 \\
\hline $11 / 17 / 2005$ & $16: 00$ & 11.09 & 10.962 & 8.059 & 6.965 & 4.452 & 4.399 & 2.652 & 2.617 & 10.746 & 6.029 & 4.847 & 6.751 & 3.729 \\
\hline $11 / 17 / 2005$ & $17: 00$ & 11.088 & 10.962 & 8.057 & 6.967 & 4.455 & 4.4 & 2.651 & 2.617 & 10.746 & 6.036 & 4.845 & 6.753 & 3.731 \\
\hline $11 / 17 / 2005$ & $18: 00$ & 11.088 & 10.962 & 8.06 & 6.971 & 4.459 & 4.397 & 2.655 & 2.621 & 10.746 & 6.033 & 4.843 & 6.751 & 3.733 \\
\hline $11 / 17 / 2005$ & $19: 00$ & 11.086 & 10.964 & 8.06 & 6.974 & 4.461 & 4.395 & 2.658 & 2.624 & 10.746 & 6.033 & 4.841 & 6.751 & 3.735 \\
\hline $11 / 17 / 2005$ & $20: 00$ & 11.088 & 10.966 & 8.062 & 6.976 & 4.462 & 4.395 & 2.657 & 2.624 & 10.748 & 6.031 & 4.835 & 6.749 & 3.735 \\
\hline $11 / 17 / 2005$ & $21: 00$ & 11.086 & 10.963 & 8.058 & 6.971 & 4.458 & 4.393 & 2.652 & 2.619 & 10.746 & 6.031 & 4.831 & 6.747 & 3.731 \\
\hline $11 / 17 / 2005$ & $22: 00$ & 11.091 & 10.972 & 8.062 & 6.98 & 4.466 & 4.395 & 2.663 & 2.628 & 10.748 & 6.035 & 4.833 & 6.753 & 3.734 \\
\hline $11 / 17 / 2005$ & $23: 00$ & 11.09 & 10.972 & 8.064 & 6.978 & 4.463 & 4.395 & 2.661 & 2.626 & 10.748 & 6.031 & 4.827 & 6.749 & 3.732 \\
\hline $11 / 18 / 2005$ & $0: 00$ & 11.086 & 10.962 & 8.06 & 6.974 & 4.457 & 4.393 & 2.655 & 2.621 & 10.746 & 6.029 & 4.821 & 6.747 & 3.724 \\
\hline $11 / 18 / 2005$ & $1: 00$ & 11.083 & 10.959 & 8.058 & 6.97 & 4.453 & 4.393 & 2.652 & 2.617 & 10.744 & 6.029 & 4.817 & 6.745 & 3.724 \\
\hline $11 / 18 / 2005$ & $2: 00$ & 11.078 & 10.954 & 8.054 & 6.963 & 4.45 & 4.391 & 2.647 & 2.611 & 10.742 & 6.029 & 4.814 & 6.742 & 3.722 \\
\hline $11 / 18 / 2005$ & $3: 00$ & 11.076 & 10.952 & 8.052 & 6.963 & 4.45 & 4.388 & 2.644 & 2.611 & 10.74 & 6.029 & 4.812 & 6.743 & 3.722 \\
\hline $11 / 18 / 2005$ & $4: 00$ & 11.073 & 10.948 & 8.05 & 6.96 & 4.447 & 4.386 & 2.643 & 2.609 & 10.74 & 6.024 & 4.81 & 6.74 & 3.722 \\
\hline $11 / 18 / 2005$ & $5: 00$ & 11.071 & 10.944 & 8.048 & 6.956 & 4.446 & 4.386 & 2.64 & 2.607 & 10.738 & 6.022 & 4.806 & 6.74 & 3.72 \\
\hline $11 / 18 / 2005$ & $6: 00$ & 11.066 & 10.941 & 8.046 & 6.954 & 4.442 & 4.382 & 2.637 & 2.603 & 10.736 & 6.022 & 4.806 & 6.739 & 3.718 \\
\hline $11 / 18 / 2005$ & $7: 00$ & 11.063 & 10.937 & 8.044 & 6.952 & 4.441 & 4.382 & 2.636 & 2.603 & 10.736 & 6.022 & 4.806 & 6.738 & 3.718 \\
\hline $11 / 18 / 2005$ & $8: 00$ & 11.063 & 10.935 & 8.044 & 6.954 & 4.442 & 4.38 & 2.636 & 2.603 & 10.732 & 6.024 & 4.806 & 6.736 & 3.72 \\
\hline $11 / 18 / 2005$ & $9: 00$ & 11.061 & 10.934 & 8.042 & 6.95 & 4.442 & 4.377 & 2.634 & 2.601 & 10.732 & 6.022 & 4.808 & 6.738 & 3.72 \\
\hline $11 / 18 / 2005$ & $10: 00$ & 11.056 & 10.93 & 8.042 & 6.947 & 4.438 & 4.375 & 2.631 & 2.599 & 10.734 & 6.022 & 4.808 & 6.736 & 3.72 \\
\hline $11 / 18 / 2005$ & $11: 00$ & 11.054 & 10.924 & 8.036 & 6.941 & 4.433 & 4.371 & 2.627 & 2.595 & 10.73 & 6.018 & 4.803 & 6.732 & 3.716 \\
\hline $11 / 18 / 2005$ & $12: 00$ & 11.048 & 10.915 & 8.032 & 6.932 & 4.423 & 4.364 & 2.616 & 2.584 & 10.727 & 6.015 & 4.797 & 6.728 & 3.709 \\
\hline $11 / 18 / 2005$ & $13: 00$ & 11.038 & 10.903 & 8.024 & 6.919 & 4.412 & 4.36 & 2.603 & 2.569 & 10.723 & 6.006 & 4.786 & 6.722 & 3.693 \\
\hline $11 / 18 / 2005$ & $14: 00$ & 11.029 & 10.886 & 8.013 & 6.901 & 4.389 & 4.351 & 2.583 & 2.55 & 10.717 & 5.982 & 4.764 & 6.711 & 3.644 \\
\hline $11 / 18 / 2005$ & $15: 00$ & 11.016 & 10.87 & 8.003 & 6.885 & 4.356 & 4.342 & 2.566 & 2.532 & 10.708 & 5.964 & 4.729 & 6.694 & 3.592 \\
\hline $11 / 18 / 2005$ & $16: 00$ & 11.004 & 10.857 & 7.991 & 6.874 & 4.322 & 4.331 & 2.551 & 2.517 & 10.702 & 5.951 & 4.701 & 6.686 & 3.562 \\
\hline $11 / 18 / 2005$ & $17: 00$ & 10.994 & 10.842 & 7.982 & 6.863 & 4.295 & 4.322 & 2.541 & 2.506 & 10.694 & 5.944 & 4.682 & 6.681 & 3.553 \\
\hline
\end{tabular}


Water Level (ft below top of casing) at Indicated Well

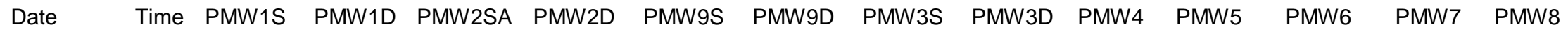

\begin{tabular}{|c|c|c|c|c|c|c|c|c|c|c|c|c|c|c|}
\hline $11 / 18 / 2005$ & $18: 00$ & 10.982 & 10.828 & 7.972 & 6.852 & 4.277 & 4.311 & 2.529 & 2.494 & 10.689 & 5.94 & 4.668 & 6.677 & 3.552 \\
\hline 11/18/2005 & $19: 00$ & 10.972 & 10.819 & 7.966 & 6.846 & 4.273 & 4.302 & 2.521 & 2.487 & 10.683 & 5.942 & 4.66 & 6.671 & 3.555 \\
\hline $11 / 18 / 2005$ & $20: 00$ & 10.965 & 10.81 & 7.96 & 6.839 & 4.274 & 4.293 & 2.519 & 2.485 & 10.679 & 5.938 & 4.654 & 6.667 & 3.563 \\
\hline $11 / 18 / 2005$ & $21: 00$ & 10.955 & 10.799 & 7.952 & 6.83 & 4.27 & 4.284 & 2.511 & 2.477 & 10.673 & 5.936 & 4.646 & 6.661 & 3.566 \\
\hline $11 / 18 / 2005$ & $22: 00$ & 10.947 & 10.793 & 7.95 & 6.826 & 4.272 & 4.278 & 2.508 & 2.473 & 10.672 & 5.94 & 4.647 & 6.658 & 3.574 \\
\hline 11/18/2005 & $23: 00$ & 10.94 & 10.786 & 7.946 & 6.821 & 4.276 & 4.269 & 2.506 & 2.471 & 10.668 & 5.936 & 4.644 & 6.652 & 3.582 \\
\hline 11/19/2005 & $0: 00$ & 10.932 & 10.78 & 7.942 & 6.819 & 4.277 & 4.265 & 2.503 & 2.466 & 10.664 & 5.937 & 4.642 & 6.65 & 3.588 \\
\hline $11 / 19 / 2005$ & $1: 00$ & 10.927 & 10.777 & 7.938 & 6.817 & 4.28 & 4.26 & 2.503 & 2.464 & 10.662 & 5.937 & 4.642 & 6.65 & 3.593 \\
\hline $11 / 19 / 2005$ & $2: 00$ & 10.927 & 10.779 & 7.938 & 6.819 & 4.29 & 4.258 & 2.508 & 2.471 & 10.66 & 5.946 & 4.65 & 6.652 & 3.607 \\
\hline 11/19/2005 & 3:00 & 10.923 & 10.78 & 7.938 & 6.823 & 4.299 & 4.256 & 2.51 & 2.475 & 10.66 & 5.946 & 4.654 & 6.65 & 3.616 \\
\hline $11 / 19 / 2005$ & $4: 00$ & 10.925 & 10.784 & 7.941 & 6.832 & 4.305 & 4.256 & 2.515 & 2.481 & 10.662 & 5.946 & 4.657 & 6.65 & 3.624 \\
\hline $11 / 19 / 2005$ & $5: 00$ & 10.925 & 10.784 & 7.941 & 6.83 & 4.31 & 4.256 & 2.515 & 2.479 & 10.662 & 5.948 & 4.66 & 6.65 & 3.63 \\
\hline 11/19/2005 & $6: 00$ & 10.927 & 10.791 & 7.945 & 6.837 & 4.318 & 4.258 & 2.523 & 2.487 & 10.662 & 5.953 & 4.667 & 6.652 & 3.64 \\
\hline $11 / 19 / 2005$ & $7: 00$ & 10.932 & 10.8 & 7.949 & 6.846 & 4.327 & 4.26 & 2.53 & 2.494 & 10.664 & 5.955 & 4.675 & 6.658 & 3.647 \\
\hline $11 / 19 / 2005$ & 8:00 & 10.94 & 10.804 & 7.955 & 6.843 & 4.334 & 4.262 & 2.537 & 2.502 & 10.666 & 5.959 & 4.683 & 6.656 & 3.653 \\
\hline $11 / 19 / 2005$ & $9: 00$ & 10.947 & 10.82 & 7.959 & 6.863 & 4.342 & 4.267 & 2.545 & 2.511 & 10.668 & 5.962 & 4.69 & 6.663 & 3.661 \\
\hline $11 / 19 / 2005$ & $10: 00$ & 10.954 & 10.83 & 7.965 & 6.87 & 4.35 & 4.269 & 2.553 & 2.519 & 10.668 & 5.964 & 4.695 & 6.667 & 3.666 \\
\hline $11 / 19 / 2005$ & $11: 00$ & 10.962 & 10.837 & 7.969 & 6.877 & 4.355 & 4.274 & 2.558 & 2.523 & 10.67 & 5.966 & 4.7 & 6.665 & 3.671 \\
\hline $11 / 19 / 2005$ & $12: 00$ & 10.972 & 10.85 & 7.977 & 6.888 & 4.363 & 4.28 & 2.568 & 2.534 & 10.672 & 5.973 & 4.706 & 6.675 & 3.677 \\
\hline $11 / 19 / 2005$ & $13: 00$ & 10.979 & 10.859 & 7.983 & 6.895 & 4.369 & 4.285 & 2.573 & 2.538 & 10.678 & 5.973 & 4.709 & 6.677 & 3.678 \\
\hline 11/19/2005 & $14: 00$ & 10.984 & 10.866 & 7.987 & 6.895 & 4.373 & 4.289 & 2.578 & 2.545 & 10.676 & 5.975 & 4.716 & 6.681 & 3.679 \\
\hline $11 / 19 / 2005$ & $15: 00$ & 10.994 & 10.877 & 7.995 & 6.906 & 4.381 & 4.296 & 2.585 & 2.553 & 10.675 & 5.981 & 4.722 & 6.686 & 3.688 \\
\hline $11 / 19 / 2005$ & $16: 00$ & 11.001 & 10.87 & 7.999 & 6.901 & 4.383 & 4.303 & 2.591 & 2.557 & 10.681 & 5.981 & 4.728 & 6.687 & 3.688 \\
\hline $11 / 19 / 2005$ & $17: 00$ & 11.011 & 10.875 & 8.003 & 6.915 & 4.391 & 4.307 & 2.598 & 2.566 & 10.685 & 5.986 & 4.733 & 6.706 & 3.692 \\
\hline $11 / 19 / 2005$ & $18: 00$ & 11.018 & 10.906 & 8.009 & 6.912 & 4.396 & 4.314 & 2.608 & 2.574 & 10.689 & 5.992 & 4.737 & 6.698 & 3.692 \\
\hline $11 / 19 / 2005$ & $19: 00$ & 11.028 & 10.913 & 8.017 & 6.937 & 4.403 & 4.318 & 2.613 & 2.58 & 10.693 & 5.997 & 4.739 & 6.704 & 3.693 \\
\hline $11 / 19 / 2005$ & $20: 00$ & 11.035 & 10.923 & 8.023 & 6.939 & 4.405 & 4.325 & 2.618 & 2.584 & 10.695 & 5.999 & 4.743 & 6.708 & 3.691 \\
\hline $11 / 19 / 2005$ & $21: 00$ & 11.043 & 10.928 & 8.025 & 6.946 & 4.407 & 4.331 & 2.623 & 2.591 & 10.699 & 6.003 & 4.747 & 6.709 & 3.693 \\
\hline $11 / 19 / 2005$ & $22: 00$ & 11.05 & 10.935 & 8.029 & 6.95 & 4.41 & 4.336 & 2.628 & 2.595 & 10.699 & 6.008 & 4.753 & 6.713 & 3.693 \\
\hline $11 / 19 / 2005$ & $23: 00$ & 11.055 & 10.943 & 8.034 & 6.954 & 4.412 & 4.342 & 2.633 & 2.601 & 10.704 & 6.012 & 4.756 & 6.717 & 3.696 \\
\hline $11 / 20 / 2005$ & $0: 00$ & 11.06 & 10.946 & 8.038 & 6.959 & 4.417 & 4.347 & 2.638 & 2.605 & 10.706 & 6.016 & 4.76 & 6.721 & 3.699 \\
\hline $11 / 20 / 2005$ & $1: 00$ & 11.065 & 10.95 & 8.042 & 6.963 & 4.419 & 4.351 & 2.64 & 2.608 & 10.708 & 6.019 & 4.764 & 6.723 & 3.698 \\
\hline $11 / 20 / 2005$ & $2: 00$ & 11.07 & 10.954 & 8.044 & 6.963 & 4.418 & 4.353 & 2.641 & 2.608 & 10.708 & 6.018 & 4.766 & 6.725 & 3.7 \\
\hline $11 / 20 / 2005$ & $3: 00$ & 11.075 & 10.959 & 8.048 & 6.97 & 4.423 & 4.358 & 2.648 & 2.612 & 10.712 & 6.027 & 4.772 & 6.727 & 3.704 \\
\hline
\end{tabular}


Water Level (ft below top of casing) at Indicated Well

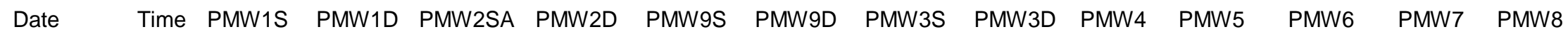

\begin{tabular}{|c|c|c|c|c|c|c|c|c|c|c|c|c|c|c|}
\hline $11 / 20 / 2005$ & $4: 00$ & 11.08 & 10.963 & 8.05 & 6.972 & 4.424 & 4.362 & 2.648 & 2.616 & 10.712 & 6.029 & 4.774 & 6.73 & 3.704 \\
\hline $11 / 20 / 2005$ & $5: 00$ & 11.082 & 10.963 & 8.052 & 6.97 & 4.421 & 4.365 & 2.648 & 2.614 & 10.714 & 6.027 & 4.774 & 6.729 & 3.703 \\
\hline $11 / 20 / 2005$ & $6: 00$ & 11.084 & 10.963 & 8.052 & 6.97 & 4.423 & 4.367 & 2.648 & 2.614 & 10.716 & 6.027 & 4.776 & 6.73 & 3.703 \\
\hline $11 / 20 / 2005$ & $7: 00$ & 11.084 & 10.959 & 8.053 & 6.963 & 4.423 & 4.369 & 2.65 & 2.614 & 10.716 & 6.027 & 4.778 & 6.721 & 3.702 \\
\hline $11 / 20 / 2005$ & $8: 00$ & 11.087 & 10.957 & 8.054 & 6.964 & 4.423 & 4.371 & 2.651 & 2.618 & 10.718 & 6.029 & 4.78 & 6.719 & 3.704 \\
\hline $11 / 20 / 2005$ & $9: 00$ & 11.087 & 10.965 & 8.053 & 6.968 & 4.425 & 4.371 & 2.651 & 2.616 & 10.718 & 6.025 & 4.78 & 6.723 & 3.702 \\
\hline $11 / 20 / 2005$ & $10: 00$ & 11.087 & 10.981 & 8.053 & 6.99 & 4.423 & 4.373 & 2.648 & 2.614 & 10.718 & 6.027 & 4.781 & 6.744 & 3.7 \\
\hline $11 / 20 / 2005$ & $11: 00$ & 11.089 & 10.966 & 8.055 & 6.975 & 4.424 & 4.373 & 2.652 & 2.616 & 10.719 & 6.031 & 4.785 & 6.736 & 3.702 \\
\hline $11 / 20 / 2005$ & $12: 00$ & 11.087 & 10.961 & 8.053 & 6.968 & 4.42 & 4.373 & 2.647 & 2.614 & 10.719 & 6.025 & 4.781 & 6.734 & 3.7 \\
\hline $11 / 20 / 2005$ & $13: 00$ & 11.082 & 10.952 & 8.049 & 6.961 & 4.415 & 4.371 & 2.64 & 2.605 & 10.718 & 6.018 & 4.779 & 6.73 & 3.696 \\
\hline $11 / 20 / 2005$ & $14: 00$ & 11.077 & 10.943 & 8.043 & 6.955 & 4.41 & 4.369 & 2.632 & 2.599 & 10.714 & 6.007 & 4.776 & 6.729 & 3.689 \\
\hline $11 / 20 / 2005$ & $15: 00$ & 11.067 & 10.932 & 8.039 & 6.946 & 4.406 & 4.367 & 2.625 & 2.591 & 10.712 & 6.002 & 4.773 & 6.727 & 3.687 \\
\hline $11 / 20 / 2005$ & $16: 00$ & 11.062 & 10.926 & 8.033 & 6.939 & 4.402 & 4.365 & 2.621 & 2.584 & 10.712 & 6.001 & 4.773 & 6.725 & 3.684 \\
\hline $11 / 20 / 2005$ & $17: 00$ & 11.059 & 10.924 & 8.031 & 6.939 & 4.403 & 4.362 & 2.619 & 2.587 & 10.712 & 5.996 & 4.773 & 6.721 & 3.689 \\
\hline $11 / 20 / 2005$ & $18: 00$ & 11.06 & 10.928 & 8.031 & 6.942 & 4.406 & 4.363 & 2.622 & 2.587 & 10.712 & 6.002 & 4.779 & 6.723 & 3.693 \\
\hline $11 / 20 / 2005$ & $19: 00$ & 11.057 & 10.926 & 8.031 & 6.942 & 4.405 & 4.36 & 2.622 & 2.587 & 10.712 & 6 & 4.778 & 6.723 & 3.694 \\
\hline $11 / 20 / 2005$ & $20: 00$ & 11.054 & 10.923 & 8.031 & 6.939 & 4.405 & 4.36 & 2.621 & 2.587 & 10.71 & 6 & 4.779 & 6.723 & 3.696 \\
\hline $11 / 20 / 2005$ & $21: 00$ & 11.054 & 10.919 & 8.029 & 6.937 & 4.402 & 4.358 & 2.617 & 2.583 & 10.71 & 5.996 & 4.777 & 6.721 & 3.694 \\
\hline $11 / 20 / 2005$ & $22: 00$ & 11.052 & 10.917 & 8.029 & 6.937 & 4.403 & 4.356 & 2.617 & 2.583 & 10.71 & 5.996 & 4.779 & 6.721 & 3.695 \\
\hline $11 / 20 / 2005$ & $23: 00$ & 11.049 & 10.91 & 8.025 & 6.93 & 4.398 & 4.354 & 2.614 & 2.579 & 10.708 & 5.991 & 4.774 & 6.717 & 3.694 \\
\hline $11 / 21 / 2005$ & $0: 00$ & 11.047 & 10.912 & 8.025 & 6.928 & 4.402 & 4.354 & 2.616 & 2.578 & 10.708 & 5.994 & 4.779 & 6.717 & 3.695 \\
\hline $11 / 21 / 2005$ & $1: 00$ & 11.042 & 10.908 & 8.023 & 6.928 & 4.398 & 4.352 & 2.611 & 2.577 & 10.706 & 5.991 & 4.776 & 6.717 & 3.697 \\
\hline $11 / 21 / 2005$ & $2: 00$ & 11.037 & 10.903 & 8.019 & 6.922 & 4.394 & 4.349 & 2.608 & 2.572 & 10.706 & 5.989 & 4.775 & 6.713 & 3.694 \\
\hline $11 / 21 / 2005$ & $3: 00$ & 11.032 & 10.897 & 8.015 & 6.917 & 4.391 & 4.347 & 2.604 & 2.566 & 10.704 & 5.987 & 4.773 & 6.709 & 3.694 \\
\hline $11 / 21 / 2005$ & $4: 00$ & 11.027 & 10.892 & 8.013 & 6.915 & 4.389 & 4.345 & 2.6 & 2.564 & 10.7 & 5.983 & 4.772 & 6.709 & 3.693 \\
\hline $11 / 21 / 2005$ & $5: 00$ & 11.022 & 10.885 & 8.009 & 6.91 & 4.385 & 4.341 & 2.596 & 2.56 & 10.699 & 5.98 & 4.771 & 6.708 & 3.693 \\
\hline $11 / 21 / 2005$ & $6: 00$ & 11.025 & 10.892 & 8.011 & 6.917 & 4.392 & 4.343 & 2.603 & 2.566 & 10.7 & 5.989 & 4.776 & 6.711 & 3.699 \\
\hline $11 / 21 / 2005$ & $7: 00$ & 11.025 & 10.894 & 8.012 & 6.917 & 4.396 & 4.341 & 2.603 & 2.568 & 10.7 & 5.987 & 4.778 & 6.709 & 3.701 \\
\hline $11 / 21 / 2005$ & $8: 00$ & 11.022 & 10.892 & 8.01 & 6.915 & 4.393 & 4.341 & 2.602 & 2.564 & 10.7 & 5.987 & 4.78 & 6.711 & 3.699 \\
\hline $11 / 21 / 2005$ & $9: 00$ & 11.027 & 10.897 & 8.014 & 6.924 & 4.4 & 4.341 & 2.61 & 2.572 & 10.702 & 5.991 & 4.786 & 6.714 & 3.705 \\
\hline $11 / 21 / 2005$ & $10: 00$ & 11.032 & 10.903 & 8.02 & 6.928 & 4.404 & 4.343 & 2.615 & 2.579 & 10.704 & 5.993 & 4.789 & 6.715 & 3.709 \\
\hline $11 / 21 / 2005$ & $11: 00$ & 11.039 & 10.914 & 8.026 & 6.935 & 4.409 & 4.345 & 2.619 & 2.583 & 10.704 & 5.998 & 4.793 & 6.715 & 3.714 \\
\hline $11 / 21 / 2005$ & $12: 00$ & 11.047 & 10.921 & 8.029 & 6.942 & 4.415 & 4.347 & 2.627 & 2.591 & 10.706 & 6 & 4.795 & 6.719 & 3.716 \\
\hline $11 / 21 / 2005$ & $13: 00$ & 11.049 & 10.925 & 8.033 & 6.946 & 4.415 & 4.349 & 2.627 & 2.593 & 10.708 & 5.999 & 4.796 & 6.719 & 3.715 \\
\hline
\end{tabular}


Water Level (ft below top of casing) at Indicated Well

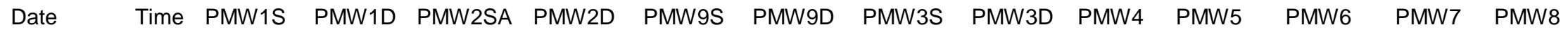

\begin{tabular}{|c|c|c|c|c|c|c|c|c|c|c|c|c|c|c|}
\hline $11 / 21 / 2005$ & $14: 00$ & 11.054 & 10.928 & 8.033 & 6.946 & 4.415 & 4.349 & 2.628 & 2.593 & 10.708 & 6.002 & 4.797 & 6.721 & 3.717 \\
\hline $11 / 21 / 2005$ & $15: 00$ & 11.054 & 10.927 & 8.033 & 6.949 & 4.416 & 4.352 & 2.63 & 2.593 & 10.706 & 6.002 & 4.799 & 6.721 & 3.716 \\
\hline $11 / 21 / 2005$ & $16: 00$ & 11.056 & 10.93 & 8.035 & 6.949 & 4.419 & 4.354 & 2.633 & 2.598 & 10.71 & 6.004 & 4.8 & 6.723 & 3.719 \\
\hline $11 / 21 / 2005$ & $17: 00$ & 11.061 & 10.936 & 8.039 & 6.953 & 4.424 & 4.358 & 2.639 & 2.602 & 10.712 & 6.008 & 4.805 & 6.727 & 3.723 \\
\hline $11 / 21 / 2005$ & $18: 00$ & 11.066 & 10.941 & 8.041 & 6.955 & 4.425 & 4.36 & 2.639 & 2.606 & 10.714 & 6.01 & 4.811 & 6.729 & 3.724 \\
\hline $11 / 21 / 2005$ & $19: 00$ & 11.068 & 10.945 & 8.043 & 6.96 & 4.427 & 4.363 & 2.643 & 2.608 & 10.716 & 6.01 & 4.811 & 6.731 & 3.725 \\
\hline $11 / 21 / 2005$ & $20: 00$ & 11.073 & 10.948 & 8.045 & 6.96 & 4.431 & 4.367 & 2.647 & 2.613 & 10.716 & 6.015 & 4.815 & 6.733 & 3.726 \\
\hline $11 / 21 / 2005$ & $21: 00$ & 11.078 & 10.952 & 8.049 & 6.966 & 4.433 & 4.372 & 2.649 & 2.614 & 10.718 & 6.017 & 4.816 & 6.735 & 3.728 \\
\hline $11 / 21 / 2005$ & $22: 00$ & 11.073 & 10.948 & 8.047 & 6.962 & 4.427 & 4.372 & 2.646 & 2.61 & 10.718 & 6.01 & 4.816 & 6.736 & 3.723 \\
\hline $11 / 21 / 2005$ & $23: 00$ & 11.071 & 10.947 & 8.049 & 6.96 & 4.426 & 4.372 & 2.642 & 2.606 & 10.718 & 6.01 & 4.818 & 6.735 & 3.72 \\
\hline $11 / 22 / 2005$ & $0: 00$ & 11.073 & 10.947 & 8.049 & 6.962 & 4.427 & 4.372 & 2.645 & 2.61 & 10.719 & 6.01 & 4.818 & 6.738 & 3.721 \\
\hline $11 / 22 / 2005$ & $1: 00$ & 11.073 & 10.945 & 8.051 & 6.96 & 4.427 & 4.374 & 2.646 & 2.61 & 10.719 & 6.008 & 4.818 & 6.736 & 3.721 \\
\hline $11 / 22 / 2005$ & $2: 00$ & 11.068 & 10.941 & 8.047 & 6.955 & 4.422 & 4.372 & 2.638 & 2.606 & 10.719 & 6.004 & 4.816 & 6.735 & 3.717 \\
\hline $11 / 22 / 2005$ & $3: 00$ & 11.071 & 10.945 & 8.049 & 6.96 & 4.424 & 4.374 & 2.643 & 2.608 & 10.719 & 6.008 & 4.818 & 6.735 & 3.722 \\
\hline $11 / 22 / 2005$ & $4: 00$ & 11.068 & 10.941 & 8.049 & 6.957 & 4.422 & 4.372 & 2.639 & 2.606 & 10.72 & 6.004 & 4.816 & 6.735 & 3.718 \\
\hline $11 / 22 / 2005$ & $5: 00$ & 11.061 & 10.936 & 8.044 & 6.949 & 4.418 & 4.372 & 2.635 & 2.6 & 10.718 & 6.001 & 4.814 & 6.731 & 3.715 \\
\hline $11 / 22 / 2005$ & $6: 00$ & 11.058 & 10.93 & 8.041 & 6.949 & 4.415 & 4.37 & 2.633 & 2.598 & 10.716 & 5.997 & 4.812 & 6.729 & 3.713 \\
\hline $11 / 22 / 2005$ & $7: 00$ & 11.058 & 10.93 & 8.043 & 6.949 & 4.416 & 4.37 & 2.632 & 2.598 & 10.716 & 5.999 & 4.812 & 6.731 & 3.714 \\
\hline $11 / 22 / 2005$ & $8: 00$ & 11.058 & 10.93 & 8.041 & 6.949 & 4.417 & 4.37 & 2.634 & 2.598 & 10.716 & 6.001 & 4.812 & 6.729 & 3.716 \\
\hline $11 / 22 / 2005$ & $9: 00$ & 11.053 & 10.927 & 8.039 & 6.944 & 4.413 & 4.368 & 2.63 & 2.594 & 10.716 & 5.997 & 4.81 & 6.725 & 3.711 \\
\hline $11 / 22 / 2005$ & $10: 00$ & 11.053 & 10.925 & 8.038 & 6.944 & 4.413 & 4.368 & 2.629 & 2.594 & 10.714 & 5.995 & 4.81 & 6.725 & 3.712 \\
\hline $11 / 22 / 2005$ & $11: 00$ & 11.048 & 10.921 & 8.037 & 6.94 & 4.411 & 4.363 & 2.627 & 2.592 & 10.714 & 5.995 & 4.807 & 6.723 & 3.711 \\
\hline $11 / 22 / 2005$ & $12: 00$ & 11.041 & 10.912 & 8.033 & 6.931 & 4.404 & 4.361 & 2.62 & 2.585 & 10.71 & 5.988 & 4.802 & 6.719 & 3.708 \\
\hline $11 / 22 / 2005$ & $13: 00$ & 11.031 & 10.899 & 8.025 & 6.918 & 4.395 & 4.354 & 2.608 & 2.573 & 10.706 & 5.983 & 4.794 & 6.716 & 3.705 \\
\hline $11 / 22 / 2005$ & $14: 00$ & 11.021 & 10.885 & 8.015 & 6.904 & 4.386 & 4.348 & 2.597 & 2.558 & 10.7 & 5.981 & 4.787 & 6.71 & 3.698 \\
\hline $11 / 22 / 2005$ & $15: 00$ & 11.011 & 10.87 & 8.007 & 6.895 & 4.375 & 4.341 & 2.587 & 2.548 & 10.699 & 5.979 & 4.78 & 6.704 & 3.694 \\
\hline $11 / 22 / 2005$ & $16: 00$ & 10.996 & 10.857 & 7.999 & 6.884 & 4.373 & 4.337 & 2.579 & 2.541 & 10.695 & 5.977 & 4.778 & 6.7 & 3.694 \\
\hline $11 / 22 / 2005$ & $17: 00$ & 10.991 & 10.85 & 7.995 & 6.882 & 4.37 & 4.33 & 2.575 & 2.537 & 10.693 & 5.972 & 4.772 & 6.695 & 3.693 \\
\hline $11 / 22 / 2005$ & $18: 00$ & 10.987 & 10.848 & 7.991 & 6.882 & 4.372 & 4.328 & 2.572 & 2.535 & 10.691 & 5.974 & 4.776 & 6.695 & 3.697 \\
\hline $11 / 22 / 2005$ & $19: 00$ & 10.979 & 10.843 & 7.989 & 6.878 & 4.369 & 4.323 & 2.567 & 2.533 & 10.691 & 5.97 & 4.77 & 6.689 & 3.695 \\
\hline $11 / 22 / 2005$ & $20: 00$ & 10.972 & 10.834 & 7.983 & 6.869 & 4.363 & 4.317 & 2.56 & 2.524 & 10.687 & 5.964 & 4.764 & 6.687 & 3.688 \\
\hline $11 / 22 / 2005$ & $21: 00$ & 10.964 & 10.823 & 7.977 & 6.86 & 4.356 & 4.312 & 2.552 & 2.516 & 10.682 & 5.961 & 4.759 & 6.681 & 3.683 \\
\hline $11 / 22 / 2005$ & $22: 00$ & 10.957 & 10.813 & 7.971 & 6.851 & 4.348 & 4.303 & 2.542 & 2.506 & 10.68 & 5.957 & 4.75 & 6.679 & 3.677 \\
\hline $11 / 22 / 2005$ & $23: 00$ & 10.95 & 10.803 & 7.965 & 6.844 & 4.343 & 4.299 & 2.535 & 2.499 & 10.676 & 5.955 & 4.747 & 6.674 & 3.675 \\
\hline
\end{tabular}


Water Level (ft below top of casing) at Indicated Well

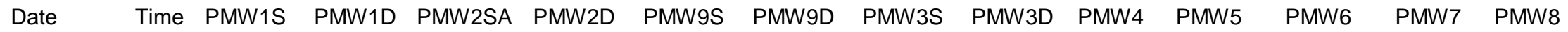

\begin{tabular}{|c|c|c|c|c|c|c|c|c|c|c|c|c|c|c|}
\hline $11 / 23 / 2005$ & $0: 00$ & 10.942 & 10.795 & 7.957 & 6.838 & 4.339 & 4.292 & 2.532 & 2.493 & 10.674 & 5.952 & 4.74 & 6.67 & 3.673 \\
\hline $11 / 23 / 2005$ & $1: 00$ & 10.935 & 10.784 & 7.953 & 6.831 & 4.335 & 4.286 & 2.524 & 2.487 & 10.67 & 5.95 & 4.736 & 6.664 & 3.671 \\
\hline $11 / 23 / 2005$ & $2: 00$ & 10.928 & 10.779 & 7.947 & 6.823 & 4.328 & 4.279 & 2.516 & 2.48 & 10.665 & 5.948 & 4.728 & 6.66 & 3.666 \\
\hline $11 / 23 / 2005$ & $3: 00$ & 10.92 & 10.773 & 7.943 & 6.82 & 4.327 & 4.272 & 2.513 & 2.476 & 10.665 & 5.948 & 4.724 & 6.656 & 3.667 \\
\hline $11 / 23 / 2005$ & $4: 00$ & 10.915 & 10.768 & 7.943 & 6.82 & 4.327 & 4.27 & 2.515 & 2.476 & 10.663 & 5.967 & 4.723 & 6.651 & 3.668 \\
\hline $11 / 23 / 2005$ & $5: 00$ & 10.91 & 10.763 & 7.939 & 6.816 & 4.325 & 4.263 & 2.508 & 2.47 & 10.657 & 5.963 & 4.718 & 6.647 & 3.666 \\
\hline $11 / 23 / 2005$ & $6: 00$ & 10.908 & 10.761 & 7.937 & 6.816 & 4.326 & 4.261 & 2.508 & 2.47 & 10.659 & 5.963 & 4.718 & 6.647 & 3.671 \\
\hline $11 / 23 / 2005$ & $7: 00$ & 10.903 & 10.761 & 7.935 & 6.816 & 4.329 & 4.259 & 2.511 & 2.47 & 10.657 & 5.963 & 4.719 & 6.645 & 3.675 \\
\hline $11 / 23 / 2005$ & $8: 00$ & 10.905 & 10.762 & 7.936 & 6.82 & 4.332 & 4.257 & 2.513 & 2.474 & 10.659 & 5.963 & 4.716 & 6.643 & 3.677 \\
\hline $11 / 23 / 2005$ & $9: 00$ & 10.903 & 10.765 & 7.936 & 6.82 & 4.334 & 4.257 & 2.515 & 2.476 & 10.655 & 5.965 & 4.717 & 6.645 & 3.681 \\
\hline $11 / 23 / 2005$ & $10: 00$ & 10.91 & 10.773 & 7.938 & 6.829 & 4.343 & 4.259 & 2.523 & 2.485 & 10.659 & 5.967 & 4.726 & 6.647 & 3.689 \\
\hline $11 / 23 / 2005$ & $11: 00$ & 10.91 & 10.781 & 7.944 & 6.838 & 4.347 & 4.259 & 2.526 & 2.489 & 10.657 & 5.969 & 4.726 & 6.647 & 3.693 \\
\hline $11 / 23 / 2005$ & $12: 00$ & 10.915 & 10.783 & 7.948 & 6.838 & 4.348 & 4.257 & 2.522 & 2.491 & 10.657 & 5.965 & 4.726 & 6.647 & 3.696 \\
\hline $11 / 23 / 2005$ & $13: 00$ & 10.917 & 10.786 & 7.95 & 6.84 & 4.351 & 4.264 & 2.532 & 2.493 & 10.657 & 5.967 & 4.73 & 6.647 & 3.7 \\
\hline $11 / 23 / 2005$ & $14: 00$ & 10.922 & 10.794 & 7.952 & 6.843 & 4.357 & 4.264 & 2.536 & 2.497 & 10.657 & 5.97 & 4.734 & 6.649 & 3.705 \\
\hline $11 / 23 / 2005$ & $15: 00$ & 10.922 & 10.79 & 7.948 & 6.84 & 4.353 & 4.266 & 2.534 & 2.493 & 10.655 & 5.967 & 4.735 & 6.651 & 3.707 \\
\hline $11 / 23 / 2005$ & $16: 00$ & 10.922 & 10.794 & 7.952 & 6.84 & 4.356 & 4.266 & 2.534 & 2.497 & 10.655 & 5.969 & 4.739 & 6.651 & 3.707 \\
\hline $11 / 23 / 2005$ & $17: 00$ & 10.927 & 10.797 & 7.955 & 6.847 & 4.359 & 4.268 & 2.539 & 2.501 & 10.655 & 5.976 & 4.743 & 6.653 & 3.712 \\
\hline $11 / 23 / 2005$ & $18: 00$ & 10.932 & 10.806 & 7.958 & 6.854 & 4.368 & 4.273 & 2.547 & 2.51 & 10.661 & 5.974 & 4.749 & 6.657 & 3.717 \\
\hline $11 / 23 / 2005$ & $19: 00$ & 10.942 & 10.814 & 7.964 & 6.86 & 4.373 & 4.275 & 2.554 & 2.516 & 10.663 & 5.974 & 4.751 & 6.658 & 3.719 \\
\hline $11 / 23 / 2005$ & $20: 00$ & 10.947 & 10.823 & 7.964 & 6.865 & 4.378 & 4.279 & 2.558 & 2.521 & 10.667 & 5.976 & 4.757 & 6.662 & 3.721 \\
\hline $11 / 23 / 2005$ & $21: 00$ & 10.949 & 10.827 & 7.966 & 6.869 & 4.378 & 4.282 & 2.561 & 2.523 & 10.667 & 5.976 & 4.757 & 6.664 & 3.719 \\
\hline $11 / 23 / 2005$ & $22: 00$ & 10.959 & 10.836 & 7.972 & 6.878 & 4.383 & 4.288 & 2.566 & 2.529 & 10.668 & 5.978 & 4.763 & 6.668 & 3.72 \\
\hline $11 / 23 / 2005$ & $23: 00$ & 10.966 & 10.845 & 7.976 & 6.881 & 4.386 & 4.29 & 2.571 & 2.535 & 10.67 & 5.978 & 4.765 & 6.67 & 3.722 \\
\hline $11 / 24 / 2005$ & $0: 00$ & 10.974 & 10.856 & 7.982 & 6.892 & 4.392 & 4.297 & 2.58 & 2.542 & 10.674 & 5.985 & 4.773 & 6.674 & 3.726 \\
\hline $11 / 24 / 2005$ & $1: 00$ & 10.981 & 10.865 & 7.988 & 6.898 & 4.396 & 4.301 & 2.587 & 2.552 & 10.676 & 5.985 & 4.773 & 6.678 & 3.729 \\
\hline $11 / 24 / 2005$ & $2: 00$ & 10.989 & 10.87 & 7.991 & 6.901 & 4.396 & 4.306 & 2.589 & 2.552 & 10.678 & 5.984 & 4.775 & 6.68 & 3.724 \\
\hline $11 / 24 / 2005$ & $3: 00$ & 10.993 & 10.878 & 7.997 & 6.907 & 4.401 & 4.31 & 2.596 & 2.561 & 10.68 & 5.985 & 4.78 & 6.685 & 3.729 \\
\hline $11 / 24 / 2005$ & $4: 00$ & 11.003 & 10.89 & 8.003 & 6.92 & 4.41 & 4.317 & 2.606 & 2.571 & 10.684 & 5.991 & 4.787 & 6.691 & 3.735 \\
\hline $11 / 24 / 2005$ & $5: 00$ & 11.013 & 10.903 & 8.011 & 6.927 & 4.416 & 4.324 & 2.615 & 2.577 & 10.686 & 5.995 & 4.792 & 6.695 & 3.739 \\
\hline $11 / 24 / 2005$ & $6: 00$ & 11.025 & 10.918 & 8.021 & 6.943 & 4.426 & 4.33 & 2.629 & 2.592 & 10.691 & 5.998 & 4.8 & 6.701 & 3.744 \\
\hline $11 / 24 / 2005$ & $7: 00$ & 11.035 & 10.929 & 8.028 & 6.951 & 4.428 & 4.339 & 2.636 & 2.598 & 10.695 & 6.002 & 4.805 & 6.704 & 3.747 \\
\hline $11 / 24 / 2005$ & $8: 00$ & 11.045 & 10.943 & 8.036 & 6.965 & 4.44 & 4.348 & 2.65 & 2.613 & 10.699 & 6.006 & 4.813 & 6.712 & 3.752 \\
\hline $11 / 24 / 2005$ & $9: 00$ & 11.06 & 10.962 & 8.046 & 6.98 & 4.451 & 4.357 & 2.665 & 2.628 & 10.705 & 6.013 & 4.822 & 6.72 & 3.763 \\
\hline
\end{tabular}


Water Level (ft below top of casing) at Indicated Well

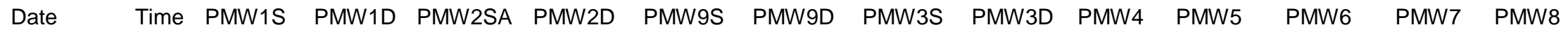

\begin{tabular}{|c|c|c|c|c|c|c|c|c|c|c|c|c|c|c|}
\hline $11 / 24 / 2005$ & $10: 00$ & 11.074 & 10.978 & 8.057 & 6.996 & 4.46 & 4.368 & 2.678 & 2.642 & 10.71 & 6.017 & 4.83 & 6.729 & 3.766 \\
\hline $11 / 24 / 2005$ & $11: 00$ & 11.087 & 10.993 & 8.067 & 7.007 & 4.468 & 4.379 & 2.69 & 2.653 & 10.716 & 6.024 & 4.837 & 6.739 & 3.77 \\
\hline $11 / 24 / 2005$ & $12: 00$ & 11.097 & 11.005 & 8.075 & 7.014 & 4.47 & 4.388 & 2.696 & 2.659 & 10.718 & 6.024 & 4.844 & 6.739 & 3.772 \\
\hline $11 / 24 / 2005$ & $13: 00$ & 11.106 & 11.011 & 8.08 & 7.018 & 4.47 & 4.39 & 2.7 & 2.661 & 10.724 & 6.025 & 4.842 & 6.743 & 3.769 \\
\hline $11 / 24 / 2005$ & $14: 00$ & 11.111 & 11.013 & 8.082 & 7.014 & 4.468 & 4.394 & 2.699 & 2.663 & 10.726 & 6.023 & 4.842 & 6.744 & 3.766 \\
\hline $11 / 24 / 2005$ & $15: 00$ & 11.116 & 11.011 & 8.082 & 7.009 & 4.462 & 4.399 & 2.693 & 2.659 & 10.726 & 6.021 & 4.842 & 6.743 & 3.758 \\
\hline $11 / 24 / 2005$ & $16: 00$ & 11.118 & 11.011 & 8.084 & 7.007 & 4.46 & 4.403 & 2.694 & 2.657 & 10.727 & 6.024 & 4.843 & 6.744 & 3.758 \\
\hline $11 / 24 / 2005$ & $17: 00$ & 11.121 & 11.013 & 8.084 & 7.009 & 4.462 & 4.408 & 2.696 & 2.659 & 10.729 & 6.026 & 4.846 & 6.746 & 3.758 \\
\hline $11 / 24 / 2005$ & $18: 00$ & 11.123 & 11.011 & 8.086 & 7.007 & 4.459 & 4.408 & 2.695 & 2.657 & 10.731 & 6.024 & 4.848 & 6.748 & 3.755 \\
\hline $11 / 24 / 2005$ & 19:00 & 11.126 & 11.018 & 8.088 & 7.016 & 4.467 & 4.412 & 2.698 & 2.663 & 10.733 & 6.03 & 4.856 & 6.754 & 3.759 \\
\hline $11 / 24 / 2005$ & $20: 00$ & 11.13 & 11.02 & 8.092 & 7.018 & 4.472 & 4.417 & 2.704 & 2.668 & 10.735 & 6.028 & 4.857 & 6.754 & 3.759 \\
\hline $11 / 24 / 2005$ & $21: 00$ & 11.133 & 11.024 & 8.094 & 7.02 & 4.471 & 4.419 & 2.705 & 2.668 & 10.735 & 6.028 & 4.859 & 6.758 & 3.758 \\
\hline $11 / 24 / 2005$ & $22: 00$ & 11.135 & 11.02 & 8.094 & 7.018 & 4.469 & 4.419 & 2.703 & 2.666 & 10.735 & 6.028 & 4.859 & 6.756 & 3.752 \\
\hline $11 / 24 / 2005$ & $23: 00$ & 11.135 & 11.016 & 8.091 & 7.014 & 4.463 & 4.419 & 2.699 & 2.661 & 10.735 & 6.028 & 4.859 & 6.754 & 3.749 \\
\hline $11 / 25 / 2005$ & $0: 00$ & 11.13 & 11.009 & 8.089 & 7.007 & 4.459 & 4.419 & 2.692 & 2.655 & 10.735 & 6.023 & 4.858 & 6.754 & 3.746 \\
\hline $11 / 25 / 2005$ & $1: 00$ & 11.125 & 11.005 & 8.089 & 7.007 & 4.458 & 4.419 & 2.69 & 2.653 & 10.733 & 6.022 & 4.857 & 6.752 & 3.743 \\
\hline $11 / 25 / 2005$ & $2: 00$ & 11.118 & 10.994 & 8.083 & 6.994 & 4.451 & 4.417 & 2.679 & 2.643 & 10.735 & 6.02 & 4.853 & 6.748 & 3.736 \\
\hline $11 / 25 / 2005$ & $3: 00$ & 11.113 & 10.987 & 8.076 & 6.99 & 4.448 & 4.415 & 2.674 & 2.638 & 10.73 & 6.021 & 4.853 & 6.746 & 3.733 \\
\hline $11 / 25 / 2005$ & $4: 00$ & 11.11 & 10.982 & 8.072 & 6.983 & 4.444 & 4.41 & 2.67 & 2.635 & 10.731 & 6.018 & 4.851 & 6.744 & 3.731 \\
\hline $11 / 25 / 2005$ & $5: 00$ & 11.105 & 10.978 & 8.068 & 6.981 & 4.444 & 4.408 & 2.67 & 2.632 & 10.726 & 6.019 & 4.855 & 6.743 & 3.731 \\
\hline $11 / 25 / 2005$ & $6: 00$ & 11.096 & 10.965 & 8.062 & 6.97 & 4.434 & 4.404 & 2.657 & 2.622 & 10.72 & 6.011 & 4.85 & 6.739 & 3.722 \\
\hline $11 / 25 / 2005$ & $7: 00$ & 11.086 & 10.951 & 8.054 & 6.956 & 4.424 & 4.399 & 2.649 & 2.609 & 10.718 & 6.009 & 4.843 & 6.735 & 3.718 \\
\hline $11 / 25 / 2005$ & $8: 00$ & 11.078 & 10.943 & 8.048 & 6.952 & 4.421 & 4.393 & 2.642 & 2.605 & 10.713 & 6.009 & 4.848 & 6.735 & 3.718 \\
\hline $11 / 25 / 2005$ & $9: 00$ & 11.071 & 10.936 & 8.046 & 6.95 & 4.419 & 4.388 & 2.639 & 2.603 & 10.712 & 6.004 & 4.845 & 6.725 & 3.713 \\
\hline $11 / 25 / 2005$ & $10: 00$ & 11.066 & 10.929 & 8.041 & 6.941 & 4.413 & 4.384 & 2.632 & 2.597 & 10.709 & 6 & 4.841 & 6.725 & 3.711 \\
\hline $11 / 25 / 2005$ & $11: 00$ & 11.058 & 10.92 & 8.036 & 6.932 & 4.413 & 4.377 & 2.627 & 2.588 & 10.707 & 5.999 & 4.844 & 6.722 & 3.712 \\
\hline $11 / 25 / 2005$ & $12: 00$ & 11.046 & 10.903 & 8.027 & 6.921 & 4.399 & 4.37 & 2.614 & 2.576 & 10.701 & 5.992 & 4.833 & 6.712 & 3.7 \\
\hline $11 / 25 / 2005$ & $13: 00$ & 11.036 & 10.885 & 8.017 & 6.903 & 4.387 & 4.359 & 2.6 & 2.557 & 10.697 & 5.992 & 4.825 & 6.712 & 3.694 \\
\hline $11 / 25 / 2005$ & $14: 00$ & 11.022 & 10.865 & 8.005 & 6.885 & 4.375 & 4.348 & 2.581 & 2.542 & 10.69 & 5.983 & 4.81 & 6.701 & 3.683 \\
\hline $11 / 25 / 2005$ & $15: 00$ & 11.009 & 10.852 & 7.997 & 6.872 & 4.366 & 4.34 & 2.57 & 2.53 & 10.686 & 5.982 & 4.803 & 6.701 & 3.681 \\
\hline $11 / 25 / 2005$ & $16: 00$ & 10.997 & 10.839 & 7.989 & 6.87 & 4.359 & 4.331 & 2.562 & 2.525 & 10.684 & 5.98 & 4.792 & 6.691 & 3.676 \\
\hline $11 / 25 / 2005$ & $17: 00$ & 10.992 & 10.843 & 7.987 & 6.874 & 4.373 & 4.327 & 2.568 & 2.53 & 10.684 & 5.987 & 4.796 & 6.693 & 3.688 \\
\hline $11 / 25 / 2005$ & $18: 00$ & 10.987 & 10.837 & 7.983 & 6.872 & 4.372 & 4.322 & 2.565 & 2.527 & 10.682 & 5.98 & 4.788 & 6.687 & 3.685 \\
\hline $11 / 25 / 2005$ & 19:00 & 10.982 & 10.834 & 7.981 & 6.872 & 4.372 & 4.32 & 2.565 & 2.527 & 10.682 & 5.981 & 4.786 & 6.685 & 3.687 \\
\hline
\end{tabular}


Water Level (ft below top of casing) at Indicated Well

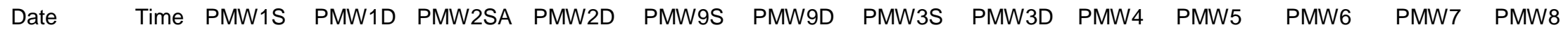

\begin{tabular}{|c|c|c|c|c|c|c|c|c|c|c|c|c|c|c|}
\hline $11 / 25 / 2005$ & $20: 00$ & 10.98 & 10.83 & 7.98 & 6.87 & 4.373 & 4.318 & 2.564 & 2.523 & 10.68 & 5.98 & 4.781 & 6.684 & 3.687 \\
\hline $11 / 25 / 2005$ & $21: 00$ & 10.977 & 10.832 & 7.98 & 6.872 & 4.376 & 4.313 & 2.565 & 2.527 & 10.68 & 5.979 & 4.782 & 6.685 & 3.693 \\
\hline $11 / 25 / 2005$ & $22: 00$ & 10.975 & 10.834 & 7.98 & 6.872 & 4.377 & 4.313 & 2.565 & 2.527 & 10.676 & 5.981 & 4.78 & 6.683 & 3.692 \\
\hline $11 / 25 / 2005$ & $23: 00$ & 10.977 & 10.837 & 7.98 & 6.879 & 4.379 & 4.311 & 2.569 & 2.532 & 10.678 & 5.979 & 4.779 & 6.682 & 3.697 \\
\hline $11 / 26 / 2005$ & $0: 00$ & 10.977 & 10.839 & 7.982 & 6.879 & 4.38 & 4.311 & 2.569 & 2.532 & 10.678 & 5.982 & 4.78 & 6.683 & 3.696 \\
\hline $11 / 26 / 2005$ & $1: 00$ & 10.979 & 10.843 & 7.982 & 6.881 & 4.382 & 4.311 & 2.571 & 2.536 & 10.676 & 5.979 & 4.781 & 6.682 & 3.7 \\
\hline $11 / 26 / 2005$ & $2: 00$ & 10.982 & 10.845 & 7.982 & 6.883 & 4.383 & 4.311 & 2.573 & 2.536 & 10.678 & 5.978 & 4.779 & 6.682 & 3.697 \\
\hline $11 / 26 / 2005$ & $3: 00$ & 10.982 & 10.841 & 7.982 & 6.881 & 4.381 & 4.311 & 2.571 & 2.534 & 10.676 & 5.975 & 4.775 & 6.68 & 3.694 \\
\hline $11 / 26 / 2005$ & $4: 00$ & 10.979 & 10.839 & 7.982 & 6.879 & 4.376 & 4.309 & 2.57 & 2.532 & 10.676 & 5.974 & 4.773 & 6.68 & 3.694 \\
\hline $11 / 26 / 2005$ & $5: 00$ & 10.974 & 10.836 & 7.978 & 6.877 & 4.373 & 4.307 & 2.565 & 2.527 & 10.675 & 5.971 & 4.768 & 6.678 & 3.69 \\
\hline $11 / 26 / 2005$ & $6: 00$ & 10.974 & 10.834 & 7.976 & 6.872 & 4.371 & 4.304 & 2.564 & 2.525 & 10.673 & 5.971 & 4.768 & 6.678 & 3.69 \\
\hline $11 / 26 / 2005$ & $7: 00$ & 10.972 & 10.832 & 7.976 & 6.872 & 4.37 & 4.304 & 2.563 & 2.523 & 10.673 & 5.969 & 4.767 & 6.678 & 3.689 \\
\hline $11 / 26 / 2005$ & $8: 00$ & 10.969 & 10.832 & 7.976 & 6.87 & 4.371 & 4.304 & 2.562 & 2.525 & 10.671 & 5.969 & 4.765 & 6.678 & 3.69 \\
\hline $11 / 26 / 2005$ & $9: 00$ & 10.969 & 10.829 & 7.974 & 6.868 & 4.371 & 4.302 & 2.561 & 2.523 & 10.671 & 5.966 & 4.765 & 6.674 & 3.688 \\
\hline $11 / 26 / 2005$ & $10: 00$ & 10.964 & 10.829 & 7.974 & 6.868 & 4.368 & 4.3 & 2.561 & 2.523 & 10.669 & 5.966 & 4.763 & 6.674 & 3.686 \\
\hline $11 / 26 / 2005$ & $11: 00$ & 10.965 & 10.827 & 7.972 & 6.864 & 4.365 & 4.298 & 2.558 & 2.519 & 10.665 & 5.967 & 4.763 & 6.674 & 3.685 \\
\hline $11 / 26 / 2005$ & $12: 00$ & 10.957 & 10.82 & 7.968 & 6.857 & 4.357 & 4.296 & 2.551 & 2.513 & 10.663 & 5.964 & 4.755 & 6.67 & 3.681 \\
\hline $11 / 26 / 2005$ & $13: 00$ & 10.952 & 10.809 & 7.962 & 6.846 & 4.349 & 4.289 & 2.541 & 2.501 & 10.665 & 5.96 & 4.745 & 6.665 & 3.672 \\
\hline $11 / 26 / 2005$ & $14: 00$ & 10.944 & 10.801 & 7.956 & 6.837 & 4.342 & 4.285 & 2.534 & 2.496 & 10.656 & 5.959 & 4.741 & 6.663 & 3.669 \\
\hline $11 / 26 / 2005$ & $15: 00$ & 10.937 & 10.794 & 7.95 & 6.835 & 4.338 & 4.278 & 2.53 & 2.49 & 10.652 & 5.957 & 4.737 & 6.661 & 3.666 \\
\hline $11 / 26 / 2005$ & $16: 00$ & 10.93 & 10.787 & 7.946 & 6.828 & 4.337 & 4.274 & 2.523 & 2.484 & 10.652 & 5.958 & 4.731 & 6.655 & 3.667 \\
\hline $11 / 26 / 2005$ & $17: 00$ & 10.925 & 10.783 & 7.942 & 6.828 & 4.336 & 4.271 & 2.523 & 2.484 & 10.65 & 5.957 & 4.727 & 6.651 & 3.667 \\
\hline $11 / 26 / 2005$ & $18: 00$ & 10.92 & 10.776 & 7.94 & 6.822 & 4.33 & 4.267 & 2.516 & 2.477 & 10.65 & 5.955 & 4.725 & 6.651 & 3.665 \\
\hline $11 / 26 / 2005$ & 19:00 & 10.913 & 10.768 & 7.936 & 6.819 & 4.329 & 4.265 & 2.513 & 2.473 & 10.65 & 5.953 & 4.72 & 6.648 & 3.661 \\
\hline $11 / 26 / 2005$ & $20: 00$ & 10.913 & 10.77 & 7.936 & 6.819 & 4.333 & 4.263 & 2.514 & 2.475 & 10.646 & 5.952 & 4.721 & 6.648 & 3.663 \\
\hline $11 / 26 / 2005$ & $21: 00$ & 10.91 & 10.768 & 7.934 & 6.819 & 4.332 & 4.261 & 2.514 & 2.475 & 10.648 & 5.951 & 4.718 & 6.64 & 3.663 \\
\hline $11 / 26 / 2005$ & $22: 00$ & 10.908 & 10.767 & 7.934 & 6.817 & 4.331 & 4.258 & 2.513 & 2.473 & 10.644 & 5.952 & 4.716 & 6.64 & 3.662 \\
\hline $11 / 26 / 2005$ & $23: 00$ & 10.905 & 10.765 & 7.932 & 6.815 & 4.333 & 4.256 & 2.51 & 2.473 & 10.642 & 5.95 & 4.711 & 6.636 & 3.663 \\
\hline $11 / 27 / 2005$ & $0: 00$ & 10.903 & 10.763 & 7.93 & 6.813 & 4.329 & 4.254 & 2.511 & 2.471 & 10.638 & 5.95 & 4.707 & 6.634 & 3.66 \\
\hline $11 / 27 / 2005$ & $1: 00$ & 10.9 & 10.759 & 7.928 & 6.81 & 4.325 & 4.252 & 2.506 & 2.467 & 10.638 & 5.948 & 4.701 & 6.632 & 3.656 \\
\hline $11 / 27 / 2005$ & $2: 00$ & 10.895 & 10.756 & 7.924 & 6.806 & 4.321 & 4.247 & 2.5 & 2.463 & 10.631 & 5.945 & 4.695 & 6.628 & 3.654 \\
\hline $11 / 27 / 2005$ & $3: 00$ & 10.897 & 10.752 & 7.924 & 6.804 & 4.318 & 4.245 & 2.498 & 2.459 & 10.631 & 5.944 & 4.693 & 6.625 & 3.65 \\
\hline $11 / 27 / 2005$ & $4: 00$ & 10.89 & 10.748 & 7.92 & 6.799 & 4.317 & 4.241 & 2.496 & 2.456 & 10.627 & 5.945 & 4.689 & 6.625 & 3.648 \\
\hline $11 / 27 / 2005$ & $5: 00$ & 10.888 & 10.745 & 7.916 & 6.797 & 4.31 & 4.238 & 2.491 & 2.45 & 10.633 & 5.945 & 4.684 & 6.625 & 3.644 \\
\hline
\end{tabular}


Water Level (ft below top of casing) at Indicated Well

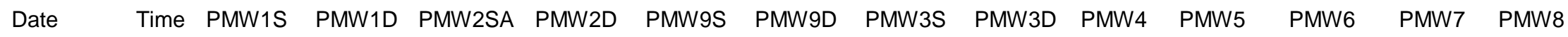

\begin{tabular}{|c|c|c|c|c|c|c|c|c|c|c|c|c|c|c|}
\hline $11 / 27 / 2005$ & $6: 00$ & 10.885 & 10.743 & 7.919 & 6.799 & 4.316 & 4.236 & 2.493 & 2.454 & 10.633 & 5.943 & 4.682 & 6.623 & 3.648 \\
\hline $11 / 27 / 2005$ & $7: 00$ & 10.883 & 10.741 & 7.917 & 6.795 & 4.313 & 4.234 & 2.488 & 2.45 & 10.631 & 5.941 & 4.676 & 6.623 & 3.648 \\
\hline $11 / 27 / 2005$ & $8: 00$ & 10.883 & 10.739 & 7.916 & 6.793 & 4.313 & 4.234 & 2.488 & 2.45 & 10.629 & 5.941 & 4.673 & 6.619 & 3.646 \\
\hline $11 / 27 / 2005$ & $9: 00$ & 10.88 & 10.736 & 7.912 & 6.79 & 4.309 & 4.232 & 2.485 & 2.446 & 10.629 & 5.94 & 4.673 & 6.619 & 3.646 \\
\hline $11 / 27 / 2005$ & $10: 00$ & 10.878 & 10.736 & 7.912 & 6.793 & 4.31 & 4.23 & 2.486 & 2.448 & 10.627 & 5.938 & 4.666 & 6.615 & 3.644 \\
\hline $11 / 27 / 2005$ & $11: 00$ & 10.88 & 10.737 & 7.912 & 6.793 & 4.311 & 4.23 & 2.486 & 2.446 & 10.627 & 5.941 & 4.667 & 6.617 & 3.647 \\
\hline $11 / 27 / 2005$ & $12: 00$ & 10.875 & 10.734 & 7.911 & 6.79 & 4.308 & 4.225 & 2.483 & 2.444 & 10.625 & 5.937 & 4.662 & 6.613 & 3.644 \\
\hline $11 / 27 / 2005$ & $13: 00$ & 10.872 & 10.727 & 7.906 & 6.782 & 4.299 & 4.223 & 2.475 & 2.438 & 10.623 & 5.933 & 4.655 & 6.609 & 3.638 \\
\hline $11 / 27 / 2005$ & $14: 00$ & 10.87 & 10.723 & 7.9 & 6.786 & 4.289 & 4.219 & 2.465 & 2.435 & 10.621 & 5.929 & 4.646 & 6.607 & 3.629 \\
\hline $11 / 27 / 2005$ & $15: 00$ & 10.863 & 10.71 & 7.894 & 6.766 & 4.281 & 4.212 & 2.459 & 2.423 & 10.619 & 5.926 & 4.642 & 6.606 & 3.625 \\
\hline $11 / 27 / 2005$ & $16: 00$ & 10.855 & 10.714 & 7.889 & 6.775 & 4.274 & 4.205 & 2.45 & 2.423 & 10.618 & 5.922 & 4.632 & 6.619 & 3.618 \\
\hline $11 / 27 / 2005$ & $17: 00$ & 10.848 & 10.708 & 7.883 & 6.784 & 4.275 & 4.207 & 2.435 & 2.419 & 10.621 & 5.879 & 4.61 & 6.613 & 3.569 \\
\hline $11 / 27 / 2005$ & $18: 00$ & 10.693 & 10.714 & 7.867 & 6.782 & 4.066 & 4.201 & 2.404 & 2.37 & 10.621 & 5.795 & 4.446 & 6.6 & 3.223 \\
\hline $11 / 27 / 2005$ & $19: 00$ & 10.608 & 10.685 & 7.856 & 6.777 & 3.94 & 4.192 & 2.387 & 2.354 & 10.623 & 5.742 & 4.32 & 6.566 & 3.074 \\
\hline $11 / 27 / 2005$ & $20: 00$ & 10.606 & 10.644 & 7.85 & 6.715 & 3.804 & 4.177 & 2.364 & 2.328 & 10.56 & 5.712 & 4.215 & 6.48 & 2.975 \\
\hline $11 / 27 / 2005$ & $21: 00$ & 10.648 & 10.674 & 7.844 & 6.696 & 3.738 & 4.163 & 2.363 & 2.328 & 10.521 & 5.714 & 4.209 & 6.447 & 2.984 \\
\hline $11 / 27 / 2005$ & $22: 00$ & 10.699 & 10.672 & 7.836 & 6.698 & 3.729 & 4.143 & 2.353 & 2.318 & 10.526 & 5.729 & 4.223 & 6.457 & 3.021 \\
\hline $11 / 27 / 2005$ & $23: 00$ & 10.753 & 10.675 & 7.834 & 6.707 & 3.757 & 4.128 & 2.359 & 2.322 & 10.465 & 5.743 & 4.242 & 6.464 & 3.061 \\
\hline $11 / 28 / 2005$ & $0: 00$ & 10.77 & 10.67 & 7.832 & 6.7 & 3.781 & 4.115 & 2.353 & 2.314 & 10.441 & 5.758 & 4.253 & 6.472 & 3.096 \\
\hline $11 / 28 / 2005$ & $1: 00$ & 10.777 & 10.666 & 7.83 & 6.698 & 3.813 & 4.101 & 2.353 & 2.316 & 10.424 & 5.762 & 4.268 & 6.472 & 3.129 \\
\hline $11 / 28 / 2005$ & $2: 00$ & 10.782 & 10.666 & 7.832 & 6.7 & 3.845 & 4.093 & 2.358 & 2.318 & 10.532 & 5.774 & 4.287 & 6.48 & 3.16 \\
\hline $11 / 28 / 2005$ & $3: 00$ & 10.782 & 10.661 & 7.83 & 6.7 & 3.872 & 4.088 & 2.359 & 2.32 & 10.53 & 5.778 & 4.301 & 6.482 & 3.186 \\
\hline $11 / 28 / 2005$ & $4: 00$ & 10.789 & 10.657 & 7.832 & 6.689 & 3.888 & 4.082 & 2.355 & 2.318 & 10.521 & 5.785 & 4.309 & 6.472 & 3.206 \\
\hline $11 / 28 / 2005$ & $5: 00$ & 10.782 & 10.657 & 7.826 & 6.702 & 3.912 & 4.073 & 2.36 & 2.318 & 10.521 & 5.795 & 4.326 & 6.485 & 3.231 \\
\hline $11 / 28 / 2005$ & $6: 00$ & 10.784 & 10.661 & 7.832 & 6.704 & 3.936 & 4.077 & 2.367 & 2.327 & 10.526 & 5.8 & 4.338 & 6.493 & 3.253 \\
\hline $11 / 28 / 2005$ & $7: 00$ & 10.787 & 10.661 & 7.832 & 6.704 & 3.952 & 4.075 & 2.367 & 2.329 & 10.524 & 5.811 & 4.354 & 6.499 & 3.278 \\
\hline $11 / 28 / 2005$ & $8: 00$ & 10.789 & 10.668 & 7.836 & 6.713 & 3.973 & 4.079 & 2.379 & 2.339 & 10.526 & 5.815 & 4.365 & 6.503 & 3.3 \\
\hline $11 / 28 / 2005$ & $9: 00$ & 10.794 & 10.668 & 7.842 & 6.716 & 3.987 & 4.084 & 2.384 & 2.346 & 10.528 & 5.821 & 4.375 & 6.508 & 3.317 \\
\hline $11 / 28 / 2005$ & $10: 00$ & 10.796 & 10.67 & 7.844 & 6.718 & 4.001 & 4.084 & 2.385 & 2.346 & 10.526 & 5.826 & 4.387 & 6.512 & 3.337 \\
\hline $11 / 28 / 2005$ & $11: 00$ & 10.804 & 10.686 & 7.852 & 6.733 & 4.023 & 4.093 & 2.402 & 2.362 & 10.53 & 5.834 & 4.406 & 6.522 & 3.362 \\
\hline $11 / 28 / 2005$ & $12: 00$ & 10.808 & 10.696 & 7.86 & 6.744 & 4.042 & 4.097 & 2.414 & 2.373 & 10.53 & 5.839 & 4.419 & 6.525 & 3.38 \\
\hline $11 / 28 / 2005$ & $13: 00$ & 10.818 & 10.71 & 7.864 & 6.755 & 4.058 & 4.106 & 2.419 & 2.381 & 10.536 & 5.845 & 4.434 & 6.533 & 3.397 \\
\hline $11 / 28 / 2005$ & $14: 00$ & 10.826 & 10.712 & 7.87 & 6.755 & 4.067 & 4.11 & 2.428 & 2.388 & 10.538 & 5.853 & 4.443 & 6.541 & 3.41 \\
\hline $11 / 28 / 2005$ & $15: 00$ & 10.838 & 10.728 & 7.878 & 6.769 & 4.083 & 4.122 & 2.439 & 2.4 & 10.542 & 5.859 & 4.457 & 6.543 & 3.429 \\
\hline
\end{tabular}


Water Level (ft below top of casing) at Indicated Well

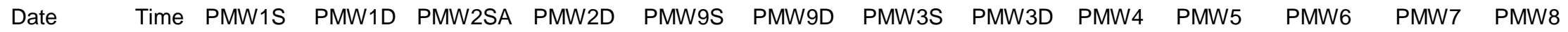

\begin{tabular}{|c|c|c|c|c|c|c|c|c|c|c|c|c|c|c|}
\hline 11/28/2005 & $16: 00$ & 10.853 & 10.747 & 7.886 & 6.786 & 4.105 & 4.133 & 2.454 & 2.419 & 10.549 & 5.87 & 4.473 & 6.556 & 3.449 \\
\hline 11/28/2005 & $17: 00$ & 10.865 & 10.767 & 7.897 & 6.8 & 4.124 & 4.141 & 2.471 & 2.432 & 10.553 & 5.877 & 4.489 & 6.562 & 3.466 \\
\hline $11 / 28 / 2005$ & $18: 00$ & 10.88 & 10.787 & 7.914 & 6.817 & 4.14 & 4.155 & 2.489 & 2.448 & 10.56 & 5.883 & 4.508 & 6.573 & 3.482 \\
\hline $11 / 28 / 2005$ & $19: 00$ & 10.894 & 10.8 & 7.924 & 6.831 & 4.148 & 4.164 & 2.496 & 2.459 & 10.566 & 5.883 & 4.519 & 6.579 & 3.495 \\
\hline $11 / 28 / 2005$ & $20: 00$ & 10.907 & 10.811 & 7.926 & 6.836 & 4.161 & 4.177 & 2.507 & 2.469 & 10.57 & 5.892 & 4.53 & 6.587 & 3.51 \\
\hline $11 / 28 / 2005$ & $21: 00$ & 10.917 & 10.821 & 7.936 & 6.844 & 4.169 & 4.184 & 2.514 & 2.478 & 10.576 & 5.9 & 4.544 & 6.594 & 3.517 \\
\hline 11/28/2005 & $22: 00$ & 10.924 & 10.833 & 7.947 & 6.86 & 4.181 & 4.199 & 2.525 & 2.484 & 10.584 & 5.906 & 4.563 & 6.606 & 3.536 \\
\hline $11 / 28 / 2005$ & $23: 00$ & 10.936 & 10.849 & 7.951 & 6.864 & 4.189 & 4.206 & 2.533 & 2.499 & 10.593 & 5.909 & 4.564 & 6.602 & 3.541 \\
\hline $11 / 29 / 2005$ & 0:00 & 10.946 & 10.858 & 7.955 & 6.873 & 4.207 & 4.217 & 2.546 & 2.507 & 10.585 & 5.918 & 4.581 & 6.617 & 3.555 \\
\hline $11 / 29 / 2005$ & $1: 00$ & 10.963 & 10.864 & 7.964 & 6.889 & 4.216 & 4.23 & 2.557 & 2.516 & 10.595 & 5.934 & 4.591 & 6.621 & 3.564 \\
\hline $11 / 29 / 2005$ & $2: 00$ & 10.973 & 10.884 & 7.976 & 6.898 & 4.229 & 4.237 & 2.569 & 2.53 & 10.601 & 5.946 & 4.601 & 6.625 & 3.576 \\
\hline 11/29/2005 & $3: 00$ & 10.98 & 10.898 & 7.981 & 6.906 & 4.24 & 4.246 & 2.583 & 2.545 & 10.612 & 5.965 & 4.617 & 6.642 & 3.591 \\
\hline 11/29/2005 & 4:00 & 10.997 & 10.916 & 7.993 & 6.922 & 4.251 & 4.261 & 2.59 & 2.553 & 10.622 & 5.985 & 4.633 & 6.642 & 3.595 \\
\hline $11 / 29 / 2005$ & $5: 00$ & 11.012 & 10.925 & 8 & 6.929 & 4.259 & 4.268 & 2.597 & 2.56 & 10.616 & 5.992 & 4.64 & 6.655 & 3.603 \\
\hline $11 / 29 / 2005$ & $6: 00$ & 11.014 & 10.931 & 8.001 & 6.935 & 4.266 & 4.277 & 2.605 & 2.568 & 10.625 & 6.005 & 4.649 & 6.661 & 3.609 \\
\hline $11 / 29 / 2005$ & $7: 00$ & 11.027 & 10.938 & 8.014 & 6.942 & 4.276 & 4.288 & 2.613 & 2.574 & 10.631 & 6.016 & 4.658 & 6.671 & 3.617 \\
\hline $11 / 29 / 2005$ & 8:00 & 11.034 & 10.951 & 8.019 & 6.951 & 4.283 & 4.294 & 2.621 & 2.585 & 10.637 & 5.975 & 4.671 & 6.676 & 3.626 \\
\hline $11 / 29 / 2005$ & $9: 00$ & 11.044 & 10.96 & 8.021 & 6.957 & 4.292 & 4.303 & 2.63 & 2.593 & 10.643 & 5.991 & 4.677 & 6.682 & 3.631 \\
\hline $11 / 29 / 2005$ & $10: 00$ & 11.054 & 10.969 & 8.031 & 6.968 & 4.299 & 4.312 & 2.637 & 2.602 & 10.648 & 6.005 & 4.691 & 6.688 & 3.642 \\
\hline $11 / 29 / 2005$ & $11: 00$ & 11.066 & 10.984 & 8.041 & 6.98 & 4.312 & 4.323 & 2.649 & 2.612 & 10.65 & 6.031 & 4.706 & 6.694 & 3.651 \\
\hline 11/29/2005 & $12: 00$ & 11.076 & 10.993 & 8.044 & 6.986 & 4.319 & 4.327 & 2.657 & 2.621 & 10.658 & 6.042 & 4.713 & 6.701 & 3.659 \\
\hline $11 / 29 / 2005$ & $13: 00$ & 11.088 & 11 & 8.05 & 6.989 & 4.321 & 4.334 & 2.658 & 2.623 & 10.656 & 6.043 & 4.717 & 6.703 & 3.656 \\
\hline $11 / 29 / 2005$ & $14: 00$ & 11.09 & 11.004 & 8.054 & 6.993 & 4.322 & 4.341 & 2.661 & 2.627 & 10.667 & 6.045 & 4.719 & 6.707 & 3.654 \\
\hline 11/29/2005 & $15: 00$ & 11.095 & 11.008 & 8.058 & 6.993 & 4.328 & 4.348 & 2.663 & 2.627 & 10.669 & 6.054 & 4.72 & 6.711 & 3.649 \\
\hline $11 / 29 / 2005$ & $16: 00$ & 11.105 & 11.019 & 8.061 & 6.999 & 4.336 & 4.354 & 2.672 & 2.637 & 10.673 & 6.065 & 4.731 & 6.716 & 3.652 \\
\hline $11 / 29 / 2005$ & $17: 00$ & 11.11 & 11.026 & 8.069 & 7.008 & 4.343 & 4.361 & 2.68 & 2.644 & 10.679 & 6.02 & 4.733 & 6.72 & 3.652 \\
\hline $11 / 29 / 2005$ & $18: 00$ & 11.119 & 11.035 & 8.075 & 7.015 & 4.35 & 4.37 & 2.685 & 2.648 & 10.683 & 6.033 & 4.738 & 6.728 & 3.657 \\
\hline $11 / 29 / 2005$ & 19:00 & 11.129 & 11.041 & 8.079 & 7.019 & 4.355 & 4.377 & 2.69 & 2.655 & 10.686 & 6.04 & 4.744 & 6.732 & 3.662 \\
\hline $11 / 29 / 2005$ & $20: 00$ & 11.134 & 11.044 & 8.083 & 7.022 & 4.356 & 4.381 & 2.695 & 2.659 & 10.69 & 6.042 & 4.749 & 6.736 & 3.663 \\
\hline $11 / 29 / 2005$ & $21: 00$ & 11.139 & 11.046 & 8.084 & 7.024 & 4.36 & 4.385 & 2.695 & 2.659 & 10.692 & 6.046 & 4.753 & 6.737 & 3.667 \\
\hline $11 / 29 / 2005$ & $22: 00$ & 11.142 & 11.05 & 8.088 & 7.026 & 4.362 & 4.39 & 2.698 & 2.663 & 10.698 & 6.054 & 4.757 & 6.741 & 3.669 \\
\hline $11 / 29 / 2005$ & $23: 00$ & 11.146 & 11.052 & 8.09 & 7.026 & 4.364 & 4.394 & 2.7 & 2.665 & 10.7 & 6.055 & 4.762 & 6.743 & 3.674 \\
\hline $11 / 30 / 2005$ & $0: 00$ & 11.149 & 11.052 & 8.092 & 7.026 & 4.368 & 4.396 & 2.7 & 2.665 & 10.702 & 6.059 & 4.768 & 6.747 & 3.675 \\
\hline $11 / 30 / 2005$ & $1: 00$ & 11.154 & 11.055 & 8.094 & 7.029 & 4.371 & 4.401 & 2.702 & 2.669 & 10.703 & 6.062 & 4.773 & 6.751 & 3.679 \\
\hline
\end{tabular}


Water Level (ft below top of casing) at Indicated Well

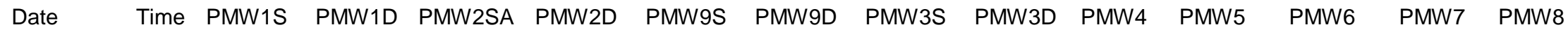

\begin{tabular}{|c|c|c|c|c|c|c|c|c|c|c|c|c|c|c|}
\hline $11 / 30 / 2005$ & $2: 00$ & 11.153 & 11.052 & 8.094 & 7.026 & 4.37 & 4.403 & 2.703 & 2.665 & 10.705 & 6.059 & 4.777 & 6.749 & 3.68 \\
\hline $11 / 30 / 2005$ & $3: 00$ & 11.151 & 11.046 & 8.094 & 7.022 & 4.371 & 4.403 & 2.699 & 2.663 & 10.707 & 6.049 & 4.78 & 6.749 & 3.681 \\
\hline $11 / 30 / 2005$ & $4: 00$ & 11.151 & 11.046 & 8.092 & 7.022 & 4.374 & 4.405 & 2.698 & 2.663 & 10.707 & 6.054 & 4.784 & 6.751 & 3.685 \\
\hline $11 / 30 / 2005$ & $5: 00$ & 11.151 & 11.042 & 8.094 & 7.02 & 4.374 & 4.405 & 2.698 & 2.661 & 10.707 & 6.052 & 4.787 & 6.751 & 3.689 \\
\hline $11 / 30 / 2005$ & $6: 00$ & 11.144 & 11.035 & 8.088 & 7.013 & 4.37 & 4.405 & 2.691 & 2.655 & 10.707 & 6.045 & 4.791 & 6.749 & 3.69 \\
\hline $11 / 30 / 2005$ & $7: 00$ & 11.144 & 11.031 & 8.088 & 7.015 & 4.375 & 4.405 & 2.693 & 2.657 & 10.709 & 6.047 & 4.796 & 6.751 & 3.694 \\
\hline $11 / 30 / 2005$ & $8: 00$ & 11.139 & 11.026 & 8.084 & 7.011 & 4.374 & 4.403 & 2.688 & 2.65 & 10.709 & 6.038 & 4.801 & 6.753 & 3.698 \\
\hline $11 / 30 / 2005$ & $9: 00$ & 11.139 & 11.024 & 8.086 & 7.009 & 4.377 & 4.405 & 2.689 & 2.653 & 10.711 & 6.042 & 4.806 & 6.753 & 3.701 \\
\hline $11 / 30 / 2005$ & $10: 00$ & 11.133 & 11.019 & 8.084 & 7.004 & 4.374 & 4.403 & 2.686 & 2.648 & 10.709 & 6.031 & 4.806 & 6.751 & 3.701 \\
\hline $11 / 30 / 2005$ & $11: 00$ & 11.128 & 11.008 & 8.078 & 6.998 & 4.373 & 4.401 & 2.681 & 2.644 & 10.709 & 6.029 & 4.806 & 6.747 & 3.701 \\
\hline $11 / 30 / 2005$ & $12: 00$ & 11.123 & 11.004 & 8.076 & 6.995 & 4.375 & 4.399 & 2.676 & 2.64 & 10.707 & 6.03 & 4.811 & 6.747 & 3.7 \\
\hline $11 / 30 / 2005$ & $13: 00$ & 11.116 & 10.995 & 8.074 & 6.989 & 4.371 & 4.394 & 2.671 & 2.634 & 10.709 & 6.018 & 4.807 & 6.743 & 3.697 \\
\hline $11 / 30 / 2005$ & $14: 00$ & 11.104 & 10.977 & 8.061 & 6.971 & 4.359 & 4.39 & 2.655 & 2.619 & 10.703 & 5.996 & 4.797 & 6.736 & 3.685 \\
\hline $11 / 30 / 2005$ & $15: 00$ & 11.096 & 10.966 & 8.055 & 6.962 & 4.356 & 4.386 & 2.648 & 2.613 & 10.7 & 5.992 & 4.796 & 6.734 & 3.682 \\
\hline $11 / 30 / 2005$ & $16: 00$ & 11.087 & 10.958 & 8.049 & 6.958 & 4.356 & 4.379 & 2.643 & 2.606 & 10.696 & 5.99 & 4.793 & 6.73 & 3.678 \\
\hline $11 / 30 / 2005$ & $17: 00$ & 11.082 & 10.953 & 8.045 & 6.953 & 4.356 & 4.377 & 2.641 & 2.6 & 10.694 & 5.988 & 4.793 & 6.728 & 3.677 \\
\hline $11 / 30 / 2005$ & $18: 00$ & 11.077 & 10.949 & 8.044 & 6.951 & 4.356 & 4.372 & 2.637 & 2.6 & 10.694 & 5.984 & 4.789 & 6.722 & 3.674 \\
\hline $11 / 30 / 2005$ & $19: 00$ & 11.079 & 10.957 & 8.045 & 6.962 & 4.366 & 4.372 & 2.646 & 2.606 & 10.696 & 5.997 & 4.796 & 6.732 & 3.68 \\
\hline $11 / 30 / 2005$ & $20: 00$ & 11.084 & 10.964 & 8.053 & 6.969 & 4.373 & 4.372 & 2.655 & 2.617 & 10.702 & 6.001 & 4.797 & 6.732 & 3.686 \\
\hline $11 / 30 / 2005$ & $21: 00$ & 11.084 & 10.964 & 8.053 & 6.973 & 4.373 & 4.372 & 2.655 & 2.619 & 10.703 & 6 & 4.795 & 6.732 & 3.685 \\
\hline $11 / 30 / 2005$ & $22: 00$ & 11.086 & 10.968 & 8.055 & 6.973 & 4.375 & 4.372 & 2.657 & 2.617 & 10.703 & 6.006 & 4.796 & 6.732 & 3.682 \\
\hline $11 / 30 / 2005$ & $23: 00$ & 11.091 & 10.975 & 8.061 & 6.982 & 4.381 & 4.375 & 2.665 & 2.625 & 10.705 & 6.018 & 4.799 & 6.734 & 3.686 \\
\hline $12 / 1 / 2005$ & $0: 00$ & 11.104 & 10.993 & 8.07 & 6.995 & 4.392 & 4.381 & 2.679 & 2.642 & 10.707 & 6.029 & 4.806 & 6.739 & 3.695 \\
\hline $12 / 1 / 2005$ & $1: 00$ & 11.118 & 11.017 & 8.087 & 7.022 & 4.405 & 4.388 & 2.7 & 2.661 & 10.709 & 6.057 & 4.815 & 6.749 & 3.705 \\
\hline $12 / 1 / 2005$ & $2: 00$ & 11.138 & 11.039 & 8.097 & 7.038 & 4.418 & 4.397 & 2.715 & 2.678 & 10.713 & 6.082 & 4.824 & 6.753 & 3.712 \\
\hline $12 / 1 / 2005$ & $3: 00$ & 11.15 & 11.057 & 8.102 & 7.049 & 4.425 & 4.408 & 2.723 & 2.688 & 10.719 & 6.098 & 4.83 & 6.764 & 3.714 \\
\hline $12 / 1 / 2005$ & $4: 00$ & 11.165 & 11.068 & 8.114 & 7.057 & 4.431 & 4.415 & 2.734 & 2.697 & 10.724 & 6.053 & 4.834 & 6.768 & 3.718 \\
\hline $12 / 1 / 2005$ & $5: 00$ & 11.172 & 11.075 & 8.116 & 7.062 & 4.429 & 4.423 & 2.738 & 2.701 & 10.73 & 6.058 & 4.837 & 6.772 & 3.716 \\
\hline $12 / 1 / 2005$ & $6: 00$ & 11.179 & 11.079 & 8.121 & 7.064 & 4.433 & 4.428 & 2.741 & 2.703 & 10.732 & 6.067 & 4.84 & 6.776 & 3.714 \\
\hline $12 / 1 / 2005$ & $7: 00$ & 11.184 & 11.084 & 8.123 & 7.069 & 4.436 & 4.434 & 2.747 & 2.708 & 10.734 & 6.075 & 4.842 & 6.779 & 3.72 \\
\hline $12 / 1 / 2005$ & $8: 00$ & 11.194 & 11.092 & 8.127 & 7.076 & 4.442 & 4.441 & 2.754 & 2.716 & 10.74 & 6.089 & 4.847 & 6.785 & 3.723 \\
\hline $12 / 1 / 2005$ & $9: 00$ & 11.197 & 11.093 & 8.129 & 7.071 & 4.436 & 4.446 & 2.748 & 2.711 & 10.741 & 6.088 & 4.847 & 6.783 & 3.719 \\
\hline $12 / 1 / 2005$ & $10: 00$ & 11.204 & 11.101 & 8.135 & 7.078 & 4.442 & 4.45 & 2.756 & 2.718 & 10.743 & 6.103 & 4.852 & 6.789 & 3.726 \\
\hline $12 / 1 / 2005$ & $11: 00$ & 11.211 & 11.106 & 8.138 & 7.084 & 4.449 & 4.457 & 2.762 & 2.724 & 10.747 & 6.107 & 4.855 & 6.791 & 3.732 \\
\hline
\end{tabular}


Water Level (ft below top of casing) at Indicated Well

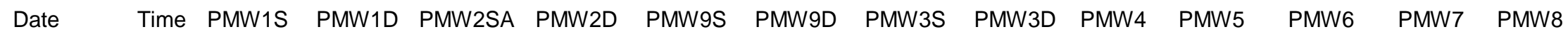

\begin{tabular}{|c|c|c|c|c|c|c|c|c|c|c|c|c|c|c|}
\hline $12 / 1 / 2005$ & $12: 00$ & 11.216 & 11.108 & 8.142 & 7.087 & 4.45 & 4.461 & 2.766 & 2.729 & 10.749 & 6.05 & 4.857 & 6.812 & 3.733 \\
\hline $12 / 1 / 2005$ & $13: 00$ & 11.216 & 11.104 & 8.143 & 7.102 & 4.442 & 4.461 & 2.759 & 2.72 & 10.749 & 6.046 & 4.853 & 6.793 & 3.728 \\
\hline $12 / 1 / 2005$ & $14: 00$ & 11.211 & 11.095 & 8.138 & 7.069 & 4.438 & 4.459 & 2.75 & 2.712 & 10.749 & 6.043 & 4.849 & 6.791 & 3.719 \\
\hline $12 / 1 / 2005$ & $15: 00$ & 11.209 & 11.093 & 8.136 & 7.069 & 4.435 & 4.461 & 2.749 & 2.709 & 10.751 & 6.037 & 4.849 & 6.791 & 3.717 \\
\hline $12 / 1 / 2005$ & $16: 00$ & 11.206 & 11.09 & 8.136 & 7.067 & 4.436 & 4.459 & 2.746 & 2.708 & 10.751 & 6.039 & 4.848 & 6.791 & 3.713 \\
\hline $12 / 1 / 2005$ & $17: 00$ & 11.206 & 11.088 & 8.135 & 7.064 & 4.436 & 4.459 & 2.744 & 2.706 & 10.751 & 6.04 & 4.848 & 6.791 & 3.708 \\
\hline $12 / 1 / 2005$ & $18: 00$ & 11.199 & 11.077 & 8.129 & 7.058 & 4.43 & 4.459 & 2.734 & 2.699 & 10.751 & 6.031 & 4.842 & 6.789 & 3.699 \\
\hline $12 / 1 / 2005$ & $19: 00$ & 11.191 & 11.07 & 8.125 & 7.051 & 4.425 & 4.457 & 2.73 & 2.693 & 10.749 & 6.028 & 4.84 & 6.785 & 3.695 \\
\hline $12 / 1 / 2005$ & $20: 00$ & 11.194 & 11.072 & 8.123 & 7.053 & 4.429 & 4.454 & 2.731 & 2.693 & 10.749 & 6.036 & 4.846 & 6.787 & 3.699 \\
\hline $12 / 1 / 2005$ & $21: 00$ & 11.191 & 11.07 & 8.123 & 7.051 & 4.43 & 4.454 & 2.73 & 2.693 & 10.751 & 6.036 & 4.846 & 6.787 & 3.699 \\
\hline $12 / 1 / 2005$ & $22: 00$ & 11.194 & 11.073 & 8.127 & 7.06 & 4.436 & 4.457 & 2.739 & 2.701 & 10.753 & 6.04 & 4.851 & 6.789 & 3.705 \\
\hline $12 / 1 / 2005$ & $23: 00$ & 11.191 & 11.072 & 8.127 & 7.056 & 4.433 & 4.455 & 2.733 & 2.697 & 10.753 & 6.037 & 4.849 & 6.789 & 3.702 \\
\hline $12 / 2 / 2005$ & 0:00 & 11.193 & 11.073 & 8.127 & 7.06 & 4.436 & 4.455 & 2.737 & 2.699 & 10.753 & 6.043 & 4.852 & 6.789 & 3.708 \\
\hline $12 / 2 / 2005$ & $1: 00$ & 11.191 & 11.068 & 8.125 & 7.051 & 4.433 & 4.454 & 2.734 & 2.695 & 10.753 & 6.036 & 4.851 & 6.787 & 3.704 \\
\hline $12 / 2 / 2005$ & $2: 00$ & 11.183 & 11.057 & 8.121 & 7.042 & 4.429 & 4.452 & 2.726 & 2.689 & 10.753 & 6.027 & 4.846 & 6.784 & 3.699 \\
\hline $12 / 2 / 2005$ & $3: 00$ & 11.181 & 11.057 & 8.119 & 7.047 & 4.428 & 4.45 & 2.726 & 2.689 & 10.751 & 6.029 & 4.85 & 6.785 & 3.704 \\
\hline $12 / 2 / 2005$ & $4: 00$ & 11.186 & 11.065 & 8.122 & 7.051 & 4.435 & 4.453 & 2.733 & 2.695 & 10.755 & 6.037 & 4.854 & 6.789 & 3.71 \\
\hline $12 / 2 / 2005$ & $5: 00$ & 11.174 & 11.048 & 8.115 & 7.038 & 4.425 & 4.448 & 2.72 & 2.682 & 10.753 & 6.021 & 4.845 & 6.782 & 3.7 \\
\hline $12 / 2 / 2005$ & $6: 00$ & 11.163 & 11.037 & 8.109 & 7.029 & 4.417 & 4.446 & 2.71 & 2.672 & 10.745 & 6.018 & 4.841 & 6.774 & 3.695 \\
\hline $12 / 2 / 2005$ & $7: 00$ & 11.163 & 11.035 & 8.107 & 7.029 & 4.421 & 4.444 & 2.712 & 2.674 & 10.745 & 6.019 & 4.844 & 6.776 & 3.698 \\
\hline $12 / 2 / 2005$ & $8: 00$ & 11.161 & 11.032 & 8.105 & 7.027 & 4.419 & 4.441 & 2.712 & 2.674 & 10.748 & 6.017 & 4.841 & 6.776 & 3.7 \\
\hline $12 / 2 / 2005$ & $9: 00$ & 11.149 & 11.021 & 8.097 & 7.016 & 4.412 & 4.437 & 2.704 & 2.666 & 10.744 & 6.009 & 4.835 & 6.768 & 3.694 \\
\hline $12 / 2 / 2005$ & $10: 00$ & 11.141 & 11.012 & 8.092 & 7.009 & 4.407 & 4.433 & 2.696 & 2.659 & 10.741 & 6.008 & 4.831 & 6.766 & 3.693 \\
\hline $12 / 2 / 2005$ & $11: 00$ & 11.139 & 11.011 & 8.092 & 7.012 & 4.411 & 4.433 & 2.696 & 2.659 & 10.74 & 6.012 & 4.834 & 6.766 & 3.697 \\
\hline $12 / 2 / 2005$ & $12: 00$ & 11.131 & 10.997 & 8.084 & 6.994 & 4.4 & 4.424 & 2.682 & 2.645 & 10.734 & 6.001 & 4.824 & 6.757 & 3.688 \\
\hline $12 / 2 / 2005$ & $13: 00$ & 11.122 & 10.986 & 8.08 & 6.989 & 4.393 & 4.417 & 2.676 & 2.636 & 10.73 & 5.999 & 4.818 & 6.755 & 3.684 \\
\hline $12 / 2 / 2005$ & $14: 00$ & 11.109 & 10.97 & 8.07 & 6.972 & 4.381 & 4.41 & 2.66 & 2.624 & 10.727 & 5.99 & 4.81 & 6.747 & 3.675 \\
\hline $12 / 2 / 2005$ & $15: 00$ & 11.097 & 10.952 & 8.059 & 6.96 & 4.374 & 4.404 & 2.649 & 2.613 & 10.721 & 5.987 & 4.801 & 6.742 & 3.666 \\
\hline $12 / 2 / 2005$ & $16: 00$ & 11.087 & 10.942 & 8.053 & 6.954 & 4.371 & 4.397 & 2.644 & 2.607 & 10.717 & 5.985 & 4.8 & 6.738 & 3.666 \\
\hline $12 / 2 / 2005$ & $17: 00$ & 11.079 & 10.937 & 8.048 & 6.949 & 4.37 & 4.388 & 2.641 & 2.603 & 10.713 & 5.987 & 4.797 & 6.736 & 3.661 \\
\hline $12 / 2 / 2005$ & $18: 00$ & 11.075 & 10.935 & 8.047 & 6.954 & 4.372 & 4.386 & 2.64 & 2.603 & 10.711 & 5.985 & 4.797 & 6.734 & 3.662 \\
\hline $12 / 2 / 2005$ & $19: 00$ & 11.072 & 10.939 & 8.047 & 6.954 & 4.377 & 4.384 & 2.646 & 2.605 & 10.711 & 5.987 & 4.799 & 6.732 & 3.668 \\
\hline $12 / 2 / 2005$ & $20: 00$ & 11.07 & 10.937 & 8.049 & 6.956 & 4.38 & 4.382 & 2.643 & 2.607 & 10.711 & 5.985 & 4.797 & 6.732 & 3.666 \\
\hline $12 / 2 / 2005$ & $21: 00$ & 11.07 & 10.935 & 8.049 & 6.956 & 4.38 & 4.38 & 2.644 & 2.605 & 10.709 & 5.987 & 4.797 & 6.73 & 3.669 \\
\hline
\end{tabular}


TABLE S1.2 (Cont.)

Water Level (ft below top of casing) at Indicated Well

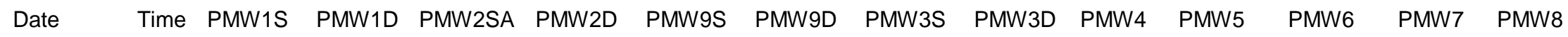

\begin{tabular}{|c|c|c|c|c|c|c|c|c|c|c|c|c|c|c|}
\hline $12 / 2 / 2005$ & $22: 00$ & 11.067 & 10.933 & 8.045 & 6.952 & 4.379 & 4.38 & 2.643 & 2.603 & 10.709 & 5.984 & 4.797 & 6.73 & 3.668 \\
\hline $12 / 2 / 2005$ & $23: 00$ & 11.065 & 10.928 & 8.043 & 6.95 & 4.377 & 4.375 & 2.64 & 2.603 & 10.708 & 5.98 & 4.793 & 6.728 & 3.668 \\
\hline $12 / 3 / 2005$ & $0: 00$ & 11.065 & 10.933 & 8.045 & 6.954 & 4.382 & 4.375 & 2.643 & 2.603 & 10.708 & 5.983 & 4.799 & 6.728 & 3.674 \\
\hline $12 / 3 / 2005$ & $1: 00$ & 11.065 & 10.935 & 8.043 & 6.954 & 4.382 & 4.375 & 2.645 & 2.605 & 10.708 & 5.986 & 4.803 & 6.73 & 3.677 \\
\hline $12 / 3 / 2005$ & $2: 00$ & 11.065 & 10.933 & 8.044 & 6.954 & 4.382 & 4.373 & 2.642 & 2.605 & 10.708 & 5.984 & 4.801 & 6.73 & 3.681 \\
\hline $12 / 3 / 2005$ & 3:00 & 11.062 & 10.93 & 8.043 & 6.95 & 4.382 & 4.373 & 2.638 & 2.601 & 10.706 & 5.981 & 4.801 & 6.728 & 3.681 \\
\hline $12 / 3 / 2005$ & $4: 00$ & 11.059 & 10.926 & 8.041 & 6.945 & 4.38 & 4.371 & 2.639 & 2.601 & 10.706 & 5.979 & 4.801 & 6.726 & 3.683 \\
\hline $12 / 3 / 2005$ & $5: 00$ & 11.057 & 10.922 & 8.037 & 6.941 & 4.375 & 4.368 & 2.635 & 2.594 & 10.702 & 5.975 & 4.797 & 6.724 & 3.681 \\
\hline $12 / 3 / 2005$ & $6: 00$ & 11.057 & 10.922 & 8.037 & 6.943 & 4.378 & 4.368 & 2.635 & 2.596 & 10.702 & 5.977 & 4.802 & 6.726 & 3.685 \\
\hline $12 / 3 / 2005$ & $7: 00$ & 11.057 & 10.927 & 8.041 & 6.95 & 4.384 & 4.368 & 2.64 & 2.601 & 10.704 & 5.979 & 4.808 & 6.726 & 3.691 \\
\hline $12 / 3 / 2005$ & $8: 00$ & 11.062 & 10.935 & 8.044 & 6.956 & 4.391 & 4.371 & 2.648 & 2.609 & 10.704 & 5.984 & 4.815 & 6.73 & 3.702 \\
\hline $12 / 3 / 2005$ & $9: 00$ & 11.064 & 10.937 & 8.043 & 6.956 & 4.391 & 4.371 & 2.648 & 2.607 & 10.704 & 5.983 & 4.821 & 6.734 & 3.704 \\
\hline $12 / 3 / 2005$ & $10: 00$ & 11.069 & 10.944 & 8.046 & 6.965 & 4.398 & 4.373 & 2.656 & 2.615 & 10.706 & 5.987 & 4.831 & 6.738 & 3.71 \\
\hline $12 / 3 / 2005$ & $11: 00$ & 11.074 & 10.952 & 8.052 & 6.97 & 4.403 & 4.375 & 2.661 & 2.619 & 10.708 & 5.987 & 4.835 & 6.74 & 3.709 \\
\hline $12 / 3 / 2005$ & $12: 00$ & 11.077 & 10.953 & 8.052 & 6.972 & 4.402 & 4.375 & 2.662 & 2.622 & 10.708 & 5.986 & 4.84 & 6.74 & 3.709 \\
\hline $12 / 3 / 2005$ & $13: 00$ & 11.074 & 10.946 & 8.05 & 6.963 & 4.395 & 4.373 & 2.654 & 2.615 & 10.706 & 5.978 & 4.835 & 6.74 & 3.702 \\
\hline $12 / 3 / 2005$ & $14: 00$ & 11.077 & 10.948 & 8.05 & 6.965 & 4.397 & 4.373 & 2.655 & 2.616 & 10.706 & 5.982 & 4.838 & 6.74 & 3.701 \\
\hline $12 / 3 / 2005$ & $15: 00$ & 11.077 & 10.952 & 8.05 & 6.967 & 4.399 & 4.375 & 2.657 & 2.617 & 10.704 & 5.984 & 4.839 & 6.743 & 3.701 \\
\hline $12 / 3 / 2005$ & $16: 00$ & 11.079 & 10.957 & 8.054 & 6.972 & 4.403 & 4.377 & 2.661 & 2.622 & 10.706 & 5.986 & 4.842 & 6.745 & 3.701 \\
\hline $12 / 3 / 2005$ & $17: 00$ & 11.089 & 10.97 & 8.06 & 6.985 & 4.411 & 4.382 & 2.672 & 2.632 & 10.708 & 5.992 & 4.847 & 6.749 & 3.705 \\
\hline $12 / 3 / 2005$ & $18: 00$ & 11.093 & 10.977 & 8.065 & 6.992 & 4.417 & 4.384 & 2.678 & 2.638 & 10.713 & 5.997 & 4.851 & 6.749 & 3.706 \\
\hline $12 / 3 / 2005$ & $19: 00$ & 11.103 & 10.99 & 8.073 & 7.003 & 4.422 & 4.391 & 2.687 & 2.651 & 10.719 & 6.001 & 4.854 & 6.755 & 3.709 \\
\hline $12 / 3 / 2005$ & $20: 00$ & 11.108 & 10.992 & 8.077 & 7.005 & 4.422 & 4.395 & 2.689 & 2.651 & 10.721 & 5.998 & 4.85 & 6.755 & 3.702 \\
\hline $12 / 3 / 2005$ & $21: 00$ & 11.123 & 11.01 & 8.086 & 7.021 & 4.434 & 4.402 & 2.703 & 2.666 & 10.725 & 6.007 & 4.857 & 6.763 & 3.708 \\
\hline $12 / 3 / 2005$ & $22: 00$ & 11.13 & 11.023 & 8.092 & 7.028 & 4.44 & 4.409 & 2.713 & 2.674 & 10.73 & 6.009 & 4.857 & 6.768 & 3.711 \\
\hline $12 / 3 / 2005$ & $23: 00$ & 11.14 & 11.034 & 8.1 & 7.036 & 4.441 & 4.415 & 2.72 & 2.681 & 10.734 & 6.014 & 4.863 & 6.772 & 3.713 \\
\hline $12 / 4 / 2005$ & $0: 00$ & 11.147 & 11.041 & 8.105 & 7.041 & 4.443 & 4.422 & 2.726 & 2.687 & 10.736 & 6.02 & 4.864 & 6.776 & 3.712 \\
\hline $12 / 4 / 2005$ & $1: 00$ & 11.155 & 11.045 & 8.109 & 7.045 & 4.443 & 4.426 & 2.729 & 2.691 & 10.738 & 6.019 & 4.862 & 6.776 & 3.71 \\
\hline $12 / 4 / 2005$ & $2: 00$ & 11.16 & 11.052 & 8.113 & 7.047 & 4.444 & 4.433 & 2.733 & 2.695 & 10.742 & 6.021 & 4.865 & 6.78 & 3.714 \\
\hline $12 / 4 / 2005$ & $3: 00$ & 11.164 & 11.057 & 8.117 & 7.052 & 4.447 & 4.435 & 2.736 & 2.698 & 10.742 & 6.028 & 4.868 & 6.784 & 3.715 \\
\hline $12 / 4 / 2005$ & $4: 00$ & 11.172 & 11.065 & 8.122 & 7.059 & 4.449 & 4.442 & 2.741 & 2.704 & 10.746 & 6.03 & 4.87 & 6.787 & 3.718 \\
\hline $12 / 4 / 2005$ & $5: 00$ & 11.179 & 11.07 & 8.126 & 7.067 & 4.454 & 4.446 & 2.748 & 2.71 & 10.749 & 6.036 & 4.874 & 6.791 & 3.723 \\
\hline $12 / 4 / 2005$ & $6: 00$ & 11.187 & 11.081 & 8.132 & 7.074 & 4.459 & 4.453 & 2.756 & 2.719 & 10.751 & 6.038 & 4.879 & 6.795 & 3.728 \\
\hline $12 / 4 / 2005$ & $7: 00$ & 11.191 & 11.088 & 8.137 & 7.078 & 4.463 & 4.457 & 2.759 & 2.723 & 10.755 & 6.047 & 4.882 & 6.799 & 3.732 \\
\hline
\end{tabular}


TABLE S1.2 (Cont.)

Water Level (ft below top of casing) at Indicated Well

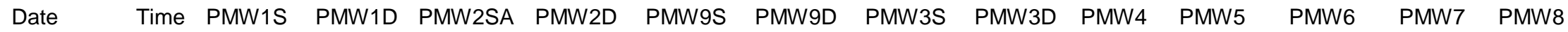

\begin{tabular}{|c|c|c|c|c|c|c|c|c|c|c|c|c|c|c|}
\hline $12 / 4 / 2005$ & 8:00 & 11.198 & 11.094 & 8.141 & 7.083 & 4.463 & 4.462 & 2.767 & 2.729 & 10.757 & 6.053 & 4.886 & 6.799 & 3.733 \\
\hline $12 / 4 / 2005$ & $9: 00$ & 11.209 & 11.103 & 8.147 & 7.092 & 4.47 & 4.468 & 2.772 & 2.735 & 10.759 & 6.059 & 4.891 & 6.805 & 3.738 \\
\hline $12 / 4 / 2005$ & $10: 00$ & 11.213 & 11.11 & 8.151 & 7.094 & 4.472 & 4.473 & 2.776 & 2.74 & 10.763 & 6.06 & 4.894 & 6.809 & 3.743 \\
\hline $12 / 4 / 2005$ & $11: 00$ & 11.218 & 11.117 & 8.154 & 7.098 & 4.472 & 4.479 & 2.78 & 2.742 & 10.763 & 6.076 & 4.899 & 6.812 & 3.743 \\
\hline $12 / 4 / 2005$ & $12: 00$ & 11.223 & 11.121 & 8.158 & 7.098 & 4.475 & 4.479 & 2.784 & 2.746 & 10.765 & 6.076 & 4.9 & 6.814 & 3.742 \\
\hline $12 / 4 / 2005$ & $13: 00$ & 11.228 & 11.123 & 8.16 & 7.101 & 4.476 & 4.484 & 2.784 & 2.744 & 10.767 & 6.079 & 4.902 & 6.814 & 3.738 \\
\hline $12 / 4 / 2005$ & $14: 00$ & 11.225 & 11.117 & 8.158 & 7.094 & 4.47 & 4.484 & 2.778 & 2.74 & 10.767 & 6.077 & 4.9 & 6.814 & 3.733 \\
\hline $12 / 4 / 2005$ & $15: 00$ & 11.228 & 11.117 & 8.158 & 7.096 & 4.468 & 4.486 & 2.779 & 2.74 & 10.768 & 6.073 & 4.901 & 6.814 & 3.73 \\
\hline $12 / 4 / 2005$ & $16: 00$ & 11.23 & 11.12 & 8.16 & 7.096 & 4.473 & 4.488 & 2.779 & 2.74 & 10.768 & 6.071 & 4.903 & 6.814 & 3.729 \\
\hline $12 / 4 / 2005$ & $17: 00$ & 11.233 & 11.125 & 8.162 & 7.105 & 4.476 & 4.491 & 2.784 & 2.746 & 10.77 & 6.08 & 4.903 & 6.816 & 3.729 \\
\hline $12 / 4 / 2005$ & $18: 00$ & 11.235 & 11.128 & 8.164 & 7.108 & 4.479 & 4.495 & 2.787 & 2.75 & 10.772 & 6.088 & 4.905 & 6.82 & 3.727 \\
\hline $12 / 4 / 2005$ & $19: 00$ & 11.238 & 11.128 & 8.166 & 7.108 & 4.476 & 4.497 & 2.788 & 2.75 & 10.774 & 6.093 & 4.903 & 6.82 & 3.724 \\
\hline $12 / 4 / 2005$ & $20: 00$ & 11.238 & 11.129 & 8.168 & 7.103 & 4.476 & 4.497 & 2.785 & 2.748 & 10.774 & 6.085 & 4.899 & 6.818 & 3.721 \\
\hline $12 / 4 / 2005$ & $21: 00$ & 11.243 & 11.134 & 8.169 & 7.108 & 4.479 & 4.499 & 2.788 & 2.752 & 10.776 & 6.096 & 4.905 & 6.824 & 3.724 \\
\hline $12 / 4 / 2005$ & $22: 00$ & 11.245 & 11.138 & 8.171 & 7.112 & 4.481 & 4.502 & 2.793 & 2.754 & 10.778 & 6.104 & 4.905 & 6.824 & 3.725 \\
\hline $12 / 4 / 2005$ & $23: 00$ & 11.247 & 11.138 & 8.171 & 7.112 & 4.481 & 4.504 & 2.791 & 2.756 & 10.78 & 6.112 & 4.906 & 6.826 & 3.726 \\
\hline $12 / 5 / 2005$ & 0:00 & 11.247 & 11.136 & 8.173 & 7.112 & 4.481 & 4.504 & 2.793 & 2.754 & 10.78 & 6.116 & 4.906 & 6.826 & 3.725 \\
\hline $12 / 5 / 2005$ & $1: 00$ & 11.247 & 11.136 & 8.173 & 7.11 & 4.482 & 4.506 & 2.791 & 2.754 & 10.78 & 6.113 & 4.906 & 6.826 & 3.724 \\
\hline $12 / 5 / 2005$ & $2: 00$ & 11.245 & 11.127 & 8.169 & 7.103 & 4.472 & 4.504 & 2.785 & 2.748 & 10.78 & 6.096 & 4.901 & 6.824 & 3.719 \\
\hline $12 / 5 / 2005$ & $3: 00$ & 11.242 & 11.125 & 8.169 & 7.101 & 4.474 & 4.504 & 2.783 & 2.746 & 10.78 & 6.093 & 4.901 & 6.822 & 3.719 \\
\hline $12 / 5 / 2005$ & $4: 00$ & 11.24 & 11.121 & 8.167 & 7.099 & 4.47 & 4.504 & 2.782 & 2.744 & 10.78 & 6.097 & 4.901 & 6.822 & 3.722 \\
\hline $12 / 5 / 2005$ & $5: 00$ & 11.232 & 11.114 & 8.161 & 7.092 & 4.466 & 4.502 & 2.776 & 2.735 & 10.778 & 6.082 & 4.899 & 6.818 & 3.718 \\
\hline $12 / 5 / 2005$ & $6: 00$ & 11.224 & 11.103 & 8.155 & 7.081 & 4.461 & 4.498 & 2.767 & 2.727 & 10.776 & 6.072 & 4.895 & 6.814 & 3.715 \\
\hline $12 / 5 / 2005$ & $7: 00$ & 11.22 & 11.101 & 8.154 & 7.079 & 4.458 & 4.498 & 2.762 & 2.725 & 10.774 & 6.069 & 4.897 & 6.814 & 3.719 \\
\hline $12 / 5 / 2005$ & 8:00 & 11.212 & 11.09 & 8.148 & 7.07 & 4.452 & 4.493 & 2.756 & 2.716 & 10.772 & 6.068 & 4.894 & 6.811 & 3.718 \\
\hline $12 / 5 / 2005$ & $9: 00$ & 11.205 & 11.078 & 8.14 & 7.059 & 4.445 & 4.487 & 2.747 & 2.708 & 10.768 & 6.049 & 4.888 & 6.807 & 3.714 \\
\hline $12 / 5 / 2005$ & $10: 00$ & 11.195 & 11.069 & 8.136 & 7.054 & 4.442 & 4.482 & 2.743 & 2.702 & 10.767 & 6.048 & 4.89 & 6.805 & 3.715 \\
\hline $12 / 5 / 2005$ & $11: 00$ & 11.19 & 11.061 & 8.13 & 7.046 & 4.442 & 4.478 & 2.739 & 2.698 & 10.763 & 6.042 & 4.889 & 6.799 & 3.716 \\
\hline $12 / 5 / 2005$ & $12: 00$ & 11.183 & 11.05 & 8.124 & 7.037 & 4.432 & 4.469 & 2.731 & 2.689 & 10.759 & 6.03 & 4.885 & 6.795 & 3.711 \\
\hline $12 / 5 / 2005$ & $13: 00$ & 11.168 & 11.028 & 8.113 & 7.019 & 4.419 & 4.46 & 2.71 & 2.67 & 10.751 & 6.017 & 4.872 & 6.784 & 3.703 \\
\hline $12 / 5 / 2005$ & $14: 00$ & 11.153 & 11.012 & 8.102 & 7.006 & 4.409 & 4.451 & 2.7 & 2.656 & 10.746 & 6.014 & 4.865 & 6.782 & 3.696 \\
\hline $12 / 5 / 2005$ & $15: 00$ & 11.141 & 10.992 & 8.09 & 6.986 & 4.399 & 4.44 & 2.682 & 2.641 & 10.742 & 6.01 & 4.855 & 6.772 & 3.685 \\
\hline $12 / 5 / 2005$ & $16: 00$ & 11.126 & 10.979 & 8.081 & 6.979 & 4.393 & 4.431 & 2.671 & 2.632 & 10.736 & 6.003 & 4.849 & 6.767 & 3.681 \\
\hline $12 / 5 / 2005$ & $17: 00$ & 11.114 & 10.966 & 8.071 & 6.968 & 4.384 & 4.422 & 2.664 & 2.62 & 10.73 & 6 & 4.842 & 6.759 & 3.677 \\
\hline
\end{tabular}


TABLE S1.2 (Cont.)

Water Level (ft below top of casing) at Indicated Well

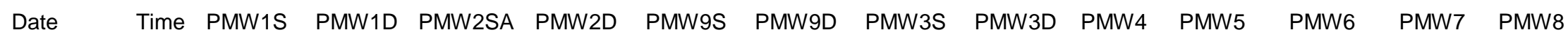

\begin{tabular}{|c|c|c|c|c|c|c|c|c|c|c|c|c|c|c|}
\hline $12 / 5 / 2005$ & $18: 00$ & 11.106 & 10.961 & 8.067 & 6.966 & 4.385 & 4.416 & 2.66 & 2.62 & 10.729 & 6 & 4.836 & 6.755 & 3.676 \\
\hline $12 / 5 / 2005$ & 19:00 & 11.099 & 10.954 & 8.061 & 6.961 & 4.385 & 4.409 & 2.654 & 2.616 & 10.725 & 5.998 & 4.828 & 6.751 & 3.672 \\
\hline $12 / 5 / 2005$ & $20: 00$ & 11.091 & 10.955 & 8.067 & 6.973 & 4.394 & 4.407 & 2.662 & 2.622 & 10.723 & 6.005 & 4.83 & 6.751 & 3.679 \\
\hline $12 / 5 / 2005$ & $21: 00$ & 11.094 & 10.961 & 8.067 & 6.977 & 4.396 & 4.405 & 2.666 & 2.624 & 10.725 & 6.004 & 4.829 & 6.751 & 3.682 \\
\hline $12 / 5 / 2005$ & $22: 00$ & 11.101 & 10.977 & 8.074 & 6.995 & 4.411 & 4.409 & 2.681 & 2.641 & 10.723 & 6.017 & 4.84 & 6.751 & 3.691 \\
\hline $12 / 5 / 2005$ & $23: 00$ & 11.121 & 11.007 & 8.087 & 7.022 & 4.431 & 4.411 & 2.707 & 2.666 & 10.732 & 6.026 & 4.849 & 6.757 & 3.708 \\
\hline $12 / 6 / 2005$ & $0: 00$ & 11.133 & 11.021 & 8.101 & 7.033 & 4.443 & 4.42 & 2.72 & 2.681 & 10.742 & 6.035 & 4.851 & 6.765 & 3.711 \\
\hline $12 / 6 / 2005$ & $1: 00$ & 11.15 & 11.047 & 8.113 & 7.055 & 4.453 & 4.427 & 2.736 & 2.698 & 10.746 & 6.049 & 4.863 & 6.776 & 3.721 \\
\hline $12 / 6 / 2005$ & $2: 00$ & 11.16 & 11.056 & 8.12 & 7.057 & 4.453 & 4.433 & 2.743 & 2.704 & 10.753 & 6.05 & 4.862 & 6.78 & 3.717 \\
\hline $12 / 6 / 2005$ & $3: 00$ & 11.17 & 11.061 & 8.124 & 7.062 & 4.454 & 4.44 & 2.746 & 2.706 & 10.755 & 6.056 & 4.862 & 6.784 & 3.716 \\
\hline $12 / 6 / 2005$ & $4: 00$ & 11.177 & 11.069 & 8.129 & 7.064 & 4.455 & 4.444 & 2.746 & 2.708 & 10.757 & 6.058 & 4.863 & 6.788 & 3.713 \\
\hline $12 / 6 / 2005$ & $5: 00$ & 11.184 & 11.076 & 8.132 & 7.07 & 4.459 & 4.451 & 2.756 & 2.715 & 10.761 & 6.066 & 4.87 & 6.793 & 3.716 \\
\hline $12 / 6 / 2005$ & $6: 00$ & 11.194 & 11.089 & 8.141 & 7.084 & 4.469 & 4.46 & 2.765 & 2.725 & 10.765 & 6.081 & 4.876 & 6.799 & 3.723 \\
\hline $12 / 6 / 2005$ & $7: 00$ & 11.201 & 11.098 & 8.149 & 7.09 & 4.474 & 4.464 & 2.774 & 2.734 & 10.768 & 6.09 & 4.881 & 6.805 & 3.726 \\
\hline $12 / 6 / 2005$ & $8: 00$ & 11.214 & 11.109 & 8.158 & 7.099 & 4.478 & 4.473 & 2.782 & 2.742 & 10.774 & 6.094 & 4.885 & 6.809 & 3.73 \\
\hline $12 / 6 / 2005$ & $9: 00$ & 11.221 & 11.12 & 8.164 & 7.108 & 4.486 & 4.48 & 2.79 & 2.75 & 10.778 & 6.113 & 4.896 & 6.814 & 3.733 \\
\hline $12 / 6 / 2005$ & $10: 00$ & 11.233 & 11.132 & 8.173 & 7.121 & 4.491 & 4.489 & 2.801 & 2.763 & 10.782 & 6.119 & 4.901 & 6.82 & 3.741 \\
\hline $12 / 6 / 2005$ & $11: 00$ & 11.241 & 11.143 & 8.179 & 7.126 & 4.496 & 4.493 & 2.809 & 2.767 & 10.784 & 6.136 & 4.909 & 6.826 & 3.744 \\
\hline $12 / 6 / 2005$ & $12: 00$ & 11.251 & 11.149 & 8.185 & 7.13 & 4.495 & 4.5 & 2.813 & 2.771 & 10.787 & 6.078 & 4.91 & 6.828 & 3.748 \\
\hline $12 / 6 / 2005$ & $13: 00$ & 11.253 & 11.151 & 8.185 & 7.126 & 4.491 & 4.502 & 2.809 & 2.767 & 10.787 & 6.076 & 4.909 & 6.828 & 3.742 \\
\hline $12 / 6 / 2005$ & $14: 00$ & 11.255 & 11.151 & 8.187 & 7.124 & 4.488 & 4.506 & 2.808 & 2.767 & 10.787 & 6.068 & 4.909 & 6.826 & 3.738 \\
\hline $12 / 6 / 2005$ & $15: 00$ & 11.255 & 11.149 & 8.187 & 7.121 & 4.489 & 4.509 & 2.806 & 2.765 & 10.787 & 6.073 & 4.908 & 6.826 & 3.739 \\
\hline $12 / 6 / 2005$ & $16: 00$ & 11.258 & 11.151 & 8.185 & 7.121 & 4.489 & 4.511 & 2.805 & 2.763 & 10.789 & 6.071 & 4.909 & 6.83 & 3.737 \\
\hline $12 / 6 / 2005$ & $17: 00$ & 11.262 & 11.156 & 8.189 & 7.128 & 4.493 & 4.515 & 2.813 & 2.771 & 10.791 & 6.079 & 4.915 & 6.83 & 3.743 \\
\hline $12 / 6 / 2005$ & $18: 00$ & 11.265 & 11.156 & 8.192 & 7.13 & 4.496 & 4.518 & 2.813 & 2.774 & 10.793 & 6.08 & 4.913 & 6.832 & 3.74 \\
\hline $12 / 6 / 2005$ & $19: 00$ & 11.27 & 11.165 & 8.194 & 7.137 & 4.497 & 4.522 & 2.819 & 2.78 & 10.795 & 6.093 & 4.918 & 6.837 & 3.742 \\
\hline $12 / 6 / 2005$ & $20: 00$ & 11.275 & 11.171 & 8.2 & 7.146 & 4.509 & 4.529 & 2.827 & 2.788 & 10.799 & 6.108 & 4.925 & 6.841 & 3.75 \\
\hline $12 / 6 / 2005$ & $21: 00$ & 11.28 & 11.176 & 8.202 & 7.146 & 4.51 & 4.533 & 2.828 & 2.788 & 10.799 & 6.114 & 4.926 & 6.843 & 3.75 \\
\hline $12 / 6 / 2005$ & $22: 00$ & 11.285 & 11.182 & 8.206 & 7.15 & 4.511 & 4.536 & 2.833 & 2.792 & 10.801 & 6.119 & 4.929 & 6.845 & 3.751 \\
\hline $12 / 6 / 2005$ & $23: 00$ & 11.289 & 11.187 & 8.211 & 7.155 & 4.517 & 4.54 & 2.839 & 2.799 & 10.805 & 6.136 & 4.934 & 6.849 & 3.756 \\
\hline $12 / 7 / 2005$ & $0: 00$ & 11.294 & 11.194 & 8.213 & 7.162 & 4.518 & 4.544 & 2.843 & 2.803 & 10.806 & 6.136 & 4.938 & 6.852 & 3.76 \\
\hline $12 / 7 / 2005$ & $1: 00$ & 11.297 & 11.194 & 8.219 & 7.159 & 4.513 & 4.547 & 2.839 & 2.799 & 10.808 & 6.144 & 4.937 & 6.851 & 3.757 \\
\hline $12 / 7 / 2005$ & $2: 00$ & 11.299 & 11.193 & 8.219 & 7.155 & 4.513 & 4.549 & 2.841 & 2.801 & 10.808 & 6.145 & 4.936 & 6.852 & 3.757 \\
\hline $12 / 7 / 2005$ & $3: 00$ & 11.301 & 11.194 & 8.22 & 7.157 & 4.513 & 4.551 & 2.84 & 2.801 & 10.81 & 6.139 & 4.94 & 6.854 & 3.757 \\
\hline
\end{tabular}


TABLE S1.2 (Cont.)

Water Level (ft below top of casing) at Indicated Well

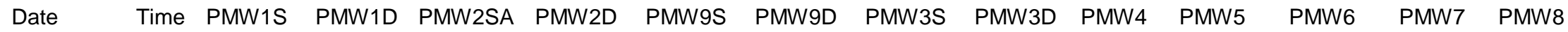

\begin{tabular}{|c|c|c|c|c|c|c|c|c|c|c|c|c|c|c|}
\hline $12 / 7 / 2005$ & $4: 00$ & 11.304 & 11.197 & 8.222 & 7.162 & 4.516 & 4.551 & 2.845 & 2.803 & 10.812 & 6.15 & 4.941 & 6.854 & 3.76 \\
\hline $12 / 7 / 2005$ & $5: 00$ & 11.306 & 11.197 & 8.222 & 7.159 & 4.517 & 4.553 & 2.841 & 2.801 & 10.812 & 6.14 & 4.943 & 6.854 & 3.758 \\
\hline $12 / 7 / 2005$ & $6: 00$ & 11.309 & 11.2 & 8.224 & 7.164 & 4.517 & 4.556 & 2.846 & 2.805 & 10.812 & 6.152 & 4.948 & 6.858 & 3.763 \\
\hline $12 / 7 / 2005$ & $7: 00$ & 11.311 & 11.209 & 8.23 & 7.173 & 4.526 & 4.558 & 2.856 & 2.816 & 10.818 & 6.099 & 4.953 & 6.862 & 3.771 \\
\hline $12 / 7 / 2005$ & 8:00 & 11.316 & 11.209 & 8.232 & 7.173 & 4.524 & 4.562 & 2.857 & 2.816 & 10.818 & 6.102 & 4.954 & 6.864 & 3.771 \\
\hline $12 / 7 / 2005$ & $9: 00$ & 11.318 & 11.209 & 8.234 & 7.173 & 4.523 & 4.562 & 2.856 & 2.816 & 10.818 & 6.102 & 4.956 & 6.864 & 3.77 \\
\hline $12 / 7 / 2005$ & $10: 00$ & 11.323 & 11.218 & 8.237 & 7.179 & 4.53 & 4.567 & 2.864 & 2.824 & 10.822 & 6.113 & 4.964 & 6.87 & 3.776 \\
\hline $12 / 7 / 2005$ & $11: 00$ & 11.326 & 11.218 & 8.24 & 7.177 & 4.529 & 4.569 & 2.861 & 2.822 & 10.824 & 6.113 & 4.965 & 6.87 & 3.775 \\
\hline $12 / 7 / 2005$ & $12: 00$ & 11.326 & 11.217 & 8.239 & 7.175 & 4.525 & 4.569 & 2.858 & 2.82 & 10.824 & 6.113 & 4.962 & 6.87 & 3.772 \\
\hline $12 / 7 / 2005$ & $13: 00$ & 11.323 & 11.211 & 8.237 & 7.17 & 4.523 & 4.567 & 2.854 & 2.813 & 10.824 & 6.103 & 4.963 & 6.87 & 3.766 \\
\hline $12 / 7 / 2005$ & $14: 00$ & 11.316 & 11.2 & 8.233 & 7.159 & 4.514 & 4.564 & 2.845 & 2.805 & 10.822 & 6.094 & 4.958 & 6.866 & 3.759 \\
\hline $12 / 7 / 2005$ & $15: 00$ & 11.311 & 11.193 & 8.23 & 7.155 & 4.511 & 4.56 & 2.839 & 2.799 & 10.824 & 6.094 & 4.957 & 6.864 & 3.756 \\
\hline $12 / 7 / 2005$ & $16: 00$ & 11.308 & 11.187 & 8.228 & 7.153 & 4.509 & 4.558 & 2.839 & 2.797 & 10.822 & 6.088 & 4.958 & 6.866 & 3.752 \\
\hline $12 / 7 / 2005$ & $17: 00$ & 11.308 & 11.191 & 8.23 & 7.155 & 4.515 & 4.558 & 2.84 & 2.799 & 10.824 & 6.092 & 4.964 & 6.872 & 3.754 \\
\hline $12 / 7 / 2005$ & $18: 00$ & 11.308 & 11.189 & 8.228 & 7.157 & 4.516 & 4.558 & 2.842 & 2.799 & 10.825 & 6.099 & 4.966 & 6.872 & 3.752 \\
\hline $12 / 7 / 2005$ & $19: 00$ & 11.306 & 11.187 & 8.228 & 7.155 & 4.514 & 4.558 & 2.839 & 2.799 & 10.824 & 6.094 & 4.967 & 6.872 & 3.752 \\
\hline $12 / 7 / 2005$ & $20: 00$ & 11.308 & 11.191 & 8.229 & 7.159 & 4.518 & 4.558 & 2.843 & 2.803 & 10.826 & 6.097 & 4.967 & 6.873 & 3.752 \\
\hline $12 / 7 / 2005$ & $21: 00$ & 11.306 & 11.187 & 8.23 & 7.153 & 4.511 & 4.556 & 2.841 & 2.799 & 10.825 & 6.096 & 4.967 & 6.873 & 3.747 \\
\hline $12 / 7 / 2005$ & $22: 00$ & 11.303 & 11.182 & 8.226 & 7.15 & 4.511 & 4.556 & 2.836 & 2.797 & 10.824 & 6.093 & 4.964 & 6.872 & 3.744 \\
\hline $12 / 7 / 2005$ & 23:00 & 11.298 & 11.176 & 8.224 & 7.146 & 4.507 & 4.553 & 2.833 & 2.792 & 10.822 & 6.088 & 4.96 & 6.868 & 3.741 \\
\hline $12 / 8 / 2005$ & $0: 00$ & 11.291 & 11.167 & 8.218 & 7.139 & 4.504 & 4.549 & 2.826 & 2.784 & 10.82 & 6.083 & 4.956 & 6.866 & 3.732 \\
\hline $12 / 8 / 2005$ & $1: 00$ & 11.286 & 11.16 & 8.212 & 7.133 & 4.499 & 4.545 & 2.819 & 2.778 & 10.818 & 6.075 & 4.953 & 6.862 & 3.73 \\
\hline $12 / 8 / 2005$ & $2: 00$ & 11.278 & 11.151 & 8.209 & 7.126 & 4.491 & 4.542 & 2.813 & 2.771 & 10.814 & 6.072 & 4.947 & 6.858 & 3.725 \\
\hline $12 / 8 / 2005$ & $3: 00$ & 11.271 & 11.144 & 8.204 & 7.119 & 4.488 & 4.538 & 2.805 & 2.765 & 10.812 & 6.07 & 4.943 & 6.854 & 3.722 \\
\hline $12 / 8 / 2005$ & $4: 00$ & 11.266 & 11.138 & 8.202 & 7.115 & 4.488 & 4.534 & 2.805 & 2.763 & 10.81 & 6.067 & 4.941 & 6.852 & 3.721 \\
\hline $12 / 8 / 2005$ & $5: 00$ & 11.259 & 11.127 & 8.194 & 7.107 & 4.479 & 4.529 & 2.796 & 2.753 & 10.808 & 6.061 & 4.93 & 6.845 & 3.712 \\
\hline $12 / 8 / 2005$ & $6: 00$ & 11.253 & 11.122 & 8.191 & 7.107 & 4.478 & 4.525 & 2.792 & 2.75 & 10.806 & 6.062 & 4.931 & 6.845 & 3.711 \\
\hline $12 / 8 / 2005$ & $7: 00$ & 11.246 & 11.116 & 8.187 & 7.097 & 4.475 & 4.522 & 2.789 & 2.746 & 10.803 & 6.061 & 4.929 & 6.843 & 3.71 \\
\hline $12 / 8 / 2005$ & $8: 00$ & 11.243 & 11.113 & 8.185 & 7.095 & 4.474 & 4.521 & 2.786 & 2.745 & 10.801 & 6.063 & 4.925 & 6.839 & 3.709 \\
\hline $12 / 8 / 2005$ & 9:00 & 11.238 & 11.109 & 8.181 & 7.095 & 4.473 & 4.516 & 2.785 & 2.742 & 10.799 & 6.058 & 4.922 & 6.837 & 3.71 \\
\hline $12 / 8 / 2005$ & $10: 00$ & 11.236 & 11.104 & 8.179 & 7.091 & 4.47 & 4.514 & 2.782 & 2.738 & 10.797 & 6.056 & 4.918 & 6.835 & 3.706 \\
\hline $12 / 8 / 2005$ & $11: 00$ & 11.234 & 11.104 & 8.179 & 7.091 & 4.472 & 4.509 & 2.783 & 2.74 & 10.797 & 6.058 & 4.918 & 6.837 & 3.709 \\
\hline $12 / 8 / 2005$ & $12: 00$ & 11.229 & 11.098 & 8.175 & 7.087 & 4.465 & 4.505 & 2.778 & 2.734 & 10.795 & 6.055 & 4.912 & 6.83 & 3.705 \\
\hline $12 / 8 / 2005$ & $13: 00$ & 11.221 & 11.091 & 8.17 & 7.08 & 4.462 & 4.503 & 2.77 & 2.727 & 10.793 & 6.052 & 4.908 & 6.826 & 3.702 \\
\hline
\end{tabular}


Water Level (ft below top of casing) at Indicated Well

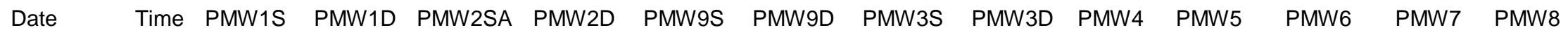

\begin{tabular}{|c|c|c|c|c|c|c|c|c|c|c|c|c|c|c|}
\hline $12 / 8 / 2005$ & $14: 00$ & 11.216 & 11.08 & 8.163 & 7.073 & 4.456 & 4.496 & 2.762 & 2.719 & 10.79 & 6.046 & 4.901 & 6.824 & 3.698 \\
\hline $12 / 8 / 2005$ & $15: 00$ & 11.209 & 11.073 & 8.16 & 7.067 & 4.449 & 4.494 & 2.758 & 2.713 & 10.786 & 6.046 & 4.895 & 6.82 & 3.692 \\
\hline $12 / 8 / 2005$ & $16: 00$ & 11.207 & 11.073 & 8.156 & 7.064 & 4.451 & 4.49 & 2.758 & 2.711 & 10.784 & 6.045 & 4.897 & 6.82 & 3.696 \\
\hline $12 / 8 / 2005$ & $17: 00$ & 11.204 & 11.073 & 8.156 & 7.069 & 4.453 & 4.487 & 2.758 & 2.717 & 10.784 & 6.048 & 4.896 & 6.818 & 3.698 \\
\hline $12 / 8 / 2005$ & $18: 00$ & 11.204 & 11.074 & 8.156 & 7.071 & 4.455 & 4.487 & 2.762 & 2.719 & 10.786 & 6.05 & 4.895 & 6.818 & 3.7 \\
\hline $12 / 8 / 2005$ & $19: 00$ & 11.206 & 11.078 & 8.156 & 7.071 & 4.458 & 4.487 & 2.76 & 2.719 & 10.786 & 6.05 & 4.893 & 6.818 & 3.702 \\
\hline $12 / 8 / 2005$ & $20: 00$ & 11.206 & 11.08 & 8.159 & 7.078 & 4.459 & 4.487 & 2.767 & 2.724 & 10.788 & 6.051 & 4.893 & 6.818 & 3.704 \\
\hline $12 / 8 / 2005$ & $21: 00$ & 11.209 & 11.083 & 8.161 & 7.08 & 4.461 & 4.49 & 2.768 & 2.725 & 10.787 & 6.051 & 4.893 & 6.82 & 3.703 \\
\hline $12 / 8 / 2005$ & $22: 00$ & 11.206 & 11.08 & 8.159 & 7.076 & 4.455 & 4.487 & 2.766 & 2.721 & 10.786 & 6.047 & 4.906 & 6.817 & 3.699 \\
\hline $12 / 8 / 2005$ & $23: 00$ & 11.209 & 11.082 & 8.16 & 7.078 & 4.459 & 4.487 & 2.767 & 2.724 & 10.786 & 6.049 & 4.918 & 6.818 & 3.702 \\
\hline $12 / 9 / 2005$ & $0: 00$ & 11.211 & 11.089 & 8.163 & 7.082 & 4.464 & 4.49 & 2.776 & 2.732 & 10.788 & 6.051 & 4.919 & 6.82 & 3.706 \\
\hline $12 / 9 / 2005$ & $1: 00$ & 11.216 & 11.093 & 8.165 & 7.084 & 4.463 & 4.49 & 2.778 & 2.732 & 10.788 & 6.052 & 4.92 & 6.82 & 3.707 \\
\hline $12 / 9 / 2005$ & $2: 00$ & 11.213 & 11.091 & 8.165 & 7.084 & 4.46 & 4.49 & 2.774 & 2.732 & 10.788 & 6.051 & 4.916 & 6.82 & 3.706 \\
\hline $12 / 9 / 2005$ & 3:00 & 11.215 & 11.089 & 8.165 & 7.082 & 4.461 & 4.49 & 2.775 & 2.73 & 10.786 & 6.051 & 4.916 & 6.82 & 3.709 \\
\hline $12 / 9 / 2005$ & $4: 00$ & 11.213 & 11.087 & 8.165 & 7.08 & 4.46 & 4.49 & 2.774 & 2.73 & 10.786 & 6.051 & 4.914 & 6.82 & 3.707 \\
\hline $12 / 9 / 2005$ & $5: 00$ & 11.211 & 11.08 & 8.159 & 7.076 & 4.453 & 4.487 & 2.767 & 2.721 & 10.784 & 6.047 & 4.911 & 6.818 & 3.705 \\
\hline $12 / 9 / 2005$ & $6: 00$ & 11.206 & 11.074 & 8.157 & 7.069 & 4.451 & 4.485 & 2.762 & 2.719 & 10.782 & 6.045 & 4.906 & 6.816 & 3.705 \\
\hline $12 / 9 / 2005$ & $7: 00$ & 11.208 & 11.078 & 8.157 & 7.073 & 4.454 & 4.485 & 2.764 & 2.721 & 10.784 & 6.047 & 4.909 & 6.815 & 3.707 \\
\hline $12 / 9 / 2005$ & $8: 00$ & 11.206 & 11.073 & 8.157 & 7.069 & 4.451 & 4.485 & 2.763 & 2.717 & 10.78 & 6.045 & 4.907 & 6.814 & 3.709 \\
\hline $12 / 9 / 2005$ & 9:00 & 11.203 & 11.074 & 8.155 & 7.069 & 4.453 & 4.483 & 2.762 & 2.719 & 10.78 & 6.046 & 4.904 & 6.817 & 3.711 \\
\hline $12 / 9 / 2005$ & $10: 00$ & 11.203 & 11.074 & 8.155 & 7.067 & 4.453 & 4.483 & 2.765 & 2.719 & 10.778 & 6.049 & 4.904 & 6.817 & 3.713 \\
\hline $12 / 9 / 2005$ & $11: 00$ & 11.203 & 11.076 & 8.155 & 7.071 & 4.454 & 4.481 & 2.764 & 2.719 & 10.78 & 6.048 & 4.895 & 6.818 & 3.716 \\
\hline $12 / 9 / 2005$ & $12: 00$ & 11.201 & 11.071 & 8.155 & 7.067 & 4.451 & 4.481 & 2.758 & 2.715 & 10.78 & 6.043 & 4.89 & 6.812 & 3.713 \\
\hline $12 / 9 / 2005$ & $13: 00$ & 11.198 & 11.065 & 8.151 & 7.062 & 4.444 & 4.476 & 2.753 & 2.709 & 10.78 & 6.037 & 4.886 & 6.811 & 3.71 \\
\hline $12 / 9 / 2005$ & $14: 00$ & 11.193 & 11.054 & 8.145 & 7.051 & 4.438 & 4.472 & 2.744 & 2.7 & 10.776 & 6.037 & 4.881 & 6.807 & 3.705 \\
\hline $12 / 9 / 2005$ & $15: 00$ & 11.186 & 11.045 & 8.14 & 7.038 & 4.431 & 4.461 & 2.736 & 2.69 & 10.771 & 6.032 & 4.874 & 6.803 & 3.698 \\
\hline $12 / 9 / 2005$ & $16: 00$ & 11.181 & 11.034 & 8.133 & 7.033 & 4.423 & 4.456 & 2.73 & 2.684 & 10.767 & 6.03 & 4.868 & 6.796 & 3.694 \\
\hline $12 / 9 / 2005$ & $17: 00$ & 11.173 & 11.027 & 8.127 & 7.027 & 4.419 & 4.454 & 2.723 & 2.677 & 10.765 & 6.031 & 4.866 & 6.795 & 3.69 \\
\hline $12 / 9 / 2005$ & $18: 00$ & 11.168 & 11.02 & 8.122 & 7.022 & 4.414 & 4.45 & 2.717 & 2.673 & 10.761 & 6.022 & 4.859 & 6.788 & 3.686 \\
\hline $12 / 9 / 2005$ & $19: 00$ & 11.161 & 11.01 & 8.118 & 7.016 & 4.411 & 4.445 & 2.712 & 2.667 & 10.759 & 6.019 & 4.852 & 6.786 & 3.682 \\
\hline $12 / 9 / 2005$ & $20: 00$ & 11.156 & 11.001 & 8.112 & 7.005 & 4.405 & 4.441 & 2.702 & 2.656 & 10.754 & 6.019 & 4.848 & 6.784 & 3.675 \\
\hline $12 / 9 / 2005$ & $21: 00$ & 11.146 & 10.992 & 8.107 & 7 & 4.401 & 4.437 & 2.697 & 2.65 & 10.752 & 6.014 & 4.846 & 6.78 & 3.673 \\
\hline $12 / 9 / 2005$ & $22: 00$ & 11.143 & 10.985 & 8.099 & 6.991 & 4.398 & 4.43 & 2.691 & 2.644 & 10.748 & 6.011 & 4.842 & 6.775 & 3.668 \\
\hline $12 / 9 / 2005$ & $23: 00$ & 11.136 & 10.979 & 8.097 & 6.992 & 4.399 & 4.426 & 2.689 & 2.642 & 10.746 & 6.01 & 4.841 & 6.773 & 3.669 \\
\hline
\end{tabular}


Water Level (ft below top of casing) at Indicated Well

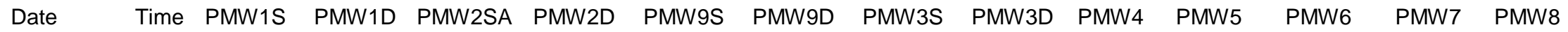

\begin{tabular}{|c|c|c|c|c|c|c|c|c|c|c|c|c|c|c|}
\hline $12 / 10 / 2005$ & 0:00 & 11.129 & 10.976 & 8.089 & 6.99 & 4.395 & 4.424 & 2.686 & 2.638 & 10.742 & 6.012 & 4.839 & 6.771 & 3.669 \\
\hline $12 / 10 / 2005$ & $1: 00$ & 11.121 & 10.967 & 8.083 & 6.978 & 4.389 & 4.417 & 2.679 & 2.631 & 10.742 & 6.005 & 4.83 & 6.765 & 3.662 \\
\hline $12 / 10 / 2005$ & $2: 00$ & 11.109 & 10.954 & 8.076 & 6.967 & 4.382 & 4.408 & 2.668 & 2.619 & 10.736 & 5.999 & 4.822 & 6.757 & 3.656 \\
\hline 12/10/2005 & 3:00 & 11.097 & 10.943 & 8.068 & 6.958 & 4.374 & 4.401 & 2.661 & 2.61 & 10.731 & 6.014 & 4.812 & 6.751 & 3.65 \\
\hline $12 / 10 / 2005$ & 4:00 & 11.092 & 10.936 & 8.066 & 6.954 & 4.373 & 4.397 & 2.656 & 2.608 & 10.727 & 6.013 & 4.808 & 6.75 & 3.654 \\
\hline 12/10/2005 & $5: 00$ & 11.081 & 10.93 & 8.063 & 6.947 & 4.367 & 4.39 & 2.651 & 2.602 & 10.725 & 6.009 & 4.8 & 6.742 & 3.653 \\
\hline $12 / 10 / 2005$ & 6:00 & 11.074 & 10.921 & 8.056 & 6.945 & 4.367 & 4.386 & 2.648 & 2.602 & 10.721 & 6.01 & 4.794 & 6.738 & 3.653 \\
\hline 12/10/2005 & $7: 00$ & 11.072 & 10.917 & 8.054 & 6.945 & 4.367 & 4.384 & 2.648 & 2.598 & 10.721 & 6.011 & 4.792 & 6.737 & 3.657 \\
\hline $12 / 10 / 2005$ & 8:00 & 11.069 & 10.921 & 8.054 & 6.945 & 4.369 & 4.381 & 2.65 & 2.6 & 10.721 & 6.01 & 4.792 & 6.734 & 3.659 \\
\hline $12 / 10 / 2005$ & $9: 00$ & 11.067 & 10.919 & 8.056 & 6.947 & 4.37 & 4.379 & 2.647 & 2.598 & 10.719 & 6.011 & 4.786 & 6.731 & 3.66 \\
\hline $12 / 10 / 2005$ & $10: 00$ & 11.062 & 10.921 & 8.058 & 6.954 & 4.375 & 4.379 & 2.652 & 2.602 & 10.717 & 6.012 & 4.786 & 6.732 & 3.661 \\
\hline $12 / 10 / 2005$ & $11: 00$ & 11.064 & 10.928 & 8.06 & 6.961 & 4.381 & 4.379 & 2.657 & 2.606 & 10.716 & 6.012 & 4.791 & 6.733 & 3.666 \\
\hline $12 / 10 / 2005$ & $12: 00$ & 11.072 & 10.934 & 8.062 & 6.965 & 4.381 & 4.377 & 2.662 & 2.612 & 10.719 & 6.012 & 4.795 & 6.736 & 3.668 \\
\hline $12 / 10 / 2005$ & $13: 00$ & 11.069 & 10.934 & 8.062 & 6.963 & 4.378 & 4.375 & 2.659 & 2.612 & 10.717 & 6.012 & 4.794 & 6.734 & 3.662 \\
\hline $12 / 10 / 2005$ & $14: 00$ & 11.072 & 10.936 & 8.062 & 6.965 & 4.376 & 4.377 & 2.66 & 2.612 & 10.716 & 6.01 & 4.79 & 6.73 & 3.646 \\
\hline 12/10/2005 & $15: 00$ & 11.077 & 10.943 & 8.063 & 6.967 & 4.373 & 4.375 & 2.66 & 2.612 & 10.714 & 6.004 & 4.791 & 6.729 & 3.608 \\
\hline $12 / 10 / 2005$ & $16: 00$ & 11.086 & 10.954 & 8.067 & 6.976 & 4.356 & 4.379 & 2.664 & 2.619 & 10.716 & 6.001 & 4.782 & 6.731 & 3.536 \\
\hline $12 / 10 / 2005$ & $17: 00$ & 11.074 & 10.965 & 8.069 & 6.985 & 4.314 & 4.386 & 2.671 & 2.625 & 10.716 & 5.992 & 4.773 & 6.729 & 3.449 \\
\hline $12 / 10 / 2005$ & 18:00 & 11.084 & 10.976 & 8.078 & 6.992 & 4.249 & 4.393 & 2.671 & 2.629 & 10.714 & 5.98 & 4.754 & 6.729 & 3.372 \\
\hline $12 / 10 / 2005$ & $19: 00$ & 11.093 & 10.987 & 8.078 & 6.998 & 4.175 & 4.395 & 2.675 & 2.629 & 10.712 & 5.963 & 4.73 & 6.725 & 3.327 \\
\hline 12/10/2005 & 20:00 & 11.103 & 10.989 & 8.077 & 6.994 & 4.102 & 4.397 & 2.673 & 2.627 & 10.708 & 5.952 & 4.704 & 6.725 & 3.298 \\
\hline $12 / 10 / 2005$ & 21:00 & 11.108 & 10.993 & 8.074 & 6.994 & 4.047 & 4.393 & 2.669 & 2.627 & 10.706 & 5.944 & 4.681 & 6.725 & 3.281 \\
\hline $12 / 10 / 2005$ & $22: 00$ & 11.11 & 10.994 & 8.072 & 6.992 & 4.004 & 4.388 & 2.668 & 2.623 & 10.702 & 5.935 & 4.663 & 6.721 & 3.273 \\
\hline $12 / 10 / 2005$ & 23:00 & 11.115 & 11 & 8.071 & 6.994 & 3.973 & 4.386 & 2.668 & 2.625 & 10.698 & 5.93 & 4.648 & 6.723 & 3.27 \\
\hline $12 / 11 / 2005$ & $0: 00$ & 11.118 & 11 & 8.07 & 6.992 & 3.947 & 4.379 & 2.667 & 2.623 & 10.697 & 5.924 & 4.634 & 6.721 & 3.264 \\
\hline $12 / 11 / 2005$ & 1:00 & 11.12 & 11 & 8.068 & 6.99 & 3.922 & 4.373 & 2.664 & 2.619 & 10.695 & 5.918 & 4.62 & 6.721 & 3.258 \\
\hline $12 / 11 / 2005$ & $2: 00$ & 11.118 & 10.996 & 8.064 & 6.983 & 3.9 & 4.368 & 2.658 & 2.614 & 10.689 & 5.911 & 4.608 & 6.719 & 3.255 \\
\hline $12 / 11 / 2005$ & 3:00 & 11.117 & 10.996 & 8.063 & 6.981 & 3.885 & 4.362 & 2.657 & 2.612 & 10.683 & 5.91 & 4.6 & 6.717 & 3.253 \\
\hline $12 / 11 / 2005$ & $4: 00$ & 11.12 & 10.996 & 8.062 & 6.983 & 3.874 & 4.355 & 2.657 & 2.614 & 10.681 & 5.905 & 4.598 & 6.719 & 3.253 \\
\hline $12 / 11 / 2005$ & 5:00 & 11.122 & 11.002 & 8.062 & 6.983 & 3.874 & 4.35 & 2.658 & 2.614 & 10.681 & 5.903 & 4.6 & 6.721 & 3.261 \\
\hline $12 / 11 / 2005$ & 6:00 & 11.115 & 10.991 & 8.056 & 6.977 & 3.872 & 4.342 & 2.65 & 2.606 & 10.678 & 5.899 & 4.598 & 6.721 & 3.268 \\
\hline 12/11/2005 & $7: 00$ & 11.117 & 10.993 & 8.056 & 6.977 & 3.885 & 4.337 & 2.65 & 2.606 & 10.676 & 5.899 & 4.605 & 6.721 & 3.283 \\
\hline $12 / 11 / 2005$ & $8: 00$ & 11.11 & 10.985 & 8.054 & 6.97 & 3.894 & 4.331 & 2.647 & 2.602 & 10.672 & 5.894 & 4.603 & 6.719 & 3.292 \\
\hline $12 / 11 / 2005$ & 9:00 & 11.108 & 10.982 & 8.05 & 6.968 & 3.908 & 4.327 & 2.645 & 2.6 & 10.67 & 5.9 & 4.611 & 6.721 & 3.308 \\
\hline
\end{tabular}


Water Level (ft below top of casing) at Indicated Well

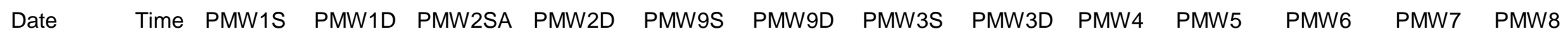

\begin{tabular}{|c|c|c|c|c|c|c|c|c|c|c|c|c|c|c|}
\hline $12 / 11 / 2005$ & $10: 00$ & 11.103 & 10.976 & 8.049 & 6.963 & 3.927 & 4.32 & 2.638 & 2.593 & 10.666 & 5.899 & 4.617 & 6.719 & 3.323 \\
\hline $12 / 11 / 2005$ & $11: 00$ & 11.095 & 10.969 & 8.047 & 6.959 & 3.942 & 4.315 & 2.637 & 2.591 & 10.664 & 5.903 & 4.623 & 6.717 & 3.338 \\
\hline $12 / 11 / 2005$ & $12: 00$ & 11.09 & 10.962 & 8.044 & 6.952 & 3.954 & 4.309 & 2.628 & 2.583 & 10.66 & 5.9 & 4.626 & 6.716 & 3.349 \\
\hline $12 / 11 / 2005$ & $13: 00$ & 11.08 & 10.947 & 8.036 & 6.939 & 3.959 & 4.304 & 2.618 & 2.572 & 10.66 & 5.9 & 4.626 & 6.713 & 3.35 \\
\hline $12 / 11 / 2005$ & $14: 00$ & 11.068 & 10.932 & 8.032 & 6.932 & 3.965 & 4.3 & 2.609 & 2.564 & 10.657 & 5.89 & 4.62 & 6.71 & 3.337 \\
\hline $12 / 11 / 2005$ & $15: 00$ & 11.051 & 10.929 & 8.027 & 6.926 & 3.941 & 4.3 & 2.602 & 2.558 & 10.653 & 5.88 & 4.577 & 6.702 & 3.282 \\
\hline $12 / 11 / 2005$ & $16: 00$ & 11.029 & 10.92 & 8.018 & 6.921 & 3.784 & 4.304 & 2.596 & 2.55 & 10.651 & 5.841 & 4.392 & 6.672 & 3.122 \\
\hline $12 / 11 / 2005$ & $17: 00$ & 11.031 & 10.931 & 8.01 & 6.921 & 3.608 & 4.304 & 2.59 & 2.543 & 10.645 & 5.817 & 4.302 & 6.647 & 3.017 \\
\hline $12 / 11 / 2005$ & $18: 00$ & 11.036 & 10.936 & 8 & 6.917 & 3.494 & 4.3 & 2.581 & 2.537 & 10.636 & 5.803 & 4.278 & 6.632 & 2.977 \\
\hline $12 / 11 / 2005$ & $19: 00$ & 11.041 & 10.941 & 7.996 & 6.917 & 3.456 & 4.287 & 2.581 & 2.537 & 10.628 & 5.804 & 4.293 & 6.63 & 2.978 \\
\hline $12 / 11 / 2005$ & $20: 00$ & 11.051 & 10.947 & 7.994 & 6.921 & 3.463 & 4.276 & 2.582 & 2.539 & 10.621 & 5.809 & 4.318 & 6.632 & 2.994 \\
\hline $12 / 11 / 2005$ & $21: 00$ & 11.058 & 10.952 & 7.994 & 6.921 & 3.489 & 4.265 & 2.582 & 2.539 & 10.613 & 5.829 & 4.342 & 6.635 & 3.015 \\
\hline $12 / 11 / 2005$ & $22: 00$ & 11.062 & 10.962 & 7.997 & 6.926 & 3.52 & 4.253 & 2.589 & 2.543 & 10.607 & 5.829 & 4.365 & 6.641 & 3.037 \\
\hline $12 / 11 / 2005$ & $23: 00$ & 11.068 & 10.962 & 7.997 & 6.926 & 3.547 & 4.245 & 2.587 & 2.543 & 10.603 & 5.833 & 4.381 & 6.643 & 3.056 \\
\hline $12 / 12 / 2005$ & $0: 00$ & 11.07 & 10.962 & 7.999 & 6.926 & 3.573 & 4.238 & 2.585 & 2.541 & 10.602 & 5.833 & 4.398 & 6.645 & 3.08 \\
\hline $12 / 12 / 2005$ & $1: 00$ & 11.07 & 10.962 & 8.001 & 6.928 & 3.601 & 4.234 & 2.59 & 2.543 & 10.596 & 5.835 & 4.414 & 6.649 & 3.105 \\
\hline $12 / 12 / 2005$ & $2: 00$ & 11.072 & 10.962 & 8.001 & 6.928 & 3.631 & 4.231 & 2.59 & 2.548 & 10.594 & 5.854 & 4.43 & 6.653 & 3.128 \\
\hline $12 / 12 / 2005$ & 3:00 & 11.077 & 10.972 & 8.005 & 6.937 & 3.663 & 4.229 & 2.598 & 2.554 & 10.596 & 5.858 & 4.453 & 6.658 & 3.154 \\
\hline $12 / 12 / 2005$ & $4: 00$ & 11.085 & 10.982 & 8.015 & 6.948 & 3.696 & 4.234 & 2.609 & 2.564 & 10.596 & 5.869 & 4.475 & 6.666 & 3.179 \\
\hline $12 / 12 / 2005$ & $5: 00$ & 11.092 & 10.991 & 8.02 & 6.955 & 3.723 & 4.236 & 2.618 & 2.575 & 10.598 & 5.885 & 4.492 & 6.672 & 3.205 \\
\hline $12 / 12 / 2005$ & $6: 00$ & 11.102 & 11.002 & 8.028 & 6.966 & 3.755 & 4.242 & 2.629 & 2.585 & 10.603 & 5.893 & 4.513 & 6.681 & 3.231 \\
\hline $12 / 12 / 2005$ & $7: 00$ & 11.111 & 11.011 & 8.038 & 6.975 & 3.784 & 4.247 & 2.639 & 2.594 & 10.605 & 5.859 & 4.532 & 6.687 & 3.257 \\
\hline $12 / 12 / 2005$ & $8: 00$ & 11.123 & 11.022 & 8.045 & 6.984 & 3.813 & 4.256 & 2.651 & 2.606 & 10.611 & 5.874 & 4.552 & 6.695 & 3.282 \\
\hline $12 / 12 / 2005$ & $9: 00$ & 11.131 & 11.031 & 8.053 & 6.992 & 3.841 & 4.265 & 2.66 & 2.617 & 10.617 & 5.9 & 4.573 & 6.704 & 3.306 \\
\hline $12 / 12 / 2005$ & $10: 00$ & 11.141 & 11.04 & 8.06 & 7.001 & 3.868 & 4.271 & 2.668 & 2.623 & 10.621 & 5.904 & 4.587 & 6.71 & 3.329 \\
\hline $12 / 12 / 2005$ & $11: 00$ & 11.15 & 11.051 & 8.067 & 7.01 & 3.895 & 4.28 & 2.678 & 2.634 & 10.626 & 5.918 & 4.607 & 6.716 & 3.353 \\
\hline $12 / 12 / 2005$ & $12: 00$ & 11.158 & 11.055 & 8.072 & 7.015 & 3.917 & 4.287 & 2.682 & 2.638 & 10.63 & 5.937 & 4.619 & 6.721 & 3.363 \\
\hline $12 / 12 / 2005$ & $13: 00$ & 11.163 & 11.056 & 8.075 & 7.012 & 3.929 & 4.293 & 2.681 & 2.638 & 10.636 & 5.917 & 4.624 & 6.723 & 3.339 \\
\hline $12 / 12 / 2005$ & $14: 00$ & 11.163 & 11.055 & 8.077 & 7.012 & 3.909 & 4.3 & 2.675 & 2.634 & 10.636 & 5.917 & 4.592 & 6.708 & 3.251 \\
\hline $12 / 12 / 2005$ & $15: 00$ & 11.16 & 11.051 & 8.071 & 7.001 & 3.832 & 4.307 & 2.665 & 2.619 & 10.632 & 5.887 & 4.499 & 6.675 & 3.131 \\
\hline $12 / 12 / 2005$ & $16: 00$ & 11.162 & 11.053 & 8.066 & 6.999 & 3.747 & 4.311 & 2.657 & 2.613 & 10.626 & 5.872 & 4.428 & 6.643 & 3.049 \\
\hline $12 / 12 / 2005$ & $17: 00$ & 11.165 & 11.055 & 8.064 & 6.997 & 3.687 & 4.314 & 2.651 & 2.609 & 10.621 & 5.792 & 4.396 & 6.624 & 3.011 \\
\hline $12 / 12 / 2005$ & $18: 00$ & 11.167 & 11.058 & 8.06 & 6.997 & 3.674 & 4.311 & 2.652 & 2.609 & 10.617 & 5.788 & 4.394 & 6.62 & 3.011 \\
\hline $12 / 12 / 2005$ & $19: 00$ & 11.167 & 11.06 & 8.06 & 6.997 & 3.692 & 4.309 & 2.651 & 2.606 & 10.611 & 5.796 & 4.397 & 6.626 & 3.03 \\
\hline
\end{tabular}


Water Level (ft below top of casing) at Indicated Well

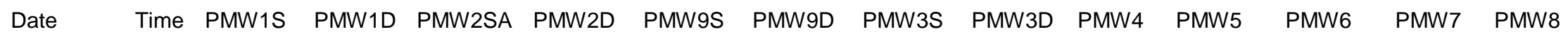

\begin{tabular}{|c|c|c|c|c|c|c|c|c|c|c|c|c|c|c|}
\hline $12 / 12 / 2005$ & $20: 00$ & 11.17 & 11.06 & 8.062 & 6.999 & 3.724 & 4.304 & 2.654 & 2.611 & 10.607 & 5.806 & 4.408 & 6.632 & 3.057 \\
\hline $12 / 12 / 2005$ & $21: 00$ & 11.17 & 11.06 & 8.064 & 6.999 & 3.759 & 4.302 & 2.653 & 2.613 & 10.607 & 5.822 & 4.426 & 6.641 & 3.093 \\
\hline $12 / 12 / 2005$ & $22: 00$ & 11.167 & 11.057 & 8.064 & 6.997 & 3.792 & 4.298 & 2.653 & 2.611 & 10.607 & 5.817 & 4.443 & 6.649 & 3.129 \\
\hline $12 / 12 / 2005$ & $23: 00$ & 11.162 & 11.051 & 8.064 & 6.995 & 3.826 & 4.296 & 2.655 & 2.609 & 10.605 & 5.824 & 4.462 & 6.656 & 3.162 \\
\hline $12 / 13 / 2005$ & $0: 00$ & 11.159 & 11.047 & 8.063 & 6.992 & 3.862 & 4.296 & 2.656 & 2.611 & 10.609 & 5.831 & 4.485 & 6.664 & 3.2 \\
\hline $12 / 13 / 2005$ & $1: 00$ & 11.152 & 11.037 & 8.061 & 6.986 & 3.887 & 4.294 & 2.654 & 2.609 & 10.611 & 5.837 & 4.502 & 6.668 & 3.229 \\
\hline $12 / 13 / 2005$ & $2: 00$ & 11.147 & 11.033 & 8.059 & 6.986 & 3.915 & 4.296 & 2.654 & 2.609 & 10.611 & 5.843 & 4.521 & 6.675 & 3.263 \\
\hline $12 / 13 / 2005$ & $3: 00$ & 11.145 & 11.029 & 8.058 & 6.984 & 3.937 & 4.296 & 2.654 & 2.609 & 10.613 & 5.854 & 4.538 & 6.679 & 3.292 \\
\hline $12 / 13 / 2005$ & $4: 00$ & 11.14 & 11.026 & 8.059 & 6.984 & 3.958 & 4.3 & 2.657 & 2.613 & 10.617 & 5.86 & 4.556 & 6.683 & 3.323 \\
\hline $12 / 13 / 2005$ & $5: 00$ & 11.132 & 11.017 & 8.059 & 6.979 & 3.972 & 4.298 & 2.653 & 2.606 & 10.617 & 5.865 & 4.571 & 6.685 & 3.343 \\
\hline $12 / 13 / 2005$ & $6: 00$ & 11.124 & 11.006 & 8.057 & 6.97 & 3.985 & 4.3 & 2.65 & 2.604 & 10.617 & 5.868 & 4.583 & 6.687 & 3.366 \\
\hline $12 / 13 / 2005$ & $7: 00$ & 11.12 & 11.002 & 8.056 & 6.97 & 4.001 & 4.3 & 2.649 & 2.602 & 10.619 & 5.876 & 4.598 & 6.693 & 3.386 \\
\hline $12 / 13 / 2005$ & $8: 00$ & 11.112 & 10.995 & 8.05 & 6.966 & 4.014 & 4.298 & 2.644 & 2.602 & 10.619 & 5.88 & 4.61 & 6.695 & 3.404 \\
\hline $12 / 13 / 2005$ & $9: 00$ & 11.105 & 10.987 & 8.05 & 6.959 & 4.022 & 4.3 & 2.642 & 2.596 & 10.619 & 5.882 & 4.619 & 6.696 & 3.419 \\
\hline $12 / 13 / 2005$ & $10: 00$ & 11.102 & 10.98 & 8.048 & 6.957 & 4.038 & 4.3 & 2.639 & 2.596 & 10.621 & 5.889 & 4.632 & 6.698 & 3.434 \\
\hline $12 / 13 / 2005$ & $11: 00$ & 11.095 & 10.975 & 8.042 & 6.953 & 4.045 & 4.298 & 2.638 & 2.592 & 10.621 & 5.893 & 4.644 & 6.698 & 3.445 \\
\hline $12 / 13 / 2005$ & $12: 00$ & 11.087 & 10.964 & 8.038 & 6.944 & 4.051 & 4.298 & 2.631 & 2.585 & 10.622 & 5.897 & 4.645 & 6.702 & 3.446 \\
\hline 12/13/2005 & $13: 00$ & 11.075 & 10.944 & 8.03 & 6.93 & 4.05 & 4.294 & 2.617 & 2.571 & 10.619 & 5.887 & 4.635 & 6.693 & 3.415 \\
\hline $12 / 13 / 2005$ & $14: 00$ & 11.065 & 10.936 & 8.023 & 6.924 & 4.047 & 4.289 & 2.605 & 2.56 & 10.619 & 5.886 & 4.625 & 6.679 & 3.347 \\
\hline $12 / 13 / 2005$ & $15: 00$ & 11.055 & 10.925 & 8.013 & 6.909 & 4.02 & 4.283 & 2.588 & 2.541 & 10.615 & 5.864 & 4.595 & 6.653 & 3.285 \\
\hline $12 / 13 / 2005$ & $16: 00$ & 11.047 & 10.896 & 8.006 & 6.888 & 3.989 & 4.283 & 2.58 & 2.527 & 10.607 & 5.844 & 4.565 & 6.609 & 3.248 \\
\hline $12 / 13 / 2005$ & $17: 00$ & 11.045 & 10.911 & 8.004 & 6.882 & 3.952 & 4.278 & 2.573 & 2.529 & 10.605 & 5.818 & 4.532 & 6.588 & 3.213 \\
\hline $12 / 13 / 2005$ & $18: 00$ & 11.038 & 10.918 & 7.993 & 6.902 & 3.891 & 4.278 & 2.552 & 2.525 & 10.607 & 5.746 & 4.454 & 6.576 & 3.097 \\
\hline $12 / 13 / 2005$ & 19:00 & 11.035 & 10.916 & 7.982 & 6.898 & 3.731 & 4.278 & 2.539 & 2.497 & 10.598 & 5.692 & 4.308 & 6.55 & 2.973 \\
\hline $12 / 13 / 2005$ & $20: 00$ & 11.023 & 10.924 & 7.967 & 6.899 & 3.582 & 4.276 & 2.516 & 2.501 & 10.602 & 5.623 & 4.172 & 6.529 & 2.879 \\
\hline $12 / 13 / 2005$ & $21: 00$ & 10.979 & 10.929 & 7.949 & 6.844 & 3.424 & 4.263 & 2.491 & 2.445 & 10.607 & 5.562 & 4.046 & 6.363 & 2.816 \\
\hline $12 / 13 / 2005$ & $22: 00$ & 10.917 & 10.887 & 7.939 & 6.842 & 3.368 & 4.241 & 2.487 & 2.445 & 10.539 & 5.567 & 4.049 & 6.369 & 2.817 \\
\hline $12 / 13 / 2005$ & $23: 00$ & 10.924 & 10.882 & 7.93 & 6.836 & 3.373 & 4.218 & 2.483 & 2.438 & 10.527 & 5.583 & 4.064 & 6.375 & 2.832 \\
\hline $12 / 14 / 2005$ & $0: 00$ & 10.948 & 10.88 & 7.926 & 6.833 & 3.402 & 4.199 & 2.485 & 2.438 & 10.52 & 5.595 & 4.087 & 6.386 & 2.856 \\
\hline $12 / 14 / 2005$ & $1: 00$ & 10.951 & 10.878 & 7.922 & 6.836 & 3.439 & 4.183 & 2.486 & 2.441 & 10.512 & 5.617 & 4.108 & 6.401 & 2.882 \\
\hline $12 / 14 / 2005$ & $2: 00$ & 10.946 & 10.878 & 7.92 & 6.844 & 3.447 & 4.174 & 2.479 & 2.432 & 10.514 & 5.605 & 4.094 & 6.407 & 2.842 \\
\hline $12 / 14 / 2005$ & $3: 00$ & 10.941 & 10.872 & 7.916 & 6.84 & 3.415 & 4.165 & 2.479 & 2.432 & 10.503 & 5.595 & 4.059 & 6.399 & 2.835 \\
\hline $12 / 14 / 2005$ & $4: 00$ & 10.943 & 10.882 & 7.914 & 6.838 & 3.419 & 4.159 & 2.485 & 2.438 & 10.497 & 5.601 & 4.072 & 6.409 & 2.857 \\
\hline $12 / 14 / 2005$ & $5: 00$ & 10.948 & 10.889 & 7.916 & 6.842 & 3.444 & 4.15 & 2.493 & 2.447 & 10.493 & 5.619 & 4.096 & 6.424 & 2.886 \\
\hline
\end{tabular}


Water Level (ft below top of casing) at Indicated Well

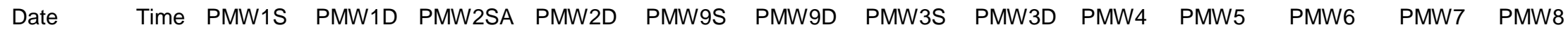

\begin{tabular}{|c|c|c|c|c|c|c|c|c|c|c|c|c|c|c|}
\hline $12 / 14 / 2005$ & $6: 00$ & 10.963 & 10.9 & 7.924 & 6.851 & 3.482 & 4.145 & 2.504 & 2.457 & 10.493 & 5.635 & 4.128 & 6.445 & 2.917 \\
\hline $12 / 14 / 2005$ & $7: 00$ & 10.972 & 10.909 & 7.929 & 6.86 & 3.518 & 4.145 & 2.514 & 2.468 & 10.493 & 5.652 & 4.157 & 6.468 & 2.948 \\
\hline $12 / 14 / 2005$ & $8: 00$ & 10.985 & 10.922 & 7.939 & 6.873 & 3.558 & 4.148 & 2.53 & 2.483 & 10.495 & 5.669 & 4.19 & 6.491 & 2.98 \\
\hline $12 / 14 / 2005$ & $9: 00$ & 10.992 & 10.927 & 7.946 & 6.88 & 3.589 & 4.148 & 2.535 & 2.491 & 10.497 & 5.685 & 4.216 & 6.506 & 3.007 \\
\hline $12 / 14 / 2005$ & $10: 00$ & 11.002 & 10.938 & 7.954 & 6.889 & 3.629 & 4.152 & 2.548 & 2.499 & 10.499 & 5.701 & 4.244 & 6.525 & 3.038 \\
\hline $12 / 14 / 2005$ & $11: 00$ & 11.009 & 10.942 & 7.963 & 6.893 & 3.658 & 4.159 & 2.556 & 2.51 & 10.503 & 5.713 & 4.266 & 6.536 & 3.059 \\
\hline $12 / 14 / 2005$ & $12: 00$ & 11.012 & 10.944 & 7.967 & 6.895 & 3.681 & 4.161 & 2.559 & 2.512 & 10.505 & 5.719 & 4.285 & 6.548 & 3.082 \\
\hline $12 / 14 / 2005$ & $13: 00$ & 11.017 & 10.944 & 7.966 & 6.9 & 3.714 & 4.168 & 2.563 & 2.514 & 10.508 & 5.73 & 4.302 & 6.553 & 3.104 \\
\hline $12 / 14 / 2005$ & $14: 00$ & 11.016 & 10.944 & 7.971 & 6.9 & 3.734 & 4.172 & 2.563 & 2.516 & 10.508 & 5.742 & 4.321 & 6.557 & 3.127 \\
\hline $12 / 14 / 2005$ & $15: 00$ & 11.014 & 10.947 & 7.971 & 6.902 & 3.763 & 4.179 & 2.567 & 2.52 & 10.514 & 5.747 & 4.339 & 6.565 & 3.148 \\
\hline $12 / 14 / 2005$ & $16: 00$ & 11.021 & 10.953 & 7.976 & 6.909 & 3.792 & 4.183 & 2.573 & 2.527 & 10.52 & 5.76 & 4.357 & 6.573 & 3.172 \\
\hline $12 / 14 / 2005$ & $17: 00$ & 11.021 & 10.953 & 7.98 & 6.911 & 3.819 & 4.192 & 2.58 & 2.533 & 10.525 & 5.77 & 4.374 & 6.582 & 3.193 \\
\hline $12 / 14 / 2005$ & $18: 00$ & 11.028 & 10.956 & 7.984 & 6.916 & 3.843 & 4.199 & 2.584 & 2.539 & 10.529 & 5.779 & 4.394 & 6.592 & 3.215 \\
\hline $12 / 14 / 2005$ & $19: 00$ & 11.03 & 10.956 & 7.988 & 6.92 & 3.864 & 4.206 & 2.588 & 2.541 & 10.535 & 5.784 & 4.408 & 6.599 & 3.235 \\
\hline $12 / 14 / 2005$ & $20: 00$ & 11.03 & 10.955 & 7.99 & 6.918 & 3.885 & 4.21 & 2.59 & 2.541 & 10.537 & 5.793 & 4.421 & 6.607 & 3.251 \\
\hline $12 / 14 / 2005$ & $21: 00$ & 11.03 & 10.953 & 7.992 & 6.918 & 3.903 & 4.217 & 2.591 & 2.543 & 10.541 & 5.801 & 4.436 & 6.613 & 3.267 \\
\hline $12 / 14 / 2005$ & $22: 00$ & 11.033 & 10.953 & 7.994 & 6.922 & 3.924 & 4.221 & 2.595 & 2.546 & 10.544 & 5.807 & 4.452 & 6.618 & 3.286 \\
\hline $12 / 14 / 2005$ & $23: 00$ & 11.035 & 10.958 & 7.999 & 6.929 & 3.945 & 4.228 & 2.599 & 2.554 & 10.548 & 5.815 & 4.47 & 6.626 & 3.306 \\
\hline $12 / 15 / 2005$ & $0: 00$ & 11.038 & 10.96 & 8.001 & 6.929 & 3.961 & 4.234 & 2.605 & 2.556 & 10.554 & 5.818 & 4.481 & 6.63 & 3.32 \\
\hline $12 / 15 / 2005$ & $1: 00$ & 11.04 & 10.958 & 8.003 & 6.929 & 3.977 & 4.239 & 2.606 & 2.558 & 10.556 & 5.825 & 4.495 & 6.635 & 3.337 \\
\hline $12 / 15 / 2005$ & $2: 00$ & 11.035 & 10.951 & 8.003 & 6.927 & 3.991 & 4.243 & 2.604 & 2.556 & 10.558 & 5.829 & 4.506 & 6.639 & 3.349 \\
\hline $12 / 15 / 2005$ & $3: 00$ & 11.035 & 10.951 & 8.005 & 6.927 & 4.004 & 4.245 & 2.606 & 2.558 & 10.562 & 5.84 & 4.52 & 6.645 & 3.365 \\
\hline $12 / 15 / 2005$ & $4: 00$ & 11.035 & 10.949 & 8.005 & 6.927 & 4.02 & 4.252 & 2.607 & 2.56 & 10.565 & 5.842 & 4.531 & 6.647 & 3.378 \\
\hline $12 / 15 / 2005$ & $5: 00$ & 11.032 & 10.938 & 8.003 & 6.922 & 4.029 & 4.254 & 2.605 & 2.556 & 10.567 & 5.844 & 4.539 & 6.653 & 3.39 \\
\hline $12 / 15 / 2005$ & $6: 00$ & 11.032 & 10.944 & 8.006 & 6.927 & 4.043 & 4.261 & 2.609 & 2.56 & 10.567 & 5.851 & 4.555 & 6.656 & 3.41 \\
\hline $12 / 15 / 2005$ & $7: 00$ & 11.037 & 10.948 & 8.007 & 6.931 & 4.058 & 4.263 & 2.615 & 2.567 & 10.573 & 5.862 & 4.568 & 6.658 & 3.429 \\
\hline $12 / 15 / 2005$ & $8: 00$ & 11.032 & 10.944 & 8.01 & 6.931 & 4.068 & 4.268 & 2.614 & 2.567 & 10.575 & 5.861 & 4.581 & 6.662 & 3.443 \\
\hline $12 / 15 / 2005$ & $9: 00$ & 11.035 & 10.948 & 8.01 & 6.933 & 4.081 & 4.272 & 2.621 & 2.573 & 10.577 & 5.871 & 4.594 & 6.668 & 3.459 \\
\hline $12 / 15 / 2005$ & $10: 00$ & 11.039 & 10.951 & 8.01 & 6.938 & 4.09 & 4.274 & 2.626 & 2.575 & 10.581 & 5.873 & 4.612 & 6.668 & 3.471 \\
\hline $12 / 15 / 2005$ & $11: 00$ & 11.042 & 10.958 & 8.017 & 6.947 & 4.103 & 4.281 & 2.631 & 2.583 & 10.583 & 5.881 & 4.631 & 6.677 & 3.487 \\
\hline $12 / 15 / 2005$ & $12: 00$ & 11.047 & 10.96 & 8.023 & 6.951 & 4.113 & 4.285 & 2.635 & 2.588 & 10.586 & 5.884 & 4.636 & 6.683 & 3.497 \\
\hline $12 / 15 / 2005$ & $13: 00$ & 11.049 & 10.96 & 8.024 & 6.947 & 4.12 & 4.292 & 2.636 & 2.59 & 10.59 & 5.888 & 4.649 & 6.687 & 3.507 \\
\hline $12 / 15 / 2005$ & $14: 00$ & 11.047 & 10.958 & 8.024 & 6.947 & 4.125 & 4.296 & 2.635 & 2.588 & 10.594 & 5.892 & 4.657 & 6.685 & 3.512 \\
\hline $12 / 15 / 2005$ & $15: 00$ & 11.051 & 10.96 & 8.025 & 6.949 & 4.135 & 4.299 & 2.639 & 2.592 & 10.596 & 5.892 & 4.661 & 6.687 & 3.517 \\
\hline
\end{tabular}


Water Level (ft below top of casing) at Indicated Well

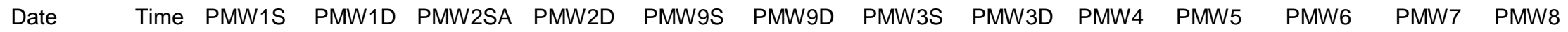

\begin{tabular}{|c|c|c|c|c|c|c|c|c|c|c|c|c|c|c|}
\hline $12 / 15 / 2005$ & $16: 00$ & 11.054 & 10.964 & 8.029 & 6.956 & 4.147 & 4.303 & 2.646 & 2.596 & 10.6 & 5.9 & 4.671 & 6.695 & 3.525 \\
\hline $12 / 15 / 2005$ & $17: 00$ & 11.058 & 10.971 & 8.034 & 6.962 & 4.155 & 4.305 & 2.648 & 2.601 & 10.605 & 5.902 & 4.681 & 6.7 & 3.532 \\
\hline $12 / 15 / 2005$ & $18: 00$ & 11.063 & 10.977 & 8.035 & 6.964 & 4.163 & 4.312 & 2.652 & 2.605 & 10.609 & 5.906 & 4.689 & 6.702 & 3.539 \\
\hline $12 / 15 / 2005$ & 19:00 & 11.068 & 10.98 & 8.041 & 6.969 & 4.172 & 4.316 & 2.656 & 2.611 & 10.613 & 5.916 & 4.695 & 6.706 & 3.547 \\
\hline $12 / 15 / 2005$ & $20: 00$ & 11.071 & 10.982 & 8.044 & 6.973 & 4.18 & 4.321 & 2.663 & 2.615 & 10.619 & 5.915 & 4.705 & 6.71 & 3.558 \\
\hline $12 / 15 / 2005$ & $21: 00$ & 11.073 & 10.982 & 8.046 & 6.971 & 4.185 & 4.325 & 2.664 & 2.615 & 10.619 & 5.919 & 4.714 & 6.714 & 3.566 \\
\hline $12 / 15 / 2005$ & $22: 00$ & 11.075 & 10.986 & 8.048 & 6.975 & 4.191 & 4.33 & 2.666 & 2.617 & 10.622 & 5.919 & 4.724 & 6.717 & 3.577 \\
\hline $12 / 15 / 2005$ & $23: 00$ & 11.078 & 10.984 & 8.048 & 6.978 & 4.197 & 4.334 & 2.668 & 2.619 & 10.624 & 5.924 & 4.733 & 6.718 & 3.582 \\
\hline $12 / 16 / 2005$ & $0: 00$ & 11.078 & 10.986 & 8.053 & 6.982 & 4.206 & 4.338 & 2.671 & 2.623 & 10.626 & 5.929 & 4.741 & 6.719 & 3.592 \\
\hline $12 / 16 / 2005$ & $1: 00$ & 11.08 & 10.99 & 8.055 & 6.982 & 4.212 & 4.343 & 2.676 & 2.627 & 10.628 & 5.928 & 4.752 & 6.723 & 3.601 \\
\hline $12 / 16 / 2005$ & $2: 00$ & 11.085 & 10.997 & 8.061 & 6.989 & 4.224 & 4.35 & 2.683 & 2.634 & 10.632 & 5.935 & 4.766 & 6.729 & 3.61 \\
\hline $12 / 16 / 2005$ & $3: 00$ & 11.09 & 11.002 & 8.063 & 6.995 & 4.231 & 4.356 & 2.691 & 2.64 & 10.634 & 5.938 & 4.774 & 6.733 & 3.619 \\
\hline $12 / 16 / 2005$ & $4: 00$ & 11.095 & 11.008 & 8.07 & 7 & 4.239 & 4.361 & 2.696 & 2.647 & 10.64 & 5.942 & 4.786 & 6.737 & 3.628 \\
\hline $12 / 16 / 2005$ & $5: 00$ & 11.099 & 11.008 & 8.072 & 7.002 & 4.246 & 4.365 & 2.699 & 2.651 & 10.641 & 5.946 & 4.793 & 6.74 & 3.634 \\
\hline $12 / 16 / 2005$ & $6: 00$ & 11.107 & 11.017 & 8.074 & 7.004 & 4.252 & 4.368 & 2.704 & 2.653 & 10.645 & 5.945 & 4.805 & 6.742 & 3.64 \\
\hline $12 / 16 / 2005$ & $7: 00$ & 11.112 & 11.021 & 8.078 & 7.013 & 4.259 & 4.374 & 2.709 & 2.661 & 10.647 & 5.952 & 4.815 & 6.742 & 3.65 \\
\hline $12 / 16 / 2005$ & $8: 00$ & 11.114 & 11.031 & 8.09 & 7.022 & 4.27 & 4.381 & 2.72 & 2.669 & 10.651 & 5.958 & 4.826 & 6.752 & 3.658 \\
\hline $12 / 16 / 2005$ & $9: 00$ & 11.124 & 11.037 & 8.09 & 7.027 & 4.281 & 4.387 & 2.723 & 2.676 & 10.655 & 5.964 & 4.835 & 6.757 & 3.667 \\
\hline $12 / 16 / 2005$ & $10: 00$ & 11.134 & 11.048 & 8.097 & 7.04 & 4.293 & 4.396 & 2.736 & 2.689 & 10.659 & 5.969 & 4.855 & 6.767 & 3.675 \\
\hline $12 / 16 / 2005$ & $11: 00$ & 11.146 & 11.06 & 8.102 & 7.047 & 4.302 & 4.403 & 2.743 & 2.695 & 10.662 & 5.978 & 4.868 & 6.777 & 3.688 \\
\hline $12 / 16 / 2005$ & $12: 00$ & 11.153 & 11.068 & 8.11 & 7.053 & 4.306 & 4.407 & 2.751 & 2.701 & 10.666 & 5.976 & 4.879 & 6.782 & 3.689 \\
\hline $12 / 16 / 2005$ & $13: 00$ & 11.155 & 11.072 & 8.112 & 7.056 & 4.309 & 4.412 & 2.754 & 2.703 & 10.672 & 5.99 & 4.893 & 6.784 & 3.689 \\
\hline $12 / 16 / 2005$ & $14: 00$ & 11.16 & 11.073 & 8.118 & 7.056 & 4.313 & 4.416 & 2.756 & 2.706 & 10.676 & 5.981 & 4.9 & 6.792 & 3.694 \\
\hline $12 / 16 / 2005$ & $15: 00$ & 11.165 & 11.077 & 8.121 & 7.06 & 4.322 & 4.423 & 2.754 & 2.705 & 10.678 & 5.992 & 4.909 & 6.796 & 3.696 \\
\hline $12 / 16 / 2005$ & $16: 00$ & 11.173 & 11.084 & 8.125 & 7.067 & 4.329 & 4.427 & 2.76 & 2.712 & 10.683 & 5.991 & 4.916 & 6.804 & 3.699 \\
\hline $12 / 16 / 2005$ & $17: 00$ & 11.177 & 11.09 & 8.129 & 7.069 & 4.334 & 4.432 & 2.767 & 2.716 & 10.685 & 6.001 & 4.921 & 6.803 & 3.701 \\
\hline $12 / 16 / 2005$ & $18: 00$ & 11.187 & 11.097 & 8.133 & 7.078 & 4.343 & 4.438 & 2.774 & 2.724 & 10.689 & 6.003 & 4.926 & 6.807 & 3.706 \\
\hline $12 / 16 / 2005$ & $19: 00$ & 11.194 & 11.106 & 8.138 & 7.082 & 4.349 & 4.445 & 2.781 & 2.735 & 10.693 & 6.014 & 4.934 & 6.811 & 3.71 \\
\hline $12 / 16 / 2005$ & $20: 00$ & 11.204 & 11.117 & 8.146 & 7.093 & 4.36 & 4.452 & 2.792 & 2.743 & 10.698 & 6.032 & 4.941 & 6.818 & 3.712 \\
\hline $12 / 16 / 2005$ & $21: 00$ & 11.211 & 11.124 & 8.152 & 7.1 & 4.365 & 4.46 & 2.797 & 2.75 & 10.702 & 6.044 & 4.95 & 6.823 & 3.717 \\
\hline $12 / 16 / 2005$ & $22: 00$ & 11.219 & 11.132 & 8.157 & 7.107 & 4.374 & 4.467 & 2.805 & 2.758 & 10.706 & 6.056 & 4.956 & 6.828 & 3.724 \\
\hline $12 / 16 / 2005$ & $23: 00$ & 11.229 & 11.139 & 8.163 & 7.113 & 4.379 & 4.474 & 2.813 & 2.764 & 10.71 & 6.047 & 4.966 & 6.832 & 3.73 \\
\hline $12 / 17 / 2005$ & $0: 00$ & 11.238 & 11.152 & 8.171 & 7.122 & 4.388 & 4.483 & 2.821 & 2.775 & 10.714 & 6.077 & 4.973 & 6.84 & 3.736 \\
\hline $12 / 17 / 2005$ & $1: 00$ & 11.246 & 11.156 & 8.177 & 7.124 & 4.39 & 4.487 & 2.824 & 2.777 & 10.719 & 6.071 & 4.98 & 6.842 & 3.739 \\
\hline
\end{tabular}


Water Level (ft below top of casing) at Indicated Well

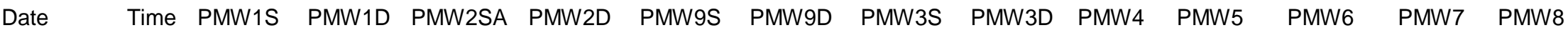

\begin{tabular}{|c|c|c|c|c|c|c|c|c|c|c|c|c|c|c|}
\hline $12 / 17 / 2005$ & $2: 00$ & 11.253 & 11.165 & 8.188 & 7.133 & 4.398 & 4.494 & 2.832 & 2.783 & 10.721 & 6.084 & 4.986 & 6.847 & 3.746 \\
\hline $12 / 17 / 2005$ & $3: 00$ & 11.26 & 11.17 & 8.186 & 7.135 & 4.403 & 4.5 & 2.838 & 2.787 & 10.725 & 6.069 & 4.99 & 6.849 & 3.748 \\
\hline $12 / 17 / 2005$ & $4: 00$ & 11.267 & 11.181 & 8.193 & 7.144 & 4.412 & 4.507 & 2.844 & 2.798 & 10.731 & 6.036 & 5 & 6.859 & 3.758 \\
\hline $12 / 17 / 2005$ & $5: 00$ & 11.275 & 11.188 & 8.198 & 7.153 & 4.419 & 4.514 & 2.851 & 2.804 & 10.735 & 6.05 & 5.004 & 6.859 & 3.76 \\
\hline $12 / 17 / 2005$ & $6: 00$ & 11.282 & 11.194 & 8.205 & 7.155 & 4.42 & 4.518 & 2.854 & 2.808 & 10.74 & 6.043 & 5.01 & 6.864 & 3.765 \\
\hline $12 / 17 / 2005$ & $7: 00$ & 11.29 & 11.203 & 8.211 & 7.164 & 4.426 & 4.525 & 2.861 & 2.815 & 10.744 & 6.066 & 5.017 & 6.87 & 3.77 \\
\hline $12 / 17 / 2005$ & $8: 00$ & 11.297 & 11.21 & 8.217 & 7.169 & 4.433 & 4.529 & 2.869 & 2.821 & 10.748 & 6.069 & 5.02 & 6.874 & 3.774 \\
\hline $12 / 17 / 2005$ & $9: 00$ & 11.304 & 11.214 & 8.22 & 7.173 & 4.439 & 4.536 & 2.873 & 2.825 & 10.75 & 6.081 & 5.024 & 6.874 & 3.772 \\
\hline $12 / 17 / 2005$ & $10: 00$ & 11.312 & 11.223 & 8.228 & 7.177 & 4.448 & 4.542 & 2.879 & 2.832 & 10.755 & 6.079 & 5.03 & 6.882 & 3.78 \\
\hline $12 / 17 / 2005$ & $11: 00$ & 11.321 & 11.23 & 8.232 & 7.189 & 4.454 & 4.549 & 2.885 & 2.838 & 10.759 & 6.097 & 5.036 & 6.886 & 3.789 \\
\hline $12 / 17 / 2005$ & $12: 00$ & 11.314 & 11.232 & 8.237 & 7.186 & 4.451 & 4.554 & 2.885 & 2.838 & 10.763 & 6.096 & 5.036 & 6.887 & 3.787 \\
\hline $12 / 17 / 2005$ & $13: 00$ & 11.316 & 11.23 & 8.237 & 7.184 & 4.452 & 4.554 & 2.879 & 2.834 & 10.765 & 6.098 & 5.035 & 6.887 & 3.783 \\
\hline $12 / 17 / 2005$ & $14: 00$ & 11.319 & 11.228 & 8.239 & 7.182 & 4.454 & 4.554 & 2.88 & 2.832 & 10.769 & 6.088 & 5.036 & 6.887 & 3.78 \\
\hline $12 / 17 / 2005$ & $15: 00$ & 11.319 & 11.223 & 8.239 & 7.18 & 4.455 & 4.556 & 2.875 & 2.829 & 10.769 & 6.105 & 5.037 & 6.889 & 3.777 \\
\hline $12 / 17 / 2005$ & $16: 00$ & 11.321 & 11.227 & 8.239 & 7.182 & 4.459 & 4.558 & 2.88 & 2.832 & 10.769 & 6.105 & 5.041 & 6.891 & 3.777 \\
\hline $12 / 17 / 2005$ & $17: 00$ & 11.326 & 11.232 & 8.243 & 7.189 & 4.466 & 4.56 & 2.885 & 2.838 & 10.773 & 6.123 & 5.043 & 6.893 & 3.781 \\
\hline $12 / 17 / 2005$ & $18: 00$ & 11.331 & 11.236 & 8.247 & 7.193 & 4.47 & 4.565 & 2.891 & 2.842 & 10.774 & 6.114 & 5.045 & 6.893 & 3.781 \\
\hline $12 / 17 / 2005$ & $19: 00$ & 11.335 & 11.241 & 8.25 & 7.197 & 4.478 & 4.572 & 2.893 & 2.848 & 10.778 & 6.12 & 5.047 & 6.897 & 3.78 \\
\hline $12 / 17 / 2005$ & $20: 00$ & 11.338 & 11.243 & 8.254 & 7.202 & 4.481 & 4.576 & 2.897 & 2.85 & 10.78 & 6.073 & 5.048 & 6.897 & 3.779 \\
\hline $12 / 17 / 2005$ & $21: 00$ & 11.343 & 11.249 & 8.258 & 7.204 & 4.484 & 4.58 & 2.901 & 2.853 & 10.782 & 6.081 & 5.049 & 6.899 & 3.782 \\
\hline $12 / 17 / 2005$ & $22: 00$ & 11.347 & 11.252 & 8.262 & 7.209 & 4.49 & 4.587 & 2.906 & 2.859 & 10.786 & 6.078 & 5.053 & 6.903 & 3.787 \\
\hline $12 / 17 / 2005$ & $23: 00$ & 11.35 & 11.259 & 8.265 & 7.213 & 4.495 & 4.591 & 2.911 & 2.863 & 10.79 & 6.088 & 5.054 & 6.904 & 3.789 \\
\hline $12 / 18 / 2005$ & $0: 00$ & 11.357 & 11.263 & 8.267 & 7.215 & 4.497 & 4.596 & 2.912 & 2.867 & 10.792 & 6.106 & 5.06 & 6.906 & 3.795 \\
\hline $12 / 18 / 2005$ & $1: 00$ & 11.362 & 11.267 & 8.272 & 7.222 & 4.5 & 4.6 & 2.919 & 2.871 & 10.794 & 6.102 & 5.061 & 6.91 & 3.798 \\
\hline $12 / 18 / 2005$ & $2: 00$ & 11.362 & 11.269 & 8.275 & 7.222 & 4.505 & 4.605 & 2.922 & 2.874 & 10.795 & 6.101 & 5.063 & 6.912 & 3.799 \\
\hline $12 / 18 / 2005$ & $3: 00$ & 11.367 & 11.272 & 8.279 & 7.227 & 4.509 & 4.607 & 2.925 & 2.878 & 10.797 & 6.116 & 5.069 & 6.916 & 3.803 \\
\hline $12 / 18 / 2005$ & $4: 00$ & 11.372 & 11.276 & 8.28 & 7.227 & 4.511 & 4.611 & 2.927 & 2.878 & 10.801 & 6.12 & 5.071 & 6.916 & 3.803 \\
\hline $12 / 18 / 2005$ & $5: 00$ & 11.372 & 11.276 & 8.286 & 7.229 & 4.513 & 4.614 & 2.928 & 2.88 & 10.801 & 6.132 & 5.073 & 6.918 & 3.803 \\
\hline $12 / 18 / 2005$ & $6: 00$ & 11.376 & 11.276 & 8.284 & 7.229 & 4.513 & 4.618 & 2.927 & 2.88 & 10.805 & 6.117 & 5.075 & 6.918 & 3.805 \\
\hline $12 / 18 / 2005$ & $7: 00$ & 11.377 & 11.28 & 8.288 & 7.233 & 4.52 & 4.62 & 2.931 & 2.884 & 10.807 & 6.13 & 5.08 & 6.922 & 3.811 \\
\hline $12 / 18 / 2005$ & $8: 00$ & 11.381 & 11.283 & 8.291 & 7.233 & 4.522 & 4.625 & 2.934 & 2.888 & 10.813 & 6.148 & 5.084 & 6.924 & 3.814 \\
\hline $12 / 18 / 2005$ & $9: 00$ & 11.384 & 11.289 & 8.294 & 7.242 & 4.528 & 4.627 & 2.942 & 2.895 & 10.813 & 6.15 & 5.087 & 6.929 & 3.819 \\
\hline $12 / 18 / 2005$ & $10: 00$ & 11.389 & 11.291 & 8.297 & 7.242 & 4.533 & 4.631 & 2.943 & 2.897 & 10.816 & 6.165 & 5.086 & 6.933 & 3.824 \\
\hline $12 / 18 / 2005$ & $11: 00$ & 11.388 & 11.294 & 8.301 & 7.244 & 4.531 & 4.631 & 2.943 & 2.897 & 10.82 & 6.165 & 5.09 & 6.935 & 3.831 \\
\hline
\end{tabular}


Water Level (ft below top of casing) at Indicated Well

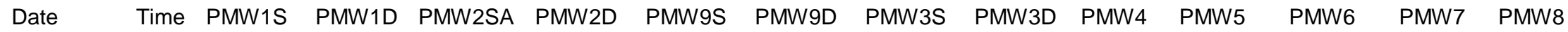

\begin{tabular}{|c|c|c|c|c|c|c|c|c|c|c|c|c|c|c|}
\hline $12 / 18 / 2005$ & $12: 00$ & 11.39 & 11.29 & 8.301 & 7.24 & 4.524 & 4.627 & 2.94 & 2.892 & 10.82 & 6.165 & 5.085 & 6.931 & 3.831 \\
\hline 12/18/2005 & $13: 00$ & 11.386 & 11.28 & 8.296 & 7.233 & 4.517 & 4.622 & 2.932 & 2.884 & 10.82 & 6.143 & 5.085 & 6.927 & 3.825 \\
\hline 12/18/2005 & $14: 00$ & 11.378 & 11.271 & 8.293 & 7.229 & 4.517 & 4.62 & 2.928 & 2.88 & 10.82 & 6.139 & 5.087 & 6.927 & 3.82 \\
\hline $12 / 18 / 2005$ & $15: 00$ & 11.376 & 11.265 & 8.288 & 7.222 & 4.519 & 4.616 & 2.924 & 2.874 & 10.82 & 6.143 & 5.09 & 6.927 & 3.816 \\
\hline $12 / 18 / 2005$ & $16: 00$ & 11.376 & 11.263 & 8.292 & 7.222 & 4.526 & 4.616 & 2.924 & 2.876 & 10.822 & 6.127 & 5.089 & 6.927 & 3.817 \\
\hline 12/18/2005 & $17: 00$ & 11.376 & 11.263 & 8.292 & 7.224 & 4.532 & 4.618 & 2.927 & 2.876 & 10.822 & 6.135 & 5.092 & 6.927 & 3.815 \\
\hline $12 / 18 / 2005$ & $18: 00$ & 11.376 & 11.263 & 8.292 & 7.222 & 4.536 & 4.62 & 2.927 & 2.878 & 10.824 & 6.153 & 5.093 & 6.929 & 3.811 \\
\hline 12/18/2005 & $19: 00$ & 11.376 & 11.262 & 8.293 & 7.224 & 4.538 & 4.625 & 2.926 & 2.878 & 10.826 & 6.147 & 5.091 & 6.927 & 3.81 \\
\hline $12 / 18 / 2005$ & $20: 00$ & 11.373 & 11.26 & 8.291 & 7.224 & 4.538 & 4.629 & 2.926 & 2.876 & 10.826 & 6.141 & 5.092 & 6.929 & 3.812 \\
\hline $12 / 18 / 2005$ & $21: 00$ & 11.376 & 11.262 & 8.293 & 7.224 & 4.54 & 4.631 & 2.928 & 2.878 & 10.826 & 6.148 & 5.094 & 6.931 & 3.817 \\
\hline $12 / 18 / 2005$ & $22: 00$ & 11.373 & 11.258 & 8.293 & 7.224 & 4.54 & 4.631 & 2.926 & 2.878 & 10.828 & 6.157 & 5.096 & 6.931 & 3.818 \\
\hline $12 / 18 / 2005$ & $23: 00$ & 11.373 & 11.256 & 8.291 & 7.224 & 4.54 & 4.634 & 2.927 & 2.878 & 10.828 & 6.137 & 5.095 & 6.931 & 3.823 \\
\hline $12 / 19 / 2005$ & $0: 00$ & 11.37 & 11.256 & 8.292 & 7.222 & 4.543 & 4.634 & 2.927 & 2.876 & 10.83 & 6.147 & 5.097 & 6.931 & 3.827 \\
\hline $12 / 19 / 2005$ & $1: 00$ & 11.37 & 11.252 & 8.29 & 7.22 & 4.542 & 4.634 & 2.924 & 2.876 & 10.83 & 6.134 & 5.098 & 6.931 & 3.828 \\
\hline $12 / 19 / 2005$ & $2: 00$ & 11.365 & 11.249 & 8.29 & 7.215 & 4.543 & 4.634 & 2.924 & 2.874 & 10.83 & 6.137 & 5.097 & 6.931 & 3.83 \\
\hline $12 / 19 / 2005$ & $3: 00$ & 11.363 & 11.245 & 8.287 & 7.215 & 4.543 & 4.631 & 2.923 & 2.874 & 10.83 & 6.142 & 5.099 & 6.933 & 3.833 \\
\hline 12/19/2005 & 4:00 & 11.362 & 11.245 & 8.289 & 7.218 & 4.546 & 4.634 & 2.925 & 2.874 & 10.833 & 6.132 & 5.102 & 6.933 & 3.836 \\
\hline 12/19/2005 & $5: 00$ & 11.36 & 11.24 & 8.286 & 7.215 & 4.544 & 4.631 & 2.921 & 2.869 & 10.83 & 6.138 & 5.103 & 6.929 & 3.837 \\
\hline $12 / 19 / 2005$ & $6: 00$ & 11.355 & 11.236 & 8.283 & 7.209 & 4.544 & 4.632 & 2.918 & 2.867 & 10.832 & 6.119 & 5.105 & 6.929 & 3.84 \\
\hline $12 / 19 / 2005$ & 7:00 & 11.35 & 11.232 & 8.282 & 7.209 & 4.544 & 4.629 & 2.917 & 2.865 & 10.832 & 6.128 & 5.105 & 6.927 & 3.842 \\
\hline $12 / 19 / 2005$ & $8: 00$ & 11.35 & 11.229 & 8.28 & 7.207 & 4.546 & 4.627 & 2.914 & 2.865 & 10.832 & 6.118 & 5.106 & 6.927 & 3.845 \\
\hline $12 / 19 / 2005$ & 9:00 & 11.347 & 11.229 & 8.281 & 7.204 & 4.548 & 4.627 & 2.916 & 2.865 & 10.832 & 6.135 & 5.108 & 6.927 & 3.848 \\
\hline $12 / 19 / 2005$ & $10: 00$ & 11.348 & 11.229 & 8.281 & 7.207 & 4.549 & 4.625 & 2.917 & 2.863 & 10.833 & 6.127 & 5.108 & 6.931 & 3.858 \\
\hline $12 / 19 / 2005$ & $11: 00$ & 11.347 & 11.229 & 8.281 & 7.209 & 4.546 & 4.623 & 2.917 & 2.865 & 10.833 & 6.123 & 5.107 & 6.933 & 3.864 \\
\hline $12 / 19 / 2005$ & $12: 00$ & 11.342 & 11.221 & 8.279 & 7.202 & 4.532 & 4.614 & 2.912 & 2.861 & 10.833 & 6.134 & 5.104 & 6.929 & 3.863 \\
\hline $12 / 19 / 2005$ & $13: 00$ & 11.332 & 11.203 & 8.27 & 7.184 & 4.518 & 4.599 & 2.896 & 2.844 & 10.828 & 6.103 & 5.097 & 6.921 & 3.853 \\
\hline $12 / 19 / 2005$ & $14: 00$ & 11.32 & 11.185 & 8.258 & 7.169 & 4.512 & 4.59 & 2.885 & 2.829 & 10.82 & 6.092 & 5.089 & 6.912 & 3.844 \\
\hline $12 / 19 / 2005$ & $15: 00$ & 11.31 & 11.17 & 8.25 & 7.156 & 4.51 & 4.581 & 2.871 & 2.819 & 10.814 & 6.091 & 5.086 & 6.908 & 3.835 \\
\hline $12 / 19 / 2005$ & $16: 00$ & 11.3 & 11.163 & 8.243 & 7.153 & 4.513 & 4.576 & 2.867 & 2.813 & 10.811 & 6.088 & 5.087 & 6.902 & 3.832 \\
\hline $12 / 19 / 2005$ & $17: 00$ & 11.295 & 11.156 & 8.239 & 7.149 & 4.514 & 4.57 & 2.862 & 2.808 & 10.809 & 6.088 & 5.083 & 6.899 & 3.825 \\
\hline $12 / 19 / 2005$ & $18: 00$ & 11.29 & 11.15 & 8.237 & 7.147 & 4.514 & 4.568 & 2.859 & 2.804 & 10.807 & 6.081 & 5.08 & 6.895 & 3.821 \\
\hline $12 / 19 / 2005$ & $19: 00$ & 11.283 & 11.147 & 8.233 & 7.142 & 4.521 & 4.568 & 2.857 & 2.802 & 10.803 & 6.08 & 5.078 & 6.893 & 3.814 \\
\hline $12 / 19 / 2005$ & $20: 00$ & 11.28 & 11.147 & 8.233 & 7.142 & 4.523 & 4.57 & 2.857 & 2.802 & 10.803 & 6.083 & 5.075 & 6.889 & 3.812 \\
\hline $12 / 19 / 2005$ & $21: 00$ & 11.282 & 11.143 & 8.232 & 7.142 & 4.524 & 4.568 & 2.854 & 2.8 & 10.803 & 6.082 & 5.072 & 6.887 & 3.805 \\
\hline
\end{tabular}


Water Level (ft below top of casing) at Indicated Well

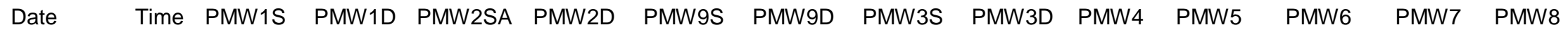

\begin{tabular}{|c|c|c|c|c|c|c|c|c|c|c|c|c|c|c|}
\hline $12 / 19 / 2005$ & $22: 00$ & 11.278 & 11.138 & 8.227 & 7.136 & 4.519 & 4.57 & 2.847 & 2.796 & 10.801 & 6.075 & 5.067 & 6.883 & 3.798 \\
\hline $12 / 19 / 2005$ & $23: 00$ & 11.273 & 11.136 & 8.225 & 7.136 & 4.521 & 4.572 & 2.844 & 2.792 & 10.799 & 6.076 & 5.065 & 6.883 & 3.796 \\
\hline $12 / 20 / 2005$ & $0: 00$ & 11.272 & 11.136 & 8.225 & 7.138 & 4.529 & 4.574 & 2.849 & 2.796 & 10.799 & 6.078 & 5.065 & 6.882 & 3.799 \\
\hline $12 / 20 / 2005$ & $1: 00$ & 11.27 & 11.132 & 8.223 & 7.131 & 4.523 & 4.574 & 2.846 & 2.792 & 10.797 & 6.073 & 5.061 & 6.88 & 3.798 \\
\hline $12 / 20 / 2005$ & $2: 00$ & 11.265 & 11.127 & 8.219 & 7.127 & 4.519 & 4.572 & 2.842 & 2.787 & 10.795 & 6.072 & 5.059 & 6.88 & 3.799 \\
\hline $12 / 20 / 2005$ & 3:00 & 11.264 & 11.13 & 8.22 & 7.132 & 4.521 & 4.572 & 2.846 & 2.79 & 10.795 & 6.076 & 5.061 & 6.88 & 3.804 \\
\hline $12 / 20 / 2005$ & 4:00 & 11.262 & 11.128 & 8.219 & 7.132 & 4.524 & 4.572 & 2.849 & 2.792 & 10.795 & 6.074 & 5.06 & 6.88 & 3.81 \\
\hline $12 / 20 / 2005$ & $5: 00$ & 11.262 & 11.127 & 8.219 & 7.132 & 4.521 & 4.572 & 2.845 & 2.792 & 10.795 & 6.078 & 5.06 & 6.88 & 3.818 \\
\hline $12 / 20 / 2005$ & $6: 00$ & 11.26 & 11.128 & 8.219 & 7.133 & 4.524 & 4.574 & 2.848 & 2.792 & 10.795 & 6.079 & 5.061 & 6.878 & 3.823 \\
\hline $12 / 20 / 2005$ & 7:00 & 11.262 & 11.127 & 8.219 & 7.131 & 4.527 & 4.572 & 2.85 & 2.794 & 10.795 & 6.075 & 5.066 & 6.88 & 3.83 \\
\hline $12 / 20 / 2005$ & $8: 00$ & 11.259 & 11.123 & 8.217 & 7.127 & 4.522 & 4.57 & 2.845 & 2.792 & 10.794 & 6.073 & 5.066 & 6.878 & 3.833 \\
\hline $12 / 20 / 2005$ & 9:00 & 11.257 & 11.125 & 8.217 & 7.127 & 4.523 & 4.568 & 2.845 & 2.792 & 10.794 & 6.076 & 5.07 & 6.878 & 3.837 \\
\hline $12 / 20 / 2005$ & $10: 00$ & 11.26 & 11.128 & 8.22 & 7.136 & 4.531 & 4.57 & 2.854 & 2.798 & 10.795 & 6.078 & 5.072 & 6.884 & 3.851 \\
\hline $12 / 20 / 2005$ & $11: 00$ & 11.264 & 11.136 & 8.226 & 7.143 & 4.531 & 4.568 & 2.858 & 2.804 & 10.799 & 6.089 & 5.079 & 6.887 & 3.861 \\
\hline $12 / 20 / 2005$ & $12: 00$ & 11.267 & 11.138 & 8.225 & 7.143 & 4.524 & 4.561 & 2.86 & 2.804 & 10.801 & 6.085 & 5.08 & 6.889 & 3.863 \\
\hline $12 / 20 / 2005$ & $13: 00$ & 11.262 & 11.13 & 8.222 & 7.136 & 4.512 & 4.552 & 2.852 & 2.796 & 10.799 & 6.081 & 5.078 & 6.885 & 3.859 \\
\hline $12 / 20 / 2005$ & $14: 00$ & 11.259 & 11.121 & 8.219 & 7.129 & 4.511 & 4.543 & 2.848 & 2.792 & 10.795 & 6.077 & 5.076 & 6.883 & 3.853 \\
\hline $12 / 20 / 2005$ & $15: 00$ & 11.254 & 11.118 & 8.215 & 7.123 & 4.511 & 4.539 & 2.842 & 2.785 & 10.794 & 6.078 & 5.073 & 6.88 & 3.847 \\
\hline $12 / 20 / 2005$ & $16: 00$ & 11.254 & 11.116 & 8.213 & 7.123 & 4.513 & 4.534 & 2.839 & 2.783 & 10.792 & 6.077 & 5.074 & 6.878 & 3.843 \\
\hline $12 / 20 / 2005$ & $17: 00$ & 11.255 & 11.118 & 8.214 & 7.125 & 4.521 & 4.537 & 2.843 & 2.787 & 10.792 & 6.077 & 5.078 & 6.878 & 3.84 \\
\hline $12 / 20 / 2005$ & $18: 00$ & 11.254 & 11.118 & 8.214 & 7.127 & 4.524 & 4.537 & 2.842 & 2.787 & 10.792 & 6.072 & 5.072 & 6.878 & 3.833 \\
\hline $12 / 20 / 2005$ & $19: 00$ & 11.256 & 11.118 & 8.214 & 7.125 & 4.525 & 4.537 & 2.843 & 2.785 & 10.792 & 6.071 & 5.07 & 6.878 & 3.825 \\
\hline $12 / 20 / 2005$ & $20: 00$ & 11.251 & 11.114 & 8.212 & 7.123 & 4.525 & 4.539 & 2.839 & 2.783 & 10.792 & 6.068 & 5.066 & 6.872 & 3.818 \\
\hline $12 / 20 / 2005$ & $21: 00$ & 11.256 & 11.119 & 8.214 & 7.125 & 4.529 & 4.543 & 2.843 & 2.787 & 10.79 & 6.065 & 5.066 & 6.874 & 3.813 \\
\hline $12 / 20 / 2005$ & $22: 00$ & 11.257 & 11.119 & 8.215 & 7.127 & 4.532 & 4.548 & 2.84 & 2.787 & 10.79 & 6.065 & 5.064 & 6.87 & 3.807 \\
\hline $12 / 20 / 2005$ & $23: 00$ & 11.256 & 11.119 & 8.214 & 7.125 & 4.532 & 4.554 & 2.837 & 2.783 & 10.788 & 6.062 & 5.059 & 6.87 & 3.803 \\
\hline $12 / 21 / 2005$ & $0: 00$ & 11.254 & 11.118 & 8.214 & 7.121 & 4.531 & 4.554 & 2.837 & 2.781 & 10.788 & 6.062 & 5.056 & 6.868 & 3.802 \\
\hline $12 / 21 / 2005$ & $1: 00$ & 11.251 & 11.112 & 8.21 & 7.116 & 4.528 & 4.556 & 2.831 & 2.777 & 10.784 & 6.06 & 5.05 & 6.866 & 3.8 \\
\hline $12 / 21 / 2005$ & $2: 00$ & 11.249 & 11.108 & 8.209 & 7.112 & 4.525 & 4.556 & 2.828 & 2.773 & 10.782 & 6.055 & 5.047 & 6.864 & 3.803 \\
\hline $12 / 21 / 2005$ & $3: 00$ & 11.246 & 11.105 & 8.207 & 7.109 & 4.524 & 4.557 & 2.828 & 2.773 & 10.782 & 6.058 & 5.046 & 6.863 & 3.808 \\
\hline $12 / 21 / 2005$ & $4: 00$ & 11.243 & 11.101 & 8.205 & 7.109 & 4.521 & 4.556 & 2.827 & 2.771 & 10.78 & 6.056 & 5.043 & 6.861 & 3.813 \\
\hline $12 / 21 / 2005$ & $5: 00$ & 11.241 & 11.101 & 8.204 & 7.109 & 4.524 & 4.557 & 2.826 & 2.773 & 10.78 & 6.058 & 5.048 & 6.862 & 3.824 \\
\hline $12 / 21 / 2005$ & $6: 00$ & 11.238 & 11.097 & 8.202 & 7.105 & 4.521 & 4.557 & 2.829 & 2.771 & 10.778 & 6.061 & 5.048 & 6.86 & 3.827 \\
\hline $12 / 21 / 2005$ & $7: 00$ & 11.236 & 11.097 & 8.204 & 7.107 & 4.522 & 4.555 & 2.825 & 2.769 & 10.778 & 6.063 & 5.05 & 6.862 & 3.835 \\
\hline
\end{tabular}


Water Level (ft below top of casing) at Indicated Well

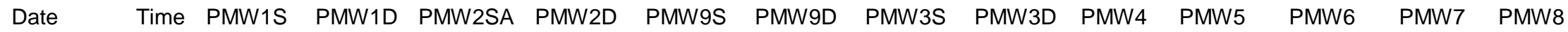

\begin{tabular}{|c|c|c|c|c|c|c|c|c|c|c|c|c|c|c|}
\hline $12 / 21 / 2005$ & $8: 00$ & 11.235 & 11.097 & 8.203 & 7.107 & 4.524 & 4.552 & 2.83 & 2.771 & 10.778 & 6.064 & 5.052 & 6.863 & 3.84 \\
\hline $12 / 21 / 2005$ & $9: 00$ & 11.238 & 11.099 & 8.204 & 7.112 & 4.53 & 4.552 & 2.831 & 2.777 & 10.778 & 6.064 & 5.058 & 6.862 & 3.849 \\
\hline $12 / 21 / 2005$ & $10: 00$ & 11.237 & 11.101 & 8.206 & 7.112 & 4.53 & 4.552 & 2.835 & 2.775 & 10.78 & 6.072 & 5.063 & 6.866 & 3.861 \\
\hline $12 / 21 / 2005$ & $11: 00$ & 11.243 & 11.11 & 8.212 & 7.121 & 4.531 & 4.55 & 2.84 & 2.783 & 10.784 & 6.076 & 5.07 & 6.87 & 3.871 \\
\hline $12 / 21 / 2005$ & $12: 00$ & 11.245 & 11.112 & 8.214 & 7.121 & 4.523 & 4.546 & 2.844 & 2.785 & 10.786 & 6.077 & 5.071 & 6.87 & 3.874 \\
\hline $12 / 21 / 2005$ & $13: 00$ & 11.245 & 11.109 & 8.213 & 7.118 & 4.515 & 4.535 & 2.84 & 2.781 & 10.786 & 6.071 & 5.069 & 6.868 & 3.87 \\
\hline $12 / 21 / 2005$ & $14: 00$ & 11.243 & 11.103 & 8.21 & 7.112 & 4.514 & 4.528 & 2.837 & 2.777 & 10.784 & 6.07 & 5.068 & 6.866 & 3.863 \\
\hline $12 / 21 / 2005$ & $15: 00$ & 11.241 & 11.1 & 8.208 & 7.107 & 4.515 & 4.521 & 2.83 & 2.773 & 10.782 & 6.072 & 5.067 & 6.864 & 3.859 \\
\hline $12 / 21 / 2005$ & $16: 00$ & 11.24 & 11.096 & 8.202 & 7.107 & 4.517 & 4.519 & 2.829 & 2.771 & 10.78 & 6.069 & 5.065 & 6.86 & 3.852 \\
\hline $12 / 21 / 2005$ & $17: 00$ & 11.238 & 11.097 & 8.201 & 7.107 & 4.519 & 4.519 & 2.828 & 2.771 & 10.78 & 6.066 & 5.066 & 6.859 & 3.845 \\
\hline $12 / 21 / 2005$ & $18: 00$ & 11.235 & 11.09 & 8.199 & 7.101 & 4.518 & 4.517 & 2.82 & 2.764 & 10.778 & 6.059 & 5.058 & 6.857 & 3.835 \\
\hline $12 / 21 / 2005$ & $19: 00$ & 11.238 & 11.096 & 8.2 & 7.107 & 4.524 & 4.519 & 2.825 & 2.769 & 10.778 & 6.067 & 5.058 & 6.859 & 3.833 \\
\hline $12 / 21 / 2005$ & $20: 00$ & 11.235 & 11.094 & 8.2 & 7.105 & 4.526 & 4.521 & 2.823 & 2.766 & 10.778 & 6.064 & 5.052 & 6.853 & 3.822 \\
\hline $12 / 21 / 2005$ & $21: 00$ & 11.237 & 11.096 & 8.199 & 7.107 & 4.525 & 4.524 & 2.823 & 2.769 & 10.778 & 6.065 & 5.046 & 6.853 & 3.816 \\
\hline $12 / 21 / 2005$ & $22: 00$ & 11.235 & 11.088 & 8.196 & 7.098 & 4.521 & 4.521 & 2.816 & 2.76 & 10.776 & 6.053 & 5.039 & 6.847 & 3.805 \\
\hline $12 / 21 / 2005$ & 23:00 & 11.23 & 11.09 & 8.196 & 7.101 & 4.52 & 4.524 & 2.816 & 2.758 & 10.774 & 6.059 & 5.037 & 6.851 & 3.798 \\
\hline $12 / 22 / 2005$ & $0: 00$ & 11.227 & 11.083 & 8.192 & 7.094 & 4.516 & 4.524 & 2.806 & 2.752 & 10.773 & 6.051 & 5.029 & 6.845 & 3.786 \\
\hline 12/22/2005 & $1: 00$ & 11.225 & 11.076 & 8.189 & 7.085 & 4.508 & 4.521 & 2.801 & 2.745 & 10.769 & 6.049 & 5.019 & 6.839 & 3.777 \\
\hline $12 / 22 / 2005$ & $2: 00$ & 11.222 & 11.072 & 8.187 & 7.081 & 4.505 & 4.522 & 2.796 & 2.741 & 10.769 & 6.044 & 5.012 & 6.839 & 3.768 \\
\hline $12 / 22 / 2005$ & $3: 00$ & 11.215 & 11.069 & 8.182 & 7.076 & 4.506 & 4.521 & 2.794 & 2.735 & 10.765 & 6.046 & 5.009 & 6.838 & 3.762 \\
\hline $12 / 22 / 2005$ & $4: 00$ & 11.212 & 11.061 & 8.178 & 7.07 & 4.498 & 4.519 & 2.785 & 2.729 & 10.763 & 6.044 & 5 & 6.832 & 3.758 \\
\hline $12 / 22 / 2005$ & $5: 00$ & 11.208 & 11.058 & 8.176 & 7.067 & 4.495 & 4.517 & 2.781 & 2.724 & 10.761 & 6.058 & 4.996 & 6.832 & 3.75 \\
\hline $12 / 22 / 2005$ & $6: 00$ & 11.203 & 11.048 & 8.172 & 7.056 & 4.486 & 4.515 & 2.773 & 2.716 & 10.757 & 6.052 & 4.985 & 6.826 & 3.744 \\
\hline $12 / 22 / 2005$ & $7: 00$ & 11.195 & 11.038 & 8.165 & 7.05 & 4.477 & 4.509 & 2.766 & 2.708 & 10.754 & 6.049 & 4.975 & 6.822 & 3.74 \\
\hline $12 / 22 / 2005$ & $8: 00$ & 11.19 & 11.036 & 8.162 & 7.052 & 4.48 & 4.506 & 2.765 & 2.71 & 10.754 & 6.047 & 4.977 & 6.818 & 3.742 \\
\hline $12 / 22 / 2005$ & $9: 00$ & 11.185 & 11.034 & 8.159 & 7.05 & 4.476 & 4.502 & 2.763 & 2.708 & 10.752 & 6.046 & 4.972 & 6.817 & 3.742 \\
\hline $12 / 22 / 2005$ & $10: 00$ & 11.183 & 11.032 & 8.158 & 7.048 & 4.476 & 4.5 & 2.761 & 2.706 & 10.75 & 6.051 & 4.968 & 6.817 & 3.747 \\
\hline $12 / 22 / 2005$ & $11: 00$ & 11.181 & 11.032 & 8.158 & 7.05 & 4.472 & 4.495 & 2.763 & 2.708 & 10.75 & 6.052 & 4.963 & 6.813 & 3.751 \\
\hline $12 / 22 / 2005$ & $12: 00$ & 11.18 & 11.028 & 8.155 & 7.045 & 4.462 & 4.486 & 2.756 & 2.703 & 10.748 & 6.043 & 4.957 & 6.809 & 3.746 \\
\hline $12 / 22 / 2005$ & $13: 00$ & 11.17 & 11.017 & 8.149 & 7.034 & 4.45 & 4.473 & 2.748 & 2.691 & 10.744 & 6.044 & 4.948 & 6.805 & 3.735 \\
\hline $12 / 22 / 2005$ & $14: 00$ & 11.163 & 11.003 & 8.14 & 7.019 & 4.44 & 4.464 & 2.735 & 2.678 & 10.738 & 6.034 & 4.94 & 6.794 & 3.724 \\
\hline $12 / 22 / 2005$ & $15: 00$ & 11.156 & 10.996 & 8.134 & 7.012 & 4.433 & 4.451 & 2.726 & 2.672 & 10.735 & 6.036 & 4.935 & 6.796 & 3.72 \\
\hline $12 / 22 / 2005$ & $16: 00$ & 11.148 & 10.988 & 8.129 & 7.008 & 4.435 & 4.447 & 2.724 & 2.666 & 10.733 & 6.034 & 4.929 & 6.791 & 3.716 \\
\hline $12 / 22 / 2005$ & $17: 00$ & 11.141 & 10.985 & 8.126 & 7.008 & 4.438 & 4.442 & 2.723 & 2.668 & 10.731 & 6.034 & 4.924 & 6.786 & 3.713 \\
\hline
\end{tabular}


Water Level (ft below top of casing) at Indicated Well

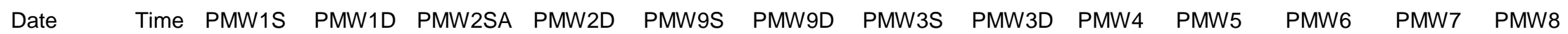

\begin{tabular}{|c|c|c|c|c|c|c|c|c|c|c|c|c|c|c|}
\hline $12 / 22 / 2005$ & $18: 00$ & 11.141 & 10.988 & 8.125 & 7.015 & 4.443 & 4.444 & 2.727 & 2.67 & 10.731 & 6.033 & 4.92 & 6.784 & 3.71 \\
\hline $12 / 22 / 2005$ & $19: 00$ & 11.138 & 10.985 & 8.123 & 7.01 & 4.439 & 4.442 & 2.724 & 2.666 & 10.727 & 6.029 & 4.913 & 6.778 & 3.705 \\
\hline $12 / 22 / 2005$ & $20: 00$ & 11.135 & 10.983 & 8.122 & 7.01 & 4.438 & 4.442 & 2.724 & 2.666 & 10.725 & 6.026 & 4.908 & 6.777 & 3.703 \\
\hline $12 / 22 / 2005$ & $21: 00$ & 11.133 & 10.983 & 8.121 & 7.01 & 4.439 & 4.442 & 2.724 & 2.666 & 10.725 & 6.027 & 4.903 & 6.775 & 3.702 \\
\hline $12 / 22 / 2005$ & $22: 00$ & 11.133 & 10.983 & 8.118 & 7.01 & 4.436 & 4.442 & 2.721 & 2.666 & 10.723 & 6.023 & 4.899 & 6.773 & 3.697 \\
\hline $12 / 22 / 2005$ & $23: 00$ & 11.131 & 10.977 & 8.117 & 7.003 & 4.432 & 4.438 & 2.716 & 2.661 & 10.723 & 6.022 & 4.89 & 6.769 & 3.689 \\
\hline $12 / 23 / 2005$ & 0:00 & 11.126 & 10.976 & 8.113 & 7.004 & 4.428 & 4.438 & 2.71 & 2.657 & 10.719 & 6.019 & 4.883 & 6.769 & 3.683 \\
\hline $12 / 23 / 2005$ & $1: 00$ & 11.126 & 10.977 & 8.115 & 7.003 & 4.429 & 4.436 & 2.716 & 2.661 & 10.719 & 6.02 & 4.877 & 6.765 & 3.683 \\
\hline $12 / 23 / 2005$ & $2: 00$ & 11.122 & 10.972 & 8.113 & 7.001 & 4.426 & 4.433 & 2.711 & 2.655 & 10.716 & 6.018 & 4.87 & 6.763 & 3.677 \\
\hline $12 / 23 / 2005$ & 3:00 & 11.125 & 10.977 & 8.112 & 7.008 & 4.428 & 4.436 & 2.716 & 2.659 & 10.716 & 6.021 & 4.871 & 6.765 & 3.679 \\
\hline $12 / 23 / 2005$ & $4: 00$ & 11.128 & 10.985 & 8.116 & 7.012 & 4.43 & 4.436 & 2.72 & 2.666 & 10.717 & 6.018 & 4.869 & 6.767 & 3.679 \\
\hline $12 / 23 / 2005$ & $5: 00$ & 11.13 & 10.988 & 8.118 & 7.012 & 4.432 & 4.438 & 2.722 & 2.668 & 10.717 & 6.017 & 4.867 & 6.765 & 3.678 \\
\hline $12 / 23 / 2005$ & $6: 00$ & 11.13 & 10.988 & 8.117 & 7.012 & 4.429 & 4.438 & 2.721 & 2.666 & 10.717 & 6.018 & 4.863 & 6.763 & 3.672 \\
\hline $12 / 23 / 2005$ & 7:00 & 11.132 & 10.992 & 8.117 & 7.015 & 4.427 & 4.438 & 2.722 & 2.668 & 10.717 & 6.018 & 4.861 & 6.765 & 3.67 \\
\hline $12 / 23 / 2005$ & $8: 00$ & 11.136 & 10.996 & 8.121 & 7.017 & 4.425 & 4.438 & 2.725 & 2.67 & 10.716 & 6.015 & 4.861 & 6.763 & 3.668 \\
\hline $12 / 23 / 2005$ & $9: 00$ & 11.141 & 11.001 & 8.121 & 7.021 & 4.428 & 4.44 & 2.728 & 2.674 & 10.716 & 6.017 & 4.86 & 6.765 & 3.67 \\
\hline $12 / 23 / 2005$ & $10: 00$ & 11.146 & 11.008 & 8.125 & 7.028 & 4.431 & 4.444 & 2.73 & 2.678 & 10.716 & 6.018 & 4.857 & 6.765 & 3.671 \\
\hline $12 / 23 / 2005$ & $11: 00$ & 11.149 & 11.014 & 8.128 & 7.03 & 4.431 & 4.444 & 2.736 & 2.682 & 10.717 & 6.019 & 4.859 & 6.769 & 3.671 \\
\hline $12 / 23 / 2005$ & $12: 00$ & 11.154 & 11.014 & 8.129 & 7.03 & 4.431 & 4.444 & 2.736 & 2.682 & 10.719 & 6.015 & 4.856 & 6.767 & 3.667 \\
\hline $12 / 23 / 2005$ & $13: 00$ & 11.147 & 11.001 & 8.127 & 7.019 & 4.418 & 4.442 & 2.725 & 2.672 & 10.714 & 6.012 & 4.85 & 6.764 & 3.655 \\
\hline $12 / 23 / 2005$ & $14: 00$ & 11.135 & 10.986 & 8.115 & 7.001 & 4.407 & 4.438 & 2.71 & 2.655 & 10.712 & 6.009 & 4.845 & 6.759 & 3.644 \\
\hline $12 / 23 / 2005$ & $15: 00$ & 11.132 & 10.985 & 8.11 & 7.004 & 4.409 & 4.436 & 2.709 & 2.655 & 10.71 & 6.01 & 4.841 & 6.759 & 3.644 \\
\hline $12 / 23 / 2005$ & $16: 00$ & 11.13 & 10.983 & 8.11 & 7.001 & 4.407 & 4.433 & 2.709 & 2.655 & 10.708 & 6.008 & 4.838 & 6.757 & 3.643 \\
\hline $12 / 23 / 2005$ & $17: 00$ & 11.13 & 10.986 & 8.109 & 7.008 & 4.408 & 4.433 & 2.711 & 2.657 & 10.71 & 6.011 & 4.836 & 6.756 & 3.642 \\
\hline $12 / 23 / 2005$ & $18: 00$ & 11.131 & 10.99 & 8.11 & 7.01 & 4.409 & 4.433 & 2.714 & 2.659 & 10.708 & 6.007 & 4.828 & 6.756 & 3.642 \\
\hline $12 / 23 / 2005$ & $19: 00$ & 11.134 & 10.996 & 8.113 & 7.012 & 4.409 & 4.433 & 2.715 & 2.664 & 10.71 & 6.008 & 4.827 & 6.754 & 3.639 \\
\hline $12 / 23 / 2005$ & $20: 00$ & 11.14 & 11.001 & 8.115 & 7.019 & 4.411 & 4.434 & 2.72 & 2.668 & 10.71 & 6.008 & 4.824 & 6.756 & 3.639 \\
\hline $12 / 23 / 2005$ & $21: 00$ & 11.143 & 11.008 & 8.118 & 7.023 & 4.414 & 4.438 & 2.721 & 2.67 & 10.71 & 6.011 & 4.825 & 6.757 & 3.641 \\
\hline $12 / 23 / 2005$ & $22: 00$ & 11.15 & 11.017 & 8.126 & 7.035 & 4.418 & 4.44 & 2.734 & 2.68 & 10.712 & 6.008 & 4.827 & 6.761 & 3.644 \\
\hline $12 / 23 / 2005$ & $23: 00$ & 11.155 & 11.023 & 8.127 & 7.032 & 4.416 & 4.442 & 2.732 & 2.68 & 10.712 & 6.01 & 4.826 & 6.763 & 3.641 \\
\hline $12 / 24 / 2005$ & $0: 00$ & 11.158 & 11.025 & 8.128 & 7.035 & 4.416 & 4.445 & 2.735 & 2.68 & 10.71 & 6.012 & 4.826 & 6.761 & 3.639 \\
\hline $12 / 24 / 2005$ & $1: 00$ & 11.159 & 11.027 & 8.13 & 7.035 & 4.412 & 4.443 & 2.732 & 2.68 & 10.712 & 6.01 & 4.827 & 6.765 & 3.635 \\
\hline $12 / 24 / 2005$ & $2: 00$ & 11.158 & 11.016 & 8.13 & 7.028 & 4.409 & 4.444 & 2.729 & 2.676 & 10.71 & 6.006 & 4.823 & 6.763 & 3.631 \\
\hline $12 / 24 / 2005$ & $3: 00$ & 11.161 & 11.01 & 8.128 & 7.023 & 4.408 & 4.445 & 2.727 & 2.676 & 10.71 & 6.008 & 4.823 & 6.757 & 3.632 \\
\hline
\end{tabular}


Water Level (ft below top of casing) at Indicated Well

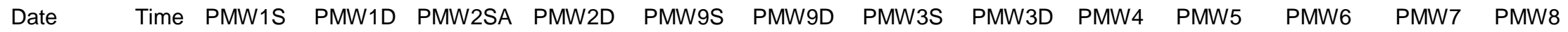

\begin{tabular}{|c|c|c|c|c|c|c|c|c|c|c|c|c|c|c|}
\hline $12 / 24 / 2005$ & $4: 00$ & 11.165 & 11.03 & 8.13 & 7.041 & 4.414 & 4.447 & 2.738 & 2.685 & 10.71 & 6.005 & 4.826 & 6.763 & 3.631 \\
\hline $12 / 24 / 2005$ & $5: 00$ & 11.17 & 11.038 & 8.133 & 7.046 & 4.415 & 4.449 & 2.74 & 2.687 & 10.71 & 6.006 & 4.823 & 6.765 & 3.632 \\
\hline $12 / 24 / 2005$ & $6: 00$ & 11.175 & 11.045 & 8.134 & 7.052 & 4.419 & 4.451 & 2.745 & 2.693 & 10.71 & 6.008 & 4.825 & 6.763 & 3.635 \\
\hline $12 / 24 / 2005$ & $7: 00$ & 11.183 & 11.056 & 8.143 & 7.059 & 4.424 & 4.456 & 2.754 & 2.701 & 10.716 & 6.014 & 4.829 & 6.769 & 3.638 \\
\hline $12 / 24 / 2005$ & $8: 00$ & 11.19 & 11.067 & 8.147 & 7.068 & 4.43 & 4.46 & 2.763 & 2.71 & 10.719 & 6.017 & 4.828 & 6.771 & 3.643 \\
\hline $12 / 24 / 2005$ & 9:00 & 11.202 & 11.08 & 8.158 & 7.079 & 4.435 & 4.469 & 2.77 & 2.72 & 10.725 & 6.017 & 4.834 & 6.778 & 3.646 \\
\hline $12 / 24 / 2005$ & $10: 00$ & 11.209 & 11.089 & 8.163 & 7.09 & 4.442 & 4.469 & 2.779 & 2.727 & 10.723 & 6.017 & 4.831 & 6.778 & 3.647 \\
\hline $12 / 24 / 2005$ & $11: 00$ & 11.217 & 11.103 & 8.17 & 7.099 & 4.445 & 4.478 & 2.789 & 2.737 & 10.727 & 6.024 & 4.837 & 6.788 & 3.65 \\
\hline $12 / 24 / 2005$ & $12: 00$ & 11.229 & 11.109 & 8.174 & 7.103 & 4.446 & 4.482 & 2.794 & 2.743 & 10.735 & 6.023 & 4.839 & 6.782 & 3.649 \\
\hline $12 / 24 / 2005$ & $13: 00$ & 11.231 & 11.111 & 8.179 & 7.099 & 4.445 & 4.487 & 2.793 & 2.741 & 10.733 & 6.023 & 4.837 & 6.79 & 3.645 \\
\hline $12 / 24 / 2005$ & $14: 00$ & 11.234 & 11.109 & 8.174 & 7.097 & 4.439 & 4.491 & 2.787 & 2.737 & 10.731 & 6.02 & 4.833 & 6.79 & 3.639 \\
\hline $12 / 24 / 2005$ & $15: 00$ & 11.238 & 11.113 & 8.179 & 7.097 & 4.442 & 4.491 & 2.792 & 2.739 & 10.735 & 6.022 & 4.835 & 6.792 & 3.64 \\
\hline $12 / 24 / 2005$ & $16: 00$ & 11.241 & 11.112 & 8.182 & 7.104 & 4.439 & 4.493 & 2.794 & 2.741 & 10.736 & 6.024 & 4.838 & 6.792 & 3.639 \\
\hline $12 / 24 / 2005$ & $17: 00$ & 11.243 & 11.114 & 8.183 & 7.104 & 4.444 & 4.496 & 2.794 & 2.741 & 10.738 & 6.022 & 4.836 & 6.796 & 3.636 \\
\hline $12 / 24 / 2005$ & $18: 00$ & 11.246 & 11.118 & 8.184 & 7.106 & 4.445 & 4.498 & 2.797 & 2.745 & 10.74 & 6.026 & 4.837 & 6.797 & 3.638 \\
\hline $12 / 24 / 2005$ & $19: 00$ & 11.251 & 11.122 & 8.188 & 7.11 & 4.446 & 4.5 & 2.8 & 2.75 & 10.744 & 6.027 & 4.838 & 6.798 & 3.638 \\
\hline $12 / 24 / 2005$ & $20: 00$ & 11.253 & 11.125 & 8.188 & 7.112 & 4.446 & 4.502 & 2.806 & 2.752 & 10.744 & 6.028 & 4.836 & 6.797 & 3.638 \\
\hline $12 / 24 / 2005$ & $21: 00$ & 11.256 & 11.131 & 8.193 & 7.117 & 4.452 & 4.507 & 2.808 & 2.756 & 10.746 & 6.031 & 4.841 & 6.798 & 3.644 \\
\hline $12 / 24 / 2005$ & $22: 00$ & 11.258 & 11.133 & 8.193 & 7.117 & 4.45 & 4.509 & 2.811 & 2.758 & 10.748 & 6.027 & 4.841 & 6.801 & 3.644 \\
\hline $12 / 24 / 2005$ & $23: 00$ & 11.262 & 11.133 & 8.194 & 7.117 & 4.452 & 4.511 & 2.811 & 2.758 & 10.748 & 6.024 & 4.839 & 6.801 & 3.644 \\
\hline $12 / 25 / 2005$ & $0: 00$ & 11.264 & 11.136 & 8.197 & 7.119 & 4.452 & 4.513 & 2.813 & 2.76 & 10.75 & 6.028 & 4.841 & 6.806 & 3.648 \\
\hline $12 / 25 / 2005$ & $1: 00$ & 11.264 & 11.131 & 8.195 & 7.115 & 4.448 & 4.513 & 2.808 & 2.758 & 10.75 & 6.025 & 4.838 & 6.804 & 3.649 \\
\hline $12 / 25 / 2005$ & $2: 00$ & 11.26 & 11.127 & 8.193 & 7.11 & 4.443 & 4.513 & 2.805 & 2.754 & 10.75 & 6.023 & 4.838 & 6.804 & 3.651 \\
\hline $12 / 25 / 2005$ & $3: 00$ & 11.26 & 11.127 & 8.194 & 7.112 & 4.447 & 4.513 & 2.809 & 2.756 & 10.75 & 6.028 & 4.845 & 6.805 & 3.656 \\
\hline $12 / 25 / 2005$ & $4: 00$ & 11.265 & 11.131 & 8.195 & 7.115 & 4.451 & 4.516 & 2.812 & 2.76 & 10.75 & 6.03 & 4.848 & 6.807 & 3.666 \\
\hline $12 / 25 / 2005$ & $5: 00$ & 11.26 & 11.125 & 8.193 & 7.11 & 4.446 & 4.516 & 2.808 & 2.754 & 10.75 & 6.03 & 4.844 & 6.803 & 3.667 \\
\hline $12 / 25 / 2005$ & $6: 00$ & 11.259 & 11.124 & 8.193 & 7.112 & 4.449 & 4.516 & 2.808 & 2.756 & 10.752 & 6.031 & 4.849 & 6.805 & 3.671 \\
\hline $12 / 25 / 2005$ & $7: 00$ & 11.261 & 11.129 & 8.195 & 7.115 & 4.453 & 4.516 & 2.812 & 2.76 & 10.752 & 6.034 & 4.854 & 6.809 & 3.68 \\
\hline $12 / 25 / 2005$ & $8: 00$ & 11.264 & 11.133 & 8.198 & 7.119 & 4.458 & 4.52 & 2.819 & 2.766 & 10.754 & 6.036 & 4.857 & 6.812 & 3.688 \\
\hline $12 / 25 / 2005$ & $9: 00$ & 11.267 & 11.138 & 8.2 & 7.121 & 4.459 & 4.52 & 2.82 & 2.769 & 10.755 & 6.036 & 4.863 & 6.812 & 3.692 \\
\hline $12 / 25 / 2005$ & $10: 00$ & 11.269 & 11.14 & 8.202 & 7.124 & 4.461 & 4.522 & 2.823 & 2.771 & 10.755 & 6.037 & 4.867 & 6.814 & 3.698 \\
\hline $12 / 25 / 2005$ & $11: 00$ & 11.274 & 11.145 & 8.206 & 7.128 & 4.467 & 4.527 & 2.83 & 2.777 & 10.757 & 6.043 & 4.871 & 6.817 & 3.704 \\
\hline $12 / 25 / 2005$ & $12: 00$ & 11.273 & 11.142 & 8.206 & 7.126 & 4.463 & 4.527 & 2.825 & 2.773 & 10.757 & 6.036 & 4.871 & 6.813 & 3.701 \\
\hline $12 / 25 / 2005$ & $13: 00$ & 11.268 & 11.135 & 8.204 & 7.119 & 4.46 & 4.524 & 2.82 & 2.766 & 10.757 & 6.036 & 4.87 & 6.813 & 3.697 \\
\hline
\end{tabular}


Water Level (ft below top of casing) at Indicated Well

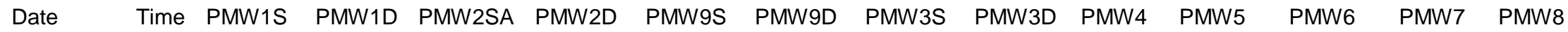

\begin{tabular}{|c|c|c|c|c|c|c|c|c|c|c|c|c|c|c|}
\hline $12 / 25 / 2005$ & $14: 00$ & 11.264 & 11.125 & 8.199 & 7.115 & 4.457 & 4.524 & 2.815 & 2.762 & 10.755 & 6.031 & 4.868 & 6.812 & 3.695 \\
\hline $12 / 25 / 2005$ & $15: 00$ & 11.259 & 11.122 & 8.196 & 7.108 & 4.455 & 4.522 & 2.808 & 2.756 & 10.755 & 6.033 & 4.869 & 6.811 & 3.692 \\
\hline $12 / 25 / 2005$ & $16: 00$ & 11.261 & 11.122 & 8.195 & 7.11 & 4.456 & 4.52 & 2.81 & 2.756 & 10.753 & 6.032 & 4.868 & 6.809 & 3.691 \\
\hline $12 / 25 / 2005$ & $17: 00$ & 11.261 & 11.125 & 8.196 & 7.115 & 4.461 & 4.522 & 2.814 & 2.76 & 10.755 & 6.04 & 4.869 & 6.811 & 3.692 \\
\hline $12 / 25 / 2005$ & $18: 00$ & 11.261 & 11.125 & 8.198 & 7.112 & 4.46 & 4.522 & 2.811 & 2.758 & 10.757 & 6.04 & 4.866 & 6.809 & 3.688 \\
\hline $12 / 25 / 2005$ & $19: 00$ & 11.263 & 11.127 & 8.198 & 7.115 & 4.462 & 4.522 & 2.814 & 2.76 & 10.757 & 6.038 & 4.866 & 6.811 & 3.685 \\
\hline $12 / 25 / 2005$ & $20: 00$ & 11.263 & 11.127 & 8.198 & 7.115 & 4.462 & 4.522 & 2.811 & 2.76 & 10.757 & 6.036 & 4.863 & 6.809 & 3.687 \\
\hline $12 / 25 / 2005$ & $21: 00$ & 11.262 & 11.129 & 8.2 & 7.117 & 4.461 & 4.522 & 2.815 & 2.76 & 10.757 & 6.037 & 4.863 & 6.808 & 3.686 \\
\hline $12 / 25 / 2005$ & $22: 00$ & 11.265 & 11.129 & 8.198 & 7.115 & 4.463 & 4.524 & 2.814 & 2.762 & 10.757 & 6.038 & 4.863 & 6.808 & 3.683 \\
\hline $12 / 25 / 2005$ & $23: 00$ & 11.26 & 11.125 & 8.196 & 7.112 & 4.46 & 4.523 & 2.813 & 2.76 & 10.757 & 6.037 & 4.861 & 6.809 & 3.685 \\
\hline $12 / 26 / 2005$ & $0: 00$ & 11.26 & 11.124 & 8.196 & 7.112 & 4.459 & 4.523 & 2.812 & 2.76 & 10.757 & 6.036 & 4.862 & 6.806 & 3.684 \\
\hline $12 / 26 / 2005$ & $1: 00$ & 11.256 & 11.116 & 8.192 & 7.106 & 4.458 & 4.52 & 2.805 & 2.754 & 10.753 & 6.036 & 4.856 & 6.805 & 3.682 \\
\hline $12 / 26 / 2005$ & $2: 00$ & 11.256 & 11.118 & 8.192 & 7.106 & 4.459 & 4.52 & 2.806 & 2.751 & 10.755 & 6.033 & 4.857 & 6.803 & 3.681 \\
\hline $12 / 26 / 2005$ & 3:00 & 11.253 & 11.116 & 8.191 & 7.104 & 4.456 & 4.52 & 2.807 & 2.752 & 10.754 & 6.033 & 4.856 & 6.803 & 3.684 \\
\hline $12 / 26 / 2005$ & $4: 00$ & 11.251 & 11.113 & 8.19 & 7.101 & 4.455 & 4.52 & 2.804 & 2.75 & 10.752 & 6.035 & 4.853 & 6.8 & 3.683 \\
\hline $12 / 26 / 2005$ & $5: 00$ & 11.245 & 11.104 & 8.183 & 7.093 & 4.45 & 4.516 & 2.794 & 2.741 & 10.749 & 6.032 & 4.85 & 6.798 & 3.68 \\
\hline $12 / 26 / 2005$ & $6: 00$ & 11.239 & 11.098 & 8.18 & 7.09 & 4.446 & 4.514 & 2.79 & 2.739 & 10.748 & 6.032 & 4.847 & 6.797 & 3.681 \\
\hline $12 / 26 / 2005$ & $7: 00$ & 11.236 & 11.093 & 8.177 & 7.088 & 4.445 & 4.512 & 2.786 & 2.735 & 10.746 & 6.028 & 4.846 & 6.795 & 3.679 \\
\hline $12 / 26 / 2005$ & $8: 00$ & 11.234 & 11.093 & 8.177 & 7.086 & 4.448 & 4.509 & 2.788 & 2.737 & 10.744 & 6.032 & 4.848 & 6.795 & 3.684 \\
\hline $12 / 26 / 2005$ & $9: 00$ & 11.227 & 11.087 & 8.174 & 7.084 & 4.444 & 4.507 & 2.788 & 2.733 & 10.744 & 6.027 & 4.848 & 6.792 & 3.683 \\
\hline $12 / 26 / 2005$ & $10: 00$ & 11.228 & 11.087 & 8.171 & 7.082 & 4.446 & 4.505 & 2.786 & 2.733 & 10.744 & 6.029 & 4.848 & 6.794 & 3.687 \\
\hline $12 / 26 / 2005$ & $11: 00$ & 11.228 & 11.085 & 8.17 & 7.081 & 4.444 & 4.505 & 2.786 & 2.733 & 10.74 & 6.024 & 4.847 & 6.784 & 3.684 \\
\hline $12 / 26 / 2005$ & $12: 00$ & 11.223 & 11.078 & 8.169 & 7.075 & 4.437 & 4.498 & 2.778 & 2.724 & 10.738 & 6.028 & 4.849 & 6.786 & 3.681 \\
\hline $12 / 26 / 2005$ & $13: 00$ & 11.215 & 11.071 & 8.164 & 7.068 & 4.436 & 4.496 & 2.772 & 2.718 & 10.738 & 6.021 & 4.842 & 6.782 & 3.675 \\
\hline $12 / 26 / 2005$ & $14: 00$ & 11.208 & 11.062 & 8.157 & 7.057 & 4.426 & 4.492 & 2.763 & 2.708 & 10.734 & 6.018 & 4.834 & 6.781 & 3.658 \\
\hline $12 / 26 / 2005$ & $15: 00$ & 11.2 & 11.051 & 8.151 & 7.05 & 4.421 & 4.483 & 2.753 & 2.697 & 10.734 & 6.017 & 4.822 & 6.776 & 3.639 \\
\hline $12 / 26 / 2005$ & $16: 00$ & 11.195 & 11.045 & 8.146 & 7.046 & 4.419 & 4.481 & 2.747 & 2.693 & 10.73 & 6.017 & 4.819 & 6.773 & 3.625 \\
\hline $12 / 26 / 2005$ & $17: 00$ & 11.19 & 11.045 & 8.142 & 7.049 & 4.419 & 4.476 & 2.747 & 2.693 & 10.73 & 6.018 & 4.814 & 6.771 & 3.616 \\
\hline $12 / 26 / 2005$ & $18: 00$ & 11.188 & 11.043 & 8.142 & 7.048 & 4.42 & 4.476 & 2.743 & 2.693 & 10.729 & 6.014 & 4.809 & 6.77 & 3.61 \\
\hline $12 / 26 / 2005$ & $19: 00$ & 11.188 & 11.047 & 8.142 & 7.053 & 4.418 & 4.474 & 2.751 & 2.695 & 10.729 & 6.014 & 4.808 & 6.772 & 3.61 \\
\hline 12/26/2005 & $20: 00$ & 11.188 & 11.049 & 8.142 & 7.053 & 4.418 & 4.474 & 2.751 & 2.697 & 10.729 & 6.012 & 4.806 & 6.771 & 3.611 \\
\hline $12 / 26 / 2005$ & $21: 00$ & 11.194 & 11.054 & 8.145 & 7.057 & 4.418 & 4.474 & 2.755 & 2.701 & 10.731 & 6.013 & 4.807 & 6.771 & 3.619 \\
\hline $12 / 26 / 2005$ & $22: 00$ & 11.19 & 11.051 & 8.143 & 7.055 & 4.407 & 4.472 & 2.751 & 2.697 & 10.729 & 6.009 & 4.806 & 6.77 & 3.622 \\
\hline $12 / 26 / 2005$ & $23: 00$ & 11.19 & 11.049 & 8.141 & 7.051 & 4.409 & 4.472 & 2.749 & 2.695 & 10.727 & 6.008 & 4.806 & 6.77 & 3.626 \\
\hline
\end{tabular}


Water Level (ft below top of casing) at Indicated Well

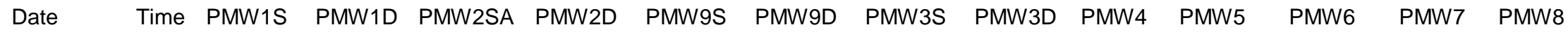

\begin{tabular}{|c|c|c|c|c|c|c|c|c|c|c|c|c|c|c|}
\hline $12 / 27 / 2005$ & $0: 00$ & 11.188 & 11.051 & 8.141 & 7.053 & 4.413 & 4.472 & 2.753 & 2.697 & 10.727 & 6.011 & 4.809 & 6.771 & 3.635 \\
\hline $12 / 27 / 2005$ & $1: 00$ & 11.189 & 11.047 & 8.138 & 7.049 & 4.409 & 4.469 & 2.749 & 2.695 & 10.723 & 6.009 & 4.809 & 6.768 & 3.642 \\
\hline $12 / 27 / 2005$ & $2: 00$ & 11.183 & 11.042 & 8.137 & 7.044 & 4.404 & 4.467 & 2.749 & 2.693 & 10.723 & 6.01 & 4.811 & 6.768 & 3.648 \\
\hline $12 / 27 / 2005$ & $3: 00$ & 11.177 & 11.036 & 8.135 & 7.042 & 4.402 & 4.465 & 2.745 & 2.687 & 10.721 & 6.006 & 4.815 & 6.769 & 3.657 \\
\hline $12 / 27 / 2005$ & $4: 00$ & 11.175 & 11.032 & 8.131 & 7.037 & 4.399 & 4.463 & 2.737 & 2.685 & 10.719 & 6.007 & 4.814 & 6.765 & 3.661 \\
\hline $12 / 27 / 2005$ & $5: 00$ & 11.174 & 11.031 & 8.129 & 7.035 & 4.4 & 4.461 & 2.74 & 2.685 & 10.719 & 6.004 & 4.817 & 6.764 & 3.67 \\
\hline $12 / 27 / 2005$ & $6: 00$ & 11.167 & 11.021 & 8.125 & 7.026 & 4.397 & 4.456 & 2.733 & 2.678 & 10.715 & 6.006 & 4.815 & 6.764 & 3.676 \\
\hline $12 / 27 / 2005$ & $7: 00$ & 11.167 & 11.027 & 8.126 & 7.035 & 4.405 & 4.456 & 2.74 & 2.685 & 10.717 & 6.007 & 4.824 & 6.766 & 3.691 \\
\hline $12 / 27 / 2005$ & $8: 00$ & 11.167 & 11.029 & 8.128 & 7.035 & 4.409 & 4.456 & 2.743 & 2.689 & 10.717 & 6.012 & 4.828 & 6.767 & 3.703 \\
\hline $12 / 27 / 2005$ & 9:00 & 11.165 & 11.027 & 8.127 & 7.033 & 4.407 & 4.456 & 2.741 & 2.684 & 10.715 & 6.008 & 4.831 & 6.763 & 3.71 \\
\hline $12 / 27 / 2005$ & $10: 00$ & 11.164 & 11.031 & 8.129 & 7.037 & 4.412 & 4.456 & 2.743 & 2.687 & 10.715 & 6.013 & 4.838 & 6.764 & 3.72 \\
\hline $12 / 27 / 2005$ & $11: 00$ & 11.166 & 11.031 & 8.127 & 7.037 & 4.413 & 4.456 & 2.742 & 2.688 & 10.717 & 6.013 & 4.839 & 6.76 & 3.722 \\
\hline $12 / 27 / 2005$ & $12: 00$ & 11.17 & 11.033 & 8.129 & 7.04 & 4.417 & 4.456 & 2.747 & 2.691 & 10.715 & 6.014 & 4.841 & 6.764 & 3.72 \\
\hline $12 / 27 / 2005$ & $13: 00$ & 11.172 & 11.038 & 8.132 & 7.044 & 4.42 & 4.458 & 2.749 & 2.695 & 10.717 & 6.013 & 4.842 & 6.764 & 3.713 \\
\hline $12 / 27 / 2005$ & $14: 00$ & 11.169 & 11.033 & 8.13 & 7.042 & 4.415 & 4.457 & 2.743 & 2.687 & 10.713 & 6.007 & 4.833 & 6.761 & 3.691 \\
\hline $12 / 27 / 2005$ & $15: 00$ & 11.164 & 11.023 & 8.123 & 7.026 & 4.407 & 4.452 & 2.729 & 2.676 & 10.711 & 6.002 & 4.821 & 6.755 & 3.661 \\
\hline $12 / 27 / 2005$ & $16: 00$ & 11.167 & 11.029 & 8.126 & 7.035 & 4.413 & 4.454 & 2.738 & 2.68 & 10.713 & 6.007 & 4.82 & 6.756 & 3.648 \\
\hline $12 / 27 / 2005$ & $17: 00$ & 11.177 & 11.051 & 8.136 & 7.055 & 4.425 & 4.458 & 2.752 & 2.699 & 10.715 & 6.018 & 4.822 & 6.763 & 3.648 \\
\hline $12 / 27 / 2005$ & $18: 00$ & 11.187 & 11.058 & 8.141 & 7.06 & 4.426 & 4.461 & 2.758 & 2.706 & 10.721 & 6.015 & 4.817 & 6.763 & 3.642 \\
\hline $12 / 27 / 2005$ & 19:00 & 11.194 & 11.069 & 8.146 & 7.069 & 4.429 & 4.465 & 2.764 & 2.71 & 10.723 & 6.016 & 4.817 & 6.767 & 3.644 \\
\hline $12 / 27 / 2005$ & $20: 00$ & 11.206 & 11.082 & 8.153 & 7.077 & 4.431 & 4.47 & 2.775 & 2.72 & 10.727 & 6.016 & 4.816 & 6.77 & 3.649 \\
\hline $12 / 27 / 2005$ & $21: 00$ & 11.216 & 11.097 & 8.161 & 7.093 & 4.439 & 4.477 & 2.788 & 2.733 & 10.729 & 6.02 & 4.821 & 6.774 & 3.658 \\
\hline $12 / 27 / 2005$ & $22: 00$ & 11.223 & 11.1 & 8.166 & 7.088 & 4.434 & 4.481 & 2.786 & 2.733 & 10.729 & 6.016 & 4.818 & 6.774 & 3.657 \\
\hline $12 / 27 / 2005$ & $23: 00$ & 11.223 & 11.097 & 8.164 & 7.082 & 4.43 & 4.481 & 2.783 & 2.729 & 10.73 & 6.017 & 4.816 & 6.774 & 3.656 \\
\hline $12 / 28 / 2005$ & $0: 00$ & 11.228 & 11.102 & 8.168 & 7.091 & 4.432 & 4.485 & 2.788 & 2.735 & 10.732 & 6.02 & 4.818 & 6.776 & 3.662 \\
\hline $12 / 28 / 2005$ & $1: 00$ & 11.233 & 11.106 & 8.172 & 7.093 & 4.433 & 4.488 & 2.792 & 2.735 & 10.732 & 6.018 & 4.821 & 6.779 & 3.669 \\
\hline $12 / 28 / 2005$ & $2: 00$ & 11.233 & 11.106 & 8.17 & 7.091 & 4.433 & 4.49 & 2.79 & 2.735 & 10.736 & 6.021 & 4.823 & 6.779 & 3.677 \\
\hline $12 / 28 / 2005$ & $3: 00$ & 11.233 & 11.106 & 8.172 & 7.093 & 4.434 & 4.494 & 2.791 & 2.737 & 10.732 & 6.022 & 4.828 & 6.781 & 3.686 \\
\hline $12 / 28 / 2005$ & $4: 00$ & 11.24 & 11.111 & 8.177 & 7.097 & 4.438 & 4.496 & 2.795 & 2.743 & 10.736 & 6.022 & 4.835 & 6.785 & 3.699 \\
\hline $12 / 28 / 2005$ & $5: 00$ & 11.242 & 11.117 & 8.18 & 7.102 & 4.442 & 4.498 & 2.804 & 2.749 & 10.738 & 6.026 & 4.841 & 6.788 & 3.707 \\
\hline $12 / 28 / 2005$ & $6: 00$ & 11.247 & 11.12 & 8.184 & 7.104 & 4.444 & 4.501 & 2.807 & 2.75 & 10.738 & 6.028 & 4.848 & 6.791 & 3.706 \\
\hline $12 / 28 / 2005$ & $7: 00$ & 11.25 & 11.122 & 8.183 & 7.106 & 4.445 & 4.503 & 2.804 & 2.758 & 10.74 & 6.028 & 4.849 & 6.791 & 3.703 \\
\hline $12 / 28 / 2005$ & $8: 00$ & 11.256 & 11.131 & 8.19 & 7.115 & 4.454 & 4.508 & 2.814 & 2.76 & 10.742 & 6.032 & 4.853 & 6.794 & 3.703 \\
\hline $12 / 28 / 2005$ & $9: 00$ & 11.262 & 11.135 & 8.194 & 7.122 & 4.453 & 4.51 & 2.816 & 2.751 & 10.744 & 6.034 & 4.856 & 6.799 & 3.692 \\
\hline
\end{tabular}


Water Level (ft below top of casing) at Indicated Well

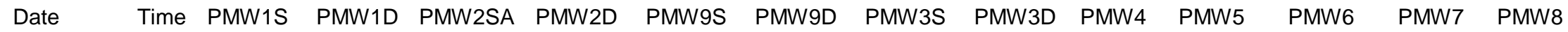

\begin{tabular}{|c|c|c|c|c|c|c|c|c|c|c|c|c|c|c|}
\hline $12 / 28 / 2005$ & $10: 00$ & 11.267 & 11.14 & 8.198 & 7.124 & 4.455 & 4.512 & 2.82 & 2.766 & 10.746 & 6.03 & 4.855 & 6.801 & 3.685 \\
\hline $12 / 28 / 2005$ & $11: 00$ & 11.27 & 11.148 & 8.201 & 7.131 & 4.459 & 4.514 & 2.824 & 2.771 & 10.75 & 6.035 & 4.856 & 6.802 & 3.681 \\
\hline $12 / 28 / 2005$ & $12: 00$ & 11.272 & 11.146 & 8.203 & 7.124 & 4.453 & 4.519 & 2.822 & 2.766 & 10.749 & 6.031 & 4.851 & 6.801 & 3.671 \\
\hline $12 / 28 / 2005$ & $13: 00$ & 11.272 & 11.144 & 8.2 & 7.124 & 4.448 & 4.519 & 2.819 & 2.764 & 10.749 & 6.033 & 4.848 & 6.8 & 3.66 \\
\hline $12 / 28 / 2005$ & $14: 00$ & 11.269 & 11.135 & 8.198 & 7.115 & 4.444 & 4.519 & 2.812 & 2.756 & 10.744 & 6.029 & 4.843 & 6.8 & 3.654 \\
\hline $12 / 28 / 2005$ & $15: 00$ & 11.266 & 11.135 & 8.196 & 7.115 & 4.442 & 4.516 & 2.807 & 2.754 & 10.746 & 6.031 & 4.839 & 6.801 & 3.647 \\
\hline $12 / 28 / 2005$ & $16: 00$ & 11.271 & 11.14 & 8.197 & 7.118 & 4.447 & 4.519 & 2.812 & 2.758 & 10.748 & 6.032 & 4.84 & 6.8 & 3.653 \\
\hline $12 / 28 / 2005$ & $17: 00$ & 11.276 & 11.148 & 8.201 & 7.124 & 4.449 & 4.521 & 2.82 & 2.766 & 10.751 & 6.033 & 4.836 & 6.802 & 3.656 \\
\hline $12 / 28 / 2005$ & $18: 00$ & 11.276 & 11.148 & 8.2 & 7.124 & 4.447 & 4.523 & 2.819 & 2.764 & 10.751 & 6.032 & 4.834 & 6.802 & 3.656 \\
\hline $12 / 28 / 2005$ & $19: 00$ & 11.278 & 11.148 & 8.201 & 7.126 & 4.448 & 4.523 & 2.819 & 2.766 & 10.751 & 6.031 & 4.833 & 6.806 & 3.662 \\
\hline $12 / 28 / 2005$ & $20: 00$ & 11.281 & 11.151 & 8.203 & 7.129 & 4.448 & 4.525 & 2.824 & 2.77 & 10.753 & 6.032 & 4.837 & 6.807 & 3.67 \\
\hline $12 / 28 / 2005$ & $21: 00$ & 11.281 & 11.153 & 8.207 & 7.133 & 4.451 & 4.53 & 2.825 & 2.774 & 10.755 & 6.034 & 4.838 & 6.809 & 3.678 \\
\hline $12 / 28 / 2005$ & $22: 00$ & 11.281 & 11.155 & 8.207 & 7.133 & 4.451 & 4.53 & 2.828 & 2.774 & 10.755 & 6.034 & 4.842 & 6.811 & 3.687 \\
\hline $12 / 28 / 2005$ & $23: 00$ & 11.285 & 11.159 & 8.209 & 7.14 & 4.456 & 4.534 & 2.832 & 2.781 & 10.757 & 6.041 & 4.852 & 6.814 & 3.704 \\
\hline $12 / 29 / 2005$ & $0: 00$ & 11.289 & 11.166 & 8.216 & 7.146 & 4.463 & 4.536 & 2.84 & 2.787 & 10.759 & 6.041 & 4.861 & 6.818 & 3.714 \\
\hline $12 / 29 / 2005$ & $1: 00$ & 11.293 & 11.164 & 8.216 & 7.142 & 4.461 & 4.538 & 2.84 & 2.785 & 10.761 & 6.041 & 4.863 & 6.816 & 3.71 \\
\hline $12 / 29 / 2005$ & $2: 00$ & 11.29 & 11.159 & 8.212 & 7.135 & 4.457 & 4.538 & 2.833 & 2.779 & 10.759 & 6.04 & 4.865 & 6.815 & 3.708 \\
\hline $12 / 29 / 2005$ & 3:00 & 11.288 & 11.155 & 8.21 & 7.131 & 4.455 & 4.536 & 2.828 & 2.774 & 10.759 & 6.039 & 4.866 & 6.813 & 3.705 \\
\hline $12 / 29 / 2005$ & $4: 00$ & 11.289 & 11.159 & 8.213 & 7.138 & 4.462 & 4.538 & 2.833 & 2.779 & 10.759 & 6.04 & 4.869 & 6.816 & 3.708 \\
\hline $12 / 29 / 2005$ & $5: 00$ & 11.29 & 11.16 & 8.216 & 7.138 & 4.461 & 4.538 & 2.836 & 2.781 & 10.759 & 6.042 & 4.871 & 6.816 & 3.707 \\
\hline $12 / 29 / 2005$ & $6: 00$ & 11.288 & 11.155 & 8.212 & 7.135 & 4.46 & 4.538 & 2.831 & 2.779 & 10.759 & 6.041 & 4.871 & 6.813 & 3.706 \\
\hline $12 / 29 / 2005$ & $7: 00$ & 11.285 & 11.151 & 8.212 & 7.131 & 4.457 & 4.538 & 2.829 & 2.774 & 10.759 & 6.037 & 4.868 & 6.811 & 3.705 \\
\hline $12 / 29 / 2005$ & $8: 00$ & 11.287 & 11.153 & 8.212 & 7.133 & 4.461 & 4.538 & 2.828 & 2.777 & 10.759 & 6.036 & 4.868 & 6.812 & 3.709 \\
\hline $12 / 29 / 2005$ & $9: 00$ & 11.286 & 11.153 & 8.211 & 7.133 & 4.461 & 4.538 & 2.829 & 2.777 & 10.759 & 6.043 & 4.868 & 6.811 & 3.713 \\
\hline $12 / 29 / 2005$ & $10: 00$ & 11.283 & 11.148 & 8.209 & 7.126 & 4.46 & 4.539 & 2.829 & 2.774 & 10.759 & 6.038 & 4.868 & 6.808 & 3.713 \\
\hline $12 / 29 / 2005$ & $11: 00$ & 11.288 & 11.153 & 8.213 & 7.133 & 4.464 & 4.538 & 2.835 & 2.779 & 10.759 & 6.042 & 4.873 & 6.811 & 3.716 \\
\hline $12 / 29 / 2005$ & $12: 00$ & 11.283 & 11.148 & 8.206 & 7.126 & 4.459 & 4.536 & 2.824 & 2.777 & 10.759 & 6.036 & 4.863 & 6.808 & 3.704 \\
\hline $12 / 29 / 2005$ & $13: 00$ & 11.272 & 11.131 & 8.201 & 7.115 & 4.446 & 4.53 & 2.814 & 2.762 & 10.753 & 6.03 & 4.852 & 6.798 & 3.687 \\
\hline $12 / 29 / 2005$ & $14: 00$ & 11.263 & 11.12 & 8.193 & 7.104 & 4.443 & 4.528 & 2.802 & 2.749 & 10.749 & 6.03 & 4.841 & 6.796 & 3.678 \\
\hline $12 / 29 / 2005$ & $15: 00$ & 11.26 & 11.12 & 8.19 & 7.104 & 4.441 & 4.525 & 2.802 & 2.749 & 10.749 & 6.033 & 4.833 & 6.782 & 3.676 \\
\hline $12 / 29 / 2005$ & $16: 00$ & 11.25 & 11.107 & 8.183 & 7.093 & 4.433 & 4.519 & 2.793 & 2.739 & 10.744 & 6.028 & 4.822 & 6.788 & 3.667 \\
\hline $12 / 29 / 2005$ & $17: 00$ & 11.243 & 11.109 & 8.176 & 7.1 & 4.426 & 4.514 & 2.785 & 2.737 & 10.744 & 6.02 & 4.809 & 6.801 & 3.66 \\
\hline $12 / 29 / 2005$ & $18: 00$ & 11.232 & 11.093 & 8.168 & 7.104 & 4.412 & 4.508 & 2.769 & 2.716 & 10.738 & 6.012 & 4.799 & 6.808 & 3.645 \\
\hline $12 / 29 / 2005$ & $19: 00$ & 11.232 & 11.091 & 8.167 & 7.095 & 4.422 & 4.505 & 2.779 & 2.724 & 10.736 & 6.027 & 4.807 & 6.798 & 3.649 \\
\hline
\end{tabular}


Water Level (ft below top of casing) at Indicated Well

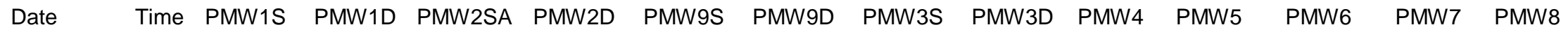

\begin{tabular}{|c|c|c|c|c|c|c|c|c|c|c|c|c|c|c|}
\hline $12 / 29 / 2005$ & $20: 00$ & 11.227 & 11.084 & 8.164 & 7.076 & 4.417 & 4.501 & 2.779 & 2.724 & 10.732 & 6.018 & 4.797 & 6.778 & 3.645 \\
\hline $12 / 29 / 2005$ & $21: 00$ & 11.223 & 11.076 & 8.159 & 7.071 & 4.413 & 4.497 & 2.769 & 2.716 & 10.73 & 6.017 & 4.792 & 6.773 & 3.642 \\
\hline $12 / 29 / 2005$ & $22: 00$ & 11.215 & 11.067 & 8.155 & 7.062 & 4.405 & 4.495 & 2.763 & 2.709 & 10.727 & 6.008 & 4.787 & 6.768 & 3.636 \\
\hline $12 / 29 / 2005$ & $23: 00$ & 11.215 & 11.075 & 8.158 & 7.071 & 4.413 & 4.492 & 2.77 & 2.716 & 10.73 & 6.013 & 4.796 & 6.772 & 3.646 \\
\hline $12 / 30 / 2005$ & $0: 00$ & 11.212 & 11.067 & 8.154 & 7.064 & 4.407 & 4.488 & 2.764 & 2.709 & 10.729 & 6.011 & 4.791 & 6.77 & 3.645 \\
\hline $12 / 30 / 2005$ & $1: 00$ & 11.21 & 11.071 & 8.154 & 7.069 & 4.411 & 4.488 & 2.77 & 2.714 & 10.73 & 6.014 & 4.795 & 6.772 & 3.653 \\
\hline $12 / 30 / 2005$ & $2: 00$ & 11.212 & 11.071 & 8.154 & 7.069 & 4.409 & 4.486 & 2.768 & 2.711 & 10.73 & 6.014 & 4.796 & 6.773 & 3.659 \\
\hline $12 / 30 / 2005$ & $3: 00$ & 11.213 & 11.075 & 8.155 & 7.071 & 4.415 & 4.488 & 2.774 & 2.716 & 10.729 & 6.019 & 4.804 & 6.773 & 3.669 \\
\hline $12 / 30 / 2005$ & 4:00 & 11.22 & 11.082 & 8.161 & 7.08 & 4.42 & 4.49 & 2.782 & 2.724 & 10.732 & 6.019 & 4.81 & 6.777 & 3.68 \\
\hline $12 / 30 / 2005$ & $5: 00$ & 11.222 & 11.084 & 8.163 & 7.082 & 4.424 & 4.49 & 2.78 & 2.726 & 10.732 & 6.021 & 4.818 & 6.778 & 3.687 \\
\hline $12 / 30 / 2005$ & $6: 00$ & 11.222 & 11.087 & 8.165 & 7.084 & 4.425 & 4.492 & 2.786 & 2.728 & 10.732 & 6.021 & 4.823 & 6.78 & 3.696 \\
\hline $12 / 30 / 2005$ & $7: 00$ & 11.231 & 11.098 & 8.171 & 7.093 & 4.432 & 4.495 & 2.793 & 2.737 & 10.734 & 6.024 & 4.831 & 6.785 & 3.704 \\
\hline $12 / 30 / 2005$ & $8: 00$ & 11.234 & 11.1 & 8.173 & 7.095 & 4.429 & 4.497 & 2.796 & 2.737 & 10.736 & 6.025 & 4.834 & 6.784 & 3.704 \\
\hline $12 / 30 / 2005$ & $9: 00$ & 11.237 & 11.102 & 8.172 & 7.098 & 4.434 & 4.499 & 2.798 & 2.741 & 10.734 & 6.026 & 4.837 & 6.784 & 3.705 \\
\hline $12 / 30 / 2005$ & $10: 00$ & 11.241 & 11.111 & 8.179 & 7.1 & 4.437 & 4.501 & 2.8 & 2.743 & 10.736 & 6.026 & 4.841 & 6.786 & 3.709 \\
\hline $12 / 30 / 2005$ & $11: 00$ & 11.249 & 11.119 & 8.184 & 7.111 & 4.443 & 4.503 & 2.808 & 2.751 & 10.738 & 6.033 & 4.845 & 6.789 & 3.71 \\
\hline $12 / 30 / 2005$ & $12: 00$ & 11.255 & 11.128 & 8.188 & 7.118 & 4.446 & 4.508 & 2.814 & 2.758 & 10.742 & 6.03 & 4.844 & 6.789 & 3.7 \\
\hline $12 / 30 / 2005$ & $13: 00$ & 11.258 & 11.126 & 8.192 & 7.113 & 4.444 & 4.508 & 2.811 & 2.756 & 10.742 & 6.03 & 4.838 & 6.787 & 3.687 \\
\hline $12 / 30 / 2005$ & $14: 00$ & 11.255 & 11.126 & 8.186 & 7.111 & 4.442 & 4.512 & 2.813 & 2.753 & 10.742 & 6.031 & 4.833 & 6.787 & 3.683 \\
\hline $12 / 30 / 2005$ & $15: 00$ & 11.258 & 11.128 & 8.192 & 7.114 & 4.444 & 4.515 & 2.813 & 2.756 & 10.742 & 6.029 & 4.828 & 6.787 & 3.685 \\
\hline $12 / 30 / 2005$ & $16: 00$ & 11.264 & 11.129 & 8.191 & 7.118 & 4.446 & 4.517 & 2.814 & 2.756 & 10.74 & 6.028 & 4.827 & 6.795 & 3.687 \\
\hline $12 / 30 / 2005$ & $17: 00$ & 11.264 & 11.135 & 8.194 & 7.12 & 4.452 & 4.517 & 2.817 & 2.76 & 10.746 & 6.031 & 4.833 & 6.793 & 3.691 \\
\hline $12 / 30 / 2005$ & $18: 00$ & 11.271 & 11.141 & 8.2 & 7.127 & 4.453 & 4.519 & 2.824 & 2.768 & 10.746 & 6.034 & 4.834 & 6.796 & 3.7 \\
\hline $12 / 30 / 2005$ & 19:00 & 11.274 & 11.148 & 8.202 & 7.131 & 4.459 & 4.526 & 2.83 & 2.772 & 10.749 & 6.037 & 4.837 & 6.801 & 3.703 \\
\hline $12 / 30 / 2005$ & $20: 00$ & 11.28 & 11.152 & 8.208 & 7.138 & 4.463 & 4.528 & 2.835 & 2.779 & 10.751 & 6.038 & 4.842 & 6.803 & 3.71 \\
\hline $12 / 30 / 2005$ & $21: 00$ & 11.288 & 11.164 & 8.212 & 7.145 & 4.47 & 4.532 & 2.84 & 2.785 & 10.753 & 6.037 & 4.848 & 6.807 & 3.713 \\
\hline $12 / 30 / 2005$ & $22: 00$ & 11.293 & 11.164 & 8.214 & 7.145 & 4.469 & 4.534 & 2.842 & 2.785 & 10.755 & 6.04 & 4.855 & 6.81 & 3.717 \\
\hline $12 / 30 / 2005$ & $23: 00$ & 11.297 & 11.172 & 8.219 & 7.153 & 4.476 & 4.539 & 2.847 & 2.793 & 10.757 & 6.046 & 4.865 & 6.818 & 3.726 \\
\hline $12 / 31 / 2005$ & $0: 00$ & 11.3 & 11.177 & 8.222 & 7.156 & 4.48 & 4.546 & 2.851 & 2.797 & 10.761 & 6.045 & 4.875 & 6.819 & 3.734 \\
\hline $12 / 31 / 2005$ & $1: 00$ & 11.304 & 11.181 & 8.226 & 7.162 & 4.484 & 4.55 & 2.857 & 2.802 & 10.763 & 6.044 & 4.883 & 6.825 & 3.745 \\
\hline $12 / 31 / 2005$ & $2: 00$ & 11.309 & 11.184 & 8.227 & 7.16 & 4.483 & 4.552 & 2.858 & 2.804 & 10.767 & 6.047 & 4.892 & 6.825 & 3.757 \\
\hline $12 / 31 / 2005$ & $3: 00$ & 11.312 & 11.184 & 8.231 & 7.165 & 4.49 & 4.557 & 2.862 & 2.808 & 10.765 & 6.051 & 4.903 & 6.827 & 3.769 \\
\hline $12 / 31 / 2005$ & $4: 00$ & 11.315 & 11.19 & 8.234 & 7.169 & 4.493 & 4.561 & 2.866 & 2.812 & 10.768 & 6.052 & 4.91 & 6.83 & 3.781 \\
\hline $12 / 31 / 2005$ & $5: 00$ & 11.317 & 11.194 & 8.236 & 7.169 & 4.5 & 4.563 & 2.869 & 2.814 & 10.768 & 6.055 & 4.92 & 6.834 & 3.791 \\
\hline
\end{tabular}


TABLE S1.2 (Cont.)

Water Level (ft below top of casing) at Indicated Well

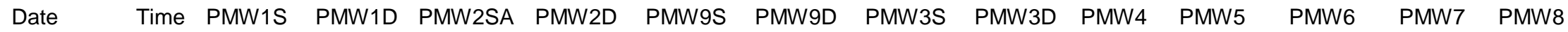

\begin{tabular}{|c|c|c|c|c|c|c|c|c|c|c|c|c|c|c|}
\hline $12 / 31 / 2005$ & $6: 00$ & 11.32 & 11.195 & 8.24 & 7.173 & 4.501 & 4.568 & 2.872 & 2.818 & 10.77 & 6.057 & 4.925 & 6.835 & 3.8 \\
\hline $12 / 31 / 2005$ & $7: 00$ & 11.321 & 11.194 & 8.241 & 7.169 & 4.501 & 4.568 & 2.87 & 2.814 & 10.772 & 6.055 & 4.93 & 6.834 & 3.807 \\
\hline $12 / 31 / 2005$ & $8: 00$ & 11.324 & 11.197 & 8.245 & 7.176 & 4.508 & 4.572 & 2.877 & 2.821 & 10.774 & 6.056 & 4.938 & 6.837 & 3.817 \\
\hline $12 / 31 / 2005$ & $9: 00$ & 11.331 & 11.204 & 8.247 & 7.18 & 4.515 & 4.574 & 2.882 & 2.827 & 10.776 & 6.061 & 4.949 & 6.841 & 3.829 \\
\hline $12 / 31 / 2005$ & $10: 00$ & 11.334 & 11.21 & 8.251 & 7.184 & 4.52 & 4.579 & 2.885 & 2.831 & 10.78 & 6.064 & 4.957 & 6.846 & 3.837 \\
\hline $12 / 31 / 2005$ & $11: 00$ & 11.336 & 11.208 & 8.255 & 7.184 & 4.522 & 4.579 & 2.887 & 2.833 & 10.78 & 6.063 & 4.958 & 6.845 & 3.836 \\
\hline $12 / 31 / 2005$ & $12: 00$ & 11.338 & 11.21 & 8.254 & 7.184 & 4.521 & 4.581 & 2.888 & 2.831 & 10.78 & 6.068 & 4.966 & 6.845 & 3.835 \\
\hline $12 / 31 / 2005$ & $13: 00$ & 11.327 & 11.192 & 8.247 & 7.167 & 4.507 & 4.577 & 2.867 & 2.812 & 10.778 & 6.058 & 4.95 & 6.836 & 3.806 \\
\hline $12 / 31 / 2005$ & $14: 00$ & 11.311 & 11.166 & 8.233 & 7.145 & 4.492 & 4.57 & 2.844 & 2.791 & 10.772 & 6.045 & 4.934 & 6.829 & 3.765 \\
\hline $12 / 31 / 2005$ & $15: 00$ & 11.299 & 11.153 & 8.221 & 7.131 & 4.484 & 4.564 & 2.829 & 2.774 & 10.767 & 6.048 & 4.92 & 6.82 & 3.726 \\
\hline $12 / 31 / 2005$ & $16: 00$ & 11.291 & 11.146 & 8.217 & 7.127 & 4.478 & 4.557 & 2.827 & 2.772 & 10.763 & 6.04 & 4.901 & 6.812 & 3.696 \\
\hline $12 / 31 / 2005$ & $17: 00$ & 11.287 & 11.141 & 8.211 & 7.122 & 4.478 & 4.555 & 2.822 & 2.768 & 10.763 & 6.038 & 4.892 & 6.811 & 3.681 \\
\hline $12 / 31 / 2005$ & $18: 00$ & 11.281 & 11.139 & 8.208 & 7.122 & 4.477 & 4.553 & 2.821 & 2.766 & 10.759 & 6.038 & 4.883 & 6.808 & 3.677 \\
\hline $12 / 31 / 2005$ & $19: 00$ & 11.279 & 11.135 & 8.205 & 7.12 & 4.473 & 4.55 & 2.817 & 2.764 & 10.759 & 6.037 & 4.876 & 6.804 & 3.677 \\
\hline $12 / 31 / 2005$ & $20: 00$ & 11.273 & 11.13 & 8.201 & 7.118 & 4.467 & 4.546 & 2.815 & 2.76 & 10.757 & 6.035 & 4.868 & 6.806 & 3.677 \\
\hline $12 / 31 / 2005$ & $21: 00$ & 11.272 & 11.13 & 8.2 & 7.114 & 4.463 & 4.541 & 2.815 & 2.76 & 10.753 & 6.031 & 4.865 & 6.8 & 3.679 \\
\hline $12 / 31 / 2005$ & $22: 00$ & 11.269 & 11.126 & 8.196 & 7.114 & 4.46 & 4.541 & 2.812 & 2.758 & 10.753 & 6.032 & 4.863 & 6.8 & 3.679 \\
\hline $12 / 31 / 2005$ & $23: 00$ & 11.264 & 11.119 & 8.192 & 7.107 & 4.456 & 4.535 & 2.806 & 2.751 & 10.749 & 6.028 & 4.859 & 6.802 & 3.682 \\
\hline $1 / 1 / 2006$ & $0: 00$ & 11.26 & 11.121 & 8.193 & 7.109 & 4.458 & 4.535 & 2.811 & 2.756 & 10.751 & 6.033 & 4.861 & 6.804 & 3.686 \\
\hline $1 / 1 / 2006$ & $1: 00$ & 11.257 & 11.115 & 8.191 & 7.105 & 4.455 & 4.533 & 2.807 & 2.751 & 10.751 & 6.026 & 4.857 & 6.801 & 3.686 \\
\hline $1 / 1 / 2006$ & $2: 00$ & 11.254 & 11.111 & 8.188 & 7.102 & 4.45 & 4.528 & 2.802 & 2.747 & 10.749 & 6.026 & 4.855 & 6.797 & 3.686 \\
\hline $1 / 1 / 2006$ & $3: 00$ & 11.25 & 11.106 & 8.185 & 7.096 & 4.448 & 4.524 & 2.798 & 2.743 & 10.748 & 6.028 & 4.854 & 6.797 & 3.685 \\
\hline $1 / 1 / 2006$ & $4: 00$ & 11.247 & 11.106 & 8.184 & 7.096 & 4.45 & 4.524 & 2.8 & 2.743 & 10.746 & 6.022 & 4.852 & 6.797 & 3.687 \\
\hline $1 / 1 / 2006$ & $5: 00$ & 11.244 & 11.099 & 8.18 & 7.091 & 4.445 & 4.519 & 2.794 & 2.739 & 10.744 & 6.021 & 4.85 & 6.793 & 3.686 \\
\hline $1 / 1 / 2006$ & $6: 00$ & 11.239 & 11.097 & 8.179 & 7.089 & 4.442 & 4.517 & 2.792 & 2.735 & 10.744 & 6.023 & 4.852 & 6.794 & 3.684 \\
\hline $1 / 1 / 2006$ & $7: 00$ & 11.238 & 11.093 & 8.177 & 7.087 & 4.442 & 4.515 & 2.792 & 2.735 & 10.742 & 6.022 & 4.848 & 6.792 & 3.685 \\
\hline $1 / 1 / 2006$ & $8: 00$ & 11.236 & 11.095 & 8.176 & 7.089 & 4.443 & 4.513 & 2.789 & 2.737 & 10.742 & 6.025 & 4.853 & 6.792 & 3.689 \\
\hline $1 / 1 / 2006$ & 9:00 & 11.228 & 11.084 & 8.172 & 7.078 & 4.434 & 4.508 & 2.781 & 2.728 & 10.738 & 6.022 & 4.845 & 6.789 & 3.683 \\
\hline $1 / 1 / 2006$ & $10: 00$ & 11.229 & 11.086 & 8.17 & 7.081 & 4.437 & 4.508 & 2.785 & 2.728 & 10.738 & 6.024 & 4.849 & 6.79 & 3.687 \\
\hline $1 / 1 / 2006$ & $11: 00$ & 11.226 & 11.084 & 8.168 & 7.083 & 4.438 & 4.504 & 2.788 & 2.73 & 10.738 & 6.024 & 4.849 & 6.788 & 3.689 \\
\hline $1 / 1 / 2006$ & $12: 00$ & 11.223 & 11.079 & 8.167 & 7.078 & 4.432 & 4.502 & 2.781 & 2.726 & 10.736 & 6.022 & 4.845 & 6.784 & 3.685 \\
\hline $1 / 1 / 2006$ & $13: 00$ & 11.214 & 11.062 & 8.158 & 7.058 & 4.417 & 4.495 & 2.765 & 2.709 & 10.732 & 6.014 & 4.836 & 6.781 & 3.673 \\
\hline $1 / 1 / 2006$ & $14: 00$ & 11.208 & 11.057 & 8.153 & 7.052 & 4.418 & 4.491 & 2.762 & 2.705 & 10.73 & 6.016 & 4.834 & 6.779 & 3.675 \\
\hline $1 / 1 / 2006$ & $15: 00$ & 11.199 & 11.049 & 8.148 & 7.047 & 4.416 & 4.486 & 2.755 & 2.699 & 10.727 & 6.017 & 4.833 & 6.774 & 3.675 \\
\hline
\end{tabular}


Water Level (ft below top of casing) at Indicated Well

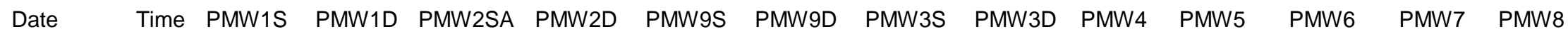

\begin{tabular}{|c|c|c|c|c|c|c|c|c|c|c|c|c|c|c|}
\hline $1 / 1 / 2006$ & $16: 00$ & 11.196 & 11.048 & 8.145 & 7.049 & 4.419 & 4.484 & 2.755 & 2.701 & 10.724 & 6.017 & 4.836 & 6.775 & 3.681 \\
\hline $1 / 1 / 2006$ & $17: 00$ & 11.198 & 11.053 & 8.149 & 7.058 & 4.427 & 4.486 & 2.767 & 2.707 & 10.726 & 6.013 & 4.844 & 6.776 & 3.69 \\
\hline $1 / 1 / 2006$ & $18: 00$ & 11.199 & 11.057 & 8.15 & 7.06 & 4.429 & 4.486 & 2.765 & 2.707 & 10.725 & 6.018 & 4.847 & 6.778 & 3.694 \\
\hline $1 / 1 / 2006$ & $19: 00$ & 11.213 & 11.08 & 8.161 & 7.085 & 4.445 & 4.493 & 2.789 & 2.732 & 10.73 & 6.042 & 4.858 & 6.783 & 3.712 \\
\hline $1 / 1 / 2006$ & $20: 00$ & 11.215 & 11.084 & 8.165 & 7.083 & 4.447 & 4.493 & 2.79 & 2.73 & 10.732 & 6.026 & 4.86 & 6.786 & 3.712 \\
\hline $1 / 1 / 2006$ & $21: 00$ & 11.223 & 11.091 & 8.172 & 7.087 & 4.448 & 4.495 & 2.792 & 2.732 & 10.734 & 6.033 & 4.865 & 6.784 & 3.714 \\
\hline $1 / 1 / 2006$ & $22: 00$ & 11.227 & 11.099 & 8.173 & 7.094 & 4.452 & 4.499 & 2.796 & 2.741 & 10.734 & 6.048 & 4.868 & 6.785 & 3.717 \\
\hline $1 / 1 / 2006$ & $23: 00$ & 11.233 & 11.108 & 8.178 & 7.103 & 4.457 & 4.504 & 2.805 & 2.747 & 10.738 & 6.046 & 4.873 & 6.786 & 3.722 \\
\hline $1 / 2 / 2006$ & 0:00 & 11.238 & 11.106 & 8.182 & 7.107 & 4.457 & 4.506 & 2.805 & 2.747 & 10.736 & 6.045 & 4.874 & 6.789 & 3.722 \\
\hline $1 / 2 / 2006$ & $1: 00$ & 11.247 & 11.095 & 8.188 & 7.096 & 4.459 & 4.511 & 2.813 & 2.758 & 10.725 & 6.043 & 4.873 & 6.785 & 3.705 \\
\hline $1 / 2 / 2006$ & $2: 00$ & 11.25 & 11.131 & 8.187 & 7.123 & 4.407 & 4.513 & 2.785 & 2.747 & 10.743 & 6.024 & 4.79 & 6.792 & 3.38 \\
\hline $1 / 2 / 2006$ & $3: 00$ & 11.261 & 11.128 & 8.181 & 7.114 & 4.176 & 4.515 & 2.766 & 2.714 & 10.732 & 5.947 & 4.583 & 6.766 & 3.098 \\
\hline $1 / 2 / 2006$ & $4: 00$ & 11.249 & 11.141 & 8.177 & 7.105 & 4.004 & 4.515 & 2.757 & 2.707 & 10.713 & 5.914 & 4.519 & 6.693 & 3.048 \\
\hline $1 / 2 / 2006$ & $5: 00$ & 11.256 & 11.155 & 8.176 & 7.112 & 3.941 & 4.508 & 2.764 & 2.711 & 10.707 & 5.933 & 4.503 & 6.701 & 3.055 \\
\hline $1 / 2 / 2006$ & $6: 00$ & 11.275 & 11.166 & 8.181 & 7.114 & 3.92 & 4.497 & 2.766 & 2.716 & 10.7 & 5.943 & 4.5 & 6.708 & 3.074 \\
\hline $1 / 2 / 2006$ & $7: 00$ & 11.293 & 11.181 & 8.184 & 7.129 & 3.934 & 4.491 & 2.782 & 2.73 & 10.69 & 5.949 & 4.519 & 6.722 & 3.103 \\
\hline $1 / 2 / 2006$ & $8: 00$ & 11.307 & 11.204 & 8.196 & 7.147 & 3.955 & 4.486 & 2.798 & 2.747 & 10.692 & 5.913 & 4.533 & 6.729 & 3.132 \\
\hline $1 / 2 / 2006$ & $9: 00$ & 11.317 & 11.208 & 8.202 & 7.149 & 3.97 & 4.482 & 2.8 & 2.751 & 10.692 & 5.924 & 4.543 & 6.733 & 3.154 \\
\hline $1 / 2 / 2006$ & $10: 00$ & 11.328 & 11.206 & 8.209 & 7.16 & 3.993 & 4.48 & 2.814 & 2.762 & 10.694 & 5.926 & 4.561 & 6.747 & 3.181 \\
\hline $1 / 2 / 2006$ & $11: 00$ & 11.336 & 11.228 & 8.213 & 7.167 & 4.018 & 4.48 & 2.82 & 2.77 & 10.696 & 5.93 & 4.576 & 6.753 & 3.205 \\
\hline $1 / 2 / 2006$ & $12: 00$ & 11.337 & 11.235 & 8.219 & 7.167 & 4.031 & 4.477 & 2.821 & 2.77 & 10.694 & 5.937 & 4.59 & 6.758 & 3.224 \\
\hline $1 / 2 / 2006$ & $13: 00$ & 11.346 & 11.234 & 8.221 & 7.165 & 4.048 & 4.477 & 2.824 & 2.772 & 10.694 & 5.938 & 4.598 & 6.763 & 3.242 \\
\hline $1 / 2 / 2006$ & $14: 00$ & 11.347 & 11.235 & 8.227 & 7.165 & 4.063 & 4.48 & 2.826 & 2.774 & 10.696 & 5.957 & 4.612 & 6.769 & 3.263 \\
\hline $1 / 2 / 2006$ & $15: 00$ & 11.352 & 11.241 & 8.231 & 7.172 & 4.08 & 4.482 & 2.833 & 2.779 & 10.7 & 5.948 & 4.627 & 6.772 & 3.285 \\
\hline $1 / 2 / 2006$ & $16: 00$ & 11.359 & 11.25 & 8.238 & 7.185 & 4.107 & 4.486 & 2.845 & 2.791 & 10.703 & 5.987 & 4.648 & 6.784 & 3.311 \\
\hline $1 / 2 / 2006$ & $17: 00$ & 11.371 & 11.263 & 8.247 & 7.2 & 4.132 & 4.495 & 2.863 & 2.808 & 10.711 & 5.994 & 4.672 & 6.798 & 3.338 \\
\hline $1 / 2 / 2006$ & $18: 00$ & 11.375 & 11.272 & 8.253 & 7.205 & 4.149 & 4.499 & 2.867 & 2.814 & 10.717 & 6.027 & 4.689 & 6.805 & 3.355 \\
\hline $1 / 2 / 2006$ & $19: 00$ & 11.383 & 11.281 & 8.26 & 7.214 & 4.164 & 4.509 & 2.874 & 2.825 & 10.722 & 6.01 & 4.707 & 6.813 & 3.372 \\
\hline $1 / 2 / 2006$ & $20: 00$ & 11.39 & 11.285 & 8.264 & 7.211 & 4.178 & 4.513 & 2.877 & 2.825 & 10.724 & 6.009 & 4.719 & 6.816 & 3.386 \\
\hline $1 / 2 / 2006$ & $21: 00$ & 11.394 & 11.287 & 8.266 & 7.214 & 4.19 & 4.517 & 2.88 & 2.826 & 10.73 & 6.025 & 4.729 & 6.818 & 3.403 \\
\hline $1 / 2 / 2006$ & $22: 00$ & 11.397 & 11.287 & 8.269 & 7.214 & 4.199 & 4.522 & 2.881 & 2.829 & 10.732 & 6.031 & 4.735 & 6.823 & 3.414 \\
\hline $1 / 2 / 2006$ & $23: 00$ & 11.401 & 11.29 & 8.271 & 7.216 & 4.213 & 4.526 & 2.883 & 2.831 & 10.736 & 6.032 & 4.748 & 6.827 & 3.43 \\
\hline $1 / 3 / 2006$ & $0: 00$ & 11.401 & 11.288 & 8.271 & 7.214 & 4.221 & 4.528 & 2.88 & 2.829 & 10.738 & 6.038 & 4.753 & 6.829 & 3.442 \\
\hline $1 / 3 / 2006$ & $1: 00$ & 11.401 & 11.283 & 8.271 & 7.209 & 4.231 & 4.531 & 2.879 & 2.826 & 10.74 & 6.013 & 4.76 & 6.831 & 3.453 \\
\hline
\end{tabular}


TABLE S1.2 (Cont.)

Water Level (ft below top of casing) at Indicated Well

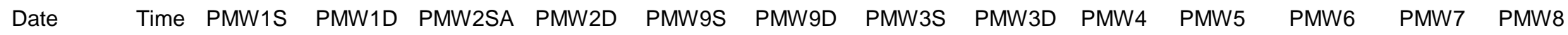

\begin{tabular}{|c|c|c|c|c|c|c|c|c|c|c|c|c|c|c|}
\hline $1 / 3 / 2006$ & $2: 00$ & 11.403 & 11.288 & 8.274 & 7.218 & 4.245 & 4.537 & 2.889 & 2.835 & 10.743 & 6.019 & 4.769 & 6.834 & 3.465 \\
\hline $1 / 3 / 2006$ & $3: 00$ & 11.402 & 11.28 & 8.269 & 7.211 & 4.249 & 4.537 & 2.881 & 2.829 & 10.745 & 6.024 & 4.773 & 6.836 & 3.471 \\
\hline $1 / 3 / 2006$ & $4: 00$ & 11.401 & 11.28 & 8.27 & 7.209 & 4.257 & 4.54 & 2.883 & 2.831 & 10.745 & 6.029 & 4.78 & 6.836 & 3.482 \\
\hline $1 / 3 / 2006$ & $5: 00$ & 11.393 & 11.265 & 8.265 & 7.198 & 4.262 & 4.54 & 2.875 & 2.823 & 10.745 & 6.011 & 4.781 & 6.834 & 3.486 \\
\hline $1 / 3 / 2006$ & $6: 00$ & 11.388 & 11.258 & 8.262 & 7.194 & 4.265 & 4.54 & 2.871 & 2.818 & 10.743 & 6.016 & 4.781 & 6.831 & 3.494 \\
\hline $1 / 3 / 2006$ & $7: 00$ & 11.382 & 11.254 & 8.258 & 7.191 & 4.274 & 4.54 & 2.867 & 2.814 & 10.745 & 6.017 & 4.791 & 6.833 & 3.505 \\
\hline $1 / 3 / 2006$ & $8: 00$ & 11.385 & 11.256 & 8.26 & 7.196 & 4.287 & 4.542 & 2.875 & 2.82 & 10.747 & 6.027 & 4.798 & 6.836 & 3.518 \\
\hline $1 / 3 / 2006$ & $9: 00$ & 11.377 & 11.247 & 8.256 & 7.185 & 4.284 & 4.542 & 2.866 & 2.812 & 10.745 & 6.006 & 4.799 & 6.832 & 3.522 \\
\hline $1 / 3 / 2006$ & $10: 00$ & 11.371 & 11.243 & 8.252 & 7.185 & 4.29 & 4.54 & 2.863 & 2.812 & 10.743 & 6.009 & 4.805 & 6.832 & 3.532 \\
\hline $1 / 3 / 2006$ & $11: 00$ & 11.368 & 11.234 & 8.249 & 7.178 & 4.29 & 4.54 & 2.86 & 2.805 & 10.747 & 6 & 4.804 & 6.83 & 3.534 \\
\hline $1 / 3 / 2006$ & $12: 00$ & 11.36 & 11.217 & 8.242 & 7.165 & 4.293 & 4.535 & 2.851 & 2.799 & 10.738 & 6.005 & 4.802 & 6.826 & 3.531 \\
\hline $1 / 3 / 2006$ & $13: 00$ & 11.345 & 11.203 & 8.231 & 7.147 & 4.288 & 4.529 & 2.836 & 2.782 & 10.74 & 5.995 & 4.791 & 6.816 & 3.528 \\
\hline $1 / 3 / 2006$ & $14: 00$ & 11.33 & 11.179 & 8.22 & 7.134 & 4.28 & 4.522 & 2.821 & 2.766 & 10.732 & 5.986 & 4.782 & 6.809 & 3.527 \\
\hline $1 / 3 / 2006$ & $15: 00$ & 11.316 & 11.165 & 8.208 & 7.116 & 4.275 & 4.515 & 2.808 & 2.753 & 10.73 & 5.979 & 4.778 & 6.8 & 3.529 \\
\hline $1 / 3 / 2006$ & $16: 00$ & 11.304 & 11.148 & 8.199 & 7.107 & 4.275 & 4.506 & 2.798 & 2.74 & 10.724 & 5.982 & 4.779 & 6.794 & 3.532 \\
\hline $1 / 3 / 2006$ & $17: 00$ & 11.296 & 11.148 & 8.196 & 7.109 & 4.285 & 4.504 & 2.798 & 2.742 & 10.724 & 5.988 & 4.787 & 6.801 & 3.545 \\
\hline $1 / 3 / 2006$ & $18: 00$ & 11.29 & 11.144 & 8.195 & 7.114 & 4.296 & 4.504 & 2.803 & 2.747 & 10.726 & 5.989 & 4.794 & 6.8 & 3.555 \\
\hline $1 / 3 / 2006$ & 19:00 & 11.284 & 11.135 & 8.191 & 7.103 & 4.292 & 4.498 & 2.798 & 2.74 & 10.726 & 5.98 & 4.788 & 6.795 & 3.555 \\
\hline $1 / 3 / 2006$ & $20: 00$ & 11.281 & 11.135 & 8.19 & 7.107 & 4.302 & 4.498 & 2.797 & 2.742 & 10.724 & 5.983 & 4.798 & 6.797 & 3.566 \\
\hline $1 / 3 / 2006$ & $21: 00$ & 11.276 & 11.134 & 8.189 & 7.107 & 4.304 & 4.498 & 2.799 & 2.742 & 10.724 & 5.985 & 4.8 & 6.797 & 3.572 \\
\hline $1 / 3 / 2006$ & $22: 00$ & 11.28 & 11.143 & 8.193 & 7.116 & 4.317 & 4.5 & 2.808 & 2.753 & 10.728 & 5.992 & 4.808 & 6.801 & 3.583 \\
\hline $1 / 3 / 2006$ & $23: 00$ & 11.284 & 11.156 & 8.199 & 7.13 & 4.327 & 4.502 & 2.822 & 2.763 & 10.73 & 6 & 4.817 & 6.806 & 3.594 \\
\hline $1 / 4 / 2006$ & $0: 00$ & 11.299 & 11.176 & 8.212 & 7.152 & 4.344 & 4.509 & 2.84 & 2.784 & 10.736 & 6.007 & 4.827 & 6.812 & 3.61 \\
\hline $1 / 4 / 2006$ & $1: 00$ & 11.316 & 11.203 & 8.226 & 7.172 & 4.358 & 4.52 & 2.86 & 2.805 & 10.745 & 6.019 & 4.839 & 6.819 & 3.624 \\
\hline $1 / 4 / 2006$ & $2: 00$ & 11.334 & 11.221 & 8.24 & 7.19 & 4.365 & 4.526 & 2.875 & 2.818 & 10.747 & 6.031 & 4.847 & 6.826 & 3.632 \\
\hline $1 / 4 / 2006$ & $3: 00$ & 11.348 & 11.239 & 8.244 & 7.203 & 4.379 & 4.537 & 2.885 & 2.833 & 10.755 & 6.04 & 4.853 & 6.832 & 3.639 \\
\hline $1 / 4 / 2006$ & $4: 00$ & 11.362 & 11.256 & 8.26 & 7.216 & 4.386 & 4.546 & 2.902 & 2.843 & 10.757 & 6.067 & 4.864 & 6.842 & 3.649 \\
\hline $1 / 4 / 2006$ & $5: 00$ & 11.375 & 11.27 & 8.271 & 7.225 & 4.394 & 4.555 & 2.909 & 2.854 & 10.766 & 6.08 & 4.869 & 6.846 & 3.652 \\
\hline $1 / 4 / 2006$ & $6: 00$ & 11.39 & 11.283 & 8.279 & 7.236 & 4.403 & 4.564 & 2.922 & 2.864 & 10.77 & 6.095 & 4.876 & 6.856 & 3.658 \\
\hline $1 / 4 / 2006$ & $7: 00$ & 11.403 & 11.294 & 8.287 & 7.243 & 4.408 & 4.575 & 2.925 & 2.873 & 10.776 & 6.098 & 4.879 & 6.858 & 3.661 \\
\hline $1 / 4 / 2006$ & $8: 00$ & 11.41 & 11.301 & 8.292 & 7.249 & 4.412 & 4.58 & 2.931 & 2.877 & 10.779 & 6.092 & 4.887 & 6.864 & 3.667 \\
\hline $1 / 4 / 2006$ & $9: 00$ & 11.416 & 11.307 & 8.298 & 7.254 & 4.42 & 4.588 & 2.933 & 2.879 & 10.783 & 6.109 & 4.889 & 6.867 & 3.675 \\
\hline $1 / 4 / 2006$ & $10: 00$ & 11.421 & 11.312 & 8.3 & 7.254 & 4.424 & 4.595 & 2.937 & 2.885 & 10.787 & 6.132 & 4.897 & 6.872 & 3.682 \\
\hline $1 / 4 / 2006$ & $11: 00$ & 11.427 & 11.32 & 8.306 & 7.261 & 4.43 & 4.602 & 2.946 & 2.892 & 10.791 & 6.068 & 4.903 & 6.875 & 3.685 \\
\hline
\end{tabular}


TABLE S1.2 (Cont.)

Water Level (ft below top of casing) at Indicated Well

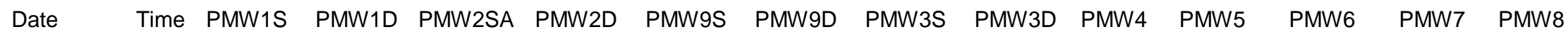

\begin{tabular}{|c|c|c|c|c|c|c|c|c|c|c|c|c|c|c|}
\hline $1 / 4 / 2006$ & $12: 00$ & 11.43 & 11.325 & 8.31 & 7.265 & 4.434 & 4.606 & 2.947 & 2.894 & 10.793 & 6.075 & 4.903 & 6.873 & 3.677 \\
\hline $1 / 4 / 2006$ & $13: 00$ & 11.432 & 11.318 & 8.308 & 7.256 & 4.43 & 4.608 & 2.942 & 2.887 & 10.795 & 6.071 & 4.903 & 6.874 & 3.67 \\
\hline $1 / 4 / 2006$ & $14: 00$ & 11.434 & 11.318 & 8.309 & 7.254 & 4.433 & 4.61 & 2.942 & 2.885 & 10.797 & 6.075 & 4.906 & 6.873 & 3.673 \\
\hline $1 / 4 / 2006$ & $15: 00$ & 11.436 & 11.316 & 8.312 & 7.254 & 4.437 & 4.613 & 2.939 & 2.885 & 10.797 & 6.071 & 4.906 & 6.874 & 3.679 \\
\hline $1 / 4 / 2006$ & $16: 00$ & 11.435 & 11.32 & 8.312 & 7.256 & 4.442 & 4.617 & 2.945 & 2.887 & 10.797 & 6.071 & 4.909 & 6.876 & 3.688 \\
\hline $1 / 4 / 2006$ & $17: 00$ & 11.442 & 11.323 & 8.313 & 7.263 & 4.448 & 4.619 & 2.947 & 2.894 & 10.802 & 6.077 & 4.915 & 6.881 & 3.698 \\
\hline $1 / 4 / 2006$ & $18: 00$ & 11.444 & 11.331 & 8.317 & 7.269 & 4.455 & 4.628 & 2.955 & 2.9 & 10.804 & 6.077 & 4.922 & 6.883 & 3.705 \\
\hline $1 / 4 / 2006$ & $19: 00$ & 11.449 & 11.336 & 8.32 & 7.276 & 4.461 & 4.633 & 2.96 & 2.906 & 10.808 & 6.101 & 4.927 & 6.886 & 3.712 \\
\hline $1 / 4 / 2006$ & $20: 00$ & 11.452 & 11.34 & 8.325 & 7.28 & 4.466 & 4.637 & 2.964 & 2.91 & 10.81 & 6.096 & 4.928 & 6.888 & 3.717 \\
\hline $1 / 4 / 2006$ & $21: 00$ & 11.458 & 11.342 & 8.326 & 7.28 & 4.468 & 4.639 & 2.968 & 2.913 & 10.814 & 6.11 & 4.93 & 6.892 & 3.72 \\
\hline $1 / 4 / 2006$ & $22: 00$ & 11.46 & 11.347 & 8.33 & 7.285 & 4.472 & 4.644 & 2.968 & 2.915 & 10.816 & 6.1 & 4.933 & 6.893 & 3.725 \\
\hline $1 / 4 / 2006$ & $23: 00$ & 11.464 & 11.349 & 8.334 & 7.285 & 4.475 & 4.649 & 2.973 & 2.917 & 10.819 & 6.113 & 4.936 & 6.894 & 3.728 \\
\hline $1 / 5 / 2006$ & $0: 00$ & 11.465 & 11.351 & 8.334 & 7.287 & 4.477 & 4.651 & 2.973 & 2.919 & 10.819 & 6.108 & 4.938 & 6.894 & 3.73 \\
\hline $1 / 5 / 2006$ & $1: 00$ & 11.468 & 11.352 & 8.336 & 7.289 & 4.481 & 4.653 & 2.973 & 2.921 & 10.821 & 6.123 & 4.939 & 6.894 & 3.732 \\
\hline $1 / 5 / 2006$ & $2: 00$ & 11.47 & 11.354 & 8.338 & 7.287 & 4.484 & 4.655 & 2.978 & 2.921 & 10.823 & 6.113 & 4.94 & 6.895 & 3.737 \\
\hline $1 / 5 / 2006$ & $3: 00$ & 11.469 & 11.354 & 8.338 & 7.289 & 4.488 & 4.657 & 2.976 & 2.923 & 10.823 & 6.144 & 4.942 & 6.898 & 3.741 \\
\hline $1 / 5 / 2006$ & $4: 00$ & 11.475 & 11.356 & 8.342 & 7.294 & 4.492 & 4.661 & 2.981 & 2.927 & 10.825 & 6.153 & 4.947 & 6.898 & 3.748 \\
\hline $1 / 5 / 2006$ & $5: 00$ & 11.477 & 11.36 & 8.344 & 7.298 & 4.496 & 4.664 & 2.984 & 2.931 & 10.827 & 6.122 & 4.95 & 6.9 & 3.759 \\
\hline $1 / 5 / 2006$ & $6: 00$ & 11.478 & 11.36 & 8.344 & 7.294 & 4.497 & 4.666 & 2.983 & 2.929 & 10.829 & 6.141 & 4.955 & 6.899 & 3.765 \\
\hline $1 / 5 / 2006$ & $7: 00$ & 11.478 & 11.362 & 8.345 & 7.296 & 4.501 & 4.668 & 2.987 & 2.931 & 10.831 & 6.161 & 4.96 & 6.903 & 3.774 \\
\hline $1 / 5 / 2006$ & 8:00 & 11.482 & 11.365 & 8.35 & 7.303 & 4.507 & 4.671 & 2.992 & 2.936 & 10.831 & 6.171 & 4.968 & 6.906 & 3.787 \\
\hline $1 / 5 / 2006$ & $9: 00$ & 11.485 & 11.369 & 8.35 & 7.305 & 4.513 & 4.675 & 2.995 & 2.94 & 10.833 & 6.154 & 4.973 & 6.907 & 3.795 \\
\hline $1 / 5 / 2006$ & $10: 00$ & 11.488 & 11.373 & 8.353 & 7.307 & 4.516 & 4.677 & 2.998 & 2.944 & 10.833 & 6.159 & 4.978 & 6.911 & 3.805 \\
\hline $1 / 5 / 2006$ & $11: 00$ & 11.489 & 11.374 & 8.356 & 7.309 & 4.52 & 4.682 & 2.998 & 2.946 & 10.835 & 6.163 & 4.985 & 6.911 & 3.809 \\
\hline $1 / 5 / 2006$ & $12: 00$ & 11.492 & 11.376 & 8.358 & 7.311 & 4.523 & 4.682 & 3.002 & 2.946 & 10.835 & 6.167 & 4.989 & 6.91 & 3.8 \\
\hline $1 / 5 / 2006$ & $13: 00$ & 11.494 & 11.374 & 8.358 & 7.309 & 4.52 & 4.684 & 3 & 2.944 & 10.837 & 6.176 & 4.984 & 6.91 & 3.783 \\
\hline $1 / 5 / 2006$ & $14: 00$ & 11.493 & 11.369 & 8.356 & 7.305 & 4.52 & 4.682 & 2.991 & 2.936 & 10.836 & 6.169 & 4.976 & 6.906 & 3.754 \\
\hline $1 / 5 / 2006$ & $15: 00$ & 11.487 & 11.365 & 8.356 & 7.3 & 4.517 & 4.682 & 2.989 & 2.931 & 10.838 & 6.179 & 4.968 & 6.905 & 3.733 \\
\hline $1 / 5 / 2006$ & $16: 00$ & 11.49 & 11.367 & 8.359 & 7.301 & 4.519 & 4.682 & 2.988 & 2.931 & 10.837 & 6.167 & 4.961 & 6.903 & 3.717 \\
\hline $1 / 5 / 2006$ & $17: 00$ & 11.486 & 11.365 & 8.356 & 7.303 & 4.521 & 4.68 & 2.99 & 2.931 & 10.84 & 6.17 & 4.953 & 6.902 & 3.709 \\
\hline $1 / 5 / 2006$ & $18: 00$ & 11.49 & 11.365 & 8.355 & 7.3 & 4.519 & 4.68 & 2.992 & 2.934 & 10.842 & 6.171 & 4.949 & 6.904 & 3.708 \\
\hline $1 / 5 / 2006$ & $19: 00$ & 11.489 & 11.362 & 8.354 & 7.299 & 4.518 & 4.68 & 2.987 & 2.934 & 10.842 & 6.16 & 4.944 & 6.906 & 3.712 \\
\hline $1 / 5 / 2006$ & $20: 00$ & 11.489 & 11.367 & 8.356 & 7.305 & 4.525 & 4.684 & 2.994 & 2.938 & 10.842 & 6.17 & 4.946 & 6.908 & 3.726 \\
\hline $1 / 5 / 2006$ & $21: 00$ & 11.493 & 11.371 & 8.358 & 7.31 & 4.528 & 4.686 & 2.998 & 2.942 & 10.844 & 6.168 & 4.957 & 6.913 & 3.743 \\
\hline
\end{tabular}


TABLE S1.2 (Cont.)

Water Level (ft below top of casing) at Indicated Well

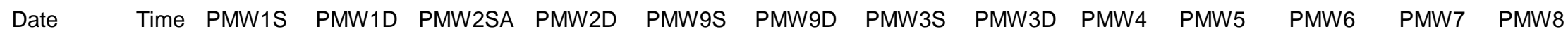

\begin{tabular}{|c|c|c|c|c|c|c|c|c|c|c|c|c|c|c|}
\hline 1/5/2006 & $22: 00$ & 11.493 & 11.369 & 8.36 & 7.307 & 4.528 & 4.688 & 3.002 & 2.944 & 10.846 & 6.171 & 4.962 & 6.912 & 3.758 \\
\hline $1 / 5 / 2006$ & 23:00 & 11.495 & 11.367 & 8.361 & 7.307 & 4.531 & 4.688 & 3.002 & 2.944 & 10.846 & 6.162 & 4.97 & 6.913 & 3.77 \\
\hline $1 / 6 / 2006$ & 0:00 & 11.494 & 11.369 & 8.361 & 7.31 & 4.535 & 4.691 & 3.004 & 2.946 & 10.846 & 6.178 & 4.978 & 6.916 & 3.783 \\
\hline 1/6/2006 & $1: 00$ & 11.495 & 11.367 & 8.362 & 7.307 & 4.535 & 4.691 & 3.003 & 2.946 & 10.848 & 6.162 & 4.982 & 6.915 & 3.787 \\
\hline $1 / 6 / 2006$ & $2: 00$ & 11.49 & 11.363 & 8.361 & 7.307 & 4.536 & 4.691 & 3.001 & 2.944 & 10.848 & 6.182 & 4.989 & 6.918 & 3.788 \\
\hline 1/6/2006 & $3: 00$ & 11.491 & 11.366 & 8.362 & 7.307 & 4.54 & 4.693 & 3.002 & 2.946 & 10.851 & 6.173 & 4.992 & 6.919 & 3.795 \\
\hline $1 / 6 / 2006$ & $4: 00$ & 11.487 & 11.354 & 8.358 & 7.299 & 4.536 & 4.691 & 2.996 & 2.939 & 10.848 & 6.163 & 4.995 & 6.917 & 3.803 \\
\hline $1 / 6 / 2006$ & $5: 00$ & 11.483 & 11.351 & 8.356 & 7.296 & 4.54 & 4.691 & 2.994 & 2.938 & 10.848 & 6.171 & 5 & 6.919 & 3.813 \\
\hline $1 / 6 / 2006$ & $6: 00$ & 11.48 & 11.347 & 8.356 & 7.296 & 4.542 & 4.691 & 2.996 & 2.939 & 10.849 & 6.164 & 5.004 & 6.919 & 3.828 \\
\hline $1 / 6 / 2006$ & $7: 00$ & 11.469 & 11.333 & 8.348 & 7.28 & 4.535 & 4.686 & 2.981 & 2.923 & 10.846 & 6.139 & 5.007 & 6.915 & 3.829 \\
\hline $1 / 6 / 2006$ & 8:00 & 11.469 & 11.338 & 8.349 & 7.29 & 4.545 & 4.688 & 2.991 & 2.929 & 10.848 & 6.169 & 5.018 & 6.919 & 3.847 \\
\hline 1/6/2006 & 9:00 & 11.464 & 11.331 & 8.348 & 7.285 & 4.545 & 4.686 & 2.988 & 2.931 & 10.848 & 6.153 & 5.022 & 6.917 & 3.855 \\
\hline $1 / 6 / 2006$ & $10: 00$ & 11.464 & 11.331 & 8.346 & 7.287 & 4.549 & 4.686 & 2.987 & 2.929 & 10.846 & 6.154 & 5.032 & 6.92 & 3.863 \\
\hline $1 / 6 / 2006$ & $11: 00$ & 11.457 & 11.315 & 8.341 & 7.27 & 4.543 & 4.682 & 2.978 & 2.918 & 10.844 & 6.13 & 5.03 & 6.912 & 3.858 \\
\hline $1 / 6 / 2006$ & $12: 00$ & 11.444 & 11.298 & 8.333 & 7.256 & 4.538 & 4.675 & 2.964 & 2.906 & 10.838 & 6.109 & 5.027 & 6.906 & 3.85 \\
\hline $1 / 6 / 2006$ & $13: 00$ & 11.432 & 11.283 & 8.322 & 7.243 & 4.533 & 4.671 & 2.949 & 2.889 & 10.832 & 6.111 & 5.026 & 6.902 & 3.841 \\
\hline $1 / 6 / 2006$ & $14: 00$ & 11.415 & 11.262 & 8.308 & 7.221 & 4.516 & 4.66 & 2.929 & 2.868 & 10.827 & 6.091 & 5.008 & 6.891 & 3.811 \\
\hline $1 / 6 / 2006$ & $15: 00$ & 11.397 & 11.24 & 8.295 & 7.203 & 4.507 & 4.646 & 2.908 & 2.847 & 10.819 & 6.07 & 4.987 & 6.879 & 3.771 \\
\hline $1 / 6 / 2006$ & $16: 00$ & 11.384 & 11.225 & 8.283 & 7.197 & 4.504 & 4.64 & 2.897 & 2.839 & 10.813 & 6.068 & 4.97 & 6.873 & 3.734 \\
\hline $1 / 6 / 2006$ & $17: 00$ & 11.375 & 11.22 & 8.277 & 7.19 & 4.502 & 4.629 & 2.895 & 2.832 & 10.81 & 6.064 & 4.954 & 6.866 & 3.704 \\
\hline 1/6/2006 & $18: 00$ & 11.368 & 11.218 & 8.275 & 7.19 & 4.503 & 4.626 & 2.894 & 2.832 & 10.81 & 6.069 & 4.945 & 6.865 & 3.691 \\
\hline $1 / 6 / 2006$ & $19: 00$ & 11.358 & 11.205 & 8.266 & 7.181 & 4.489 & 4.618 & 2.882 & 2.822 & 10.806 & 6.055 & 4.931 & 6.856 & 3.676 \\
\hline 1/6/2006 & $20: 00$ & 11.353 & 11.201 & 8.26 & 7.177 & 4.487 & 4.613 & 2.879 & 2.82 & 10.804 & 6.05 & 4.926 & 6.852 & 3.677 \\
\hline 1/6/2006 & $21: 00$ & 11.346 & 11.198 & 8.258 & 7.177 & 4.487 & 4.611 & 2.879 & 2.818 & 10.8 & 6.049 & 4.921 & 6.851 & 3.681 \\
\hline $1 / 6 / 2006$ & $22: 00$ & 11.345 & 11.198 & 8.258 & 7.179 & 4.487 & 4.606 & 2.881 & 2.82 & 10.8 & 6.056 & 4.92 & 6.852 & 3.689 \\
\hline 1/6/2006 & 23:00 & 11.341 & 11.196 & 8.254 & 7.179 & 4.483 & 4.604 & 2.881 & 2.82 & 10.798 & 6.053 & 4.918 & 6.852 & 3.698 \\
\hline 1/7/2006 & $0: 00$ & 11.339 & 11.192 & 8.252 & 7.172 & 4.48 & 4.6 & 2.875 & 2.816 & 10.796 & 6.051 & 4.919 & 6.852 & 3.704 \\
\hline $1 / 7 / 2006$ & 1:00 & 11.336 & 11.19 & 8.25 & 7.172 & 4.479 & 4.598 & 2.875 & 2.816 & 10.794 & 6.051 & 4.921 & 6.85 & 3.715 \\
\hline 1/7/2006 & $2: 00$ & 11.33 & 11.185 & 8.245 & 7.166 & 4.475 & 4.593 & 2.87 & 2.811 & 10.792 & 6.046 & 4.921 & 6.851 & 3.725 \\
\hline $1 / 7 / 2006$ & 3:00 & 11.326 & 11.183 & 8.244 & 7.168 & 4.476 & 4.591 & 2.871 & 2.811 & 10.791 & 6.052 & 4.925 & 6.85 & 3.737 \\
\hline 1/7/2006 & $4: 00$ & 11.324 & 11.181 & 8.243 & 7.166 & 4.477 & 4.589 & 2.87 & 2.811 & 10.791 & 6.048 & 4.929 & 6.849 & 3.749 \\
\hline 1/7/2006 & 5:00 & 11.321 & 11.174 & 8.241 & 7.159 & 4.475 & 4.587 & 2.867 & 2.807 & 10.787 & 6.047 & 4.929 & 6.846 & 3.755 \\
\hline $1 / 7 / 2006$ & $6: 00$ & 11.317 & 11.172 & 8.238 & 7.159 & 4.474 & 4.582 & 2.863 & 2.805 & 10.787 & 6.04 & 4.934 & 6.846 & 3.759 \\
\hline $1 / 7 / 2006$ & 7:00 & 11.317 & 11.172 & 8.238 & 7.159 & 4.476 & 4.583 & 2.869 & 2.805 & 10.785 & 6.042 & 4.939 & 6.844 & 3.77 \\
\hline
\end{tabular}


Water Level (ft below top of casing) at Indicated Well

$\begin{array}{lllllllllllll}\text { Date } & \text { Time } & \text { PMW1S } & \text { PMW1D } & \text { PMW2SA } & \text { PMW2D } & \text { PMW9S } & \text { PMW9D } & \text { PMW3S } & \text { PMW3D } & \text { PMW4 } & \text { PMW5 } & \text { PMW6 }\end{array}$

\begin{tabular}{|c|c|c|c|c|c|c|c|c|c|c|c|c|c|c|}
\hline $1 / 7 / 2006$ & 8:00 & 11.313 & 11.169 & 8.234 & 7.155 & 4.476 & 4.58 & 2.861 & 2.803 & 10.785 & 6.046 & 4.942 & 6.843 & 3.771 \\
\hline $1 / 7 / 2006$ & $9: 00$ & 11.311 & 11.165 & 8.233 & 7.152 & 4.478 & 4.578 & 2.863 & 2.801 & 10.783 & 6.044 & 4.943 & 6.843 & 3.774 \\
\hline $1 / 7 / 2006$ & $10: 00$ & 11.307 & 11.161 & 8.231 & 7.15 & 4.479 & 4.575 & 2.859 & 2.801 & 10.781 & 6.044 & 4.944 & 6.838 & 3.78 \\
\hline $1 / 7 / 2006$ & $11: 00$ & 11.307 & 11.163 & 8.232 & 7.152 & 4.48 & 4.576 & 2.862 & 2.803 & 10.781 & 6.04 & 4.946 & 6.84 & 3.781 \\
\hline $1 / 7 / 2006$ & $12: 00$ & 11.293 & 11.156 & 8.229 & 7.146 & 4.472 & 4.571 & 2.853 & 2.795 & 10.777 & 6.043 & 4.94 & 6.834 & 3.761 \\
\hline $1 / 7 / 2006$ & $13: 00$ & 11.283 & 11.147 & 8.225 & 7.137 & 4.465 & 4.563 & 2.846 & 2.784 & 10.773 & 6.028 & 4.924 & 6.83 & 3.726 \\
\hline $1 / 7 / 2006$ & $14: 00$ & 11.276 & 11.136 & 8.216 & 7.121 & 4.453 & 4.556 & 2.829 & 2.767 & 10.77 & 6.029 & 4.909 & 6.825 & 3.68 \\
\hline $1 / 7 / 2006$ & $15: 00$ & 11.266 & 11.128 & 8.21 & 7.117 & 4.45 & 4.552 & 2.824 & 2.763 & 10.768 & 6.022 & 4.897 & 6.819 & 3.66 \\
\hline $1 / 7 / 2006$ & $16: 00$ & 11.258 & 11.118 & 8.207 & 7.11 & 4.441 & 4.545 & 2.814 & 2.757 & 10.764 & 6.015 & 4.886 & 6.815 & 3.651 \\
\hline $1 / 7 / 2006$ & $17: 00$ & 11.25 & 11.112 & 8.202 & 7.104 & 4.439 & 4.54 & 2.813 & 2.753 & 10.762 & 6.015 & 4.877 & 6.811 & 3.649 \\
\hline $1 / 7 / 2006$ & $18: 00$ & 11.245 & 11.108 & 8.199 & 7.104 & 4.433 & 4.536 & 2.809 & 2.748 & 10.758 & 6.014 & 4.87 & 6.808 & 3.65 \\
\hline $1 / 7 / 2006$ & $19: 00$ & 11.239 & 11.103 & 8.194 & 7.099 & 4.433 & 4.534 & 2.807 & 2.746 & 10.756 & 6.014 & 4.868 & 6.805 & 3.652 \\
\hline $1 / 7 / 2006$ & $20: 00$ & 11.232 & 11.096 & 8.19 & 7.095 & 4.427 & 4.529 & 2.801 & 2.742 & 10.754 & 6.008 & 4.859 & 6.802 & 3.654 \\
\hline $1 / 7 / 2006$ & $21: 00$ & 11.228 & 11.092 & 8.187 & 7.09 & 4.425 & 4.525 & 2.801 & 2.74 & 10.752 & 6.007 & 4.855 & 6.801 & 3.657 \\
\hline $1 / 7 / 2006$ & $22: 00$ & 11.22 & 11.081 & 8.182 & 7.079 & 4.412 & 4.518 & 2.79 & 2.729 & 10.749 & 6.004 & 4.848 & 6.797 & 3.652 \\
\hline $1 / 7 / 2006$ & 23:00 & 11.213 & 11.076 & 8.176 & 7.077 & 4.414 & 4.516 & 2.785 & 2.725 & 10.745 & 6.004 & 4.846 & 6.796 & 3.654 \\
\hline $1 / 8 / 2006$ & $0: 00$ & 11.205 & 11.068 & 8.173 & 7.07 & 4.407 & 4.512 & 2.78 & 2.721 & 10.743 & 6 & 4.841 & 6.791 & 3.655 \\
\hline $1 / 8 / 2006$ & $1: 00$ & 11.196 & 11.057 & 8.165 & 7.059 & 4.4 & 4.503 & 2.772 & 2.711 & 10.737 & 6.001 & 4.836 & 6.787 & 3.651 \\
\hline $1 / 8 / 2006$ & $2: 00$ & 11.191 & 11.056 & 8.162 & 7.064 & 4.401 & 4.498 & 2.773 & 2.713 & 10.737 & 5.997 & 4.836 & 6.788 & 3.657 \\
\hline $1 / 8 / 2006$ & $3: 00$ & 11.185 & 11.046 & 8.156 & 7.053 & 4.395 & 4.494 & 2.767 & 2.704 & 10.733 & 6.011 & 4.831 & 6.781 & 3.657 \\
\hline $1 / 8 / 2006$ & $4: 00$ & 11.186 & 11.052 & 8.155 & 7.059 & 4.402 & 4.494 & 2.772 & 2.706 & 10.732 & 6.018 & 4.838 & 6.785 & 3.668 \\
\hline $1 / 8 / 2006$ & $5: 00$ & 11.18 & 11.045 & 8.154 & 7.057 & 4.401 & 4.49 & 2.766 & 2.706 & 10.73 & 6.008 & 4.836 & 6.779 & 3.675 \\
\hline $1 / 8 / 2006$ & $6: 00$ & 11.173 & 11.041 & 8.152 & 7.055 & 4.398 & 4.487 & 2.766 & 2.706 & 10.73 & 6.01 & 4.837 & 6.778 & 3.683 \\
\hline $1 / 8 / 2006$ & 7:00 & 11.175 & 11.046 & 8.152 & 7.059 & 4.404 & 4.487 & 2.771 & 2.711 & 10.728 & 6.01 & 4.847 & 6.781 & 3.699 \\
\hline $1 / 8 / 2006$ & $8: 00$ & 11.169 & 11.039 & 8.15 & 7.053 & 4.399 & 4.483 & 2.764 & 2.706 & 10.726 & 6.009 & 4.848 & 6.778 & 3.707 \\
\hline $1 / 8 / 2006$ & $9: 00$ & 11.177 & 11.054 & 8.153 & 7.066 & 4.412 & 4.487 & 2.78 & 2.716 & 10.726 & 6.018 & 4.863 & 6.783 & 3.727 \\
\hline $1 / 8 / 2006$ & $10: 00$ & 11.18 & 11.057 & 8.158 & 7.07 & 4.418 & 4.49 & 2.786 & 2.723 & 10.728 & 6.016 & 4.872 & 6.784 & 3.741 \\
\hline $1 / 8 / 2006$ & $11: 00$ & 11.193 & 11.076 & 8.166 & 7.088 & 4.431 & 4.494 & 2.8 & 2.737 & 10.73 & 6.025 & 4.886 & 6.788 & 3.757 \\
\hline $1 / 8 / 2006$ & $12: 00$ & 11.206 & 11.092 & 8.176 & 7.101 & 4.438 & 4.501 & 2.812 & 2.75 & 10.732 & 6.025 & 4.895 & 6.79 & 3.763 \\
\hline $1 / 8 / 2006$ & $13: 00$ & 11.222 & 11.11 & 8.187 & 7.115 & 4.442 & 4.505 & 2.821 & 2.761 & 10.737 & 6.029 & 4.897 & 6.794 & 3.749 \\
\hline $1 / 8 / 2006$ & $14: 00$ & 11.233 & 11.125 & 8.194 & 7.128 & 4.453 & 4.512 & 2.829 & 2.771 & 10.739 & 6.03 & 4.9 & 6.796 & 3.727 \\
\hline $1 / 8 / 2006$ & $15: 00$ & 11.252 & 11.147 & 8.205 & 7.143 & 4.466 & 4.521 & 2.844 & 2.784 & 10.743 & 6.037 & 4.9 & 6.805 & 3.722 \\
\hline $1 / 8 / 2006$ & $16: 00$ & 11.275 & 11.17 & 8.216 & 7.166 & 4.479 & 4.529 & 2.866 & 2.805 & 10.748 & 6.041 & 4.906 & 6.807 & 3.729 \\
\hline $1 / 8 / 2006$ & $17: 00$ & 11.297 & 11.196 & 8.232 & 7.183 & 4.49 & 4.545 & 2.884 & 2.824 & 10.752 & 6.048 & 4.913 & 6.821 & 3.731 \\
\hline
\end{tabular}


Water Level (ft below top of casing) at Indicated Well

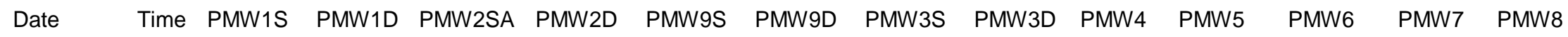

\begin{tabular}{|c|c|c|c|c|c|c|c|c|c|c|c|c|c|c|}
\hline $1 / 8 / 2006$ & $18: 00$ & 11.315 & 11.212 & 8.242 & 7.199 & 4.498 & 4.556 & 2.895 & 2.838 & 10.762 & 6.05 & 4.914 & 6.83 & 3.735 \\
\hline $1 / 8 / 2006$ & $19: 00$ & 11.362 & 11.242 & 8.263 & 7.225 & 4.516 & 4.571 & 2.922 & 2.861 & 10.772 & 6.063 & 4.928 & 6.844 & 3.752 \\
\hline $1 / 8 / 2006$ & $20: 00$ & 11.356 & 11.262 & 8.277 & 7.241 & 4.525 & 4.585 & 2.937 & 2.88 & 10.779 & 6.065 & 4.935 & 6.853 & 3.758 \\
\hline $1 / 8 / 2006$ & $21: 00$ & 11.375 & 11.282 & 8.29 & 7.256 & 4.532 & 4.598 & 2.95 & 2.891 & 10.787 & 6.072 & 4.942 & 6.86 & 3.762 \\
\hline $1 / 8 / 2006$ & $22: 00$ & 11.393 & 11.298 & 8.304 & 7.268 & 4.539 & 4.611 & 2.963 & 2.903 & 10.794 & 6.075 & 4.949 & 6.868 & 3.767 \\
\hline $1 / 8 / 2006$ & $23: 00$ & 11.408 & 11.311 & 8.313 & 7.279 & 4.542 & 4.62 & 2.969 & 2.912 & 10.798 & 6.075 & 4.952 & 6.874 & 3.768 \\
\hline $1 / 9 / 2006$ & $0: 00$ & 11.422 & 11.329 & 8.323 & 7.292 & 4.552 & 4.636 & 2.984 & 2.926 & 10.803 & 6.085 & 4.964 & 6.88 & 3.776 \\
\hline $1 / 9 / 2006$ & $1: 00$ & 11.44 & 11.339 & 8.332 & 7.297 & 4.551 & 4.642 & 2.988 & 2.933 & 10.809 & 6.089 & 4.966 & 6.886 & 3.776 \\
\hline $1 / 9 / 2006$ & $2: 00$ & 11.45 & 11.348 & 8.34 & 7.301 & 4.555 & 4.651 & 2.995 & 2.937 & 10.815 & 6.089 & 4.971 & 6.889 & 3.775 \\
\hline $1 / 9 / 2006$ & $3: 00$ & 11.458 & 11.356 & 8.344 & 7.307 & 4.558 & 4.66 & 3 & 2.941 & 10.817 & 6.094 & 4.977 & 6.896 & 3.776 \\
\hline $1 / 9 / 2006$ & $4: 00$ & 11.47 & 11.37 & 8.354 & 7.321 & 4.568 & 4.669 & 3.012 & 2.954 & 10.822 & 6.1 & 4.984 & 6.907 & 3.786 \\
\hline $1 / 9 / 2006$ & $5: 00$ & 11.478 & 11.379 & 8.36 & 7.323 & 4.568 & 4.678 & 3.016 & 2.958 & 10.828 & 6.099 & 4.988 & 6.909 & 3.787 \\
\hline $1 / 9 / 2006$ & $6: 00$ & 11.492 & 11.391 & 8.366 & 7.334 & 4.577 & 4.687 & 3.023 & 2.966 & 10.83 & 6.107 & 4.997 & 6.913 & 3.793 \\
\hline $1 / 9 / 2006$ & $7: 00$ & 11.501 & 11.397 & 8.373 & 7.341 & 4.578 & 4.695 & 3.029 & 2.973 & 10.834 & 6.108 & 4.999 & 6.918 & 3.797 \\
\hline $1 / 9 / 2006$ & $8: 00$ & 11.505 & 11.415 & 8.382 & 7.359 & 4.589 & 4.702 & 3.045 & 2.987 & 10.843 & 6.116 & 5.012 & 6.929 & 3.806 \\
\hline $1 / 9 / 2006$ & 9:00 & 11.516 & 11.428 & 8.391 & 7.368 & 4.597 & 4.713 & 3.056 & 2.998 & 10.849 & 6.123 & 5.021 & 6.935 & 3.814 \\
\hline $1 / 9 / 2006$ & $10: 00$ & 11.529 & 11.439 & 8.399 & 7.374 & 4.601 & 4.724 & 3.063 & 3.006 & 10.853 & 6.125 & 5.028 & 6.941 & 3.823 \\
\hline $1 / 9 / 2006$ & $11: 00$ & 11.538 & 11.448 & 8.403 & 7.383 & 4.604 & 4.731 & 3.069 & 3.012 & 10.857 & 6.129 & 5.037 & 6.951 & 3.829 \\
\hline $1 / 9 / 2006$ & $12: 00$ & 11.542 & 11.452 & 8.409 & 7.379 & 4.603 & 4.736 & 3.072 & 3.012 & 10.86 & 6.127 & 5.039 & 6.947 & 3.827 \\
\hline $1 / 9 / 2006$ & $13: 00$ & 11.548 & 11.45 & 8.407 & 7.372 & 4.597 & 4.737 & 3.061 & 3.006 & 10.862 & 6.124 & 5.038 & 6.949 & 3.815 \\
\hline $1 / 9 / 2006$ & $14: 00$ & 11.55 & 11.442 & 8.405 & 7.365 & 4.593 & 4.737 & 3.055 & 2.996 & 10.86 & 6.125 & 5.03 & 6.945 & 3.804 \\
\hline $1 / 9 / 2006$ & $15: 00$ & 11.55 & 11.441 & 8.406 & 7.363 & 4.595 & 4.738 & 3.055 & 2.996 & 10.862 & 6.125 & 5.026 & 6.947 & 3.798 \\
\hline $1 / 9 / 2006$ & $16: 00$ & 11.552 & 11.437 & 8.404 & 7.361 & 4.596 & 4.74 & 3.054 & 2.994 & 10.862 & 6.127 & 5.028 & 6.948 & 3.799 \\
\hline $1 / 9 / 2006$ & $17: 00$ & 11.553 & 11.437 & 8.407 & 7.361 & 4.598 & 4.742 & 3.055 & 2.996 & 10.864 & 6.125 & 5.029 & 6.95 & 3.801 \\
\hline $1 / 9 / 2006$ & $18: 00$ & 11.549 & 11.433 & 8.406 & 7.361 & 4.6 & 4.742 & 3.053 & 2.994 & 10.866 & 6.127 & 5.029 & 6.948 & 3.799 \\
\hline $1 / 9 / 2006$ & $19: 00$ & 11.549 & 11.433 & 8.405 & 7.359 & 4.599 & 4.742 & 3.052 & 2.994 & 10.866 & 6.129 & 5.03 & 6.95 & 3.8 \\
\hline $1 / 9 / 2006$ & $20: 00$ & 11.547 & 11.426 & 8.405 & 7.354 & 4.598 & 4.742 & 3.048 & 2.989 & 10.868 & 6.128 & 5.031 & 6.95 & 3.799 \\
\hline $1 / 9 / 2006$ & $21: 00$ & 11.546 & 11.422 & 8.403 & 7.354 & 4.599 & 4.74 & 3.047 & 2.989 & 10.866 & 6.129 & 5.033 & 6.95 & 3.799 \\
\hline $1 / 9 / 2006$ & $22: 00$ & 11.545 & 11.421 & 8.404 & 7.354 & 4.601 & 4.742 & 3.048 & 2.989 & 10.868 & 6.13 & 5.036 & 6.954 & 3.802 \\
\hline $1 / 9 / 2006$ & $23: 00$ & 11.545 & 11.419 & 8.403 & 7.352 & 4.602 & 4.744 & 3.048 & 2.989 & 10.868 & 6.13 & 5.043 & 6.953 & 3.814 \\
\hline $1 / 10 / 2006$ & $0: 00$ & 11.541 & 11.417 & 8.402 & 7.352 & 4.602 & 4.744 & 3.048 & 2.989 & 10.87 & 6.129 & 5.049 & 6.954 & 3.824 \\
\hline $1 / 10 / 2006$ & $1: 00$ & 11.537 & 11.41 & 8.399 & 7.348 & 4.601 & 4.742 & 3.045 & 2.985 & 10.87 & 6.129 & 5.052 & 6.956 & 3.832 \\
\hline $1 / 10 / 2006$ & $2: 00$ & 11.53 & 11.397 & 8.394 & 7.334 & 4.594 & 4.74 & 3.035 & 2.974 & 10.866 & 6.129 & 5.059 & 6.95 & 3.838 \\
\hline $1 / 10 / 2006$ & $3: 00$ & 11.521 & 11.388 & 8.39 & 7.328 & 4.594 & 4.736 & 3.029 & 2.97 & 10.866 & 6.127 & 5.063 & 6.95 & 3.848 \\
\hline
\end{tabular}


Water Level (ft below top of casing) at Indicated Well

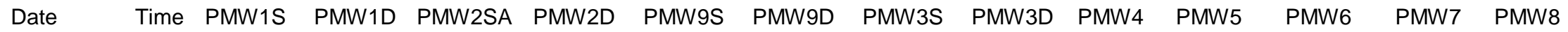

\begin{tabular}{|c|c|c|c|c|c|c|c|c|c|c|c|c|c|c|}
\hline $1 / 10 / 2006$ & $4: 00$ & 11.514 & 11.379 & 8.385 & 7.323 & 4.596 & 4.733 & 3.024 & 2.968 & 10.864 & 6.125 & 5.067 & 6.947 & 3.856 \\
\hline $1 / 10 / 2006$ & $5: 00$ & 11.51 & 11.375 & 8.381 & 7.321 & 4.597 & 4.733 & 3.022 & 2.964 & 10.864 & 6.13 & 5.074 & 6.947 & 3.867 \\
\hline $1 / 10 / 2006$ & $6: 00$ & 11.5 & 11.362 & 8.377 & 7.312 & 4.594 & 4.727 & 3.014 & 2.955 & 10.86 & 6.122 & 5.074 & 6.942 & 3.87 \\
\hline $1 / 10 / 2006$ & 7:00 & 11.494 & 11.353 & 8.371 & 7.303 & 4.592 & 4.722 & 3.007 & 2.949 & 10.859 & 6.122 & 5.076 & 6.939 & 3.874 \\
\hline $1 / 10 / 2006$ & $8: 00$ & 11.486 & 11.346 & 8.368 & 7.299 & 4.592 & 4.72 & 3.006 & 2.945 & 10.857 & 6.124 & 5.081 & 6.939 & 3.88 \\
\hline $1 / 10 / 2006$ & 9:00 & 11.482 & 11.344 & 8.365 & 7.301 & 4.596 & 4.72 & 3.009 & 2.947 & 10.857 & 6.122 & 5.087 & 6.939 & 3.888 \\
\hline $1 / 10 / 2006$ & $10: 00$ & 11.482 & 11.342 & 8.365 & 7.301 & 4.599 & 4.718 & 3.008 & 2.947 & 10.859 & 6.124 & 5.092 & 6.938 & 3.893 \\
\hline $1 / 10 / 2006$ & $11: 00$ & 11.477 & 11.337 & 8.363 & 7.297 & 4.599 & 4.713 & 3.005 & 2.945 & 10.857 & 6.127 & 5.094 & 6.936 & 3.896 \\
\hline $1 / 10 / 2006$ & $12: 00$ & 11.47 & 11.328 & 8.359 & 7.288 & 4.594 & 4.711 & 2.996 & 2.936 & 10.855 & 6.121 & 5.094 & 6.931 & 3.892 \\
\hline $1 / 10 / 2006$ & $13: 00$ & 11.459 & 11.309 & 8.349 & 7.268 & 4.58 & 4.702 & 2.979 & 2.918 & 10.847 & 6.11 & 5.082 & 6.919 & 3.874 \\
\hline $1 / 10 / 2006$ & $14: 00$ & 11.447 & 11.293 & 8.338 & 7.252 & 4.572 & 4.694 & 2.964 & 2.903 & 10.841 & 6.101 & 5.076 & 6.915 & 3.859 \\
\hline $1 / 10 / 2006$ & $15: 00$ & 11.431 & 11.278 & 8.326 & 7.239 & 4.56 & 4.682 & 2.946 & 2.886 & 10.836 & 6.101 & 5.061 & 6.906 & 3.837 \\
\hline $1 / 10 / 2006$ & $16: 00$ & 11.423 & 11.266 & 8.317 & 7.235 & 4.559 & 4.676 & 2.939 & 2.878 & 10.83 & 6.101 & 5.05 & 6.902 & 3.814 \\
\hline $1 / 10 / 2006$ & $17: 00$ & 11.412 & 11.258 & 8.312 & 7.228 & 4.554 & 4.669 & 2.931 & 2.871 & 10.83 & 6.095 & 5.036 & 6.895 & 3.789 \\
\hline $1 / 10 / 2006$ & $18: 00$ & 11.403 & 11.249 & 8.305 & 7.221 & 4.55 & 4.663 & 2.926 & 2.863 & 10.826 & 6.086 & 5.023 & 6.89 & 3.769 \\
\hline $1 / 10 / 2006$ & $19: 00$ & 11.396 & 11.242 & 8.298 & 7.217 & 4.547 & 4.656 & 2.921 & 2.861 & 10.822 & 6.083 & 5.011 & 6.886 & 3.76 \\
\hline $1 / 10 / 2006$ & $20: 00$ & 11.387 & 11.233 & 8.292 & 7.208 & 4.541 & 4.652 & 2.918 & 2.855 & 10.817 & 6.08 & 5.002 & 6.877 & 3.755 \\
\hline $1 / 10 / 2006$ & $21: 00$ & 11.381 & 11.227 & 8.289 & 7.206 & 4.538 & 4.647 & 2.912 & 2.85 & 10.815 & 6.081 & 5.001 & 6.876 & 3.758 \\
\hline $1 / 10 / 2006$ & $22: 00$ & 11.378 & 11.222 & 8.282 & 7.201 & 4.533 & 4.643 & 2.906 & 2.846 & 10.813 & 6.073 & 4.994 & 6.876 & 3.762 \\
\hline $1 / 10 / 2006$ & $23: 00$ & 11.372 & 11.22 & 8.282 & 7.199 & 4.533 & 4.638 & 2.906 & 2.846 & 10.811 & 6.079 & 4.996 & 6.876 & 3.771 \\
\hline $1 / 11 / 2006$ & $0: 00$ & 11.366 & 11.216 & 8.278 & 7.199 & 4.531 & 4.634 & 2.904 & 2.844 & 10.809 & 6.074 & 4.994 & 6.871 & 3.779 \\
\hline $1 / 11 / 2006$ & $1: 00$ & 11.361 & 11.207 & 8.273 & 7.193 & 4.527 & 4.632 & 2.901 & 2.84 & 10.805 & 6.074 & 4.994 & 6.869 & 3.785 \\
\hline $1 / 11 / 2006$ & $2: 00$ & 11.354 & 11.198 & 8.267 & 7.184 & 4.522 & 4.625 & 2.894 & 2.834 & 10.802 & 6.069 & 4.992 & 6.864 & 3.787 \\
\hline $1 / 11 / 2006$ & $3: 00$ & 11.347 & 11.191 & 8.263 & 7.177 & 4.521 & 4.621 & 2.891 & 2.827 & 10.8 & 6.066 & 4.993 & 6.862 & 3.793 \\
\hline $1 / 11 / 2006$ & $4: 00$ & 11.342 & 11.183 & 8.258 & 7.173 & 4.517 & 4.614 & 2.884 & 2.823 & 10.798 & 6.063 & 4.995 & 6.861 & 3.799 \\
\hline $1 / 11 / 2006$ & $5: 00$ & 11.336 & 11.18 & 8.254 & 7.17 & 4.519 & 4.612 & 2.882 & 2.821 & 10.796 & 6.065 & 4.998 & 6.86 & 3.808 \\
\hline $1 / 11 / 2006$ & $6: 00$ & 11.328 & 11.171 & 8.25 & 7.162 & 4.514 & 4.605 & 2.872 & 2.813 & 10.792 & 6.062 & 5 & 6.858 & 3.809 \\
\hline $1 / 11 / 2006$ & $7: 00$ & 11.323 & 11.169 & 8.248 & 7.159 & 4.516 & 4.603 & 2.876 & 2.813 & 10.79 & 6.06 & 5.005 & 6.856 & 3.815 \\
\hline $1 / 11 / 2006$ & 8:00 & 11.32 & 11.165 & 8.246 & 7.162 & 4.52 & 4.601 & 2.878 & 2.815 & 10.788 & 6.061 & 5.008 & 6.857 & 3.826 \\
\hline $1 / 11 / 2006$ & $9: 00$ & 11.314 & 11.163 & 8.243 & 7.159 & 4.52 & 4.598 & 2.876 & 2.812 & 10.786 & 6.062 & 5.012 & 6.854 & 3.831 \\
\hline $1 / 11 / 2006$ & $10: 00$ & 11.316 & 11.165 & 8.245 & 7.162 & 4.528 & 4.596 & 2.877 & 2.816 & 10.788 & 6.065 & 5.022 & 6.858 & 3.84 \\
\hline $1 / 11 / 2006$ & $11: 00$ & 11.314 & 11.167 & 8.244 & 7.164 & 4.532 & 4.596 & 2.881 & 2.818 & 10.786 & 6.067 & 5.027 & 6.857 & 3.844 \\
\hline $1 / 11 / 2006$ & $12: 00$ & 11.312 & 11.165 & 8.245 & 7.162 & 4.532 & 4.596 & 2.881 & 2.818 & 10.786 & 6.064 & 5.036 & 6.863 & 3.843 \\
\hline $1 / 11 / 2006$ & $13: 00$ & 11.31 & 11.162 & 8.243 & 7.159 & 4.529 & 4.592 & 2.872 & 2.81 & 10.784 & 6.059 & 5.04 & 6.866 & 3.834 \\
\hline
\end{tabular}


Water Level (ft below top of casing) at Indicated Well

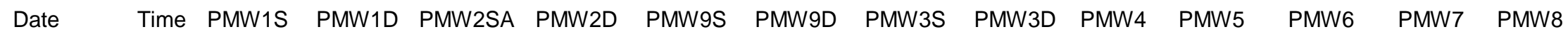

\begin{tabular}{|c|c|c|c|c|c|c|c|c|c|c|c|c|c|c|}
\hline $1 / 11 / 2006$ & $14: 00$ & 11.306 & 11.156 & 8.239 & 7.15 & 4.523 & 4.59 & 2.866 & 2.802 & 10.782 & 6.059 & 5.037 & 6.862 & 3.814 \\
\hline $1 / 11 / 2006$ & $15: 00$ & 11.301 & 11.149 & 8.233 & 7.146 & 4.515 & 4.583 & 2.857 & 2.793 & 10.779 & 6.054 & 5.025 & 6.856 & 3.78 \\
\hline $1 / 11 / 2006$ & $16: 00$ & 11.301 & 11.151 & 8.233 & 7.146 & 4.516 & 4.581 & 2.858 & 2.795 & 10.776 & 6.055 & 5.014 & 6.854 & 3.748 \\
\hline $1 / 11 / 2006$ & $17: 00$ & 11.298 & 11.149 & 8.232 & 7.148 & 4.515 & 4.579 & 2.856 & 2.795 & 10.776 & 6.052 & 5.001 & 6.85 & 3.723 \\
\hline $1 / 11 / 2006$ & $18: 00$ & 11.294 & 11.149 & 8.229 & 7.148 & 4.511 & 4.578 & 2.858 & 2.793 & 10.775 & 6.049 & 4.989 & 6.847 & 3.707 \\
\hline $1 / 11 / 2006$ & $19: 00$ & 11.297 & 11.151 & 8.228 & 7.148 & 4.508 & 4.576 & 2.859 & 2.793 & 10.775 & 6.048 & 4.977 & 6.845 & 3.697 \\
\hline $1 / 11 / 2006$ & $20: 00$ & 11.296 & 11.149 & 8.227 & 7.148 & 4.506 & 4.576 & 2.856 & 2.793 & 10.771 & 6.046 & 4.973 & 6.842 & 3.693 \\
\hline $1 / 11 / 2006$ & $21: 00$ & 11.293 & 11.149 & 8.225 & 7.149 & 4.503 & 4.574 & 2.854 & 2.793 & 10.771 & 6.045 & 4.964 & 6.841 & 3.693 \\
\hline $1 / 11 / 2006$ & $22: 00$ & 11.292 & 11.147 & 8.224 & 7.144 & 4.496 & 4.572 & 2.852 & 2.791 & 10.771 & 6.041 & 4.957 & 6.838 & 3.691 \\
\hline $1 / 11 / 2006$ & $23: 00$ & 11.293 & 11.145 & 8.223 & 7.144 & 4.495 & 4.572 & 2.849 & 2.789 & 10.767 & 6.04 & 4.952 & 6.838 & 3.693 \\
\hline $1 / 12 / 2006$ & $0: 00$ & 11.29 & 11.147 & 8.223 & 7.144 & 4.49 & 4.572 & 2.852 & 2.789 & 10.767 & 6.039 & 4.946 & 6.835 & 3.695 \\
\hline $1 / 12 / 2006$ & $1: 00$ & 11.29 & 11.147 & 8.223 & 7.142 & 4.488 & 4.57 & 2.85 & 2.789 & 10.763 & 6.042 & 4.942 & 6.836 & 3.697 \\
\hline $1 / 12 / 2006$ & $2: 00$ & 11.29 & 11.145 & 8.221 & 7.139 & 4.484 & 4.57 & 2.847 & 2.787 & 10.763 & 6.037 & 4.939 & 6.834 & 3.698 \\
\hline $1 / 12 / 2006$ & $3: 00$ & 11.289 & 11.142 & 8.221 & 7.139 & 4.482 & 4.568 & 2.846 & 2.785 & 10.763 & 6.034 & 4.936 & 6.832 & 3.702 \\
\hline $1 / 12 / 2006$ & $4: 00$ & 11.285 & 11.14 & 8.219 & 7.137 & 4.476 & 4.566 & 2.845 & 2.783 & 10.761 & 6.033 & 4.933 & 6.831 & 3.704 \\
\hline $1 / 12 / 2006$ & $5: 00$ & 11.283 & 11.14 & 8.219 & 7.137 & 4.476 & 4.563 & 2.845 & 2.783 & 10.761 & 6.035 & 4.932 & 6.829 & 3.703 \\
\hline $1 / 12 / 2006$ & $6: 00$ & 11.28 & 11.131 & 8.214 & 7.128 & 4.467 & 4.559 & 2.835 & 2.774 & 10.757 & 6.028 & 4.925 & 6.826 & 3.699 \\
\hline $1 / 12 / 2006$ & $7: 00$ & 11.28 & 11.136 & 8.213 & 7.133 & 4.474 & 4.559 & 2.844 & 2.783 & 10.757 & 6.035 & 4.928 & 6.83 & 3.71 \\
\hline $1 / 12 / 2006$ & $8: 00$ & 11.279 & 11.136 & 8.215 & 7.133 & 4.475 & 4.559 & 2.844 & 2.783 & 10.757 & 6.029 & 4.927 & 6.827 & 3.714 \\
\hline $1 / 12 / 2006$ & $9: 00$ & 11.281 & 11.143 & 8.216 & 7.142 & 4.478 & 4.561 & 2.849 & 2.789 & 10.757 & 6.034 & 4.934 & 6.827 & 3.727 \\
\hline $1 / 12 / 2006$ & $10: 00$ & 11.287 & 11.151 & 8.219 & 7.146 & 4.482 & 4.563 & 2.857 & 2.795 & 10.759 & 6.037 & 4.94 & 6.829 & 3.74 \\
\hline $1 / 12 / 2006$ & $11: 00$ & 11.292 & 11.154 & 8.225 & 7.153 & 4.484 & 4.563 & 2.86 & 2.797 & 10.761 & 6.033 & 4.941 & 6.828 & 3.743 \\
\hline $1 / 12 / 2006$ & $12: 00$ & 11.291 & 11.156 & 8.226 & 7.148 & 4.481 & 4.563 & 2.858 & 2.795 & 10.757 & 6.031 & 4.942 & 6.826 & 3.734 \\
\hline $1 / 12 / 2006$ & $13: 00$ & 11.295 & 11.156 & 8.229 & 7.15 & 4.482 & 4.563 & 2.858 & 2.795 & 10.756 & 6.034 & 4.938 & 6.826 & 3.722 \\
\hline $1 / 12 / 2006$ & $14: 00$ & 11.296 & 11.154 & 8.228 & 7.15 & 4.48 & 4.563 & 2.856 & 2.793 & 10.756 & 6.033 & 4.934 & 6.824 & 3.716 \\
\hline $1 / 12 / 2006$ & $15: 00$ & 11.296 & 11.16 & 8.23 & 7.155 & 4.484 & 4.563 & 2.857 & 2.795 & 10.746 & 6.039 & 4.93 & 6.823 & 3.717 \\
\hline $1 / 12 / 2006$ & $16: 00$ & 11.304 & 11.178 & 8.235 & 7.173 & 4.497 & 4.57 & 2.875 & 2.808 & 10.754 & 6.04 & 4.943 & 6.827 & 3.729 \\
\hline $1 / 12 / 2006$ & $17: 00$ & 11.316 & 11.193 & 8.244 & 7.181 & 4.503 & 4.574 & 2.885 & 2.822 & 10.765 & 6.047 & 4.945 & 6.838 & 3.742 \\
\hline $1 / 12 / 2006$ & $18: 00$ & 11.329 & 11.208 & 8.251 & 7.197 & 4.512 & 4.583 & 2.897 & 2.837 & 10.767 & 6.05 & 4.95 & 6.841 & 3.752 \\
\hline $1 / 12 / 2006$ & $19: 00$ & 11.344 & 11.228 & 8.264 & 7.213 & 4.523 & 4.592 & 2.91 & 2.85 & 10.769 & 6.053 & 4.959 & 6.852 & 3.759 \\
\hline $1 / 12 / 2006$ & $20: 00$ & 11.358 & 11.244 & 8.276 & 7.226 & 4.53 & 4.601 & 2.928 & 2.864 & 10.773 & 6.061 & 4.969 & 6.856 & 3.77 \\
\hline $1 / 12 / 2006$ & $21: 00$ & 11.372 & 11.251 & 8.284 & 7.237 & 4.538 & 4.608 & 2.937 & 2.875 & 10.78 & 6.059 & 4.977 & 6.856 & 3.774 \\
\hline $1 / 12 / 2006$ & $22: 00$ & 11.381 & 11.239 & 8.289 & 7.22 & 4.537 & 4.616 & 2.938 & 2.878 & 10.786 & 6.061 & 4.977 & 6.851 & 3.77 \\
\hline $1 / 12 / 2006$ & $23: 00$ & 11.388 & 11.273 & 8.294 & 7.246 & 4.539 & 4.619 & 2.941 & 2.881 & 10.786 & 6.061 & 4.977 & 6.869 & 3.765 \\
\hline
\end{tabular}


TABLE S1.2 (Cont.)

Water Level (ft below top of casing) at Indicated Well

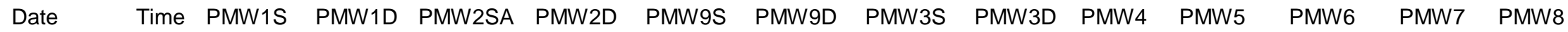

\begin{tabular}{|c|c|c|c|c|c|c|c|c|c|c|c|c|c|c|}
\hline $1 / 13 / 2006$ & $0: 00$ & 11.396 & 11.279 & 8.301 & 7.248 & 4.541 & 4.625 & 2.944 & 2.885 & 10.792 & 6.061 & 4.978 & 6.871 & 3.763 \\
\hline $1 / 13 / 2006$ & $1: 00$ & 11.404 & 11.286 & 8.309 & 7.253 & 4.544 & 4.63 & 2.949 & 2.887 & 10.792 & 6.063 & 4.982 & 6.869 & 3.763 \\
\hline $1 / 13 / 2006$ & $2: 00$ & 11.411 & 11.293 & 8.311 & 7.259 & 4.55 & 4.636 & 2.957 & 2.894 & 10.791 & 6.066 & 4.985 & 6.877 & 3.768 \\
\hline $1 / 13 / 2006$ & $3: 00$ & 11.418 & 11.301 & 8.32 & 7.27 & 4.551 & 4.643 & 2.963 & 2.902 & 10.794 & 6.071 & 4.992 & 6.882 & 3.774 \\
\hline $1 / 13 / 2006$ & $4: 00$ & 11.426 & 11.315 & 8.328 & 7.282 & 4.561 & 4.652 & 2.974 & 2.913 & 10.797 & 6.078 & 4.999 & 6.889 & 3.781 \\
\hline $1 / 13 / 2006$ & $5: 00$ & 11.436 & 11.326 & 8.336 & 7.29 & 4.566 & 4.661 & 2.984 & 2.925 & 10.814 & 6.074 & 5.002 & 6.892 & 3.785 \\
\hline $1 / 13 / 2006$ & $6: 00$ & 11.446 & 11.333 & 8.342 & 7.299 & 4.571 & 4.665 & 2.989 & 2.929 & 10.807 & 6.08 & 5.003 & 6.896 & 3.789 \\
\hline $1 / 13 / 2006$ & $7: 00$ & 11.454 & 11.346 & 8.347 & 7.308 & 4.573 & 4.672 & 3.002 & 2.94 & 10.816 & 6.084 & 5.017 & 6.899 & 3.795 \\
\hline $1 / 13 / 2006$ & $8: 00$ & 11.468 & 11.359 & 8.354 & 7.319 & 4.588 & 4.683 & 3.011 & 2.95 & 10.818 & 6.092 & 5.021 & 6.909 & 3.804 \\
\hline $1 / 13 / 2006$ & $9: 00$ & 11.475 & 11.368 & 8.366 & 7.326 & 4.591 & 4.69 & 3.018 & 2.958 & 10.82 & 6.091 & 5.03 & 6.909 & 3.811 \\
\hline $1 / 13 / 2006$ & $10: 00$ & 11.487 & 11.381 & 8.373 & 7.337 & 4.597 & 4.698 & 3.028 & 2.967 & 10.826 & 6.099 & 5.038 & 6.92 & 3.819 \\
\hline $1 / 13 / 2006$ & $11: 00$ & 11.494 & 11.388 & 8.377 & 7.339 & 4.601 & 4.703 & 3.032 & 2.971 & 10.83 & 6.101 & 5.042 & 6.924 & 3.824 \\
\hline $1 / 13 / 2006$ & $12: 00$ & 11.502 & 11.394 & 8.382 & 7.346 & 4.603 & 4.707 & 3.037 & 2.977 & 10.835 & 6.105 & 5.047 & 6.927 & 3.821 \\
\hline $1 / 13 / 2006$ & $13: 00$ & 11.505 & 11.394 & 8.387 & 7.344 & 4.598 & 4.709 & 3.035 & 2.975 & 10.839 & 6.1 & 5.043 & 6.926 & 3.806 \\
\hline $1 / 13 / 2006$ & $14: 00$ & 11.509 & 11.394 & 8.388 & 7.337 & 4.593 & 4.714 & 3.031 & 2.973 & 10.833 & 6.103 & 5.043 & 6.929 & 3.803 \\
\hline $1 / 13 / 2006$ & $15: 00$ & 11.512 & 11.399 & 8.39 & 7.344 & 4.604 & 4.716 & 3.034 & 2.977 & 10.841 & 6.103 & 5.05 & 6.935 & 3.803 \\
\hline $1 / 13 / 2006$ & $16: 00$ & 11.517 & 11.401 & 8.392 & 7.346 & 4.607 & 4.721 & 3.041 & 2.981 & 10.843 & 6.106 & 5.051 & 6.943 & 3.809 \\
\hline $1 / 13 / 2006$ & $17: 00$ & 11.522 & 11.404 & 8.394 & 7.35 & 4.609 & 4.727 & 3.045 & 2.983 & 10.848 & 6.112 & 5.054 & 6.946 & 3.811 \\
\hline $1 / 13 / 2006$ & $18: 00$ & 11.524 & 11.404 & 8.396 & 7.348 & 4.609 & 4.727 & 3.041 & 2.984 & 10.85 & 6.108 & 5.057 & 6.949 & 3.811 \\
\hline $1 / 13 / 2006$ & 19:00 & 11.528 & 11.408 & 8.397 & 7.352 & 4.612 & 4.732 & 3.044 & 2.984 & 10.852 & 6.113 & 5.061 & 6.952 & 3.814 \\
\hline $1 / 13 / 2006$ & $20: 00$ & 11.53 & 11.408 & 8.399 & 7.352 & 4.612 & 4.734 & 3.046 & 2.986 & 10.854 & 6.111 & 5.063 & 6.951 & 3.816 \\
\hline $1 / 13 / 2006$ & $21: 00$ & 11.531 & 11.41 & 8.402 & 7.355 & 4.615 & 4.738 & 3.048 & 2.989 & 10.854 & 6.113 & 5.068 & 6.952 & 3.825 \\
\hline $1 / 13 / 2006$ & $22: 00$ & 11.533 & 11.408 & 8.402 & 7.352 & 4.615 & 4.738 & 3.046 & 2.989 & 10.856 & 6.115 & 5.07 & 6.953 & 3.83 \\
\hline $1 / 13 / 2006$ & $23: 00$ & 11.533 & 11.41 & 8.404 & 7.352 & 4.616 & 4.74 & 3.047 & 2.989 & 10.856 & 6.112 & 5.074 & 6.956 & 3.836 \\
\hline $1 / 14 / 2006$ & $0: 00$ & 11.535 & 11.41 & 8.403 & 7.355 & 4.619 & 4.743 & 3.047 & 2.989 & 10.86 & 6.117 & 5.079 & 6.957 & 3.845 \\
\hline $1 / 14 / 2006$ & $1: 00$ & 11.538 & 11.414 & 8.407 & 7.359 & 4.622 & 4.747 & 3.053 & 2.996 & 10.86 & 6.119 & 5.086 & 6.956 & 3.852 \\
\hline $1 / 14 / 2006$ & $2: 00$ & 11.536 & 11.408 & 8.407 & 7.355 & 4.619 & 4.747 & 3.051 & 2.99 & 10.86 & 6.118 & 5.088 & 6.955 & 3.855 \\
\hline $1 / 14 / 2006$ & $3: 00$ & 11.538 & 11.408 & 8.405 & 7.352 & 4.623 & 4.747 & 3.049 & 2.991 & 10.862 & 6.118 & 5.093 & 6.959 & 3.863 \\
\hline $1 / 14 / 2006$ & $4: 00$ & 11.535 & 11.404 & 8.404 & 7.35 & 4.622 & 4.747 & 3.048 & 2.989 & 10.862 & 6.118 & 5.097 & 6.954 & 3.867 \\
\hline $1 / 14 / 2006$ & $5: 00$ & 11.527 & 11.392 & 8.398 & 7.339 & 4.616 & 4.745 & 3.039 & 2.982 & 10.858 & 6.118 & 5.095 & 6.952 & 3.868 \\
\hline $1 / 14 / 2006$ & $6: 00$ & 11.522 & 11.383 & 8.393 & 7.332 & 4.616 & 4.743 & 3.033 & 2.976 & 10.854 & 6.115 & 5.098 & 6.948 & 3.869 \\
\hline $1 / 14 / 2006$ & $7: 00$ & 11.521 & 11.384 & 8.393 & 7.335 & 4.621 & 4.743 & 3.035 & 2.977 & 10.856 & 6.117 & 5.105 & 6.951 & 3.877 \\
\hline $1 / 14 / 2006$ & $8: 00$ & 11.52 & 11.384 & 8.396 & 7.335 & 4.622 & 4.743 & 3.036 & 2.977 & 10.858 & 6.116 & 5.109 & 6.953 & 3.882 \\
\hline $1 / 14 / 2006$ & $9: 00$ & 11.515 & 11.377 & 8.393 & 7.33 & 4.618 & 4.74 & 3.034 & 2.975 & 10.856 & 6.116 & 5.111 & 6.948 & 3.882 \\
\hline
\end{tabular}


Water Level (ft below top of casing) at Indicated Well

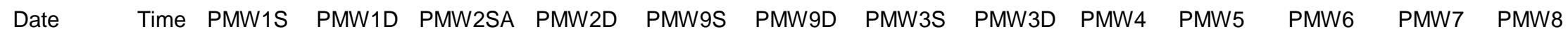

\begin{tabular}{|c|c|c|c|c|c|c|c|c|c|c|c|c|c|c|}
\hline $1 / 14 / 2006$ & $10: 00$ & 11.512 & 11.375 & 8.389 & 7.33 & 4.623 & 4.739 & 3.033 & 2.974 & 10.856 & 6.119 & 5.113 & 6.948 & 3.887 \\
\hline $1 / 14 / 2006$ & $11: 00$ & 11.507 & 11.368 & 8.388 & 7.324 & 4.621 & 4.736 & 3.027 & 2.968 & 10.858 & 6.115 & 5.113 & 6.947 & 3.886 \\
\hline $1 / 14 / 2006$ & $12: 00$ & 11.502 & 11.359 & 8.383 & 7.313 & 4.616 & 4.734 & 3.019 & 2.96 & 10.848 & 6.112 & 5.107 & 6.938 & 3.878 \\
\hline $1 / 14 / 2006$ & $13: 00$ & 11.491 & 11.344 & 8.374 & 7.301 & 4.606 & 4.725 & 3.006 & 2.945 & 10.848 & 6.102 & 5.102 & 6.934 & 3.86 \\
\hline $1 / 14 / 2006$ & $14: 00$ & 11.477 & 11.321 & 8.358 & 7.277 & 4.591 & 4.714 & 2.986 & 2.922 & 10.839 & 6.095 & 5.086 & 6.914 & 3.826 \\
\hline $1 / 14 / 2006$ & $15: 00$ & 11.463 & 11.304 & 8.35 & 7.27 & 4.585 & 4.707 & 2.97 & 2.91 & 10.831 & 6.09 & 5.067 & 6.911 & 3.792 \\
\hline $1 / 14 / 2006$ & $16: 00$ & 11.45 & 11.293 & 8.344 & 7.257 & 4.579 & 4.701 & 2.959 & 2.899 & 10.827 & 6.085 & 5.051 & 6.908 & 3.765 \\
\hline $1 / 14 / 2006$ & $17: 00$ & 11.441 & 11.286 & 8.337 & 7.253 & 4.575 & 4.692 & 2.955 & 2.894 & 10.826 & 6.081 & 5.034 & 6.896 & 3.754 \\
\hline $1 / 14 / 2006$ & $18: 00$ & 11.432 & 11.277 & 8.33 & 7.248 & 4.568 & 4.688 & 2.947 & 2.888 & 10.824 & 6.079 & 5.021 & 6.895 & 3.746 \\
\hline $1 / 14 / 2006$ & $19: 00$ & 11.423 & 11.271 & 8.326 & 7.242 & 4.561 & 4.681 & 2.942 & 2.881 & 10.82 & 6.078 & 5.014 & 6.892 & 3.746 \\
\hline $1 / 14 / 2006$ & $20: 00$ & 11.416 & 11.262 & 8.318 & 7.237 & 4.558 & 4.676 & 2.937 & 2.878 & 10.818 & 6.074 & 5.006 & 6.888 & 3.745 \\
\hline $1 / 14 / 2006$ & $21: 00$ & 11.411 & 11.255 & 8.314 & 7.231 & 4.551 & 4.672 & 2.931 & 2.872 & 10.812 & 6.074 & 4.998 & 6.884 & 3.744 \\
\hline $1 / 14 / 2006$ & $22: 00$ & 11.404 & 11.249 & 8.309 & 7.226 & 4.548 & 4.666 & 2.928 & 2.867 & 10.812 & 6.065 & 4.99 & 6.883 & 3.744 \\
\hline $1 / 14 / 2006$ & $23: 00$ & 11.394 & 11.24 & 8.302 & 7.217 & 4.54 & 4.661 & 2.92 & 2.86 & 10.808 & 6.068 & 4.982 & 6.876 & 3.744 \\
\hline $1 / 15 / 2006$ & $0: 00$ & 11.386 & 11.231 & 8.297 & 7.206 & 4.533 & 4.652 & 2.911 & 2.852 & 10.805 & 6.061 & 4.976 & 6.873 & 3.737 \\
\hline $1 / 15 / 2006$ & $1: 00$ & 11.379 & 11.22 & 8.289 & 7.2 & 4.528 & 4.647 & 2.906 & 2.844 & 10.799 & 6.058 & 4.972 & 6.87 & 3.735 \\
\hline $1 / 15 / 2006$ & $2: 00$ & 11.371 & 11.209 & 8.283 & 7.191 & 4.522 & 4.637 & 2.899 & 2.836 & 10.795 & 6.057 & 4.966 & 6.864 & 3.735 \\
\hline $1 / 15 / 2006$ & $3: 00$ & 11.359 & 11.2 & 8.276 & 7.184 & 4.517 & 4.632 & 2.89 & 2.829 & 10.791 & 6.052 & 4.961 & 6.86 & 3.735 \\
\hline $1 / 15 / 2006$ & $4: 00$ & 11.35 & 11.195 & 8.272 & 7.182 & 4.517 & 4.625 & 2.886 & 2.825 & 10.789 & 6.054 & 4.96 & 6.858 & 3.739 \\
\hline $1 / 15 / 2006$ & $5: 00$ & 11.344 & 11.184 & 8.262 & 7.171 & 4.507 & 4.619 & 2.878 & 2.816 & 10.786 & 6.052 & 4.953 & 6.849 & 3.733 \\
\hline $1 / 15 / 2006$ & $6: 00$ & 11.336 & 11.176 & 8.257 & 7.164 & 4.506 & 4.612 & 2.874 & 2.81 & 10.784 & 6.048 & 4.95 & 6.847 & 3.734 \\
\hline $1 / 15 / 2006$ & $7: 00$ & 11.332 & 11.175 & 8.254 & 7.169 & 4.509 & 4.61 & 2.874 & 2.813 & 10.782 & 6.051 & 4.949 & 6.847 & 3.741 \\
\hline $1 / 15 / 2006$ & $8: 00$ & 11.326 & 11.169 & 8.251 & 7.162 & 4.504 & 4.606 & 2.873 & 2.81 & 10.78 & 6.04 & 4.946 & 6.841 & 3.743 \\
\hline $1 / 15 / 2006$ & $9: 00$ & 11.323 & 11.167 & 8.249 & 7.16 & 4.505 & 4.604 & 2.869 & 2.809 & 10.78 & 6.04 & 4.947 & 6.842 & 3.747 \\
\hline $1 / 15 / 2006$ & $10: 00$ & 11.321 & 11.169 & 8.248 & 7.166 & 4.508 & 4.601 & 2.873 & 2.812 & 10.776 & 6.041 & 4.947 & 6.84 & 3.755 \\
\hline $1 / 15 / 2006$ & $11: 00$ & 11.317 & 11.166 & 8.246 & 7.16 & 4.503 & 4.597 & 2.869 & 2.807 & 10.776 & 6.041 & 4.946 & 6.839 & 3.756 \\
\hline $1 / 15 / 2006$ & $12: 00$ & 11.314 & 11.162 & 8.242 & 7.155 & 4.5 & 4.593 & 2.866 & 2.802 & 10.772 & 6.038 & 4.943 & 6.835 & 3.749 \\
\hline $1 / 15 / 2006$ & $13: 00$ & 11.31 & 11.155 & 8.24 & 7.149 & 4.495 & 4.586 & 2.859 & 2.795 & 10.772 & 6.037 & 4.937 & 6.833 & 3.738 \\
\hline $1 / 15 / 2006$ & $14: 00$ & 11.303 & 11.145 & 8.232 & 7.14 & 4.489 & 4.581 & 2.853 & 2.788 & 10.769 & 6.033 & 4.93 & 6.83 & 3.733 \\
\hline $1 / 15 / 2006$ & $15: 00$ & 11.295 & 11.138 & 8.229 & 7.133 & 4.486 & 4.577 & 2.847 & 2.781 & 10.766 & 6.033 & 4.925 & 6.826 & 3.731 \\
\hline $1 / 15 / 2006$ & $16: 00$ & 11.293 & 11.138 & 8.225 & 7.135 & 4.491 & 4.575 & 2.846 & 2.782 & 10.763 & 6.036 & 4.924 & 6.825 & 3.736 \\
\hline $1 / 15 / 2006$ & $17: 00$ & 11.288 & 11.139 & 8.226 & 7.14 & 4.493 & 4.575 & 2.849 & 2.787 & 10.762 & 6.035 & 4.922 & 6.821 & 3.745 \\
\hline $1 / 15 / 2006$ & $18: 00$ & 11.288 & 11.139 & 8.225 & 7.142 & 4.494 & 4.573 & 2.851 & 2.786 & 10.762 & 6.035 & 4.922 & 6.82 & 3.75 \\
\hline $1 / 15 / 2006$ & $19: 00$ & 11.287 & 11.14 & 8.224 & 7.142 & 4.494 & 4.573 & 2.851 & 2.787 & 10.762 & 6.036 & 4.92 & 6.815 & 3.751 \\
\hline
\end{tabular}


Water Level (ft below top of casing) at Indicated Well

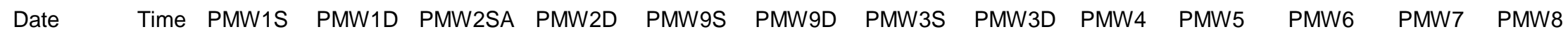

\begin{tabular}{|c|c|c|c|c|c|c|c|c|c|c|c|c|c|c|}
\hline $1 / 15 / 2006$ & $20: 00$ & 11.287 & 11.142 & 8.225 & 7.146 & 4.498 & 4.575 & 2.855 & 2.791 & 10.761 & 6.033 & 4.921 & 6.818 & 3.756 \\
\hline $1 / 15 / 2006$ & $21: 00$ & 11.287 & 11.142 & 8.225 & 7.144 & 4.496 & 4.573 & 2.853 & 2.79 & 10.761 & 6.036 & 4.919 & 6.816 & 3.754 \\
\hline $1 / 15 / 2006$ & $22: 00$ & 11.287 & 11.144 & 8.225 & 7.146 & 4.495 & 4.573 & 2.853 & 2.789 & 10.759 & 6.037 & 4.918 & 6.817 & 3.755 \\
\hline $1 / 15 / 2006$ & $23: 00$ & 11.29 & 11.149 & 8.227 & 7.151 & 4.498 & 4.575 & 2.857 & 2.795 & 10.759 & 6.036 & 4.918 & 6.815 & 3.758 \\
\hline $1 / 16 / 2006$ & $0: 00$ & 11.289 & 11.148 & 8.226 & 7.149 & 4.495 & 4.575 & 2.857 & 2.793 & 10.759 & 6.034 & 4.914 & 6.815 & 3.756 \\
\hline $1 / 16 / 2006$ & 1:00 & 11.291 & 11.151 & 8.228 & 7.151 & 4.497 & 4.575 & 2.857 & 2.795 & 10.759 & 6.035 & 4.914 & 6.815 & 3.757 \\
\hline $1 / 16 / 2006$ & $2: 00$ & 11.292 & 11.155 & 8.23 & 7.155 & 4.501 & 4.575 & 2.86 & 2.797 & 10.759 & 6.034 & 4.914 & 6.816 & 3.761 \\
\hline $1 / 16 / 2006$ & $3: 00$ & 11.301 & 11.17 & 8.233 & 7.164 & 4.508 & 4.579 & 2.872 & 2.806 & 10.759 & 6.042 & 4.919 & 6.819 & 3.766 \\
\hline $1 / 16 / 2006$ & $4: 00$ & 11.309 & 11.179 & 8.242 & 7.175 & 4.513 & 4.581 & 2.879 & 2.817 & 10.761 & 6.041 & 4.918 & 6.821 & 3.774 \\
\hline $1 / 16 / 2006$ & $5: 00$ & 11.322 & 11.191 & 8.249 & 7.184 & 4.519 & 4.588 & 2.885 & 2.825 & 10.762 & 6.041 & 4.924 & 6.824 & 3.778 \\
\hline $1 / 16 / 2006$ & $6: 00$ & 11.328 & 11.206 & 8.261 & 7.197 & 4.526 & 4.595 & 2.901 & 2.836 & 10.762 & 6.046 & 4.935 & 6.822 & 3.783 \\
\hline $1 / 16 / 2006$ & $7: 00$ & 11.337 & 11.217 & 8.269 & 7.206 & 4.535 & 4.599 & 2.911 & 2.847 & 10.757 & 6.048 & 4.933 & 6.825 & 3.785 \\
\hline $1 / 16 / 2006$ & 8:00 & 11.353 & 11.23 & 8.277 & 7.215 & 4.537 & 4.604 & 2.916 & 2.853 & 10.77 & 6.051 & 4.935 & 6.834 & 3.789 \\
\hline $1 / 16 / 2006$ & $9: 00$ & 11.363 & 11.242 & 8.284 & 7.226 & 4.543 & 4.61 & 2.924 & 2.862 & 10.774 & 6.055 & 4.941 & 6.842 & 3.793 \\
\hline $1 / 16 / 2006$ & $10: 00$ & 11.37 & 11.252 & 8.291 & 7.231 & 4.547 & 4.615 & 2.933 & 2.866 & 10.774 & 6.056 & 4.946 & 6.842 & 3.793 \\
\hline $1 / 16 / 2006$ & $11: 00$ & 11.38 & 11.264 & 8.296 & 7.242 & 4.552 & 4.624 & 2.939 & 2.878 & 10.778 & 6.058 & 4.947 & 6.846 & 3.794 \\
\hline $1 / 16 / 2006$ & $12: 00$ & 11.391 & 11.273 & 8.307 & 7.253 & 4.558 & 4.63 & 2.947 & 2.885 & 10.783 & 6.059 & 4.954 & 6.85 & 3.799 \\
\hline $1 / 16 / 2006$ & $13: 00$ & 11.398 & 11.283 & 8.311 & 7.255 & 4.562 & 4.635 & 2.954 & 2.891 & 10.778 & 6.065 & 4.959 & 6.852 & 3.798 \\
\hline $1 / 16 / 2006$ & $14: 00$ & 11.404 & 11.283 & 8.309 & 7.259 & 4.558 & 4.639 & 2.953 & 2.891 & 10.783 & 6.065 & 4.958 & 6.854 & 3.796 \\
\hline $1 / 16 / 2006$ & $15: 00$ & 11.411 & 11.255 & 8.316 & 7.226 & 4.563 & 4.643 & 2.956 & 2.895 & 10.789 & 6.068 & 4.964 & 6.854 & 3.795 \\
\hline $1 / 16 / 2006$ & $16: 00$ & 11.418 & 11.299 & 8.326 & 7.27 & 4.572 & 4.65 & 2.964 & 2.904 & 10.789 & 6.072 & 4.969 & 6.867 & 3.802 \\
\hline $1 / 16 / 2006$ & $17: 00$ & 11.428 & 11.315 & 8.332 & 7.284 & 4.579 & 4.661 & 2.98 & 2.914 & 10.795 & 6.072 & 4.979 & 6.866 & 3.808 \\
\hline $1 / 16 / 2006$ & $18: 00$ & 11.439 & 11.326 & 8.343 & 7.295 & 4.589 & 4.666 & 2.986 & 2.925 & 10.802 & 6.079 & 4.983 & 6.875 & 3.815 \\
\hline $1 / 16 / 2006$ & 19:00 & 11.449 & 11.334 & 8.349 & 7.301 & 4.589 & 4.672 & 2.995 & 2.933 & 10.802 & 6.081 & 4.986 & 6.881 & 3.818 \\
\hline $1 / 16 / 2006$ & $20: 00$ & 11.459 & 11.343 & 8.351 & 7.308 & 4.596 & 4.683 & 3.003 & 2.941 & 10.808 & 6.081 & 4.99 & 6.882 & 3.822 \\
\hline $1 / 16 / 2006$ & $21: 00$ & 11.464 & 11.35 & 8.36 & 7.313 & 4.598 & 4.685 & 3.003 & 2.945 & 10.812 & 6.084 & 4.997 & 6.887 & 3.824 \\
\hline $1 / 16 / 2006$ & $22: 00$ & 11.473 & 11.361 & 8.365 & 7.321 & 4.606 & 4.692 & 3.012 & 2.951 & 10.819 & 6.087 & 5.008 & 6.89 & 3.83 \\
\hline $1 / 16 / 2006$ & $23: 00$ & 11.48 & 11.366 & 8.372 & 7.324 & 4.612 & 4.701 & 3.017 & 2.957 & 10.823 & 6.09 & 5.012 & 6.895 & 3.834 \\
\hline $1 / 17 / 2006$ & $0: 00$ & 11.485 & 11.37 & 8.382 & 7.328 & 4.609 & 4.703 & 3.015 & 2.958 & 10.825 & 6.093 & 5.017 & 6.899 & 3.836 \\
\hline $1 / 17 / 2006$ & $1: 00$ & 11.491 & 11.372 & 8.378 & 7.326 & 4.611 & 4.708 & 3.019 & 2.959 & 10.827 & 6.091 & 5.02 & 6.905 & 3.839 \\
\hline $1 / 17 / 2006$ & $2: 00$ & 11.496 & 11.374 & 8.381 & 7.326 & 4.609 & 4.71 & 3.02 & 2.958 & 10.829 & 6.094 & 5.026 & 6.904 & 3.838 \\
\hline $1 / 17 / 2006$ & $3: 00$ & 11.498 & 11.374 & 8.382 & 7.328 & 4.611 & 4.714 & 3.022 & 2.961 & 10.833 & 6.098 & 5.031 & 6.906 & 3.842 \\
\hline $1 / 17 / 2006$ & $4: 00$ & 11.501 & 11.379 & 8.385 & 7.33 & 4.613 & 4.716 & 3.022 & 2.963 & 10.832 & 6.099 & 5.035 & 6.912 & 3.842 \\
\hline $1 / 17 / 2006$ & $5: 00$ & 11.502 & 11.377 & 8.384 & 7.328 & 4.614 & 4.719 & 3.023 & 2.962 & 10.834 & 6.096 & 5.038 & 6.912 & 3.847 \\
\hline
\end{tabular}


TABLE S1.2 (Cont.)

Water Level (ft below top of casing) at Indicated Well

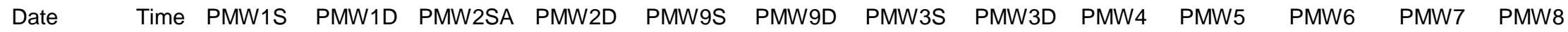

\begin{tabular}{|c|c|c|c|c|c|c|c|c|c|c|c|c|c|c|}
\hline $1 / 17 / 2006$ & $6: 00$ & 11.503 & 11.377 & 8.384 & 7.326 & 4.615 & 4.721 & 3.024 & 2.962 & 10.835 & 6.1 & 5.045 & 6.913 & 3.853 \\
\hline $1 / 17 / 2006$ & $7: 00$ & 11.501 & 11.376 & 8.386 & 7.328 & 4.616 & 4.721 & 3.023 & 2.962 & 10.837 & 6.098 & 5.048 & 6.913 & 3.857 \\
\hline $1 / 17 / 2006$ & $8: 00$ & 11.501 & 11.37 & 8.387 & 7.326 & 4.618 & 4.723 & 3.021 & 2.959 & 10.836 & 6.1 & 5.055 & 6.915 & 3.864 \\
\hline $1 / 17 / 2006$ & 9:00 & 11.501 & 11.372 & 8.387 & 7.326 & 4.62 & 4.725 & 3.026 & 2.962 & 10.838 & 6.101 & 5.061 & 6.917 & 3.874 \\
\hline $1 / 17 / 2006$ & $10: 00$ & 11.501 & 11.368 & 8.386 & 7.326 & 4.623 & 4.725 & 3.025 & 2.962 & 10.836 & 6.101 & 5.064 & 6.916 & 3.881 \\
\hline $1 / 17 / 2006$ & $11: 00$ & 11.5 & 11.366 & 8.389 & 7.321 & 4.618 & 4.726 & 3.02 & 2.962 & 10.838 & 6.102 & 5.069 & 6.915 & 3.884 \\
\hline $1 / 17 / 2006$ & $12: 00$ & 11.495 & 11.357 & 8.382 & 7.319 & 4.618 & 4.723 & 3.016 & 2.954 & 10.834 & 6.098 & 5.072 & 6.911 & 3.88 \\
\hline $1 / 17 / 2006$ & $13: 00$ & 11.486 & 11.345 & 8.373 & 7.304 & 4.612 & 4.719 & 3.005 & 2.942 & 10.829 & 6.096 & 5.068 & 6.906 & 3.867 \\
\hline $1 / 17 / 2006$ & $14: 00$ & 11.475 & 11.334 & 8.366 & 7.293 & 4.605 & 4.716 & 2.991 & 2.93 & 10.827 & 6.093 & 5.065 & 6.904 & 3.852 \\
\hline $1 / 17 / 2006$ & $15: 00$ & 11.471 & 11.326 & 8.358 & 7.288 & 4.605 & 4.715 & 2.986 & 2.923 & 10.827 & 6.091 & 5.059 & 6.901 & 3.835 \\
\hline $1 / 17 / 2006$ & $16: 00$ & 11.465 & 11.326 & 8.36 & 7.293 & 4.609 & 4.71 & 2.986 & 2.925 & 10.827 & 6.09 & 5.052 & 6.898 & 3.818 \\
\hline $1 / 17 / 2006$ & $17: 00$ & 11.469 & 11.332 & 8.359 & 7.297 & 4.607 & 4.71 & 2.991 & 2.93 & 10.827 & 6.091 & 5.044 & 6.9 & 3.806 \\
\hline $1 / 17 / 2006$ & $18: 00$ & 11.471 & 11.335 & 8.363 & 7.299 & 4.61 & 4.708 & 2.996 & 2.933 & 10.829 & 6.09 & 5.037 & 6.899 & 3.797 \\
\hline $1 / 17 / 2006$ & $19: 00$ & 11.469 & 11.334 & 8.359 & 7.297 & 4.608 & 4.708 & 2.989 & 2.93 & 10.829 & 6.088 & 5.028 & 6.899 & 3.795 \\
\hline $1 / 17 / 2006$ & $20: 00$ & 11.472 & 11.337 & 8.361 & 7.299 & 4.608 & 4.708 & 2.995 & 2.933 & 10.831 & 6.086 & 5.027 & 6.899 & 3.802 \\
\hline $1 / 17 / 2006$ & $21: 00$ & 11.47 & 11.334 & 8.359 & 7.295 & 4.606 & 4.708 & 2.991 & 2.93 & 10.829 & 6.09 & 5.024 & 6.9 & 3.809 \\
\hline $1 / 17 / 2006$ & $22: 00$ & 11.467 & 11.332 & 8.357 & 7.293 & 4.605 & 4.708 & 2.993 & 2.93 & 10.829 & 6.087 & 5.026 & 6.899 & 3.819 \\
\hline $1 / 17 / 2006$ & $23: 00$ & 11.468 & 11.335 & 8.361 & 7.297 & 4.609 & 4.71 & 2.997 & 2.935 & 10.829 & 6.092 & 5.03 & 6.902 & 3.835 \\
\hline $1 / 18 / 2006$ & $0: 00$ & 11.47 & 11.335 & 8.362 & 7.299 & 4.612 & 4.71 & 3.001 & 2.937 & 10.831 & 6.09 & 5.038 & 6.903 & 3.846 \\
\hline $1 / 18 / 2006$ & $1: 00$ & 11.468 & 11.33 & 8.359 & 7.297 & 4.61 & 4.71 & 2.998 & 2.936 & 10.831 & 6.089 & 5.039 & 6.904 & 3.855 \\
\hline $1 / 18 / 2006$ & $2: 00$ & 11.463 & 11.325 & 8.358 & 7.29 & 4.61 & 4.708 & 2.994 & 2.93 & 10.827 & 6.091 & 5.048 & 6.902 & 3.865 \\
\hline $1 / 18 / 2006$ & $3: 00$ & 11.463 & 11.326 & 8.359 & 7.293 & 4.615 & 4.71 & 2.999 & 2.934 & 10.829 & 6.092 & 5.06 & 6.904 & 3.877 \\
\hline $1 / 18 / 2006$ & $4: 00$ & 11.46 & 11.325 & 8.356 & 7.29 & 4.616 & 4.708 & 2.995 & 2.933 & 10.827 & 6.094 & 5.066 & 6.902 & 3.883 \\
\hline $1 / 18 / 2006$ & $5: 00$ & 11.455 & 11.312 & 8.354 & 7.279 & 4.61 & 4.706 & 2.987 & 2.925 & 10.825 & 6.086 & 5.067 & 6.899 & 3.881 \\
\hline $1 / 18 / 2006$ & $6: 00$ & 11.448 & 11.304 & 8.348 & 7.275 & 4.608 & 4.701 & 2.983 & 2.917 & 10.824 & 6.088 & 5.067 & 6.896 & 3.883 \\
\hline $1 / 18 / 2006$ & $7: 00$ & 11.447 & 11.308 & 8.348 & 7.277 & 4.614 & 4.704 & 2.985 & 2.923 & 10.821 & 6.092 & 5.078 & 6.901 & 3.892 \\
\hline $1 / 18 / 2006$ & $8: 00$ & 11.45 & 11.312 & 8.352 & 7.284 & 4.618 & 4.704 & 2.99 & 2.927 & 10.823 & 6.091 & 5.079 & 6.898 & 3.898 \\
\hline $1 / 18 / 2006$ & $9: 00$ & 11.451 & 11.314 & 8.352 & 7.286 & 4.62 & 4.704 & 2.992 & 2.93 & 10.823 & 6.092 & 5.084 & 6.904 & 3.9 \\
\hline $1 / 18 / 2006$ & $10: 00$ & 11.455 & 11.319 & 8.358 & 7.293 & 4.623 & 4.706 & 2.999 & 2.934 & 10.827 & 6.097 & 5.088 & 6.906 & 3.905 \\
\hline $1 / 18 / 2006$ & $11: 00$ & 11.459 & 11.325 & 8.358 & 7.295 & 4.626 & 4.705 & 3.001 & 2.938 & 10.827 & 6.098 & 5.093 & 6.907 & 3.908 \\
\hline $1 / 18 / 2006$ & $12: 00$ & 11.458 & 11.321 & 8.36 & 7.29 & 4.621 & 4.706 & 2.998 & 2.934 & 10.827 & 6.093 & 5.09 & 6.904 & 3.9 \\
\hline $1 / 18 / 2006$ & $13: 00$ & 11.449 & 11.31 & 8.354 & 7.282 & 4.614 & 4.701 & 2.988 & 2.923 & 10.824 & 6.089 & 5.084 & 6.902 & 3.887 \\
\hline $1 / 18 / 2006$ & $14: 00$ & 11.442 & 11.297 & 8.347 & 7.268 & 4.607 & 4.697 & 2.975 & 2.909 & 10.821 & 6.084 & 5.077 & 6.895 & 3.872 \\
\hline $1 / 18 / 2006$ & $15: 00$ & 11.436 & 11.292 & 8.341 & 7.264 & 4.606 & 4.695 & 2.968 & 2.903 & 10.817 & 6.086 & 5.07 & 6.892 & 3.856 \\
\hline
\end{tabular}


TABLE S1.2 (Cont.)

Water Level (ft below top of casing) at Indicated Well

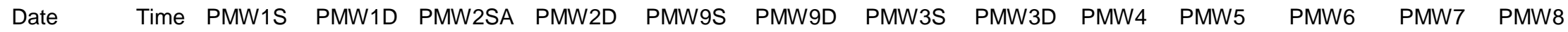

\begin{tabular}{|c|c|c|c|c|c|c|c|c|c|c|c|c|c|c|}
\hline $1 / 18 / 2006$ & $16: 00$ & 11.428 & 11.284 & 8.334 & 7.257 & 4.602 & 4.688 & 2.96 & 2.893 & 10.814 & 6.079 & 5.06 & 6.886 & 3.83 \\
\hline $1 / 18 / 2006$ & $17: 00$ & 11.425 & 11.281 & 8.329 & 7.253 & 4.597 & 4.686 & 2.953 & 2.889 & 10.812 & 6.078 & 5.042 & 6.88 & 3.803 \\
\hline 1/18/2006 & $18: 00$ & 11.419 & 11.272 & 8.323 & 7.246 & 4.588 & 4.679 & 2.944 & 2.882 & 10.808 & 6.071 & 5.026 & 6.879 & 3.777 \\
\hline 1/18/2006 & 19:00 & 11.414 & 11.27 & 8.32 & 7.246 & 4.587 & 4.677 & 2.942 & 2.88 & 10.809 & 6.072 & 5.016 & 6.875 & 3.761 \\
\hline $1 / 18 / 2006$ & $20: 00$ & 11.417 & 11.276 & 8.322 & 7.253 & 4.592 & 4.677 & 2.95 & 2.885 & 10.809 & 6.073 & 5.006 & 6.875 & 3.757 \\
\hline $1 / 18 / 2006$ & $21: 00$ & 11.418 & 11.277 & 8.321 & 7.253 & 4.587 & 4.675 & 2.949 & 2.886 & 10.809 & 6.069 & 4.998 & 6.872 & 3.752 \\
\hline 1/18/2006 & $22: 00$ & 11.412 & 11.272 & 8.318 & 7.248 & 4.579 & 4.673 & 2.944 & 2.882 & 10.805 & 6.064 & 4.989 & 6.867 & 3.747 \\
\hline 1/18/2006 & 23:00 & 11.414 & 11.272 & 8.316 & 7.248 & 4.577 & 4.673 & 2.945 & 2.882 & 10.805 & 6.066 & 4.981 & 6.867 & 3.745 \\
\hline 1/19/2006 & 0:00 & 11.41 & 11.27 & 8.317 & 7.246 & 4.573 & 4.67 & 2.943 & 2.879 & 10.803 & 6.066 & 4.978 & 6.866 & 3.746 \\
\hline $1 / 19 / 2006$ & $1: 00$ & 11.41 & 11.27 & 8.315 & 7.246 & 4.572 & 4.668 & 2.942 & 2.881 & 10.804 & 6.062 & 4.974 & 6.868 & 3.748 \\
\hline 1/19/2006 & $2: 00$ & 11.407 & 11.263 & 8.312 & 7.237 & 4.563 & 4.666 & 2.938 & 2.873 & 10.802 & 6.059 & 4.964 & 6.864 & 3.744 \\
\hline $1 / 19 / 2006$ & $3: 00$ & 11.402 & 11.261 & 8.31 & 7.237 & 4.563 & 4.664 & 2.933 & 2.869 & 10.8 & 6.062 & 4.966 & 6.864 & 3.747 \\
\hline 1/19/2006 & $4: 00$ & 11.404 & 11.264 & 8.311 & 7.244 & 4.567 & 4.664 & 2.94 & 2.878 & 10.801 & 6.059 & 4.966 & 6.863 & 3.757 \\
\hline $1 / 19 / 2006$ & $5: 00$ & 11.404 & 11.265 & 8.31 & 7.244 & 4.566 & 4.664 & 2.939 & 2.877 & 10.798 & 6.063 & 4.963 & 6.863 & 3.762 \\
\hline 1/19/2006 & $6: 00$ & 11.403 & 11.263 & 8.31 & 7.239 & 4.563 & 4.662 & 2.94 & 2.878 & 10.799 & 6.061 & 4.963 & 6.862 & 3.768 \\
\hline $1 / 19 / 2006$ & $7: 00$ & 11.399 & 11.259 & 8.306 & 7.235 & 4.558 & 4.659 & 2.935 & 2.87 & 10.796 & 6.062 & 4.964 & 6.862 & 3.772 \\
\hline $1 / 19 / 2006$ & $8: 00$ & 11.401 & 11.265 & 8.308 & 7.242 & 4.566 & 4.662 & 2.941 & 2.877 & 10.797 & 6.062 & 4.968 & 6.863 & 3.784 \\
\hline $1 / 19 / 2006$ & $9: 00$ & 11.406 & 11.27 & 8.314 & 7.251 & 4.568 & 4.662 & 2.946 & 2.885 & 10.797 & 6.066 & 4.97 & 6.866 & 3.791 \\
\hline $1 / 19 / 2006$ & $10: 00$ & 11.421 & 11.294 & 8.326 & 7.273 & 4.588 & 4.67 & 2.969 & 2.906 & 10.804 & 6.076 & 4.984 & 6.876 & 3.81 \\
\hline $1 / 19 / 2006$ & $11: 00$ & 11.433 & 11.308 & 8.336 & 7.282 & 4.592 & 4.673 & 2.979 & 2.916 & 10.805 & 6.077 & 4.991 & 6.876 & 3.815 \\
\hline $1 / 19 / 2006$ & $12: 00$ & 11.445 & 11.321 & 8.344 & 7.29 & 4.594 & 4.677 & 2.986 & 2.923 & 10.809 & 6.077 & 4.993 & 6.881 & 3.813 \\
\hline 1/19/2006 & $13: 00$ & 11.45 & 11.325 & 8.346 & 7.29 & 4.593 & 4.679 & 2.984 & 2.922 & 10.811 & 6.075 & 4.989 & 6.882 & 3.794 \\
\hline 1/19/2006 & $14: 00$ & 11.455 & 11.332 & 8.351 & 7.297 & 4.599 & 4.684 & 2.988 & 2.925 & 10.812 & 6.079 & 4.985 & 6.884 & 3.777 \\
\hline $1 / 19 / 2006$ & $15: 00$ & 11.466 & 11.339 & 8.355 & 7.304 & 4.6 & 4.686 & 2.994 & 2.93 & 10.814 & 6.082 & 4.984 & 6.883 & 3.768 \\
\hline $1 / 19 / 2006$ & $16: 00$ & 11.474 & 11.352 & 8.361 & 7.313 & 4.604 & 4.693 & 3.005 & 2.938 & 10.82 & 6.083 & 4.98 & 6.886 & 3.765 \\
\hline 1/19/2006 & $17: 00$ & 11.482 & 11.361 & 8.369 & 7.319 & 4.608 & 4.699 & 3.014 & 2.948 & 10.82 & 6.083 & 4.979 & 6.892 & 3.766 \\
\hline $1 / 19 / 2006$ & $18: 00$ & 11.492 & 11.372 & 8.374 & 7.33 & 4.612 & 4.704 & 3.02 & 2.958 & 10.824 & 6.086 & 4.977 & 6.898 & 3.771 \\
\hline 1/19/2006 & 19:00 & 11.507 & 11.391 & 8.386 & 7.348 & 4.625 & 4.715 & 3.033 & 2.972 & 10.831 & 6.095 & 4.984 & 6.905 & 3.783 \\
\hline $1 / 19 / 2006$ & $20: 00$ & 11.516 & 11.401 & 8.393 & 7.357 & 4.626 & 4.719 & 3.041 & 2.982 & 10.835 & 6.093 & 4.986 & 6.909 & 3.787 \\
\hline 1/19/2006 & $21: 00$ & 11.525 & 11.414 & 8.401 & 7.366 & 4.633 & 4.73 & 3.056 & 2.992 & 10.841 & 6.098 & 4.988 & 6.916 & 3.794 \\
\hline $1 / 19 / 2006$ & $22: 00$ & 11.533 & 11.423 & 8.408 & 7.375 & 4.636 & 4.737 & 3.062 & 2.998 & 10.845 & 6.102 & 4.993 & 6.92 & 3.797 \\
\hline $1 / 19 / 2006$ & $23: 00$ & 11.543 & 11.432 & 8.414 & 7.379 & 4.639 & 4.746 & 3.066 & 3.005 & 10.849 & 6.106 & 4.996 & 6.923 & 3.803 \\
\hline $1 / 20 / 2006$ & $0: 00$ & 11.55 & 11.442 & 8.42 & 7.388 & 4.645 & 4.75 & 3.073 & 3.011 & 10.85 & 6.11 & 4.997 & 6.928 & 3.804 \\
\hline $1 / 20 / 2006$ & $1: 00$ & 11.555 & 11.44 & 8.423 & 7.382 & 4.638 & 4.755 & 3.066 & 3.007 & 10.854 & 6.106 & 4.993 & 6.926 & 3.8 \\
\hline
\end{tabular}


Water Level (ft below top of casing) at Indicated Well

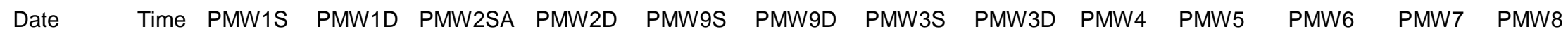

\begin{tabular}{|c|c|c|c|c|c|c|c|c|c|c|c|c|c|c|}
\hline $1 / 20 / 2006$ & $2: 00$ & 11.559 & 11.443 & 8.423 & 7.382 & 4.638 & 4.759 & 3.067 & 3.007 & 10.856 & 6.107 & 4.996 & 6.929 & 3.803 \\
\hline $1 / 20 / 2006$ & $3: 00$ & 11.562 & 11.438 & 8.422 & 7.377 & 4.634 & 4.761 & 3.063 & 2.998 & 10.856 & 6.103 & 4.995 & 6.928 & 3.801 \\
\hline $1 / 20 / 2006$ & $4: 00$ & 11.563 & 11.445 & 8.425 & 7.384 & 4.638 & 4.763 & 3.068 & 3.009 & 10.86 & 6.11 & 4.999 & 6.932 & 3.806 \\
\hline $1 / 20 / 2006$ & $5: 00$ & 11.57 & 11.454 & 8.433 & 7.392 & 4.646 & 4.77 & 3.077 & 3.018 & 10.864 & 6.111 & 5.006 & 6.936 & 3.812 \\
\hline $1 / 20 / 2006$ & $6: 00$ & 11.572 & 11.451 & 8.431 & 7.386 & 4.641 & 4.77 & 3.071 & 3.01 & 10.864 & 6.109 & 5.002 & 6.934 & 3.803 \\
\hline $1 / 20 / 2006$ & $7: 00$ & 11.573 & 11.451 & 8.431 & 7.386 & 4.642 & 4.77 & 3.072 & 3.009 & 10.864 & 6.113 & 5.001 & 6.935 & 3.8 \\
\hline $1 / 20 / 2006$ & 8:00 & 11.576 & 11.458 & 8.431 & 7.393 & 4.648 & 4.774 & 3.077 & 3.015 & 10.866 & 6.117 & 5.006 & 6.939 & 3.809 \\
\hline $1 / 20 / 2006$ & $9: 00$ & 11.58 & 11.463 & 8.439 & 7.399 & 4.651 & 4.777 & 3.086 & 3.023 & 10.868 & 6.116 & 5.011 & 6.941 & 3.811 \\
\hline $1 / 20 / 2006$ & $10: 00$ & 11.585 & 11.465 & 8.44 & 7.401 & 4.654 & 4.781 & 3.087 & 3.024 & 10.869 & 6.119 & 5.014 & 6.945 & 3.811 \\
\hline $1 / 20 / 2006$ & $11: 00$ & 11.585 & 11.463 & 8.441 & 7.397 & 4.652 & 4.783 & 3.083 & 3.022 & 10.87 & 6.115 & 5.012 & 6.943 & 3.808 \\
\hline $1 / 20 / 2006$ & $12: 00$ & 11.586 & 11.46 & 8.44 & 7.395 & 4.649 & 4.781 & 3.08 & 3.017 & 10.871 & 6.115 & 5.011 & 6.939 & 3.805 \\
\hline $1 / 20 / 2006$ & $13: 00$ & 11.577 & 11.447 & 8.434 & 7.381 & 4.64 & 4.779 & 3.066 & 3.006 & 10.866 & 6.11 & 5.003 & 6.936 & 3.799 \\
\hline $1 / 20 / 2006$ & $14: 00$ & 11.573 & 11.434 & 8.43 & 7.37 & 4.636 & 4.781 & 3.065 & 3.001 & 10.865 & 6.112 & 5.003 & 6.932 & 3.796 \\
\hline $1 / 20 / 2006$ & $15: 00$ & 11.57 & 11.436 & 8.427 & 7.352 & 4.638 & 4.779 & 3.061 & 2.998 & 10.864 & 6.11 & 5 & 6.929 & 3.792 \\
\hline $1 / 20 / 2006$ & $16: 00$ & 11.572 & 11.44 & 8.431 & 7.381 & 4.642 & 4.781 & 3.067 & 3.005 & 10.869 & 6.111 & 5.001 & 6.935 & 3.797 \\
\hline $1 / 20 / 2006$ & $17: 00$ & 11.579 & 11.453 & 8.436 & 7.393 & 4.651 & 4.783 & 3.074 & 3.016 & 10.869 & 6.12 & 5.007 & 6.938 & 3.802 \\
\hline $1 / 20 / 2006$ & $18: 00$ & 11.586 & 11.462 & 8.442 & 7.397 & 4.653 & 4.785 & 3.084 & 3.022 & 10.872 & 6.121 & 5.008 & 6.941 & 3.804 \\
\hline $1 / 20 / 2006$ & 19:00 & 11.589 & 11.467 & 8.443 & 7.401 & 4.656 & 4.788 & 3.088 & 3.027 & 10.873 & 6.123 & 5.009 & 6.945 & 3.808 \\
\hline $1 / 20 / 2006$ & $20: 00$ & 11.592 & 11.471 & 8.445 & 7.406 & 4.66 & 4.792 & 3.092 & 3.032 & 10.875 & 6.125 & 5.012 & 6.948 & 3.811 \\
\hline $1 / 20 / 2006$ & $21: 00$ & 11.592 & 11.471 & 8.448 & 7.406 & 4.658 & 4.794 & 3.092 & 3.032 & 10.875 & 6.124 & 5.015 & 6.948 & 3.811 \\
\hline $1 / 20 / 2006$ & $22: 00$ & 11.596 & 11.474 & 8.45 & 7.41 & 4.661 & 4.797 & 3.098 & 3.035 & 10.877 & 6.126 & 5.02 & 6.951 & 3.82 \\
\hline $1 / 20 / 2006$ & $23: 00$ & 11.598 & 11.478 & 8.453 & 7.415 & 4.664 & 4.801 & 3.102 & 3.04 & 10.877 & 6.129 & 5.026 & 6.952 & 3.83 \\
\hline $1 / 21 / 2006$ & $0: 00$ & 11.602 & 11.482 & 8.455 & 7.417 & 4.666 & 4.803 & 3.104 & 3.044 & 10.879 & 6.126 & 5.036 & 6.956 & 3.844 \\
\hline $1 / 21 / 2006$ & $1: 00$ & 11.608 & 11.485 & 8.456 & 7.419 & 4.668 & 4.808 & 3.111 & 3.045 & 10.882 & 6.131 & 5.044 & 6.96 & 3.853 \\
\hline $1 / 21 / 2006$ & $2: 00$ & 11.61 & 11.487 & 8.46 & 7.421 & 4.669 & 4.81 & 3.114 & 3.049 & 10.884 & 6.133 & 5.05 & 6.961 & 3.862 \\
\hline $1 / 21 / 2006$ & $3: 00$ & 11.611 & 11.491 & 8.46 & 7.421 & 4.672 & 4.812 & 3.113 & 3.049 & 10.885 & 6.135 & 5.06 & 6.963 & 3.872 \\
\hline $1 / 21 / 2006$ & $4: 00$ & 11.613 & 11.491 & 8.462 & 7.423 & 4.671 & 4.814 & 3.115 & 3.051 & 10.884 & 6.133 & 5.066 & 6.964 & 3.879 \\
\hline $1 / 21 / 2006$ & $5: 00$ & 11.614 & 11.489 & 8.462 & 7.419 & 4.672 & 4.817 & 3.113 & 3.05 & 10.885 & 6.135 & 5.071 & 6.966 & 3.884 \\
\hline $1 / 21 / 2006$ & $6: 00$ & 11.615 & 11.491 & 8.464 & 7.424 & 4.676 & 4.816 & 3.117 & 3.053 & 10.886 & 6.139 & 5.08 & 6.966 & 3.891 \\
\hline $1 / 21 / 2006$ & $7: 00$ & 11.616 & 11.491 & 8.464 & 7.421 & 4.677 & 4.819 & 3.114 & 3.053 & 10.886 & 6.138 & 5.087 & 6.969 & 3.896 \\
\hline $1 / 21 / 2006$ & $8: 00$ & 11.62 & 11.494 & 8.467 & 7.428 & 4.681 & 4.821 & 3.121 & 3.058 & 10.89 & 6.138 & 5.095 & 6.972 & 3.905 \\
\hline $1 / 21 / 2006$ & $9: 00$ & 11.621 & 11.496 & 8.468 & 7.428 & 4.683 & 4.823 & 3.123 & 3.061 & 10.89 & 6.142 & 5.102 & 6.974 & 3.91 \\
\hline $1 / 21 / 2006$ & $10: 00$ & 11.624 & 11.502 & 8.473 & 7.437 & 4.691 & 4.828 & 3.128 & 3.067 & 10.89 & 6.145 & 5.112 & 6.979 & 3.918 \\
\hline $1 / 21 / 2006$ & $11: 00$ & 11.63 & 11.505 & 8.475 & 7.439 & 4.691 & 4.828 & 3.135 & 3.071 & 10.892 & 6.147 & 5.118 & 6.981 & 3.922 \\
\hline
\end{tabular}


Water Level (ft below top of casing) at Indicated Well

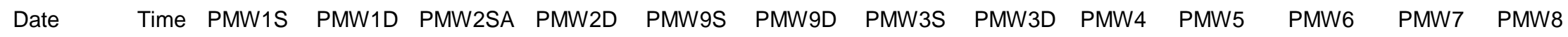

\begin{tabular}{|c|c|c|c|c|c|c|c|c|c|c|c|c|c|c|}
\hline $1 / 21 / 2006$ & $12: 00$ & 11.629 & 11.502 & 8.478 & 7.433 & 4.688 & 4.828 & 3.128 & 3.066 & 10.892 & 6.146 & 5.121 & 7.009 & 3.921 \\
\hline $1 / 21 / 2006$ & $13: 00$ & 11.626 & 11.493 & 8.47 & 7.421 & 4.683 & 4.828 & 3.119 & 3.057 & 10.89 & 6.143 & 5.118 & 6.974 & 3.912 \\
\hline $1 / 21 / 2006$ & $14: 00$ & 11.619 & 11.485 & 8.47 & 7.419 & 4.682 & 4.824 & 3.112 & 3.049 & 10.892 & 6.144 & 5.115 & 6.974 & 3.901 \\
\hline $1 / 21 / 2006$ & $15: 00$ & 11.612 & 11.476 & 8.464 & 7.408 & 4.681 & 4.821 & 3.102 & 3.038 & 10.891 & 6.143 & 5.106 & 6.971 & 3.88 \\
\hline $1 / 21 / 2006$ & $16: 00$ & 11.608 & 11.471 & 8.46 & 7.408 & 4.679 & 4.817 & 3.098 & 3.031 & 10.89 & 6.138 & 5.094 & 6.967 & 3.853 \\
\hline $1 / 21 / 2006$ & $17: 00$ & 11.608 & 11.473 & 8.459 & 7.406 & 4.678 & 4.815 & 3.097 & 3.035 & 10.892 & 6.134 & 5.079 & 6.964 & 3.826 \\
\hline $1 / 21 / 2006$ & $18: 00$ & 11.61 & 11.478 & 8.46 & 7.412 & 4.681 & 4.817 & 3.102 & 3.039 & 10.89 & 6.14 & 5.07 & 6.96 & 3.811 \\
\hline $1 / 21 / 2006$ & $19: 00$ & 11.612 & 11.478 & 8.462 & 7.415 & 4.682 & 4.817 & 3.102 & 3.038 & 10.894 & 6.138 & 5.061 & 6.963 & 3.804 \\
\hline $1 / 21 / 2006$ & $20: 00$ & 11.614 & 11.482 & 8.462 & 7.417 & 4.678 & 4.815 & 3.103 & 3.039 & 10.894 & 6.138 & 5.055 & 6.959 & 3.8 \\
\hline $1 / 21 / 2006$ & $21: 00$ & 11.617 & 11.487 & 8.466 & 7.424 & 4.681 & 4.817 & 3.109 & 3.046 & 10.896 & 6.14 & 5.05 & 6.966 & 3.802 \\
\hline $1 / 21 / 2006$ & $22: 00$ & 11.622 & 11.489 & 8.47 & 7.426 & 4.68 & 4.819 & 3.111 & 3.049 & 10.894 & 6.139 & 5.047 & 6.964 & 3.803 \\
\hline $1 / 21 / 2006$ & $23: 00$ & 11.623 & 11.493 & 8.468 & 7.424 & 4.681 & 4.819 & 3.117 & 3.053 & 10.896 & 6.141 & 5.043 & 6.97 & 3.81 \\
\hline $1 / 22 / 2006$ & 0:00 & 11.624 & 11.491 & 8.47 & 7.426 & 4.68 & 4.821 & 3.114 & 3.051 & 10.896 & 6.14 & 5.044 & 6.966 & 3.814 \\
\hline $1 / 22 / 2006$ & $1: 00$ & 11.624 & 11.493 & 8.472 & 7.424 & 4.68 & 4.821 & 3.116 & 3.053 & 10.895 & 6.142 & 5.046 & 6.97 & 3.82 \\
\hline $1 / 22 / 2006$ & $2: 00$ & 11.627 & 11.494 & 8.474 & 7.43 & 4.683 & 4.826 & 3.118 & 3.055 & 10.897 & 6.139 & 5.049 & 6.971 & 3.827 \\
\hline $1 / 22 / 2006$ & 3:00 & 11.63 & 11.498 & 8.475 & 7.433 & 4.685 & 4.826 & 3.123 & 3.059 & 10.901 & 6.146 & 5.052 & 6.971 & 3.834 \\
\hline $1 / 22 / 2006$ & $4: 00$ & 11.631 & 11.5 & 8.475 & 7.434 & 4.688 & 4.828 & 3.125 & 3.063 & 10.899 & 6.146 & 5.057 & 6.972 & 3.84 \\
\hline $1 / 22 / 2006$ & $5: 00$ & 11.627 & 11.494 & 8.477 & 7.43 & 4.685 & 4.828 & 3.121 & 3.058 & 10.899 & 6.14 & 5.057 & 6.972 & 3.843 \\
\hline $1 / 22 / 2006$ & $6: 00$ & 11.627 & 11.493 & 8.474 & 7.428 & 4.685 & 4.828 & 3.118 & 3.056 & 10.901 & 6.146 & 5.061 & 6.977 & 3.848 \\
\hline $1 / 22 / 2006$ & $7: 00$ & 11.627 & 11.495 & 8.477 & 7.433 & 4.689 & 4.83 & 3.124 & 3.06 & 10.902 & 6.145 & 5.066 & 6.978 & 3.854 \\
\hline $1 / 22 / 2006$ & 8:00 & 11.633 & 11.509 & 8.481 & 7.444 & 4.7 & 4.835 & 3.135 & 3.073 & 10.905 & 6.155 & 5.078 & 6.986 & 3.869 \\
\hline $1 / 22 / 2006$ & $9: 00$ & 11.635 & 11.511 & 8.483 & 7.444 & 4.7 & 4.837 & 3.137 & 3.073 & 10.907 & 6.152 & 5.082 & 6.985 & 3.878 \\
\hline $1 / 22 / 2006$ & $10: 00$ & 11.638 & 11.515 & 8.486 & 7.45 & 4.703 & 4.841 & 3.144 & 3.079 & 10.909 & 6.155 & 5.092 & 6.989 & 3.885 \\
\hline $1 / 22 / 2006$ & $11: 00$ & 11.642 & 11.518 & 8.49 & 7.452 & 4.704 & 4.841 & 3.144 & 3.082 & 10.909 & 6.156 & 5.102 & 6.99 & 3.893 \\
\hline $1 / 22 / 2006$ & $12: 00$ & 11.645 & 11.522 & 8.493 & 7.454 & 4.703 & 4.846 & 3.147 & 3.081 & 10.909 & 6.159 & 5.105 & 6.991 & 3.896 \\
\hline $1 / 22 / 2006$ & $13: 00$ & 11.643 & 11.513 & 8.49 & 7.446 & 4.697 & 4.843 & 3.135 & 3.074 & 10.91 & 6.154 & 5.099 & 6.985 & 3.883 \\
\hline $1 / 22 / 2006$ & $14: 00$ & 11.633 & 11.5 & 8.485 & 7.433 & 4.689 & 4.839 & 3.122 & 3.06 & 10.909 & 6.146 & 5.087 & 6.98 & 3.859 \\
\hline $1 / 22 / 2006$ & $15: 00$ & 11.631 & 11.498 & 8.48 & 7.43 & 4.688 & 4.837 & 3.118 & 3.054 & 10.905 & 6.143 & 5.075 & 6.977 & 3.834 \\
\hline $1 / 22 / 2006$ & $16: 00$ & 11.629 & 11.494 & 8.479 & 7.43 & 4.686 & 4.837 & 3.118 & 3.053 & 10.906 & 6.143 & 5.065 & 6.978 & 3.813 \\
\hline $1 / 22 / 2006$ & $17: 00$ & 11.633 & 11.5 & 8.481 & 7.437 & 4.692 & 4.837 & 3.124 & 3.059 & 10.909 & 6.147 & 5.064 & 6.98 & 3.809 \\
\hline $1 / 22 / 2006$ & $18: 00$ & 11.638 & 11.509 & 8.485 & 7.444 & 4.693 & 4.837 & 3.131 & 3.066 & 10.912 & 6.144 & 5.06 & 6.984 & 3.806 \\
\hline $1 / 22 / 2006$ & $19: 00$ & 11.639 & 11.506 & 8.486 & 7.443 & 4.69 & 4.837 & 3.131 & 3.064 & 10.912 & 6.146 & 5.053 & 6.982 & 3.804 \\
\hline $1 / 22 / 2006$ & $20: 00$ & 11.639 & 11.511 & 8.488 & 7.443 & 4.693 & 4.839 & 3.131 & 3.068 & 10.912 & 6.146 & 5.052 & 6.986 & 3.809 \\
\hline $1 / 22 / 2006$ & $21: 00$ & 11.639 & 11.506 & 8.486 & 7.441 & 4.687 & 4.839 & 3.126 & 3.064 & 10.911 & 6.144 & 5.048 & 6.981 & 3.807 \\
\hline
\end{tabular}


TABLE S1.2 (Cont.)

Water Level (ft below top of casing) at Indicated Well

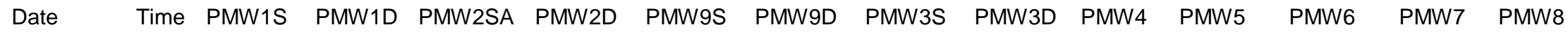

\begin{tabular}{|c|c|c|c|c|c|c|c|c|c|c|c|c|c|c|}
\hline $1 / 22 / 2006$ & $22: 00$ & 11.635 & 11.5 & 8.484 & 7.434 & 4.685 & 4.837 & 3.125 & 3.061 & 10.911 & 6.145 & 5.049 & 6.983 & 3.811 \\
\hline $1 / 22 / 2006$ & $23: 00$ & 11.634 & 11.5 & 8.482 & 7.435 & 4.686 & 4.837 & 3.126 & 3.061 & 10.912 & 6.146 & 5.048 & 6.984 & 3.816 \\
\hline $1 / 23 / 2006$ & $0: 00$ & 11.632 & 11.493 & 8.48 & 7.43 & 4.682 & 4.837 & 3.119 & 3.058 & 10.91 & 6.145 & 5.045 & 6.982 & 3.817 \\
\hline $1 / 23 / 2006$ & $1: 00$ & 11.625 & 11.486 & 8.475 & 7.424 & 4.679 & 4.832 & 3.116 & 3.052 & 10.91 & 6.142 & 5.045 & 6.979 & 3.818 \\
\hline $1 / 23 / 2006$ & $2: 00$ & 11.617 & 11.478 & 8.472 & 7.419 & 4.676 & 4.832 & 3.111 & 3.046 & 10.908 & 6.139 & 5.045 & 6.978 & 3.818 \\
\hline $1 / 23 / 2006$ & 3:00 & 11.616 & 11.478 & 8.471 & 7.421 & 4.677 & 4.832 & 3.112 & 3.048 & 10.907 & 6.141 & 5.049 & 6.978 & 3.827 \\
\hline $1 / 23 / 2006$ & 4:00 & 11.613 & 11.475 & 8.468 & 7.417 & 4.675 & 4.83 & 3.107 & 3.047 & 10.906 & 6.138 & 5.047 & 6.977 & 3.828 \\
\hline $1 / 23 / 2006$ & $5: 00$ & 11.606 & 11.466 & 8.463 & 7.41 & 4.672 & 4.826 & 3.102 & 3.04 & 10.904 & 6.139 & 5.046 & 6.975 & 3.828 \\
\hline $1 / 23 / 2006$ & $6: 00$ & 11.601 & 11.456 & 8.459 & 7.402 & 4.667 & 4.821 & 3.097 & 3.032 & 10.901 & 6.137 & 5.048 & 6.973 & 3.829 \\
\hline $1 / 23 / 2006$ & 7:00 & 11.595 & 11.453 & 8.454 & 7.399 & 4.667 & 4.819 & 3.095 & 3.03 & 10.901 & 6.138 & 5.05 & 6.97 & 3.834 \\
\hline $1 / 23 / 2006$ & $8: 00$ & 11.592 & 11.449 & 8.453 & 7.395 & 4.668 & 4.817 & 3.093 & 3.029 & 10.897 & 6.135 & 5.052 & 6.97 & 3.839 \\
\hline $1 / 23 / 2006$ & $9: 00$ & 11.586 & 11.447 & 8.45 & 7.395 & 4.668 & 4.815 & 3.093 & 3.029 & 10.897 & 6.132 & 5.057 & 6.971 & 3.843 \\
\hline $1 / 23 / 2006$ & $10: 00$ & 11.585 & 11.446 & 8.449 & 7.395 & 4.67 & 4.815 & 3.09 & 3.028 & 10.897 & 6.137 & 5.061 & 6.969 & 3.851 \\
\hline $1 / 23 / 2006$ & $11: 00$ & 11.583 & 11.444 & 8.449 & 7.395 & 4.671 & 4.812 & 3.093 & 3.029 & 10.895 & 6.137 & 5.06 & 6.967 & 3.856 \\
\hline $1 / 23 / 2006$ & $12: 00$ & 11.578 & 11.431 & 8.442 & 7.383 & 4.664 & 4.808 & 3.084 & 3.02 & 10.893 & 6.13 & 5.055 & 6.96 & 3.853 \\
\hline $1 / 23 / 2006$ & $13: 00$ & 11.57 & 11.418 & 8.434 & 7.368 & 4.65 & 4.801 & 3.068 & 3.003 & 10.889 & 6.123 & 5.044 & 6.952 & 3.841 \\
\hline $1 / 23 / 2006$ & $14: 00$ & 11.552 & 11.396 & 8.424 & 7.346 & 4.637 & 4.793 & 3.052 & 2.982 & 10.879 & 6.117 & 5.033 & 6.946 & 3.821 \\
\hline $1 / 23 / 2006$ & $15: 00$ & 11.543 & 11.387 & 8.415 & 7.344 & 4.632 & 4.784 & 3.046 & 2.979 & 10.874 & 6.118 & 5.025 & 6.942 & 3.797 \\
\hline $1 / 23 / 2006$ & $16: 00$ & 11.529 & 11.376 & 8.41 & 7.33 & 4.628 & 4.779 & 3.036 & 2.968 & 10.872 & 6.111 & 5.011 & 6.935 & 3.782 \\
\hline $1 / 23 / 2006$ & $17: 00$ & 11.52 & 11.363 & 8.399 & 7.324 & 4.619 & 4.768 & 3.025 & 2.959 & 10.868 & 6.107 & 5.002 & 6.93 & 3.775 \\
\hline $1 / 23 / 2006$ & $18: 00$ & 11.514 & 11.358 & 8.394 & 7.321 & 4.622 & 4.764 & 3.022 & 2.955 & 10.864 & 6.107 & 4.996 & 6.928 & 3.779 \\
\hline $1 / 23 / 2006$ & $19: 00$ & 11.51 & 11.356 & 8.391 & 7.324 & 4.624 & 4.762 & 3.026 & 2.958 & 10.864 & 6.106 & 4.995 & 6.928 & 3.786 \\
\hline $1 / 23 / 2006$ & $20: 00$ & 11.506 & 11.354 & 8.39 & 7.321 & 4.622 & 4.759 & 3.024 & 2.959 & 10.862 & 6.104 & 4.992 & 6.926 & 3.789 \\
\hline $1 / 23 / 2006$ & $21: 00$ & 11.5 & 11.353 & 8.386 & 7.321 & 4.62 & 4.757 & 3.023 & 2.956 & 10.862 & 6.107 & 4.987 & 6.921 & 3.792 \\
\hline $1 / 23 / 2006$ & $22: 00$ & 11.499 & 11.349 & 8.383 & 7.317 & 4.619 & 4.753 & 3.022 & 2.954 & 10.86 & 6.105 & 4.987 & 6.92 & 3.792 \\
\hline $1 / 23 / 2006$ & $23: 00$ & 11.496 & 11.349 & 8.388 & 7.319 & 4.618 & 4.753 & 3.024 & 2.955 & 10.858 & 6.105 & 4.987 & 6.92 & 3.795 \\
\hline $1 / 24 / 2006$ & $0: 00$ & 11.494 & 11.349 & 8.385 & 7.321 & 4.618 & 4.748 & 3.023 & 2.957 & 10.859 & 6.104 & 4.989 & 6.92 & 3.8 \\
\hline $1 / 24 / 2006$ & $1: 00$ & 11.491 & 11.345 & 8.385 & 7.319 & 4.617 & 4.746 & 3.022 & 2.955 & 10.856 & 6.102 & 4.988 & 6.918 & 3.804 \\
\hline $1 / 24 / 2006$ & $2: 00$ & 11.491 & 11.345 & 8.385 & 7.319 & 4.616 & 4.746 & 3.022 & 2.953 & 10.856 & 6.106 & 4.991 & 6.92 & 3.81 \\
\hline $1 / 24 / 2006$ & $3: 00$ & 11.496 & 11.354 & 8.388 & 7.326 & 4.622 & 4.746 & 3.028 & 2.962 & 10.856 & 6.105 & 5 & 6.922 & 3.82 \\
\hline $1 / 24 / 2006$ & $4: 00$ & 11.5 & 11.363 & 8.394 & 7.337 & 4.629 & 4.75 & 3.04 & 2.971 & 10.858 & 6.11 & 5.006 & 6.926 & 3.829 \\
\hline $1 / 24 / 2006$ & $5: 00$ & 11.505 & 11.365 & 8.396 & 7.335 & 4.627 & 4.75 & 3.039 & 2.972 & 10.858 & 6.109 & 5.01 & 6.927 & 3.833 \\
\hline $1 / 24 / 2006$ & $6: 00$ & 11.506 & 11.373 & 8.399 & 7.341 & 4.634 & 4.753 & 3.042 & 2.975 & 10.859 & 6.115 & 5.018 & 6.931 & 3.843 \\
\hline $1 / 24 / 2006$ & $7: 00$ & 11.516 & 11.382 & 8.405 & 7.352 & 4.64 & 4.755 & 3.054 & 2.985 & 10.862 & 6.113 & 5.027 & 6.935 & 3.853 \\
\hline
\end{tabular}


TABLE S1.2 (Cont.)

Water Level (ft below top of casing) at Indicated Well

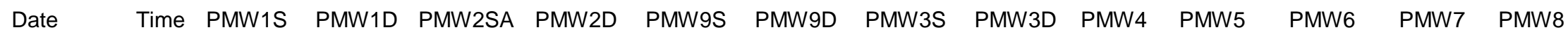

\begin{tabular}{|c|c|c|c|c|c|c|c|c|c|c|c|c|c|c|}
\hline $1 / 24 / 2006$ & $8: 00$ & 11.525 & 11.396 & 8.413 & 7.366 & 4.649 & 4.762 & 3.064 & 2.998 & 10.866 & 6.122 & 5.04 & 6.942 & 3.865 \\
\hline $1 / 24 / 2006$ & $9: 00$ & 11.537 & 11.404 & 8.419 & 7.372 & 4.657 & 4.766 & 3.073 & 3.006 & 10.871 & 6.124 & 5.047 & 6.945 & 3.869 \\
\hline $1 / 24 / 2006$ & $10: 00$ & 11.545 & 11.418 & 8.43 & 7.386 & 4.666 & 4.773 & 3.085 & 3.018 & 10.874 & 6.125 & 5.058 & 6.951 & 3.878 \\
\hline $1 / 24 / 2006$ & $11: 00$ & 11.55 & 11.435 & 8.438 & 7.395 & 4.668 & 4.779 & 3.091 & 3.024 & 10.873 & 6.128 & 5.066 & 6.95 & 3.877 \\
\hline $1 / 24 / 2006$ & $12: 00$ & 11.564 & 11.442 & 8.444 & 7.401 & 4.672 & 4.781 & 3.092 & 3.027 & 10.875 & 6.128 & 5.064 & 6.954 & 3.87 \\
\hline $1 / 24 / 2006$ & $13: 00$ & 11.567 & 11.444 & 8.449 & 7.399 & 4.667 & 4.78 & 3.095 & 3.028 & 10.873 & 6.13 & 5.058 & 6.947 & 3.849 \\
\hline $1 / 24 / 2006$ & $14: 00$ & 11.572 & 11.444 & 8.442 & 7.399 & 4.667 & 4.788 & 3.094 & 3.028 & 10.872 & 6.13 & 5.043 & 6.952 & 3.838 \\
\hline $1 / 24 / 2006$ & $15: 00$ & 11.573 & 11.44 & 8.455 & 7.397 & 4.668 & 4.79 & 3.09 & 3.023 & 10.873 & 6.126 & 5.038 & 6.948 & 3.834 \\
\hline $1 / 24 / 2006$ & $16: 00$ & 11.58 & 11.451 & & 7.403 & & 4.799 & 3.1 & 3.032 & 10.881 & 6.13 & 5.04 & 6.951 & 3.841 \\
\hline $1 / 24 / 2006$ & $17: 00$ & 11.588 & 11.466 & & 7.412 & & 4.801 & 3.111 & 3.045 & 10.879 & 6.139 & 5.051 & 6.965 & 3.854 \\
\hline $1 / 24 / 2006$ & $18: 00$ & 11.601 & 11.48 & & 7.43 & & 4.808 & 3.124 & 3.061 & 10.887 & 6.145 & 5.056 & 6.97 & 3.864 \\
\hline $1 / 24 / 2006$ & $19: 00$ & 11.611 & 11.497 & & 7.443 & & 4.817 & 3.136 & 3.072 & 10.896 & 6.145 & 5.071 & 6.979 & 3.877 \\
\hline $1 / 24 / 2006$ & $20: 00$ & 11.623 & 11.508 & & 7.454 & & 4.826 & 3.142 & 3.082 & 10.902 & 6.149 & 5.079 & 6.987 & 3.879 \\
\hline $1 / 24 / 2006$ & $21: 00$ & 11.631 & 11.515 & & 7.454 & & 4.83 & 3.15 & 3.085 & 10.907 & 6.151 & 5.085 & 6.988 & 3.883 \\
\hline $1 / 24 / 2006$ & $22: 00$ & 11.642 & 11.526 & & 7.465 & & 4.839 & 3.158 & 3.095 & 10.911 & 6.152 & 5.089 & 6.992 & 3.891 \\
\hline $1 / 24 / 2006$ & $23: 00$ & 11.648 & 11.533 & & 7.47 & & 4.846 & 3.164 & 3.098 & 10.913 & 6.159 & 5.095 & 6.996 & 3.897 \\
\hline $1 / 25 / 2006$ & $0: 00$ & 11.653 & 11.539 & & 7.474 & & 4.85 & 3.166 & 3.103 & 10.915 & 6.158 & 5.102 & 6.999 & 3.904 \\
\hline $1 / 25 / 2006$ & $1: 00$ & 11.658 & 11.54 & & 7.474 & & 4.854 & 3.167 & 3.106 & 10.917 & 6.159 & 5.103 & 6.998 & 3.909 \\
\hline $1 / 25 / 2006$ & $2: 00$ & 11.661 & 11.542 & & 7.476 & & 4.861 & 3.17 & 3.106 & 10.917 & 6.158 & 5.109 & 7.001 & 3.914 \\
\hline $1 / 25 / 2006$ & $3: 00$ & 11.663 & 11.544 & & 7.474 & & 4.861 & 3.17 & 3.105 & 10.919 & 6.159 & 5.114 & 7.002 & 3.918 \\
\hline $1 / 25 / 2006$ & $4: 00$ & 11.668 & 11.548 & & 7.481 & & 4.868 & 3.174 & 3.111 & 10.92 & 6.165 & 5.121 & 7.006 & 3.927 \\
\hline $1 / 25 / 2006$ & $5: 00$ & 11.668 & 11.548 & & 7.476 & & 4.868 & 3.173 & 3.109 & 10.922 & 6.162 & 5.126 & 7.006 & 3.928 \\
\hline $1 / 25 / 2006$ & $6: 00$ & 11.67 & 11.544 & & 7.474 & & 4.868 & 3.17 & 3.107 & 10.921 & 6.16 & 5.131 & 7.007 & 3.93 \\
\hline $1 / 25 / 2006$ & $7: 00$ & 11.671 & 11.544 & & 7.474 & & 4.87 & 3.17 & 3.107 & 10.921 & 6.164 & 5.132 & 7.007 & 3.934 \\
\hline $1 / 25 / 2006$ & $8: 00$ & 11.673 & 11.548 & & 7.481 & & 4.872 & 3.176 & 3.111 & 10.924 & 6.163 & 5.137 & 7.009 & 3.94 \\
\hline $1 / 25 / 2006$ & $9: 00$ & 11.675 & 11.551 & & 7.481 & & 4.874 & 3.179 & 3.114 & 10.924 & 6.166 & 5.144 & 7.012 & 3.946 \\
\hline $1 / 25 / 2006$ & $10: 00$ & 11.675 & 11.555 & & 7.485 & & 4.877 & 3.181 & 3.118 & 10.926 & 6.17 & 5.15 & 7.015 & 3.95 \\
\hline $1 / 25 / 2006$ & $11: 00$ & 11.68 & 11.559 & & 7.488 & & 4.879 & 3.186 & 3.123 & 10.928 & 6.174 & 5.153 & 7.016 & 3.952 \\
\hline $1 / 25 / 2006$ & $12: 00$ & 11.681 & 11.56 & & 7.488 & & 4.881 & 3.185 & 3.123 & 10.93 & 6.171 & 5.156 & 7.018 & 3.948 \\
\hline $1 / 25 / 2006$ & $13: 00$ & 11.68 & 11.553 & & 7.483 & & 4.879 & 3.178 & 3.113 & 10.929 & 6.168 & 5.151 & 7.015 & 3.938 \\
\hline $1 / 25 / 2006$ & $14: 00$ & 11.675 & 11.539 & & 7.468 & & 4.875 & 3.164 & 3.098 & 10.926 & 6.166 & 5.139 & 7.007 & 3.915 \\
\hline $1 / 25 / 2006$ & $15: 00$ & 11.662 & 11.524 & & 7.457 & & 4.87 & 3.151 & 3.087 & 10.924 & 6.162 & 5.122 & 7.002 & 3.89 \\
\hline $1 / 25 / 2006$ & $16: 00$ & 11.654 & 11.513 & & 7.445 & & 4.866 & 3.142 & 3.077 & 10.92 & 6.155 & 5.105 & 6.991 & 3.865 \\
\hline $1 / 25 / 2006$ & $17: 00$ & 11.648 & 11.506 & & 7.441 & & 4.861 & 3.133 & 3.07 & 10.917 & 6.148 & 5.087 & 6.985 & 3.848 \\
\hline
\end{tabular}


TABLE S1.2 (Cont.)

Water Level (ft below top of casing) at Indicated Well

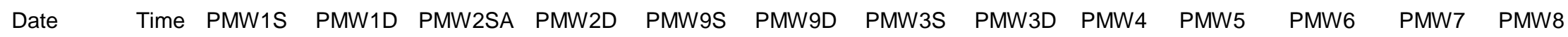

\begin{tabular}{|c|c|c|c|c|c|c|c|c|c|c|c|c|}
\hline $1 / 25 / 2006$ & $18: 00$ & 11.638 & 11.498 & 7.437 & 4.859 & 3.13 & 3.066 & 10.917 & 6.146 & 5.082 & 6.986 & 3.839 \\
\hline $1 / 25 / 2006$ & $19: 00$ & 11.635 & 11.491 & 7.428 & 4.855 & 3.125 & 3.058 & 10.913 & 6.147 & 5.069 & 6.982 & 3.834 \\
\hline $1 / 25 / 2006$ & $20: 00$ & 11.627 & 11.482 & 7.423 & 4.85 & 3.118 & 3.054 & 10.911 & 6.144 & 5.061 & 6.978 & 3.83 \\
\hline $1 / 25 / 2006$ & $21: 00$ & 11.622 & 11.475 & 7.421 & 4.846 & 3.115 & 3.048 & 10.907 & 6.144 & 5.055 & 6.975 & 3.831 \\
\hline $1 / 25 / 2006$ & $22: 00$ & 11.616 & 11.471 & 7.417 & 4.842 & 3.112 & 3.046 & 10.906 & 6.141 & 5.051 & 6.971 & 3.834 \\
\hline $1 / 25 / 2006$ & $23: 00$ & 11.611 & 11.466 & 7.412 & 4.839 & 3.109 & 3.045 & 10.905 & 6.141 & 5.053 & 6.969 & 3.833 \\
\hline $1 / 26 / 2006$ & $0: 00$ & 11.608 & 11.462 & 7.408 & 4.837 & 3.107 & 3.042 & 10.904 & 6.14 & 5.052 & 6.972 & 3.837 \\
\hline $1 / 26 / 2006$ & $1: 00$ & 11.602 & 11.459 & 7.406 & 4.833 & 3.103 & 3.04 & 10.901 & 6.139 & 5.051 & 6.97 & 3.838 \\
\hline $1 / 26 / 2006$ & $2: 00$ & 11.6 & 11.453 & 7.403 & 4.831 & 3.101 & 3.038 & 10.901 & 6.136 & 5.05 & 6.97 & 3.839 \\
\hline $1 / 26 / 2006$ & $3: 00$ & 11.595 & 11.449 & 7.401 & 4.826 & 3.097 & 3.035 & 10.901 & 6.135 & 5.054 & 6.969 & 3.842 \\
\hline $1 / 26 / 2006$ & $4: 00$ & 11.591 & 11.447 & 7.399 & 4.824 & 3.099 & 3.035 & 10.898 & 6.139 & 5.056 & 6.968 & 3.847 \\
\hline $1 / 26 / 2006$ & $5: 00$ & 11.588 & 11.444 & 7.395 & 4.822 & 3.093 & 3.03 & 10.898 & 6.135 & 5.055 & 6.964 & 3.848 \\
\hline $1 / 26 / 2006$ & $6: 00$ & 11.585 & 11.439 & 7.395 & 4.819 & 3.092 & 3.028 & 10.896 & 6.138 & 5.056 & 6.965 & 3.85 \\
\hline $1 / 26 / 2006$ & $7: 00$ & 11.58 & 11.435 & 7.39 & 4.815 & 3.09 & 3.026 & 10.893 & 6.133 & 5.057 & 6.964 & 3.856 \\
\hline $1 / 26 / 2006$ & $8: 00$ & 11.577 & 11.431 & 7.388 & 4.813 & 3.088 & 3.024 & 10.892 & 6.133 & 5.058 & 6.961 & 3.857 \\
\hline $1 / 26 / 2006$ & $9: 00$ & 11.572 & 11.429 & 7.383 & 4.811 & 3.085 & 3.022 & 10.892 & 6.131 & 5.061 & 6.962 & 3.862 \\
\hline $1 / 26 / 2006$ & $10: 00$ & 11.57 & 11.427 & 7.383 & 4.811 & 3.086 & 3.021 & 10.892 & 6.135 & 5.066 & 6.959 & 3.868 \\
\hline $1 / 26 / 2006$ & $11: 00$ & 11.569 & 11.425 & 7.383 & 4.804 & 3.088 & 3.023 & 10.89 & 6.132 & 5.065 & 6.957 & 3.865 \\
\hline $1 / 26 / 2006$ & $12: 00$ & 11.57 & 11.426 & 7.381 & 4.802 & 3.084 & 3.018 & 10.885 & 6.131 & 5.06 & 6.951 & 3.858 \\
\hline $1 / 26 / 2006$ & $13: 00$ & 11.563 & 11.417 & 7.37 & 4.797 & 3.075 & 3.008 & 10.881 & 6.122 & 5.054 & 6.957 & 3.837 \\
\hline $1 / 26 / 2006$ & $14: 00$ & 11.556 & 11.404 & 7.361 & 4.793 & 3.068 & 2.993 & 10.877 & 6.119 & 5.044 & 6.951 & 3.822 \\
\hline $1 / 26 / 2006$ & $15: 00$ & 11.548 & 11.393 & 7.348 & 4.786 & 3.06 & 2.983 & 10.868 & 6.118 & 5.034 & 6.94 & 3.815 \\
\hline $1 / 26 / 2006$ & $16: 00$ & 11.541 & 11.389 & 7.348 & 4.786 & 3.057 & 2.99 & 10.871 & 6.115 & 5.028 & 6.934 & 3.822 \\
\hline $1 / 26 / 2006$ & $17: 00$ & 11.534 & 11.384 & 7.346 & 4.78 & 3.052 & 2.987 & 10.871 & 6.113 & 5.023 & 6.929 & 3.826 \\
\hline $1 / 26 / 2006$ & $18: 00$ & 11.532 & 11.378 & 7.339 & 4.78 & 3.047 & 2.98 & 10.868 & 6.114 & 5.017 & 6.932 & 3.826 \\
\hline $1 / 26 / 2006$ & $19: 00$ & 11.529 & 11.378 & 7.346 & 4.777 & 3.05 & 2.983 & 10.868 & 6.115 & 5.016 & 6.926 & 3.83 \\
\hline $1 / 26 / 2006$ & $20: 00$ & 11.528 & 11.373 & 7.339 & 4.775 & 3.048 & 2.981 & 10.863 & 6.114 & 5.013 & 6.925 & 3.835 \\
\hline $1 / 26 / 2006$ & $21: 00$ & 11.524 & 11.382 & 7.346 & 4.773 & 3.054 & 2.985 & 10.866 & 6.116 & 5.017 & 6.929 & 3.841 \\
\hline $1 / 26 / 2006$ & $22: 00$ & 11.522 & 11.38 & 7.348 & 4.773 & 3.054 & 2.987 & 10.866 & 6.116 & 5.014 & 6.927 & 3.844 \\
\hline $1 / 26 / 2006$ & $23: 00$ & 11.524 & 11.378 & 7.352 & 4.771 & 3.054 & 2.989 & 10.866 & 6.116 & 5.016 & 6.929 & 3.85 \\
\hline $1 / 27 / 2006$ & 0:00 & 11.523 & 11.377 & 7.348 & 4.771 & 3.052 & 2.988 & 10.862 & 6.117 & 5.018 & 6.925 & 3.852 \\
\hline $1 / 27 / 2006$ & $1: 00$ & 11.525 & 11.38 & 7.348 & 4.773 & 3.054 & 2.987 & 10.865 & 6.115 & 5.019 & 6.927 & 3.851 \\
\hline $1 / 27 / 2006$ & $2: 00$ & 11.522 & 11.378 & 7.346 & 4.771 & 3.054 & 2.986 & 10.869 & 6.113 & 5.02 & 6.926 & 3.857 \\
\hline $1 / 27 / 2006$ & $3: 00$ & 11.526 & 11.387 & 7.352 & 4.773 & 3.058 & 2.993 & 10.866 & 6.119 & 5.021 & 6.929 & 3.861 \\
\hline
\end{tabular}


TABLE S1.2 (Cont.)

Water Level (ft below top of casing) at Indicated Well

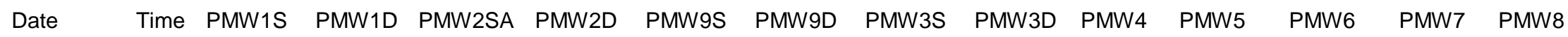

\begin{tabular}{|c|c|c|c|c|c|c|c|c|c|c|c|c|}
\hline $1 / 27 / 2006$ & $4: 00$ & 11.528 & 11.389 & 7.357 & 4.777 & 3.062 & 2.998 & 10.869 & 6.119 & 5.019 & 6.928 & 3.859 \\
\hline $1 / 27 / 2006$ & $5: 00$ & 11.528 & 11.389 & 7.357 & 4.773 & 3.06 & 2.993 & 10.868 & 6.119 & 5.02 & 6.931 & 3.86 \\
\hline $1 / 27 / 2006$ & $6: 00$ & 11.531 & 11.393 & 7.357 & 4.775 & 3.062 & 2.996 & 10.868 & 6.117 & 5.022 & 6.93 & 3.86 \\
\hline $1 / 27 / 2006$ & $7: 00$ & 11.536 & 11.398 & 7.366 & 4.777 & 3.068 & 3.002 & 10.87 & 6.121 & 5.026 & 6.935 & 3.865 \\
\hline $1 / 27 / 2006$ & $8: 00$ & 11.538 & 11.404 & 7.368 & 4.777 & 3.071 & 3.004 & 10.871 & 6.119 & 5.026 & 6.936 & 3.865 \\
\hline $1 / 27 / 2006$ & 9:00 & 11.543 & 11.409 & 7.37 & 4.78 & 3.073 & 3.008 & 10.871 & 6.122 & 5.03 & 6.938 & 3.865 \\
\hline $1 / 27 / 2006$ & $10: 00$ & 11.549 & 11.418 & 7.379 & 4.784 & 3.082 & 3.016 & 10.874 & 6.124 & 5.034 & 6.941 & 3.867 \\
\hline $1 / 27 / 2006$ & $11: 00$ & 11.553 & 11.422 & 7.383 & 4.784 & 3.087 & 3.019 & 10.874 & 6.124 & 5.034 & 6.943 & 3.868 \\
\hline $1 / 27 / 2006$ & $12: 00$ & 11.561 & 11.428 & 7.386 & 4.788 & 3.089 & 3.023 & 10.876 & 6.123 & 5.034 & 6.944 & 3.867 \\
\hline $1 / 27 / 2006$ & $13: 00$ & 11.558 & 11.422 & 7.379 & 4.788 & 3.081 & 3.016 & 10.873 & 6.121 & 5.032 & 6.943 & 3.861 \\
\hline $1 / 27 / 2006$ & $14: 00$ & 11.552 & 11.413 & 7.37 & 4.786 & 3.074 & 3.008 & 10.874 & 6.118 & 5.031 & 6.941 & 3.856 \\
\hline $1 / 27 / 2006$ & $15: 00$ & 11.548 & 11.404 & 7.364 & 4.784 & 3.07 & 3.002 & 10.874 & 6.117 & 5.031 & 6.939 & 3.853 \\
\hline $1 / 27 / 2006$ & $16: 00$ & 11.541 & 11.398 & 7.357 & 4.782 & 3.063 & 2.994 & 10.87 & 6.114 & 5.028 & 6.936 & 3.852 \\
\hline $1 / 27 / 2006$ & $17: 00$ & 11.54 & 11.395 & 7.357 & 4.782 & 3.063 & 2.995 & 10.868 & 6.112 & 5.028 & 6.934 & 3.853 \\
\hline $1 / 27 / 2006$ & $18: 00$ & 11.537 & 11.395 & 7.357 & 4.78 & 3.061 & 2.995 & 10.868 & 6.115 & 5.03 & 6.936 & 3.855 \\
\hline $1 / 27 / 2006$ & $19: 00$ & 11.537 & 11.397 & 7.359 & 4.78 & 3.063 & 2.996 & 10.868 & 6.114 & 5.033 & 6.939 & 3.857 \\
\hline $1 / 27 / 2006$ & $20: 00$ & 11.537 & 11.395 & 7.359 & 4.78 & 3.061 & 2.996 & 10.868 & 6.114 & 5.035 & 6.939 & 3.858 \\
\hline $1 / 27 / 2006$ & $21: 00$ & 11.532 & 11.391 & 7.357 & 4.777 & 3.061 & 2.993 & 10.868 & 6.11 & 5.037 & 6.936 & 3.856 \\
\hline $1 / 27 / 2006$ & $22: 00$ & 11.53 & 11.387 & 7.35 & 4.777 & 3.055 & 2.987 & 10.866 & 6.108 & 5.032 & 6.935 & 3.853 \\
\hline $1 / 27 / 2006$ & $23: 00$ & 11.53 & 11.387 & 7.352 & 4.777 & 3.056 & 2.989 & 10.865 & 6.108 & 5.03 & 6.936 & 3.853 \\
\hline $1 / 28 / 2006$ & $0: 00$ & 11.53 & 11.386 & 7.352 & 4.777 & 3.057 & 2.991 & 10.866 & 6.11 & 5.032 & 6.933 & 3.852 \\
\hline $1 / 28 / 2006$ & $1: 00$ & 11.527 & 11.386 & 7.352 & 4.775 & 3.056 & 2.989 & 10.862 & 6.111 & 5.03 & 6.932 & 3.849 \\
\hline $1 / 28 / 2006$ & $2: 00$ & 11.526 & 11.384 & 7.35 & 4.773 & 3.052 & 2.987 & 10.864 & 6.105 & 5.029 & 6.93 & 3.844 \\
\hline $1 / 28 / 2006$ & $3: 00$ & 11.522 & 11.38 & 7.348 & 4.771 & 3.051 & 2.984 & 10.86 & 6.107 & 5.024 & 6.929 & 3.84 \\
\hline $1 / 28 / 2006$ & $4: 00$ & 11.519 & 11.375 & 7.341 & 4.769 & 3.044 & 2.978 & 10.86 & 6.104 & 5.02 & 6.925 & 3.835 \\
\hline $1 / 28 / 2006$ & $5: 00$ & 11.516 & 11.366 & 7.332 & 4.762 & 3.035 & 2.969 & 10.858 & 6.099 & 5.012 & 6.921 & 3.828 \\
\hline $1 / 28 / 2006$ & $6: 00$ & 11.509 & 11.362 & 7.328 & 4.76 & 3.032 & 2.965 & 10.853 & 6.098 & 5.011 & 6.924 & 3.829 \\
\hline $1 / 28 / 2006$ & $7: 00$ & 11.507 & 11.353 & 7.321 & 4.755 & 3.027 & 2.96 & 10.851 & 6.096 & 5.006 & 6.915 & 3.822 \\
\hline $1 / 28 / 2006$ & 8:00 & 11.5 & 11.356 & 7.326 & 4.751 & 3.022 & 2.954 & 10.849 & 6.093 & 5 & 6.922 & 3.815 \\
\hline $1 / 28 / 2006$ & 9:00 & 11.495 & 11.344 & 7.339 & 4.746 & 3.015 & 2.948 & 10.847 & 6.091 & 4.997 & 6.936 & 3.811 \\
\hline $1 / 28 / 2006$ & $10: 00$ & 11.49 & 11.353 & 7.346 & 4.74 & 3.01 & 2.958 & 10.859 & 6.088 & 4.987 & 6.949 & 3.797 \\
\hline $1 / 28 / 2006$ & $11: 00$ & 11.481 & 11.36 & 7.352 & 4.733 & 2.996 & 2.964 & 10.869 & 6.082 & 4.976 & 6.956 & 3.753 \\
\hline $1 / 28 / 2006$ & $12: 00$ & 11.477 & 11.369 & 7.344 & 4.729 & 2.99 & 2.928 & 10.877 & 6.084 & 4.96 & 6.962 & 3.725 \\
\hline $1 / 28 / 2006$ & $13: 00$ & 11.47 & 11.389 & 7.357 & 4.72 & 2.979 & 2.915 & 10.906 & 6.075 & 4.948 & 6.894 & 3.704 \\
\hline
\end{tabular}


TABLE S1.2 (Cont.)

Water Level (ft below top of casing) at Indicated Well

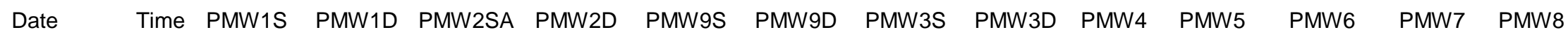

\begin{tabular}{|c|c|c|c|c|c|c|c|c|c|c|c|c|}
\hline $1 / 28 / 2006$ & $14: 00$ & 11.46 & 11.397 & 7.355 & 4.711 & 2.966 & 2.911 & 10.927 & 6.069 & 4.935 & 6.91 & 3.692 \\
\hline $1 / 28 / 2006$ & $15: 00$ & 11.451 & 11.391 & 7.357 & 4.709 & 2.967 & 2.896 & 10.929 & 6.073 & 4.928 & 6.912 & 3.691 \\
\hline $1 / 28 / 2006$ & $16: 00$ & 11.444 & 11.389 & 7.359 & 4.702 & 2.958 & 2.903 & 10.934 & 6.066 & 4.916 & 6.92 & 3.674 \\
\hline $1 / 28 / 2006$ & $17: 00$ & 11.44 & 11.387 & 7.361 & 4.693 & 2.949 & 2.889 & 10.934 & 6.059 & 4.901 & 6.92 & 3.614 \\
\hline $1 / 28 / 2006$ & $18: 00$ & 11.43 & 11.391 & 7.332 & 4.687 & 2.926 & 2.867 & 10.943 & 6.053 & 4.877 & 6.928 & 3.424 \\
\hline $1 / 28 / 2006$ & $19: 00$ & 11.423 & 11.371 & 7.317 & 4.68 & 2.923 & 2.863 & 10.927 & 6.045 & 4.855 & 6.911 & 3.344 \\
\hline $1 / 28 / 2006$ & $20: 00$ & 11.42 & 11.362 & 7.315 & 4.678 & 2.915 & 2.856 & 10.92 & 6.035 & 4.828 & 6.906 & 3.256 \\
\hline $1 / 28 / 2006$ & $21: 00$ & 11.419 & 11.36 & 7.317 & 4.671 & 2.909 & 2.846 & 10.922 & 6.021 & 4.772 & 6.906 & 3.117 \\
\hline $1 / 28 / 2006$ & $22: 00$ & 11.417 & 11.347 & 7.308 & 4.669 & 2.904 & 2.84 & 10.913 & 6.001 & 4.707 & 6.894 & 3.054 \\
\hline $1 / 28 / 2006$ & $23: 00$ & 11.415 & 11.333 & 7.239 & 4.667 & 2.898 & 2.836 & 10.894 & 5.983 & 4.659 & 6.827 & 3.036 \\
\hline $1 / 29 / 2006$ & $0: 00$ & 11.414 & 11.269 & 7.239 & 4.66 & 2.896 & 2.834 & 10.814 & 5.977 & 4.628 & 6.827 & 3.047 \\
\hline $1 / 29 / 2006$ & $1: 00$ & 11.416 & 11.269 & 7.237 & 4.651 & 2.895 & 2.833 & 10.773 & 5.965 & 4.614 & 6.823 & 3.067 \\
\hline $1 / 29 / 2006$ & $2: 00$ & 11.414 & 11.271 & 7.233 & 4.645 & 2.895 & 2.831 & 10.766 & 5.957 & 4.607 & 6.821 & 3.095 \\
\hline $1 / 29 / 2006$ & $3: 00$ & 11.418 & 11.278 & 7.242 & 4.636 & 2.896 & 2.838 & 10.765 & 5.961 & 4.614 & 6.821 & 3.127 \\
\hline $1 / 29 / 2006$ & $4: 00$ & 11.416 & 11.276 & 7.239 & 4.629 & 2.901 & 2.837 & 10.759 & 5.957 & 4.616 & 6.821 & 3.154 \\
\hline $1 / 29 / 2006$ & $5: 00$ & 11.417 & 11.278 & 7.239 & 4.622 & 2.9 & 2.839 & 10.755 & 5.957 & 4.621 & 6.82 & 3.179 \\
\hline $1 / 29 / 2006$ & $6: 00$ & 11.423 & 11.289 & 7.246 & 4.618 & 2.909 & 2.846 & 10.753 & 5.961 & 4.634 & 6.824 & 3.209 \\
\hline $1 / 29 / 2006$ & $7: 00$ & 11.428 & 11.293 & 7.253 & 4.618 & 2.916 & 2.853 & 10.751 & 5.964 & 4.645 & 6.826 & 3.232 \\
\hline $1 / 29 / 2006$ & $8: 00$ & 11.438 & 11.307 & 7.259 & 4.616 & 2.924 & 2.863 & 10.75 & 5.969 & 4.66 & 6.829 & 3.261 \\
\hline $1 / 29 / 2006$ & $9: 00$ & 11.442 & 11.313 & 7.268 & 4.618 & 2.932 & 2.869 & 10.749 & 5.971 & 4.669 & 6.835 & 3.284 \\
\hline $1 / 29 / 2006$ & $10: 00$ & 11.45 & 11.318 & 7.27 & 4.618 & 2.94 & 2.875 & 10.752 & 5.967 & 4.68 & 6.836 & 3.305 \\
\hline $1 / 29 / 2006$ & $11: 00$ & 11.454 & 11.329 & 7.279 & 4.621 & 2.947 & 2.885 & 10.749 & 5.977 & 4.693 & 6.842 & 3.327 \\
\hline $1 / 29 / 2006$ & $12: 00$ & 11.463 & 11.335 & 7.284 & 4.623 & 2.95 & 2.889 & 10.751 & 5.979 & 4.705 & 6.846 & 3.345 \\
\hline $1 / 29 / 2006$ & $13: 00$ & 11.463 & 11.333 & 7.279 & 4.622 & 2.947 & 2.886 & 10.751 & 5.981 & 4.709 & 6.846 & 3.358 \\
\hline $1 / 29 / 2006$ & $14: 00$ & 11.464 & 11.331 & 7.279 & 4.622 & 2.947 & 2.883 & 10.752 & 5.979 & 4.713 & 6.845 & 3.376 \\
\hline $1 / 29 / 2006$ & $15: 00$ & 11.461 & 11.329 & 7.277 & 4.623 & 2.95 & 2.887 & 10.751 & 5.981 & 4.721 & 6.848 & 3.393 \\
\hline $1 / 29 / 2006$ & $16: 00$ & 11.467 & 11.338 & 7.284 & 4.627 & 2.956 & 2.896 & 10.753 & 5.989 & 4.734 & 6.853 & 3.414 \\
\hline $1 / 29 / 2006$ & $17: 00$ & 11.475 & 11.347 & 7.297 & 4.631 & 2.967 & 2.906 & 10.759 & 5.993 & 4.746 & 6.857 & 3.435 \\
\hline $1 / 29 / 2006$ & $18: 00$ & 11.482 & 11.356 & 7.304 & 4.638 & 2.976 & 2.911 & 10.76 & 5.991 & 4.753 & 6.861 & 3.454 \\
\hline $1 / 29 / 2006$ & $19: 00$ & 11.488 & 11.364 & 7.313 & 4.645 & 2.983 & 2.921 & 10.763 & 6 & 4.764 & 6.865 & 3.473 \\
\hline $1 / 29 / 2006$ & $20: 00$ & 11.497 & 11.375 & 7.319 & 4.651 & 2.991 & 2.93 & 10.766 & 6.008 & 4.776 & 6.869 & 3.488 \\
\hline $1 / 29 / 2006$ & $21: 00$ & 11.502 & 11.38 & 7.324 & 4.656 & 2.999 & 2.932 & 10.77 & 6.009 & 4.781 & 6.873 & 3.504 \\
\hline $1 / 29 / 2006$ & $22: 00$ & 11.509 & 11.384 & 7.326 & 4.661 & 3 & 2.937 & 10.773 & 6.013 & 4.786 & 6.874 & 3.515 \\
\hline $1 / 29 / 2006$ & $23: 00$ & 11.513 & 11.389 & 7.332 & 4.665 & 3.005 & 2.942 & 10.776 & 6.016 & 4.796 & 6.878 & 3.526 \\
\hline
\end{tabular}


TABLE S1.2 (Cont.)

Water Level (ft below top of casing) at Indicated Well

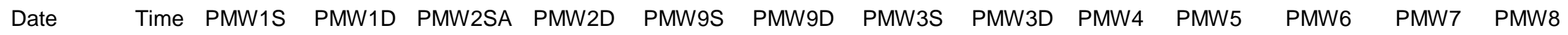

\begin{tabular}{|c|c|c|c|c|c|c|c|c|c|c|c|c|}
\hline $1 / 30 / 2006$ & $0: 00$ & 11.52 & 11.395 & 7.335 & 4.669 & 3.008 & 2.946 & 10.778 & 6.022 & 4.8 & 6.88 & 3.539 \\
\hline $1 / 30 / 2006$ & $1: 00$ & 11.522 & 11.397 & 7.341 & 4.676 & 3.012 & 2.95 & 10.782 & 6.017 & 4.809 & 6.883 & 3.551 \\
\hline $1 / 30 / 2006$ & $2: 00$ & 11.524 & 11.395 & 7.337 & 4.678 & 3.014 & 2.95 & 10.783 & 6.017 & 4.812 & 6.884 & 3.564 \\
\hline $1 / 30 / 2006$ & $3: 00$ & 11.528 & 11.402 & 7.341 & 4.683 & 3.018 & 2.954 & 10.785 & 6.028 & 4.822 & 6.89 & 3.58 \\
\hline $1 / 30 / 2006$ & $4: 00$ & 11.531 & 11.402 & 7.344 & 4.687 & 3.021 & 2.957 & 10.789 & 6.027 & 4.827 & 6.89 & 3.594 \\
\hline $1 / 30 / 2006$ & $5: 00$ & 11.533 & 11.402 & 7.344 & 4.689 & 3.022 & 2.958 & 10.791 & 6.028 & 4.836 & 6.892 & 3.609 \\
\hline $1 / 30 / 2006$ & $6: 00$ & 11.535 & 11.408 & 7.352 & 4.696 & 3.029 & 2.965 & 10.793 & 6.036 & 4.847 & 6.897 & 3.629 \\
\hline $1 / 30 / 2006$ & $7: 00$ & 11.54 & 11.415 & 7.357 & 4.7 & 3.037 & 2.97 & 10.796 & 6.042 & 4.855 & 6.899 & 3.642 \\
\hline $1 / 30 / 2006$ & $8: 00$ & 11.549 & 11.422 & 7.366 & 4.705 & 3.042 & 2.979 & 10.8 & 6.045 & 4.866 & 6.905 & 3.66 \\
\hline $1 / 30 / 2006$ & 9:00 & 11.554 & 11.433 & 7.375 & 4.711 & 3.053 & 2.988 & 10.804 & 6.049 & 4.88 & 6.909 & 3.677 \\
\hline $1 / 30 / 2006$ & $10: 00$ & 11.561 & 11.44 & 7.381 & 4.718 & 3.059 & 2.997 & 10.808 & 6.055 & 4.891 & 6.913 & 3.692 \\
\hline $1 / 30 / 2006$ & $11: 00$ & 11.568 & 11.45 & 7.39 & 4.724 & 3.065 & 3.002 & 10.809 & 6.06 & 4.9 & 6.917 & 3.703 \\
\hline $1 / 30 / 2006$ & $12: 00$ & 11.576 & 11.46 & 7.397 & 4.731 & 3.073 & 3.01 & 10.813 & 6.064 & 4.907 & 6.919 & 3.705 \\
\hline $1 / 30 / 2006$ & $13: 00$ & 11.584 & 11.464 & 7.399 & 4.736 & 3.076 & 3.011 & 10.819 & 6.066 & 4.909 & 6.922 & 3.691 \\
\hline $1 / 30 / 2006$ & $14: 00$ & 11.587 & 11.466 & 7.401 & 4.74 & 3.077 & 3.011 & 10.822 & 6.065 & 4.904 & 6.923 & 3.671 \\
\hline $1 / 30 / 2006$ & $15: 00$ & 11.589 & 11.464 & 7.397 & 4.742 & 3.072 & 3.01 & 10.824 & 6.065 & 4.897 & 6.923 & 3.647 \\
\hline $1 / 30 / 2006$ & $16: 00$ & 11.59 & 11.466 & 7.399 & 4.745 & 3.077 & 3.01 & 10.827 & 6.068 & 4.894 & 6.923 & 3.636 \\
\hline $1 / 30 / 2006$ & $17: 00$ & 11.591 & 11.464 & 7.395 & 4.747 & 3.074 & 3.008 & 10.828 & 6.066 & 4.893 & 6.924 & 3.632 \\
\hline $1 / 30 / 2006$ & $18: 00$ & 11.592 & 11.464 & 7.395 & 4.749 & 3.073 & 3.008 & 10.831 & 6.066 & 4.89 & 6.924 & 3.637 \\
\hline $1 / 30 / 2006$ & $19: 00$ & 11.595 & 11.464 & 7.397 & 4.751 & 3.076 & 3.013 & 10.832 & 6.066 & 4.892 & 6.926 & 3.643 \\
\hline $1 / 30 / 2006$ & $20: 00$ & 11.591 & 11.46 & 7.395 & 4.751 & 3.073 & 3.009 & 10.832 & 6.065 & 4.889 & 6.925 & 3.65 \\
\hline $1 / 30 / 2006$ & $21: 00$ & 11.588 & 11.453 & 7.39 & 4.753 & 3.071 & 3.005 & 10.834 & 6.065 & 4.89 & 6.925 & 3.658 \\
\hline $1 / 30 / 2006$ & $22: 00$ & 11.585 & 11.452 & 7.388 & 4.753 & 3.068 & 3.006 & 10.833 & 6.067 & 4.894 & 6.925 & 3.675 \\
\hline $1 / 30 / 2006$ & $23: 00$ & 11.581 & 11.446 & 7.383 & 4.756 & 3.065 & 3.001 & 10.832 & 6.071 & 4.897 & 6.923 & 3.692 \\
\hline $1 / 31 / 2006$ & $0: 00$ & 11.575 & 11.437 & 7.379 & 4.753 & 3.063 & 2.998 & 10.832 & 6.069 & 4.904 & 6.923 & 3.705 \\
\hline $1 / 31 / 2006$ & $1: 00$ & 11.572 & 11.433 & 7.375 & 4.753 & 3.061 & 2.995 & 10.83 & 6.068 & 4.91 & 6.921 & 3.717 \\
\hline $1 / 31 / 2006$ & $2: 00$ & 11.57 & 11.43 & 7.372 & 4.753 & 3.06 & 2.994 & 10.832 & 6.072 & 4.915 & 6.925 & 3.729 \\
\hline $1 / 31 / 2006$ & $3: 00$ & 11.565 & 11.426 & 7.37 & 4.753 & 3.058 & 2.992 & 10.832 & 6.074 & 4.921 & 6.924 & 3.74 \\
\hline $1 / 31 / 2006$ & $4: 00$ & 11.559 & 11.417 & 7.364 & 4.751 & 3.054 & 2.988 & 10.83 & 6.068 & 4.925 & 6.919 & 3.745 \\
\hline $1 / 31 / 2006$ & $5: 00$ & 11.553 & 11.404 & 7.352 & 4.747 & 3.044 & 2.979 & 10.827 & 6.068 & 4.927 & 6.915 & 3.75 \\
\hline $1 / 31 / 2006$ & $6: 00$ & 11.543 & 11.393 & 7.346 & 4.745 & 3.037 & 2.97 & 10.824 & 6.069 & 4.93 & 6.913 & 3.755 \\
\hline $1 / 31 / 2006$ & $7: 00$ & 11.536 & 11.39 & 7.344 & 4.742 & 3.039 & 2.968 & 10.822 & 6.069 & 4.937 & 6.91 & 3.765 \\
\hline $1 / 31 / 2006$ & $8: 00$ & 11.529 & 11.384 & 7.337 & 4.738 & 3.03 & 2.964 & 10.821 & 6.068 & 4.941 & 6.91 & 3.771 \\
\hline $1 / 31 / 2006$ & $9: 00$ & 11.523 & 11.377 & 7.335 & 4.736 & 3.031 & 2.963 & 10.819 & 6.069 & 4.947 & 6.907 & 3.777 \\
\hline
\end{tabular}


TABLE S1.2 (Cont.)

Water Level (ft below top of casing) at Indicated Well

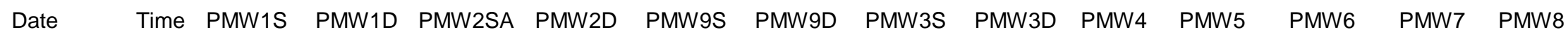

\begin{tabular}{|c|c|c|c|c|c|c|c|c|c|c|c|c|}
\hline $1 / 31 / 2006$ & $10: 00$ & 11.521 & 11.373 & 7.33 & 4.734 & 3.027 & 2.96 & 10.819 & 6.068 & 4.953 & 6.91 & 3.784 \\
\hline $1 / 31 / 2006$ & $11: 00$ & 11.516 & 11.371 & 7.328 & 4.731 & 3.028 & 2.962 & 10.818 & 6.071 & 4.956 & 6.905 & 3.789 \\
\hline $1 / 31 / 2006$ & $12: 00$ & 11.512 & 11.368 & 7.328 & 4.729 & 3.023 & 2.957 & 10.821 & 6.068 & 4.956 & 6.906 & 3.785 \\
\hline $1 / 31 / 2006$ & $13: 00$ & 11.508 & 11.359 & 7.319 & 4.725 & 3.015 & 2.948 & 10.817 & 6.065 & 4.95 & 6.9 & 3.763 \\
\hline $1 / 31 / 2006$ & $14: 00$ & 11.498 & 11.348 & 7.308 & 4.718 & 3.003 & 2.937 & 10.816 & 6.061 & 4.931 & 6.894 & 3.72 \\
\hline $1 / 31 / 2006$ & $15: 00$ & 11.488 & 11.337 & 7.299 & 4.711 & 2.995 & 2.925 & 10.811 & 6.053 & 4.914 & 6.889 & 3.688 \\
\hline $1 / 31 / 2006$ & $16: 00$ & 11.482 & 11.329 & 7.293 & 4.707 & 2.987 & 2.921 & 10.81 & 6.052 & 4.903 & 6.883 & 3.675 \\
\hline $1 / 31 / 2006$ & $17: 00$ & 11.475 & 11.32 & 7.286 & 4.703 & 2.982 & 2.914 & 10.805 & 6.048 & 4.89 & 6.881 & 3.673 \\
\hline $1 / 31 / 2006$ & $18: 00$ & 11.472 & 11.324 & 7.29 & 4.703 & 2.988 & 2.919 & 10.806 & 6.051 & 4.887 & 6.882 & 3.679 \\
\hline $1 / 31 / 2006$ & 19:00 & 11.471 & 11.328 & 7.297 & 4.703 & 2.991 & 2.924 & 10.807 & 6.052 & 4.886 & 6.881 & 3.688 \\
\hline $1 / 31 / 2006$ & $20: 00$ & 11.476 & 11.335 & 7.304 & 4.705 & 2.998 & 2.932 & 10.807 & 6.055 & 4.887 & 6.884 & 3.696 \\
\hline $1 / 31 / 2006$ & $21: 00$ & 11.478 & 11.339 & 7.308 & 4.703 & 3 & 2.933 & 10.807 & 6.055 & 4.885 & 6.884 & 3.702 \\
\hline $1 / 31 / 2006$ & $22: 00$ & 11.485 & 11.359 & 7.328 & 4.711 & 3.019 & 2.95 & 10.809 & 6.061 & 4.897 & 6.888 & 3.718 \\
\hline $1 / 31 / 2006$ & $23: 00$ & 11.498 & 11.371 & 7.337 & 4.714 & 3.027 & 2.959 & 10.815 & 6.06 & 4.899 & 6.892 & 3.724 \\
\hline 2/1/2006 & $0: 00$ & 11.507 & 11.38 & 7.346 & 4.72 & 3.035 & 2.967 & 10.816 & 6.066 & 4.904 & 6.895 & 3.732 \\
\hline $2 / 1 / 2006$ & $1: 00$ & 11.517 & 11.39 & 7.352 & 4.722 & 3.039 & 2.973 & 10.82 & 6.066 & 4.906 & 6.899 & 3.735 \\
\hline $2 / 1 / 2006$ & $2: 00$ & 11.522 & 11.395 & 7.355 & 4.727 & 3.042 & 2.975 & 10.822 & 6.068 & 4.908 & 6.903 & 3.74 \\
\hline $2 / 1 / 2006$ & $3: 00$ & 11.526 & 11.397 & 7.357 & 4.729 & 3.046 & 2.98 & 10.824 & 6.067 & 4.91 & 6.904 & 3.742 \\
\hline 2/1/2006 & $4: 00$ & 11.527 & 11.397 & 7.352 & 4.731 & 3.042 & 2.976 & 10.824 & 6.061 & 4.912 & 6.903 & 3.742 \\
\hline $2 / 1 / 2006$ & $5: 00$ & 11.531 & 11.399 & 7.352 & 4.734 & 3.045 & 2.976 & 10.824 & 6.066 & 4.914 & 6.906 & 3.744 \\
\hline 2/1/2006 & $6: 00$ & 11.537 & 11.408 & 7.364 & 4.738 & 3.052 & 2.986 & 10.827 & 6.069 & 4.919 & 6.908 & 3.754 \\
\hline $2 / 1 / 2006$ & $7: 00$ & 11.541 & 11.41 & 7.366 & 4.74 & 3.054 & 2.988 & 10.83 & 6.068 & 4.922 & 6.911 & 3.757 \\
\hline $2 / 1 / 2006$ & $8: 00$ & 11.545 & 11.413 & 7.368 & 4.742 & 3.057 & 2.99 & 10.83 & 6.067 & 4.927 & 6.912 & 3.763 \\
\hline 2/1/2006 & $9: 00$ & 11.543 & 11.411 & 7.366 & 4.745 & 3.057 & 2.988 & 10.829 & 6.067 & 4.93 & 6.913 & 3.771 \\
\hline $2 / 1 / 2006$ & $10: 00$ & 11.545 & 11.417 & 7.368 & 4.747 & 3.058 & 2.992 & 10.831 & 6.073 & 4.937 & 6.916 & 3.777 \\
\hline 2/1/2006 & $11: 00$ & 11.552 & 11.422 & 7.375 & 4.751 & 3.063 & 2.998 & 10.833 & 6.074 & 4.943 & 6.916 & 3.783 \\
\hline $2 / 1 / 2006$ & $12: 00$ & 11.556 & 11.424 & 7.377 & 4.751 & 3.067 & 2.998 & 10.835 & 6.074 & 4.945 & 6.919 & 3.777 \\
\hline 2/1/2006 & $13: 00$ & 11.554 & 11.419 & 7.37 & 4.751 & 3.062 & 2.995 & 10.835 & 6.071 & 4.94 & 6.915 & 3.763 \\
\hline $2 / 1 / 2006$ & $14: 00$ & 11.547 & 11.411 & 7.364 & 4.751 & 3.055 & 2.989 & 10.834 & 6.071 & 4.934 & 6.913 & 3.749 \\
\hline 2/1/2006 & $15: 00$ & 11.541 & 11.401 & 7.352 & 4.749 & 3.044 & 2.978 & 10.832 & 6.068 & 4.927 & 6.912 & 3.745 \\
\hline $2 / 1 / 2006$ & $16: 00$ & 11.533 & 11.393 & 7.344 & 4.747 & 3.039 & 2.971 & 10.828 & 6.067 & 4.923 & 6.908 & 3.746 \\
\hline 2/1/2006 & $17: 00$ & 11.527 & 11.384 & 7.339 & 4.747 & 3.032 & 2.968 & 10.826 & 6.064 & 4.925 & 6.907 & 3.748 \\
\hline $2 / 1 / 2006$ & $18: 00$ & 11.524 & 11.38 & 7.341 & 4.745 & 3.033 & 2.967 & 10.826 & 6.063 & 4.924 & 6.905 & 3.753 \\
\hline 2/1/2006 & 19:00 & 11.521 & 11.38 & 7.339 & 4.745 & 3.035 & 2.964 & 10.824 & 6.066 & 4.927 & 6.905 & 3.756 \\
\hline
\end{tabular}


TABLE S1.2 (Cont.)

Water Level (ft below top of casing) at Indicated Well

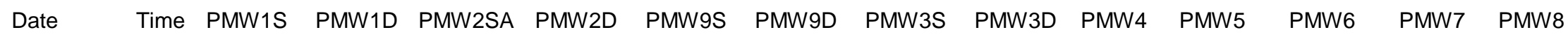

\begin{tabular}{|c|c|c|c|c|c|c|c|c|c|c|c|c|}
\hline 2/1/2006 & $20: 00$ & 11.52 & 11.379 & 7.337 & 4.742 & 3.032 & 2.965 & 10.824 & 6.066 & 4.924 & 6.901 & 3.76 \\
\hline $2 / 1 / 2006$ & $21: 00$ & 11.521 & 11.384 & 7.344 & 4.742 & 3.036 & 2.968 & 10.824 & 6.068 & 4.93 & 6.902 & 3.763 \\
\hline $2 / 1 / 2006$ & $22: 00$ & 11.518 & 11.377 & 7.335 & 4.74 & 3.033 & 2.965 & 10.822 & 6.063 & 4.925 & 6.901 & 3.763 \\
\hline 2/1/2006 & $23: 00$ & 11.514 & 11.371 & 7.33 & 4.738 & 3.027 & 2.958 & 10.821 & 6.064 & 4.925 & 6.899 & 3.763 \\
\hline 2/2/2006 & 0:00 & 11.507 & 11.366 & 7.326 & 4.736 & 3.022 & 2.957 & 10.822 & 6.062 & 4.926 & 6.898 & 3.764 \\
\hline 2/2/2006 & $1: 00$ & 11.503 & 11.359 & 7.319 & 4.734 & 3.019 & 2.951 & 10.818 & 6.06 & 4.922 & 6.894 & 3.763 \\
\hline $2 / 2 / 2006$ & $2: 00$ & 11.5 & 11.355 & 7.319 & 4.731 & 3.017 & 2.95 & 10.816 & 6.06 & 4.923 & 6.893 & 3.767 \\
\hline 2/2/2006 & $3: 00$ & 11.499 & 11.355 & 7.319 & 4.729 & 3.015 & 2.948 & 10.816 & 6.057 & 4.923 & 6.894 & 3.768 \\
\hline $2 / 2 / 2006$ & $4: 00$ & 11.496 & 11.353 & 7.319 & 4.729 & 3.016 & 2.95 & 10.814 & 6.062 & 4.925 & 6.895 & 3.77 \\
\hline $2 / 2 / 2006$ & $5: 00$ & 11.496 & 11.353 & 7.323 & 4.727 & 3.018 & 2.948 & 10.816 & 6.058 & 4.927 & 6.895 & 3.774 \\
\hline $2 / 2 / 2006$ & $6: 00$ & 11.493 & 11.348 & 7.314 & 4.725 & 3.013 & 2.944 & 10.814 & 6.056 & 4.923 & 6.891 & 3.77 \\
\hline $2 / 2 / 2006$ & $7: 00$ & 11.49 & 11.346 & 7.312 & 4.725 & 3.011 & 2.943 & 10.814 & 6.056 & 4.922 & 6.892 & 3.772 \\
\hline $2 / 2 / 2006$ & $8: 00$ & 11.487 & 11.346 & 7.31 & 4.722 & 3.012 & 2.943 & 10.813 & 6.055 & 4.925 & 6.894 & 3.774 \\
\hline $2 / 2 / 2006$ & 9:00 & 11.486 & 11.348 & 7.315 & 4.722 & 3.016 & 2.946 & 10.814 & 6.059 & 4.931 & 6.893 & 3.78 \\
\hline 2/2/2006 & $10: 00$ & 11.489 & 11.348 & 7.317 & 4.722 & 3.015 & 2.947 & 10.814 & 6.06 & 4.937 & 6.894 & 3.786 \\
\hline 2/2/2006 & $11: 00$ & 11.487 & 11.348 & 7.315 & 4.72 & 3.015 & 2.947 & 10.813 & 6.057 & 4.939 & 6.895 & 3.784 \\
\hline $2 / 2 / 2006$ & $12: 00$ & 11.49 & 11.351 & 7.319 & 4.72 & 3.018 & 2.949 & 10.814 & 6.058 & 4.941 & 6.894 & 3.781 \\
\hline $2 / 2 / 2006$ & $13: 00$ & 11.489 & 11.349 & 7.317 & 4.72 & 3.014 & 2.948 & 10.814 & 6.056 & 4.934 & 6.893 & 3.773 \\
\hline 2/2/2006 & $14: 00$ & 11.488 & 11.351 & 7.317 & 4.72 & 3.018 & 2.948 & 10.813 & 6.061 & 4.935 & 6.895 & 3.773 \\
\hline $2 / 2 / 2006$ & $15: 00$ & 11.483 & 11.339 & 7.306 & 4.718 & 3.006 & 2.938 & 10.81 & 6.056 & 4.929 & 6.891 & 3.767 \\
\hline 2/2/2006 & $16: 00$ & 11.481 & 11.339 & 7.306 & 4.718 & 3.007 & 2.936 & 10.811 & 6.055 & 4.931 & 6.893 & 3.769 \\
\hline $2 / 2 / 2006$ & $17: 00$ & 11.486 & 11.346 & 7.314 & 4.718 & 3.013 & 2.945 & 10.81 & 6.062 & 4.938 & 6.893 & 3.777 \\
\hline 2/2/2006 & $18: 00$ & 11.489 & 11.353 & 7.321 & 4.72 & 3.022 & 2.951 & 10.81 & 6.065 & 4.945 & 6.896 & 3.783 \\
\hline $2 / 2 / 2006$ & $19: 00$ & 11.493 & 11.36 & 7.328 & 4.722 & 3.026 & 2.956 & 10.814 & 6.059 & 4.947 & 6.899 & 3.792 \\
\hline 2/2/2006 & $20: 00$ & 11.501 & 11.368 & 7.335 & 4.727 & 3.033 & 2.963 & 10.816 & 6.063 & 4.955 & 6.902 & 3.797 \\
\hline $2 / 2 / 2006$ & $21: 00$ & 11.507 & 11.379 & 7.343 & 4.731 & 3.039 & 2.971 & 10.819 & 6.066 & 4.958 & 6.903 & 3.8 \\
\hline 2/2/2006 & $22: 00$ & 11.511 & 11.384 & 7.346 & 4.734 & 3.043 & 2.973 & 10.821 & 6.067 & 4.961 & 6.906 & 3.802 \\
\hline $2 / 2 / 2006$ & $23: 00$ & 11.519 & 11.388 & 7.35 & 4.738 & 3.046 & 2.977 & 10.821 & 6.069 & 4.966 & 6.91 & 3.805 \\
\hline 2/3/2006 & $0: 00$ & 11.527 & 11.399 & 7.359 & 4.742 & 3.054 & 2.985 & 10.823 & 6.069 & 4.97 & 6.912 & 3.812 \\
\hline $2 / 3 / 2006$ & $1: 00$ & 11.536 & 11.41 & 7.37 & 4.747 & 3.063 & 2.994 & 10.827 & 6.075 & 4.978 & 6.918 & 3.816 \\
\hline 2/3/2006 & $2: 00$ & 11.542 & 11.417 & 7.372 & 4.751 & 3.067 & 3 & 10.829 & 6.076 & 4.979 & 6.919 & 3.822 \\
\hline 2/3/2006 & $3: 00$ & 11.551 & 11.428 & 7.383 & 4.758 & 3.076 & 3.006 & 10.833 & 6.076 & 4.985 & 6.923 & 3.826 \\
\hline $2 / 3 / 2006$ & $4: 00$ & 11.558 & 11.435 & 7.39 & 4.762 & 3.082 & 3.013 & 10.836 & 6.081 & 4.992 & 6.926 & 3.833 \\
\hline 2/3/2006 & $5: 00$ & 11.567 & 11.446 & 7.396 & 4.769 & 3.086 & 3.021 & 10.837 & 6.081 & 4.998 & 6.929 & 3.839 \\
\hline
\end{tabular}


TABLE S1.2 (Cont.)

Water Level (ft below top of casing) at Indicated Well

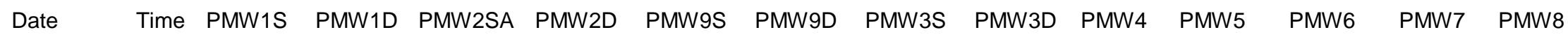

\begin{tabular}{|c|c|c|c|c|c|c|c|c|c|c|c|c|}
\hline $2 / 3 / 2006$ & $6: 00$ & 11.576 & 11.455 & 7.407 & 4.773 & 3.098 & 3.029 & 10.835 & 6.083 & 5.005 & 6.93 & 3.843 \\
\hline $2 / 3 / 2006$ & $7: 00$ & 11.587 & 11.468 & 7.416 & 4.782 & 3.105 & 3.039 & 10.838 & 6.09 & 5.013 & 6.94 & 3.847 \\
\hline $2 / 3 / 2006$ & $8: 00$ & 11.594 & 11.481 & 7.428 & 4.789 & 3.116 & 3.047 & 10.845 & 6.092 & 5.02 & 6.944 & 3.851 \\
\hline 2/3/2006 & 9:00 & 11.604 & 11.492 & 7.436 & 4.798 & 3.128 & 3.057 & 10.845 & 6.094 & 5.027 & 6.947 & 3.855 \\
\hline 2/3/2006 & $10: 00$ & 11.614 & 11.505 & 7.445 & 4.804 & 3.136 & 3.064 & 10.85 & 6.101 & 5.034 & 6.951 & 3.859 \\
\hline 2/3/2006 & $11: 00$ & 11.623 & 11.514 & 7.456 & 4.811 & 3.14 & 3.074 & 10.858 & 6.101 & 5.037 & 6.956 & 3.862 \\
\hline $2 / 3 / 2006$ & $12: 00$ & 11.632 & 11.521 & 7.461 & 4.818 & 3.148 & 3.078 & 10.861 & 6.105 & 5.041 & 6.961 & 3.861 \\
\hline 2/3/2006 & $13: 00$ & 11.64 & 11.523 & 7.461 & 4.82 & 3.146 & 3.075 & 10.863 & 6.102 & 5.041 & 6.962 & 3.848 \\
\hline 2/3/2006 & $14: 00$ & 11.643 & 11.523 & 7.456 & 4.822 & 3.14 & 3.075 & 10.864 & 6.104 & 5.036 & 6.959 & 3.832 \\
\hline $2 / 3 / 2006$ & $15: 00$ & 11.644 & 11.525 & 7.454 & 4.824 & 3.141 & 3.071 & 10.865 & 6.102 & 5.031 & 6.96 & 3.822 \\
\hline $2 / 3 / 2006$ & $16: 00$ & 11.648 & 11.526 & 7.454 & 4.827 & 3.141 & 3.072 & 10.869 & 6.105 & 5.029 & 6.961 & 3.82 \\
\hline $2 / 3 / 2006$ & $17: 00$ & 11.651 & 11.529 & 7.458 & 4.831 & 3.145 & 3.077 & 10.87 & 6.107 & 5.03 & 6.964 & 3.825 \\
\hline $2 / 3 / 2006$ & $18: 00$ & 11.655 & 11.534 & 7.465 & 4.835 & 3.153 & 3.082 & 10.872 & 6.107 & 5.032 & 6.969 & 3.831 \\
\hline $2 / 3 / 2006$ & $19: 00$ & 11.659 & 11.543 & 7.474 & 4.842 & 3.159 & 3.093 & 10.876 & 6.113 & 5.039 & 6.973 & 3.84 \\
\hline 2/3/2006 & $20: 00$ & 11.667 & 11.549 & 7.478 & 4.846 & 3.162 & 3.096 & 10.881 & 6.112 & 5.042 & 6.975 & 3.846 \\
\hline 2/3/2006 & $21: 00$ & 11.673 & 11.554 & 7.483 & 4.851 & 3.17 & 3.1 & 10.882 & 6.115 & 5.049 & 6.98 & 3.854 \\
\hline $2 / 3 / 2006$ & $22: 00$ & 11.676 & 11.56 & 7.487 & 4.855 & 3.174 & 3.103 & 10.884 & 6.116 & 5.051 & 6.982 & 3.861 \\
\hline $2 / 3 / 2006$ & $23: 00$ & 11.682 & 11.565 & 7.492 & 4.86 & 3.177 & 3.11 & 10.886 & 6.119 & 5.059 & 6.985 & 3.869 \\
\hline 2/4/2006 & $0: 00$ & 11.687 & 11.57 & 7.496 & 4.864 & 3.181 & 3.114 & 10.889 & 6.119 & 5.066 & 6.99 & 3.877 \\
\hline $2 / 4 / 2006$ & $1: 00$ & 11.692 & 11.574 & 7.5 & 4.871 & 3.187 & 3.119 & 10.891 & 6.122 & 5.073 & 6.991 & 3.885 \\
\hline 2/4/2006 & $2: 00$ & 11.694 & 11.576 & 7.5 & 4.873 & 3.187 & 3.12 & 10.892 & 6.126 & 5.079 & 6.993 & 3.891 \\
\hline $2 / 4 / 2006$ & $3: 00$ & 11.697 & 11.578 & 7.5 & 4.875 & 3.188 & 3.121 & 10.896 & 6.128 & 5.086 & 6.997 & 3.894 \\
\hline 2/4/2006 & $4: 00$ & 11.701 & 11.581 & 7.507 & 4.877 & 3.193 & 3.125 & 10.897 & 6.129 & 5.092 & 6.999 & 3.901 \\
\hline $2 / 4 / 2006$ & $5: 00$ & 11.702 & 11.581 & 7.505 & 4.882 & 3.19 & 3.123 & 10.899 & 6.131 & 5.099 & 7.001 & 3.907 \\
\hline 2/4/2006 & $6: 00$ & 11.703 & 11.583 & 7.507 & 4.884 & 3.193 & 3.124 & 10.901 & 6.131 & 5.106 & 7.002 & 3.913 \\
\hline $2 / 4 / 2006$ & $7: 00$ & 11.707 & 11.585 & 7.507 & 4.886 & 3.197 & 3.128 & 10.902 & 6.133 & 5.11 & 7.005 & 3.915 \\
\hline $2 / 4 / 2006$ & $8: 00$ & 11.708 & 11.589 & 7.512 & 4.888 & 3.201 & 3.132 & 10.905 & 6.134 & 5.119 & 7.01 & 3.921 \\
\hline $2 / 4 / 2006$ & $9: 00$ & 11.711 & 11.592 & 7.516 & 4.893 & 3.206 & 3.135 & 10.907 & 6.138 & 5.125 & 7.01 & 3.927 \\
\hline $2 / 4 / 2006$ & $10: 00$ & 11.714 & 11.592 & 7.516 & 4.895 & 3.204 & 3.137 & 10.907 & 6.141 & 5.134 & 7.013 & 3.931 \\
\hline $2 / 4 / 2006$ & $11: 00$ & 11.716 & 11.594 & 7.514 & 4.895 & 3.203 & 3.135 & 10.908 & 6.141 & 5.14 & 7.013 & 3.931 \\
\hline 2/4/2006 & $12: 00$ & 11.716 & 11.591 & 7.514 & 4.895 & 3.202 & 3.132 & 10.909 & 6.141 & 5.142 & 7.013 & 3.932 \\
\hline 2/4/2006 & $13: 00$ & 11.708 & 11.58 & 7.5 & 4.893 & 3.194 & 3.123 & 10.911 & 6.138 & 5.139 & 7.007 & 3.921 \\
\hline $2 / 4 / 2006$ & $14: 00$ & 11.7 & 11.567 & 7.492 & 4.891 & 3.18 & 3.11 & 10.909 & 6.132 & 5.139 & 7.003 & 3.913 \\
\hline 2/4/2006 & $15: 00$ & 11.69 & 11.552 & 7.478 & 4.886 & 3.167 & 3.098 & 10.903 & 6.131 & 5.131 & 7.001 & 3.898 \\
\hline
\end{tabular}


TABLE S1.2 (Cont.)

Water Level (ft below top of casing) at Indicated Well

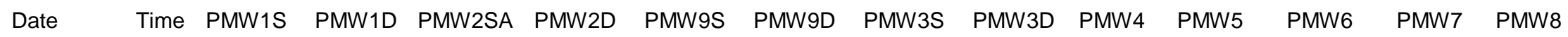

\begin{tabular}{|c|c|c|c|c|c|c|c|c|c|c|c|c|}
\hline 2/4/2006 & $16: 00$ & 11.683 & 11.543 & 7.474 & 4.882 & 3.16 & 3.09 & 10.902 & 6.13 & 5.124 & 6.994 & 3.882 \\
\hline $2 / 4 / 2006$ & $17: 00$ & 11.677 & 11.538 & 7.467 & 4.877 & 3.156 & 3.085 & 10.903 & 6.128 & 5.118 & 6.994 & 3.868 \\
\hline $2 / 4 / 2006$ & $18: 00$ & 11.672 & 11.53 & 7.461 & 4.875 & 3.149 & 3.078 & 10.9 & 6.126 & 5.107 & 6.988 & 3.852 \\
\hline 2/4/2006 & $19: 00$ & 11.668 & 11.527 & 7.463 & 4.873 & 3.152 & 3.08 & 10.9 & 6.125 & 5.101 & 6.988 & 3.846 \\
\hline 2/4/2006 & $20: 00$ & 11.664 & 11.521 & 7.458 & 4.871 & 3.148 & 3.076 & 10.899 & 6.123 & 5.095 & 6.985 & 3.839 \\
\hline 2/4/2006 & $21: 00$ & 11.659 & 11.518 & 7.456 & 4.869 & 3.141 & 3.074 & 10.897 & 6.12 & 5.087 & 6.983 & 3.84 \\
\hline $2 / 4 / 2006$ & $22: 00$ & 11.657 & 11.516 & 7.454 & 4.866 & 3.147 & 3.075 & 10.896 & 6.12 & 5.084 & 6.98 & 3.845 \\
\hline 2/4/2006 & $23: 00$ & 11.651 & 11.512 & 7.452 & 4.864 & 3.14 & 3.072 & 10.895 & 6.122 & 5.085 & 6.981 & 3.851 \\
\hline 2/5/2006 & $0: 00$ & 11.648 & 11.507 & 7.447 & 4.862 & 3.139 & 3.069 & 10.893 & 6.119 & 5.086 & 6.981 & 3.857 \\
\hline $2 / 5 / 2006$ & $1: 00$ & 11.644 & 11.503 & 7.445 & 4.86 & 3.141 & 3.07 & 10.895 & 6.12 & 5.088 & 6.981 & 3.869 \\
\hline $2 / 5 / 2006$ & $2: 00$ & 11.64 & 11.496 & 7.441 & 4.857 & 3.135 & 3.065 & 10.891 & 6.119 & 5.09 & 6.979 & 3.875 \\
\hline $2 / 5 / 2006$ & $3: 00$ & 11.635 & 11.494 & 7.441 & 4.855 & 3.136 & 3.065 & 10.891 & 6.12 & 5.095 & 6.977 & 3.887 \\
\hline $2 / 5 / 2006$ & $4: 00$ & 11.63 & 11.488 & 7.434 & 4.853 & 3.134 & 3.062 & 10.889 & 6.118 & 5.1 & 6.977 & 3.895 \\
\hline $2 / 5 / 2006$ & $5: 00$ & 11.625 & 11.477 & 7.427 & 4.849 & 3.127 & 3.058 & 10.888 & 6.113 & 5.1 & 6.974 & 3.897 \\
\hline $2 / 5 / 2006$ & $6: 00$ & 11.619 & 11.47 & 7.419 & 4.844 & 3.119 & 3.05 & 10.885 & 6.112 & 5.104 & 6.972 & 3.9 \\
\hline $2 / 5 / 2006$ & $7: 00$ & 11.616 & 11.468 & 7.421 & 4.844 & 3.122 & 3.053 & 10.883 & 6.114 & 5.113 & 6.974 & 3.909 \\
\hline $2 / 5 / 2006$ & $8: 00$ & 11.618 & 11.479 & 7.432 & 4.846 & 3.133 & 3.062 & 10.885 & 6.122 & 5.125 & 6.978 & 3.921 \\
\hline $2 / 5 / 2006$ & $9: 00$ & 11.624 & 11.488 & 7.441 & 4.849 & 3.143 & 3.071 & 10.889 & 6.127 & 5.133 & 6.983 & 3.932 \\
\hline $2 / 5 / 2006$ & $10: 00$ & 11.63 & 11.498 & 7.452 & 4.851 & 3.152 & 3.079 & 10.891 & 6.127 & 5.142 & 6.987 & 3.936 \\
\hline $2 / 5 / 2006$ & $11: 00$ & 11.635 & 11.501 & 7.452 & 4.853 & 3.153 & 3.084 & 10.893 & 6.128 & 5.142 & 6.988 & 3.938 \\
\hline $2 / 5 / 2006$ & $12: 00$ & 11.64 & 11.508 & 7.458 & 4.855 & 3.156 & 3.086 & 10.895 & 6.13 & 5.146 & 6.991 & 3.94 \\
\hline $2 / 5 / 2006$ & $13: 00$ & 11.645 & 11.508 & 7.456 & 4.855 & 3.158 & 3.086 & 10.897 & 6.125 & 5.145 & 6.99 & 3.93 \\
\hline 2/5/2006 & $14: 00$ & 11.638 & 11.499 & 7.447 & 4.855 & 3.148 & 3.077 & 10.894 & 6.123 & 5.14 & 6.99 & 3.919 \\
\hline $2 / 5 / 2006$ & $15: 00$ & 11.63 & 11.487 & 7.436 & 4.853 & 3.135 & 3.066 & 10.891 & 6.12 & 5.134 & 6.985 & 3.905 \\
\hline $2 / 5 / 2006$ & $16: 00$ & 11.629 & 11.49 & 7.441 & 4.851 & 3.136 & 3.064 & 10.889 & 6.121 & 5.132 & 6.981 & 3.899 \\
\hline $2 / 5 / 2006$ & $17: 00$ & 11.633 & 11.498 & 7.447 & 4.853 & 3.143 & 3.071 & 10.89 & 6.123 & 5.13 & 6.985 & 3.895 \\
\hline $2 / 5 / 2006$ & $18: 00$ & 11.638 & 11.507 & 7.458 & 4.855 & 3.151 & 3.08 & 10.892 & 6.127 & 5.125 & 6.986 & 3.887 \\
\hline $2 / 5 / 2006$ & $19: 00$ & 11.648 & 11.521 & 7.467 & 4.86 & 3.16 & 3.089 & 10.897 & 6.128 & 5.127 & 6.989 & 3.882 \\
\hline $2 / 5 / 2006$ & $20: 00$ & 11.66 & 11.536 & 7.481 & 4.864 & 3.169 & 3.101 & 10.9 & 6.133 & 5.127 & 6.994 & 3.879 \\
\hline $2 / 5 / 2006$ & $21: 00$ & 11.67 & 11.547 & 7.489 & 4.871 & 3.18 & 3.11 & 10.904 & 6.135 & 5.124 & 6.998 & 3.879 \\
\hline $2 / 5 / 2006$ & $22: 00$ & 11.679 & 11.556 & 7.496 & 4.875 & 3.184 & 3.118 & 10.906 & 6.134 & 5.123 & 7.001 & 3.875 \\
\hline $2 / 5 / 2006$ & $23: 00$ & 11.689 & 11.567 & 7.503 & 4.88 & 3.19 & 3.124 & 10.91 & 6.137 & 5.123 & 7.003 & 3.878 \\
\hline $2 / 6 / 2006$ & $0: 00$ & 11.697 & 11.574 & 7.512 & 4.884 & 3.198 & 3.128 & 10.912 & 6.136 & 5.124 & 7.008 & 3.88 \\
\hline 2/6/2006 & $1: 00$ & 11.704 & 11.578 & 7.512 & 4.888 & 3.2 & 3.133 & 10.913 & 6.137 & 5.121 & 7.009 & 3.877 \\
\hline
\end{tabular}


TABLE S1.2 (Cont.)

Water Level (ft below top of casing) at Indicated Well

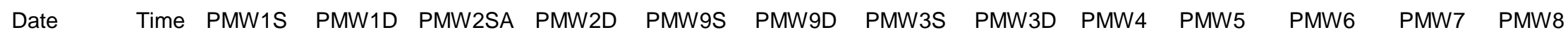

\begin{tabular}{|c|c|c|c|c|c|c|c|c|c|c|c|c|}
\hline $2 / 6 / 2006$ & $2: 00$ & 11.709 & 11.583 & 7.516 & 4.893 & 3.203 & 3.136 & 10.913 & 6.139 & 5.122 & 7.01 & 3.881 \\
\hline $2 / 6 / 2006$ & $3: 00$ & 11.713 & 11.587 & 7.516 & 4.897 & 3.207 & 3.136 & 10.917 & 6.141 & 5.12 & 7.011 & 3.886 \\
\hline $2 / 6 / 2006$ & $4: 00$ & 11.718 & 11.591 & 7.518 & 4.9 & 3.209 & 3.139 & 10.917 & 6.141 & 5.121 & 7.013 & 3.893 \\
\hline 2/6/2006 & $5: 00$ & 11.719 & 11.591 & 7.518 & 4.902 & 3.209 & 3.14 & 10.919 & 6.141 & 5.12 & 7.015 & 3.9 \\
\hline 2/6/2006 & $6: 00$ & 11.721 & 11.594 & 7.522 & 4.906 & 3.214 & 3.144 & 10.919 & 6.146 & 5.127 & 7.017 & 3.911 \\
\hline 2/6/2006 & $7: 00$ & 11.724 & 11.598 & 7.524 & 4.908 & 3.218 & 3.146 & 10.921 & 6.146 & 5.134 & 7.019 & 3.923 \\
\hline $2 / 6 / 2006$ & $8: 00$ & 11.727 & 11.6 & 7.527 & 4.913 & 3.223 & 3.153 & 10.922 & 6.151 & 5.145 & 7.024 & 3.935 \\
\hline 2/6/2006 & $9: 00$ & 11.731 & 11.607 & 7.536 & 4.917 & 3.229 & 3.157 & 10.924 & 6.151 & 5.155 & 7.028 & 3.948 \\
\hline $2 / 6 / 2006$ & $10: 00$ & 11.734 & 11.612 & 7.54 & 4.922 & 3.231 & 3.164 & 10.927 & 6.157 & 5.163 & 7.032 & 3.958 \\
\hline $2 / 6 / 2006$ & $11: 00$ & 11.737 & 11.618 & 7.542 & 4.926 & 3.236 & 3.166 & 10.929 & 6.156 & 5.17 & 7.033 & 3.96 \\
\hline $2 / 6 / 2006$ & $12: 00$ & 11.742 & 11.622 & 7.544 & 4.929 & 3.239 & 3.17 & 10.93 & 6.16 & 5.175 & 7.037 & 3.96 \\
\hline $2 / 6 / 2006$ & $13: 00$ & 11.745 & 11.616 & 7.54 & 4.931 & 3.235 & 3.167 & 10.933 & 6.157 & 5.174 & 7.034 & 3.953 \\
\hline $2 / 6 / 2006$ & $14: 00$ & 11.74 & 11.609 & 7.533 & 4.928 & 3.224 & 3.156 & 10.931 & 6.155 & 5.169 & 7.031 & 3.942 \\
\hline $2 / 6 / 2006$ & $15: 00$ & 11.737 & 11.603 & 7.527 & 4.928 & 3.219 & 3.149 & 10.93 & 6.152 & 5.168 & 7.029 & 3.932 \\
\hline $2 / 6 / 2006$ & $16: 00$ & 11.736 & 11.598 & 7.522 & 4.926 & 3.214 & 3.143 & 10.93 & 6.153 & 5.166 & 7.028 & 3.924 \\
\hline 2/6/2006 & $17: 00$ & 11.731 & 11.596 & 7.522 & 4.924 & 3.211 & 3.14 & 10.93 & 6.151 & 5.159 & 7.028 & 3.914 \\
\hline $2 / 6 / 2006$ & $18: 00$ & 11.732 & 11.596 & 7.522 & 4.924 & 3.213 & 3.14 & 10.93 & 6.154 & 5.155 & 7.027 & 3.906 \\
\hline $2 / 6 / 2006$ & $19: 00$ & 11.731 & 11.594 & 7.524 & 4.922 & 3.211 & 3.139 & 10.93 & 6.154 & 5.148 & 7.024 & 3.893 \\
\hline $2 / 6 / 2006$ & $20: 00$ & 11.73 & 11.596 & 7.524 & 4.924 & 3.211 & 3.14 & 10.93 & 6.155 & 5.144 & 7.024 & 3.885 \\
\hline $2 / 6 / 2006$ & $21: 00$ & 11.736 & 11.6 & 7.529 & 4.926 & 3.212 & 3.144 & 10.931 & 6.153 & 5.137 & 7.025 & 3.879 \\
\hline 2/6/2006 & $22: 00$ & 11.737 & 11.6 & 7.531 & 4.924 & 3.213 & 3.142 & 10.931 & 6.152 & 5.132 & 7.026 & 3.871 \\
\hline $2 / 6 / 2006$ & $23: 00$ & 11.736 & 11.601 & 7.529 & 4.924 & 3.214 & 3.145 & 10.932 & 6.153 & 5.126 & 7.028 & 3.866 \\
\hline 2/7/2006 & $0: 00$ & 11.735 & 11.601 & 7.529 & 4.924 & 3.215 & 3.144 & 10.931 & 6.151 & 5.123 & 7.025 & 3.863 \\
\hline $2 / 7 / 2006$ & $1: 00$ & 11.735 & 11.6 & 7.527 & 4.924 & 3.211 & 3.143 & 10.933 & 6.151 & 5.12 & 7.027 & 3.859 \\
\hline 2/7/2006 & $2: 00$ & 11.738 & 11.603 & 7.529 & 4.924 & 3.215 & 3.147 & 10.934 & 6.152 & 5.116 & 7.025 & 3.859 \\
\hline $2 / 7 / 2006$ & $3: 00$ & 11.736 & 11.603 & 7.531 & 4.926 & 3.216 & 3.147 & 10.933 & 6.151 & 5.114 & 7.025 & 3.858 \\
\hline 2/7/2006 & $4: 00$ & 11.735 & 11.601 & 7.529 & 4.926 & 3.215 & 3.146 & 10.934 & 6.149 & 5.11 & 7.023 & 3.857 \\
\hline $2 / 7 / 2006$ & $5: 00$ & 11.733 & 11.596 & 7.524 & 4.924 & 3.21 & 3.143 & 10.932 & 6.149 & 5.108 & 7.023 & 3.853 \\
\hline 2/7/2006 & $6: 00$ & 11.733 & 11.596 & 7.524 & 4.924 & 3.211 & 3.142 & 10.934 & 6.149 & 5.105 & 7.022 & 3.856 \\
\hline 2/7/2006 & $7: 00$ & 11.733 & 11.594 & 7.527 & 4.924 & 3.214 & 3.144 & 10.934 & 6.151 & 5.103 & 7.021 & 3.856 \\
\hline 2/7/2006 & $8: 00$ & 11.734 & 11.598 & 7.529 & 4.924 & 3.217 & 3.147 & 10.934 & 6.153 & 5.103 & 7.025 & 3.861 \\
\hline 2/7/2006 & $9: 00$ & 11.738 & 11.605 & 7.531 & 4.926 & 3.222 & 3.152 & 10.934 & 6.153 & 5.105 & 7.025 & 3.868 \\
\hline $2 / 7 / 2006$ & $10: 00$ & 11.741 & 11.607 & 7.538 & 4.926 & 3.224 & 3.157 & 10.934 & 6.153 & 5.107 & 7.026 & 3.873 \\
\hline 2/7/2006 & $11: 00$ & 11.738 & 11.603 & 7.536 & 4.929 & 3.223 & 3.153 & 10.935 & 6.155 & 5.102 & 7.024 & 3.873 \\
\hline
\end{tabular}


TABLE S1.2 (Cont.)

Water Level (ft below top of casing) at Indicated Well

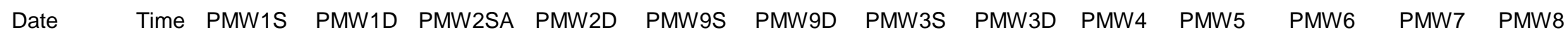

\begin{tabular}{|c|c|c|c|c|c|c|c|c|c|c|c|c|}
\hline 2/7/2006 & $12: 00$ & 11.741 & 11.609 & 7.538 & 4.931 & 3.226 & 3.155 & 10.935 & 6.156 & 5.106 & 7.026 & 3.875 \\
\hline $2 / 7 / 2006$ & $13: 00$ & 11.738 & 11.6 & 7.531 & 4.929 & 3.22 & 3.149 & 10.935 & 6.152 & 5.1 & 7.023 & 3.869 \\
\hline $2 / 7 / 2006$ & $14: 00$ & 11.731 & 11.591 & 7.52 & 4.924 & 3.206 & 3.14 & 10.932 & 6.149 & 5.092 & 7.019 & 3.856 \\
\hline 2/7/2006 & $15: 00$ & 11.72 & 11.578 & 7.507 & 4.92 & 3.198 & 3.127 & 10.93 & 6.146 & 5.083 & 7.014 & 3.842 \\
\hline 2/7/2006 & $16: 00$ & 11.713 & 11.569 & 7.502 & 4.915 & 3.187 & 3.12 & 10.927 & 6.141 & 5.076 & 7.008 & 3.827 \\
\hline 2/7/2006 & $17: 00$ & 11.707 & 11.563 & 7.498 & 4.913 & 3.183 & 3.114 & 10.924 & 6.141 & 5.066 & 7.004 & 3.812 \\
\hline $2 / 7 / 2006$ & $18: 00$ & 11.7 & 11.556 & 7.491 & 4.911 & 3.178 & 3.107 & 10.924 & 6.135 & 5.058 & 7.002 & 3.798 \\
\hline 2/7/2006 & $19: 00$ & 11.697 & 11.55 & 7.489 & 4.906 & 3.176 & 3.106 & 10.92 & 6.138 & 5.051 & 7 & 3.79 \\
\hline 2/7/2006 & $20: 00$ & 11.691 & 11.549 & 7.489 & 4.904 & 3.174 & 3.103 & 10.921 & 6.134 & 5.048 & 6.998 & 3.786 \\
\hline 2/7/2006 & $21: 00$ & 11.689 & 11.545 & 7.482 & 4.9 & 3.172 & 3.101 & 10.918 & 6.132 & 5.039 & 6.996 & 3.783 \\
\hline $2 / 7 / 2006$ & $22: 00$ & 11.689 & 11.547 & 7.485 & 4.9 & 3.171 & 3.104 & 10.919 & 6.133 & 5.04 & 6.999 & 3.786 \\
\hline 2/7/2006 & $23: 00$ & 11.69 & 11.551 & 7.491 & 4.898 & 3.176 & 3.107 & 10.919 & 6.135 & 5.036 & 6.996 & 3.789 \\
\hline $2 / 8 / 2006$ & $0: 00$ & 11.692 & 11.556 & 7.496 & 4.898 & 3.182 & 3.111 & 10.918 & 6.137 & 5.038 & 6.998 & 3.796 \\
\hline 2/8/2006 & $1: 00$ & 11.695 & 11.56 & 7.5 & 4.9 & 3.186 & 3.114 & 10.92 & 6.138 & 5.039 & 7 & 3.8 \\
\hline $2 / 8 / 2006$ & $2: 00$ & 11.696 & 11.56 & 7.498 & 4.898 & 3.187 & 3.115 & 10.921 & 6.138 & 5.04 & 7 & 3.804 \\
\hline $2 / 8 / 2006$ & $3: 00$ & 11.699 & 11.561 & 7.5 & 4.9 & 3.188 & 3.117 & 10.92 & 6.14 & 5.041 & 7 & 3.812 \\
\hline $2 / 8 / 2006$ & $4: 00$ & 11.704 & 11.57 & 7.509 & 4.902 & 3.196 & 3.125 & 10.922 & 6.145 & 5.047 & 7.004 & 3.825 \\
\hline $2 / 8 / 2006$ & $5: 00$ & 11.706 & 11.572 & 7.509 & 4.902 & 3.199 & 3.129 & 10.922 & 6.143 & 5.051 & 7.007 & 3.835 \\
\hline 2/8/2006 & $6: 00$ & 11.714 & 11.582 & 7.518 & 4.904 & 3.206 & 3.137 & 10.926 & 6.148 & 5.06 & 7.01 & 3.846 \\
\hline 2/8/2006 & $7: 00$ & 11.722 & 11.591 & 7.527 & 4.908 & 3.213 & 3.145 & 10.928 & 6.152 & 5.067 & 7.014 & 3.857 \\
\hline 2/8/2006 & $8: 00$ & 11.728 & 11.603 & 7.538 & 4.915 & 3.224 & 3.153 & 10.931 & 6.155 & 5.078 & 7.019 & 3.868 \\
\hline 2/8/2006 & $9: 00$ & 11.739 & 11.614 & 7.547 & 4.92 & 3.233 & 3.165 & 10.937 & 6.158 & 5.086 & 7.024 & 3.878 \\
\hline 2/8/2006 & $10: 00$ & 11.748 & 11.624 & 7.553 & 4.924 & 3.242 & 3.172 & 10.936 & 6.16 & 5.097 & 7.028 & 3.892 \\
\hline $2 / 8 / 2006$ & $11: 00$ & 11.751 & 11.626 & 7.553 & 4.929 & 3.243 & 3.175 & 10.94 & 6.159 & 5.102 & 7.028 & 3.898 \\
\hline 2/8/2006 & $12: 00$ & 11.754 & 11.631 & 7.56 & 4.933 & 3.249 & 3.179 & 10.937 & 6.166 & 5.112 & 7.033 & 3.905 \\
\hline $2 / 8 / 2006$ & $13: 00$ & 11.756 & 11.629 & 7.555 & 4.935 & 3.246 & 3.174 & 10.94 & 6.165 & 5.115 & 7.034 & 3.905 \\
\hline 2/8/2006 & $14: 00$ & 11.756 & 11.626 & 7.549 & 4.935 & 3.239 & 3.168 & 10.939 & 6.163 & 5.113 & 7.031 & 3.897 \\
\hline $2 / 8 / 2006$ & $15: 00$ & 11.754 & 11.622 & 7.547 & 4.935 & 3.234 & 3.165 & 10.943 & 6.161 & 5.113 & 7.031 & 3.889 \\
\hline 2/8/2006 & $16: 00$ & 11.752 & 11.614 & 7.542 & 4.935 & 3.227 & 3.156 & 10.939 & 6.159 & 5.108 & 7.028 & 3.873 \\
\hline 2/8/2006 & $17: 00$ & 11.754 & 11.618 & 7.542 & 4.935 & 3.232 & 3.16 & 10.944 & 6.16 & 5.104 & 7.028 & 3.861 \\
\hline 2/8/2006 & $18: 00$ & 11.751 & 11.618 & 7.542 & 4.935 & 3.228 & 3.158 & 10.942 & 6.159 & 5.097 & 7.026 & 3.854 \\
\hline 2/8/2006 & $19: 00$ & 11.751 & 11.618 & 7.544 & 4.938 & 3.231 & 3.16 & 10.941 & 6.158 & 5.093 & 7.027 & 3.849 \\
\hline $2 / 8 / 2006$ & $20: 00$ & 11.755 & 11.626 & 7.551 & 4.94 & 3.235 & 3.168 & 10.942 & 6.163 & 5.095 & 7.03 & 3.85 \\
\hline 2/8/2006 & $21: 00$ & 11.76 & 11.631 & 7.557 & 4.942 & 3.243 & 3.175 & 10.944 & 6.163 & 5.097 & 7.035 & 3.861 \\
\hline
\end{tabular}


TABLE S1.2 (Cont.)

Water Level (ft below top of casing) at Indicated Well

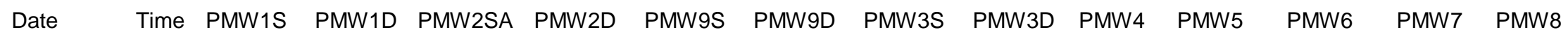

\begin{tabular}{|c|c|c|c|c|c|c|c|c|c|c|c|c|}
\hline 2/8/2006 & $22: 00$ & 11.762 & 11.635 & 7.562 & 4.946 & 3.249 & 3.18 & 10.947 & 6.163 & 5.103 & 7.036 & 3.872 \\
\hline 2/8/2006 & $23: 00$ & 11.763 & 11.635 & 7.56 & 4.946 & 3.248 & 3.179 & 10.947 & 6.161 & 5.106 & 7.037 & 3.878 \\
\hline $2 / 9 / 2006$ & $0: 00$ & 11.762 & 11.635 & 7.557 & 4.949 & 3.251 & 3.179 & 10.945 & 6.165 & 5.111 & 7.037 & 3.89 \\
\hline 2/9/2006 & $1: 00$ & 11.762 & 11.629 & 7.555 & 4.949 & 3.246 & 3.175 & 10.947 & 6.163 & 5.116 & 7.038 & 3.898 \\
\hline $2 / 9 / 2006$ & $2: 00$ & 11.764 & 11.627 & 7.553 & 4.949 & 3.246 & 3.175 & 10.947 & 6.166 & 5.124 & 7.038 & 3.91 \\
\hline 2/9/2006 & 3:00 & 11.758 & 11.622 & 7.546 & 4.949 & 3.244 & 3.171 & 10.946 & 6.165 & 5.128 & 7.038 & 3.918 \\
\hline $2 / 9 / 2006$ & $4: 00$ & 11.75 & 11.611 & 7.54 & 4.946 & 3.238 & 3.167 & 10.945 & 6.162 & 5.132 & 7.037 & 3.922 \\
\hline 2/9/2006 & $5: 00$ & 11.739 & 11.593 & 7.524 & 4.942 & 3.224 & 3.154 & 10.941 & 6.158 & 5.132 & 7.032 & 3.924 \\
\hline $2 / 9 / 2006$ & $6: 00$ & 11.728 & 11.578 & 7.513 & 4.938 & 3.214 & 3.142 & 10.937 & 6.155 & 5.134 & 7.027 & 3.923 \\
\hline 2/9/2006 & $7: 00$ & 11.715 & 11.567 & 7.504 & 4.933 & 3.205 & 3.133 & 10.932 & 6.156 & 5.142 & 7.026 & 3.927 \\
\hline $2 / 9 / 2006$ & $8: 00$ & 11.708 & 11.56 & 7.5 & 4.929 & 3.204 & 3.131 & 10.933 & 6.156 & 5.149 & 7.028 & 3.932 \\
\hline $2 / 9 / 2006$ & $9: 00$ & 11.701 & 11.551 & 7.493 & 4.924 & 3.199 & 3.124 & 10.928 & 6.157 & 5.156 & 7.024 & 3.938 \\
\hline 2/9/2006 & $10: 00$ & 11.695 & 11.543 & 7.486 & 4.92 & 3.191 & 3.12 & 10.929 & 6.153 & 5.155 & 7.019 & 3.94 \\
\hline 2/9/2006 & $11: 00$ & 11.686 & 11.534 & 7.478 & 4.913 & 3.189 & 3.11 & 10.928 & 6.152 & 5.159 & 7.015 & 3.939 \\
\hline 2/9/2006 & $12: 00$ & 11.675 & 11.523 & 7.464 & 4.907 & 3.172 & 3.097 & 10.918 & 6.144 & 5.157 & 7.01 & 3.932 \\
\hline $2 / 9 / 2006$ & $13: 00$ & 11.664 & 11.505 & 7.453 & 4.9 & 3.163 & 3.086 & 10.914 & 6.14 & 5.148 & 7.008 & 3.924 \\
\hline 2/9/2006 & $14: 00$ & 11.649 & 11.491 & 7.44 & 4.891 & 3.152 & 3.074 & 10.908 & 6.136 & 5.142 & 6.998 & 3.915 \\
\hline $2 / 9 / 2006$ & $15: 00$ & 11.641 & 11.48 & 7.433 & 4.884 & 3.141 & 3.067 & 10.907 & 6.137 & 5.139 & 6.996 & 3.91 \\
\hline 2/9/2006 & $16: 00$ & 11.632 & 11.472 & 7.433 & 4.878 & 3.143 & 3.065 & 10.903 & 6.133 & 5.133 & 6.991 & 3.904 \\
\hline $2 / 9 / 2006$ & $17: 00$ & 11.627 & 11.469 & 7.429 & 4.871 & 3.133 & 3.06 & 10.899 & 6.133 & 5.126 & 6.99 & 3.897 \\
\hline 2/9/2006 & $18: 00$ & 11.625 & 11.469 & 7.433 & 4.869 & 3.137 & 3.06 & 10.901 & 6.131 & 5.126 & 6.988 & 3.895 \\
\hline $2 / 9 / 2006$ & $19: 00$ & 11.622 & 11.476 & 7.438 & 4.869 & 3.139 & 3.065 & 10.898 & 6.135 & 5.125 & 6.987 & 3.893 \\
\hline 2/9/2006 & $20: 00$ & 11.629 & 11.485 & 7.447 & 4.871 & 3.149 & 3.078 & 10.901 & 6.133 & 5.122 & 6.99 & 3.895 \\
\hline 2/9/2006 & $21: 00$ & 11.634 & 11.491 & 7.451 & 4.871 & 3.152 & 3.082 & 10.903 & 6.131 & 5.116 & 6.989 & 3.892 \\
\hline 2/9/2006 & $22: 00$ & 11.638 & 11.498 & 7.455 & 4.871 & 3.155 & 3.082 & 10.903 & 6.135 & 5.115 & 6.991 & 3.89 \\
\hline $2 / 9 / 2006$ & $23: 00$ & 11.636 & 11.498 & 7.453 & 4.871 & 3.155 & 3.081 & 10.902 & 6.132 & 5.112 & 6.989 & 3.883 \\
\hline $2 / 10 / 2006$ & $0: 00$ & 11.643 & 11.5 & 7.46 & 4.873 & 3.159 & 3.084 & 10.9 & 6.13 & 5.109 & 6.99 & 3.88 \\
\hline 2/10/2006 & $1: 00$ & 11.646 & 11.511 & 7.467 & 4.876 & 3.167 & 3.092 & 10.904 & 6.134 & 5.107 & 6.993 & 3.882 \\
\hline $2 / 10 / 2006$ & $2: 00$ & 11.649 & 11.512 & 7.467 & 4.876 & 3.166 & 3.093 & 10.904 & 6.134 & 5.103 & 6.99 & 3.878 \\
\hline $2 / 10 / 2006$ & $3: 00$ & 11.653 & 11.514 & 7.469 & 4.876 & 3.167 & 3.094 & 10.905 & 6.128 & 5.1 & 6.993 & 3.876 \\
\hline $2 / 10 / 2006$ & $4: 00$ & 11.655 & 11.516 & 7.471 & 4.878 & 3.171 & 3.095 & 10.903 & 6.132 & 5.097 & 6.992 & 3.876 \\
\hline 2/10/2006 & $5: 00$ & 11.654 & 11.514 & 7.469 & 4.878 & 3.167 & 3.094 & 10.904 & 6.128 & 5.093 & 6.991 & 3.871 \\
\hline $2 / 10 / 2006$ & $6: 00$ & 11.655 & 11.516 & 7.469 & 4.876 & 3.166 & 3.094 & 10.903 & 6.134 & 5.09 & 6.99 & 3.871 \\
\hline $2 / 10 / 2006$ & $7: 00$ & 11.657 & 11.518 & 7.473 & 4.878 & 3.169 & 3.096 & 10.904 & 6.133 & 5.091 & 6.992 & 3.878 \\
\hline
\end{tabular}


TABLE S1.2 (Cont.)

Water Level (ft below top of casing) at Indicated Well

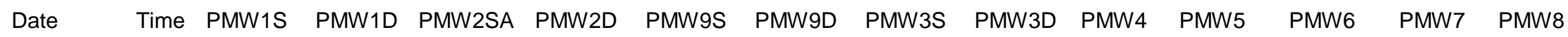

\begin{tabular}{|c|c|c|c|c|c|c|c|c|c|c|c|c|}
\hline 2/10/2006 & $8: 00$ & 11.662 & 11.523 & 7.475 & 4.88 & 3.175 & 3.102 & 10.907 & 6.134 & 5.09 & 6.995 & 3.884 \\
\hline $2 / 10 / 2006$ & $9: 00$ & 11.665 & 11.529 & 7.484 & 4.882 & 3.18 & 3.105 & 10.906 & 6.136 & 5.092 & 6.994 & 3.893 \\
\hline $2 / 10 / 2006$ & $10: 00$ & 11.669 & 11.534 & 7.486 & 4.884 & 3.183 & 3.11 & 10.907 & 6.138 & 5.094 & 6.997 & 3.907 \\
\hline $2 / 10 / 2006$ & $11: 00$ & 11.673 & 11.538 & 7.489 & 4.884 & 3.19 & 3.113 & 10.908 & 6.14 & 5.095 & 6.998 & 3.909 \\
\hline 2/10/2006 & $12: 00$ & 11.678 & 11.545 & 7.491 & 4.891 & 3.191 & 3.118 & 10.908 & 6.141 & 5.102 & 7.003 & 3.91 \\
\hline 2/10/2006 & $13: 00$ & 11.679 & 11.545 & 7.493 & 4.891 & 3.192 & 3.118 & 10.91 & 6.141 & 5.102 & 7 & 3.909 \\
\hline $2 / 10 / 2006$ & $14: 00$ & 11.675 & 11.538 & 7.484 & 4.891 & 3.184 & 3.109 & 10.909 & 6.139 & 5.1 & 6.998 & 3.901 \\
\hline 2/10/2006 & $15: 00$ & 11.673 & 11.54 & 7.482 & 4.891 & 3.181 & 3.108 & 10.907 & 6.137 & 5.097 & 6.993 & 3.898 \\
\hline $2 / 10 / 2006$ & $16: 00$ & 11.673 & 11.532 & 7.486 & 4.891 & 3.18 & 3.107 & 10.906 & 6.138 & 5.095 & 6.998 & 3.89 \\
\hline 2/10/2006 & $17: 00$ & 11.678 & 11.54 & 7.489 & 4.891 & 3.184 & 3.11 & 10.907 & 6.138 & 5.095 & 6.998 & 3.891 \\
\hline $2 / 10 / 2006$ & $18: 00$ & 11.677 & 11.545 & 7.488 & 4.891 & 3.185 & 3.113 & 10.908 & 6.139 & 5.098 & 6.994 & 3.886 \\
\hline 2/10/2006 & $19: 00$ & 11.688 & 11.556 & 7.504 & 4.896 & 3.194 & 3.122 & 10.915 & 6.144 & 5.096 & 7.002 & 3.889 \\
\hline $2 / 10 / 2006$ & $20: 00$ & 11.695 & 11.569 & 7.515 & 4.902 & 3.204 & 3.133 & 10.915 & 6.149 & 5.099 & 7.008 & 3.89 \\
\hline 2/10/2006 & $21: 00$ & 11.703 & 11.573 & 7.515 & 4.904 & 3.207 & 3.136 & 10.92 & 6.148 & 5.094 & 7.008 & 3.885 \\
\hline $2 / 10 / 2006$ & $22: 00$ & 11.706 & 11.576 & 7.517 & 4.909 & 3.21 & 3.135 & 10.923 & 6.148 & 5.092 & 7.009 & 3.879 \\
\hline $2 / 10 / 2006$ & $23: 00$ & 11.711 & 11.582 & 7.524 & 4.907 & 3.213 & 3.142 & 10.92 & 6.147 & 5.093 & 7.009 & 3.88 \\
\hline $2 / 11 / 2006$ & $0: 00$ & 11.714 & 11.58 & 7.524 & 4.911 & 3.214 & 3.138 & 10.92 & 6.146 & 5.091 & 7.012 & 3.874 \\
\hline $2 / 11 / 2006$ & $1: 00$ & 11.716 & 11.582 & 7.522 & 4.913 & 3.213 & 3.141 & 10.921 & 6.147 & 5.091 & 7.012 & 3.87 \\
\hline $2 / 11 / 2006$ & $2: 00$ & 11.719 & 11.584 & 7.522 & 4.915 & 3.213 & 3.141 & 10.923 & 6.146 & 5.088 & 7.014 & 3.866 \\
\hline $2 / 11 / 2006$ & $3: 00$ & 11.719 & 11.585 & 7.529 & 4.915 & 3.217 & 3.144 & 10.923 & 6.146 & 5.086 & 7.012 & 3.864 \\
\hline $2 / 11 / 2006$ & $4: 00$ & 11.72 & 11.587 & 7.528 & 4.918 & 3.218 & 3.144 & 10.923 & 6.148 & 5.086 & 7.014 & 3.863 \\
\hline $2 / 11 / 2006$ & $5: 00$ & 11.719 & 11.582 & 7.522 & 4.918 & 3.212 & 3.14 & 10.923 & 6.144 & 5.083 & 7.013 & 3.861 \\
\hline $2 / 11 / 2006$ & $6: 00$ & 11.724 & 11.589 & 7.53 & 4.918 & 3.22 & 3.146 & 10.923 & 6.149 & 5.085 & 7.015 & 3.865 \\
\hline $2 / 11 / 2006$ & $7: 00$ & 11.728 & 11.594 & 7.533 & 4.922 & 3.222 & 3.149 & 10.925 & 6.151 & 5.086 & 7.016 & 3.869 \\
\hline $2 / 11 / 2006$ & $8: 00$ & 11.731 & 11.6 & 7.539 & 4.924 & 3.225 & 3.154 & 10.931 & 6.15 & 5.088 & 7.021 & 3.873 \\
\hline $2 / 11 / 2006$ & $9: 00$ & 11.733 & 11.607 & 7.546 & 4.926 & 3.231 & 3.16 & 10.931 & 6.155 & 5.091 & 7.022 & 3.875 \\
\hline $2 / 11 / 2006$ & $10: 00$ & 11.743 & 11.615 & 7.55 & 4.931 & 3.238 & 3.169 & 10.932 & 6.161 & 5.097 & 7.026 & 3.882 \\
\hline $2 / 11 / 2006$ & $11: 00$ & 11.748 & 11.624 & 7.559 & 4.935 & 3.248 & 3.175 & 10.933 & 6.162 & 5.101 & 7.03 & 3.886 \\
\hline $2 / 11 / 2006$ & $12: 00$ & 11.755 & 11.631 & 7.566 & 4.94 & 3.25 & 3.18 & 10.936 & 6.164 & 5.102 & 7.03 & 3.888 \\
\hline $2 / 11 / 2006$ & $13: 00$ & 11.759 & 11.633 & 7.568 & 4.942 & 3.253 & 3.183 & 10.937 & 6.163 & 5.109 & 7.036 & 3.885 \\
\hline $2 / 11 / 2006$ & $14: 00$ & 11.765 & 11.636 & 7.566 & 4.942 & 3.251 & 3.18 & 10.942 & 6.163 & 5.106 & 7.037 & 3.884 \\
\hline $2 / 11 / 2006$ & $15: 00$ & 11.761 & 11.633 & 7.564 & 4.944 & 3.251 & 3.177 & 10.94 & 6.165 & 5.105 & 7.038 & 3.88 \\
\hline $2 / 11 / 2006$ & $16: 00$ & 11.765 & 11.633 & 7.561 & 4.942 & 3.246 & 3.173 & 10.942 & 6.164 & 5.104 & 7.035 & 3.878 \\
\hline $2 / 11 / 2006$ & $17: 00$ & 11.765 & 11.631 & 7.557 & 4.944 & 3.243 & 3.173 & 10.943 & 6.16 & 5.103 & 7.035 & 3.874 \\
\hline
\end{tabular}


TABLE S1.2 (Cont.)

Water Level (ft below top of casing) at Indicated Well

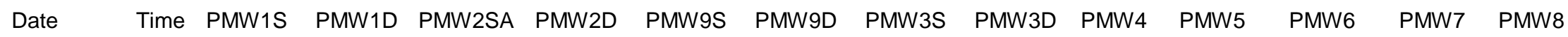

\begin{tabular}{|c|c|c|c|c|c|c|c|c|c|c|c|c|}
\hline $2 / 11 / 2006$ & $18: 00$ & 11.763 & 11.627 & 7.555 & 4.946 & 3.238 & 3.168 & 10.944 & 6.159 & 5.099 & 7.034 & 3.869 \\
\hline $2 / 11 / 2006$ & $19: 00$ & 11.761 & 11.622 & 7.55 & 4.946 & 3.239 & 3.166 & 10.945 & 6.159 & 5.096 & 7.035 & 3.866 \\
\hline $2 / 11 / 2006$ & $20: 00$ & 11.76 & 11.622 & 7.553 & 4.946 & 3.235 & 3.165 & 10.945 & 6.162 & 5.095 & 7.035 & 3.861 \\
\hline $2 / 11 / 2006$ & $21: 00$ & 11.757 & 11.616 & 7.548 & 4.944 & 3.234 & 3.163 & 10.945 & 6.161 & 5.093 & 7.032 & 3.858 \\
\hline $2 / 11 / 2006$ & $22: 00$ & 11.75 & 11.607 & 7.539 & 4.942 & 3.226 & 3.154 & 10.944 & 6.157 & 5.089 & 7.031 & 3.852 \\
\hline $2 / 11 / 2006$ & $23: 00$ & 11.743 & 11.602 & 7.537 & 4.942 & 3.222 & 3.149 & 10.94 & 6.157 & 5.086 & 7.029 & 3.849 \\
\hline $2 / 12 / 2006$ & $0: 00$ & 11.735 & 11.591 & 7.526 & 4.938 & 3.215 & 3.143 & 10.94 & 6.151 & 5.078 & 7.023 & 3.842 \\
\hline 2/12/2006 & $1: 00$ & 11.726 & 11.578 & 7.515 & 4.931 & 3.205 & 3.132 & 10.934 & 6.148 & 5.073 & 7.02 & 3.839 \\
\hline 2/12/2006 & $2: 00$ & 11.718 & 11.567 & 7.506 & 4.929 & 3.2 & 3.127 & 10.93 & 6.148 & 5.071 & 7.016 & 3.837 \\
\hline 2/12/2006 & $3: 00$ & 11.708 & 11.556 & 7.497 & 4.922 & 3.19 & 3.118 & 10.928 & 6.14 & 5.064 & 7.01 & 3.832 \\
\hline $2 / 12 / 2006$ & $4: 00$ & 11.698 & 11.545 & 7.488 & 4.915 & 3.187 & 3.11 & 10.925 & 6.141 & 5.063 & 7.008 & 3.837 \\
\hline 2/12/2006 & $5: 00$ & 11.689 & 11.536 & 7.482 & 4.911 & 3.18 & 3.106 & 10.922 & 6.136 & 5.062 & 7.007 & 3.842 \\
\hline $2 / 12 / 2006$ & $6: 00$ & 11.683 & 11.531 & 7.475 & 4.907 & 3.177 & 3.102 & 10.921 & 6.137 & 5.061 & 7.002 & 3.854 \\
\hline 2/12/2006 & $7: 00$ & 11.681 & 11.529 & 7.482 & 4.902 & 3.178 & 3.105 & 10.919 & 6.139 & 5.066 & 7.002 & 3.866 \\
\hline 2/12/2006 & $8: 00$ & 11.682 & 11.536 & 7.486 & 4.902 & 3.187 & 3.111 & 10.919 & 6.142 & 5.072 & 7.004 & 3.88 \\
\hline 2/12/2006 & $9: 00$ & 11.691 & 11.551 & 7.502 & 4.904 & 3.198 & 3.125 & 10.922 & 6.149 & 5.081 & 7.009 & 3.895 \\
\hline $2 / 12 / 2006$ & $10: 00$ & 11.698 & 11.563 & 7.513 & 4.907 & 3.211 & 3.136 & 10.923 & 6.152 & 5.09 & 7.013 & 3.908 \\
\hline 2/12/2006 & $11: 00$ & 11.71 & 11.584 & 7.53 & 4.913 & 3.224 & 3.151 & 10.928 & 6.156 & 5.103 & 7.016 & 3.922 \\
\hline 2/12/2006 & $12: 00$ & 11.728 & 11.598 & 7.544 & 4.915 & 3.237 & 3.161 & 10.932 & 6.164 & 5.114 & 7.023 & 3.93 \\
\hline 2/12/2006 & $13: 00$ & 11.739 & 11.609 & 7.553 & 4.926 & 3.244 & 3.173 & 10.938 & 6.163 & 5.12 & 7.028 & 3.933 \\
\hline 2/12/2006 & $14: 00$ & 11.744 & 11.618 & 7.559 & 4.928 & 3.25 & 3.175 & 10.936 & 6.165 & 5.125 & 7.036 & 3.93 \\
\hline 2/12/2006 & $15: 00$ & 11.754 & 11.624 & 7.561 & 4.933 & 3.253 & 3.179 & 10.941 & 6.167 & 5.131 & 7.035 & 3.93 \\
\hline 2/12/2006 & $16: 00$ & 11.759 & 11.631 & 7.568 & 4.938 & 3.256 & 3.182 & 10.944 & 6.169 & 5.128 & 7.038 & 3.927 \\
\hline $2 / 12 / 2006$ & $17: 00$ & 11.766 & 11.638 & 7.572 & 4.944 & 3.261 & 3.186 & 10.945 & 6.169 & 5.13 & 7.042 & 3.925 \\
\hline 2/12/2006 & $18: 00$ & 11.768 & 11.64 & 7.572 & 4.944 & 3.26 & 3.185 & 10.948 & 6.17 & 5.131 & 7.04 & 3.919 \\
\hline $2 / 12 / 2006$ & $19: 00$ & 11.772 & 11.642 & 7.572 & 4.948 & 3.262 & 3.187 & 10.949 & 6.17 & 5.127 & 7.042 & 3.914 \\
\hline 2/12/2006 & $20: 00$ & 11.774 & 11.642 & 7.574 & 4.953 & 3.265 & 3.191 & 10.953 & 6.171 & 5.127 & 7.042 & 3.909 \\
\hline $2 / 12 / 2006$ & $21: 00$ & 11.774 & 11.642 & 7.57 & 4.953 & 3.262 & 3.186 & 10.953 & 6.17 & 5.12 & 7.043 & 3.902 \\
\hline 2/12/2006 & $22: 00$ & 11.772 & 11.635 & 7.563 & 4.953 & 3.254 & 3.181 & 10.953 & 6.165 & 5.114 & 7.04 & 3.894 \\
\hline 2/12/2006 & $23: 00$ & 11.765 & 11.626 & 7.554 & 4.953 & 3.245 & 3.171 & 10.951 & 6.162 & 5.11 & 7.037 & 3.891 \\
\hline 2/13/2006 & $0: 00$ & 11.757 & 11.615 & 7.546 & 4.95 & 3.237 & 3.162 & 10.949 & 6.161 & 5.107 & 7.034 & 3.889 \\
\hline 2/13/2006 & $1: 00$ & 11.748 & 11.6 & 7.534 & 4.949 & 3.227 & 3.153 & 10.945 & 6.16 & 5.104 & 7.03 & 3.89 \\
\hline $2 / 13 / 2006$ & $2: 00$ & 11.739 & 11.589 & 7.526 & 4.944 & 3.224 & 3.147 & 10.943 & 6.156 & 5.105 & 7.028 & 3.897 \\
\hline 2/13/2006 & $3: 00$ & 11.728 & 11.574 & 7.515 & 4.937 & 3.214 & 3.138 & 10.938 & 6.151 & 5.103 & 7.025 & 3.902 \\
\hline
\end{tabular}


TABLE S1.2 (Cont.)

Water Level (ft below top of casing) at Indicated Well

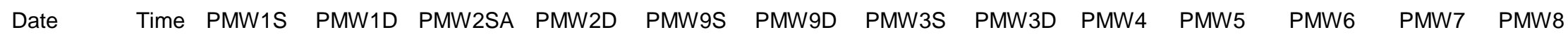

\begin{tabular}{|c|c|c|c|c|c|c|c|c|c|c|c|c|}
\hline 2/13/2006 & $4: 00$ & 11.716 & 11.563 & 7.506 & 4.933 & 3.207 & 3.131 & 10.936 & 6.152 & 5.106 & 7.023 & 3.909 \\
\hline $2 / 13 / 2006$ & $5: 00$ & 11.705 & 11.545 & 7.492 & 4.926 & 3.194 & 3.119 & 10.931 & 6.148 & 5.106 & 7.018 & 3.91 \\
\hline 2/13/2006 & $6: 00$ & 11.69 & 11.531 & 7.479 & 4.92 & 3.184 & 3.105 & 10.928 & 6.146 & 5.105 & 7.012 & 3.913 \\
\hline 2/13/2006 & $7: 00$ & 11.677 & 11.516 & 7.466 & 4.911 & 3.177 & 3.097 & 10.922 & 6.145 & 5.104 & 7.008 & 3.914 \\
\hline 2/13/2006 & 8:00 & 11.668 & 11.505 & 7.457 & 4.904 & 3.17 & 3.089 & 10.918 & 6.142 & 5.106 & 7.002 & 3.92 \\
\hline 2/13/2006 & $9: 00$ & 11.656 & 11.487 & 7.446 & 4.893 & 3.155 & 3.077 & 10.915 & 6.14 & 5.105 & 7.002 & 3.921 \\
\hline $2 / 13 / 2006$ & $10: 00$ & 11.647 & 11.485 & 7.448 & 4.888 & 3.158 & 3.078 & 10.909 & 6.141 & 5.11 & 6.997 & 3.931 \\
\hline 2/13/2006 & $11: 00$ & 11.644 & 11.482 & 7.45 & 4.887 & 3.161 & 3.078 & 10.91 & 6.14 & 5.116 & 6.994 & 3.934 \\
\hline 2/13/2006 & $12: 00$ & 11.64 & 11.485 & 7.453 & 4.884 & 3.162 & 3.081 & 10.909 & 6.141 & 5.121 & 6.998 & 3.934 \\
\hline 2/13/2006 & $13: 00$ & 11.64 & 11.483 & 7.448 & 4.882 & 3.159 & 3.079 & 10.907 & 6.137 & 5.123 & 6.995 & 3.93 \\
\hline $2 / 13 / 2006$ & $14: 00$ & 11.635 & 11.476 & 7.446 & 4.877 & 3.149 & 3.071 & 10.904 & 6.136 & 5.114 & 6.987 & 3.919 \\
\hline 2/13/2006 & $15: 00$ & 11.627 & 11.471 & 7.437 & 4.869 & 3.143 & 3.064 & 10.899 & 6.133 & 5.101 & 6.98 & 3.909 \\
\hline $2 / 13 / 2006$ & $16: 00$ & 11.622 & 11.469 & 7.439 & 4.866 & 3.137 & 3.061 & 10.897 & 6.13 & 5.09 & 6.978 & 3.901 \\
\hline 2/13/2006 & $17: 00$ & 11.622 & 11.467 & 7.435 & 4.864 & 3.135 & 3.059 & 10.899 & 6.128 & 5.083 & 6.977 & 3.891 \\
\hline 2/13/2006 & $18: 00$ & 11.624 & 11.471 & 7.437 & 4.862 & 3.136 & 3.061 & 10.896 & 6.127 & 5.072 & 6.974 & 3.884 \\
\hline 2/13/2006 & $19: 00$ & 11.627 & 11.474 & 7.441 & 4.862 & 3.139 & 3.061 & 10.897 & 6.127 & 5.06 & 6.971 & 3.874 \\
\hline 2/13/2006 & $20: 00$ & 11.641 & 11.484 & 7.45 & 4.862 & 3.145 & 3.067 & 10.895 & 6.127 & 5.056 & 6.971 & 3.868 \\
\hline 2/13/2006 & $21: 00$ & 11.652 & 11.487 & 7.45 & 4.865 & 3.145 & 3.07 & 10.895 & 6.125 & 5.047 & 6.972 & 3.86 \\
\hline 2/13/2006 & $22: 00$ & 11.652 & 11.487 & 7.448 & 4.862 & 3.142 & 3.068 & 10.894 & 6.121 & 5.04 & 6.968 & 3.85 \\
\hline 2/13/2006 & $23: 00$ & 11.65 & 11.485 & 7.448 & 4.862 & 3.14 & 3.066 & 10.893 & 6.117 & 5.034 & 6.969 & 3.845 \\
\hline 2/14/2006 & $0: 00$ & 11.645 & 11.485 & 7.446 & 4.862 & 3.138 & 3.067 & 10.89 & 6.121 & 5.029 & 6.968 & 3.842 \\
\hline $2 / 14 / 2006$ & $1: 00$ & 11.634 & 11.478 & 7.439 & 4.858 & 3.135 & 3.059 & 10.888 & 6.119 & 5.027 & 6.964 & 3.837 \\
\hline 2/14/2006 & $2: 00$ & 11.624 & 11.473 & 7.435 & 4.855 & 3.131 & 3.057 & 10.888 & 6.114 & 5.02 & 6.964 & 3.835 \\
\hline $2 / 14 / 2006$ & $3: 00$ & 11.617 & 11.469 & 7.43 & 4.853 & 3.128 & 3.054 & 10.886 & 6.113 & 5.016 & 6.961 & 3.833 \\
\hline 2/14/2006 & $4: 00$ & 11.612 & 11.465 & 7.428 & 4.851 & 3.124 & 3.05 & 10.884 & 6.108 & 5.012 & 6.959 & 3.831 \\
\hline $2 / 14 / 2006$ & $5: 00$ & 11.597 & 11.456 & 7.417 & 4.847 & 3.118 & 3.042 & 10.88 & 6.11 & 5.008 & 6.956 & 3.825 \\
\hline 2/14/2006 & $6: 00$ & 11.592 & 11.454 & 7.417 & 4.844 & 3.115 & 3.041 & 10.88 & 6.111 & 5.004 & 6.955 & 3.827 \\
\hline $2 / 14 / 2006$ & $7: 00$ & 11.587 & 11.449 & 7.412 & 4.84 & 3.112 & 3.037 & 10.878 & 6.108 & 5.003 & 6.954 & 3.825 \\
\hline 2/14/2006 & $8: 00$ & 11.584 & 11.443 & 7.408 & 4.838 & 3.11 & 3.032 & 10.875 & 6.107 & 4.999 & 6.949 & 3.823 \\
\hline 2/14/2006 & 9:00 & 11.582 & 11.447 & 7.413 & 4.838 & 3.115 & 3.038 & 10.876 & 6.108 & 5 & 6.951 & 3.825 \\
\hline 2/14/2006 & $10: 00$ & 11.584 & 11.453 & 7.419 & 4.838 & 3.121 & 3.046 & 10.874 & 6.108 & 5.003 & 6.948 & 3.831 \\
\hline 2/14/2006 & $11: 00$ & 11.583 & 11.445 & 7.408 & 4.833 & 3.112 & 3.036 & 10.872 & 6.107 & 4.996 & 6.946 & 3.824 \\
\hline $2 / 14 / 2006$ & $12: 00$ & 11.58 & 11.443 & 7.408 & 4.831 & 3.112 & 3.034 & 10.872 & 6.108 & 4.995 & 6.946 & 3.824 \\
\hline 2/14/2006 & $13: 00$ & 11.574 & 11.44 & 7.404 & 4.827 & 3.106 & 3.028 & 10.872 & 6.103 & 4.99 & 6.943 & 3.817 \\
\hline
\end{tabular}


TABLE S1.2 (Cont.)

Water Level (ft below top of casing) at Indicated Well

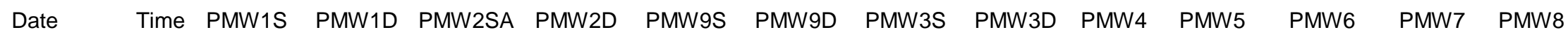

\begin{tabular}{|c|c|c|c|c|c|c|c|c|c|c|c|c|}
\hline 2/14/2006 & $14: 00$ & 11.569 & 11.434 & 7.397 & 4.822 & 3.099 & 3.023 & 10.865 & 6.099 & 4.98 & 6.938 & 3.805 \\
\hline $2 / 14 / 2006$ & $15: 00$ & 11.559 & 11.423 & 7.388 & 4.82 & 3.089 & 3.014 & 10.867 & 6.097 & 4.97 & 6.935 & 3.79 \\
\hline $2 / 14 / 2006$ & $16: 00$ & 11.557 & 11.423 & 7.388 & 4.813 & 3.087 & 3.01 & 10.863 & 6.098 & 4.963 & 6.934 & 3.771 \\
\hline 2/14/2006 & $17: 00$ & 11.559 & 11.427 & 7.397 & 4.813 & 3.089 & 3.014 & 10.861 & 6.097 & 4.955 & 6.93 & 3.759 \\
\hline 2/14/2006 & $18: 00$ & 11.571 & 11.445 & 7.412 & 4.822 & 3.106 & 3.03 & 10.861 & 6.101 & 4.958 & 6.932 & 3.751 \\
\hline 2/14/2006 & $19: 00$ & 11.587 & 11.463 & 7.43 & 4.822 & 3.117 & 3.042 & 10.866 & 6.106 & 4.952 & 6.934 & 3.748 \\
\hline $2 / 14 / 2006$ & $20: 00$ & 11.607 & 11.487 & 7.45 & 4.829 & 3.136 & 3.061 & 10.873 & 6.106 & 4.955 & 6.947 & 3.753 \\
\hline 2/14/2006 & $21: 00$ & 11.628 & 11.505 & 7.463 & 4.838 & 3.15 & 3.075 & 10.876 & 6.111 & 4.955 & 6.953 & 3.754 \\
\hline 2/14/2006 & $22: 00$ & 11.661 & 11.524 & 7.477 & 4.844 & 3.161 & 3.086 & 10.879 & 6.113 & 4.961 & 6.96 & 3.753 \\
\hline 2/14/2006 & $23: 00$ & 11.69 & 11.54 & 7.49 & 4.853 & 3.172 & 3.098 & 10.884 & 6.122 & 4.967 & 6.968 & 3.763 \\
\hline $2 / 15 / 2006$ & $0: 00$ & 11.675 & 11.557 & 7.503 & 4.862 & 3.187 & 3.111 & 10.89 & 6.124 & 4.973 & 6.975 & 3.767 \\
\hline 2/15/2006 & $1: 00$ & 11.685 & 11.566 & 7.51 & 4.869 & 3.192 & 3.118 & 10.894 & 6.122 & 4.974 & 6.977 & 3.768 \\
\hline $2 / 15 / 2006$ & $2: 00$ & 11.692 & 11.571 & 7.512 & 4.875 & 3.195 & 3.121 & 10.895 & 6.121 & 4.975 & 6.981 & 3.772 \\
\hline 2/15/2006 & $3: 00$ & 11.7 & 11.577 & 7.514 & 4.88 & 3.199 & 3.125 & 10.899 & 6.125 & 4.979 & 6.981 & 3.78 \\
\hline $2 / 15 / 2006$ & $4: 00$ & 11.702 & 11.58 & 7.516 & 4.884 & 3.199 & 3.127 & 10.902 & 6.124 & 4.984 & 6.988 & 3.783 \\
\hline $2 / 15 / 2006$ & $5: 00$ & 11.705 & 11.578 & 7.514 & 4.886 & 3.194 & 3.123 & 10.902 & 6.125 & 4.986 & 6.985 & 3.785 \\
\hline $2 / 15 / 2006$ & $6: 00$ & 11.707 & 11.577 & 7.51 & 4.888 & 3.196 & 3.124 & 10.904 & 6.122 & 4.987 & 6.988 & 3.79 \\
\hline $2 / 15 / 2006$ & $7: 00$ & 11.716 & 11.588 & 7.521 & 4.893 & 3.205 & 3.134 & 10.907 & 6.13 & 4.995 & 6.992 & 3.802 \\
\hline $2 / 15 / 2006$ & $8: 00$ & 11.718 & 11.589 & 7.521 & 4.895 & 3.205 & 3.134 & 10.907 & 6.133 & 5 & 6.995 & 3.805 \\
\hline $2 / 15 / 2006$ & $9: 00$ & 11.724 & 11.595 & 7.527 & 4.902 & 3.213 & 3.139 & 10.911 & 6.134 & 5.006 & 6.999 & 3.817 \\
\hline $2 / 15 / 2006$ & $10: 00$ & 11.729 & 11.6 & 7.534 & 4.904 & 3.217 & 3.144 & 10.911 & 6.138 & 5.014 & 7.003 & 3.822 \\
\hline $2 / 15 / 2006$ & $11: 00$ & 11.731 & 11.602 & 7.532 & 4.908 & 3.218 & 3.144 & 10.915 & 6.133 & 5.019 & 7.006 & 3.826 \\
\hline 2/15/2006 & $12: 00$ & 11.737 & 11.606 & 7.539 & 4.908 & 3.223 & 3.145 & 10.918 & 6.139 & 5.025 & 7.011 & 3.828 \\
\hline $2 / 15 / 2006$ & $13: 00$ & 11.734 & 11.602 & 7.53 & 4.911 & 3.217 & 3.146 & 10.918 & 6.138 & 5.023 & 7.009 & 3.82 \\
\hline 2/15/2006 & $14: 00$ & 11.723 & 11.586 & 7.514 & 4.906 & 3.2 & 3.128 & 10.914 & 6.132 & 5.014 & 7.002 & 3.799 \\
\hline $2 / 15 / 2006$ & $15: 00$ & 11.712 & 11.571 & 7.503 & 4.904 & 3.194 & 3.117 & 10.912 & 6.126 & 5.008 & 6.996 & 3.784 \\
\hline 2/15/2006 & $16: 00$ & 11.702 & 11.56 & 7.492 & 4.902 & 3.183 & 3.111 & 10.911 & 6.125 & 5 & 6.993 & 3.777 \\
\hline $2 / 15 / 2006$ & $17: 00$ & 11.693 & 11.547 & 7.487 & 4.897 & 3.176 & 3.104 & 10.908 & 6.12 & 4.99 & 6.986 & 3.769 \\
\hline 2/15/2006 & $18: 00$ & 11.692 & 11.551 & 7.492 & 4.895 & 3.179 & 3.106 & 10.907 & 6.121 & 4.996 & 6.993 & 3.777 \\
\hline $2 / 15 / 2006$ & $19: 00$ & 11.694 & 11.555 & 7.496 & 4.897 & 3.181 & 3.109 & 10.907 & 6.124 & 4.998 & 6.997 & 3.785 \\
\hline $2 / 15 / 2006$ & $20: 00$ & 11.704 & 11.567 & 7.507 & 4.9 & 3.194 & 3.119 & 10.911 & 6.127 & 5.007 & 6.997 & 3.8 \\
\hline $2 / 15 / 2006$ & $21: 00$ & 11.706 & 11.575 & 7.516 & 4.902 & 3.203 & 3.128 & 10.91 & 6.133 & 5.013 & 7 & 3.815 \\
\hline $2 / 15 / 2006$ & $22: 00$ & 11.711 & 11.578 & 7.512 & 4.904 & 3.205 & 3.128 & 10.912 & 6.135 & 5.018 & 7 & 3.821 \\
\hline $2 / 15 / 2006$ & $23: 00$ & 11.702 & 11.564 & 7.498 & 4.902 & 3.194 & 3.124 & 10.91 & 6.13 & 5.017 & 6.996 & 3.825 \\
\hline
\end{tabular}


TABLE S1.2 (Cont.)

Water Level (ft below top of casing) at Indicated Well

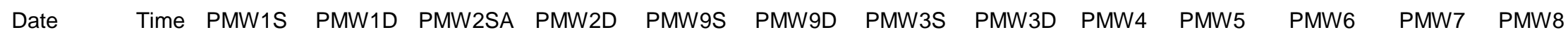

\begin{tabular}{|c|c|c|c|c|c|c|c|c|c|c|c|c|}
\hline 2/16/2006 & $0: 00$ & 11.708 & 11.573 & 7.509 & 4.904 & 3.2 & 3.125 & 10.912 & 6.136 & 5.03 & 7 & 3.84 \\
\hline $2 / 16 / 2006$ & $1: 00$ & 11.707 & 11.575 & 7.512 & 4.904 & 3.2 & 3.128 & 10.911 & 6.135 & 5.034 & 7 & 3.847 \\
\hline $2 / 16 / 2006$ & $2: 00$ & 11.703 & 11.562 & 7.505 & 4.9 & 3.195 & 3.117 & 10.912 & 6.133 & 5.039 & 6.998 & 3.85 \\
\hline 2/16/2006 & 3:00 & 11.691 & 11.553 & 7.492 & 4.897 & 3.187 & 3.111 & 10.907 & 6.132 & 5.038 & 6.996 & 3.852 \\
\hline $2 / 16 / 2006$ & $4: 00$ & 11.687 & 11.553 & 7.494 & 4.9 & 3.193 & 3.115 & 10.908 & 6.135 & 5.046 & 6.999 & 3.864 \\
\hline 2/16/2006 & $5: 00$ & 11.682 & 11.546 & 7.487 & 4.9 & 3.185 & 3.106 & 10.906 & 6.131 & 5.047 & 6.996 & 3.865 \\
\hline $2 / 16 / 2006$ & $6: 00$ & 11.684 & 11.547 & 7.494 & 4.895 & 3.188 & 3.107 & 10.906 & 6.137 & 5.057 & 6.995 & 3.874 \\
\hline 2/16/2006 & $7: 00$ & 11.683 & 11.542 & 7.489 & 4.895 & 3.188 & 3.107 & 10.906 & 6.134 & 5.061 & 6.993 & 3.877 \\
\hline $2 / 16 / 2006$ & $8: 00$ & 11.68 & 11.538 & 7.489 & 4.895 & 3.186 & 3.106 & 10.906 & 6.132 & 5.065 & 6.997 & 3.885 \\
\hline 2/16/2006 & 9:00 & 11.689 & 11.555 & 7.503 & 4.9 & 3.195 & 3.121 & 10.907 & 6.136 & 5.074 & 6.997 & 3.895 \\
\hline $2 / 16 / 2006$ & $10: 00$ & 11.692 & 11.558 & 7.507 & 4.9 & 3.2 & 3.125 & 10.907 & 6.139 & 5.081 & 6.996 & 3.9 \\
\hline 2/16/2006 & $11: 00$ & 11.704 & 11.575 & 7.523 & 4.902 & 3.216 & 3.139 & 10.912 & 6.142 & 5.088 & 7.002 & 3.91 \\
\hline $2 / 16 / 2006$ & $12: 00$ & 11.717 & 11.589 & 7.529 & 4.908 & 3.224 & 3.15 & 10.917 & 6.146 & 5.097 & 7.007 & 3.918 \\
\hline 2/16/2006 & $13: 00$ & 11.729 & 11.606 & 7.543 & 4.913 & 3.237 & 3.162 & 10.918 & 6.151 & 5.107 & 7.004 & 3.926 \\
\hline 2/16/2006 & $14: 00$ & 11.743 & 11.617 & 7.558 & 4.919 & 3.248 & 3.172 & 10.921 & 6.159 & 5.112 & 7.012 & 3.932 \\
\hline $2 / 16 / 2006$ & $15: 00$ & 11.753 & 11.629 & 7.565 & 4.931 & 3.256 & 3.183 & 10.925 & 6.161 & 5.12 & 7.022 & 3.934 \\
\hline $2 / 16 / 2006$ & $16: 00$ & 11.765 & 11.642 & 7.582 & 4.937 & 3.272 & 3.196 & 10.93 & 6.17 & 5.128 & 7.03 & 3.939 \\
\hline $2 / 16 / 2006$ & $17: 00$ & 11.778 & 11.659 & 7.596 & 4.946 & 3.28 & 3.206 & 10.93 & 6.173 & 5.136 & 7.037 & 3.944 \\
\hline 2/16/2006 & $18: 00$ & 11.793 & 11.675 & 7.611 & 4.959 & 3.293 & 3.222 & 10.936 & 6.175 & 5.147 & 7.047 & 3.951 \\
\hline $2 / 16 / 2006$ & $19: 00$ & 11.807 & 11.691 & 7.62 & 4.968 & 3.307 & 3.233 & 10.949 & 6.181 & 5.151 & 7.053 & 3.952 \\
\hline 2/16/2006 & $20: 00$ & 11.805 & 11.706 & 7.631 & 4.981 & 3.316 & 3.242 & 10.951 & 6.185 & 5.158 & 7.061 & 3.954 \\
\hline $2 / 16 / 2006$ & $21: 00$ & 11.819 & 11.721 & 7.642 & 4.99 & 3.328 & 3.253 & 10.955 & 6.19 & 5.164 & 7.067 & 3.956 \\
\hline 2/16/2006 & $22: 00$ & 11.827 & 11.728 & 7.645 & 4.999 & 3.329 & 3.257 & 10.959 & 6.188 & 5.165 & 7.07 & 3.953 \\
\hline $2 / 16 / 2006$ & $23: 00$ & 11.838 & 11.737 & 7.651 & 5.004 & 3.335 & 3.263 & 10.961 & 6.189 & 5.168 & 7.075 & 3.953 \\
\hline $2 / 17 / 2006$ & $0: 00$ & 11.844 & 11.744 & 7.651 & 5.01 & 3.338 & 3.265 & 10.966 & 6.194 & 5.173 & 7.078 & 3.956 \\
\hline $2 / 17 / 2006$ & $1: 00$ & 11.853 & 11.748 & 7.656 & 5.017 & 3.341 & 3.27 & 10.968 & 6.195 & 5.176 & 7.08 & 3.958 \\
\hline $2 / 17 / 2006$ & $2: 00$ & 11.858 & 11.757 & 7.66 & 5.021 & 3.349 & 3.276 & 10.97 & 6.201 & 5.184 & 7.089 & 3.965 \\
\hline $2 / 17 / 2006$ & $3: 00$ & 11.868 & 11.768 & 7.673 & 5.03 & 3.36 & 3.285 & 10.976 & 6.203 & 5.192 & 7.096 & 3.975 \\
\hline $2 / 17 / 2006$ & $4: 00$ & 11.873 & 11.77 & 7.669 & 5.035 & 3.36 & 3.287 & 10.978 & 6.203 & 5.194 & 7.095 & 3.98 \\
\hline $2 / 17 / 2006$ & $5: 00$ & 11.88 & 11.772 & 7.671 & 5.037 & 3.359 & 3.286 & 10.982 & 6.204 & 5.195 & 7.097 & 3.982 \\
\hline $2 / 17 / 2006$ & $6: 00$ & 11.885 & 11.777 & 7.675 & 5.043 & 3.365 & 3.293 & 10.982 & 6.211 & 5.202 & 7.102 & 3.987 \\
\hline 2/17/2006 & 7:00 & 11.89 & 11.777 & 7.673 & 5.046 & 3.362 & 3.287 & 10.983 & 6.213 & 5.204 & 7.102 & 3.986 \\
\hline $2 / 17 / 2006$ & $8: 00$ & 11.892 & 11.786 & 7.684 & 5.05 & 3.373 & 3.297 & 10.986 & 6.216 & 5.214 & 7.104 & 3.996 \\
\hline $2 / 17 / 2006$ & $9: 00$ & 11.903 & 11.801 & 7.698 & 5.059 & 3.388 & 3.312 & 10.989 & 6.224 & 5.222 & 7.117 & 4.005 \\
\hline
\end{tabular}


TABLE S1.2 (Cont.)

Water Level (ft below top of casing) at Indicated Well

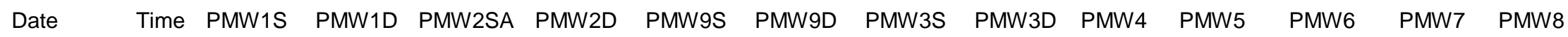

\begin{tabular}{|c|c|c|c|c|c|c|c|c|c|c|c|c|}
\hline 2/17/2006 & $10: 00$ & 11.908 & 11.805 & 7.702 & 5.063 & 3.389 & 3.317 & 10.995 & 6.22 & 5.225 & 7.12 & 4.007 \\
\hline $2 / 17 / 2006$ & $11: 00$ & 11.913 & 11.806 & 7.702 & 5.068 & 3.392 & 3.317 & 11.003 & 6.225 & 5.229 & 7.124 & 4.01 \\
\hline 2/17/2006 & $12: 00$ & 11.922 & 11.819 & 7.711 & 5.072 & 3.399 & 3.322 & 11 & 6.229 & 5.238 & 7.13 & 4.015 \\
\hline 2/17/2006 & $13: 00$ & 11.922 & 11.815 & 7.706 & 5.074 & 3.396 & 3.322 & 11.004 & 6.231 & 5.239 & 7.128 & 4.014 \\
\hline 2/17/2006 & $14: 00$ & 11.923 & 11.805 & 7.695 & 5.072 & 3.384 & 3.31 & 11.006 & 6.23 & 5.239 & 7.126 & 4.006 \\
\hline 2/17/2006 & $15: 00$ & 11.92 & 11.794 & 7.686 & 5.07 & 3.379 & 3.306 & 11.008 & 6.228 & 5.234 & 7.124 & 3.998 \\
\hline $2 / 17 / 2006$ & $16: 00$ & 11.916 & 11.786 & 7.682 & 5.07 & 3.371 & 3.295 & 11.01 & 6.228 & 5.237 & 7.127 & 3.996 \\
\hline 2/17/2006 & $17: 00$ & 11.915 & 11.784 & 7.682 & 5.068 & 3.377 & 3.299 & 11.01 & 6.23 & 5.238 & 7.123 & 3.993 \\
\hline 2/17/2006 & $18: 00$ & 11.913 & 11.784 & 7.68 & 5.07 & 3.373 & 3.298 & 11.011 & 6.23 & 5.239 & 7.125 & 3.991 \\
\hline 2/17/2006 & $19: 00$ & 11.914 & 11.784 & 7.682 & 5.07 & 3.374 & 3.298 & 11.016 & 6.232 & 5.241 & 7.128 & 3.989 \\
\hline $2 / 17 / 2006$ & $20: 00$ & 11.916 & 11.784 & 7.686 & 5.072 & 3.381 & 3.304 & 11.016 & 6.235 & 5.243 & 7.131 & 3.993 \\
\hline 2/17/2006 & $21: 00$ & 11.917 & 11.786 & 7.686 & 5.072 & 3.378 & 3.304 & 11.016 & 6.237 & 5.248 & 7.134 & 3.999 \\
\hline $2 / 17 / 2006$ & $22: 00$ & 11.913 & 11.781 & 7.686 & 5.074 & 3.379 & 3.302 & 11.017 & 6.237 & 5.25 & 7.133 & 4.001 \\
\hline 2/17/2006 & $23: 00$ & 11.914 & 11.783 & 7.688 & 5.074 & 3.381 & 3.305 & 11.018 & 6.24 & 5.253 & 7.135 & 4.008 \\
\hline 2/18/2006 & $0: 00$ & 11.918 & 11.781 & 7.686 & 5.074 & 3.383 & 3.309 & 11.022 & 6.238 & 5.255 & 7.137 & 4.01 \\
\hline 2/18/2006 & $1: 00$ & 11.912 & 11.775 & 7.682 & 5.074 & 3.379 & 3.301 & 11.022 & 6.235 & 5.254 & 7.135 & 4.01 \\
\hline $2 / 18 / 2006$ & $2: 00$ & 11.912 & 11.775 & 7.682 & 5.072 & 3.379 & 3.301 & 11.025 & 6.242 & 5.259 & 7.139 & 4.015 \\
\hline 2/18/2006 & $3: 00$ & 11.91 & 11.774 & 7.682 & 5.074 & 3.384 & 3.304 & 11.025 & 6.242 & 5.263 & 7.139 & 4.016 \\
\hline 2/18/2006 & $4: 00$ & 11.91 & 11.77 & 7.679 & 5.074 & 3.38 & 3.303 & 11.027 & 6.238 & 5.261 & 7.139 & 4.017 \\
\hline 2/18/2006 & $5: 00$ & 11.907 & 11.77 & 7.679 & 5.074 & 3.381 & 3.302 & 11.027 & 6.243 & 5.265 & 7.142 & 4.02 \\
\hline 2/18/2006 & $6: 00$ & 11.904 & 11.766 & 7.682 & 5.074 & 3.38 & 3.303 & 11.029 & 6.244 & 5.267 & 7.143 & 4.022 \\
\hline 2/18/2006 & $7: 00$ & 11.902 & 11.763 & 7.677 & 5.072 & 3.376 & 3.299 & 11.029 & 6.242 & 5.27 & 7.143 & 4.022 \\
\hline 2/18/2006 & 8:00 & 11.901 & 11.763 & 7.677 & 5.074 & 3.378 & 3.302 & 11.031 & 6.242 & 5.271 & 7.145 & 4.025 \\
\hline 2/18/2006 & $9: 00$ & 11.902 & 11.763 & 7.682 & 5.076 & 3.381 & 3.303 & 11.033 & 6.238 & 5.275 & 7.146 & 4.03 \\
\hline 2/18/2006 & $10: 00$ & 11.903 & 11.767 & 7.684 & 5.076 & 3.386 & 3.304 & 11.032 & 6.242 & 5.277 & 7.148 & 4.031 \\
\hline 2/18/2006 & $11: 00$ & 11.904 & 11.766 & 7.684 & 5.077 & 3.384 & 3.306 & 11.035 & 6.244 & 5.279 & 7.149 & 4.036 \\
\hline 2/18/2006 & $12: 00$ & 11.9 & 11.763 & 7.682 & 5.072 & 3.384 & 3.303 & 11.037 & 6.244 & 5.279 & 7.151 & 4.035 \\
\hline $2 / 18 / 2006$ & $13: 00$ & 11.896 & 11.755 & 7.675 & 5.07 & 3.378 & 3.296 & 11.035 & 6.242 & 5.276 & 7.147 & 4.031 \\
\hline 2/18/2006 & $14: 00$ & 11.887 & 11.743 & 7.66 & 5.063 & 3.365 & 3.284 & 11.032 & 6.233 & 5.272 & 7.143 & 4.02 \\
\hline 2/18/2006 & $15: 00$ & 11.876 & 11.726 & 7.646 & 5.057 & 3.352 & 3.271 & 11.03 & 6.233 & 5.267 & 7.134 & 4.011 \\
\hline 2/18/2006 & $16: 00$ & 11.869 & 11.717 & 7.639 & 5.052 & 3.342 & 3.262 & 11.027 & 6.232 & 5.262 & 7.131 & 4.003 \\
\hline 2/18/2006 & $17: 00$ & 11.86 & 11.704 & 7.63 & 5.05 & 3.335 & 3.254 & 11.024 & 6.226 & 5.259 & 7.124 & 3.992 \\
\hline 2/18/2006 & $18: 00$ & 11.852 & 11.699 & 7.624 & 5.046 & 3.331 & 3.25 & 11.021 & 6.224 & 5.253 & 7.124 & 3.989 \\
\hline 2/18/2006 & $19: 00$ & 11.847 & 11.692 & 7.622 & 5.041 & 3.327 & 3.245 & 11.019 & 6.221 & 5.249 & 7.121 & 3.983 \\
\hline
\end{tabular}


TABLE S1.2 (Cont.)

Water Level (ft below top of casing) at Indicated Well

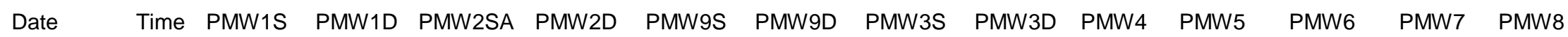

\begin{tabular}{|c|c|c|c|c|c|c|c|c|c|c|c|c|}
\hline 2/18/2006 & $20: 00$ & 11.84 & 11.686 & 7.618 & 5.039 & 3.324 & 3.241 & 11.017 & 6.223 & 5.245 & 7.117 & 3.978 \\
\hline $2 / 18 / 2006$ & $21: 00$ & 11.835 & 11.684 & 7.619 & 5.039 & 3.32 & 3.24 & 11.015 & 6.22 & 5.24 & 7.116 & 3.976 \\
\hline 2/18/2006 & $22: 00$ & 11.833 & 11.679 & 7.613 & 5.037 & 3.318 & 3.237 & 11.015 & 6.217 & 5.234 & 7.113 & 3.972 \\
\hline 2/18/2006 & $23: 00$ & 11.829 & 11.679 & 7.613 & 5.037 & 3.32 & 3.237 & 11.015 & 6.223 & 5.236 & 7.112 & 3.977 \\
\hline 2/19/2006 & $0: 00$ & 11.825 & 11.675 & 7.613 & 5.035 & 3.319 & 3.238 & 11.014 & 6.223 & 5.232 & 7.111 & 3.983 \\
\hline 2/19/2006 & $1: 00$ & 11.825 & 11.673 & 7.608 & 5.035 & 3.318 & 3.238 & 11.013 & 6.221 & 5.233 & 7.11 & 3.992 \\
\hline 2/19/2006 & $2: 00$ & 11.819 & 11.668 & 7.602 & 5.03 & 3.315 & 3.234 & 11.011 & 6.219 & 5.232 & 7.109 & 3.996 \\
\hline 2/19/2006 & $3: 00$ & 11.816 & 11.664 & 7.602 & 5.03 & 3.312 & 3.231 & 11.011 & 6.218 & 5.233 & 7.106 & 4.001 \\
\hline 2/19/2006 & $4: 00$ & 11.811 & 11.657 & 7.595 & 5.026 & 3.306 & 3.226 & 11.007 & 6.215 & 5.234 & 7.104 & 4.003 \\
\hline 2/19/2006 & $5: 00$ & 11.804 & 11.646 & 7.586 & 5.019 & 3.3 & 3.218 & 11.004 & 6.212 & 5.232 & 7.1 & 4.003 \\
\hline $2 / 19 / 2006$ & $6: 00$ & 11.795 & 11.641 & 7.582 & 5.015 & 3.297 & 3.213 & 11.003 & 6.211 & 5.235 & 7.1 & 4.007 \\
\hline 2/19/2006 & $7: 00$ & 11.792 & 11.639 & 7.58 & 5.012 & 3.297 & 3.214 & 11 & 6.216 & 5.24 & 7.1 & 4.018 \\
\hline 2/19/2006 & $8: 00$ & 11.789 & 11.633 & 7.577 & 5.008 & 3.292 & 3.209 & 11 & 6.213 & 5.24 & 7.097 & 4.018 \\
\hline 2/19/2006 & $9: 00$ & 11.786 & 11.631 & 7.577 & 5.005 & 3.293 & 3.212 & 11 & 6.212 & 5.244 & 7.097 & 4.021 \\
\hline 2/19/2006 & $10: 00$ & 11.785 & 11.631 & 7.58 & 5.003 & 3.298 & 3.214 & 10.998 & 6.213 & 5.252 & 7.098 & 4.028 \\
\hline 2/19/2006 & $11: 00$ & 11.78 & 11.628 & 7.577 & 4.999 & 3.294 & 3.206 & 10.998 & 6.214 & 5.25 & 7.099 & 4.031 \\
\hline 2/19/2006 & $12: 00$ & 11.774 & 11.622 & 7.568 & 4.992 & 3.285 & 3.201 & 10.996 & 6.207 & 5.247 & 7.096 & 4.026 \\
\hline 2/19/2006 & $13: 00$ & 11.771 & 11.617 & 7.566 & 4.988 & 3.285 & 3.198 & 10.996 & 6.205 & 5.246 & 7.094 & 4.022 \\
\hline 2/19/2006 & $14: 00$ & 11.766 & 11.604 & 7.553 & 4.979 & 3.271 & 3.188 & 10.993 & 6.2 & 5.236 & 7.088 & 4.011 \\
\hline 2/19/2006 & $15: 00$ & 11.755 & 11.586 & 7.54 & 4.972 & 3.257 & 3.173 & 10.986 & 6.195 & 5.227 & 7.083 & 3.997 \\
\hline 2/19/2006 & $16: 00$ & 11.747 & 11.581 & 7.535 & 4.966 & 3.255 & 3.169 & 10.982 & 6.196 & 5.224 & 7.079 & 3.994 \\
\hline 2/19/2006 & $17: 00$ & 11.743 & 11.573 & 7.526 & 4.962 & 3.25 & 3.162 & 10.982 & 6.191 & 5.217 & 7.072 & 3.985 \\
\hline 2/19/2006 & $18: 00$ & 11.737 & 11.566 & 7.522 & 4.957 & 3.241 & 3.157 & 10.977 & 6.187 & 5.211 & 7.071 & 3.979 \\
\hline 2/19/2006 & $19: 00$ & 11.728 & 11.56 & 7.517 & 4.952 & 3.236 & 3.152 & 10.976 & 6.186 & 5.203 & 7.067 & 3.971 \\
\hline 2/19/2006 & 20:00 & 11.727 & 11.56 & 7.519 & 4.95 & 3.239 & 3.154 & 10.974 & 6.186 & 5.198 & 7.066 & 3.969 \\
\hline $2 / 19 / 2006$ & $21: 00$ & 11.725 & 11.56 & 7.519 & 4.95 & 3.238 & 3.152 & 10.971 & 6.183 & 5.196 & 7.061 & 3.964 \\
\hline 2/19/2006 & $22: 00$ & 11.72 & 11.558 & 7.52 & 4.95 & 3.236 & 3.153 & 10.97 & 6.181 & 5.193 & 7.062 & 3.957 \\
\hline $2 / 19 / 2006$ & $23: 00$ & 11.719 & 11.559 & 7.52 & 4.95 & 3.238 & 3.153 & 10.968 & 6.18 & 5.186 & 7.057 & 3.954 \\
\hline 2/20/2006 & $0: 00$ & 11.718 & 11.557 & 7.517 & 4.948 & 3.235 & 3.15 & 10.967 & 6.179 & 5.182 & 7.057 & 3.948 \\
\hline 2/20/2006 & $1: 00$ & 11.717 & 11.559 & 7.522 & 4.948 & 3.236 & 3.151 & 10.965 & 6.179 & 5.177 & 7.055 & 3.945 \\
\hline 2/20/2006 & $2: 00$ & 11.714 & 11.553 & 7.515 & 4.948 & 3.233 & 3.148 & 10.963 & 6.174 & 5.17 & 7.051 & 3.945 \\
\hline 2/20/2006 & 3:00 & 11.71 & 11.549 & 7.51 & 4.946 & 3.23 & 3.142 & 10.962 & 6.176 & 5.166 & 7.049 & 3.946 \\
\hline 2/20/2006 & $4: 00$ & 11.707 & 11.544 & 7.504 & 4.942 & 3.223 & 3.14 & 10.959 & 6.167 & 5.16 & 7.046 & 3.95 \\
\hline 2/20/2006 & 5:00 & 11.702 & 11.538 & 7.497 & 4.939 & 3.221 & 3.135 & 10.958 & 6.169 & 5.159 & 7.046 & 3.955 \\
\hline
\end{tabular}


TABLE S1.2 (Cont.)

Water Level (ft below top of casing) at Indicated Well

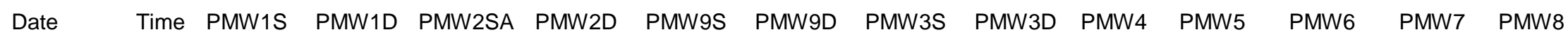

\begin{tabular}{|c|c|c|c|c|c|c|c|c|c|c|c|c|}
\hline 2/20/2006 & $6: 00$ & 11.698 & 11.535 & 7.497 & 4.937 & 3.219 & 3.133 & 10.956 & 6.172 & 5.159 & 7.043 & 3.961 \\
\hline $2 / 20 / 2006$ & $7: 00$ & 11.695 & 11.531 & 7.495 & 4.935 & 3.22 & 3.133 & 10.954 & 6.168 & 5.158 & 7.041 & 3.968 \\
\hline $2 / 20 / 2006$ & $8: 00$ & 11.693 & 11.528 & 7.493 & 4.933 & 3.217 & 3.134 & 10.952 & 6.166 & 5.16 & 7.039 & 3.972 \\
\hline 2/20/2006 & 9:00 & 11.69 & 11.528 & 7.491 & 4.931 & 3.216 & 3.13 & 10.949 & 6.172 & 5.163 & 7.04 & 3.979 \\
\hline 2/20/2006 & $10: 00$ & 11.687 & 11.524 & 7.493 & 4.928 & 3.218 & 3.13 & 10.948 & 6.172 & 5.161 & 7.036 & 3.989 \\
\hline 2/20/2006 & $11: 00$ & 11.683 & 11.519 & 7.488 & 4.924 & 3.209 & 3.124 & 10.945 & 6.172 & 5.162 & 7.038 & 3.997 \\
\hline $2 / 20 / 2006$ & $12: 00$ & 11.677 & 11.515 & 7.479 & 4.915 & 3.206 & 3.118 & 10.946 & 6.167 & 5.163 & 7.041 & 3.996 \\
\hline 2/20/2006 & $13: 00$ & 11.677 & 11.511 & 7.477 & 4.908 & 3.21 & 3.115 & 10.94 & 6.167 & 5.165 & 7.037 & 3.996 \\
\hline 2/20/2006 & $14: 00$ & 11.672 & 11.504 & 7.475 & 4.902 & 3.203 & 3.111 & 10.939 & 6.161 & 5.164 & 7.033 & 3.988 \\
\hline 2/20/2006 & $15: 00$ & 11.665 & 11.493 & 7.466 & 4.895 & 3.196 & 3.107 & 10.938 & 6.165 & 5.166 & 7.032 & 3.983 \\
\hline $2 / 20 / 2006$ & $16: 00$ & 11.663 & 11.493 & 7.462 & 4.893 & 3.194 & 3.101 & 10.936 & 6.164 & 5.162 & 7.028 & 3.976 \\
\hline 2/20/2006 & $17: 00$ & 11.659 & 11.493 & 7.464 & 4.891 & 3.191 & 3.102 & 10.936 & 6.161 & 5.162 & 7.027 & 3.97 \\
\hline $2 / 20 / 2006$ & $18: 00$ & 11.656 & 11.497 & 7.471 & 4.891 & 3.193 & 3.104 & 10.934 & 6.158 & 5.158 & 7.026 & 3.971 \\
\hline 2/20/2006 & $19: 00$ & 11.66 & 11.497 & 7.468 & 4.888 & 3.19 & 3.104 & 10.933 & 6.156 & 5.153 & 7.024 & 3.964 \\
\hline 2/20/2006 & $20: 00$ & 11.661 & 11.5 & 7.471 & 4.891 & 3.193 & 3.106 & 10.932 & 6.154 & 5.15 & 7.022 & 3.957 \\
\hline 2/20/2006 & $21: 00$ & 11.664 & 11.508 & 7.479 & 4.893 & 3.2 & 3.113 & 10.933 & 6.155 & 5.149 & 7.023 & 3.958 \\
\hline $2 / 20 / 2006$ & $22: 00$ & 11.667 & 11.513 & 7.481 & 4.895 & 3.199 & 3.115 & 10.932 & 6.152 & 5.146 & 7.023 & 3.951 \\
\hline 2/20/2006 & $23: 00$ & 11.67 & 11.52 & 7.488 & 4.897 & 3.202 & 3.118 & 10.931 & 6.157 & 5.145 & 7.027 & 3.947 \\
\hline $2 / 21 / 2006$ & $0: 00$ & 11.677 & 11.528 & 7.493 & 4.899 & 3.207 & 3.125 & 10.934 & 6.153 & 5.143 & 7.024 & 3.946 \\
\hline $2 / 21 / 2006$ & $1: 00$ & 11.679 & 11.527 & 7.491 & 4.899 & 3.205 & 3.122 & 10.933 & 6.152 & 5.138 & 7.024 & 3.94 \\
\hline $2 / 21 / 2006$ & $2: 00$ & 11.681 & 11.529 & 7.491 & 4.899 & 3.204 & 3.122 & 10.933 & 6.153 & 5.135 & 7.025 & 3.932 \\
\hline $2 / 21 / 2006$ & $3: 00$ & 11.687 & 11.537 & 7.499 & 4.904 & 3.211 & 3.128 & 10.933 & 6.154 & 5.136 & 7.028 & 3.931 \\
\hline $2 / 21 / 2006$ & $4: 00$ & 11.689 & 11.539 & 7.499 & 4.906 & 3.21 & 3.129 & 10.933 & 6.149 & 5.132 & 7.027 & 3.924 \\
\hline $2 / 21 / 2006$ & $5: 00$ & 11.691 & 11.54 & 7.499 & 4.91 & 3.213 & 3.127 & 10.933 & 6.151 & 5.13 & 7.026 & 3.918 \\
\hline 2/21/2006 & $6: 00$ & 11.694 & 11.542 & 7.501 & 4.912 & 3.209 & 3.128 & 10.933 & 6.151 & 5.129 & 7.027 & 3.918 \\
\hline $2 / 21 / 2006$ & $7: 00$ & 11.691 & 11.54 & 7.497 & 4.915 & 3.206 & 3.125 & 10.933 & 6.147 & 5.123 & 7.026 & 3.914 \\
\hline 2/21/2006 & $8: 00$ & 11.691 & 11.533 & 7.49 & 4.915 & 3.203 & 3.121 & 10.932 & 6.145 & 5.12 & 7.023 & 3.91 \\
\hline $2 / 21 / 2006$ & $9: 00$ & 11.69 & 11.539 & 7.495 & 4.915 & 3.207 & 3.122 & 10.931 & 6.151 & 5.123 & 7.026 & 3.919 \\
\hline 2/21/2006 & $10: 00$ & 11.693 & 11.538 & 7.497 & 4.917 & 3.206 & 3.124 & 10.931 & 6.15 & 5.12 & 7.025 & 3.93 \\
\hline 2/21/2006 & $11: 00$ & 11.689 & 11.538 & 7.495 & 4.915 & 3.209 & 3.124 & 10.931 & 6.151 & 5.119 & 7.025 & 3.937 \\
\hline 2/21/2006 & $12: 00$ & 11.689 & 11.535 & 7.492 & 4.91 & 3.206 & 3.123 & 10.931 & 6.15 & 5.12 & 7.026 & 3.941 \\
\hline $2 / 21 / 2006$ & $13: 00$ & 11.685 & 11.528 & 7.486 & 4.906 & 3.202 & 3.118 & 10.93 & 6.15 & 5.12 & 7.022 & 3.943 \\
\hline $2 / 21 / 2006$ & $14: 00$ & 11.676 & 11.517 & 7.473 & 4.899 & 3.193 & 3.108 & 10.927 & 6.147 & 5.114 & 7.018 & 3.936 \\
\hline $2 / 21 / 2006$ & $15: 00$ & 11.665 & 11.502 & 7.464 & 4.893 & 3.182 & 3.096 & 10.925 & 6.143 & 5.112 & 7.018 & 3.929 \\
\hline
\end{tabular}


TABLE S1.2 (Cont.)

Water Level (ft below top of casing) at Indicated Well

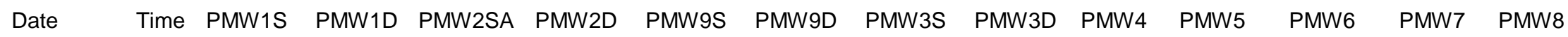

\begin{tabular}{|c|c|c|c|c|c|c|c|c|c|c|c|c|}
\hline $2 / 21 / 2006$ & $16: 00$ & 11.659 & 11.497 & 7.461 & 4.89 & 3.182 & 3.091 & 10.921 & 6.142 & 5.113 & 7.015 & 3.927 \\
\hline 2/21/2006 & $17: 00$ & 11.653 & 11.489 & 7.455 & 4.886 & 3.172 & 3.086 & 10.92 & 6.144 & 5.109 & 7.012 & 3.922 \\
\hline 2/21/2006 & $18: 00$ & 11.65 & 11.487 & 7.453 & 4.881 & 3.168 & 3.086 & 10.918 & 6.141 & 5.104 & 7.009 & 3.917 \\
\hline 2/21/2006 & 19:00 & 11.647 & 11.486 & 7.453 & 4.877 & 3.166 & 3.083 & 10.918 & 6.14 & 5.1 & 7.005 & 3.91 \\
\hline 2/21/2006 & $20: 00$ & 11.644 & 11.487 & 7.457 & 4.877 & 3.168 & 3.088 & 10.917 & 6.138 & 5.099 & 7.005 & 3.911 \\
\hline 2/21/2006 & $21: 00$ & 11.654 & 11.5 & 7.47 & 4.881 & 3.18 & 3.098 & 10.917 & 6.144 & 5.101 & 7.006 & 3.917 \\
\hline $2 / 21 / 2006$ & $22: 00$ & 11.661 & 11.509 & 7.477 & 4.884 & 3.184 & 3.104 & 10.919 & 6.144 & 5.1 & 7.008 & 3.915 \\
\hline $2 / 21 / 2006$ & $23: 00$ & 11.668 & 11.519 & 7.485 & 4.885 & 3.191 & 3.11 & 10.92 & 6.142 & 5.099 & 7.008 & 3.914 \\
\hline $2 / 22 / 2006$ & $0: 00$ & 11.676 & 11.529 & 7.49 & 4.89 & 3.198 & 3.117 & 10.922 & 6.147 & 5.1 & 7.01 & 3.914 \\
\hline 2/22/2006 & $1: 00$ & 11.678 & 11.529 & 7.49 & 4.89 & 3.195 & 3.114 & 10.921 & 6.144 & 5.095 & 7.009 & 3.906 \\
\hline $2 / 22 / 2006$ & $2: 00$ & 11.681 & 11.533 & 7.492 & 4.892 & 3.196 & 3.117 & 10.922 & 6.139 & 5.091 & 7.009 & 3.902 \\
\hline $2 / 22 / 2006$ & $3: 00$ & 11.685 & 11.539 & 7.497 & 4.895 & 3.197 & 3.119 & 10.921 & 6.14 & 5.092 & 7.01 & 3.899 \\
\hline $2 / 22 / 2006$ & $4: 00$ & 11.679 & 11.528 & 7.484 & 4.892 & 3.186 & 3.111 & 10.921 & 6.131 & 5.081 & 7.004 & 3.888 \\
\hline $2 / 22 / 2006$ & $5: 00$ & 11.683 & 11.535 & 7.49 & 4.895 & 3.194 & 3.114 & 10.92 & 6.139 & 5.08 & 7.007 & 3.887 \\
\hline $2 / 22 / 2006$ & $6: 00$ & 11.683 & 11.535 & 7.49 & 4.895 & 3.192 & 3.113 & 10.92 & 6.135 & 5.076 & 7.005 & 3.881 \\
\hline $2 / 22 / 2006$ & $7: 00$ & 11.688 & 11.54 & 7.495 & 4.899 & 3.196 & 3.117 & 10.921 & 6.137 & 5.079 & 7.007 & 3.88 \\
\hline 2/22/2006 & $8: 00$ & 11.692 & 11.546 & 7.499 & 4.903 & 3.202 & 3.122 & 10.921 & 6.141 & 5.077 & 7.01 & 3.881 \\
\hline 2/22/2006 & $9: 00$ & 11.695 & 11.55 & 7.503 & 4.906 & 3.204 & 3.126 & 10.922 & 6.136 & 5.075 & 7.01 & 3.883 \\
\hline $2 / 22 / 2006$ & $10: 00$ & 11.7 & 11.557 & 7.508 & 4.908 & 3.208 & 3.13 & 10.924 & 6.141 & 5.079 & 7.012 & 3.89 \\
\hline $2 / 22 / 2006$ & $11: 00$ & 11.703 & 11.555 & 7.508 & 4.91 & 3.204 & 3.127 & 10.924 & 6.139 & 5.076 & 7.011 & 3.89 \\
\hline 2/22/2006 & $12: 00$ & 11.702 & 11.55 & 7.503 & 4.908 & 3.206 & 3.126 & 10.92 & 6.14 & 5.071 & 7.01 & 3.89 \\
\hline $2 / 22 / 2006$ & $13: 00$ & 11.693 & 11.539 & 7.492 & 4.903 & 3.196 & 3.115 & 10.922 & 6.137 & 5.07 & 7.004 & 3.888 \\
\hline 2/22/2006 & $14: 00$ & 11.685 & 11.524 & 7.481 & 4.899 & 3.19 & 3.106 & 10.917 & 6.131 & 5.066 & 7.006 & 3.884 \\
\hline $2 / 22 / 2006$ & $15: 00$ & 11.675 & 11.515 & 7.472 & 4.893 & 3.18 & 3.096 & 10.911 & 6.132 & 5.063 & 7 & 3.878 \\
\hline 2/22/2006 & $16: 00$ & 11.67 & 11.513 & 7.475 & 4.894 & 3.178 & 3.093 & 10.911 & 6.136 & 5.062 & 7.001 & 3.881 \\
\hline $2 / 22 / 2006$ & $17: 00$ & 11.67 & 11.513 & 7.477 & 4.89 & 3.184 & 3.102 & 10.913 & 6.133 & 5.062 & 6.997 & 3.883 \\
\hline $2 / 22 / 2006$ & $18: 00$ & 11.675 & 11.522 & 7.483 & 4.892 & 3.186 & 3.105 & 10.913 & 6.134 & 5.061 & 6.997 & 3.884 \\
\hline $2 / 22 / 2006$ & $19: 00$ & 11.682 & 11.537 & 7.494 & 4.895 & 3.196 & 3.116 & 10.917 & 6.137 & 5.065 & 7.005 & 3.884 \\
\hline $2 / 22 / 2006$ & $20: 00$ & 11.691 & 11.55 & 7.505 & 4.896 & 3.206 & 3.126 & 10.92 & 6.139 & 5.066 & 7.007 & 3.887 \\
\hline $2 / 22 / 2006$ & $21: 00$ & 11.702 & 11.56 & 7.512 & 4.901 & 3.212 & 3.132 & 10.921 & 6.137 & 5.068 & 7.008 & 3.886 \\
\hline $2 / 22 / 2006$ & $22: 00$ & 11.705 & 11.566 & 7.519 & 4.905 & 3.214 & 3.137 & 10.924 & 6.138 & 5.067 & 7.012 & 3.884 \\
\hline 2/22/2006 & $23: 00$ & 11.715 & 11.575 & 7.523 & 4.907 & 3.221 & 3.142 & 10.925 & 6.141 & 5.072 & 7.015 & 3.882 \\
\hline $2 / 23 / 2006$ & $0: 00$ & 11.719 & 11.582 & 7.532 & 4.914 & 3.224 & 3.148 & 10.927 & 6.143 & 5.073 & 7.018 & 3.882 \\
\hline $2 / 23 / 2006$ & $1: 00$ & 11.724 & 11.584 & 7.532 & 4.914 & 3.227 & 3.148 & 10.928 & 6.142 & 5.071 & 7.02 & 3.879 \\
\hline
\end{tabular}


TABLE S1.2 (Cont.)

Water Level (ft below top of casing) at Indicated Well

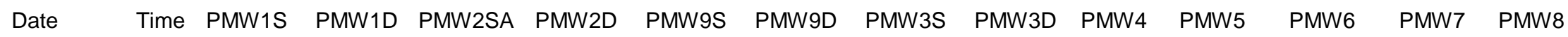

\begin{tabular}{|c|c|c|c|c|c|c|c|c|c|c|c|c|}
\hline 2/23/2006 & $2: 00$ & 11.727 & 11.586 & 7.529 & 4.916 & 3.228 & 3.148 & 10.928 & 6.143 & 5.071 & 7.02 & 3.875 \\
\hline $2 / 23 / 2006$ & $3: 00$ & 11.731 & 11.59 & 7.534 & 4.919 & 3.227 & 3.15 & 10.932 & 6.14 & 5.072 & 7.021 & 3.872 \\
\hline $2 / 23 / 2006$ & $4: 00$ & 11.73 & 11.586 & 7.532 & 4.918 & 3.224 & 3.15 & 10.931 & 6.14 & 5.068 & 7.021 & 3.868 \\
\hline 2/23/2006 & $5: 00$ & 11.733 & 11.593 & 7.536 & 4.923 & 3.233 & 3.157 & 10.931 & 6.146 & 5.071 & 7.024 & 3.868 \\
\hline 2/23/2006 & $6: 00$ & 11.741 & 11.602 & 7.545 & 4.928 & 3.241 & 3.165 & 10.933 & 6.148 & 5.073 & 7.026 & 3.87 \\
\hline 2/23/2006 & $7: 00$ & 11.752 & 11.619 & 7.56 & 4.932 & 3.251 & 3.174 & 10.938 & 6.154 & 5.079 & 7.032 & 3.875 \\
\hline $2 / 23 / 2006$ & $8: 00$ & 11.764 & 11.63 & 7.569 & 4.938 & 3.263 & 3.187 & 10.942 & 6.154 & 5.082 & 7.036 & 3.876 \\
\hline 2/23/2006 & $9: 00$ & 11.773 & 11.646 & 7.585 & 4.947 & 3.273 & 3.197 & 10.948 & 6.159 & 5.09 & 7.043 & 3.881 \\
\hline 2/23/2006 & $10: 00$ & 11.784 & 11.661 & 7.598 & 4.956 & 3.287 & 3.211 & 10.949 & 6.163 & 5.097 & 7.049 & 3.885 \\
\hline 2/23/2006 & $11: 00$ & 11.799 & 11.675 & 7.609 & 4.963 & 3.3 & 3.221 & 10.956 & 6.169 & 5.103 & 7.054 & 3.889 \\
\hline $2 / 23 / 2006$ & $12: 00$ & 11.805 & 11.683 & 7.609 & 4.967 & 3.297 & 3.223 & 10.96 & 6.168 & 5.104 & 7.057 & 3.885 \\
\hline 2/23/2006 & $13: 00$ & 11.814 & 11.686 & 7.616 & 4.974 & 3.303 & 3.227 & 10.96 & 6.169 & 5.107 & 7.059 & 3.886 \\
\hline $2 / 23 / 2006$ & $14: 00$ & 11.817 & 11.681 & 7.607 & 4.976 & 3.294 & 3.22 & 10.964 & 6.164 & 5.097 & 7.057 & 3.882 \\
\hline 2/23/2006 & $15: 00$ & 11.812 & 11.672 & 7.596 & 4.974 & 3.285 & 3.211 & 10.961 & 6.162 & 5.094 & 7.055 & 3.871 \\
\hline 2/23/2006 & $16: 00$ & 11.81 & 11.668 & 7.594 & 4.974 & 3.281 & 3.205 & 10.964 & 6.164 & 5.09 & 7.054 & 3.87 \\
\hline 2/23/2006 & $17: 00$ & 11.815 & 11.672 & 7.598 & 4.976 & 3.287 & 3.211 & 10.966 & 6.168 & 5.088 & 7.059 & 3.871 \\
\hline 2/23/2006 & $18: 00$ & 11.809 & 11.666 & 7.594 & 4.976 & 3.282 & 3.207 & 10.966 & 6.162 & 5.082 & 7.055 & 3.865 \\
\hline 2/23/2006 & $19: 00$ & 11.807 & 11.661 & 7.589 & 4.976 & 3.277 & 3.201 & 10.964 & 6.161 & 5.08 & 7.053 & 3.862 \\
\hline 2/23/2006 & $20: 00$ & 11.803 & 11.659 & 7.589 & 4.976 & 3.275 & 3.201 & 10.964 & 6.16 & 5.076 & 7.051 & 3.86 \\
\hline 2/23/2006 & $21: 00$ & 11.802 & 11.657 & 7.587 & 4.976 & 3.273 & 3.198 & 10.964 & 6.159 & 5.074 & 7.051 & 3.856 \\
\hline 2/23/2006 & $22: 00$ & 11.798 & 11.652 & 7.583 & 4.974 & 3.269 & 3.196 & 10.964 & 6.156 & 5.07 & 7.047 & 3.852 \\
\hline $2 / 23 / 2006$ & $23: 00$ & 11.798 & 11.652 & 7.583 & 4.974 & 3.268 & 3.195 & 10.964 & 6.158 & 5.073 & 7.049 & 3.85 \\
\hline 2/24/2006 & $0: 00$ & 11.795 & 11.65 & 7.581 & 4.974 & 3.268 & 3.194 & 10.964 & 6.157 & 5.073 & 7.049 & 3.849 \\
\hline $2 / 24 / 2006$ & $1: 00$ & 11.795 & 11.648 & 7.578 & 4.972 & 3.268 & 3.191 & 10.963 & 6.158 & 5.071 & 7.049 & 3.848 \\
\hline 2/24/2006 & $2: 00$ & 11.788 & 11.639 & 7.572 & 4.969 & 3.258 & 3.185 & 10.961 & 6.154 & 5.067 & 7.044 & 3.843 \\
\hline $2 / 24 / 2006$ & $3: 00$ & 11.78 & 11.632 & 7.565 & 4.967 & 3.253 & 3.18 & 10.958 & 6.152 & 5.066 & 7.042 & 3.838 \\
\hline 2/24/2006 & $4: 00$ & 11.775 & 11.624 & 7.558 & 4.963 & 3.25 & 3.175 & 10.955 & 6.146 & 5.062 & 7.04 & 3.835 \\
\hline $2 / 24 / 2006$ & $5: 00$ & 11.768 & 11.612 & 7.547 & 4.958 & 3.24 & 3.167 & 10.952 & 6.145 & 5.059 & 7.037 & 3.831 \\
\hline 2/24/2006 & $6: 00$ & 11.76 & 11.608 & 7.543 & 4.954 & 3.242 & 3.165 & 10.952 & 6.142 & 5.063 & 7.037 & 3.828 \\
\hline 2/24/2006 & $7: 00$ & 11.754 & 11.601 & 7.541 & 4.952 & 3.236 & 3.159 & 10.946 & 6.141 & 5.062 & 7.035 & 3.829 \\
\hline 2/24/2006 & $8: 00$ & 11.75 & 11.595 & 7.534 & 4.947 & 3.233 & 3.153 & 10.945 & 6.142 & 5.062 & 7.034 & 3.825 \\
\hline 2/24/2006 & $9: 00$ & 11.741 & 11.588 & 7.53 & 4.943 & 3.228 & 3.149 & 10.941 & 6.141 & 5.061 & 7.031 & 3.82 \\
\hline $2 / 24 / 2006$ & $10: 00$ & 11.739 & 11.586 & 7.527 & 4.94 & 3.227 & 3.149 & 10.941 & 6.139 & 5.065 & 7.034 & 3.823 \\
\hline $2 / 24 / 2006$ & $11: 00$ & 11.735 & 11.582 & 7.525 & 4.936 & 3.224 & 3.146 & 10.94 & 6.137 & 5.063 & 7.031 & 3.821 \\
\hline
\end{tabular}


TABLE S1.2 (Cont.)

Water Level (ft below top of casing) at Indicated Well

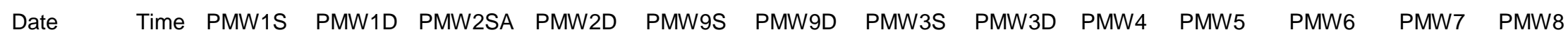

\begin{tabular}{|c|c|c|c|c|c|c|c|c|c|c|c|c|}
\hline 2/24/2006 & $12: 00$ & 11.73 & 11.575 & 7.518 & 4.932 & 3.218 & 3.139 & 10.941 & 6.134 & 5.062 & 7.031 & 3.818 \\
\hline 2/24/2006 & $13: 00$ & 11.724 & 11.57 & 7.516 & 4.929 & 3.216 & 3.136 & 10.937 & 6.137 & 5.059 & 7.027 & 3.816 \\
\hline $2 / 24 / 2006$ & $14: 00$ & 11.714 & 11.56 & 7.509 & 4.923 & 3.206 & 3.127 & 10.935 & 6.134 & 5.053 & 7.022 & 3.813 \\
\hline 2/24/2006 & $15: 00$ & 11.707 & 11.55 & 7.502 & 4.919 & 3.201 & 3.121 & 10.932 & 6.133 & 5.048 & 7.018 & 3.808 \\
\hline $2 / 24 / 2006$ & $16: 00$ & 11.704 & 11.55 & 7.503 & 4.916 & 3.196 & 3.119 & 10.927 & 6.133 & 5.044 & 7.015 & 3.806 \\
\hline 2/24/2006 & $17: 00$ & 11.701 & 11.55 & 7.503 & 4.913 & 3.198 & 3.119 & 10.927 & 6.133 & 5.042 & 7.016 & 3.809 \\
\hline $2 / 24 / 2006$ & $18: 00$ & 11.7 & 11.551 & 7.503 & 4.914 & 3.197 & 3.121 & 10.928 & 6.127 & 5.036 & 7.013 & 3.805 \\
\hline 2/24/2006 & $19: 00$ & 11.706 & 11.562 & 7.518 & 4.916 & 3.208 & 3.131 & 10.929 & 6.133 & 5.041 & 7.019 & 3.81 \\
\hline $2 / 24 / 2006$ & $20: 00$ & 11.719 & 11.582 & 7.534 & 4.918 & 3.223 & 3.146 & 10.935 & 6.138 & 5.041 & 7.024 & 3.812 \\
\hline 2/24/2006 & $21: 00$ & 11.733 & 11.601 & 7.551 & 4.925 & 3.237 & 3.159 & 10.937 & 6.14 & 5.045 & 7.025 & 3.818 \\
\hline $2 / 24 / 2006$ & $22: 00$ & 11.752 & 11.63 & 7.576 & 4.933 & 3.256 & 3.182 & 10.941 & 6.148 & 5.059 & 7.032 & 3.828 \\
\hline 2/24/2006 & $23: 00$ & 11.772 & 11.655 & 7.598 & 4.94 & 3.278 & 3.202 & 10.948 & 6.153 & 5.063 & 7.045 & 3.837 \\
\hline 2/25/2006 & 0:00 & 11.789 & 11.677 & 7.611 & 4.954 & 3.293 & 3.221 & 10.957 & 6.159 & 5.074 & 7.055 & 3.844 \\
\hline 2/25/2006 & $1: 00$ & 11.807 & 11.694 & 7.625 & 4.962 & 3.304 & 3.233 & 10.963 & 6.162 & 5.08 & 7.059 & 3.846 \\
\hline $2 / 25 / 2006$ & $2: 00$ & 11.815 & 11.703 & 7.631 & 4.971 & 3.311 & 3.237 & 10.965 & 6.166 & 5.085 & 7.066 & 3.849 \\
\hline $2 / 25 / 2006$ & $3: 00$ & 11.828 & 11.717 & 7.645 & 4.982 & 3.322 & 3.247 & 10.967 & 6.173 & 5.092 & 7.071 & 3.855 \\
\hline $2 / 25 / 2006$ & $4: 00$ & 11.841 & 11.726 & 7.653 & 4.991 & 3.332 & 3.258 & 10.972 & 6.176 & 5.101 & 7.079 & 3.86 \\
\hline $2 / 25 / 2006$ & $5: 00$ & 11.85 & 11.737 & 7.66 & 4.998 & 3.341 & 3.268 & 10.978 & 6.177 & 5.11 & 7.087 & 3.866 \\
\hline $2 / 25 / 2006$ & $6: 00$ & 11.858 & 11.748 & 7.669 & 5.006 & 3.348 & 3.276 & 10.984 & 6.178 & 5.116 & 7.092 & 3.872 \\
\hline $2 / 25 / 2006$ & $7: 00$ & 11.87 & 11.761 & 7.68 & 5.017 & 3.359 & 3.287 & 10.987 & 6.19 & 5.127 & 7.099 & 3.879 \\
\hline 2/25/2006 & $8: 00$ & 11.88 & 11.772 & 7.687 & 5.022 & 3.365 & 3.295 & 10.993 & 6.193 & 5.135 & 7.105 & 3.883 \\
\hline $2 / 25 / 2006$ & $9: 00$ & 11.89 & 11.779 & 7.689 & 5.031 & 3.374 & 3.301 & 10.997 & 6.194 & 5.143 & 7.11 & 3.885 \\
\hline 2/25/2006 & $10: 00$ & 11.898 & 11.79 & 7.702 & 5.039 & 3.386 & 3.31 & 11.003 & 6.199 & 5.154 & 7.118 & 3.891 \\
\hline $2 / 25 / 2006$ & $11: 00$ & 11.908 & 11.799 & 7.709 & 5.048 & 3.39 & 3.315 & 11.005 & 6.205 & 5.163 & 7.137 & 3.895 \\
\hline 2/25/2006 & $12: 00$ & 11.915 & 11.803 & 7.709 & 5.053 & 3.391 & 3.317 & 11.011 & 6.21 & 5.17 & 7.139 & 3.894 \\
\hline $2 / 25 / 2006$ & $13: 00$ & 11.922 & 11.798 & 7.704 & 5.057 & 3.394 & 3.314 & 11.015 & 6.206 & 5.175 & 7.143 & 3.89 \\
\hline 2/25/2006 & $14: 00$ & 11.919 & 11.796 & 7.698 & 5.058 & 3.382 & 3.307 & 11.016 & 6.201 & 5.17 & 7.139 & 3.882 \\
\hline $2 / 25 / 2006$ & $15: 00$ & 11.92 & 11.792 & 7.693 & 5.057 & 3.376 & 3.302 & 11.017 & 6.204 & 5.171 & 7.139 & 3.878 \\
\hline 2/25/2006 & $16: 00$ & 11.92 & 11.792 & 7.691 & 5.06 & 3.372 & 3.3 & 11.019 & 6.203 & 5.167 & 7.138 & 3.872 \\
\hline $2 / 25 / 2006$ & $17: 00$ & 11.919 & 11.79 & 7.693 & 5.06 & 3.375 & 3.298 & 11.019 & 6.205 & 5.165 & 7.138 & 3.867 \\
\hline $2 / 25 / 2006$ & $18: 00$ & 11.922 & 11.79 & 7.693 & 5.061 & 3.373 & 3.299 & 11.02 & 6.204 & 5.161 & 7.136 & 3.864 \\
\hline $2 / 25 / 2006$ & $19: 00$ & 11.919 & 11.787 & 7.693 & 5.061 & 3.374 & 3.298 & 11.021 & 6.202 & 5.157 & 7.137 & 3.858 \\
\hline $2 / 25 / 2006$ & $20: 00$ & 11.922 & 11.79 & 7.695 & 5.064 & 3.377 & 3.301 & 11.023 & 6.206 & 5.158 & 7.14 & 3.862 \\
\hline $2 / 25 / 2006$ & $21: 00$ & 11.924 & 11.792 & 7.698 & 5.064 & 3.381 & 3.304 & 11.025 & 6.207 & 5.159 & 7.14 & 3.862 \\
\hline
\end{tabular}


TABLE S1.2 (Cont.)

Water Level (ft below top of casing) at Indicated Well

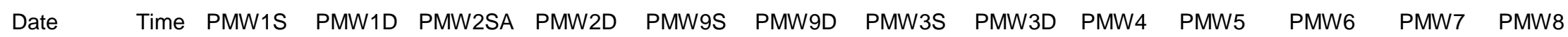

\begin{tabular}{|c|c|c|c|c|c|c|c|c|c|c|c|c|}
\hline $2 / 25 / 2006$ & $22: 00$ & 11.924 & 11.794 & 7.698 & 5.066 & 3.377 & 3.304 & 11.024 & 6.21 & 5.16 & 7.14 & 3.864 \\
\hline $2 / 25 / 2006$ & $23: 00$ & 11.923 & 11.792 & 7.7 & 5.068 & 3.38 & 3.306 & 11.026 & 6.208 & 5.163 & 7.142 & 3.867 \\
\hline $2 / 26 / 2006$ & $0: 00$ & 11.927 & 11.794 & 7.7 & 5.068 & 3.381 & 3.309 & 11.029 & 6.211 & 5.166 & 7.144 & 3.869 \\
\hline 2/26/2006 & $1: 00$ & 11.926 & 11.796 & 7.702 & 5.071 & 3.383 & 3.31 & 11.029 & 6.214 & 5.166 & 7.145 & 3.869 \\
\hline 2/26/2006 & $2: 00$ & 11.928 & 11.798 & 7.704 & 5.073 & 3.386 & 3.311 & 11.03 & 6.209 & 5.171 & 7.144 & 3.874 \\
\hline 2/26/2006 & $3: 00$ & 11.926 & 11.79 & 7.7 & 5.073 & 3.381 & 3.307 & 11.03 & 6.213 & 5.168 & 7.143 & 3.874 \\
\hline $2 / 26 / 2006$ & $4: 00$ & 11.923 & 11.785 & 7.691 & 5.071 & 3.379 & 3.305 & 11.029 & 6.21 & 5.172 & 7.143 & 3.874 \\
\hline 2/26/2006 & $5: 00$ & 11.913 & 11.774 & 7.684 & 5.068 & 3.372 & 3.295 & 11.026 & 6.204 & 5.166 & 7.141 & 3.873 \\
\hline 2/26/2006 & $6: 00$ & 11.912 & 11.768 & 7.682 & 5.067 & 3.369 & 3.293 & 11.026 & 6.209 & 5.169 & 7.139 & 3.873 \\
\hline 2/26/2006 & $7: 00$ & 11.907 & 11.767 & 7.678 & 5.063 & 3.368 & 3.291 & 11.026 & 6.21 & 5.171 & 7.14 & 3.876 \\
\hline $2 / 26 / 2006$ & $8: 00$ & 11.904 & 11.761 & 7.675 & 5.064 & 3.366 & 3.29 & 11.025 & 6.209 & 5.176 & 7.138 & 3.879 \\
\hline 2/26/2006 & $9: 00$ & 11.899 & 11.754 & 7.669 & 5.061 & 3.359 & 3.285 & 11.022 & 6.211 & 5.175 & 7.137 & 3.878 \\
\hline $2 / 26 / 2006$ & $10: 00$ & 11.897 & 11.754 & 7.673 & 5.062 & 3.363 & 3.285 & 11.023 & 6.211 & 5.177 & 7.134 & 3.881 \\
\hline 2/26/2006 & $11: 00$ & 11.892 & 11.745 & 7.664 & 5.057 & 3.356 & 3.279 & 11.023 & 6.205 & 5.177 & 7.132 & 3.88 \\
\hline 2/26/2006 & $12: 00$ & 11.882 & 11.732 & 7.653 & 5.053 & 3.345 & 3.267 & 11.017 & 6.203 & 5.174 & 7.128 & 3.879 \\
\hline 2/26/2006 & $13: 00$ & 11.872 & 11.717 & 7.637 & 5.045 & 3.335 & 3.257 & 11.015 & 6.198 & 5.169 & 7.123 & 3.874 \\
\hline $2 / 26 / 2006$ & $14: 00$ & 11.856 & 11.699 & 7.621 & 5.037 & 3.32 & 3.238 & 11.012 & 6.193 & 5.158 & 7.112 & 3.863 \\
\hline 2/26/2006 & $15: 00$ & 11.84 & 11.675 & 7.601 & 5.026 & 3.3 & 3.22 & 11 & 6.184 & 5.149 & 7.106 & 3.854 \\
\hline 2/26/2006 & $16: 00$ & 11.826 & 11.661 & 7.591 & 5.019 & 3.289 & 3.208 & 10.995 & 6.181 & 5.136 & 7.098 & 3.847 \\
\hline 2/26/2006 & $17: 00$ & 11.813 & 11.644 & 7.578 & 5.009 & 3.277 & 3.194 & 10.993 & 6.176 & 5.126 & 7.087 & 3.839 \\
\hline 2/26/2006 & $18: 00$ & 11.8 & 11.633 & 7.567 & 5 & 3.265 & 3.189 & 10.986 & 6.169 & 5.117 & 7.085 & 3.832 \\
\hline $2 / 26 / 2006$ & $19: 00$ & 11.788 & 11.624 & 7.564 & 4.995 & 3.262 & 3.183 & 10.984 & 6.164 & 5.112 & 7.081 & 3.827 \\
\hline 2/26/2006 & $20: 00$ & 11.78 & 11.617 & 7.558 & 4.988 & 3.257 & 3.179 & 10.983 & 6.162 & 5.109 & 7.075 & 3.825 \\
\hline $2 / 26 / 2006$ & $21: 00$ & 11.773 & 11.61 & 7.556 & 4.981 & 3.253 & 3.172 & 10.979 & 6.162 & 5.104 & 7.071 & 3.825 \\
\hline 2/26/2006 & $22: 00$ & 11.763 & 11.599 & 7.547 & 4.975 & 3.244 & 3.166 & 10.974 & 6.16 & 5.099 & 7.065 & 3.822 \\
\hline $2 / 26 / 2006$ & $23: 00$ & 11.756 & 11.591 & 7.538 & 4.968 & 3.236 & 3.159 & 10.969 & 6.159 & 5.096 & 7.064 & 3.824 \\
\hline 2/27/2006 & $0: 00$ & 11.745 & 11.581 & 7.527 & 4.959 & 3.231 & 3.151 & 10.967 & 6.15 & 5.091 & 7.062 & 3.823 \\
\hline $2 / 27 / 2006$ & $1: 00$ & 11.736 & 11.571 & 7.52 & 4.953 & 3.224 & 3.144 & 10.962 & 6.15 & 5.089 & 7.056 & 3.823 \\
\hline 2/27/2006 & $2: 00$ & 11.728 & 11.562 & 7.513 & 4.946 & 3.216 & 3.138 & 10.961 & 6.151 & 5.09 & 7.056 & 3.825 \\
\hline 2/27/2006 & $3: 00$ & 11.718 & 11.553 & 7.507 & 4.939 & 3.211 & 3.13 & 10.957 & 6.146 & 5.087 & 7.051 & 3.826 \\
\hline 2/27/2006 & $4: 00$ & 11.71 & 11.546 & 7.502 & 4.935 & 3.207 & 3.125 & 10.953 & 6.143 & 5.086 & 7.049 & 3.827 \\
\hline 2/27/2006 & $5: 00$ & 11.702 & 11.537 & 7.496 & 4.926 & 3.2 & 3.119 & 10.949 & 6.141 & 5.086 & 7.045 & 3.827 \\
\hline $2 / 27 / 2006$ & $6: 00$ & 11.696 & 11.535 & 7.496 & 4.924 & 3.2 & 3.119 & 10.945 & 6.145 & 5.088 & 7.042 & 3.833 \\
\hline $2 / 27 / 2006$ & $7: 00$ & 11.695 & 11.535 & 7.496 & 4.92 & 3.201 & 3.12 & 10.944 & 6.143 & 5.089 & 7.04 & 3.837 \\
\hline
\end{tabular}


TABLE S1.2 (Cont.)

Water Level (ft below top of casing) at Indicated Well

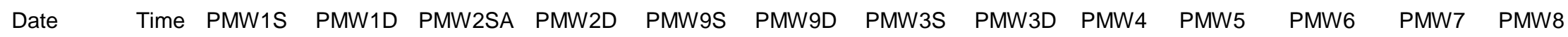

\begin{tabular}{|c|c|c|c|c|c|c|c|c|c|c|c|c|}
\hline 2/27/2006 & $8: 00$ & 11.69 & 11.533 & 7.498 & 4.917 & 3.202 & 3.121 & 10.943 & 6.139 & 5.092 & 7.042 & 3.836 \\
\hline $2 / 27 / 2006$ & $9: 00$ & 11.691 & 11.533 & 7.499 & 4.915 & 3.201 & 3.124 & 10.943 & 6.142 & 5.093 & 7.039 & 3.841 \\
\hline $2 / 27 / 2006$ & $10: 00$ & 11.688 & 11.535 & 7.499 & 4.915 & 3.2 & 3.125 & 10.94 & 6.143 & 5.095 & 7.037 & 3.844 \\
\hline 2/27/2006 & $11: 00$ & 11.686 & 11.532 & 7.495 & 4.911 & 3.202 & 3.122 & 10.94 & 6.139 & 5.094 & 7.037 & 3.841 \\
\hline 2/27/2006 & $12: 00$ & 11.686 & 11.535 & 7.498 & 4.909 & 3.2 & 3.121 & 10.936 & 6.14 & 5.091 & 7.037 & 3.841 \\
\hline 2/27/2006 & $13: 00$ & 11.684 & 11.531 & 7.495 & 4.906 & 3.198 & 3.12 & 10.936 & 6.14 & 5.084 & 7.034 & 3.83 \\
\hline $2 / 27 / 2006$ & $14: 00$ & 11.681 & 11.526 & 7.486 & 4.902 & 3.191 & 3.112 & 10.935 & 6.131 & 5.077 & 7.031 & 3.815 \\
\hline 2/27/2006 & $15: 00$ & 11.679 & 11.524 & 7.486 & 4.9 & 3.187 & 3.109 & 10.933 & 6.131 & 5.069 & 7.027 & 3.806 \\
\hline 2/27/2006 & $16: 00$ & 11.676 & 11.522 & 7.484 & 4.897 & 3.185 & 3.106 & 10.931 & 6.131 & 5.064 & 7.025 & 3.8 \\
\hline 2/27/2006 & $17: 00$ & 11.676 & 11.522 & 7.486 & 4.895 & 3.185 & 3.108 & 10.929 & 6.129 & 5.059 & 7.025 & 3.794 \\
\hline $2 / 27 / 2006$ & $18: 00$ & 11.675 & 11.524 & 7.489 & 4.895 & 3.187 & 3.109 & 10.93 & 6.127 & 5.052 & 7.023 & 3.79 \\
\hline 2/27/2006 & $19: 00$ & 11.675 & 11.526 & 7.491 & 4.895 & 3.191 & 3.111 & 10.928 & 6.129 & 5.048 & 7.023 & 3.789 \\
\hline $2 / 27 / 2006$ & $20: 00$ & 11.68 & 11.535 & 7.498 & 4.897 & 3.193 & 3.115 & 10.927 & 6.128 & 5.046 & 7.021 & 3.787 \\
\hline 2/27/2006 & $21: 00$ & 11.68 & 11.537 & 7.498 & 4.897 & 3.193 & 3.115 & 10.928 & 6.126 & 5.044 & 7.022 & 3.787 \\
\hline 2/27/2006 & $22: 00$ & 11.685 & 11.542 & 7.499 & 4.899 & 3.197 & 3.12 & 10.927 & 6.124 & 5.042 & 7.024 & 3.791 \\
\hline $2 / 27 / 2006$ & $23: 00$ & 11.692 & 11.553 & 7.511 & 4.903 & 3.209 & 3.13 & 10.928 & 6.129 & 5.046 & 7.027 & 3.798 \\
\hline 2/28/2006 & $0: 00$ & 11.695 & 11.555 & 7.515 & 4.903 & 3.206 & 3.132 & 10.927 & 6.128 & 5.043 & 7.026 & 3.799 \\
\hline 2/28/2006 & $1: 00$ & 11.698 & 11.559 & 7.515 & 4.906 & 3.21 & 3.13 & 10.927 & 6.129 & 5.043 & 7.027 & 3.801 \\
\hline 2/28/2006 & $2: 00$ & 11.699 & 11.562 & 7.515 & 4.907 & 3.213 & 3.134 & 10.93 & 6.129 & 5.045 & 7.03 & 3.805 \\
\hline $2 / 28 / 2006$ & $3: 00$ & 11.703 & 11.564 & 7.515 & 4.908 & 3.215 & 3.135 & 10.928 & 6.13 & 5.047 & 7.029 & 3.808 \\
\hline 2/28/2006 & $4: 00$ & 11.702 & 11.559 & 7.513 & 4.908 & 3.208 & 3.133 & 10.928 & 6.124 & 5.043 & 7.027 & 3.807 \\
\hline $2 / 28 / 2006$ & $5: 00$ & 11.7 & 11.553 & 7.508 & 4.908 & 3.205 & 3.125 & 10.926 & 6.127 & 5.046 & 7.03 & 3.811 \\
\hline 2/28/2006 & $6: 00$ & 11.702 & 11.562 & 7.515 & 4.91 & 3.21 & 3.135 & 10.924 & 6.129 & 5.055 & 7.031 & 3.819 \\
\hline $2 / 28 / 2006$ & $7: 00$ & 11.707 & 11.568 & 7.522 & 4.91 & 3.218 & 3.14 & 10.926 & 6.131 & 5.058 & 7.032 & 3.827 \\
\hline 2/28/2006 & $8: 00$ & 11.705 & 11.563 & 7.515 & 4.91 & 3.215 & 3.135 & 10.926 & 6.127 & 5.06 & 7.032 & 3.831 \\
\hline $2 / 28 / 2006$ & $9: 00$ & 11.709 & 11.57 & 7.52 & 4.913 & 3.218 & 3.139 & 10.928 & 6.133 & 5.066 & 7.035 & 3.841 \\
\hline 2/28/2006 & $10: 00$ & 11.707 & 11.568 & 7.52 & 4.912 & 3.218 & 3.141 & 10.928 & 6.127 & 5.07 & 7.033 & 3.841 \\
\hline $2 / 28 / 2006$ & $11: 00$ & 11.711 & 11.568 & 7.52 & 4.912 & 3.219 & 3.14 & 10.927 & 6.129 & 5.071 & 7.032 & 3.842 \\
\hline 2/28/2006 & $12: 00$ & 11.709 & 11.568 & 7.518 & 4.912 & 3.216 & 3.136 & 10.927 & 6.129 & 5.07 & 7.031 & 3.835 \\
\hline 2/28/2006 & $13: 00$ & 11.711 & 11.568 & 7.52 & 4.91 & 3.214 & 3.137 & 10.927 & 6.13 & 5.064 & 7.034 & 3.825 \\
\hline 2/28/2006 & $14: 00$ & 11.704 & 11.56 & 7.51 & 4.91 & 3.208 & 3.129 & 10.926 & 6.129 & 5.059 & 7.031 & 3.816 \\
\hline 2/28/2006 & $15: 00$ & 11.692 & 11.542 & 7.493 & 4.901 & 3.195 & 3.112 & 10.923 & 6.122 & 5.047 & 7.024 & 3.8 \\
\hline $2 / 28 / 2006$ & $16: 00$ & 11.685 & 11.537 & 7.489 & 4.899 & 3.19 & 3.109 & 10.922 & 6.122 & 5.039 & 7.021 & 3.794 \\
\hline 2/28/2006 & $17: 00$ & 11.68 & 11.533 & 7.489 & 4.897 & 3.186 & 3.107 & 10.92 & 6.123 & 5.039 & 7.015 & 3.794 \\
\hline
\end{tabular}


TABLE S1.2 (Cont.)

Water Level (ft below top of casing) at Indicated Well

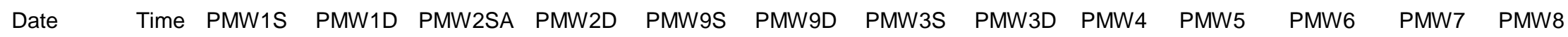

\begin{tabular}{|c|c|c|c|c|c|c|c|c|c|c|c|c|}
\hline 2/28/2006 & $18: 00$ & 11.677 & 11.53 & 7.486 & 4.895 & 3.183 & 3.105 & 10.921 & 6.118 & 5.034 & 7.016 & 3.787 \\
\hline $2 / 28 / 2006$ & $19: 00$ & 11.67 & 11.522 & 7.482 & 4.892 & 3.179 & 3.098 & 10.917 & 6.115 & 5.029 & 7.015 & 3.785 \\
\hline $2 / 28 / 2006$ & $20: 00$ & 11.671 & 11.524 & 7.484 & 4.892 & 3.182 & 3.102 & 10.915 & 6.119 & 5.03 & 7.013 & 3.789 \\
\hline 2/28/2006 & $21: 00$ & 11.669 & 11.522 & 7.484 & 4.892 & 3.18 & 3.103 & 10.916 & 6.117 & 5.029 & 7.01 & 3.79 \\
\hline 2/28/2006 & $22: 00$ & 11.666 & 11.52 & 7.482 & 4.888 & 3.178 & 3.098 & 10.913 & 6.113 & 5.024 & 7.008 & 3.787 \\
\hline 2/28/2006 & $23: 00$ & 11.667 & 11.521 & 7.482 & 4.886 & 3.176 & 3.097 & 10.912 & 6.115 & 5.027 & 7.011 & 3.789 \\
\hline $3 / 1 / 2006$ & $0: 00$ & 11.667 & 11.519 & 7.479 & 4.884 & 3.179 & 3.099 & 10.912 & 6.116 & 5.024 & 7.008 & 3.789 \\
\hline 3/1/2006 & $1: 00$ & 11.659 & 11.515 & 7.473 & 4.881 & 3.171 & 3.095 & 10.91 & 6.113 & 5.023 & 7.008 & 3.786 \\
\hline 3/1/2006 & $2: 00$ & 11.656 & 11.511 & 7.471 & 4.879 & 3.171 & 3.089 & 10.909 & 6.108 & 5.023 & 7.005 & 3.785 \\
\hline $3 / 1 / 2006$ & $3: 00$ & 11.652 & 11.506 & 7.466 & 4.876 & 3.167 & 3.087 & 10.907 & 6.108 & 5.022 & 7.005 & 3.785 \\
\hline $3 / 1 / 2006$ & $4: 00$ & 11.647 & 11.502 & 7.461 & 4.873 & 3.164 & 3.084 & 10.904 & 6.108 & 5.019 & 7.002 & 3.786 \\
\hline $3 / 1 / 2006$ & $5: 00$ & 11.646 & 11.502 & 7.461 & 4.872 & 3.163 & 3.084 & 10.903 & 6.11 & 5.021 & 7.004 & 3.788 \\
\hline $3 / 1 / 2006$ & $6: 00$ & 11.644 & 11.499 & 7.461 & 4.87 & 3.165 & 3.083 & 10.903 & 6.11 & 5.021 & 7.002 & 3.788 \\
\hline $3 / 1 / 2006$ & $7: 00$ & 11.646 & 11.506 & 7.468 & 4.87 & 3.17 & 3.089 & 10.904 & 6.109 & 5.024 & 7.003 & 3.793 \\
\hline $3 / 1 / 2006$ & $8: 00$ & 11.647 & 11.508 & 7.471 & 4.872 & 3.172 & 3.091 & 10.904 & 6.108 & 5.026 & 7.002 & 3.795 \\
\hline 3/1/2006 & $9: 00$ & 11.656 & 11.517 & 7.477 & 4.872 & 3.178 & 3.097 & 10.902 & 6.113 & 5.031 & 7.005 & 3.803 \\
\hline $3 / 1 / 2006$ & $10: 00$ & 11.664 & 11.524 & 7.484 & 4.875 & 3.184 & 3.104 & 10.904 & 6.112 & 5.036 & 7.006 & 3.807 \\
\hline $3 / 1 / 2006$ & $11: 00$ & 11.671 & 11.533 & 7.495 & 4.879 & 3.19 & 3.111 & 10.905 & 6.118 & 5.041 & 7.009 & 3.81 \\
\hline $3 / 1 / 2006$ & $12: 00$ & 11.676 & 11.541 & 7.499 & 4.879 & 3.195 & 3.116 & 10.908 & 6.119 & 5.044 & 7.011 & 3.812 \\
\hline $3 / 1 / 2006$ & $13: 00$ & 11.684 & 11.548 & 7.503 & 4.883 & 3.199 & 3.12 & 10.905 & 6.117 & 5.046 & 7.012 & 3.815 \\
\hline 3/1/2006 & $14: 00$ & 11.69 & 11.552 & 7.504 & 4.887 & 3.204 & 3.123 & 10.908 & 6.115 & 5.047 & 7.013 & 3.814 \\
\hline $3 / 1 / 2006$ & $15: 00$ & 11.69 & 11.553 & 7.506 & 4.886 & 3.201 & 3.122 & 10.906 & 6.118 & 5.048 & 7.012 & 3.812 \\
\hline $3 / 1 / 2006$ & $16: 00$ & 11.702 & 11.568 & 7.519 & 4.892 & 3.214 & 3.134 & 10.91 & 6.123 & 5.056 & 7.02 & 3.822 \\
\hline $3 / 1 / 2006$ & $17: 00$ & 11.713 & 11.584 & 7.535 & 4.902 & 3.228 & 3.146 & 10.913 & 6.125 & 5.071 & 7.027 & 3.832 \\
\hline $3 / 1 / 2006$ & $18: 00$ & 11.728 & 11.601 & 7.55 & 4.908 & 3.239 & 3.158 & 10.915 & 6.127 & 5.079 & 7.032 & 3.837 \\
\hline $3 / 1 / 2006$ & $19: 00$ & 11.745 & 11.625 & 7.572 & 4.92 & 3.26 & 3.18 & 10.922 & 6.138 & 5.091 & 7.043 & 3.849 \\
\hline 3/1/2006 & $20: 00$ & 11.762 & 11.643 & 7.585 & 4.929 & 3.275 & 3.194 & 10.931 & 6.142 & 5.101 & 7.054 & 3.854 \\
\hline $3 / 1 / 2006$ & $21: 00$ & 11.776 & 11.661 & 7.601 & 4.938 & 3.287 & 3.208 & 10.935 & 6.148 & 5.11 & 7.063 & 3.861 \\
\hline $3 / 1 / 2006$ & $22: 00$ & 11.789 & 11.676 & 7.607 & 4.948 & 3.297 & 3.22 & 10.943 & 6.152 & 5.117 & 7.07 & 3.865 \\
\hline $3 / 1 / 2006$ & $23: 00$ & 11.803 & 11.687 & 7.616 & 4.958 & 3.307 & 3.226 & 10.948 & 6.152 & 5.122 & 7.073 & 3.867 \\
\hline $3 / 2 / 2006$ & $0: 00$ & 11.811 & 11.694 & 7.623 & 4.965 & 3.309 & 3.232 & 10.95 & 6.154 & 5.126 & 7.076 & 3.868 \\
\hline 3/2/2006 & $1: 00$ & 11.82 & 11.699 & 7.623 & 4.97 & 3.311 & 3.236 & 10.954 & 6.154 & 5.126 & 7.079 & 3.865 \\
\hline $3 / 2 / 2006$ & $2: 00$ & 11.826 & 11.703 & 7.63 & 4.976 & 3.315 & 3.236 & 10.956 & 6.157 & 5.13 & 7.083 & 3.868 \\
\hline 3/2/2006 & $3: 00$ & 11.831 & 11.703 & 7.625 & 4.981 & 3.313 & 3.236 & 10.958 & 6.159 & 5.13 & 7.081 & 3.866 \\
\hline
\end{tabular}


TABLE S1.2 (Cont.)

Water Level (ft below top of casing) at Indicated Well

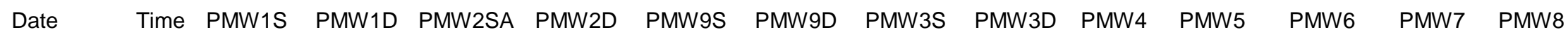

\begin{tabular}{|c|c|c|c|c|c|c|c|c|c|c|c|c|}
\hline $3 / 2 / 2006$ & $4: 00$ & 11.833 & 11.707 & 7.63 & 4.985 & 3.313 & 3.237 & 10.961 & 6.157 & 5.129 & 7.084 & 3.867 \\
\hline $3 / 2 / 2006$ & $5: 00$ & 11.839 & 11.712 & 7.634 & 4.99 & 3.32 & 3.242 & 10.963 & 6.16 & 5.133 & 7.084 & 3.872 \\
\hline $3 / 2 / 2006$ & $6: 00$ & 11.843 & 11.718 & 7.641 & 4.994 & 3.328 & 3.247 & 10.967 & 6.163 & 5.135 & 7.088 & 3.875 \\
\hline 3/2/2006 & $7: 00$ & 11.853 & 11.727 & 7.648 & 4.999 & 3.333 & 3.255 & 10.969 & 6.17 & 5.142 & 7.093 & 3.883 \\
\hline 3/2/2006 & 8:00 & 11.858 & 11.736 & 7.654 & 5.005 & 3.339 & 3.263 & 10.969 & 6.172 & 5.147 & 7.098 & 3.888 \\
\hline 3/2/2006 & $9: 00$ & 11.86 & 11.743 & 7.665 & 5.012 & 3.349 & 3.272 & 10.975 & 6.174 & 5.152 & 7.102 & 3.897 \\
\hline $3 / 2 / 2006$ & $10: 00$ & 11.872 & 11.754 & 7.676 & 5.019 & 3.36 & 3.281 & 10.975 & 6.177 & 5.16 & 7.104 & 3.905 \\
\hline 3/2/2006 & $11: 00$ & 11.882 & 11.765 & 7.681 & 5.025 & 3.366 & 3.286 & 10.981 & 6.182 & 5.163 & 7.107 & 3.904 \\
\hline 3/2/2006 & $12: 00$ & 11.89 & 11.774 & 7.687 & 5.031 & 3.374 & 3.294 & 10.986 & 6.188 & 5.167 & 7.111 & 3.901 \\
\hline $3 / 2 / 2006$ & $13: 00$ & 11.897 & 11.78 & 7.692 & 5.034 & 3.377 & 3.297 & 10.989 & 6.187 & 5.169 & 7.117 & 3.902 \\
\hline $3 / 2 / 2006$ & $14: 00$ & 11.9 & 11.78 & 7.692 & 5.04 & 3.375 & 3.298 & 10.992 & 6.188 & 5.167 & 7.118 & 3.9 \\
\hline $3 / 2 / 2006$ & $15: 00$ & 11.905 & 11.776 & 7.687 & 5.044 & 3.369 & 3.293 & 10.99 & 6.184 & 5.167 & 7.117 & 3.896 \\
\hline $3 / 2 / 2006$ & $16: 00$ & 11.907 & 11.78 & 7.687 & 5.045 & 3.373 & 3.293 & 10.997 & 6.187 & 5.169 & 7.118 & 3.898 \\
\hline $3 / 2 / 2006$ & $17: 00$ & 11.91 & 11.781 & 7.691 & 5.047 & 3.374 & 3.297 & 11 & 6.192 & 5.176 & 7.122 & 3.904 \\
\hline $3 / 2 / 2006$ & $18: 00$ & 11.915 & 11.787 & 7.696 & 5.051 & 3.38 & 3.299 & 11.001 & 6.194 & 5.178 & 7.123 & 3.903 \\
\hline 3/2/2006 & $19: 00$ & 11.917 & 11.791 & 7.703 & 5.058 & 3.386 & 3.308 & 11.007 & 6.198 & 5.182 & 7.13 & 3.909 \\
\hline $3 / 2 / 2006$ & $20: 00$ & 11.922 & 11.798 & 7.707 & 5.062 & 3.395 & 3.314 & 11.009 & 6.198 & 5.186 & 7.132 & 3.913 \\
\hline $3 / 2 / 2006$ & $21: 00$ & 11.925 & 11.798 & 7.707 & 5.064 & 3.392 & 3.314 & 11.012 & 6.2 & 5.189 & 7.132 & 3.913 \\
\hline $3 / 2 / 2006$ & $22: 00$ & 11.929 & 11.803 & 7.714 & 5.069 & 3.396 & 3.319 & 11.016 & 6.201 & 5.191 & 7.136 & 3.916 \\
\hline $3 / 2 / 2006$ & $23: 00$ & 11.932 & 11.807 & 7.711 & 5.073 & 3.397 & 3.321 & 11.016 & 6.203 & 5.192 & 7.138 & 3.919 \\
\hline 3/3/2006 & $0: 00$ & 11.937 & 11.807 & 7.716 & 5.075 & 3.4 & 3.323 & 11.019 & 6.206 & 5.195 & 7.141 & 3.923 \\
\hline $3 / 3 / 2006$ & $1: 00$ & 11.939 & 11.809 & 7.716 & 5.078 & 3.402 & 3.322 & 11.021 & 6.207 & 5.199 & 7.14 & 3.925 \\
\hline 3/3/2006 & $2: 00$ & 11.939 & 11.809 & 7.716 & 5.08 & 3.401 & 3.323 & 11.022 & 6.208 & 5.201 & 7.141 & 3.927 \\
\hline $3 / 3 / 2006$ & $3: 00$ & 11.944 & 11.816 & 7.725 & 5.085 & 3.412 & 3.334 & 11.026 & 6.215 & 5.208 & 7.147 & 3.94 \\
\hline $3 / 3 / 2006$ & $4: 00$ & 11.946 & 11.818 & 7.725 & 5.086 & 3.411 & 3.332 & 11.026 & 6.211 & 5.209 & 7.146 & 3.942 \\
\hline $3 / 3 / 2006$ & $5: 00$ & 11.948 & 11.82 & 7.725 & 5.089 & 3.411 & 3.334 & 11.028 & 6.213 & 5.213 & 7.149 & 3.947 \\
\hline $3 / 3 / 2006$ & $6: 00$ & 11.95 & 11.823 & 7.727 & 5.091 & 3.414 & 3.336 & 11.03 & 6.215 & 5.22 & 7.149 & 3.954 \\
\hline $3 / 3 / 2006$ & $7: 00$ & 11.954 & 11.825 & 7.729 & 5.095 & 3.42 & 3.338 & 11.031 & 6.217 & 5.221 & 7.153 & 3.958 \\
\hline $3 / 3 / 2006$ & $8: 00$ & 11.954 & 11.829 & 7.731 & 5.098 & 3.42 & 3.34 & 11.032 & 6.22 & 5.227 & 7.155 & 3.964 \\
\hline $3 / 3 / 2006$ & 9:00 & 11.957 & 11.831 & 7.735 & 5.1 & 3.422 & 3.343 & 11.033 & 6.222 & 5.231 & 7.154 & 3.968 \\
\hline $3 / 3 / 2006$ & $10: 00$ & 11.961 & 11.834 & 7.738 & 5.102 & 3.428 & 3.348 & 11.035 & 6.223 & 5.236 & 7.159 & 3.975 \\
\hline 3/3/2006 & $11: 00$ & 11.959 & 11.827 & 7.731 & 5.102 & 3.422 & 3.34 & 11.035 & 6.219 & 5.236 & 7.155 & 3.969 \\
\hline $3 / 3 / 2006$ & $12: 00$ & 11.959 & 11.823 & 7.725 & 5.1 & 3.413 & 3.336 & 11.035 & 6.219 & 5.234 & 7.152 & 3.962 \\
\hline 3/3/2006 & $13: 00$ & 11.956 & 11.818 & 7.72 & 5.099 & 3.41 & 3.33 & 11.036 & 6.22 & 5.229 & 7.15 & 3.947 \\
\hline
\end{tabular}


TABLE S1.2 (Cont.)

Water Level (ft below top of casing) at Indicated Well

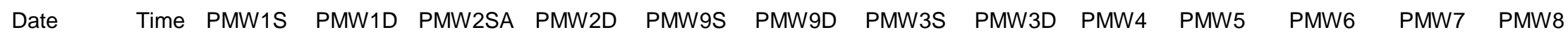

\begin{tabular}{|c|c|c|c|c|c|c|c|c|c|c|c|c|}
\hline $3 / 3 / 2006$ & $14: 00$ & 11.949 & 11.807 & 7.711 & 5.096 & 3.399 & 3.321 & 11.037 & 6.218 & 5.215 & 7.147 & 3.93 \\
\hline $3 / 3 / 2006$ & $15: 00$ & 11.937 & 11.791 & 7.698 & 5.091 & 3.386 & 3.306 & 11.033 & 6.209 & 5.199 & 7.139 & 3.91 \\
\hline 3/3/2006 & $16: 00$ & 11.929 & 11.785 & 7.694 & 5.087 & 3.38 & 3.301 & 11.03 & 6.212 & 5.192 & 7.135 & 3.904 \\
\hline 3/3/2006 & $17: 00$ & 11.921 & 11.772 & 7.684 & 5.082 & 3.373 & 3.293 & 11.03 & 6.205 & 5.179 & 7.134 & 3.894 \\
\hline $3 / 3 / 2006$ & $18: 00$ & 11.914 & 11.763 & 7.678 & 5.081 & 3.364 & 3.288 & 11.027 & 6.202 & 5.175 & 7.13 & 3.891 \\
\hline $3 / 3 / 2006$ & $19: 00$ & 11.906 & 11.76 & 7.676 & 5.076 & 3.363 & 3.283 & 11.025 & 6.204 & 5.174 & 7.129 & 3.889 \\
\hline 3/3/2006 & $20: 00$ & 11.904 & 11.758 & 7.676 & 5.075 & 3.361 & 3.286 & 11.024 & 6.206 & 5.168 & 7.128 & 3.891 \\
\hline 3/3/2006 & $21: 00$ & 11.9 & 11.752 & 7.671 & 5.071 & 3.359 & 3.281 & 11.023 & 6.199 & 5.165 & 7.124 & 3.886 \\
\hline 3/3/2006 & $22: 00$ & 11.896 & 11.747 & 7.669 & 5.067 & 3.36 & 3.279 & 11.023 & 6.193 & 5.163 & 7.123 & 3.886 \\
\hline $3 / 3 / 2006$ & $23: 00$ & 11.894 & 11.749 & 7.669 & 5.064 & 3.355 & 3.282 & 11.022 & 6.2 & 5.162 & 7.125 & 3.889 \\
\hline $3 / 4 / 2006$ & $0: 00$ & 11.893 & 11.749 & 7.673 & 5.067 & 3.361 & 3.284 & 11.021 & 6.2 & 5.165 & 7.125 & 3.892 \\
\hline $3 / 4 / 2006$ & $1: 00$ & 11.893 & 11.754 & 7.676 & 5.064 & 3.361 & 3.283 & 11.022 & 6.203 & 5.167 & 7.125 & 3.893 \\
\hline $3 / 4 / 2006$ & $2: 00$ & 11.896 & 11.754 & 7.676 & 5.064 & 3.363 & 3.285 & 11.022 & 6.199 & 5.166 & 7.125 & 3.892 \\
\hline $3 / 4 / 2006$ & $3: 00$ & 11.891 & 11.747 & 7.669 & 5.06 & 3.354 & 3.279 & 11.02 & 6.2 & 5.162 & 7.122 & 3.889 \\
\hline $3 / 4 / 2006$ & $4: 00$ & 11.89 & 11.749 & 7.673 & 5.06 & 3.358 & 3.282 & 11.018 & 6.196 & 5.158 & 7.119 & 3.89 \\
\hline $3 / 4 / 2006$ & $5: 00$ & 11.893 & 11.754 & 7.676 & 5.062 & 3.362 & 3.282 & 11.02 & 6.206 & 5.162 & 7.124 & 3.886 \\
\hline $3 / 4 / 2006$ & $6: 00$ & 11.894 & 11.754 & 7.671 & 5.06 & 3.358 & 3.286 & 11.019 & 6.198 & 5.153 & 7.124 & 3.879 \\
\hline $3 / 4 / 2006$ & $7: 00$ & 11.889 & 11.74 & 7.667 & 5.057 & 3.354 & 3.278 & 11.018 & 6.195 & 5.146 & 7.11 & 3.87 \\
\hline $3 / 4 / 2006$ & $8: 00$ & 11.889 & 11.738 & 7.66 & 5.056 & 3.35 & 3.271 & 11.014 & 6.196 & 5.139 & 7.115 & 3.861 \\
\hline $3 / 4 / 2006$ & $9: 00$ & 11.886 & 11.741 & 7.663 & 5.054 & 3.351 & 3.281 & 11.016 & 6.192 & 5.135 & 7.116 & 3.855 \\
\hline $3 / 4 / 2006$ & $10: 00$ & 11.883 & 11.74 & 7.663 & 5.051 & 3.346 & 3.271 & 11.015 & 6.192 & 5.131 & 7.114 & 3.853 \\
\hline 3/4/2006 & $11: 00$ & 11.881 & 11.732 & 7.658 & 5.047 & 3.345 & 3.267 & 11.01 & 6.189 & 5.124 & 7.113 & 3.846 \\
\hline 3/4/2006 & $12: 00$ & 11.876 & 11.723 & 7.649 & 5.045 & 3.337 & 3.262 & 11.01 & 6.184 & 5.122 & 7.112 & 3.841 \\
\hline $3 / 4 / 2006$ & $13: 00$ & 11.868 & 11.719 & 7.642 & 5.042 & 3.332 & 3.259 & 11.005 & 6.184 & 5.122 & 7.111 & 3.84 \\
\hline $3 / 4 / 2006$ & $14: 00$ & 11.863 & 11.708 & 7.638 & 5.035 & 3.324 & 3.248 & 11.006 & 6.18 & 5.114 & 7.106 & 3.836 \\
\hline $3 / 4 / 2006$ & $15: 00$ & 11.854 & 11.696 & 7.623 & 5.029 & 3.312 & 3.235 & 10.998 & 6.177 & 5.107 & 7.101 & 3.832 \\
\hline $3 / 4 / 2006$ & $16: 00$ & 11.844 & 11.685 & 7.616 & 5.025 & 3.307 & 3.231 & 10.995 & 6.172 & 5.112 & 7.102 & 3.834 \\
\hline $3 / 4 / 2006$ & $17: 00$ & 11.835 & 11.676 & 7.611 & 5.022 & 3.304 & 3.224 & 10.993 & 6.173 & 5.108 & 7.097 & 3.832 \\
\hline $3 / 4 / 2006$ & $18: 00$ & 11.834 & 11.67 & 7.603 & 5.015 & 3.298 & 3.22 & 10.996 & 6.17 & 5.107 & 7.09 & 3.834 \\
\hline $3 / 4 / 2006$ & 19:00 & 11.824 & 11.67 & 7.605 & 5.013 & 3.296 & 3.219 & 10.987 & 6.172 & 5.108 & 7.093 & 3.833 \\
\hline $3 / 4 / 2006$ & $20: 00$ & 11.823 & 11.674 & 7.611 & 5.013 & 3.303 & 3.223 & 10.988 & 6.175 & 5.113 & 7.093 & 3.841 \\
\hline $3 / 4 / 2006$ & $21: 00$ & 11.824 & 11.674 & 7.614 & 5.011 & 3.306 & 3.228 & 10.987 & 6.173 & 5.111 & 7.094 & 3.841 \\
\hline $3 / 4 / 2006$ & $22: 00$ & 11.819 & 11.665 & 7.602 & 5.004 & 3.298 & 3.219 & 10.987 & 6.168 & 5.108 & 7.09 & 3.84 \\
\hline $3 / 4 / 2006$ & $23: 00$ & 11.817 & 11.667 & 7.605 & 5.009 & 3.298 & 3.222 & 10.984 & 6.168 & 5.111 & 7.09 & 3.843 \\
\hline
\end{tabular}


TABLE S1.2 (Cont.)

Water Level (ft below top of casing) at Indicated Well

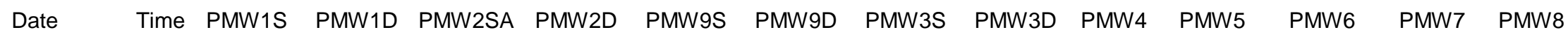

\begin{tabular}{|c|c|c|c|c|c|c|c|c|c|c|c|c|}
\hline $3 / 5 / 2006$ & $0: 00$ & 11.813 & 11.667 & 7.606 & 5.004 & 3.298 & 3.221 & 10.985 & 6.17 & 5.112 & 7.091 & 3.84 \\
\hline $3 / 5 / 2006$ & $1: 00$ & 11.81 & 11.657 & 7.598 & 5 & 3.292 & 3.212 & 10.982 & 6.162 & 5.108 & 7.085 & 3.838 \\
\hline $3 / 5 / 2006$ & $2: 00$ & 11.807 & 11.652 & 7.589 & 4.997 & 3.285 & 3.207 & 10.98 & 6.159 & 5.103 & 7.082 & 3.835 \\
\hline $3 / 5 / 2006$ & $3: 00$ & 11.801 & 11.648 & 7.589 & 4.995 & 3.284 & 3.207 & 10.982 & 6.159 & 5.106 & 7.082 & 3.837 \\
\hline $3 / 5 / 2006$ & $4: 00$ & 11.811 & 11.67 & 7.614 & 5.002 & 3.308 & 3.228 & 10.983 & 6.171 & 5.118 & 7.093 & 3.854 \\
\hline $3 / 5 / 2006$ & $5: 00$ & 11.808 & 11.661 & 7.607 & 4.995 & 3.292 & 3.212 & 10.978 & 6.164 & 5.106 & 7.089 & 3.829 \\
\hline $3 / 5 / 2006$ & $6: 00$ & 11.801 & 11.65 & 7.622 & 4.989 & 3.278 & 3.222 & 10.989 & 6.154 & 5.09 & 7.075 & 3.783 \\
\hline $3 / 5 / 2006$ & $7: 00$ & 11.803 & 11.656 & 7.598 & 4.989 & 3.278 & 3.203 & 10.975 & 6.16 & 5.084 & 7.075 & 3.764 \\
\hline $3 / 5 / 2006$ & $8: 00$ & 11.798 & 11.65 & 7.587 & 4.986 & 3.271 & 3.195 & 10.973 & 6.156 & 5.075 & 7.072 & 3.75 \\
\hline $3 / 5 / 2006$ & 9:00 & 11.813 & 11.672 & 7.614 & 4.991 & 3.295 & 3.216 & 10.977 & 6.163 & 5.085 & 7.085 & 3.762 \\
\hline $3 / 5 / 2006$ & $10: 00$ & 11.816 & 11.677 & 7.618 & 4.993 & 3.298 & 3.222 & 10.977 & 6.158 & 5.079 & 7.082 & 3.76 \\
\hline $3 / 5 / 2006$ & $11: 00$ & 11.818 & 11.677 & 7.616 & 4.993 & 3.297 & 3.219 & 10.976 & 6.158 & 5.075 & 7.083 & 3.758 \\
\hline $3 / 5 / 2006$ & $12: 00$ & 11.826 & 11.685 & 7.622 & 4.997 & 3.302 & 3.223 & 10.978 & 6.156 & 5.075 & 7.085 & 3.763 \\
\hline $3 / 5 / 2006$ & $13: 00$ & 11.826 & 11.688 & 7.62 & 4.995 & 3.306 & 3.226 & 10.978 & 6.159 & 5.076 & 7.084 & 3.764 \\
\hline $3 / 5 / 2006$ & $14: 00$ & 11.833 & 11.696 & 7.624 & 4.997 & 3.307 & 3.227 & 10.974 & 6.16 & 5.076 & 7.086 & 3.769 \\
\hline $3 / 5 / 2006$ & $15: 00$ & 11.836 & 11.698 & 7.631 & 5.002 & 3.313 & 3.234 & 10.975 & 6.166 & 5.077 & 7.089 & 3.779 \\
\hline $3 / 5 / 2006$ & $16: 00$ & 11.84 & 11.705 & 7.637 & 5.004 & 3.317 & 3.24 & 10.977 & 6.164 & 5.084 & 7.092 & 3.784 \\
\hline $3 / 5 / 2006$ & $17: 00$ & 11.849 & 11.714 & 7.642 & 5.007 & 3.325 & 3.245 & 10.981 & 6.17 & 5.087 & 7.095 & 3.793 \\
\hline $3 / 5 / 2006$ & $18: 00$ & 11.855 & 11.716 & 7.646 & 5.012 & 3.329 & 3.249 & 10.984 & 6.171 & 5.092 & 7.097 & 3.802 \\
\hline $3 / 5 / 2006$ & $19: 00$ & 11.858 & 11.723 & 7.651 & 5.015 & 3.329 & 3.254 & 10.987 & 6.172 & 5.098 & 7.102 & 3.805 \\
\hline $3 / 5 / 2006$ & $20: 00$ & 11.865 & 11.729 & 7.657 & 5.02 & 3.339 & 3.259 & 10.989 & 6.177 & 5.107 & 7.106 & 3.815 \\
\hline $3 / 5 / 2006$ & $21: 00$ & 11.872 & 11.736 & 7.662 & 5.021 & 3.343 & 3.265 & 10.992 & 6.173 & 5.108 & 7.109 & 3.82 \\
\hline $3 / 5 / 2006$ & $22: 00$ & 11.877 & 11.74 & 7.664 & 5.026 & 3.343 & 3.269 & 10.994 & 6.173 & 5.115 & 7.109 & 3.825 \\
\hline $3 / 5 / 2006$ & $23: 00$ & 11.88 & 11.741 & 7.666 & 5.028 & 3.347 & 3.269 & 10.995 & 6.175 & 5.119 & 7.112 & 3.828 \\
\hline $3 / 6 / 2006$ & $0: 00$ & 11.879 & 11.741 & 7.664 & 5.03 & 3.347 & 3.269 & 10.997 & 6.174 & 5.12 & 7.111 & 3.829 \\
\hline $3 / 6 / 2006$ & $1: 00$ & 11.879 & 11.74 & 7.662 & 5.032 & 3.345 & 3.268 & 10.996 & 6.176 & 5.122 & 7.111 & 3.833 \\
\hline $3 / 6 / 2006$ & $2: 00$ & 11.879 & 11.74 & 7.662 & 5.031 & 3.343 & 3.269 & 10.996 & 6.175 & 5.123 & 7.112 & 3.836 \\
\hline $3 / 6 / 2006$ & $3: 00$ & 11.881 & 11.741 & 7.664 & 5.035 & 3.346 & 3.269 & 10.998 & 6.179 & 5.126 & 7.115 & 3.841 \\
\hline $3 / 6 / 2006$ & $4: 00$ & 11.884 & 11.747 & 7.673 & 5.037 & 3.353 & 3.276 & 10.998 & 6.183 & 5.133 & 7.117 & 3.849 \\
\hline $3 / 6 / 2006$ & $5: 00$ & 11.889 & 11.754 & 7.677 & 5.04 & 3.358 & 3.282 & 11.002 & 6.183 & 5.135 & 7.114 & 3.853 \\
\hline $3 / 6 / 2006$ & $6: 00$ & 11.894 & 11.76 & 7.682 & 5.044 & 3.365 & 3.289 & 11.004 & 6.183 & 5.138 & 7.114 & 3.861 \\
\hline 3/6/2006 & 7:00 & 11.901 & 11.765 & 7.686 & 5.046 & 3.369 & 3.291 & 11.006 & 6.184 & 5.138 & 7.116 & 3.869 \\
\hline $3 / 6 / 2006$ & $8: 00$ & 11.906 & 11.771 & 7.693 & 5.05 & 3.375 & 3.297 & 11.007 & 6.186 & 5.143 & 7.119 & 3.876 \\
\hline 3/6/2006 & $9: 00$ & 11.909 & 11.778 & 7.697 & 5.055 & 3.38 & 3.302 & 11.01 & 6.19 & 5.147 & 7.12 & 3.884 \\
\hline
\end{tabular}


TABLE S1.2 (Cont.)

Water Level (ft below top of casing) at Indicated Well

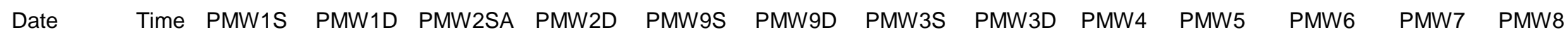

\begin{tabular}{|c|c|c|c|c|c|c|c|c|c|c|c|c|}
\hline $3 / 6 / 2006$ & $10: 00$ & 11.914 & 11.783 & 7.702 & 5.059 & 3.387 & 3.307 & 11.013 & 6.189 & 5.154 & 7.124 & 3.888 \\
\hline $3 / 6 / 2006$ & $11: 00$ & 11.921 & 11.789 & 7.706 & 5.062 & 3.385 & 3.307 & 11.014 & 6.19 & 5.152 & 7.128 & 3.884 \\
\hline $3 / 6 / 2006$ & $12: 00$ & 11.925 & 11.787 & 7.704 & 5.064 & 3.386 & 3.309 & 11.015 & 6.194 & 5.151 & 7.127 & 3.876 \\
\hline 3/6/2006 & $13: 00$ & 11.926 & 11.787 & 7.703 & 5.063 & 3.382 & 3.305 & 11.015 & 6.189 & 5.147 & 7.129 & 3.868 \\
\hline $3 / 6 / 2006$ & $14: 00$ & 11.918 & 11.776 & 7.69 & 5.061 & 3.376 & 3.295 & 11.017 & 6.188 & 5.141 & 7.122 & 3.863 \\
\hline 3/6/2006 & $15: 00$ & 11.911 & 11.761 & 7.679 & 5.062 & 3.366 & 3.287 & 11.013 & 6.184 & 5.134 & 7.12 & 3.858 \\
\hline $3 / 6 / 2006$ & $16: 00$ & 11.904 & 11.754 & 7.674 & 5.057 & 3.362 & 3.284 & 11.011 & 6.183 & 5.132 & 7.116 & 3.859 \\
\hline 3/6/2006 & $17: 00$ & 11.9 & 11.75 & 7.67 & 5.059 & 3.359 & 3.279 & 11.012 & 6.184 & 5.132 & 7.113 & 3.861 \\
\hline 3/6/2006 & $18: 00$ & 11.896 & 11.749 & 7.672 & 5.057 & 3.358 & 3.279 & 11.01 & 6.185 & 5.13 & 7.11 & 3.866 \\
\hline $3 / 6 / 2006$ & $19: 00$ & 11.895 & 11.75 & 7.674 & 5.057 & 3.36 & 3.282 & 11.012 & 6.185 & 5.134 & 7.113 & 3.872 \\
\hline $3 / 6 / 2006$ & $20: 00$ & 11.896 & 11.75 & 7.674 & 5.057 & 3.361 & 3.281 & 11.012 & 6.183 & 5.137 & 7.112 & 3.874 \\
\hline $3 / 6 / 2006$ & $21: 00$ & 11.898 & 11.756 & 7.679 & 5.059 & 3.365 & 3.285 & 11.01 & 6.188 & 5.142 & 7.116 & 3.879 \\
\hline $3 / 6 / 2006$ & $22: 00$ & 11.901 & 11.758 & 7.681 & 5.061 & 3.37 & 3.29 & 11.011 & 6.189 & 5.143 & 7.113 & 3.881 \\
\hline $3 / 6 / 2006$ & $23: 00$ & 11.898 & 11.75 & 7.677 & 5.059 & 3.362 & 3.286 & 11.011 & 6.184 & 5.142 & 7.113 & 3.878 \\
\hline 3/7/2006 & $0: 00$ & 11.889 & 11.745 & 7.67 & 5.056 & 3.357 & 3.279 & 11.01 & 6.18 & 5.138 & 7.109 & 3.874 \\
\hline 3/7/2006 & $1: 00$ & 11.888 & 11.741 & 7.668 & 5.055 & 3.356 & 3.275 & 11.009 & 6.184 & 5.137 & 7.108 & 3.874 \\
\hline $3 / 7 / 2006$ & $2: 00$ & 11.883 & 11.734 & 7.659 & 5.052 & 3.35 & 3.269 & 11.006 & 6.18 & 5.136 & 7.103 & 3.873 \\
\hline 3/7/2006 & $3: 00$ & 11.878 & 11.725 & 7.653 & 5.05 & 3.342 & 3.268 & 11.003 & 6.176 & 5.129 & 7.104 & 3.873 \\
\hline $3 / 7 / 2006$ & $4: 00$ & 11.871 & 11.716 & 7.646 & 5.048 & 3.337 & 3.256 & 10.998 & 6.172 & 5.13 & 7.1 & 3.869 \\
\hline $3 / 7 / 2006$ & $5: 00$ & 11.861 & 11.709 & 7.635 & 5.041 & 3.331 & 3.25 & 10.999 & 6.172 & 5.126 & 7.096 & 3.866 \\
\hline 3/7/2006 & $6: 00$ & 11.856 & 11.703 & 7.637 & 5.039 & 3.328 & 3.25 & 10.994 & 6.173 & 5.12 & 7.093 & 3.868 \\
\hline 3/7/2006 & $7: 00$ & 11.851 & 11.694 & 7.628 & 5.032 & 3.322 & 3.242 & 10.992 & 6.166 & 5.121 & 7.089 & 3.867 \\
\hline 3/7/2006 & $8: 00$ & 11.839 & 11.683 & 7.615 & 5.028 & 3.31 & 3.232 & 10.99 & 6.161 & 5.113 & 7.084 & 3.861 \\
\hline $3 / 7 / 2006$ & $9: 00$ & 11.827 & 11.665 & 7.599 & 5.019 & 3.296 & 3.222 & 10.986 & 6.154 & 5.102 & 7.073 & 3.854 \\
\hline 3/7/2006 & $10: 00$ & 11.819 & 11.656 & 7.591 & 5.013 & 3.288 & 3.211 & 10.979 & 6.152 & 5.103 & 7.069 & 3.852 \\
\hline $3 / 7 / 2006$ & $11: 00$ & 11.808 & 11.641 & 7.579 & 5.004 & 3.278 & 3.199 & 10.976 & 6.15 & 5.098 & 7.059 & 3.846 \\
\hline 3/7/2006 & $12: 00$ & 11.802 & 11.637 & 7.581 & 4.999 & 3.277 & 3.197 & 10.971 & 6.155 & 5.094 & 7.065 & 3.848 \\
\hline $3 / 7 / 2006$ & $13: 00$ & 11.792 & 11.63 & 7.57 & 4.994 & 3.272 & 3.187 & 10.964 & 6.148 & 5.093 & 7.064 & 3.845 \\
\hline 3/7/2006 & $14: 00$ & 11.781 & 11.615 & 7.559 & 4.986 & 3.26 & 3.18 & 10.962 & 6.145 & 5.092 & 7.06 & 3.839 \\
\hline 3/7/2006 & $15: 00$ & 11.771 & 11.608 & 7.555 & 4.979 & 3.254 & 3.171 & 10.959 & 6.146 & 5.092 & 7.057 & 3.841 \\
\hline 3/7/2006 & $16: 00$ & 11.759 & 11.59 & 7.539 & 4.968 & 3.242 & 3.159 & 10.952 & 6.14 & 5.081 & 7.051 & 3.833 \\
\hline 3/7/2006 & $17: 00$ & 11.752 & 11.59 & 7.542 & 4.966 & 3.245 & 3.161 & 10.954 & 6.146 & 5.088 & 7.051 & 3.844 \\
\hline $3 / 7 / 2006$ & $18: 00$ & 11.746 & 11.585 & 7.539 & 4.961 & 3.24 & 3.16 & 10.953 & 6.14 & 5.085 & 7.047 & 3.844 \\
\hline 3/7/2006 & $19: 00$ & 11.742 & 11.586 & 7.544 & 4.959 & 3.246 & 3.163 & 10.953 & 6.146 & 5.089 & 7.046 & 3.851 \\
\hline
\end{tabular}


TABLE S1.2 (Cont.)

Water Level (ft below top of casing) at Indicated Well

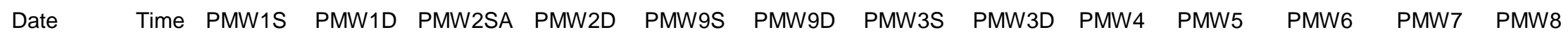

\begin{tabular}{|c|c|c|c|c|c|c|c|c|c|c|c|c|}
\hline 3/7/2006 & $20: 00$ & 11.741 & 11.59 & 7.55 & 4.959 & 3.249 & 3.169 & 10.952 & 6.145 & 5.095 & 7.047 & 3.858 \\
\hline $3 / 7 / 2006$ & $21: 00$ & 11.744 & 11.597 & 7.554 & 4.961 & 3.256 & 3.175 & 10.954 & 6.146 & 5.098 & 7.05 & 3.86 \\
\hline $3 / 7 / 2006$ & $22: 00$ & 11.746 & 11.597 & 7.555 & 4.961 & 3.254 & 3.172 & 10.95 & 6.145 & 5.101 & 7.049 & 3.858 \\
\hline 3/7/2006 & $23: 00$ & 11.746 & 11.599 & 7.554 & 4.959 & 3.254 & 3.174 & 10.952 & 6.14 & 5.104 & 7.048 & 3.86 \\
\hline 3/8/2006 & 0:00 & 11.744 & 11.595 & 7.553 & 4.957 & 3.249 & 3.169 & 10.95 & 6.141 & 5.101 & 7.047 & 3.855 \\
\hline 3/8/2006 & $1: 00$ & 11.739 & 11.59 & 7.544 & 4.955 & 3.245 & 3.163 & 10.948 & 6.14 & 5.099 & 7.044 & 3.851 \\
\hline $3 / 8 / 2006$ & $2: 00$ & 11.738 & 11.588 & 7.546 & 4.953 & 3.246 & 3.165 & 10.947 & 6.138 & 5.097 & 7.044 & 3.853 \\
\hline 3/8/2006 & $3: 00$ & 11.739 & 11.592 & 7.548 & 4.952 & 3.251 & 3.17 & 10.946 & 6.135 & 5.098 & 7.044 & 3.855 \\
\hline 3/8/2006 & $4: 00$ & 11.736 & 11.59 & 7.546 & 4.952 & 3.248 & 3.166 & 10.945 & 6.135 & 5.093 & 7.042 & 3.853 \\
\hline $3 / 8 / 2006$ & $5: 00$ & 11.729 & 11.583 & 7.539 & 4.948 & 3.24 & 3.157 & 10.942 & 6.135 & 5.088 & 7.041 & 3.847 \\
\hline $3 / 8 / 2006$ & $6: 00$ & 11.725 & 11.579 & 7.532 & 4.946 & 3.234 & 3.154 & 10.94 & 6.134 & 5.087 & 7.038 & 3.849 \\
\hline $3 / 8 / 2006$ & $7: 00$ & 11.719 & 11.57 & 7.523 & 4.942 & 3.225 & 3.145 & 10.938 & 6.129 & 5.078 & 7.036 & 3.843 \\
\hline $3 / 8 / 2006$ & $8: 00$ & 11.725 & 11.581 & 7.537 & 4.941 & 3.235 & 3.16 & 10.938 & 6.137 & 5.087 & 7.039 & 3.852 \\
\hline $3 / 8 / 2006$ & 9:00 & 11.715 & 11.594 & 7.55 & 4.939 & 3.228 & 3.184 & 10.952 & 6.128 & 5.076 & 7.033 & 3.845 \\
\hline $3 / 8 / 2006$ & $10: 00$ & 11.713 & 11.57 & 7.568 & 4.939 & 3.229 & 3.16 & 10.976 & 6.13 & 5.077 & 7.043 & 3.84 \\
\hline $3 / 8 / 2006$ & $11: 00$ & 11.709 & 11.605 & 7.599 & 4.934 & 3.224 & 3.145 & 11.005 & 6.12 & 5.068 & 7.086 & 3.825 \\
\hline $3 / 8 / 2006$ & $12: 00$ & 11.701 & 11.574 & 7.535 & 4.925 & 3.209 & 3.148 & 11.036 & 6.116 & 5.055 & 7.109 & 3.8 \\
\hline $3 / 8 / 2006$ & $13: 00$ & 11.686 & 11.546 & 7.5 & 4.921 & 3.198 & 3.117 & 10.928 & 6.119 & 5.044 & 7.027 & 3.782 \\
\hline $3 / 8 / 2006$ & $14: 00$ & 11.68 & 11.541 & 7.5 & 4.914 & 3.196 & 3.115 & 10.925 & 6.112 & 5.038 & 7.018 & 3.777 \\
\hline $3 / 8 / 2006$ & $15: 00$ & 11.679 & 11.536 & 7.495 & 4.91 & 3.191 & 3.109 & 10.921 & 6.115 & 5.03 & 7.01 & 3.771 \\
\hline $3 / 8 / 2006$ & $16: 00$ & 11.669 & 11.532 & 7.493 & 4.906 & 3.19 & 3.105 & 10.914 & 6.114 & 5.025 & 7.013 & 3.771 \\
\hline $3 / 8 / 2006$ & $17: 00$ & 11.659 & 11.53 & 7.497 & 4.906 & 3.186 & 3.106 & 10.915 & 6.111 & 5.017 & 7.007 & 3.771 \\
\hline $3 / 8 / 2006$ & $18: 00$ & 11.662 & 11.508 & 7.477 & 4.901 & 3.193 & 3.114 & 10.914 & 6.109 & 5.011 & 6.993 & 3.777 \\
\hline $3 / 8 / 2006$ & $19: 00$ & 11.662 & 11.51 & 7.484 & 4.902 & 3.193 & 3.111 & 10.912 & 6.107 & 5.011 & 7.002 & 3.773 \\
\hline $3 / 8 / 2006$ & $20: 00$ & 11.67 & 11.51 & 7.486 & 4.904 & 3.201 & 3.116 & 10.91 & 6.113 & 5.012 & 7.007 & 3.779 \\
\hline $3 / 8 / 2006$ & $21: 00$ & 11.688 & 11.51 & 7.495 & 4.907 & 3.208 & 3.13 & 10.913 & 6.111 & 5.01 & 7.016 & 3.783 \\
\hline $3 / 8 / 2006$ & $22: 00$ & 11.693 & 11.514 & 7.494 & 4.91 & 3.211 & 3.13 & 10.904 & 6.112 & 5.005 & 7.016 & 3.772 \\
\hline $3 / 8 / 2006$ & $23: 00$ & 11.705 & 11.568 & 7.494 & 4.908 & 3.214 & 3.134 & 10.906 & 6.111 & 5.002 & 7.004 & 3.761 \\
\hline $3 / 9 / 2006$ & $0: 00$ & 11.708 & 11.575 & 7.532 & 4.913 & 3.223 & 3.141 & 10.912 & 6.11 & 5 & 7.001 & 3.759 \\
\hline $3 / 9 / 2006$ & $1: 00$ & 11.711 & 11.577 & 7.528 & 4.915 & 3.218 & 3.14 & 10.912 & 6.106 & 4.995 & 7.001 & 3.754 \\
\hline 3/9/2006 & $2: 00$ & 11.711 & 11.579 & 7.53 & 4.915 & 3.218 & 3.125 & 10.911 & 6.104 & 4.993 & 7.008 & 3.754 \\
\hline $3 / 9 / 2006$ & $3: 00$ & 11.703 & 11.57 & 7.521 & 4.912 & 3.212 & 3.134 & 10.908 & 6.101 & 4.988 & 7.006 & 3.749 \\
\hline $3 / 9 / 2006$ & $4: 00$ & 11.671 & 11.543 & 7.495 & 4.903 & 3.186 & 3.098 & 10.905 & 6.098 & 4.978 & 7.001 & 3.737 \\
\hline $3 / 9 / 2006$ & $5: 00$ & 11.684 & 11.559 & 7.515 & 4.907 & 3.206 & 3.109 & 10.907 & 6.106 & 4.986 & 7.007 & 3.755 \\
\hline
\end{tabular}


TABLE S1.2 (Cont.)

Water Level (ft below top of casing) at Indicated Well

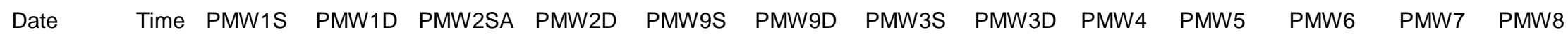

\begin{tabular}{|c|c|c|c|c|c|c|c|c|c|c|c|c|}
\hline 3/9/2006 & $6: 00$ & 11.685 & 11.563 & 7.517 & 4.908 & 3.205 & 3.125 & 10.905 & 6.1 & 4.984 & 7.004 & 3.759 \\
\hline 3/9/2006 & $7: 00$ & 11.688 & 11.564 & 7.519 & 4.91 & 3.209 & 3.15 & 10.906 & 6.101 & 4.982 & 7.004 & 3.761 \\
\hline $3 / 9 / 2006$ & $8: 00$ & 11.699 & 11.57 & 7.523 & 4.91 & 3.213 & 3.158 & 10.907 & 6.104 & 4.985 & 7.004 & 3.768 \\
\hline 3/9/2006 & 9:00 & 11.69 & 11.567 & 7.521 & 4.91 & 3.211 & 3.138 & 10.906 & 6.1 & 4.982 & 7.002 & 3.767 \\
\hline 3/9/2006 & $10: 00$ & 11.688 & 11.57 & 7.521 & 4.91 & 3.211 & 3.13 & 10.906 & 6.101 & 4.985 & 7.003 & 3.771 \\
\hline 3/9/2006 & $11: 00$ & 11.698 & 11.572 & 7.521 & 4.91 & 3.212 & 3.132 & 10.907 & 6.097 & 4.985 & 7.003 & 3.773 \\
\hline $3 / 9 / 2006$ & $12: 00$ & 11.693 & 11.568 & 7.517 & 4.908 & 3.209 & 3.13 & 10.904 & 6.102 & 4.984 & 7.006 & 3.77 \\
\hline 3/9/2006 & $13: 00$ & 11.688 & 11.567 & 7.519 & 4.907 & 3.209 & 3.127 & 10.905 & 6.101 & 4.983 & 7.003 & 3.771 \\
\hline 3/9/2006 & $14: 00$ & 11.671 & 11.553 & 7.503 & 4.904 & 3.2 & 3.116 & 10.903 & 6.096 & 4.981 & 6.997 & 3.764 \\
\hline $3 / 9 / 2006$ & $15: 00$ & 11.656 & 11.544 & 7.497 & 4.901 & 3.191 & 3.109 & 10.901 & 6.097 & 4.977 & 6.999 & 3.765 \\
\hline $3 / 9 / 2006$ & $16: 00$ & 11.651 & 11.537 & 7.495 & 4.899 & 3.187 & 3.106 & 10.899 & 6.112 & 4.978 & 6.995 & 3.763 \\
\hline $3 / 9 / 2006$ & $17: 00$ & 11.651 & 11.537 & 7.495 & 4.895 & 3.19 & 3.11 & 10.897 & 6.097 & 4.983 & 6.997 & 3.771 \\
\hline $3 / 9 / 2006$ & $18: 00$ & 11.655 & 11.545 & 7.501 & 4.897 & 3.195 & 3.114 & 10.898 & 6.098 & 4.986 & 6.996 & 3.781 \\
\hline $3 / 9 / 2006$ & $19: 00$ & 11.676 & 11.557 & 7.514 & 4.9 & 3.204 & 3.125 & 10.9 & 6.1 & 4.997 & 7 & 3.791 \\
\hline $3 / 9 / 2006$ & $20: 00$ & 11.69 & 11.57 & 7.525 & 4.903 & 3.218 & 3.135 & 10.901 & 6.105 & 5.001 & 7.003 & 3.801 \\
\hline 3/9/2006 & $21: 00$ & 11.705 & 11.584 & 7.536 & 4.91 & 3.225 & 3.145 & 10.904 & 6.107 & 5.013 & 7.006 & 3.809 \\
\hline $3 / 9 / 2006$ & $22: 00$ & 11.717 & 11.594 & 7.543 & 4.913 & 3.233 & 3.152 & 10.905 & 6.105 & 5.015 & 7.009 & 3.811 \\
\hline $3 / 9 / 2006$ & $23: 00$ & 11.722 & 11.596 & 7.543 & 4.917 & 3.235 & 3.153 & 10.905 & 6.105 & 5.017 & 7.012 & 3.815 \\
\hline $3 / 10 / 2006$ & 0:00 & 11.731 & 11.605 & 7.55 & 4.921 & 3.24 & 3.162 & 10.907 & 6.108 & 5.021 & 7.013 & 3.817 \\
\hline $3 / 10 / 2006$ & $1: 00$ & 11.737 & 11.61 & 7.554 & 4.923 & 3.243 & 3.163 & 10.908 & 6.108 & 5.023 & 7.014 & 3.82 \\
\hline $3 / 10 / 2006$ & $2: 00$ & 11.742 & 11.615 & 7.557 & 4.928 & 3.246 & 3.168 & 10.91 & 6.107 & 5.028 & 7.017 & 3.822 \\
\hline $3 / 10 / 2006$ & $3: 00$ & 11.747 & 11.623 & 7.564 & 4.93 & 3.25 & 3.171 & 10.913 & 6.106 & 5.032 & 7.02 & 3.824 \\
\hline 3/10/2006 & $4: 00$ & 11.753 & 11.625 & 7.563 & 4.932 & 3.252 & 3.173 & 10.914 & 6.109 & 5.036 & 7.021 & 3.826 \\
\hline $3 / 10 / 2006$ & $5: 00$ & 11.756 & 11.629 & 7.567 & 4.937 & 3.254 & 3.177 & 10.914 & 6.108 & 5.037 & 7.022 & 3.828 \\
\hline 3/10/2006 & $6: 00$ & 11.766 & 11.638 & 7.577 & 4.94 & 3.264 & 3.185 & 10.917 & 6.114 & 5.044 & 7.026 & 3.833 \\
\hline $3 / 10 / 2006$ & $7: 00$ & 11.776 & 11.647 & 7.583 & 4.945 & 3.269 & 3.19 & 10.919 & 6.117 & 5.046 & 7.028 & 3.835 \\
\hline 3/10/2006 & $8: 00$ & 11.783 & 11.654 & 7.588 & 4.95 & 3.275 & 3.197 & 10.92 & 6.117 & 5.051 & 7.033 & 3.84 \\
\hline $3 / 10 / 2006$ & $9: 00$ & 11.788 & 11.663 & 7.594 & 4.956 & 3.281 & 3.204 & 10.924 & 6.121 & 5.057 & 7.038 & 3.843 \\
\hline 3/10/2006 & $10: 00$ & 11.799 & 11.672 & 7.605 & 4.959 & 3.291 & 3.211 & 10.926 & 6.125 & 5.062 & 7.042 & 3.845 \\
\hline 3/10/2006 & $11: 00$ & 11.806 & 11.68 & 7.609 & 4.966 & 3.296 & 3.216 & 10.931 & 6.123 & 5.066 & 7.046 & 3.848 \\
\hline $3 / 10 / 2006$ & $12: 00$ & 11.81 & 11.681 & 7.608 & 4.965 & 3.292 & 3.215 & 10.93 & 6.124 & 5.064 & 7.045 & 3.843 \\
\hline $3 / 10 / 2006$ & $13: 00$ & 11.807 & 11.677 & 7.605 & 4.97 & 3.29 & 3.213 & 10.931 & 6.124 & 5.06 & 7.045 & 3.841 \\
\hline $3 / 10 / 2006$ & $14: 00$ & 11.808 & 11.668 & 7.594 & 4.969 & 3.282 & 3.205 & 10.928 & 6.115 & 5.06 & 7.043 & 3.836 \\
\hline $3 / 10 / 2006$ & $15: 00$ & 11.796 & 11.656 & 7.583 & 4.965 & 3.272 & 3.194 & 10.928 & 6.116 & 5.056 & 7.039 & 3.833 \\
\hline
\end{tabular}


TABLE S1.2 (Cont.)

Water Level (ft below top of casing) at Indicated Well

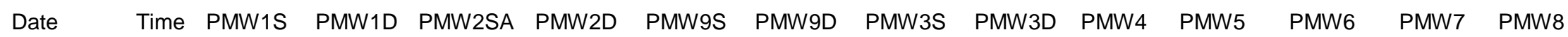

\begin{tabular}{|c|c|c|c|c|c|c|c|c|c|c|c|c|}
\hline 3/10/2006 & $16: 00$ & 11.778 & 11.646 & 7.579 & 4.965 & 3.265 & 3.188 & 10.924 & 6.117 & 5.054 & 7.034 & 3.832 \\
\hline 3/10/2006 & 17:00 & 11.776 & 11.641 & 7.574 & 4.965 & 3.262 & 3.186 & 10.925 & 6.117 & 5.06 & 7.035 & 3.839 \\
\hline 3/10/2006 & $18: 00$ & 11.781 & 11.645 & 7.579 & 4.965 & 3.271 & 3.187 & 10.921 & 6.117 & 5.067 & 7.038 & 3.843 \\
\hline 3/10/2006 & 19:00 & 11.78 & 11.645 & 7.579 & 4.965 & 3.269 & 3.191 & 10.926 & 6.122 & 5.071 & 7.038 & 3.847 \\
\hline $3 / 10 / 2006$ & 20:00 & 11.788 & 11.652 & 7.588 & 4.967 & 3.276 & 3.197 & 10.931 & 6.124 & 5.077 & 7.043 & 3.855 \\
\hline 3/10/2006 & $21: 00$ & 11.8 & 11.663 & 7.599 & 4.972 & 3.284 & 3.206 & 10.93 & 6.123 & 5.083 & 7.046 & 3.859 \\
\hline $3 / 10 / 2006$ & $22: 00$ & 11.8 & 11.665 & 7.598 & 4.971 & 3.285 & 3.207 & 10.933 & 6.123 & 5.084 & 7.046 & 3.855 \\
\hline 3/10/2006 & 23:00 & 11.797 & 11.661 & 7.597 & 4.972 & 3.279 & 3.202 & 10.934 & 6.121 & 5.082 & 7.043 & 3.852 \\
\hline $3 / 11 / 2006$ & $0: 00$ & 11.79 & 11.654 & 7.588 & 4.972 & 3.275 & 3.198 & 10.933 & 6.127 & 5.081 & 7.042 & 3.847 \\
\hline $3 / 11 / 2006$ & 1:00 & 11.78 & 11.645 & 7.581 & 4.969 & 3.269 & 3.19 & 10.93 & 6.125 & 5.078 & 7.041 & 3.843 \\
\hline $3 / 11 / 2006$ & $2: 00$ & 11.773 & 11.638 & 7.576 & 4.969 & 3.263 & 3.184 & 10.925 & 6.122 & 5.077 & 7.037 & 3.839 \\
\hline 3/11/2006 & 3:00 & 11.768 & 11.634 & 7.572 & 4.967 & 3.262 & 3.184 & 10.927 & 6.124 & 5.074 & 7.036 & 3.84 \\
\hline $3 / 11 / 2006$ & $4: 00$ & 11.773 & 11.639 & 7.581 & 4.967 & 3.273 & 3.195 & 10.928 & 6.121 & 5.075 & 7.038 & 3.845 \\
\hline $3 / 11 / 2006$ & 5:00 & 11.78 & 11.645 & 7.585 & 4.967 & 3.273 & 3.193 & 10.928 & 6.13 & 5.079 & 7.041 & 3.845 \\
\hline 3/11/2006 & 6:00 & 11.79 & 11.658 & 7.592 & 4.972 & 3.278 & 3.199 & 10.93 & 6.132 & 5.083 & 7.045 & 3.849 \\
\hline $3 / 11 / 2006$ & $7: 00$ & 11.796 & 11.665 & 7.598 & 4.973 & 3.287 & 3.208 & 10.933 & 6.131 & 5.085 & 7.045 & 3.852 \\
\hline 3/11/2006 & 8:00 & 11.815 & 11.683 & 7.616 & 4.979 & 3.301 & 3.222 & 10.936 & 6.14 & 5.092 & 7.053 & 3.861 \\
\hline 3/11/2006 & $9: 00$ & 11.827 & 11.703 & 7.634 & 4.987 & 3.318 & 3.239 & 10.941 & 6.149 & 5.101 & 7.059 & 3.87 \\
\hline 3/11/2006 & $10: 00$ & 11.841 & 11.722 & 7.654 & 4.996 & 3.334 & 3.256 & 10.946 & 6.155 & 5.111 & 7.068 & 3.879 \\
\hline 3/11/2006 & $11: 00$ & 11.854 & 11.736 & 7.663 & 5.004 & 3.347 & 3.268 & 10.95 & 6.155 & 5.117 & 7.071 & 3.882 \\
\hline 3/11/2006 & $12: 00$ & 11.866 & 11.747 & 7.671 & 5.012 & 3.351 & 3.275 & 10.949 & 6.156 & 5.119 & 7.077 & 3.881 \\
\hline $3 / 11 / 2006$ & $13: 00$ & 11.876 & 11.756 & 7.676 & 5.018 & 3.356 & 3.278 & 10.956 & 6.157 & 5.121 & 7.08 & 3.88 \\
\hline 3/11/2006 & $14: 00$ & 11.881 & 11.762 & 7.68 & 5.022 & 3.358 & 3.28 & 10.962 & 6.156 & 5.124 & 7.085 & 3.878 \\
\hline 3/11/2006 & $15: 00$ & 11.888 & 11.76 & 7.678 & 5.027 & 3.358 & 3.278 & 10.964 & 6.157 & 5.125 & 7.087 & 3.879 \\
\hline $3 / 11 / 2006$ & $16: 00$ & 11.891 & 11.767 & 7.68 & 5.033 & 3.362 & 3.285 & 10.966 & 6.163 & 5.13 & 7.088 & 3.882 \\
\hline $3 / 11 / 2006$ & $17: 00$ & 11.898 & 11.773 & 7.683 & 5.036 & 3.365 & 3.285 & 10.97 & 6.163 & 5.138 & 7.093 & 3.886 \\
\hline 3/11/2006 & $18: 00$ & 11.903 & 11.774 & 7.689 & 5.042 & 3.368 & 3.288 & 10.97 & 6.165 & 5.137 & 7.094 & 3.888 \\
\hline $3 / 11 / 2006$ & 19:00 & 11.907 & 11.782 & 7.694 & 5.046 & 3.372 & 3.294 & 10.975 & 6.167 & 5.144 & 7.099 & 3.891 \\
\hline $3 / 11 / 2006$ & 20:00 & 11.916 & 11.793 & 7.707 & 5.053 & 3.383 & 3.305 & 10.979 & 6.173 & 5.151 & 7.106 & 3.897 \\
\hline $3 / 11 / 2006$ & 21:00 & 11.923 & 11.804 & 7.716 & 5.06 & 3.392 & 3.314 & 10.982 & 6.173 & 5.153 & 7.109 & 3.902 \\
\hline $3 / 11 / 2006$ & $22: 00$ & 11.927 & 11.803 & 7.716 & 5.064 & 3.393 & 3.314 & 10.985 & 6.173 & 5.155 & 7.111 & 3.899 \\
\hline 3/11/2006 & 23:00 & 11.933 & 11.812 & 7.72 & 5.071 & 3.399 & 3.32 & 10.987 & 6.172 & 5.159 & 7.115 & 3.902 \\
\hline 3/12/2006 & 0:00 & 11.938 & 11.815 & 7.72 & 5.073 & 3.399 & 3.319 & 10.988 & 6.176 & 5.161 & 7.116 & 3.9 \\
\hline 3/12/2006 & 1:00 & 11.943 & 11.813 & 7.718 & 5.076 & 3.397 & 3.318 & 10.992 & 6.174 & 5.158 & 7.115 & 3.897 \\
\hline
\end{tabular}


TABLE S1.2 (Cont.)

Water Level (ft below top of casing) at Indicated Well

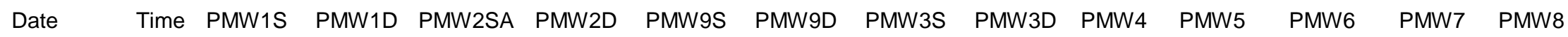

\begin{tabular}{|c|c|c|c|c|c|c|c|c|c|c|c|c|}
\hline 3/12/2006 & $2: 00$ & 11.935 & 11.802 & 7.707 & 5.073 & 3.385 & 3.306 & 10.991 & 6.172 & 5.153 & 7.11 & 3.89 \\
\hline $3 / 12 / 2006$ & $3: 00$ & 11.937 & 11.804 & 7.711 & 5.076 & 3.39 & 3.315 & 10.992 & 6.167 & 5.151 & 7.112 & 3.889 \\
\hline $3 / 12 / 2006$ & $4: 00$ & 11.93 & 11.793 & 7.7 & 5.073 & 3.381 & 3.302 & 10.99 & 6.167 & 5.148 & 7.111 & 3.884 \\
\hline 3/12/2006 & $5: 00$ & 11.928 & 11.785 & 7.694 & 5.072 & 3.374 & 3.295 & 10.991 & 6.166 & 5.145 & 7.108 & 3.88 \\
\hline $3 / 12 / 2006$ & $6: 00$ & 11.927 & 11.787 & 7.698 & 5.073 & 3.383 & 3.301 & 10.992 & 6.169 & 5.146 & 7.108 & 3.88 \\
\hline 3/12/2006 & $7: 00$ & 11.925 & 11.784 & 7.695 & 5.073 & 3.383 & 3.298 & 10.991 & 6.17 & 5.142 & 7.107 & 3.88 \\
\hline $3 / 12 / 2006$ & $8: 00$ & 11.913 & 11.761 & 7.675 & 5.066 & 3.361 & 3.281 & 10.988 & 6.159 & 5.132 & 7.097 & 3.867 \\
\hline 3/12/2006 & $9: 00$ & 11.89 & 11.734 & 7.649 & 5.058 & 3.339 & 3.258 & 10.983 & 6.149 & 5.119 & 7.088 & 3.856 \\
\hline $3 / 12 / 2006$ & $10: 00$ & 11.883 & 11.729 & 7.669 & 5.056 & 3.339 & 3.254 & 10.98 & 6.153 & 5.12 & 7.087 & 3.859 \\
\hline 3/12/2006 & $11: 00$ & 11.883 & 11.714 & 7.605 & 5.053 & 3.332 & 3.249 & 10.973 & 6.18 & 5.135 & 7.095 & 3.86 \\
\hline $3 / 12 / 2006$ & $12: 00$ & 11.861 & 11.718 & 7.642 & 5.04 & 3.324 & 3.239 & 10.992 & 6.145 & 5.104 & 7.078 & 3.834 \\
\hline 3/12/2006 & $13: 00$ & 11.843 & 11.689 & 7.616 & 5.031 & 3.31 & 3.226 & 10.97 & 6.141 & 5.092 & 7.071 & 3.819 \\
\hline $3 / 12 / 2006$ & $14: 00$ & 11.827 & 11.67 & 7.6 & 5.024 & 3.287 & 3.21 & 10.962 & 6.133 & 5.081 & 7.063 & 3.802 \\
\hline 3/12/2006 & $15: 00$ & 11.812 & 11.658 & 7.587 & 5.013 & 3.281 & 3.197 & 10.957 & 6.132 & 5.072 & 7.054 & 3.792 \\
\hline 3/12/2006 & $16: 00$ & 11.801 & 11.646 & 7.58 & 5.007 & 3.268 & 3.191 & 10.953 & 6.133 & 5.069 & 7.051 & 3.794 \\
\hline 3/12/2006 & $17: 00$ & 11.794 & 11.643 & 7.58 & 5.002 & 3.278 & 3.189 & 10.951 & 6.133 & 5.064 & 7.049 & 3.797 \\
\hline $3 / 12 / 2006$ & $18: 00$ & 11.784 & 11.634 & 7.573 & 4.996 & 3.269 & 3.183 & 10.945 & 6.131 & 5.06 & 7.047 & 3.796 \\
\hline $3 / 12 / 2006$ & $19: 00$ & 11.777 & 11.632 & 7.573 & 4.991 & 3.272 & 3.182 & 10.945 & 6.131 & 5.059 & 7.047 & 3.8 \\
\hline 3/12/2006 & $20: 00$ & 11.784 & 11.639 & 7.585 & 4.989 & 3.279 & 3.191 & 10.944 & 6.137 & 5.062 & 7.046 & 3.811 \\
\hline $3 / 12 / 2006$ & $21: 00$ & 11.776 & 11.634 & 7.576 & 4.987 & 3.271 & 3.183 & 10.941 & 6.13 & 5.057 & 7.041 & 3.808 \\
\hline 3/12/2006 & $22: 00$ & 11.777 & 11.638 & 7.582 & 4.984 & 3.277 & 3.188 & 10.94 & 6.132 & 5.059 & 7.037 & 3.811 \\
\hline $3 / 12 / 2006$ & $23: 00$ & 11.776 & 11.639 & 7.58 & 4.982 & 3.266 & 3.185 & 10.94 & 6.134 & 5.058 & 7.037 & 3.813 \\
\hline 3/13/2006 & $0: 00$ & 11.764 & 11.623 & 7.567 & 4.977 & 3.258 & 3.18 & 10.939 & 6.123 & 5.048 & 7.036 & 3.808 \\
\hline $3 / 13 / 2006$ & $1: 00$ & 11.759 & 11.625 & 7.571 & 4.976 & 3.268 & 3.18 & 10.938 & 6.127 & 5.047 & 7.036 & 3.812 \\
\hline $3 / 13 / 2006$ & $2: 00$ & 11.771 & 11.623 & 7.557 & 4.978 & 3.275 & 3.191 & 10.935 & 6.13 & 5.051 & 7.021 & 3.816 \\
\hline 3/13/2006 & $3: 00$ & 11.781 & 11.597 & 7.588 & 4.975 & 3.278 & 3.184 & 10.902 & 6.135 & 5.055 & 6.988 & 3.817 \\
\hline 3/13/2006 & $4: 00$ & 11.784 & 11.634 & 7.566 & 4.98 & 3.285 & 3.196 & 10.879 & 6.129 & 5.05 & 6.966 & 3.807 \\
\hline $3 / 13 / 2006$ & $5: 00$ & 11.794 & 11.661 & 7.602 & 4.98 & 3.291 & 3.202 & 10.931 & 6.136 & 5.045 & 7.043 & 3.802 \\
\hline $3 / 13 / 2006$ & $6: 00$ & 11.808 & 11.663 & 7.617 & 4.984 & 3.304 & 3.218 & 10.921 & 6.139 & 5.051 & 7.038 & 3.8 \\
\hline 3/13/2006 & $7: 00$ & 11.831 & 11.705 & 7.582 & 4.982 & 3.331 & 3.241 & 10.941 & 6.144 & 5.053 & 7.04 & 3.805 \\
\hline $3 / 13 / 2006$ & $8: 00$ & 11.85 & 11.734 & 7.555 & 5.004 & 3.346 & 3.258 & 10.946 & 6.152 & 5.062 & 7.047 & 3.805 \\
\hline 3/13/2006 & $9: 00$ & 11.868 & 11.751 & 7.551 & 5.012 & 3.36 & 3.272 & 10.954 & 6.155 & 5.062 & 7.072 & 3.811 \\
\hline $3 / 13 / 2006$ & $10: 00$ & 11.884 & 11.771 & 7.694 & 5.022 & 3.373 & 3.286 & 10.958 & 6.161 & 5.073 & 7.074 & 3.816 \\
\hline $3 / 13 / 2006$ & $11: 00$ & 11.902 & 11.789 & 7.708 & 5.031 & 3.378 & 3.299 & 10.968 & 6.165 & 5.078 & 7.082 & 3.823 \\
\hline
\end{tabular}


TABLE S1.2 (Cont.)

Water Level (ft below top of casing) at Indicated Well

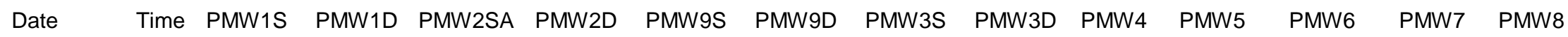

\begin{tabular}{|c|c|c|c|c|c|c|c|c|c|c|c|c|}
\hline $3 / 13 / 2006$ & $12: 00$ & 11.914 & 11.8 & 7.717 & 5.039 & 3.384 & 3.305 & 10.969 & 6.165 & 5.085 & 7.093 & 3.826 \\
\hline $3 / 13 / 2006$ & $13: 00$ & 11.927 & 11.813 & 7.724 & 5.049 & 3.399 & 3.314 & 10.98 & 6.17 & 5.089 & 7.1 & 3.829 \\
\hline $3 / 13 / 2006$ & $14: 00$ & 11.941 & 11.82 & 7.73 & 5.057 & 3.406 & 3.321 & 10.981 & 6.171 & 5.094 & 7.102 & 3.835 \\
\hline 3/13/2006 & $15: 00$ & 11.946 & 11.826 & 7.733 & 5.064 & 3.405 & 3.322 & 10.981 & 6.17 & 5.094 & 7.106 & 3.837 \\
\hline 3/13/2006 & $16: 00$ & 11.954 & 11.831 & 7.742 & 5.07 & 3.407 & 3.325 & 10.99 & 6.174 & 5.103 & 7.11 & 3.84 \\
\hline 3/13/2006 & $17: 00$ & 11.958 & 11.835 & 7.742 & 5.077 & 3.411 & 3.329 & 10.992 & 6.178 & 5.108 & 7.116 & 3.848 \\
\hline $3 / 13 / 2006$ & $18: 00$ & 11.966 & 11.844 & 7.746 & 5.081 & 3.423 & 3.336 & 10.996 & 6.183 & 5.115 & 7.123 & 3.854 \\
\hline 3/13/2006 & $19: 00$ & 11.971 & 11.847 & 7.748 & 5.086 & 3.427 & 3.342 & 11 & 6.185 & 5.12 & 7.122 & 3.861 \\
\hline 3/13/2006 & $20: 00$ & 11.976 & 11.857 & 7.757 & 5.093 & 3.43 & 3.348 & 11.005 & 6.188 & 5.127 & 7.128 & 3.87 \\
\hline 3/13/2006 & $21: 00$ & 11.983 & 11.86 & 7.759 & 5.1 & 3.439 & 3.35 & 11.008 & 6.19 & 5.132 & 7.132 & 3.874 \\
\hline $3 / 13 / 2006$ & $22: 00$ & 11.986 & 11.864 & 7.764 & 5.106 & 3.439 & 3.353 & 11.01 & 6.193 & 5.138 & 7.135 & 3.879 \\
\hline $3 / 13 / 2006$ & $23: 00$ & 11.991 & 11.867 & 7.763 & 5.108 & 3.442 & 3.355 & 11.012 & 6.192 & 5.141 & 7.136 & 3.881 \\
\hline $3 / 14 / 2006$ & $0: 00$ & 11.995 & 11.871 & 7.768 & 5.112 & 3.441 & 3.36 & 11.016 & 6.193 & 5.146 & 7.137 & 3.89 \\
\hline $3 / 14 / 2006$ & $1: 00$ & 11.996 & 11.869 & 7.763 & 5.115 & 3.441 & 3.357 & 11.018 & 6.194 & 5.147 & 7.138 & 3.893 \\
\hline $3 / 14 / 2006$ & $2: 00$ & 11.996 & 11.871 & 7.763 & 5.117 & 3.443 & 3.357 & 11.017 & 6.197 & 5.151 & 7.14 & 3.897 \\
\hline $3 / 14 / 2006$ & $3: 00$ & 12.001 & 11.869 & 7.763 & 5.119 & 3.444 & 3.356 & 11.021 & 6.197 & 5.159 & 7.139 & 3.902 \\
\hline $3 / 14 / 2006$ & $4: 00$ & 12.001 & 11.871 & 7.763 & 5.121 & 3.438 & 3.36 & 11.022 & 6.199 & 5.161 & 7.14 & 3.907 \\
\hline $3 / 14 / 2006$ & $5: 00$ & 12.003 & 11.871 & 7.763 & 5.123 & 3.447 & 3.358 & 11.023 & 6.2 & 5.166 & 7.142 & 3.915 \\
\hline $3 / 14 / 2006$ & $6: 00$ & 12.006 & 11.875 & 7.77 & 5.126 & 3.452 & 3.366 & 11.026 & 6.201 & 5.171 & 7.148 & 3.922 \\
\hline $3 / 14 / 2006$ & $7: 00$ & 12.005 & 11.873 & 7.768 & 5.128 & 3.447 & 3.363 & 11.025 & 6.202 & 5.175 & 7.147 & 3.924 \\
\hline $3 / 14 / 2006$ & $8: 00$ & 12.008 & 11.877 & 7.77 & 5.128 & 3.454 & 3.367 & 11.027 & 6.206 & 5.18 & 7.15 & 3.929 \\
\hline $3 / 14 / 2006$ & $9: 00$ & 12.008 & 11.878 & 7.772 & 5.133 & 3.457 & 3.367 & 11.029 & 6.207 & 5.185 & 7.151 & 3.932 \\
\hline $3 / 14 / 2006$ & $10: 00$ & 12.01 & 11.877 & 7.772 & 5.133 & 3.456 & 3.369 & 11.031 & 6.206 & 5.187 & 7.152 & 3.934 \\
\hline $3 / 14 / 2006$ & $11: 00$ & 12.01 & 11.877 & 7.768 & 5.132 & 3.445 & 3.364 & 11.03 & 6.209 & 5.19 & 7.151 & 3.93 \\
\hline $3 / 14 / 2006$ & $12: 00$ & 12.008 & 11.869 & 7.763 & 5.13 & 3.441 & 3.358 & 11.026 & 6.202 & 5.188 & 7.149 & 3.913 \\
\hline $3 / 14 / 2006$ & $13: 00$ & 12.002 & 11.86 & 7.757 & 5.128 & 3.437 & 3.353 & 11.029 & 6.2 & 5.177 & 7.144 & 3.894 \\
\hline $3 / 14 / 2006$ & $14: 00$ & 11.995 & 11.851 & 7.75 & 5.123 & 3.424 & 3.345 & 11.028 & 6.2 & 5.167 & 7.139 & 3.878 \\
\hline $3 / 14 / 2006$ & $15: 00$ & 11.983 & 11.836 & 7.736 & 5.121 & 3.421 & 3.331 & 11.025 & 6.195 & 5.154 & 7.135 & 3.866 \\
\hline $3 / 14 / 2006$ & $16: 00$ & 11.975 & 11.831 & 7.734 & 5.119 & 3.412 & 3.328 & 11.022 & 6.193 & 5.151 & 7.132 & 3.864 \\
\hline $3 / 14 / 2006$ & $17: 00$ & 11.975 & 11.831 & 7.739 & 5.119 & 3.423 & 3.332 & 11.023 & 6.192 & 5.149 & 7.133 & 3.868 \\
\hline $3 / 14 / 2006$ & $18: 00$ & 11.975 & 11.835 & 7.741 & 5.117 & 3.416 & 3.334 & 11.024 & 6.195 & 5.148 & 7.133 & 3.872 \\
\hline $3 / 14 / 2006$ & $19: 00$ & 11.98 & 11.838 & 7.746 & 5.117 & 3.426 & 3.339 & 11.026 & 6.195 & 5.153 & 7.136 & 3.878 \\
\hline $3 / 14 / 2006$ & $20: 00$ & 11.983 & 11.842 & 7.748 & 5.119 & 3.426 & 3.342 & 11.028 & 6.197 & 5.153 & 7.137 & 3.884 \\
\hline $3 / 14 / 2006$ & $21: 00$ & 11.99 & 11.851 & 7.757 & 5.121 & 3.431 & 3.351 & 11.03 & 6.198 & 5.159 & 7.141 & 3.887 \\
\hline
\end{tabular}


TABLE S1.2 (Cont.)

Water Level (ft below top of casing) at Indicated Well

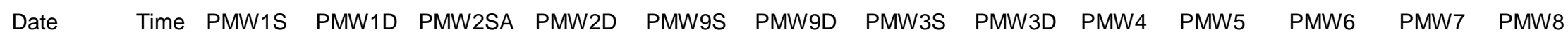

\begin{tabular}{|c|c|c|c|c|c|c|c|c|c|c|c|c|}
\hline $3 / 14 / 2006$ & $22: 00$ & 11.994 & 11.853 & 7.759 & 5.125 & 3.442 & 3.356 & 11.031 & 6.193 & 5.161 & 7.144 & 3.892 \\
\hline $3 / 14 / 2006$ & $23: 00$ & 11.997 & 11.855 & 7.759 & 5.126 & 3.436 & 3.356 & 11.03 & 6.192 & 5.162 & 7.144 & 3.893 \\
\hline $3 / 15 / 2006$ & $0: 00$ & 11.997 & 11.857 & 7.763 & 5.126 & 3.444 & 3.358 & 11.034 & 6.193 & 5.164 & 7.146 & 3.895 \\
\hline $3 / 15 / 2006$ & $1: 00$ & 11.999 & 11.857 & 7.763 & 5.128 & 3.438 & 3.358 & 11.033 & 6.195 & 5.166 & 7.146 & 3.897 \\
\hline $3 / 15 / 2006$ & $2: 00$ & 11.997 & 11.855 & 7.759 & 5.129 & 3.443 & 3.358 & 11.033 & 6.195 & 5.167 & 7.148 & 3.901 \\
\hline $3 / 15 / 2006$ & $3: 00$ & 11.995 & 11.851 & 7.756 & 5.128 & 3.439 & 3.355 & 11.033 & 6.19 & 5.166 & 7.146 & 3.903 \\
\hline $3 / 15 / 2006$ & $4: 00$ & 11.99 & 11.846 & 7.749 & 5.128 & 3.437 & 3.349 & 11.033 & 6.191 & 5.166 & 7.145 & 3.904 \\
\hline $3 / 15 / 2006$ & $5: 00$ & 11.992 & 11.851 & 7.759 & 5.13 & 3.442 & 3.354 & 11.033 & 6.195 & 5.172 & 7.148 & 3.913 \\
\hline 3/15/2006 & $6: 00$ & 11.99 & 11.846 & 7.752 & 5.129 & 3.438 & 3.353 & 11.033 & 6.191 & 5.172 & 7.146 & 3.916 \\
\hline $3 / 15 / 2006$ & $7: 00$ & 11.985 & 11.838 & 7.748 & 5.128 & 3.428 & 3.35 & 11.031 & 6.19 & 5.175 & 7.143 & 3.915 \\
\hline $3 / 15 / 2006$ & $8: 00$ & 11.982 & 11.834 & 7.744 & 5.126 & 3.425 & 3.344 & 11.031 & 6.19 & 5.177 & 7.141 & 3.917 \\
\hline $3 / 15 / 2006$ & $9: 00$ & 11.977 & 11.829 & 7.739 & 5.124 & 3.422 & 3.341 & 11.03 & 6.191 & 5.176 & 7.137 & 3.916 \\
\hline $3 / 15 / 2006$ & $10: 00$ & 11.968 & 11.818 & 7.73 & 5.119 & 3.422 & 3.332 & 11.028 & 6.184 & 5.17 & 7.134 & 3.915 \\
\hline $3 / 15 / 2006$ & $11: 00$ & 11.955 & 11.8 & 7.712 & 5.112 & 3.402 & 3.317 & 11.02 & 6.177 & 5.166 & 7.128 & 3.901 \\
\hline $3 / 15 / 2006$ & $12: 00$ & 11.94 & 11.782 & 7.697 & 5.106 & 3.387 & 3.302 & 11.016 & 6.183 & 5.155 & 7.119 & 3.883 \\
\hline $3 / 15 / 2006$ & $13: 00$ & 11.923 & 11.753 & 7.668 & 5.099 & 3.365 & 3.28 & 11.011 & 6.165 & 5.135 & 7.104 & 3.86 \\
\hline $3 / 15 / 2006$ & $14: 00$ & 11.904 & 11.731 & 7.65 & 5.079 & 3.342 & 3.271 & 10.98 & 6.157 & 5.124 & 7.098 & 3.848 \\
\hline $3 / 15 / 2006$ & $15: 00$ & 11.888 & 11.712 & 7.635 & 5.073 & 3.328 & 3.25 & 10.987 & 6.151 & 5.11 & 7.083 & 3.841 \\
\hline $3 / 15 / 2006$ & $16: 00$ & 11.871 & 11.698 & 7.626 & 5.061 & 3.317 & 3.236 & 10.985 & 6.143 & 5.109 & 7.08 & 3.84 \\
\hline $3 / 15 / 2006$ & $17: 00$ & 11.859 & 11.685 & 7.615 & 5.054 & 3.314 & 3.229 & 10.976 & 6.157 & 5.109 & 7.08 & 3.846 \\
\hline $3 / 15 / 2006$ & $18: 00$ & 11.849 & 11.676 & 7.61 & 5.048 & 3.308 & 3.226 & 10.974 & 6.149 & 5.106 & 7.068 & 3.85 \\
\hline $3 / 15 / 2006$ & $19: 00$ & 11.848 & 11.685 & 7.626 & 5.043 & 3.322 & 3.233 & 10.979 & 6.156 & 5.117 & 7.082 & 3.863 \\
\hline $3 / 15 / 2006$ & $20: 00$ & 11.838 & 11.678 & 7.619 & 5.039 & 3.31 & 3.227 & 10.976 & 6.153 & 5.115 & 7.079 & 3.864 \\
\hline $3 / 15 / 2006$ & $21: 00$ & 11.834 & 11.674 & 7.617 & 5.035 & 3.316 & 3.226 & 10.976 & 6.149 & 5.114 & 7.075 & 3.867 \\
\hline $3 / 15 / 2006$ & $22: 00$ & 11.831 & 11.672 & 7.619 & 5.032 & 3.317 & 3.229 & 10.974 & 6.148 & 5.114 & 7.075 & 3.87 \\
\hline $3 / 15 / 2006$ & $23: 00$ & 11.824 & 11.67 & 7.614 & 5.028 & 3.313 & 3.225 & 10.974 & 6.146 & 5.115 & 7.074 & 3.869 \\
\hline 3/16/2006 & $0: 00$ & 11.825 & 11.678 & 7.621 & 5.028 & 3.32 & 3.227 & 10.971 & 6.151 & 5.124 & 7.077 & 3.875 \\
\hline $3 / 16 / 2006$ & $1: 00$ & 11.821 & 11.665 & 7.61 & 5.025 & 3.304 & 3.221 & 10.971 & 6.142 & 5.113 & 7.071 & 3.869 \\
\hline $3 / 16 / 2006$ & $2: 00$ & 11.816 & 11.661 & 7.603 & 5.021 & 3.308 & 3.216 & 10.967 & 6.141 & 5.108 & 7.071 & 3.864 \\
\hline 3/16/2006 & $3: 00$ & 11.808 & 11.654 & 7.597 & 5.017 & 3.294 & 3.21 & 10.964 & 6.139 & 5.105 & 7.067 & 3.858 \\
\hline $3 / 16 / 2006$ & $4: 00$ & 11.806 & 11.652 & 7.597 & 5.012 & 3.299 & 3.21 & 10.962 & 6.135 & 5.103 & 7.065 & 3.86 \\
\hline 3/16/2006 & $5: 00$ & 11.811 & 11.661 & 7.612 & 5.014 & 3.31 & 3.218 & 10.962 & 6.143 & 5.108 & 7.07 & 3.868 \\
\hline $3 / 16 / 2006$ & $6: 00$ & 11.819 & 11.674 & 7.63 & 5.019 & 3.322 & 3.233 & 10.96 & 6.151 & 5.114 & 7.065 & 3.878 \\
\hline 3/16/2006 & $7: 00$ & 11.84 & 11.703 & 7.652 & 5.028 & 3.349 & 3.257 & 10.963 & 6.157 & 5.125 & 7.082 & 3.892 \\
\hline
\end{tabular}


TABLE S1.2 (Cont.)

Water Level (ft below top of casing) at Indicated Well

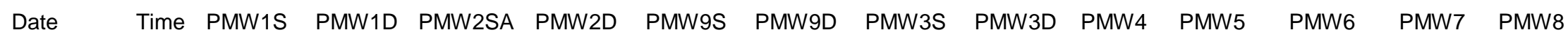

\begin{tabular}{|c|c|c|c|c|c|c|c|c|c|c|c|c|}
\hline $3 / 16 / 2006$ & $8: 00$ & 11.858 & 11.729 & 7.672 & 5.03 & 3.364 & 3.276 & 10.965 & 6.16 & 5.137 & 7.084 & 3.894 \\
\hline 3/16/2006 & $9: 00$ & 11.875 & 11.754 & 7.696 & 5.041 & 3.377 & 3.292 & 10.981 & 6.169 & 5.148 & 7.092 & 3.906 \\
\hline $3 / 16 / 2006$ & $10: 00$ & 11.897 & 11.778 & 7.71 & 5.053 & 3.395 & 3.309 & 10.978 & 6.171 & 5.153 & 7.102 & 3.913 \\
\hline 3/16/2006 & $11: 00$ & 11.912 & 11.791 & 7.725 & 5.063 & 3.409 & 3.321 & 10.989 & 6.176 & 5.16 & 7.109 & 3.914 \\
\hline 3/16/2006 & $12: 00$ & 11.929 & 11.809 & 7.736 & 5.065 & 3.424 & 3.337 & 10.992 & 6.176 & 5.164 & 7.117 & 3.921 \\
\hline 3/16/2006 & $13: 00$ & 11.939 & 11.824 & 7.747 & 5.081 & 3.432 & 3.344 & 10.992 & 6.182 & 5.169 & 7.123 & 3.922 \\
\hline $3 / 16 / 2006$ & $14: 00$ & 11.954 & 11.835 & 7.756 & 5.088 & 3.439 & 3.351 & 11.002 & 6.185 & 5.173 & 7.127 & 3.928 \\
\hline 3/16/2006 & $15: 00$ & 11.966 & 11.846 & 7.765 & 5.096 & 3.45 & 3.36 & 11.008 & 6.189 & 5.18 & 7.134 & 3.93 \\
\hline 3/16/2006 & $16: 00$ & 11.97 & 11.858 & 7.774 & 5.105 & 3.451 & 3.365 & 11.014 & 6.191 & 5.183 & 7.139 & 3.929 \\
\hline 3/16/2006 & $17: 00$ & 11.986 & 11.869 & 7.78 & 5.116 & 3.464 & 3.374 & 11.016 & 6.193 & 5.189 & 7.146 & 3.935 \\
\hline $3 / 16 / 2006$ & $18: 00$ & 11.997 & 11.878 & 7.787 & 5.121 & 3.467 & 3.381 & 11.02 & 6.198 & 5.194 & 7.15 & 3.937 \\
\hline $3 / 16 / 2006$ & $19: 00$ & 12.005 & 11.888 & 7.791 & 5.13 & 3.474 & 3.384 & 11.024 & 6.197 & 5.199 & 7.149 & 3.94 \\
\hline $3 / 16 / 2006$ & $20: 00$ & 12.013 & 11.897 & 7.798 & 5.135 & 3.48 & 3.392 & 11.031 & 6.202 & 5.2 & 7.158 & 3.941 \\
\hline 3/16/2006 & $21: 00$ & 12.02 & 11.902 & 7.803 & 5.14 & 3.484 & 3.397 & 11.033 & 6.204 & 5.204 & 7.16 & 3.942 \\
\hline 3/16/2006 & $22: 00$ & 12.025 & 11.908 & 7.805 & 5.147 & 3.487 & 3.404 & 11.038 & 6.205 & 5.207 & 7.161 & 3.943 \\
\hline 3/16/2006 & $23: 00$ & 12.03 & 11.911 & 7.809 & 5.154 & 3.483 & 3.403 & 11.039 & 6.21 & 5.209 & 7.164 & 3.943 \\
\hline $3 / 17 / 2006$ & $0: 00$ & 12.036 & 11.915 & 7.811 & 5.156 & 3.492 & 3.404 & 11.04 & 6.209 & 5.211 & 7.166 & 3.943 \\
\hline $3 / 17 / 2006$ & $1: 00$ & 12.04 & 11.915 & 7.809 & 5.161 & 3.484 & 3.402 & 11.042 & 6.208 & 5.212 & 7.166 & 3.94 \\
\hline $3 / 17 / 2006$ & $2: 00$ & 12.043 & 11.919 & 7.811 & 5.162 & 3.493 & 3.405 & 11.043 & 6.211 & 5.211 & 7.167 & 3.94 \\
\hline $3 / 17 / 2006$ & $3: 00$ & 12.047 & 11.92 & 7.809 & 5.167 & 3.494 & 3.405 & 11.046 & 6.212 & 5.214 & 7.17 & 3.939 \\
\hline $3 / 17 / 2006$ & $4: 00$ & 12.048 & 11.92 & 7.811 & 5.17 & 3.492 & 3.406 & 11.046 & 6.214 & 5.215 & 7.17 & 3.941 \\
\hline 3/17/2006 & $5: 00$ & 12.05 & 11.922 & 7.814 & 5.171 & 3.492 & 3.408 & 11.047 & 6.213 & 5.215 & 7.17 & 3.939 \\
\hline 3/17/2006 & $6: 00$ & 12.05 & 11.926 & 7.816 & 5.171 & 3.49 & 3.41 & 11.048 & 6.213 & 5.217 & 7.175 & 3.939 \\
\hline $3 / 17 / 2006$ & $7: 00$ & 12.052 & 11.924 & 7.814 & 5.174 & 3.49 & 3.409 & 11.05 & 6.21 & 5.216 & 7.177 & 3.939 \\
\hline $3 / 17 / 2006$ & $8: 00$ & 12.055 & 11.926 & 7.814 & 5.176 & 3.495 & 3.41 & 11.051 & 6.214 & 5.219 & 7.177 & 3.938 \\
\hline $3 / 17 / 2006$ & $9: 00$ & 12.057 & 11.929 & 7.821 & 5.178 & 3.496 & 3.414 & 11.052 & 6.218 & 5.22 & 7.178 & 3.944 \\
\hline $3 / 17 / 2006$ & $10: 00$ & 12.058 & 11.929 & 7.821 & 5.18 & 3.495 & 3.413 & 11.053 & 6.215 & 5.218 & 7.179 & 3.938 \\
\hline $3 / 17 / 2006$ & $11: 00$ & 12.062 & 11.931 & 7.825 & 5.182 & 3.504 & 3.418 & 11.055 & 6.217 & 5.222 & 7.183 & 3.941 \\
\hline $3 / 17 / 2006$ & $12: 00$ & 12.062 & 11.933 & 7.823 & 5.182 & 3.503 & 3.418 & 11.056 & 6.218 & 5.219 & 7.181 & 3.937 \\
\hline $3 / 17 / 2006$ & $13: 00$ & 12.059 & 11.924 & 7.814 & 5.181 & 3.497 & 3.411 & 11.056 & 6.215 & 5.215 & 7.181 & 3.927 \\
\hline $3 / 17 / 2006$ & $14: 00$ & 12.052 & 11.911 & 7.803 & 5.178 & 3.488 & 3.399 & 11.055 & 6.212 & 5.205 & 7.179 & 3.92 \\
\hline $3 / 17 / 2006$ & $15: 00$ & 12.037 & 11.891 & 7.785 & 5.174 & 3.473 & 3.385 & 11.052 & 6.205 & 5.196 & 7.173 & 3.91 \\
\hline $3 / 17 / 2006$ & $16: 00$ & 12.027 & 11.878 & 7.776 & 5.169 & 3.463 & 3.373 & 11.049 & 6.204 & 5.194 & 7.168 & 3.906 \\
\hline $3 / 17 / 2006$ & $17: 00$ & 12.018 & 11.867 & 7.769 & 5.165 & 3.459 & 3.369 & 11.048 & 6.203 & 5.189 & 7.166 & 3.905 \\
\hline
\end{tabular}


TABLE S1.2 (Cont.)

Water Level (ft below top of casing) at Indicated Well

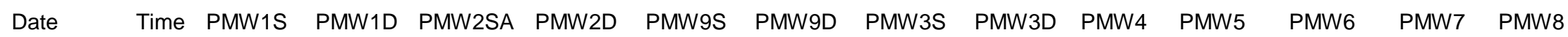

\begin{tabular}{|c|c|c|c|c|c|c|c|c|c|c|c|c|}
\hline $3 / 17 / 2006$ & $18: 00$ & 12.008 & 11.858 & 7.76 & 5.163 & 3.444 & 3.363 & 11.046 & 6.202 & 5.189 & 7.163 & 3.905 \\
\hline $3 / 17 / 2006$ & $19: 00$ & 12.003 & 11.851 & 7.756 & 5.156 & 3.44 & 3.358 & 11.043 & 6.198 & 5.187 & 7.16 & 3.904 \\
\hline $3 / 17 / 2006$ & $20: 00$ & 11.998 & 11.849 & 7.756 & 5.154 & 3.439 & 3.356 & 11.041 & 6.196 & 5.187 & 7.158 & 3.907 \\
\hline 3/17/2006 & $21: 00$ & 11.998 & 11.849 & 7.763 & 5.154 & 3.449 & 3.359 & 11.042 & 6.2 & 5.191 & 7.161 & 3.911 \\
\hline 3/17/2006 & $22: 00$ & 11.995 & 11.847 & 7.758 & 5.151 & 3.442 & 3.357 & 11.043 & 6.199 & 5.191 & 7.159 & 3.911 \\
\hline 3/17/2006 & $23: 00$ & 11.997 & 11.853 & 7.767 & 5.151 & 3.451 & 3.365 & 11.044 & 6.201 & 5.197 & 7.163 & 3.915 \\
\hline $3 / 18 / 2006$ & $0: 00$ & 11.998 & 11.851 & 7.763 & 5.15 & 3.447 & 3.364 & 11.043 & 6.203 & 5.195 & 7.161 & 3.913 \\
\hline 3/18/2006 & $1: 00$ & 11.997 & 11.853 & 7.767 & 5.15 & 3.449 & 3.367 & 11.044 & 6.201 & 5.197 & 7.163 & 3.915 \\
\hline 3/18/2006 & $2: 00$ & 11.995 & 11.849 & 7.762 & 5.147 & 3.45 & 3.362 & 11.045 & 6.196 & 5.19 & 7.159 & 3.91 \\
\hline 3/18/2006 & $3: 00$ & 11.993 & 11.844 & 7.758 & 5.147 & 3.441 & 3.358 & 11.041 & 6.2 & 5.189 & 7.16 & 3.908 \\
\hline $3 / 18 / 2006$ & $4: 00$ & 11.987 & 11.842 & 7.753 & 5.143 & 3.436 & 3.356 & 11.04 & 6.196 & 5.186 & 7.159 & 3.906 \\
\hline $3 / 18 / 2006$ & $5: 00$ & 11.987 & 11.84 & 7.753 & 5.142 & 3.444 & 3.355 & 11.04 & 6.196 & 5.188 & 7.158 & 3.907 \\
\hline $3 / 18 / 2006$ & $6: 00$ & 11.99 & 11.844 & 7.762 & 5.145 & 3.451 & 3.339 & 11.042 & 6.201 & 5.19 & 7.159 & 3.911 \\
\hline 3/18/2006 & $7: 00$ & 11.988 & 11.846 & 7.76 & 5.142 & 3.447 & 3.327 & 11.039 & 6.194 & 5.186 & 7.158 & 3.908 \\
\hline $3 / 18 / 2006$ & $8: 00$ & 11.993 & 11.849 & 7.766 & 5.143 & 3.454 & 3.336 & 11.042 & 6.197 & 5.185 & 7.159 & 3.907 \\
\hline 3/18/2006 & $9: 00$ & 11.995 & 11.855 & 7.769 & 5.144 & 3.451 & 3.369 & 11.043 & 6.202 & 5.189 & 7.161 & 3.91 \\
\hline $3 / 18 / 2006$ & $10: 00$ & 11.995 & 11.857 & 7.767 & 5.143 & 3.447 & 3.392 & 11.041 & 6.198 & 5.182 & 7.161 & 3.904 \\
\hline $3 / 18 / 2006$ & $11: 00$ & 11.995 & 11.849 & 7.763 & 5.142 & 3.454 & 3.365 & 11.043 & 6.198 & 5.18 & 7.156 & 3.897 \\
\hline 3/18/2006 & $12: 00$ & 11.991 & 11.846 & 7.763 & 5.142 & 3.444 & 3.364 & 11.042 & 6.196 & 5.173 & 7.159 & 3.894 \\
\hline $3 / 18 / 2006$ & $13: 00$ & 11.989 & 11.84 & 7.754 & 5.14 & 3.445 & 3.36 & 11.04 & 6.192 & 5.168 & 7.15 & 3.888 \\
\hline $3 / 18 / 2006$ & $14: 00$ & 11.98 & 11.833 & 7.747 & 5.136 & 3.438 & 3.35 & 11.038 & 6.194 & 5.165 & 7.151 & 3.884 \\
\hline $3 / 18 / 2006$ & $15: 00$ & 11.975 & 11.826 & 7.74 & 5.133 & 3.432 & 3.342 & 11.035 & 6.188 & 5.162 & 7.149 & 3.88 \\
\hline 3/18/2006 & $16: 00$ & 11.97 & 11.816 & 7.734 & 5.129 & 3.424 & 3.337 & 11.033 & 6.188 & 5.156 & 7.146 & 3.877 \\
\hline $3 / 18 / 2006$ & $17: 00$ & 11.962 & 11.813 & 7.731 & 5.129 & 3.425 & 3.334 & 11.032 & 6.189 & 5.157 & 7.144 & 3.881 \\
\hline $3 / 18 / 2006$ & $18: 00$ & 11.963 & 11.813 & 7.736 & 5.127 & 3.427 & 3.336 & 11.03 & 6.187 & 5.158 & 7.145 & 3.882 \\
\hline $3 / 18 / 2006$ & $19: 00$ & 11.96 & 11.811 & 7.734 & 5.125 & 3.418 & 3.337 & 11.03 & 6.183 & 5.159 & 7.144 & 3.886 \\
\hline 3/18/2006 & $20: 00$ & 11.96 & 11.809 & 7.731 & 5.125 & 3.424 & 3.332 & 11.028 & 6.187 & 5.157 & 7.14 & 3.886 \\
\hline $3 / 18 / 2006$ & $21: 00$ & 11.955 & 11.805 & 7.727 & 5.123 & 3.419 & 3.329 & 11.028 & 6.181 & 5.159 & 7.14 & 3.888 \\
\hline $3 / 18 / 2006$ & $22: 00$ & 11.954 & 11.805 & 7.727 & 5.12 & 3.42 & 3.333 & 11.027 & 6.189 & 5.16 & 7.142 & 3.889 \\
\hline 3/18/2006 & $23: 00$ & 11.952 & 11.802 & 7.729 & 5.123 & 3.415 & 3.336 & 11.027 & 6.183 & 5.161 & 7.138 & 3.891 \\
\hline 3/19/2006 & 0:00 & 11.954 & 11.811 & 7.736 & 5.12 & 3.422 & 3.338 & 11.027 & 6.185 & 5.166 & 7.144 & 3.897 \\
\hline $3 / 19 / 2006$ & $1: 00$ & 11.96 & 11.815 & 7.74 & 5.121 & 3.423 & 3.341 & 11.027 & 6.188 & 5.171 & 7.146 & 3.897 \\
\hline $3 / 19 / 2006$ & $2: 00$ & 11.962 & 11.818 & 7.742 & 5.121 & 3.434 & 3.342 & 11.029 & 6.19 & 5.172 & 7.148 & 3.901 \\
\hline 3/19/2006 & $3: 00$ & 11.965 & 11.82 & 7.74 & 5.123 & 3.433 & 3.347 & 11.027 & 6.185 & 5.173 & 7.146 & 3.903 \\
\hline
\end{tabular}


TABLE S1.2 (Cont.)

Water Level (ft below top of casing) at Indicated Well

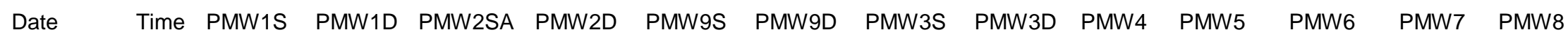

\begin{tabular}{|c|c|c|c|c|c|c|c|c|c|c|c|c|}
\hline 3/19/2006 & $4: 00$ & 11.959 & 11.815 & 7.715 & 5.121 & 3.418 & 3.323 & 11.022 & 6.18 & 5.169 & 7.145 & 3.896 \\
\hline 3/19/2006 & $5: 00$ & 11.95 & 11.8 & 7.72 & 5.118 & 3.412 & 3.331 & 11.027 & 6.18 & 5.163 & 7.143 & 3.888 \\
\hline 3/19/2006 & $6: 00$ & 11.949 & 11.816 & 7.72 & 5.113 & 3.409 & 3.327 & 11.023 & 6.182 & 5.162 & 7.136 & 3.886 \\
\hline 3/19/2006 & $7: 00$ & 11.949 & 11.795 & 7.728 & 5.116 & 3.414 & 3.328 & 11.022 & 6.182 & 5.158 & 7.125 & 3.89 \\
\hline $3 / 19 / 2006$ & $8: 00$ & 11.947 & 11.802 & 7.729 & 5.115 & 3.413 & 3.331 & 11.023 & 6.184 & 5.159 & 7.137 & 3.884 \\
\hline $3 / 19 / 2006$ & 9:00 & 11.951 & 11.811 & 7.736 & 5.118 & 3.425 & 3.336 & 11.022 & 6.185 & 5.157 & 7.137 & 3.885 \\
\hline $3 / 19 / 2006$ & $10: 00$ & 11.954 & 11.815 & 7.738 & 5.114 & 3.43 & 3.337 & 11.025 & 6.182 & 5.154 & 7.143 & 3.883 \\
\hline 3/19/2006 & $11: 00$ & 11.954 & 11.82 & 7.738 & 5.113 & 3.429 & 3.34 & 11.032 & 6.182 & 5.148 & 7.146 & 3.88 \\
\hline $3 / 19 / 2006$ & $12: 00$ & 11.954 & 11.82 & 7.76 & 5.111 & 3.427 & 3.338 & 11.048 & 6.182 & 5.144 & 7.141 & 3.872 \\
\hline 3/19/2006 & $13: 00$ & 11.952 & 11.827 & 7.769 & 5.109 & 3.421 & 3.333 & 11.023 & 6.177 & 5.139 & 7.145 & 3.868 \\
\hline $3 / 19 / 2006$ & $14: 00$ & 11.952 & 11.82 & 7.762 & 5.112 & 3.421 & 3.329 & 11.018 & 6.179 & 5.134 & 7.14 & 3.859 \\
\hline $3 / 19 / 2006$ & $15: 00$ & 11.947 & 11.835 & 7.753 & 5.105 & 3.408 & 3.333 & 11.016 & 6.17 & 5.121 & 7.136 & 3.845 \\
\hline $3 / 19 / 2006$ & $16: 00$ & 11.942 & 11.851 & 7.769 & 5.101 & 3.406 & 3.319 & 11.014 & 6.168 & 5.115 & 7.149 & 3.834 \\
\hline $3 / 19 / 2006$ & $17: 00$ & 11.935 & 11.853 & 7.773 & 5.1 & 3.4 & 3.316 & 11.013 & 6.168 & 5.109 & 7.153 & 3.822 \\
\hline $3 / 19 / 2006$ & $18: 00$ & 11.935 & 11.84 & 7.706 & 5.096 & 3.386 & 3.305 & 11.011 & 6.167 & 5.101 & 7.135 & 3.811 \\
\hline $3 / 19 / 2006$ & $19: 00$ & 11.93 & 11.784 & 7.707 & 5.093 & 3.387 & 3.303 & 11.012 & 6.167 & 5.096 & 7.125 & 3.805 \\
\hline 3/19/2006 & $20: 00$ & 11.929 & 11.789 & 7.711 & 5.093 & 3.388 & 3.305 & 11.012 & 6.167 & 5.095 & 7.126 & 3.8 \\
\hline $3 / 19 / 2006$ & $21: 00$ & 11.928 & 11.785 & 7.707 & 5.091 & 3.387 & 3.303 & 11.011 & 6.162 & 5.085 & 7.124 & 3.792 \\
\hline $3 / 19 / 2006$ & $22: 00$ & 11.927 & 11.784 & 7.707 & 5.09 & 3.382 & 3.305 & 11.008 & 6.162 & 5.081 & 7.121 & 3.789 \\
\hline $3 / 19 / 2006$ & $23: 00$ & 11.927 & 11.784 & 7.704 & 5.087 & 3.393 & 3.304 & 11.007 & 6.163 & 5.078 & 7.115 & 3.785 \\
\hline 3/20/2006 & $0: 00$ & 11.917 & 11.765 & 7.687 & 5.084 & 3.372 & 3.29 & 11.006 & 6.156 & 5.064 & 7.1 & 3.773 \\
\hline $3 / 20 / 2006$ & $1: 00$ & 11.919 & 11.774 & 7.698 & 5.084 & 3.383 & 3.292 & 11.003 & 6.16 & 5.07 & 7.116 & 3.781 \\
\hline $3 / 20 / 2006$ & $2: 00$ & 11.925 & 11.78 & 7.705 & 5.083 & 3.387 & 3.302 & 11.005 & 6.158 & 5.063 & 7.114 & 3.782 \\
\hline $3 / 20 / 2006$ & $3: 00$ & 11.919 & 11.769 & 7.693 & 5.08 & 3.377 & 3.29 & 10.999 & 6.153 & 5.059 & 7.11 & 3.776 \\
\hline $3 / 20 / 2006$ & $4: 00$ & 11.917 & 11.765 & 7.689 & 5.078 & 3.367 & 3.285 & 11.003 & 6.152 & 5.055 & 7.103 & 3.77 \\
\hline $3 / 20 / 2006$ & $5: 00$ & 11.912 & 11.762 & 7.684 & 5.075 & 3.362 & 3.281 & 10.999 & 6.15 & 5.049 & 7.102 & 3.771 \\
\hline $3 / 20 / 2006$ & $6: 00$ & 11.907 & 11.76 & 7.684 & 5.071 & 3.37 & 3.28 & 10.998 & 6.15 & 5.044 & 7.096 & 3.767 \\
\hline $3 / 20 / 2006$ & $7: 00$ & 11.905 & 11.758 & 7.684 & 5.069 & 3.369 & 3.281 & 10.997 & 6.147 & 5.039 & 7.094 & 3.767 \\
\hline $3 / 20 / 2006$ & $8: 00$ & 11.902 & 11.754 & 7.673 & 5.067 & 3.363 & 3.276 & 10.995 & 6.141 & 5.032 & 7.091 & 3.759 \\
\hline $3 / 20 / 2006$ & $9: 00$ & 11.899 & 11.747 & 7.673 & 5.063 & 3.354 & 3.272 & 10.992 & 6.141 & 5.026 & 7.083 & 3.759 \\
\hline $3 / 20 / 2006$ & $10: 00$ & 11.894 & 11.749 & 7.667 & 5.059 & 3.356 & 3.269 & 10.987 & 6.143 & 5.026 & 7.088 & 3.754 \\
\hline 3/20/2006 & $11: 00$ & 11.887 & 11.736 & 7.657 & 5.053 & 3.343 & 3.253 & 10.985 & 6.136 & 5.017 & 7.084 & 3.741 \\
\hline $3 / 20 / 2006$ & $12: 00$ & 11.88 & 11.72 & 7.651 & 5.048 & 3.336 & 3.25 & 10.984 & 6.134 & 5.011 & 7.083 & 3.735 \\
\hline $3 / 20 / 2006$ & $13: 00$ & 11.872 & 11.725 & 7.642 & 5.047 & 3.336 & 3.252 & 10.984 & 6.127 & 5.003 & 7.089 & 3.731 \\
\hline
\end{tabular}


TABLE S1.2 (Cont.)

Water Level (ft below top of casing) at Indicated Well

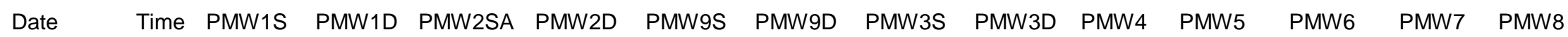

\begin{tabular}{|c|c|c|c|c|c|c|c|c|c|c|c|c|}
\hline $3 / 20 / 2006$ & $14: 00$ & 11.865 & 11.712 & 7.631 & 5.038 & 3.313 & 3.23 & 10.973 & 6.129 & 4.999 & 7.091 & 3.721 \\
\hline 3/20/2006 & $15: 00$ & 11.86 & 11.712 & 7.638 & 5.036 & 3.327 & 3.24 & 10.983 & 6.129 & 4.996 & 7.063 & 3.722 \\
\hline $3 / 20 / 2006$ & $16: 00$ & 11.862 & 11.712 & 7.64 & 5.036 & 3.327 & 3.237 & 10.976 & 6.126 & 4.992 & 7.083 & 3.717 \\
\hline $3 / 20 / 2006$ & $17: 00$ & 11.858 & 11.714 & 7.642 & 5.031 & 3.32 & 3.238 & 10.975 & 6.123 & 4.987 & 7.077 & 3.71 \\
\hline 3/20/2006 & $18: 00$ & 11.858 & 11.711 & 7.642 & 5.031 & 3.325 & 3.237 & 10.971 & 6.124 & 4.985 & 7.081 & 3.706 \\
\hline $3 / 20 / 2006$ & $19: 00$ & 11.86 & 11.725 & 7.651 & 5.031 & 3.329 & 3.248 & 10.976 & 6.126 & 4.989 & 7.073 & 3.705 \\
\hline $3 / 20 / 2006$ & $20: 00$ & 11.862 & 11.733 & 7.655 & 5.031 & 3.335 & 3.251 & 10.973 & 6.125 & 4.984 & 7.074 & 3.7 \\
\hline $3 / 20 / 2006$ & $21: 00$ & 11.87 & 11.74 & 7.66 & 5.033 & 3.336 & 3.256 & 10.976 & 6.121 & 4.982 & 7.075 & 3.698 \\
\hline 3/20/2006 & $22: 00$ & 11.881 & 11.747 & 7.673 & 5.035 & 3.343 & 3.262 & 10.976 & 6.126 & 4.985 & 7.078 & 3.697 \\
\hline $3 / 20 / 2006$ & $23: 00$ & 11.887 & 11.749 & 7.673 & 5.036 & 3.343 & 3.263 & 10.975 & 6.12 & 4.984 & 7.078 & 3.693 \\
\hline $3 / 21 / 2006$ & $0: 00$ & 11.892 & 11.753 & 7.678 & 5.038 & 3.352 & 3.264 & 10.976 & 6.121 & 4.984 & 7.079 & 3.687 \\
\hline $3 / 21 / 2006$ & $1: 00$ & 11.891 & 11.753 & 7.675 & 5.038 & 3.342 & 3.262 & 10.975 & 6.12 & 4.982 & 7.077 & 3.684 \\
\hline $3 / 21 / 2006$ & $2: 00$ & 11.894 & 11.76 & 7.677 & 5.037 & 3.354 & 3.265 & 10.975 & 6.121 & 4.98 & 7.078 & 3.683 \\
\hline $3 / 21 / 2006$ & $3: 00$ & 11.898 & 11.765 & 7.686 & 5.042 & 3.354 & 3.271 & 10.978 & 6.12 & 4.982 & 7.081 & 3.683 \\
\hline $3 / 21 / 2006$ & $4: 00$ & 11.905 & 11.773 & 7.693 & 5.044 & 3.358 & 3.277 & 10.978 & 6.123 & 4.986 & 7.086 & 3.683 \\
\hline $3 / 21 / 2006$ & $5: 00$ & 11.916 & 11.784 & 7.702 & 5.048 & 3.367 & 3.289 & 10.98 & 6.124 & 4.992 & 7.091 & 3.686 \\
\hline $3 / 21 / 2006$ & $6: 00$ & 11.928 & 11.795 & 7.715 & 5.053 & 3.376 & 3.295 & 10.983 & 6.126 & 4.996 & 7.094 & 3.687 \\
\hline $3 / 21 / 2006$ & $7: 00$ & 11.938 & 11.809 & 7.722 & 5.06 & 3.387 & 3.305 & 10.988 & 6.128 & 4.997 & 7.098 & 3.687 \\
\hline $3 / 21 / 2006$ & $8: 00$ & 11.948 & 11.824 & 7.735 & 5.064 & 3.399 & 3.316 & 10.99 & 6.131 & 4.999 & 7.104 & 3.687 \\
\hline $3 / 21 / 2006$ & $9: 00$ & 11.96 & 11.838 & 7.742 & 5.071 & 3.406 & 3.323 & 10.994 & 6.132 & 5.003 & 7.109 & 3.69 \\
\hline $3 / 21 / 2006$ & $10: 00$ & 11.971 & 11.849 & 7.755 & 5.075 & 3.412 & 3.333 & 10.997 & 6.133 & 5.007 & 7.113 & 3.692 \\
\hline $3 / 21 / 2006$ & $11: 00$ & 11.98 & 11.855 & 7.761 & 5.082 & 3.419 & 3.339 & 11.002 & 6.137 & 5.009 & 7.116 & 3.691 \\
\hline $3 / 21 / 2006$ & $12: 00$ & 11.991 & 11.866 & 7.768 & 5.09 & 3.434 & 3.351 & 11.007 & 6.139 & 5.015 & 7.126 & 3.687 \\
\hline $3 / 21 / 2006$ & $13: 00$ & 12 & 11.877 & 7.775 & 5.094 & 3.439 & 3.355 & 11.009 & 6.139 & 5.017 & 7.127 & 3.685 \\
\hline $3 / 21 / 2006$ & $14: 00$ & 12.008 & 11.882 & 7.78 & 5.101 & 3.443 & 3.358 & 11.013 & 6.135 & 5.015 & 7.129 & 3.678 \\
\hline $3 / 21 / 2006$ & $15: 00$ & 12.012 & 11.884 & 7.777 & 5.104 & 3.438 & 3.355 & 11.013 & 6.13 & 5.011 & 7.132 & 3.665 \\
\hline $3 / 21 / 2006$ & $16: 00$ & 12.018 & 11.891 & 7.786 & 5.108 & 3.444 & 3.358 & 11.014 & 6.136 & 5.014 & 7.135 & 3.658 \\
\hline $3 / 21 / 2006$ & $17: 00$ & 12.022 & 11.897 & 7.788 & 5.113 & 3.438 & 3.36 & 11.017 & 6.132 & 5.013 & 7.132 & 3.648 \\
\hline $3 / 21 / 2006$ & $18: 00$ & 12.03 & 11.9 & 7.793 & 5.117 & 3.44 & 3.365 & 11.018 & 6.128 & 5.01 & 7.137 & 3.64 \\
\hline $3 / 21 / 2006$ & $19: 00$ & 12.034 & 11.906 & 7.793 & 5.122 & 3.447 & 3.364 & 11.019 & 6.125 & 5.009 & 7.137 & 3.628 \\
\hline $3 / 21 / 2006$ & $20: 00$ & 12.037 & 11.902 & 7.79 & 5.122 & 3.437 & 3.361 & 11.02 & 6.119 & 5.004 & 7.136 & 3.616 \\
\hline $3 / 21 / 2006$ & $21: 00$ & 12.039 & 11.908 & 7.795 & 5.126 & 3.447 & 3.362 & 11.022 & 6.116 & 5.004 & 7.138 & 3.611 \\
\hline $3 / 21 / 2006$ & $22: 00$ & 12.042 & 11.913 & 7.799 & 5.128 & 3.45 & 3.365 & 11.023 & 6.116 & 5.004 & 7.141 & 3.608 \\
\hline $3 / 21 / 2006$ & $23: 00$ & 12.047 & 11.919 & 7.808 & 5.131 & 3.457 & 3.373 & 11.025 & 6.123 & 5.007 & 7.143 & 3.612 \\
\hline
\end{tabular}


TABLE S1.2 (Cont.)

Water Level (ft below top of casing) at Indicated Well

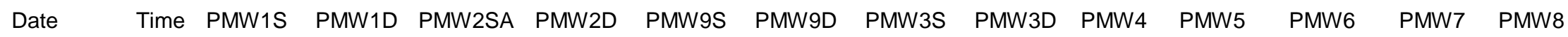

\begin{tabular}{|c|c|c|c|c|c|c|c|c|c|c|c|c|}
\hline 3/22/2006 & $0: 00$ & 12.052 & 11.92 & 7.807 & 5.133 & 3.455 & 3.373 & 11.025 & 6.123 & 5.006 & 7.145 & 3.607 \\
\hline 3/22/2006 & $1: 00$ & 12.055 & 11.924 & 7.81 & 5.134 & 3.46 & 3.378 & 11.028 & 6.127 & 5.005 & 7.147 & 3.608 \\
\hline $3 / 22 / 2006$ & $2: 00$ & 12.059 & 11.931 & 7.816 & 5.14 & 3.456 & 3.383 & 11.032 & 6.128 & 5.008 & 7.15 & 3.614 \\
\hline 3/22/2006 & $3: 00$ & 12.064 & 11.935 & 7.816 & 5.14 & 3.468 & 3.384 & 11.032 & 6.133 & 5.008 & 7.15 & 3.618 \\
\hline $3 / 22 / 2006$ & $4: 00$ & 12.067 & 11.933 & 7.812 & 5.141 & 3.457 & 3.383 & 11.032 & 6.129 & 5.004 & 7.151 & 3.62 \\
\hline 3/22/2006 & $5: 00$ & 12.067 & 11.933 & 7.812 & 5.142 & 3.464 & 3.381 & 11.033 & 6.132 & 5.002 & 7.151 & 3.629 \\
\hline 3/22/2006 & 6:00 & 12.067 & 11.935 & 7.812 & 5.144 & 3.458 & 3.385 & 11.035 & 6.14 & 5.002 & 7.153 & 3.638 \\
\hline 3/22/2006 & 7:00 & 12.069 & 11.935 & 7.816 & 5.145 & 3.462 & 3.386 & 11.037 & 6.141 & 5.003 & 7.153 & 3.646 \\
\hline 3/22/2006 & 8:00 & 12.069 & 11.937 & 7.816 & 5.146 & 3.463 & 3.386 & 11.038 & 6.146 & 5.003 & 7.154 & 3.655 \\
\hline $3 / 22 / 2006$ & $9: 00$ & 12.071 & 11.94 & 7.821 & 5.148 & 3.475 & 3.392 & 11.04 & 6.152 & 5.005 & 7.156 & 3.667 \\
\hline 3/22/2006 & $10: 00$ & 12.074 & 11.94 & 7.823 & 5.148 & 3.469 & 3.393 & 11.041 & 6.154 & 5.007 & 7.154 & 3.677 \\
\hline $3 / 22 / 2006$ & $11: 00$ & 12.074 & 11.94 & 7.819 & 5.148 & 3.476 & 3.39 & 11.044 & 6.154 & 5.005 & 7.154 & 3.684 \\
\hline 3/22/2006 & $12: 00$ & 12.071 & 11.937 & 7.816 & 5.15 & 3.467 & 3.389 & 11.043 & 6.155 & 5.006 & 7.155 & 3.69 \\
\hline $3 / 22 / 2006$ & $13: 00$ & 12.073 & 11.933 & 7.817 & 5.15 & 3.462 & 3.389 & 11.043 & 6.156 & 5.005 & 7.155 & 3.694 \\
\hline 3/22/2006 & $14: 00$ & 12.067 & 11.926 & 7.81 & 5.148 & 3.457 & 3.378 & 11.041 & 6.153 & 5.002 & 7.153 & 3.684 \\
\hline $3 / 22 / 2006$ & $15: 00$ & 12.061 & 11.919 & 7.798 & 5.146 & 3.446 & 3.368 & 11.041 & 6.143 & 4.999 & 7.148 & 3.661 \\
\hline 3/22/2006 & $16: 00$ & 12.055 & 11.911 & 7.794 & 5.145 & 3.443 & 3.359 & 11.037 & 6.123 & 4.995 & 7.145 & 3.62 \\
\hline $3 / 22 / 2006$ & $17: 00$ & 12.056 & 11.911 & 7.794 & 5.146 & 3.437 & 3.353 & 11.037 & 6.104 & 4.993 & 7.144 & 3.563 \\
\hline 3/22/2006 & $18: 00$ & 12.051 & 11.909 & 7.789 & 5.143 & 3.422 & 3.349 & 11.032 & 6.083 & 4.986 & 7.142 & 3.499 \\
\hline $3 / 22 / 2006$ & $19: 00$ & 12.049 & 11.909 & 7.79 & 5.141 & 3.428 & 3.348 & 11.029 & 6.071 & 4.982 & 7.141 & 3.456 \\
\hline 3/22/2006 & $20: 00$ & 12.052 & 11.909 & 7.791 & 5.139 & 3.427 & 3.348 & 11.026 & 6.063 & 4.977 & 7.141 & 3.432 \\
\hline 3/22/2006 & 21:00 & 12.051 & 11.909 & 7.788 & 5.134 & 3.428 & 3.347 & 11.023 & 6.064 & 4.971 & 7.141 & 3.426 \\
\hline 3/22/2006 & 22:00 & 12.052 & 11.913 & 7.791 & 5.133 & 3.427 & 3.351 & 11.02 & 6.068 & 4.966 & 7.141 & 3.433 \\
\hline $3 / 22 / 2006$ & $23: 00$ & 12.054 & 11.917 & 7.794 & 5.128 & 3.429 & 3.354 & 11.017 & 6.075 & 4.965 & 7.141 & 3.452 \\
\hline 3/23/2006 & $0: 00$ & 12.056 & 11.917 & 7.794 & 5.121 & 3.425 & 3.355 & 11.018 & 6.08 & 4.962 & 7.142 & 3.474 \\
\hline 3/23/2006 & $1: 00$ & 12.056 & 11.913 & 7.794 & 5.116 & 3.437 & 3.355 & 11.015 & 6.09 & 4.958 & 7.141 & 3.498 \\
\hline $3 / 23 / 2006$ & $2: 00$ & 12.056 & 11.915 & 7.794 & 5.115 & 3.435 & 3.357 & 11.018 & 6.098 & 4.959 & 7.144 & 3.525 \\
\hline 3/23/2006 & 3:00 & 12.054 & 11.911 & 7.792 & 5.11 & 3.435 & 3.356 & 11.016 & 6.103 & 4.956 & 7.141 & 3.55 \\
\hline $3 / 23 / 2006$ & $4: 00$ & 12.051 & 11.908 & 7.788 & 5.108 & 3.432 & 3.355 & 11.019 & 6.105 & 4.959 & 7.142 & 3.573 \\
\hline 3/23/2006 & 5:00 & 12.049 & 11.906 & 7.787 & 5.104 & 3.437 & 3.353 & 11.019 & 6.116 & 4.962 & 7.143 & 3.598 \\
\hline $3 / 23 / 2006$ & $6: 00$ & 12.046 & 11.904 & 7.787 & 5.102 & 3.439 & 3.353 & 11.019 & 6.122 & 4.964 & 7.145 & 3.62 \\
\hline 3/23/2006 & 7:00 & 12.047 & 11.908 & 7.792 & 5.103 & 3.442 & 3.358 & 11.022 & 6.129 & 4.971 & 7.146 & 3.645 \\
\hline $3 / 23 / 2006$ & $8: 00$ & 12.05 & 11.913 & 7.796 & 5.105 & 3.449 & 3.366 & 11.024 & 6.138 & 4.978 & 7.148 & 3.668 \\
\hline 3/23/2006 & 9:00 & 12.053 & 11.915 & 7.8 & 5.105 & 3.457 & 3.371 & 11.027 & 6.143 & 4.986 & 7.151 & 3.69 \\
\hline
\end{tabular}


TABLE S1.2 (Cont.)

Water Level (ft below top of casing) at Indicated Well

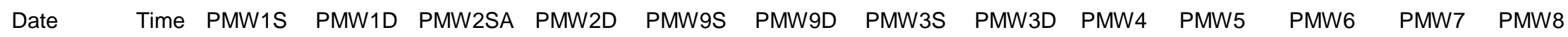

\begin{tabular}{|c|c|c|c|c|c|c|c|c|c|c|c|c|}
\hline $3 / 23 / 2006$ & $10: 00$ & 12.056 & 11.915 & 7.798 & 5.101 & 3.447 & 3.371 & 11.027 & 6.149 & 4.992 & 7.152 & 3.708 \\
\hline $3 / 23 / 2006$ & $11: 00$ & 12.055 & 11.913 & 7.799 & 5.105 & 3.459 & 3.373 & 11.028 & 6.151 & 4.997 & 7.154 & 3.724 \\
\hline $3 / 23 / 2006$ & $12: 00$ & 12.058 & 11.917 & 7.805 & 5.106 & 3.454 & 3.374 & 11.031 & 6.156 & 5.006 & 7.153 & 3.736 \\
\hline 3/23/2006 & $13: 00$ & 12.053 & 11.913 & 7.801 & 5.11 & 3.457 & 3.374 & 11.033 & 6.16 & 5.011 & 7.155 & 3.74 \\
\hline $3 / 23 / 2006$ & $14: 00$ & 12.051 & 11.909 & 7.799 & 5.11 & 3.452 & 3.368 & 11.032 & 6.155 & 5.013 & 7.155 & 3.727 \\
\hline 3/23/2006 & $15: 00$ & 12.045 & 11.898 & 7.787 & 5.11 & 3.442 & 3.356 & 11.032 & 6.147 & 5.013 & 7.153 & 3.671 \\
\hline $3 / 23 / 2006$ & $16: 00$ & 12.04 & 11.895 & 7.781 & 5.11 & 3.428 & 3.348 & 11.029 & 6.132 & 5.015 & 7.148 & 3.572 \\
\hline 3/23/2006 & $17: 00$ & 12.038 & 11.891 & 7.779 & 5.112 & 3.421 & 3.341 & 11.027 & 6.112 & 5.012 & 7.145 & 3.453 \\
\hline $3 / 23 / 2006$ & $18: 00$ & 12.033 & 11.888 & 7.774 & 5.112 & 3.41 & 3.333 & 11.023 & 6.09 & 5.003 & 7.142 & 3.363 \\
\hline $3 / 23 / 2006$ & $19: 00$ & 12.032 & 11.888 & 7.772 & 5.11 & 3.409 & 3.329 & 11.016 & 6.072 & 4.992 & 7.141 & 3.314 \\
\hline $3 / 23 / 2006$ & $20: 00$ & 12.031 & 11.884 & 7.768 & 5.104 & 3.396 & 3.327 & 11.014 & 6.062 & 4.979 & 7.137 & 3.296 \\
\hline $3 / 23 / 2006$ & $21: 00$ & 12.026 & 11.882 & 7.768 & 5.096 & 3.402 & 3.323 & 11.008 & 6.058 & 4.968 & 7.136 & 3.303 \\
\hline 3/23/2006 & $22: 00$ & 12.027 & 11.882 & 7.767 & 5.088 & 3.393 & 3.323 & 11.005 & 6.064 & 4.958 & 7.134 & 3.324 \\
\hline $3 / 23 / 2006$ & $23: 00$ & 12.024 & 11.875 & 7.762 & 5.079 & 3.392 & 3.323 & 11.002 & 6.066 & 4.95 & 7.131 & 3.352 \\
\hline $3 / 24 / 2006$ & $0: 00$ & 12.018 & 11.873 & 7.756 & 5.07 & 3.391 & 3.318 & 10.997 & 6.068 & 4.944 & 7.131 & 3.386 \\
\hline $3 / 24 / 2006$ & $1: 00$ & 12.017 & 11.866 & 7.752 & 5.061 & 3.388 & 3.317 & 10.997 & 6.071 & 4.937 & 7.127 & 3.421 \\
\hline $3 / 24 / 2006$ & $2: 00$ & 12.011 & 11.862 & 7.749 & 5.054 & 3.386 & 3.313 & 10.993 & 6.078 & 4.934 & 7.127 & 3.456 \\
\hline $3 / 24 / 2006$ & $3: 00$ & 12.004 & 11.857 & 7.747 & 5.048 & 3.384 & 3.312 & 10.993 & 6.083 & 4.933 & 7.123 & 3.49 \\
\hline $3 / 24 / 2006$ & $4: 00$ & 12.004 & 11.855 & 7.743 & 5.047 & 3.393 & 3.313 & 10.992 & 6.086 & 4.934 & 7.123 & 3.524 \\
\hline $3 / 24 / 2006$ & $5: 00$ & 11.999 & 11.851 & 7.741 & 5.042 & 3.386 & 3.312 & 10.991 & 6.096 & 4.937 & 7.125 & 3.557 \\
\hline 3/24/2006 & $6: 00$ & 11.997 & 11.849 & 7.743 & 5.039 & 3.391 & 3.313 & 10.992 & 6.1 & 4.941 & 7.124 & 3.586 \\
\hline $3 / 24 / 2006$ & $7: 00$ & 11.994 & 11.847 & 7.741 & 5.039 & 3.399 & 3.315 & 10.992 & 6.105 & 4.95 & 7.127 & 3.615 \\
\hline 3/24/2006 & $8: 00$ & 11.994 & 11.849 & 7.743 & 5.038 & 3.401 & 3.315 & 10.993 & 6.113 & 4.959 & 7.129 & 3.644 \\
\hline $3 / 24 / 2006$ & 9:00 & 11.996 & 11.853 & 7.75 & 5.04 & 3.401 & 3.322 & 10.996 & 6.119 & 4.971 & 7.133 & 3.673 \\
\hline 3/24/2006 & $10: 00$ & 11.998 & 11.857 & 7.752 & 5.041 & 3.414 & 3.328 & 10.997 & 6.126 & 4.982 & 7.133 & 3.696 \\
\hline $3 / 24 / 2006$ & $11: 00$ & 12.001 & 11.86 & 7.756 & 5.043 & 3.41 & 3.331 & 10.998 & 6.127 & 4.993 & 7.136 & 3.713 \\
\hline 3/24/2006 & $12: 00$ & 12.003 & 11.858 & 7.756 & 5.046 & 3.417 & 3.332 & 11 & 6.133 & 4.997 & 7.139 & 3.718 \\
\hline $3 / 24 / 2006$ & $13: 00$ & 12.001 & 11.857 & 7.753 & 5.047 & 3.415 & 3.329 & 11.001 & 6.135 & 5.003 & 7.138 & 3.69 \\
\hline 3/24/2006 & $14: 00$ & 11.999 & 11.849 & 7.747 & 5.05 & 3.403 & 3.32 & 11.001 & 6.129 & 5.005 & 7.136 & 3.603 \\
\hline $3 / 24 / 2006$ & $15: 00$ & 11.994 & 11.846 & 7.743 & 5.052 & 3.382 & 3.312 & 10.999 & 6.105 & 5.003 & 7.135 & 3.451 \\
\hline $3 / 24 / 2006$ & $16: 00$ & 11.992 & 11.842 & 7.736 & 5.055 & 3.375 & 3.298 & 10.995 & 6.068 & 4.982 & 7.13 & 3.267 \\
\hline $3 / 24 / 2006$ & $17: 00$ & 11.986 & 11.838 & 7.732 & 5.054 & 3.366 & 3.289 & 10.989 & 6.045 & 4.945 & 7.122 & 3.145 \\
\hline $3 / 24 / 2006$ & $18: 00$ & 11.986 & 11.838 & 7.729 & 5.051 & 3.359 & 3.284 & 10.98 & 6.029 & 4.899 & 7.116 & 3.08 \\
\hline $3 / 24 / 2006$ & $19: 00$ & 11.986 & 11.842 & 7.731 & 5.042 & 3.354 & 3.284 & 10.973 & 6.021 & 4.849 & 7.109 & 3.064 \\
\hline
\end{tabular}


TABLE S1.2 (Cont.)

Water Level (ft below top of casing) at Indicated Well

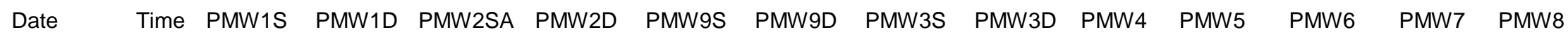

\begin{tabular}{|c|c|c|c|c|c|c|c|c|c|c|c|c|}
\hline $3 / 24 / 2006$ & $20: 00$ & 11.986 & 11.842 & 7.727 & 5.029 & 3.357 & 3.281 & 10.965 & 6.019 & 4.802 & 7.1 & 3.068 \\
\hline $3 / 24 / 2006$ & $21: 00$ & 11.989 & 11.846 & 7.729 & 5.018 & 3.354 & 3.279 & 10.959 & 6.016 & 4.772 & 7.095 & 3.086 \\
\hline $3 / 24 / 2006$ & $22: 00$ & 11.988 & 11.846 & 7.729 & 5.01 & 3.353 & 3.279 & 10.952 & 6.016 & 4.751 & 7.09 & 3.115 \\
\hline 3/24/2006 & $23: 00$ & 11.993 & 11.849 & 7.731 & 5.004 & 3.36 & 3.281 & 10.949 & 6.019 & 4.744 & 7.088 & 3.157 \\
\hline $3 / 25 / 2006$ & $0: 00$ & 11.993 & 11.851 & 7.731 & 4.998 & 3.361 & 3.282 & 10.945 & 6.023 & 4.738 & 7.084 & 3.2 \\
\hline $3 / 25 / 2006$ & $1: 00$ & 11.993 & 11.851 & 7.729 & 4.993 & 3.362 & 3.282 & 10.942 & 6.027 & 4.739 & 7.083 & 3.238 \\
\hline $3 / 25 / 2006$ & $2: 00$ & 11.991 & 11.849 & 7.725 & 4.988 & 3.362 & 3.281 & 10.938 & 6.03 & 4.742 & 7.082 & 3.271 \\
\hline $3 / 25 / 2006$ & $3: 00$ & 11.993 & 11.851 & 7.727 & 4.987 & 3.357 & 3.282 & 10.937 & 6.037 & 4.751 & 7.081 & 3.303 \\
\hline $3 / 25 / 2006$ & $4: 00$ & 11.993 & 11.851 & 7.727 & 4.988 & 3.358 & 3.284 & 10.937 & 6.036 & 4.759 & 7.082 & 3.33 \\
\hline $3 / 25 / 2006$ & $5: 00$ & 11.993 & 11.853 & 7.73 & 4.986 & 3.364 & 3.287 & 10.938 & 6.047 & 4.771 & 7.083 & 3.36 \\
\hline $3 / 25 / 2006$ & $6: 00$ & 11.995 & 11.857 & 7.731 & 4.988 & 3.363 & 3.288 & 10.938 & 6.047 & 4.782 & 7.085 & 3.386 \\
\hline $3 / 25 / 2006$ & $7: 00$ & 12.001 & 11.86 & 7.736 & 4.992 & 3.376 & 3.293 & 10.938 & 6.054 & 4.794 & 7.087 & 3.413 \\
\hline $3 / 25 / 2006$ & $8: 00$ & 12.001 & 11.864 & 7.743 & 4.995 & 3.379 & 3.3 & 10.943 & 6.059 & 4.805 & 7.089 & 3.437 \\
\hline $3 / 25 / 2006$ & $9: 00$ & 12.006 & 11.867 & 7.743 & 4.999 & 3.385 & 3.303 & 10.946 & 6.065 & 4.817 & 7.093 & 3.455 \\
\hline $3 / 25 / 2006$ & $10: 00$ & 12.008 & 11.871 & 7.746 & 5.001 & 3.39 & 3.307 & 10.947 & 6.067 & 4.828 & 7.098 & 3.464 \\
\hline $3 / 25 / 2006$ & $11: 00$ & 12.011 & 11.873 & 7.751 & 5.006 & 3.387 & 3.31 & 10.95 & 6.074 & 4.839 & 7.099 & 3.439 \\
\hline $3 / 25 / 2006$ & $12: 00$ & 12.015 & 11.877 & 7.751 & 5.01 & 3.389 & 3.312 & 10.952 & 6.075 & 4.844 & 7.101 & 3.401 \\
\hline $3 / 25 / 2006$ & $13: 00$ & 12.015 & 11.873 & 7.749 & 5.014 & 3.384 & 3.308 & 10.953 & 6.077 & 4.843 & 7.1 & 3.344 \\
\hline $3 / 25 / 2006$ & $14: 00$ & 12.01 & 11.869 & 7.745 & 5.015 & 3.382 & 3.304 & 10.952 & 6.074 & 4.82 & 7.096 & 3.287 \\
\hline $3 / 25 / 2006$ & $15: 00$ & 12.005 & 11.86 & 7.734 & 5.017 & 3.37 & 3.293 & 10.951 & 6.065 & 4.744 & 7.087 & 3.24 \\
\hline $3 / 25 / 2006$ & $16: 00$ & 12 & 11.855 & 7.727 & 5.017 & 3.363 & 3.281 & 10.945 & 6.054 & 4.603 & 7.071 & 3.201 \\
\hline $3 / 25 / 2006$ & $17: 00$ & 11.993 & 11.845 & 7.718 & 5.011 & 3.35 & 3.269 & 10.936 & 6.04 & 4.406 & 7.045 & 3.181 \\
\hline $3 / 25 / 2006$ & $18: 00$ & 11.986 & 11.838 & 7.707 & 5.003 & 3.327 & 3.257 & 10.926 & 6.027 & 4.306 & 7.015 & 3.185 \\
\hline $3 / 25 / 2006$ & 19:00 & 11.983 & 11.831 & 7.698 & 4.995 & 3.328 & 3.245 & 10.917 & 6.018 & 4.253 & 6.989 & 3.207 \\
\hline $3 / 25 / 2006$ & $20: 00$ & 11.978 & 11.827 & 7.691 & 4.988 & 3.32 & 3.237 & 10.906 & 6.014 & 4.243 & 6.967 & 3.237 \\
\hline $3 / 25 / 2006$ & $21: 00$ & 11.973 & 11.824 & 7.687 & 4.982 & 3.312 & 3.232 & 10.896 & 6.015 & 4.261 & 6.956 & 3.267 \\
\hline $3 / 25 / 2006$ & $22: 00$ & 11.97 & 11.82 & 7.684 & 4.975 & 3.304 & 3.227 & 10.889 & 6.015 & 4.289 & 6.947 & 3.298 \\
\hline $3 / 25 / 2006$ & $23: 00$ & 11.966 & 11.82 & 7.682 & 4.972 & 3.308 & 3.225 & 10.885 & 6.015 & 4.323 & 6.944 & 3.331 \\
\hline $3 / 26 / 2006$ & $0: 00$ & 11.965 & 11.816 & 7.677 & 4.967 & 3.304 & 3.221 & 10.877 & 6.012 & 4.357 & 6.943 & 3.364 \\
\hline $3 / 26 / 2006$ & $1: 00$ & 11.961 & 11.811 & 7.671 & 4.963 & 3.301 & 3.216 & 10.872 & 6.016 & 4.391 & 6.944 & 3.394 \\
\hline $3 / 26 / 2006$ & $2: 00$ & 11.955 & 11.804 & 7.667 & 4.96 & 3.29 & 3.212 & 10.87 & 6.015 & 4.424 & 6.949 & 3.425 \\
\hline $3 / 26 / 2006$ & $3: 00$ & 11.95 & 11.8 & 7.664 & 4.957 & 3.293 & 3.211 & 10.867 & 6.019 & 4.461 & 6.954 & 3.455 \\
\hline $3 / 26 / 2006$ & $4: 00$ & 11.945 & 11.791 & 7.653 & 4.952 & 3.282 & 3.202 & 10.863 & 6.015 & 4.488 & 6.956 & 3.48 \\
\hline $3 / 26 / 2006$ & $5: 00$ & 11.94 & 11.789 & 7.653 & 4.95 & 3.286 & 3.204 & 10.864 & 6.024 & 4.525 & 6.964 & 3.509 \\
\hline
\end{tabular}


TABLE S1.2 (Cont.)

Water Level (ft below top of casing) at Indicated Well

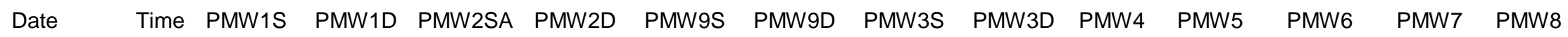

\begin{tabular}{|c|c|c|c|c|c|c|c|c|c|c|c|c|}
\hline 3/26/2006 & $6: 00$ & 11.938 & 11.785 & 7.654 & 4.95 & 3.294 & 3.206 & 10.863 & 6.028 & 4.557 & 6.97 & 3.537 \\
\hline 3/26/2006 & 7:00 & 11.933 & 11.78 & 7.651 & 4.95 & 3.285 & 3.207 & 10.864 & 6.031 & 4.584 & 6.975 & 3.56 \\
\hline 3/26/2006 & 8:00 & 11.928 & 11.778 & 7.651 & 4.95 & 3.295 & 3.21 & 10.862 & 6.034 & 4.613 & 6.982 & 3.583 \\
\hline 3/26/2006 & 9:00 & 11.926 & 11.773 & 7.647 & 4.948 & 3.295 & 3.209 & 10.864 & 6.038 & 4.636 & 6.988 & 3.602 \\
\hline $3 / 26 / 2006$ & $10: 00$ & 11.921 & 11.767 & 7.645 & 4.946 & 3.286 & 3.205 & 10.862 & 6.038 & 4.657 & 6.989 & 3.608 \\
\hline 3/26/2006 & 11:00 & 11.918 & 11.76 & 7.638 & 4.946 & 3.284 & 3.203 & 10.864 & 6.038 & 4.676 & 6.992 & 3.581 \\
\hline 3/26/2006 & $12: 00$ & 11.912 & 11.754 & 7.629 & 4.941 & 3.282 & 3.197 & 10.863 & 6.037 & 4.674 & 6.99 & 3.521 \\
\hline 3/26/2006 & $13: 00$ & 11.903 & 11.743 & 7.621 & 4.937 & 3.266 & 3.186 & 10.861 & 6.032 & 4.572 & 6.977 & 3.507 \\
\hline 3/26/2006 & $14: 00$ & 11.89 & 11.729 & 7.605 & 4.93 & 3.252 & 3.169 & 10.857 & 6.028 & 4.347 & 6.924 & 3.505 \\
\hline $3 / 26 / 2006$ & $15: 00$ & 11.886 & 11.716 & 7.583 & 4.919 & 3.217 & 3.131 & 10.845 & 6.023 & 4.099 & 6.793 & 3.512 \\
\hline 3/26/2006 & $16: 00$ & 11.869 & 11.7 & 7.545 & 4.906 & 3.138 & 3.056 & 10.837 & 6.015 & 3.926 & 6.627 & 3.52 \\
\hline $3 / 26 / 2006$ & $17: 00$ & 11.856 & 11.692 & 7.489 & 4.895 & 3.041 & 2.959 & 10.819 & 6.013 & 3.773 & 6.47 & 3.535 \\
\hline 3/26/2006 & 18:00 & 11.847 & 11.687 & 7.443 & 4.879 & 2.981 & 2.887 & 10.804 & 6.013 & 3.657 & 6.36 & 3.55 \\
\hline $3 / 26 / 2006$ & $19: 00$ & 11.846 & 11.685 & 7.417 & 4.863 & 2.947 & 2.846 & 10.795 & 6.012 & 3.591 & 6.3 & 3.574 \\
\hline 3/26/2006 & $20: 00$ & 11.844 & 11.687 & 7.396 & 4.844 & 2.92 & 2.819 & 10.787 & 6.009 & 3.571 & 6.278 & 3.593 \\
\hline $3 / 26 / 2006$ & $21: 00$ & 11.837 & 11.674 & 7.374 & 4.82 & 2.901 & 2.8 & 10.777 & 6.003 & 3.571 & 6.263 & 3.599 \\
\hline 3/26/2006 & $22: 00$ & 11.832 & 11.674 & 7.371 & 4.805 & 2.902 & 2.8 & 10.767 & 6.005 & 3.593 & 6.271 & 3.613 \\
\hline $3 / 26 / 2006$ & $23: 00$ & 11.829 & 11.669 & 7.364 & 4.784 & 2.891 & 2.798 & 10.761 & 6 & 3.616 & 6.267 & 3.619 \\
\hline 3/27/2006 & $0: 00$ & 11.822 & 11.669 & 7.362 & 4.771 & 2.903 & 2.8 & 10.753 & 5.998 & 3.647 & 6.264 & 3.633 \\
\hline $3 / 27 / 2006$ & $1: 00$ & 11.816 & 11.634 & 7.328 & 4.755 & 2.887 & 2.79 & 10.724 & 5.993 & 3.645 & 6.232 & 3.627 \\
\hline 3/27/2006 & $2: 00$ & 11.807 & 11.65 & 7.326 & 4.74 & 2.868 & 2.768 & 10.74 & 5.985 & 3.575 & 6.236 & 3.582 \\
\hline 3/27/2006 & 3:00 & 11.795 & 11.638 & 7.312 & 4.725 & 2.856 & 2.753 & 10.736 & 5.985 & 3.585 & 6.238 & 3.555 \\
\hline $3 / 27 / 2006$ & $4: 00$ & 11.787 & 11.63 & 7.307 & 4.713 & 2.846 & 2.751 & 10.73 & 5.982 & 3.62 & 6.254 & 3.552 \\
\hline 3/27/2006 & 5:00 & 11.777 & 11.621 & 7.304 & 4.701 & 2.853 & 2.753 & 10.727 & 5.976 & 3.652 & 6.273 & 3.557 \\
\hline 3/27/2006 & $6: 00$ & 11.771 & 11.619 & 7.312 & 4.695 & 2.87 & 2.77 & 10.726 & 5.977 & 3.692 & 6.297 & 3.565 \\
\hline 3/27/2006 & 7:00 & 11.765 & 11.614 & 7.315 & 4.688 & 2.883 & 2.78 & 10.725 & 5.976 & 3.725 & 6.32 & 3.573 \\
\hline $3 / 27 / 2006$ & $8: 00$ & 11.762 & 11.612 & 7.317 & 4.682 & 2.891 & 2.789 & 10.722 & 5.975 & 3.76 & 6.341 & 3.58 \\
\hline 3/27/2006 & 9:00 & 11.757 & 11.61 & 7.324 & 4.68 & 2.901 & 2.8 & 10.722 & 5.978 & 3.794 & 6.361 & 3.59 \\
\hline $3 / 27 / 2006$ & $10: 00$ & 11.754 & 11.608 & 7.33 & 4.679 & 2.904 & 2.808 & 10.721 & 5.972 & 3.823 & 6.379 & 3.599 \\
\hline 3/27/2006 & 11:00 & 11.752 & 11.619 & 7.352 & 4.678 & 2.914 & 2.818 & 10.729 & 5.973 & 3.813 & 6.399 & 3.583 \\
\hline $3 / 27 / 2006$ & $12: 00$ & 11.754 & 11.656 & 7.385 & 4.676 & 2.905 & 2.81 & 10.77 & 5.976 & 3.713 & 6.431 & 3.462 \\
\hline 3/27/2006 & $13: 00$ & 11.757 & 11.649 & 7.368 & 4.678 & 2.903 & 2.806 & 10.748 & 5.966 & 3.646 & 6.406 & 3.337 \\
\hline $3 / 27 / 2006$ & $14: 00$ & 11.757 & 11.63 & 7.339 & 4.68 & 2.884 & 2.793 & 10.72 & 5.958 & 3.636 & 6.335 & 3.29 \\
\hline 3/27/2006 & $15: 00$ & 11.765 & 11.636 & 7.337 & 4.68 & 2.894 & 2.798 & 10.717 & 5.951 & 3.672 & 6.346 & 3.29 \\
\hline
\end{tabular}


TABLE S1.2 (Cont.)

Water Level (ft below top of casing) at Indicated Well

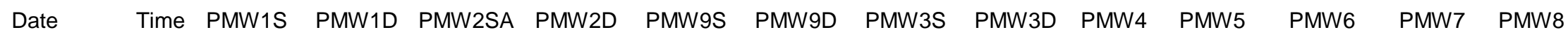

\begin{tabular}{|c|c|c|c|c|c|c|c|c|c|c|c|c|}
\hline $3 / 27 / 2006$ & $16: 00$ & 11.767 & 11.643 & 7.341 & 4.68 & 2.901 & 2.802 & 10.715 & 5.948 & 3.714 & 6.367 & 3.306 \\
\hline 3/27/2006 & $17: 00$ & 11.773 & 11.647 & 7.348 & 4.678 & 2.912 & 2.816 & 10.716 & 5.941 & 3.76 & 6.387 & 3.326 \\
\hline $3 / 27 / 2006$ & $18: 00$ & 11.777 & 11.656 & 7.354 & 4.675 & 2.918 & 2.829 & 10.716 & 5.944 & 3.804 & 6.41 & 3.349 \\
\hline 3/27/2006 & $19: 00$ & 11.784 & 11.663 & 7.369 & 4.677 & 2.939 & 2.847 & 10.717 & 5.943 & 3.848 & 6.439 & 3.373 \\
\hline 3/27/2006 & $20: 00$ & 11.79 & 11.676 & 7.382 & 4.678 & 2.963 & 2.867 & 10.717 & 5.948 & 3.892 & 6.469 & 3.398 \\
\hline $3 / 27 / 2006$ & $21: 00$ & 11.796 & 11.681 & 7.392 & 4.68 & 2.969 & 2.879 & 10.721 & 5.944 & 3.926 & 6.495 & 3.417 \\
\hline $3 / 27 / 2006$ & $22: 00$ & 11.801 & 11.685 & 7.401 & 4.682 & 2.991 & 2.893 & 10.723 & 5.948 & 3.964 & 6.52 & 3.435 \\
\hline $3 / 27 / 2006$ & $23: 00$ & 11.806 & 11.689 & 7.412 & 4.688 & 3.002 & 2.908 & 10.724 & 5.949 & 3.997 & 6.546 & 3.456 \\
\hline 3/28/2006 & $0: 00$ & 11.811 & 11.696 & 7.42 & 4.693 & 3.011 & 2.922 & 10.724 & 5.951 & 4.029 & 6.574 & 3.471 \\
\hline $3 / 28 / 2006$ & $1: 00$ & 11.816 & 11.7 & 7.43 & 4.698 & 3.03 & 2.937 & 10.729 & 5.952 & 4.063 & 6.598 & 3.486 \\
\hline $3 / 28 / 2006$ & $2: 00$ & 11.818 & 11.705 & 7.438 & 4.704 & 3.043 & 2.947 & 10.732 & 5.956 & 4.098 & 6.624 & 3.501 \\
\hline $3 / 28 / 2006$ & $3: 00$ & 11.82 & 11.705 & 7.445 & 4.71 & 3.043 & 2.955 & 10.734 & 5.957 & 4.124 & 6.645 & 3.515 \\
\hline $3 / 28 / 2006$ & $4: 00$ & 11.823 & 11.707 & 7.449 & 4.717 & 3.062 & 2.964 & 10.737 & 5.96 & 4.151 & 6.668 & 3.527 \\
\hline $3 / 28 / 2006$ & $5: 00$ & 11.82 & 11.705 & 7.454 & 4.722 & 3.067 & 2.973 & 10.74 & 5.962 & 4.182 & 6.688 & 3.537 \\
\hline $3 / 28 / 2006$ & $6: 00$ & 11.828 & 11.712 & 7.467 & 4.729 & 3.083 & 2.988 & 10.744 & 5.965 & 4.213 & 6.712 & 3.555 \\
\hline 3/28/2006 & $7: 00$ & 11.832 & 11.718 & 7.476 & 4.737 & 3.092 & 3.003 & 10.747 & 5.97 & 4.243 & 6.73 & 3.568 \\
\hline $3 / 28 / 2006$ & $8: 00$ & 11.835 & 11.723 & 7.485 & 4.745 & 3.106 & 3.012 & 10.752 & 5.973 & 4.269 & 6.747 & 3.578 \\
\hline $3 / 28 / 2006$ & $9: 00$ & 11.843 & 11.729 & 7.495 & 4.753 & 3.11 & 3.026 & 10.755 & 5.978 & 4.292 & 6.764 & 3.588 \\
\hline $3 / 28 / 2006$ & $10: 00$ & 11.847 & 11.734 & 7.503 & 4.761 & 3.127 & 3.036 & 10.761 & 5.983 & 4.315 & 6.777 & 3.598 \\
\hline 3/28/2006 & $11: 00$ & 11.853 & 11.74 & 7.51 & 4.768 & 3.132 & 3.045 & 10.766 & 5.982 & 4.337 & 6.792 & 3.607 \\
\hline $3 / 28 / 2006$ & $12: 00$ & 11.855 & 11.74 & 7.513 & 4.775 & 3.138 & 3.052 & 10.77 & 5.988 & 4.358 & 6.804 & 3.614 \\
\hline $3 / 28 / 2006$ & $13: 00$ & 11.855 & 11.736 & 7.512 & 4.781 & 3.143 & 3.054 & 10.772 & 5.989 & 4.373 & 6.813 & 3.615 \\
\hline $3 / 28 / 2006$ & $14: 00$ & 11.852 & 11.731 & 7.512 & 4.784 & 3.148 & 3.057 & 10.774 & 5.991 & 4.39 & 6.823 & 3.621 \\
\hline $3 / 28 / 2006$ & $15: 00$ & 11.846 & 11.722 & 7.507 & 4.79 & 3.151 & 3.056 & 10.776 & 5.99 & 4.406 & 6.834 & 3.625 \\
\hline $3 / 28 / 2006$ & $16: 00$ & 11.84 & 11.714 & 7.507 & 4.794 & 3.15 & 3.058 & 10.777 & 5.992 & 4.428 & 6.841 & 3.631 \\
\hline $3 / 28 / 2006$ & $17: 00$ & 11.836 & 11.709 & 7.504 & 4.796 & 3.151 & 3.057 & 10.78 & 5.994 & 4.447 & 6.852 & 3.638 \\
\hline $3 / 28 / 2006$ & $18: 00$ & 11.832 & 11.702 & 7.502 & 4.799 & 3.144 & 3.058 & 10.78 & 5.995 & 4.466 & 6.858 & 3.642 \\
\hline $3 / 28 / 2006$ & $19: 00$ & 11.825 & 11.696 & 7.502 & 4.802 & 3.143 & 3.058 & 10.784 & 5.996 & 4.484 & 6.865 & 3.648 \\
\hline $3 / 28 / 2006$ & $20: 00$ & 11.823 & 11.692 & 7.505 & 4.804 & 3.152 & 3.06 & 10.784 & 5.998 & 4.503 & 6.874 & 3.659 \\
\hline $3 / 28 / 2006$ & $21: 00$ & 11.82 & 11.694 & 7.509 & 4.809 & 3.154 & 3.067 & 10.786 & 5.998 & 4.528 & 6.882 & 3.668 \\
\hline $3 / 28 / 2006$ & $22: 00$ & 11.823 & 11.696 & 7.513 & 4.812 & 3.166 & 3.073 & 10.79 & 6.003 & 4.548 & 6.887 & 3.676 \\
\hline $3 / 28 / 2006$ & $23: 00$ & 11.822 & 11.692 & 7.513 & 4.815 & 3.171 & 3.076 & 10.791 & 6.004 & 4.564 & 6.894 & 3.68 \\
\hline $3 / 29 / 2006$ & $0: 00$ & 11.82 & 11.691 & 7.511 & 4.818 & 3.167 & 3.078 & 10.791 & 6.002 & 4.584 & 6.897 & 3.684 \\
\hline $3 / 29 / 2006$ & $1: 00$ & 11.812 & 11.683 & 7.509 & 4.817 & 3.169 & 3.077 & 10.79 & 6.005 & 4.596 & 6.903 & 3.687 \\
\hline
\end{tabular}


TABLE S1.2 (Cont.)

Water Level (ft below top of casing) at Indicated Well

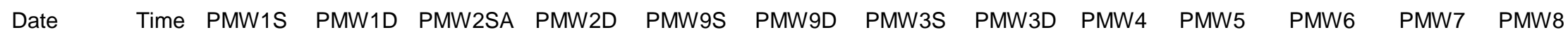

\begin{tabular}{|c|c|c|c|c|c|c|c|c|c|c|c|c|}
\hline 3/29/2006 & $2: 00$ & 11.815 & 11.685 & 7.513 & 4.823 & 3.173 & 3.081 & 10.793 & 6.006 & 4.618 & 6.909 & 3.693 \\
\hline $3 / 29 / 2006$ & $3: 00$ & 11.81 & 11.68 & 7.511 & 4.821 & 3.175 & 3.081 & 10.796 & 6.008 & 4.63 & 6.915 & 3.694 \\
\hline $3 / 29 / 2006$ & $4: 00$ & 11.807 & 11.672 & 7.509 & 4.823 & 3.171 & 3.079 & 10.794 & 6.009 & 4.642 & 6.917 & 3.697 \\
\hline 3/29/2006 & $5: 00$ & 11.805 & 11.67 & 7.507 & 4.826 & 3.176 & 3.083 & 10.794 & 6.006 & 4.659 & 6.923 & 3.701 \\
\hline 3/29/2006 & $6: 00$ & 11.802 & 11.669 & 7.509 & 4.827 & 3.178 & 3.084 & 10.796 & 6.012 & 4.668 & 6.926 & 3.706 \\
\hline 3/29/2006 & $7: 00$ & 11.802 & 11.665 & 7.509 & 4.828 & 3.171 & 3.089 & 10.796 & 6.013 & 4.678 & 6.929 & 3.708 \\
\hline $3 / 29 / 2006$ & $8: 00$ & 11.8 & 11.667 & 7.513 & 4.832 & 3.176 & 3.092 & 10.797 & 6.014 & 4.693 & 6.934 & 3.713 \\
\hline 3/29/2006 & $9: 00$ & 11.8 & 11.667 & 7.511 & 4.832 & 3.179 & 3.092 & 10.793 & 6.014 & 4.707 & 6.935 & 3.716 \\
\hline 3/29/2006 & $10: 00$ & 11.8 & 11.667 & 7.514 & 4.837 & 3.183 & 3.095 & 10.797 & 6.022 & 4.719 & 6.934 & 3.72 \\
\hline 3/29/2006 & $11: 00$ & 11.798 & 11.665 & 7.513 & 4.836 & 3.189 & 3.096 & 10.801 & 6.018 & 4.729 & 6.944 & 3.723 \\
\hline 3/29/2006 & $12: 00$ & 11.793 & 11.658 & 7.507 & 4.835 & 3.178 & 3.091 & 10.812 & 6.015 & 4.736 & 6.947 & 3.713 \\
\hline 3/29/2006 & $13: 00$ & 11.788 & 11.647 & 7.499 & 4.832 & 3.171 & 3.084 & 10.798 & 6.012 & 4.739 & 6.948 & 3.713 \\
\hline 3/29/2006 & $14: 00$ & 11.785 & 11.636 & 7.491 & 4.83 & 3.163 & 3.078 & 10.797 & 6.01 & 4.743 & 6.943 & 3.712 \\
\hline 3/29/2006 & $15: 00$ & 11.771 & 11.621 & 7.48 & 4.825 & 3.158 & 3.068 & 10.796 & 6.009 & 4.747 & 6.941 & 3.723 \\
\hline $3 / 29 / 2006$ & $16: 00$ & 11.763 & 11.618 & 7.477 & 4.826 & 3.16 & 3.067 & 10.791 & 6.011 & 4.763 & 6.947 & 3.731 \\
\hline 3/29/2006 & $17: 00$ & 11.758 & 11.607 & 7.468 & 4.825 & 3.16 & 3.063 & 10.79 & 6.013 & 4.773 & 6.94 & 3.744 \\
\hline $3 / 29 / 2006$ & $18: 00$ & 11.75 & 11.601 & 7.467 & 4.823 & 3.156 & 3.06 & 10.793 & 6.013 & 4.783 & 6.951 & 3.751 \\
\hline 3/29/2006 & $19: 00$ & 11.746 & 11.598 & 7.471 & 4.823 & 3.152 & 3.061 & 10.791 & 6.015 & 4.793 & 6.952 & 3.76 \\
\hline $3 / 29 / 2006$ & $20: 00$ & 11.738 & 11.598 & 7.471 & 4.823 & 3.16 & 3.063 & 10.793 & 6.02 & 4.805 & 6.956 & 3.768 \\
\hline 3/29/2006 & $21: 00$ & 11.737 & 11.599 & 7.476 & 4.826 & 3.166 & 3.069 & 10.796 & 6.02 & 4.817 & 6.958 & 3.776 \\
\hline $3 / 29 / 2006$ & $22: 00$ & 11.738 & 11.601 & 7.485 & 4.827 & 3.175 & 3.078 & 10.801 & 6.024 & 4.828 & 6.96 & 3.781 \\
\hline $3 / 29 / 2006$ & $23: 00$ & 11.738 & 11.599 & 7.484 & 4.827 & 3.173 & 3.081 & 10.796 & 6.024 & 4.834 & 6.962 & 3.782 \\
\hline 3/30/2006 & $0: 00$ & 11.738 & 11.601 & 7.488 & 4.83 & 3.181 & 3.082 & 10.797 & 6.021 & 4.843 & 6.964 & 3.787 \\
\hline $3 / 30 / 2006$ & $1: 00$ & 11.735 & 11.598 & 7.482 & 4.833 & 3.172 & 3.077 & 10.797 & 6.024 & 4.854 & 6.968 & 3.78 \\
\hline $3 / 30 / 2006$ & $2: 00$ & 11.736 & 11.596 & 7.484 & 4.832 & 3.169 & 3.079 & 10.797 & 6.019 & 4.858 & 6.964 & 3.783 \\
\hline $3 / 30 / 2006$ & $3: 00$ & 11.728 & 11.59 & 7.476 & 4.83 & 3.172 & 3.075 & 10.796 & 6.023 & 4.861 & 6.969 & 3.78 \\
\hline 3/30/2006 & $4: 00$ & 11.728 & 11.581 & 7.46 & 4.827 & 3.153 & 3.067 & 10.788 & 6.023 & 4.861 & 6.944 & 3.774 \\
\hline $3 / 30 / 2006$ & $5: 00$ & 11.719 & 11.579 & 7.466 & 4.825 & 3.151 & 3.063 & 10.798 & 6.017 & 4.86 & 6.959 & 3.769 \\
\hline 3/30/2006 & $6: 00$ & 11.718 & 11.579 & 7.467 & 4.826 & 3.159 & 3.066 & 10.796 & 6.023 & 4.859 & 6.962 & 3.765 \\
\hline $3 / 30 / 2006$ & $7: 00$ & 11.727 & 11.588 & 7.48 & 4.828 & 3.164 & 3.077 & 10.803 & 6.029 & 4.869 & 6.974 & 3.772 \\
\hline 3/30/2006 & $8: 00$ & 11.715 & 11.581 & 7.506 & 4.83 & 3.169 & 3.079 & 10.826 & 6.021 & 4.864 & 6.99 & 3.76 \\
\hline $3 / 30 / 2006$ & $9: 00$ & 11.703 & 11.579 & 7.485 & 4.826 & 3.161 & 3.069 & 10.841 & 6.019 & 4.856 & 7.001 & 3.737 \\
\hline $3 / 30 / 2006$ & $10: 00$ & 11.701 & 11.574 & 7.482 & 4.823 & 3.161 & 3.067 & 10.837 & 6.018 & 4.85 & 6.965 & 3.721 \\
\hline $3 / 30 / 2006$ & $11: 00$ & 11.698 & 11.574 & 7.471 & 4.823 & 3.161 & 3.065 & 10.798 & 6.018 & 4.845 & 6.962 & 3.715 \\
\hline
\end{tabular}


TABLE S1.2 (Cont.)

Water Level (ft below top of casing) at Indicated Well

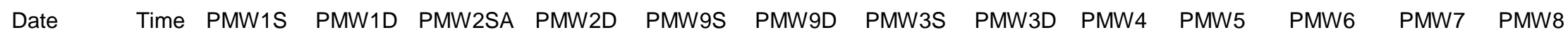

\begin{tabular}{|c|c|c|c|c|c|c|c|c|c|c|c|c|}
\hline 3/30/2006 & $12: 00$ & 11.691 & 11.57 & 7.464 & 4.824 & 3.155 & 3.059 & 10.796 & 6.018 & 4.838 & 6.954 & 3.708 \\
\hline $3 / 30 / 2006$ & $13: 00$ & 11.679 & 11.561 & 7.451 & 4.823 & 3.135 & 3.048 & 10.791 & 6.012 & 4.827 & 6.949 & 3.698 \\
\hline $3 / 30 / 2006$ & $14: 00$ & 11.662 & 11.548 & 7.437 & 4.812 & 3.13 & 3.037 & 10.79 & 6.01 & 4.821 & 6.954 & 3.697 \\
\hline 3/30/2006 & $15: 00$ & 11.664 & 11.461 & 7.394 & 4.808 & 3.055 & 2.984 & 10.739 & 5.979 & 4.796 & 6.89 & 3.633 \\
\hline 3/30/2006 & $16: 00$ & 11.602 & 11.519 & 7.354 & 4.823 & 2.945 & 2.87 & 10.752 & 5.358 & 4.04 & 6.528 & 2.797 \\
\hline 3/30/2006 & $17: 00$ & 11.604 & 11.464 & 7.259 & 4.79 & 2.879 & 2.792 & 10.62 & 5.206 & 3.76 & 6.345 & 2.58 \\
\hline $3 / 30 / 2006$ & $18: 00$ & 11.52 & 11.499 & 7.274 & 4.73 & 2.78 & 2.696 & 10.613 & 5.044 & 3.583 & 6.203 & 2.505 \\
\hline 3/30/2006 & $19: 00$ & 11.447 & 11.492 & 7.214 & 4.664 & 2.744 & 2.662 & 10.549 & 5.08 & 3.554 & 6.153 & 2.53 \\
\hline 3/30/2006 & $20: 00$ & 11.384 & 11.475 & 7.194 & 4.595 & 2.734 & 2.653 & 10.505 & 5.127 & 3.554 & 6.133 & 2.566 \\
\hline 3/30/2006 & $21: 00$ & 11.257 & 11.47 & 7.196 & 4.542 & 2.752 & 2.66 & 10.472 & 5.178 & 3.583 & 6.142 & 2.605 \\
\hline $3 / 30 / 2006$ & $22: 00$ & 11.26 & 11.475 & 7.213 & 4.502 & 2.777 & 2.684 & 10.447 & 5.233 & 3.621 & 6.169 & 2.641 \\
\hline $3 / 30 / 2006$ & $23: 00$ & 11.307 & 11.488 & 7.218 & 4.471 & 2.803 & 2.71 & 10.421 & 5.277 & 3.659 & 6.184 & 2.667 \\
\hline $3 / 31 / 2006$ & $0: 00$ & 11.348 & 11.501 & 7.243 & 4.446 & 2.811 & 2.727 & 10.39 & 5.311 & 3.694 & 6.235 & 2.695 \\
\hline $3 / 31 / 2006$ & $1: 00$ & 11.383 & 11.505 & 7.248 & 4.429 & 2.83 & 2.739 & 10.399 & 5.34 & 3.729 & 6.273 & 2.719 \\
\hline $3 / 31 / 2006$ & $2: 00$ & 11.418 & 11.512 & 7.26 & 4.418 & 2.837 & 2.755 & 10.389 & 5.366 & 3.768 & 6.318 & 2.746 \\
\hline 3/31/2006 & $3: 00$ & 11.448 & 11.521 & 7.268 & 4.409 & 2.856 & 2.766 & 10.382 & 5.391 & 3.813 & 6.369 & 2.774 \\
\hline $3 / 31 / 2006$ & $4: 00$ & 11.478 & 11.525 & 7.275 & 4.409 & 2.866 & 2.78 & 10.376 & 5.416 & 3.855 & 6.424 & 2.803 \\
\hline $3 / 31 / 2006$ & $5: 00$ & 11.498 & 11.528 & 7.282 & 4.407 & 2.881 & 2.793 & 10.375 & 5.437 & 3.898 & 6.467 & 2.829 \\
\hline $3 / 31 / 2006$ & $6: 00$ & 11.523 & 11.536 & 7.293 & 4.411 & 2.894 & 2.808 & 10.374 & 5.46 & 3.943 & 6.502 & 2.862 \\
\hline 3/31/2006 & $7: 00$ & 11.523 & 11.546 & 7.307 & 4.418 & 2.905 & 2.822 & 10.37 & 5.478 & 3.983 & 6.531 & 2.889 \\
\hline $3 / 31 / 2006$ & $8: 00$ & 11.535 & 11.548 & 7.311 & 4.422 & 2.919 & 2.83 & 10.372 & 5.496 & 4.017 & 6.558 & 2.913 \\
\hline 3/31/2006 & $9: 00$ & 11.546 & 11.546 & 7.32 & 4.431 & 2.929 & 2.844 & 10.37 & 5.516 & 4.06 & 6.586 & 2.936 \\
\hline 3/31/2006 & $10: 00$ & 11.562 & 11.565 & 7.331 & 4.442 & 2.947 & 2.857 & 10.374 & 5.532 & 4.1 & 6.61 & 2.965 \\
\hline $3 / 31 / 2006$ & $11: 00$ & 11.575 & 11.574 & 7.346 & 4.456 & 2.956 & 2.875 & 10.379 & 5.549 & 4.139 & 6.632 & 2.991 \\
\hline $3 / 31 / 2006$ & $12: 00$ & 11.594 & 11.579 & 7.358 & 4.467 & 2.979 & 2.889 & 10.383 & 5.566 & 4.178 & 6.656 & 3.02 \\
\hline $3 / 31 / 2006$ & $13: 00$ & 11.605 & 11.588 & 7.369 & 4.48 & 2.987 & 2.9 & 10.389 & 5.579 & 4.212 & 6.676 & 3.046 \\
\hline $3 / 31 / 2006$ & $14: 00$ & 11.615 & 11.598 & 7.375 & 4.492 & 2.996 & 2.914 & 10.391 & 5.596 & 4.249 & 6.699 & 3.08 \\
\hline $3 / 31 / 2006$ & $15: 00$ & 11.622 & 11.599 & 7.376 & 4.504 & 3.004 & 2.92 & 10.397 & 5.607 & 4.28 & 6.714 & 3.105 \\
\hline $3 / 31 / 2006$ & $16: 00$ & 11.63 & 11.601 & 7.39 & 4.519 & 3.016 & 2.927 & 10.403 & 5.621 & 4.313 & 6.733 & 3.138 \\
\hline $3 / 31 / 2006$ & $17: 00$ & 11.644 & 11.608 & 7.395 & 4.53 & 3.028 & 2.942 & 10.412 & 5.637 & 4.346 & 6.751 & 3.17 \\
\hline $3 / 31 / 2006$ & $18: 00$ & 11.652 & 11.616 & 7.403 & 4.546 & 3.041 & 2.953 & 10.421 & 5.649 & 4.379 & 6.766 & 3.202 \\
\hline $3 / 31 / 2006$ & $19: 00$ & 11.661 & 11.625 & 7.413 & 4.558 & 3.041 & 2.963 & 10.427 & 5.665 & 4.413 & 6.784 & 3.23 \\
\hline $3 / 31 / 2006$ & $20: 00$ & 11.669 & 11.629 & 7.421 & 4.572 & 3.064 & 2.975 & 10.437 & 5.676 & 4.442 & 6.797 & 3.254 \\
\hline $3 / 31 / 2006$ & $21: 00$ & 11.674 & 11.638 & 7.432 & 4.588 & 3.073 & 2.986 & 10.446 & 5.69 & 4.471 & 6.811 & 3.276 \\
\hline
\end{tabular}


TABLE S1.2 (Cont.)

Water Level (ft below top of casing) at Indicated Well

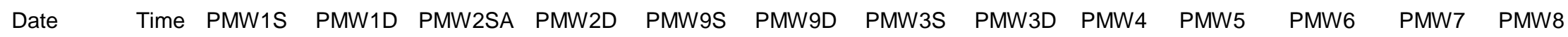

\begin{tabular}{|c|c|c|c|c|c|c|c|c|c|c|c|c|}
\hline 3/31/2006 & $22: 00$ & 11.683 & 11.643 & 7.439 & 4.6 & 3.08 & 2.995 & 10.454 & 5.699 & 4.495 & 6.824 & 3.296 \\
\hline 3/31/2006 & 23:00 & 11.686 & 11.643 & 7.441 & 4.612 & 3.076 & 2.998 & 10.459 & 5.712 & 4.516 & 6.832 & 3.309 \\
\hline $4 / 1 / 2006$ & 0:00 & 11.693 & 11.649 & 7.452 & 4.626 & 3.098 & 3.01 & 10.468 & 5.723 & 4.542 & 6.845 & 3.33 \\
\hline 4/1/2006 & $1: 00$ & 11.699 & 11.652 & 7.457 & 4.634 & 3.095 & 3.015 & 10.472 & 5.731 & 4.558 & 6.853 & 3.345 \\
\hline $4 / 1 / 2006$ & $2: 00$ & 11.706 & 11.654 & 7.459 & 4.646 & 3.102 & 3.022 & 10.48 & 5.738 & 4.576 & 6.861 & 3.36 \\
\hline 4/1/2006 & $3: 00$ & 11.708 & 11.652 & 7.46 & 4.654 & 3.109 & 3.024 & 10.487 & 5.746 & 4.591 & 6.868 & 3.372 \\
\hline $4 / 1 / 2006$ & $4: 00$ & 11.708 & 11.649 & 7.459 & 4.663 & 3.113 & 3.026 & 10.493 & 5.752 & 4.605 & 6.874 & 3.384 \\
\hline $4 / 1 / 2006$ & $5: 00$ & 11.709 & 11.647 & 7.459 & 4.672 & 3.116 & 3.026 & 10.499 & 5.76 & 4.618 & 6.88 & 3.398 \\
\hline $4 / 1 / 2006$ & $6: 00$ & 11.71 & 11.645 & 7.461 & 4.679 & 3.11 & 3.029 & 10.503 & 5.77 & 4.635 & 6.886 & 3.413 \\
\hline 4/1/2006 & $7: 00$ & 11.713 & 11.65 & 7.47 & 4.688 & 3.126 & 3.037 & 10.51 & 5.78 & 4.651 & 6.895 & 3.429 \\
\hline $4 / 1 / 2006$ & 8:00 & 11.718 & 11.656 & 7.477 & 4.696 & 3.134 & 3.047 & 10.55 & 5.785 & 4.668 & 6.903 & 3.444 \\
\hline 4/1/2006 & 9:00 & 11.721 & 11.656 & 7.477 & 4.703 & 3.135 & 3.05 & 10.525 & 5.792 & 4.681 & 6.908 & 3.451 \\
\hline 4/1/2006 & $10: 00$ & 11.721 & 11.65 & 7.476 & 4.709 & 3.14 & 3.052 & 10.528 & 5.796 & 4.689 & 6.912 & 3.457 \\
\hline 4/1/2006 & $11: 00$ & 11.723 & 11.65 & 7.478 & 4.715 & 3.138 & 3.052 & 10.531 & 5.804 & 4.704 & 6.917 & 3.465 \\
\hline $4 / 1 / 2006$ & $12: 00$ & 11.718 & 11.641 & 7.472 & 4.719 & 3.138 & 3.05 & 10.539 & 5.806 & 4.711 & 6.921 & 3.476 \\
\hline $4 / 1 / 2006$ & $13: 00$ & 11.713 & 11.632 & 7.468 & 4.725 & 3.135 & 3.048 & 10.54 & 5.809 & 4.72 & 6.922 & 3.489 \\
\hline $4 / 1 / 2006$ & $14: 00$ & 11.698 & 11.614 & 7.452 & 4.725 & 3.116 & 3.035 & 10.54 & 5.811 & 4.725 & 6.923 & 3.498 \\
\hline $4 / 1 / 2006$ & $15: 00$ & 11.683 & 11.594 & 7.437 & 4.723 & 3.104 & 3.022 & 10.542 & 5.81 & 4.728 & 6.92 & 3.512 \\
\hline $4 / 1 / 2006$ & $16: 00$ & 11.671 & 11.581 & 7.43 & 4.723 & 3.106 & 3.019 & 10.541 & 5.813 & 4.741 & 6.919 & 3.532 \\
\hline $4 / 1 / 2006$ & $17: 00$ & 11.666 & 11.574 & 7.43 & 4.723 & 3.108 & 3.018 & 10.547 & 5.818 & 4.752 & 6.926 & 3.553 \\
\hline $4 / 1 / 2006$ & $18: 00$ & 11.654 & 11.563 & 7.42 & 4.722 & 3.103 & 3.013 & 10.544 & 5.818 & 4.761 & 6.92 & 3.566 \\
\hline $4 / 1 / 2006$ & $19: 00$ & 11.646 & 11.554 & 7.416 & 4.723 & 3.09 & 3.007 & 10.546 & 5.824 & 4.77 & 6.927 & 3.584 \\
\hline 4/1/2006 & $20: 00$ & 11.643 & 11.55 & 7.419 & 4.723 & 3.096 & 3.011 & 10.55 & 5.829 & 4.784 & 6.931 & 3.6 \\
\hline $4 / 1 / 2006$ & $21: 00$ & 11.629 & 11.539 & 7.41 & 4.721 & 3.096 & 3.003 & 10.553 & 5.827 & 4.788 & 6.925 & 3.606 \\
\hline 4/1/2006 & $22: 00$ & 11.626 & 11.534 & 7.408 & 4.722 & 3.097 & 3 & 10.555 & 5.83 & 4.798 & 6.925 & 3.614 \\
\hline $4 / 1 / 2006$ & 23:00 & 11.628 & 11.534 & 7.41 & 4.724 & 3.093 & 3.008 & 10.558 & 5.835 & 4.808 & 6.933 & 3.625 \\
\hline $4 / 2 / 2006$ & $0: 00$ & 11.636 & 11.505 & 7.375 & 4.723 & 3.102 & 3.004 & 10.525 & 5.836 & 4.818 & 6.9 & 3.631 \\
\hline $4 / 2 / 2006$ & $1: 00$ & 11.589 & 11.563 & 7.441 & 4.725 & 3.04 & 2.99 & 10.585 & 5.698 & 4.728 & 6.961 & 3.29 \\
\hline $4 / 2 / 2006$ & $2: 00$ & 11.431 & 11.616 & 7.468 & 4.73 & 2.985 & 2.916 & 10.634 & 5.62 & 4.411 & 6.928 & 2.985 \\
\hline $4 / 2 / 2006$ & 3:00 & 11.351 & 11.598 & 7.427 & 4.709 & 2.96 & 2.888 & 10.62 & 5.596 & 4.259 & 6.845 & 2.885 \\
\hline $4 / 2 / 2006$ & $4: 00$ & 11.29 & 11.479 & 7.319 & 4.685 & 2.96 & 2.879 & 10.596 & 5.592 & 4.211 & 6.784 & 2.856 \\
\hline $4 / 2 / 2006$ & 5:00 & 11.194 & 11.49 & 7.334 & 4.66 & 2.949 & 2.87 & 10.574 & 5.552 & 4.121 & 6.795 & 2.797 \\
\hline $4 / 2 / 2006$ & $6: 00$ & 11.127 & 11.453 & 7.308 & 4.628 & 2.94 & 2.856 & 10.543 & 5.532 & 4.101 & 6.727 & 2.796 \\
\hline $4 / 2 / 2006$ & 7:00 & 11.089 & 11.452 & 7.31 & 4.601 & 2.935 & 2.858 & 10.452 & 5.534 & 4.121 & 6.733 & 2.821 \\
\hline
\end{tabular}


TABLE S1.2 (Cont.)

Water Level (ft below top of casing) at Indicated Well

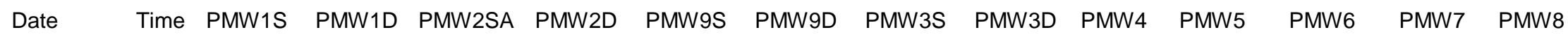

\begin{tabular}{|c|c|c|c|c|c|c|c|c|c|c|c|c|c|c|}
\hline $4 / 2 / 2006$ & 8:00 & 11.072 & 11.443 & & 7.306 & & 4.577 & 2.933 & 2.856 & 10.443 & 5.531 & 4.146 & 6.738 & 2.847 \\
\hline 4/2/2006 & 9:00 & 11.054 & 11.433 & & 7.296 & & 4.552 & 2.929 & 2.849 & 10.433 & 5.532 & 4.165 & 6.739 & 2.874 \\
\hline $4 / 2 / 2006$ & $10: 00$ & 11.041 & 11.428 & & 7.289 & & 4.537 & 2.925 & 2.846 & 10.427 & 5.534 & 4.186 & 6.743 & 2.902 \\
\hline $4 / 2 / 2006$ & $11: 00$ & 11.058 & 11.421 & & 7.288 & & 4.522 & 2.924 & 2.845 & 10.423 & 5.536 & 4.211 & 6.746 & 2.93 \\
\hline $4 / 2 / 2006$ & $12: 00$ & 11.195 & 11.424 & & 7.288 & & 4.513 & 2.934 & 2.849 & 10.418 & 5.547 & 4.241 & 6.752 & 2.962 \\
\hline $4 / 2 / 2006$ & $13: 00$ & 11.269 & 11.43 & & 7.296 & & 4.507 & 2.934 & 2.855 & 10.416 & 5.554 & 4.268 & 6.757 & 2.986 \\
\hline $4 / 2 / 2006$ & $14: 00$ & 11.299 & 11.419 & & 7.281 & & 4.499 & 2.942 & 2.851 & 10.391 & 5.556 & 4.26 & 6.74 & 2.928 \\
\hline $4 / 2 / 2006$ & $15: 00$ & 11.084 & 11.443 & & 7.294 & & 4.512 & 2.849 & 2.767 & 10.399 & 5.159 & 3.999 & 6.71 & 2.638 \\
\hline 4/2/2006 & $16: 00$ & 10.806 & 11.392 & & 7.239 & & 4.503 & 2.834 & 2.746 & 10.378 & 5.108 & 3.887 & 6.527 & 2.567 \\
\hline $4 / 2 / 2006$ & $17: 00$ & 10.701 & 11.375 & & 7.23 & & 4.479 & 2.828 & 2.744 & 10.325 & 5.132 & 3.86 & 6.508 & 2.582 \\
\hline $4 / 2 / 2006$ & $18: 00$ & 10.666 & 11.363 & & 7.23 & & 4.456 & 2.824 & 2.743 & 10.299 & 5.151 & 3.845 & 6.487 & 2.595 \\
\hline $4 / 2 / 2006$ & 19:00 & 10.664 & 11.377 & & 7.219 & & 4.438 & 2.781 & 2.72 & 10.274 & 4.966 & 3.718 & 6.46 & 2.492 \\
\hline $4 / 2 / 2006$ & 20:00 & 10.604 & 11.348 & & 7.16 & & 4.421 & 2.688 & 2.617 & 10.21 & 4.75 & 3.597 & 6.36 & 2.433 \\
\hline $4 / 2 / 2006$ & $21: 00$ & 10.574 & 11.342 & & 7.132 & & 4.387 & 2.649 & 2.564 & 10.177 & 4.666 & 3.525 & 6.305 & 2.422 \\
\hline 4/2/2006 & $22: 00$ & 10.583 & 11.375 & & 7.095 & & 4.351 & 2.629 & 2.538 & 10.144 & 4.678 & 3.502 & 6.203 & 2.448 \\
\hline $4 / 2 / 2006$ & $23: 00$ & 10.588 & 11.375 & & 7.081 & & 4.316 & 2.63 & 2.538 & 10.113 & 4.732 & 3.512 & 6.198 & 2.488 \\
\hline $4 / 3 / 2006$ & $0: 00$ & 10.598 & 11.374 & & 7.083 & & 4.287 & 2.629 & 2.547 & 10.089 & 4.786 & 3.542 & 6.214 & 2.53 \\
\hline 4/3/2006 & 1:00 & 10.615 & 11.377 & & 7.094 & & 4.265 & 2.653 & 2.568 & 10.065 & 4.835 & 3.59 & 6.24 & 2.568 \\
\hline $4 / 3 / 2006$ & $2: 00$ & 10.615 & 11.381 & & 7.101 & & 4.25 & 2.677 & 2.589 & 10.048 & 4.879 & 3.637 & 6.275 & 2.602 \\
\hline 4/3/2006 & 3:00 & 10.733 & 11.401 & & 7.112 & & 4.24 & 2.687 & 2.605 & 10.025 & 4.923 & 3.685 & 6.307 & 2.636 \\
\hline $4 / 3 / 2006$ & $4: 00$ & 10.804 & 11.412 & & 7.121 & & 4.236 & 2.714 & 2.625 & 10.013 & 4.959 & 3.729 & 6.345 & 2.666 \\
\hline 4/3/2006 & 5:00 & 10.844 & 11.423 & & 7.135 & & 4.236 & 2.731 & 2.645 & 10 & 4.995 & 3.777 & 6.379 & 2.698 \\
\hline $4 / 3 / 2006$ & $6: 00$ & 10.884 & 11.43 & & 7.148 & & 4.24 & 2.749 & 2.662 & 9.991 & 5.031 & 3.821 & 6.41 & 2.729 \\
\hline 4/3/2006 & 7:00 & 10.956 & 11.445 & & 7.167 & & 4.247 & 2.768 & 2.683 & 9.988 & 5.066 & 3.87 & 6.446 & 2.763 \\
\hline 4/3/2006 & $8: 00$ & 11.033 & 11.456 & & 7.18 & & 4.258 & 2.789 & 2.705 & 9.984 & 5.096 & 3.917 & 6.483 & 2.796 \\
\hline $4 / 3 / 2006$ & $9: 00$ & 11.082 & 11.465 & & 7.19 & & 4.267 & 2.8 & 2.721 & 9.979 & 5.124 & 3.959 & 6.514 & 2.826 \\
\hline $4 / 3 / 2006$ & $10: 00$ & 11.121 & 11.474 & & 7.203 & & 4.282 & 2.816 & 2.735 & 9.981 & 5.155 & 4.005 & 6.543 & 2.857 \\
\hline $4 / 3 / 2006$ & $11: 00$ & 11.146 & 11.477 & & 7.214 & & 4.295 & 2.837 & 2.75 & 9.984 & 5.181 & 4.044 & 6.57 & 2.889 \\
\hline $4 / 3 / 2006$ & $12: 00$ & 11.169 & 11.481 & & 7.219 & & 4.307 & 2.847 & 2.761 & 9.983 & 5.204 & 4.081 & 6.595 & 2.924 \\
\hline $4 / 3 / 2006$ & $13: 00$ & 11.186 & 11.479 & & 7.223 & & 4.318 & 2.859 & 2.771 & 9.985 & 5.228 & 4.118 & 6.612 & 2.962 \\
\hline $4 / 3 / 2006$ & $14: 00$ & 11.194 & 11.472 & & 7.223 & & 4.333 & 2.855 & 2.777 & 9.99 & 5.247 & 4.154 & 6.629 & 3.005 \\
\hline $4 / 3 / 2006$ & $15: 00$ & & & & & & & & & & & & & \\
\hline 6/30/06 & 20:00 & 12.242 & 12.314 & 9.201 & 8.276 & 5.829 & 5.852 & 3.781 & 3.898 & 10.609 & 6.75 & 5.37 & 7.376 & 4.721 \\
\hline $7 / 1 / 06$ & $0: 00$ & 12.251 & 12.33 & 9.184 & 8.271 & 5.794 & 5.857 & 3.762 & 3.88 & 10.584 & 6.694 & 5.258 & 7.343 & 4.64 \\
\hline
\end{tabular}


Water Level (ft below top of casing) at Indicated Well

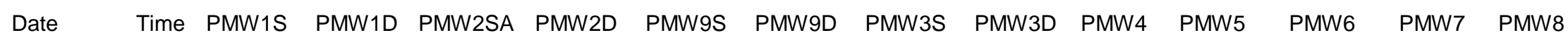

\begin{tabular}{|c|c|c|c|c|c|c|c|c|c|c|c|c|c|c|}
\hline 7/1/06 & 4:00 & 12.254 & 12.335 & 9.177 & 8.267 & 5.768 & 5.852 & 3.748 & 3.868 & 10.565 & 6.663 & 5.191 & 7.327 & 4.588 \\
\hline $7 / 1 / 06$ & $8: 00$ & 12.263 & 12.344 & 9.191 & 8.281 & 5.78 & 5.843 & 3.776 & 3.891 & 10.57 & 6.71 & 5.251 & 7.348 & 4.637 \\
\hline $7 / 1 / 06$ & $12: 00$ & 12.279 & 12.353 & 9.226 & 8.309 & 5.852 & 5.855 & 3.83 & 3.94 & 10.621 & 6.827 & 5.44 & 7.406 & 4.796 \\
\hline $7 / 1 / 06$ & $16: 00$ & 12.284 & 12.349 & 9.24 & 8.313 & 5.883 & 5.874 & 3.844 & 3.952 & 10.64 & 6.862 & 5.529 & 7.422 & 4.868 \\
\hline $7 / 1 / 06$ & $20: 00$ & 12.291 & 12.363 & 9.24 & 8.318 & 5.873 & 5.892 & 3.82 & 3.928 & 10.637 & 6.785 & 5.405 & 7.381 & 4.815 \\
\hline $7 / 2 / 06$ & $0: 00$ & 12.298 & 12.381 & 9.231 & 8.318 & 5.843 & 5.899 & 3.802 & 3.914 & 10.612 & 6.719 & 5.284 & 7.355 & 4.717 \\
\hline $7 / 2 / 06$ & $4: 00$ & 12.305 & 12.381 & 9.217 & 8.288 & 5.801 & 5.892 & 3.778 & 3.877 & 10.589 & 6.666 & 5.205 & 7.308 & 4.635 \\
\hline $7 / 2 / 06$ & $8: 00$ & 12.305 & 12.383 & 9.203 & 8.295 & 5.777 & 5.878 & 3.767 & 3.88 & 10.563 & 6.647 & 5.167 & 7.325 & 4.584 \\
\hline 7/2/06 & $12: 00$ & 12.31 & 12.381 & 9.226 & 8.309 & 5.806 & 5.864 & 3.797 & 3.907 & 10.582 & 6.722 & 5.286 & 7.364 & 4.644 \\
\hline $7 / 2 / 06$ & $16: 00$ & 12.307 & 12.367 & 9.247 & 8.316 & 5.862 & 5.869 & 3.827 & 3.936 & 10.635 & 6.815 & 5.433 & 7.406 & 4.768 \\
\hline $7 / 2 / 06$ & $20: 00$ & 12.307 & 12.369 & 9.24 & 8.313 & 5.866 & 5.883 & 3.806 & 3.915 & 10.633 & 6.757 & 5.34 & 7.371 & 4.742 \\
\hline $7 / 3 / 06$ & $0: 00$ & 12.298 & 12.363 & 9.212 & 8.288 & 5.813 & 5.881 & 3.767 & 3.875 & 10.596 & 6.68 & 5.212 & 7.329 & 4.635 \\
\hline $7 / 3 / 06$ & $4: 00$ & 12.296 & 12.365 & 9.196 & 8.281 & 5.782 & 5.871 & 3.751 & 3.858 & 10.568 & 6.64 & 5.137 & 7.313 & 4.565 \\
\hline $7 / 3 / 06$ & $8: 00$ & 12.305 & 12.381 & 9.203 & 8.288 & 5.77 & 5.862 & 3.762 & 3.87 & 10.554 & 6.647 & 5.156 & 7.322 & 4.544 \\
\hline $7 / 3 / 06$ & $12: 00$ & 12.319 & 12.388 & 9.226 & 8.306 & 5.803 & 5.86 & 3.788 & 3.872 & 10.579 & 6.691 & 5.209 & 7.343 & 4.605 \\
\hline $7 / 3 / 06$ & $16: 00$ & 12.317 & 12.379 & 9.219 & 8.297 & 5.796 & 5.862 & 3.769 & 3.879 & 10.577 & 6.677 & 5.158 & 7.329 & 4.577 \\
\hline $7 / 3 / 06$ & $20: 00$ & 12.321 & 12.393 & 9.212 & 8.299 & 5.777 & 5.867 & 3.764 & 3.873 & 10.558 & 6.64 & 5.102 & 7.315 & 4.528 \\
\hline $7 / 4 / 06$ & $0: 00$ & 12.324 & 12.393 & 9.201 & 8.292 & 5.749 & 5.86 & 3.75 & 3.858 & 10.54 & 6.607 & 5.053 & 7.301 & 4.479 \\
\hline $7 / 4 / 06$ & $4: 00$ & 12.324 & 12.397 & 9.194 & 8.29 & 5.728 & 5.852 & 3.746 & 3.854 & 10.523 & 6.589 & 5.023 & 7.297 & 4.444 \\
\hline $7 / 4 / 06$ & 8:00 & 12.333 & 12.411 & 9.21 & 8.306 & 5.747 & 5.85 & 3.776 & 3.882 & 10.526 & 6.624 & 5.13 & 7.329 & 4.472 \\
\hline $7 / 4 / 06$ & $12: 00$ & 12.347 & 12.416 & 9.25 & 8.337 & 5.817 & 5.857 & 3.827 & 3.929 & 10.584 & 6.75 & 5.33 & 7.388 & 4.637 \\
\hline $7 / 4 / 06$ & $16: 00$ & 12.347 & 12.402 & 9.269 & 8.342 & 5.878 & 5.874 & 3.848 & 3.95 & 10.637 & 6.824 & 5.449 & 7.422 & 4.759 \\
\hline $7 / 4 / 06$ & $20: 00$ & 12.356 & 12.421 & 9.271 & 8.351 & 5.882 & 5.902 & 3.834 & 3.938 & 10.635 & 6.761 & 5.337 & 7.383 & 4.719 \\
\hline $7 / 5 / 06$ & $0: 00$ & 12.363 & 12.428 & 9.259 & 8.342 & 5.843 & 5.913 & 3.811 & 3.917 & 10.609 & 6.691 & 5.225 & 7.355 & 4.626 \\
\hline $7 / 5 / 06$ & $4: 00$ & 12.359 & 12.428 & 9.24 & 8.327 & 5.81 & 5.904 & 3.792 & 3.896 & 10.584 & 6.652 & 5.151 & 7.334 & 4.556 \\
\hline $7 / 5 / 06$ & 8:00 & 12.368 & 12.437 & 9.25 & 8.339 & 5.805 & 5.897 & 3.806 & 3.912 & 10.577 & 6.666 & 5.17 & 7.348 & 4.539 \\
\hline $7 / 5 / 06$ & $12: 00$ & 12.37 & 12.43 & 9.278 & 8.351 & 5.868 & 5.895 & 3.848 & 3.947 & 10.628 & 6.792 & 5.365 & 7.409 & 4.698 \\
\hline $7 / 5 / 06$ & $16: 00$ & 12.366 & 12.416 & 9.29 & 8.356 & 5.917 & 5.906 & 3.867 & 3.966 & 10.658 & 6.85 & 5.494 & 7.436 & 4.822 \\
\hline $7 / 5 / 06$ & $20: 00$ & 12.375 & 12.432 & 9.292 & 8.367 & 5.917 & 5.93 & 3.855 & 3.954 & 10.658 & 6.789 & 5.389 & 7.402 & 4.784 \\
\hline $7 / 6 / 06$ & $0: 00$ & 12.382 & 12.444 & 9.28 & 8.358 & 5.875 & 5.934 & 3.832 & 3.933 & 10.635 & 6.717 & 5.265 & 7.369 & 4.682 \\
\hline $7 / 6 / 06$ & $4: 00$ & 12.375 & 12.439 & 9.261 & 8.339 & 5.838 & 5.925 & 3.809 & 3.91 & 10.609 & 6.673 & 5.181 & 7.346 & 4.605 \\
\hline $7 / 6 / 06$ & $8: 00$ & 12.384 & 12.451 & 9.278 & 8.358 & 5.852 & 5.918 & 3.834 & 3.935 & 10.609 & 6.703 & 5.235 & 7.369 & 4.605 \\
\hline $7 / 6 / 06$ & $12: 00$ & 12.389 & 12.444 & 9.304 & 8.372 & 5.917 & 5.918 & 3.874 & 3.968 & 10.663 & 6.829 & 5.419 & 7.427 & 4.768 \\
\hline $7 / 6 / 06$ & $16: 00$ & 12.384 & 12.43 & 9.313 & 8.374 & 5.95 & 5.932 & 3.893 & 3.985 & 10.684 & 6.878 & 5.533 & 7.45 & 4.88 \\
\hline
\end{tabular}


TABLE S1.2 (Cont.)

Water Level (ft below top of casing) at Indicated Well

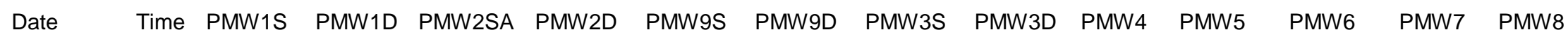

\begin{tabular}{|c|c|c|c|c|c|c|c|c|c|c|c|c|c|c|}
\hline 7/6/06 & 20:00 & 12.384 & 12.434 & 9.306 & 8.37 & 5.943 & 5.948 & 3.865 & 3.961 & 10.684 & 6.813 & 5.414 & 7.411 & 4.831 \\
\hline 7/7/06 & $0: 00$ & 12.384 & 12.439 & 9.287 & 8.358 & 5.901 & 5.948 & 3.839 & 3.935 & 10.661 & 6.743 & 5.286 & 7.374 & 4.731 \\
\hline 7/7/06 & 4:00 & 12.375 & 12.432 & 9.269 & 8.339 & 5.866 & 5.934 & 3.813 & 3.91 & 10.635 & 6.703 & 5.202 & 7.35 & 4.658 \\
\hline 7/7/06 & 8:00 & 12.377 & 12.437 & 9.273 & 8.349 & 5.868 & 5.923 & 3.83 & 3.926 & 10.633 & 6.731 & 5.246 & 7.369 & 4.668 \\
\hline 7/7/06 & $12: 00$ & 12.382 & 12.425 & 9.308 & 8.363 & 5.934 & 5.918 & 3.874 & 3.966 & 10.686 & 6.857 & 5.438 & 7.432 & 4.833 \\
\hline 7/7/06 & $16: 00$ & 12.373 & 12.411 & 9.318 & 8.367 & 5.973 & 5.932 & 3.893 & 3.982 & 10.71 & 6.904 & 5.559 & 7.46 & 4.929 \\
\hline 7/7/06 & 20:00 & 12.37 & 12.414 & 9.306 & 8.363 & 5.968 & 5.946 & 3.869 & 3.957 & 10.714 & 6.85 & 5.454 & 7.418 & 4.889 \\
\hline 7/8/06 & $0: 00$ & 12.366 & 12.411 & 9.283 & 8.342 & 5.922 & 5.946 & 3.83 & 3.924 & 10.684 & 6.775 & 5.314 & 7.374 & 4.791 \\
\hline 7/8/06 & 4:00 & 12.356 & 12.411 & 9.261 & 8.327 & 5.894 & 5.934 & 3.806 & 3.898 & 10.654 & 6.731 & 5.225 & 7.348 & 4.714 \\
\hline $7 / 8 / 06$ & $8: 00$ & 12.354 & 12.407 & 9.25 & 8.311 & 5.852 & 5.913 & 3.788 & 3.884 & 10.63 & 6.687 & 5.158 & 7.329 & 4.642 \\
\hline 7/8/06 & $12: 00$ & 12.359 & 12.407 & 9.273 & 8.332 & 5.896 & 5.904 & 3.832 & 3.924 & 10.658 & 6.799 & 5.302 & 7.383 & 4.745 \\
\hline $7 / 8 / 06$ & $16: 00$ & 12.363 & 12.404 & 9.292 & 8.344 & 5.933 & 5.911 & 3.844 & 3.935 & 10.696 & 6.831 & 5.312 & 7.383 & 4.81 \\
\hline 7/8/06 & 20:00 & 12.359 & 12.402 & 9.28 & 8.332 & 5.929 & 5.918 & 3.82 & 3.91 & 10.691 & 6.785 & 5.239 & 7.355 & 4.766 \\
\hline $7 / 9 / 06$ & $0: 00$ & 12.347 & 12.395 & 9.252 & 8.311 & 5.875 & 5.913 & 3.783 & 3.875 & 10.649 & 6.71 & 5.132 & 7.32 & 4.665 \\
\hline 7/9/06 & $4: 00$ & 12.328 & 12.381 & 9.226 & 8.288 & 5.837 & 5.892 & 3.755 & 3.849 & 10.616 & 6.67 & 5.072 & 7.299 & 4.595 \\
\hline $7 / 9 / 06$ & $8: 00$ & 12.331 & 12.388 & 9.226 & 8.295 & 5.835 & 5.878 & 3.774 & 3.865 & 10.607 & 6.694 & 5.125 & 7.318 & 4.595 \\
\hline 7/9/06 & $12: 00$ & 12.347 & 12.393 & 9.266 & 8.323 & 5.901 & 5.876 & 3.827 & 3.917 & 10.658 & 6.817 & 5.305 & 7.385 & 4.745 \\
\hline $7 / 9 / 06$ & $16: 00$ & 12.354 & 12.39 & 9.29 & 8.339 & 5.954 & 5.895 & 3.848 & 3.938 & 10.707 & 6.871 & 5.363 & 7.401 & 4.845 \\
\hline 7/9/06 & 20:00 & 12.354 & 12.397 & 9.276 & 8.33 & 5.94 & 5.913 & 3.818 & 3.91 & 10.693 & 6.792 & 5.244 & 7.355 & 4.773 \\
\hline $7 / 10 / 06$ & $0: 00$ & 12.347 & 12.393 & 9.252 & 8.311 & 5.889 & 5.911 & 3.785 & 3.875 & 10.658 & 6.722 & 5.134 & 7.318 & 4.675 \\
\hline $7 / 10 / 06$ & $4: 00$ & 12.338 & 12.388 & 9.231 & 8.285 & 5.851 & 5.897 & 3.762 & 3.854 & 10.628 & 6.68 & 5.058 & 7.285 & 4.607 \\
\hline 7/10/06 & 8:00 & 12.335 & 12.39 & 9.217 & 8.323 & 5.821 & 5.878 & 3.748 & 3.868 & 10.602 & 6.647 & 5.011 & 7.308 & 4.549 \\
\hline $7 / 10 / 06$ & $12: 00$ & 12.326 & 12.379 & 9.203 & 8.269 & 5.793 & 5.86 & 3.732 & 3.823 & 10.582 & 6.631 & 4.992 & 7.276 & 4.507 \\
\hline $7 / 10 / 06$ & $16: 00$ & 12.307 & 12.358 & 9.189 & 8.252 & 5.774 & 5.838 & 3.715 & 3.807 & 10.568 & 6.619 & 4.985 & 7.273 & 4.483 \\
\hline $7 / 10 / 06$ & $20: 00$ & 12.298 & 12.353 & 9.175 & 8.222 & 5.758 & 5.824 & 3.706 & 3.746 & 10.551 & 6.603 & 4.95 & 7.231 & 4.453 \\
\hline $7 / 11 / 06$ & 0:00 & 12.296 & 12.351 & 9.165 & 8.238 & 5.734 & 5.813 & 3.694 & 3.786 & 10.535 & 6.584 & 4.927 & 7.252 & 4.423 \\
\hline $7 / 11 / 06$ & $4: 00$ & 12.293 & 12.356 & 9.156 & 8.236 & 5.723 & 5.803 & 3.692 & 3.784 & 10.521 & 6.57 & 4.915 & 7.245 & 4.402 \\
\hline $7 / 11 / 06$ & 8:00 & 12.305 & 12.372 & 9.163 & 8.245 & 5.716 & 5.803 & 3.701 & 3.795 & 10.514 & 6.568 & 4.918 & 7.25 & 4.388 \\
\hline $7 / 11 / 06$ & $12: 00$ & 12.326 & 12.388 & 9.193 & 8.276 & 5.753 & 5.808 & 3.75 & 3.84 & 10.537 & 6.649 & 5.086 & 7.306 & 4.502 \\
\hline $7 / 11 / 06$ & $16: 00$ & 12.352 & 12.402 & 9.231 & 8.306 & 5.804 & 5.827 & 3.788 & 3.875 & 10.584 & 6.71 & 5.186 & 7.341 & 4.581 \\
\hline $7 / 11 / 06$ & $20: 00$ & 12.366 & 12.418 & 9.243 & 8.313 & 5.816 & 5.85 & 3.778 & 3.868 & 10.596 & 6.675 & 5.111 & 7.32 & 4.532 \\
\hline $7 / 12 / 06$ & $0: 00$ & 12.373 & 12.428 & 9.231 & 8.306 & 5.786 & 5.862 & 3.764 & 3.854 & 10.577 & 6.624 & 5.011 & 7.294 & 4.453 \\
\hline $7 / 12 / 06$ & $4: 00$ & 12.368 & 12.43 & 9.219 & 8.302 & 5.765 & 5.857 & 3.753 & 3.842 & 10.556 & 6.598 & 4.946 & 7.278 & 4.402 \\
\hline $7 / 12 / 06$ & $8: 00$ & 12.38 & 12.444 & 9.231 & 8.313 & 5.762 & 5.855 & 3.767 & 3.858 & 10.549 & 6.603 & 4.962 & 7.292 & 4.39 \\
\hline
\end{tabular}


Water Level (ft below top of casing) at Indicated Well

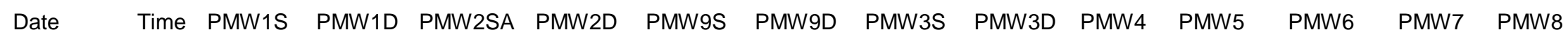

\begin{tabular}{|c|c|c|c|c|c|c|c|c|c|c|c|c|c|c|}
\hline $7 / 12 / 06$ & $12: 00$ & 12.384 & 12.439 & 9.259 & 8.33 & 5.814 & 5.857 & 3.806 & 3.893 & 10.591 & 6.705 & 5.141 & 7.348 & 4.542 \\
\hline $7 / 12 / 06$ & $16: 00$ & 12.389 & 12.43 & 9.285 & 8.342 & 5.874 & 5.869 & 3.834 & 3.917 & 10.64 & 6.771 & 5.256 & 7.385 & 4.64 \\
\hline $7 / 12 / 06$ & $20: 00$ & 12.394 & 12.439 & 9.287 & 8.346 & 5.884 & 5.892 & 3.818 & 3.903 & 10.647 & 6.719 & 5.179 & 7.353 & 4.586 \\
\hline $7 / 13 / 06$ & $0: 00$ & 12.38 & 12.428 & 9.259 & 8.32 & 5.842 & 5.895 & 3.783 & 3.868 & 10.619 & 6.659 & 5.067 & 7.315 & 4.495 \\
\hline $7 / 13 / 06$ & $4: 00$ & 12.366 & 12.418 & 9.238 & 8.302 & 5.811 & 5.883 & 3.76 & 3.844 & 10.593 & 6.626 & 4.985 & 7.29 & 4.437 \\
\hline $7 / 13 / 06$ & $8: 00$ & 12.361 & 12.411 & 9.236 & 8.302 & 5.809 & 5.867 & 3.769 & 3.849 & 10.582 & 6.64 & 5.018 & 7.306 & 4.434 \\
\hline $7 / 13 / 06$ & $12: 00$ & 12.368 & 12.409 & 9.271 & 8.323 & 5.881 & 5.867 & 3.82 & 3.898 & 10.63 & 6.768 & 5.221 & 7.374 & 4.619 \\
\hline $7 / 13 / 06$ & $16: 00$ & 11.635 & 12.337 & 9.165 & 8.297 & 5.213 & 5.86 & 3.601 & 3.695 & 10.57 & 6.372 & 4.496 & 7.052 & 3.972 \\
\hline $7 / 13 / 06$ & $20: 00$ & 9.608 & 12.195 & 9.1 & 8.196 & 5.257 & 5.745 & 3.622 & 3.709 & 10.519 & 6.486 & 4.624 & 7.11 & 4.129 \\
\hline $7 / 14 / 06$ & $0: 00$ & 10.913 & 12.256 & 9.072 & 8.189 & 5.39 & 5.705 & 3.631 & 3.718 & 10.507 & 6.509 & 4.675 & 7.159 & 4.18 \\
\hline $7 / 14 / 06$ & $4: 00$ & 11.757 & 12.318 & 9.086 & 8.215 & 5.463 & 5.705 & 3.659 & 3.746 & 10.498 & 6.526 & 4.71 & 7.194 & 4.21 \\
\hline $7 / 14 / 06$ & $8: 00$ & 12.049 & 12.36 & 9.114 & 8.234 & 5.51 & 5.717 & 3.685 & 3.77 & 10.498 & 6.544 & 4.757 & 7.222 & 4.233 \\
\hline $7 / 14 / 06$ & $12: 00$ & 12.142 & 12.386 & 9.156 & 8.262 & 5.58 & 5.738 & 3.736 & 3.819 & 10.533 & 6.626 & 4.894 & 7.276 & 4.404 \\
\hline $7 / 14 / 06$ & $16: 00$ & 12.21 & 12.4 & 9.196 & 8.292 & 5.647 & 5.771 & 3.774 & 3.854 & 10.584 & 6.696 & 5.039 & 7.32 & 4.525 \\
\hline $7 / 14 / 06$ & $20: 00$ & 12.254 & 12.416 & 9.21 & 8.299 & 5.664 & 5.799 & 3.767 & 3.849 & 10.598 & 6.654 & 4.981 & 7.301 & 4.46 \\
\hline $7 / 15 / 06$ & 0:00 & 12.28 & 12.428 & 9.207 & 8.297 & 5.657 & 5.813 & 3.755 & 3.835 & 10.582 & 6.617 & 4.908 & 7.285 & 4.38 \\
\hline $7 / 15 / 06$ & $4: 00$ & 12.296 & 12.437 & 9.205 & 8.299 & 5.654 & 5.817 & 3.753 & 3.835 & 10.568 & 6.598 & 4.864 & 7.276 & 4.329 \\
\hline $7 / 15 / 06$ & $8: 00$ & 12.31 & 12.441 & 9.217 & 8.309 & 5.675 & 5.82 & 3.771 & 3.854 & 10.57 & 6.624 & 4.89 & 7.292 & 4.338 \\
\hline $7 / 15 / 06$ & $12: 00$ & 12.319 & 12.441 & 9.245 & 8.325 & 5.736 & 5.829 & 3.811 & 3.891 & 10.616 & 6.724 & 5.048 & 7.341 & 4.532 \\
\hline $7 / 15 / 06$ & $16: 00$ & 12.322 & 12.43 & 9.268 & 8.332 & 5.778 & 5.848 & 3.829 & 3.91 & 10.658 & 6.782 & 5.174 & 7.376 & 4.632 \\
\hline $7 / 15 / 06$ & $20: 00$ & 12.329 & 12.432 & 9.271 & 8.332 & 5.785 & 5.867 & 3.811 & 3.891 & 10.668 & 6.733 & 5.106 & 7.346 & 4.569 \\
\hline $7 / 16 / 06$ & $0: 00$ & 12.319 & 12.423 & 9.245 & 8.309 & 5.748 & 5.867 & 3.778 & 3.858 & 10.637 & 6.668 & 4.997 & 7.308 & 4.471 \\
\hline $7 / 16 / 06$ & $4: 00$ & 12.312 & 12.418 & 9.226 & 8.295 & 5.725 & 5.855 & 3.757 & 3.837 & 10.612 & 6.638 & 4.932 & 7.29 & 4.413 \\
\hline $7 / 16 / 06$ & $8: 00$ & 12.315 & 12.416 & 9.233 & 8.302 & 5.736 & 5.841 & 3.774 & 3.851 & 10.607 & 6.666 & 4.966 & 7.304 & 4.418 \\
\hline $7 / 16 / 06$ & $12: 00$ & 12.319 & 12.409 & 9.268 & 8.32 & 5.802 & 5.845 & 3.816 & 3.889 & 10.658 & 6.778 & 5.137 & 7.364 & 4.586 \\
\hline $7 / 16 / 06$ & $16: 00$ & 12.329 & 12.414 & 9.289 & 8.339 & 5.858 & 5.867 & 3.848 & 3.919 & 10.703 & 6.838 & 5.27 & 7.406 & 4.695 \\
\hline $7 / 16 / 06$ & $20: 00$ & 12.326 & 12.409 & 9.282 & 8.327 & 5.855 & 5.883 & 3.818 & 3.889 & 10.7 & 6.775 & 5.181 & 7.362 & 4.63 \\
\hline $7 / 17 / 06$ & $0: 00$ & 12.326 & 12.411 & 9.259 & 8.313 & 5.818 & 5.888 & 3.79 & 3.863 & 10.672 & 6.71 & 5.069 & 7.325 & 4.541 \\
\hline $7 / 17 / 06$ & $4: 00$ & 12.324 & 12.416 & 9.245 & 8.304 & 5.788 & 5.876 & 3.774 & 3.847 & 10.649 & 6.677 & 4.992 & 7.304 & 4.485 \\
\hline $7 / 17 / 06$ & $8: 00$ & 12.333 & 12.428 & 9.266 & 8.325 & 5.813 & 5.869 & 3.806 & 3.877 & 10.656 & 6.731 & 5.041 & 7.329 & 4.534 \\
\hline $7 / 17 / 06$ & $12: 00$ & 12.364 & 12.453 & 9.315 & 8.367 & 5.893 & 5.888 & 3.869 & 3.938 & 10.714 & 6.85 & 5.232 & 7.399 & 4.679 \\
\hline $7 / 17 / 06$ & $16: 00$ & 12.385 & 12.469 & 9.341 & 8.393 & 5.944 & 5.92 & 3.899 & 3.966 & 10.74 & 6.887 & 5.354 & 7.434 & 4.761 \\
\hline $7 / 17 / 06$ & $20: 00$ & 12.403 & 12.49 & 9.35 & 8.405 & 5.951 & 5.951 & 3.888 & 3.959 & 10.742 & 6.829 & 5.263 & 7.404 & 4.707 \\
\hline $7 / 18 / 06$ & $0: 00$ & 12.41 & 12.499 & 9.334 & 8.388 & 5.907 & 5.958 & 3.86 & 3.933 & 10.717 & 6.757 & 5.139 & 7.367 & 4.609 \\
\hline
\end{tabular}


TABLE S1.2 (Cont.)

Water Level (ft below top of casing) at Indicated Well

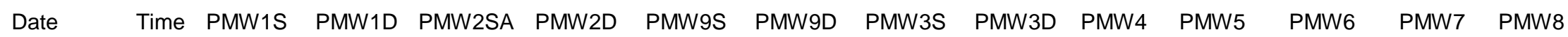

\begin{tabular}{|c|c|c|c|c|c|c|c|c|c|c|c|c|c|c|}
\hline $7 / 18 / 06$ & $4: 00$ & 12.41 & 12.497 & 9.315 & 8.372 & 5.865 & 5.951 & 3.834 & 3.907 & 10.689 & 6.708 & 5.048 & 7.339 & 4.534 \\
\hline $7 / 18 / 06$ & $8: 00$ & 12.41 & 12.497 & 9.32 & 8.372 & 5.86 & 5.934 & 3.841 & 3.912 & 10.679 & 6.717 & 5.067 & 7.353 & 4.518 \\
\hline $7 / 18 / 06$ & $12: 00$ & 12.415 & 12.49 & 9.353 & 8.391 & 5.939 & 5.932 & 3.885 & 3.957 & 10.733 & 6.836 & 5.242 & 7.418 & 4.658 \\
\hline $7 / 18 / 06$ & $16: 00$ & 12.41 & 12.476 & 9.364 & 8.398 & 5.984 & 5.946 & 3.909 & 3.977 & 10.766 & 6.901 & 5.379 & 7.453 & 4.772 \\
\hline $7 / 18 / 06$ & $20: 00$ & 12.408 & 12.474 & 9.353 & 8.386 & 5.97 & 5.96 & 3.874 & 3.945 & 10.763 & 6.827 & 5.258 & 7.404 & 4.705 \\
\hline $7 / 19 / 06$ & $0: 00$ & 12.389 & 12.46 & 9.322 & 8.358 & 5.918 & 5.953 & 3.834 & 3.907 & 10.728 & 6.757 & 5.134 & 7.362 & 4.607 \\
\hline $7 / 19 / 06$ & $4: 00$ & 12.368 & 12.439 & 9.292 & 8.33 & 5.879 & 5.932 & 3.801 & 3.875 & 10.7 & 6.715 & 5.046 & 7.329 & 4.544 \\
\hline $7 / 19 / 06$ & 8:00 & 12.378 & 12.453 & 9.301 & 8.346 & 5.888 & 5.918 & 3.827 & 3.9 & 10.696 & 6.752 & 5.083 & 7.346 & 4.576 \\
\hline $7 / 19 / 06$ & $12: 00$ & 12.394 & 12.458 & 9.346 & 8.377 & 5.96 & 5.92 & 3.885 & 3.954 & 10.756 & 6.885 & 5.281 & 7.427 & 4.756 \\
\hline $7 / 19 / 06$ & $16: 00$ & 12.406 & 12.462 & 9.369 & 8.398 & 6.005 & 5.944 & 3.916 & 3.98 & 10.789 & 6.929 & 5.412 & 7.467 & 4.856 \\
\hline 7/19/06 & $20: 00$ & 12.406 & 12.465 & 9.362 & 8.391 & 6.002 & 5.96 & 3.89 & 3.956 & 10.789 & 6.873 & 5.309 & 7.42 & 4.814 \\
\hline $7 / 20 / 06$ & $0: 00$ & 12.417 & 12.488 & 9.35 & 8.395 & 5.972 & 5.969 & 3.876 & 3.945 & 10.768 & 6.815 & 5.2 & 7.387 & 4.73 \\
\hline $7 / 20 / 06$ & $4: 00$ & 12.434 & 12.513 & 9.348 & 8.4 & 5.944 & 5.974 & 3.867 & 3.935 & 10.745 & 6.768 & 5.118 & 7.362 & 4.653 \\
\hline $7 / 20 / 06$ & $8: 00$ & 12.455 & 12.534 & 9.362 & 8.414 & 5.937 & 5.974 & 3.883 & 3.952 & 10.735 & 6.773 & 5.125 & 7.376 & 4.639 \\
\hline $7 / 20 / 06$ & $12: 00$ & 12.469 & 12.544 & 9.393 & 8.44 & 6.002 & 5.979 & 3.939 & 4.003 & 10.789 & 6.901 & 5.309 & 7.453 & 4.784 \\
\hline $7 / 20 / 06$ & $16: 00$ & 12.478 & 12.544 & 9.416 & 8.449 & 6.042 & 5.997 & 3.958 & 4.022 & 10.815 & 6.936 & 5.419 & 7.481 & 4.877 \\
\hline $7 / 20 / 06$ & $20: 00$ & 12.483 & 12.546 & 9.407 & 8.445 & 6.026 & 6.014 & 3.927 & 3.994 & 10.805 & 6.859 & 5.295 & 7.432 & 4.807 \\
\hline $7 / 21 / 06$ & $0: 00$ & 12.485 & 12.553 & 9.397 & 8.445 & 5.998 & 6.014 & 3.918 & 3.989 & 10.784 & 6.808 & 5.202 & 7.406 & 4.728 \\
\hline $7 / 21 / 06$ & $4: 00$ & 12.478 & 12.539 & 9.376 & 8.416 & 5.951 & 6 & 3.881 & 3.942 & 10.759 & 6.761 & 5.109 & 7.378 & 4.644 \\
\hline $7 / 21 / 06$ & $8: 00$ & 12.49 & 12.551 & 9.367 & 8.449 & 5.911 & 5.981 & 3.871 & 3.956 & 10.733 & 6.717 & 5.011 & 7.353 & 4.576 \\
\hline $7 / 21 / 06$ & $12: 00$ & 12.485 & 12.555 & 9.369 & 8.414 & 5.89 & 5.967 & 3.876 & 3.947 & 10.728 & 6.754 & 5.062 & 7.367 & 4.588 \\
\hline $7 / 21 / 06$ & $16: 00$ & 12.485 & 12.546 & 9.393 & 8.428 & 5.937 & 5.962 & 3.906 & 3.975 & 10.77 & 6.836 & 5.207 & 7.413 & 4.691 \\
\hline $7 / 21 / 06$ & $20: 00$ & 12.487 & 12.551 & 9.395 & 8.428 & 5.951 & 5.974 & 3.895 & 3.966 & 10.782 & 6.803 & 5.148 & 7.387 & 4.653 \\
\hline $7 / 22 / 06$ & $0: 00$ & 12.483 & 12.548 & 9.371 & 8.409 & 5.911 & 5.976 & 3.867 & 3.935 & 10.752 & 6.743 & 5.036 & 7.355 & 4.569 \\
\hline $7 / 22 / 06$ & $4: 00$ & 12.473 & 12.544 & 9.35 & 8.398 & 5.883 & 5.965 & 3.848 & 3.919 & 10.724 & 6.71 & 4.971 & 7.336 & 4.518 \\
\hline $7 / 22 / 06$ & 8:00 & 12.471 & 12.544 & 9.35 & 8.398 & 5.873 & 5.951 & 3.855 & 3.924 & 10.71 & 6.71 & 4.987 & 7.343 & 4.506 \\
\hline $7 / 22 / 06$ & $12: 00$ & 12.48 & 12.539 & 9.383 & 8.419 & 5.93 & 5.948 & 3.899 & 3.966 & 10.752 & 6.815 & 5.174 & 7.406 & 4.644 \\
\hline $7 / 22 / 06$ & $16: 00$ & 12.48 & 12.53 & 9.409 & 8.428 & 5.986 & 5.96 & 3.925 & 3.987 & 10.801 & 6.887 & 5.297 & 7.448 & 4.74 \\
\hline $7 / 22 / 06$ & $20: 00$ & 12.478 & 12.53 & 9.397 & 8.423 & 5.988 & 5.979 & 3.899 & 3.963 & 10.803 & 6.834 & 5.2 & 7.404 & 4.684 \\
\hline 7/23/06 & $0: 00$ & 12.466 & 12.523 & 9.369 & 8.402 & 5.941 & 5.979 & 3.864 & 3.931 & 10.768 & 6.766 & 5.081 & 7.364 & 4.593 \\
\hline $7 / 23 / 06$ & $4: 00$ & 12.452 & 12.516 & 9.346 & 8.384 & 5.908 & 5.962 & 3.843 & 3.91 & 10.74 & 6.729 & 5.001 & 7.339 & 4.537 \\
\hline 7/23/06 & $8: 00$ & 12.455 & 12.518 & 9.35 & 8.391 & 5.908 & 5.951 & 3.857 & 3.921 & 10.731 & 6.747 & 5.018 & 7.35 & 4.551 \\
\hline $7 / 23 / 06$ & $12: 00$ & 12.462 & 12.516 & 9.388 & 8.412 & 5.976 & 5.948 & 3.904 & 3.966 & 10.782 & 6.869 & 5.195 & 7.418 & 4.705 \\
\hline $7 / 23 / 06$ & $16: 00$ & 12.466 & 12.509 & 9.411 & 8.428 & 6.035 & 5.965 & 3.937 & 3.994 & 10.831 & 6.943 & 5.335 & 7.464 & 4.831 \\
\hline
\end{tabular}


TABLE S1.2 (Cont.)

Water Level (ft below top of casing) at Indicated Well

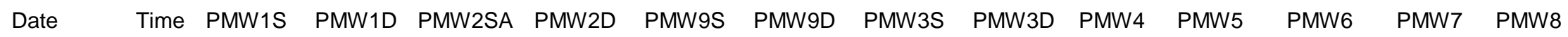

\begin{tabular}{|c|c|c|c|c|c|c|c|c|c|c|c|c|c|c|}
\hline $7 / 23 / 06$ & $20: 00$ & 12.466 & 12.513 & 9.397 & 8.419 & 6.021 & 5.986 & 3.904 & 3.966 & 10.824 & 6.873 & 5.216 & 7.411 & 4.779 \\
\hline $7 / 24 / 06$ & $0: 00$ & 12.455 & 12.506 & 9.369 & 8.395 & 5.971 & 5.983 & 3.867 & 3.931 & 10.791 & 6.803 & 5.099 & 7.366 & 4.679 \\
\hline $7 / 24 / 06$ & $4: 00$ & 12.445 & 12.502 & 9.348 & 8.381 & 5.936 & 5.969 & 3.848 & 3.912 & 10.768 & 6.768 & 5.025 & 7.343 & 4.621 \\
\hline $7 / 24 / 06$ & $8: 00$ & 12.448 & 12.506 & 9.357 & 8.391 & 5.948 & 5.958 & 3.869 & 3.931 & 10.768 & 6.808 & 5.064 & 7.364 & 4.667 \\
\hline $7 / 24 / 06$ & $12: 00$ & 12.464 & 12.511 & 9.4 & 8.421 & 6.018 & 5.96 & 3.927 & 3.984 & 10.831 & 6.936 & 5.265 & 7.446 & 4.835 \\
\hline $7 / 24 / 06$ & $16: 00$ & 12.471 & 12.511 & 9.421 & 8.435 & 6.06 & 5.983 & 3.955 & 4.01 & 10.857 & 6.976 & 5.409 & 7.488 & 4.929 \\
\hline $7 / 24 / 06$ & $20: 00$ & 12.469 & 12.511 & 9.411 & 8.428 & 6.051 & 6 & 3.923 & 3.98 & 10.857 & 6.92 & 5.316 & 7.436 & 4.88 \\
\hline $7 / 25 / 06$ & $0: 00$ & 12.466 & 12.513 & 9.386 & 8.409 & 6.004 & 6.002 & 3.89 & 3.947 & 10.829 & 6.845 & 5.188 & 7.39 & 4.775 \\
\hline $7 / 25 / 06$ & $4: 00$ & 12.455 & 12.509 & 9.364 & 8.395 & 5.969 & 5.988 & 3.867 & 3.926 & 10.798 & 6.799 & 5.097 & 7.362 & 4.702 \\
\hline $7 / 25 / 06$ & $8: 00$ & 12.462 & 12.52 & 9.376 & 8.409 & 5.974 & 5.976 & 3.892 & 3.949 & 10.801 & 6.838 & 5.141 & 7.385 & 4.73 \\
\hline $7 / 25 / 06$ & $12: 00$ & 12.478 & 12.53 & 9.407 & 8.428 & 6.02 & 5.981 & 3.92 & 3.977 & 10.843 & 6.906 & 5.214 & 7.422 & 4.817 \\
\hline $7 / 25 / 06$ & $16: 00$ & 12.478 & 12.518 & 9.423 & 8.433 & 6.055 & 5.986 & 3.939 & 3.991 & 10.868 & 6.955 & 5.307 & 7.46 & 4.884 \\
\hline $7 / 25 / 06$ & $20: 00$ & 12.464 & 12.506 & 9.4 & 8.412 & 6.041 & 5.995 & 3.902 & 3.959 & 10.861 & 6.892 & 5.225 & 7.411 & 4.831 \\
\hline $7 / 26 / 06$ & 0:00 & 12.45 & 12.497 & 9.371 & 8.391 & 5.995 & 5.988 & 3.867 & 3.921 & 10.829 & 6.827 & 5.111 & 7.369 & 4.735 \\
\hline $7 / 26 / 06$ & $4: 00$ & 12.445 & 12.502 & 9.35 & 8.381 & 5.962 & 5.974 & 3.848 & 3.905 & 10.798 & 6.782 & 5.022 & 7.339 & 4.663 \\
\hline $7 / 26 / 06$ & $8: 00$ & 12.473 & 12.534 & 9.364 & 8.405 & 5.957 & 5.972 & 3.871 & 3.928 & 10.782 & 6.787 & 5.036 & 7.352 & 4.637 \\
\hline $7 / 26 / 06$ & $12: 00$ & 12.497 & 12.553 & 9.416 & 8.442 & 6.018 & 5.976 & 3.936 & 3.989 & 10.836 & 6.911 & 5.237 & 7.443 & 4.77 \\
\hline $7 / 26 / 06$ & $16: 00$ & 12.508 & 12.557 & 9.442 & 8.463 & 6.079 & 5.997 & 3.971 & 4.024 & 10.882 & 6.983 & 5.379 & 7.495 & 4.903 \\
\hline 7/26/06 & $20: 00$ & 12.518 & 12.567 & 9.442 & 8.463 & 6.074 & 6.023 & 3.95 & 4.003 & 10.88 & 6.915 & 5.276 & 7.441 & 4.852 \\
\hline $7 / 27 / 06$ & 0:00 & 12.518 & 12.571 & 9.423 & 8.447 & 6.027 & 6.028 & 3.918 & 3.975 & 10.85 & 6.843 & 5.153 & 7.397 & 4.747 \\
\hline $7 / 27 / 06$ & $4: 00$ & 12.515 & 12.574 & 9.404 & 8.435 & 5.992 & 6.018 & 3.899 & 3.956 & 10.822 & 6.801 & 5.069 & 7.366 & 4.674 \\
\hline $7 / 27 / 06$ & $8: 00$ & 12.522 & 12.585 & 9.404 & 8.442 & 5.976 & 6.011 & 3.904 & 3.959 & 10.801 & 6.789 & 5.039 & 7.369 & 4.628 \\
\hline $7 / 27 / 06$ & $12: 00$ & 12.534 & 12.595 & 9.432 & 8.463 & 6.006 & 6.009 & 3.934 & 3.989 & 10.824 & 6.845 & 5.132 & 7.408 & 4.677 \\
\hline $7 / 27 / 06$ & $16: 00$ & 12.548 & 12.602 & 9.463 & 8.487 & 6.076 & 6.021 & 3.981 & 4.034 & 10.887 & 6.969 & 5.307 & 7.483 & 4.84 \\
\hline $7 / 27 / 06$ & $20: 00$ & 12.555 & 12.609 & 9.465 & 8.491 & 6.083 & 6.044 & 3.964 & 4.019 & 10.889 & 6.913 & 5.218 & 7.439 & 4.812 \\
\hline $7 / 28 / 06$ & $0: 00$ & 12.56 & 12.613 & 9.449 & 8.477 & 6.039 & 6.049 & 3.939 & 3.994 & 10.859 & 6.845 & 5.109 & 7.401 & 4.719 \\
\hline $7 / 28 / 06$ & $4: 00$ & 12.555 & 12.613 & 9.432 & 8.466 & 6.006 & 6.039 & 3.918 & 3.975 & 10.831 & 6.806 & 5.027 & 7.373 & 4.649 \\
\hline $7 / 28 / 06$ & $8: 00$ & 12.562 & 12.625 & 9.444 & 8.477 & 6.008 & 6.03 & 3.939 & 3.994 & 10.824 & 6.824 & 5.06 & 7.394 & 4.646 \\
\hline $7 / 28 / 06$ & $12: 00$ & 12.574 & 12.629 & 9.479 & 8.503 & 6.083 & 6.035 & 3.995 & 4.045 & 10.887 & 6.96 & 5.265 & 7.485 & 4.814 \\
\hline $7 / 28 / 06$ & $16: 00$ & 12.576 & 12.622 & 9.496 & 8.515 & 6.128 & 6.054 & 4.02 & 4.071 & 10.922 & 7.018 & 5.428 & 7.534 & 4.95 \\
\hline $7 / 28 / 06$ & $20: 00$ & 12.578 & 12.627 & 9.496 & 8.515 & 6.125 & 6.075 & 3.999 & 4.05 & 10.924 & 6.962 & 5.349 & 7.488 & 4.908 \\
\hline 7/29/06 & $0: 00$ & 12.567 & 12.615 & 9.47 & 8.487 & 6.074 & 6.072 & 3.957 & 4.01 & 10.898 & 6.887 & 5.216 & 7.439 & 4.8 \\
\hline $7 / 29 / 06$ & $4: 00$ & 12.557 & 12.613 & 9.449 & 8.475 & 6.041 & 6.061 & 3.936 & 3.989 & 10.873 & 6.845 & 5.125 & 7.406 & 4.725 \\
\hline 7/29/06 & $8: 00$ & 12.56 & 12.618 & 9.456 & 8.482 & 6.041 & 6.044 & 3.953 & 4.003 & 10.866 & 6.871 & 5.15 & 7.425 & 4.728 \\
\hline
\end{tabular}


Water Level (ft below top of casing) at Indicated Well

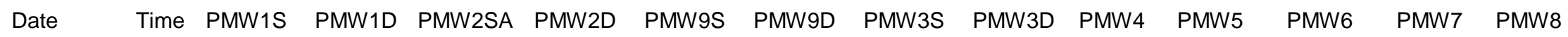

\begin{tabular}{|c|c|c|c|c|c|c|c|c|c|c|c|c|c|c|}
\hline $7 / 29 / 06$ & $12: 00$ & 12.581 & 12.627 & 9.503 & 8.515 & 6.113 & 6.049 & 4.016 & 4.062 & 10.929 & 7.006 & 5.353 & 7.52 & 4.901 \\
\hline 7/29/06 & $16: 00$ & 12.578 & 12.618 & 9.512 & 8.524 & 6.153 & 6.068 & 4.037 & 4.082 & 10.957 & 7.055 & 5.5 & 7.562 & 5.005 \\
\hline $7 / 29 / 06$ & $20: 00$ & 12.581 & 12.625 & 9.512 & 8.524 & 6.156 & 6.086 & 4.013 & 4.061 & 10.961 & 7.002 & 5.412 & 7.513 & 4.963 \\
\hline $7 / 30 / 06$ & $0: 00$ & 12.578 & 12.625 & 9.489 & 8.505 & 6.111 & 6.089 & 3.981 & 4.031 & 10.938 & 6.929 & 5.283 & 7.462 & 4.865 \\
\hline $7 / 30 / 06$ & $4: 00$ & 12.56 & 12.609 & 9.463 & 8.477 & 6.069 & 6.072 & 3.946 & 3.996 & 10.91 & 6.885 & 5.185 & 7.427 & 4.786 \\
\hline $7 / 30 / 06$ & $8: 00$ & 12.562 & 12.615 & 9.474 & 8.494 & 6.083 & 6.058 & 3.976 & 4.024 & 10.915 & 6.934 & 5.241 & 7.457 & 4.819 \\
\hline $7 / 30 / 06$ & $12: 00$ & 12.583 & 12.62 & 9.517 & 8.522 & 6.148 & 6.065 & 4.034 & 4.08 & 10.975 & 7.06 & 5.451 & 7.557 & 4.982 \\
\hline $7 / 30 / 06$ & $16: 00$ & 12.576 & 12.611 & 9.526 & 8.526 & 6.179 & 6.082 & 4.051 & 4.092 & 10.996 & 7.095 & 5.568 & 7.59 & 5.054 \\
\hline $7 / 30 / 06$ & $20: 00$ & 12.583 & 12.625 & 9.524 & 8.531 & 6.181 & 6.1 & 4.027 & 4.073 & 11.001 & 7.041 & 5.479 & 7.536 & 5.015 \\
\hline $7 / 31 / 06$ & $0: 00$ & 12.581 & 12.627 & 9.503 & 8.513 & 6.137 & 6.105 & 3.992 & 4.04 & 10.975 & 6.967 & 5.346 & 7.483 & 4.917 \\
\hline 7/31/06 & $4: 00$ & 12.564 & 12.613 & 9.474 & 8.489 & 6.102 & 6.086 & 3.96 & 4.008 & 10.947 & 6.922 & 5.255 & 7.446 & 4.844 \\
\hline $7 / 31 / 06$ & $8: 00$ & 12.578 & 12.632 & 9.498 & 8.515 & 6.118 & 6.075 & 4.004 & 4.05 & 10.959 & 6.978 & 5.323 & 7.485 & 4.882 \\
\hline $7 / 31 / 06$ & $12: 00$ & 12.595 & 12.641 & 9.535 & 8.545 & 6.179 & 6.089 & 4.062 & 4.101 & 11.013 & 7.093 & 5.512 & 7.581 & 5.022 \\
\hline $7 / 31 / 06$ & $16: 00$ & 12.604 & 12.643 & 9.549 & 8.557 & 6.214 & 6.112 & 4.079 & 4.12 & 11.038 & 7.123 & 5.614 & 7.611 & 5.092 \\
\hline 7/31/06 & $20: 00$ & 12.616 & 12.662 & 9.556 & 8.566 & 6.216 & 6.133 & 4.065 & 4.108 & 11.041 & 7.074 & 5.533 & 7.564 & 5.057 \\
\hline $8 / 1 / 06$ & $0: 00$ & 12.62 & 12.667 & 9.542 & 8.552 & 6.179 & 6.138 & 4.032 & 4.075 & 11.02 & 7.011 & 5.409 & 7.511 & 4.973 \\
\hline $8 / 1 / 06$ & $4: 00$ & 12.623 & 12.66 & 9.528 & 8.548 & 6.153 & 6.131 & 4.018 & 4.057 & 10.999 & 6.967 & 5.316 & 7.457 & 4.907 \\
\hline $8 / 1 / 06$ & $8: 00$ & 12.627 & 12.683 & 9.524 & 8.545 & 6.13 & 6.121 & 4.011 & 4.057 & 10.975 & 6.934 & 5.255 & 7.467 & 4.847 \\
\hline $8 / 1 / 06$ & $12: 00$ & 12.634 & 12.683 & 9.556 & 8.564 & 6.169 & 6.114 & 4.058 & 4.099 & 11.015 & 7.048 & 5.407 & 7.548 & 4.933 \\
\hline $8 / 1 / 06$ & $16: 00$ & 12.632 & 12.674 & 9.571 & 8.573 & 6.223 & 6.124 & 4.088 & 4.129 & 11.059 & 7.135 & 5.579 & 7.616 & 5.064 \\
\hline $8 / 1 / 06$ & $20: 00$ & 12.641 & 12.697 & 9.575 & 8.587 & 6.242 & 6.145 & 4.081 & 4.122 & 11.066 & 7.1 & 5.533 & 7.576 & 5.064 \\
\hline $8 / 2 / 06$ & $0: 00$ & 12.648 & 12.699 & 9.559 & 8.571 & 6.195 & 6.152 & 4.046 & 4.089 & 11.041 & 7.016 & 5.381 & 7.518 & 4.963 \\
\hline $8 / 2 / 06$ & $4: 00$ & 12.653 & 12.711 & 9.552 & 8.573 & 6.169 & 6.152 & 4.037 & 4.08 & 11.015 & 6.974 & 5.295 & 7.487 & 4.893 \\
\hline $8 / 2 / 06$ & $8: 00$ & 12.66 & 12.72 & 9.554 & 8.576 & 6.155 & 6.145 & 4.041 & 4.085 & 11.001 & 6.969 & 5.262 & 7.487 & 4.861 \\
\hline $8 / 2 / 06$ & $12: 00$ & 12.676 & 12.736 & 9.575 & 8.594 & 6.181 & 6.147 & 4.06 & 4.104 & 11.02 & 7.002 & 5.283 & 7.506 & 4.875 \\
\hline $8 / 2 / 06$ & $16: 00$ & 12.679 & 12.734 & 9.568 & 8.583 & 6.174 & 6.149 & 4.041 & 4.085 & 11.015 & 6.971 & 5.234 & 7.483 & 4.837 \\
\hline $8 / 2 / 06$ & $20: 00$ & 12.681 & 12.738 & 9.556 & 8.58 & 6.155 & 6.147 & 4.032 & 4.075 & 10.994 & 6.934 & 5.188 & 7.464 & 4.795 \\
\hline $8 / 3 / 06$ & $0: 00$ & 12.669 & 12.732 & 9.538 & 8.562 & 6.12 & 6.135 & 4.006 & 4.052 & 10.968 & 6.899 & 5.141 & 7.441 & 4.742 \\
\hline $8 / 3 / 06$ & $4: 00$ & 12.674 & 12.741 & 9.533 & 8.566 & 6.106 & 6.128 & 4.009 & 4.054 & 10.947 & 6.878 & 5.104 & 7.432 & 4.707 \\
\hline $8 / 3 / 06$ & $8: 00$ & 12.686 & 12.755 & 9.545 & 8.578 & 6.108 & 6.126 & 4.023 & 4.068 & 10.94 & 6.88 & 5.113 & 7.446 & 4.695 \\
\hline $8 / 3 / 06$ & $12: 00$ & 12.695 & 12.757 & 9.582 & 8.604 & 6.174 & 6.126 & 4.078 & 4.12 & 10.996 & 7.02 & 5.321 & 7.546 & 4.833 \\
\hline $8 / 3 / 06$ & $16: 00$ & 12.704 & 12.759 & 9.606 & 8.623 & 6.244 & 6.149 & 4.118 & 4.155 & 11.064 & 7.107 & 5.467 & 7.611 & 4.952 \\
\hline $8 / 3 / 06$ & $20: 00$ & 12.716 & 12.771 & 9.617 & 8.634 & 6.263 & 6.175 & 4.106 & 4.146 & 11.069 & 7.055 & 5.405 & 7.564 & 4.933 \\
\hline $8 / 4 / 06$ & $0: 00$ & 12.718 & 12.773 & 9.603 & 8.618 & 6.216 & 6.184 & 4.076 & 4.115 & 11.043 & 6.983 & 5.288 & 7.518 & 4.842 \\
\hline
\end{tabular}


TABLE S1.2 (Cont.)

Water Level (ft below top of casing) at Indicated Well

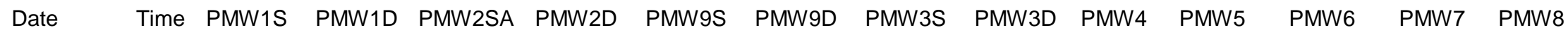

\begin{tabular}{|c|c|c|c|c|c|c|c|c|c|c|c|c|c|c|}
\hline $8 / 4 / 06$ & $4: 00$ & 12.709 & 12.769 & 9.585 & 8.602 & 6.178 & 6.175 & 4.053 & 4.094 & 11.017 & 6.941 & 5.213 & 7.487 & 4.774 \\
\hline $8 / 4 / 06$ & $8: 00$ & 12.714 & 12.776 & 9.592 & 8.611 & 6.178 & 6.166 & 4.069 & 4.11 & 11.006 & 6.952 & 5.23 & 7.501 & 4.765 \\
\hline $8 / 4 / 06$ & $12: 00$ & 12.718 & 12.764 & 9.62 & 8.627 & 6.244 & 6.166 & 4.113 & 4.153 & 11.069 & 7.088 & 5.414 & 7.599 & 4.914 \\
\hline $8 / 4 / 06$ & $16: 00$ & 12.707 & 12.745 & 9.627 & 8.627 & 6.281 & 6.177 & 4.13 & 4.169 & 11.099 & 7.153 & 5.57 & 7.643 & 5.038 \\
\hline $8 / 4 / 06$ & $20: 00$ & 12.711 & 12.755 & 9.624 & 8.63 & 6.281 & 6.194 & 4.111 & 4.15 & 11.106 & 7.095 & 5.493 & 7.588 & 5.015 \\
\hline $8 / 5 / 06$ & $0: 00$ & 12.688 & 12.736 & 9.594 & 8.597 & 6.227 & 6.187 & 4.064 & 4.104 & 11.078 & 7.023 & 5.36 & 7.532 & 4.919 \\
\hline $8 / 5 / 06$ & $4: 00$ & 12.662 & 12.706 & 9.563 & 8.571 & 6.185 & 6.168 & 4.032 & 4.043 & 11.045 & 6.976 & 5.274 & 7.492 & 4.844 \\
\hline $8 / 5 / 06$ & 8:00 & 12.653 & 12.706 & 9.554 & 8.562 & 6.169 & 6.145 & 4.027 & 4.066 & 11.027 & 6.973 & 5.262 & 7.492 & 4.826 \\
\hline $8 / 5 / 06$ & $12: 00$ & 12.665 & 12.713 & 9.587 & 8.594 & 6.237 & 6.14 & 4.088 & 4.122 & 11.085 & 7.114 & 5.454 & 7.588 & 4.987 \\
\hline $8 / 5 / 06$ & $16: 00$ & 12.681 & 12.722 & 9.624 & 8.618 & 6.29 & 6.156 & 4.13 & 4.159 & 11.129 & 7.181 & 5.614 & 7.653 & 5.115 \\
\hline $8 / 5 / 06$ & $20: 00$ & 12.7 & 12.743 & 9.627 & 8.627 & 6.295 & 6.184 & 4.113 & 4.148 & 11.132 & 7.118 & 5.523 & 7.597 & 5.071 \\
\hline $8 / 6 / 06$ & $0: 00$ & 12.723 & 12.752 & 9.627 & 8.62 & 6.272 & 6.203 & 4.111 & 4.122 & 11.108 & 7.053 & 5.402 & 7.518 & 4.984 \\
\hline $8 / 6 / 06$ & $4: 00$ & 12.714 & 12.752 & 9.601 & 8.604 & 6.22 & 6.191 & 4.071 & 4.099 & 11.073 & 6.999 & 5.297 & 7.499 & 4.896 \\
\hline 8/6/06 & 8:00 & 12.718 & 12.783 & 9.594 & 8.618 & 6.197 & 6.18 & 4.071 & 4.108 & 11.05 & 6.978 & 5.253 & 7.499 & 4.844 \\
\hline 8/6/06 & $12: 00$ & 12.73 & 12.794 & 9.627 & 8.625 & 6.223 & 6.18 & 4.102 & 4.124 & 11.071 & 7.022 & 5.311 & 7.522 & 4.863 \\
\hline 8/6/06 & $16: 00$ & 12.749 & 12.815 & 9.641 & 8.651 & 6.262 & 6.196 & 4.113 & 4.138 & 11.083 & 7.015 & 5.304 & 7.536 & 4.854 \\
\hline $8 / 6 / 06$ & $20: 00$ & 12.733 & 12.794 & 9.603 & 8.62 & 6.197 & 6.187 & 4.062 & 4.094 & 11.045 & 6.948 & 5.202 & 7.487 & 4.779 \\
\hline 8/7/06 & $0: 00$ & 12.737 & 12.806 & 9.599 & 8.637 & 6.171 & 6.177 & 4.057 & 4.089 & 11.022 & 6.922 & 5.153 & 7.48 & 4.732 \\
\hline 8/7/06 & $4: 00$ & 12.74 & 12.808 & 9.589 & 8.576 & 6.15 & 6.168 & 4.05 & 4.061 & 10.999 & 6.896 & 5.111 & 7.439 & 4.695 \\
\hline $8 / 7 / 06$ & $8: 00$ & 12.744 & 12.815 & 9.589 & 8.62 & 6.136 & 6.161 & 4.05 & 4.087 & 10.982 & 6.88 & 5.076 & 7.448 & 4.667 \\
\hline 8/7/06 & $12: 00$ & 12.742 & 12.81 & 9.598 & 8.62 & 6.15 & 6.154 & 4.067 & 4.101 & 10.987 & 6.915 & 5.15 & 7.48 & 4.697 \\
\hline $8 / 7 / 06$ & $16: 00$ & 12.737 & 12.799 & 9.603 & 8.616 & 6.169 & 6.152 & 4.06 & 4.096 & 10.996 & 6.917 & 5.164 & 7.483 & 4.693 \\
\hline 8/7/06 & $20: 00$ & 12.735 & 12.799 & 9.591 & 8.613 & 6.162 & 6.156 & 4.05 & 4.087 & 10.985 & 6.892 & 5.106 & 7.459 & 4.66 \\
\hline $8 / 8 / 06$ & $0: 00$ & 12.714 & 12.787 & 9.568 & 8.601 & 6.12 & 6.138 & 4.018 & 4.022 & 10.964 & 6.84 & 5.001 & 7.432 & 4.613 \\
\hline 8/8/06 & $4: 00$ & 7.927 & 12.404 & 9.17 & 8.269 & 3.993 & 6.093 & 3.465 & 3.554 & 10.64 & 5.701 & 3.861 & 7.001 & 3.147 \\
\hline $8 / 8 / 06$ & $8: 00$ & 7.394 & 11.877 & 8.626 & 7.913 & 3.16 & 5.675 & 3.162 & 3.234 & 10.262 & 5.188 & 3.318 & 6.719 & 2.557 \\
\hline 8/8/06 & $12: 00$ & 8.145 & 11.727 & 8.513 & 7.87 & 3.343 & 5.345 & 3.288 & 3.342 & 10.211 & 5.736 & 3.387 & 6.747 & 2.65 \\
\hline $8 / 8 / 06$ & $16: 00$ & 9.581 & 11.866 & 8.518 & 7.852 & 3.618 & 5.189 & 3.328 & 3.37 & 10.206 & 5.916 & 3.6 & 6.81 & 2.83 \\
\hline 8/8/06 & 20:00 & 10.99 & 12.005 & 8.591 & 7.896 & 3.981 & 5.161 & 3.372 & 3.414 & 10.225 & 6.052 & 3.761 & 6.863 & 2.991 \\
\hline 8/9/06 & $0: 00$ & 11.598 & 12.086 & 8.654 & 7.922 & 4.224 & 5.186 & 3.395 & 3.435 & 10.239 & 6.122 & 3.851 & 6.901 & 3.096 \\
\hline 8/9/06 & $4: 00$ & 11.794 & 12.135 & 8.708 & 7.948 & 4.418 & 5.228 & 3.421 & 3.461 & 10.255 & 6.18 & 3.935 & 6.94 & 3.196 \\
\hline 8/9/06 & 8:00 & 11.878 & 12.163 & 8.757 & 7.971 & 4.588 & 5.268 & 3.444 & 3.487 & 10.276 & 6.227 & 4.029 & 6.98 & 3.299 \\
\hline 8/9/06 & $12: 00$ & 11.941 & 12.191 & 8.818 & 8.016 & 4.808 & 5.32 & 3.51 & 3.543 & 10.323 & 6.336 & 4.274 & 7.068 & 3.649 \\
\hline 8/9/06 & $16: 00$ & 11.979 & 12.2 & 8.877 & 8.046 & 5.025 & 5.378 & 3.556 & 3.585 & 10.386 & 6.432 & 4.486 & 7.145 & 3.971 \\
\hline
\end{tabular}


TABLE S1.2 (Cont.)

Water Level (ft below top of casing) at Indicated Well

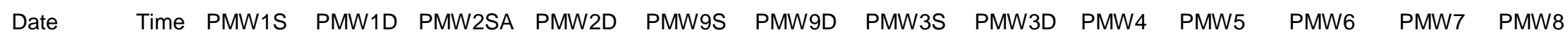

\begin{tabular}{|c|c|c|c|c|c|c|c|c|c|c|c|c|c|c|}
\hline $8 / 9 / 06$ & $20: 00$ & 12.023 & 12.226 & 8.919 & 8.079 & 5.152 & 5.439 & 3.575 & 3.606 & 10.425 & 6.451 & 4.504 & 7.164 & 3.918 \\
\hline $8 / 10 / 06$ & $0: 00$ & 12.065 & 12.26 & 8.947 & 8.102 & 5.212 & 5.49 & 3.587 & 3.62 & 10.446 & 6.455 & 4.479 & 7.173 & 3.852 \\
\hline $8 / 10 / 06$ & $4: 00$ & 10.504 & 12.202 & 8.909 & 8.053 & 4.612 & 5.502 & 3.505 & 3.559 & 10.388 & 6.203 & 3.721 & 6.959 & 2.998 \\
\hline $8 / 10 / 06$ & 8:00 & 11.326 & 12.226 & 8.888 & 8.053 & 4.52 & 5.453 & 3.486 & 3.526 & 10.346 & 6.178 & 3.8 & 6.97 & 3.026 \\
\hline $8 / 10 / 06$ & $12: 00$ & 11.841 & 12.272 & 8.94 & 8.093 & 4.705 & 5.436 & 3.549 & 3.585 & 10.369 & 6.28 & 4.096 & 7.068 & 3.355 \\
\hline $8 / 10 / 06$ & $16: 00$ & 12.013 & 12.291 & 8.97 & 8.109 & 4.901 & 5.453 & 3.575 & 3.608 & 10.411 & 6.35 & 4.288 & 7.133 & 3.549 \\
\hline $8 / 10 / 06$ & $20: 00$ & 12.081 & 12.316 & 8.994 & 8.133 & 5.035 & 5.488 & 3.594 & 3.627 & 10.437 & 6.376 & 4.334 & 7.154 & 3.572 \\
\hline $8 / 11 / 06$ & $0: 00$ & 12.128 & 12.342 & 9.012 & 8.149 & 5.1 & 5.523 & 3.603 & 3.636 & 10.444 & 6.381 & 4.327 & 7.159 & 3.565 \\
\hline $8 / 11 / 06$ & $4: 00$ & 10.357 & 12.209 & 8.914 & 8.114 & 4.102 & 5.56 & 3.447 & 3.498 & 10.348 & 5.876 & 3.653 & 6.957 & 2.87 \\
\hline $8 / 11 / 06$ & $8: 00$ & 9.905 & 12.082 & 8.827 & 8.009 & 3.889 & 5.408 & 3.43 & 3.47 & 10.258 & 5.907 & 3.665 & 6.849 & 2.802 \\
\hline $8 / 11 / 06$ & $12: 00$ & 10.94 & 12.151 & 8.834 & 8.02 & 4.212 & 5.324 & 3.47 & 3.505 & 10.26 & 6.061 & 3.959 & 6.959 & 3.115 \\
\hline 8/11/06 & $16: 00$ & 11.581 & 12.198 & 8.876 & 8.041 & 4.509 & 5.324 & 3.507 & 3.54 & 10.309 & 6.199 & 4.227 & 7.052 & 3.428 \\
\hline $8 / 11 / 06$ & $20: 00$ & 11.77 & 12.233 & 8.907 & 8.065 & 4.698 & 5.364 & 3.521 & 3.554 & 10.341 & 6.245 & 4.257 & 7.075 & 3.432 \\
\hline $8 / 12 / 06$ & 0:00 & 11.908 & 12.272 & 8.928 & 8.084 & 4.815 & 5.406 & 3.538 & 3.571 & 10.355 & 6.269 & 4.248 & 7.096 & 3.43 \\
\hline $8 / 12 / 06$ & $4: 00$ & 11.985 & 12.272 & 8.933 & 8.079 & 4.88 & 5.427 & 3.528 & 3.561 & 10.36 & 6.278 & 4.241 & 7.101 & 3.439 \\
\hline $8 / 12 / 06$ & $8: 00$ & 12.044 & 12.3 & 8.954 & 8.1 & 4.955 & 5.455 & 3.552 & 3.582 & 10.369 & 6.306 & 4.269 & 7.122 & 3.481 \\
\hline $8 / 12 / 06$ & $12: 00$ & 12.079 & 12.305 & 8.977 & 8.112 & 5.032 & 5.478 & 3.582 & 3.608 & 10.395 & 6.36 & 4.374 & 7.159 & 3.654 \\
\hline $8 / 12 / 06$ & $16: 00$ & 12.09 & 12.293 & 9.001 & 8.119 & 5.14 & 5.5 & 3.608 & 3.631 & 10.439 & 6.434 & 4.551 & 7.21 & 3.908 \\
\hline $8 / 12 / 06$ & $20: 00$ & 12.116 & 12.311 & 9.022 & 8.142 & 5.219 & 5.53 & 3.615 & 3.641 & 10.465 & 6.446 & 4.553 & 7.203 & 3.855 \\
\hline $8 / 13 / 06$ & $0: 00$ & 12.144 & 12.325 & 9.031 & 8.147 & 5.243 & 5.558 & 3.615 & 3.643 & 10.474 & 6.432 & 4.504 & 7.192 & 3.78 \\
\hline $8 / 13 / 06$ & $4: 00$ & 12.142 & 12.314 & 9.024 & 8.133 & 5.243 & 5.563 & 3.596 & 3.624 & 10.47 & 6.42 & 4.46 & 7.173 & 3.731 \\
\hline $8 / 13 / 06$ & $8: 00$ & 11.116 & 12.288 & 8.996 & 8.112 & 5.086 & 5.565 & 3.54 & 3.578 & 10.444 & 6.313 & 3.926 & 7.061 & 3.182 \\
\hline $8 / 13 / 06$ & $12: 00$ & 11.7 & 12.305 & 8.984 & 8.105 & 4.817 & 5.542 & 3.53 & 3.561 & 10.416 & 6.278 & 3.905 & 7.029 & 3.091 \\
\hline $8 / 13 / 06$ & $16: 00$ & 11.967 & 12.305 & 8.982 & 8.1 & 4.763 & 5.502 & 3.526 & 3.559 & 10.404 & 6.285 & 3.998 & 7.059 & 3.199 \\
\hline $8 / 13 / 06$ & $20: 00$ & 12.079 & 12.332 & 8.994 & 8.119 & 4.819 & 5.49 & 3.542 & 3.573 & 10.402 & 6.287 & 4.047 & 7.075 & 3.257 \\
\hline $8 / 14 / 06$ & $0: 00$ & 12.132 & 12.363 & 9.012 & 8.144 & 4.882 & 5.5 & 3.57 & 3.601 & 10.402 & 6.297 & 4.096 & 7.094 & 3.311 \\
\hline $8 / 14 / 06$ & 4:00 & 12.168 & 12.381 & 9.026 & 8.159 & 4.934 & 5.511 & 3.582 & 3.615 & 10.404 & 6.306 & 4.134 & 7.11 & 3.353 \\
\hline $8 / 14 / 06$ & 8:00 & 12.2 & 12.4 & 9.05 & 8.18 & 4.999 & 5.53 & 3.612 & 3.643 & 10.418 & 6.339 & 4.208 & 7.14 & 3.451 \\
\hline $8 / 14 / 06$ & $12: 00$ & 12.221 & 12.407 & 9.083 & 8.203 & 5.119 & 5.553 & 3.659 & 3.685 & 10.467 & 6.432 & 4.425 & 7.208 & 3.787 \\
\hline $8 / 14 / 06$ & $16: 00$ & 12.231 & 12.4 & 9.111 & 8.217 & 5.228 & 5.584 & 3.691 & 3.713 & 10.512 & 6.5 & 4.609 & & 4.03 \\
\hline $8 / 14 / 06$ & $20: 00$ & 12.245 & 12.409 & 9.127 & 8.229 & 5.289 & 5.616 & 3.691 & 3.713 & 10.535 & 6.5 & 4.6 & 7.336 & 3.922 \\
\hline $8 / 15 / 06$ & $0: 00$ & 12.266 & 12.425 & 9.132 & 8.234 & 5.31 & 5.644 & 3.689 & 3.713 & 10.54 & 6.483 & 4.549 & 7.322 & 3.834 \\
\hline $8 / 15 / 06$ & $4: 00$ & 12.27 & 12.428 & 9.13 & 8.231 & 5.317 & 5.656 & 3.684 & 3.708 & 10.537 & 6.472 & 4.514 & 7.312 & 3.789 \\
\hline $8 / 15 / 06$ & $8: 00$ & 12.282 & 12.439 & 9.141 & 8.245 & 5.341 & 5.663 & 3.701 & 3.722 & 10.542 & 6.488 & 4.523 & 7.324 & 3.829 \\
\hline
\end{tabular}


Water Level (ft below top of casing) at Indicated Well

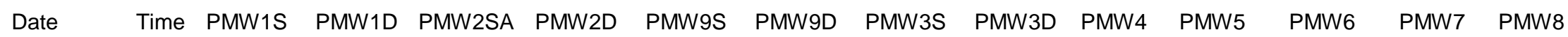

\begin{tabular}{|c|c|c|c|c|c|c|c|c|c|c|c|c|c|c|}
\hline $8 / 15 / 06$ & $12: 00$ & 12.298 & 12.444 & 9.174 & 8.269 & 5.408 & 5.677 & 3.752 & 3.769 & 10.575 & 6.57 & 4.719 & 7.389 & 4.191 \\
\hline $8 / 15 / 06$ & $16: 00$ & 12.301 & 12.439 & 9.202 & 8.283 & 5.469 & 5.703 & 3.784 & 3.797 & 10.619 & 6.628 & 4.873 & 7.426 & 4.347 \\
\hline $8 / 15 / 06$ & $20: 00$ & 12.315 & 12.453 & 9.214 & 8.294 & 5.499 & 5.729 & 3.775 & 3.781 & 10.64 & 6.614 & 4.838 & 7.403 & 4.249 \\
\hline 8/16/06 & $0: 00$ & 12.324 & 12.46 & 9.209 & 8.287 & 5.497 & 5.743 & 3.757 & 3.762 & 10.637 & 6.588 & 4.758 & 7.38 & 4.139 \\
\hline 8/16/06 & 4:00 & 12.324 & 12.46 & 9.2 & 8.28 & 5.49 & 5.745 & 3.745 & 3.748 & 10.63 & 6.572 & 4.705 & 7.361 & 4.067 \\
\hline 8/16/06 & 8:00 & 12.331 & 12.465 & 9.209 & 8.287 & 5.495 & 5.745 & 3.752 & 3.755 & 10.63 & 6.572 & 4.686 & 7.363 & 4.053 \\
\hline $8 / 16 / 06$ & $12: 00$ & 12.336 & 12.46 & 9.219 & 8.287 & 5.511 & 5.745 & 3.761 & 3.762 & 10.64 & 6.6 & 4.73 & 7.382 & 4.114 \\
\hline 8/16/06 & $16: 00$ & 12.329 & 12.448 & 9.233 & 8.297 & & & 3.785 & 3.783 & 10.665 & 6.649 & 4.866 & 7.419 & 4.279 \\
\hline 8/16/06 & $20: 00$ & 12.34 & 12.416 & 9.235 & 8.259 & & & 3.775 & 3.741 & 10.637 & 6.621 & 4.807 & 7.359 & 4.188 \\
\hline $8 / 17 / 06$ & $0: 00$ & 12.326 & 12.425 & 9.195 & 8.294 & 5.558 & & 3.687 & 3.744 & 10.621 & 6.432 & 4.234 & 7.333 & 3.404 \\
\hline $8 / 17 / 06$ & $4: 00$ & 11.162 & 12.423 & 9.043 & 8.154 & 3.928 & & 3.547 & 3.587 & 10.554 & 5.914 & 3.569 & 6.998 & 2.676 \\
\hline $8 / 17 / 06$ & 8:00 & 11.817 & 12.497 & & 8.144 & 4.141 & & 3.528 & 3.54 & 10.383 & 5.998 & 3.688 & 7.042 & 2.771 \\
\hline $8 / 17 / 06$ & $12: 00$ & 12.034 & & 9.019 & 8.163 & 4.377 & & 3.561 & 3.568 & 10.369 & 6.131 & 3.945 & 7.138 & 3.068 \\
\hline $8 / 17 / 06$ & $16: 00$ & 11.873 & 12.381 & 9.045 & 8.161 & 4.609 & & 3.589 & 3.594 & 10.397 & 6.245 & 4.178 & 7.205 & 3.334 \\
\hline $8 / 17 / 06$ & $20: 00$ & 11.75 & 12.393 & 9.057 & 8.17 & 4.775 & & 3.598 & 3.606 & 10.414 & 6.278 & 4.21 & 7.212 & 3.36 \\
\hline $8 / 18 / 06$ & $0: 00$ & 11.824 & 12.381 & 9.043 & 8.151 & 4.852 & & 3.582 & 3.587 & 10.407 & 6.278 & 4.18 & 7.203 & 3.353 \\
\hline $8 / 18 / 06$ & $4: 00$ & 9.844 & 12.135 & 8.668 & 7.87 & 3.917 & & 3.057 & 3.113 & 10.155 & 4.576 & 3.324 & 6.625 & 2.466 \\
\hline $8 / 18 / 06$ & $8: 00$ & 10.194 & 11.831 & 8.391 & 7.613 & 3.274 & & 2.992 & 3.003 & 9.75 & 4.817 & 3.056 & 6.171 & 2.319 \\
\hline $8 / 18 / 06$ & $12: 00$ & 10.993 & 11.833 & 8.344 & 7.594 & 3.491 & & 3.011 & 3.019 & 9.626 & 5.123 & 3.149 & 6.25 & 2.503 \\
\hline $8 / 18 / 06$ & $16: 00$ & 11.289 & 11.852 & 8.382 & 7.61 & 3.783 & & 3.043 & 3.052 & 9.607 & 5.344 & 3.261 & 6.376 & 2.657 \\
\hline $8 / 18 / 06$ & $20: 00$ & 11.163 & 11.88 & 8.419 & 7.641 & 3.991 & & 3.081 & 3.089 & 9.624 & 5.491 & 3.315 & 6.469 & 2.734 \\
\hline $8 / 19 / 06$ & $0: 00$ & 11.259 & 11.912 & 8.457 & 7.673 & 4.137 & & 3.116 & 3.122 & 9.652 & 5.592 & 3.371 & 6.562 & 2.804 \\
\hline $8 / 19 / 06$ & $4: 00$ & 11.383 & 11.922 & 8.485 & 7.688 & 4.228 & 5.443 & 3.132 & 3.138 & 9.682 & 5.659 & 3.417 & 6.644 & 2.853 \\
\hline $8 / 19 / 06$ & $8: 00$ & 11.535 & 11.942 & 8.515 & 7.709 & 4.312 & 5.441 & 3.158 & 3.164 & 9.715 & 5.725 & 3.487 & 6.73 & 2.911 \\
\hline 8/19/06 & $12: 00$ & 11.726 & 11.966 & 8.565 & 7.746 & 4.443 & 5.457 & 3.213 & 3.215 & 9.771 & 5.818 & 3.672 & 6.847 & 3.126 \\
\hline $8 / 19 / 06$ & $16: 00$ & 11.799 & 11.977 & 8.609 & 7.777 & 4.576 & 5.329 & 3.255 & 3.257 & 9.831 & 5.9 & 3.83 & 6.928 & 3.297 \\
\hline 8/19/06 & $20: 00$ & 11.521 & 11.996 & 8.635 & 7.8 & 4.669 & 5.268 & 3.274 & 3.274 & 9.875 & 5.937 & 3.856 & 6.956 & 3.29 \\
\hline $8 / 20 / 06$ & $0: 00$ & 11.526 & 12.012 & 8.654 & 7.812 & 4.712 & 5.242 & 3.286 & 3.286 & 9.903 & 5.956 & 3.863 & 6.965 & 3.29 \\
\hline 8/20/06 & $4: 00$ & 11.635 & 12.021 & 8.668 & 7.823 & 4.747 & 5.231 & 3.295 & 3.295 & 9.948 & 5.979 & 3.877 & 6.977 & 3.311 \\
\hline 8/20/06 & $8: 00$ & 11.838 & 12.042 & 8.691 & 7.845 & 4.791 & 5.235 & 3.316 & 3.314 & 9.952 & 6.005 & 3.9 & 6.991 & 3.336 \\
\hline 8/20/06 & $12: 00$ & 12.041 & 12.049 & 8.719 & 7.863 & 4.88 & 5.287 & 3.356 & 3.349 & 9.987 & 6.072 & 4.061 & 7.049 & 3.558 \\
\hline $8 / 20 / 06$ & $16: 00$ & 12.083 & 12.049 & 8.754 & 7.884 & 4.997 & 5.28 & 3.393 & 3.386 & 10.043 & 6.152 & 4.248 & 7.11 & 3.787 \\
\hline $8 / 20 / 06$ & $20: 00$ & 11.733 & 12.07 & 8.78 & 7.912 & 5.064 & 5.301 & 3.409 & 3.402 & 10.08 & 6.17 & 4.236 & 7.11 & 3.705 \\
\hline $8 / 21 / 06$ & $0: 00$ & 11.743 & 12.086 & 8.79 & 7.915 & 5.067 & 5.317 & 3.407 & 3.402 & 10.099 & 6.161 & 4.18 & 7.096 & 3.626 \\
\hline
\end{tabular}


TABLE S1.2 (Cont.)

Water Level (ft below top of casing) at Indicated Well

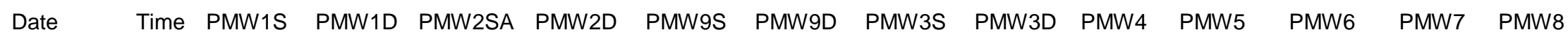

\begin{tabular}{|c|c|c|c|c|c|c|c|c|c|c|c|c|c|c|}
\hline 8/21/06 & $4: 00$ & 11.75 & 12.091 & 8.792 & 7.915 & 5.057 & 5.322 & 3.4 & 3.393 & 10.106 & 6.166 & 4.143 & 7.086 & 3.593 \\
\hline $8 / 21 / 06$ & $8: 00$ & 11.999 & 12.121 & 8.813 & 7.938 & 5.083 & 5.341 & 3.426 & 3.419 & 10.125 & 6.18 & 4.154 & 7.098 & 3.6 \\
\hline $8 / 21 / 06$ & $12: 00$ & 12.392 & 12.133 & 8.846 & 7.962 & 5.149 & 5.352 & 3.47 & 3.458 & & 6.252 & 4.311 & 7.156 & 3.882 \\
\hline $8 / 21 / 06$ & $16: 00$ & 12.226 & 12.137 & 8.876 & 7.983 & 5.219 & 5.371 & 3.5 & 3.491 & 10.216 & 6.338 & 4.45 & 7.2 & \\
\hline $8 / 21 / 06$ & $20: 00$ & 11.817 & 12.163 & 8.897 & 8.009 & 5.254 & 5.404 & 3.512 & 3.5 & 10.232 & 6.327 & 4.427 & 7.191 & 3.924 \\
\hline $8 / 22 / 06$ & $0: 00$ & 11.803 & 12.175 & 8.904 & 8.006 & 5.242 & 5.422 & 3.503 & 3.493 & 10.239 & 6.294 & 4.359 & 7.175 & 3.819 \\
\hline 8/22/06 & $4: 00$ & 11.838 & 12.184 & 8.904 & 8.011 & 5.233 & 5.432 & 3.498 & 3.491 & 10.244 & 6.289 & 4.32 & 7.165 & 3.766 \\
\hline $8 / 22 / 06$ & $8: 00$ & 12.1 & 12.205 & 8.921 & 8.03 & 5.249 & 5.443 & 3.519 & 3.51 & 10.251 & 6.303 & 4.322 & 7.177 & 3.784 \\
\hline $8 / 22 / 06$ & $12: 00$ & 12.483 & 12.209 & 8.951 & 8.046 & 5.307 & 5.45 & 3.561 & 3.547 & 10.283 & 6.373 & 4.488 & 7.231 & 4.067 \\
\hline $8 / 22 / 06$ & $16: 00$ & 12.217 & 12.195 & 8.975 & 8.053 & 5.361 & 5.46 & 3.582 & 3.564 & 10.323 & 6.432 & 4.628 & 7.268 & 4.232 \\
\hline $8 / 22 / 06$ & $20: 00$ & 11.915 & 12.207 & 8.982 & 8.062 & 5.378 & 5.483 & 3.57 & 3.557 & 10.344 & 6.415 & 4.576 & 7.245 & 4.106 \\
\hline 8/23/06 & $0: 00$ & 11.885 & 12.212 & 8.975 & 8.053 & 5.359 & 5.495 & 3.551 & 3.54 & 10.346 & 6.392 & 4.499 & 7.219 & 3.994 \\
\hline 8/23/06 & $4: 00$ & 11.906 & 12.198 & 8.956 & 8.034 & 5.336 & 5.49 & 3.53 & 3.519 & 10.337 & 6.369 & 4.434 & 7.196 & 3.91 \\
\hline 8/23/06 & 8:00 & 12.163 & 12.202 & 8.965 & 8.039 & 5.338 & 5.485 & 3.54 & 3.524 & 10.337 & 6.38 & 4.427 & & 3.938 \\
\hline 8/23/06 & $12: 00$ & 12.527 & 12.2 & 8.996 & 8.058 & 5.401 & 5.481 & 3.584 & 3.566 & 10.369 & 6.462 & 4.704 & 7.252 & 4.225 \\
\hline $8 / 23 / 06$ & $16: 00$ & 12.296 & 12.186 & 9.015 & 8.067 & 5.455 & 5.493 & 3.603 & 3.582 & 10.409 & 6.516 & 4.721 & 7.284 & 4.365 \\
\hline 8/23/06 & $20: 00$ & 11.967 & 12.202 & 9.017 & 8.072 & 5.469 & 5.516 & 3.589 & 3.571 & 10.425 & 6.492 & 4.649 & 7.249 & 4.265 \\
\hline $8 / 24 / 06$ & $0: 00$ & 11.964 & 12.205 & 9.005 & 8.06 & 5.445 & 5.523 & 3.568 & 3.55 & 10.423 & 6.457 & 4.562 & 7.219 & 4.146 \\
\hline $8 / 24 / 06$ & $4: 00$ & 11.985 & 12.209 & 8.993 & 8.055 & 5.427 & 5.525 & 3.556 & 3.538 & 10.411 & 6.441 & 4.504 & 7.2 & 4.064 \\
\hline $8 / 24 / 06$ & $8: 00$ & 12.238 & 12.214 & 8.998 & 8.058 & 5.422 & 5.518 & 3.558 & 3.54 & 10.407 & 6.441 & 4.481 & 7.198 & 4.055 \\
\hline $8 / 24 / 06$ & $12: 00$ & 12.564 & 12.214 & 9.033 & 8.081 & 5.48 & 5.514 & 3.605 & 3.585 & 10.439 & 6.523 & 4.625 & 7.254 & 4.295 \\
\hline $8 / 24 / 06$ & $16: 00$ & 12.277 & 12.221 & 9.061 & 8.102 & 5.536 & 5.53 & 3.633 & 3.61 & 10.477 & 6.574 & 4.756 & 7.287 & 4.431 \\
\hline $8 / 24 / 06$ & $20: 00$ & 12.046 & 12.233 & 9.059 & 8.1 & 5.541 & 5.553 & 3.614 & 3.592 & 10.491 & 6.546 & 4.693 & 7.254 & 4.335 \\
\hline $8 / 25 / 06$ & $0: 00$ & 12.002 & 12.253 & 9.057 & 8.109 & 5.529 & 5.57 & 3.61 & 3.589 & 10.486 & 6.518 & 4.621 & 7.233 & 4.235 \\
\hline $8 / 25 / 06$ & $4: 00$ & 11.957 & 12.274 & 9.059 & 8.119 & 5.52 & 5.579 & 3.612 & 3.591 & 10.481 & 6.499 & 4.567 & 7.219 & 4.162 \\
\hline $8 / 25 / 06$ & 8:00 & 15.301 & & 9.073 & 8.133 & 5.522 & 5.586 & 3.626 & 3.605 & 10.481 & 6.499 & 4.553 & 7.226 & 4.144 \\
\hline $8 / 25 / 06$ & $12: 00$ & 12.368 & 12.302 & 9.099 & 8.151 & 5.592 & 5.918 & 3.67 & 3.641 & 10.488 & 6.569 & 4.683 & 7.273 & 4.321 \\
\hline $8 / 25 / 06$ & $16: 00$ & 12.116 & 12.316 & 9.129 & 8.163 & 5.618 & 5.939 & 3.696 & 3.662 & 10.516 & 6.597 & 4.77 & 7.291 & 4.391 \\
\hline $8 / 25 / 06$ & $20: 00$ & 12.004 & 12.335 & 9.136 & 8.175 & 5.621 & 5.789 & 3.693 & 3.662 & 10.528 & 6.576 & 4.723 & 7.275 & 4.312 \\
\hline $8 / 26 / 06$ & $0: 00$ & 12.058 & 12.332 & 9.12 & 8.151 & 5.586 & 5.71 & 3.663 & 3.634 & 10.516 & 6.541 & 4.639 & 7.247 & 4.211 \\
\hline 8/26/06 & $4: 00$ & 12.105 & 12.337 & 9.115 & 8.149 & 5.564 & 5.668 & 3.656 & 3.627 & 10.505 & 6.52 & 4.583 & 7.231 & 4.141 \\
\hline 8/26/06 & $8: 00$ & 12.21 & 12.351 & 9.122 & 8.156 & 5.56 & 5.644 & 3.663 & 3.634 & 10.498 & 6.518 & 4.555 & 7.228 & 4.109 \\
\hline $8 / 26 / 06$ & $12: 00$ & 12.413 & 12.356 & 9.143 & 8.17 & 5.59 & 5.63 & 3.689 & 3.655 & 10.523 & 6.565 & 4.632 & 7.261 & 4.228 \\
\hline 8/26/06 & $16: 00$ & 12.305 & 12.36 & 9.169 & 8.189 & 5.639 & 5.63 & 3.715 & 3.68 & 10.547 & 6.6 & 4.725 & 7.289 & 4.328 \\
\hline
\end{tabular}


TABLE S1.2 (Cont.)

Water Level (ft below top of casing) at Indicated Well

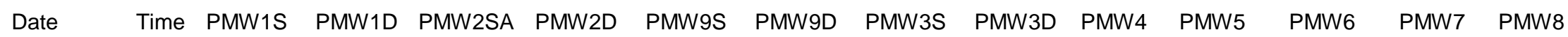

\begin{tabular}{|c|c|c|c|c|c|c|c|c|c|c|c|c|c|c|}
\hline 8/26/06 & $20: 00$ & 12.102 & 12.374 & 9.174 & 8.196 & 5.649 & 5.644 & 3.71 & 3.678 & 10.556 & 6.586 & 4.693 & 7.275 & 4.265 \\
\hline $8 / 27 / 06$ & $0: 00$ & 12.07 & 12.374 & 9.157 & 8.18 & 5.616 & 5.649 & 3.686 & 3.655 & 10.542 & 6.551 & 4.621 & 7.249 & 4.169 \\
\hline $8 / 27 / 06$ & $4: 00$ & 12.144 & 12.374 & 9.148 & 8.18 & 5.597 & 5.644 & 3.675 & 3.643 & 10.528 & 6.532 & 4.569 & 7.235 & 4.109 \\
\hline $8 / 27 / 06$ & $8: 00$ & 12.294 & 12.374 & 9.141 & 8.168 & 5.579 & 5.635 & 3.666 & 3.634 & 10.514 & 6.518 & 4.53 & 7.221 & 4.06 \\
\hline $8 / 27 / 06$ & $12: 00$ & 12.305 & 12.367 & 9.153 & 8.172 & 5.595 & 5.623 & 3.679 & 3.645 & 10.521 & 6.546 & 4.569 & 7.24 & 4.139 \\
\hline $8 / 27 / 06$ & $16: 00$ & 12.252 & 12.332 & 9.125 & 8.142 & 5.546 & 5.612 & 3.628 & 3.601 & 10.502 & 6.495 & 4.359 & 7.179 & 3.952 \\
\hline $8 / 27 / 06$ & $20: 00$ & 12.2 & 12.339 & 9.108 & 8.133 & 5.501 & 5.598 & 3.61 & 3.58 & 10.481 & 6.467 & 4.175 & 7.135 & 3.749 \\
\hline 8/28/06 & $0: 00$ & 12.231 & 12.321 & 9.089 & 8.109 & 5.462 & 5.579 & 3.584 & 3.556 & 10.46 & 6.453 & 4.119 & 7.114 & 3.7 \\
\hline $8 / 28 / 06$ & $4: 00$ & 12.21 & 12.332 & 9.085 & 8.116 & 5.464 & 5.57 & 3.591 & 3.561 & 10.449 & 6.446 & 4.11 & 7.11 & 3.702 \\
\hline 8/28/06 & $8: 00$ & 12.254 & 12.349 & 9.089 & 8.126 & 5.466 & 5.567 & 3.598 & 3.568 & 10.442 & 6.443 & 4.117 & 7.11 & 3.707 \\
\hline 8/28/06 & $12: 00$ & 12.235 & 12.374 & 9.113 & 8.149 & 5.485 & 5.572 & 3.626 & 3.598 & 10.449 & 6.46 & 4.173 & 7.131 & 3.768 \\
\hline 8/28/06 & $16: 00$ & 12.2 & 12.379 & 9.118 & 8.151 & 5.487 & 5.577 & 3.628 & 3.598 & 10.453 & 6.457 & 4.184 & 7.135 & 3.772 \\
\hline 8/28/06 & $20: 00$ & 12.193 & 12.381 & 9.118 & 8.154 & 5.483 & 5.581 & 3.63 & 3.601 & 10.453 & 6.45 & 4.184 & 7.133 & 3.761 \\
\hline 8/29/06 & 0:00 & 12.233 & 12.379 & 9.113 & 8.147 & 5.471 & 5.579 & 3.621 & 3.591 & 10.446 & 6.443 & 4.175 & 7.128 & 3.749 \\
\hline 8/29/06 & $4: 00$ & 12.217 & 12.379 & 9.108 & 8.144 & 5.464 & 5.577 & 3.619 & 3.589 & 10.439 & 6.439 & 4.17 & 7.126 & 3.744 \\
\hline $8 / 29 / 06$ & $8: 00$ & 12.422 & 12.383 & 9.115 & 8.151 & 5.466 & 5.574 & 3.626 & 3.596 & 10.439 & 6.446 & 4.184 & 7.131 & 3.751 \\
\hline 8/29/06 & $12: 00$ & 12.793 & 12.379 & 9.15 & 8.168 & 5.525 & 5.567 & 3.67 & 3.636 & 10.474 & 6.525 & 4.348 & 7.186 & 4.022 \\
\hline 8/29/06 & $16: 00$ & 12.499 & 12.379 & 9.181 & 8.191 & 5.595 & 5.579 & 3.705 & 3.669 & 10.533 & 6.597 & 4.516 & 7.238 & 4.207 \\
\hline 8/29/06 & $20: 00$ & 12.1 & 12.393 & 9.183 & 8.193 & 5.609 & 5.602 & 3.696 & 3.664 & 10.554 & 6.579 & 4.495 & 7.219 & 4.097 \\
\hline $8 / 30 / 06$ & $0: 00$ & 12.056 & 12.395 & 9.171 & 8.184 & 5.585 & 5.612 & 3.679 & 3.648 & 10.542 & 6.544 & 4.434 & 7.198 & 4.004 \\
\hline $8 / 30 / 06$ & $4: 00$ & 12.067 & 12.397 & 9.162 & 8.182 & 5.569 & 5.612 & 3.672 & 3.64 & 10.53 & 6.525 & 4.394 & 7.184 & 3.95 \\
\hline $8 / 30 / 06$ & $8: 00$ & 12.445 & 12.404 & 9.164 & 8.182 & 5.562 & 5.614 & 3.672 & 3.64 & 10.519 & 6.516 & 4.371 & 7.182 & 3.919 \\
\hline $8 / 30 / 06$ & $12: 00$ & 12.768 & 12.404 & 9.197 & 8.203 & 5.623 & 5.605 & 3.721 & 3.683 & 10.554 & 6.6 & 4.534 & 7.242 & 4.193 \\
\hline $8 / 30 / 06$ & $16: 00$ & 12.548 & 12.386 & 9.228 & 8.208 & 5.679 & 5.572 & 3.733 & 3.682 & 10.577 & 6.642 & 4.679 & 7.273 & 4.333 \\
\hline 8/30/06 & $20: 00$ & 12.137 & 12.407 & 9.235 & 8.217 & 5.695 & 5.593 & 3.726 & 3.68 & 10.593 & 6.618 & 4.655 & 7.252 & 4.244 \\
\hline $8 / 31 / 06$ & $0: 00$ & 12.128 & 12.411 & 9.225 & 8.21 & 5.672 & 5.602 & 3.71 & 3.661 & 10.582 & 6.586 & 4.578 & 7.228 & 4.139 \\
\hline 8/31/06 & $4: 00$ & 12.149 & 12.418 & 9.216 & 8.205 & 5.651 & 5.602 & 3.698 & 3.652 & 10.568 & 6.562 & 4.523 & 7.21 & 4.067 \\
\hline $8 / 31 / 06$ & 8:00 & 12.464 & 12.425 & 9.218 & 8.208 & 5.644 & 5.598 & 3.703 & 3.654 & 10.561 & 6.558 & 4.497 & 7.205 & 4.034 \\
\hline $8 / 31 / 06$ & $12: 00$ & 12.737 & 12.434 & 9.251 & 8.236 & 5.705 & 5.595 & 3.752 & 3.699 & 10.596 & 6.637 & 4.648 & 7.266 & 4.265 \\
\hline $8 / 31 / 06$ & $16: 00$ & 12.511 & 12.437 & 9.282 & 8.254 & 5.758 & 5.612 & 3.782 & 3.729 & 10.633 & 6.691 & 4.795 & 7.305 & 4.412 \\
\hline $8 / 31 / 06$ & $20: 00$ & 12.163 & 12.455 & 9.289 & 8.261 & 5.765 & 5.637 & 3.773 & 3.722 & 10.644 & 6.663 & 4.756 & 7.284 & 4.326 \\
\hline $9 / 1 / 06$ & $0: 00$ & 12.165 & 12.469 & 9.279 & 8.261 & 5.742 & 5.651 & 3.761 & 3.713 & 10.637 & 6.63 & 4.679 & 7.263 & 4.228 \\
\hline $9 / 1 / 06$ & $4: 00$ & 12.221 & 12.472 & 9.27 & 8.25 & 5.716 & 5.651 & 3.747 & 3.699 & 10.621 & 6.604 & 4.616 & 7.245 & 4.151 \\
\hline $9 / 1 / 06$ & $8: 00$ & 12.41 & 12.474 & 9.263 & 8.25 & 5.702 & 5.647 & 3.74 & 3.692 & 10.607 & 6.593 & 4.574 & 7.231 & 4.099 \\
\hline
\end{tabular}


Water Level (ft below top of casing) at Indicated Well

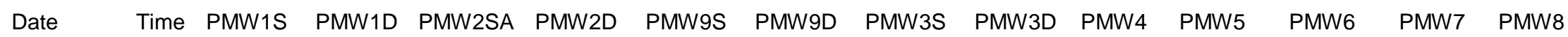

\begin{tabular}{|c|c|c|c|c|c|c|c|c|c|c|c|c|c|c|}
\hline 9/1/06 & $12: 00$ & 12.728 & 12.474 & 9.291 & 8.266 & 5.742 & 5.619 & 3.78 & 3.727 & 10.637 & 6.665 & 4.686 & 7.277 & 4.281 \\
\hline $9 / 1 / 06$ & $16: 00$ & 12.438 & 12.476 & 9.314 & 8.285 & 5.8 & 5.616 & 3.805 & 3.753 & 10.67 & 6.714 & 4.816 & 7.314 & 4.414 \\
\hline $9 / 1 / 06$ & $20: 00$ & 12.186 & 12.488 & 9.319 & 8.287 & 5.8 & 5.63 & 3.791 & 3.741 & 10.677 & 6.679 & 4.765 & 7.293 & 4.328 \\
\hline $9 / 2 / 06$ & $0: 00$ & 12.163 & 12.49 & 9.3 & 8.273 & 5.768 & 5.63 & 3.77 & 3.72 & 10.661 & 6.644 & 4.686 & 7.266 & 4.23 \\
\hline $9 / 2 / 06$ & 4:00 & 12.27 & 12.497 & 9.296 & 8.273 & 5.747 & 5.623 & 3.766 & 3.718 & 10.647 & 6.621 & 4.632 & 7.252 & 4.162 \\
\hline $9 / 2 / 06$ & $8: 00$ & 12.282 & 12.499 & 9.289 & 8.268 & 5.73 & 5.616 & 3.756 & 3.708 & 10.633 & 6.604 & 4.585 & 7.238 & 4.108 \\
\hline $9 / 2 / 06$ & $12: 00$ & 12.34 & 12.495 & 9.279 & 8.257 & 5.705 & 5.602 & 3.731 & 3.687 & 10.64 & 6.569 & 4.406 & 7.191 & 3.966 \\
\hline $9 / 2 / 06$ & $16: 00$ & 12.469 & 12.469 & 9.253 & 8.226 & 5.609 & 5.577 & 3.691 & 3.647 & 10.586 & 6.527 & 4.166 & 7.254 & 3.681 \\
\hline $9 / 2 / 06$ & $20: 00$ & 12.168 & 12.467 & 9.242 & 8.222 & 5.599 & 5.558 & 3.686 & 3.643 & 10.568 & 6.52 & 4.152 & 7.105 & 3.698 \\
\hline $9 / 3 / 06$ & $0: 00$ & 12.219 & 12.46 & 9.23 & 8.21 & 5.588 & 5.544 & 3.677 & 3.631 & 10.551 & 6.506 & 4.145 & 7.07 & 3.705 \\
\hline $9 / 3 / 06$ & $4: 00$ & 12.191 & 12.446 & 9.216 & 8.201 & 5.581 & 5.53 & 3.668 & 3.622 & 10.537 & 6.499 & 4.145 & 7.063 & 3.714 \\
\hline $9 / 3 / 06$ & $8: 00$ & 12.599 & 12.446 & 9.211 & 8.196 & 5.576 & 5.523 & 3.665 & 3.619 & 10.528 & 6.495 & 4.149 & 7.21 & 3.723 \\
\hline $9 / 3 / 06$ & $12: 00$ & 12.873 & 12.434 & 9.232 & 8.205 & 5.623 & 5.509 & 3.698 & 3.647 & 10.549 & 6.569 & 4.289 & 7.154 & 3.985 \\
\hline $9 / 3 / 06$ & $16: 00$ & 12.501 & 12.421 & 9.253 & 8.217 & 5.681 & 5.511 & 3.719 & 3.666 & 10.593 & 6.623 & 4.385 & 7.177 & 4.134 \\
\hline $9 / 3 / 06$ & $20: 00$ & 12.256 & 12.455 & 9.265 & 8.238 & 5.695 & 5.539 & 3.724 & 3.675 & 10.607 & 6.604 & 4.364 & 7.17 & 4.034 \\
\hline $9 / 4 / 06$ & $0: 00$ & 12.259 & 12.46 & 9.251 & 8.226 & 5.669 & 5.549 & 3.71 & 3.659 & 10.593 & 6.574 & 4.317 & 7.156 & 3.952 \\
\hline $9 / 4 / 06$ & $4: 00$ & 12.268 & 12.465 & 9.244 & 8.224 & 5.653 & 5.549 & 3.703 & 3.654 & 10.579 & 6.555 & 4.292 & 7.147 & 3.91 \\
\hline $9 / 4 / 06$ & $8: 00$ & 12.613 & 12.49 & 9.253 & 8.24 & 5.653 & 5.556 & 3.717 & 3.668 & 10.575 & 6.551 & 4.289 & 7.154 & 3.889 \\
\hline $9 / 4 / 06$ & $12: 00$ & 12.796 & 12.492 & 9.284 & 8.257 & 5.698 & 5.551 & 3.756 & 3.701 & 10.6 & 6.618 & 4.422 & 7.203 & 4.101 \\
\hline $9 / 4 / 06$ & $16: 00$ & 12.497 & 12.492 & 9.314 & 8.276 & 5.754 & 5.56 & 3.784 & 3.727 & 10.647 & 6.679 & 4.55 & 7.238 & 4.241 \\
\hline $9 / 4 / 06$ & $20: 00$ & 12.191 & 12.509 & 9.319 & 8.285 & 5.758 & 5.586 & 3.78 & 3.725 & 10.658 & 6.651 & 4.522 & 7.224 & 4.141 \\
\hline $9 / 5 / 06$ & $0: 00$ & 12.219 & 12.504 & 9.298 & 8.268 & 5.728 & 5.591 & 3.754 & 3.701 & 10.642 & 6.614 & 4.459 & 7.203 & 4.048 \\
\hline $9 / 5 / 06$ & 4:00 & 12.247 & 12.504 & 9.289 & 8.259 & 5.707 & 5.591 & 3.745 & 3.692 & 10.621 & 6.595 & 4.42 & 7.191 & 3.992 \\
\hline $9 / 5 / 06$ & $8: 00$ & 12.698 & 12.511 & 9.286 & 8.264 & 5.7 & 5.588 & 3.747 & 3.694 & 10.612 & 6.586 & 4.394 & 7.189 & 3.957 \\
\hline $9 / 5 / 06$ & $12: 00$ & 13.108 & 12.504 & 9.317 & 8.28 & 5.747 & 5.579 & 3.787 & 3.729 & 10.644 & 6.667 & 4.534 & 7.24 & 4.197 \\
\hline 9/5/06 & $16: 00$ & 12.7 & 12.495 & 9.338 & 8.289 & 5.8 & 5.586 & 3.807 & 3.748 & 10.689 & 6.726 & 4.669 & 7.275 & 4.339 \\
\hline 9/5/06 & $20: 00$ & 12.163 & 12.511 & 9.335 & 8.297 & 5.807 & 5.607 & 3.796 & 3.741 & 10.693 & 6.691 & 4.627 & 7.252 & 4.239 \\
\hline 9/6/06 & $0: 00$ & 12.172 & 12.504 & 9.317 & 8.275 & 5.77 & 5.614 & 3.773 & 3.715 & 10.672 & 6.646 & 4.55 & 7.226 & 4.139 \\
\hline 9/6/06 & $4: 00$ & 12.231 & 12.497 & 9.298 & 8.261 & 5.744 & 5.607 & 3.752 & 3.696 & 10.654 & 6.621 & 4.497 & 7.205 & 4.069 \\
\hline 9/6/06 & $8: 00$ & 12.679 & 12.506 & 9.296 & 8.264 & 5.735 & 5.6 & 3.754 & 3.699 & 10.637 & 6.609 & 4.464 & 7.2 & 4.022 \\
\hline 9/6/06 & $12: 00$ & 13.055 & 12.499 & 9.331 & 8.282 & 5.789 & 5.588 & 3.798 & 3.738 & 10.675 & 6.702 & 4.604 & 7.249 & 4.281 \\
\hline 9/6/06 & $16: 00$ & 12.684 & 12.49 & 9.352 & 8.297 & 5.845 & 5.598 & 3.821 & 3.759 & 10.719 & 6.763 & 4.742 & 7.286 & 4.428 \\
\hline $9 / 6 / 06$ & $20: 00$ & 12.226 & 12.497 & 9.345 & 8.292 & 5.84 & 5.616 & 3.803 & 3.741 & 10.721 & 6.716 & 4.693 & 7.258 & 4.316 \\
\hline $9 / 7 / 06$ & $0: 00$ & 12.221 & 12.486 & 9.321 & 8.271 & 5.805 & 5.612 & 3.773 & 3.713 & 10.703 & 6.674 & 4.611 & 7.228 & 4.218 \\
\hline
\end{tabular}


TABLE S1.2 (Cont.)

Water Level (ft below top of casing) at Indicated Well

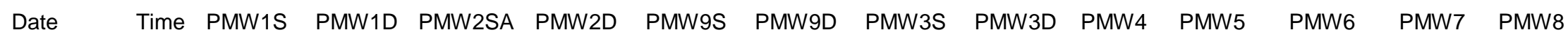

\begin{tabular}{|c|c|c|c|c|c|c|c|c|c|c|c|c|c|c|}
\hline 9/7/06 & $4: 00$ & 12.231 & 12.476 & 9.3 & 8.254 & 5.779 & 5.6 & 3.752 & 3.694 & 10.677 & 6.646 & 4.546 & 7.205 & 4.146 \\
\hline $9 / 7 / 06$ & $8: 00$ & 12.653 & 12.488 & 9.3 & 8.259 & 5.775 & 5.588 & 3.759 & 3.699 & 10.665 & 6.646 & 4.52 & 7.203 & 4.146 \\
\hline 9/7/06 & $12: 00$ & 12.952 & 12.483 & 9.34 & 8.282 & 5.838 & 5.581 & 3.807 & 3.743 & 10.71 & 6.754 & 4.662 & 7.256 & 4.4 \\
\hline 9/7/06 & $16: 00$ & 12.611 & 12.479 & 9.364 & 8.294 & 5.882 & 5.593 & 3.828 & 3.762 & 10.752 & 6.803 & 4.779 & 7.286 & 4.521 \\
\hline 9/7/06 & $20: 00$ & 12.268 & 12.492 & 9.357 & 8.294 & 5.88 & 5.616 & 3.81 & 3.745 & 10.752 & 6.751 & 4.716 & 7.254 & 4.421 \\
\hline $9 / 8 / 06$ & $0: 00$ & 12.228 & 12.497 & 9.338 & 8.28 & 5.85 & 5.621 & 3.784 & 3.724 & 10.731 & 6.707 & 4.637 & 7.228 & 4.321 \\
\hline $9 / 8 / 06$ & $4: 00$ & 12.249 & 12.495 & 9.324 & 8.268 & 5.821 & 5.614 & 3.768 & 3.708 & 10.705 & 6.677 & 4.576 & 7.21 & 4.248 \\
\hline $9 / 8 / 06$ & 8:00 & 12.7 & 12.506 & 9.326 & 8.278 & 5.819 & 5.607 & 3.777 & 3.715 & 10.696 & 6.672 & 4.548 & 7.207 & 4.23 \\
\hline $9 / 8 / 06$ & $12: 00$ & 12.999 & 12.511 & 9.371 & 8.306 & 5.885 & 5.605 & 3.833 & 3.769 & 10.74 & 6.782 & 4.7 & 7.268 & 4.47 \\
\hline 9/8/06 & $16: 00$ & 12.522 & 12.516 & 9.394 & 8.327 & 5.936 & 5.621 & 3.859 & 3.792 & 10.782 & 6.831 & 4.823 & 7.3 & 4.584 \\
\hline 9/8/06 & $20: 00$ & 12.277 & 12.532 & 9.389 & 8.329 & 5.927 & 5.644 & 3.84 & 3.776 & 10.782 & 6.779 & 4.758 & 7.275 & 4.489 \\
\hline 9/9/06 & $0: 00$ & 12.308 & 12.537 & 9.373 & 8.313 & 5.892 & 5.651 & 3.817 & 3.755 & 10.759 & 6.733 & 4.681 & 7.249 & 4.391 \\
\hline 9/9/06 & $4: 00$ & 12.345 & 12.537 & 9.359 & 8.306 & 5.866 & 5.644 & 3.8 & 3.738 & 10.738 & 6.7 & 4.623 & 7.231 & 4.318 \\
\hline 9/9/06 & $8: 00$ & 12.574 & 12.541 & 9.354 & 8.301 & 5.852 & 5.64 & 3.796 & 3.736 & 10.719 & 6.686 & 4.581 & 7.221 & 4.262 \\
\hline 9/9/06 & $12: 00$ & 12.396 & 12.537 & 9.354 & 8.299 & 5.847 & 5.628 & 3.793 & 3.734 & 10.707 & 6.681 & 4.567 & 7.217 & 4.255 \\
\hline 9/9/06 & $16: 00$ & 12.385 & 12.504 & 9.331 & 8.278 & 5.822 & 5.607 & 3.768 & 3.708 & 10.67 & 6.658 & 4.504 & 7.189 & 4.188 \\
\hline 9/9/06 & $20: 00$ & 12.371 & 12.481 & 9.319 & 8.271 & 5.805 & 5.591 & 3.756 & 3.696 & 10.654 & 6.637 & 4.429 & 7.165 & 4.125 \\
\hline $9 / 10 / 06$ & $0: 00$ & 12.357 & 12.534 & 9.3 & 8.282 & 5.695 & 5.574 & 3.693 & 3.645 & 10.644 & 6.474 & 3.942 & 7.058 & 3.646 \\
\hline 9/10/06 & $4: 00$ & 12.329 & 12.49 & 9.178 & 8.254 & 4.499 & 5.5 & 3.553 & 3.507 & 10.523 & 6.035 & 3.254 & 6.574 & 2.712 \\
\hline $9 / 10 / 06$ & $8: 00$ & 12.354 & 12.444 & 9.125 & 8.135 & 4.41 & 5.383 & 3.507 & 3.458 & 10.418 & 6.025 & 3.242 & 6.534 & 2.689 \\
\hline $9 / 10 / 06$ & $12: 00$ & 12.452 & 12.421 & 9.099 & 8.081 & 4.55 & 5.298 & 3.49 & 3.439 & 10.367 & 6.098 & 3.324 & 6.588 & 2.775 \\
\hline 9/10/06 & $16: 00$ & 12.406 & 12.4 & 9.08 & 8.065 & 4.718 & 5.254 & 3.486 & 3.432 & 10.346 & 6.163 & 3.433 & 6.646 & 2.901 \\
\hline 9/10/06 & $20: 00$ & 12.277 & 12.397 & 9.075 & 8.069 & 4.861 & 5.245 & 3.497 & 3.444 & 10.344 & 6.21 & 3.515 & 6.702 & 3.004 \\
\hline $9 / 11 / 06$ & $0: 00$ & 12.298 & 12.386 & 9.068 & 8.062 & 4.95 & 5.242 & 3.495 & 3.444 & 10.339 & 6.235 & 3.564 & 6.735 & 3.074 \\
\hline $9 / 11 / 06$ & $4: 00$ & 12.207 & 12.332 & 9.066 & 8.015 & 5.02 & 5.249 & 3.504 & 3.451 & 10.341 & 6.259 & 3.613 & 6.765 & 3.142 \\
\hline $9 / 11 / 06$ & $8: 00$ & 12.2 & 12.404 & 9.08 & 8.088 & 5.085 & 5.263 & 3.53 & 3.477 & 10.348 & 6.284 & 3.662 & 6.802 & 3.205 \\
\hline $9 / 11 / 06$ & $12: 00$ & 12.284 & 12.414 & 9.089 & 8.095 & 5.13 & 5.277 & 3.544 & 3.491 & 10.355 & 6.305 & 3.711 & 6.832 & 3.263 \\
\hline $9 / 11 / 06$ & $16: 00$ & 12.31 & 12.397 & 9.092 & 8.09 & 5.167 & 5.284 & 3.544 & 3.488 & 10.367 & 6.326 & 3.762 & 6.858 & 3.336 \\
\hline $9 / 11 / 06$ & $20: 00$ & 12.21 & 12.409 & 9.101 & 8.107 & 5.209 & 5.298 & 3.563 & 3.507 & 10.376 & 6.34 & 3.806 & 6.879 & 3.382 \\
\hline $9 / 12 / 06$ & $0: 00$ & 12.245 & 12.404 & 9.101 & 8.1 & 5.223 & 5.308 & 3.56 & 3.505 & 10.383 & 6.343 & 3.818 & 6.888 & 3.406 \\
\hline $9 / 12 / 06$ & $4: 00$ & 12.245 & 12.4 & 9.096 & 8.097 & 5.237 & 5.31 & 3.56 & 3.502 & 10.381 & 6.352 & 3.837 & 6.897 & 3.434 \\
\hline $9 / 12 / 06$ & $8: 00$ & 12.319 & 12.4 & 9.101 & 8.102 & 5.256 & 5.317 & 3.565 & 3.512 & 10.386 & 6.361 & 3.86 & 6.909 & 3.459 \\
\hline $9 / 12 / 06$ & $12: 00$ & 12.712 & 12.39 & 9.11 & 8.102 & 5.286 & 5.317 & 3.577 & 3.519 & 10.397 & 6.392 & 3.925 & 6.937 & 3.571 \\
\hline $9 / 12 / 06$ & $16: 00$ & 12.599 & 12.376 & 9.124 & 8.107 & 5.347 & 5.32 & 3.595 & 3.533 & 10.425 & 6.443 & 4.042 & 6.977 & 3.746 \\
\hline
\end{tabular}


TABLE S1.2 (Cont.)

Water Level (ft below top of casing) at Indicated Well

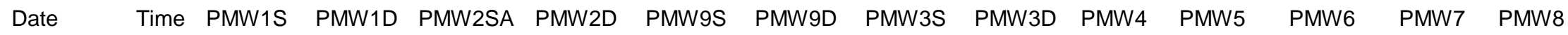

\begin{tabular}{|c|c|c|c|c|c|c|c|c|c|c|c|c|c|c|}
\hline $9 / 12 / 06$ & $20: 00$ & 12.053 & 12.381 & 9.127 & 8.111 & 5.38 & 5.334 & 3.595 & 3.533 & 10.439 & 6.439 & 4.037 & 6.974 & 3.693 \\
\hline $9 / 13 / 06$ & $0: 00$ & 12.13 & 12.374 & 9.115 & 8.102 & 5.375 & 5.341 & 3.581 & 3.519 & 10.432 & 6.422 & 4.009 & 6.963 & 3.655 \\
\hline $9 / 13 / 06$ & $4: 00$ & 12.1 & 12.365 & 9.103 & 8.09 & 5.366 & 5.338 & 3.57 & 3.509 & 10.423 & 6.41 & 3.988 & 6.951 & 3.641 \\
\hline $9 / 13 / 06$ & $8: 00$ & 12.672 & 12.363 & 9.099 & 8.088 & 5.366 & 5.336 & 3.57 & 3.507 & 10.418 & 6.408 & 3.986 & 6.949 & 3.644 \\
\hline $9 / 13 / 06$ & $12: 00$ & 13.057 & 12.351 & 9.127 & 8.097 & 5.424 & 5.327 & 3.602 & 3.533 & 10.446 & 6.478 & 4.121 & 7 & 3.907 \\
\hline $9 / 13 / 06$ & $16: 00$ & 12.674 & 12.337 & 9.146 & 8.107 & 5.483 & 5.336 & 3.626 & 3.551 & 10.481 & 6.532 & 4.231 & 7.03 & 4.057 \\
\hline $9 / 13 / 06$ & $20: 00$ & 12.074 & 12.346 & 9.148 & 8.114 & 5.502 & 5.357 & 3.619 & 3.547 & 10.495 & 6.513 & 4.203 & 7.021 & 3.942 \\
\hline $9 / 14 / 06$ & $0: 00$ & 12.109 & 12.351 & 9.132 & 8.104 & 5.488 & 5.364 & 3.602 & 3.533 & 10.486 & 6.488 & 4.147 & 7.005 & 3.861 \\
\hline $9 / 14 / 06$ & $4: 00$ & 12.149 & 12.349 & 9.122 & 8.097 & 5.474 & 5.359 & 3.591 & 3.521 & 10.474 & 6.466 & 4.105 & 6.986 & 3.809 \\
\hline $9 / 14 / 06$ & $8: 00$ & 12.585 & 12.358 & 9.129 & 8.109 & 5.483 & 5.357 & 3.607 & 3.535 & 10.47 & 6.478 & 4.114 & 6.998 & 3.851 \\
\hline $9 / 14 / 06$ & $12: 00$ & 12.861 & 12.363 & 9.174 & 8.137 & 5.555 & 5.359 & 3.663 & 3.589 & 10.512 & 6.569 & 4.305 & 7.07 & 4.181 \\
\hline $9 / 14 / 06$ & $16: 00$ & 12.431 & 12.369 & 9.202 & 8.163 & 5.618 & 5.38 & 3.695 & 3.619 & 10.551 & 6.621 & 4.436 & 7.109 & 4.311 \\
\hline $9 / 14 / 06$ & $20: 00$ & 12.116 & 12.39 & 9.216 & 8.175 & 5.63 & 5.413 & 3.693 & 3.617 & 10.568 & 6.602 & 4.422 & 7.102 & 4.213 \\
\hline $9 / 15 / 06$ & 0:00 & 12.147 & 12.397 & 9.207 & 8.165 & 5.614 & 5.427 & 3.675 & 3.6 & 10.563 & 6.572 & 4.359 & 7.084 & 4.113 \\
\hline $9 / 15 / 06$ & $4: 00$ & 12.13 & 12.39 & 9.188 & 8.151 & 5.593 & 5.425 & 3.656 & 3.582 & 10.547 & 6.546 & 4.303 & 7.067 & 4.031 \\
\hline $9 / 15 / 06$ & $8: 00$ & 12.375 & 12.4 & 9.19 & 8.156 & 5.593 & 5.42 & 3.663 & 3.589 & 10.537 & 6.544 & 4.287 & 7.067 & 4.01 \\
\hline $9 / 15 / 06$ & $12: 00$ & 12.651 & 12.367 & 9.185 & 8.132 & 5.597 & 5.401 & 3.649 & 3.575 & 10.535 & 6.555 & 4.287 & 7.063 & 4.064 \\
\hline $9 / 15 / 06$ & $16: 00$ & 12.609 & 12.346 & 9.204 & 8.146 & 5.656 & 5.394 & 3.684 & 3.603 & 10.575 & 6.63 & 4.434 & 7.105 & 4.288 \\
\hline $9 / 15 / 06$ & $20: 00$ & 12.242 & 12.358 & 9.211 & 8.161 & 5.677 & 5.413 & 3.684 & 3.603 & 10.584 & 6.623 & 4.448 & 7.1 & 4.246 \\
\hline $9 / 16 / 06$ & $0: 00$ & 12.284 & 12.356 & 9.178 & 8.13 & 5.548 & 5.406 & 3.619 & 3.547 & 10.551 & 6.534 & 4.054 & 6.977 & 3.819 \\
\hline 9/16/06 & $4: 00$ & 12.303 & 12.349 & 9.16 & 8.111 & 5.52 & 5.385 & 3.593 & 3.521 & 10.526 & 6.508 & 3.988 & 6.932 & 3.767 \\
\hline $9 / 16 / 06$ & $8: 00$ & 12.59 & 12.349 & 9.157 & 8.111 & 5.53 & 5.369 & 3.6 & 3.526 & 10.516 & 6.513 & 4.009 & 6.935 & 3.84 \\
\hline 9/16/06 & $12: 00$ & 12.821 & 12.339 & 9.183 & 8.123 & 5.595 & 5.359 & 3.637 & 3.558 & 10.547 & 6.59 & 4.158 & 6.991 & 4.115 \\
\hline 9/16/06 & $16: 00$ & 12.518 & 12.337 & 9.207 & 8.142 & 5.663 & 5.371 & 3.665 & 3.584 & 10.584 & 6.644 & 4.291 & 7.04 & 4.29 \\
\hline 9/16/06 & $20: 00$ & 12.177 & 12.358 & 9.197 & 8.144 & 5.686 & 5.397 & 3.651 & 3.57 & 10.586 & 6.646 & 4.298 & 7.042 & 4.225 \\
\hline 9/17/06 & $0: 00$ & 11.953 & 12.383 & 9.183 & 8.146 & 5.523 & 5.404 & 3.609 & 3.542 & 10.542 & 6.469 & 3.713 & 6.76 & 3.548 \\
\hline $9 / 17 / 06$ & $4: 00$ & 11.873 & 12.423 & 9.188 & 8.168 & 5.45 & 5.408 & 3.618 & 3.549 & 10.514 & 6.459 & 3.739 & 6.774 & 3.515 \\
\hline 9/17/06 & 8:00 & 12.298 & 12.432 & 9.19 & 8.151 & 5.434 & 5.401 & 3.614 & 3.544 & 10.502 & 6.462 & 3.795 & 6.8 & 3.574 \\
\hline $9 / 17 / 06$ & $12: 00$ & 12.506 & 12.428 & 9.202 & 8.158 & 5.483 & 5.392 & 3.637 & 3.565 & 10.512 & 6.506 & 3.93 & 6.856 & 3.784 \\
\hline $9 / 17 / 06$ & $16: 00$ & 12.282 & 12.407 & 9.214 & 8.16 & 5.537 & 5.39 & 3.651 & 3.575 & 10.537 & 6.548 & 4.054 & 6.904 & 3.963 \\
\hline $9 / 17 / 06$ & $20: 00$ & 11.939 & 12.407 & 9.202 & 8.153 & 5.546 & 5.401 & 3.637 & 3.563 & 10.533 & 6.529 & 4.056 & 6.907 & 3.884 \\
\hline 9/18/06 & $0: 00$ & 12.046 & 12.411 & 9.19 & 8.151 & 5.539 & 5.401 & 3.63 & 3.556 & 10.516 & 6.506 & 4.035 & 6.904 & 3.837 \\
\hline $9 / 18 / 06$ & $4: 00$ & 12.023 & 12.411 & 9.181 & 8.146 & 5.532 & 5.399 & 3.625 & 3.549 & 10.502 & 6.492 & 4.025 & 6.907 & 3.816 \\
\hline $9 / 18 / 06$ & $8: 00$ & 12.436 & 12.411 & 9.176 & 8.142 & 5.53 & 5.394 & 3.623 & 3.549 & 10.495 & 6.494 & 4.03 & 6.911 & 3.828 \\
\hline
\end{tabular}


TABLE S1.2 (Cont.)

Water Level (ft below top of casing) at Indicated Well

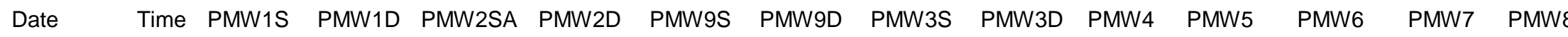

\begin{tabular}{|c|c|c|c|c|c|c|c|c|c|c|c|c|c|c|}
\hline $9 / 18 / 06$ & $12: 00$ & 12.555 & 12.416 & 9.197 & 8.158 & 5.581 & 5.39 & 3.653 & 3.579 & 10.507 & 6.536 & 4.126 & 6.958 & 4.001 \\
\hline 9/18/06 & $16: 00$ & 12.095 & 12.432 & 9.223 & 8.184 & 5.63 & 5.404 & 3.681 & 3.605 & 10.533 & 6.564 & 4.205 & 6.998 & 4.078 \\
\hline $9 / 18 / 06$ & $20: 00$ & 12.121 & 12.451 & 9.232 & 8.196 & 5.637 & 5.425 & 3.691 & 3.612 & 10.54 & 6.555 & 4.212 & 7.009 & 4.033 \\
\hline 9/19/06 & $0: 00$ & 12.123 & 12.451 & 9.223 & 8.189 & 5.623 & 5.434 & 3.677 & 3.6 & 10.528 & 6.534 & 4.186 & 7.007 & 3.973 \\
\hline $9 / 19 / 06$ & $4: 00$ & 12.242 & 12.455 & 9.221 & 8.189 & 5.612 & 5.439 & 3.674 & 3.6 & 10.519 & 6.522 & 4.168 & 7.005 & 3.931 \\
\hline 9/19/06 & $8: 00$ & 12.471 & 12.472 & 9.235 & 8.205 & 5.621 & 5.443 & 3.693 & 3.614 & 10.521 & 6.534 & 4.189 & 7.023 & 3.952 \\
\hline $9 / 19 / 06$ & $12: 00$ & 12.702 & 12.467 & 9.249 & 8.21 & 5.663 & 5.441 & 3.714 & 3.633 & 10.54 & 6.571 & 4.259 & 7.051 & 4.087 \\
\hline 9/19/06 & $16: 00$ & 12.452 & 12.444 & 9.258 & 8.205 & 5.696 & 5.443 & 3.716 & 3.633 & 10.563 & 6.604 & 4.322 & 7.072 & 4.178 \\
\hline $9 / 19 / 06$ & $20: 00$ & 11.979 & 12.458 & 9.256 & 8.212 & 5.696 & 5.46 & 3.712 & 3.626 & 10.561 & 6.576 & 4.305 & 7.065 & 4.078 \\
\hline 9/20/06 & 0:00 & 12.123 & 12.451 & 9.237 & 8.196 & 5.663 & 5.462 & 3.691 & 3.607 & 10.54 & 6.546 & 4.256 & 7.049 & 3.996 \\
\hline 9/20/06 & $4: 00$ & 12.189 & 12.441 & 9.221 & 8.184 & 5.637 & 5.453 & 3.674 & 3.593 & 10.521 & 6.522 & 4.217 & 7.035 & 3.94 \\
\hline 9/20/06 & $8: 00$ & 12.649 & 12.437 & 9.214 & 8.174 & 5.626 & 5.443 & 3.67 & 3.586 & 10.507 & 6.513 & 4.191 & 7.025 & 3.907 \\
\hline 9/20/06 & $12: 00$ & 12.852 & 12.388 & 9.207 & 8.144 & 5.649 & 5.415 & 3.665 & 3.575 & 10.512 & 6.548 & 4.259 & 7.039 & 4.075 \\
\hline 9/20/06 & $16: 00$ & 12.548 & 12.36 & 9.211 & 8.146 & 5.703 & 5.404 & 3.674 & 3.584 & 10.535 & 6.592 & 4.359 & 7.063 & 4.218 \\
\hline 9/20/06 & $20: 00$ & 12.14 & 12.349 & 9.197 & 8.135 & 5.696 & 5.408 & 3.653 & 3.561 & 10.526 & 6.567 & 4.34 & 7.046 & 4.138 \\
\hline $9 / 21 / 06$ & 0:00 & 12.303 & 12.321 & 9.167 & 8.102 & 5.656 & 5.397 & 3.616 & 3.526 & 10.502 & 6.532 & 4.27 & 7.012 & 4.052 \\
\hline $9 / 21 / 06$ & $4: 00$ & 12.42 & 12.3 & 9.139 & 8.081 & 5.633 & 5.376 & 3.591 & 3.5 & 10.481 & 6.508 & 4.214 & 6.979 & 3.994 \\
\hline $9 / 21 / 06$ & $8: 00$ & 12.322 & 12.3 & 9.127 & 8.097 & 5.621 & 5.359 & 3.581 & 3.491 & 10.474 & 6.494 & 4.161 & 6.949 & 3.949 \\
\hline $9 / 21 / 06$ & $12: 00$ & 12.375 & 12.342 & 9.094 & 8.125 & 5.544 & 5.331 & 3.518 & 3.43 & 10.46 & 6.429 & 3.683 & 6.804 & 3.641 \\
\hline $9 / 21 / 06$ & $16: 00$ & 11.733 & 12.17 & 8.979 & 7.933 & 4.31 & 5.287 & 3.36 & 3.283 & 10.311 & 5.965 & 3.135 & 6.306 & 2.682 \\
\hline $9 / 21 / 06$ & $20: 00$ & 11.158 & 12.112 & 8.829 & 7.889 & 3.782 & 5.147 & 3.271 & 3.11 & 10.167 & 5.519 & 2.916 & 6.047 & 2.411 \\
\hline $9 / 22 / 06$ & $0: 00$ & 10.743 & 11.926 & 8.644 & 7.659 & 3.496 & 4.964 & 3.236 & 2.981 & 9.922 & 5.325 & 2.89 & 5.824 & 2.395 \\
\hline 9/22/06 & $4: 00$ & 10.656 & 11.905 & 8.595 & 7.65 & 3.735 & 4.833 & 3.192 & 2.991 & 9.831 & 5.512 & 2.955 & 5.847 & 2.523 \\
\hline 9/22/06 & $8: 00$ & 11.434 & 11.97 & 8.595 & 7.659 & 3.978 & 4.777 & 3.388 & 3.009 & 9.787 & 5.626 & 3.009 & 5.905 & 2.645 \\
\hline $9 / 22 / 06$ & $12: 00$ & 11.941 & 12 & 8.625 & 7.68 & 4.247 & 4.766 & 3.504 & 3.044 & 9.782 & 5.748 & 3.102 & 5.987 & 2.897 \\
\hline $9 / 22 / 06$ & $16: 00$ & 11.749 & 12.026 & 8.665 & 7.706 & 4.504 & 4.791 & 3.269 & 3.079 & 9.801 & 5.848 & 3.198 & 6.061 & 3.125 \\
\hline $9 / 22 / 06$ & $20: 00$ & 11.565 & 12.059 & 8.691 & 7.732 & 4.647 & 4.836 & 3.551 & 3.103 & 9.819 & 5.897 & 3.233 & 6.115 & 3.17 \\
\hline 9/23/06 & $0: 00$ & 11.609 & 12.052 & 8.691 & 7.722 & 4.705 & 4.861 & 3.472 & 3.096 & 9.826 & 5.916 & 3.249 & 6.152 & 3.195 \\
\hline 9/23/06 & $4: 00$ & 11.698 & 12.049 & 8.696 & 7.727 & 4.759 & 4.875 & 3.399 & 3.103 & 9.836 & 5.944 & 3.279 & 6.206 & 3.237 \\
\hline 9/23/06 & $8: 00$ & 12.128 & 12.052 & 8.703 & 7.729 & 4.808 & 4.887 & 3.705 & 3.107 & 9.85 & 5.972 & 3.31 & 6.255 & 3.279 \\
\hline $9 / 23 / 06$ & $12: 00$ & 11.922 & 12.061 & 8.726 & 7.75 & 4.906 & 4.901 & 3.707 & 3.138 & 9.875 & 6.021 & 3.382 & 6.313 & 3.415 \\
\hline 9/23/06 & $16: 00$ & 11.773 & 12.07 & 8.747 & 7.771 & 4.983 & 4.927 & 3.472 & 3.166 & 9.906 & 6.058 & 3.433 & 6.357 & 3.466 \\
\hline $9 / 23 / 06$ & $20: 00$ & 11.782 & 12.107 & 8.768 & 7.799 & 5.018 & 4.957 & 3.416 & 3.187 & 9.927 & 6.079 & 3.461 & 6.397 & 3.48 \\
\hline $9 / 24 / 06$ & $0: 00$ & 11.82 & 12.133 & 8.787 & 7.821 & 5.046 & 4.983 & 3.306 & 3.21 & 9.948 & 6.1 & 3.498 & 6.434 & 3.501 \\
\hline
\end{tabular}


TABLE S1.2 (Cont.)

Water Level (ft below top of casing) at Indicated Well

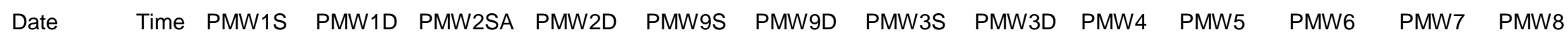

\begin{tabular}{|c|c|c|c|c|c|c|c|c|c|c|c|c|c|c|}
\hline $9 / 24 / 06$ & $4: 00$ & 11.689 & 12.149 & 8.806 & 7.839 & 5.07 & 5.006 & 3.183 & 3.231 & 9.969 & 6.119 & 3.533 & 6.464 & 3.522 \\
\hline $9 / 24 / 06$ & $8: 00$ & 12.198 & 12.165 & 8.824 & 7.856 & 5.088 & 5.025 & 3.532 & 3.248 & 9.985 & 6.137 & 3.564 & 6.492 & 3.539 \\
\hline $9 / 24 / 06$ & $12: 00$ & 12.399 & 12.158 & 8.843 & 7.863 & 5.152 & 5.03 & 3.882 & 3.269 & 10.011 & 6.186 & 3.662 & 6.534 & 3.723 \\
\hline $9 / 24 / 06$ & $16: 00$ & 12.018 & 12.142 & 8.862 & 7.867 & 5.231 & 5.041 & 3.721 & 3.283 & 10.043 & 6.231 & 3.762 & 6.571 & 3.875 \\
\hline $9 / 24 / 06$ & $20: 00$ & 11.642 & 12.149 & 8.869 & 7.874 & 5.247 & 5.065 & 3.185 & 3.278 & 10.059 & 6.228 & 3.748 & 6.569 & 3.765 \\
\hline $9 / 25 / 06$ & $0: 00$ & 11.82 & 12.13 & 8.853 & 7.853 & 5.226 & 5.067 & 3.127 & 3.257 & 10.062 & 6.214 & 3.711 & 6.557 & 3.704 \\
\hline $9 / 25 / 06$ & $4: 00$ & 11.901 & 12.119 & 8.839 & 7.846 & 5.21 & 5.065 & 3.145 & 3.248 & 10.062 & 6.212 & 3.69 & 6.553 & 3.683 \\
\hline $9 / 25 / 06$ & 8:00 & 12.431 & 12.126 & 8.848 & 7.853 & 5.217 & 5.062 & 3.684 & 3.257 & 10.066 & 6.219 & 3.697 & 6.564 & 3.702 \\
\hline $9 / 25 / 06$ & $12: 00$ & 12.548 & 12.126 & 8.876 & 7.872 & 5.29 & 5.06 & 4.21 & 3.292 & 10.09 & 6.277 & 3.834 & 6.616 & 3.961 \\
\hline $9 / 25 / 06$ & $16: 00$ & 12.161 & 12.119 & 8.897 & 7.886 & 5.355 & 5.076 & 3.891 & 3.313 & 10.122 & 6.322 & 3.939 & 6.653 & 4.082 \\
\hline $9 / 25 / 06$ & $20: 00$ & 11.619 & 12.135 & 8.9 & 7.893 & 5.357 & 5.1 & 3.481 & 3.308 & 10.136 & 6.308 & 3.9 & 6.641 & 3.935 \\
\hline 9/26/06 & $0: 00$ & 11.754 & 12.135 & 8.89 & 7.884 & 5.334 & 5.111 & 3.288 & 3.292 & 10.134 & 6.289 & 3.844 & 6.63 & 3.849 \\
\hline 9/26/06 & $4: 00$ & 11.855 & 12.121 & 8.871 & 7.867 & 5.311 & 5.104 & 3.215 & 3.273 & 10.125 & 6.273 & 3.799 & 6.611 & 3.795 \\
\hline 9/26/06 & $8: 00$ & 12.553 & 12.114 & 8.864 & 7.856 & 5.294 & 5.093 & 3.952 & 3.262 & 10.118 & 6.263 & 3.771 & 6.602 & 3.76 \\
\hline 9/26/06 & $12: 00$ & 12.728 & 12.093 & 8.895 & 7.865 & 5.369 & 5.076 & 4.133 & 3.294 & 10.143 & 6.336 & 3.904 & 6.648 & 4.075 \\
\hline $9 / 26 / 06$ & $16: 00$ & 12.298 & 12.075 & 8.909 & 7.872 & 5.432 & 5.081 & 4.117 & 3.313 & 10.169 & 6.382 & 4.011 & 6.678 & 4.229 \\
\hline 9/26/06 & $20: 00$ & 11.757 & 12.075 & 8.904 & 7.87 & 5.43 & 5.1 & 3.791 & 3.294 & 10.181 & 6.364 & 3.955 & 6.657 & 4.103 \\
\hline $9 / 27 / 06$ & $0: 00$ & 11.855 & 12.105 & 8.909 & 7.886 & 5.421 & 5.118 & 3.679 & 3.304 & 10.183 & 6.345 & 3.916 & 6.655 & 4.005 \\
\hline 9/27/06 & $4: 00$ & 11.859 & 12.123 & 8.907 & 7.891 & 5.409 & 5.123 & 3.528 & 3.303 & 10.181 & 6.336 & 3.89 & 6.65 & 3.942 \\
\hline $9 / 27 / 06$ & $8: 00$ & 12.018 & 12.147 & 8.916 & 7.905 & 5.407 & 5.13 & 3.542 & 3.313 & 10.181 & 6.336 & 3.879 & 6.66 & 3.907 \\
\hline 9/27/06 & $12: 00$ & 12.385 & 12.147 & 8.928 & 7.907 & 5.421 & 5.128 & 3.847 & 3.325 & 10.19 & 6.357 & 3.932 & 6.683 & 3.998 \\
\hline $9 / 27 / 06$ & $16: 00$ & 11.81 & 12.112 & 8.937 & 7.921 & 5.451 & 5.133 & 3.523 & 3.327 & 10.199 & 6.364 & 3.955 & 6.685 & 4.019 \\
\hline 9/27/06 & $20: 00$ & 11.752 & 12.177 & 8.944 & 7.933 & 5.442 & 5.151 & 3.674 & 3.339 & 10.204 & 6.354 & 3.855 & 6.674 & 3.931 \\
\hline 9/28/06 & 0:00 & 11.768 & 12.193 & 8.949 & 7.94 & 5.425 & 5.158 & 3.388 & 3.339 & 10.204 & 6.347 & 3.827 & 6.674 & 3.879 \\
\hline 9/28/06 & $4: 00$ & 11.806 & 12.202 & 8.946 & 7.94 & 5.411 & 5.163 & 3.583 & 3.339 & 10.199 & 6.338 & 3.82 & 6.676 & 3.844 \\
\hline 9/28/06 & 8:00 & 12.289 & 12.212 & 8.951 & 7.942 & 5.404 & 5.161 & 3.847 & 3.339 & 10.199 & 6.338 & 3.818 & 6.681 & 3.821 \\
\hline 9/28/06 & $12: 00$ & 12.434 & 12.181 & 8.953 & 7.928 & 5.423 & 5.151 & 4.061 & 3.339 & 10.204 & 6.361 & 3.876 & 6.695 & 3.938 \\
\hline 9/28/06 & $16: 00$ & 11.995 & 12.156 & 8.956 & 7.924 & 5.465 & 5.142 & 3.996 & 3.339 & 10.218 & 6.392 & 3.946 & 6.709 & 4.05 \\
\hline $9 / 28 / 06$ & $20: 00$ & 11.815 & 12.126 & 8.93 & 7.891 & 5.439 & 5.137 & 3.607 & 3.301 & 10.211 & 6.361 & 3.886 & 6.674 & 3.94 \\
\hline 9/29/06 & $0: 00$ & 11.988 & 12.098 & 8.9 & 7.863 & 5.407 & 5.118 & 3.597 & 3.268 & 10.19 & 6.338 & 3.834 & 6.643 & 3.886 \\
\hline $9 / 29 / 06$ & $4: 00$ & 12.053 & 12.07 & 8.869 & 7.839 & 5.381 & 5.095 & 3.597 & 3.245 & 10.171 & 6.324 & 3.795 & 6.618 & 3.858 \\
\hline 9/29/06 & $8: 00$ & 12.382 & 12.07 & 8.867 & 7.842 & 5.383 & 5.081 & 3.917 & 3.25 & 10.167 & 6.329 & 3.792 & 6.618 & 3.877 \\
\hline $9 / 29 / 06$ & $12: 00$ & 12.525 & 12.084 & 8.904 & 7.872 & 5.454 & 5.081 & 4.469 & 3.294 & 10.197 & 6.385 & 3.927 & 6.671 & 4.089 \\
\hline 9/29/06 & $16: 00$ & 12.161 & 12.098 & 8.939 & 7.898 & 5.514 & 5.1 & 4.378 & 3.322 & 10.23 & 6.424 & 4.035 & 6.716 & 4.204 \\
\hline
\end{tabular}


TABLE S1.2 (Cont.)

Water Level (ft below top of casing) at Indicated Well

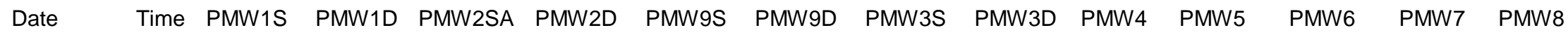

\begin{tabular}{|c|c|c|c|c|c|c|c|c|c|c|c|c|c|c|}
\hline 9/29/06 & $20: 00$ & 11.831 & 12.121 & 8.942 & 7.91 & 5.51 & 5.128 & 3.938 & 3.322 & 10.237 & 6.403 & 3.997 & 6.709 & 4.087 \\
\hline 9/30/06 & $0: 00$ & 11.925 & 12.14 & 8.942 & 7.912 & 5.489 & 5.142 & 3.786 & 3.32 & 10.234 & 6.387 & 3.948 & 6.697 & 4.001 \\
\hline $9 / 30 / 06$ & $4: 00$ & 11.892 & 12.142 & 8.93 & 7.907 & 5.468 & 5.142 & 3.583 & 3.308 & 10.223 & 6.368 & 3.904 & 6.683 & 3.938 \\
\hline 9/30/06 & $8: 00$ & 12.343 & 12.163 & 8.939 & 7.921 & 5.463 & 5.147 & 3.807 & 3.32 & 10.223 & 6.371 & 3.892 & 6.688 & 3.914 \\
\hline 9/30/06 & $12: 00$ & 12.723 & 12.165 & 8.975 & 7.94 & 5.54 & 5.142 & 4.511 & 3.36 & 10.251 & 6.438 & 4.03 & 6.744 & 4.18 \\
\hline $9 / 30 / 06$ & $16: 00$ & 12.375 & 12.163 & 8.998 & 7.956 & 5.599 & 5.154 & 4.553 & 3.383 & 10.279 & 6.48 & 4.137 & 6.788 & 4.313 \\
\hline $9 / 30 / 06$ & $20: 00$ & 11.803 & 12.177 & 9.003 & 7.961 & 5.589 & 5.179 & 3.868 & 3.374 & 10.286 & 6.455 & 4.095 & 6.769 & 4.176 \\
\hline $10 / 1 / 06$ & $0: 00$ & 11.92 & 12.179 & 8.991 & 7.954 & 5.564 & 5.184 & 3.686 & 3.357 & 10.279 & 6.434 & 4.032 & 6.748 & 4.094 \\
\hline $10 / 1 / 06$ & $4: 00$ & 12.037 & 12.175 & 8.982 & 7.945 & 5.54 & 5.182 & 3.66 & 3.346 & 10.272 & 6.417 & 3.988 & 6.732 & 4.045 \\
\hline $10 / 1 / 06$ & $8: 00$ & 12.55 & 12.177 & 8.986 & 7.947 & 5.547 & 5.172 & 4.14 & 3.353 & 10.269 & 6.424 & 3.983 & 6.737 & 4.075 \\
\hline $10 / 1 / 06$ & $12: 00$ & 12.821 & 12.161 & 9.012 & 7.959 & 5.627 & 5.165 & 4.863 & 3.388 & 10.3 & 6.49 & 4.149 & 6.802 & 4.332 \\
\hline $10 / 1 / 06$ & $16: 00$ & 12.399 & 12.154 & 9.033 & 7.973 & 5.683 & 5.179 & 4.171 & 3.409 & 10.323 & 6.527 & 4.252 & 6.841 & 4.449 \\
\hline $10 / 1 / 06$ & $20: 00$ & 11.866 & 12.175 & 9.04 & 7.985 & 5.68 & 5.207 & 3.702 & 3.404 & 10.33 & 6.506 & 4.226 & 6.83 & 4.353 \\
\hline $10 / 2 / 06$ & $0: 00$ & 12.037 & 12.177 & 9.029 & 7.973 & 5.652 & 5.217 & 3.747 & 3.385 & 10.325 & 6.483 & 4.163 & 6.806 & 4.271 \\
\hline $10 / 2 / 06$ & $4: 00$ & 11.892 & 12.181 & 9.019 & 7.968 & 5.631 & 5.214 & 3.646 & 3.376 & 10.316 & 6.462 & 4.112 & 6.786 & 4.204 \\
\hline $10 / 2 / 06$ & $8: 00$ & 12.231 & 12.198 & 9.021 & 7.98 & 5.627 & 5.214 & 3.826 & 3.38 & 10.309 & 6.455 & 4.081 & 6.779 & 4.164 \\
\hline $10 / 2 / 06$ & $12: 00$ & 12.59 & 12.198 & 9.045 & 7.992 & 5.678 & 5.207 & 4.357 & 3.411 & 10.33 & 6.504 & 4.149 & 6.813 & 4.33 \\
\hline $10 / 2 / 06$ & $16: 00$ & 12.175 & 12.209 & 9.073 & 8.017 & 5.744 & 5.224 & 4.043 & 3.446 & 10.355 & 6.55 & 4.263 & 6.867 & 4.477 \\
\hline $10 / 2 / 06$ & $20: 00$ & 11.836 & 12.223 & 9.073 & 8.017 & 5.716 & 5.247 & 3.642 & 3.43 & 10.355 & 6.513 & 4.214 & 6.844 & 4.341 \\
\hline $10 / 3 / 06$ & $0: 00$ & 11.99 & 12.212 & 9.05 & 7.992 & 5.674 & 5.247 & 3.539 & 3.399 & 10.337 & 6.476 & 4.133 & 6.806 & 4.232 \\
\hline $10 / 3 / 06$ & $4: 00$ & 11.957 & 12.202 & 9.031 & 7.977 & 5.641 & 5.238 & 3.353 & 3.378 & 10.316 & 6.452 & 4.074 & 6.781 & 4.148 \\
\hline $10 / 3 / 06$ & $8: 00$ & 12.354 & 12.212 & 9.024 & 7.98 & 5.624 & 5.226 & 3.702 & 3.376 & 10.304 & 6.441 & 4.037 & 6.767 & 4.089 \\
\hline $10 / 3 / 06$ & $12: 00$ & 12.651 & 12.202 & 9.05 & 7.987 & 5.681 & 5.21 & 4.418 & 3.404 & 10.32 & 6.494 & 4.13 & 6.811 & 4.299 \\
\hline $10 / 3 / 06$ & $16: 00$ & 12.135 & 12.207 & 9.08 & 8.013 & 5.76 & 5.219 & 4.098 & 3.441 & 10.358 & 6.548 & 4.263 & 6.869 & 4.463 \\
\hline $10 / 3 / 06$ & $20: 00$ & 11.965 & 12.219 & 9.08 & 8.015 & 5.739 & 5.245 & 3.747 & 3.427 & 10.355 & 6.513 & 4.221 & 6.848 & 4.341 \\
\hline $10 / 4 / 06$ & $0: 00$ & 11.768 & 12.263 & 9.089 & 8.041 & 5.73 & 5.273 & 3.448 & 3.446 & 10.353 & 6.504 & 4.196 & 6.855 & 4.269 \\
\hline $10 / 4 / 06$ & $4: 00$ & 11.899 & 12.295 & 9.096 & 8.059 & 5.716 & 5.287 & 3.292 & 3.458 & 10.351 & 6.492 & 4.168 & 6.86 & 4.204 \\
\hline $10 / 4 / 06$ & $8: 00$ & 11.948 & 12.328 & 9.11 & 8.078 & 5.709 & 5.301 & 3.486 & 3.476 & 10.351 & 6.487 & 4.161 & 6.869 & 4.159 \\
\hline $10 / 4 / 06$ & $12: 00$ & 12.177 & 12.342 & 9.122 & 8.088 & 5.709 & 5.308 & 3.497 & 3.481 & 10.355 & 6.49 & 4.163 & 6.883 & 4.152 \\
\hline $10 / 4 / 06$ & $16: 00$ & 12.144 & 12.339 & 9.127 & 8.085 & 5.723 & 5.31 & 3.402 & 3.486 & 10.358 & 6.497 & 4.182 & 6.895 & 4.164 \\
\hline $10 / 4 / 06$ & $20: 00$ & 12.079 & 12.344 & 9.127 & 8.088 & 5.713 & 5.315 & 3.621 & 3.481 & 10.355 & 6.49 & 4.172 & 6.89 & 4.122 \\
\hline $10 / 5 / 06$ & $0: 00$ & 12.119 & 12.335 & 9.113 & 8.074 & 5.685 & 5.31 & 3.495 & 3.465 & 10.346 & 6.473 & 4.133 & 6.874 & 4.068 \\
\hline $10 / 5 / 06$ & $4: 00$ & 12.058 & 12.323 & 9.099 & 8.059 & 5.66 & 5.298 & 3.865 & 3.448 & 10.334 & 6.459 & 4.095 & 6.855 & 4.019 \\
\hline $10 / 5 / 06$ & $8: 00$ & 12.527 & 12.325 & 9.096 & 8.059 & 5.648 & 5.291 & 4.066 & 3.448 & 10.327 & 6.452 & 4.072 & 6.851 & 3.984 \\
\hline
\end{tabular}


TABLE S1.2 (Cont.)

Water Level (ft below top of casing) at Indicated Well

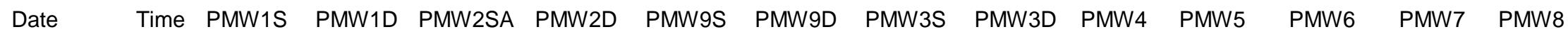

\begin{tabular}{|c|c|c|c|c|c|c|c|c|c|c|c|c|c|c|}
\hline $10 / 5 / 06$ & $12: 00$ & 12.674 & 12.311 & 9.115 & 8.064 & 5.697 & 5.275 & 4.315 & 3.465 & 10.339 & 6.492 & 4.158 & 6.881 & 4.157 \\
\hline $10 / 5 / 06$ & $16: 00$ & 12.231 & 12.291 & 9.122 & 8.064 & 5.753 & 5.27 & 4.178 & 3.472 & 10.358 & 6.522 & 4.242 & 6.907 & 4.264 \\
\hline $10 / 5 / 06$ & $20: 00$ & 11.864 & 12.302 & 9.12 & 8.069 & 5.744 & 5.284 & 3.644 & 3.467 & 10.36 & 6.501 & 4.224 & 6.9 & 4.162 \\
\hline $10 / 6 / 06$ & 0:00 & 11.988 & 12.307 & 9.113 & 8.062 & 5.716 & 5.289 & 3.472 & 3.458 & 10.348 & 6.48 & 4.175 & 6.883 & 4.087 \\
\hline $10 / 6 / 06$ & 4:00 & 12.067 & 12.298 & 9.099 & 8.05 & 5.686 & 5.282 & 3.84 & 3.439 & 10.334 & 6.462 & 4.126 & 6.862 & 4.028 \\
\hline $10 / 6 / 06$ & $8: 00$ & 12.504 & 12.3 & 9.094 & 8.05 & 5.683 & 5.273 & 4.133 & 3.441 & 10.327 & 6.459 & 4.109 & 6.858 & 4.024 \\
\hline $10 / 6 / 06$ & $12: 00$ & 12.737 & 12.274 & 9.108 & 8.043 & 5.742 & 5.256 & 4.574 & 3.451 & 10.337 & 6.494 & 4.189 & 6.886 & 4.211 \\
\hline $10 / 6 / 06$ & $16: 00$ & 12.345 & 12.251 & 9.118 & 8.043 & 5.793 & 5.254 & 4.541 & 3.458 & 10.358 & 6.534 & 4.282 & 6.914 & 4.344 \\
\hline $10 / 6 / 06$ & $20: 00$ & 11.993 & 12.263 & 9.115 & 8.048 & 5.784 & 5.273 & 4.08 & 3.455 & 10.365 & 6.52 & 4.273 & 6.907 & 4.274 \\
\hline $10 / 7 / 06$ & $0: 00$ & 12.077 & 12.256 & 9.099 & 8.034 & 5.753 & 5.275 & 3.905 & 3.437 & 10.348 & 6.497 & 4.224 & 6.886 & 4.201 \\
\hline $10 / 7 / 06$ & $4: 00$ & 12.095 & 12.246 & 9.082 & 8.024 & 5.732 & 5.268 & 3.793 & 3.42 & 10.334 & 6.48 & 4.184 & 6.865 & 4.157 \\
\hline $10 / 7 / 06$ & $8: 00$ & 12.504 & 12.251 & 9.082 & 8.027 & 5.737 & 5.259 & 4.117 & 3.427 & 10.327 & 6.48 & 4.175 & 6.862 & 4.159 \\
\hline $10 / 7 / 06$ & $12: 00$ & 12.917 & 12.237 & 9.111 & 8.036 & 5.812 & 5.249 & 4.835 & 3.451 & 10.351 & 6.534 & 4.277 & 6.911 & 4.374 \\
\hline $10 / 7 / 06$ & $16: 00$ & 12.361 & 12.237 & 9.127 & 8.05 & 5.859 & 5.261 & 4.723 & 3.474 & 10.374 & 6.567 & 4.366 & 6.946 & 4.486 \\
\hline $10 / 7 / 06$ & $20: 00$ & 12.014 & 12.256 & 9.129 & 8.057 & 5.845 & 5.284 & 4.273 & 3.467 & 10.379 & 6.548 & 4.349 & 6.934 & 4.411 \\
\hline $10 / 8 / 06$ & $0: 00$ & 12.056 & 12.26 & 9.118 & 8.052 & 5.812 & 5.294 & 4.049 & 3.453 & 10.369 & 6.525 & 4.298 & 6.916 & 4.332 \\
\hline $10 / 8 / 06$ & $4: 00$ & 11.934 & 12.279 & 9.118 & 8.059 & 5.798 & 5.296 & 3.719 & 3.455 & 10.36 & 6.513 & 4.261 & 6.904 & 4.274 \\
\hline $10 / 8 / 06$ & $8: 00$ & 12.168 & 12.314 & 9.134 & 8.083 & 5.788 & 5.303 & 3.702 & 3.474 & 10.36 & 6.506 & 4.24 & 6.911 & 4.222 \\
\hline $10 / 8 / 06$ & $12: 00$ & 12.672 & 12.316 & 9.143 & 8.088 & 5.819 & 5.301 & 4.042 & 3.486 & 10.367 & 6.527 & 4.27 & 6.93 & 4.295 \\
\hline $10 / 8 / 06$ & $16: 00$ & 12.184 & 12.316 & 9.16 & 8.099 & 5.868 & 5.308 & 3.949 & 3.504 & 10.381 & 6.555 & 4.349 & 6.962 & 4.397 \\
\hline $10 / 8 / 06$ & $20: 00$ & 11.934 & 12.337 & 9.167 & 8.111 & 5.852 & 5.329 & 3.53 & 3.509 & 10.383 & 6.539 & 4.34 & 6.965 & 4.325 \\
\hline $10 / 9 / 06$ & $0: 00$ & 12.088 & 12.332 & 9.155 & 8.099 & 5.819 & 5.334 & 3.469 & 3.49 & 10.374 & 6.518 & 4.296 & 6.944 & 4.253 \\
\hline $10 / 9 / 06$ & $4: 00$ & 12.114 & 12.339 & 9.155 & 8.102 & 5.8 & 5.331 & 3.392 & 3.488 & 10.367 & 6.506 & 4.266 & 6.937 & 4.206 \\
\hline $10 / 9 / 06$ & $8: 00$ & 12.207 & 12.346 & 9.155 & 8.104 & 5.793 & 5.329 & 3.378 & 3.49 & 10.362 & 6.501 & 4.245 & 6.932 & 4.173 \\
\hline $10 / 9 / 06$ & $12: 00$ & 12.462 & 12.339 & 9.153 & 8.095 & 5.793 & 5.322 & 3.6 & 3.486 & 10.36 & 6.501 & 4.24 & 6.934 & 4.183 \\
\hline $10 / 9 / 06$ & $16: 00$ & 12.261 & 12.323 & 9.148 & 8.09 & 5.817 & 5.313 & 3.539 & 3.486 & 10.36 & 6.513 & 4.261 & 6.937 & 4.222 \\
\hline $10 / 9 / 06$ & $20: 00$ & 12.067 & 12.33 & 9.148 & 8.092 & 5.81 & 5.317 & 3.327 & 3.481 & 10.358 & 6.504 & 4.254 & 6.934 & 4.178 \\
\hline $10 / 10 / 06$ & $0: 00$ & 12.161 & 12.311 & 9.129 & 8.073 & 5.779 & 5.31 & 3.357 & 3.46 & 10.346 & 6.485 & 4.21 & 6.911 & 4.131 \\
\hline $10 / 10 / 06$ & $4: 00$ & 12.175 & 12.277 & 9.104 & 8.041 & 5.746 & 5.291 & 3.355 & 3.429 & 10.327 & 6.469 & 4.161 & 6.876 & 4.078 \\
\hline $10 / 10 / 06$ & $8: 00$ & 12.245 & 12.295 & 9.083 & 8.055 & 5.714 & 5.27 & 3.404 & 3.406 & 10.311 & 6.448 & 4.105 & 6.844 & 4.019 \\
\hline $10 / 10 / 06$ & $12: 00$ & 12.387 & 12.235 & 9.047 & 8.001 & 5.669 & 5.235 & 3.574 & 3.362 & 10.302 & 6.429 & 4.032 & 6.799 & 3.958 \\
\hline $10 / 10 / 06$ & $16: 00$ & 12.291 & 12.212 & 9.017 & 8.01 & 5.637 & 5.203 & 3.632 & 3.329 & 10.281 & 6.406 & 3.939 & 6.744 & 3.909 \\
\hline $10 / 10 / 06$ & $20: 00$ & 12.24 & 12.158 & 8.991 & 7.926 & 5.611 & 5.177 & 3.632 & 3.308 & 10.246 & 6.392 & 3.855 & 6.709 & 3.872 \\
\hline $10 / 11 / 06$ & $0: 00$ & 12.182 & 12.13 & 8.965 & 7.9 & 5.581 & 5.149 & 3.614 & 3.28 & 10.227 & 6.378 & 3.813 & 6.683 & 3.837 \\
\hline
\end{tabular}


Water Level (ft below top of casing) at Indicated Well

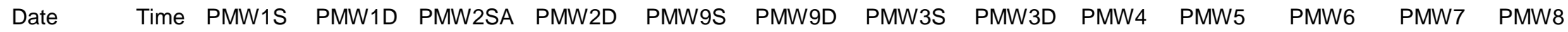

\begin{tabular}{|c|c|c|c|c|c|c|c|c|c|c|c|c|c|c|}
\hline $10 / 11 / 06$ & $4: 00$ & 12.147 & 12.117 & 8.947 & 7.886 & 5.562 & 5.128 & 3.56 & 3.266 & 10.211 & 6.368 & 3.795 & 6.664 & 3.816 \\
\hline $10 / 11 / 06$ & $8: 00$ & 12.109 & 12.158 & 8.963 & 7.926 & 5.581 & 5.133 & 3.527 & 3.301 & 10.211 & 6.371 & 3.829 & 6.688 & 3.837 \\
\hline $10 / 11 / 06$ & $12: 00$ & 12.217 & 12.202 & 8.989 & 7.959 & 5.597 & 5.149 & 3.558 & 3.336 & 10.22 & 6.389 & 3.883 & 6.727 & 3.893 \\
\hline 10/11/06 & $16: 00$ & 12.116 & 12.205 & 9.005 & 7.961 & 5.625 & 5.154 & 3.509 & 3.35 & 10.232 & 6.403 & 3.925 & 6.746 & 3.954 \\
\hline 10/11/06 & $20: 00$ & 11.717 & 12.209 & 9.008 & 7.963 & 5.616 & 5.168 & 3.089 & 3.348 & 10.237 & 6.399 & 3.93 & 6.746 & 3.907 \\
\hline 10/12/06 & $0: 00$ & 11.979 & 12.219 & 9.003 & 7.963 & 5.592 & 5.172 & 3.124 & 3.341 & 10.232 & 6.392 & 3.916 & 6.743 & 3.865 \\
\hline $10 / 12 / 06$ & $4: 00$ & 11.913 & 12.235 & 9.01 & 7.975 & 5.583 & 5.177 & 3.036 & 3.35 & 10.234 & 6.387 & 3.913 & 6.751 & 3.849 \\
\hline $10 / 12 / 06$ & 8:00 & 12.45 & 12.228 & 9.003 & 7.963 & 5.567 & 5.175 & 3.413 & 3.338 & 10.227 & 6.38 & 3.899 & 6.743 & 3.823 \\
\hline 10/12/06 & $12: 00$ & 12.684 & 12.2 & 8.991 & 7.94 & 5.557 & 5.158 & 3.83 & 3.32 & 10.22 & 6.375 & 3.878 & 6.73 & 3.83 \\
\hline $10 / 12 / 06$ & $16: 00$ & 12.282 & 12.186 & 8.977 & 7.935 & 5.567 & 5.149 & 3.814 & 3.317 & 10.216 & 6.378 & 3.888 & 6.73 & 3.87 \\
\hline 10/12/06 & $20: 00$ & 11.64 & 12.209 & 8.989 & 7.956 & 5.571 & 5.154 & 3.183 & 3.336 & 10.22 & 6.385 & 3.911 & 6.743 & 3.863 \\
\hline $10 / 13 / 06$ & $0: 00$ & 11.897 & 12.216 & 8.991 & 7.959 & 5.555 & 5.156 & 3.164 & 3.334 & 10.22 & 6.378 & 3.904 & 6.743 & 3.832 \\
\hline $10 / 13 / 06$ & $4: 00$ & 11.932 & 12.228 & 8.996 & 7.966 & 5.548 & 5.161 & 3.129 & 3.341 & 10.22 & 6.378 & 3.902 & 6.746 & 3.818 \\
\hline 10/13/06 & 8:00 & 12.555 & 12.242 & 9.005 & 7.973 & 5.548 & 5.163 & 3.532 & 3.345 & 10.223 & 6.38 & 3.906 & 6.755 & 3.811 \\
\hline 10/13/06 & $12: 00$ & 12.723 & 12.223 & 8.996 & 7.959 & 5.546 & 5.158 & 4.028 & 3.338 & 10.223 & 6.378 & 3.89 & 6.748 & 3.825 \\
\hline 10/13/06 & $16: 00$ & 12.294 & 12.209 & 8.989 & 7.949 & 5.555 & 5.154 & 3.977 & 3.331 & 10.22 & 6.38 & 3.897 & 6.746 & 3.856 \\
\hline $10 / 13 / 06$ & $20: 00$ & 11.782 & 12.23 & 9 & 7.97 & 5.56 & 5.158 & 3.362 & 3.348 & 10.225 & 6.387 & 3.916 & 6.76 & 3.851 \\
\hline $10 / 14 / 06$ & $0: 00$ & 11.782 & 12.253 & 9.012 & 7.987 & 5.555 & 5.168 & 3.143 & 3.362 & 10.232 & 6.389 & 3.925 & 6.771 & 3.835 \\
\hline $10 / 14 / 06$ & $4: 00$ & 11.855 & 12.27 & 9.024 & 7.998 & 5.553 & 5.179 & 3.763 & 3.371 & 10.237 & 6.392 & 3.93 & 6.783 & 3.821 \\
\hline 10/14/06 & 8:00 & 12.693 & 12.286 & 9.033 & 8.008 & 5.555 & 5.189 & 4.061 & 3.38 & 10.241 & 6.396 & 3.934 & 6.792 & 3.816 \\
\hline $10 / 14 / 06$ & $12: 00$ & 12.985 & 12.26 & 9.024 & 7.987 & 5.55 & 5.184 & 4.474 & 3.362 & 10.241 & 6.389 & 3.911 & 6.778 & 3.821 \\
\hline $10 / 14 / 06$ & $16: 00$ & 12.406 & 12.23 & 9.005 & 7.968 & 5.555 & 5.17 & 4.406 & 3.343 & 10.232 & 6.389 & 3.902 & 6.767 & 3.849 \\
\hline $10 / 14 / 06$ & $20: 00$ & 11.974 & 12.212 & 8.991 & 7.952 & 5.539 & 5.158 & 3.986 & 3.327 & 10.227 & 6.382 & 3.883 & 6.748 & 3.825 \\
\hline $10 / 15 / 06$ & $0: 00$ & 12.186 & 12.191 & 8.977 & 7.933 & 5.525 & 5.144 & 4.063 & 3.31 & 10.22 & 6.373 & 3.862 & 6.732 & 3.807 \\
\hline $10 / 15 / 06$ & $4: 00$ & 12.07 & 12.179 & 8.97 & 7.926 & 5.515 & 5.135 & 3.928 & 3.301 & 10.213 & 6.366 & 3.848 & 6.72 & 3.795 \\
\hline $10 / 15 / 06$ & $8: 00$ & 12.231 & 12.172 & 8.961 & 7.919 & 5.506 & 5.121 & 3.968 & 3.291 & 10.206 & 6.361 & 3.767 & 6.688 & 3.774 \\
\hline $10 / 15 / 06$ & $12: 00$ & 12.396 & 12.161 & 8.951 & 7.902 & 5.488 & 5.111 & 4.129 & 3.275 & 10.199 & 6.354 & 3.734 & 6.671 & 3.751 \\
\hline $10 / 15 / 06$ & $16: 00$ & 12.415 & 12.144 & 8.937 & 7.893 & 5.481 & 5.097 & 4.299 & 3.261 & 10.19 & 6.35 & 3.727 & 6.66 & 3.744 \\
\hline $10 / 15 / 06$ & 20:00 & 12.254 & 12.142 & 8.933 & 7.893 & 5.476 & 5.09 & 4.227 & 3.261 & 10.185 & 6.347 & 3.736 & 6.657 & 3.741 \\
\hline $10 / 16 / 06$ & $0: 00$ & 12.217 & 12.154 & 8.926 & 7.895 & 5.462 & 5.083 & 4.219 & 3.249 & 10.178 & 6.34 & 3.729 & 6.648 & 3.732 \\
\hline $10 / 16 / 06$ & $4: 00$ & 12.196 & 12.121 & 8.914 & 7.867 & 5.448 & 5.067 & 4.203 & 3.235 & 10.169 & 6.333 & 3.615 & 6.618 & 3.709 \\
\hline 10/16/06 & 8:00 & 12.158 & 12.121 & 8.909 & 7.867 & 5.443 & 5.062 & 4.173 & 3.233 & 10.164 & 6.331 & 3.641 & 6.615 & 3.704 \\
\hline $10 / 16 / 06$ & $12: 00$ & 12.261 & 12.103 & 8.897 & 7.848 & 5.425 & 5.051 & 4.285 & 3.217 & 10.16 & 6.322 & 3.645 & 6.606 & 3.692 \\
\hline $10 / 16 / 06$ & $16: 00$ & 12.172 & 12.091 & 8.888 & 7.844 & 5.42 & 5.039 & 4.238 & 3.21 & 10.15 & 6.319 & 3.659 & 6.601 & 3.697 \\
\hline
\end{tabular}


Water Level (ft below top of casing) at Indicated Well

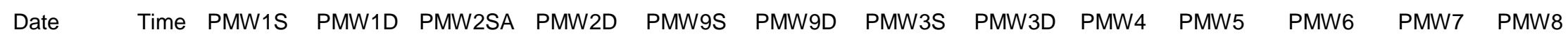

\begin{tabular}{|c|c|c|c|c|c|c|c|c|c|c|c|c|c|c|}
\hline $10 / 16 / 06$ & $20: 00$ & 12.121 & 12.091 & 8.888 & 7.844 & 5.413 & 5.034 & 4.175 & 3.21 & 10.148 & 6.317 & 3.671 & 6.599 & 3.695 \\
\hline $10 / 17 / 06$ & $0: 00$ & 12.098 & 12.089 & 8.888 & 7.851 & 5.413 & 5.037 & 4.129 & 3.214 & 10.146 & 6.317 & 3.683 & 6.601 & 3.699 \\
\hline $10 / 17 / 06$ & $4: 00$ & 12.077 & 12.086 & 8.893 & 7.851 & 5.411 & 5.039 & 4.082 & 3.222 & 10.146 & 6.319 & 3.692 & 6.608 & 3.702 \\
\hline $10 / 17 / 06$ & $8: 00$ & 12.179 & 12.158 & 8.919 & 7.898 & 5.429 & 5.055 & 4.117 & 3.259 & 10.155 & 6.331 & 3.725 & 6.634 & 3.725 \\
\hline $10 / 17 / 06$ & $12: 00$ & 12.151 & 12.181 & 8.937 & 7.912 & 5.434 & 5.074 & 4.122 & 3.273 & 10.167 & 6.333 & 3.734 & 6.65 & 3.732 \\
\hline $10 / 17 / 06$ & $16: 00$ & 12.095 & 12.205 & 8.956 & 7.933 & 5.46 & 5.088 & 3.942 & 3.294 & 10.176 & 6.347 & 3.764 & 6.671 & 3.758 \\
\hline $10 / 17 / 06$ & $20: 00$ & 11.829 & 12.244 & 8.984 & 7.97 & 5.488 & 5.111 & 3.632 & 3.329 & 10.195 & 6.364 & 3.809 & 6.706 & 3.786 \\
\hline $10 / 18 / 06$ & $0: 00$ & 11.862 & 12.212 & 8.993 & 7.942 & 5.469 & 5.123 & 3.516 & 3.327 & 10.202 & 6.366 & 3.815 & 6.711 & 3.774 \\
\hline $10 / 18 / 06$ & $4: 00$ & 11.885 & 12.237 & 9.003 & 7.968 & 5.469 & 5.13 & 3.385 & 3.338 & 10.188 & 6.371 & 3.804 & 6.715 & 3.769 \\
\hline $10 / 18 / 06$ & $8: 00$ & 11.948 & 12.244 & 9.017 & 7.975 & 5.462 & 5.14 & 3.273 & 3.343 & 10.174 & 6.359 & 3.445 & 6.657 & 3.706 \\
\hline $10 / 18 / 06$ & $12: 00$ & 12.207 & 12.281 & 9.017 & 7.984 & 5.446 & 5.142 & 3.425 & 3.331 & 10.216 & 6.357 & 3.529 & 6.657 & 3.676 \\
\hline $10 / 18 / 06$ & $16: 00$ & 12.116 & 12.279 & 9.017 & 7.987 & 5.451 & 5.137 & 3.367 & 3.331 & 10.218 & 6.366 & 3.617 & 6.674 & 3.695 \\
\hline $10 / 18 / 06$ & $20: 00$ & 12.077 & 12.293 & 9.026 & 7.998 & 5.458 & 5.144 & 3.287 & 3.348 & 10.225 & 6.375 & 3.69 & 6.697 & 3.716 \\
\hline $10 / 19 / 06$ & $0: 00$ & 12.044 & 12.274 & 9.017 & 7.982 & 5.446 & 5.142 & 3.252 & 3.336 & 10.223 & 6.371 & 3.72 & 6.699 & 3.716 \\
\hline $10 / 19 / 06$ & $4: 00$ & 11.923 & 12.253 & 9.003 & 7.965 & 5.432 & 5.133 & 3.124 & 3.32 & 10.216 & 6.366 & 3.745 & 6.697 & 3.713 \\
\hline $10 / 19 / 06$ & 8:00 & 12.483 & 12.249 & 9.001 & 7.963 & 5.427 & 5.128 & 3.392 & 3.32 & 10.216 & 6.366 & 3.764 & 6.699 & 3.72 \\
\hline $10 / 19 / 06$ & $12: 00$ & 12.693 & 12.212 & 8.977 & 7.928 & 5.411 & 5.107 & 3.896 & 3.289 & 10.204 & 6.359 & 3.741 & 6.681 & 3.718 \\
\hline $10 / 19 / 06$ & $16: 00$ & 12.431 & 12.186 & 8.958 & 7.914 & 5.418 & 5.093 & 3.968 & 3.277 & 10.197 & 6.357 & 3.743 & 6.671 & 3.746 \\
\hline $10 / 19 / 06$ & $20: 00$ & 11.885 & 12.188 & 8.958 & 7.921 & 5.42 & 5.088 & 3.581 & 3.28 & 10.197 & 6.357 & 3.76 & 6.674 & 3.748 \\
\hline $10 / 20 / 06$ & $0: 00$ & 11.927 & 12.172 & 8.947 & 7.905 & 5.397 & 5.079 & 3.474 & 3.266 & 10.188 & 6.347 & 3.755 & 6.662 & 3.73 \\
\hline $10 / 20 / 06$ & $4: 00$ & 12.109 & 12.165 & 8.937 & 7.902 & 5.386 & 5.067 & 3.541 & 3.259 & 10.183 & 6.345 & 3.755 & 6.657 & 3.725 \\
\hline $10 / 20 / 06$ & $8: 00$ & 12.373 & 12.158 & 8.933 & 7.893 & 5.379 & 5.062 & 3.728 & 3.254 & 10.178 & 6.34 & 3.746 & 6.653 & 3.725 \\
\hline $10 / 20 / 06$ & $12: 00$ & 12.674 & 12.142 & 8.921 & 7.879 & 5.381 & 5.051 & 4.157 & 3.242 & 10.174 & 6.338 & 3.727 & 6.643 & 3.739 \\
\hline $10 / 20 / 06$ & $16: 00$ & 12.464 & 12.142 & 8.923 & 7.883 & 5.409 & 5.046 & 4.285 & 3.247 & 10.176 & 6.347 & 3.743 & 6.648 & 3.779 \\
\hline $10 / 20 / 06$ & $20: 00$ & 11.986 & 12.168 & 8.937 & 7.905 & 5.416 & 5.06 & 3.933 & 3.266 & 10.183 & 6.352 & 3.75 & 6.662 & 3.772 \\
\hline $10 / 21 / 06$ & $0: 00$ & 11.99 & 12.195 & 8.954 & 7.933 & 5.416 & 5.069 & 3.812 & 3.284 & 10.185 & 6.354 & 3.745 & 6.669 & 3.762 \\
\hline $10 / 21 / 06$ & $4: 00$ & 11.782 & 12.212 & 8.977 & 7.935 & 5.407 & 5.086 & 3.527 & 3.298 & 10.192 & 6.345 & 3.489 & 6.636 & 3.667 \\
\hline $10 / 21 / 06$ & $8: 00$ & 11.902 & 12.195 & 9.001 & 7.909 & 5.411 & 5.104 & 3.402 & 3.322 & 10.139 & 6.354 & 3.503 & 6.648 & 3.648 \\
\hline $10 / 21 / 06$ & $12: 00$ & 12.074 & 12.281 & 9.008 & 7.98 & 5.397 & 5.109 & 3.451 & 3.322 & 10.209 & 6.354 & 3.531 & 6.648 & 3.629 \\
\hline $10 / 21 / 06$ & $16: 00$ & 12.13 & 12.295 & 9.019 & 7.991 & 5.397 & 5.118 & 3.434 & 3.333 & 10.213 & 6.361 & 3.596 & 6.669 & 3.643 \\
\hline $10 / 21 / 06$ & $20: 00$ & 11.918 & 12.307 & 9.029 & 8.003 & 5.407 & 5.133 & 3.22 & 3.345 & 10.223 & 6.368 & 3.662 & 6.692 & 3.664 \\
\hline $10 / 22 / 06$ & $0: 00$ & 12.011 & 12.311 & 9.036 & 8.008 & 5.404 & 5.14 & 3.185 & 3.352 & 10.227 & 6.375 & 3.713 & 6.709 & 3.678 \\
\hline $10 / 22 / 06$ & $4: 00$ & 12.233 & 12.304 & 9.036 & 8.003 & 5.397 & 5.14 & 3.332 & 3.348 & 10.23 & 6.375 & 3.739 & 6.715 & 3.688 \\
\hline $10 / 22 / 06$ & $8: 00$ & 12.387 & 12.316 & 9.043 & 8.015 & 5.407 & 5.144 & 3.451 & 3.361 & 10.234 & 6.385 & 3.767 & 6.729 & 3.706 \\
\hline
\end{tabular}


Water Level (ft below top of casing) at Indicated Well

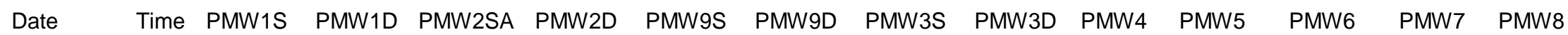

\begin{tabular}{|c|c|c|c|c|c|c|c|c|c|c|c|c|c|c|}
\hline $10 / 22 / 06$ & $12: 00$ & 12.494 & 12.311 & 9.043 & 8.01 & 5.409 & 5.144 & 3.679 & 3.357 & 10.241 & 6.389 & 3.769 & 6.732 & 3.716 \\
\hline $10 / 22 / 06$ & $16: 00$ & 12.34 & 12.291 & 9.033 & 7.996 & 5.421 & 5.142 & 3.684 & 3.35 & 10.239 & 6.387 & 3.783 & 6.734 & 3.732 \\
\hline $10 / 22 / 06$ & $20: 00$ & 11.934 & 12.304 & 9.043 & 8.012 & 5.433 & 5.147 & 3.346 & 3.361 & 10.244 & 6.394 & 3.806 & 6.746 & 3.746 \\
\hline $10 / 23 / 06$ & 0:00 & 11.925 & 12.295 & 9.038 & 8.003 & 5.416 & 5.147 & 3.287 & 3.354 & 10.239 & 6.394 & 3.813 & 6.746 & 3.737 \\
\hline $10 / 23 / 06$ & $4: 00$ & 12.023 & 12.298 & 9.038 & 8.005 & 5.409 & 5.149 & 3.218 & 3.359 & 10.244 & 6.394 & 3.829 & 6.755 & 3.741 \\
\hline $10 / 23 / 06$ & 8:00 & 12.553 & 12.3 & 9.04 & 8.008 & 5.405 & 5.149 & 3.523 & 3.362 & 10.241 & 6.394 & 3.836 & 6.757 & 3.744 \\
\hline $10 / 23 / 06$ & $12: 00$ & 12.758 & 12.284 & 9.033 & 7.991 & 5.4 & 5.144 & 3.965 & 3.345 & 10.239 & 6.392 & 3.818 & 6.748 & 3.746 \\
\hline $10 / 23 / 06$ & $16: 00$ & 12.357 & 12.267 & 9.022 & 7.984 & 5.412 & 5.137 & 3.952 & 3.343 & 10.239 & 6.392 & 3.827 & 6.746 & 3.765 \\
\hline $10 / 23 / 06$ & $20: 00$ & 12.004 & 12.279 & 9.029 & 7.998 & 5.416 & 5.137 & 3.423 & 3.352 & 10.239 & 6.396 & 3.846 & 6.757 & 3.767 \\
\hline $10 / 24 / 06$ & $0: 00$ & 12.098 & 12.281 & 9.031 & 7.998 & 5.409 & 5.14 & 3.409 & 3.352 & 10.239 & 6.394 & 3.848 & 6.76 & 3.758 \\
\hline $10 / 24 / 06$ & $4: 00$ & 12.07 & 12.277 & 9.026 & 7.991 & 5.4 & 5.14 & 3.385 & 3.345 & 10.239 & 6.392 & 3.848 & 6.76 & 3.751 \\
\hline $10 / 24 / 06$ & $8: 00$ & 12.513 & 12.277 & 9.026 & 7.994 & 5.393 & 5.135 & 3.653 & 3.347 & 10.237 & 6.394 & 3.85 & 6.76 & 3.751 \\
\hline $10 / 24 / 06$ & $12: 00$ & 12.812 & 12.242 & 9.008 & 7.961 & 5.379 & 5.123 & 4.208 & 3.319 & 10.227 & 6.378 & 3.813 & 6.736 & 3.741 \\
\hline $10 / 24 / 06$ & $16: 00$ & 12.34 & 12.219 & 8.989 & 7.947 & 5.389 & 5.111 & 4.131 & 3.305 & 10.22 & 6.38 & 3.811 & 6.727 & 3.751 \\
\hline $10 / 24 / 06$ & $20: 00$ & 12.081 & 12.219 & 8.989 & 7.949 & 5.391 & 5.104 & 3.842 & 3.308 & 10.218 & 6.378 & 3.818 & 6.727 & 3.751 \\
\hline $10 / 25 / 06$ & $0: 00$ & 12.063 & 12.209 & 8.982 & 7.94 & 5.375 & 5.1 & 3.742 & 3.296 & 10.211 & 6.373 & 3.809 & 6.72 & 3.739 \\
\hline $10 / 25 / 06$ & 4:00 & 11.981 & 12.202 & 8.973 & 7.935 & 5.365 & 5.093 & 3.597 & 3.289 & 10.206 & 6.371 & 3.809 & 6.713 & 3.734 \\
\hline $10 / 25 / 06$ & $8: 00$ & 12.504 & 12.205 & 8.973 & 7.935 & 5.361 & 5.088 & 3.861 & 3.291 & 10.204 & 6.366 & 3.809 & 6.711 & 3.734 \\
\hline $10 / 25 / 06$ & $12: 00$ & 12.389 & 12.191 & 8.966 & 7.921 & 5.351 & 5.079 & 4.066 & 3.28 & 10.199 & 6.361 & 3.788 & 6.701 & 3.727 \\
\hline $10 / 25 / 06$ & $16: 00$ & 12.214 & 12.181 & 8.961 & 7.923 & 5.351 & 5.076 & 3.977 & 3.275 & 10.19 & 6.361 & 3.776 & 6.694 & 3.727 \\
\hline $10 / 25 / 06$ & $20: 00$ & 12.203 & 12.186 & 8.958 & 7.94 & 5.344 & 5.072 & 3.963 & 3.277 & 10.19 & 6.357 & 3.734 & 6.683 & 3.716 \\
\hline $10 / 26 / 06$ & $0: 00$ & 12.196 & 12.219 & 8.963 & 7.961 & 5.34 & 5.069 & 3.945 & 3.273 & 10.188 & 6.352 & 3.491 & 6.643 & 3.657 \\
\hline $10 / 26 / 06$ & $4: 00$ & 12.163 & 12.212 & 8.956 & 7.949 & 5.291 & 5.062 & 3.882 & 3.247 & 10.167 & 6.308 & 3.249 & 6.557 & 3.328 \\
\hline $10 / 26 / 06$ & $8: 00$ & 12.17 & 12.209 & 8.961 & 7.928 & 5.251 & 5.06 & 3.849 & 3.256 & 10.171 & 6.305 & 3.372 & 6.571 & 3.382 \\
\hline $10 / 26 / 06$ & $12: 00$ & 12.301 & 12.226 & 8.963 & 7.928 & 5.23 & 5.058 & 3.961 & 3.259 & 10.169 & 6.31 & 3.468 & 6.59 & 3.438 \\
\hline $10 / 26 / 06$ & $16: 00$ & 12.158 & 12.228 & 8.966 & 7.935 & 5.232 & 5.053 & 3.884 & 3.266 & 10.171 & 6.317 & 3.54 & 6.611 & 3.489 \\
\hline $10 / 26 / 06$ & $20: 00$ & 12.126 & 12.244 & 8.977 & 7.947 & 5.244 & 5.06 & 3.812 & 3.282 & 10.178 & 6.326 & 3.599 & 6.634 & 3.536 \\
\hline $10 / 27 / 06$ & $0: 00$ & 12.142 & 12.239 & 8.977 & 7.942 & 5.246 & 5.062 & 3.784 & 3.28 & 10.181 & 6.329 & 3.634 & 6.646 & 3.562 \\
\hline $10 / 27 / 06$ & $4: 00$ & 12.135 & 12.251 & 8.984 & 7.954 & 5.26 & 5.069 & 3.746 & 3.294 & 10.185 & 6.34 & 3.673 & 6.662 & 3.594 \\
\hline $10 / 27 / 06$ & $8: 00$ & 12.221 & 12.267 & 8.998 & 7.97 & 5.277 & 5.076 & 3.779 & 3.31 & 10.195 & 6.352 & 3.704 & 6.681 & 3.622 \\
\hline $10 / 27 / 06$ & $12: 00$ & 12.765 & 12.246 & 8.991 & 7.951 & 5.282 & 5.079 & 4.264 & 3.294 & 10.197 & 6.352 & 3.701 & 6.678 & 3.629 \\
\hline $10 / 27 / 06$ & $16: 00$ & 12.41 & 12.226 & 8.98 & 7.942 & 5.3 & 5.072 & 4.364 & 3.284 & 10.195 & 6.354 & 3.718 & 6.678 & 3.65 \\
\hline $10 / 27 / 06$ & $20: 00$ & 11.967 & 12.256 & 8.996 & 7.965 & 5.324 & 5.081 & 3.833 & 3.305 & 10.204 & 6.366 & 3.753 & 6.697 & 3.676 \\
\hline $10 / 28 / 06$ & $0: 00$ & 11.799 & 12.27 & 9.005 & 7.977 & 5.324 & 5.093 & 3.558 & 3.317 & 10.211 & 6.368 & 3.769 & 6.708 & 3.685 \\
\hline
\end{tabular}


Water Level (ft below top of casing) at Indicated Well

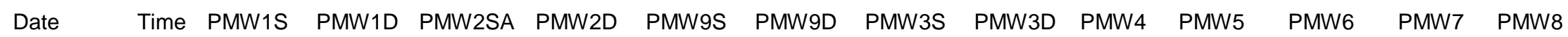

\begin{tabular}{|c|c|c|c|c|c|c|c|c|c|c|c|c|c|c|}
\hline $10 / 28 / 06$ & $4: 00$ & 11.955 & 12.267 & 9.01 & 7.975 & 5.317 & 5.095 & 3.479 & 3.319 & 10.213 & 6.371 & 3.781 & 6.715 & 3.69 \\
\hline $10 / 28 / 06$ & $8: 00$ & 12.455 & 12.258 & 9.005 & 7.968 & 5.307 & 5.093 & 3.763 & 3.31 & 10.211 & 6.368 & 3.783 & 6.713 & 3.692 \\
\hline $10 / 28 / 06$ & $12: 00$ & 12.807 & 12.219 & 8.982 & 7.935 & 5.296 & 5.079 & 4.385 & 3.28 & 10.204 & 6.359 & 3.748 & 6.69 & 3.685 \\
\hline $10 / 28 / 06$ & $16: 00$ & 12.499 & 12.198 & 8.966 & 7.923 & 5.314 & 5.065 & 4.525 & 3.27 & 10.197 & 6.361 & 3.748 & 6.681 & 3.699 \\
\hline $10 / 28 / 06$ & $20: 00$ & 11.965 & 12.2 & 8.966 & 7.926 & 5.317 & 5.06 & 4.024 & 3.27 & 10.197 & 6.361 & 3.762 & 6.681 & 3.704 \\
\hline $10 / 29 / 06$ & $0: 00$ & 11.86 & 12.205 & 8.966 & 7.928 & 5.31 & 5.06 & 3.702 & 3.273 & 10.195 & 6.359 & 3.771 & 6.683 & 3.709 \\
\hline $10 / 29 / 06$ & $4: 00$ & 11.899 & 12.2 & 8.963 & 7.926 & 5.296 & 5.058 & 3.581 & 3.27 & 10.192 & 6.352 & 3.774 & 6.68 & 3.706 \\
\hline $10 / 29 / 06$ & 8:00 & 12.546 & 12.2 & 8.961 & 7.923 & 5.289 & 5.053 & 3.907 & 3.268 & 10.188 & 6.352 & 3.774 & 6.683 & 3.709 \\
\hline $10 / 29 / 06$ & $12: 00$ & 13.213 & 12.181 & 8.954 & 7.904 & 5.293 & 5.044 & 4.774 & 3.252 & 10.188 & 6.35 & 3.743 & 6.669 & 3.709 \\
\hline $10 / 29 / 06$ & $16: 00$ & 12.49 & 12.163 & 8.94 & 7.893 & 5.315 & 5.034 & 4.427 & 3.245 & 10.181 & 6.352 & 3.741 & 6.662 & 3.725 \\
\hline $10 / 29 / 06$ & $20: 00$ & 11.944 & 12.154 & 8.933 & 7.888 & 5.31 & 5.027 & 3.889 & 3.235 & 10.174 & 6.345 & 3.741 & 6.65 & 3.72 \\
\hline $10 / 30 / 06$ & $0: 00$ & 12.107 & 12.135 & 8.921 & 7.872 & 5.287 & 5.013 & 4.014 & 3.217 & 10.167 & 6.336 & 3.725 & 6.639 & 3.706 \\
\hline $10 / 30 / 06$ & $4: 00$ & 11.965 & 12.121 & 8.907 & 7.858 & 5.268 & 4.995 & 3.807 & 3.202 & 10.155 & 6.331 & 3.711 & 6.627 & 3.697 \\
\hline $10 / 30 / 06$ & 8:00 & 12.203 & 12.116 & 8.902 & 7.858 & 5.261 & 4.985 & 3.973 & 3.2 & 10.148 & 6.326 & 3.711 & 6.622 & 3.699 \\
\hline $10 / 30 / 06$ & $12: 00$ & 12.56 & 12.135 & 8.909 & 7.872 & 5.284 & 4.985 & 4.56 & 3.217 & 10.153 & 6.338 & 3.722 & 6.632 & 3.725 \\
\hline $10 / 30 / 06$ & $16: 00$ & 11.526 & 12.235 & 8.973 & 7.963 & 5.357 & 5.025 & 3.775 & 3.305 & 10.178 & 6.371 & 3.813 & 6.699 & 3.786 \\
\hline $10 / 30 / 06$ & $20: 00$ & 11.605 & 12.293 & 9.015 & 8.001 & 5.371 & 5.065 & 3.248 & 3.34 & 10.202 & 6.385 & 3.851 & 6.739 & 3.793 \\
\hline $10 / 31 / 06$ & $0: 00$ & 11.719 & 12.346 & 9.057 & 8.047 & 5.396 & 5.102 & 3.043 & 3.382 & 10.225 & 6.406 & 3.888 & 6.778 & 3.807 \\
\hline $10 / 31 / 06$ & $4: 00$ & 11.897 & 12.376 & 9.083 & 8.068 & 5.399 & 5.128 & 3.565 & 3.403 & 10.241 & 6.415 & 3.909 & 6.802 & 3.814 \\
\hline $10 / 31 / 06$ & $8: 00$ & 12.21 & 12.39 & 9.097 & 8.078 & 5.399 & 5.144 & 3.569 & 3.413 & 10.255 & 6.424 & 3.921 & 6.823 & 3.807 \\
\hline $10 / 31 / 06$ & $12: 00$ & 12.593 & 12.362 & 9.09 & 8.054 & 5.38 & 5.14 & 3.835 & 3.387 & 10.255 & 6.415 & 3.907 & 6.811 & 3.788 \\
\hline $10 / 31 / 06$ & $16: 00$ & 12.163 & 12.337 & 9.073 & 8.038 & 5.373 & 5.125 & 3.751 & 3.373 & 10.251 & 6.413 & 3.897 & 6.804 & 3.781 \\
\hline $10 / 31 / 06$ & $20: 00$ & 11.736 & 12.346 & 9.08 & 8.05 & 5.38 & 5.123 & 3.35 & 3.385 & 10.253 & 6.417 & 3.916 & 6.813 & 3.788 \\
\hline $11 / 1 / 06$ & $0: 00$ & 12.028 & 12.353 & 9.087 & 8.054 & 5.38 & 5.125 & 3.311 & 3.392 & 10.255 & 6.42 & 3.925 & 6.825 & 3.79 \\
\hline $11 / 1 / 06$ & $4: 00$ & 12.24 & 12.36 & 9.092 & 8.061 & 5.383 & 5.13 & 3.455 & 3.396 & 10.258 & 6.422 & 3.923 & 6.83 & 3.788 \\
\hline 11/1/06 & $8: 00$ & 12.562 & 12.383 & 9.106 & 8.08 & 5.397 & 5.142 & 3.49 & 3.415 & 10.267 & 6.434 & 3.942 & 6.843 & 3.8 \\
\hline $11 / 1 / 06$ & $12: 00$ & 12.565 & 12.374 & 9.109 & 8.071 & 5.392 & 5.142 & 3.861 & 3.406 & 10.267 & 6.431 & 3.914 & 6.836 & 3.788 \\
\hline $11 / 1 / 06$ & $16: 00$ & 12.301 & 12.367 & 9.101 & 8.068 & 5.394 & 5.139 & 3.684 & 3.403 & 10.265 & 6.431 & 3.916 & 6.834 & 3.786 \\
\hline $11 / 1 / 06$ & $20: 00$ & 12.077 & 12.376 & 9.109 & 8.075 & 5.399 & 5.147 & 3.155 & 3.413 & 10.269 & 6.434 & 3.935 & 6.846 & 3.788 \\
\hline $11 / 2 / 06$ & $0: 00$ & 12.119 & 12.383 & 9.113 & 8.085 & 5.404 & 5.151 & 3.19 & 3.42 & 10.274 & 6.436 & 3.946 & 6.857 & 3.793 \\
\hline $11 / 2 / 06$ & $4: 00$ & 12.074 & 12.386 & 9.116 & 8.087 & 5.402 & 5.154 & 3.157 & 3.42 & 10.274 & 6.438 & 3.96 & 6.867 & 3.795 \\
\hline $11 / 2 / 06$ & $8: 00$ & 12.649 & 12.402 & 9.127 & 8.104 & 5.415 & 5.165 & 3.448 & 3.441 & 10.279 & 6.445 & 3.986 & 6.881 & 3.811 \\
\hline $11 / 2 / 06$ & $12: 00$ & 13.09 & 12.386 & 9.125 & 8.085 & 5.404 & 5.163 & 3.835 & 3.42 & 10.279 & 6.438 & 3.921 & 6.86 & 3.788 \\
\hline $11 / 2 / 06$ & $16: 00$ & 12.343 & 12.362 & 9.106 & 8.071 & 5.395 & 5.151 & 3.793 & 3.406 & 10.272 & 6.434 & 3.9 & 6.843 & 3.772 \\
\hline
\end{tabular}


TABLE S1.2 (Cont.)

Water Level (ft below top of casing) at Indicated Well

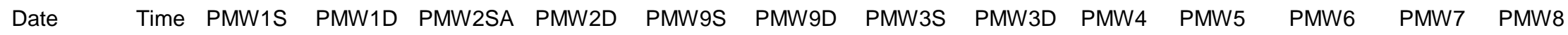

\begin{tabular}{|c|c|c|c|c|c|c|c|c|c|c|c|c|c|c|}
\hline $11 / 2 / 06$ & $20: 00$ & 12.084 & 12.353 & 9.102 & 8.064 & 5.39 & 5.144 & 3.309 & 3.401 & 10.267 & 6.431 & 3.907 & 6.839 & 3.767 \\
\hline $11 / 3 / 06$ & $0: 00$ & 12.168 & 12.349 & 9.097 & 8.059 & 5.385 & 5.139 & 3.353 & 3.394 & 10.265 & 6.427 & 3.911 & 6.841 & 3.765 \\
\hline $11 / 3 / 06$ & $4: 00$ & 12.242 & 12.33 & 9.085 & 8.043 & 5.371 & 5.13 & 3.369 & 3.382 & 10.255 & 6.42 & 3.904 & 6.836 & 3.755 \\
\hline $11 / 3 / 06$ & $8: 00$ & 12.63 & 12.316 & 9.076 & 8.036 & 5.364 & 5.121 & 3.637 & 3.373 & 10.251 & 6.417 & 3.902 & 6.83 & 3.755 \\
\hline $11 / 3 / 06$ & $12: 00$ & 12.824 & 12.279 & 9.05 & 8 & 5.339 & 5.097 & 4.397 & 3.338 & 10.237 & 6.403 & 3.853 & 6.795 & 3.732 \\
\hline $11 / 3 / 06$ & $16: 00$ & 12.373 & 12.251 & 9.029 & 7.979 & 5.329 & 5.079 & 4.255 & 3.319 & 10.225 & 6.396 & 3.837 & 6.776 & 3.727 \\
\hline $11 / 3 / 06$ & $20: 00$ & 12.261 & 12.249 & 9.022 & 7.977 & 5.327 & 5.069 & 3.975 & 3.319 & 10.22 & 6.392 & 3.842 & 6.771 & 3.73 \\
\hline $11 / 4 / 06$ & $0: 00$ & 12.27 & 12.249 & 9.02 & 7.977 & 5.325 & 5.062 & 3.966 & 3.319 & 10.216 & 6.389 & 3.839 & 6.767 & 3.73 \\
\hline $11 / 4 / 06$ & $4: 00$ & 12.233 & 12.244 & 9.015 & 7.975 & 5.318 & 5.058 & 3.886 & 3.315 & 10.211 & 6.385 & 3.837 & 6.762 & 3.73 \\
\hline $11 / 4 / 06$ & $8: 00$ & 12.333 & 12.26 & 9.024 & 7.989 & 5.325 & 5.06 & 3.877 & 3.326 & 10.213 & 6.389 & 3.848 & 6.771 & 3.739 \\
\hline $11 / 4 / 06$ & $12: 00$ & 12.688 & 12.26 & 9.024 & 7.984 & 5.322 & 5.06 & 4.373 & 3.322 & 10.213 & 6.389 & 3.837 & 6.769 & 3.737 \\
\hline $11 / 4 / 06$ & $16: 00$ & 12.221 & 12.27 & 9.029 & 7.996 & 5.329 & 5.06 & 4.147 & 3.331 & 10.216 & 6.394 & 3.846 & 6.774 & 3.746 \\
\hline $11 / 4 / 06$ & $20: 00$ & 12.116 & 12.288 & 9.041 & 8.01 & 5.334 & 5.069 & 3.6 & 3.343 & 10.22 & 6.396 & 3.86 & 6.783 & 3.751 \\
\hline $11 / 5 / 06$ & 0:00 & 12.1 & 12.293 & 9.045 & 8.012 & 5.334 & 5.076 & 3.544 & 3.345 & 10.22 & 6.394 & 3.867 & 6.79 & 3.746 \\
\hline $11 / 5 / 06$ & $4: 00$ & 12.084 & 12.291 & 9.045 & 8.01 & 5.332 & 5.076 & 3.444 & 3.343 & 10.223 & 6.394 & 3.867 & 6.788 & 3.746 \\
\hline $11 / 5 / 06$ & $8: 00$ & 12.322 & 12.302 & 9.055 & 8.022 & 5.339 & 5.083 & 3.733 & 3.352 & 10.225 & 6.399 & 3.877 & 6.799 & 3.753 \\
\hline $11 / 5 / 06$ & $12: 00$ & 12.87 & 12.277 & 9.041 & 7.996 & 5.323 & 5.074 & 4.613 & 3.329 & 10.22 & 6.392 & 3.839 & 6.781 & 3.735 \\
\hline $11 / 5 / 06$ & $16: 00$ & 12.333 & 12.251 & 9.02 & 7.977 & 5.311 & 5.06 & 4.593 & 3.31 & 10.209 & 6.387 & 3.823 & 6.762 & 3.725 \\
\hline $11 / 5 / 06$ & $20: 00$ & 12.135 & 12.258 & 9.022 & 7.984 & 5.316 & 5.058 & 3.975 & 3.317 & 10.206 & 6.389 & 3.832 & 6.762 & 3.73 \\
\hline $11 / 6 / 06$ & 0:00 & 12.121 & 12.265 & 9.027 & 7.989 & 5.316 & 5.055 & 3.877 & 3.317 & 10.204 & 6.387 & 3.83 & 6.762 & 3.73 \\
\hline $11 / 6 / 06$ & $4: 00$ & 12.17 & 12.258 & 9.022 & 7.979 & 5.306 & 5.053 & 3.905 & 3.315 & 10.202 & 6.382 & 3.821 & 6.76 & 3.723 \\
\hline $11 / 6 / 06$ & $8: 00$ & 12.256 & 12.27 & 9.029 & 7.991 & 5.316 & 5.055 & 3.973 & 3.322 & 10.204 & 6.387 & 3.828 & 6.762 & 3.728 \\
\hline $11 / 6 / 06$ & $12: 00$ & 12.698 & 12.258 & 9.022 & 7.977 & 5.304 & 5.048 & 4.583 & 3.31 & 10.199 & 6.383 & 3.807 & 6.753 & 3.718 \\
\hline $11 / 6 / 06$ & $16: 00$ & 12.263 & 12.246 & 9.015 & 7.975 & 5.299 & 5.041 & 4.56 & 3.305 & 10.197 & 6.38 & 3.807 & 6.75 & 3.721 \\
\hline $11 / 6 / 06$ & $20: 00$ & 11.983 & 12.256 & 9.017 & 7.979 & 5.304 & 5.041 & 3.761 & 3.31 & 10.195 & 6.383 & 3.823 & 6.75 & 3.725 \\
\hline $11 / 7 / 06$ & $0: 00$ & 12.018 & 12.249 & 9.015 & 7.975 & 5.297 & 5.041 & 3.586 & 3.303 & 10.19 & 6.378 & 3.823 & 6.75 & 3.721 \\
\hline $11 / 7 / 06$ & $4: 00$ & 12.079 & 12.226 & 9.003 & 7.951 & 5.278 & 5.03 & 3.602 & 3.282 & 10.181 & 6.366 & 3.804 & 6.732 & 3.709 \\
\hline $11 / 7 / 06$ & $8: 00$ & 12.235 & 12.23 & 9.001 & 7.958 & 5.285 & 5.027 & 3.831 & 3.286 & 10.181 & 6.371 & 3.814 & 6.736 & 3.718 \\
\hline $11 / 7 / 06$ & $12: 00$ & 12.88 & 12.202 & 8.987 & 7.93 & 5.264 & 5.011 & 4.751 & 3.261 & 10.171 & 6.362 & 3.774 & 6.715 & 3.702 \\
\hline $11 / 7 / 06$ & $16: 00$ & 12.233 & 12.193 & 8.975 & 7.925 & 5.264 & 5.002 & 4.537 & 3.256 & 10.162 & 6.359 & 3.774 & 6.708 & 3.709 \\
\hline $11 / 7 / 06$ & $20: 00$ & 12.081 & 12.191 & 8.97 & 7.925 & 5.264 & 4.997 & 3.975 & 3.256 & 10.155 & 6.355 & 3.776 & 6.704 & 3.709 \\
\hline $11 / 8 / 06$ & $0: 00$ & 12.081 & 12.179 & 8.961 & 7.914 & 5.255 & 4.99 & 3.935 & 3.245 & 10.15 & 6.352 & 3.765 & 6.694 & 3.702 \\
\hline $11 / 8 / 06$ & $4: 00$ & 12.098 & 12.168 & 8.954 & 7.904 & 5.243 & 4.978 & 3.863 & 3.233 & 10.143 & 6.345 & 3.755 & 6.685 & 3.695 \\
\hline $11 / 8 / 06$ & $8: 00$ & 12.317 & 12.165 & 8.949 & 7.904 & 5.244 & 4.974 & 4.124 & 3.231 & 10.139 & 6.345 & 3.753 & 6.68 & 3.695 \\
\hline
\end{tabular}


TABLE S1.2 (Cont.)

Water Level (ft below top of casing) at Indicated Well

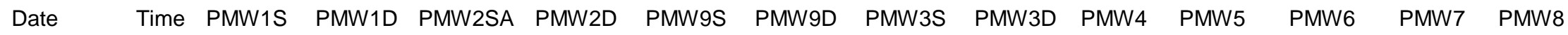

\begin{tabular}{|c|c|c|c|c|c|c|c|c|c|c|c|c|c|c|}
\hline $11 / 8 / 06$ & $12: 00$ & 13.064 & 12.147 & 8.935 & 7.883 & 5.23 & 4.96 & 5.117 & 3.212 & 10.129 & 6.341 & 3.725 & 6.669 & 3.688 \\
\hline $11 / 8 / 06$ & $16: 00$ & 12.275 & 12.142 & 8.926 & 7.886 & 5.232 & 4.952 & 5.014 & 3.214 & 10.122 & 6.338 & 3.73 & 6.664 & 3.695 \\
\hline $11 / 8 / 06$ & $20: 00$ & 11.981 & 12.184 & 8.949 & 7.921 & 5.255 & 4.967 & 4.015 & 3.247 & 10.125 & 6.348 & 3.765 & 6.685 & 3.718 \\
\hline $11 / 9 / 06$ & $0: 00$ & 11.878 & 12.23 & 8.978 & 7.958 & 5.281 & 4.99 & 3.677 & 3.284 & 10.139 & 6.359 & 3.793 & 6.715 & 3.735 \\
\hline $11 / 9 / 06$ & $4: 00$ & 11.839 & 12.26 & 9.003 & 7.982 & 5.293 & 5.013 & 3.32 & 3.303 & 10.15 & 6.364 & 3.814 & 6.736 & 3.744 \\
\hline $11 / 9 / 06$ & $8: 00$ & 12.17 & 12.284 & 9.022 & 7.996 & 5.304 & 5.03 & 3.516 & 3.319 & 10.16 & 6.373 & 3.832 & 6.753 & 3.751 \\
\hline $11 / 9 / 06$ & $12: 00$ & 12.38 & 12.281 & 9.031 & 7.991 & 5.302 & 5.039 & 4.094 & 3.317 & 10.164 & 6.373 & 3.823 & 6.757 & 3.746 \\
\hline $11 / 9 / 06$ & $16: 00$ & 12.063 & 12.274 & 9.024 & 7.989 & 5.3 & 5.041 & 3.847 & 3.312 & 10.164 & 6.376 & 3.828 & 6.757 & 3.746 \\
\hline $11 / 9 / 06$ & $20: 00$ & 11.983 & 12.284 & 9.034 & 7.998 & 5.309 & 5.044 & 3.351 & 3.319 & 10.164 & 6.378 & 3.842 & 6.764 & 3.751 \\
\hline $11 / 10 / 06$ & 0:00 & 12.067 & 12.302 & 9.045 & 8.017 & 5.321 & 5.051 & 3.332 & 3.336 & 10.169 & 6.387 & 3.858 & 6.778 & 3.758 \\
\hline $11 / 10 / 06$ & $4: 00$ & 12.002 & 12.316 & 9.057 & 8.026 & 5.328 & 5.06 & 3.176 & 3.347 & 10.178 & 6.392 & 3.867 & 6.79 & 3.76 \\
\hline $11 / 10 / 06$ & $8: 00$ & 12.032 & 12.339 & 9.071 & 8.052 & 5.349 & 5.076 & 3.09 & 3.373 & 10.188 & 6.404 & 3.877 & 6.806 & 3.772 \\
\hline $11 / 10 / 06$ & $12: 00$ & 12.105 & 12.367 & 9.092 & 8.066 & 5.354 & 5.09 & 3.078 & 3.385 & 10.199 & 6.408 & 3.884 & 6.822 & 3.772 \\
\hline $11 / 10 / 06$ & $16: 00$ & 12.046 & 12.402 & 9.116 & 8.099 & 5.377 & 5.111 & 3.518 & 3.417 & 10.216 & 6.425 & 3.916 & 6.85 & 3.788 \\
\hline $11 / 10 / 06$ & $20: 00$ & 12.044 & 12.421 & 9.132 & 8.115 & 5.389 & 5.128 & 3.271 & 3.434 & 10.227 & 6.436 & 3.933 & 6.869 & 3.793 \\
\hline $11 / 11 / 06$ & $0: 00$ & 12.037 & 12.425 & 9.142 & 8.115 & 5.391 & 5.135 & 3.178 & 3.434 & 10.234 & 6.439 & 3.935 & 6.878 & 3.788 \\
\hline $11 / 11 / 06$ & $4: 00$ & 12.053 & 12.425 & 9.146 & 8.115 & 5.393 & 5.139 & 3.034 & 3.434 & 10.239 & 6.441 & 3.944 & 6.883 & 3.786 \\
\hline $11 / 11 / 06$ & $8: 00$ & 12.572 & 12.414 & 9.149 & 8.108 & 5.391 & 5.137 & 3.29 & 3.431 & 10.241 & 6.441 & 3.949 & 6.888 & 3.784 \\
\hline $11 / 11 / 06$ & $12: 00$ & 12.42 & 12.379 & 9.127 & 8.08 & 5.377 & 5.125 & 3.661 & 3.399 & 10.232 & 6.432 & 3.909 & 6.864 & 3.765 \\
\hline $11 / 11 / 06$ & $16: 00$ & 12.289 & 12.33 & 9.095 & 8.04 & 5.356 & 5.102 & 3.682 & 3.364 & 10.216 & 6.415 & 3.872 & 6.829 & 3.744 \\
\hline $11 / 11 / 06$ & $20: 00$ & 12.21 & 12.307 & 9.074 & 8.024 & 5.347 & 5.083 & 3.418 & 3.347 & 10.204 & 6.411 & 3.867 & 6.813 & 3.739 \\
\hline $11 / 12 / 06$ & $0: 00$ & 12.245 & 12.293 & 9.067 & 8.012 & 5.335 & 5.067 & 3.376 & 3.338 & 10.195 & 6.399 & 3.856 & 6.804 & 3.735 \\
\hline $11 / 12 / 06$ & $4: 00$ & 12.277 & 12.263 & 9.043 & 7.984 & 5.314 & 5.048 & 3.507 & 3.31 & 10.181 & 6.39 & 3.837 & 6.783 & 3.721 \\
\hline $11 / 12 / 06$ & $8: 00$ & 12.312 & 12.26 & 9.036 & 7.986 & 5.316 & 5.039 & 3.572 & 3.315 & 10.176 & 6.392 & 3.844 & 6.783 & 3.73 \\
\hline $11 / 12 / 06$ & $12: 00$ & 12.579 & 12.253 & 9.029 & 7.982 & 5.312 & 5.032 & 4.008 & 3.305 & 10.174 & 6.385 & 3.832 & 6.776 & 3.728 \\
\hline $11 / 12 / 06$ & $16: 00$ & 12.275 & 12.235 & 9.029 & 7.972 & 5.316 & 5.027 & 3.898 & 3.31 & 10.169 & 6.387 & 3.832 & 6.774 & 3.735 \\
\hline $11 / 12 / 06$ & $20: 00$ & 12.231 & 12.272 & 9.053 & 7.998 & 5.335 & 5.041 & 3.642 & 3.338 & 10.164 & 6.392 & 3.749 & 6.769 & 3.714 \\
\hline $11 / 13 / 06$ & $0: 00$ & 12.205 & 12.323 & 9.071 & 8.04 & 5.34 & 5.053 & 3.486 & 3.357 & 10.185 & 6.399 & 3.767 & 6.785 & 3.711 \\
\hline $11 / 13 / 06$ & $4: 00$ & 12.135 & 12.33 & 9.081 & 8.043 & 5.337 & 5.062 & 3.269 & 3.357 & 10.19 & 6.399 & 3.793 & 6.795 & 3.711 \\
\hline $11 / 13 / 06$ & $8: 00$ & 12.254 & 12.349 & 9.092 & 8.059 & 5.351 & 5.072 & 3.288 & 3.375 & 10.197 & 6.408 & 3.833 & 6.811 & 3.728 \\
\hline $11 / 13 / 06$ & $12: 00$ & 12.7 & 12.304 & 9.071 & 8.017 & 5.319 & 5.06 & 3.968 & 3.333 & 10.19 & 6.392 & 3.798 & 6.788 & 3.704 \\
\hline $11 / 13 / 06$ & $16: 00$ & 12.252 & 12.274 & 9.046 & 7.996 & 5.309 & 5.044 & 3.793 & 3.315 & 10.176 & 6.39 & 3.795 & 6.771 & 3.704 \\
\hline $11 / 13 / 06$ & $20: 00$ & 12.219 & 12.27 & 9.041 & 7.993 & 5.307 & 5.037 & 3.616 & 3.312 & 10.174 & 6.385 & 3.8 & 6.769 & 3.707 \\
\hline $11 / 14 / 06$ & $0: 00$ & 12.231 & 12.258 & 9.034 & 7.979 & 5.295 & 5.027 & 3.56 & 3.301 & 10.169 & 6.383 & 3.795 & 6.762 & 3.702 \\
\hline
\end{tabular}


Water Level (ft below top of casing) at Indicated Well

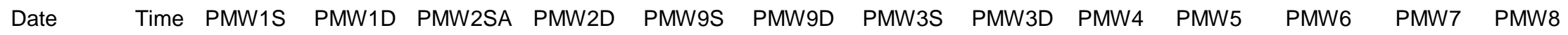

\begin{tabular}{|c|c|c|c|c|c|c|c|c|c|c|c|c|c|c|}
\hline $11 / 14 / 06$ & $4: 00$ & 12.2 & 12.237 & 9.02 & 7.965 & 5.284 & 5.011 & 3.519 & 3.284 & 10.157 & 6.371 & 3.788 & 6.748 & 3.697 \\
\hline $11 / 14 / 06$ & $8: 00$ & 12.254 & 12.228 & 9.008 & 7.958 & 5.282 & 5.002 & 3.609 & 3.277 & 10.15 & 6.373 & 3.788 & 6.741 & 3.7 \\
\hline $11 / 14 / 06$ & $12: 00$ & 12.688 & 12.202 & 8.994 & 7.932 & 5.258 & 4.983 & 4.162 & 3.254 & 10.141 & 6.357 & 3.753 & 6.72 & 3.683 \\
\hline $11 / 14 / 06$ & $16: 00$ & 12.231 & 12.209 & 8.989 & 7.946 & 5.272 & 4.978 & 3.964 & 3.265 & 10.139 & 6.364 & 3.772 & 6.722 & 3.702 \\
\hline $11 / 14 / 06$ & $20: 00$ & 12.133 & 12.239 & 9.008 & 7.97 & 5.286 & 4.99 & 3.621 & 3.289 & 10.143 & 6.366 & 3.793 & 6.739 & 3.714 \\
\hline $11 / 15 / 06$ & $0: 00$ & 12.086 & 12.277 & 9.034 & 8 & 5.302 & 5.009 & 3.393 & 3.315 & 10.155 & 6.376 & 3.819 & 6.762 & 3.73 \\
\hline $11 / 15 / 06$ & $4: 00$ & 12.168 & 12.298 & 9.048 & 8.014 & 5.314 & 5.023 & 3.379 & 3.329 & 10.162 & 6.383 & 3.835 & 6.776 & 3.737 \\
\hline $11 / 15 / 06$ & $8: 00$ & 12.165 & 12.337 & 9.083 & 8.057 & 5.34 & 5.051 & 3.348 & 3.368 & 10.181 & 6.397 & 3.863 & 6.808 & 3.756 \\
\hline $11 / 15 / 06$ & $12: 00$ & 12.207 & 12.358 & 9.099 & 8.059 & 5.345 & 5.067 & 3.337 & 3.373 & 10.19 & 6.401 & 3.861 & 6.818 & 3.753 \\
\hline $11 / 15 / 06$ & $16: 00$ & 12.161 & 12.36 & 9.102 & 8.061 & 5.345 & 5.072 & 3.276 & 3.375 & 10.195 & 6.406 & 3.872 & 6.825 & 3.751 \\
\hline $11 / 15 / 06$ & $20: 00$ & 12.158 & 12.372 & 9.113 & 8.073 & 5.356 & 5.083 & 3.183 & 3.385 & 10.202 & 6.411 & 3.886 & 6.839 & 3.756 \\
\hline $11 / 16 / 06$ & $0: 00$ & 12.2 & 12.358 & 9.109 & 8.064 & 5.352 & 5.083 & 3.211 & 3.378 & 10.204 & 6.411 & 3.884 & 6.839 & 3.749 \\
\hline $11 / 16 / 06$ & $4: 00$ & 12.217 & 12.332 & 9.092 & 8.04 & 5.34 & 5.074 & 3.269 & 3.354 & 10.197 & 6.404 & 3.872 & 6.825 & 3.74 \\
\hline $11 / 16 / 06$ & 8:00 & 12.235 & 12.335 & 9.095 & 8.05 & 5.345 & 5.074 & 3.295 & 3.364 & 10.197 & 6.406 & 3.879 & 6.829 & 3.744 \\
\hline $11 / 16 / 06$ & $12: 00$ & 12.389 & 12.304 & 9.078 & 8.019 & 5.326 & 5.062 & 3.598 & 3.336 & 10.19 & 6.394 & 3.849 & 6.808 & 3.728 \\
\hline $11 / 16 / 06$ & $16: 00$ & 12.282 & 12.274 & 9.053 & 7.996 & 5.314 & 5.044 & 3.717 & 3.315 & 10.176 & 6.387 & 3.83 & 6.79 & 3.721 \\
\hline $11 / 16 / 06$ & $20: 00$ & 12.217 & 12.265 & 9.048 & 7.991 & 5.31 & 5.032 & 3.474 & 3.31 & 10.169 & 6.38 & 3.83 & 6.778 & 3.721 \\
\hline $11 / 17 / 06$ & $0: 00$ & 12.247 & 12.26 & 9.041 & 7.984 & 5.303 & 5.025 & 3.486 & 3.303 & 10.164 & 6.378 & 3.826 & 6.774 & 3.721 \\
\hline $11 / 17 / 06$ & $4: 00$ & 12.172 & 12.281 & 9.048 & 8.005 & 5.319 & 5.027 & 3.383 & 3.322 & 10.167 & 6.385 & 3.844 & 6.785 & 3.735 \\
\hline $11 / 17 / 06$ & $8: 00$ & 12.352 & 12.328 & 9.081 & 8.042 & 5.343 & 5.046 & 3.505 & 3.359 & 10.181 & 6.399 & 3.877 & 6.818 & 3.756 \\
\hline $11 / 17 / 06$ & $12: 00$ & 12.656 & 12.365 & 9.109 & 8.073 & 5.361 & 5.069 & 4.071 & 3.385 & 10.192 & 6.413 & 3.889 & 6.841 & 3.768 \\
\hline $11 / 17 / 06$ & $16: 00$ & 12.256 & 12.397 & 9.13 & 8.099 & 5.38 & 5.09 & 3.691 & 3.41 & 10.211 & 6.425 & 3.914 & 6.864 & 3.779 \\
\hline $11 / 17 / 06$ & $20: 00$ & 12.126 & 12.421 & 9.151 & 8.122 & 5.396 & 5.114 & 3.118 & 3.431 & 10.223 & 6.434 & 3.935 & 6.888 & 3.786 \\
\hline $11 / 18 / 06$ & $0: 00$ & 12.165 & 12.434 & 9.163 & 8.127 & 5.401 & 5.13 & 3.006 & 3.438 & 10.232 & 6.436 & 3.947 & 6.897 & 3.786 \\
\hline $11 / 18 / 06$ & $4: 00$ & 12.119 & 12.441 & 9.172 & 8.136 & 5.408 & 5.139 & 2.966 & 3.445 & 10.239 & 6.441 & 3.963 & 6.911 & 3.791 \\
\hline $11 / 18 / 06$ & 8:00 & 12.579 & 12.448 & 9.181 & 8.146 & 5.415 & 5.149 & 3.234 & 3.452 & 10.244 & 6.448 & 3.989 & 6.925 & 3.8 \\
\hline $11 / 18 / 06$ & $12: 00$ & 12.611 & 12.441 & 9.181 & 8.134 & 5.413 & 5.154 & 3.712 & 3.443 & 10.248 & 6.446 & 3.942 & 6.916 & 3.786 \\
\hline $11 / 18 / 06$ & $16: 00$ & 12.277 & 12.437 & 9.181 & 8.139 & 5.42 & 5.154 & 3.568 & 3.448 & 10.251 & 6.448 & 3.938 & 6.913 & 3.779 \\
\hline $11 / 18 / 06$ & $20: 00$ & 12.191 & 12.455 & 9.193 & 8.155 & 5.432 & 5.161 & 3.12 & 3.464 & 10.255 & 6.455 & 3.963 & 6.93 & 3.786 \\
\hline $11 / 19 / 06$ & $0: 00$ & 12.109 & 12.46 & 9.196 & 8.157 & 5.434 & 5.168 & 2.957 & 3.466 & 10.258 & 6.46 & 3.982 & 6.939 & 3.789 \\
\hline $11 / 19 / 06$ & $4: 00$ & 12.219 & 12.462 & 9.2 & 8.157 & 5.436 & 5.172 & 2.978 & 3.469 & 10.262 & 6.46 & 4.005 & 6.951 & 3.796 \\
\hline $11 / 19 / 06$ & $8: 00$ & 12.686 & 12.467 & 9.207 & 8.162 & 5.441 & 5.175 & 3.293 & 3.476 & 10.265 & 6.464 & 4.029 & 6.962 & 3.805 \\
\hline $11 / 19 / 06$ & $12: 00$ & 12.593 & 12.441 & 9.196 & 8.141 & 5.432 & 5.17 & 4.024 & 3.452 & 10.26 & 6.457 & 3.947 & 6.937 & 3.782 \\
\hline $11 / 19 / 06$ & $16: 00$ & 12.357 & 12.448 & 9.196 & 8.155 & 5.443 & 5.168 & 3.915 & 3.464 & 10.262 & 6.462 & 3.938 & 6.934 & 3.782 \\
\hline
\end{tabular}


Water Level (ft below top of casing) at Indicated Well

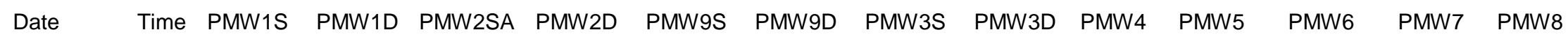

\begin{tabular}{|c|c|c|c|c|c|c|c|c|c|c|c|c|c|c|}
\hline $11 / 19 / 06$ & $20: 00$ & 12.107 & 12.474 & 9.212 & 8.176 & 5.455 & 5.177 & 3.183 & 3.485 & 10.269 & 6.469 & 3.97 & 6.953 & 3.789 \\
\hline $11 / 20 / 06$ & 0:00 & 12.203 & 12.476 & 9.217 & 8.176 & 5.455 & 5.184 & 3.076 & 3.487 & 10.274 & 6.469 & 3.991 & 6.962 & 3.791 \\
\hline $11 / 20 / 06$ & $4: 00$ & 12.249 & 12.455 & 9.21 & 8.162 & 5.446 & 5.182 & 3.055 & 3.473 & 10.269 & 6.467 & 4.008 & 6.964 & 3.789 \\
\hline $11 / 20 / 06$ & $8: 00$ & 12.674 & 12.446 & 9.205 & 8.155 & 5.443 & 5.177 & 3.384 & 3.469 & 10.269 & 6.464 & 4.031 & 6.967 & 3.793 \\
\hline $11 / 20 / 06$ & $12: 00$ & 12.628 & 12.418 & 9.189 & 8.127 & 5.432 & 5.168 & 4.16 & 3.443 & 10.262 & 6.453 & 3.942 & 6.937 & 3.77 \\
\hline $11 / 20 / 06$ & $16: 00$ & 12.396 & 12.376 & 9.158 & 8.094 & 5.411 & 5.144 & 4.171 & 3.41 & 10.246 & 6.443 & 3.898 & 6.902 & 3.747 \\
\hline $11 / 20 / 06$ & $20: 00$ & 12.296 & 12.353 & 9.137 & 8.078 & 5.399 & 5.121 & 3.889 & 3.392 & 10.234 & 6.436 & 3.893 & 6.881 & 3.74 \\
\hline $11 / 21 / 06$ & $0: 00$ & 12.366 & 12.337 & 9.123 & 8.061 & 5.385 & 5.104 & 3.959 & 3.38 & 10.22 & 6.425 & 3.886 & 6.869 & 3.733 \\
\hline $11 / 21 / 06$ & $4: 00$ & 12.317 & 12.323 & 9.111 & 8.052 & 5.376 & 5.09 & 3.948 & 3.368 & 10.216 & 6.422 & 3.882 & 6.86 & 3.733 \\
\hline $11 / 21 / 06$ & $8: 00$ & 12.375 & 12.328 & 9.111 & 8.059 & 5.376 & 5.083 & 3.968 & 3.373 & 10.213 & 6.422 & 3.889 & 6.86 & 3.742 \\
\hline $11 / 21 / 06$ & $12: 00$ & 12.905 & 12.323 & 9.107 & 8.052 & 5.371 & 5.079 & 4.586 & 3.363 & 10.211 & 6.42 & 3.877 & 6.853 & 3.742 \\
\hline $11 / 21 / 06$ & $16: 00$ & 12.343 & 12.323 & 9.104 & 8.054 & 5.369 & 5.074 & 4.567 & 3.363 & 10.209 & 6.418 & 3.877 & 6.853 & 3.744 \\
\hline $11 / 21 / 06$ & $20: 00$ & 12.179 & 12.339 & 9.114 & 8.068 & 5.373 & 5.076 & 3.889 & 3.375 & 10.209 & 6.418 & 3.891 & 6.86 & 3.754 \\
\hline $11 / 22 / 06$ & $0: 00$ & 12.219 & 12.332 & 9.111 & 8.059 & 5.366 & 5.074 & 3.908 & 3.368 & 10.209 & 6.413 & 3.884 & 6.855 & 3.744 \\
\hline $11 / 22 / 06$ & $4: 00$ & 12.165 & 12.332 & 9.107 & 8.056 & 5.364 & 5.072 & 3.805 & 3.366 & 10.204 & 6.413 & 3.882 & 6.85 & 3.744 \\
\hline $11 / 22 / 06$ & $8: 00$ & 12.284 & 12.346 & 9.118 & 8.071 & 5.373 & 5.074 & 3.85 & 3.38 & 10.209 & 6.418 & 3.896 & 6.86 & 3.754 \\
\hline $11 / 22 / 06$ & $12: 00$ & 12.852 & 12.346 & 9.118 & 8.064 & 5.371 & 5.076 & 4.642 & 3.373 & 10.211 & 6.418 & 3.882 & 6.86 & 3.747 \\
\hline $11 / 22 / 06$ & $16: 00$ & 12.357 & 12.344 & 9.116 & 8.066 & 5.371 & 5.074 & 4.635 & 3.375 & 10.211 & 6.418 & 3.877 & 6.86 & 3.749 \\
\hline $11 / 22 / 06$ & $20: 00$ & 12.196 & 12.365 & 9.128 & 8.085 & 5.381 & 5.081 & 3.999 & 3.389 & 10.216 & 6.422 & 3.898 & 6.874 & 3.756 \\
\hline $11 / 23 / 06$ & $0: 00$ & 12.179 & 12.372 & 9.135 & 8.085 & 5.381 & 5.088 & 3.715 & 3.389 & 10.218 & 6.422 & 3.905 & 6.876 & 3.756 \\
\hline $11 / 23 / 06$ & $4: 00$ & 12.074 & 12.36 & 9.13 & 8.075 & 5.374 & 5.083 & 3.503 & 3.382 & 10.216 & 6.418 & 3.9 & 6.869 & 3.751 \\
\hline $11 / 23 / 06$ & $8: 00$ & 12.28 & 12.356 & 9.128 & 8.075 & 5.374 & 5.083 & 3.763 & 3.382 & 10.216 & 6.42 & 3.903 & 6.869 & 3.751 \\
\hline $11 / 23 / 06$ & $12: 00$ & 12.903 & 12.335 & 9.116 & 8.056 & 5.362 & 5.074 & 4.663 & 3.361 & 10.209 & 6.415 & 3.872 & 6.857 & 3.738 \\
\hline $11 / 23 / 06$ & $16: 00$ & 12.422 & 12.309 & 9.095 & 8.035 & 5.35 & 5.06 & 4.64 & 3.342 & 10.199 & 6.406 & 3.859 & 6.841 & 3.731 \\
\hline $11 / 23 / 06$ & $20: 00$ & 12.224 & 12.302 & 9.088 & 8.031 & 5.348 & 5.048 & 4.185 & 3.338 & 10.192 & 6.406 & 3.858 & 6.834 & 3.728 \\
\hline $11 / 24 / 06$ & $0: 00$ & 12.193 & 12.311 & 9.09 & 8.04 & 5.35 & 5.048 & 3.983 & 3.345 & 10.192 & 6.406 & 3.866 & 6.834 & 3.735 \\
\hline $11 / 24 / 06$ & $4: 00$ & 12.163 & 12.344 & 9.109 & 8.068 & 5.367 & 5.06 & 3.794 & 3.37 & 10.199 & 6.415 & 3.891 & 6.853 & 3.751 \\
\hline $11 / 24 / 06$ & $8: 00$ & 12.266 & 12.386 & 9.137 & 8.103 & 5.385 & 5.079 & 3.738 & 3.403 & 10.213 & 6.427 & 3.912 & 6.878 & 3.768 \\
\hline $11 / 24 / 06$ & $12: 00$ & 12.504 & 12.381 & 9.144 & 8.094 & 5.381 & 5.088 & 4.25 & 3.394 & 10.218 & 6.425 & 3.903 & 6.881 & 3.759 \\
\hline $11 / 24 / 06$ & $16: 00$ & 12.298 & 12.376 & 9.137 & 8.092 & 5.381 & 5.086 & 4.115 & 3.392 & 10.218 & 6.425 & 3.907 & 6.883 & 3.759 \\
\hline $11 / 24 / 06$ & $20: 00$ & 12.056 & 12.379 & 9.142 & 8.099 & 5.383 & 5.088 & 3.393 & 3.394 & 10.22 & 6.425 & 3.919 & 6.885 & 3.761 \\
\hline $11 / 25 / 06$ & $0: 00$ & 12.137 & 12.369 & 9.137 & 8.087 & 5.378 & 5.086 & 3.367 & 3.385 & 10.218 & 6.425 & 3.919 & 6.881 & 3.759 \\
\hline $11 / 25 / 06$ & $4: 00$ & 12.156 & 12.367 & 9.137 & 8.085 & 5.378 & 5.086 & 3.528 & 3.385 & 10.216 & 6.422 & 3.922 & 6.881 & 3.756 \\
\hline $11 / 25 / 06$ & $8: 00$ & 12.228 & 12.376 & 9.142 & 8.094 & 5.386 & 5.086 & 3.528 & 3.394 & 10.218 & 6.425 & 3.929 & 6.888 & 3.763 \\
\hline
\end{tabular}


Water Level (ft below top of casing) at Indicated Well

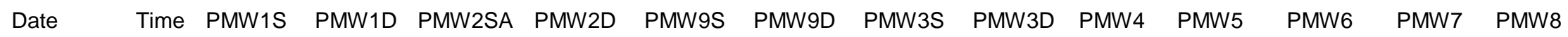

\begin{tabular}{|c|c|c|c|c|c|c|c|c|c|c|c|c|c|c|}
\hline $11 / 25 / 06$ & $12: 00$ & 12.847 & 12.36 & 9.132 & 8.078 & 5.376 & 5.081 & 4.167 & 3.375 & 10.216 & 6.422 & 3.903 & 6.878 & 3.754 \\
\hline $11 / 25 / 06$ & $16: 00$ & 12.359 & 12.353 & 9.123 & 8.071 & 5.376 & 5.074 & 4.069 & 3.373 & 10.211 & 6.42 & 3.891 & 6.867 & 3.747 \\
\hline $11 / 25 / 06$ & $20: 00$ & 12.301 & 12.356 & 9.128 & 8.078 & 5.376 & 5.069 & 3.838 & 3.375 & 10.211 & 6.418 & 3.884 & 6.864 & 3.747 \\
\hline $11 / 26 / 06$ & $0: 00$ & 12.198 & 12.351 & 9.125 & 8.073 & 5.371 & 5.069 & 3.54 & 3.37 & 10.209 & 6.415 & 3.884 & 6.862 & 3.742 \\
\hline $11 / 26 / 06$ & $4: 00$ & 12.245 & 12.349 & 9.121 & 8.071 & 5.369 & 5.065 & 3.61 & 3.368 & 10.206 & 6.413 & 3.882 & 6.857 & 3.742 \\
\hline 11/26/06 & 8:00 & 12.301 & 12.372 & 9.135 & 8.089 & 5.379 & 5.069 & 3.563 & 3.387 & 10.211 & 6.42 & 3.898 & 6.871 & 3.754 \\
\hline $11 / 26 / 06$ & $12: 00$ & 12.49 & 12.358 & 9.13 & 8.078 & 5.372 & 5.067 & 3.983 & 3.373 & 10.211 & 6.413 & 3.877 & 6.862 & 3.74 \\
\hline $11 / 26 / 06$ & $16: 00$ & 12.275 & 12.379 & 9.139 & 8.096 & 5.381 & 5.069 & 3.717 & 3.387 & 10.213 & 6.423 & 3.889 & 6.871 & 3.747 \\
\hline $11 / 26 / 06$ & 20:00 & 12.2 & 12.404 & 9.158 & 8.117 & 5.395 & 5.081 & 3.409 & 3.41 & 10.223 & 6.427 & 3.912 & 6.89 & 3.759 \\
\hline $11 / 27 / 06$ & $0: 00$ & 12.217 & 12.416 & 9.17 & 8.129 & 5.402 & 5.09 & 3.302 & 3.422 & 10.23 & 6.43 & 3.926 & 6.904 & 3.766 \\
\hline $11 / 27 / 06$ & $4: 00$ & 12.254 & 12.418 & 9.175 & 8.127 & 5.402 & 5.097 & 3.244 & 3.42 & 10.234 & 6.434 & 3.931 & 6.909 & 3.763 \\
\hline $11 / 27 / 06$ & $8: 00$ & 12.352 & 12.418 & 9.177 & 8.127 & 5.404 & 5.102 & 3.288 & 3.42 & 10.234 & 6.437 & 3.933 & 6.911 & 3.761 \\
\hline $11 / 27 / 06$ & $12: 00$ & 12.41 & 12.381 & 9.153 & 8.094 & 5.383 & 5.088 & 3.449 & 3.389 & 10.227 & 6.423 & 3.903 & 6.89 & 3.742 \\
\hline $11 / 27 / 06$ & $16: 00$ & 12.329 & 12.351 & 9.132 & 8.073 & 5.374 & 5.069 & 3.512 & 3.368 & 10.216 & 6.413 & 3.887 & 6.871 & 3.735 \\
\hline $11 / 27 / 06$ & 20:00 & 12.352 & 12.33 & 9.116 & 8.052 & 5.36 & 5.053 & 3.621 & 3.349 & 10.206 & 6.409 & 3.868 & 6.855 & 3.726 \\
\hline $11 / 28 / 06$ & $0: 00$ & 12.378 & 12.335 & 9.09 & 8.024 & 5.339 & 5.032 & 3.771 & 3.324 & 10.192 & 6.397 & 3.84 & 6.829 & 3.712 \\
\hline $11 / 28 / 06$ & $4: 00$ & 12.824 & 12.323 & 9.067 & 8.052 & 5.32 & 5.004 & 4.272 & 3.293 & 10.178 & 6.388 & 3.805 & 6.801 & 3.698 \\
\hline $11 / 28 / 06$ & $8: 00$ & 12.768 & 12.256 & 9.055 & 7.993 & 5.313 & 4.992 & 4.484 & 3.286 & 10.169 & 6.381 & 3.728 & 6.774 & 3.672 \\
\hline $11 / 28 / 06$ & $12: 00$ & 12.805 & 12.258 & 9.055 & 7.995 & 5.304 & 4.985 & 4.786 & 3.286 & 10.164 & 6.378 & 3.73 & 6.769 & 3.665 \\
\hline $11 / 28 / 06$ & $16: 00$ & 11.843 & 12.321 & 9.086 & 8.049 & 5.341 & 5.002 & 4.045 & 3.34 & 10.176 & 6.395 & 3.796 & 6.804 & 3.703 \\
\hline $11 / 28 / 06$ & $20: 00$ & 11.729 & 12.376 & 9.125 & 8.094 & 5.367 & 5.034 & 3.633 & 3.385 & 10.192 & 6.406 & 3.847 & 6.843 & 3.728 \\
\hline $11 / 29 / 06$ & $0: 00$ & 11.652 & 12.404 & 9.149 & 8.115 & 5.379 & 5.055 & 3.489 & 3.403 & 10.206 & 6.416 & 3.882 & 6.871 & 3.74 \\
\hline $11 / 29 / 06$ & $4: 00$ & 11.825 & 12.432 & 9.17 & 8.131 & 5.388 & 5.081 & 3.344 & 3.42 & 10.218 & 6.423 & 3.915 & 6.899 & 3.754 \\
\hline $11 / 29 / 06$ & 8:00 & 12.049 & 12.462 & 9.196 & 8.169 & 5.416 & 5.102 & 3.265 & 3.45 & 10.232 & 6.439 & 3.966 & 6.932 & 3.78 \\
\hline $11 / 29 / 06$ & $12: 00$ & 12.193 & 12.467 & 9.207 & 8.162 & 5.416 & 5.114 & 3.305 & 3.448 & 10.244 & 6.444 & 3.975 & 6.944 & 3.775 \\
\hline $11 / 29 / 06$ & $16: 00$ & 12.168 & 12.485 & 9.221 & 8.183 & 5.435 & 5.13 & 3.151 & 3.469 & 10.253 & 6.458 & 4.001 & 6.967 & 3.791 \\
\hline $11 / 29 / 06$ & $20: 00$ & 12.196 & 12.502 & 9.236 & 8.199 & 5.449 & 5.146 & 3.004 & 3.487 & 10.262 & 6.465 & 4.031 & 6.985 & 3.801 \\
\hline $11 / 30 / 06$ & $0: 00$ & 12.189 & 12.502 & 9.243 & 8.199 & 5.451 & 5.156 & 2.918 & 3.485 & 10.269 & 6.465 & 4.062 & 6.999 & 3.803 \\
\hline $11 / 30 / 06$ & $4: 00$ & 12.095 & 12.509 & 9.247 & 8.204 & 5.458 & 5.163 & 2.685 & 3.492 & 10.274 & 6.472 & 4.104 & 7.016 & 3.815 \\
\hline $11 / 30 / 06$ & 8:00 & 12.27 & 12.504 & 9.252 & 8.206 & 5.461 & 5.17 & 2.724 & 3.494 & 10.279 & 6.469 & 4.136 & 7.025 & 3.822 \\
\hline $11 / 30 / 06$ & $12: 00$ & 12.747 & 12.472 & 9.236 & 8.176 & 5.449 & 5.163 & 3.174 & 3.469 & 10.274 & 6.462 & 4.125 & 7.018 & 3.808 \\
\hline $11 / 30 / 06$ & $16: 00$ & 12.569 & 12.418 & 9.198 & 8.134 & 5.433 & 5.139 & 3.179 & 3.431 & 10.253 & 6.451 & 4.069 & 6.988 & 3.78 \\
\hline $11 / 30 / 06$ & $20: 00$ & 12.291 & 12.397 & 9.179 & 8.12 & 5.426 & 5.118 & 2.939 & 3.417 & 10.241 & 6.444 & 4.069 & 6.976 & 3.777 \\
\hline $12 / 1 / 06$ & $0: 00$ & 12.2 & 12.379 & 9.168 & 8.106 & 5.414 & 5.102 & 2.86 & 3.406 & 10.234 & 6.434 & 4.118 & 6.978 & 3.791 \\
\hline
\end{tabular}


TABLE S1.2 (Cont.)

Water Level (ft below top of casing) at Indicated Well

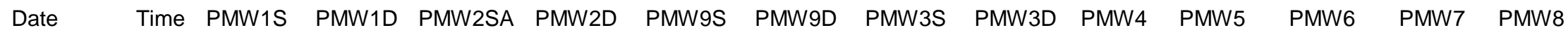

\begin{tabular}{|c|c|c|c|c|c|c|c|c|c|c|c|c|c|c|}
\hline $12 / 1 / 06$ & $4: 00$ & 12.177 & 12.379 & 9.161 & 8.108 & 5.419 & 5.097 & 2.834 & 3.41 & 10.23 & 6.439 & 4.157 & 6.99 & 3.812 \\
\hline $12 / 1 / 06$ & 8:00 & 12.562 & 12.395 & 9.172 & 8.124 & 5.43 & 5.1 & 3.079 & 3.424 & 10.234 & 6.444 & 4.19 & 7.006 & 3.831 \\
\hline $12 / 1 / 06$ & $12: 00$ & 13.162 & 12.404 & 9.182 & 8.134 & 5.433 & 5.107 & 3.503 & 3.429 & 10.237 & 6.446 & 4.174 & 7.004 & 3.826 \\
\hline $12 / 1 / 06$ & $16: 00$ & 12.644 & 12.423 & 9.191 & 8.148 & 5.442 & 5.114 & 3.552 & 3.441 & 10.241 & 6.446 & 4.125 & 6.999 & 3.815 \\
\hline $12 / 1 / 06$ & $20: 00$ & 12.24 & 12.451 & 9.21 & 8.169 & 5.456 & 5.128 & 3.251 & 3.459 & 10.251 & 6.453 & 4.087 & 6.999 & 3.808 \\
\hline $12 / 2 / 06$ & 0:00 & 12.27 & 12.465 & 9.224 & 8.181 & 5.463 & 5.139 & 3.239 & 3.471 & 10.255 & 6.458 & 4.099 & 7.006 & 3.81 \\
\hline $12 / 2 / 06$ & $4: 00$ & 12.268 & 12.499 & 9.247 & 8.211 & 5.482 & 5.158 & 3.156 & 3.501 & 10.269 & 6.474 & 4.153 & 7.034 & 3.836 \\
\hline $12 / 2 / 06$ & 8:00 & 12.294 & 12.543 & 9.275 & 8.251 & 5.508 & 5.189 & 3.102 & 3.539 & 10.288 & 6.49 & 4.202 & 7.062 & 3.861 \\
\hline $12 / 2 / 06$ & $12: 00$ & 12.737 & 12.546 & 9.287 & 8.246 & 5.501 & 5.203 & 3.326 & 3.532 & 10.292 & 6.49 & 4.197 & 7.062 & 3.852 \\
\hline $12 / 2 / 06$ & $16: 00$ & 12.268 & 12.55 & 9.289 & 8.253 & 5.512 & 5.207 & 3.186 & 3.539 & 10.297 & 6.495 & 4.167 & 7.065 & 3.84 \\
\hline $12 / 2 / 06$ & $20: 00$ & 11.878 & 12.567 & 9.303 & 8.267 & 5.522 & 5.221 & 2.771 & 3.555 & 10.306 & 6.502 & 4.181 & 7.076 & 3.845 \\
\hline $12 / 3 / 06$ & $0: 00$ & 12.133 & 12.571 & 9.311 & 8.274 & 5.526 & 5.231 & 2.804 & 3.56 & 10.313 & 6.507 & 4.232 & 7.093 & 3.861 \\
\hline $12 / 3 / 06$ & $4: 00$ & 12.172 & 12.569 & 9.315 & 8.272 & 5.531 & 5.235 & 2.752 & 3.562 & 10.316 & 6.514 & 4.267 & 7.102 & 3.871 \\
\hline $12 / 3 / 06$ & $8: 00$ & 12.45 & 12.581 & 9.325 & 8.286 & 5.54 & 5.245 & 2.864 & 3.574 & 10.325 & 6.518 & 4.302 & 7.12 & 3.885 \\
\hline $12 / 3 / 06$ & $12: 00$ & 13.064 & 12.555 & 9.318 & 8.265 & 5.529 & 5.24 & 3.431 & 3.555 & 10.325 & 6.516 & 4.302 & 7.116 & 3.873 \\
\hline $12 / 3 / 06$ & $16: 00$ & 12.572 & 12.516 & 9.289 & 8.232 & 5.517 & 5.221 & 3.431 & 3.525 & 10.311 & 6.504 & 4.246 & 7.09 & 3.848 \\
\hline $12 / 3 / 06$ & $20: 00$ & 12.382 & 12.472 & 9.264 & 8.195 & 5.498 & 5.2 & 3.153 & 3.49 & 10.297 & 6.49 & 4.227 & 7.072 & 3.831 \\
\hline $12 / 4 / 06$ & 0:00 & 12.84 & 12.425 & 9.229 & 8.157 & 5.477 & 5.172 & 3.643 & 3.452 & 10.279 & 6.479 & 4.232 & 7.055 & 3.827 \\
\hline $12 / 4 / 06$ & $4: 00$ & 12.824 & 12.397 & 9.205 & 8.136 & 5.463 & 5.149 & 3.822 & 3.436 & 10.265 & 6.469 & 4.204 & 7.034 & 3.82 \\
\hline $12 / 4 / 06$ & $8: 00$ & 12.735 & 12.407 & 9.207 & 8.152 & 5.471 & 5.139 & 3.836 & 3.445 & 10.26 & 6.469 & 4.171 & 7.027 & 3.822 \\
\hline $12 / 4 / 06$ & $12: 00$ & 12.971 & 12.423 & 9.212 & 8.159 & 5.468 & 5.142 & 4.183 & 3.452 & 10.262 & 6.465 & 4.132 & 7.023 & 3.813 \\
\hline $12 / 4 / 06$ & $16: 00$ & 12.427 & 12.42 & 9.21 & 8.157 & 5.466 & 5.135 & 3.987 & 3.448 & 10.258 & 6.462 & 4.085 & 7.009 & 3.796 \\
\hline $12 / 4 / 06$ & $20: 00$ & 11.96 & 12.43 & 9.214 & 8.164 & 5.466 & 5.139 & 3.48 & 3.45 & 10.255 & 6.46 & 4.045 & 7.002 & 3.785 \\
\hline $12 / 5 / 06$ & $0: 00$ & 12.45 & 12.407 & 9.2 & 8.141 & 5.45 & 5.132 & 3.829 & 3.431 & 10.253 & 6.451 & 4.001 & 6.981 & 3.761 \\
\hline $12 / 5 / 06$ & $4: 00$ & 12.392 & 12.379 & 9.182 & 8.113 & 5.431 & 5.114 & 3.757 & 3.403 & 10.241 & 6.441 & 3.971 & 6.96 & 3.745 \\
\hline $12 / 5 / 06$ & $8: 00$ & 12.607 & 12.351 & 9.161 & 8.092 & 5.419 & 5.093 & 3.964 & 3.382 & 10.227 & 6.434 & 3.964 & 6.944 & 3.74 \\
\hline $12 / 5 / 06$ & $12: 00$ & 13.407 & 12.318 & 9.14 & 8.061 & 5.396 & 5.072 & 4.754 & 3.352 & 10.216 & 6.423 & 3.938 & 6.92 & 3.726 \\
\hline $12 / 5 / 06$ & $16: 00$ & 12.574 & 12.311 & 9.125 & 8.061 & 5.396 & 5.055 & 4.682 & 3.349 & 10.206 & 6.416 & 3.906 & 6.899 & 3.717 \\
\hline $12 / 5 / 06$ & $20: 00$ & 12.088 & 12.351 & 9.144 & 8.094 & 5.41 & 5.065 & 4.043 & 3.378 & 10.211 & 6.42 & 3.896 & 6.902 & 3.719 \\
\hline $12 / 6 / 06$ & $0: 00$ & 11.972 & 12.383 & 9.165 & 8.117 & 5.422 & 5.081 & 3.803 & 3.401 & 10.218 & 6.425 & 3.901 & 6.913 & 3.726 \\
\hline $12 / 6 / 06$ & $4: 00$ & 11.99 & 12.404 & 9.177 & 8.134 & 5.426 & 5.093 & 3.817 & 3.415 & 10.225 & 6.432 & 3.903 & 6.923 & 3.731 \\
\hline $12 / 6 / 06$ & $8: 00$ & 12.287 & 12.427 & 9.196 & 8.152 & 5.438 & 5.107 & 3.915 & 3.431 & 10.234 & 6.439 & 3.915 & 6.937 & 3.74 \\
\hline $12 / 6 / 06$ & $12: 00$ & 12.74 & 12.425 & 9.203 & 8.148 & 5.438 & 5.111 & 4.37 & 3.427 & 10.239 & 6.439 & 3.91 & 6.939 & 3.736 \\
\hline $12 / 6 / 06$ & $16: 00$ & 12.401 & 12.441 & 9.203 & 8.162 & 5.447 & 5.114 & 4.209 & 3.438 & 10.241 & 6.444 & 3.901 & 6.937 & 3.726 \\
\hline
\end{tabular}


TABLE S1.2 (Cont.)

Water Level (ft below top of casing) at Indicated Well

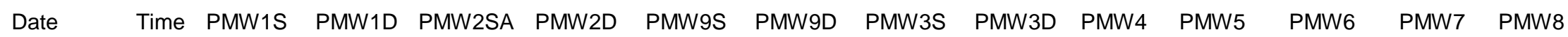

\begin{tabular}{|c|c|c|c|c|c|c|c|c|c|c|c|c|c|c|}
\hline $12 / 6 / 06$ & $20: 00$ & 12.466 & 12.518 & 9.254 & 8.234 & 5.487 & 5.146 & 3.647 & 3.504 & 10.265 & 6.472 & 3.95 & 6.983 & 3.757 \\
\hline $12 / 7 / 06$ & 0:00 & 12.527 & 12.578 & 9.301 & 8.284 & 5.515 & 5.189 & 3.104 & 3.553 & 10.292 & 6.495 & 3.994 & 7.03 & 3.785 \\
\hline $12 / 7 / 06$ & $4: 00$ & 12.562 & 12.608 & 9.329 & 8.307 & 5.534 & 5.224 & 2.797 & 3.576 & 10.316 & 6.511 & 4.057 & 7.062 & 3.808 \\
\hline $12 / 7 / 06$ & $8: 00$ & 12.583 & 12.627 & 9.348 & 8.326 & 5.55 & 5.245 & 2.83 & 3.597 & 10.332 & 6.53 & 4.155 & 7.102 & 3.841 \\
\hline $12 / 7 / 06$ & $12: 00$ & 12.576 & 12.608 & 9.353 & 8.309 & 5.546 & 5.252 & 3.351 & 3.583 & 10.337 & 6.53 & 4.209 & 7.116 & 3.85 \\
\hline $12 / 7 / 06$ & $16: 00$ & 12.529 & 12.564 & 9.329 & 8.272 & 5.534 & 5.24 & 3.324 & 3.553 & 10.33 & 6.521 & 4.206 & 7.102 & 3.838 \\
\hline $12 / 7 / 06$ & $20: 00$ & 12.499 & 12.525 & 9.304 & 8.244 & 5.525 & 5.221 & 3.023 & 3.527 & 10.32 & 6.516 & 4.225 & 7.097 & 3.838 \\
\hline $12 / 8 / 06$ & $0: 00$ & 12.471 & 12.49 & 9.282 & 8.218 & 5.511 & 5.2 & 3.191 & 3.501 & 10.309 & 6.507 & 4.253 & 7.093 & 3.843 \\
\hline $12 / 8 / 06$ & 4:00 & 12.436 & 12.453 & 9.257 & 8.185 & 5.494 & 5.177 & 3.445 & 3.473 & 10.297 & 6.497 & 4.265 & 7.081 & 3.838 \\
\hline $12 / 8 / 06$ & $8: 00$ & 12.42 & 12.432 & 9.238 & 8.171 & 5.49 & 5.16 & 3.626 & 3.464 & 10.286 & 6.49 & 4.274 & 7.074 & 3.845 \\
\hline $12 / 8 / 06$ & $12: 00$ & 12.394 & 12.409 & 9.219 & 8.148 & 5.469 & 5.137 & 4.016 & 3.436 & 10.274 & 6.479 & 4.227 & 7.051 & 3.831 \\
\hline $12 / 8 / 06$ & $16: 00$ & 12.368 & 12.386 & 9.2 & 8.134 & 5.46 & 5.118 & 4.074 & 3.42 & 10.262 & 6.469 & 4.153 & 7.025 & 3.81 \\
\hline $12 / 8 / 06$ & $20: 00$ & 12.359 & 12.379 & 9.191 & 8.124 & 5.45 & 5.107 & 3.783 & 3.41 & 10.251 & 6.46 & 4.088 & 7.006 & 3.794 \\
\hline $12 / 9 / 06$ & $0: 00$ & 12.347 & 12.362 & 9.177 & 8.11 & 5.438 & 5.095 & 3.901 & 3.396 & 10.244 & 6.451 & 4.032 & 6.983 & 3.773 \\
\hline $12 / 9 / 06$ & $4: 00$ & 12.336 & 12.353 & 9.168 & 8.101 & 5.429 & 5.083 & 3.885 & 3.385 & 10.234 & 6.448 & 4.001 & 6.964 & 3.761 \\
\hline $12 / 9 / 06$ & $8: 00$ & 12.336 & 12.356 & 9.163 & 8.103 & 5.429 & 5.079 & 3.96 & 3.387 & 10.232 & 6.444 & 3.997 & 6.955 & 3.764 \\
\hline $12 / 9 / 06$ & $12: 00$ & 12.329 & 12.346 & 9.154 & 8.089 & 5.415 & 5.067 & 4.493 & 3.373 & 10.227 & 6.437 & 3.973 & 6.944 & 3.752 \\
\hline $12 / 9 / 06$ & $16: 00$ & 12.315 & 12.335 & 9.144 & 8.08 & 5.408 & 5.058 & 4.654 & 3.363 & 10.218 & 6.43 & 3.936 & 6.925 & 3.738 \\
\hline $12 / 9 / 06$ & $20: 00$ & 12.324 & 12.353 & 9.149 & 8.094 & 5.413 & 5.06 & 4.223 & 3.375 & 10.216 & 6.432 & 3.92 & 6.92 & 3.736 \\
\hline $12 / 10 / 06$ & $0: 00$ & 12.324 & 12.349 & 9.147 & 8.087 & 5.404 & 5.055 & 4.132 & 3.368 & 10.216 & 6.425 & 3.892 & 6.904 & 3.717 \\
\hline $12 / 10 / 06$ & 4:00 & 12.319 & 12.344 & 9.142 & 8.085 & 5.401 & 5.053 & 3.999 & 3.361 & 10.213 & 6.423 & 3.875 & 6.897 & 3.71 \\
\hline $12 / 10 / 06$ & $8: 00$ & 12.329 & 12.358 & 9.144 & 8.092 & 5.404 & 5.053 & 3.981 & 3.368 & 10.211 & 6.423 & 3.873 & 6.897 & 3.71 \\
\hline $12 / 10 / 06$ & $12: 00$ & 12.326 & 12.351 & 9.142 & 8.085 & 5.397 & 5.051 & 4.468 & 3.359 & 10.211 & 6.42 & 3.852 & 6.888 & 3.698 \\
\hline $12 / 10 / 06$ & $16: 00$ & 12.326 & 12.353 & 9.142 & 8.092 & 5.397 & 5.046 & 4.412 & 3.363 & 10.209 & 6.418 & 3.826 & 6.876 & 3.677 \\
\hline $12 / 10 / 06$ & $20: 00$ & 12.343 & 12.372 & 9.156 & 8.106 & 5.401 & 5.053 & 3.887 & 3.375 & 10.211 & 6.42 & 3.829 & 6.883 & 3.673 \\
\hline $12 / 11 / 06$ & $0: 00$ & 12.35 & 12.376 & 9.158 & 8.108 & 5.401 & 5.055 & 3.757 & 3.378 & 10.211 & 6.42 & 3.833 & 6.885 & 3.675 \\
\hline $12 / 11 / 06$ & $4: 00$ & 12.35 & 12.379 & 9.161 & 8.103 & 5.399 & 5.058 & 3.752 & 3.378 & 10.213 & 6.423 & 3.84 & 6.89 & 3.684 \\
\hline $12 / 11 / 06$ & $8: 00$ & 12.354 & 12.381 & 9.163 & 8.11 & 5.401 & 5.06 & 3.962 & 3.38 & 10.216 & 6.425 & 3.831 & 6.888 & 3.684 \\
\hline $12 / 11 / 06$ & $12: 00$ & 12.345 & 12.369 & 9.156 & 8.096 & 5.392 & 5.055 & 4.232 & 3.366 & 10.213 & 6.418 & 3.792 & 6.874 & 3.661 \\
\hline $12 / 11 / 06$ & $16: 00$ & 12.338 & 12.346 & 9.151 & 8.096 & 5.392 & 5.048 & 4.162 & 3.363 & 10.209 & 6.418 & 3.75 & 6.839 & 3.645 \\
\hline $12 / 11 / 06$ & $20: 00$ & 12.34 & 12.367 & 9.149 & 8.092 & 5.383 & 5.048 & 4.109 & 3.356 & 10.209 & 6.413 & 3.724 & 6.825 & 3.633 \\
\hline $12 / 12 / 06$ & $0: 00$ & 12.352 & 12.381 & 9.156 & 8.103 & 5.387 & 5.048 & 3.766 & 3.366 & 10.211 & 6.418 & 3.694 & 6.848 & 3.619 \\
\hline $12 / 12 / 06$ & $4: 00$ & 12.366 & 12.395 & 9.165 & 8.117 & 5.39 & 5.053 & 3.685 & 3.378 & 10.216 & 6.418 & 3.719 & 6.857 & 3.629 \\
\hline $12 / 12 / 06$ & $8: 00$ & 12.382 & 12.411 & 9.177 & 8.134 & 5.399 & 5.062 & 3.58 & 3.394 & 10.22 & 6.425 & 3.759 & 6.876 & 3.647 \\
\hline
\end{tabular}


Water Level (ft below top of casing) at Indicated Well

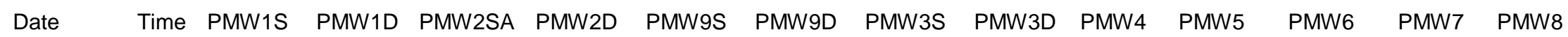

\begin{tabular}{|c|c|c|c|c|c|c|c|c|c|c|c|c|c|c|}
\hline $12 / 12 / 06$ & $12: 00$ & 12.38 & 12.407 & 9.179 & 8.124 & 5.392 & 5.065 & 3.624 & 3.389 & 10.223 & 6.423 & 3.768 & 6.878 & 3.652 \\
\hline $12 / 12 / 06$ & $16: 00$ & 12.378 & 12.407 & 9.177 & 8.124 & 5.395 & 5.065 & 3.652 & 3.389 & 10.223 & 6.427 & 3.775 & 6.881 & 3.661 \\
\hline $12 / 12 / 06$ & $20: 00$ & 12.378 & 12.409 & 9.177 & 8.124 & 5.397 & 5.065 & 3.468 & 3.389 & 10.225 & 6.43 & 3.817 & 6.892 & 3.673 \\
\hline $12 / 13 / 06$ & $0: 00$ & 12.378 & 12.404 & 9.179 & 8.124 & 5.399 & 5.065 & 3.578 & 3.389 & 10.225 & 6.434 & 3.859 & 6.906 & 3.687 \\
\hline $12 / 13 / 06$ & $4: 00$ & 12.389 & 12.418 & 9.186 & 8.136 & 5.409 & 5.072 & 3.512 & 3.401 & 10.227 & 6.437 & 3.889 & 6.923 & 3.703 \\
\hline $12 / 13 / 06$ & $8: 00$ & 12.401 & 12.434 & 9.198 & 8.15 & 5.418 & 5.081 & 3.501 & 3.415 & 10.234 & 6.444 & 3.924 & 6.941 & 3.722 \\
\hline $12 / 13 / 06$ & $12: 00$ & 12.389 & 12.414 & 9.194 & 8.129 & 5.409 & 5.079 & 4.221 & 3.394 & 10.237 & 6.437 & 3.876 & 6.927 & 3.71 \\
\hline $12 / 13 / 06$ & $16: 00$ & 12.357 & 12.376 & 9.168 & 8.103 & 5.392 & 5.062 & 4.253 & 3.368 & 10.225 & 6.43 & 3.792 & 6.89 & 3.685 \\
\hline $12 / 13 / 06$ & $20: 00$ & 12.343 & 12.365 & 9.154 & 8.092 & 5.385 & 5.048 & 3.846 & 3.356 & 10.218 & 6.425 & 3.803 & 6.876 & 3.68 \\
\hline $12 / 14 / 06$ & $0: 00$ & 12.319 & 12.344 & 9.137 & 8.068 & 5.371 & 5.034 & 4.246 & 3.338 & 10.211 & 6.413 & 3.796 & 6.862 & 3.673 \\
\hline $12 / 14 / 06$ & $4: 00$ & 12.31 & 12.332 & 9.128 & 8.066 & 5.371 & 5.025 & 4.179 & 3.333 & 10.202 & 6.413 & 3.794 & 6.853 & 3.678 \\
\hline $12 / 14 / 06$ & $8: 00$ & 12.336 & 12.367 & 9.144 & 8.096 & 5.388 & 5.034 & 3.964 & 3.361 & 10.206 & 6.423 & 3.831 & 6.876 & 3.696 \\
\hline $12 / 14 / 06$ & $12: 00$ & 12.347 & 12.376 & 9.154 & 8.098 & 5.385 & 5.039 & 4.442 & 3.361 & 10.209 & 6.42 & 3.806 & 6.871 & 3.694 \\
\hline $12 / 14 / 06$ & $16: 00$ & 12.345 & 12.374 & 9.154 & 8.098 & 5.385 & 5.041 & 4.305 & 3.359 & 10.209 & 6.42 & 3.794 & 6.869 & 3.694 \\
\hline $12 / 14 / 06$ & $20: 00$ & 12.378 & 12.411 & 9.177 & 8.129 & 5.404 & 5.055 & 3.99 & 3.389 & 10.22 & 6.43 & 3.843 & 6.895 & 3.71 \\
\hline $12 / 15 / 06$ & 0:00 & 12.396 & 12.43 & 9.191 & 8.145 & 5.416 & 5.069 & 3.487 & 3.406 & 10.227 & 6.437 & 3.88 & 6.916 & 3.72 \\
\hline $12 / 15 / 06$ & $4: 00$ & 12.403 & 12.434 & 9.198 & 8.148 & 5.416 & 5.074 & 3.345 & 3.408 & 10.232 & 6.437 & 3.911 & 6.93 & 3.724 \\
\hline $12 / 15 / 06$ & $8: 00$ & 12.406 & 12.434 & 9.203 & 8.148 & 5.423 & 5.081 & 3.405 & 3.41 & 10.234 & 6.442 & 3.95 & 6.941 & 3.734 \\
\hline $12 / 15 / 06$ & $12: 00$ & 12.389 & 12.414 & 9.191 & 8.127 & 5.411 & 5.076 & 4.116 & 3.389 & 10.234 & 6.432 & 3.892 & 6.925 & 3.72 \\
\hline $12 / 15 / 06$ & $16: 00$ & 12.357 & 12.379 & 9.168 & 8.101 & 5.397 & 5.06 & 4.034 & 3.363 & 10.218 & 6.423 & 3.85 & 6.897 & 3.699 \\
\hline $12 / 15 / 06$ & $20: 00$ & 12.336 & 12.36 & 9.151 & 8.082 & 5.385 & 5.046 & 3.974 & 3.349 & 10.211 & 6.416 & 3.855 & 6.885 & 3.692 \\
\hline $12 / 16 / 06$ & $0: 00$ & 12.319 & 12.342 & 9.14 & 8.07 & 5.374 & 5.032 & 3.953 & 3.335 & 10.199 & 6.411 & 3.852 & 6.874 & 3.692 \\
\hline $12 / 16 / 06$ & $4: 00$ & 12.308 & 12.332 & 9.13 & 8.061 & 5.369 & 5.02 & 3.874 & 3.326 & 10.192 & 6.407 & 3.855 & 6.864 & 3.692 \\
\hline $12 / 16 / 06$ & $8: 00$ & 12.329 & 12.36 & 9.14 & 8.089 & 5.386 & 5.025 & 3.771 & 3.352 & 10.195 & 6.416 & 3.883 & 6.881 & 3.708 \\
\hline $12 / 16 / 06$ & $12: 00$ & 12.352 & 12.381 & 9.156 & 8.101 & 5.39 & 5.037 & 4.067 & 3.366 & 10.199 & 6.416 & 3.878 & 6.89 & 3.713 \\
\hline $12 / 16 / 06$ & $16: 00$ & 12.392 & 12.43 & 9.189 & 8.148 & 5.418 & 5.058 & 3.778 & 3.41 & 10.213 & 6.432 & 3.913 & 6.92 & 3.736 \\
\hline $12 / 16 / 06$ & $20: 00$ & 12.443 & 12.483 & 9.226 & 8.19 & 5.444 & 5.09 & 3.317 & 3.45 & 10.232 & 6.451 & 3.955 & 6.955 & 3.755 \\
\hline $12 / 17 / 06$ & $0: 00$ & 12.473 & 12.511 & 9.25 & 8.211 & 5.456 & 5.114 & 3.191 & 3.469 & 10.248 & 6.46 & 3.978 & 6.979 & 3.762 \\
\hline $12 / 17 / 06$ & $4: 00$ & 12.492 & 12.53 & 9.268 & 8.225 & 5.47 & 5.132 & 3.035 & 3.485 & 10.26 & 6.467 & 4.004 & 6.997 & 3.769 \\
\hline $12 / 17 / 06$ & $8: 00$ & 12.515 & 12.555 & 9.29 & 8.253 & 5.488 & 5.153 & 2.977 & 3.506 & 10.274 & 6.484 & 4.046 & 7.023 & 3.785 \\
\hline $12 / 17 / 06$ & $12: 00$ & 12.515 & 12.548 & 9.294 & 8.242 & 5.488 & 5.163 & 3.505 & 3.499 & 10.281 & 6.481 & 4.006 & 7.023 & 3.771 \\
\hline $12 / 17 / 06$ & $16: 00$ & 12.52 & 12.553 & 9.301 & 8.251 & 5.498 & 5.167 & 3.398 & 3.508 & 10.286 & 6.488 & 4.004 & 7.025 & 3.766 \\
\hline $12 / 17 / 06$ & $20: 00$ & 12.539 & 12.576 & 9.315 & 8.272 & 5.514 & 5.182 & 3.224 & 3.529 & 10.295 & 6.498 & 4.032 & 7.041 & 3.776 \\
\hline $12 / 18 / 06$ & $0: 00$ & 12.55 & 12.585 & 9.325 & 8.281 & 5.519 & 5.193 & 2.928 & 3.536 & 10.304 & 6.5 & 4.048 & 7.051 & 3.78 \\
\hline
\end{tabular}


Water Level (ft below top of casing) at Indicated Well

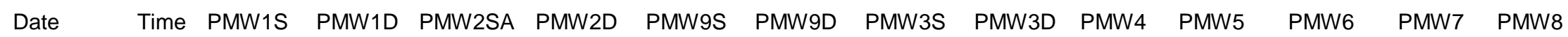

\begin{tabular}{|c|c|c|c|c|c|c|c|c|c|c|c|c|c|c|}
\hline $12 / 18 / 06$ & $4: 00$ & 12.562 & 12.599 & 9.336 & 8.295 & 5.531 & 5.203 & 2.702 & 3.55 & 10.309 & 6.509 & 4.102 & 7.069 & 3.797 \\
\hline $12 / 18 / 06$ & $8: 00$ & 12.578 & 12.618 & 9.353 & 8.316 & 5.545 & 5.219 & 2.739 & 3.569 & 10.32 & 6.521 & 4.155 & 7.09 & 3.822 \\
\hline $12 / 18 / 06$ & $12: 00$ & 12.585 & 12.618 & 9.362 & 8.314 & 5.549 & 5.228 & 3.585 & 3.565 & 10.327 & 6.523 & 4.13 & 7.093 & 3.818 \\
\hline $12 / 18 / 06$ & $16: 00$ & 12.569 & 12.602 & 9.353 & 8.3 & 5.549 & 5.226 & 3.464 & 3.553 & 10.325 & 6.521 & 4.044 & 7.079 & 3.787 \\
\hline $12 / 18 / 06$ & $20: 00$ & 12.574 & 12.604 & 9.358 & 8.305 & 5.552 & 5.228 & 2.867 & 3.56 & 10.33 & 6.523 & 4.081 & 7.088 & 3.79 \\
\hline $12 / 19 / 06$ & $0: 00$ & 12.571 & 12.602 & 9.358 & 8.305 & 5.554 & 5.231 & 2.865 & 3.562 & 10.33 & 6.526 & 4.158 & 7.1 & 3.808 \\
\hline $12 / 19 / 06$ & $4: 00$ & 12.555 & 12.585 & 9.35 & 8.293 & 5.554 & 5.228 & 2.907 & 3.553 & 10.33 & 6.526 & 4.2 & 7.107 & 3.822 \\
\hline $12 / 19 / 06$ & 8:00 & 12.548 & 12.576 & 9.346 & 8.288 & 5.556 & 5.224 & 3.175 & 3.548 & 10.327 & 6.528 & 4.23 & 7.114 & 3.834 \\
\hline $12 / 19 / 06$ & $12: 00$ & 12.532 & 12.553 & 9.334 & 8.267 & 5.545 & 5.214 & 3.829 & 3.53 & 10.323 & 6.519 & 4.2 & 7.1 & 3.822 \\
\hline $12 / 19 / 06$ & $16: 00$ & 12.501 & 12.527 & 9.311 & 8.246 & 5.538 & 5.2 & 3.839 & 3.508 & 10.309 & 6.507 & 4.118 & 7.072 & 3.794 \\
\hline $12 / 19 / 06$ & $20: 00$ & 12.485 & 12.506 & 9.297 & 8.227 & 5.524 & 5.184 & 3.781 & 3.49 & 10.299 & 6.498 & 4.06 & 7.053 & 3.766 \\
\hline $12 / 20 / 06$ & $0: 00$ & 12.457 & 12.476 & 9.276 & 8.202 & 5.505 & 5.163 & 3.857 & 3.462 & 10.286 & 6.484 & 4.004 & 7.027 & 3.741 \\
\hline $12 / 20 / 06$ & $4: 00$ & 12.434 & 12.453 & 9.254 & 8.183 & 5.491 & 5.142 & 3.862 & 3.441 & 10.274 & 6.477 & 3.962 & 7.009 & 3.724 \\
\hline $12 / 20 / 06$ & $8: 00$ & 12.415 & 12.455 & 9.236 & 8.166 & 5.477 & 5.125 & 3.762 & 3.424 & 10.262 & 6.465 & 3.906 & 7.009 & 3.703 \\
\hline $12 / 20 / 06$ & $12: 00$ & 12.396 & 12.488 & 9.219 & 8.138 & 5.449 & 5.097 & 3.899 & 3.385 & 10.248 & 6.444 & 3.456 & 6.992 & 3.466 \\
\hline $12 / 20 / 06$ & $16: 00$ & 12.38 & 12.455 & 9.198 & 8.131 & 5.4 & 5.079 & 3.913 & 3.366 & 10.23 & 6.421 & 3.377 & 6.892 & 3.314 \\
\hline $12 / 20 / 06$ & $20: 00$ & 12.378 & 12.434 & 9.194 & 8.122 & 5.344 & 5.062 & 3.904 & 3.357 & 10.22 & 6.402 & 3.405 & 6.862 & 3.293 \\
\hline $12 / 21 / 06$ & $0: 00$ & 12.375 & 12.46 & 9.184 & 8.12 & 5.311 & 5.048 & 3.874 & 3.345 & 10.209 & 6.395 & 3.363 & 6.806 & 3.232 \\
\hline $12 / 21 / 06$ & $4: 00$ & 12.378 & 12.453 & 9.177 & 8.115 & 5.26 & 5.034 & 3.883 & 3.34 & 10.197 & 6.381 & 3.435 & 6.785 & 3.232 \\
\hline $12 / 21 / 06$ & $8: 00$ & 12.392 & 12.46 & 9.18 & 8.129 & 5.25 & 5.027 & 3.862 & 3.357 & 10.195 & 6.386 & 3.554 & 6.804 & 3.321 \\
\hline $12 / 21 / 06$ & $12: 00$ & 12.382 & 12.407 & 9.175 & 8.113 & 5.251 & 5.02 & 4.067 & 3.345 & 10.192 & 6.381 & 3.617 & 6.823 & 3.377 \\
\hline $12 / 21 / 06$ & $16: 00$ & 12.375 & 12.402 & 9.168 & 8.11 & 5.264 & 5.013 & 3.995 & 3.35 & 10.19 & 6.388 & 3.671 & 6.834 & 3.431 \\
\hline $12 / 21 / 06$ & $20: 00$ & 12.378 & 12.404 & 9.168 & 8.115 & 5.276 & 5.013 & 3.781 & 3.354 & 10.192 & 6.393 & 3.72 & 6.85 & 3.477 \\
\hline $12 / 22 / 06$ & $0: 00$ & 12.371 & 12.393 & 9.163 & 8.106 & 5.285 & 5.009 & 3.902 & 3.347 & 10.19 & 6.393 & 3.745 & 6.853 & 3.51 \\
\hline $12 / 22 / 06$ & $4: 00$ & 12.378 & 12.376 & 9.17 & 8.12 & 5.304 & 5.016 & 3.79 & 3.361 & 10.195 & 6.4 & 3.778 & 6.867 & 3.545 \\
\hline $12 / 22 / 06$ & 8:00 & 12.394 & 12.418 & 9.182 & 8.134 & 5.321 & 5.023 & 3.692 & 3.38 & 10.204 & 6.407 & 3.803 & 6.885 & 3.573 \\
\hline $12 / 22 / 06$ & $12: 00$ & 12.413 & 12.444 & 9.198 & 8.15 & 5.337 & 5.037 & 3.711 & 3.396 & 10.211 & 6.418 & 3.829 & 6.904 & 3.599 \\
\hline $12 / 22 / 06$ & $16: 00$ & 12.434 & 12.467 & 9.215 & 8.173 & 5.356 & 5.053 & 3.617 & 3.417 & 10.22 & 6.423 & 3.852 & 6.923 & 3.62 \\
\hline $12 / 22 / 06$ & $20: 00$ & 12.452 & 12.483 & 9.233 & 8.188 & 5.372 & 5.069 & 3.489 & 3.432 & 10.232 & 6.432 & 3.876 & 6.939 & 3.636 \\
\hline $12 / 23 / 06$ & $0: 00$ & 12.459 & 12.492 & 9.24 & 8.195 & 5.381 & 5.079 & 3.38 & 3.438 & 10.237 & 6.439 & 3.897 & 6.951 & 3.652 \\
\hline $12 / 23 / 06$ & $4: 00$ & 12.478 & 12.511 & 9.257 & 8.213 & 5.398 & 5.095 & 3.231 & 3.457 & 10.248 & 6.451 & 3.939 & 6.974 & 3.678 \\
\hline $12 / 23 / 06$ & $8: 00$ & 12.504 & 12.539 & 9.276 & 8.239 & 5.421 & 5.114 & 3.247 & 3.483 & 10.26 & 6.463 & 3.99 & 7.002 & 3.706 \\
\hline $12 / 23 / 06$ & $12: 00$ & 12.508 & 12.537 & 9.287 & 8.237 & 5.428 & 5.123 & 3.627 & 3.478 & 10.269 & 6.467 & 3.955 & 7.004 & 3.699 \\
\hline $12 / 23 / 06$ & $16: 00$ & 12.511 & 12.543 & 9.29 & 8.244 & 5.438 & 5.13 & 3.697 & 3.488 & 10.274 & 6.472 & 3.929 & 7.004 & 3.69 \\
\hline
\end{tabular}


Water Level (ft below top of casing) at Indicated Well

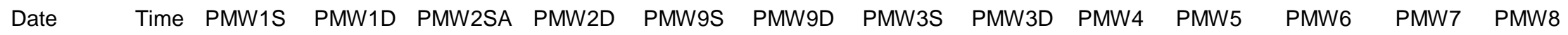

\begin{tabular}{|c|c|c|c|c|c|c|c|c|c|c|c|c|c|c|}
\hline $12 / 23 / 06$ & $20: 00$ & 12.511 & 12.537 & 9.292 & 8.241 & 5.44 & 5.132 & 3.147 & 3.485 & 10.276 & 6.47 & 3.955 & 7.006 & 3.699 \\
\hline $12 / 24 / 06$ & 0:00 & 12.506 & 12.532 & 9.292 & 8.239 & 5.447 & 5.137 & 3.158 & 3.485 & 10.279 & 6.474 & 4.013 & 7.02 & 3.72 \\
\hline $12 / 24 / 06$ & $4: 00$ & 12.48 & 12.504 & 9.276 & 8.216 & 5.442 & 5.128 & 3.273 & 3.467 & 10.272 & 6.47 & 4.044 & 7.023 & 3.732 \\
\hline $12 / 24 / 06$ & $8: 00$ & 12.478 & 12.506 & 9.276 & 8.22 & 5.452 & 5.125 & 3.401 & 3.469 & 10.272 & 6.474 & 4.067 & 7.03 & 3.746 \\
\hline $12 / 24 / 06$ & $12: 00$ & 12.471 & 12.495 & 9.271 & 8.209 & 5.452 & 5.123 & 4.032 & 3.457 & 10.272 & 6.47 & 4.013 & 7.016 & 3.732 \\
\hline $12 / 24 / 06$ & $16: 00$ & 12.478 & 12.509 & 9.273 & 8.225 & 5.459 & 5.123 & 3.899 & 3.469 & 10.272 & 6.474 & 3.925 & 7.002 & 3.706 \\
\hline $12 / 24 / 06$ & $20: 00$ & 12.518 & 12.546 & 9.299 & 8.255 & 5.47 & 5.137 & 3.361 & 3.497 & 10.281 & 6.481 & 3.95 & 7.018 & 3.72 \\
\hline $12 / 25 / 06$ & $0: 00$ & 12.527 & 12.555 & 9.311 & 8.26 & 5.477 & 5.151 & 3.496 & 3.504 & 10.288 & 6.484 & 3.967 & 7.027 & 3.727 \\
\hline $12 / 25 / 06$ & $4: 00$ & 12.504 & 12.53 & 9.297 & 8.237 & 5.468 & 5.146 & 3.424 & 3.483 & 10.283 & 6.479 & 3.964 & 7.02 & 3.718 \\
\hline $12 / 25 / 06$ & 8:00 & 12.518 & 12.543 & 9.306 & 8.253 & 5.48 & 5.151 & 3.408 & 3.497 & 10.29 & 6.484 & 4.002 & 7.034 & 3.734 \\
\hline $12 / 25 / 06$ & $12: 00$ & 12.492 & 12.513 & 9.29 & 8.223 & 5.47 & 5.144 & 3.923 & 3.471 & 10.281 & 6.479 & 3.985 & 7.023 & 3.725 \\
\hline $12 / 25 / 06$ & $16: 00$ & 12.487 & 12.513 & 9.283 & 8.227 & 5.47 & 5.139 & 3.699 & 3.474 & 10.281 & 6.479 & 3.976 & 7.016 & 3.727 \\
\hline $12 / 25 / 06$ & $20: 00$ & 12.49 & 12.513 & 9.287 & 8.227 & 5.473 & 5.135 & 3.317 & 3.478 & 10.281 & 6.477 & 4.004 & 7.023 & 3.736 \\
\hline $12 / 26 / 06$ & $0: 00$ & 12.487 & 12.513 & 9.287 & 8.23 & 5.473 & 5.137 & 3.212 & 3.478 & 10.281 & 6.477 & 4.065 & 7.037 & 3.76 \\
\hline $12 / 26 / 06$ & $4: 00$ & 12.487 & 12.513 & 9.285 & 8.23 & 5.477 & 5.135 & 3.168 & 3.481 & 10.281 & 6.481 & 4.114 & 7.048 & 3.778 \\
\hline $12 / 26 / 06$ & $8: 00$ & 12.485 & 12.509 & 9.283 & 8.227 & 5.48 & 5.137 & 3.406 & 3.481 & 10.281 & 6.479 & 4.153 & 7.06 & 3.792 \\
\hline $12 / 26 / 06$ & $12: 00$ & 12.476 & 12.495 & 9.278 & 8.216 & 5.477 & 5.132 & 4.114 & 3.467 & 10.276 & 6.477 & 4.123 & 7.051 & 3.788 \\
\hline $12 / 26 / 06$ & $16: 00$ & 12.462 & 12.485 & 9.266 & 8.211 & 5.478 & 5.128 & 4.046 & 3.46 & 10.272 & 6.481 & 4.018 & 7.02 & 3.75 \\
\hline $12 / 26 / 06$ & $20: 00$ & 12.462 & 12.488 & 9.266 & 8.211 & 5.475 & 5.123 & 3.715 & 3.457 & 10.267 & 6.481 & 3.969 & 7.011 & 3.727 \\
\hline $12 / 27 / 06$ & $0: 00$ & 12.45 & 12.472 & 9.257 & 8.195 & 5.461 & 5.116 & 3.802 & 3.441 & 10.262 & 6.472 & 3.944 & 6.997 & 3.711 \\
\hline $12 / 27 / 06$ & 4:00 & 12.429 & 12.448 & 9.24 & 8.173 & 5.447 & 5.102 & 3.662 & 3.42 & 10.255 & 6.465 & 3.929 & 6.983 & 3.701 \\
\hline $12 / 27 / 06$ & $8: 00$ & 12.415 & 12.437 & 9.229 & 8.164 & 5.438 & 5.088 & 3.746 & 3.413 & 10.248 & 6.463 & 3.946 & 6.974 & 3.708 \\
\hline $12 / 27 / 06$ & $12: 00$ & 12.401 & 12.418 & 9.215 & 8.145 & 5.426 & 5.074 & 4.242 & 3.394 & 10.239 & 6.456 & 3.916 & 6.96 & 3.699 \\
\hline $12 / 27 / 06$ & $16: 00$ & 12.385 & 12.407 & 9.201 & 8.136 & 5.419 & 5.065 & 4.195 & 3.385 & 10.232 & 6.451 & 3.878 & 6.944 & 3.69 \\
\hline $12 / 27 / 06$ & $20: 00$ & 12.403 & 12.43 & 9.212 & 8.159 & 5.428 & 5.067 & 3.883 & 3.404 & 10.234 & 6.453 & 3.899 & 6.948 & 3.701 \\
\hline $12 / 28 / 06$ & $0: 00$ & 12.415 & 12.441 & 9.222 & 8.166 & 5.428 & 5.069 & 3.648 & 3.411 & 10.237 & 6.453 & 3.916 & 6.953 & 3.708 \\
\hline $12 / 28 / 06$ & $4: 00$ & 12.431 & 12.46 & 9.231 & 8.18 & 5.436 & 5.079 & 3.559 & 3.425 & 10.239 & 6.458 & 3.934 & 6.962 & 3.718 \\
\hline $12 / 28 / 06$ & $8: 00$ & 12.466 & 12.502 & 9.259 & 8.218 & 5.459 & 5.097 & 3.482 & 3.457 & 10.251 & 6.47 & 3.971 & 6.99 & 3.741 \\
\hline $12 / 28 / 06$ & $12: 00$ & 12.499 & 12.532 & 9.285 & 8.241 & 5.475 & 5.118 & 3.816 & 3.481 & 10.265 & 6.479 & 3.981 & 7.011 & 3.75 \\
\hline $12 / 28 / 06$ & $16: 00$ & 12.527 & 12.564 & 9.311 & 8.272 & 5.494 & 5.139 & 3.725 & 3.509 & 10.279 & 6.493 & 3.993 & 7.03 & 3.76 \\
\hline $12 / 28 / 06$ & $20: 00$ & 12.555 & 12.59 & 9.329 & 8.291 & 5.506 & 5.158 & 3.641 & 3.527 & 10.293 & 6.502 & 4.009 & 7.048 & 3.767 \\
\hline $12 / 29 / 06$ & $0: 00$ & 12.574 & 12.606 & 9.346 & 8.305 & 5.515 & 5.177 & 3.592 & 3.539 & 10.304 & 6.507 & 4.02 & 7.058 & 3.769 \\
\hline $12 / 29 / 06$ & $4: 00$ & 12.581 & 12.613 & 9.355 & 8.307 & 5.522 & 5.189 & 3.634 & 3.544 & 10.311 & 6.512 & 4.025 & 7.067 & 3.769 \\
\hline $12 / 29 / 06$ & $8: 00$ & 12.59 & 12.648 & 9.365 & 8.319 & 5.532 & 5.196 & 3.655 & 3.553 & 10.316 & 6.516 & 4.02 & 7.097 & 3.769 \\
\hline
\end{tabular}


TABLE S1.2 (Cont.)

Water Level (ft below top of casing) at Indicated Well

\begin{tabular}{|c|c|c|c|c|c|c|c|c|c|c|c|c|c|c|}
\hline Date & Time & PMW1S & PMW1D & PMW2SA & PMW2D & PMW9S & PMW9D & PMW3S & PMW3D & PMW4 & PMW5 & PMW6 & PMW7 & PMW8 \\
\hline $12 / 29 / 06$ & $12: 00$ & 12.578 & 12.697 & 9.358 & 8.298 & 5.513 & 5.193 & 3.753 & 3.523 & 10.313 & 6.491 & 3.624 & 7.104 & 3.494 \\
\hline $12 / 29 / 06$ & $16: 00$ & 12.562 & 12.62 & 9.329 & 8.255 & 5.031 & 5.17 & 3.536 & 3.446 & 10.272 & 6.355 & 3.258 & 6.888 & 2.874 \\
\hline $12 / 29 / 06$ & $20: 00$ & 12.553 & 12.578 & 9.301 & 8.204 & 4.868 & 5.109 & 3.448 & 3.427 & 10.237 & 6.33 & 3.403 & 6.841 & 2.965 \\
\hline $12 / 30 / 06$ & $0: 00$ & 12.525 & 12.602 & 9.269 & 8.23 & 4.912 & 5.055 & 3.543 & 3.404 & 10.218 & 6.332 & 3.489 & 6.879 & 3.044 \\
\hline $12 / 30 / 06$ & 4:00 & 12.504 & 12.571 & 9.247 & 8.232 & 4.7 & 5.025 & 3.536 & 3.366 & 10.204 & 6.264 & 3.233 & 6.837 & 2.734 \\
\hline $12 / 30 / 06$ & $8: 00$ & 12.485 & 12.59 & 9.184 & 8.248 & 3.919 & 4.962 & 3.55 & 3.284 & 10.139 & 6.012 & 3.041 & 6.629 & 2.496 \\
\hline $12 / 30 / 06$ & $12: 00$ & 12.448 & 12.497 & 9.088 & 8.141 & 3.786 & 4.829 & 3.867 & 3.2 & 10.036 & 5.891 & 3.06 & 6.431 & 2.505 \\
\hline $12 / 30 / 06$ & $16: 00$ & 12.41 & 12.458 & 9.039 & 8.089 & 3.936 & 4.733 & 3.758 & 3.172 & 9.978 & 5.926 & 3.081 & 6.425 & 2.543 \\
\hline $12 / 30 / 06$ & $20: 00$ & 12.382 & 12.383 & 8.943 & 7.953 & 3.116 & 4.693 & 3.424 & 3.063 & 9.899 & 5.361 & 2.862 & 6.222 & 2.33 \\
\hline $12 / 31 / 06$ & $0: 00$ & 12.326 & 12.316 & 8.73 & 7.834 & 2.978 & 4.525 & 3.124 & 2.897 & 9.712 & 5.132 & 2.822 & 5.98 & 2.293 \\
\hline $12 / 31 / 06$ & $4: 00$ & 12.245 & 12.302 & 8.573 & 7.749 & 2.885 & 4.366 & 2.951 & 2.754 & 9.558 & 4.81 & 2.755 & 5.84 & 2.237 \\
\hline $12 / 31 / 06$ & 8:00 & 11.682 & 12.186 & 8.247 & 7.466 & 2.887 & 4.179 & 2.66 & 2.493 & 9.325 & 4.521 & 2.783 & 5.558 & 2.243 \\
\hline $12 / 31 / 06$ & $12: 00$ & 11.733 & 12.121 & 8.233 & 7.438 & 3.069 & 4.027 & 2.63 & 2.504 & 9.197 & 4.827 & 2.85 & 5.568 & 2.318 \\
\hline $12 / 31 / 06$ & $16: 00$ & 11.838 & 12.054 & 8.287 & 7.402 & 3.296 & 3.98 & 2.602 & 2.558 & 9.127 & 5.03 & 2.904 & 5.551 & 2.386 \\
\hline $12 / 31 / 06$ & $20: 00$ & 11.859 & 12.033 & 8.343 & 7.412 & 3.516 & 4.001 & 2.593 & 2.612 & 9.088 & 5.181 & 2.946 & 5.586 & 2.449 \\
\hline
\end{tabular}


Argonne

Environmental Science Division

Argonne National Laboratory

9700 South Cass Avenue, Bldg. 203

Argonne, IL 60439-4843

www.anl.gov

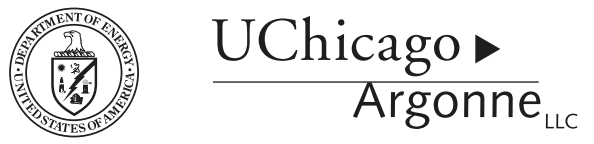

A U.S. Department of Energy laboratory

managed by UChicago Argonne, LLC 